WSRC-TR-2001-00400

SRT-RPP-2001-000134

\title{
Preliminary Ion Exchange Modeling for Removal of Cesium from Hanford Waste Using Hydrous Crystalline Silicotitanate Material
}

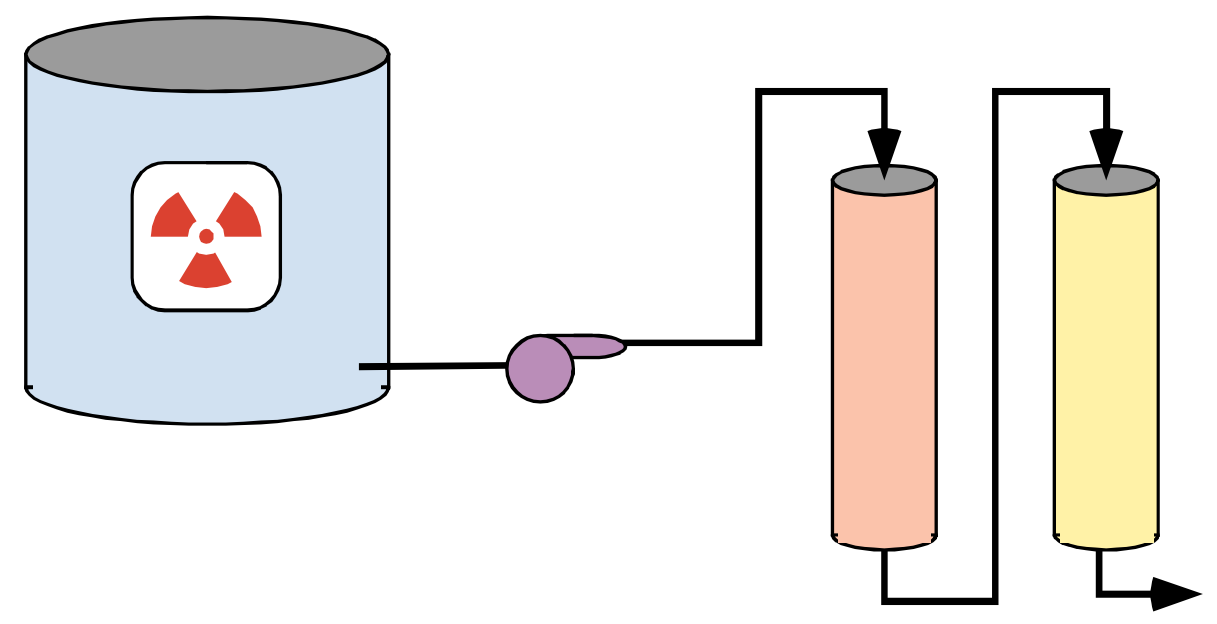

Westinghouse Savannah River Company

Savannah River Site

Aiken, SC 29808

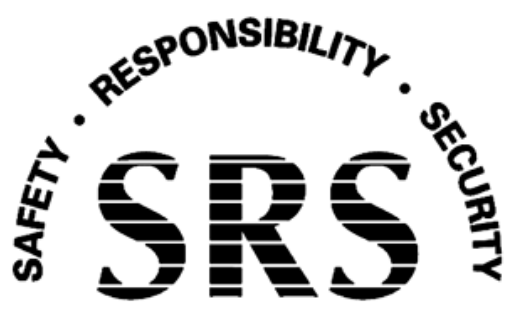

Prepared for the U.S. Department of Energy under Contract No. DE-AC09-96SR18500 
This document was prepared in conjunction with work accomplished under Contract No. DE-AC09-96SR18500 with the U. S. Department of Energy.

\section{DISCLAIMER}

This report was prepared as an account of work sponsored by an agency of the United States Government. Neither the United States Government nor any agency thereof, nor any of their employees, makes any warranty, express or implied, or assumes any legal liability or responsibility for the accuracy, completeness, or usefulness of any information, apparatus, product or process disclosed, or represents that its use would not infringe privately owned rights. Reference herein to any specific commercial product, process or service by trade name, trademark, manufacturer, or otherwise does not necessarily constitute or imply its endorsement, recommendation, or favoring by the United States Government or any agency thereof. The views and opinions of authors expressed herein do not necessarily state or reflect those of the United States Government or any agency thereof.

This report has been reproduced directly from the best available copy.

Available for sale to the public, in paper, from: U.S. Department of Commerce, National Technical Information Service, 5285 Port Royal Road, Springfield, VA 22161, phone: (800) 553-6847, fax: (703) 605-6900

email: orders@ntis.fedworld.gov

online ordering: http://www.ntis.gov/help/index.asp

Available electronically at http://www.osti.gov/bridge

Available for a processing fee to U.S. Department of Energy and its contractors, in paper, from: U.S. Department of Energy, Office of Scientific and Technical Information, P.O. Box 62, Oak Ridge, TN 37831-0062,

phone: (865)576-8401,

fax: (865)576-5728

email: $\underline{\text { reports@ adonis.osti.gov }}$ 
WSRC-TR-2001-00400

SRT-RPP-2001-00134

KEYWORDS:

Hanford River Protection Project Ion Exchange Technology

Crystalline Silicotanate Resins

Technetium

Cesium

VERSE Code

Column Modeling

RETENTION - Permanent

\title{
Preliminary Ion Exchange Modeling for Removal of Cesium from Hanford Waste Using Hydrous Crystalline Silicotitanate Material
}

SAVANNAH RIVER TECHNOLOGY CENTER

\author{
L. Larry Hamm \\ Thong Hang \\ Daniel J. McCabe \\ William D. King
}

Publication Date: July 2002

Westinghouse Savannah River Company

Savannah River Site

Aiken, SC 29808

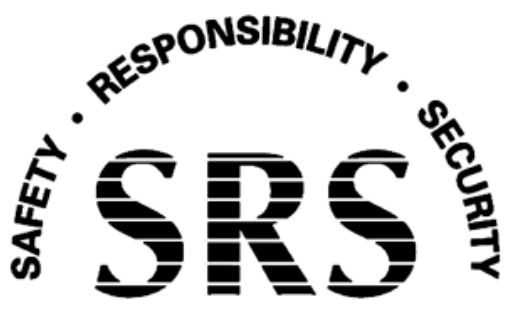


This page was intentionally left blank 


\section{TABLE OF CONTENTS}

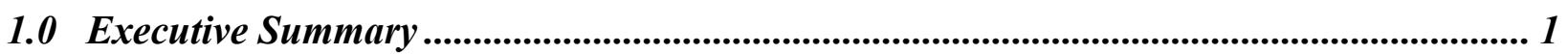

2.0 Introduction and Background ............................................................................................... 11

2.1 Test Specification Objectives ........................................................................................................... 13

2.2 IONSIV $^{\circledR}$ IE-910/IE-911 CST Versus SuperLig ${ }^{\circledR}$ 644 ...................................................................13

2.3 Ion Exchange Modeling.................................................................................................................. 14

2.4 Report Overview ................................................................................................................................ 15

3.0 Column Model Formulations............................................................................................. 19

3.1 The Multi-Component Model .............................................................................................................20

3.2 The Single-Component Model .......................................................................................................22

3.3 The Cesium-IONSIV ${ }^{\circledR}$ IE-911 CST System .............................................................................22

4.0 Equilibrium Cesium Isotherms ................................................................................................. 27

4.1 The Isotherm Model ................................................................................................................... 28

4.2 Batch Feed Compositions.................................................................................................. 29

4.3 The Beta Parameter Values ...............................................................................................30

4.4 Isotherm Model for VERSE-LC Application ..............................................................................31

5.0 Column Properties ........................................................................................................ 39

5.1 Basic Constraint Functions ..........................................................................................................39

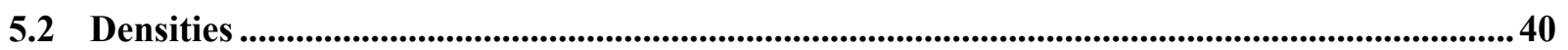

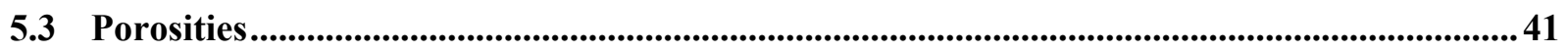

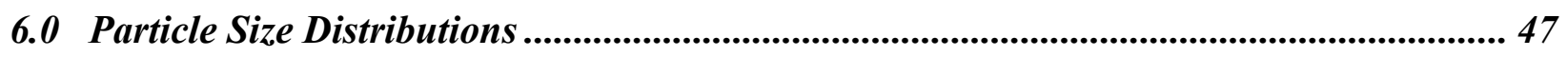

6.1 MicroTrac ${ }^{\circledast}$ Laser Technology Data..........................................................................................4

6.2 Lasentec ${ }^{\otimes}$ Laser Technology Data ...............................................................................................48

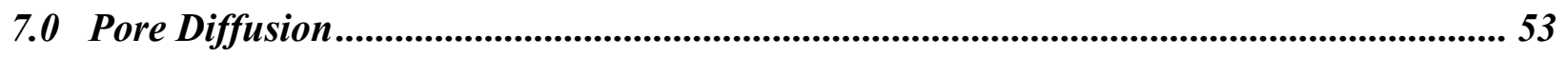

7.1 Waste Solution Density and Viscosity ........................................................................................5

7.2 Molecular Diffusion Coefficients ..........................................................................................5

7.3 Pore Diffusion Coefficients................................................................................................................55

7.3.1 CST Conceptual Model …………………………………………………………………...5

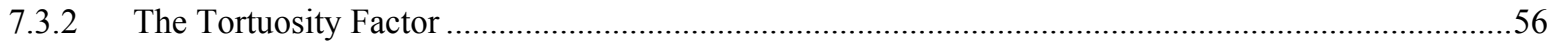

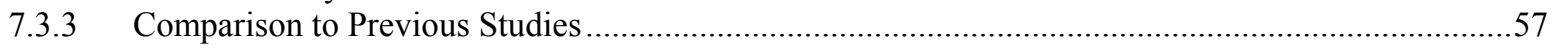

7.4 VERSE-LC Simulations for Batch Kinetics Tests ..........................................................................58

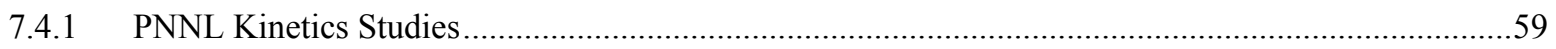

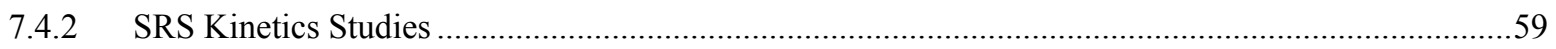

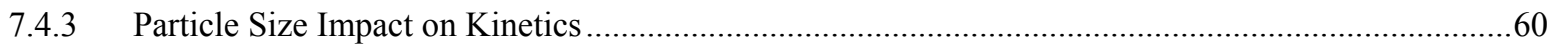

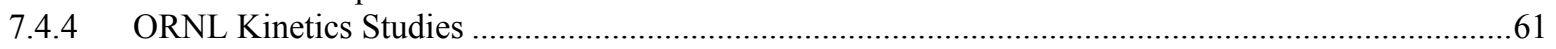

8.0 Axial Dispersion and Film Diffusion..................................................................................... 75 


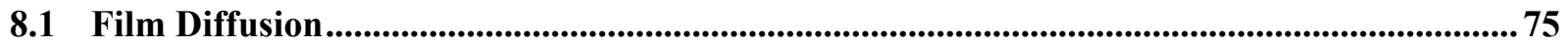

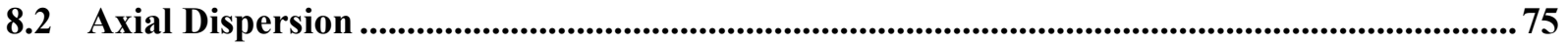

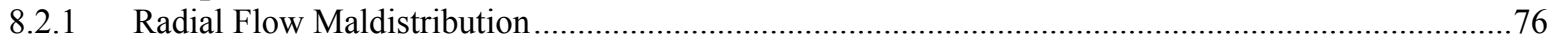

8.2.2 Headspace and Short Column Impacts ………..............................................................................

9.0 Laboratory-Scale Column Assessments .................................................................... 77

9.1 CST Pore Diffusion Coefficient .................................................................................................. 78

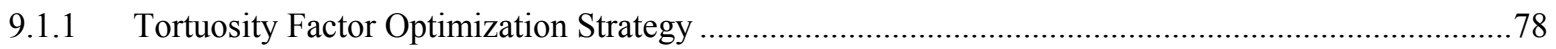

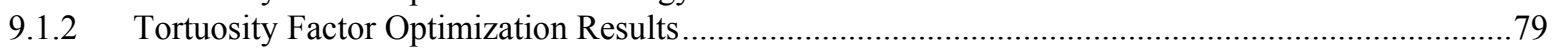

9.1.3 Tortuosity Factor Coefficient Recommendation.......................................................................8

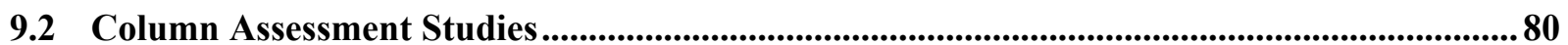

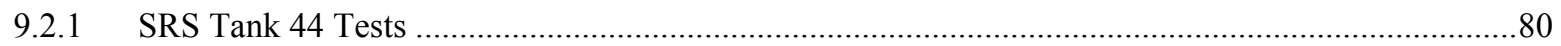

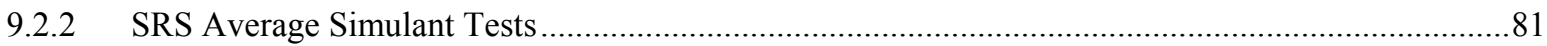

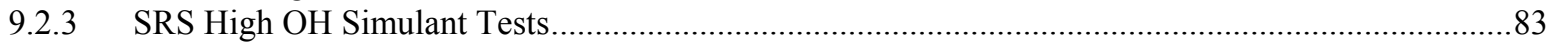

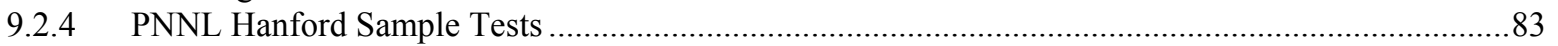

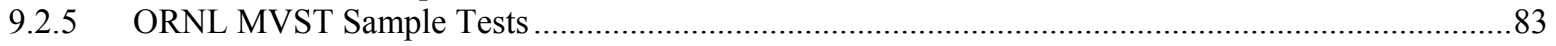

$10.0 \quad$ Full-Scale Column Predictions and Design ........................................................... 101

10.1 IONSIV ${ }^{\circledR}$ IE-911 CST versus SuperLig $^{\circledR}$ 644 Ion-Exchanger Performance ..................... 101

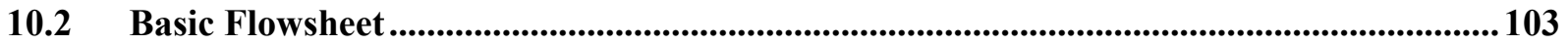

10.3 VERSE-LC Modeling of the Full-Scale Facility ...................................................................... 104

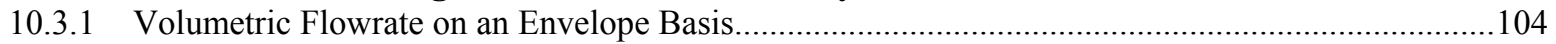

10.3.2 Cesium-137 Exit Concentration Criterion on an Envelope Basis..................................................105

10.3.3 Input Concentration and Flowrate Boundary Conditions ................................................................106

10.4 Mass Transfer Zone Concept for Column Design ................................................................... 106

10.5 The Global Optimization Strategy …............................................................................................ 107

10.6 Theoretical Minimum Spent CST Material ................................................................................. 108

10.7 Spent CST Material Dependency on Geometry....................................................................... 109

10.8 CST Column Design and Performance..................................................................................... 111

10.8.1 Nominal Case Results for 2-Column Carousels .......................................................................111

10.8.2 Nominal Case Results for 3-Column Carousels .....................................................................113

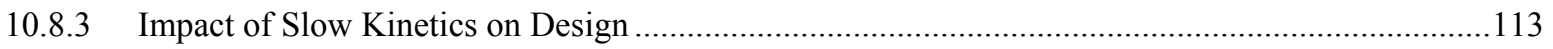

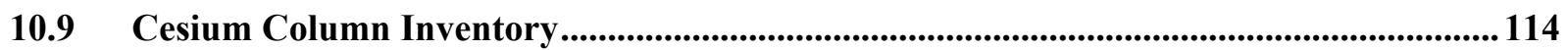

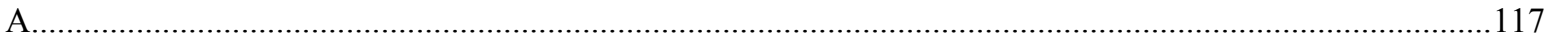

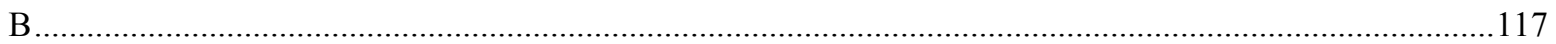

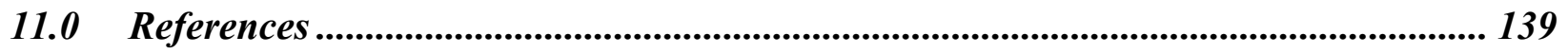

Appendix A (BBI Phase 1 LAW Feed Solution Definitions) ................................................. 145

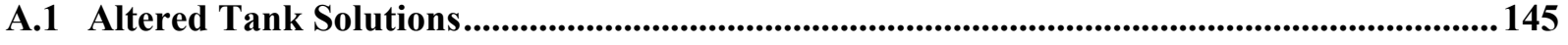

A.2 Isotopic Dilution Process for Strontium-90 ........................................................................ 147

A.3 Adjusted Tank Solutions for $K_{d}$ Modeling.................................................................................... 148

A.4 Feed Solution Densities........................................................................................................................149

Appendix B (Cesium Isotherms for LAW Phase 1 Batch Feeds) ........................................... 167

B.1 Molar Ratios of $\mathrm{Na} / \mathrm{Cs}, \mathrm{K} / \mathrm{Cs}$, and $\mathrm{SrOH} / \mathrm{Cs}$................................................................................. 168 
B.2 Isotherm Predictions using the ZAM Model ............................................................................ 168

B.3 Isotherm Model for VERSE-LC Application ................................................................................... 169

B.4 Isotherm Parameter Estimation Technique ................................................................................... 171

B.5 The Least Favorable Isotherm........................................................................................................ 172

B.6 "Effective" Single-Component Isotherm Models ......................................................................... 173

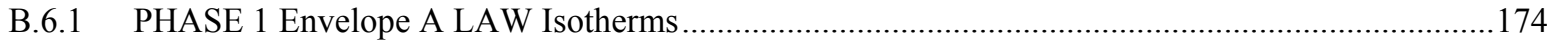

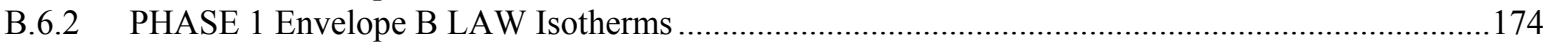

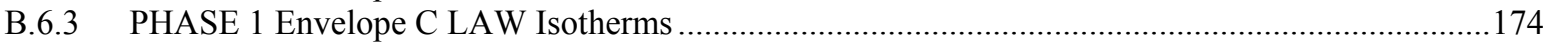

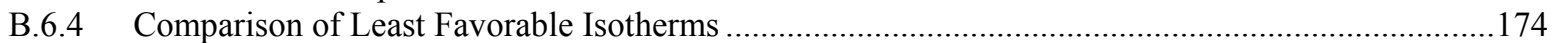

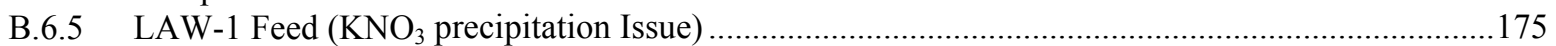

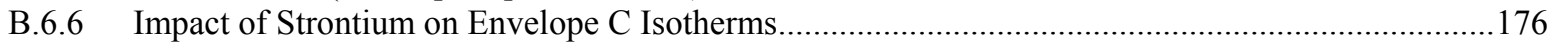

B.7 Cesium Isotherm Sensitivity Study and Error Analysis.............................................................. 177

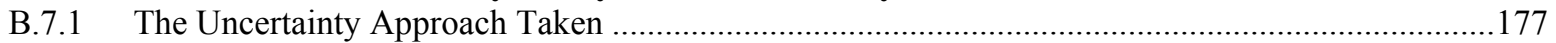

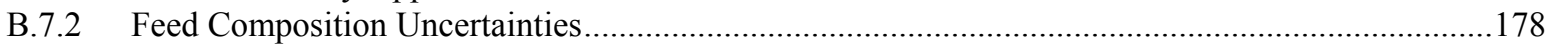

B.7.3 Feed Density and Process Temperature Uncertainties...................................................................179

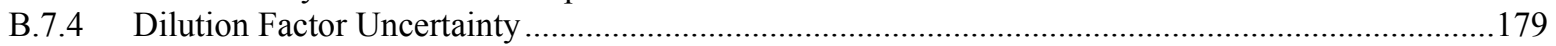

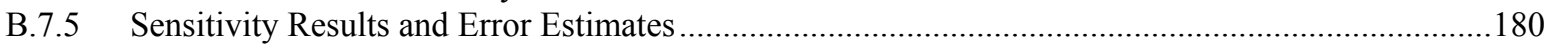

Appendix C (Dilution Factor for IONSIV ${ }^{\circledR}$ IE-911) .................................................... 217

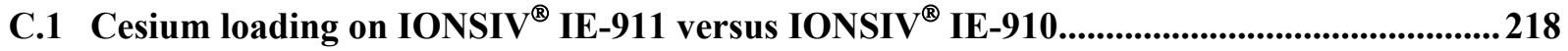

C.2 Averaged Dilution Factor for Baseline IE-911 CST Material ..................................................219

C.3 Dilution Factor Variability ............................................................................................................221

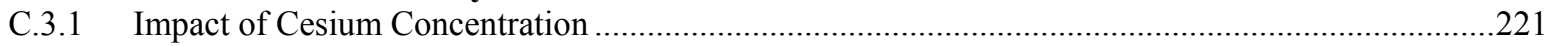

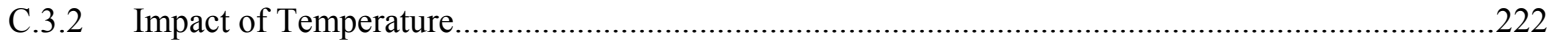

C.4 Cesium Loading Curve Comparisons ................................................................................................. 223

C.5 Strontium Loading Curve Comparisons............................................................................................224

Appendix D (VERSE-LC Input Files for Phase 1 Batch Feeds) ............................................ 239

Makefile for NMAKE Utility (2 nominal case runs of VERSE-LC) .........................................................243

VERSE-LC Input for Phase 1 LAW-1 Batch Feed (nominal case; 2000 L) ..................................................249

VERSE-LC Input for Phase 1 LAW-2a Batch Feed (nominal case; 2000 L) ................................................249

VERSE-LC Input for Phase 1 LAW-2b Batch Feed (nominal case; 2000 L) .................................................250

VERSE-LC Input for Phase 1 LAW-3 Batch Feed (nominal case; 2000 L) .....................................................250

VERSE-LC Input for Phase 1 LAW-4 Batch Feed (nominal case; 2000 L) ..................................................251

VERSE-LC Input for Phase 1 LAW-5 Batch Feed (nominal case; 2000 L) ..................................................251

VERSE-LC Input for Phase 1 LAW-6 Batch Feed (nominal case; 2000 L) ................................................252

VERSE-LC Input for Phase 1 LAW-7 Batch Feed (nominal case; 2000 L)..................................................252

VERSE-LC Input for Phase 1 LAW-8 Batch Feed (nominal case; 2000 L) ..................................................253

VERSE-LC Input for Phase 1 LAW-9 Batch Feed (nominal case; 2000 L) ................................................253

VERSE-LC Input for Phase 1 LAW-10 Batch Feed (nominal case; 2000 L) ................................................254

VERSE-LC Input for Phase 1 LAW-11 Batch Feed (nominal case; 2000 L) ..............................................254

VERSE-LC Input for Phase 1 LAW-12 Batch Feed (nominal case; 2000 L) ...............................................25

VERSE-LC Input for Phase 1 LAW-13 Batch Feed (nominal case; 2000 L) ..................................................25

VERSE-LC Input for Phase 1 LAW-14 Batch Feed (nominal case; 2000 L) .................................................256

VERSE-LC Input for Phase 1 LAW-15 Batch Feed (nominal case; 2000 L)................................................256

VERSE-LC Output for Phase 1 LAW-1 Batch Feed (nominal case; 2000 L) ...............................................257

VERSE-LC Output for Phase 1 LAW-2b Batch Feed (nominal case; 2000 L) ...............................................258

VERSE-LC Output for Phase 1 LAW-3 Batch Feed (nominal case; 2000 L) ..................................................259 
E.1 PNNL Kinetics Studies ..................................................................................................................261

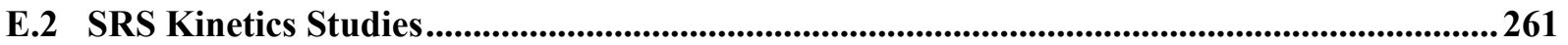

E.3 Particle Size Impact on Kinetics ........................................................................................................261

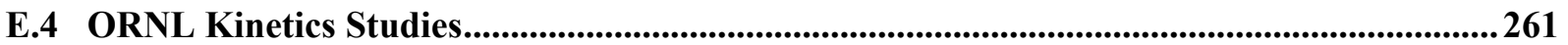

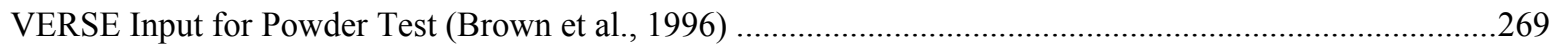

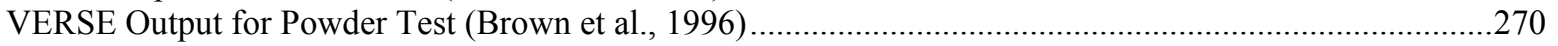

VERSE Input for Engineered -08 Test (Brown et al., 1996) .....................................................................227

VERSE Input for Engineered -38b Test (Brown et al., 1996)...................................................................22

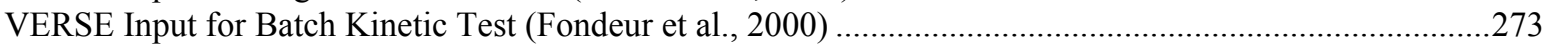

VERSE Output for Batch Kinetic Test (Fondeur et al., 2000)......................................................................274

VERSE Input for Cs Uptake Test (Anthony et al., 1996; $112 \mu \mathrm{m}$ ) ............................................................276

VERSE Input for Cs Uptake Test (Anthony et al., 1996; $334 \mu \mathrm{m}$ ) .................................................................277

Appendix F (ZAM Code Description) ............................................................................... 279

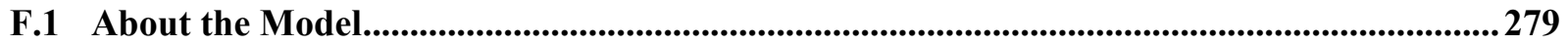

F.2 I/O Files and Running the ZAM Code .........................................................................282

F.3 Input File Structure .....................................................................................................................283

F.4 Installation, Verification \& Validation ...........................................................................285

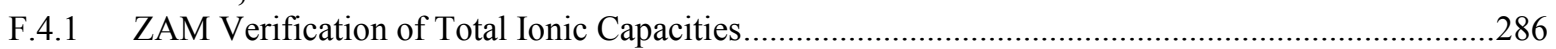

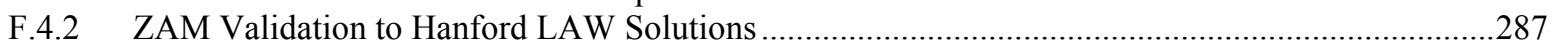

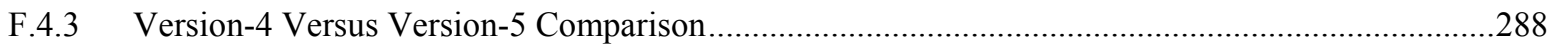

F.5 ZAM Version-4 or Version-5 Input for 5 M Na AW-101 Feed) .........................................302

F.6 ZAM Version-4 Output for 5 M Na AW-101 Feed).............................................................302

F.7 ZAM Version-4 or Version-5 Input for Phase 1 LAW-1 Batch Feed) .................................. 302

F.8 ZAM Version-4 Output for Phase 1 LAW-1 Batch Feed) .....................................................303

F.9 ZAM Version-5 Output for Phase 1 LAW-1 Batch Feed) .....................................................303

Appendix G (ZAM Input Files for Phase 1 Batch Feeds)....................................................... 305

ZAM Input for Phase 1 LAW-1 Batch Feed (Envelope A; nominal case) ....................................................306

ZAM Input for Phase 1 LAW-5 Batch Feed (Envelope A; nominal case) ......................................................306

ZAM Input for Phase 1 LAW-6 Batch Feed (Envelope A; nominal case) ....................................................306

ZAM Input for Phase 1 LAW-8 Batch Feed (Envelope A; nominal case) .......................................................306

ZAM Input for Phase 1 LAW-9 Batch Feed (Envelope A; nominal case) ......................................................307

ZAM Input for Phase 1 LAW-10 Batch Feed (Envelope A; nominal case) ...................................................307

ZAM Input for Phase 1 LAW-11 Batch Feed (Envelope A; nominal case) ....................................................307

ZAM Input for Phase 1 LAW-12 Batch Feed (Envelope A; nominal case) ....................................................307

ZAM Input for Phase 1 LAW-13 Batch Feed (Envelope A; nominal case) ....................................................308

ZAM Input for Phase 1 LAW-14 Batch Feed (Envelope A; nominal case) ....................................................308

ZAM Input for Phase 1 LAW-15 Batch Feed (Envelope A; nominal case) ....................................................308

ZAM Input for Phase 1 LAW-2a Batch Feed (Envelope B; nominal case).....................................................308

ZAM Input for Phase 1 LAW-2b Batch Feed (Envelope B; nominal case) .....................................................309

ZAM Input for Phase 1 LAW-3 Batch Feed (Envelope C; nominal case) .....................................................309

ZAM Input for Phase 1 LAW-4 Batch Feed (Envelope C; nominal case) ......................................................309

ZAM Input for Phase 1 LAW-7 Batch Feed (Envelope C; nominal case) .....................................................309

ZAM Input for Phase $1 \mathrm{LAW}-1$ Batch Feed (Envelope A; with $4 \mathrm{M} \mathrm{Na}^{+}$) ..................................................310

ZAM Input for Phase 1 LAW-1 Batch Feed (Envelope A; with $6 \mathrm{M} \mathrm{Na}^{+}$) ...................................................310 


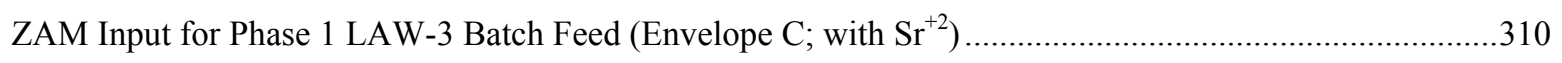

Appendix H (Column Test Input Files) ................................................................... 311

H.1 SRS Tank 44 Studies......................................................................................................................311

H.2 SRS Average Simulant Studies .....................................................................................................311

H.3 SRS High OH Simulant Studies.........................................................................................................312

H.4 PNNL AW-101 Sample Studies ...................................................................................................312

H.5 ORNL MVST Sample Tests ........................................................................................................312

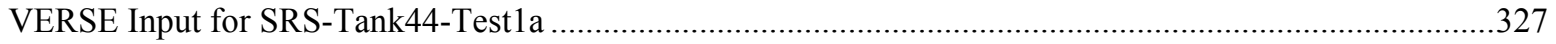

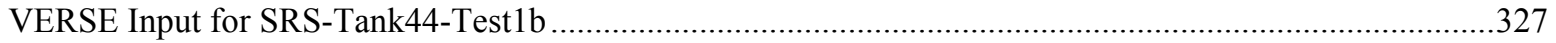

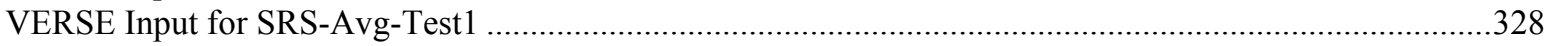

VERSE Input for SRS-Avg-Test2 (single-component approach) .............................................................328

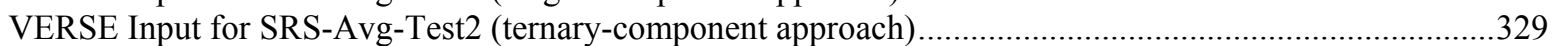

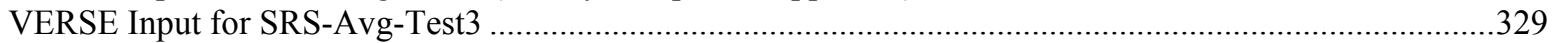

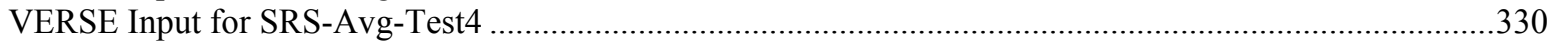

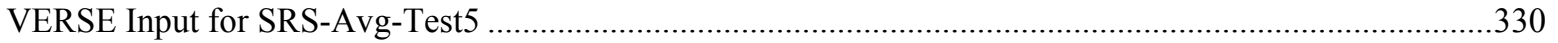

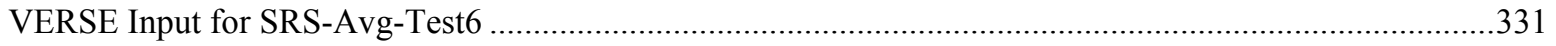

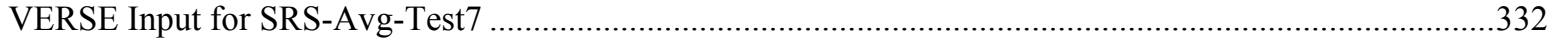

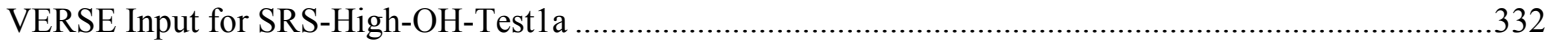

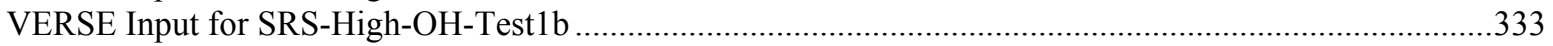

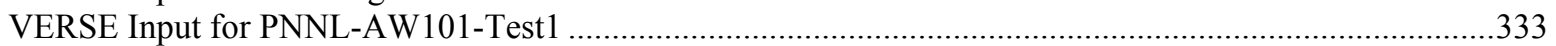

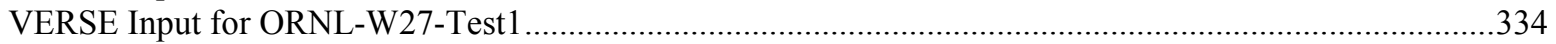

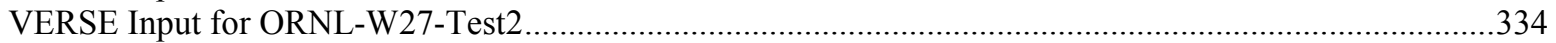

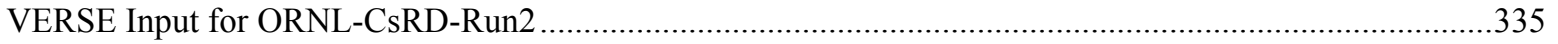

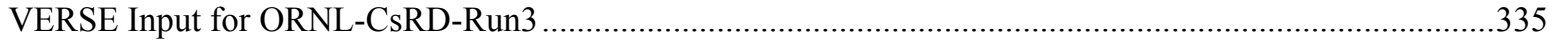

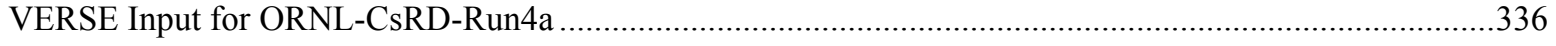

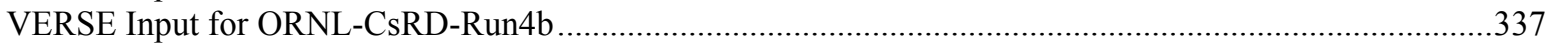


(This Page Intentionally Left Blank) 


\section{LIST OF TABLES}

Table 2-1. Key batch processing information on the Phase 1 Low activity waste (LAW) feeds listed in their scheduled order to be processed.

Table 4-1. Summary of the Phase 1 batch feed compositions in molarity units (on a 5M sodium basis) highlighting those ionic species who are dominant contributors to the effectiveness of CST material for cesium loading. ${ }^{a}$

Table 4-2. Estimated beta parameter values and error estimate for a one-component (total cesium) homovalent algebraic isotherm model based on CST in its powder-form (IE-910) where the data sets used were created using the ZAM code.

Table 4-3. Parameter settings for an "effective" single component Freundlich/Langmuir Hybrid equilibrium isotherm model for total cesium on CST based on the 1-component homovalent model....................35

Table 5-1. Key CST exchange properties taken from literature for various ion exchangers.................................42

Table 5-2. Estimated particle density based on measured densities taken from slurry mixtures of CST and water by Qureshi (1999).

Table 5-3. "Best estimate” and upper/lower bounds of total porosity or particle density based on available measured densities or bed/particle porosities for IE-911 CST material.....

Table 5-4. Particle porosity as a function of bed porosity at three different total porosity values for the IONSIV ${ }^{\circledR}$ IE-911 CST material.

Table 6-1. Particle size distributions ${ }^{a}$ of CST material in its engineered-form (batch number not recorded)

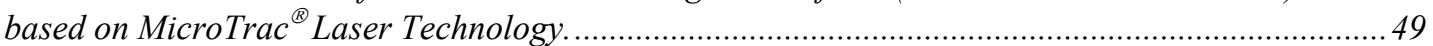

Table 6-2. Chord size distributions ${ }^{a}$ of CST material in its engineered-form (Baseline Lot \#9090-76) based on Lasentec $^{\circledR}$ Laser Technology.

Table 6-3. Estimated average particle radius of CST material in its engineered-form (Baseline Lot \#9090-76) based on Lasentec ${ }^{\circledR}$ Laser Technology.

Table 7-1. Fluid density and dynamic viscosity for water and simulated Envelope A 5 M sodium waste solution. .. 61

Table 7-2. Measured fluid density and dynamic viscosity for various $S R S$ waste solutions. .................................. 62

Table 7-3. Limiting ionic conductances in water at $25{ }^{\circ} \mathrm{C}$ (Reid et al., 1977; Perry, 1973, Glasstone and Lewis, 1960).

Table 7-4. Best estimate binary molecular ${ }^{a}$ diffusion coefficients at $25{ }^{\circ} \mathrm{C}$ for a solution containing essentially only cesium cations and anion of one particular species.

Table 7-5. Phase $1 \mathrm{LAW}$ feed anion concentrations and relative mole fractions ${ }^{a}$ for Envelope A used in determining "effective" binary molecular diffusion coefficients with cesium. ................................64

Table 7-6. Phase 1 LAW feed anion concentrations and relative mole fractions ${ }^{a}$ for Envelopes $B$ and $C$ used in determining "effective" binary molecular diffusion coefficients with cesium. ...............................65

Table 7-7. Simulant or sample anion concentrations and relative mole fractions ${ }^{a}$ for various solutions ${ }^{b}$ used in $^{-}$ determining "effective" binary molecular diffusion coefficients with cesium. ...............................66

Table 7-8. Best estimate cesium effective molecular and pore diffusion coefficients at $25{ }^{\circ} \mathrm{C}$ for each Phase 1 LAW batch feed.

Table 7-9. Best Estimate cesium effective molecular and pore diffusion coefficients at $25^{\circ} \mathrm{C}$ for various solutions used in column transport assessments. .........................................................................6...6

Table 9-1. Key features of Cesium-CST IE-911 fixed bed small-scale columns considered ...................................85

Table 9-2. Summary of recommended nominal parameter settings for Cesium-IONSIV ${ }^{\circledR}$ IE-911 CST system

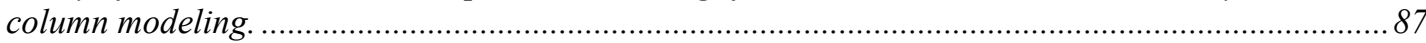

Table 9-2. Summary of recommended nominal parameter settings for Cesium-IONSIV ${ }^{\circledR}$ IE-911 CST system column modeling (continued).

Table 9-3. Root-mean-square residuals for each column study as a function of the ratio of pore to free diffusion coefficients $(\eta)$......

Table 10-1. Comparison of key parameters ${ }^{a}$ between IONSIV ${ }^{\circledR}$ IE-911 CST material and SuperLig ${ }^{\circledR} 644$ resin for removal of cesium from $L A W$ by ion exchange........................................................... 117

Table 10-2. Ion-exchange facility cesium exit concentration criterion on an envelope basis................................ 117

Table 10-3. Processing information on the Phase 1 Low activity waste (LAW) feeds listed in their scheduled order to be processed.

Table 10-4. Ion-exchange facility total cesium exit concentration criterion on a batch feed basis. 119 
Table 10-5. Estimate of the theoretical minimum amount of spent CST material required to process each Phase 1 LAW batch feed.

Table 10-6. Impact of L/D geometry on estimated amount of total spent CST material generated to process the Phase 1 LAW inventory and the number of cycles required per batch of feed (i.e., based on a 2000 L 2-column carousel facility operating at 25 C under nominal parameter settings ${ }^{a}$, except for those parameters explicitly listed in this table).

Table 10-7. Geometric L/D ratios and resulting geometries considered in CST column design simulations using VERSE-LC based on a 2-column carousel configuration. ...............................................122

Table 10-8. Geometric L/D ratios and resulting geometries considered in CST column design simulations using VERSE-LC based on a 3-column carousel configuration. ${ }^{a}$.....

Table 10-9. Estimated amount of total spent CST material generated to process the Phase 1 LAW inventory and the number of cycles required per batch of feed (i.e., based on a 2-column carousel facility operating at 25 C under nominal parameter settings ${ }^{a}$ ).

Table 10-10. Estimated amount of total spent CST material generated to process the Phase 1 LAW inventory and the number of cycles required per batch offeed (i.e., based on a 3-column carousel facility operating at $25 \mathrm{C}$ under nominal parameter settings ${ }^{a}$ ).....

Table 10-11. Impact of mass transfer limitations on estimated amount of total spent CST material generated to process the Phase 1 LAW inventory and the number of cycles required per batch of feed (i.e., based on a 2-column carousel facility operating at $25 \mathrm{C}$ under nominal parameter settings ${ }^{a}$, except for those parameters explicitly listed in this table).

Table 10-12. Estimated amount of total spent CST material generated to process the Phase 1 LAW inventory and the number of cycles required per batch of feed (i.e., based on a 2-column carousel facility operating at $25 \mathrm{C}$ under nominal parameter settings ${ }^{a}$, except for the cesium pore diffusivity coefficient).

Table 10-13. Estimated amount of total spent CST material generated to process the Phase 1 LAW inventory and the number of cycles required per batch of feed (i.e., based on a 2-column carousel facility operating at $25 \mathrm{C}$ under nominal parameter settings ${ }^{a}$, except for the cesium pore diffusivity coefficient)....

Table A-1. Best Basis Inventory (BBI) Phase 1 LAW feed solution data for Envelope A where stated alterations were made to minimize inventory inconsistencies and to establish an ionic charge balance (a value of zero implies that no information was provided on species).....

Table A-2. Best Basis Inventory (BBI) Phase 1 LAW feed solution data for Envelopes B and C where stated alterations were made to minimize inventory inconsistencies and to establish an ionic charge balance (a value of zero implies that no information was provided on species).

Table A-3. Best Basis Inventory (BBI) Phase 1 LAW feed solution data for Envelope C where the impact from the isotopic dilution pretreatment process for Strontium-90 and permanganate addition for TRUs separation have been taken into account. ....

Table A-4. Ionic species available within the ZAM CST ion-exchange equilibrium model. ................................. 159

Table A-5. Best estimate Phase $1 \mathrm{LAW}$ feed solution data for Envelope A adjusted to $5 \mathrm{M} \mathrm{Na}{ }^{a}$ and used as input to the CST equilibrium model. ................................................................................. 160

Table A-6. Best estimate Phase 1 LAW feed solution data for Envelopes B and C adjusted to $5 \mathrm{M} \mathrm{Na}^{a}$ and used as input to the CST equilibrium model.

Table A-7. Hanford and SRS measured liquid-phase densities for several LAW liquid samples and simulants considered.

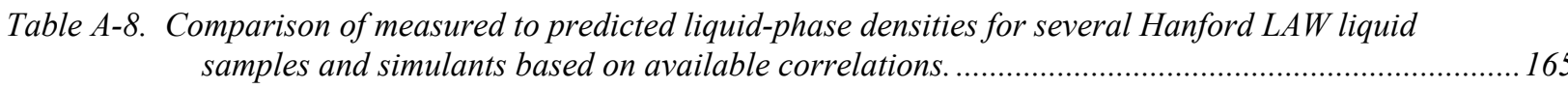

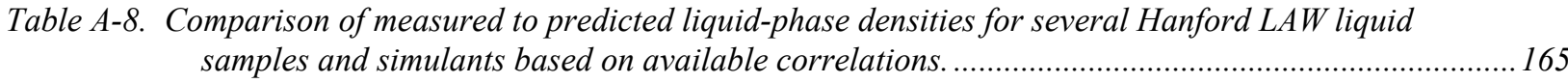

Table B-1. Key CST exchange properties taken from literature ................................................................. 181

Table B-2. Key molar ratios for the various $L A W$ batch feed solutions........................................................ 181

Table B-3. "Extrapolated" ZAM equilibrium isotherm model predictions for CST material in the powder form

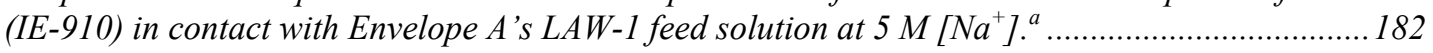

Table B-4. ZAM equilibrium isotherm model predictions for CST material in the powder form (IE-910) in contact with Envelope A's LAW-5 feed solution at $5 \mathrm{M}\left[\mathrm{Na}^{+}\right]{ }^{a}$

Table B-5. ZAM equilibrium isotherm model predictions for CST material in the powder form (IE-910) in contact with Envelope A's LAW-6 feed solution at $5 \mathrm{M}\left[\mathrm{Na}^{+}\right] .^{a}$ 
Table B-6. ZAM equilibrium isotherm model predictions for CST material in the powder form (IE-910) in contact with Envelope A's LAW-8 feed solution at $5 \mathrm{M}\left[\mathrm{Na}^{+}\right]^{a}{ }^{a}$

Table B-7. ZAM equilibrium isotherm model predictions for CST material in the powder form (IE-910) in contact with Envelope A's LAW-9 feed solution at $5 \mathrm{M}\left[\mathrm{Na}^{+}\right] .^{a}$

Table B-8. ZAM equilibrium isotherm model predictions for CST material in the powder form (IE-910) in contact with Envelope A's LAW-10 feed solution at $5 \mathrm{M}\left[\mathrm{Na}^{+}\right]^{a}$

Table B-9. ZAM equilibrium isotherm model predictions for CST material in the powder form (IE-910) in

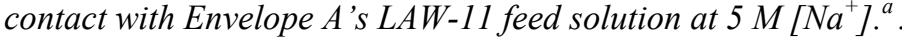

Table B-10. ZAM equilibrium isotherm model predictions for CST material in the powder form (IE-910) in contact with Envelope A's LAW-12 feed solution at $5 \mathrm{M}\left[\mathrm{Na}^{+}\right] .^{a}$

Table B-11. ZAM equilibrium isotherm model predictions for CST material in the powder form (IE-910) in contact with Envelope A's LAW-13 feed solution at $5 \mathrm{M}\left[\mathrm{Na}^{+}\right] .^{a}$

Table B-12. ZAM equilibrium isotherm model predictions for CST material in the powder form (IE-910) in contact with Envelope A's LAW-14 feed solution at $5 \mathrm{M}\left[\mathrm{Na}^{+}\right]^{a}$

Table B-13. ZAM equilibrium isotherm model predictions for CST material in the powder form (IE-910) in contact with Envelope A's LAW-15 feed solution at $5 \mathrm{M}\left[\mathrm{Na}^{+}\right] .^{a}$

Table B-14. ZAM equilibrium isotherm model predictions for CST material in the powder form (IE-910) in

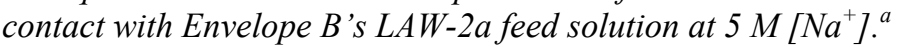

Table B-15. ZAM equilibrium isotherm model predictions for CST material in the powder form (IE-910) in contact with Envelope B's LAW-2b feed solution at $5 \mathrm{M}\left[\mathrm{Na}^{+}\right] .^{a}$

Table B-16. ZAM equilibrium isotherm model predictions for CST material in the powder form (IE-910) in contact with Envelope C's LAW-3 feed solution at $5 \mathrm{M}\left[\mathrm{Na}^{+}\right]^{a}{ }^{a}$

Table B-17. ZAM equilibrium isotherm model predictions for CST material in the powder form (IE-910) in contact with Envelope C's $\mathrm{LAW}-4$ feed solution at $5 \mathrm{M}\left[\mathrm{Na}^{+}\right] .^{a}$......

Table B-18. ZAM equilibrium isotherm model predictions for CST material in the powder form (IE-910) in contact with Envelope C's LAW-7 feed solution at $5 \mathrm{M}\left[\mathrm{Na}^{+}\right]^{a}{ }^{a}$

Table B-19. Parameter identities between a 4-component VERSE-LC Freundlich/Langmuir Hybrid equilibrium isotherm model and the "effective" single-component homovalent isotherm model.

Table B-20. Estimated selectivity coefficients for the Cesium-CST system based on ZAM predictions and the 4component homovalent isotherm model for the Envelope A feed solutions.

Table B-21. The resulting correlation coefficient matrix for the fit of the selectivity coefficients for the CesiumCST system, ZAM produced data, and the 4-component homovalent isotherm model for the Envelope A feed solutions.

Table B-22. Estimated beta parameter values and error estimate for a one-component (total cesium) homovalent algebraic isotherm model based on CST in its powder-form (IE-910) where the data sets used were created using the ZAM code.

Table B-23. Parameter settings for an "effective" single component Freundlich/Langmuir Hybrid equilibrium isotherm model for total cesium on CST based on the 1-component homovalent model................... 193

Table B-24. "Extrapolated" ZAM equilibrium model versus algebraic model cesium loading predictions for

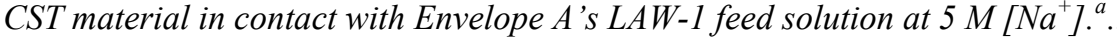

Table B-25. ZAM equilibrium model versus algebraic model cesium loading predictions for CST material in

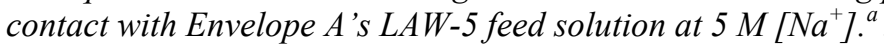

Table B-26. ZAM equilibrium model versus algebraic model cesium loading predictions for CST material in contact with Envelope A's LAW-6 feed solution at $5 \mathrm{M}\left[\mathrm{Na}^{+}\right]^{a}{ }^{a}$

Table B-27. ZAM equilibrium model versus algebraic model cesium loading predictions for CST material in contact with Envelope A's LAW-8 feed solution at $5 \mathrm{M}\left[\mathrm{Na}^{+}\right] .^{a}$

Table B-28. ZAM equilibrium model versus algebraic model cesium loading predictions for CST material in contact with Envelope A's LAW-9 feed solution at $5 \mathrm{M}\left[\mathrm{Na}^{+}\right]{ }^{a}{ }^{a}$

Table B-29. ZAM equilibrium model versus algebraic model cesium loading predictions for CST material in

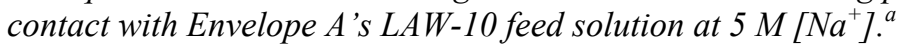

Table B-30. ZAM equilibrium model versus algebraic model cesium loading predictions for CST material in

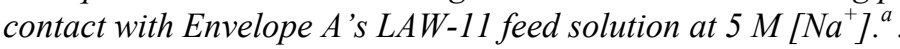

Table B-31. ZAM equilibrium model versus algebraic model cesium loading predictions for CST material in

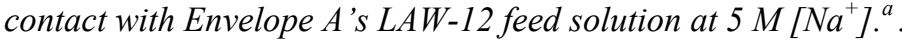


Table B-32. ZAM equilibrium model versus algebraic model cesium loading predictions for CST material in contact with Envelope A's LAW-13 feed solution at $5 \mathrm{M}\left[\mathrm{Na}^{+}\right] .^{a}$

Table B-33. ZAM equilibrium model versus algebraic model cesium loading predictions for CST material in

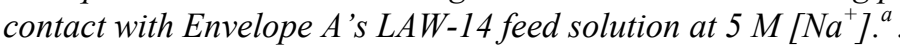

Table B-34. ZAM equilibrium model versus algebraic model cesium loading predictions for CST material in contact with Envelope A's LAW-15 feed solution at $5 \mathrm{M}\left[\mathrm{Na}^{+}\right]^{a}{ }^{a}$

Table B-35. ZAM equilibrium model versus algebraic model cesium loading predictions for CST material in contact with Envelope B's LAW-2a feed solution at $5 \mathrm{M}\left[\mathrm{Na}^{+}\right] .^{a}$

Table B-36. ZAM equilibrium model versus algebraic model cesium loading predictions for CST material in contact with Envelope B's LAW-2b feed solution at $5 \mathrm{M}\left[\mathrm{Na}^{+}\right] .^{a}$

Table B-37. ZAM equilibrium model versus algebraic model cesium loading predictions for CST material in contact with Envelope C's LAW-3 feed solution at $5 \mathrm{M}\left[\mathrm{Na}^{+}\right] .^{a}$

Table B-38. ZAM equilibrium model versus algebraic model cesium loading predictions for CST material in

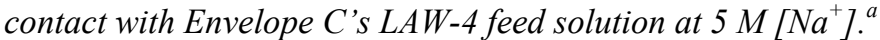

Table B-39. ZAM equilibrium model versus algebraic model cesium loading predictions for CST material in contact with Envelope C's LAW-7 feed solution at $\left.5 \mathrm{M}^{\mathrm{N}} \mathrm{Na}^{+}\right]{ }^{a}{ }^{a}$

Table B-40. Cesium isotherm (engineered-form) sensitivity results based on the ZAM model for deviations about the nominal settings for the Phase $1 \mathrm{LAW}-15$ feed solution at $5.0 \mathrm{M} \mathrm{Na}^{+}, 25 \mathrm{C}$, and $4.552 \times 10^{-5} \mathrm{MCs}^{+}$(100\% of feed value).

Table B-41. Cesium isotherm (engineered-form) sensitivity results based on the ZAM model for deviations about the nominal settings for the Phase $1 \mathrm{LAW}-15$ feed solution at $5.0 \mathrm{M} \mathrm{Na}^{+}, 25 \mathrm{C}$, and $2.276 \times 10^{-5} \mathrm{MCs}^{+}$(50\% of feed value).

Table B-42. Error estimates associated with feed compositions for the key species of interest............................205

Table B-43. Phase 1 LAW-15 feed solution data used as input to the CST equilibrium model sensitivity study.....205

Table C-1. Ionic species molar concentrations for simulated waste solutions used in ZAM batch contact

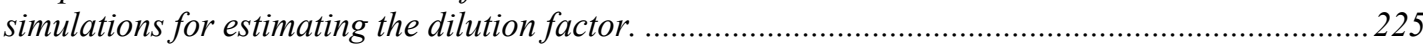

Table C-2. Cesium batch contact test data taken by Walker et al. (2001) for various batches of CST material in contact with SRS-average simulated waste samples at 36.2 C. ....................................................226

Table C-3. Cesium batch contact test data taken by Walker et al. (2001) for various batches of CST material in contact with SRS-average simulated waste samples at different temperatures................................227

Table C-4. Cesium batch contact test data taken by Fondeur et al. (2000) for two batches of CST material in contact with SRS-average simulated waste samples at 25 C . .....................................................227

Table C-5. Power-law coefficients based on cesium loading data taken by Walker et al. (2001) for various batches of CST material in contact with SRS-average simulated waste samples at 36.2 C.............. 228

Table C-6. Estimated dilution factors based on an "effective" single component homovalent isotherm model of the cesium loading curve computed for selected SRS batch contact data ${ }^{a}$.....................................228

Table C-7. Cesium batch contact test data taken by Walker et al. (1997) for the Baseline CST material in contact with SRS Tank 44 actual waste samples at 31 C. ${ }^{a}$.

Table C-8. Cesium batch contact test data taken by McCabe (1995 \& 1997) for two CST materials in contact

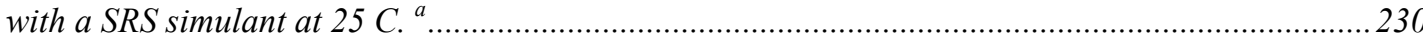

Table C-9. Key CST exchange properties taken from literature. ................................................................2230

Table C-10. Strontium batch contact test data taken by Walker et al. (2001) for various batches of CST material in contact with SRS-average simulated waste samples at 36.2 C .....................................231

Table D-1. Listing of batch feed sequence and VERSE-LC I/O transferring..................................................240

Table D-2. VERSE-LC nominal input parameters settings used in the Envelope A batch feed simulations at 25

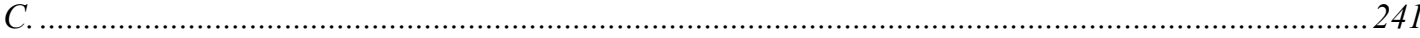

Table D-3. VERSE-LC nominal input parameters settings used in the Envelopes B and C batch feed simulations at 25 C.......

Table E-1. Cesium uptake measurements made at 25 C during the batch kinetics tests of Brown et al., 1996 (initial cesium concentration of $1.0 \times 10^{-4} \mathrm{M}$ ).

Table E-2. Key parameters measured or specified during the batch kinetics tests of Brown et al. (1996) and used to establish the cesium isotherms used in VERSE-LC kinetic modeling.

Table E-3. Cesium uptake measurements made at 25 C during the batch kinetics tests of Fondeur et al., 2000. Also included are estimated conditions at earlier contact times. 
Table E-4. Key parameters measured or specified during the batch kinetics tests of Fondeur et al. (2000) and used to establish the cesium isotherm used in VERSE-LC kinetic modeling..................................264

Table E-5. Cesium uptake measurements made at 25 C during the transient cesium uptake tests of Miller and Brown (1997) and Anthony et al., 1996.

Table E-6. Key parameters measured or specified during the transient cesium uptake tests of Miller and Brown (1997) and Anthony et al. (1996) and used to establish the cesium isotherm used in VERSE-LC kinetic modeling.

Table E-7. Cesium uptake measurements made at 25 C during the batch kinetics tests of Davidson et al., $1998 . . .267$

Table E-8. Key parameters measured or specified during the batch kinetics tests of Davidson et al. (1998) and used to establish the cesium isotherm used in VERSE-LC kinetic modeling..................................268

Table F-1. Ionic species available within the ZAM CST ion-exchange equilibrium model. ..................................28

Table F-2. ZAM ionic species used in CST modeling of Phase 1 LAW batch feeds.............................................290

Table F-3. Equilibrium data for cesium on CST powder (IE-910) based on batch contact tests performed at 25 $C$ in simulated and actual AW-101 solutions (Brown et al. (1996), 0.475 M potassium). ${ }^{a}$................290

Table F-4. Equilibrium data for cesium on CST engineered-form (IE-911-38b) based on batch contact tests performed at $25 \mathrm{C}$ in simulated and actual AW-101 solutions (Brown et al. (1996), $0.475 \mathrm{M}$ potassium). ${ }^{a}$.....

Table F-5. Equilibrium data for cesium on CST engineered-form (IE-911 -08) based on batch contact tests performed at $25 \mathrm{C}$ in simulated AW-101 solutions (Brown et al. (1996), $0.475 \mathrm{M}$ potassium). ${ }^{a} \ldots . . . .293$

Table F-6. ZAM predictions for cesium on CST powder (IE-910) based on simulated batch contact tests performed at $25 \mathrm{C}$ in $\mathrm{AW}-101$ solutions. ${ }^{a}$.

Table F-7. AW-101 simulant and actual solution compositions used to perform ZAM predictions for cesium on

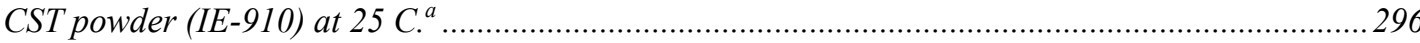

Table E-1. Listing of ZAM input files provided in this appendix for reference...............................................305

Table H-1. Ionic species molar concentrations for various simulated and actual waste solutions used in ZAM batch contact simulations to generate a cesium isotherm data used for estimating the beta factor..313

Table H-2. Key column parameters for CST IE-911 packed columns using SRS high OH simulant and SRS Tank 44 supernate waste taken by Walker et al. (1999) at 31. ${ }^{\text {a.b }}$....

Table H-3. Cesium breakthrough data for CST IE-911 (Lot 98-05) and actual SRS Tank 44 waste taken by

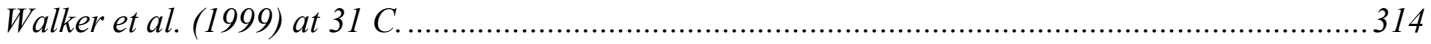

Table H-4. Key column parameters for CST IE-911 packed columns using SRS-Avg simulant taken by Wilmarth et al. (1999) at $25 C( \pm 5 C){ }^{a}$

Table H-5. Cesium breakthrough data for CST IE-911 and SRS-Avg waste simulant taken by Wilmarth et al. (1999) at $\sim 25$ C.

Table H-6. Cesium breakthrough data for CST IE-911 (with and without prior exposure to humid air) and SRS-Avg waste simulant taken by Wilmarth et al. (1999) at $\sim 25$ C.

Table H-7. Key column parameters for CST IE-911 packed columns using SRS-Avg simulant taken by Walker et al. (1998) at $22 C^{a, b}$

Table H-8. Cesium breakthrough data for CST IE-911 and SRS-Avg waste simulant taken by Walker et al.

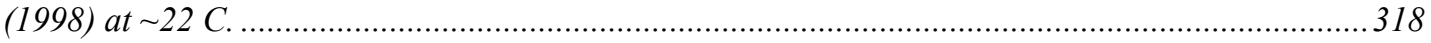

Table H-9. Cesium breakthrough data for CST IE-911 (Lot 98-05) and SRS high OH simulant taken by Walker et al. (1999) at $31 \mathrm{C}$.

Table H-10. Key column parameters for CST IE-911 packed columns using PNNL diluted AW-101 sample taken by Hendrickson (1997) at $25 \mathrm{C}^{\mathrm{a}}{ }^{\mathrm{a}}$.

Table H-11. Cesium breakthrough data for CST IE-911 and PNNL diluted AW-101 sample taken by Hendrickson (1997) at 25 C.

Table H-12. Key column parameters for CST IE-911 packed columns using MVST W-27 sample taken by Lee et al. (1997b) at $25 C^{a, b}$

Table H-13. Cesium breakthrough data for CST IE-911 and MVST W-27 waste sample taken by Lee et al. (1997b) at $\sim 25$ C.

Table H-14. Key column parameters for CST IE-911 packed pilot-scale two-column carousel facility using MVST W-29 waste streams taken by Walker, Jr., et al. (1998) at $25 \mathrm{C}^{a}{ }^{a}$.....

Table H-15. Cycle 1 ORNL-CSRD-Run2 cesium breakthrough data for CST IE-911 and MVST W-29 waste streams taken by Walker, Jr., et al. (1998) at 25 C. 
Table H-16. Cycle 1 ORNL-CSRD-Run3 cesium breakthrough data for CST IE-911 and MVST W-29 waste streams taken by Walker, Jr., et al. (1998) at $\sim 25$ C.....

Table H-17. Cycle 1 ORNL-CSRD-Run4 cesium breakthrough data for CST IE-911 and MVST W-29 waste streams taken by Walker, Jr., et al. (1998) at 25 C..

Table H-18. Cycle 2 ORNL-CSRD-Run 4 cesium breakthrough data for CST IE-911 and MVST W-29 waste streams taken by Walker, Jr., et al. (1998) at 25 C. 326 


\section{LIST OF FIGURES}

Figure 1-1. Computed total spent CST material required to process the entire Phase 1 LAW inventory based on a two-column carousel configuration at $25 \mathrm{C}$ and nominal parameter settings (solid circles are VERSE-LC results while the solid line represents its average behavior).

Figure 1-2. Computed lead column exit cesium concentration breakthrough curves during the processing of the entire Phase 1 LAW inventory based on a two-column carousel configuration at $25 C$ and nominal parameter settings (2000 L bed volumes).

Figure 1-3. Normalized (to feed inlet conditions; $c / c_{o}$ ) lead column exit cesium concentration breakthrough curves during the processing of the entire Phase 1 LAW inventory based on a two-column carousel configuration at $25 \mathrm{C}$ and nominal parameter settings (2000 L bed volumes).

Figure 2-1. Simplified material flowsheet overview highlighting the ion-exchange units used for removal of cesium from a candidate $L A W$ stream.....

Figure 2-2. Cesium loading performance comparison between IONSIV ${ }^{\circledR}$ IE-911 CST and SuperLig ${ }^{\circledR} 644$

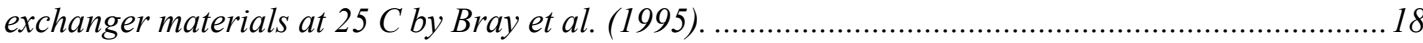

Figure 3-1. The basic building blocks of a porous particle ion exchange column model.......................................24

Figure 3-2. Graphical representation of the various mass transport mechanisms considered important for Cesium-IONSIV ${ }^{\circledR} I E-911$ CST system ion exchange column modeling.....

Figure 3-3. Estimated $\mathrm{Cs}^{+}, \mathrm{K}^{+}$, and $\mathrm{Na}^{+}$exit breakthrough curves for the test SRS-Avg-Test 2 performed by Wilmarth et al. (1999) based on a porous particle multi-component (i.e., ternary) ion exchange column model for SRS Average simulant using IONSIV ${ }^{\circledR} I E-911$ CST.

Figure 3-4. Measured versus predicted $\mathrm{Cs}^{+}$column exit breakthrough curves based on the ternary and "effective" single-component ion exchange column models (test SRS-Avg-Test2 performed by Wilmarth et al. (1999) in a SRS Average simulant liquid at $1.24 \times 10^{-4} \mathrm{M}$ Cs and at $25 \mathrm{C}$ ).

Figure 4-1. The estimated Phase 1 feed beta values used in the cesium effective single-component isotherm model for CST powder-form and engineered-form materials. The beta values are grouped by envelope and are based on feeds assuming zero $\mathrm{Rb}^{+}$and $\mathrm{SrOH}^{+}$present.

Figure 4-2. Comparison of the ZAM generated database for cesium loading onto IE-910 CST (powder) versus the algebraic model fit for the Phase $1 \mathrm{LAW}-15 \mathrm{feed}$. Also shown is the algebraic model when applied to the engineered-form using a dilution factor of $68 \%$.

Figure 4-3. Comparison of Envelope A isotherms for the CST material in its engineered-form (IE-911). The lines represent predictions based on the single-component Freundlich/Langmuir Hybrid isotherm model and symbols indicate the feed concentrations of cesium.

Figure 4-4. Comparison of Envelope B isotherms for the CST material in its engineered-form (IE-911). The lines represent predictions based on the single-component Freundlich/Langmuir Hybrid isotherm model and symbols indicate the feed concentrations of cesium.

Figure 4-5. Comparison of Envelope C isotherms for the CST material in its engineered-form (IE-911). The lines represent predictions based on the "effective" single-component Freundlich/Langmuir Hybrid isotherm model and symbols indicate the feed concentrations of cesium.

Figure 4-6. Comparison of the least favorable isotherms for Envelope A, B, and C feeds for the CST material in its engineered-form (IE-911). The lines represent predictions based on the "effective" singlecomponent Freundlich/Langmuir Hybrid isotherm model.

Figure 5-1. Functional behavior between bed porosity and particle porosity for the IONSIV ${ }^{\circledast}$ IE-911 CST material for various assumed total porosity values. The nominal settings used for the majority of column studies performed are shown by the solid circle.

Figure 6-1. Volume percent of particles as a function of particle size for one sample of the CST material in its IE-911 engineered-form (batch \# not recorded) based on MicroTrac ${ }^{\circledR}$ laser technology (data from Qureshi, 1999).

Figure 6-2. Chord length percent of particles as a function of chord size for one sample of the CST material in its IE-911 engineered-form (Baseline Lot \# 9090-76) based on Lasentec ${ }^{\circledR}$ laser technology...............52

Figure 7-1. Cesium $K_{d}$ measurements obtained from batch kinetics tests performed by Brown et al. (1996) at $25 \mathrm{C}$ and in a $70 \% \mathrm{AW}-101 \mathrm{DSSF}$ simulant liquid at $1 \times 10^{-4} \mathrm{MCs}$ and $5 \mathrm{M} \mathrm{Na}$.

Figure 7-2. Estimation of the cesium pore diffusion coefficient based on batch kinetics tests performed by Brown et al. (1996) at 25 C for cesium uptake on IONSIV ${ }^{\circledR} I E-910$ CST (powder-form). 
Figure 7-3. Estimation of the cesium pore diffusion coefficient based on batch kinetics tests performed by Brown et al. (1996) at 25 C for cesium uptake on IONSIV ${ }^{\circledR}$ IE-911 CST (engineered-form 08)......... 70

Figure 7-4. Estimation of the cesium pore diffusion coefficient based on batch kinetics tests performed by Brown et al. (1996) at 25 C for cesium uptake on IONSIV ${ }^{\circledast} I E-911$ CST (engineered-form 38b)....... 70

Figure 7-5. Cesium $K_{d}$ measurements obtained from batch kinetics tests performed by Fondeur et al. (2000) at $25 \mathrm{C}$ and in a SRS average simulant liquid at $1.4 \times 10^{-4} \mathrm{M}$ Cs and $5.6 \mathrm{M} \mathrm{Na}$. Also shown is an estimated behavior at early times for the Fondeur et al. (2000) data.

Figure 7-6. A comparison of the cesium $K_{d}$ measurements obtained from batch kinetics tests performed by Brown et al. (1996) and Fondeur et al. (2000) on various CST materials at 25 C.

Figure 7-7. Estimation of the cesium pore diffusion coefficient based on batch kinetics tests performed by Fondeur et al. (2000) at 25 C for cesium uptake on IONSIV ${ }^{\circledR} I E-911$ CST (engineered-form Baseline).

Figure 7-8. Comparison of VERSE-LC predictions to measured cesium concentrations during approach to equilibrium based on batch kinetics tests performed by Fondeur et al. (2000) at 25 C for the Baseline form of CST material (IONSIV ${ }^{\circledR}$ IE-911).

Figure 7-9. The effect particle size has on rates of cesium uptake by IONSIV ${ }^{\circledR} I E-910$ (Miller and Brown, 1997) and by IONSIV ${ }^{\circledR} I E-911$ (Anthony et al., 1996) CST materials based on transient cesium uptake testing.

Figure 7-10. The effect phase ratio has on rates of cesium uptake by IONSIV ${ }^{\circledR}$ IE-910 CST material (Davidson et al., 1998) based on batch kinetics testing.

Figure 9-1. Measure of error for individual tests in predicting column exit cesium breakthrough as a function of the ratio of pore to free stream diffusion coefficients. Average behavior of the top eight tests is also plotted......

Figure 9-2. Overall measure of error in predicting column exit cesium breakthrough as a function of the pore to free stream diffusion coefficient ratio (8 column tests considered in summation).

Figure 9-3. Measured versus predicted cesium column exit breakthrough for three assumed pore diffusion coefficient values (test SRS-Avg-Test2 performed by Wilmarth et al. (1999) in a SRS Average simulant liquid at $1.24 \times 10^{-4} \mathrm{M}$ Cs and at $25 \mathrm{C}$ ).

Figure 9-4. Measured versus predicted cesium column exit breakthrough for two assumed pore diffusion coefficient values (test SRS-Avg-Test2 performed by Wilmarth et al. (1999) in a SRS Average simulant liquid at $1.24 \times 10^{-4} \mathrm{MCs}$ and at $25 \mathrm{C}$ ).....

Figure 9-5. A direct comparison of predicted versus measured cesium loadings on IONSIV ${ }^{\circledR} I E-911$ CST material for diluted SRS Tank 44 waste (Walker et al., 1999). The algebraic model is plotted for both the powder (solid-curve) and engineered (dashed-curve) forms.

Figure 9-6. VERSE-LC cesium exit breakthrough curves compared to data from Walker et al. (1999) for SRS Tank 44 waste: $D=1.59 \mathrm{~cm}, L=10 \mathrm{~cm}$ and $85 \mathrm{~cm}, U=5.319 \mathrm{~cm} / \mathrm{min}, T=31{ }^{\circ} \mathrm{C} \ldots \ldots \ldots \ldots \ldots \ldots \ldots \ldots . . .92$

Figure 9-7. VERSE-LC cesium exit breakthrough curve compared to data from SRS-Avg-Test1 (Wilmarth et al., 1999): $D=1.5 \mathrm{~cm}, L=10 \mathrm{~cm}, U=5.5 \mathrm{~cm} / \mathrm{min}, T=\sim 25{ }^{\circ} \mathrm{C}$.

Figure 9-8. VERSE-LC cesium exit breakthrough curve compared to data from SRS-Avg-Test2 (Wilmarth et al., 1999): $D=1.5 \mathrm{~cm}, L=10 \mathrm{~cm}, U=7.0 \mathrm{~cm} / \mathrm{min}, T=\sim 25{ }^{\circ} \mathrm{C}$.

Figure 9-9. VERSE-LC cesium exit breakthrough curve compared to data from SRS-Avg-Test 3 a and Test $3 b$ (Wilmarth et al., 1999): $D=1.5 \mathrm{~cm}, L=10 \mathrm{~cm}, U=4.1 \mathrm{~cm} / \mathrm{min}, T=\sim 25{ }^{\circ} \mathrm{C}$.

Figure 9-10. VERSE-LC cesium exit breakthrough curve compared to data from Wilmarth et al. (1999) for 9805 CST material and Walker et al. (1998) for 96-04 CST material: $D=1.5 \mathrm{~cm}, L=10 \mathrm{~cm}, U=$ $\sim 4.1 \mathrm{~cm} / \mathrm{min}, T=\sim 25^{\circ} \mathrm{C}$

Figure 9-11. VERSE-LC cesium exit breakthrough curve compared to data from SRS-Avg-Test4 (Wilmarth et al., 1999): $D=2.5 \mathrm{~cm}, L=10 \mathrm{~cm}, U=4.1 \mathrm{~cm} / \mathrm{min}, T=\sim 25{ }^{\circ} \mathrm{C}$.

Figure 9-12. VERSE-LC cesium exit breakthrough curve compared to data from SRS-Avg-Test5 (Walker et al., 1998): $D=1.5 \mathrm{~cm}, L=10 \mathrm{~cm}, U=0.47 \mathrm{~cm} / \mathrm{min}, T=\sim 22{ }^{\circ} \mathrm{C}$.

Figure 9-13. VERSE-LC cesium exit breakthrough curve compared to data from SRS-Avg-Test6 (Walker et al., 1998): $D=1.5 \mathrm{~cm}, L=10 \mathrm{~cm}, U=0.98 \mathrm{~cm} / \mathrm{min}, T=\sim 22{ }^{\circ} \mathrm{C}$.

Figure 9-14. VERSE-LC cesium exit breakthrough curve compared to data from SRS-Avg-Test7 (Walker et al., 1998): $D=1.43 \mathrm{~cm}, L=11 \mathrm{~cm}, U=4.1 \mathrm{~cm} / \mathrm{min}, T=\sim 22{ }^{\circ} \mathrm{C}$. 
Figure 9-15. VERSE-LC cesium exit breakthrough curve compared to data from SRS-High-OH-Test1 (Walker et al., 1999): $D=1.5 \mathrm{~cm}, L=10 \mathrm{~cm}$ and $85 \mathrm{~cm}, U=5.43 \mathrm{~cm} / \mathrm{min}, T=\sim 31{ }^{\circ} \mathrm{C}$.

Figure 9-16. VERSE-LC cesium exit breakthrough curve compared to data from PNNL-AW101-Test1 (Hendrickson, 1997): $D=1.5 \mathrm{~cm}, L=10 \mathrm{~cm}$ and $85 \mathrm{~cm}, U=5.43 \mathrm{~cm} / \mathrm{min}, T=\sim 31{ }^{\circ} \mathrm{C}$..............97

Figure 9-17. VERSE-LC cesium exit breakthrough curve compared to data from ORNL-W27-Test1 (Lee et al., 1997b): $D=1.5 \mathrm{~cm}, L=5.659 \mathrm{~cm}, U=0.283 \mathrm{~cm} / \mathrm{min}, T=\sim 25{ }^{\circ} \mathrm{C}$.

Figure 9-18. VERSE-LC cesium exit breakthrough curve compared to data from ORNL-W27-Test2 (Lee et al., 1997b): $D=1.5 \mathrm{~cm}, L=5.659 \mathrm{~cm}, U=0.566 \mathrm{~cm} / \mathrm{min}, T=\sim 25{ }^{\circ} \mathrm{C}$......

Figure 9-19. VERSE-LC cesium exit breakthrough curve compared to data from ORNL-CsRD-Run2 (Walker, $J r$, et al., 1998): $D=30.6 \mathrm{~cm}, L=51.67 \mathrm{~cm}$ per column, $U=2.584 \mathrm{~cm} / \mathrm{min}, T=\sim 25{ }^{\circ} \mathrm{C}$............98

Figure 9-20. VERSE-LC cesium exit breakthrough curve compared to data from ORNL-CsRD-Run3 (Walker, $J r$., et al., 1998): $D=30.6 \mathrm{~cm}, L=51.67 \mathrm{~cm}$ per column, $U=5.167 \mathrm{~cm} / \mathrm{min}, T=\sim 25{ }^{\circ} \mathrm{C}$...........99

Figure 9-21. VERSE-LC cesium exit breakthrough curve compared to data from ORNL-CsRD-Run4a (Walker, $J r$., et al., 1998): $D=30.6 \mathrm{~cm}, L=51.67 \mathrm{~cm}$ per column, $U=5.167 \mathrm{~cm} / \mathrm{min}, T=\sim 25{ }^{\circ} \mathrm{C}$...........99

Figure 9-22. VERSE-LC cesium exit breakthrough curve compared to data from ORNL-CsRD-Run4b (Walker, $J r$., et al., 1998): $D=30.6 \mathrm{~cm}, L=51.67 \mathrm{~cm}$ per column, $U=5.167 \mathrm{~cm} / \mathrm{min}, T=\sim 25{ }^{\circ} \mathrm{C}$.

Figure 10-1. Comparison of cesium loading curves for IONSIV ${ }^{\circledR}$ IE-911 CST material and SuperLig ${ }^{\circledR} 644$ resin in contact with LAW-12 feed solution (241-AN-103) both on a mass and bed volume basis.... 128

Figure 10-2. Comparison of normalized cesium breakthrough curves for IONSIV ${ }^{\circledR}$ IE-911 CST packed and SuperLig ${ }^{\circledR} 644$ packed columns at 25 C using LAW-12 feed solution (i.e., 241-AN-103, identical 2-column carousel configuration, 1000 L columns, $L / D=3$ ).

Figure 10-3. Basic flowsheet for a full-scale (two-column carousel configuration) ion-exchange facility for removal of cesium using IONSIV ${ }^{\circledR} I E-911$ CST material.

Figure 10-4. VERSE-LC model representing a (two-column or three-column carousel configuration) full-scale facility for removal of cesium using IONSIV ${ }^{\circledR}$ IE-911 CST material. The locations where the inlet feed conditions are applied and the exit breakthrough curves are monitored is shown.

Figure 10-5. Phase 1 LAW batch feed inlet flowrates and cesium concentrations used as input boundary conditions for VERSE-LC CST column design simulations. Constant values are applied over each batch process period.

Figure 10-6. The conceptual model defining the length of the mass transfer zone based on a 2-column carousel configuration with specified exit criteria for both columns.

Figure 10-7. Typical impact on mass transfer zone length (i.e., concentration profiles) due to envelope cesium concentration and flowrate differences based on VERSE-LC model predictions when using the IONSIV ${ }^{\circledR}$ IE-911 material.

Figure 10-8. Sensitivity of spent CST to the bed L/D geometric ratio for the total processing of the Phase 1 LAW inventory (i.e., a 2-column carousel configuration operating at $25 \mathrm{C}$ with $2000 \mathrm{~L}$ columns of varying $L / D$ geometries).....

Figure 10-9. VERSE-LC cesium concentration predictions at the exit of the lead column based on CST and the Phase 1 LAW inventory (i.e., a 2-column carousel configuration operating at $25 \mathrm{C}$ with $1000 \mathrm{~L}$ columns for a total of 78 carousel cycles performed).

Figure 10-10. VERSE-LC normalized cesium concentration predictions at the exit of the lead column based on CST and the Phase 1 LAW inventory (i.e., a 2-column carousel configuration operating at 25 C with $1000 \mathrm{~L}$ columns for a total of 78 carousel cycles performed).

Figure 10-11. VERSE-LC cesium concentration predictions at the exit of the lead column based on CST and the Phase 1 LAW inventory (i.e., a 2-column carousel configuration operating at 25 C with 2000 $L$ columns for a total of 31 carousel cycles performed).

Figure 10-12. VERSE-LC normalized cesium concentration predictions at the exit of the lead column based on CST and the Phase 1 LAW inventory (i.e., a 2-column carousel configuration operating at 25 C with 2000 L columns for a total of 31 carousel cycles performed)

Figure 10-13. VERSE-LC normalized cesium concentration predictions at the exit of the lead column based on CST and the Phase 1 LAW inventory (i.e., a 2-column carousel configuration operating at 25 C with 3000 L columns for a total of 20 carousel cycles performed). 
Figure 10-14. VERSE-LC cesium concentration predictions at the exit of the lead column based on CST and the Phase 1 LAW inventory (i.e., a 2-column carousel configuration operating at 25 C with 3000 $L$ columns for a total of 20 carousel cycles performed).

Figure 10-15. VERSE-LC cesium concentration predictions at the exit of the lead column based on CST and the Phase 1 LAW inventory (i.e., a 2-column carousel configuration operating at 25 C with 4000 $L$ columns for a total of 15 carousel cycles performed).

Figure 10-16. VERSE-LC normalized cesium concentration predictions at the exit of the lead column based on CST and the Phase 1 LAW inventory (i.e., a 2-column carousel configuration operating at 25 C with 4000 L columns for a total of 15 carousel cycles performed).

Figure 10-17. Computed total spent CST material required to process the entire Phase 1 LAW inventory based on a two-column carousel configuration at $25 \mathrm{C}$ and nominal parameter settings (solid circles are VERSE-LC results while the solid line represents its average behavior).

Figure 10-18. Computed total spent CST material required to process the entire Phase 1 LAW inventory at 25 $C$ and nominal parameter settings (VERSE-LC results for 2-column and 3-column carousels and limiting cases).

Figure 10-19. Computed total spent CST material required to process the entire Phase 1 LAW inventory based on a two-column carousel configuration at 25 C and nominal parameter settings (VERSE-LC results for various pore diffusivity coefficients and limiting cases).

Figure 10-20. Estimated total cesium inventory contained within a lead column during the worst case process cycle for a two-column carousel configuration (results for 3 differing column sizes are shown; worst case cycles occur primarily in the LAW-2a processing period).

Figure B-1. Molar ratios of Na/Cs versus K/Cs for the Envelope A candidate feed solutions...............................208

Figure B-2. Molar ratios of Na/Cs versus K/Cs for the Envelope B candidate feed solutions................................208

Figure B-3. Molar ratios of Na/Cs versus K/Cs for the Envelope $C$ candidate feed solutions. ............................209

Figure B-4. Molar ratios of $\mathrm{Na} / \mathrm{Cs}$ versus $\mathrm{K} / \mathrm{Cs}$ for all Envelope A, B, and C candidate feed solutions. The least favorable feed solutions for each envelope are highlighted by open symbols...........................209

Figure B-5. LAW molar ratios of Na/Cs versus K/Cs for all 177 Hanford waste tanks. The Phase 1 LAW batch feeds are highlighted using larger symbols.

Figure B-6. Comparison of ZAM model versus "effective" single-component Freundlich/Langmuir Hybrid isotherm model predictions for cesium loadings on CST material in the powder-form (IE-910) for the eleven Envelope A candidate feed solutions.

Figure B-7. Comparison of ZAM model versus "effective" single-component Freundlich/Langmuir Hybrid isotherm model predictions for cesium loadings on CST material in the powder-form (IE-910) for the two Envelope B candidate feed solutions......

Figure B-8. Comparison of ZAM model versus "effective" single-component Freundlich/Langmuir Hybrid isotherm model predictions for cesium loadings on CST material in the powder-form (IE-910) for the three Envelope $C$ candidate feed solutions.

Figure B-9. The estimated Phase 1 feed beta values used in the cesium effective single-component isotherm model for CST powder-form and engineered-form materials. The beta values are grouped by envelope and are based on feeds assuming zero $\mathrm{Rb}^{+}$and $\mathrm{SrOH}^{+}$present.

Figure B-10. Comparison of Envelope A isotherms for the CST material in its engineered-form (IE-911). The lines represent predictions based on the "effective" single-component Freundlich/Langmuir Hybrid isotherm model and symbols indicate the feed concentrations of cesium.

Figure B-11. Comparison of Envelope B isotherms for the CST material in its engineered-form (IE-911). The lines represent predictions based on the "effective" single-component Freundlich/Langmuir Hybrid isotherm model and symbols indicate cesium feed concentrations.

Figure B-12. Comparison of Envelope C isotherms for the CST material in its engineered-form (IE-911). The lines represent predictions based on the "effective" single-component Freundlich/Langmuir Hybrid isotherm model and symbols indicate cesium feed concentrations.

Figure B-13. Comparison of the least favorable isotherms for Envelope A, B, and C feeds for the CST material in its engineered-form (IE-911). The lines represent predictions based on the "effective" singlecomponent Freundlich/Langmuir Hybrid isotherm model.

Figure B-14. Comparison of ZAM model predicted versus experimental (Washburn et al., 1928) $\mathrm{KNO}_{3}$ solubility limit at $25 \mathrm{C}$ (also shown is OLI version 6.5 prediction). For ZAM predictions $\mathrm{NaOH}$ was added to see the ionic strength effect. 
Figure B-15. Predicted impact for cesium loading on CST engineered-form material upon a swapping of nitrate with nitrite starting with a nominal solution of LAW-1 feed at $5.0 \mathrm{M} \mathrm{Na}^{+}$and $25 \mathrm{C}$ (the effect with [solid line] and without [dashed line] $\mathrm{KNO}_{3}$ precipitation is shown).

Figure B-16. Estimated impact of aqueous strontium hydroxide on cesium loadings for CST engineered-form material starting with a nominal solution of $\mathrm{LAW}-3$ feed at $5.0 \mathrm{M} \mathrm{Na}^{+}$and $25 \mathrm{C}$ (zero $\mathrm{SrOH}$ present [solid line] and upper bound of $\mathrm{SrOH}^{+}$present [dashed line]).

Figure B-17. Predicted impact of solution density on ZAM prediction of cesium loading on CST material based on LAW-2a and LAW-2b feeds at $5.0 \mathrm{M} \mathrm{Na}^{+}$and $25 \mathrm{C}$ (the modified HTWOS density model values represent nominal conditions)

Figure B-18. Predicted impact for cesium loading on CST engineered-form material upon an increase in potassium concentration starting with a nominal solution of $\mathrm{LAW}-15$ feed at $5.0 \mathrm{M} \mathrm{Na}$ and $25 \mathrm{C}$ (the effect with [solid line] and without [dashed line] $\mathrm{KNO}_{3}$ precipitation is shown)..

Figure C-1. Comparison of measured cesium $K_{d}$ values for several CST batches taken by Walker et al. (2001) in contact with a SRS-average simulated waste sample at $36.2 \mathrm{C}$.

Figure C-2. Comparison of measured cesium loading values for several CST batches taken by Walker et al. (2001) in contact with a SRS-average simulated waste sample at 36.2 C .....................................232

Figure C-3. Comparison of SRS-Avg simulant cesium CST loading data recently taken by Walker et al. (2001)

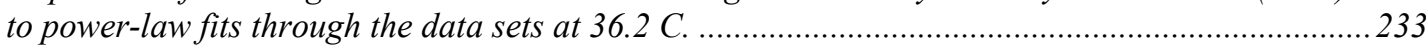

Figure C-4. Comparison of SRS-Avg simulant cesium CST loading data recently taken by Walker et al. (2001) to both best estimate and adjusted ZAM predictions at 36.2 C.....

Figure C-5. Comparison of dilution factor for several CST batch contact data sets with respect to equilibrium

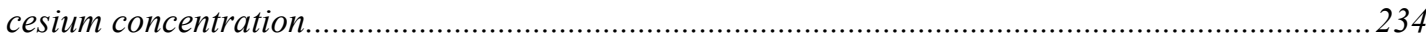

Figure C-6. Comparison of SRS-Avg simulant cesium CST (new powder-form) loading data taken by Fondeur et al. (2000) and Walker et al. (2001) to best estimate ZAM predictions at 26.7, 30.2, and 36.2 C..234

Figure C-7. Comparison of dilution factor for several CST batch contact data sets with respect to equilibrium

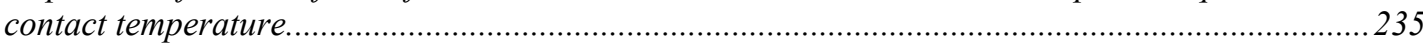

Figure C-8. Comparison of UOP simulant cesium CST loading data recently taken by Walker et al. (2001) to both best estimate and adjusted ZAM predictions at $36.2 \mathrm{C}$.

Figure C-9. A close-up comparison of UOP simulant cesium CST loading data recently taken by Walker et al. (2001) to both best estimate and adjusted ZAM predictions at 36.2 C ..........................................2. 236

Figure C-10. Comparison of SRS-Avg simulant cesium CST loading data taken by Fondeur et al. (2000) to both best estimate and adjusted ZAM predictions at 25 C........................................................2. 236

Figure C-11. Comparison of SRS-Tank 44 sample cesium CST loading data taken by Walker et al. (1997) to both best estimate and adjusted ZAM predictions at 31 C.....

Figure C-12. Comparison of SRS simulant cesium loading data on CST powder and an early on engineeredform material taken by McCabe (1995 [powder data] and 1997 [engineered-form data]) to both best estimate and adjusted ZAM predictions at $\sim 25 \mathrm{C}$......

Figure C-13. Comparison of SRS-Avg simulant strontium CST loading data recently taken by Walker et al. (2001) to both best estimate and adjusted ZAM predictions at $36.2 \mathrm{C}$.

Figure F-1. A comparison of measured versus ZAM predicted cesium $K_{d}$ values for simulated $A W$-101 feed in contact with CST in its power-form (IE-910) over a range of sodium concentration levels [data by Brown et al. (1996)].

Figure F-2. A comparison of measured versus $Z A M$ predicted cesium $K_{d}$ values for $5 M$ sodium simulated and actual AW-101 feeds in contact with CST in its power-form (IE-910) [data by Brown et al. (1996)].

Figure F-3. A comparison of measured versus ZAM predicted cesium $K_{d}$ values for simulated $A W-101$ feed in contact with CST in one of its engineered-forms referred to as "08" (IE-911) over a range of sodium concentration levels [data by Brown et al. (1996)]

Figure F-4. A comparison of measured versus ZAM predicted cesium $K_{d}$ values for simulated $A W$-101 feed in contact with CST in one of its engineered-forms referred to as "38b" (IE-911) over a range of sodium concentration levels [data by Brown et al. (1996)]

Figure F-5. A comparison of measured versus ZAM predicted cesium $K_{d}$ values for $5 M$ sodium simulated and actual AW-101 feeds in contact with CST in one of its engineered-forms referred to as "038b" (IE-911) [data by Brown et al. (1996)]. 
Figure F-6. A comparison of measured versus ZAM predicted cesium loadings for simulated AW-101 feed in contact with CST in its power-form (IE-910) over a range of sodium concentration levels [data by Brown et al. (1996)].

Figure F-7. A comparison of measured versus ZAM predicted cesium loadings for simulated AW-101 feed in contact with CST in one of its engineered-forms referred to as "08" (IE-911) over a range of sodium concentration levels [data by Brown et al. (1996)].

Figure F-8. A comparison of measured versus ZAM predicted cesium loadings for simulated $A W$-101 feed in contact with CST in one of its engineered-forms referred to as " $38 b$ " (IE-911) over a range of sodium concentration levels [data by Brown et al. (1996)].

Figure F-9. A comparison of measured versus ZAM predicted cesium loading values for 5 M sodium simulated and actual AW-101 feeds in contact with CST in its power-form (IE-910) [data by Brown et al. (1996)]. 


\section{TABLE OF NOTATION}

A, B Binding constants for Langmuir isotherm, $\mathrm{M}^{-1}$.

$\mathrm{CV}, \mathrm{BV} \quad$ Active column (bed) volume, $\mathrm{ml}$.

$\mathrm{c}_{\mathrm{bi}}$

Species i conc. in bed fluid, $M$.

$\mathrm{c}_{\mathrm{pi}}$

Species i conc. in pore fluid, M.

$\overline{\mathrm{c}}_{\mathrm{pi}}$

Species i solid surface conc. (or loadings), gmole $/ \mathrm{g}_{\mathrm{resin}}$ or mmole $/ \mathrm{g}_{\mathrm{g} e s i n}$.

$\overline{\mathrm{C}}_{\mathrm{pi}}$

Species i solid surface conc. based on column volume, gmole/ $\mathrm{L}_{\mathrm{CV}}$ or $\mathrm{M}$.

$\overline{\mathrm{C}}_{\mathrm{T}}$

$\overline{\mathrm{C}}_{\mathrm{Ti}}$

Total ion-exchange capacity of resin, mmole $/ \mathrm{g}_{\text {resin }}$.

$\mathrm{C}_{\mathrm{T}}$

Species i ion-exchange capacity of resin, mmole $/ \mathrm{g}_{\text {resin }}$.

$\operatorname{cdf}(\mathrm{x})$

Total cationic strength of solution, $\mathrm{M}$.

D Column diameter, $\mathrm{cm}$.

Cumulative distribution function for the variable $\mathrm{x}$.

D.F. Decontamination factor.

$\mathrm{D}_{\mathrm{pi}} \quad$ Species i pore diffusion coefficient, $\mathrm{cm}^{2} / \mathrm{min}$.

$\widetilde{\mathrm{D}}_{\mathrm{pi}}$

Species i "overall” pore diffusion coefficient, $\mathrm{cm} 2 / \mathrm{min}$.

$\mathrm{D}_{ \pm}^{\infty}$

Binary diffusion coefficient at infinite dilution, $\mathrm{cm}^{2} / \mathrm{s}$.

$\mathrm{D}_{\mathrm{AB}}$

Binary diffusion coefficient for A diffusing through solvent $\mathrm{B}, \mathrm{cm}^{2} / \mathrm{s}$.

$\mathrm{D}_{\infty \mathrm{i}}$

Species i diffusion coefficient, $\mathrm{cm}^{2} / \mathrm{min}$.

$\mathrm{E}_{\mathrm{bi}}$

Species i axial dispersivity, $\mathrm{cm}^{2} / \mathrm{min}$.

$\mathrm{F}$

F

$\mathrm{J}$

$\mathrm{k}_{\mathrm{f}}$

$\mathrm{K}_{\mathrm{di}}$

$\mathrm{K}_{\mathrm{ji}}$

$\widetilde{\mathrm{K}}_{\mathrm{ji}}$

$\mathrm{L}$

$\mathrm{m}_{\mathrm{i}}$

$\mathrm{m}_{\text {resin }}$

$\mathrm{M}_{\mathrm{i}}$

$\mathrm{M}_{\mathrm{ai}}, \mathrm{M}_{\mathrm{bi}}$
Ratio of dry-to-"as received" resin mass

Faraday constant, 96,500 C/g-equiv.

"J factor" analogy dimensionless number.

Liquid film mass transfer coefficient, $\mathrm{cm} / \mathrm{min}$.

Species i distribution coefficient, $\mathrm{M}^{-1}$ or $\mathrm{ml} / \mathrm{g}$.

Equilibrium constant between species $\mathrm{j}$ and $\mathrm{i}$.

Selectivity coefficient between species $j$ and $i$.

Axial length of active bed of column, $\mathrm{cm}$.

Mass of species i, $g$.

Mass of resin, $\mathrm{g}$.

Species i molecular weight, g/gmole.

Freundlich/Langmuir Hybrid model exponents for species i. 


\begin{tabular}{|c|c|}
\hline $\mathrm{N}_{\mathrm{s}}$ & Total number of cations of interest. \\
\hline $\mathrm{N}_{\mathrm{c}}$ & Total number of chemical reactions. \\
\hline $\operatorname{pdf}(x)$ & Probability distribution function for the variable $\mathrm{x}$. \\
\hline Q & Column volumetric flow rate, $\mathrm{ml} / \mathrm{min}$. \\
\hline $\mathrm{q}_{\mathrm{i}}$ & Species i fractional surface site loading. \\
\hline $\mathrm{r}$ & Radial coordinate within avg. size particle, $\mathrm{cm}$. \\
\hline $\mathrm{R}$ & Ideal gas constant, $8.314 \mathrm{~J} /$ gmole-K. \\
\hline $\mathrm{R}_{\mathrm{i}}$ & Isotherm model residual for $i^{\text {th }}$ data point. \\
\hline $\mathrm{R}_{\mathrm{A}}$ & Radius of diffusing particle, $\mathrm{cm}$. \\
\hline $\operatorname{Re}$ & Reynolds Number. \\
\hline$<\mathrm{R}_{\mathrm{p}}>$ & Average particle radius, $\mu \mathrm{m}$. \\
\hline $\mathrm{Sc}_{\mathrm{i}}$ & Species i Schmidt Number. \\
\hline $\mathrm{t}$ & Time. \\
\hline $\mathrm{T}$ & Absolute temperature, $\mathrm{K}$. \\
\hline $\mathrm{u}$ & Linear interstitial velocity, $\mathrm{cm} / \mathrm{min}$. \\
\hline $\mathrm{u}^{\prime}$ & Retarded linear interstitial velocity, $\mathrm{cm} / \mathrm{min}$. \\
\hline $\mathrm{u}_{\text {peak }}$ & Peak radial velocity, $\mathrm{cm} / \mathrm{min}$. \\
\hline $\mathrm{u}_{\mathrm{CL}}$ & Column centerline velocity, $\mathrm{cm} / \mathrm{min}$. \\
\hline $\mathrm{U}$ & Superficial (Darcy) velocity, $\mathrm{cm} / \mathrm{min}$. \\
\hline$V_{\text {bed }}$ & Total volume of active column, $\mathrm{ml}$. \\
\hline $\mathrm{V}_{\text {void }}$ & Total volume of voids within active column, ml. \\
\hline $\mathrm{V}_{\text {pore }}$ & Total volume of pores within particles, ml. \\
\hline $\mathrm{V}_{\text {part }}$ & Total volume of particles within active column, ml. \\
\hline $\mathrm{V}_{\text {sld }}$ & Total volume of solid resin within active column, ml. \\
\hline $\mathrm{V}_{\mathrm{CSTR}}$ & Volume of inlet/outlet headspaces, ml. \\
\hline $\mathrm{Z}$ & Axial coordinate, $\mathrm{cm}$. \\
\hline $\mathrm{z}_{+}, \mathrm{z}_{-}$ & valences of cation and anion, respectively. \\
\hline
\end{tabular}

\section{Greek}

$\beta \quad$ Isotherm parameter constant.

$\hat{\beta}_{\mathrm{i}} \quad$ Langmuir "effective" single isotherm model constant.

$\beta_{\mathrm{i}}, \mathrm{a}_{\mathrm{i}}, \mathrm{b}_{\mathrm{i}} \quad$ Freundlich/Langmuir Hybrid model coefficients for species i.

$\eta_{\mathrm{df}} \quad$ Dilution factor for converting form IE-910 cesium loading to IE-911 value. 


$\begin{array}{ll}\kappa & \text { Boltzmann's constant. } \\ \tau_{\mathrm{p}} & \text { Particle tortuosity. } \\ \lambda & \text { Lambda value }\left(\equiv \mathrm{K}_{\mathrm{d}} \rho_{\mathrm{b}}\right), \mathrm{ml}_{\mathrm{resin}} / \mathrm{ml}_{\mathrm{BV}} . \\ v_{\mathrm{j}} & \text { Species j stoichiometric coefficient. } \\ \varepsilon_{\mathrm{b}} & \text { Bed porosity. } \\ \varepsilon_{\mathrm{p}} & \text { Particle porosity. } \\ \varepsilon_{\mathrm{T}} & \text { Total porosity within column bed. } \\ \sigma_{\mathrm{q}} & \text { Isotherm model standard deviation. } \\ \sigma_{\mathrm{K}_{\mathrm{ji}}} & \text { Selectivity coefficient standard deviation. } \\ \mu_{\mathrm{B}} & \text { Dynamic viscosity of solvent mixture. } \\ \mu_{\mathrm{w}} & \text { Dynamic viscosity of feed solution, centi-poise. } \\ \rho_{\mathrm{w}} & \text { Density of feed solution, } \mathrm{g} / \mathrm{ml} . \\ \rho_{\mathrm{b}} & \text { Bed density of active column, } \mathrm{g} / \mathrm{ml} . \\ \rho_{\mathrm{s}} & \text { Solid (particle) density of resin, } \mathrm{g} / \mathrm{ml} . \\ \lambda_{+}^{\mathrm{o}}, \lambda_{-}^{\mathrm{o}} & \text { Limiting ionic conductance for cation and anion, mhos/equiv. }\end{array}$

\section{Data Set Labeling}

SRS-Avg-Test1

SRS-Avg-Test2

SRS-Avg-Test3

SRS-Avg-Test 4

SRS-Avg-Test5

SRS-Avg-Test6

SRS-Avg-Test7

SRS-High-OH-Test1

PNNL-AW101-Test1

ORNL-W27-Test1

ORNL-W27-Test2

ORNL-CsRD-Test 1

ORNL-CsRD-Test2

ORNL-CsRD-Test3
Test 1 reported by Wilmarth et al., 1999 .

Test 2 reported by Wilmarth et al., 1999.

Test 3 reported by Wilmarth et al., 1999.

Test 4 reported by Wilmarth et al., 1999 .

Test 5 reported by Walker et al., 1998.

Test 6 reported by Walker et al., 1998 .

Test 7 reported by Walker et al., 1998.

Test 1 reported by Walker et al., 1999.

Test 1 reported by Hendrickson, 1997.

Test 1 reported by Lee et al., $1997 \mathrm{~b}$.

Test 2 reported by Lee et al., 1997b.

Test 1 reported by Walker, Jr., et al., 19978.

Test 2 reported by Walker, Jr., et al., 19978.

Test 3 reported by Walker, Jr., et al., 19978. 
(This Page Intentionally Left Blank)

xxiv 


\subsection{Executive Summary}

For the current pretreatment facility design of the River Protection Project (RPP) Waste Treatment Plant (WTP), the removal of cesium from low activity waste (LAW) is achieved by ion-exchange technology based on SuperLig ${ }^{\circledR} 644$ resin. Due to recent concerns over potential radiological and chemical degradation of SuperLig ${ }^{\circledR} 644$ resin and increased pressure drops observed during pilot-scale column studies, an increased interest in developing a potential backup ion-exchanger material has resulted. Ideally, a backup ion-exchanger material would replace the SuperLig ${ }^{\circledR} 644$ resin and have no other major impacts on the pretreatment facility flowsheet. Such an ideal exchanger has not been identified to date.

However, Crystalline Silicotitanate (CST) ion-exchanger materials have been studied for the removal of cesium from a variety of DOE wastes over the last decade. CST ion-exchanger materials demonstrate a high affinity for cesium under high alkalinity conditions and have been under investigation for cesium removal specifically at Hanford and SRS during the last six years. Since CST is an inorganic based material (with excellent properties in regard to chemical, radiological, and thermal stability) that is considered to be practically non-elutable (while SuperLig $^{\circledR} 644$ is an organic based elutable resin), the overall pretreatment facility flowsheet would be impacted in various ways. However, the CST material is still being considered as a potential backup ion-exchanger material. The performance of a proposed backup ion-exchange column using IONSIV IE-911 (CST in its engineered-form) material for the removal of cesium from Hanford high level radioactive alkaline waste is discussed. This report focuses attention only on the ion-exchange aspects and only addresses the loading phase of the process cycle.

Available bench-scale column tests, batch kinetics tests, and batch equilibrium experiments using CST materials were utilized (wherever possible) in the development and validation of an analysis methodology. The methodology employed and the results of the study are discussed.

The major accomplishments and conclusions are:

- The planned waste treatment processing of the Phase 1 LAW tank inventory consists of processing 16 separate batches of feeds from the ten targeted waste tanks in a sequential fashion. The sequence chosen is reflected in the numbering sequence used to label each batch feed (i.e., LAW-1, LAW-2a, LAW-2b, LAW-3, ..., LAW-15). Each batch represents a fixed amount of liquid waste volume whose volume includes all planned dilution processes. The ionic composition of all 16 candidate feed solutions were determined based on the Tank Farm COUP report, Best Basis Inventory (BBI) Phase 1 database, more recent PNNL and SRTC analytical analysis of available samples. The necessary adjustments to achieve an ionic charge balance (consistent with the demands of the isotherm model used) was performed. The various estimation methods used focused on those waste feed ions that have a direct impact on cesium loading. The batch feed compositions for ${ }^{137} \mathrm{Cs}$ were decay corrected from $\sim 1999$ to the date of scheduled waste processing in the Waste Treatment Plant. 
- A sensitivity study and error analysis were performed to estimate an overall uncertainty value of $\sim 30 \%$ for predicted cesium loadings based on the engineered-form (IE-911) of CST material. The predicted cesium isotherms are based on the Texas A\&M ZAM model coupled with a dilution factor. The ZAM model solves the appropriate liquid-solid equilibrium equations for the Cesium-CST system where its modeling parameters were determined based on batch contact tests using CST powder. The dilution factor is based on the measured deviations of engineered-form contact test data when compared to ZAM predicted powder behavior. To evaluate the impact on the cesium isotherms resulting from uncertainties in the composition of the various Phase $1 \mathrm{LAW}$ feeds, a sensitivity analysis was performed. Using the sensitivity results, a "simplified" error analysis was performed where an estimate of the overall uncertainty in a cesium isotherm was computed. Much of this effort is based on a good engineering judgement approach where errors in various variables are stated based on an assumed/implied confidence level of approximately 2-sigma. Where available, supporting data were used in establishing these error estimates. Verification and validation analyses were performed for the ZAM code based on available Hanford AW-101 simulant and actual waste samples.

- To accommodate batch variability in CST engineered-form material, a dilution factor of $68 \%$ was used in the column sizing study. The $68 \%$ dilution factor is consistent with the measured impact of the inert binder for baseline IE-911 engineered-form CST material and is statistically conservative (i.e., $\sim 85 \%$ confidence) with respect to the majority of batch material data available. The use of $68 \%$ for the dilution factor sets a CST material acceptance criterion that should be reasonably achieved by the manufacturer of the CST engineered-forms.

- Originally, batch kinetics test data were going to be used to establish an acceptable value for the cesium pore diffusion coefficient; while, available laboratory-scale column test data were going to be used for assessment purposes only. Unfortunately, significant inconsistencies exist within the available batch kinetics tests such that no conclusive value for the pore diffusion coefficient could be determined. The kinetics data also indicate that simple Fickian diffusion through pores of a homogeneous material is in question. Surface diffusion and heterogeneous pores (between the powder and the binder) may play an important role in establishing the overall mass transfer rate. For this analysis effort it was decided that an "effective" value of the cesium pore diffusion coefficient (based on a purely Fickian formulation) would be determined based on assessment directly to available lab-scale column test data. Based on ten lab-scale column tests a "best estimate" value of $\sim 20 \%$ of its free (molecular) stream diffusion coefficient value was computed with a standard deviation of approximately $5 \%$.

- It is assumed that the cesium isotherms for CST material are true solid-liquid equilibrium curves that are unique and are ultimately approached either from above or from below (i.e., no form of historesis exists). Limited data exist indicating that this should be a reasonable assumption. Also, it is assumed that the kinetics involved for CST material is directional independent (i.e., diffusion rates are equal for the adsorbing and de-adsorbing processes). 
- Early column performance (say the first 5 to 10 column volumes or so) requires the use of multi-component modeling formulations (i.e., ternary component isotherms and transport equations). However, long-term performance should be adequately handled using the simpler single-component formulations. Justification for this simplification is provided. Since significant CPU savings are achieved when the single-component model is used and the differences are well within our current predictive capabilities, the majority of column analyses presented in this report were performed using the single-component approach. This simplification only applies to the loading cycle, while for future elution studies a multicomponent version would be required due to the strong concentration gradients that would be present throughout the columns. Elution of CST is not normally possible or conducted.

- Based on the specified Envelope A, B, and C flowrates (i.e., 52.6, 9.4, and $16.2 \mathrm{~L} / \mathrm{min}$, respectively) and estimated feed volumes when adjusted to a common $5 \mathrm{M} \mathrm{Na}^{+}$basis, an overall processing time of $\sim 4.2$ years is required to process the entire Phase 1 LAW inventory. This processing time yields a $42 \%$ utilization requirement for the ion-exchange facility over the scheduled 10-year project lifecycle. If the Envelope A feeds are processed at the $30 \mathrm{MT} /$ day production rate, an overall processing time of $\sim 5.8$ years is required.

- Surface film diffusion along with pore diffusion place mass transfer limitations on the kinetics of an ion-exchange material. At the flowrates of interest, the CST material is primarily pore diffusion limited. Since the CST columns are not film diffusion limited, the impact on estimated spent CST material is insensitive to geometrical variations (e.g., length to diameter ratio). Therefore, total column "bed" volume is the key parameter for design purposes. For design purposes, the bed $\mathrm{L} / \mathrm{D}$ ratio can be established based on other operational considerations than column performance in terms of the exit breakthrough curves (e.g., overall column pressure drop or sweeping out of generated gases).

- From the standpoint of minimizing the spent CST material to be sent to the melters versus the physical bed size of an individual column, the column transport results indicate that an $\sim 2$ $\mathrm{m}^{3}$ column is near the optimum value. At this column size an estimated $66,000 \mathrm{~kg}$ (66 MT) of spent CST material would be generated corresponding to an $\sim 2.5 \mathrm{wt} \%$ of waste sodium oxide in the final glass product (assuming 2651 MT of glass to be produced).

- Also, these results indicate that the current column design based on SuperLig ${ }^{\circledR}$ technology (i.e., $\sim 1 \mathrm{~m}^{3}$ in volume with an $\sim 3 \mathrm{~L} / \mathrm{D}$ ratio) is adequate, even though not optimal (i.e., $\sim 20 \%$ increase in spent CST, $\sim 80 \mathrm{MT}$ ), for use if CST material was later chosen as a backup ionexchanger. Increasing the bed volume up to its current design maximum of $\sim 1.5 \mathrm{~m}^{3}$ results in only an increase of $\sim 10 \%$ in spent CST ( $\sim 72 \mathrm{MT})$. In terms of the total number of carousel cycles required the $1 \mathrm{~m}^{3}, 1.5 \mathrm{~m}^{3}$, and $2 \mathrm{~m}^{3}$ column volume designs required 78,46 , and 31 cycles, respectively.

- Historically, is has been stated that CST overall has relatively slow kinetics. However, for the $2 \mathrm{~m}^{3}$ bed volume design using a two-column carousel, the resistances associated with mass transfer only increases the computed spent CST by $\sim 14 \%$. Impacts of this magnitude would suggest that further reduction in particle size or in bed $\mathrm{L} / \mathrm{D}$ is not warranted based on improving the "kinetics" alone. Based on the theoretical minimum in spent CST required 
(which can not be reached using practical designs, but helps gage how efficient or inefficient the current CST material and carousel designs are), the actual/practical design only increases the computed spent CST by $\sim 19 \%$. These numbers are based on a nominal value for the cesium pore diffusivity coefficient of $20 \%$ of its free diffusion value. However, uncertainty in the actual value of the pore diffusivity coefficient can result in larger impacts. For larger coefficient values its impact on spent CST becomes more aggressive. Future efforts should be focused on obtaining better confidence in the magnitude of this diffusivity coefficient.

- The CST column sizing results are based on the sixteen different Phase 1 batch feeds being processed sequentially in the order of their current schedule. However, the actual processing order of the 16 feeds has only a marginal impact on the above results.

- Under the current batch feed process schedule, Envelope B feeds (LAW-2a and LAW-2b, which refer to tank waste from AZ-101 and AZ-102, respectively) are to be processed early on. The inlet feed concentrations for the Envelope B feeds are approximately one order of magnitude greater than values for Envelopes A and C. Under this processing strategy a significant amount of cesium inventory will be held up within the columns throughout much of the total period.

- The majority of analyses performed were based on a two-column carousel configuration. A smaller subset of analyses was performed using a three-column carousel configuration to determine the potential benefit associated with more column stages. Only marginal gains can be achieved when a 3 -column versus 2 -column carousel facility is considered. Theoretically, increased column stages will reduce the amount of spent CST generated; however, the predicted gains are within the expected accuracy of the methodology and the increased staging is not warranted.

- A homovalent cation exchange process occurs between the batch feeds and the CST material where the cations $\mathrm{Cs}^{+}, \mathrm{K}^{+}, \mathrm{Na}^{+}, \mathrm{Rb}^{+}$, and $\mathrm{SrOH}^{+}$are in direct competition (i.e., the stated total ionic exchange capacities are $0.58,1.2,4.6,1.18$, and $1.0 \mathrm{mmole} / \mathrm{g}_{\mathrm{CST}}$, respectively). Only trace amounts of $\mathrm{Rb}^{+}$are present within the batch feeds and its concentration within these feeds was set to zero. Due to the complex formation of strontium with various complexants (e.g., EDTA), the actual amount of free $\mathrm{SrOH}^{+}$present within a batch feed is currently unknown and was assumed to be zero for the best estimate analyses. To a first approximation $\mathrm{SrOH}^{+}$competes equally for CST sites with $\mathrm{Cs}^{+}$and should be accounted for in any future design efforts. For example, if equal amounts of $\mathrm{SrOH}^{+}$and $\mathrm{Cs}^{+}$are present within the feed, then a column approximately twice the size of one handling $\mathrm{Cs}^{+}$alone would be required. In this work the impact of competition from $\mathrm{Cs}^{+}, \mathrm{K}^{+}$, and $\mathrm{Na}^{+}$was addressed.

- The estimated selectivity coefficients between $\mathrm{Cs}^{+}$and $\mathrm{K}^{+}$, and $\mathrm{Cs}^{+}$and $\mathrm{Na}^{+}$, for CST material are very similar in magnitude to (i.e., only slightly better than) those measured for SuperLig ${ }^{\circledR} 644$. However, on a fixed bed volume basis the CST material contains over twice the number of cesium active exchange sites than SuperLig ${ }^{\circledR} 644$, since its bed density is more than double. 
- For each of the 16 batch feeds cesium isotherms were generated using the ZAM model. For each batch feed a simple algebraic model based on the homovalent cation exchange process was found to provide an excellent prediction of the ZAM results. Based on the excellent agreement achieved with the algebraic model, the assumption that the selectivity coefficients are constants over the entire cesium concentration range appears to be reasonable.

- From an equilibrium loading consideration only, batch feeds LAW-1 and LAW-15 (i.e., feeds from tanks AP-101 and AW-101, respectively) exhibit the least favorable isotherms. The primary reason for this is due to their high potassium levels (i.e., 0.71 and $0.41 \mathrm{M}$, respectively, at $5 \mathrm{M} \mathrm{Na}^{+}$conditions).

- Cesium total inventories within the column (i.e., cesium content in the liquid-phase plus adsorbed onto the solid-phase) as a function of time were computed during the loading cycle corresponding to worst case conditions. This inventory estimate can be used in subsequent analyses (beyond the scope of this report) for estimating conservative exposure levels. For design purposes a maximum loading of $\sim 250 \mathrm{mmole}_{\mathrm{Cs}} / \mathrm{L}_{\text {bed }}$ over an approximate 150 day exposure time should be considered. At a $30 \%$ isotopic fraction of ${ }^{137} \mathrm{Cs}$ the loading becomes $\sim 75 \mathrm{mmole}_{\mathrm{Cs}-137} / \mathrm{L}_{\text {bed }}$ (or $\left.\sim 889 \mathrm{Ci} / \mathrm{L}_{\text {bed }}\right)$.

- A bed porosity of 0.50 and particle porosity of 0.24 were used for the majority of column simulations performed within this report. These values yield a total column porosity of $62 \%$. Based on recent access to measured particle and bed densities, we estimate that the total porosity is in the range of $29 \%$ to $53 \%$. The $62 \%$ we assumed earlier is outside this range indicating that the results from our column models are based on total bed voids that are larger than they should have been. This would normally imply that the column models contain less CST material by mass than they should have had. Fortunately, the manner in which VERSELC handles the isotherms enforces the bed density to be the value used in creating the isotherms (i.e., typically $1 \mathrm{~g}_{\mathrm{CST}} / \mathrm{ml}_{\text {bed }}$ ). The net effect is larger bed and/or pore volumes that can have a secondary effect on the predicted breakthrough curves (e.g., typical impacts are less than $\pm 10 \%$ ). Future design efforts should be based on more up to date porosity values based on measured bed and particle densities consistent with the engineered-form under consideration.

A few of the above listed conclusions disserve some addition clarification. The methodology used in estimating the column performance of CST in removal of cesium is not an overall bounding analysis, but better represents an approximate "best estimate" analysis for columns packed with modest performance CST material. Significant variability among the available batches in engineered-form can be seen. Therefore, the parameter settings were "nominally" established with the intent to represent in a conservative manner the majority of these CST batches.

The major objective of this study was to estimate the quantity of CST material (in its engineeredform) that would be required to process the entire Phase 1 LAW feed solution inventory. It is assumed that these feeds are all adjusted to a $5 \mathrm{M} \mathrm{Na}^{+}$basis prior to entering the ion-exchange facility and that the facility will operate at $25 \mathrm{C}$. In determining the required quantity of CST 
material (i.e., spent CST) an exit criterion for ${ }^{137}$ Cs was placed on each Envelope along with a design volumetric flowrate as:

- Envelope A

- Envelope B

- Envelope C

$$
1.75 \times 10^{-5} \mathrm{Ci} / \text { gmole of } \mathrm{Na}(0.088 \mu \mathrm{Ci} / \mathrm{ml})
$$$$
5.00 \times 10^{-5} \mathrm{Ci} / \mathrm{gmole} \text { of } \mathrm{Na}(0.250 \mu \mathrm{Ci} / \mathrm{ml})
$$$$
1.75 \times 10^{-5} \mathrm{Ci} / \mathrm{gmole} \text { of } \mathrm{Na}(0.150 \mu \mathrm{Ci} / \mathrm{ml})
$$

$52.6 \mathrm{~L} / \mathrm{min}$

$9.4 \mathrm{~L} / \mathrm{min}$

$16.2 \mathrm{~L} / \mathrm{min}$
$25 \%{ }^{137} \mathrm{Cs}$

$30 \%{ }^{137} \mathrm{Cs}$

$25 \%{ }^{137} \mathrm{Cs}$

Since these CST columns are not film diffusion limited, the bed volume of an individual column is sufficient for defining the performance of a given carousel configuration (i.e., performance is nearly independent of L/D). From a theoretical perspective the amount of spent CST will exhibit a unique minimum with respect to column volume. The minimum point corresponds to the column volume where the cesium exit criterion in the lag column is reached at the same point in time that the last drop of Phase 1 LAW inventory enters the lead column without the need to perform any carousel cycles. Unfortunately, this minimum point represents a significantly large column (i.e., probably greater than $20 \mathrm{~m}^{3}$ ) and is unpractical from a cost or operational perspective. Fortunately, for smaller column volumes the actual shape of the spent CST versus column volume curve remains relatively flat until a knee in the curve occurs as shown in Figure $1-1$.

In Figure 1-1 the solid circles represent VERSE-LC two-column carousel simulation results where the number of carousel cycles performed for each simulation case is provided. The solid line shown represents an average behavior. Carousel cycles are discrete operations that occur each time the exit cesium criterion is reached at the exit of a lag column. During a carousel cycle, the lead column is removed from the column train (i.e., adding to the total spent CST material) and replaced with the current partially loaded lag column, while a fresh column is placed into the lag column position. Therefore, if a sufficient number of simulations at varying column volumes were run the actual shape of this curve would be somewhat saw-toothed. Some of this saw-toothed appearance can be seen at the higher column volumes in Figure 1-1 (note that the magnitude of this saw-toothed behavior diminishes at the smaller column volumes). If the maximum utilization of CST material could be achieved theoretically for each batch feed, an estimated $\sim 56,000 \mathrm{~kg}$ of spent CST would be generated (see the dashed line in Figure 1-1).

Based on the location of the "knee", the optimal column size appears to be approximately $2 \mathrm{~m}^{3}$. This corresponds to the smallest column size where only a negligible increase in spent CST results. For column sizes smaller than $\sim 2 \mathrm{~m}^{3}$ a rapid increase in spent CST is observed. A very similar (i.e., only slightly reduced) curve exists for a three-column carousel configuration.

Based on the order of batch feeds to be processed and a $2 \mathrm{~m}^{3}$ in size bed volume, the total cesium concentration exiting the lead column (i.e., which provides some measured on its loading level) is shown in Figure 1-2 for the 16 feeds (i.e., each envelope is color-coded). The process time is given in months where the time interval for each batch feed is shown by the various vertical lines provided. Each carousel operation (cycle) resets the exit cesium concentration back to zero (i.e., 31 cycles in total resulting in a total of 33 bed volumes of spent CST). Since Envelope B feeds contain cesium approximately one order larger than in Envelope A and C feeds, cesium loadings within the lead columns are significantly impacted over a sizable range of the entire process 
period. As shown in Figure 1-2, for several carousel cycles after the Envelope B feeds have been processed, de-adsorption on the lead columns occur where re-adsorption then takes place on the lag columns (i.e., redistribution of cesium within the carousel). This is a direct result of the significant reduction in inlet cesium concentration after the Envelope B feeds.

By looking at the lead column loadings during each carousel operation, an indication as to the degree of utilization of the CST material can be seen. Under standard operations, a column approaches saturation with respect to its feed when its exit concentration approaches its feed value (i.e., when $\mathrm{c} / \mathrm{c}_{\mathrm{o}}$ approaches unity). For a $2 \mathrm{~m}^{3}$ in size bed volume the results shown in Figure 1-2 are also plotted in Figure 1-3 where the appropriate cesium feed concentrations have been used to normalized the breakthrough curves. Redistribution within the carousel occurs for those cycles whose normalized concentrations $\left(\mathrm{c} / \mathrm{c}_{\mathrm{o}}\right)$ exceed unity. The peak values during each cycle indicate the degree of utilization. For example, $100 \%$ utilization occurs for the Envelope B feeds, $>100 \%$ for the Envelope C feeds, and $\sim 45 \%$ for the Envelope A feeds.

If a sizing strategy based on worst case feed conditions had been used, the design would have been set based on the Envelope A's AP-101 or AW-101 feeds where a significantly larger column volume would be required to up Envelope A's utilization to the typical $90-95 \%$ goal. A mass transfer zone (MTZ) approach to sizing the columns was inappropriate here since the basic assumptions required in determining the MTZ length are invalidated/limited (i.e., inlet conditions are time varying, for many feeds the degree of non-linearity in their isotherms were marginal, and significant variability within feed isotherms).

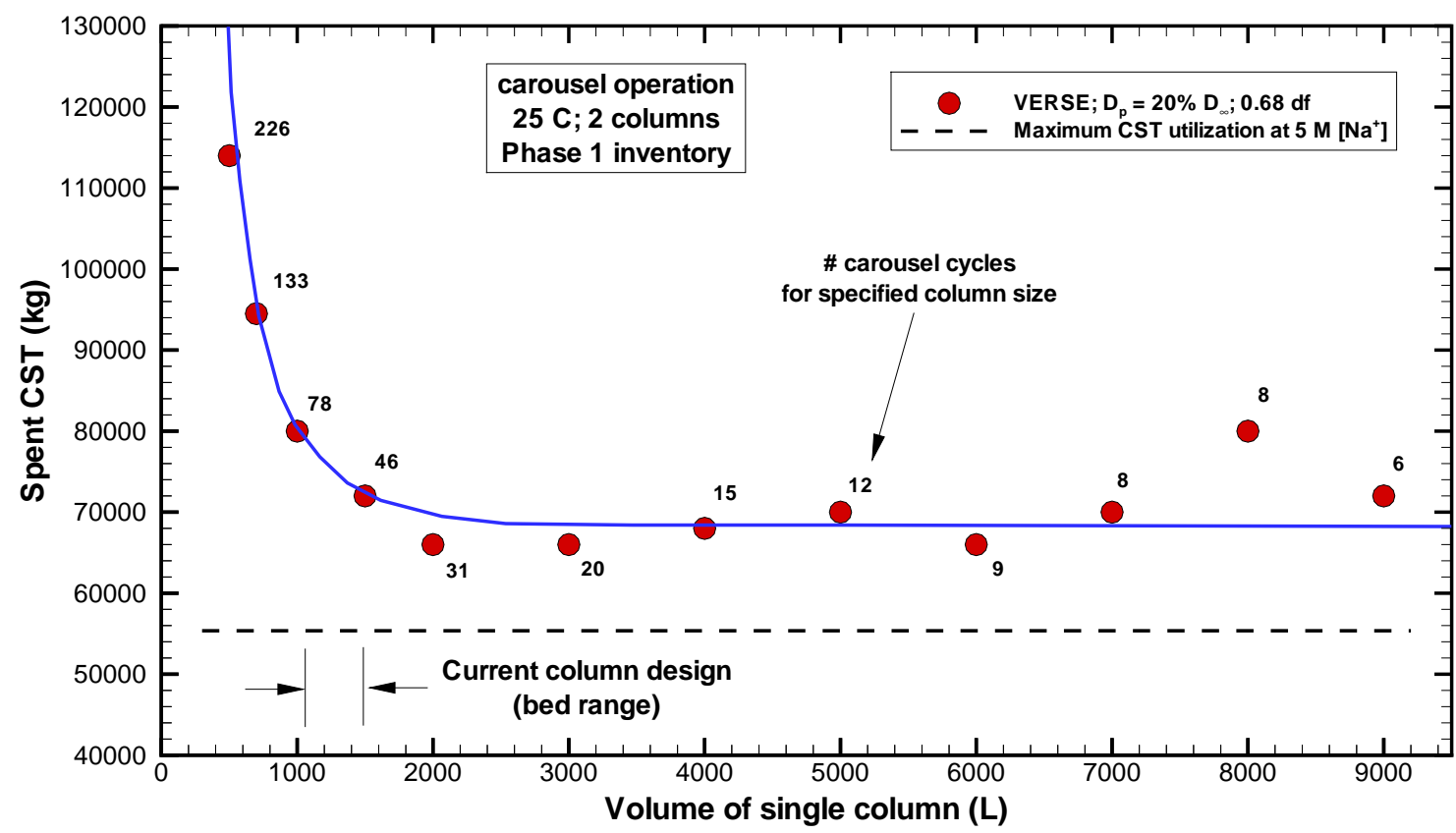

Figure 1-1. Computed total spent CST material required to process the entire Phase 1 LAW inventory based on a two-column carousel configuration at $25 \mathrm{C}$ and nominal parameter settings (solid circles are VERSE-LC results while the solid line represents its average behavior). 


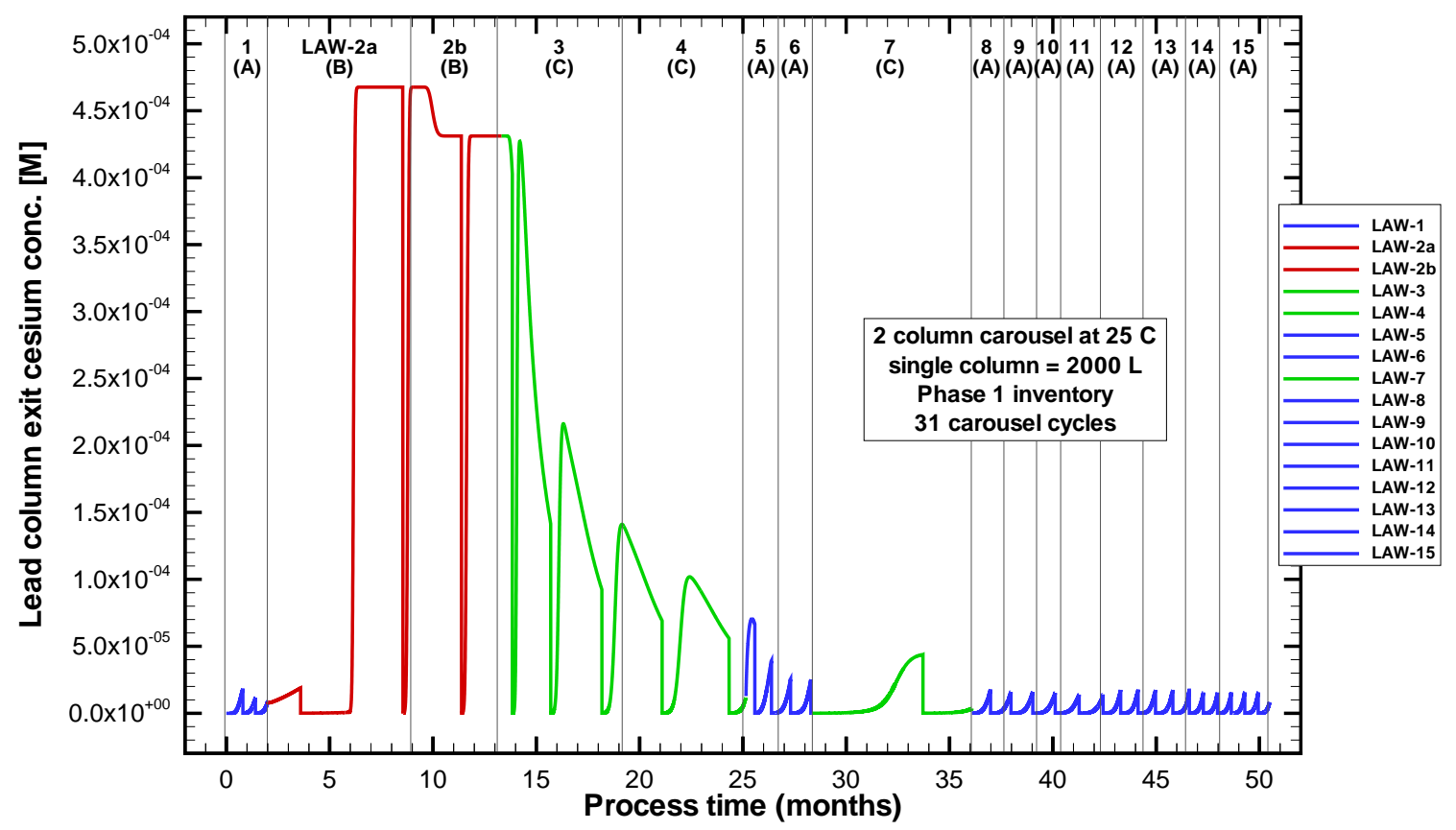

Figure 1-2. Computed lead column exit cesium concentration breakthrough curves during the processing of the entire Phase 1 LAW inventory based on a two-column carousel configuration at 25 $\mathrm{C}$ and nominal parameter settings (2000 L bed volumes). 


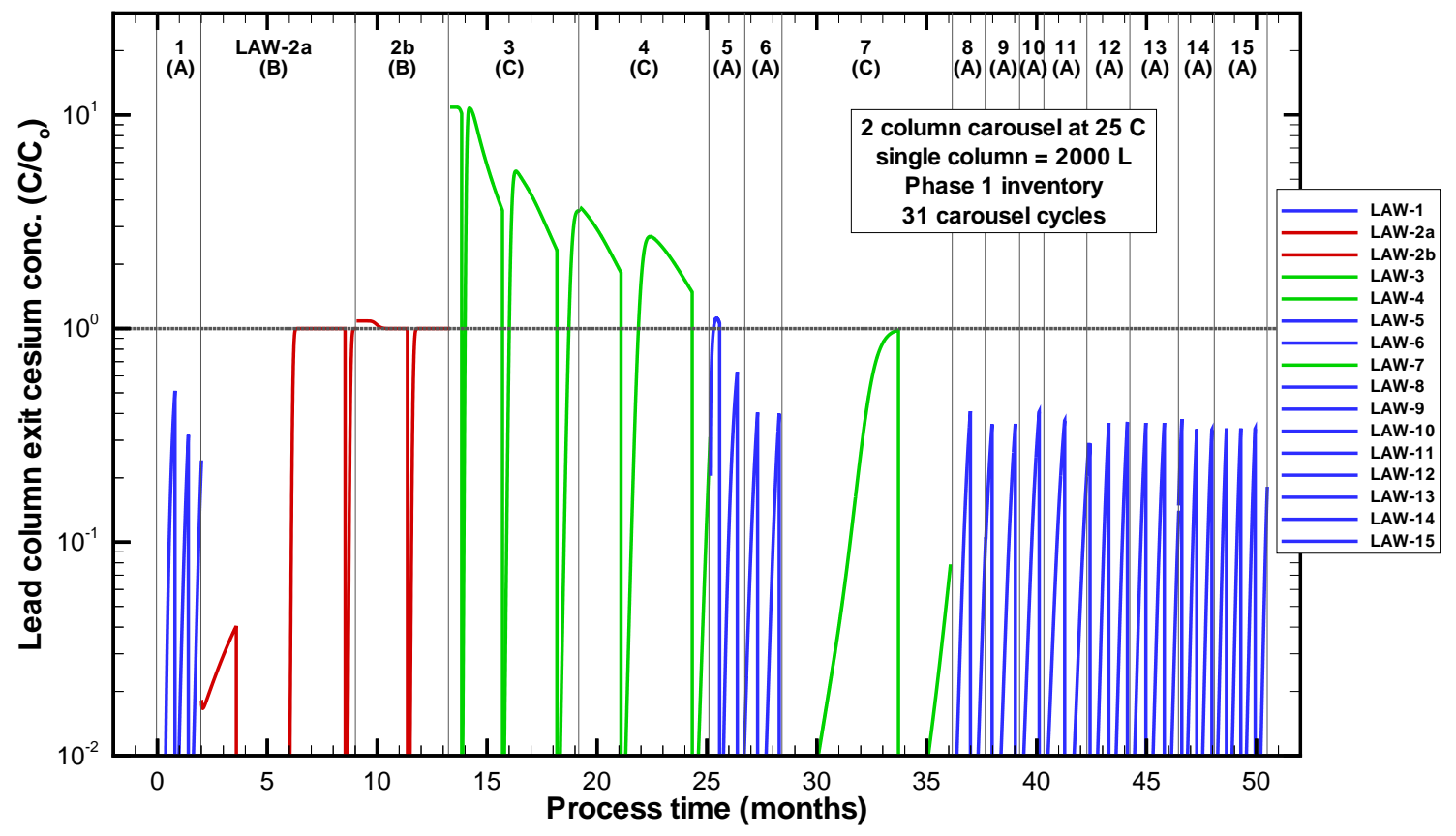

Figure 1-3. Normalized (to feed inlet conditions; $\mathrm{c} / \mathrm{c}_{\mathrm{o}}$ ) lead column exit cesium concentration breakthrough curves during the processing of the entire Phase 1 LAW inventory based on a two-column carousel configuration at $25 \mathrm{C}$ and nominal parameter settings $(2000 \mathrm{~L}$ bed volumes). 


\subsection{Introduction and Background}

A proposed facility is being designed for the immobilization of radioactive waste contained within Hanford underground storage tanks. The waste is pretreated to split it into Low Activity Waste (LAW) and High Level Waste (HLW) streams for separate vitrification. One unit process in the overall pretreatment facility is designed to remove radioactive cesium (therefore total Cs) by ion-exchange from a highly alkaline aqueous phase. A resin specifically designed with moderately high selectivity for cesium under high $\mathrm{pH}$ conditions is being investigated. For the current pretreatment facility design of the River Protection Project (RPP) Waste Treatment Plant (WTP), the removal of cesium from low activity waste (LAW) is achieved by ion-exchange technology based on SuperLig ${ }^{\circledR} 644$ resin. This resin is elutable under lower pH conditions (e.g., adequate elution occurs under water or dilute nitric acid conditions). The proposed design of the facility consists of two sets of two packed columns placed in series (i.e., a lead column followed by a lag column configuration). It should be noted that the WTP Contractor has more recently modified the design susequent to this effort to include a third column within the carousel. During operation, upon reaching a specified cesium concentration criterion at the exit of the lag column, operation is switched to the second set of lead and lag columns. The cesium-loaded lead column is processed (i.e., washed and eluted) and switched to the lag position. The previous lag column is then placed in the lead position (without eluting) and the system is ready for use in the next cycle. For a well designed process, the loading and elution processes result in significant volume reductions in aqueous high level waste. A third column is provided within the facility's footprint for the option to perform three-column carousel operations if needed.

Due to chemical and radiological degradation of the exposed resin, at some point the resin must be replaced. Current design requirements placed on the SuperLig ${ }^{\circledR} 644$ resin assume that adequate removal performance from the resin can be achieved for up to 10 process cycles. Due to recent concerns over potentially high rates of radiological and chemical degradation of SuperLig $^{\circledR} 644$ resin and increased pressure drops observed during pilot-scale column studies, an increased interest in developing a potential backup ion-exchanger material has resulted. Ideally, a backup ion-exchanger material would replace the SuperLig ${ }^{\circledR} 644$ resin and have no other major impacts on the pretreatment facility flowsheet. Such an ideal exchanger would have a high affinity for cesium under high $\mathrm{pH}$ conditions and would be elutable. No such exchanger has been identified to date.

However, Crystalline Silicotitanate (CST) ion-exchanger materials have been studied for the removal of cesium from a variety of DOE wastes over the last decade. CST ion-exchanger materials demonstrate a high affinity for cesium under high alkalinity conditions and have been under investigation for cesium removal at specifically Hanford and SRS during the last six years. Since CST is an inorganic based material (with excellent properties in regard to chemical, radiological, and thermal stability and also little swelling/shrinking) that is considered to be practically non-elutable (while SuperLig ${ }^{\circledR} 644$ is an organic based elutable resin), the overall pretreatment facility flowsheet would be impacted in various ways. However, the CST material is still being considered as a potential backup ion-exchanger material. The performance of proposed backup ion-exchange columns using IONSIV IE-911 (CST in its engineered-form) 
material for the removal of cesium from Hanford high level radioactive alkaline waste is the main purpose of this analysis report. This report focuses attention only on the ion-exchange aspects and only addresses the loading phase of the process cycle.

From the viewpoint of being an ion-exchange material that is elutable or non-elutable, here we are referring to the level of cesium loading on the exchanger over a wide range of $\mathrm{pH}$. Due to this behavior with respect to $\mathrm{pH}$, the CST material is used only once and then becomes "spent CST" material that must be disposed of by vitrification in the IHLW glass melters. A simplified material flowsheet of the proposed CST based facility is shown in Figure 2-1. As shown in Figure 2-1, a spent CST material stream leaves this facility and ultimately contributes to the composite feed entering the IHLW glass melters.

One of the major questions being answered in this analysis report is the total amount of CST material being consumed (i.e., spent CST) in processing the entire Phase 1 LAW inventory. The current design estimates for the number of glass logs (cans) to be generated based on the use of SuperLig ${ }^{\circledR}$ technology is $\sim 866$ (see Table 4.1-1 of Kirkbride et al., 2000, the COUP document). This number of glass logs corresponds to 2651 MT (metric tons) of glass product. Due to concerns as to the allowable amount of waste sodium oxides that can be placed into the glass matrix, minimization of the spent CST material is of prime importance in the optimum column design. The feasibility of using CST material rests predominately upon the amount of spent CST generated.

The maximum loadings for waste sodium oxide $\left(\mathrm{Na}_{2} \mathrm{O}\right)$ currently planned for ILAW glass forms are envelope dependent. Therefore, the volumetric flowrate of the LAW stream passing through the ion-exchange facility during the loading phase is envelope dependent. The current processing plan for the Phase 1 LAW inventory is to batch process the entire inventory. The Phase 1 campaign constitutes the processing of ten waste tanks that are broken up into 16 batch feeds (i.e., 11 are Envelope A feeds, 2 Envelope B feeds, and 3 Envelope C feeds). The source tank and volume of solution to be processed on a batch feed basis (i.e., shown here on a $5 \mathrm{M}$ sodium basis) are listed in Table 2-1. The envelope dependent flowrates used in the VERSE-LC design calculations are also listed in Table 2-1 for the 16 batch feeds. The basis for the flowrates centers on the production goals of glass and the allowable limits of waste sodium oxide loading within the glass matrix.

Constraints on the allowable levels of ${ }^{137} \mathrm{Cs}$ contained within the aqueous effluent stream exiting the ion-exchange facility are also envelope dependent. Based on these ${ }^{137} \mathrm{Cs}$ concentration limits total cesium exit criteria can be computed based on the isotopic content of cesium within each feed (i.e., it is assumed that the isotopic fraction of ${ }^{137} \mathrm{Cs}$ to ${ }^{\text {total }} \mathrm{Cs}$ is $25 \mathrm{~mole} \%$ for Envelopes A and $\mathrm{C}$ and $30 \%$ for Envelope B). These total cesium exit criteria are also listed in Table 2-1.

From the viewpoint of determining an optimal ion-exchange facility based on CST packed columns, a minimization of spent CST strategy has been chosen. In this strategy all 16 batch feeds are being addressed individually. Specifically, we are addressing the following key varying attributes: (1) volume of solutions to be processed; (2) feed volumetric flowrates; (3) composition of key constituents; (4) cesium exit criteria; (5) the geometry of the columns; and (6) the number of columns used within a carousel configuration. The methodology used to 
perform the design (i.e., minimization) is discussed in detail in Chapter 10 where the supporting bases are provided throughout the remainder of the report.

\subsection{Test Specification Objectives}

The main objectives of this report were defined in the original test specification document by Johnson (2000) and restated in the Task Technical and QA Plan by Hang et al. (2001). In summary, these objectives are:

- To predict the quantity of IONSIV ${ }^{\circledR}$ IE-911 CST needed to treat the candidate Phase 1 LAW batch feed solutions (16 feeds in total);

- To predict the number and dimensions of CST ion-exchange columns (i.e., bed geometry) required to reduce the ${ }^{137} \mathrm{Cs}$ concentrations to $1.75 \times 10^{-5} \mathrm{Ci}{ }^{137} \mathrm{Cs}$ per gmole of $\mathrm{Na}$ after treatment of a selected candidate LAW solution; and

- To compare the dimensions of the CST material bed to the RPP-WTP reference resin bed design based on SuperLig ${ }^{\circledR} 644$ resin.

Based on updated information from Mike Johnson the exit cesium criterion was made envelope dependent:

- $\quad 1.75 \times 10^{-5} \mathrm{Ci}^{137} \mathrm{Cs}$ per gmole of $\mathrm{Na}$ for Envelope $\mathrm{A}$ feeds

- $5.00 \times 10^{-5} \mathrm{Ci}^{137} \mathrm{Cs}$ per gmole of $\mathrm{Na}$ for Envelope B feeds

- $2.90 \times 10^{-5} \mathrm{Ci}^{137} \mathrm{Cs}$ per gmole of $\mathrm{Na}$ for Envelope $\mathrm{C}$ feeds

These criteria are reflected in the exit criteria listed in Table 2-1. Within the Test Specification document no specific criterion as the degree of cesium loading within the lead columns was provided. Based on discussions with Mike Johnson, and after some up front scoping analyses, the traditional $+90 \%$ loading requirement (established using a mass transfer zone (MTZ) concept) was abandoned in favor of a more robust global optimization strategy alluded to in the previous section and discussed in Chapter 10 where the justification for its use versus the MTZ approach is provided.

\section{$2.2 \quad$ IONSIV $^{\circledR}$ IE-910/IE-911 CST Versus SuperLig ${ }^{\circledR} 644$}

In Chapter 10 a detailed look at the loading performance (both from an equilibrium and a kinetics viewpoint) is provided for IONSIV ${ }^{\circledR}$ IE-911 CST material versus SuperLig ${ }^{\circledR} 644$ resin. In brief summary, we see that the IONSIV ${ }^{\circledR}$ IE-911 CST material has an increased cesium capacity of $\sim 30 \%$ on a per mass basis and $\sim 640 \%$ on a per bed volume basis. The significant difference being the higher average bed density of IONSIV ${ }^{\circledR}$ IE-911 CST material (i.e., $\sim 1.0$ $\mathrm{g} / \mathrm{ml}$ ) when compared to SuperLig ${ }^{\circledR} 644$ resin (i.e., $\sim 0.224 \mathrm{~g} / \mathrm{ml}$ ). The overall kinetics for IONSIV $^{\circledR}$ IE-911 CST material appears to be slower than for SuperLig ${ }^{\circledR} 644$ resin, due primarily to a $\sim 3.5$ factor smaller cesium pore diffusivity coefficient. 
These comparisons were made focusing on the cesium loading phase where the feed solution is near $5 \mathrm{M}$ sodium. The organic based SuperLig ${ }^{\circledR} 644$ resin is considered an elutable resin, while the inorganic based IONSIV ${ }^{\circledR}$ IE-911 CST material is considered to be a non-elutable exchanger. The definition of elutable versus non-elutable is based on the exchanger's cesium loading performance under reduced $\mathrm{pH}$ conditions. In Figure 2-2 the cesium $\mathrm{K}_{\mathrm{d}}$ values for both exchanger materials are shown as a function of $\mathrm{pH}$ for a fixed cesium concentration of $5 \times 10^{-4} \mathrm{M}$. This data was taken by Bray et al. (1995) at $25 \mathrm{C}$, where an initial screening comparison was made of the loading performance of five different exchanger materials for Hanford applications.

As shown in Figure 2-2 and stated elsewhere, at high alkalinity conditions the two exchangers have similar loading performances. However, as the $\mathrm{pH}$ of the liquid-phase is reduced the two exchangers exhibit opposite behavior. The SuperLig ${ }^{\circledR} 644$ resin shows a systematic drop in cesium loading where it bottoms out for solutions whose $\mathrm{pH}$ are at or below $\mathrm{pH}$ of $\sim 7$. For the IONSIV $^{\circledR}$ IE-910 CST material, it rises to an approximate plateau for solutions whose $\mathrm{pH}$ are at or below $\mathrm{pH}$ of $\sim 7$. As such, the pretreatment flowsheet will be impacted due to this unique $\mathrm{pH}$ effect between these two exchangers. Other factors are briefly highlighted in Chapter 10.

The ZAM model for describing the loading performance of CST material addresses the $\mathrm{pH}$ effect as shown in Figure 2-2. Its Supersite model (i.e., three neighboring surface sites) conceptually handles this effect assuming steric limitations of cations within a supersite. For example, under high $\mathrm{pH}$ conditions the supersite is fully occupied by sodium cations. At reduced $\mathrm{pH}$ conditions one or more of the sites is occupied by hydrogen cations which have a smaller radius of hydration. At these reduced $\mathrm{pH}$ conditions the ability of cesium displacing one of the remaining sodium cations is greater due to the improved steric conditions. Thus, an increased $\mathrm{K}_{\mathrm{d}}$ is observed.

Based on analytical data of the eluate retrieved from elution cycles, the major competitors for exchange sites appear to be $\mathrm{Cs}^{+}, \mathrm{K}^{+}$, and $\mathrm{Na}^{+}$for both exchanger types. For the IONSIV ${ }^{\circledR}$ IE-911 CST material $\mathrm{Rb}^{+}$and $\mathrm{SrOH}^{+}$are also potential major competitors however, their impact on performance is assumed to be small due to the low concentrations present within the feeds (i.e., further investigation is required to determined how low the $\mathrm{SrOH}^{+}$really is). The relative affinities of these two exchanger types are quite similar for the three major competitors. Our current estimates for these relative affinities are (1) 1,200 to 1,400 for cesium versus potassium and (2) 24,000 to 26,000 for cesium versus sodium.

\subsection{Ion Exchange Modeling}

This ion-exchange system is one of many unit operations within a larger process flowsheet. Experimental efforts are currently underway to characterize the exchangers and the ion-exchange process in support of the overall design. Modeling the ion-exchange process in detail provides key supporting information needed in establishing the overall flowsheet. For example, cycle (time) average decontamination factors are required at the overall flowsheet level. Separate (offline) detailed transient column modeling provides these average decontamination factors where the detail of the analysis is not restricted due to constraints imposed by the flowsheet runtime and storage requirements. 
In addition, modeling:

- Reduces the overall number of experiments required;

- Provides guidance on experimental efforts and focuses attention on the critical parameters;

- Evaluates the adequacy and consistency of multiple data sets;

- Consolidates available information on a particular ion exchange system; and

- Establishes then confirms full-scale facility design and operational requirements.

\subsection{Report Overview}

This report focuses on the cesium-loading phase of a complete cycle. An analysis methodology is developed where as much of the available and pertinent data on the Cesium-IONSIV ${ }^{\circledR}$ IE-911 CST system is incorporated. Many of the model parameters are currently defined by direct experimentation. However, some of these parameters are based on limited data or significant uncertainties with regard to the data existed. In several cases assumptions had to be made prior to the assessment efforts (e.g., column test exit breakthrough curves). The methodology can easily be updated as new information becomes available (e.g., measured bed and pore porosities or mean particle radius).

This document represents a status report on our current knowledge and capability to model the ion-exchange process for the Cesium-IONSIV ${ }^{\circledR}$ IE-911 CST system under various Hanford feed conditions. The methodology, its justification, assessment, and application to the proposed facility is discussed in the following chapters. Supporting information has also been provided in several appendices and wherever possible references to available published data/information pertinent to the discussion has been cited.

Chapter 3 briefly discusses the transport model chosen for modeling column behavior. The governing equations and an appropriate simplification is presented. For the modeling efforts presented in this report the VERSE-LC code was chosen (Berninger et al., 1991) based on its availability and widespread (and accepted) use in this field. Essentially, a very similar methodology was used in earlier analyses for the Cesium-SuperLig ${ }^{\circledR} 644$ system as presented by Hamm et al. (2000a).

Local equilibrium between the pore fluid and its neighboring surface sites is assumed where equilibrium adsorption isotherm(s) must be specified. The algebraic isotherm model(s) used for each of the 16 batch feeds and the database employed in its creation are discussed in Chapter 4. The batch contact test databases in support of isotherm model development are numerically derived based on the ZAM code. The current version of ZAM is based on CST powder and a dilution factor must be applied to address CST in its engineered-forms. Due to batch variability observed in the manufacturing of CST material in its engineered-form(s), a statistically conservative cesium isotherm is used to accommodate the production range of expected CST material. This is accomplished by using a $68 \%$ dilution factor. The methods and results for creating charge-balanced compositions of the 16 batch feeds are presented in Appendix A. With these feed compositions cesium isotherm databases were generated and are presented in 
Appendix B. The justification for using a dilution factor of $68 \%$ is discussed in Appendix C. A description, along with limited verification and validation assessments, for the ZAM code is provided in Appendix F.

Key column properties (i.e., densities and porosities) are addressed in Chapter 5 where the constraint between the porosities is highlighted. Particle size distribution and the average particle radius used for the IONSIV ${ }^{\circledR}$ IE-911 CST (i.e., the Baseline CST in its engineered-form) is addressed in Chapter 6.

Pore diffusion and Brownian motion are discussed in Chapter 7 where an assessment to batch kinetics data is provided. The VERSE-LC code input and output files for the batch kinetics simulations are provided in Appendix E. In summary, we see inconsistencies among the available batch contact test data and deficiencies with using the homogeneous particle concept (and perhaps without addressing surface diffusion) currently within VERSE-LC. As such the particle tortuosity factor chosen (i.e., 5.0) for our design efforts is not based on these batch kinetics tests, but rather is based on the assessment of available column tests as discussed in Chapter 9. Future efforts to determine the important diffusional aspects of CST in its most common engineered-forms are recommended.

In Chapter 8 the constitutive models for axial dispersion and film diffusion are presented. Headspace and short column impacts are also briefly discussed. For each column an inlet headspace of $25 \%$ based on bed volume is assumed. From the loading perspective the size of this headspace has little impact on the predicted cesium breakthrough curves.

Chapter 9 contains the laboratory-scale (including pilot-scale) column assessments (15 in total). Appendix H contains the VERSE-LC code input files for each of the laboratory-scale and pilotscale simulations. To perform column transport analyses for sizing the CST columns, a method of estimating an "effective" cesium pore diffusion coefficient value is required (i.e., the tortuosity factor approach is chosen here). We had hoped that available batch kinetics test data would provide us the appropriate values. Unfortunately, the pore diffusion values based on existing kinetics data did not compare favorably when used in laboratory-scale column performance assessments. Given this situation, we are now estimating the pore diffusion coefficient values using a tortuosity factor based on the laboratory-scale column data directly. The tortuosity factor computed and the basis behind its creation are provided in Chapter 9. Also, a brief assessment to pilot-scale experiments is provided.

Based on the test specification objectives for the design of full-scale columns packed with CST material, Chapter 10 presents full-scale column performance predictions where the entire Phase 1 Law inventory is considered. The VERSE-LC code input and output files for the full-scale facility simulations are contained in Appendix D. The basis behind the need to use a global optimization strategy versus the more traditional mass transfer zone concept is provided. A comparison is made of facility performance between columns packed with SuperLig ${ }^{\circledR} 644$ resin versus IONSIV ${ }^{\circledR}$ IE-911 CST material. Both 2-column and 3-column carousel configurations are considered. In order to bound the IONSIV ${ }^{\circledR}$ IE-911 CST material's radioactive exposure levels associated with radioactive decay of cesium-137, cesium inventories within the column (i.e., cesium content in the liquid-phase plus adsorbed onto the solid-phase) are estimated during 
the loading cycle corresponding to the worst case conditions. This inventory estimate can be used in subsequent analyses (beyond the scope of this report) for estimating conservative exposure levels. The impact of geometry (i.e. L/D, length to diameter ratio) on column performance is also addressed in Chapter 10.

A reasonable complete listing of pertinent literature references is provided in Chapter 11.

Table 2-1. Key batch processing information on the Phase 1 Low activity waste (LAW) feeds listed in their scheduled order to be processed.

\begin{tabular}{|c|c|c|c|c|c|c|}
\hline Envelope & $\begin{array}{c}\text { Source } \\
\text { Tank }\end{array}$ & $\begin{array}{c}\text { LAW } \\
\text { batch feed } \\
\text { (id) }\end{array}$ & $\begin{array}{c}\text { Flowrate } \\
\text { (L/min) }\end{array}$ & $\begin{array}{c}\text { Cesium } \\
\text { feed } \\
\text { conc. } \\
{[\mathrm{M}]}\end{array}$ & $\begin{array}{c}\text { Batch } \\
\text { volume to } \\
\text { be } \\
\text { processed a,c } \\
\left(\mathbf{m}^{3}\right)\end{array}$ & $\begin{array}{c}\text { Total Cs } \\
\text { exit } \\
\text { criterion }^{b} \\
{[\mathrm{M}]}\end{array}$ \\
\hline $\mathbf{A}$ & AP-101 & LAW-1 & 52.62 & $3.598 \mathrm{E}-05$ & 4,626 & 2.953E-08 \\
\hline B & AZ-101 & LAW-2a & 9.4 & $4.676 \mathrm{E}-04$ & 2,906 & $7.032 \mathrm{E}-08$ \\
\hline B & AZ-102 & LAW-2b & 9.4 & $4.311 \mathrm{E}-04$ & 1,755 & $7.032 \mathrm{E}-08$ \\
\hline $\mathbf{C}$ & AN-102 & LAW-3 & 16.2 & $3.967 \mathrm{E}-05$ & 4,200 & $4.894 \mathrm{E}-08$ \\
\hline $\mathbf{C}$ & AN-102 & LAW-4 & 16.2 & $3.779 \mathrm{E}-05$ & 4,200 & 4.894E-08 \\
\hline $\mathbf{A}$ & AN-104 & LAW-5 & 52.62 & $6.283 \mathrm{E}-05$ & 3,820 & $2.953 \mathrm{E}-08$ \\
\hline $\mathbf{A}$ & AN-104 & LAW-6 & 52.62 & $6.328 \mathrm{E}-05$ & 3,540 & 2.953E-08 \\
\hline $\mathrm{C}$ & AN-107 & LAW-7 & 16.2 & $4.455 \mathrm{E}-05$ & 5,498 & 4.894E-08 \\
\hline $\mathbf{A}$ & AN-105 & LAW-8 & 52.62 & $4.324 \mathrm{E}-05$ & 3,700 & $2.953 \mathrm{E}-08$ \\
\hline $\mathbf{A}$ & AN-105 & LAW-9 & 52.62 & 4.444E-05 & 3,600 & $2.953 \mathrm{E}-08$ \\
\hline $\mathbf{A}$ & SY-101 & LAW-10 & 52.62 & 3.692E-05 & 2,600 & $2.953 \mathrm{E}-08$ \\
\hline $\mathbf{A}$ & SY-101 & LAW-11 & 52.62 & $3.739 \mathrm{E}-05$ & 4,600 & $2.953 \mathrm{E}-08$ \\
\hline $\mathbf{A}$ & AN-103 & LAW-12 & 52.62 & $4.831 \mathrm{E}-05$ & 4,720 & $2.953 \mathrm{E}-08$ \\
\hline $\mathbf{A}$ & AN-103 & LAW-13 & 52.62 & $4.831 \mathrm{E}-05$ & 4,720 & $2.953 \mathrm{E}-08$ \\
\hline $\mathbf{A}$ & AW-101 & LAW-14 & 52.62 & $4.569 \mathrm{E}-05$ & 3,940 & $2.953 \mathrm{E}-08$ \\
\hline $\mathbf{A}$ & AW-101 & LAW-15 & 52.62 & $4.552 \mathrm{E}-05$ & 5,360 & $2.953 \mathrm{E}-08$ \\
\hline
\end{tabular}

${ }^{\text {a }}$ The volume of each batch feed represents the volume of solution entering the ion-exchange facility at a $5 \mathrm{M}$ sodium basis and includes the volume changes that occur upstream to this facility (i.e., pretreatment activities).

b The volumetric flowrates and batch process times are based on the $30 \mathrm{MT} /$ day operation schedule for Envelopes $\mathrm{B}$ and $\mathrm{C}$ and on the expanded capability of $60 \mathrm{MT} /$ day operation for Envelope A.

c The total amount of processing time is $\sim 4.2$ years. If the Envelope A feeds are processed at $30 \mathrm{MT} /$ day, then the total amount of processing time is $\sim 5.8$ years. 


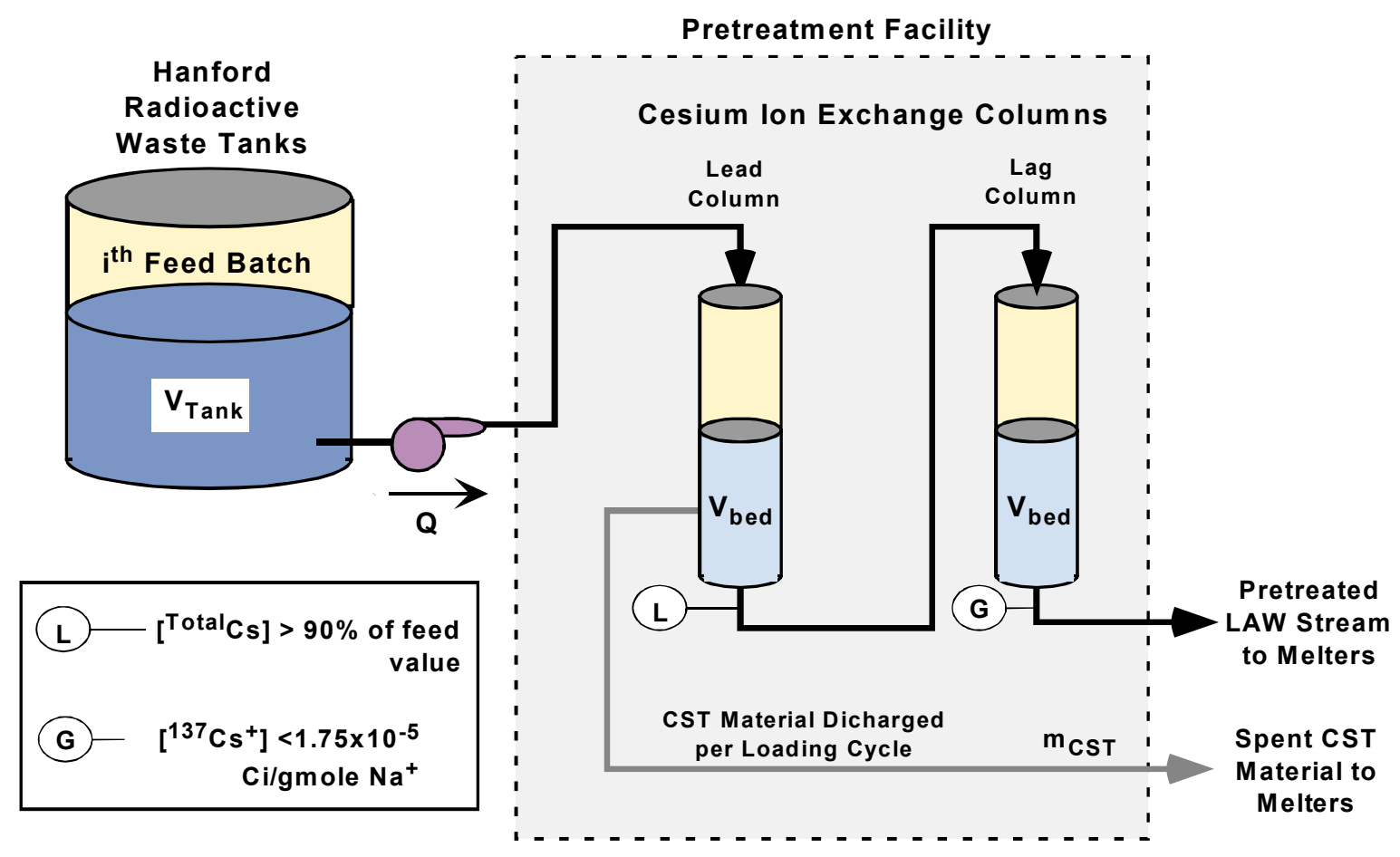

Figure 2-1. Simplified material flowsheet overview highlighting the ion-exchange units used for removal of cesium from a candidate LAW stream.

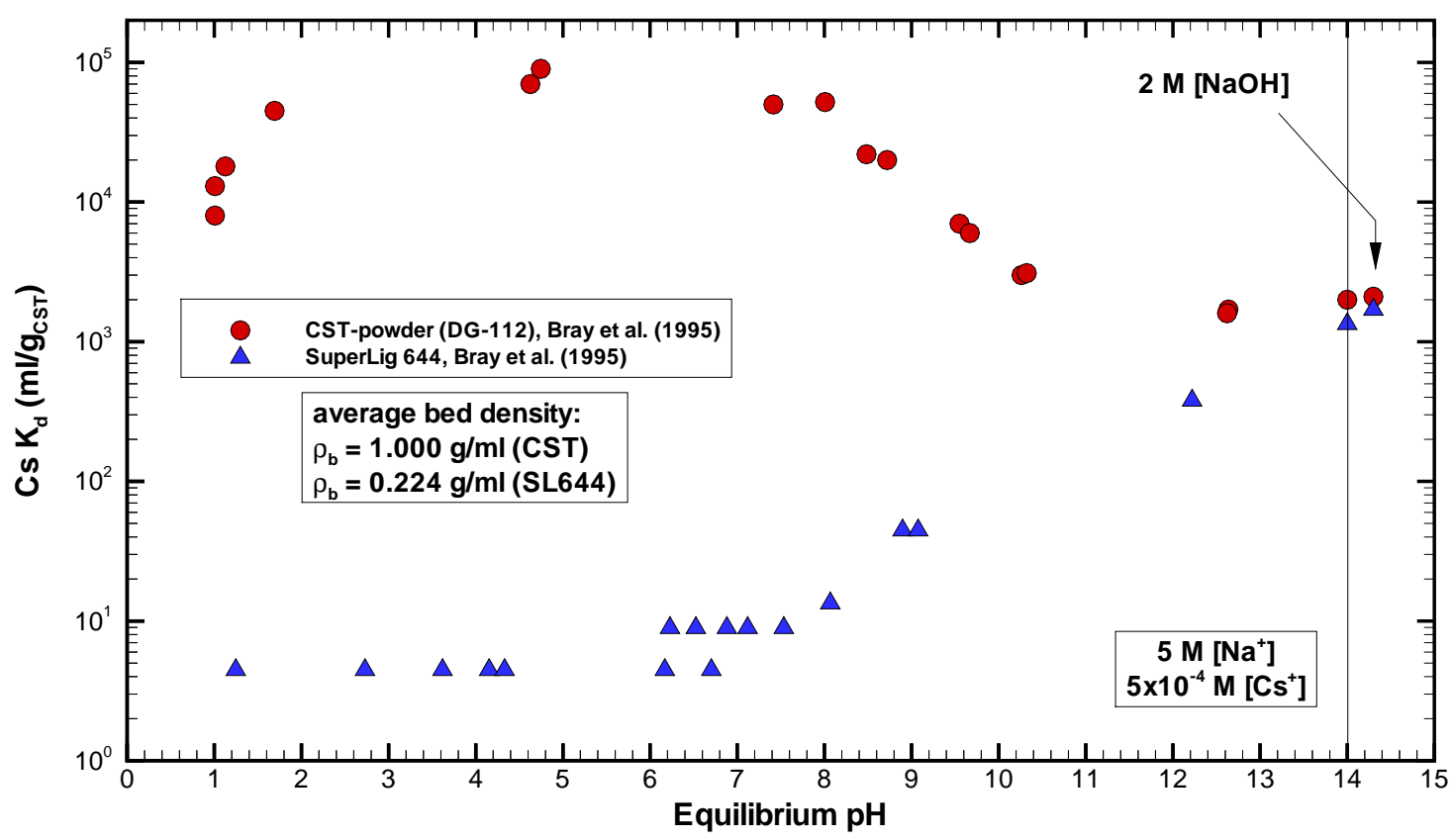

Figure 2-2. Cesium loading performance comparison between IONSIV ${ }^{\circledR}$ IE-911 CST and SuperLig ${ }^{\circledR} 644$ exchanger materials at $25 \mathrm{C}$ by Bray et al. (1995). 


\subsection{Column Model Formulations}

The modeling of ion exchange columns is typically broken up into two basic categories:

- An equilibrium model generally highly empirical in nature, and

- A column model based on one-dimensional solute transport.

In this section the equations for the multi-component and the simpler "effective" singlecomponent ion exchange column models are addressed. Justification for use of the simpler "effective" single-component column model is provided where its use is limited to the loading cycle of the process only. Chapter 4 of this report addresses both equilibrium models considered (i.e., the ternary isotherm model and the "effective" single-component isotherm model).

To take into account the various mechanisms for ion transport and adsorption as it travels down an ion exchange column, a porous particle solute transport formulation has experienced widespread use and acceptability. For this class of column models five basic aspects of the ion exchange column are addressed as highlighted in Figure 3-1. In order of their importance with respect to predicting exit breakthrough curves for the Cesium-IONSIV ${ }^{\circledR}$ IE-911 CST system, they are:

- Bed Definition (high impact) - column size, geometry and resin mass have a very direct impact on overall column performance, with particle geometry having a slightly less important impact (Shifts entire breakthrough curve with respect to number of column volumes required to reach a specified concentration level);

- Adsorption Isotherms (high impact) - resin affinities for the various competing ions of interest have a very direct impact on overall column performance (Shifts entire breakthrough curve with respect to number of column volumes required to reach a specified concentration level and for non-linear isotherms alters breakthrough curve shape as well as its sensitivity with respect to inlet feed conditions);

- Pore Diffusion (high impact) - intra-particle mass transport by pore diffusion to available surface sites has a moderate impact on overall column performance, with particle geometry having a slightly less important impact (under non-limiting mass transfer conditions it alters the shape of exit breakthrough curves typically by a rotation about the $\sim 50 \%$ relative concentration level with slight shifting; under limiting mass transfer conditions the rotation is generally at a point higher than 50\%);

- Film Diffusion (low impact) - liquid mass transport by film diffusion across the particle-tobed boundary has a low impact on overall column performance (Alters the shape of exit breakthrough curves typically by a rotation about the $\sim 50 \%$ relative concentration level with slight shifting);

- Axial Dispersion (low impact) - mass transport along the column by axial dispersion has a low impact on overall column performance (Alters the shape of exit breakthrough curves typically by a rotation about the $\sim 50 \%$ relative concentration level with slight shifting); 
The above stated levels of impact are based on sensitivity studies and are relative values. Mechanisms such as surface migration or adsorption kinetics are not included in our column model since their impacts were considered to be negligible or already indirectly incorporated into the other features during our parameter estimation process. Pore heterogeneity within the CST particles is not explicitly handled as well. A simple graphical representation of the various transport mechanisms listed above and considered important for the Cesium-IONSIV ${ }^{\circledR}$ IE-911 CST system is shown in Figure 3-2.

\subsection{The Multi-Component Model}

For the Cesium-IONSIV ${ }^{\circledR}$ IE-911 CST system a porous particle multi-component ion exchange column model was considered. In this model we assume that the kinetics associated with local ion exchange at an active resin site are very fast (faster than the various liquid mass transfer mechanisms that transport ions to that site). Assuming radial effects to be negligible within the active region of the packed bed (i.e., a large column-to-particle diameter ratio), a onedimensional species (ion) transport equation for the mobile phase (within the bed) becomes

$$
\varepsilon_{\mathrm{b}} \frac{\partial \mathrm{c}_{\mathrm{bi}}}{\partial \mathrm{t}}+\varepsilon_{\mathrm{b}} \mathrm{u} \frac{\partial \mathrm{c}_{\mathrm{bi}}}{\partial \mathrm{z}}=\varepsilon_{\mathrm{b}} \mathrm{E}_{\mathrm{bi}} \frac{\partial^{2} \mathrm{c}_{\mathrm{bi}}}{\partial \mathrm{z}^{2}}-\left(\frac{3}{<\mathrm{R}_{\mathrm{p}}>}\right)\left(1-\varepsilon_{\mathrm{b}}\right) \mathrm{k}_{\mathrm{f}}\left(\mathrm{c}_{\mathrm{bi}}-\left.\mathrm{c}_{\mathrm{pi}}\right|_{\mathrm{r}=\mathrm{R}_{\mathrm{p}}}\right)
$$

storage advection

\section{axial dispersion}

liquid film diffusion

(mass transfer)

with boundary and initial conditions

$$
\begin{array}{ll}
\mathrm{z}=0, & \varepsilon_{\mathrm{b}} \mathrm{E}_{\mathrm{b}} \frac{\partial \mathrm{c}_{\mathrm{bi}}}{\partial \mathrm{z}}=\mathrm{uL}\left[\mathrm{c}_{\mathrm{bi}}-\mathrm{c}_{\mathrm{bi}}^{\text {feed }}(\mathrm{t})\right], \\
\mathrm{z}=1, & \varepsilon_{\mathrm{b}} \mathrm{E}_{\mathrm{b}} \frac{\partial \mathrm{c}_{\mathrm{bi}}}{\partial \mathrm{z}}=0, \\
\mathrm{t}=0, & \mathrm{c}_{\mathrm{bi}}=\mathrm{c}_{\mathrm{bi}}(0, \mathrm{z}) .
\end{array}
$$

Assuming uniformly sized spherical particles with a homogeneous distribution of pores, a onedimensional species transport equation for the pore phase (within an average sized particle of resin) becomes

$$
\begin{aligned}
& \varepsilon_{\mathrm{p}} \frac{\partial \mathrm{c}_{\mathrm{pi}}}{\partial \mathrm{t}}+\left(1-\varepsilon_{\mathrm{p}}\right) \overline{\mathrm{C}}_{\mathrm{T}} \sum_{\mathrm{j}=1}^{\mathrm{N}_{\mathrm{s}}}\left[\left(\frac{\partial \mathrm{q}_{\mathrm{i}}}{\partial \mathrm{c}_{\mathrm{pj}}}\right) \frac{\partial \mathrm{c}_{\mathrm{pj}}}{\partial \mathrm{t}}\right]=\varepsilon_{\mathrm{p}} \mathrm{D}_{\mathrm{pi}} \frac{1}{\mathrm{r}^{2}} \frac{\partial}{\partial \mathrm{r}}\left[\mathrm{r}^{2} \frac{\partial \mathrm{c}_{\mathrm{pi}}}{\partial \mathrm{r}}\right] \text {, } \\
& \text { storage surface } \\
& \text { adsorption diffusion }
\end{aligned}
$$

with boundary and initial conditions

$$
\mathrm{r}=0, \quad \varepsilon_{\mathrm{p}} \mathrm{D}_{\mathrm{p}} \frac{\partial \mathrm{c}_{\mathrm{bi}}}{\partial \mathrm{r}}=0
$$




$$
\begin{array}{ll}
\mathrm{r}=<\mathrm{R}_{\mathrm{p}}>, & \varepsilon_{\mathrm{p}} \mathrm{D}_{\mathrm{p}} \frac{\partial \mathrm{c}_{\mathrm{bi}}}{\partial \mathrm{r}}=\mathrm{k}_{\mathrm{f}}\left(\mathrm{c}_{\mathrm{bi}}-\mathrm{c}_{\mathrm{pi}}\right), \\
\mathrm{t}=0, & \mathrm{c}_{\mathrm{pi}}=\mathrm{c}_{\mathrm{pi}}(0, \mathrm{r}) .
\end{array}
$$

In Eq. (3-2) it is assumed that the pore diameters are large relative to the size of migrating ions of interest. Therefore, Fickian diffusion is acceptable and surface migration is considered to be small (or incorporated into the apparent pore diffusivity coefficient) when compared to pore diffusion. Unfortunately, for high affinity exchangers such as CST, which exhibit nonlinear isotherms, an apparent pore diffusivity coefficient is composition dependent as discussed by $\mathrm{Ma}$ et al. (1996). As discussed in Chapter 7, in this report we assumed that a cesium composition independent pore diffusivity value can be adequately chosen. Future efforts addressing surface migration and pore heterogeneity should be considered.

Assuming local equilibrium between the pore fluid and its neighboring surface sites, an equilibrium isotherm model for the ion exchange between the pore and solid phases can be generically expressed as:

$$
\mathrm{q}_{\mathrm{i}}=\mathrm{F}_{\mathrm{i}}\left(\overline{\mathrm{C}}_{\mathrm{T}}, \overline{\overline{\mathrm{K}}}_{\mathrm{ij}}, \mathrm{c}_{\mathrm{p} 1}, \mathrm{c}_{\mathrm{p} 2}, \cdots, \mathrm{c}_{\mathrm{pN}_{\mathrm{s}}}\right), \quad \mathrm{i}=1, \mathrm{~N}_{\mathrm{s}},
$$

where it has been assumed that surface loadings for the $\mathrm{i}^{\text {th }}$ species can be explicitly related to the liquid concentrations locally. The $\mathrm{K}_{\mathrm{ij}}$ values are selectivity coefficients that specify the relative affinity between species I versus species $j$. The number of species required to model the behavior of the $\mathrm{i}^{\text {th }}$ species depends upon its dependence on other species through the functional form (i.e., $\mathrm{F}_{\mathrm{i}}$ ) of the isotherm model [Eq. (3-3)]. Specific application of Eq. (3-3) to the cesiumIONSIV $^{\circledR}$ IE-911 CST system is discussed in Chapter 4 and Appendices B and C. Initial and boundary conditions for Eqs. (3-1) and (3-2) must also be specified. For further details on these equations and their solution in VERSE-LC see Berninger et al. (1991). Helfferich and Carr (1993) provide an excellent review paper describing the behavior of non-linear waves in chromatography and also a brief listing of available algorithms (see their Table I.4). Their paper provides very clear insight into how the above equation set behaves for non-linear isotherms consistent with the system of interest discussed in this report.

For the modeling efforts presented in this report the VERSE-LC code was chosen (Berninger et al., 1991) based on its availability and widespread (and accepted) use in this field. Prior to applying VERSE-LC to the ion exchange modeling presented in this report a verification process was completed and the results of that effort are reported in Hamm et al. (1999). The verification process provided us quality assurance that the installed PC Window $95^{\mathrm{TM}}$ version of VERSE-LC (i.e., version 7.80) was capable of adequately solving the above mentioned equations and also helped us to better understand how to accurately use the VERSE-LC code (e.g., mesh refinement requirements and input/output options). For all column results presented in this report numerical errors associated with the results of VERSE-LC should be very small when compared to the uncertainties associated with various model input parameters (bed density, particle radius, pore diffusion, etc.). 


\subsection{The Single-Component Model}

Under certain situations the porous particle multi-component transport equations discussed in Section 3.1 can be adequately decoupled to a series of single-component transport equations. The reduction to single-component equations is:

- valid when the total ionic strength, $\mathrm{C}_{\mathrm{T}}$, is the same between the column's native and feed solutions; or

- a reasonable approximation when one ion absorbs significantly more onto the resin than others.

Making the same basic assumptions as in Section 3.1 the single-component equations can be derived. For each species a one-dimensional species (ion) transport equation for the mobile phase (within the bed) becomes

$\varepsilon_{\mathrm{b}} \frac{\partial \mathrm{c}_{\mathrm{b}}}{\partial \mathrm{t}}+\varepsilon_{\mathrm{b}} \mathrm{u} \frac{\partial \mathrm{c}_{\mathrm{b}}}{\partial \mathrm{z}}=\varepsilon_{\mathrm{b}} \mathrm{E}_{\mathrm{b}} \frac{\partial^{2} \mathrm{c}_{\mathrm{b}}}{\partial \mathrm{z}^{2}}-\left(\frac{3}{<\mathrm{R}_{\mathrm{p}}>}\right)\left(1-\varepsilon_{\mathrm{b}}\right) \mathrm{k}_{\mathrm{f}}\left(\mathrm{c}_{\mathrm{b}}-\left.\mathrm{c}_{\mathrm{p}}\right|_{\mathrm{r}=\mathrm{R}_{\mathrm{p}}}\right)$,

storage advection

axial

dispersion

liquid film diffusion

(mass transfer)

where initial and boundary conditions are consistent with Eqs. (3-1b,c,d). A one-dimensional species transport equation for the pore phase (within a particle of resin) becomes

$$
\varepsilon_{\mathrm{p}} \frac{\partial \mathrm{c}_{\mathrm{p}}}{\partial \mathrm{t}}+\left(1-\varepsilon_{\mathrm{p}}\right) \overline{\mathrm{C}}_{\mathrm{T}}\left(\frac{\partial \mathrm{q}}{\partial \mathrm{c}_{\mathrm{p}}}\right) \frac{\partial \mathrm{c}_{\mathrm{p}}}{\partial \mathrm{t}}=\varepsilon_{\mathrm{p}} \mathrm{D}_{\mathrm{p}} \frac{1}{\mathrm{r}^{2}} \frac{\partial}{\partial \mathrm{r}}\left[\mathrm{r}^{2} \frac{\partial \mathrm{c}_{\mathrm{p}}}{\partial \mathrm{r}}\right]
$$

storage

surface

adsorption
Fickian pore diffusion

where initial and boundary conditions are consistent with Eqs. (3-2b,c,d).

The equilibrium isotherm model for species i for the ion exchange between the pore and solid phases becomes:

$$
q=\frac{c_{p}}{\beta+c_{p}},
$$

\section{single-component isotherm}

where Eq. (3-6) is of the Langmuir form and $\beta$ (the beta parameter) is a function of the feed conditions.

\subsection{The Cesium-IONSIV ${ }^{\circledR}$ IE-911 CST System}

Based on our current understanding, for the Cesium-IONSIV ${ }^{\circledR}$ IE-911 CST system the competition for cation exchange loading (under high alkaline conditions) at the CST sites is 
primarily between cesium $\left(\mathrm{Cs}^{+}\right)$, strontium hydroxide $\left(\mathrm{SrOH}^{+}\right)$, rubidium $\left(\mathrm{Rb}^{+}\right)$, potassium $\left(\mathrm{K}^{+}\right)$, and sodium $\left(\mathrm{Na}^{+}\right)$. For the analyses presented in this report it is assumed that rubidium and strontium hydroxide presence within the feeds is negligible for our modeling purposes. For rubidium this is a reasonable assumption due to its trace amounts within the Phase 1 LAW feeds; however, for strontium hydroxide its concentration depends upon the concentrations of $\mathrm{Sr}^{+2}$ and potential complexants within the feeds. Further studies must be performed to determine actual levels of strontium hydroxide present. Given these assumptions a ternary component feed (i.e., component 1 being $\mathrm{Cs}^{+}$, component 2 being $\mathrm{K}^{+}$, and component 3 being $\mathrm{Na}^{+}$) must be addressed.

Prior to the loading phase the initial sodium and potassium levels in the pretreatment solution are approximately $0.25 \mathrm{M}$ and $0 \mathrm{M}$, respectively. During the loading phase these concentration levels increase to approximately $\sim 5.0 \mathrm{M}$ sodium and 0.0 -to- $1.0 \mathrm{M}$ potassium. Therefore, initially a total ionic concentration wave will pass through the column. Based on available batch equilibrium studies estimates for the relative affinities for ion-exchange with the CST material have been computed as discussed in Chapter 4 and Appendix B (i.e., the CST exchanger affinities are $\mathrm{Cs}^{+}>\mathrm{K}^{+}>\mathrm{Na}^{+}$). For the Envelope A Phase 1 LAW batch feeds, selectivity coefficients have been estimated to be $\sim 1,400$ for cesium versus potassium $\left(\mathrm{K}_{12}\right)$ and $\sim 26,000$ for cesium versus sodium $\left(\mathrm{K}_{13}\right)$. Based on this selectivity coefficient values the three ternary isotherms and the one "effective" single-component cesium isotherm at $25 \mathrm{C}$ were generated (see Chapter 4 for more details).

Given the above information, early column performance (say the first 5 to 10 column volumes or so) probably will require the use of the multi-component (i.e., ternary modeling) formulations of Section 3.1. Long-term performance should be adequately handled using the simpler singlecomponent formulations of Section 3.2. To check the validity of these statements, cesium exit breakthrough curves from several column simulations were compared where both the multicomponent and single-component formulations were used. Very similar results were obtained. To illustrate the differences in timing for the three ionic species, the exit breakthrough curves for each species is plotted in Figure 3-3 for a multi-component (i.e., ternary model) simulation of one of the experimental column tests (test SRS-Avg-Test2 performed by Wilmarth et al., 1999). The SRS-Avg-Test 2 test was performed using a packed column containing an engineered-form of CST operating at $25 \mathrm{C}$ with a feed composition of $1.24 \times 10^{-4} \mathrm{M} \mathrm{Cs}^{+}, 0.015 \mathrm{M} \mathrm{K}^{+}$, and $5.6 \mathrm{M}$ $\mathrm{Na}^{+}$. Under this feed composition the selectivity coefficients were estimated to be approximately $\mathrm{K}_{12}=1,400$ and $\mathrm{K}_{13}=24,270$ and a beta parameter of $\beta=2.4145 \times 10^{-4} \mathrm{M}$. Using these isotherms both VERSE-LC ternary and single-component transport simulations were performed. The VERSE-LC input files for both modeling approaches are provided in Appendix H.

As expected sodium breakthrough is the fastest, quickly followed by potassium, and then an order of magnitude later by cesium. Similar behavior is observed for the Cesium-SuperLig ${ }^{\circledR} 644$ resin system (an ion-exchange process, see Hamm et al., 2000a). When the single-component formulations are used, the cesium breakthrough curve is only very slightly altered. Compare the ternary system cesium breakthrough curve prediction in Figure 3-4 (open circles) to the singlecomponent result also provided in Figure 3-4 (solid curve). The VERSE-LC parameter settings chosen are all set to their nominal values (e.g., pore diffusion coefficients are $20 \%$ of their free 
molecular values and a dilution factor of $68 \%$ ). The two modeling approaches result in cesium breakthrough curves that are essentially identical.

Since significant CPU savings are achieved when the single-component model is used and the differences are well within our current predictive capabilities, the majority of column analyses presented in this report were performed using the single-component model. This simplification only applies to the loading cycle and for future elution studies a multi-component version will be required due to the short process timing and strong concentration gradients that will be present throughout the columns.

\section{Axial Dispersion \\ residence time tests \\ (end-effects \& short column impacts)}

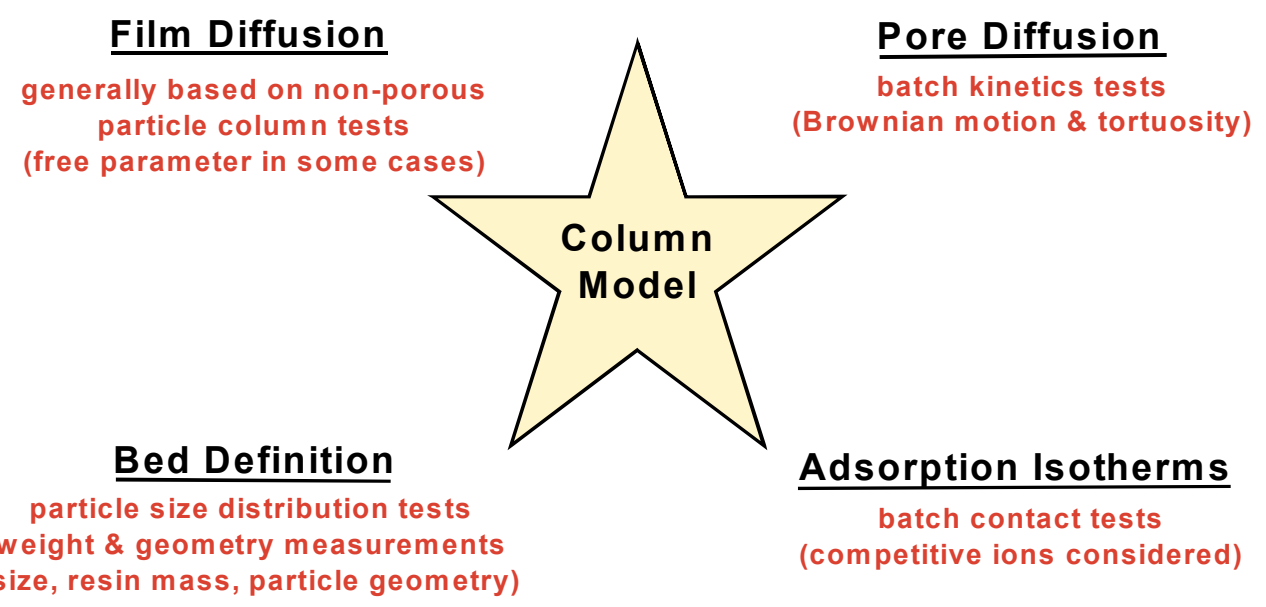

Figure 3-1. The basic building blocks of a porous particle ion exchange column model. 


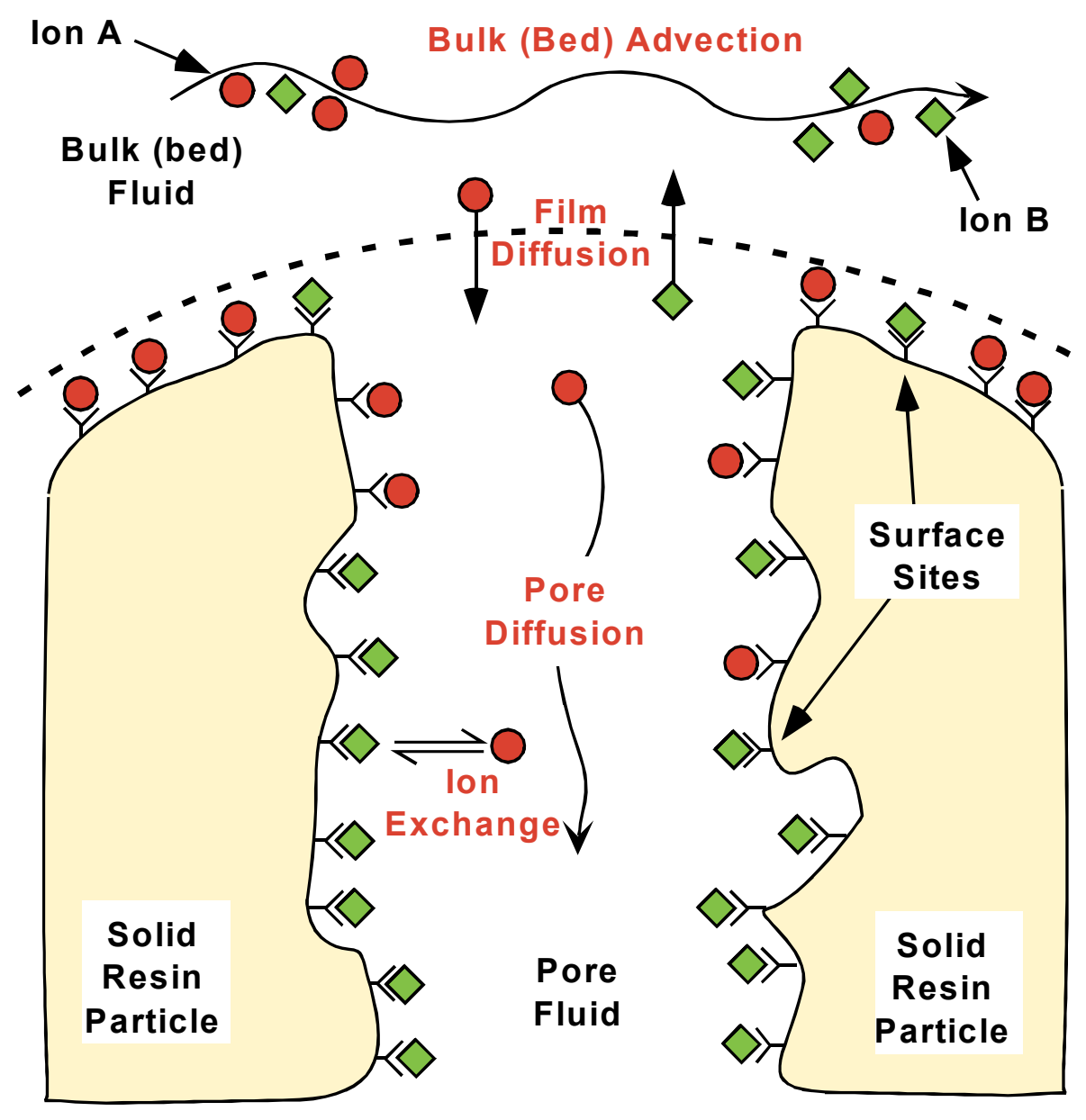

Figure 3-2. Graphical representation of the various mass transport mechanisms considered important for Cesium-IONSIV ${ }^{\circledR}$ IE-911 CST system ion exchange column modeling. 


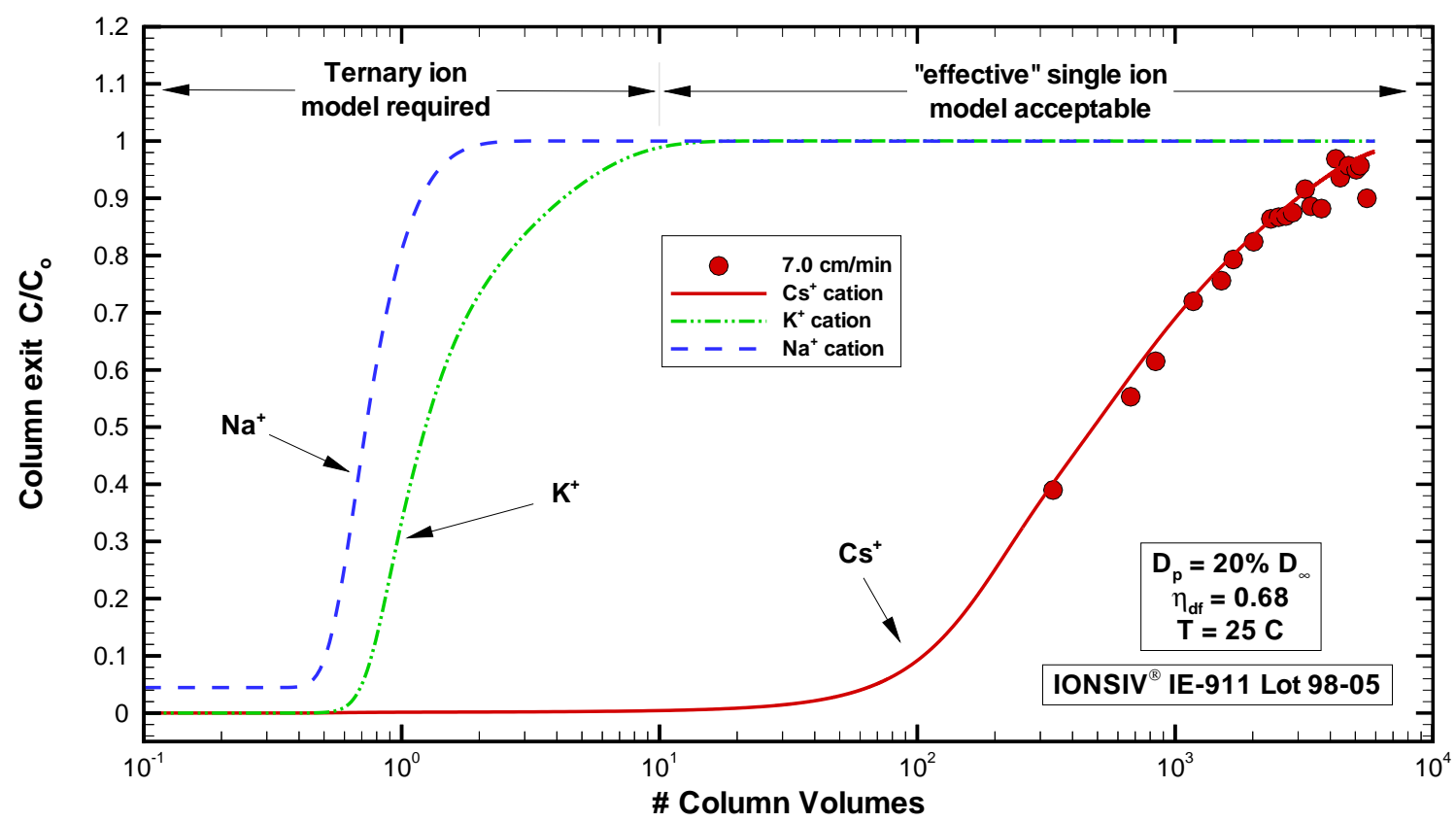

Figure 3-3. Estimated $\mathrm{Cs}^{+}, \mathrm{K}^{+}$, and $\mathrm{Na}^{+}$exit breakthrough curves for the test SRS-Avg-Test2 performed by Wilmarth et al. (1999) based on a porous particle multi-component (i.e., ternary) ion exchange column model for SRS Average simulant using IONSIV ${ }^{\circledR}$ IE-911 CST.

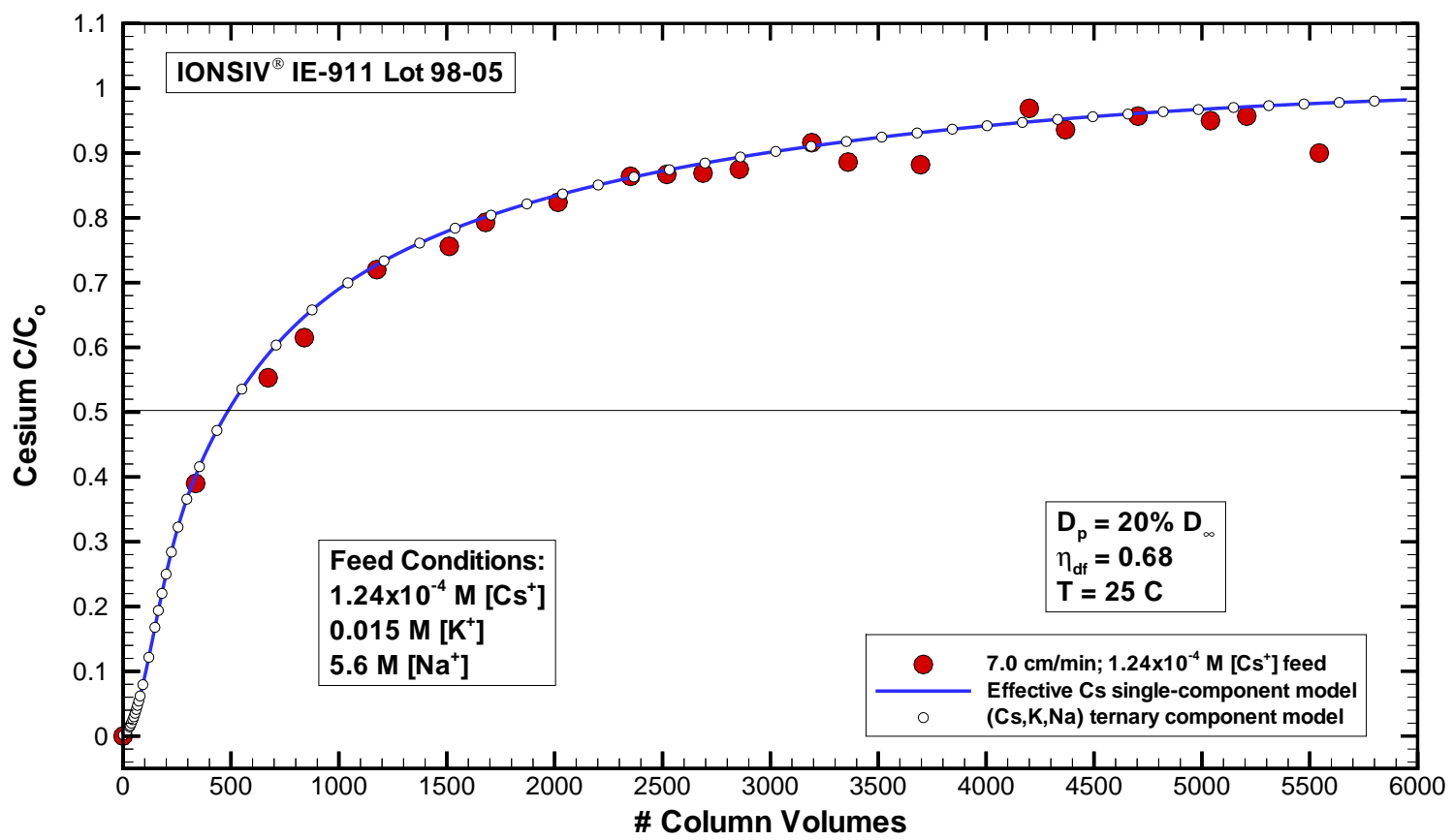

Figure 3-4. Measured versus predicted $\mathrm{Cs}^{+}$column exit breakthrough curves based on the ternary and

"effective" single-component ion exchange column models (test SRS-Avg-Test2 performed by Wilmarth et al. (1999) in a SRS Average simulant liquid at $1.24 \times 10^{-4} \mathrm{M} \mathrm{Cs}$ and at $25 \mathrm{C}$ ). 


\subsection{Equilibrium Cesium Isotherms}

In our column modeling efforts we assume that the rate of ion exchange (i.e., exchange of ions at a surface site) is very fast when compared to the rates of diffusion within the pore fluid and mass transfer across the liquid film at the outer boundaries of the particles. In other words, we assume that local equilibrium exists between the pore fluid and its neighboring surface sites. With this assumption an algebraic expression relating ionic (or species) concentrations between the pore fluid and the solid CST material (i.e., surface sites) can be established. No explicit attempt is made in this report to verify this assumption. In an indirect manner this assumption is either verified or incorporated into some of the model parameters. In addition, we assume that the cesium total ionic capacity (i.e., active sites for cesium per gram of CST material) is independent of total ionic strength or solution composition.

The total cation exchange capacity of the CST material in its powder-form (batch IE-910) is species dependent. Two types of exchange sites exist on the CST solid. The total ion exchange capacity is stated to be $\sim 4.6 \mathrm{mmole} / \mathrm{g}_{\mathrm{CST}}$, but the cesium exchange capacity is much less indicating that not all sites are available for cesium exchange (see, Zheng et al., 1996). In the ideal solid region (i.e., prior to the first step of the isotherm), the apparent total capacities are $\sim 0.58 \mathrm{mmole} / \mathrm{g}_{\text {CST }}$ for $\mathrm{Cs}^{+}, \sim 1.2 \mathrm{mmole} / \mathrm{g}_{\text {CST }}$ for $\mathrm{K}^{+}, \sim 1.18 \mathrm{mmole} / \mathrm{g}_{\text {CST }}$ for $\mathrm{Rb}^{+}$, and $\sim 1.0$ mmole $/ \mathrm{g}_{\mathrm{CST}}$ for $\mathrm{SrOH}^{+}$. For the expected feed concentrations it is anticipated that the entire columns will be operating within this ideal solid region. For additional information on this see Appendix C or Zheng et al., (1996).

In column sizing one of two possible design strategies are typically considered: (1) bounding analysis where "worst case" feed compositions are used or (2) global optimization where best estimate feed compositions for each individual batch are used. Each approach has its own advantages and disadvantages. For example, the bounding approach requires less analysis overall but it may be difficult to establish a reasonable bound that is not too excessive. Since the amount of waste to be processed, flowrate, and key feed compositions depend significantly on which envelope is being considered, the global optimization strategy is taken. To use this global optimization strategy "best estimate" cesium isotherms for each of the 16 batch feeds must be determined. In deriving an appropriate generic isotherm model for use with each batch feed the following items were considered:

- To keep reasonable runtimes an algebraic form of an isotherm model was desired that would be compatible/consistent with standard column models;

- The form of the isotherm model is to be thermodynamically consistent with the local massaction equations where appropriate;

- The effects of competing anions within possible feed solutions needed to be easily addressed (i.e., able to handle the expected range of Hanford waste feeds without major alterations required);

- Batch equilibrium " $\mathrm{K}_{\mathrm{d}}$ " databases needed to be generated for each individual batch feed; and 
- Batch variability must be handled by establishing a dilution factor that conservatively incorporates the various potential engineered forms of CST; and

Based on these considerations a binary homovalent isotherm model was developed and is presented in this section. Overall the model meets all of the above items; however, the confidence intervals for the model are higher than hoped. In Appendix C, further discussion of the isotherm model is given, where the effects associated with uncertainties and confidence levels are investigated. These isotherms are for the loading phase applications only.

The isotherm model development discussed below follows very closely to the development efforts presented by Hamm et al. (2000) for the Cesium-SuperLig ${ }^{\circledR} 644$ ion-exchange system. For the Cesium-SuperLig ${ }^{\circledR} 644$ ion-exchange system, batch equilibrium " $\mathrm{K}_{\mathrm{d}}$ " data was used in generating the isotherm models that were then used in VERSE-LC column transport simulations. For the Cesium-IONSIV ${ }^{\circledR}$ IIE-911 CST ion-exchange system, batch equilibrium " $K_{d}$ " data for each batch feed is not available and the ZAM computer code is used to numerically generate the necessary " $\mathrm{K}_{\mathrm{d}}$ " databases. A description of the ZAM code is given in Appendix F where some limited validation results are shown for use with Hanford LAW solutions. The details of generating these cesium isotherms can be found in Appendix B.

In the next subsection the "effective" cesium isotherm model is discussed based on an ionexchange process and in the following subsection its specific application to the CesiumIONSIV $^{\circledR}$ IIE-911 CST ion-exchange system is discussed.

\subsection{The Isotherm Model}

As demonstrated by Hamm et al. (2000a, Figure 3-3), for ion exchange competitors with affinities significantly less than the value for cesium, a single-component transport modeling approach is adequate for the cesium loading phase. To perform single-component transport simulations, an "effective" binary isotherm model in an algebraic form must be available for use in the VERSE-LC code. Based on our previous experience using VERSE-LC for modeling SuperLig ${ }^{\circledR} 644$ and SuperLig ${ }^{\circledR} 639$ resins (Hamm et al., 2000a and 2000b), the VERSE-LC Freundlich/Langmuir Hybrid isotherm model was chosen. As described by Hamm et al. (2000a, see Chapter 4), the cations cesium, potassium, sodium, and strontium hydroxide form a 4component homovalent system where the surface loading for cesium on the CST material can be expressed as:

$$
\mathrm{Q}_{\mathrm{Cs}}=\frac{\eta_{\mathrm{df}} \overline{\mathrm{C}}_{\mathrm{T}} \mathrm{c}_{\mathrm{Cs}}}{\mathrm{c}_{\mathrm{Cs}}+\left[\widetilde{\mathrm{K}}_{21} \mathrm{c}_{\mathrm{K}}+\widetilde{\mathrm{K}}_{31} \mathrm{c}_{\mathrm{Na}}+\widetilde{\mathrm{K}}_{41} \mathrm{c}_{\mathrm{SrOH}}+\cdots\right]} \Rightarrow \frac{\eta_{\mathrm{df}} \overline{\mathrm{C}}_{\mathrm{T}} \mathrm{c}_{\mathrm{Cs}}}{\mathrm{c}_{\mathrm{Cs}}+\beta},
$$

where the beta parameter for cesium becomes dependent upon the other ionic competitors for CST adsorption (i.e., $\mathrm{K}^{+}, \mathrm{Na}^{+}, \mathrm{SrOH}^{+}$, and $\mathrm{Rb}^{+}$). The beta parameter contains the selectivity coefficients making it dependent upon temperature and liquid composition of all of the ionic species in solution. The larger the beta parameter the less favorable (and lower loadings) an isotherm will be (have). The dilution factor $\left(\eta_{\mathrm{df}}\right)$ is unity when considering a specific powerform and is less than one upon addition of an inert binder. Based on analyses discussed in 
Appendix $\mathrm{C}$ the best estimate dilution factor for the engineered-form is set to 0.68 . The total cesium capacity term is only a function of which batch of powder-form material is being considered and is set to 0.58 mmole $_{\mathrm{Cs}} / \mathrm{g}_{\mathrm{CST}}$. Based on the work of Zheng et al., (1996). With the dilution factor and the total cesium capacity set, Eq. (4-1) contains only one free parameter (beta) that need to be specified. The beta parameter to determined through nonlinear regression using a ZAM generated database for each batch feed.

\subsection{Batch Feed Compositions}

In order to generate the cesium loading databases (i.e., " $K_{d}$ " databases) using the ZAM code, the feed compositions for the 16 candidate batch feeds were determined based on available Best Basis Inventory (BBI) Phase $1 \mathrm{LAW}$ feed solution data. Alterations and adjustments were necessary to the raw BBI data in order for it to conform to current pretreatment plans and for overall charge balancing. Charge balancing is required due to the ZAM code input limitations placed on an ionic solution. The alterations and adjustments made focused on only those ionic species who have a direct impact on cesium loading (i.e., those ionic species considered in the cesium isotherm modeling - ZAM code input options).

A homovalent cation exchange process occurs between the ionic solution and the CST material where the dominant cations competing for exchange sites are $\mathrm{Cs}^{+}, \mathrm{SrOH}^{+}, \mathrm{Rb}^{+}, \mathrm{K}^{+}, \mathrm{H}^{+}$, and $\mathrm{Na}^{+}$ (where the order presented here is somewhat consistent with the degree of selectivity). For the 16 Phase 1 batch feeds, Table 4-1 lists the key ionic species that play an important role in determining the cesium loading levels on CST material. The following basic assumptions were made in generating the batch feed compositions:

- $\mathrm{Rb}^{+}$is only present in trace amounts and was assumed to be zero in the development of cesium isotherms.

- CST has a strong affinity for $\mathrm{SrOH}^{+}$; however, the actual amount of total $\mathrm{Sr}^{+2}$ available in solution to form $\mathrm{SrOH}^{+}$is currently believed to be small but is unknown at this time. In this report the $\mathrm{Sr}^{+2}$ concentration has been set to zero. Future work will need to address this issue. Further discussion on this is given in Appendix B.

- Total $\mathrm{Cs}^{+}$concentrations estimated based on the ${ }^{137} \mathrm{Cs}^{+}$content assuming isotopic fractions of $25 \%$ for Envelope A and C feeds and 30\% for Envelope B feeds.

- Only those species that are handled within the ZAM code are charge balanced. The concentration of free $\mathrm{OH}^{-}$was allowed to vary in order to achieve charge balance.

- The ZAM generated cesium loading databases are based on $25 \mathrm{C}$ column operation. In order to generate one isotherm database, the cesium concentration within a feed solution was varied over a wide range. This was accomplished by adding or removing $\mathrm{CsCl}$ from the original LAW feed solution. The choice of $\mathrm{CsCl}$, versus some other salts, was confirmed by the excellent fits achieved using the algebraic model (i.e., selectivity coefficients remained relatively constant over the entire range considered).

The complete list of assumptions made is provided in Appendix A, along with the feed compositions of the original BBI inventories (i.e., obtained from the test specification document) 
and the final $5 \mathrm{M}$ basis batch feeds. The details and results of the creation of the 16 candidate feed compositions are provided in Appendix A. Tables A-5 and A-6 contain the feed compositions on a $5 \mathrm{M}$ sodium basis used as input to the ZAM code.

\subsection{The Beta Parameter Values}

As mentioned above, a nonlinear regression analyses was used to determine the beta value, given in Eq. (4-1), for each of the 16 batch feeds. A non-linear regression analysis based on the maximum likelihood principle (see Anderson et al., 1978) was chosen consistent with earlier work (Hamm et al., 2000a and 2000b). The details and results of the regression analyses are provided in Appendix B.

As shown by Eq. (4-1), it is assumed that the beta parameter is only temperature and composition dependent and does not depend on the CST form under consideration (i.e. powder or engineered material). The dilution factor addresses which particular form is being considered. The ZAM code performs a numerical batch contact simulation for a specific solution composition and temperature (i.e., solves the appropriate liquid-solid equilibrium and mass balance equations for each competitor). The ZAM generated loadings are for the powder-form CST material.

The results of the nonlinear regression analyses are listed in Table 4-2 for the 16 batch feeds. Table 4-2 contains the "best estimate" value of the beta parameter, the standard error in the beta parameter estimate, and the root-mean-square (rms) in cesium loading (i.e., in terms of \%). The fitting results are grouped by envelope where overall averages of the three values are also given. Variability within an envelope can be seen, as well as between envelopes. This variability can easily be seen in the barchart of the beta values shown in Figure 4-1. The least favorable isotherm corresponds to batch feed LAW-1 (AP-101 source tank) with the next being LAW-15 (source tank AW-101). As indicated in Table 4-1, these two batch feeds have the highest competitor (specifically potassium) concentrations.

As the standard errors in beta indicate in Table 4-2, the algebraic model fits the ZAM generated database quite accurately. For example, in Figure 4-2 the cesium loading curve (i.e., the $25 \mathrm{C}$ isotherm) for powder-form CST in contact with the LAW-15 batch feed is shown. The solid circles represent the ZAM generated database, while the solid curve is the algebraic model for CST powder. For application to CST column design a cesium loading curve for the engineeredform must be used and also shown in Figure 4-2 by the dashed curve is the isotherm for the LAW-15 batch feed. The location of the cesium feed concentration for LAW-15 is also shown by the gradient symbol on this dashed curve. Here a dilution factor of $68 \%$ is employed which shifts the loading curve downward. The basis for this value of the dilution factor is given in Appendix C.

The ZAM generated databases and their corresponding fits to Eq. (4-1) are provided in Appendix $\mathrm{B}$ for each of the 16 batch feeds. Also in Appendix B, are graphical comparisons of these isotherms grouped by envelope and by comparing the least favorable between envelopes. 


\subsection{Isotherm Model for VERSE-LC Application}

In order to perform column transport simulations, the algebraic model given by Eq. (4-1) above must be converted into one of the available VERSE-LC isotherm modeling options. Based on our previous experience using VERSE-LC for modeling SuperLig ${ }^{\circledR} 644$ and SuperLig ${ }^{\circledR} 639$ resins (Hamm et al., 2000a and 2000b, respectively), the VERSE-LC Freundlich/Langmuir Hybrid isotherm model was chosen. See Hamm et al. (2000a, see Chapter 4) for further descriptions of the multi-component homovalent isotherm approach.

For a 4-component homovalent isotherm, the VERSE-LC Freundlich/Langmuir Hybrid model is expressed as:

$$
\overline{\mathrm{C}}_{\mathrm{pi}}=\frac{\mathrm{a}_{\mathrm{i}} \mathrm{c}_{\mathrm{pi}}^{\mathrm{M}_{\mathrm{ai}}}}{\beta_{\mathrm{i}}+\mathrm{b}_{1} \mathrm{c}_{\mathrm{p} 1}^{\mathrm{M}_{\mathrm{b} 1}}+\mathrm{b}_{2} \mathrm{c}_{\mathrm{p} 2}^{\mathrm{M}_{\mathrm{b} 2}}+\mathrm{b}_{3} \mathrm{c}_{\mathrm{p} 3}^{\mathrm{M}_{\mathrm{b} 3}}+\mathrm{b}_{4} \mathrm{c}_{\mathrm{p} 4}^{\mathrm{M}_{\mathrm{b} 4}}} \quad \text { for } \quad \mathrm{i}=1,4
$$

where the model parameters $\left(a_{i}, b_{i}, M_{a i}, M_{b i}\right.$, and $\beta_{i}$ for $\left.i=1,4\right)$ can be determined from the parameter values associated with the 4-component homovalent model.

The Freundlich/Langmuir Hybrid model can also be used for an "effective" single-component case as well. Here the potassium, sodium, and strontium hydroxide concentrations throughout the column are assumed to be at their feed concentration levels. For an "effective" singlecomponent total cesium isotherm, Eq. (4-2) under these conditions reduces to:

$$
\overline{\mathrm{C}}_{\mathrm{p} 1}=\frac{\mathrm{a}_{1} \mathrm{c}_{\mathrm{p} 1}^{\mathrm{M}_{\mathrm{ai}}}}{\left[\beta_{\mathrm{i}}+\mathrm{b}_{2} \mathrm{c}_{\mathrm{p} 2}^{\mathrm{M}_{\mathrm{b} 2}}+\mathrm{b}_{3} \mathrm{c}_{\mathrm{p} 3}^{\mathrm{M}_{\mathrm{b}}}+\mathrm{b}_{4} \mathrm{c}_{\mathrm{p} 4}^{\mathrm{M}_{\mathrm{b} 4}}\right]+\mathrm{b}_{1} \mathrm{c}_{\mathrm{p} 1}^{\mathrm{M}_{\mathrm{b}}}} \Rightarrow \frac{\mathrm{a}_{1} \mathrm{c}_{\mathrm{p} 1}^{\mathrm{M}_{\mathrm{a}}}}{\hat{\beta}_{\mathrm{i}}+\mathrm{b}_{1} \mathrm{c}_{\mathrm{p} 1}^{\mathrm{M}_{\mathrm{b}}}},
$$

where the beta parameter for cesium becomes dependent upon the potassium, sodium, and strontium hydroxide feed concentrations. The relationship between the two models expressed by Eqs. (4-2) and (4-3) (i.e., 4-component homovalent and "effective" single-component isotherm models, respectively) is provided in Appendix B. The dilution factor, $\eta_{\mathrm{df}}$, is set to unity when CST in its powder-form is being considered.

The modeling parameter values for a cesium single-component isotherm is listed in Table 4-3 for each of the 16 batch feeds. The $a_{1}$ parameter of Eq. (4-3) is computed by:

$$
\mathrm{a}_{1}=\rho_{\mathrm{b}} \eta_{\mathrm{df}} \overline{\mathrm{C}}_{\mathrm{T}}
$$

For the CST column design analyses performed in Chapter 10, an average density of $1 \mathrm{~g} / \mathrm{ml}$, a total cesium capacity of $0.58 \mathrm{mmole} / \mathrm{g}_{\mathrm{CST}}$ for CST powder, and a dilution factor of 0.68 for CST in its engineered-form are assumed. The $a_{1}$ parameter values for both CST powder and its engineered-form are listed in Table 4-3. 
Based on CST in its engineered-form the cesium isotherms for the 16 batch feeds, using the parameter settings listed in Table 4-3, are plotted in Figure 4-3 (for Envelope A), Figure 4-4 (for Envelope B), and Figure 4-5 (for Envelope C). In Figure 4-6 the least favorable isotherm taken from each envelope is plotted together for a direct comparison. The feed concentration of cesium is also shown on the figures.

Looking at Figure 4-6 we see that Envelope A's LAW-1 isotherm is the least favorable overall. However, Envelope B's cesium feed concentration is significantly larger than the other two. In addition, in Envelope $\mathrm{C}$ we have assumed that the impact of $\mathrm{SrOH}^{+}$is negligible (an assumption that needs to be verified in the future). Therefore, a clear determination as to which feed results in the worst case isotherm for design purposes is difficult to determine based on this information alone. 
Table 4-1. Summary of the Phase 1 batch feed compositions in molarity units (on a 5M sodium basis) highlighting those ionic species who are dominant contributors to the effectiveness of CST material for cesium loading. ${ }^{a}$

\begin{tabular}{|c|c|c|c|c|c|c|c|c|c|c|c|c|}
\hline Env & $\begin{array}{l}\text { Batch } \\
\text { Name }\end{array}$ & $\begin{array}{c}\text { Staging } \\
\text { Tank }\end{array}$ & $\begin{array}{c}\mathrm{Cs}^{+} \mathrm{e} \\
\text { (total) }\end{array}$ & $\mathbf{K}^{+}$ & $\mathbf{R} \mathbf{b}^{+\mathbf{c}}$ & $\mathrm{SrOH}^{+}$ & $\mathrm{pH}^{\mathrm{d}}$ & $\begin{array}{c}\mathrm{OH}^{-} \\
\text {(free) }\end{array}$ & $\mathrm{NO}_{3}{ }^{-}$ & $\mathrm{NO}_{2}^{-}$ & $\mathrm{Al}(\mathrm{OH})_{4}^{-}$ & $\mathrm{CO}_{3}{ }^{2-}$ \\
\hline $\mathbf{A}$ & LAW-1 & AP-101 & $3.60 \mathrm{E}-05$ & 0.71 & - & - & 14.2 & 1.55 & 1.87 & 0.81 & 0.23 & 0.48 \\
\hline $\mathbf{A}$ & LAW-5 & AN-104 & $6.28 \mathrm{E}-05$ & 0.06 & - & - & 13.9 & 0.86 & 1.38 & 1.19 & 0.57 & 0.39 \\
\hline $\mathbf{A}$ & LAW-6 & AN-104 & $6.33 \mathrm{E}-05$ & 0.06 & - & - & 14.0 & 0.95 & 1.36 & 1.19 & 0.57 & 0.36 \\
\hline $\mathbf{A}$ & LAW-8 & AN-105 & 4.32E-05 & 0.07 & - & - & 14.1 & 1.34 & 1.28 & 1.20 & 0.64 & 0.20 \\
\hline $\mathbf{A}$ & LAW-9 & AN-105 & 4.44E-05 & 0.06 & - & - & 13.8 & 0.57 & 1.41 & 1.21 & 0.45 & 0.55 \\
\hline $\mathbf{A}$ & LAW-10 & SY-101 & $3.69 \mathrm{E}-05$ & 0.04 & - & - & 13.9 & 0.87 & 1.43 & 1.31 & 0.49 & 0.28 \\
\hline $\mathbf{A}$ & LAW-11 & SY-101 & $3.74 \mathrm{E}-05$ & 0.04 & - & - & 14.0 & 0.93 & 1.20 & 1.39 & 0.47 & 0.36 \\
\hline $\mathbf{A}$ & LAW-12 & AN-103 & $4.83 \mathrm{E}-05$ & 0.12 & - & - & 14.2 & 1.54 & 1.22 & 1.13 & 0.75 & 0.16 \\
\hline $\mathbf{A}$ & LAW-13 & AN-103 & 4.83E-05 & 0.13 & - & - & 14.2 & 1.54 & 1.22 & 1.13 & 0.76 & 0.16 \\
\hline $\mathbf{A}$ & LAW-14 & AW-101 & 4.57E-05 & 0.32 & - & - & 14.2 & 1.75 & 1.32 & 1.06 & 0.49 & 0.25 \\
\hline $\mathbf{A}$ & LAW-15 & AW-101 & $4.55 \mathrm{E}-05$ & 0.41 & - & - & 14.3 & 2.14 & 1.30 & 1.01 & 0.50 & 0.15 \\
\hline B & LAW-2a & AZ-101 & $4.68 \mathrm{E}-04$ & 0.12 & - & - & 13.2 & 0.16 & 1.28 & 1.48 & 0.42 & 0.59 \\
\hline B & LAW-2b & AZ-102 & $4.31 \mathrm{E}-04$ & 0.14 & - & - & 13.4 & 0.27 & 0.67 & 1.18 & 0.10 & 1.03 \\
\hline C & LAW-3 & AN-102 & $3.97 \mathrm{E}-05$ & 0.04 & - & - & 14.0 & 1.03 & 1.74 & 0.80 & 0.26 & 0.51 \\
\hline $\mathrm{C}$ & LAW-4 & $\mathrm{AN}-102$ & $3.78 \mathrm{E}-05$ & 0.04 & - & - & 14.0 & 1.03 & 1.74 & 0.80 & 0.26 & 0.51 \\
\hline $\mathrm{C}$ & LAW-7 & AN-107 & $4.45 \mathrm{E}-05$ & 0.02 & - & - & 13.9 & 0.79 & 1.97 & 0.70 & 0.14 & 0.66 \\
\hline
\end{tabular}

${ }^{\text {a }}$ For CST material homovalent cation exchange takes place where the dominant species are $\mathrm{Cs}^{+}, \mathrm{SrOH}^{+}, \mathrm{Rb}^{+}, \mathrm{K}^{+}, \mathrm{H}^{+}, \mathrm{and} \mathrm{Na}^{+}$.

${ }^{\mathrm{b}} \mathrm{CST}$ has a strong affinity for $\mathrm{SrOH}^{+}$; however, the actual amount of total $\mathrm{Sr}^{+2}$ available in solution to form $\mathrm{SrOH}^{+}$is currently believed to be small but is unknown at this time. In this report the $\mathrm{Sr}^{+2}$ concentration has been set to zero. Future work will need to address this issue.

${ }^{c} \mathrm{Rb}^{+}$is only present in trace amounts and was assumed to be zero in the development of cesium isotherms.

${ }^{\mathrm{d}} \mathrm{pH}$ is only being estimated here without properly accounting for the water activity; however, ZAM correctly computes the $\mathrm{H}^{+}$concentration based on the inputted free $\mathrm{OH}^{-}$.

${ }^{\mathrm{e}}$ Total $\mathrm{Cs}^{+}$concentrations estimated based on the ${ }^{137} \mathrm{Cs}^{+}$content assuming isotopic fractions of $25 \%$ for Envelope A and C feeds and $30 \%$ for Envelope B feeds. 
Table 4-2. Estimated beta parameter values and error estimate for a one-component (total cesium) homovalent algebraic isotherm model based on CST in its powder-form (IE-910) where the data sets used were created using the ZAM code.

\begin{tabular}{|c|c|c|c|}
\hline Feed Solution & $\begin{array}{c}\text { "Best Estimate" } \\
\beta_{\mathbf{i}} \\
{[\mathrm{M}]}\end{array}$ & $\begin{array}{c}\text { Standard Error in } \\
\qquad \beta_{\mathbf{i}} \\
{[\mathbf{M}]}\end{array}$ & $\begin{array}{l}\text { Root Mean Square } \\
\text { in Cs loading }{ }^{\text {a }} \\
\text { (\% difference) }\end{array}$ \\
\hline \multicolumn{4}{|l|}{ Envelope A } \\
\hline LAW-1 & $4.3376 \mathrm{E}-04$ & $\pm 8.3713 \mathrm{E}-07$ & 0.3912 \\
\hline LAW-5 & $2.3431 \mathrm{E}-04$ & $\pm 2.0648 \mathrm{E}-08$ & 0.0137 \\
\hline LAW-6 & $2.3668 \mathrm{E}-04$ & $\pm 2.2931 \mathrm{E}-08$ & 0.0202 \\
\hline LAW-8 & $2.5147 \mathrm{E}-04$ & $\pm 3.8060 \mathrm{E}-08$ & 0.0270 \\
\hline LAW-9 & 2.2453E-04 & $\pm 2.8251 \mathrm{E}-08$ & 0.0220 \\
\hline LAW-10 & $2.2135 \mathrm{E}-04$ & $\pm 2.2209 \mathrm{E}-08$ & 0.0161 \\
\hline LAW-11 & $2.0543 \mathrm{E}-04$ & $\pm 2.4697 \mathrm{E}-08$ & 0.0229 \\
\hline LAW-12 & $2.9196 \mathrm{E}-04$ & $\pm 3.2849 \mathrm{E}-08$ & 0.0177 \\
\hline LAW-13 & 2.9328E-04 & $\pm 3.2820 \mathrm{E}-08$ & 0.0184 \\
\hline LAW-14 & $3.6283 \mathrm{E}-04$ & $\pm 4.0017 \mathrm{E}-08$ & 0.0262 \\
\hline LAW-15 & $3.8513 \mathrm{E}-04$ & $\pm 3.7129 \mathrm{E}-08$ & 0.0219 \\
\hline Overall avg. & $2.8552 \mathrm{E}-04$ & $\pm 1.0334 \mathrm{E}-07$ & 0.0543 \\
\hline \multicolumn{4}{|l|}{ Envelope B } \\
\hline LAW-2a & $2.6230 \mathrm{E}-04$ & $\pm 2.9902 \mathrm{E}-08$ & 0.0173 \\
\hline LAW-2b & $2.1296 \mathrm{E}-04$ & $\pm 2.3999 \mathrm{E}-08$ & 0.0138 \\
\hline Overall avg. & $2.3763 \mathrm{E}-04$ & $\pm 2.6951 \mathrm{E}-08$ & 0.0156 \\
\hline \multicolumn{4}{|l|}{ Envelope C } \\
\hline LAW-3 & $2.1769 \mathrm{E}-04$ & $\pm 2.4699 \mathrm{E}-08$ & 0.0135 \\
\hline LAW-4 & $2.1769 \mathrm{E}-04$ & $\pm 2.4646 \mathrm{E}-08$ & 0.0132 \\
\hline LAW-7 & $1.9258 \mathrm{E}-04$ & $\pm 2.0511 \mathrm{E}-08$ & 0.0166 \\
\hline Overall avg. & $2.0932 \mathrm{E}-04$ & $\pm 2.3285 \mathrm{E}-08$ & 0.0144 \\
\hline
\end{tabular}

a Based on a percent difference in Cs loading [i.e., 100(algebraic-ZAM)/ZAM]. 
Preliminary Ion Exchange Modeling for Removal of Cesium from

Table 4-3. Parameter settings for an "effective" single component Freundlich/Langmuir Hybrid equilibrium isotherm model for total cesium on CST based on the 1-component homovalent model.

\begin{tabular}{|c|c|c|c|c|c|c|}
\hline Feed Solution & $\begin{array}{c}\text { "Powder-form" } \\
(\text { IE-910) } \\
\mathbf{a}_{\mathbf{i}} \\
\left(\text { gmoles } / \mathbf{L}_{\mathrm{CV}}\right)\end{array}$ & $\begin{array}{c}\text { "Engineered-form" } \\
(\text { IE-911) } \\
\mathbf{a}_{\mathrm{i}} \\
\left(\text { gmoles/L } / \mathrm{L}_{\mathrm{CV}}\right)\end{array}$ & $\left(\mathbf{b}_{\mathbf{i}}\right.$ & $\begin{array}{c}\mathbf{M}_{\mathrm{ai}} \\
(-)\end{array}$ & $\begin{array}{c}M_{b i} \\
(-)\end{array}$ & $\begin{array}{c}\text { "Effective" } \mathrm{b} \\
\beta_{\mathrm{I}} \\
(-)\end{array}$ \\
\hline \multicolumn{7}{|l|}{ Envelope A } \\
\hline LAW-1 & 0.580 & 0.3944 & 1.0 & 1.0 & 1.0 & $4.3376 \mathrm{E}-04$ \\
\hline LAW-5 & 0.580 & 0.3944 & 1.0 & 1.0 & 1.0 & $2.3431 \mathrm{E}-04$ \\
\hline LAW-6 & 0.580 & 0.3944 & 1.0 & 1.0 & 1.0 & $2.3668 \mathrm{E}-04$ \\
\hline LAW-8 & 0.580 & 0.3944 & 1.0 & 1.0 & 1.0 & $2.5147 \mathrm{E}-04$ \\
\hline LAW-9 & 0.580 & 0.3944 & 1.0 & 1.0 & 1.0 & $2.2453 \mathrm{E}-04$ \\
\hline LAW-10 & 0.580 & 0.3944 & 1.0 & 1.0 & 1.0 & $2.2135 \mathrm{E}-04$ \\
\hline LAW-11 & 0.580 & 0.3944 & 1.0 & 1.0 & 1.0 & $2.0543 \mathrm{E}-04$ \\
\hline LAW-12 & 0.580 & 0.3944 & 1.0 & 1.0 & 1.0 & $2.9196 \mathrm{E}-04$ \\
\hline LAW-13 & 0.580 & 0.3944 & 1.0 & 1.0 & 1.0 & $2.9328 \mathrm{E}-04$ \\
\hline LAW-14 & 0.580 & 0.3944 & 1.0 & 1.0 & 1.0 & $3.6283 \mathrm{E}-04$ \\
\hline LAW-15 & 0.580 & 0.3944 & 1.0 & 1.0 & 1.0 & $3.8513 \mathrm{E}-04$ \\
\hline \multicolumn{7}{|l|}{ Envelope B } \\
\hline LAW-2a & 0.580 & 0.3944 & 1.0 & 1.0 & 1.0 & $2.6230 \mathrm{E}-04$ \\
\hline LAW-2b & 0.580 & 0.3944 & 1.0 & 1.0 & 1.0 & $2.1296 \mathrm{E}-04$ \\
\hline \multicolumn{7}{|l|}{ Envelope C } \\
\hline LAW-3 & 0.580 & 0.3944 & 1.0 & 1.0 & 1.0 & $2.1769 \mathrm{E}-04$ \\
\hline LAW-4 & 0.580 & 0.3944 & 1.0 & 1.0 & 1.0 & $2.1769 \mathrm{E}-04$ \\
\hline LAW-7 & 0.580 & 0.3944 & 1.0 & 1.0 & 1.0 & $1.9258 \mathrm{E}-04$ \\
\hline
\end{tabular}

${ }^{a}$ A dilution factor of $68 \%$ is assumed when going from the powder-form to the engineered-form and a bed density of $1.0 \mathrm{~g} / \mathrm{ml}$ assumed.

${ }^{\mathrm{b}}$ These are "best estimate" values based on the maximum likelihood algorithm. 


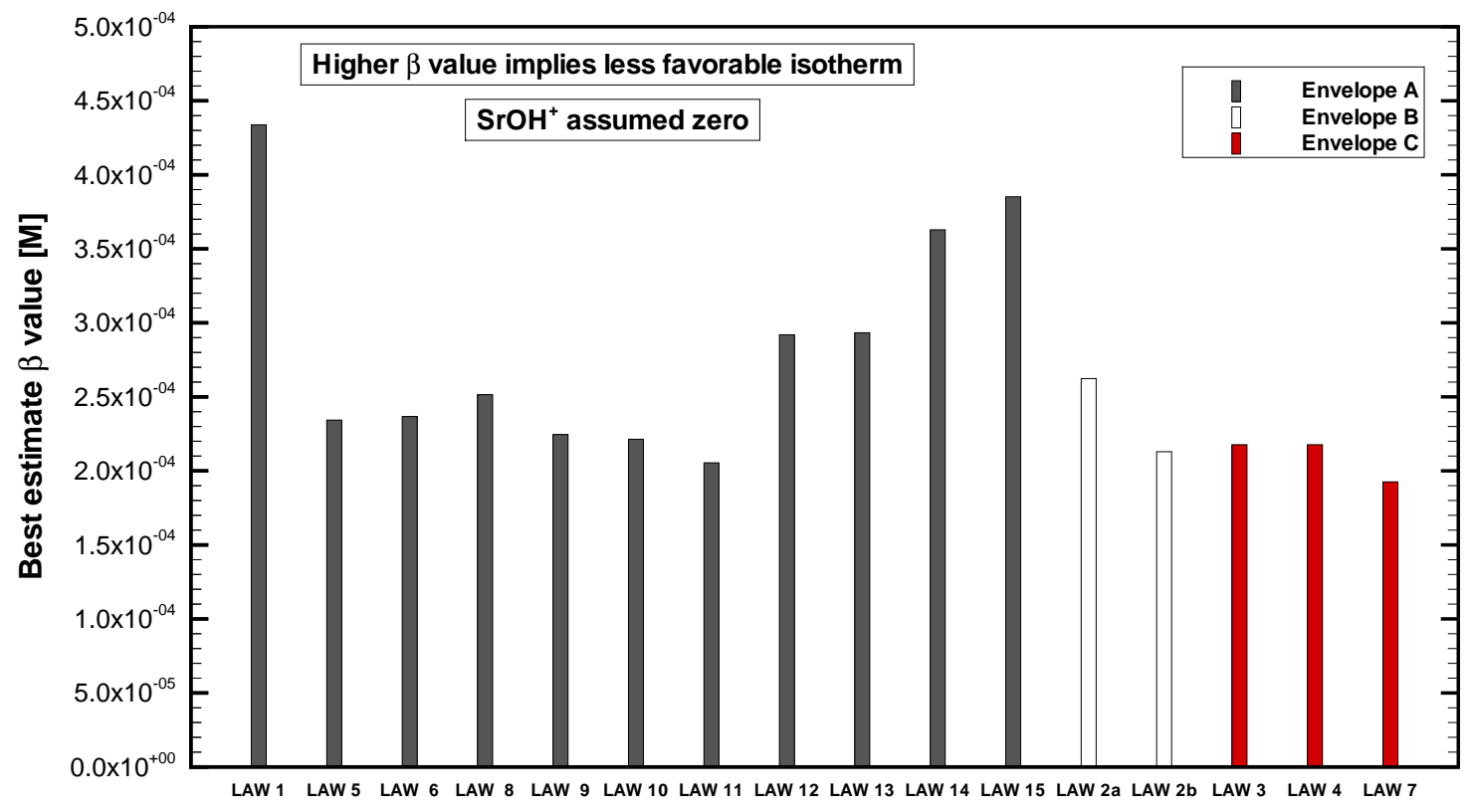

Candidate feed solutions

Figure 4-1. The estimated Phase 1 feed beta values used in the cesium effective single-component isotherm model for CST powder-form and engineered-form materials. The beta values are grouped by envelope and are based on feeds assuming zero $\mathrm{Rb}^{+}$and $\mathrm{SrOH}^{+}$present.

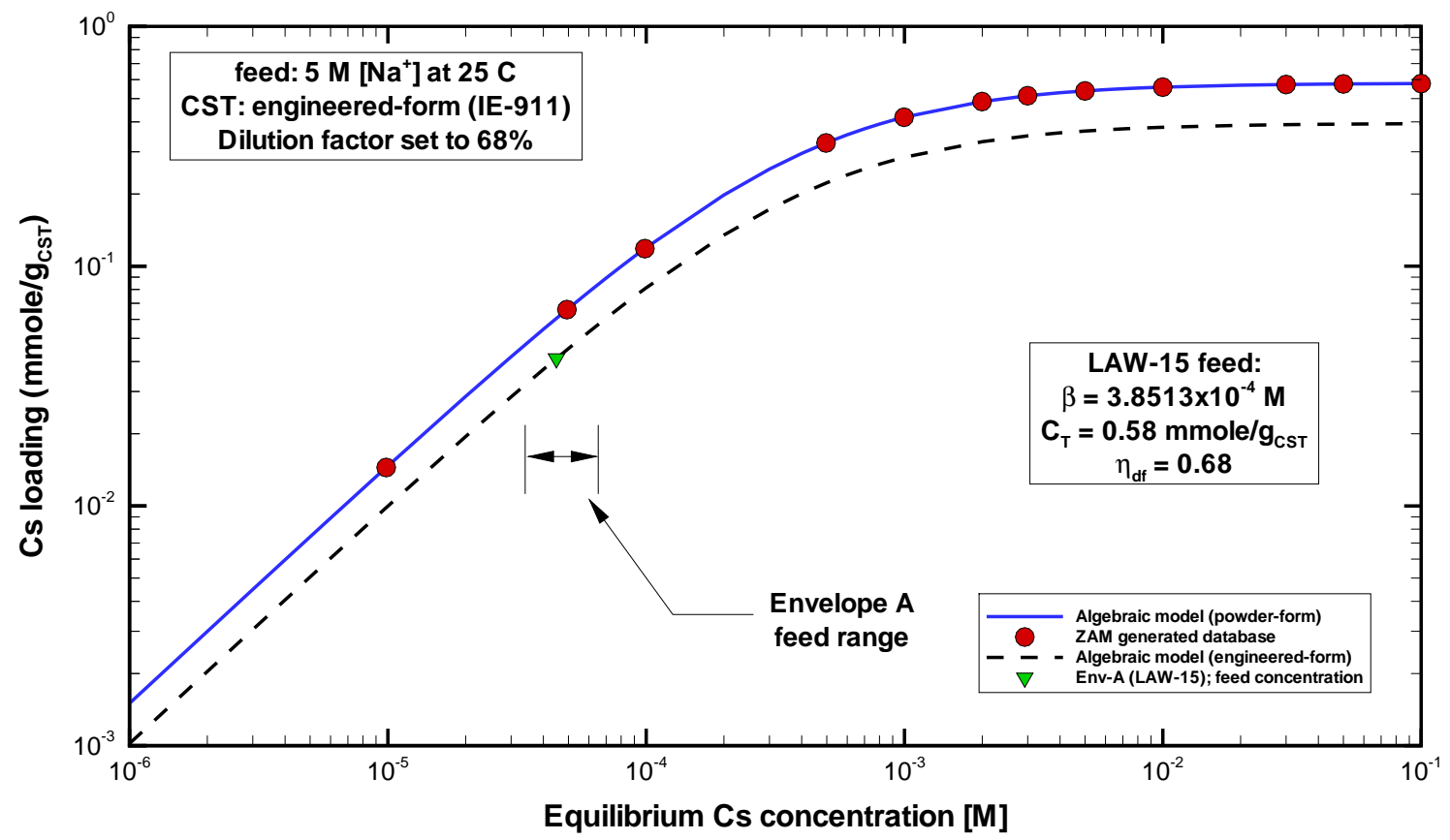

Figure 4-2. Comparison of the ZAM generated database for cesium loading onto IE-910 CST (powder) versus the algebraic model fit for the Phase $1 \mathrm{LAW}-15$ feed. Also shown is the algebraic model when applied to the engineered-form using a dilution factor of $68 \%$. 


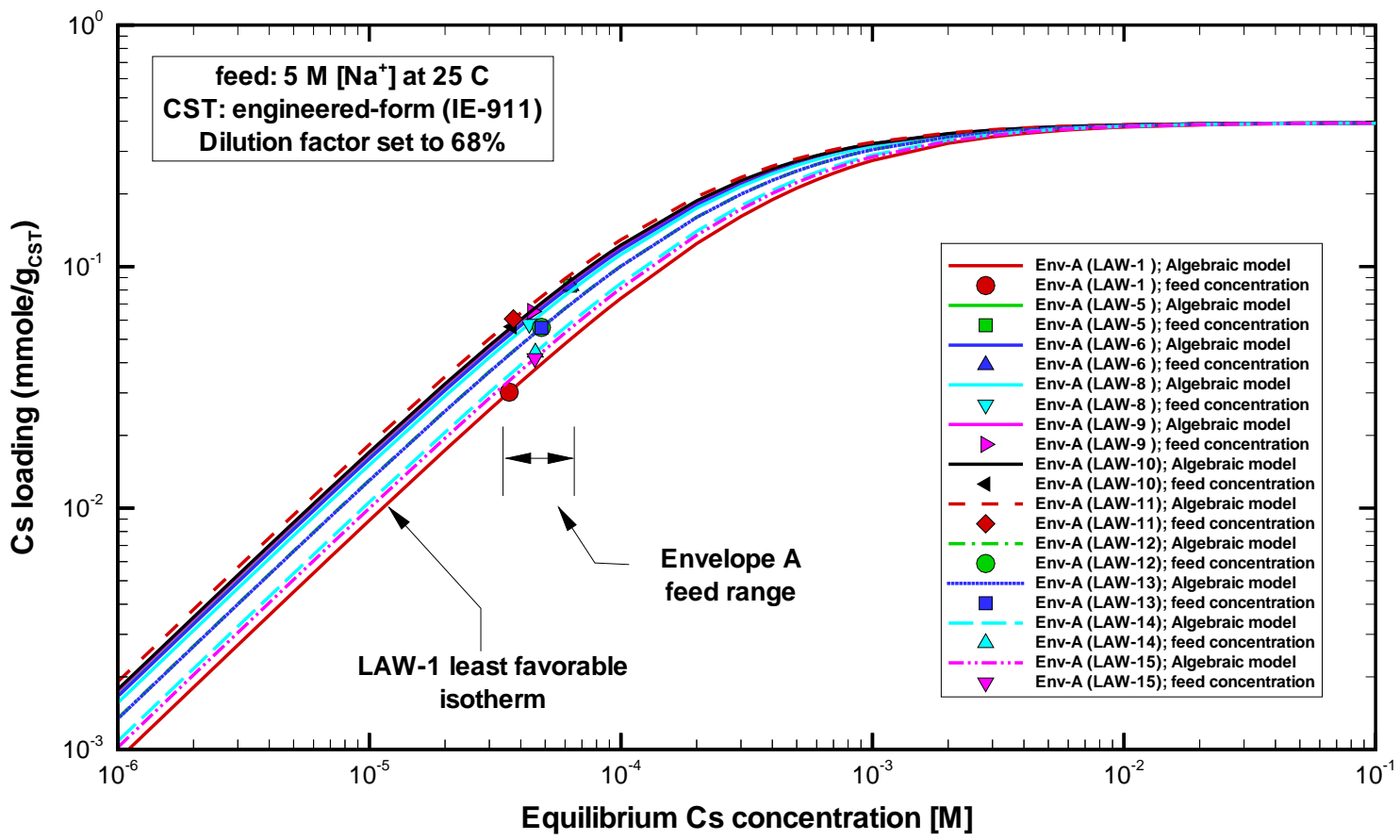

Figure 4-3. Comparison of Envelope A isotherms for the CST material in its engineered-form (IE-911). The lines represent predictions based on the single-component Freundlich/ Langmuir Hybrid isotherm model and symbols indicate the feed concentrations of cesium.

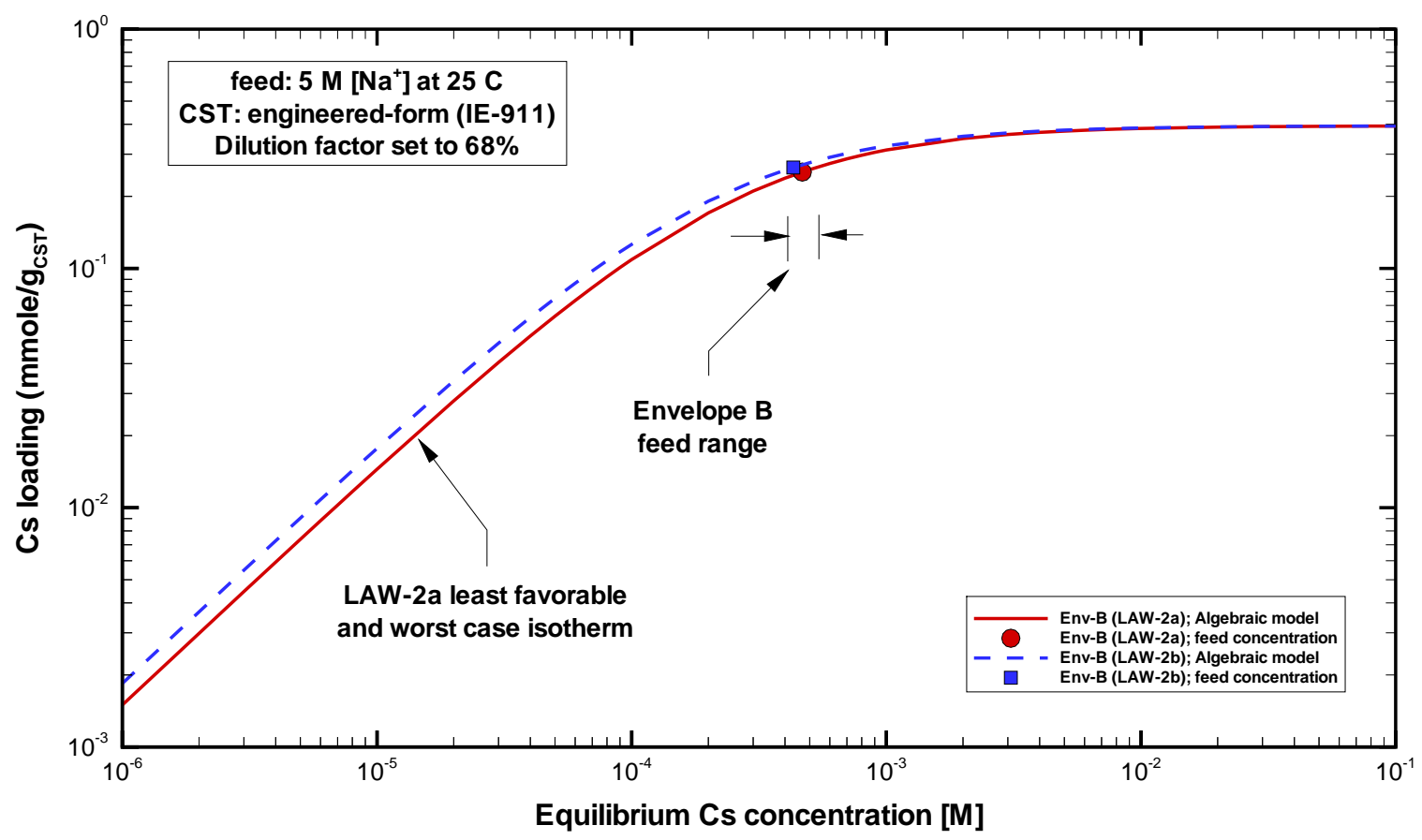

Figure 4-4. Comparison of Envelope B isotherms for the CST material in its engineered-form (IE-911). The lines represent predictions based on the single-component Freundlich/ Langmuir Hybrid isotherm model and symbols indicate the feed concentrations of cesium. 


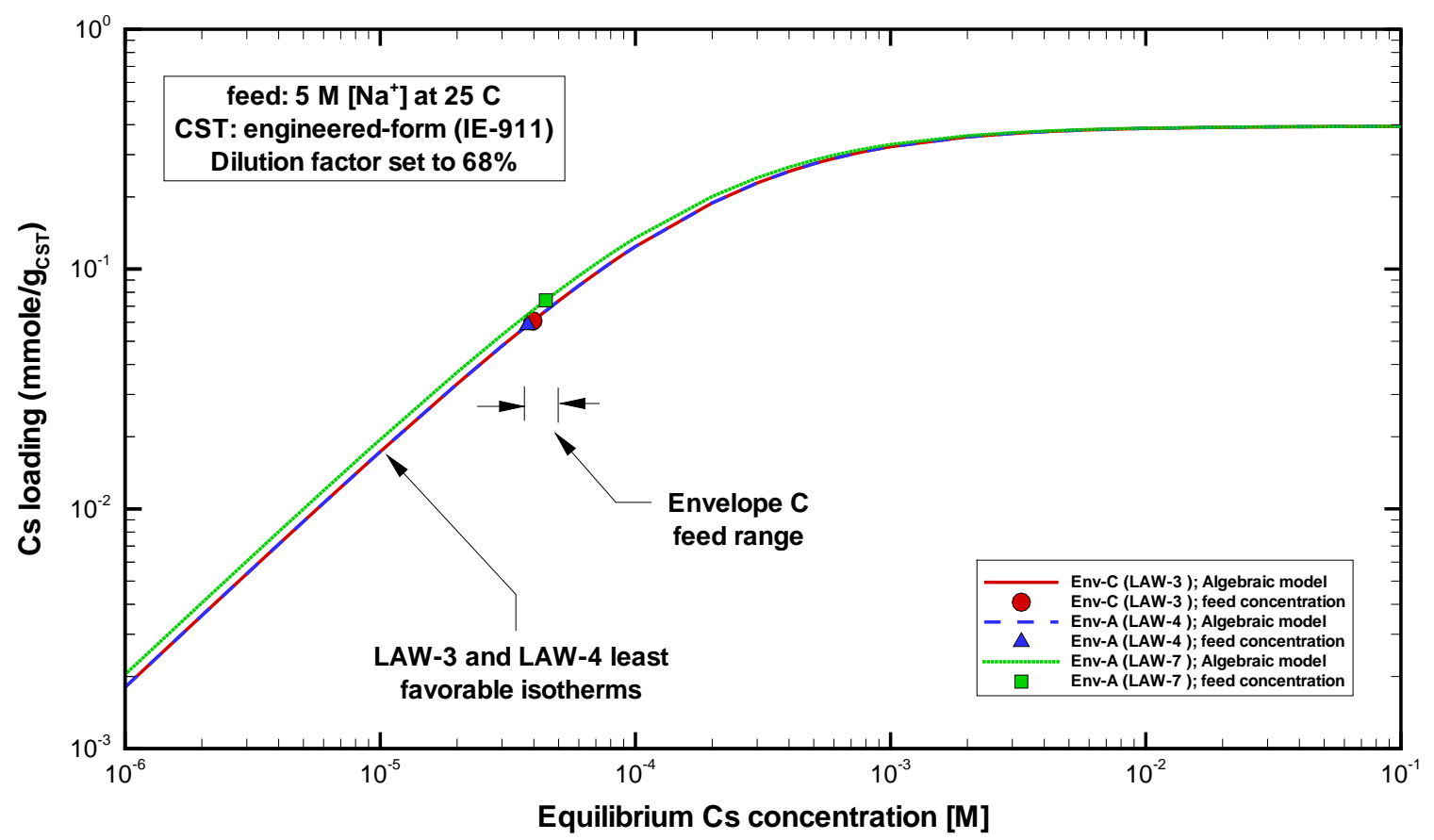

Figure 4-5. Comparison of Envelope C isotherms for the CST material in its engineered-form (IE-911). The lines represent predictions based on the "effective" single-component Freundlich/ Langmuir Hybrid isotherm model and symbols indicate the feed concentrations of cesium.

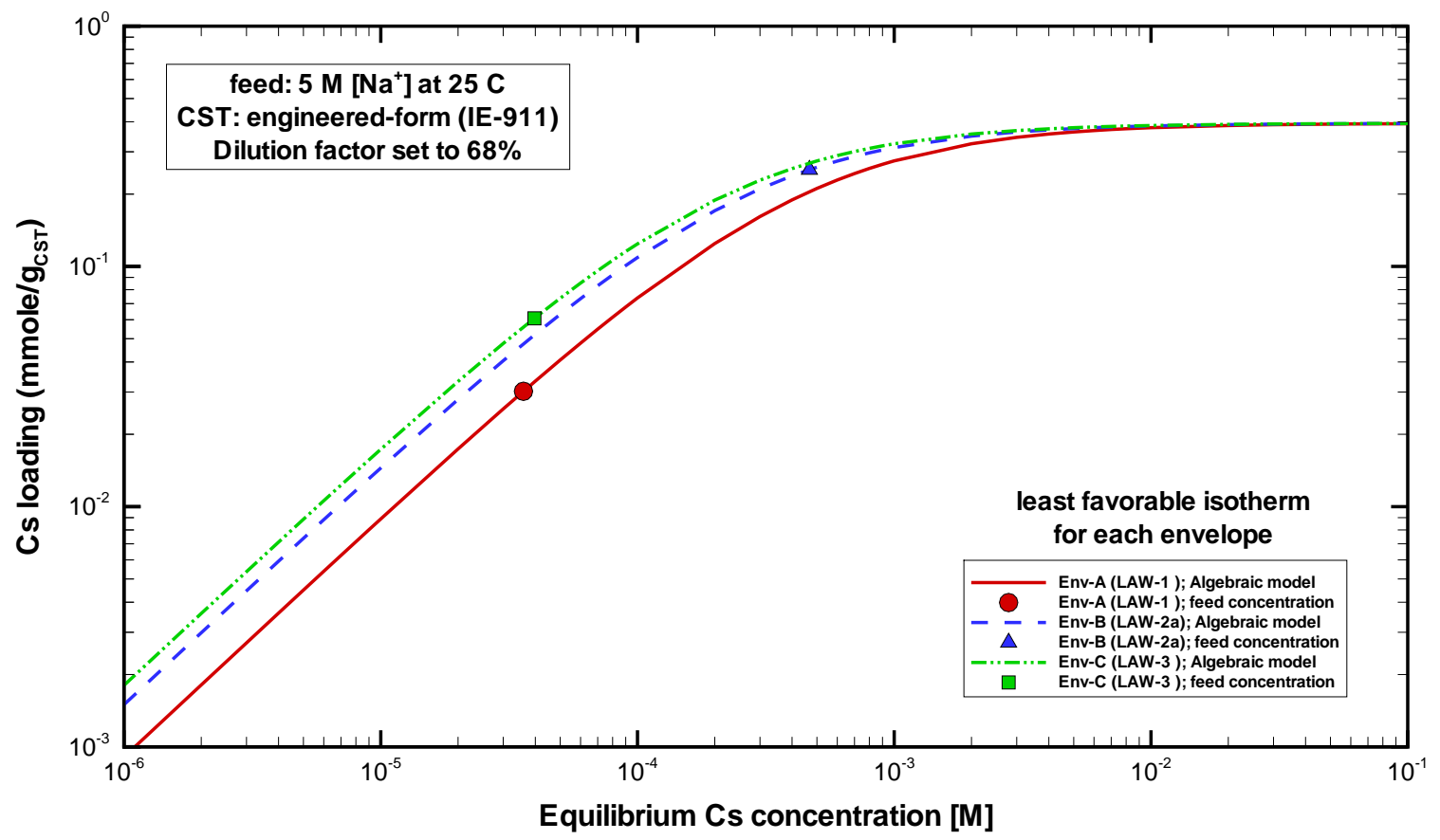

Figure 4-6. Comparison of the least favorable isotherms for Envelope A, B, and C feeds for the CST material in its engineered-form (IE-911). The lines represent predictions based on the "effective" single-component Freundlich/Langmuir Hybrid isotherm model. 


\subsection{Column Properties}

Certain material properties (such as, solid particle density and total ionic capacity) are unique to the ion-exchange material and vary only between batches. On the other hand, composite properties associated with an ion-exchange column (such as, bed density or porosities) are inherently column specific resulting from how it was packed for example. Even when different columns are made from the same batch of material, column properties can and will vary. During operation upflow and then downflow can result in changes in particle packing arrangements (see, Steimke, 2000). During operational cycles where a significant variation in total ionic strength of the feed occurs, the active bed size of a column can change. The discussion that follows focuses on the column properties required when performing column transport simulations during the loading phase of a column cycle.

UOP (1996) states that the bulk density of the IE-911 exchanger is approximately $1 \mathrm{~g} / \mathrm{ml}$ (i.e., a typical range of 0.8 to $1.13 \mathrm{~g} / \mathrm{ml}$ ) and that typically water contents are about $12 \mathrm{wt} \%$ for the IE910 powder and about $20 \mathrm{wt} \%$ for the IE-911 engineered-form exchangers. Since the IE-910 and IE-911 materials have rigid inorganic structures, they resist significant swelling or shrinkage due to exposure to changes in temperature, $\mathrm{pH}$, ionic strength, and ion exchange levels. The engineered-forms contain about $20 \%$ to $30 \%$ inert binder with the rest being CST powder.

The bed porosity of 0.50 and particle porosity of 0.24 were used for the majority of column simulations performed within this report. These porosity values have been used by numerous investigators in earlier analysis efforts (e.g., see Hritzko et al., 1998). These values yield a total bed porosity of $62 \%$. During the writing of this chapter estimates of the particle density for IE911 material were made available to the authors (i.e., actually slurry densities were provided which were then used to estimate particle density, Qureshi, 1999). Based on the limited measured particle and bed densities available, we estimate that the total porosity is in the range of $29 \%$ to $53 \%$. The $62 \%$ we assumed earlier is outside this range indicating that the results from our column models are based on total bed voids that are larger than they should have been. This would normally imply that the column models contain less CST material by mass than they should have had. Fortunately, the manner in which VERSE-LC handles the isotherms enforces the bed density to be the value used in creating the isotherms (i.e., as shown in Chapter 4 bed density is part of the isotherm itself). The net effect is larger bed and/or pore volumes that can have a secondary effect on the predicted breakthrough curves (e.g., typical impacts are less than $\pm 10 \%)$. Future design efforts should be based on more up to date porosity values based on measured bed and particle densities consistent with the engineered-form under consideration.

\subsection{Basic Constraint Functions}

Effective bed and particle porosity values for the powder-form and the engineered-forms will vary. The average particle radius for the powder-form is significantly less than for the engineered-forms (e.g., $\sim 0.8 \mu \mathrm{m}$ versus $\sim 344 \mu \mathrm{m}$ ). Based on geometrical considerations not all densities and porosities are independent. The following two expressions place constraints on the densities and porosities: 


$$
\varepsilon_{\mathrm{T}}=\varepsilon_{\mathrm{b}}+\left(1-\varepsilon_{\mathrm{b}}\right) \varepsilon_{\mathrm{p}},
$$

and

$$
\rho_{\mathrm{b}}=\rho_{\mathrm{s}}\left(1-\varepsilon_{\mathrm{T}}\right)
$$

where

$$
\begin{array}{ll}
\varepsilon_{\mathrm{T}} & - \text { total porosity of active column, [-] } \\
\varepsilon_{\mathrm{b}} & \text { - bed porosity of active column, [-] } \\
\varepsilon_{\mathrm{p}} & \text { - particle porosity of CST particles, [-] } \\
\rho_{\mathrm{b}} & \text { - bed density of active column, } \mathrm{g} / \mathrm{ml} \\
\rho_{\mathrm{s}} & \text { - solid (particle) density of CST material, g/ml }
\end{array}
$$

and

$$
\rho_{\mathrm{b}} \equiv \frac{\mathrm{m}_{\mathrm{CST}}}{\mathrm{V}_{\mathrm{bed}}} .
$$

For the five variables listed above only three are independent. The various porosities used in Eq. (5-1) are defined as:

$$
\varepsilon_{\mathrm{b}}=\frac{\mathrm{V}_{\text {void }}-\mathrm{V}_{\text {pore }}}{\mathrm{V}_{\text {bed }}} ; \quad \varepsilon_{\mathrm{p}}=\frac{\mathrm{V}_{\text {pore }}}{\mathrm{V}_{\text {part }}} ; \quad \varepsilon_{\mathrm{T}}=\frac{\mathrm{V}_{\text {void }}}{\mathrm{V}_{\text {bed }}}=\frac{\mathrm{V}_{\text {bed }}-\mathrm{V}_{\text {sld }}}{\mathrm{V}_{\text {bed }}},
$$

where

$$
\begin{array}{ll}
V_{\text {bed }} & \text { - total volume of active (bed) column, ml } \\
V_{\text {void }} & \text { - total volume of voids within active column, ml } \\
V_{\text {pore }} & \text { - total volume of pores within particles, } \mathrm{ml} \\
\mathrm{V}_{\text {part }} & \text { - total volume of particles within active column, } \mathrm{ml} \\
\mathrm{V}_{\text {sld }} & \text { - total volume of solid material within active column, ml }
\end{array}
$$

The above constraint equations provide a means to internally check for consistency between the various parameters. Below, just such a consistency check is performed where it can be seen that some of the available parameter values fall outside the limited measured data.

\subsection{Densities}

The actual amount of CST material present within a column is a parameter of prime importance with respect to column performance (i.e., exit breakthrough curves). For the column studies assessed within this report no direct measure of the total amount of CST residing within the active beds was available for most columns. For those columns whose original CST mass was not measured, the bed density was assumed to be the nominal value of $1.0 \mathrm{~g}_{\mathrm{CST}} / \mathrm{ml}_{\text {bed. }}$. The specific values for bed density from various data sources are listed in Table 5-1. 
The particle ("material") density of the CST IE-911 material can be estimated based on the measured slurry densities taken by Qureshi (1999) for CST and water mixtures. A summary of the results is given in Table 5-2. From this data an average particle density of $\sim 1.65 \mathrm{~g} / \mathrm{ml}$ is seen.

As Eq. (5-2) indicates, particle density, along with bed density, can be used to assist in determining the total porosity of a packed bed. Based on the Baseline CST material listed in Table 5-1 (and assumed properties for the majority of column simulations performed within this report), a particle density of $\sim 1.62 \mathrm{~g} / \mathrm{ml}$ can be back-calculated using Eq. (5-2). Based on these two different methods and data sources, the consistency of the estimates indicates that particle density is in the range of 1.6 to $1.7 \mathrm{~g} / \mathrm{ml}$. We assumed an average value of $1.65 \mathrm{~g} / \mathrm{ml}$ for the Baseline material.

\subsection{Porosities}

No direct measured bed, particle (i.e., sometimes referred to as pore), or total porosities have been consistently reported for the various column studies considered. Table 5-1 contains some of the values found within the literature. Values of $50 \%$ bed porosity and $24 \%$ particle porosity have been used in many of the existing column analyses to date reported by Hritzko et al. (1998) and many other sets of reported analyses throughout the literature. Within the Hritzko et al. (1998) report they reference their values to personal communications with R. A. Anthony at Texas A\&M University. These two porosity values were assumed to be our nominal values used in the majority of VERSE-LC column simulations reported within this report. As stated above, given the recent access to slurry data listed in Table 5-2, a consistency check of the porosity values chosen can be made.

Based on Eq. (5-2) the total porosity of a column is known once the particle and bed densities are specified. Using the values presented in the above subsections and listed in Tables 5-1 and 5-2, the total porosity becomes:

$$
\varepsilon_{\mathrm{T}} \equiv 1-\frac{\rho_{\mathrm{b}}}{\rho_{\mathrm{s}}},
$$

where several values are given in Table 5-3. In Table 5-3 four cases are considered. Based on the available data, the first case represents a lower bound, the second case a best estimate, and the third case an upper bound. The fourth case corresponds to the nominal value used in the column simulations reported with Chapters 9 and 10. As Table 5-3 indicates our nominal value for total porosity is greater than the expected range based on available data.

A range of possible void distributions exists between the bed and particle porosities constrained by Eq. (5-1) and any specified total porosity computed by Eq. (5-5). In Table 5-4 Eq. (5-1) is used to compute particle porosity for the four different resulting total porosity values tabulated in Table 5-3. The results provided in Table 5-4 are also plotted in Figure 5-1. In Figure 5-1 the solid curve represent our best estimate, while the solid circle represent our nominal porosity values used in the majority of VERSE-LC simulations. 
Also shown in Figure 5-1 is the average measured porosity for dense packing of mono-sized hard spheres (i.e., widely accepted value of 0.363 ) as reported by German (1989, page 106). Two thirds of the reported values lie between 0.356 and 0.364 . Greater packing fractions (or smaller bed porosities) can be achieved when multi-sized spheres are employed. Given the non-uniform sizes of the CST particles, no specific lower limit can be uniquely specified. However, the nominal bed porosity value of 0.50 versus the mono-sized value of 0.363 would appear to be somewhat high.

As stated above, the nominal bed porosity value of 0.50 and the nominal value for particle porosity of 0.24 were taken from the available literature. Based on Eq. (5-1) this corresponds to a nominal value for the total porosity of $62 \%$. However, our best estimate value for total porosity, based on measured densities, is $38 \%$. Notice that the nominal value of $38 \%$ and the best estimate value of $62 \%$ suggest that an error was made in the earlier literature work where in Eq. (5-2) the one minus total porosity term was assumed to be just total porosity. Assuming this incorrect form for Eq. (5-2) would give the $62 \%$ value based on the measured densities.

Table 5-1. Key CST exchange properties taken from literature for various ion exchangers.

\begin{tabular}{|c|c|c|c|c|c|c|}
\hline $\begin{array}{l}\text { Exchanger } \\
\text { Form }\end{array}$ & $\begin{array}{l}\text { Batch } \\
\text { Name }\end{array}$ & $\begin{array}{c}\text { F Factor }{ }^{a} \\
(-)\end{array}$ & $\begin{array}{c}\text { Bulk Dry } \\
\text { Density } \\
\left(\mathrm{g}_{\mathrm{CST}} / \mathrm{ml}_{\text {bed }}\right)\end{array}$ & $\begin{array}{l}\text { Avg. Particle } \\
\text { Diameter } \\
(\mu \mathrm{m})\end{array}$ & $\begin{array}{c}\text { Bed Porosity } \\
(-)\end{array}$ & $\begin{array}{c}\text { Particle Porosity } \\
(-)\end{array}$ \\
\hline SuperLig $^{\circledR} 644$ & $10-\mathrm{SM}-171$ & 0.9751 & 0.2238 & $664^{\mathrm{b}}$ & $0.450^{\mathrm{b}}$ & $0.614^{\mathrm{b}}$ \\
\hline Powder & IE-910 & 0.9680 & 0.7738 & 0.4 to $0.8^{\mathrm{d}}$ & $0.41^{\mathrm{e}}$ & $0.1^{\mathrm{e}}$ \\
\hline Engineered & $\begin{array}{l}\text { IE-911 } \\
(38 b)\end{array}$ & 0.8870 & 1.13 & - & - & - \\
\hline Engineered & IE-911 (08) & 0.8990 & 0.8999 & - & - & - \\
\hline Engineered & Nominal $^{\mathrm{d}}$ & 0.80 & 1.0 & 200 to 600 & 0.3 to $0.5^{\mathrm{e}}$ & $0.22^{\mathrm{e}}$ \\
\hline Engineered & Baseline & - & $\begin{array}{c}1.0 \\
(\text { avg) }\end{array}$ & $\begin{array}{c}344 \\
(\text { see Chapter 6) }\end{array}$ & $\begin{array}{c}0.50 \\
\text { (assumed) }^{\mathrm{c}}\end{array}$ & $\begin{array}{c}0.24 \\
\text { (assumed) }^{\mathrm{c}}\end{array}$ \\
\hline
\end{tabular}

${ }^{a}$ Data obtained from Brown et al. (1996, Table 3.1).

${ }^{\mathrm{b}}$ Data obtained from Hamm et al. (2000) based on available data and supporting analyses.

${ }^{c}$ These are values used in many of the existing column analyses to date reported by Hritzko et al. (1998) and many other sets of reported analyses throughout the literature. Within the Hritzko et al. (1998) report they reference their values to personal communications with R. A. Anthony at Texas A\&M University.

${ }^{d}$ The average properties of IONSIV ${ }^{\circledR}$ IE-911 exchanger as stated by UOP (1996).

${ }^{\mathrm{e}}$ Other porosity and particle sizes are given by Thibaud-Erkey and Anthony, 1999. 
Preliminary Ion Exchange Modeling for Removal of Cesium from

Revision (Date):

$0(07 / 05 / 02)$

Hanford Waste Using Hydrous Crystalline Silicotitanate Material

Page: 43 of 338

Table 5-2. Estimated particle density based on measured densities taken from slurry mixtures of CST and water by Qureshi (1999).

\begin{tabular}{|c|c|c|c|}
\hline Test ID & $\begin{array}{c}\text { CST } \\
\mathbf{w t} \%\end{array}$ & $\begin{array}{c}\text { Measured } \\
\text { slurry density } \\
(\mathbf{g} / \mathbf{m l})\end{array}$ & $\begin{array}{c}\text { Estimated } \\
\text { CST particle } \\
\mathbf{d e n s i t y} \\
\left(\mathbf{g}_{\text {CST }} / \mathbf{m l}_{\text {bed }}\right)\end{array}$ \\
\hline \hline 1 & 5 & 1.030 & 1.60 \\
\hline 2 & 10 & 1.067 & 1.67 \\
\hline 3 & 15 & 1.105 & 1.70 \\
\hline
\end{tabular}

Table 5-3. "Best estimate" and upper/lower bounds of total porosity or particle density based on available measured densities or bed/particle porosities for IE-911 CST material.

\begin{tabular}{|c|c|c|c|l|}
\hline $\begin{array}{c}\text { Parameter being } \\
\text { estimated }\end{array}$ & $\begin{array}{c}\text { Bed density } \\
\left(\mathbf{g}_{\mathbf{C S T}} / \mathbf{m l}_{\text {bed }}\right)\end{array}$ & $\begin{array}{c}\text { Particle density } \\
\left(\mathbf{g}_{\mathbf{C S T}} / \mathbf{m l}_{\text {bed }}\right)\end{array}$ & $\begin{array}{c}\text { Total } \\
\text { porosity } \\
(\%)\end{array}$ & \multicolumn{1}{|c|}{ Comments } \\
\hline \hline $\begin{array}{c}\text { Lower bound for } \\
\text { total porosity }\end{array}$ & 1.13 & 1.60 & 29.4 & $\begin{array}{l}\text { Lower estimated bound for total } \\
\text { porosity based on upper measured bed } \\
\text { density and lower measured particle } \\
\text { density. }\end{array}$ \\
\hline $\begin{array}{c}\text { Best estimate for } \\
\text { total porosity }\end{array}$ & 1.0 & 1.613 & 38.0 & $\begin{array}{l}\text { Best estimate value for total porosity } \\
\text { based on average measured bed and } \\
\text { particle densities. }\end{array}$ \\
\hline $\begin{array}{c}\text { Upper bound for } \\
\text { total porosity }\end{array}$ & 0.8 & 1.70 & 52.9 & $\begin{array}{l}\text { Upper estimated bound for total } \\
\text { porosity based on lower measured bed } \\
\text { density and upper measured particle } \\
\text { density. }\end{array}$ \\
\hline $\begin{array}{c}\text { Particle density } \\
\text { consistent with } \\
\text { current bed and } \\
\text { particle porosities }\end{array}$ & 1.0 & 2.632 & 62.0 & $\begin{array}{l}\text { Estimated value for particle density } \\
\text { based on average measured bed } \\
\text { density and a total porosity computed } \\
\text { using a bed porosity of 50\% and } \\
\text { particle porosity of 24\%. }\end{array}$ \\
\hline
\end{tabular}


Table 5-4. Particle porosity as a function of bed porosity at three different total porosity values for the IONSIV $^{\circledR}$ IE-911 CST material.

\begin{tabular}{|c|c|c|c|c|}
\hline $\begin{array}{c}\text { Bed porosity } \\
(-)\end{array}$ & $\begin{array}{c}\text { Particle porosity } \\
\text { given } \varepsilon_{\mathbf{T}}=\mathbf{0 . 2 9 4} \\
(-)\end{array}$ & $\begin{array}{c}\text { Particle porosity } \\
\text { given } \boldsymbol{\varepsilon}_{\mathbf{T}}=\mathbf{0 . 3 8 0} \\
(-)\end{array}$ & $\begin{array}{c}\text { Particle porosity } \\
\text { given } \boldsymbol{\varepsilon}_{\mathbf{T}}=\mathbf{0 . 5 2 9} \\
(-)\end{array}$ & $\begin{array}{c}\text { Particle porosity }^{\text {a }} \\
\text { given } \boldsymbol{\varepsilon}_{\mathbf{T}}=\mathbf{0 . 6 2 0} \\
(-)\end{array}$ \\
\hline \hline 0.00 & 0.294 & 0.380 & 0.529 & 0.620 \\
\hline 0.05 & 0.257 & 0.347 & 0.505 & 0.600 \\
\hline 0.15 & 0.169 & 0.271 & 0.446 & 0.553 \\
\hline 0.20 & 0.117 & 0.225 & 0.412 & 0.525 \\
\hline 0.25 & 0.058 & 0.173 & 0.373 & 0.493 \\
\hline 0.30 & na & 0.114 & 0.328 & 0.457 \\
\hline 0.35 & na & 0.046 & 0.276 & 0.415 \\
\hline 0.40 & na & na & 0.216 & 0.367 \\
\hline 0.45 & na & na & 0.144 & 0.309 \\
\hline 0.50 & na & na & 0.059 & 0.240 \\
\hline 0.55 & na & na & na & 0.156 \\
\hline 0.60 & na & na & na & 0.050 \\
\hline 0.65 & na & na & na & na \\
\hline Bed density $(\mathrm{g} / \mathrm{ml})=$ & 1.13 & 1.0 & 0.8 & 1.0 \\
\hline Particle density $(\mathrm{g} / \mathrm{ml})=$ & 1.6 & 1.613 & 1.7 & 2.632 \\
\hline
\end{tabular}

${ }^{a}$ This total porosity value corresponds to the value used in the majority of column simulations performed.

b This total porosity value corresponds to the value most consistent with the available data. 
Report:

WSRC-TR-2001-00400

Preliminary Ion Exchange Modeling for Removal of Cesium from Hanford Waste Using Hydrous Crystalline Silicotitanate Material
Revision (Date):

Page:
$0(07 / 05 / 02)$ 45 of 338

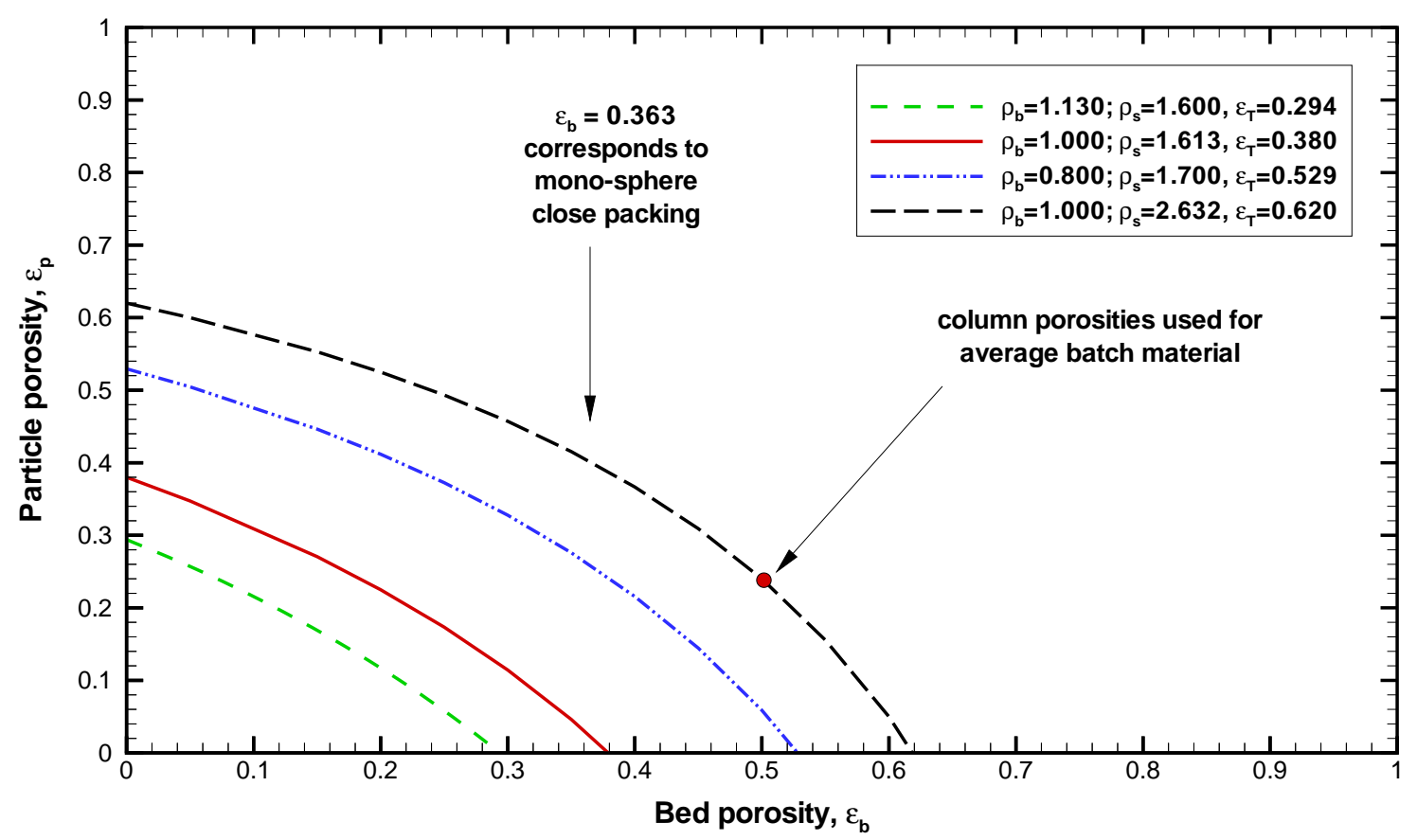

Figure 5-1. Functional behavior between bed porosity and particle porosity for the IONSIV ${ }^{\circledR}$ IE-911 CST material for various assumed total porosity values. The nominal settings used for the majority of column studies performed are shown by the solid circle. 


\subsection{Particle Size Distributions}

Since CST exchanger material has been determined to be mass transfer limited, as a result of pore diffusion, the particle size of its engineered forms is important. The rate of kinetics and particle size are inversely related. The commercial development of IE-911 exchanger has focused on achieving optimum mass transfer efficiency through varying particle size (see UOP, 1996). Based on optimization studies, where a balance between ion exchange kinetics and design engineering requirements (e.g., column pressure drop) was achieved, 30x60 mesh (i.e., 250 to $595 \mu \mathrm{m}$ diameter) particles produce an engineering optimum (see Miller and Brown, 1997). Unlike organic ion exchange resins, the rigid inorganic structure of IE-910 and IE-911 materials resist significant swelling or shrinkage due to changes in temperature, $\mathrm{pH}$, and ion exchange levels.

The particle size distribution, and therefore its average, vary between batches. For example, for cesium removal assessments the following average particle radiuses have been used in previous column performance analyses :

- $187.5 \mu \mathrm{m}$ by Hritzko et al. (1998) for the design of a carousel process for handling SRS waste; and

- $\quad 123.0 \mu \mathrm{m}$ by Thibaud-Erkey and Anthony for evaluating laboratory-scale experiments (1999).

Since the particle size distribution varies with batch ID, "as received" Baseline IE-911 material was analyzed using more recent laser technologies. Based on the laser testing a mean particle diameter of $172 \mu \mathrm{m}$ has been chosen for use in the column transport analysis efforts presented within this report. Uncertainties in this mean diameter exist resulting from potential batch variability and the issue of how representative is a particular tested sample to the entire batch population.

The CST exchanger material is approximately spherical, as assumed in the governing equations used to model the ion-exchange process. The column models also assume that the mass transfer and pore diffusion processes can be modeled using a single average size particle to represent the entire population of CST particles. Given the level of knowledge with regard to particle size and shape distributions, the above assumptions should be acceptable.

\subsection{MicroTrac ${ }^{\circledR}$ Laser Technology Data}

Based on MicroTrac ${ }^{\circledR}$ technology particle size distributions have been measured by Qureshi (1999) during a mixing and sampling study of sludge-frit-CSR slurries. In particluar, a particle size distribution is provided in Qureshi (1999) for "as received" CST in its engineered-form (batch number not recorded). This sample contained CST only. Percent by volume for a series of particle size ranges are provided by Qureshi (1999, see Figure 9). These volume fractions were first normalized where the average particle radius for each size bracket was set to the 
bracket's mid-point value. The resulting normalized volume fractions are then used as weights for estimating the mean particle radius for this sample.

The results of these conversions are tabulated in Table 6-1. The volume fraction measurements for this sample are also plotted in Figure 6-1. An average particle radius of $163 \mu \mathrm{m}$ was computed for this sample.

\section{2 $\quad$ Lasentec $^{\circledR}$ Laser Technology Data}

Chord length distributions have been measured on a single CST sample with a Lasentec ${ }^{\circledR}$ FBRM at the Savannah River Technology Center. The mean chord length measured for the "asreceived", engineered-form of CST (IE-911, Baseline Lot \#9090-76) by FBRM was $312 \mu \mathrm{m}$. Based on the probabilities of measuring chords that are not representative of the full particle diameter (such as particle edges), a standard correction of $+10 \%$ is often applied to the mean chord length value obtained for approximately spherical particles. (Note: The true-mean chord length is expected to be in the range $310-390 \mu \mathrm{m}$.) After correction, the mean chord length is estimated to be $343 \mu \mathrm{m}$, which corresponds to a mean particle radius of $172 \mu \mathrm{m}$. Chord length data below $70 \mu \mathrm{m}$ was not utilized for the calculation of the mean value, since these fine particles are generally readily washed away from the CST bed prior to or during column operation.

For plotting purposes only, the chord length data were normalized where the average particle diameter for each size bracket was set to the bracket's mid-point value. The chord length distribution for this sample is also plotted in Figure 6-2 and tabulated in Table 6-2. The results of the chord length to particle diameter conversions are tabulated in Table 6-3. The calculated mean chord length is based on the raw data prior to normalization.

From these measurements an estimate of the average particle radius of $\sim 172 \mu \mathrm{m}$ has been determined. All column assessment and column design analyses presented in this report use this particle radius value when columns packed with IE-911 material are being considered. 
Table 6-1. Particle size distributions ${ }^{\text {a }}$ of CST material in its engineered-form (batch number not recorded) based on MicroTrac ${ }^{\circledR}$ Laser Technology.

\begin{tabular}{|c|c|c|}
\hline $\begin{array}{c}\text { Particle diameter } \\
\text { range } \\
\text { [channels] } \\
(\boldsymbol{\mu m})\end{array}$ & $\begin{array}{c}\text { Particle } \\
\text { diameter } \\
\text { midpoint } \\
(\mu \mathbf{m})\end{array}$ & $\begin{array}{c}\text { Sample 1 } \\
\text { volume \% } \\
\text { [normalized] }\end{array}$ \\
\hline \hline$<22.00^{\mathrm{b}}$ & - & 0.00 \\
\hline $22.00-31.11$ & 26.6 & 0.29 \\
\hline $31.11-44.00$ & 37.6 & 0.70 \\
\hline $44.00-62.23$ & 53.1 & 1.37 \\
\hline $62.23-88.00$ & 75.1 & 2.31 \\
\hline $88.00-124.50$ & 106.3 & 4.15 \\
\hline $124.50-176.00$ & 150.3 & 5.19 \\
\hline $176.00-248.90$ & 212.5 & 13.21 \\
\hline $248.90-352.00$ & 300.5 & 30.63 \\
\hline $352.00-497.80$ & 424.9 & 35.87 \\
\hline $497.80-704.00$ & 600.9 & 6.28 \\
\hline \multicolumn{2}{|c|}{ Mean Radius $(\mu \mathbf{m})=$} & \\
\hline
\end{tabular}

${ }^{a}$ Laser analysis performed on "as received" CST material.

${ }^{\mathrm{b}}$ No particles detected where operating channels as low as $\sim 1 \mu \mathrm{m}$ were employed.

Table 6-2. Chord size distributions ${ }^{a}$ of CST material in its engineered-form (Baseline Lot \#9090-76) based on Lasentec ${ }^{\circledR}$ Laser Technology.

\begin{tabular}{|c|c|c|c|}
\hline $\begin{array}{l}\text { Chord length } \\
\text { lower channel } \\
\text { bound } \\
(\mu \mathrm{m})\end{array}$ & $\begin{array}{l}\text { Chord length } \\
\text { upper channel } \\
\text { bound } \\
(\mu \mathrm{m})\end{array}$ & $\begin{array}{l}\text { Chord } \\
\text { length } \\
\text { channel } \\
\text { midpoint } \\
(\mu \mathrm{m})\end{array}$ & $\begin{array}{c}\text { Sample } 1 \\
\text { chord \% } \\
\text { [normalized] }\end{array}$ \\
\hline 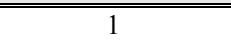 & $\begin{array}{l}1.08 \\
\end{array}$ & "1.0400 & 0.001 \\
\hline 1.08 & 1.166 & 1.1230 & 0.001 \\
\hline 1.166 & 1.259 & 1.2125 & 0.001 \\
\hline 1.259 & 1.359 & 1.3090 & 0.002 \\
\hline 1.359 & 1.468 & 1.4135 & 0.003 \\
\hline 1.468 & 1.585 & 1.5265 & 0.003 \\
\hline 1.585 & 1.711 & 1.6480 & 0.003 \\
\hline 1.711 & 1.848 & 1.7795 & 0.004 \\
\hline 1.848 & 1.995 & 1.9215 & 0.005 \\
\hline 1.995 & 2.154 & 2.0745 & 0.006 \\
\hline 2.154 & 2.326 & 2.2400 & 0.007 \\
\hline 2.326 & 2.512 & 2.4190 & 0.008 \\
\hline 2.512 & 2.712 & 2.6120 & 0.017 \\
\hline 2.712 & 2.929 & 2.8205 & 0.013 \\
\hline 2.929 & 3.162 & 3.0455 & 0.023 \\
\hline 3.162 & 3.415 & 3.2885 & 0.029 \\
\hline 3.415 & 3.687 & 3.5510 & 0.033 \\
\hline 3.687 & 3.981 & 3.8340 & 0.033 \\
\hline 3.981 & 4.299 & 4.1400 & 0.043 \\
\hline 4.299 & 4.642 & 4.4705 & 0.046 \\
\hline 4.642 & 5.012 & 4.8270 & 0.055 \\
\hline 5.012 & 5.412 & 5.2120 & 0.066 \\
\hline 5.412 & 5.844 & 5.6280 & 0.068 \\
\hline 5.844 & 6.31 & 6.0770 & 0.065 \\
\hline 6.31 & 6.813 & 6.5615 & 0.07 \\
\hline
\end{tabular}


WESTINGHOUSE SAVANNAH RIVER COMPANY

Report: WSRC-TR-2001-00400

Preliminary Ion Exchange Modeling for Removal of Cesium from

Revision (Date):

$0(07 / 05 / 02)$

Hanford Waste Using Hydrous Crystalline Silicotitanate Material

Page:

50 of 338

\begin{tabular}{|c|c|c|c|}
\hline $\begin{array}{l}\text { Chord length } \\
\text { lower channel } \\
\text { bound } \\
(\mu \mathrm{m})\end{array}$ & $\begin{array}{l}\text { Chord length } \\
\text { upper channel } \\
\text { bound } \\
(\mu \mathrm{m})\end{array}$ & $\begin{array}{c}\text { Chord } \\
\text { length } \\
\text { channel } \\
\text { midpoint } \\
(\mu \mathrm{m})\end{array}$ & $\begin{array}{c}\text { Sample } 1 \\
\text { chord \% } \\
\text { [normalized] }\end{array}$ \\
\hline 6.813 & 7.357 & 7.0850 & 0.077 \\
\hline 7.357 & 7.944 & 7.6505 & 0.088 \\
\hline 7.944 & 8.578 & 8.2610 & 0.097 \\
\hline 8.578 & 9.262 & 8.9200 & 0.123 \\
\hline 9.262 & 10.001 & 9.6315 & 0.13 \\
\hline 10.001 & 10.799 & 10.4000 & 0.143 \\
\hline 10.799 & 11.66 & 11.2295 & 0.136 \\
\hline 11.66 & 12.59 & 12.1250 & 0.173 \\
\hline 12.59 & 13.595 & 13.0925 & 0.179 \\
\hline 13.595 & 14.679 & 14.1370 & 0.195 \\
\hline 14.679 & 15.85 & 15.2645 & 0.191 \\
\hline 15.85 & 17.115 & 16.4825 & 0.221 \\
\hline 17.115 & 18.48 & 17.7975 & 0.228 \\
\hline 18.48 & 19.954 & 19.2170 & 0.235 \\
\hline 19.954 & 21.546 & 20.7500 & 0.245 \\
\hline 21.546 & 23.265 & 22.4055 & 0.268 \\
\hline 23.265 & 25.121 & 24.1930 & 0.273 \\
\hline 25.121 & 27.125 & 26.1230 & 0.314 \\
\hline 27.125 & 29.289 & 28.2070 & 0.365 \\
\hline 29.289 & 31.626 & 30.4575 & 0.33 \\
\hline 31.626 & 34.149 & 32.8875 & 0.388 \\
\hline 34.149 & 36.873 & 35.5110 & 0.376 \\
\hline 36.873 & 39.815 & 38.3440 & 0.355 \\
\hline 39.815 & 42.991 & 41.4030 & 0.347 \\
\hline 42.991 & 46.421 & 44.7060 & 0.338 \\
\hline 46.421 & 50.125 & 48.2730 & 0.317 \\
\hline 50.125 & 54.123 & 52.1240 & 0.327 \\
\hline 54.123 & 58.441 & 56.2820 & 0.36 \\
\hline 58.441 & 63.104 & 60.7725 & 0.356 \\
\hline 63.104 & 68.138 & 65.6210 & 0.37 \\
\hline 68.138 & 73.574 & 70.8560 & 0.414 \\
\hline 73.574 & 79.443 & 76.5085 & 0.457 \\
\hline 79.443 & 85.781 & 82.6120 & 0.491 \\
\hline 85.781 & 92.624 & 89.2025 & 0.569 \\
\hline 92.624 & 100.014 & 96.3190 & 0.635 \\
\hline 100.014 & 107.993 & 104.0035 & 0.677 \\
\hline 107.993 & 116.608 & 112.3005 & 0.863 \\
\hline 116.608 & 125.911 & 121.2595 & 0.948 \\
\hline 125.911 & 135.956 & 130.9335 & 1.188 \\
\hline 135.956 & 146.802 & 141.3790 & 1.317 \\
\hline 146.802 & 158.513 & 152.6575 & 1.692 \\
\hline 158.513 & 171.159 & 164.8360 & 1.919 \\
\hline 171.159 & 184.814 & 177.9865 & 2.314 \\
\hline 184.814 & 199.558 & 192.1860 & 2.934 \\
\hline 199.558 & 215.478 & 207.5180 & 3.313 \\
\hline 215.478 & 232.668 & 224.0730 & 4.057 \\
\hline 232.668 & 251.23 & 241.9490 & 4.989 \\
\hline 251.23 & 271.273 & 261.2515 & 5.996 \\
\hline 271.273 & 292.914 & 282.0935 & 6.972 \\
\hline 292.914 & 316.282 & 304.5980 & 7.876 \\
\hline 316.282 & 341.514 & 328.8980 & 8.268 \\
\hline 341.514 & 368.759 & 355.1365 & 7.978 \\
\hline 368.759 & 398.178 & 383.4685 & 7.039 \\
\hline 398.178 & 429.944 & 414.0610 & 5.847 \\
\hline 429.944 & 464.244 & 447.0940 & 4.454 \\
\hline 464.244 & 501.28 & 482.7620 & 3.219 \\
\hline 501.28 & 541.271 & 521.2755 & 2.111 \\
\hline
\end{tabular}




\begin{tabular}{|c|c|c|c|}
\hline $\begin{array}{c}\text { Chord length } \\
\text { lower channel } \\
\text { bound } \\
(\boldsymbol{\mu m})\end{array}$ & $\begin{array}{c}\text { Chord length } \\
\text { upper channel } \\
\text { bound } \\
(\boldsymbol{\mu m})\end{array}$ & $\begin{array}{c}\text { Chord } \\
\text { length } \\
\text { channel } \\
\text { midpoint } \\
(\boldsymbol{\mu m})\end{array}$ & $\begin{array}{c}\text { Sample 1 } \\
\text { chord \% } \\
\text { [normalized] }\end{array}$ \\
\hline 541.271 & 584.452 & 562.8615 & 1.3 \\
\hline 584.452 & 631.079 & 607.7655 & 0.774 \\
\hline 631.079 & 681.425 & 656.2520 & 0.487 \\
\hline 681.425 & 735.787 & 708.6060 & 0.294 \\
\hline 735.787 & 794.486 & 765.1365 & 0.205 \\
\hline 794.486 & 857.869 & 826.1775 & 0.127 \\
\hline 857.869 & 926.307 & 892.0880 & 0.063 \\
\hline 926.307 & 1000.206 & 963.2565 & 0.039 \\
\hline 1000.206 & 1080 & 1040.1030 & 0.023 \\
\hline \multicolumn{2}{|c|}{ Mean Radius $(\mu \mathbf{m}){ }^{\mathrm{b}}=$} & \multicolumn{2}{|c|}{156.0} \\
\hline
\end{tabular}

" Laser analysis performed on "as received" CST material.

${ }^{\mathrm{b}}$ No chord lengths below $\sim 73.5 \mu \mathrm{m}$ were employed in computing mean radius value.

Table 6-3. Estimated average particle radius of CST material in its engineered-form (Baseline Lot \#909076) based on Lasentec ${ }^{\circledR}$ Laser Technology.

\begin{tabular}{|c|c|c|c|}
\hline Parameter & Statistic 1 & Statistic 2 & Statistic 3 \\
\hline $\begin{array}{c}\text { Chord Mean } \\
\text { (No Wt) }\end{array}$ & $\begin{array}{c}\text { Chord Mean } \\
\text { (Sqr Wt) }\end{array}$ & $\begin{array}{c}\text { Chord Mode } \\
\text { (No Wt) }\end{array}$ \\
\hline $\begin{array}{c}\text { Micron range } \\
\text { (lower) }\end{array}$ & 73.574 & 73.574 & 73.574 \\
\hline $\begin{array}{c}\text { Micron range } \\
\text { (upper) }\end{array}$ & 1000.206 & 1000.206 & 1000.206 \\
\hline $\begin{array}{c}\text { Chord Statistic } \\
\text { (micron) }\end{array}$ & 311.576 & 402.171 & 328.656 \\
\hline $\begin{array}{c}\text { Diameter to chord } \\
\text { factor (Typical } \\
\text { value) }\end{array}$ & 1.1 & 1.1 & 1.1 \\
\hline $\begin{array}{c}\text { Diameter Statistic } \\
\text { (micron) }\end{array}$ & 342.7 & 442.4 & 361.5 \\
\hline $\begin{array}{c}\text { Avg Radius Statistic } \\
\text { (micron) }\end{array}$ & $\sim 172^{\mathrm{a}}$ & $\sim 221$ & $\sim 181$ \\
\hline
\end{tabular}

${ }^{\text {a }}$ Value used for all column assessment and design analysis for CST IE-911 material. 


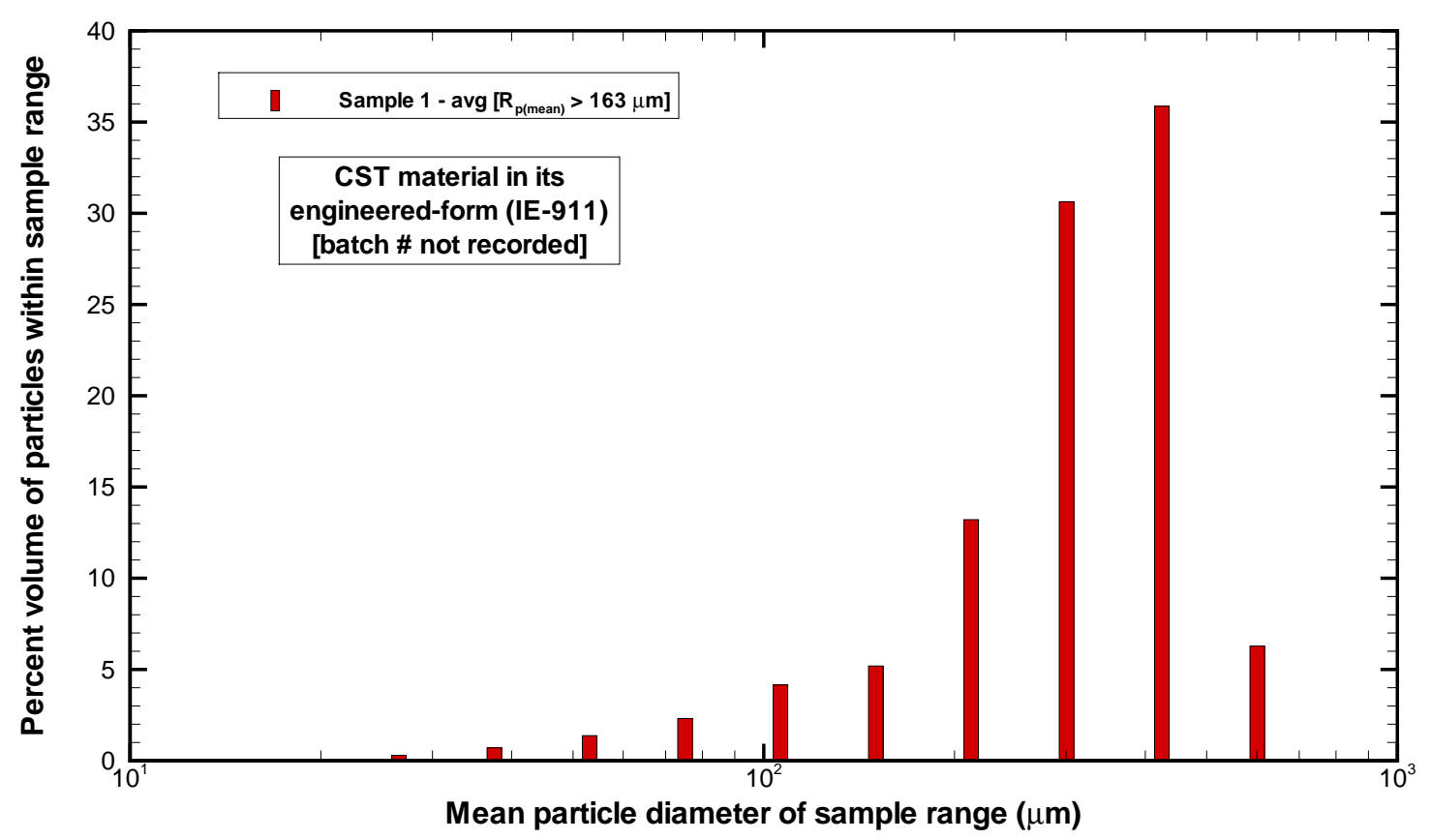

Figure 6-1. Volume percent of particles as a function of particle size for one sample of the CST material in its IE-911 engineered-form (batch \# not recorded) based on MicroTrac ${ }^{\circledR}$ laser technology (data from Qureshi, 1999).

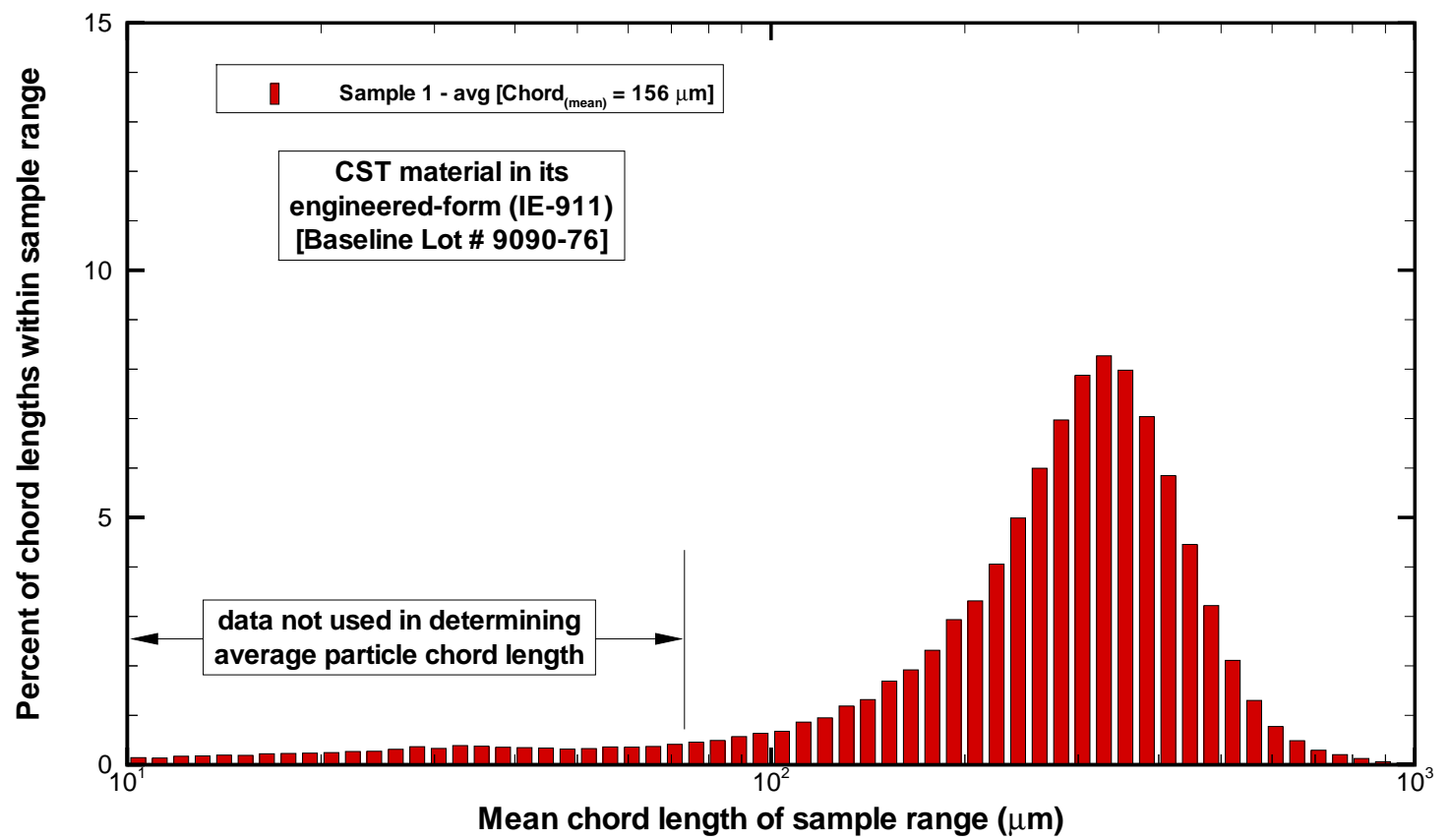

Figure 6-2. Chord length percent of particles as a function of chord size for one sample of the CST material in its IE-911 engineered-form (Baseline Lot \# 9090-76) based on Lasentec ${ }^{\circledR}$ laser technology. 


\subsection{Pore Diffusion}

As mentioned earlier we assume that the local rate of chemical adsorption (i.e., exchange of ions at a surface site) is very fast when compared to the rates of diffusion within the pore fluid and mass transfer across the liquid film at the outer boundary of the solid particles. In this section we discuss the "effective" binary molecular (Brownian motion) diffusion and pore diffusion coefficients for cesium used in the column models. In general the molecular diffusion coefficient depends upon the ionic composition of the surrounding fluid and its temperature, while the pore diffusion coefficient depends upon the internal structure of the porous solid. Here, our approach is to compute molecular diffusion coefficients based on key ionic species and then to compute pore diffusion coefficients assuming that a fixed ratio of molecular-to-pore diffusion exits. This molecular-to-pore diffusion ratio is typically referred to as a particle tortuosity factor, and for our purposes the value chosen is considered to be a true constant (i.e., in practice this is a geometric factor that probably varies depending upon which engineered-form is being considered). We also assumed that the rates of mass transfer within the CST particles are direction independent (i.e., incoming or outgoing).

In summary, we see inconsistencies among the available batch contact test data and deficiencies with using the homogeneous particle concept (and perhaps without addressing surface diffusion) currently within VERSE-LC. As such the particle tortuosity factor chosen (i.e., 5.0) for our design efforts is not based on these batch kinetics tests, but rather is based on the assessment of available column tests as discussed in Chapter 9. Future efforts to determine the important diffusional aspects of CST in its most common engineered-forms are recommended.

After writing this chapter the work of Anthony et al. (2001) was provided to the authors. Anthony et al. (2001) attempt to estimate an average value of the pore diffusivity coefficient for cesium based on their simple simulant (i.e. contains $\sim 5.7 \mathrm{M}$ sodium). The average value given is $1.9 \times 10^{-4} \mathrm{~cm}^{2} / \mathrm{min}$. This value is approximately a factor of two greater than the values estimated for the Phase 1 LAW feeds, but is within the uncertainty of the method and compositional differences of the solutions. It is interesting to note that their basic approach was to estimate a tortuosity factor of 4 (versus our value of 5) and to use viscosity corrections similar to our method. Both approaches are very similar in nature.

Future CST efforts should focus on determining a better particle diffusion model where pore heterogeneity (i.e., pore size distribution) and surface migration are accounted for along with pore diffusion.

\subsection{Waste Solution Density and Viscosity}

Waste solution density and viscosity varies depending primarily on temperature, sodium content, and composition. As discussed in Appendix A, a modified HTWOS density model is presented that estimates waste solution density typically within a few percent. This correlation is verified by comparison to measured values. For fluid dynamic viscosity few measurements exist and for the Phase 1 LAW batch feeds a constant value is employed based on a measurement of a 
Envelope A simulant. The fluid properties for this Envelope A simulated waste were measured and reported by Steimke et al. (2000). The measured values of density and dynamic viscosity for the simulated waste and for pure water are listed in Table 7-1 at $20{ }^{\circ} \mathrm{C}$. Dynamic viscosity values for the waste at $25{ }^{\circ} \mathrm{C}$ were estimated based on the measured pure water values also provided in Table 7-1.

Column transport assessments are also considered for various other waste solutions and their fluid properties are required. For the SRS solutions considered measured density and viscosity values are provided in Table 7-2.

\subsection{Molecular Diffusion Coefficients}

Binary diffusion (sometimes referred to as free stream or Brownian motion) coefficients of electrolytes originating from a single salt in solution under dilute conditions can be reasonably estimated by the Nernst-Haskell equation (Reid et al., 1977):

$$
\mathrm{D}_{ \pm}^{\infty}=\left(\frac{\mathrm{RT}}{F^{2}}\right)\left[\frac{\frac{1}{\mathrm{z}_{+}}+\frac{1}{\mathrm{z}_{-}}}{\frac{1}{\lambda_{+}^{\mathrm{o}}}+\frac{1}{\lambda_{-}^{\mathrm{o}}}}\right],
$$

where

$\mathrm{D}_{ \pm}^{\infty} \quad$ - binary diffusion coefficient at infinite dilution, $\mathrm{cm}^{2} / \mathrm{s}$

$\lambda_{+}^{\mathrm{o}}, \lambda_{-}^{\mathrm{o}}$ - limiting ionic conductance for cation and anion, mhos/equivalent

$\mathrm{z}_{+}, \mathrm{z}_{-}$- valences of cation and anion, respectively

$F \quad$ - Faraday constant, 96,500 C/g-equivalent

$\mathrm{R}$ - gas constant, $8.314 \mathrm{~J} / \mathrm{gmole}-\mathrm{K}$

$\mathrm{T} \quad$ - absolute temperature, $\mathrm{K}$

Limiting ionic conductance for the various ions of interest are tabulated in Table 7-3 (Reid et al., 1977; Perry, 1973). Using Eq. (7-1) and the limiting ionic conductances provided in Table 7-3, the binary molecular diffusion coefficient for certain single salts within an aqueous phase can be computed. To account for fluid property differences between a waste solution and pure water, a correction factor is applied. Based on a hydrodynamical theory the following expression typically referred to as the Stokes-Einstein equation is obtained (Bird et al., 1960, page 514):

$$
\frac{\mathrm{D}_{\mathrm{AB}} \mu_{\mathrm{B}}}{\kappa \mathrm{T}}=\frac{1}{6 \pi \mathrm{R}_{\mathrm{A}}},
$$

where

$$
\begin{array}{ll}
D_{A B} & - \text { binary diffusion coefficient for A diffusing through solvent } B \\
\mu_{B} & - \text { dynamic viscosity of solvent mixture } \\
R_{A} & - \text { radius of diffusing particle } \\
\kappa & - \text { Boltzmann's constant }
\end{array}
$$


Based on Eq. (7-2), the ratio of dynamic viscosities (i.e., pure water versus waste solution) is a correction factor that can be applied to the computed molecular diffusion coefficients from Eq. (7-1).

Binary pairs for the dominant cation-anion pairs (i.e., cesium paired with an individual anion) where the anions considered are based on the composition of the various wastes, and their computed binary molecular diffusion coefficients are listed in Table 7-4 at $25 \mathrm{C}$. The viscosity corrected values are also provided in Table 7-4. In Cussler (1984) the "effective" binary ionic diffusion coefficient of cesium in essentially pure water is $1.236 \times 10^{-3} \mathrm{~cm}^{2} / \mathrm{min}$ at $25^{\circ} \mathrm{C}$, which is within $16 \%$ of the estimated value computed from Eq. (7-1). Some other values based on Cussler (1984) at $25 \mathrm{C}$ are $7.98 \times 10^{-4} \mathrm{~cm}^{2} / \mathrm{min}$ for $\mathrm{Na}^{+}, 1.176 \times 10^{-3} \mathrm{~cm}^{2} / \mathrm{min}$ for $\mathrm{K}^{+}$, and $5.586 \times 10^{-}$ ${ }^{3} \mathrm{~cm}^{2} / \min$ for $\mathrm{H}^{+}$.

The ionic radii of sodium and potassium are 0.95 and 1.33 angstroms with average hydration numbers of 4 and 3, respectively. All metal cations are hydrated in aqueous media, where, for example, sodium migrates perhaps in the form $\mathrm{Na}\left(\mathrm{H}_{2} \mathrm{O}\right)_{4}{ }^{+}$. Reasonable values for the radii of the hydrated alkali metal ions sodium and potassium are $\sim 2.76$ and $\sim 2.32$ angstroms (Peters et al., 1974), respectively. Therefore, sodium is bigger and slower moving than potassium in aqueous solutions consistent with the predictions listed in Table 7-3. Anions are characteristically less heavily hydrated, suggesting that the cations set the overall diffusional pace.

Molecular diffusion coefficients are important in determining key dimensionless numbers (e.g., Schmidt Number, Sc) used in various constitutive law correlations pertinent to column transport modeling. They also provide an upper bound for pore diffusion coefficients.

\subsection{Pore Diffusion Coefficients}

Within the complicated pore structure of the CST particles we assume that net fluid motion is approximately zero resulting in equal-molar counter-diffusion. "Effective" binary diffusion coefficients based on Fick's law are assumed where surface migration is considered negligible. Surface migration generally becomes increasingly more important as the migrating ions increase in size relative to the pore aperture (Froment and Bishoff, 1979). Knudsen diffusion occurs when the molecular dimensions of the ions approach their mean free path lengths.

\subsubsection{CST Conceptual Model}

CST in its engineered-forms is made up of CST powder (i.e., very fine particles on the order of $0.8 \mu \mathrm{m}$ in diameter) packed together using an inert binder (i.e., $20 \%$ to $30 \%$ by mass) to form much larger particles (e.g., the Baseline material has an average particle diameter of $344 \mu \mathrm{m}$ ). The pore porosity of the CST powder has been estimated to be $\sim 0.1$ by ? (), while for CST in its engineered-forms a typical value of $\sim 0.24$ has been recommended by ? (). The fabrication process itself would suggest that the engineered particles are heterogeneous, potentially containing two pore size distributions. The diffusional rates within each pore distribution could be significantly different. For example, in the larger pores created by the binding material diffusion rates might be larger than the diffusional rates within the smaller pores contained 
within the CST powder itself. Below, kinetics tests comparisons suggest that this may in fact be happening.

It has been observed by Ma et al. (1996) and Robinson et al. (1994) that for ion exchange materials with high capacities and affinities for certain ions (who generally have non-linear isotherms), parallel pore and surface diffusion is occurring. Based on equilibrium ion-exchange, Ma et. al. (1996) have derived an expression for an apparent pore diffusion coefficient that handles both pore and surface diffusion given as:

$$
\mathrm{D}_{\mathrm{p}, \text { app }}=\mathrm{D}_{\mathrm{p}}+\left[\frac{1-\varepsilon_{\mathrm{p}}}{\varepsilon_{\mathrm{p}}}\right] \mathrm{D}_{\mathrm{s}}\left\{\frac{\partial \mathrm{q}}{\partial \mathrm{c}_{\mathrm{p}}}\right\},
$$

where the equilibrium isotherm is $\mathrm{q}=\mathrm{f}\left(\mathrm{c}_{\mathrm{p}}\right)$.

For linear isotherms Eq. (7-3) shows that a constant apparent pore diffusion coefficient can be used to model the parallel mechanisms. Unfortunately, when the isotherms are non-linear this apparent diffusion coefficient becomes concentration dependent. For the favorable isotherms exhibited by CST material and at low cesium concentrations the isotherm is nearly linear where the apparent diffusion coefficient is nearly constant. In this concentration range a pore diffusion model using a constant apparent diffusion coefficient value can adequately model parallel diffusion. However, at higher cesium concentrations the apparent pore diffusion coefficient decreases with increasing concentration. Surface diffusion can play a significant part when high affinity for an ion exists and the intra-particle diffusion cannot be adequately described by a constant apparent diffusion coefficient.

The VERSE-LC code is conceptually based on homogeneous particles where only a single averaged sized homogenous particle is modeled. Heterogeneity within the engineered CST particles can not be directly addressed. The VERSE-LC does have a surface migration option, but on several attempts to use this more recent option the results appeared to be in error. Therefore, for CST column modeling we have chosen to attempt to fit an average apparent diffusion coefficient to the available batch kinetics data. Below the results of this fitting effort are discussed.

\subsubsection{The Tortuosity Factor}

Specific information on the actual pore sizes was not available at the time of this report. Pore sizes within the CST powder are believed to be much smaller than pores within the binding matrix. UOP vendor information suggests that the pore sizes are large relative to the size of migrating ions of interest and that pore diffusion coefficients should not be significantly lower than their molecular values. However, some level of reduction is expected resulting from bends along the pore paths that are generally accounted for by a particle tortuosity factor (Smith, 1981; Froment and Bishoff, 1979) defined as:

$$
\mathrm{D}_{\mathrm{pi}} \cong \frac{1}{\tau_{\mathrm{p}}} \mathrm{D}_{\infty \mathrm{i}},
$$


Typical values for catalyst particle tortuosity are between 2 and 8 . For large pore diameters the tortuosity values range as low as from near 1 to 3. For small ions Mackie and Meares (1955) suggest that the intra-particle pore diffusion coefficient can be estimated from

$$
\mathrm{D}_{\mathrm{pi}} \cong\left[\frac{\varepsilon_{\mathrm{p}}}{\left(2-\varepsilon_{\mathrm{p}}\right)^{2}}\right] \mathrm{D}_{\infty \mathrm{i}} \text {. }
$$

Based on the available particle porosity values for CST powder and engineered forms, Eq. (7-4) yields particle tortuosity factors of $\sim 8.1$ and $\sim 2.4$, respectively. These particle tortuosity factors suggest pore diffusion coefficients of $\sim 12 \%$ (for powder-form) and $\sim 42 \%$ (for engineered-forms) of the molecular value. Given the heterogeneous nature of the actual engineered-forms the average percentage value probably lies within this $12 \%$ to $42 \%$ range.

For larger molecules pore diffusion coefficient experiments are generally required. Ideally, pore diffusion coefficients can be derived from batch kinetics distribution studies where the mass transfer resistance associated with film diffusion is minimized through significant mechanical mixing (note that, mixing techniques must be limited such that physical grinding of the particles does not become excessive). Various time-dependent cesium distribution studies have been performed using both CST powder and several of its engineered forms.

Batch kinetics tests, where inadequate mixing is achieved between the solid and solution, result in overall diffusion coefficients that are in effect smaller than the actual pore diffusion coefficient. Therefore, the use of the batch kinetics data can provide us with a lower bound estimate of pore diffusion, while molecular diffusion becomes our upper bound. Sufficient mechanical mixing during these tests is assumed, such that the kinetics data measured represent only the mass transfer resistance associated with pore diffusion (i.e., mass transfer resistance due to film diffusion is assumed to be small).

\subsubsection{Comparison to Previous Studies}

Pretest predictions for three SRS column experiments (Walker et al., 1998) were performed by Texas A\&M University (see Appendix B of Walker et al., 1998). An available "effective" cesium pore diffusion coefficient value of $2.7 \times 10^{-11} \mathrm{~m}^{2} / \mathrm{s}$ (i.e., $1.62 \times 10^{-5} \mathrm{~cm}^{2} / \mathrm{min}$ ) based on standard simulant was assumed to be acceptable for use with the actual SRS-averaged simulant. The "effective" diffusion coefficient is related to the pore diffusion coefficient by:

$$
\left(\mathrm{D}_{\mathrm{pi}}\right)_{\text {effective }} \equiv \varepsilon_{\mathrm{p}} \mathrm{D}_{\mathrm{pi}} \text {. }
$$

For application to CST material in its engineered forms a typical pore porosity of $24 \%$ is assumed. Based on a $24 \%$ pore porosity, the Texas A\&M pore diffusion coefficient becomes $6.75 \times 10^{-5} \mathrm{~cm}^{2} / \mathrm{min}$ as compared to the estimated value of $\sim 10.0 \times 10^{-5} \mathrm{~cm}^{2} / \mathrm{min}$ based on the approach presented in this report (see SRS simulant values in Table 7-9). Texas A\&M have also reported pore diffusion coefficient values of up to $8.75 \times 10^{-5} \mathrm{~cm}^{2} / \mathrm{min}$ for standard simulants (with $0.5 \mathrm{M} \mathrm{K}$ added) based on column studies. 


\subsection{VERSE-LC Simulations for Batch Kinetics Tests}

To estimate a cesium diffusion coefficient using batch kinetics test data, VERSE-LC models were set up consistent with the experimental conditions specified by their investigators:

- Initially CST material is placed into contact with liquid solution at a specified cesium concentration ;

- Initially the CST material is assumed to be fresh containing no cesium cations at its ion exchange sites;

- VERSE-LC geometry parameters is set consistent with the experimental setup, while other parameters are set consistent with the parameters discussed in the column modeling section of this report;

- The film mass transfer and axial dispersion coefficients are set to high values in order to appropriately model the experimental behavior using VERSE-LC (i.e., experimentally the liquid-sample remains well-mixed throughout the contact period resulting from the mechanical mixing);

- A binary transport simulation, along with a binary isotherm model, are chosen to model the cesium isotherm;

- Transient cesium concentrations are computed using VERSE-LC, while experimental values are computed from the various batch contact test data (i.e., contact tests with varying contact times were performed as discussed in Appendix E) and associated information contained within their report (and summarized in Appendix E); and

- Cesium equilibrium isotherms are used consistent with the late time (i.e., longest contact time batch kinetics test) measured $\mathrm{K}_{\mathrm{d}}$ value where complete equilibrium is assumed to have been reached given this amount of contact time.

Sample VERSE-LC input and output decks are provided in Appendix E. The measured liquid cesium concentration for various contact tests (which approximate the transient values at various times during a single contact test) and various key parameters used in this analysis are also provided in Appendix E.

To model the transient behavior occurring during a single batch contact test, special VERSE-LC parameter settings are required. To simulate a well-mixed beaker of liquid-sample one finite element representing the bed is chosen where the axial dispersion coefficient is set to a very large value to ensure negligible concentration gradients exist throughout the bed volume (i.e., liquid region outside the particles). The flowrate through this "artificial" column is set to zero (i.e., actually a very small value is necessary since the superficial velocity is used to compute various dimensionless numbers). Initially, in VERSE-LC the pore spaces are assumed to be filled with liquid free of the various ions of interest (i.e., cesium). The ionic concentrations of the liquid in the bed space are increased to account for the dilution effect (i.e., ratio of total liquid volume to liquid bed volume) where the total liquid volume (i.e., pore plus liquid bed volumes) is set to the test's initial liquid-sample volume. While the pore porosity is set to the 
CST material value, the bed porosity is based on the quantities of materials used (i.e., the majority of volume is occupied by bed liquid).

\subsubsection{PNNL Kinetics Studies}

A limited amount of batch kinetics test data was found within the literature for the Cesium-CST system. Early on Brown et al. (1996) performed a series of batch kinetics tests as part of an assessment effort. They measured cesium time-dependent $\mathrm{K}_{\mathrm{d}}$ values for a powder-form and for two early on engineered-forms (batches 08 and 38b). At $25 \mathrm{C}$ and for initial concentrations of $1.0 \times 10^{-4} \mathrm{M}[\mathrm{Cs}]$ and $5 \mathrm{M}[\mathrm{Na}]$, Brown et al. (1996) performed their tests using a 70\% AW-101 DSSF composite liquid simulant. Their experimental contact times range from 1 minute up to 120 hours. The measured $\mathrm{K}_{\mathrm{d}}$ values are shown in Figure 7-1 where impact of a dilution factor effect can clearly be seen. Their database and details are provided in Appendix E.

The adequacy in the level of mixing (i.e., agitation) used during these sets of tests is unknown. As discussed in Hamm et al. (2000a, see Chapter 7) and by Brown et al. (1996), for short contact times film diffusion resistance to mass transfer can begin to have an increased impact on the overall mass transfer resistance. However, since CST material is considered to be significantly pore diffusion limited, it will be assumed here that the mass transfer resistance inferred from these batch kinetics tests is primarily due to pore diffusion alone. The powder-form CST material has an average particle diameter of $\sim 0.8 \mu \mathrm{m}$, while for the engineered-forms the particle diameters nominally range from $250 \mu \mathrm{m}$ to $600 \mu \mathrm{m}$. Given the drastically larger particle sizes of engineered-forms when compared to its powder-forms, a significant reduction in ion pore diffusion coefficients is expected. The results shown in Figure 7-1 confirm this expectation.

To estimate the pore diffusion coefficient several VERSE-LC simulations were run where the pore diffusion coefficient was varied. The details associated with the VERSE-LC simulations are provided in Appendix E. For the CST power data significantly reduced pore diffusion coefficients (lower than $0.001 \%$ of its molecular value) would be required to fit the cesium uptake data, as shown in Figure 7-2. Similar sets of VERSE-LC runs were made for the two engineered forms of CST. The results of these simulations and comparison to the data are shown in Figures 7-3 and 7-4. The solid curves in Figures 7-3 and 7-4 represent VERSE-LC results when a pore diffusion coefficient of $5 \%$ of its molecular value is chosen. The data appears to exhibit two different decay time constants, one for early contact times (i.e., $<2$ hours) and one for later contact times (i.e., $>2$ hours). This sort of behavior may be the result of the heterogeneity present within the engineered-form particles or a shift in the effectiveness of the mechanical mixing being achieved.

\subsubsection{SRS Kinetics Studies}

More recently, Fondeur et al. (2000) measured timed $\mathrm{K}_{\mathrm{d}}$ values for a more recent engineeredform (Baseline Lot 9090-76; average particle diameter of $\sim 344 \mu \mathrm{m}$ ). At $25 \mathrm{C}$ and for initial concentrations of $1.4 \times 10^{-4} \mathrm{M}[\mathrm{Cs}]$ and $5.6 \mathrm{M}[\mathrm{Na}]$, Fondeur et al. (2000) performed their tests using a SRS average liquid simulant. The results of their tests are shown in Figure 7-5, while the 
details of their database are provided in Appendix E. Their experimental contact times range from 24 to 192 hours. Unfortunately, no short contact time data were recorded.

A rough estimate (i.e., using VERSE-LC simulation assistance) of the earlier contact time behavior is also shown in Figure 7-5 by the dashed line and open symbols. This early contact time estimate is based on the Brown et al. (1996) data for their engineered-forms. An overlay of the two data sets in provided in Figure 7-6 where it can be seen that the kinetic behavior (i.e., time rate of increase in relative $\mathrm{K}_{\mathrm{d}}$ value) of the three engineered-forms appears quite similar. The Baseline engineered-form CST data was extended to early times using the approximate average behavior of the 08 and $38 \mathrm{~b}$ engineered-form CST data.

As shown in Figure 7-6 a composite of these three data sets was chosen for establishing an "average" cesium pore diffusion coefficient value. Based on the experimental setup by Fondeur et al. (2000), VERSE-LC simulations of the batch kinetics of the Baseline CST material were performed. The VERSE-LC simulation details are provided in Appendix E. Several VERSE-LC simulations were run where the pore diffusion coefficient was varied. The results of these simulations are shown in Figure 7-7. As can be seen in Figure 7-7, a pore diffusion coefficient value of $\sim 5 \%$ of its molecular value appears to "fit" the experimental data (i.e., solid circles). The open circles in Figure 7-7 represent the estimated data points as shown in Figures 7-5 and 76. The sensitivity of the cesium uptake curves with respect to pore diffusion is also shown in Figure 7-7. The degree of mechanical mixing that might have been achieved is unknown; therefore, the $5 \%$ value probably represents an lower bound value due to possible film resistances present within the test as well. The approach to equilibrium can also be plotted as shown in Figure 7-8.

\subsubsection{Particle Size Impact on Kinetics}

The effect of particle size on the rate of cesium uptake has been measured by Anthony et al. (1996) based on TAM5 generated forms (i.e., average particle diameters of $\sim 112 \mu \mathrm{m}$ and $~ 334$ $\mu \mathrm{m})$. Miller and Brown (1997) also performed cesium uptake measured on TAM5 powder (i.e., average particle diameter of $\sim 0.8 \mu \mathrm{m}$ ). At $25 \mathrm{C}$ and for initial concentrations of $1.0 \times 10^{-4} \mathrm{M}$ [Cs] and 5.0 M [Na], these tests were performed using a DSSF5 liquid simulant. The results of their tests are shown in Figure 7-9, while the details of their database are provided in Appendix E.

VERSE-LC simulations were performed and are plotted in Figure 7-9 for comparison. A particle tortuosity factor of 10.0 was used (i.e., pore diffusion coefficient of $10 \%$ of its molecular value). Two items can be seen from the data itself. First, as expected particle size has a large impact on the kinetics but this effect appears to greatly diminish with smaller particles. No simple explanation for the diminishing impact can be given at this time, other than to suspect the data. Second, the shape of the cesium uptake (decay) curves appear to have two difference time constants. One explanation for the differing time constants is the possibility that more rapid diffusion occurs through the larger binding material pores early on, followed by much slower diffusion rates through the smaller powder pores. 


\subsubsection{ORNL Kinetics Studies}

The series of tests measuring the rate of cesium uptake have been taken by Davidson et al. (1998) based on CST powder. Tests at $25 \mathrm{C}$ and an initial concentration of $1.418 \times 10^{-6} \mathrm{M}[\mathrm{Cs}]$ were performed for four different phase ratios (i.e., 100, 200, 400, and 1000) using a W-27 supernate solution. The results of their tests are shown in Figure 7-10, while the details of their database are provided in Appendix E. Also shown in Figure 7-10 are VERSE-LC runs based on a phase ratio of 100 .

Table 7-1. Fluid density and dynamic viscosity for water and simulated Envelope A 5 M sodium waste solution.

\begin{tabular}{|c|c|c|}
\hline Fluid property & Pure water & $\begin{array}{c}\text { Simulated } 5 \mathrm{M} \mathrm{Na}^{+} \\
\text {waste solution a }\end{array}$ \\
\hline \multicolumn{3}{|l|}{ For $20^{\circ} \mathrm{C}$} \\
\hline Density, g/ml & 0.99823 & $1.225^{\mathrm{a}}$ \\
\hline Dynamic viscosity, $\mathrm{cp}$ & 1.002 & $2.94^{\mathrm{a}}$ \\
\hline \multicolumn{3}{|l|}{ For $25^{\circ} \mathrm{C}$} \\
\hline Density, $\mathrm{g} / \mathrm{ml}$ & 0.9970479 & $1.22355^{\mathrm{b}}$ \\
\hline Dynamic viscosity, $\mathrm{cp}$ & 0.8904 & $2.61255^{b}$ \\
\hline
\end{tabular}

${ }^{\text {a }}$ A 5 M sodium simulated waste consistent with Envelope A where properties were measured at $20{ }^{\circ} \mathrm{C}$ (Steimke et al., 2000).

${ }^{\mathrm{b}}$ Measured properties were adjusted to new temperature based on pure water value ratios. 
Preliminary Ion Exchange Modeling for Removal of Cesium from

Table 7-2. Measured fluid density and dynamic viscosity for various SRS waste solutions.

\begin{tabular}{|c|c|c|c|}
\hline Specific solution & $\begin{array}{c}\text { Fluid temperature } \\
(\mathrm{C})\end{array}$ & $\begin{array}{c}\text { Fluid Density } \\
(\mathrm{g} / \mathrm{ml})\end{array}$ & $\begin{array}{c}\text { Dynamic viscosity } \\
(\mathrm{cp})\end{array}$ \\
\hline \hline SRS Avg & 25 & 1.253 & 2.78 \\
\hline SRS High OH & 1.244 & 3.1 \\
\hline SRS Tank 44 & 25 & 1.2015 & 2.6 \\
\hline UOP & 31 & 1.276 & - \\
\hline
\end{tabular}

Table 7-3. Limiting ionic conductances in water at $25^{\circ} \mathrm{C}$ (Reid et al., 1977; Perry, 1973, Glasstone and Lewis, 1960).

\begin{tabular}{|c|c|c|}
\hline Ion & Ionic valance & $\begin{array}{c}\text { Limiting ionic conductance } \\
\text { MHOS/equivalent }\end{array}$ \\
\hline \hline Cations & 1 & 77.3 \\
\hline $\mathrm{Cs}^{+}$ & 1 & 73.5 \\
\hline $\mathrm{K}^{+}$ & 1 & 50.1 \\
\hline $\mathrm{Na}^{+}$ & 1 & 349.8 \\
\hline $\mathrm{H}^{+}$ & -1 & \\
\hline \hline $\mathrm{Anions}^{+}$ & -1 & 798.6 \\
\hline $\mathrm{OH}^{-}$ & -1 & 76.35 \\
\hline $\mathrm{Cl}^{--}$ & -1 & 71.46 \\
\hline $\mathrm{NO}_{3}^{-}$ & -1 & 72.0 \\
\hline $\mathrm{NO}_{2}^{-}$ & -1 & 76.8 \\
\hline $\mathrm{I}^{-}$ & -2 & 55.4 \\
\hline $\mathrm{F}^{-}$ & -2 & 69.3 \\
\hline $\mathrm{CO}_{3}^{-2}$ & -2 & 80.02 \\
\hline $\mathrm{SO}_{4}^{-2}$ & -1 & $75.0^{\mathrm{a}}$ \\
\hline $\mathrm{PO}_{4}^{-2}$ & $\mathrm{Al}^{-2}(\mathrm{OH})_{4}^{-}$ & \\
\hline
\end{tabular}

${ }^{\mathrm{a}}$ Value estimated. 
Preliminary Ion Exchange Modeling for Removal of Cesium from

Table 7-4. Best estimate binary molecular ${ }^{\mathrm{a}}$ diffusion coefficients at $25^{\circ} \mathrm{C}$ for a solution containing essentially only cesium cations and anion of one particular species.

\begin{tabular}{|c|c|c|}
\hline Ion Pair & $\begin{array}{l}\text { Molecular diffusion } \\
\text { coef. in water } \\
\left(\mathrm{cm}^{2} / \mathrm{min}\right)\end{array}$ & $\begin{array}{l}\text { Molecular diffusion coef. in } \\
\text { simulated 5M Na waste } \\
\qquad\left(\mathrm{cm}^{2} / \mathrm{min}\right)\end{array}$ \\
\hline \multicolumn{3}{|l|}{ For cesium } \\
\hline $\mathrm{Cs}^{+}-\mathrm{OH}^{-}$ & $1.778 \mathrm{E}-03$ & $6.047 \mathrm{E}-04$ \\
\hline $\mathrm{Cs}^{+}-\mathrm{Cl}^{-}$ & $1.227 \mathrm{E}-03$ & 4.175E-04 \\
\hline $\mathrm{Cs}^{+}-\mathrm{NO}_{3}^{-}$ & $1.187 \mathrm{E}-03$ & $4.036 \mathrm{E}-04$ \\
\hline $\mathrm{Cs}^{+}-\mathrm{NO}_{2}^{-}$ & $1.191 \mathrm{E}-03$ & $4.052 \mathrm{E}-04$ \\
\hline $\mathrm{Cs}^{+}-\mathrm{I}^{-}$ & $1.231 \mathrm{E}-03$ & 4.187E-04 \\
\hline $\mathrm{Cs}^{+}-\mathrm{F}^{-}$ & $1.031 \mathrm{E}-03$ & $3.507 \mathrm{E}-04$ \\
\hline $\mathrm{Cs}^{+}-\mathrm{CO}_{3}^{-2}$ & $8.757 \mathrm{E}-04$ & $2.979 \mathrm{E}-04$ \\
\hline $\mathrm{Cs}^{+}-\mathrm{SO}_{4}^{-2}$ & $9.423 \mathrm{E}-04$ & $3.205 \mathrm{E}-04$ \\
\hline $\mathrm{Cs}^{+}-\mathrm{PO}_{4}^{-2}$ & $9.123 \mathrm{E}-04$ & $3.103 \mathrm{E}-04$ \\
\hline $\mathrm{Cs}^{+}-\mathrm{Al}(\mathrm{OH})_{4}^{-}$ & $1.174 \mathrm{E}-03$ & $3.992 \mathrm{E}-04$ \\
\hline
\end{tabular}

a The molecular diffusion coefficient is sometimes referred to as the "free" or "Brownian" motion diffusion coefficient.

${ }^{b}$ Fluid viscosity correction ratio applied based on a measured simulated waste viscosity of $2.94 \mathrm{cp}$ at $20{ }^{\circ} \mathrm{C}$ (Steimke et al., 2000). 
Table 7-5. Phase 1 LAW feed anion concentrations and relative mole fractions ${ }^{\text {a }}$ for Envelope A used in determining "effective" binary molecular diffusion coefficients with cesium.

\begin{tabular}{|c|c|c|c|c|c|c|c|c|c|c|c|c|c|}
\hline $\begin{array}{c}\text { Anion } \\
\text { considered }\end{array}$ & Charge & $\begin{array}{c}\text { ZAM } \\
\text { ID }\end{array}$ & LAW-1 & LAW-5 & LAW-6 & LAW-8 & LAW-9 & LAW-10 & LAW-11 & LAW-12 & LAW-13 & LAW-14 & LAW-15 \\
\hline $\mathrm{OH}$ (free) $[\mathrm{M}]$ & -1 & 13 & $1.553 \mathrm{E}+00$ & $8.643 \mathrm{E}-01$ & $9.543 \mathrm{E}-01$ & $1.343 \mathrm{E}+00$ & $5.733 \mathrm{E}-01$ & $8.697 \mathrm{E}-01$ & $9.307 \mathrm{E}-01$ & $1.543 \mathrm{E}+00$ & $1.542 \mathrm{E}+00$ & $1.748 \mathrm{E}+00$ & $2.141 \mathrm{E}+00$ \\
\hline $\mathrm{Cl}[\mathrm{M}]$ & -1 & 2 & 4.982E-02 & $1.034 \mathrm{E}-01$ & $1.031 \mathrm{E}-01$ & $1.246 \mathrm{E}-01$ & $1.272 \mathrm{E}-01$ & $1.400 \mathrm{E}-01$ & $1.365 \mathrm{E}-01$ & $8.623 \mathrm{E}-02$ & $8.517 \mathrm{E}-02$ & $8.426 \mathrm{E}-02$ & $6.978 \mathrm{E}-02$ \\
\hline $\mathrm{NO}_{3}[\mathrm{M}]$ & -1 & 9 & $1.868 \mathrm{E}+00$ & $1.377 \mathrm{E}+00$ & $1.359 \mathrm{E}+00$ & $1.278 \mathrm{E}+00$ & $1.408 \mathrm{E}+00$ & $1.431 \mathrm{E}+00$ & $1.200 \mathrm{E}+00$ & $1.222 \mathrm{E}+00$ & $1.222 \mathrm{E}+00$ & $1.325 \mathrm{E}+00$ & $1.299 \mathrm{E}+00$ \\
\hline $\mathrm{NO}_{2}[\mathrm{M}]$ & -1 & 27 & 8.096E-01 & $1.188 \mathrm{E}+00$ & $1.192 \mathrm{E}+00$ & $1.197 \mathrm{E}+00$ & $1.208 \mathrm{E}+00$ & $1.312 \mathrm{E}+00$ & $1.387 \mathrm{E}+00$ & $1.127 \mathrm{E}+00$ & $1.127 \mathrm{E}+00$ & $1.063 \mathrm{E}+00$ & $1.015 \mathrm{E}+00$ \\
\hline 129-I [M] & -1 & 4 & $3.145 \mathrm{E}-06$ & $8.115 \mathrm{E}-06$ & $8.475 \mathrm{E}-06$ & $2.189 \mathrm{E}-05$ & $1.028 \mathrm{E}-05$ & $1.308 \mathrm{E}-05$ & $7.391 \mathrm{E}-06$ & $7.415 \mathrm{E}-06$ & $6.992 \mathrm{E}-06$ & $9.391 \mathrm{E}-06$ & $9.142 \mathrm{E}-06$ \\
\hline $\mathrm{F}[\mathrm{M}]$ & -1 & 1 & $1.335 \mathrm{E}-01$ & $1.471 \mathrm{E}-02$ & $1.336 \mathrm{E}-02$ & $1.654 \mathrm{E}-02$ & 4.472E-02 & $2.496 \mathrm{E}-02$ & $3.109 \mathrm{E}-02$ & $1.847 \mathrm{E}-02$ & $1.856 \mathrm{E}-02$ & $4.061 \mathrm{E}-02$ & 3.937E-02 \\
\hline $\mathrm{CO}_{3}[\mathrm{M}]$ & -2 & 19 & 4.804E-01 & 3.927E-01 & $3.588 \mathrm{E}-01$ & $2.014 \mathrm{E}-01$ & $5.472 \mathrm{E}-01$ & $2.785 \mathrm{E}-01$ & $3.609 \mathrm{E}-01$ & $1.614 \mathrm{E}-01$ & $1.604 \mathrm{E}-01$ & $2.530 \mathrm{E}-01$ & $1.537 \mathrm{E}-01$ \\
\hline $\mathrm{SO}_{4}[\mathrm{M}]$ & -2 & 15 & $3.737 \mathrm{E}-02$ & 4.843E-02 & 4.492E-02 & $2.170 \mathrm{E}-02$ & $3.667 \mathrm{E}-02$ & 3.727E-02 & $2.391 \mathrm{E}-02$ & $1.138 \mathrm{E}-02$ & $1.108 \mathrm{E}-02$ & $1.721 \mathrm{E}-02$ & $1.062 \mathrm{E}-02$ \\
\hline $\mathrm{PO}_{4}[\mathrm{M}]$ & -2 & 20 & $9.786 \mathrm{E}-03$ & $2.065 \mathrm{E}-02$ & $1.980 \mathrm{E}-02$ & $1.084 \mathrm{E}-02$ & $2.669 \mathrm{E}-02$ & 4.346E-02 & $3.652 \mathrm{E}-02$ & $9.386 \mathrm{E}-03$ & $9.343 \mathrm{E}-03$ & $1.099 \mathrm{E}-02$ & $5.672 \mathrm{E}-03$ \\
\hline $\mathrm{Al}(\mathrm{OH})_{4}[\mathrm{M}]$ & -1 & 28 & $2.338 \mathrm{E}-01$ & $5.707 \mathrm{E}-01$ & $5.761 \mathrm{E}-01$ & $6.375 \mathrm{E}-01$ & 4.533E-01 & $5.150 \mathrm{E}-01$ & 4.913E-01 & $7.536 \mathrm{E}-01$ & $7.600 \mathrm{E}-01$ & $4.891 \mathrm{E}-01$ & $5.013 \mathrm{E}-01$ \\
\hline Conc. sum $[\mathrm{M}]$ & - & - & $5.176 \mathrm{E}+00$ & $4.580 \mathrm{E}+00$ & $4.621 \mathrm{E}+00$ & $4.831 \mathrm{E}+00$ & $4.426 \mathrm{E}+00$ & $4.651 \mathrm{E}+00$ & $4.598 \mathrm{E}+00$ & $4.933 \mathrm{E}+00$ & $4.936 \mathrm{E}+00$ & $5.031 \mathrm{E}+00$ & $5.235 \mathrm{E}+00$ \\
\hline $\mathrm{OH}$ (free) [fraction] & -1 & 13 & $3.00 \mathrm{E}-01$ & $1.89 \mathrm{E}-01$ & 2.07E-01 & $2.78 \mathrm{E}-01$ & $1.30 \mathrm{E}-01$ & $1.87 \mathrm{E}-01$ & $2.02 \mathrm{E}-01$ & $3.13 \mathrm{E}-01$ & 3.12E-01 & 3.47E-01 & 4.09E-01 \\
\hline $\mathrm{Cl}$ [fraction] & -1 & 2 & $9.63 \mathrm{E}-03$ & $2.26 \mathrm{E}-02$ & $2.23 \mathrm{E}-02$ & $2.58 \mathrm{E}-02$ & $2.87 \mathrm{E}-02$ & $3.01 \mathrm{E}-02$ & $2.97 \mathrm{E}-02$ & $1.75 \mathrm{E}-02$ & $1.73 \mathrm{E}-02$ & $1.67 \mathrm{E}-02$ & $1.33 \mathrm{E}-02$ \\
\hline $\mathrm{NO}_{3}[$ fraction $]$ & -1 & 9 & $3.61 \mathrm{E}-01$ & $3.01 \mathrm{E}-01$ & $2.94 \mathrm{E}-01$ & $2.65 \mathrm{E}-01$ & $3.18 \mathrm{E}-01$ & $3.08 \mathrm{E}-01$ & $2.61 \mathrm{E}-01$ & $2.48 \mathrm{E}-01$ & $2.48 \mathrm{E}-01$ & $2.63 \mathrm{E}-01$ & $2.48 \mathrm{E}-01$ \\
\hline $\mathrm{NO}_{2}[$ fraction $]$ & -1 & 27 & $1.56 \mathrm{E}-01$ & $2.59 \mathrm{E}-01$ & $2.58 \mathrm{E}-01$ & $2.48 \mathrm{E}-01$ & $2.73 \mathrm{E}-01$ & $2.82 \mathrm{E}-01$ & $3.02 \mathrm{E}-01$ & $2.28 \mathrm{E}-01$ & $2.28 \mathrm{E}-01$ & $2.11 \mathrm{E}-01$ & $1.94 \mathrm{E}-01$ \\
\hline 129-I [fraction] & -1 & 4 & $6.08 \mathrm{E}-07$ & $1.77 \mathrm{E}-06$ & $1.83 \mathrm{E}-06$ & 4.53E-06 & $2.32 \mathrm{E}-06$ & $2.81 \mathrm{E}-06$ & $1.61 \mathrm{E}-06$ & $1.50 \mathrm{E}-06$ & $1.42 \mathrm{E}-06$ & $1.87 \mathrm{E}-06$ & $1.75 \mathrm{E}-06$ \\
\hline F [fraction] & -1 & 1 & $2.58 \mathrm{E}-02$ & $3.21 \mathrm{E}-03$ & $2.89 \mathrm{E}-03$ & $3.42 \mathrm{E}-03$ & $1.01 \mathrm{E}-02$ & $5.37 \mathrm{E}-03$ & $6.76 \mathrm{E}-03$ & $3.74 \mathrm{E}-03$ & $3.76 \mathrm{E}-03$ & 8.07E-03 & $7.52 \mathrm{E}-03$ \\
\hline $\mathrm{CO}_{3}$ [fraction] & -2 & 19 & $9.28 \mathrm{E}-02$ & $8.57 \mathrm{E}-02$ & $7.76 \mathrm{E}-02$ & 4.17E-02 & $1.24 \mathrm{E}-01$ & $5.99 \mathrm{E}-02$ & $7.85 \mathrm{E}-02$ & $3.27 \mathrm{E}-02$ & $3.25 \mathrm{E}-02$ & $5.03 \mathrm{E}-02$ & $2.94 \mathrm{E}-02$ \\
\hline $\mathrm{SO}_{4}$ [fraction] & -2 & 15 & $7.22 \mathrm{E}-03$ & $1.06 \mathrm{E}-02$ & $9.72 \mathrm{E}-03$ & 4.49E-03 & $8.28 \mathrm{E}-03$ & $8.01 \mathrm{E}-03$ & $5.20 \mathrm{E}-03$ & $2.31 \mathrm{E}-03$ & $2.24 \mathrm{E}-03$ & $3.42 \mathrm{E}-03$ & $2.03 \mathrm{E}-03$ \\
\hline $\mathrm{PO}_{4}$ [fraction] & -2 & 20 & $1.89 \mathrm{E}-03$ & $4.51 \mathrm{E}-03$ & $4.29 \mathrm{E}-03$ & $2.24 \mathrm{E}-03$ & $6.03 \mathrm{E}-03$ & $9.34 \mathrm{E}-03$ & $7.94 \mathrm{E}-03$ & $1.90 \mathrm{E}-03$ & $1.89 \mathrm{E}-03$ & $2.18 \mathrm{E}-03$ & $1.08 \mathrm{E}-03$ \\
\hline $\mathrm{Al}(\mathrm{OH})_{4}[$ fraction] & -1 & 28 & $4.52 \mathrm{E}-02$ & $1.25 \mathrm{E}-01$ & $1.25 \mathrm{E}-01$ & $1.32 \mathrm{E}-01$ & $1.02 \mathrm{E}-01$ & $1.11 \mathrm{E}-01$ & $1.07 \mathrm{E}-01$ & $1.53 \mathrm{E}-01$ & $1.54 \mathrm{E}-01$ & $9.72 \mathrm{E}-02$ & $9.58 \mathrm{E}-02$ \\
\hline
\end{tabular}

a The relative mole fractions refer to mole fractions for each anion where the their values are normalized to the sum of anions considered and listed in this table. The anions considered are based on the available limiting ionic conductances found within the literature as listed in Table 7-3. 
Table 7-6. Phase 1 LAW feed anion concentrations and relative mole fractions " for Envelopes B and C used in determining "effective" binary molecular diffusion coefficients with cesium.

\begin{tabular}{|c|c|c|c|c|c|c|c|}
\hline $\begin{array}{c}\text { Anion } \\
\text { considered }\end{array}$ & Charge & $\begin{array}{c}\text { ZAM } \\
\text { ID }\end{array}$ & LAW-1 & LAW-5 & LAW-6 & LAW-8 & LAW-9 \\
\hline $\mathrm{OH}$ (free) $[\mathrm{M}]$ & -1 & 13 & $1.599 \mathrm{E}-01$ & $2.720 \mathrm{E}-01$ & $1.033 \mathrm{E}+00$ & $1.033 \mathrm{E}+00$ & 7.943E-01 \\
\hline $\mathrm{Cl}[\mathrm{M}]$ & -1 & 2 & $5.834 \mathrm{E}-03$ & $4.725 \mathrm{E}-04$ & 4.794E-02 & 4.794E-02 & $2.784 \mathrm{E}-02$ \\
\hline $\mathrm{NO}_{3}[\mathrm{M}]$ & -1 & 9 & $1.277 \mathrm{E}+00$ & $6.736 \mathrm{E}-01$ & $1.737 \mathrm{E}+00$ & $1.737 \mathrm{E}+00$ & $1.967 \mathrm{E}+00$ \\
\hline $\mathrm{NO}_{2}[\mathrm{M}]$ & -1 & 27 & $1.481 \mathrm{E}+00$ & $1.182 \mathrm{E}+00$ & $7.959 \mathrm{E}-01$ & $7.959 \mathrm{E}-01$ & $6.953 \mathrm{E}-01$ \\
\hline 129-I [M] & -1 & 4 & $3.975 \mathrm{E}-02$ & $3.503 \mathrm{E}-02$ & $1.582 \mathrm{E}-05$ & $1.582 \mathrm{E}-05$ & $1.273 \mathrm{E}-05$ \\
\hline $\mathrm{F}[\mathrm{M}]$ & -1 & 1 & $1.029 \mathrm{E}-01$ & $9.867 \mathrm{E}-02$ & $4.345 \mathrm{E}-02$ & $4.345 \mathrm{E}-02$ & $9.387 \mathrm{E}-02$ \\
\hline $\mathrm{CO}_{3}[\mathrm{M}]$ & -2 & 19 & $5.907 \mathrm{E}-01$ & $1.035 \mathrm{E}+00$ & $5.101 \mathrm{E}-01$ & $5.101 \mathrm{E}-01$ & $6.555 \mathrm{E}-01$ \\
\hline $\mathrm{SO}_{4}[\mathrm{M}]$ & -2 & 15 & $1.967 \mathrm{E}-01$ & $3.369 \mathrm{E}-01$ & 7.274E-02 & 7.274E-02 & 4.693E-02 \\
\hline $\mathrm{PO}_{4}[\mathrm{M}]$ & -2 & 20 & $1.61 \mathrm{E}-02$ & $2.54 \mathrm{E}-03$ & $2.286 \mathrm{E}-02$ & $2.286 \mathrm{E}-02$ & $2.036 \mathrm{E}-02$ \\
\hline $\mathrm{Al}(\mathrm{OH})_{4}[\mathrm{M}]$ & -1 & 28 & 4.364E-01 & $1.342 \mathrm{E}-01$ & $2.610 \mathrm{E}-01$ & $2.610 \mathrm{E}-01$ & $1.391 \mathrm{E}-01$ \\
\hline Conc. sum $[\mathrm{M}]$ & - & - & $4.306 \mathrm{E}+00$ & $3.770 \mathrm{E}+00$ & $4.524 \mathrm{E}+00$ & $4.524 \mathrm{E}+00$ & $4.440 \mathrm{E}+00$ \\
\hline $\mathrm{OH}$ (free) [fraction] & -1 & 13 & $3.71 \mathrm{E}-02$ & $7.21 \mathrm{E}-02$ & $2.28 \mathrm{E}-01$ & $2.28 \mathrm{E}-01$ & $1.79 \mathrm{E}-01$ \\
\hline $\mathrm{Cl}$ [fraction] & -1 & 2 & $1.35 \mathrm{E}-03$ & $1.25 \mathrm{E}-04$ & $1.06 \mathrm{E}-02$ & $1.06 \mathrm{E}-02$ & $6.27 \mathrm{E}-03$ \\
\hline $\mathrm{NO}_{3}$ [fraction] & -1 & 9 & $2.96 \mathrm{E}-01$ & $1.79 \mathrm{E}-01$ & $3.84 \mathrm{E}-01$ & $3.84 \mathrm{E}-01$ & 4.43E-01 \\
\hline $\mathrm{NO}_{2}$ [fraction] & -1 & 27 & $3.44 \mathrm{E}-01$ & $3.13 \mathrm{E}-01$ & $1.76 \mathrm{E}-01$ & $1.76 \mathrm{E}-01$ & $1.57 \mathrm{E}-01$ \\
\hline 129-I [fraction] & -1 & 4 & $9.23 \mathrm{E}-03$ & $9.29 \mathrm{E}-03$ & $3.50 \mathrm{E}-06$ & $3.50 \mathrm{E}-06$ & $2.87 \mathrm{E}-06$ \\
\hline F [fraction] & -1 & 1 & $2.39 \mathrm{E}-02$ & 2.62E-02 & $9.61 \mathrm{E}-03$ & $9.61 \mathrm{E}-03$ & $2.11 \mathrm{E}-02$ \\
\hline $\mathrm{CO}_{3}$ [fraction] & -2 & 19 & $1.37 \mathrm{E}-01$ & $2.75 \mathrm{E}-01$ & $1.13 \mathrm{E}-01$ & $1.13 \mathrm{E}-01$ & $1.48 \mathrm{E}-01$ \\
\hline $\mathrm{SO}_{4}[$ fraction] & -2 & 15 & $4.57 \mathrm{E}-02$ & $8.94 \mathrm{E}-02$ & $1.61 \mathrm{E}-02$ & 1.61E-02 & $1.06 \mathrm{E}-02$ \\
\hline $\mathrm{PO}_{4}$ [fraction] & -2 & 20 & $3.74 \mathrm{E}-03$ & $6.73 \mathrm{E}-04$ & $5.05 \mathrm{E}-03$ & $5.05 \mathrm{E}-03$ & $4.59 \mathrm{E}-03$ \\
\hline $\mathrm{Al}(\mathrm{OH})_{4}[$ fraction $]$ & -1 & 28 & $1.01 \mathrm{E}-01$ & $3.56 \mathrm{E}-02$ & $5.77 \mathrm{E}-02$ & $5.77 \mathrm{E}-02$ & $3.13 \mathrm{E}-02$ \\
\hline
\end{tabular}

a The relative mole fractions refer to mole fractions for each anion where the their values are normalized to the sum of anions considered and listed in this table. The anions considered are based on the available limiting ionic conductances found within the literature as listed in Table 7-3. 
Table 7-7. Simulant or sample anion concentrations and relative mole fractions s for various solutions ${ }^{\mathrm{b}}$ used in determining "effective" binary molecular diffusion coefficients with cesium.

\begin{tabular}{|c|c|c|c|c|c|c|c|c|c|c|}
\hline $\begin{array}{c}\text { Anion } \\
\text { considered }\end{array}$ & Charge & $\begin{array}{c}\text { ZAM } \\
\text { ID }\end{array}$ & $\begin{array}{l}\text { SRS } \\
\text { Avg }\end{array}$ & $\begin{array}{c}\text { SRS } \\
\text { High } \text { OH }^{-}\end{array}$ & $\begin{array}{c}\text { SRS } \\
\text { Tank } 44\end{array}$ & $\begin{array}{c}\text { Brown } \\
\text { AW-101 }\end{array}$ & $\begin{array}{l}\text { ORNL } \\
\text { W27 }\end{array}$ & $\begin{array}{l}\text { Sandia } \\
\text { DSSF5 }\end{array}$ & $\begin{array}{c}\text { Texas A\&M } \\
\text { Exp1 }\end{array}$ & $\begin{array}{c}\text { Hendrickson } \\
\text { AW-101 }\end{array}$ \\
\hline & & & $\begin{array}{c}\text { Walker et } \\
\text { al. (?) }\end{array}$ & $\begin{array}{c}\text { Walker et } \\
\text { al. (?) }\end{array}$ & $\begin{array}{c}\text { Walker et } \\
\text { al. (?) }\end{array}$ & $\begin{array}{l}\text { Brown et } \\
\text { al. (1996) }\end{array}$ & Lee (1997) & $\begin{array}{c}\text { Texas A\&M } \\
\text { slides (?) }\end{array}$ & $\begin{array}{c}\text { Texas A\&M } \\
\text { report (?) }\end{array}$ & $\begin{array}{c}\text { Hendrickson } \\
\text { report (?) }\end{array}$ \\
\hline $\mathrm{OH}$ (free) [M] & -1 & 13 & $1.910 \mathrm{E}+00$ & $3.050 \mathrm{E}+00$ & $4.300 \mathrm{E}+00$ & $2.170 \mathrm{E}+00$ & $7.080 \mathrm{E}-02$ & $2.170 \mathrm{E}+00$ & $6.000 \mathrm{E}-01$ & $2.541 \mathrm{E}+00$ \\
\hline $\mathrm{Cl}[\mathrm{M}]$ & -1 & 2 & $2.500 \mathrm{E}-02$ & $3.637 \mathrm{E}-02$ & $9.000 \mathrm{E}-03$ & $6.500 \mathrm{E}-02$ & $9.000 \mathrm{E}-02$ & $2.576 \mathrm{E}-01$ & - & $8.830 \mathrm{E}-02$ \\
\hline $\mathrm{NO}_{3}[\mathrm{M}]$ & -1 & 9 & $2.180 \mathrm{E}+00$ & $1.100 \mathrm{E}+00$ & $3.700 \mathrm{E}-01$ & $1.490 \mathrm{E}+00$ & $5.194 \mathrm{E}+00$ & $1.490 \mathrm{E}+00$ & $5.100 \mathrm{E}+00$ & $1.530 \mathrm{E}+00$ \\
\hline $\mathrm{NO}_{2}[\mathrm{M}]$ & -1 & 27 & $5.100 \mathrm{E}-01$ & 7.400E-01 & $3.500 \mathrm{E}-01$ & $9.400 \mathrm{E}-01$ & - & $9.400 \mathrm{E}-01$ & - & $1.130 \mathrm{E}+00$ \\
\hline 129-I [M] & -1 & 4 & - & - & - & - & - & - & - & - \\
\hline $\mathrm{F}[\mathrm{M}]$ & -1 & 1 & $3.200 \mathrm{E}-02$ & $1.000 \mathrm{E}-02$ & - & $4.330 \mathrm{E}-02$ & - & $4.330 \mathrm{E}-02$ & - & $3.340 \mathrm{E}-02$ \\
\hline - & -2 & 19 & $1.600 \mathrm{E}-01$ & $1.700 \mathrm{E}-01$ & $1.412 \mathrm{E}-01$ & - & - & - & - & $1.100 \mathrm{E}-01$ \\
\hline $\mathrm{SO}_{4}[\mathrm{M}]$ & -2 & 15 & $1.500 \mathrm{E}-01$ & $3.000 \mathrm{E}-02$ & $1.000 \mathrm{E}-03$ & $1.260 \mathrm{E}-02$ & $1.600 \mathrm{E}-02$ & $1.260 \mathrm{E}-02$ & - & $3.280 \mathrm{E}-03$ \\
\hline $\mathrm{PO}_{4}[\mathrm{M}]$ & -2 & 20 & $1.000 \mathrm{E}-02$ & $8.000 \mathrm{E}-03$ & $4.000 \mathrm{E}-03$ & $1.750 \mathrm{E}-02$ & - & $1.750 \mathrm{E}-02$ & - & $5.530 \mathrm{E}-03$ \\
\hline $\mathrm{Al}(\mathrm{OH})_{4}[\mathrm{M}]$ & -1 & 28 & $3.100 \mathrm{E}-01$ & $2.700 \mathrm{E}-01$ & $1.260 \mathrm{E}-01$ & $4.970 \mathrm{E}-01$ & $3.140 \mathrm{E}-05$ & $4.970 \mathrm{E}-01$ & - & $5.740 \mathrm{E}-01$ \\
\hline Conc. sum [M] & - & - & $5.287 \mathrm{E}+00$ & $5.414 \mathrm{E}+00$ & $5.301 \mathrm{E}+00$ & $5.235 \mathrm{E}+00$ & $5.371 \mathrm{E}+00$ & $5.428 \mathrm{E}+00$ & $5.700 \mathrm{E}+00$ & $6.016 \mathrm{E}+00$ \\
\hline $\mathrm{OH}$ (free) [fraction] & -1 & 13 & $3.61 \mathrm{E}-01$ & $5.63 \mathrm{E}-01$ & $8.11 \mathrm{E}-01$ & 4.14E-01 & $1.32 \mathrm{E}-02$ & $4.00 \mathrm{E}-01$ & $1.05 \mathrm{E}-01$ & $4.22 \mathrm{E}-01$ \\
\hline $\mathrm{Cl}$ [fraction] & -1 & 2 & $4.73 \mathrm{E}-03$ & $6.72 \mathrm{E}-03$ & $1.70 \mathrm{E}-03$ & $1.24 \mathrm{E}-02$ & $1.68 \mathrm{E}-02$ & $4.75 \mathrm{E}-02$ & - & $1.47 \mathrm{E}-02$ \\
\hline $\mathrm{NO}_{3}$ [fraction] & -1 & 9 & 4.12E-01 & $2.03 \mathrm{E}-01$ & $6.98 \mathrm{E}-02$ & $2.85 \mathrm{E}-01$ & $9.67 \mathrm{E}-01$ & $2.75 \mathrm{E}-01$ & $8.95 \mathrm{E}-01$ & $2.54 \mathrm{E}-01$ \\
\hline $\mathrm{NO}_{2}$ [fraction] & -1 & 27 & $9.65 \mathrm{E}-02$ & $1.37 \mathrm{E}-01$ & $6.60 \mathrm{E}-02$ & $1.80 \mathrm{E}-01$ & - & $1.73 \mathrm{E}-01$ & - & $1.88 \mathrm{E}-01$ \\
\hline 129-I [fraction] & -1 & 4 & - & - & - & - & - & - & - & - \\
\hline $\mathrm{F}$ [fraction] & -1 & 1 & $6.05 \mathrm{E}-03$ & $1.85 \mathrm{E}-03$ & - & $8.27 \mathrm{E}-03$ & - & 7.98E-03 & - & $5.55 \mathrm{E}-03$ \\
\hline $\mathrm{CO}_{3}$ [fraction] & -2 & 19 & $3.03 \mathrm{E}-02$ & $3.14 \mathrm{E}-02$ & $2.66 \mathrm{E}-02$ & - & - & - & - & $1.83 \mathrm{E}-02$ \\
\hline $\mathrm{SO}_{4}[$ fraction] & -2 & 15 & $2.84 \mathrm{E}-02$ & $5.54 \mathrm{E}-03$ & $1.89 \mathrm{E}-04$ & $2.41 \mathrm{E}-03$ & $2.98 \mathrm{E}-03$ & $2.32 \mathrm{E}-03$ & - & $5.45 \mathrm{E}-04$ \\
\hline $\mathrm{PO}_{4}$ [fraction] & -2 & 20 & $1.89 \mathrm{E}-03$ & $1.48 \mathrm{E}-03$ & $7.55 \mathrm{E}-04$ & $3.34 \mathrm{E}-03$ & - & $3.22 \mathrm{E}-03$ & - & $9.19 \mathrm{E}-04$ \\
\hline $\mathrm{Al}(\mathrm{OH})_{4}[$ fraction] & -1 & 28 & $5.86 \mathrm{E}-02$ & 4.99E-02 & $2.38 \mathrm{E}-02$ & 9.49E-02 & $5.85 \mathrm{E}-06$ & $9.16 \mathrm{E}-02$ & - & $9.54 \mathrm{E}-02$ \\
\hline
\end{tabular}

a The relative mole fractions refer to mole fractions for each anion where the their values are normalized to the sum of anions considered and listed in this table

The anions considered are based on the available limiting ionic conductances found within the literature as listed in Table 7-3.

$\mathrm{b}$ These are solutions used in various CST column tests which were considered in the column transport assessment efforts. 
Table 7-8. Best estimate cesium effective molecular and pore diffusion coefficients at $25{ }^{\circ} \mathrm{C}$ for each Phase 1 LAW batch feed.

\begin{tabular}{|c|c|c|c|c|}
\hline Envelope & $\begin{array}{c}\text { Mole averaged Ion } \\
\text { Pair }^{\text {a }} \\
\text { (Cs - anion })\end{array}$ & $\begin{array}{l}\text { Molecular Diffusion } \\
\text { coef. in water } \\
\left(\mathrm{cm}^{2} / \mathrm{min}\right)\end{array}$ & $\begin{array}{c}\text { Molecular diffusion coef. } \\
\text { in simulated } 5 \mathrm{M} \mathrm{Na} \\
\text { waste }^{\mathrm{b}} \\
\left(\mathrm{cm}^{2} / \mathrm{min}\right)\end{array}$ & $\begin{array}{c}\text { Pore diffusion coef. in } \\
\text { simulated } 5 \mathrm{M} \mathrm{Na} \\
\text { waste }^{\mathrm{c}} \\
\left(\mathrm{cm}^{2} / \mathrm{min}\right)\end{array}$ \\
\hline \multirow[t]{11}{*}{$\mathbf{A}$} & LAW-1 & $1.329 \mathrm{E}-03$ & $4.522 \mathrm{E}-04$ & $9.044 \mathrm{E}-05$ \\
\hline & LAW-5 & $1.268 \mathrm{E}-03$ & $4.312 \mathrm{E}-04$ & $8.624 \mathrm{E}-05$ \\
\hline & LAW-6 & $1.281 \mathrm{E}-03$ & 4.357E-04 & $8.715 \mathrm{E}-05$ \\
\hline & LAW-8 & $1.336 \mathrm{E}-03$ & $4.545 \mathrm{E}-04$ & $9.090 \mathrm{E}-05$ \\
\hline & LAW-9 & $1.221 \mathrm{E}-03$ & $4.152 \mathrm{E}-04$ & $8.303 \mathrm{E}-05$ \\
\hline & LAW-10 & $1.274 \mathrm{E}-03$ & $4.334 \mathrm{E}-04$ & $8.669 \mathrm{E}-05$ \\
\hline & LAW-11 & $1.279 \mathrm{E}-03$ & 4.349E-04 & $8.698 \mathrm{E}-05$ \\
\hline & LAW-12 & $1.360 \mathrm{E}-03$ & $4.624 \mathrm{E}-04$ & $9.248 \mathrm{E}-05$ \\
\hline & LAW-13 & $1.359 \mathrm{E}-03$ & 4.624E-04 & $9.247 \mathrm{E}-05$ \\
\hline & LAW-14 & $1.374 \mathrm{E}-03$ & 4.674E-04 & $9.347 \mathrm{E}-05$ \\
\hline & LAW-15 & $1.418 \mathrm{E}-03$ & $4.822 \mathrm{E}-04$ & $9.643 \mathrm{E}-05$ \\
\hline \multirow[t]{2}{*}{ B } & LAW-2a & $1.151 \mathrm{E}-03$ & $3.914 \mathrm{E}-04$ & $7.828 \mathrm{E}-05$ \\
\hline & LAW-2b & $1.119 \mathrm{E}-03$ & $3.807 \mathrm{E}-04$ & 7.614E-05 \\
\hline \multirow[t]{3}{*}{$\mathbf{C}$} & LAW-3 & $1.280 \mathrm{E}-03$ & 4.354E-04 & $8.709 \mathrm{E}-05$ \\
\hline & LAW-4 & $1.280 \mathrm{E}-03$ & $4.354 \mathrm{E}-04$ & $8.709 \mathrm{E}-05$ \\
\hline & LAW-7 & $1.240 \mathrm{E}-03$ & $4.217 \mathrm{E}-04$ & $8.435 \mathrm{E}-05$ \\
\hline
\end{tabular}

${ }^{a}$ Relative anion mole fractions of each LAW candidate feed solution used based on compositions provided in Appendix Table A-5 and A-6 (and computed relative mole fractions listed in Tables 7-4 and 7-5).

${ }^{\mathrm{b}}$ Fluid viscosity correction ratio applied based on a measured simulated waste viscosity of $2.94 \mathrm{cp} \mathrm{at} 20^{\circ} \mathrm{C}$ (Steimke et al., 2000).

${ }^{\mathrm{c}}$ The cesium pore diffusion value is based on $20 \%$ of its molecular diffusion value where the tortuosity of the engineered-form of CST is assumed to independent of liquid composition. 
Preliminary Ion Exchange Modeling for Removal of Cesium from

Revision (Date):

$0(07 / 05 / 02)$

Hanford Waste Using Hydrous Crystalline Silicotitanate Material

Page:

68 of 338

Table 7-9. Best Estimate cesium effective molecular and pore diffusion coefficients at $25^{\circ} \mathrm{C}$ for various solutions used in column transport assessments.

\begin{tabular}{|c|c|c|c|}
\hline $\begin{array}{c}\text { Mole averaged Ion Pair }{ }^{\text {a }} \\
\text { (Cs - anion) }\end{array}$ & $\begin{array}{l}\text { Molecular Diffusion } \\
\text { coef. in water } \\
\left(\mathrm{cm}^{2} / \mathrm{min}\right)\end{array}$ & $\begin{array}{c}\text { Molecular diffusion coef. } \\
\text { in simulated } 5 \mathrm{M} \mathrm{Na} \\
\text { waste } \\
\left(\mathrm{cm}^{2} / \mathrm{min}\right)\end{array}$ & $\begin{array}{c}\text { Pore diffusion coef. in } \\
\text { simulated 5M Na } \\
\text { waste }^{\mathrm{d}} \\
\left(\mathrm{cm}^{2} / \mathrm{min}\right)\end{array}$ \\
\hline SRS Avg & $1.382 \mathrm{E}-03$ & $4.972 \mathrm{E}-04^{\mathrm{b}}$ & $9.944 \mathrm{E}-05$ \\
\hline SRS High $\mathrm{OH}^{-}$ & $1.508 \mathrm{E}-03$ & $5.425 \mathrm{E}-04^{b}$ & $1.085 \mathrm{E}-04$ \\
\hline SRS Tank 44 & $1.658 \mathrm{E}-03$ & $5.963 \mathrm{E}-04^{b}$ & $1.193 \mathrm{E}-04$ \\
\hline Brown AW-101 & $1.429 \mathrm{E}-03$ & $4.861 \mathrm{E}-04^{\mathrm{c}}$ & $9.721 \mathrm{E}-05$ \\
\hline ORNL W27 & $1.194 \mathrm{E}-03$ & $4.062 \mathrm{E}-04^{\mathrm{c}}$ & $8.124 \mathrm{E}-05$ \\
\hline Sandia DSSF5 & $1.422 \mathrm{E}-03$ & $4.836 \mathrm{E}-04^{\mathrm{c}}$ & $9.672 \mathrm{E}-05$ \\
\hline Texas A\&M Exp1 & $2.212 \mathrm{E}-03$ & $7.524 \mathrm{E}-04^{\mathrm{c}}$ & $1.505 \mathrm{E}-04$ \\
\hline Hendrickson AW-101 & $1.430 \mathrm{E}-03$ & $4.863 \mathrm{E}-04^{\mathrm{c}}$ & $9.726 \mathrm{E}-05$ \\
\hline
\end{tabular}

${ }^{a}$ Relative anion mole fractions of each solution used based on compositions and computed relative mole fractions provided in Table 7-7.

${ }^{\mathrm{b}}$ Fluid viscosity correction ratio applied based on a measured simulated waste viscosity of $2.94 \mathrm{cp}$ at $20{ }^{\circ} \mathrm{C}$ (Steimke et al., 2000).

${ }^{\mathrm{c}}$ Fluid viscosity correction ratio applied based on a measured simulated waste viscosity of $2.78 \mathrm{cp}$ at $20^{\circ} \mathrm{C}$ (Walker et al., ?).

${ }^{\mathrm{d}}$ The cesium pore diffusion value is based on $20 \%$ of its molecular diffusion value where the tortuosity of the engineered-form of CST is assumed to independent of liquid composition. 


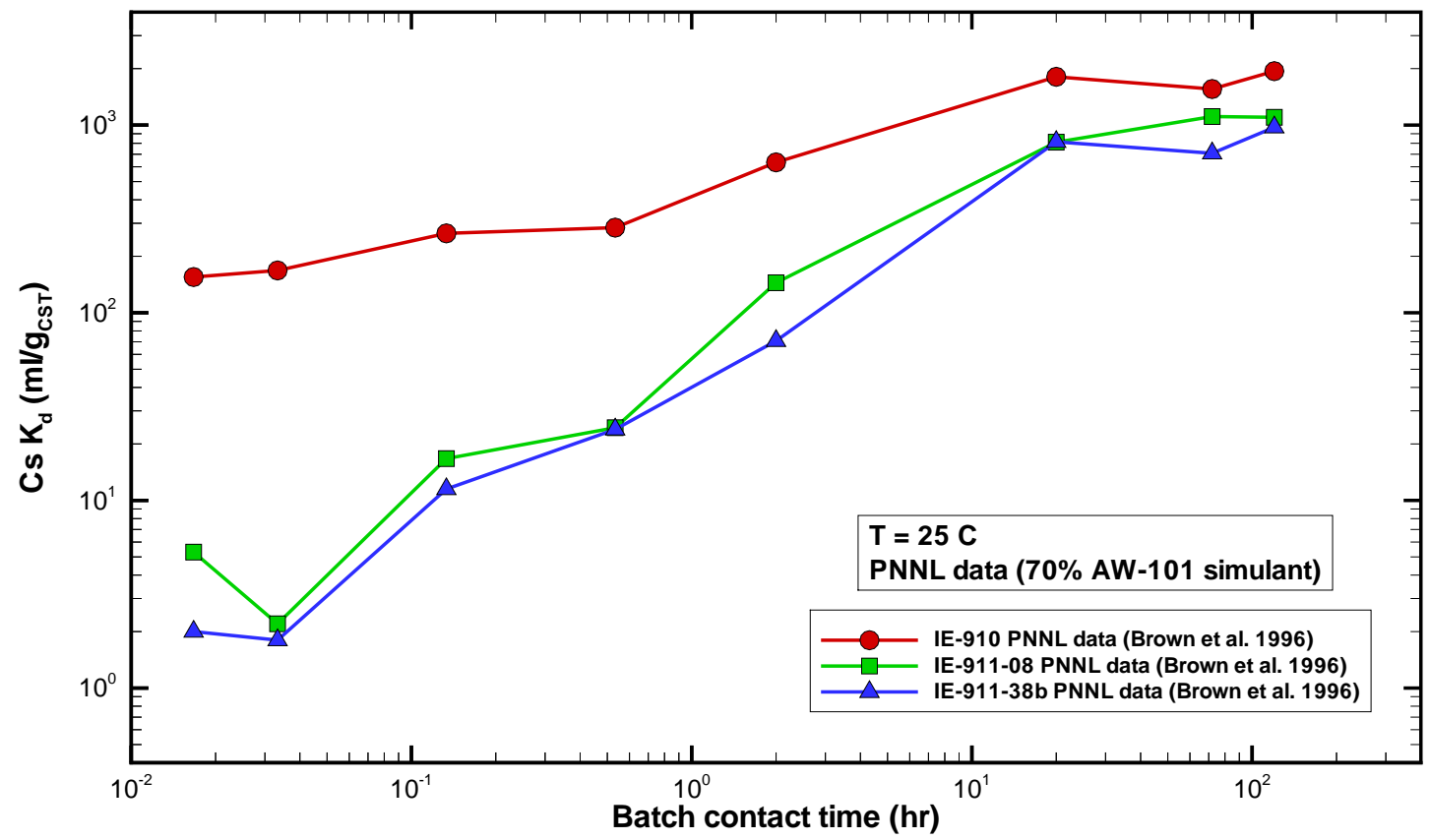

Figure 7-1. Cesium $\mathrm{K}_{\mathrm{d}}$ measurements obtained from batch kinetics tests performed by Brown et al. (1996) at $25 \mathrm{C}$ and in a $70 \% \mathrm{AW}-101 \mathrm{DSSF}$ simulant liquid at $1 \times 10^{-4} \mathrm{M} \mathrm{Cs}$ and $5 \mathrm{M} \mathrm{Na}$.

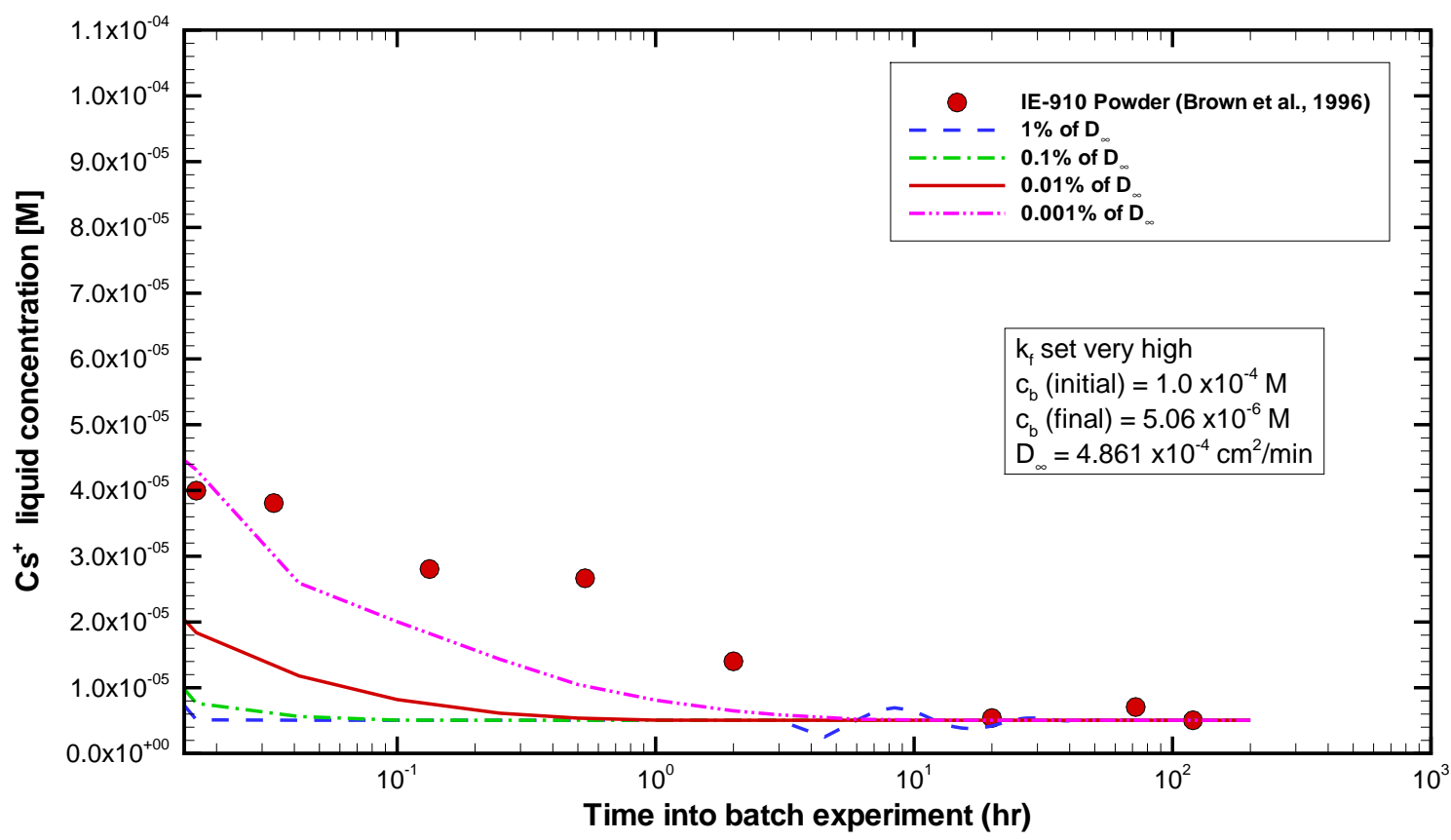

Figure 7-2. Estimation of the cesium pore diffusion coefficient based on batch kinetics tests performed by Brown et al. (1996) at $25 \mathrm{C}$ for cesium uptake on IONSIV ${ }^{\circledR}$ IE-910 CST (powderform). 


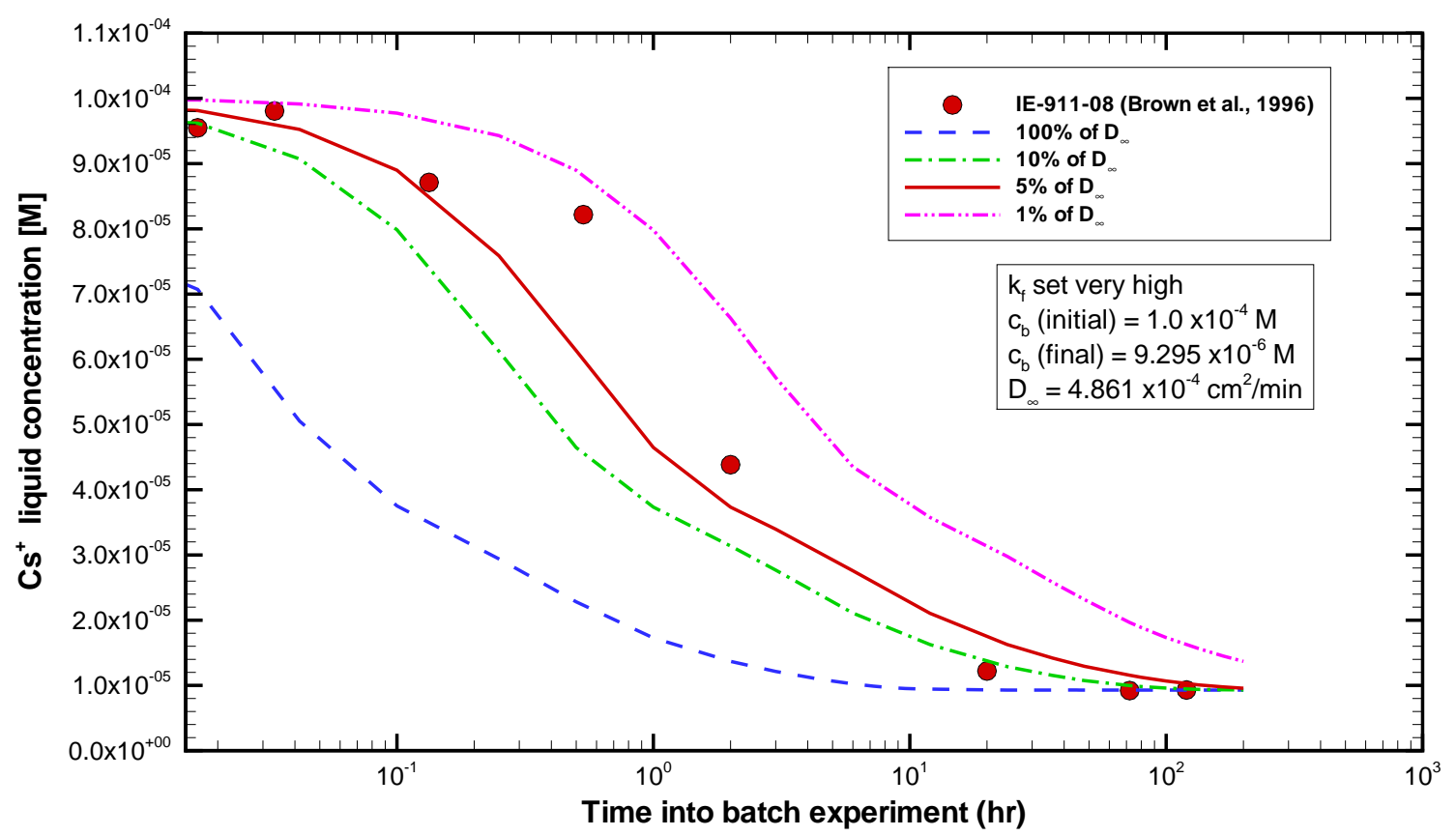

Figure 7-3. Estimation of the cesium pore diffusion coefficient based on batch kinetics tests performed by Brown et al. (1996) at $25 \mathrm{C}$ for cesium uptake on IONSIV $^{\circledR}$ IE-911 CST (engineeredform 08).

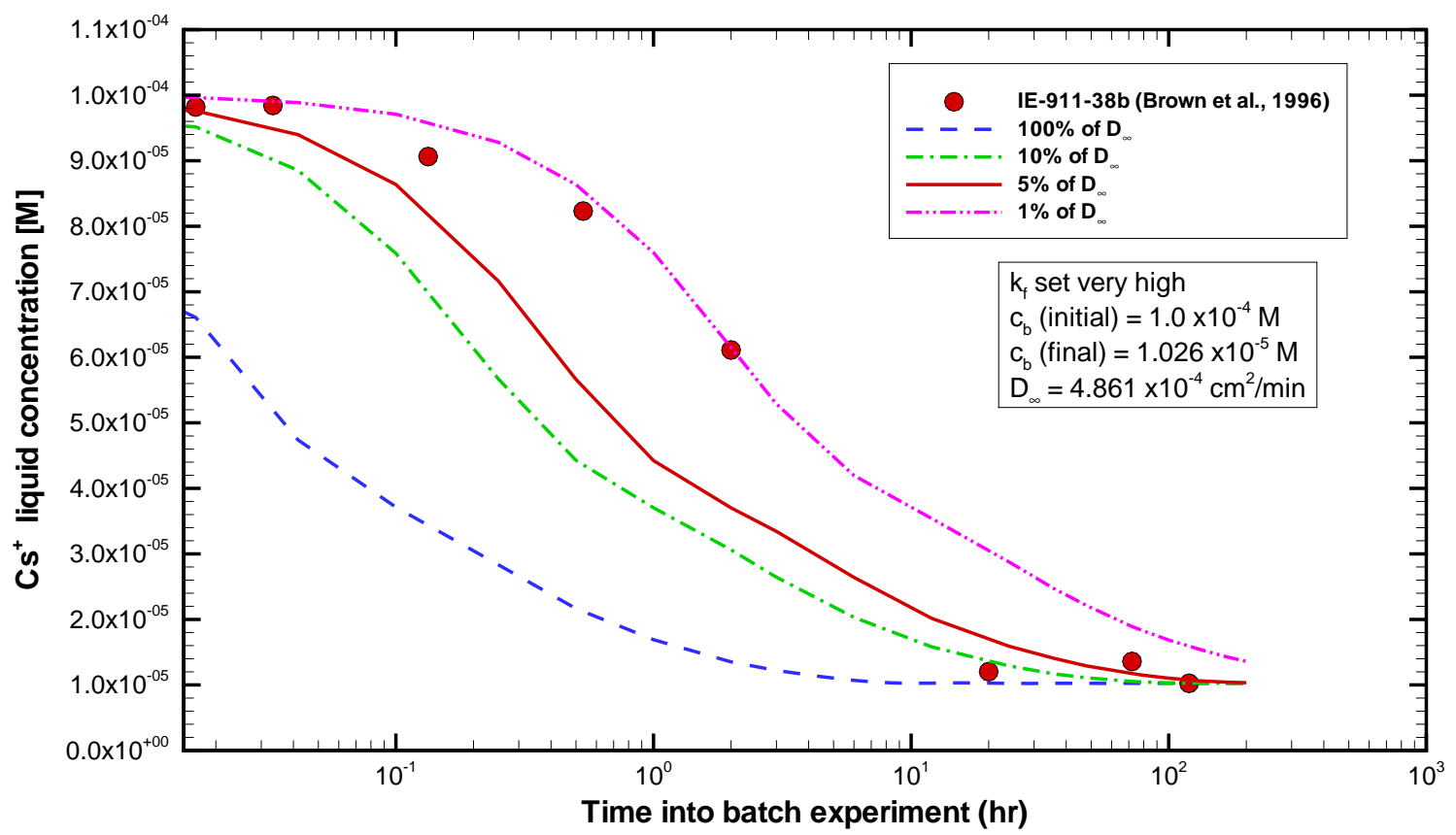

Figure 7-4. Estimation of the cesium pore diffusion coefficient based on batch kinetics tests performed by Brown et al. (1996) at $25 \mathrm{C}$ for cesium uptake on IONSIV ${ }^{\circledR}$ IE-911 CST (engineeredform $38 b)$. 


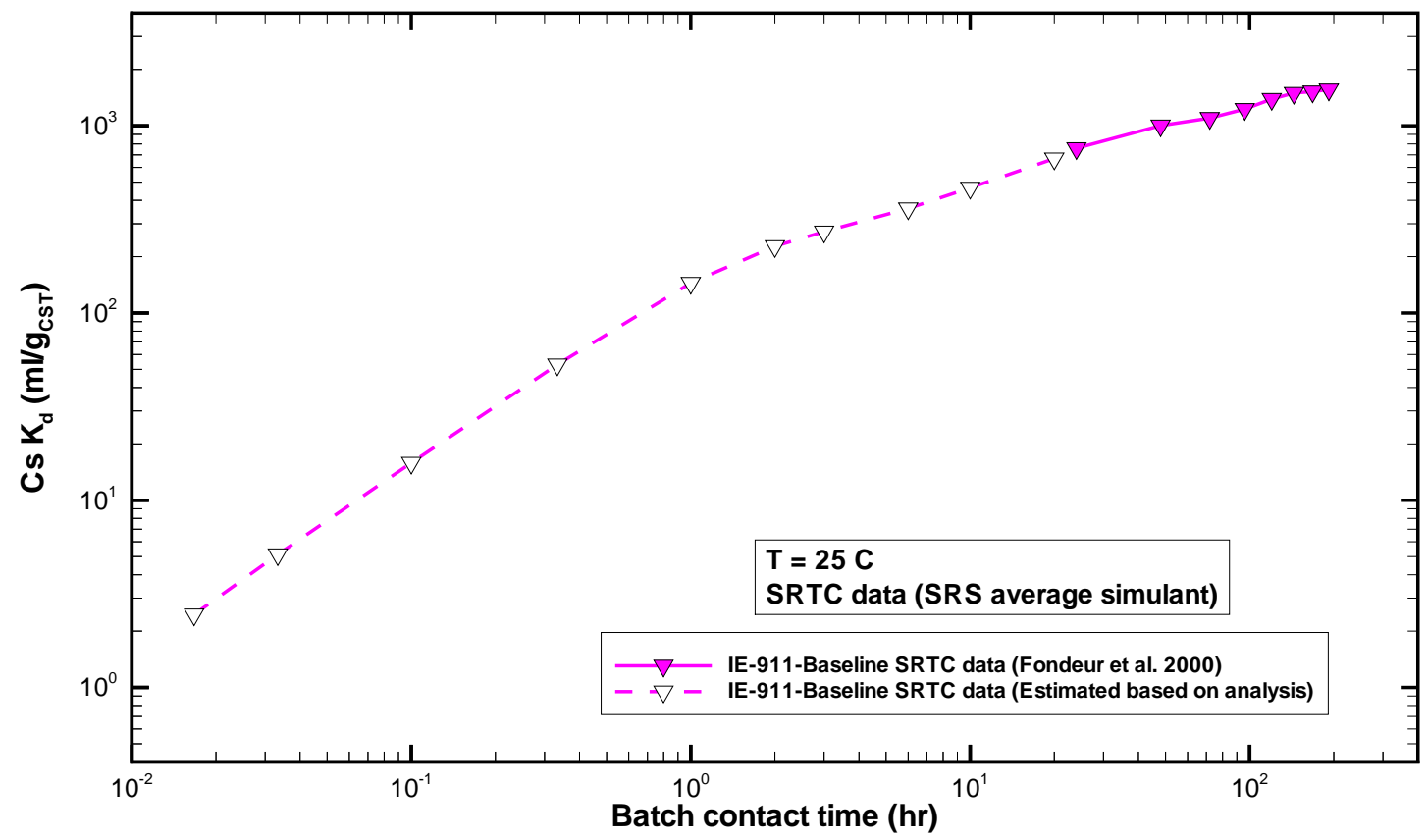

Figure 7-5. Cesium $\mathrm{K}_{\mathrm{d}}$ measurements obtained from batch kinetics tests performed by Fondeur et al. (2000) at $25 \mathrm{C}$ and in a SRS average simulant liquid at $1.4 \times 10^{-4} \mathrm{M} \mathrm{Cs}$ and $5.6 \mathrm{M} \mathrm{Na}$. Also shown is an estimated behavior at early times for the Fondeur et al. (2000) data.

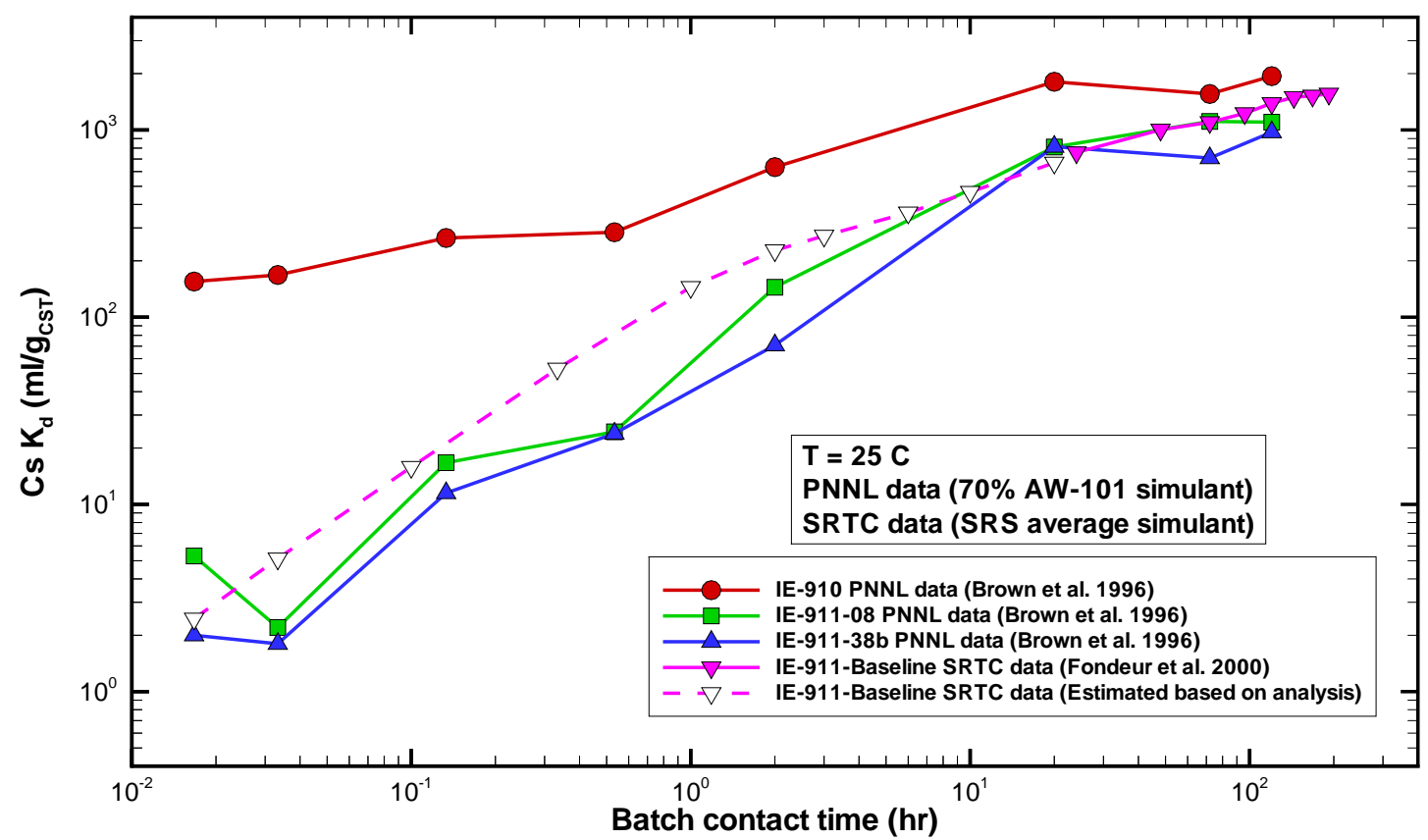

Figure 7-6. A comparison of the cesium $\mathrm{K}_{\mathrm{d}}$ measurements obtained from batch kinetics tests performed by Brown et al. (1996) and Fondeur et al. (2000) on various CST materials at 25 C. 


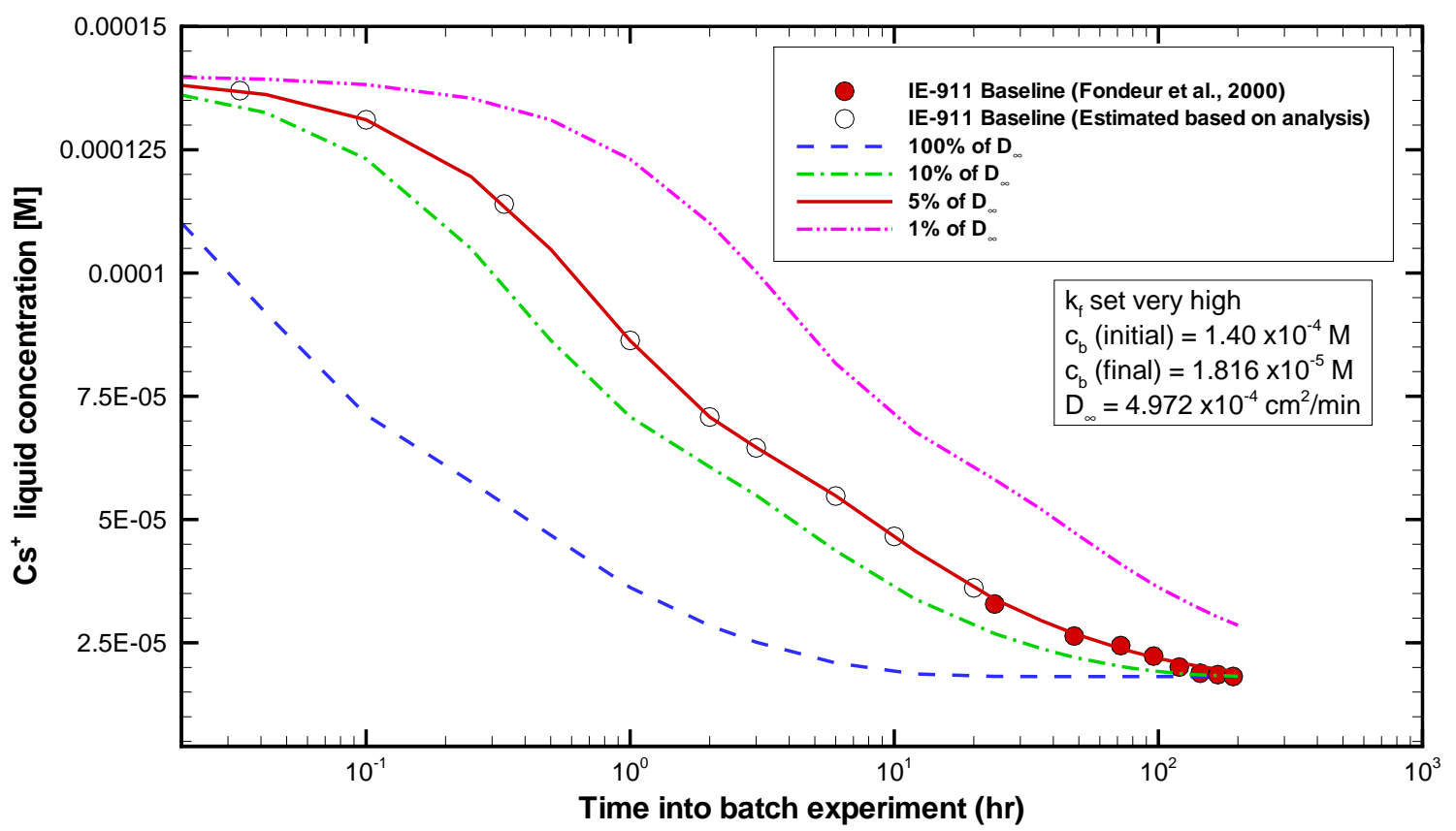

Figure 7-7. Estimation of the cesium pore diffusion coefficient based on batch kinetics tests performed by Fondeur et al. (2000) at $25 \mathrm{C}$ for cesium uptake on IONSIV ${ }^{\circledR}$ IE-911 CST (engineered-form Baseline).

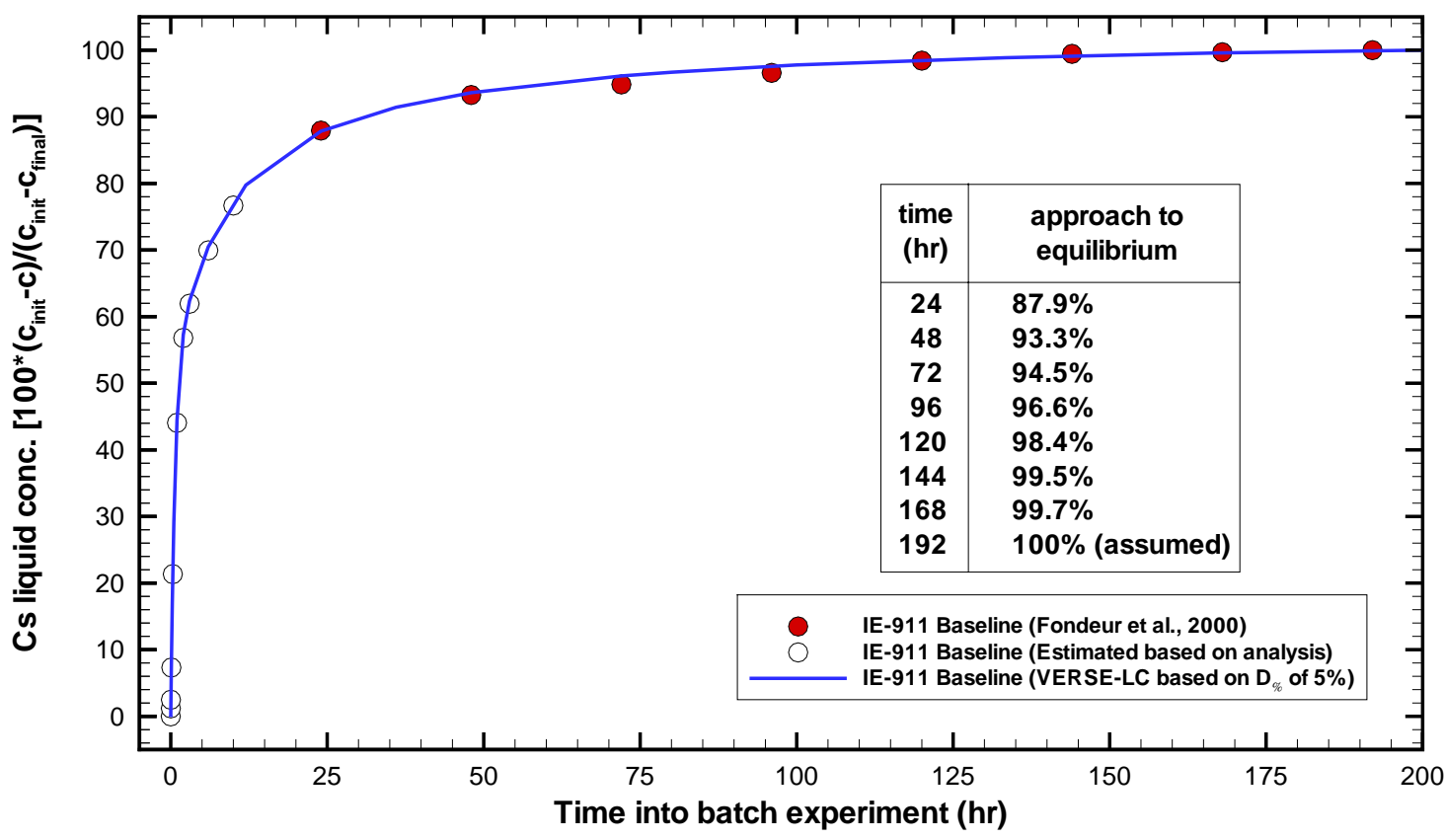

Figure 7-8. Comparison of VERSE-LC predictions to measured cesium concentrations during approach to equilibrium based on batch kinetics tests performed by Fondeur et al. (2000) at $25 \mathrm{C}$

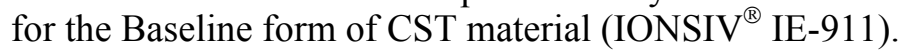




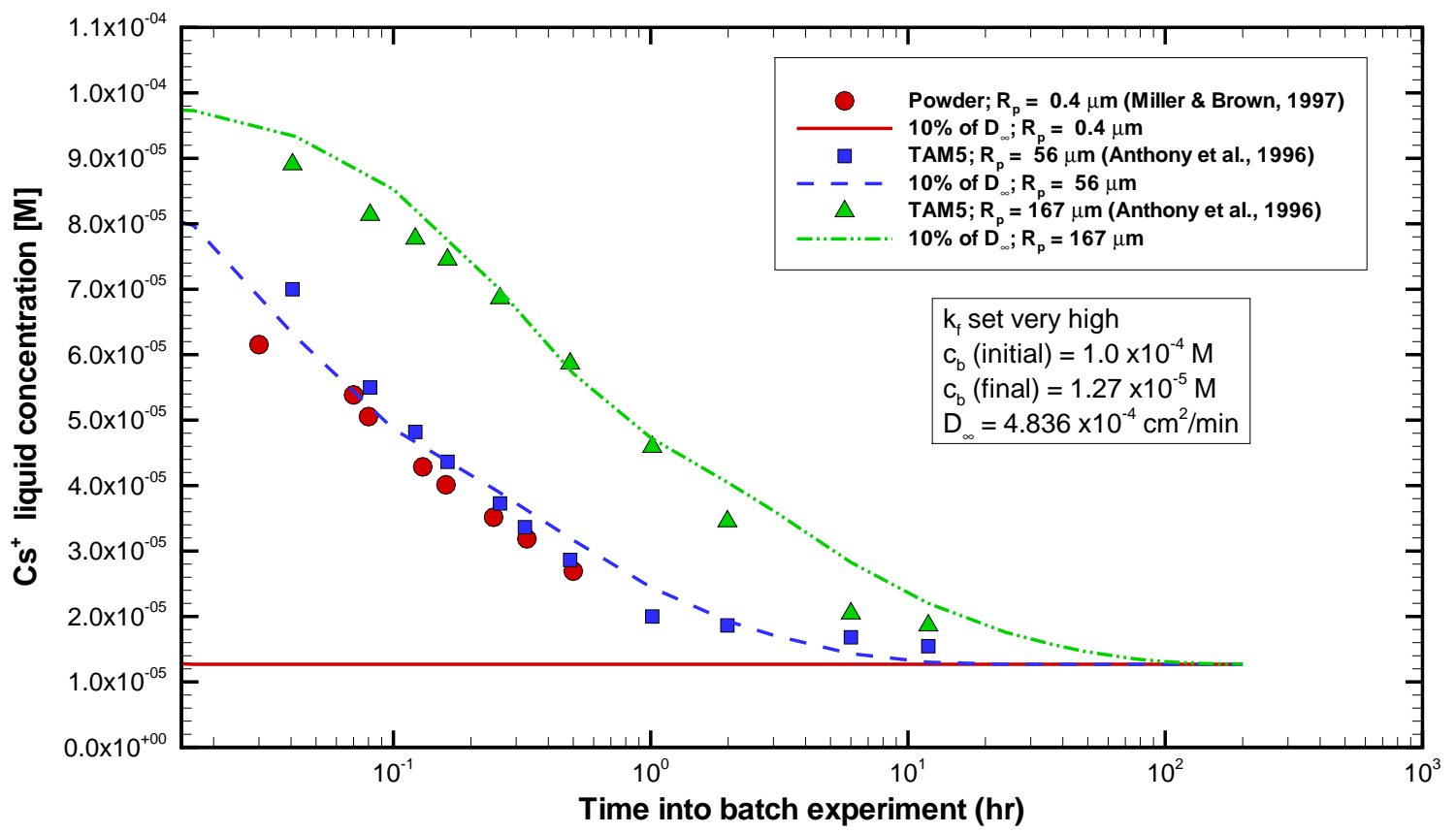

Figure 7-9. The effect particle size has on rates of cesium uptake by IONSIV ${ }^{\circledR}$ IE-910 (Miller and Brown, 1997) and by IONSIV ${ }^{\circledR}$ IE-911 (Anthony et al., 1996) CST materials based on transient cesium uptake testing.

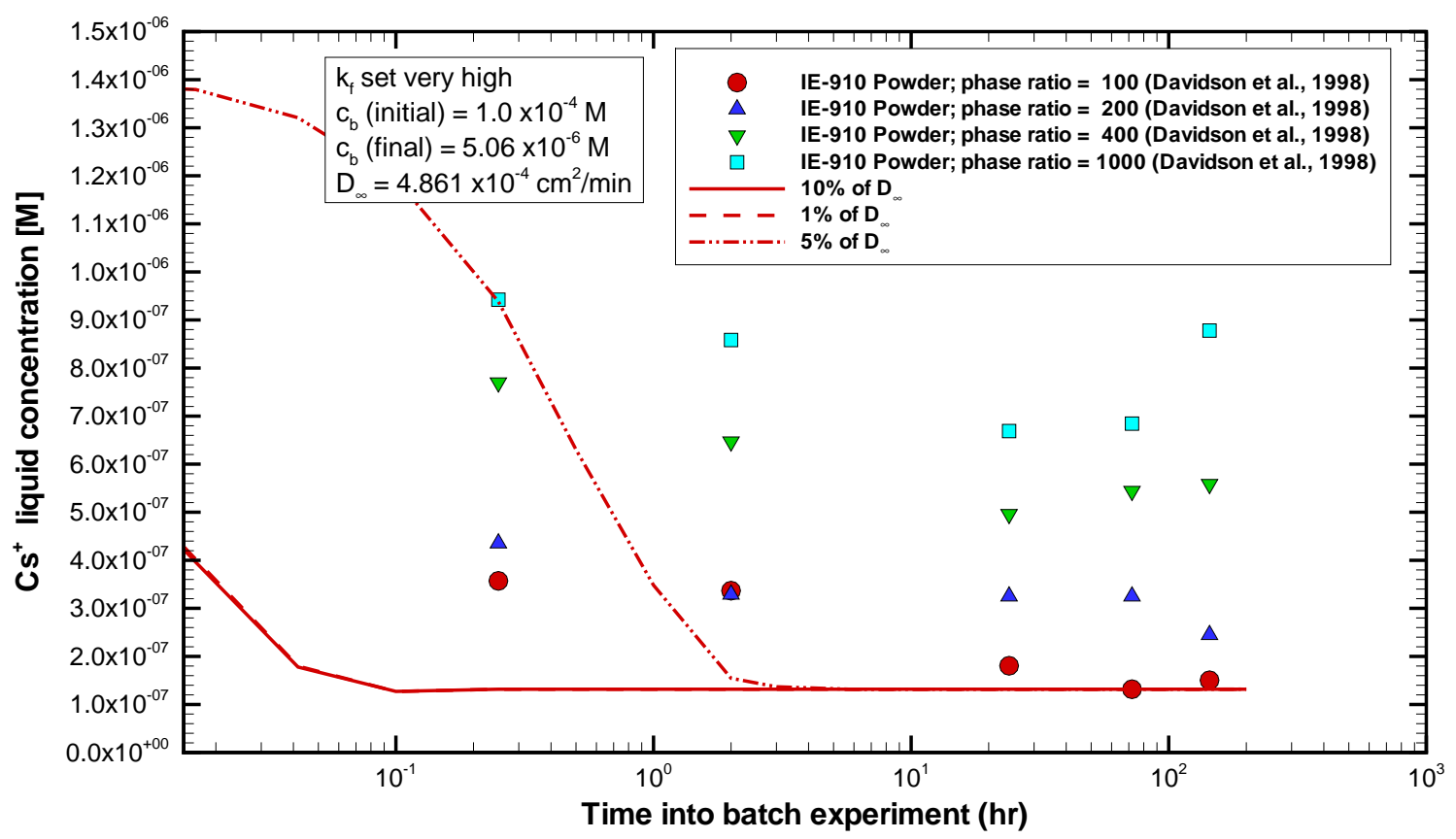

Figure 7-10. The effect phase ratio has on rates of cesium uptake by IONSIV ${ }^{\circledR}$ IE-910 CST material (Davidson et al., 1998) based on batch kinetics testing. 


\subsection{Axial Dispersion and Film Diffusion}

In this section we present the correlations used to define: (1) axial dispersion along the bed length and (2) mass transfer across the liquid film separating the bed fluid from its neighboring particle pore fluid.

\subsection{Film Diffusion}

For the laboratory-scale column tests and proposed full-scale facility, with the IONSIV ${ }^{\circledR}$ IE-911 CST particle size distributions, the Reynolds number range is approximately 0.1 to 1.0 . With respect to published literature this is a very low Reynolds number range. Numerous mass transfer correlations exist as discussed by Foo and Rice (1975, see their Figure 2). One of the correlations compared in Foo and Rice (1975) is one developed by Wilson and Geankoplis (1966) based on low Reynolds number data. Large variations between correlations can be seen; however, sensitivity to the film coefficient is low as shown in Hamm et al. (2000a and 2000b). Since VERSE-LC has the Wilson and Geankoplis (1966) correlation as an option and this correlation falls somewhat within the spread of available low Reynolds number data we have chosen it for all the column simulations in this report. For each ion species considered, the Wilson and Geankoplis (1966) correlation is expressed as:

$$
\mathrm{J} \equiv\left[\frac{\mathrm{k}_{\mathrm{fi}}}{\mathrm{u} \varepsilon_{\mathrm{b}}}\right] \mathrm{Sc}_{\mathrm{i}}^{2 / 3}=\frac{1.09}{\varepsilon_{\mathrm{b}}} \mathrm{Re}^{-2 / 3}
$$

where the Reynolds number is defined as

$$
\operatorname{Re} \equiv \frac{2 \mathrm{R}_{\mathrm{p}} \rho_{\mathrm{w}} \mathrm{u} \varepsilon_{\mathrm{b}}}{\mu_{\mathrm{w}}},
$$

and the Schmidt number for each species is defined as

$$
\mathrm{Sc}_{\mathrm{i}} \equiv \frac{\mu_{\mathrm{w}}}{\rho_{\mathrm{w}} \mathrm{D}_{\mathrm{i}}^{\infty}}
$$

A standard deviation of approximately $25 \%$ is reported for Eq. (8-1) by Wilson and Geankoplis (1966), while from comparison to the various correlations presented by Foo and Rice (1975) a standard deviation of $100 \%$ to $200 \%$ is observed.

\subsection{Axial Dispersion}

Axial dispersion in packed columns is the result of mechanical dispersion added onto molecular diffusion. For practical flowrates mechanical dispersion dominates. For well-packed columns of sufficient diameter such that wall effects (i.e., channeling) are minimal a variety of correlations exist for long column performance. A brief discussion of minimum column sizing is presented in Brooks (1994). 
In the low Reynolds number range of interest the Chung and Wen (1968) correlation is applicable for sufficiently large columns (i.e., large diameter and length) and is expressed as:

$$
\mathrm{E}_{\mathrm{b}}=\frac{2 \mathrm{R}_{\mathrm{p}} \mathrm{u} \varepsilon_{\mathrm{b}}}{0.2+0.011 \mathrm{Re}^{0.48}},
$$

where the standard deviation of this correlation based on all available data points was reported to be $46 \%$. Equation (8-2) applies for only sufficiently large columns and correction factors must be considered for columns with small diameters and/or short active bed lengths.

\subsubsection{Radial Flow Maldistribution}

Flow maldistribution is caused by packing irregularities. As such the bed porosity varies over the cross-section of a column and increases as the outer wall is approached (even for wellpacked columns). "Channeling" near the wall becomes more serious for smaller column diameters and larger particle sizes. As a "rule of thumb" Helfferich (1962) states that this effect becomes significant when the bed diameter is less than thirty times the particle diameter.

The experimental and mathematical basis for this rule of thumb stems from the work of Schwartz and Smith (1953) and Morales et al. (1951). For further discussion on the basis and potential impacts due to flow maldistribution see Hamm et al. (2000b, Chapter 8).

The impact associated with non-uniform radial velocity profiles manifests itself in spreading out the exit breakthrough curves in a manner similar to increased axial dispersion. As listed in Table 9.1 of Section 9, all of the column experiments considered have column-to-particle diameter ratios of $\sim 30$ or greater. In the simulations performed in this report no explicit account was made for any apparent increase in axial dispersion.

\subsubsection{Headspace and Short Column Impacts}

The proposed carousel column configurations (i.e., two or three column arrangements) are composed of ion exchange columns that contain headspaces. Each column in the carousel is geometrically identical where above the active bed height is a headspace void. For the design calculations presented in Chapter 10 these inlet headspaces are modeled as CSTRs whose volumes are $25 \%$ of the active bed volumes.

Liles and Geankoplis (1960) conducted experiments to ascertain the impact short column lengths and void headspaces have on axial dispersion in packed bed columns. When end effects were eliminated they concluded that no effects of length on axial dispersion were observed. However, in the presence of end effects such as void headspaces significant effects of length can result. For further discussion on the basis and potential impacts due to end effects see Hamm et al. (2000b, Chapter 8).

In the simulations performed in this report no explicit account was made for this apparent increase in axial dispersion based on end effects. For long columns exceeding $60 \mathrm{~cm}$ these effects are generally small. 


\subsection{Laboratory-Scale Column Assessments}

To perform column transport analyses for sizing the CST columns, a method of estimating an "effective" cesium pore diffusion coefficient value is required. We had hoped that available batch kinetics test data would provide us the appropriate values. Unfortunately, the pore diffusion values based on existing kinetics data did not compare favorably when used in laboratory-scale column performance assessments. Given this situation, we are now estimating the pore diffusion coefficient values based on the laboratory-scale column data directly. Originally, we had planned to use the laboratory-scale column tests in a benchmark fashion but are no longer able to do so. In this chapter the results from our pore diffusion coefficient study are discussed. Note that a pore diffusion coefficient is computed as the product of a free molecular diffusion coefficient divided by a tortuosity factor. The molecular diffusion coefficient is based on the feed solution's composition, while the tortuosity factor is based on the solid structure of the CST material. It is this tortuosity factor that is being estimated in this chapter. A brief assessment to pilot-scale experiments is also provided.

For the various column tests considered in this chapter no direct measurements were made as to the amount of CST mass that was actually packed into the columns. Unfortunately, only the bed volumes were measured. The amount of ion exchanger within the bed is the dominant factor not the space that it occupies. All future column studies should measure the amount of ion exchanger present within the test column. In the VERSE-LC simulations a constant bed density of $1.0 \mathrm{~g} / \mathrm{ml}$ is assumed. This is an average observed value for several measured packed columns.

Numerous laboratory-scale column experiments have been performed to measure the cesium removal capability of the IONSIV $^{\circledR}$ IE-911 CST material for feed conditions typical for a range of SRS and Hanford waste types. A listing of the laboratory-scale and pilot-scale column studies considered in this chapter is provided in Table 9-1. Key features associated with each column are also given in Table $9-1$.

VERSE-LC was used to model 15 of these column experiments where cesium breakthrough curves were experimentally measured for column packed with IONSIV ${ }^{\circledR}$ IE-911 CST material. Several differing batch IDs were tested and in some cases corresponding equilibrium contact tests were available. Comparisons of these model calculations to the corresponding experimental data are provided in the following subsections. The VERSE-LC input files for each of the 15 simulations are listed in Appendix H. The transport properties were established by means other than fitting to the column data directly, except for the cesium pore diffusion coefficient. Binary isotherm models for cesium were created for each unique batch ID and waste type (i.e., differing total ionic strengths and compositions) based on ZAM generated databases. A modest shifting of breakthrough prediction versus measured was observed with respect to superficial velocity and future efforts should attempt to investigate this effect.

To briefly summarize our assessments, the exit breakthrough curve model predictions for cesium are generally reasonable when compared to the available data sets. Even for sequential column tests, where the lag column in a previous test is used as the lead column in a follow-on test. No 
effort to improve these predictions, by altering the initial estimates of the various transport parameters excluding the tortuosity factor, was attempted.

A summary of recommended nominal transport parameter settings for the Cesium-IONSIV ${ }^{\circledR}$ IE911 CST system column modeling is provided in Table 9-2. The parameters in Table 9-2 are ordered into groups based on their level of importance with respect to the determination of cesium exit breakthrough curves. The ordering of these parameters is based on the numerous simulation runs made during this assessment effort and our sensitivity study for the full-scale facility as discussed in Chapter 10 and earlier modeling efforts with SuperLig ${ }^{\circledR}$ resins.

\subsection{CST Pore Diffusion Coefficient}

CST packed columns are said to be mass transfer limited giving rise to relatively slow "kinetics". Therefore, in predicting cesium breakthrough behavior for CST packed columns the pore diffusion coefficient should be an important parameter. The "effective" cesium pore diffusion coefficient can be normalized with respect to the free stream "Brownian" diffusion coefficient. The inverse of this ratio is referred to as a tortuosity factor that is primarily dependent upon the internal structure of the porous material. For materials with very large pores a tortuosity factor approaching unity is observed, while for very small pores factors approaching zero can result. The rate of "kinetics" is inversely related to the tortuosity factor. Below the "best estimate" value of this ratio, and the basis of its creation, is discussed. This value was used in all the CST design analysis presented in this report.

\subsubsection{Tortuosity Factor Optimization Strategy}

It is assumed that this ratio (i.e., pore to free stream) is independent of the aqueous phase composition. The following limited optimization strategy to determine an appropriate value for this ratio was undertaken.

- The exit breakthrough data for several representative column performance tests were utilized (i.e., 9 SRTC tests and 1 PNNL test were considered). Some of the details for each case are provided in subsequent subsections below.

- For each test 5 VERSE-LC simulations were run varying this ratio (i.e., ratio values of $10 \%$, $20 \%, 26 \%, 30 \%$, and $35 \%$ ). Preliminary assessments indicated that this range would bracket the parameter. The dilution factor was fixed to $68 \%$ for every simulation used in the optimization.

- For each ratio value an overall residual in calculated breakthrough versus measured (i.e., residual in $\mathrm{c} / \mathrm{c}_{\mathrm{o}}$ ) in the form of a root-mean-square was computed. The minimum of this residual curve provided us with a "best estimate" value of this ratio.

The performance of a column is predominately impacted by the isotherm's dilution factor and the pore diffusion coefficient value. A two-parameter optimization study (i.e., dilution and ratio factors) was not undertaken. The dilution factor was set to $68 \%$ during this optimization study. Future efforts to better estimate the cesium pore diffusion coefficients (i.e., tortuosity) is 
recommended. However, VERSE-LC simulations are shown below where a dilution factor of both $68 \%$ and $100 \%$ were used.

The cost function that was chosen to be minimized is given by:

$$
\Phi(\eta) \equiv \Phi\left(\frac{1}{\tau}\right)=\sqrt{\frac{1}{n_{\text {tests }}} \sum_{i=1}^{n_{\text {tests }}}\left[\frac{1}{n_{\text {data }, i}} \sum_{j=1}^{n_{\text {data }, i}}\left\{\left(\frac{c}{c_{o}}\right)_{i, j}^{\text {calc }}-\left(\frac{c}{c_{o}}\right)_{i, j}^{\exp }\right\}\right]},
$$

where

$$
\eta \equiv \frac{1}{\tau}=\frac{\mathrm{D}_{\mathrm{p}}}{\mathrm{D}_{\infty}}
$$

Since the data points within a given test are used to primarily specify the shape of each breakthrough curve, the overall residual based on several column tests, $\mathrm{n}_{\text {tests }}$, should be computed where equal weighting of each column test is achieved. Equation (9-1) represents a weighted root-mean-square (rms) of individual column test residuals where each set of data from a given column test is weighted by the number of data points taken during the test, $\mathrm{n}_{\text {data. }}$. In this way, uniform weighting on a column test basis is maintained. Since the cesium feed concentration can vary greatly from test to test, the data point residuals are normalized to each test's feed concentration (i.e., $\mathrm{c} / \mathrm{c}_{\mathrm{o}}$ ). Note that a value of say 0.1 for the cost function, Eq. (9-1), indicates that the VERSE-LC's predictive capability would be on average within $10 \%$ of the measured curve.

\subsubsection{Tortuosity Factor Optimization Results}

The residuals of the ten various simulations considered are plotted in Figure 9-1, along with the composite rms. The composite results of the simulations are also summarized in Figure 9-2. In Figure 9-2 the solid circles represent the computed composite rms (root-mean-square) values, the solid curve is a spline-fit through the points, and the dashed curves represent estimated behavior outside the tested range. Only 8 of the 10 data sets were used in generating the composite results. After our initial runs, two of the data sets were omitted (i.e., test SRS-Avg-Test5 contained only limited early time breakthrough data, while test PNNL-AW101-Test1 had questionable feed composition values). As shown in Figure 9-1, the rms curves for these two tests do not exhibit minima within the ratio range shown.

For theoretical considerations, as pore diffusion approaches zero the predicted breakthrough curve approaches unity at all times, resulting in a rms of $\sim 0.5$. As pore diffusion approaches infinity the predicted breakthrough curve approaches the curve dictated by film diffusion only. At some intermediate value a minimum in the rms curve is expected. For a given column performance, the area between unity and the breakthrough curve represents the total amount of cesium stored within a column as a function of time and at long times this area will be identical for any pore diffusion coefficient value assumed (i.e., capacity depend only). Due to this mass balance constraint placed on the entire exit breakthrough curve, we would expect to see a rather shallow minimum in the rms curve as seen in Figure 9-2. Based on uncertainties the minimum in 
the rms curve shown in Figure 9-2 probably exists within the range of $20 \%$ to $30 \%$. Using the data (and their individual spline-fits), as shown in Figure 9-1, an overall minimum value of $\sim 22 \%$ is computed with an $\sim 4.4 \%$ standard deviation. The individual rms values for all ten tests is given in Table 9-3.

The origin of this can further be seen in the comparison of exit breakthrough curves for one specific column study as shown in Figures 9-3 and 9-4 (SRTC data by Wilmarth et al., 1999). The total area for each VERSE-LC simulation curve will be equal. For lower pore diffusion coefficient values (e.g., 10\% in Figure 9-3) we see earlier than measured breakthrough at short process times, while for higher pore diffusion coefficient values (e.g., 35\% in Figure 9-3) we see later than measured breakthrough. For this case study the average deviations of the VERSE-LC predictions from measurement are approximately the same for ratio values between $20 \%$ to $30 \%$. Basically, values between $20 \%$ to $30 \%$ result in equally acceptable predictions when viewed on an integrated basis. However, in sizing the CST columns and holding all other parameters fixed, lower pore diffusion coefficient values result in larger column sizes.

\subsubsection{Tortuosity Factor Coefficient Recommendation}

Observable predictive improvement is obtained between ratio values of $20 \%$ and $30 \%$ as shown in Figure 9-2. Looking at one specific case study (SRTC data by Wilmarth et al., 1999), the cesium breakthrough predictions based on both the $20 \%$ and $30 \%$ ratio values are shown in Figure 9-4. Consistent with the overall rms values shown in Figure 9-2, the two breakthrough predictions in Figure 9-4 are approximately of equal quality. However, with all other parameters being fixed the lower the pore diffusion coefficient the earlier the breakthrough. From a design perspective, lower pore diffusion coefficient values provide more conservative design estimates.

Based on this, it is our recommendation that we use the lower estimated limit of the rms minimum (i.e., ratio value of $20 \%$ ). It is believed that a value lower than $\sim 20 \%$ would significantly impact column size due to its impact on the leading edge of the concentration wave and a clear systematic (on average) departure from the breakthrough data is seen for values lower than this.

\subsection{Column Assessment Studies}

Below we discuss the 15 different column tests listed in Table 9-1. Eight of these tests were used in the tortuosity factor estimation, while the remaining are provided for assessment purposes. The tests are grouped into subsections based on the feed composition type, (SRS simulant, Hanford sample, etc.).

\subsubsection{SRS Tank 44 Tests}

Walker et al. (1999) performed batch contact and column tests at $31 \mathrm{C}$ using CST IE-911 (Lot number 98-05) with actual diluted Tank 44 supernate. A single long bed column of $160 \mathrm{~cm}$ in length and $1.5 \mathrm{~cm}$ in diameter was tested where cesium concentrations at three axial sampling points were recorded (i.e., $10 \mathrm{~cm}$-lead, $85 \mathrm{~cm}$-lag, and $160 \mathrm{~cm}$-guard column locations). The sampling point at $10 \mathrm{~cm}$ represents a lead column, at $85 \mathrm{~cm}$ a lag column, and at $160 \mathrm{~cm}$ a guard 
column. The inlet feed concentration of cesium was set to $3.51 \times 10^{-4} \mathrm{M}$ with a volumetric flowrate of $9.4 \mathrm{ml} / \mathrm{min}$ (i.e., superficial velocity of $5.319 \mathrm{~cm} / \mathrm{min}$ ). The cesium breakthrough data for the lead and lag column sampling points are provided in Appendix H.

At a sodium concentration of $5.4 \mathrm{M}$ the beta value for a cesium algebraic model was computed to be $2.0486 \times 10^{-4} \mathrm{M}$. A comparison of algebraic model to the batch contact test data based on CST engineered-form material is provided in Figure 9-5. The solid-curve represent the model's prediction for CST powder-form behavior, while the dashed-curve represents the prediction for CST engineered-form behavior. Unfortunately, for the Tank 44 solution we see that the data crosses over from the powder-form predictions at lower concentrations to the engineered-form predictions at higher concentrations. This particular behavior is unique with respect to the various other data sets considered and no explanation can be given for it at this time.

VERSE-LC simulations were run based on the column test parameters provided by Walker et al. (1999). Numerous simulations varying both the cesium isotherm (i.e., the dilution factor from 0.68 to 1.0 ) and cesium pore diffusion coefficient (i.e., tortuosity factor from $10 \%$ to $30 \%$ ). A comparison of breakthrough predictions to the data is provided in Figure 9-6 for four of the cases considered (i.e., dilution factors of 0.68 and 1.0 with tortuosity factors of $10 \%$ and $26 \%$ ). This data was used in the determination of the statistically based tortuosity factor as discussed earlier.

\subsubsection{SRS Average Simulant Tests}

Numerous column studies using CST packed columns and SRS average simulant have been performed. As one of three possible salt alternatives for processing SRS salt waste, these studies were performed over a several year timeframe. Here only a subset of these experiments is addressed. Several differing engineered-forms of CST were considered, both lab-scale and production-scale forms have been tested. In most cases some level of batch contact testing was performed for each series of column tests. The feed composition of the SRS-Avg simulant used in these tests is provided in Table C-1 of Appendix C and a discussion of their preparation is provided by Walker (1999b). The cesium isotherm models used in the VERSE-LC simulations are based on fits to ZAM code generated data at a temperature of $25 \mathrm{C}$. The resulting beta parameter used is $2.4145 \times 10^{-4} \mathrm{M}$. Some of the test details and measured breakthrough data for these tests are provided in Appendix H. Example VERSE-LC input files are also provided in Appendix H.

Wilmarth et al. (1999) performed a series of column tests at $\sim 25 \mathrm{C}$ where the effects of CST pretreatment, superficial velocity, and the presence of organic constituents were considered. Four of these column tests are considered here and their test details are provided in Appendix H, along with the VERSE-LC input files. Key parameters for each column are listed in Table 9-1. For the same size column, packed with CST material taken from the same batch, the effect of superficial velocity was varied in tests SRS-Avg-Test1, SRS-Avg-Test2, and SRS-Avg-Test3 (i.e., 5.5, 7.0, and $4.1 \mathrm{~cm} / \mathrm{min}$, respectively). A comparison of the data to VERSE-LC simulations is given in Figures 9-7, 9-8, and 9-9, respectively. The VERSE-LC predictions for Test 1 and Test 2 are reasonably consistent; however, for the two Test3 runs (i.e., Test3a was conducted with CST material having prior exposure to humid air, while Test3b did not) the predictions vary significantly. The test conditions for the Test 3 column is very similar to an 
earlier test performed by Walker et al. (1998), (i.e., test labeled SRS-Avg-Test7 in Table 9-1). A comparison of these three tests (i.e., Test3a, Test3b, and Test7) is shown in Figure 9-10.

The primary difference between Test3( $a$ and $b$ ) and Test7 is the CST Lot numbers. From the measured breakthrough data the earlier CST material (Lot number 96-04) appears to have less ion-exchange capacity than the newer CST material (Lot number 98-05). Wilmarth et al. (1999) repeated the Walker et al. (1998) test using the older CST material (Lot number 96-04). Their measured breakthrough curve was very similar to the earlier Walker et al. (1998) test data. Variability between Lots of this magnitude is seen throughout the CST development history (i.e., especially when a comparison of batch contact data is viewed). At the nominal superficial velocity of $4.1 \mathrm{~cm} / \mathrm{min}$, the impact of using a larger diameter column was tested in SRS-AvgTest4 and a comparison to VERSE-LC predictions is given in Figure 9-11. Even though we see batch variability within this work, all four tests by Wilmarth et al. (1999) were used in the tortuosity factor estimation. Some of the column test variability may be the result of bed density differences due to the manner in which these columns were originally packed. In the VERSELC simulations a constant bed density of $1.0 \mathrm{~g} / \mathrm{ml}$ was assumed.

Walker et al. (1998) performed several batch contact tests and three column tests using CST IE911 (Lot numbers 96-02 and 96-04) with SRS-Avg simulant. The column tests were performed at $\sim 22 \mathrm{C}$ and the key features of these three tests are listed in Table 9-1 (i.e., tests labeled SRSAvg-Test5, SRS-Avg-Test6, and SRS-Avg-Test7). The sizes of each column are approximately the same, while the CST packing was changed for the last column test. The volumetric flowrate (i.e., thus superficial velocity) was varied to exceed the expected operating range of the proposed full-scale SRS facility. Comparisons of the data to VERSE-LC simulations are shown in Figures 9-12, 9-13, and 9-14, respectively. Several VERSE-LC simulations are shown for each test. The cesium breakthrough data for the lead and lag column locations are provided in Appendix $\mathrm{H}$.

For test SRS-Avg-Test5 the slow flowrate resulted in only a small portion of the cesium breakthrough curve being measured. This data set was considered to be insufficient for our purposes in estimating a tortuosity factor and the results shown here are for completeness. The three VERSE-LC results shown predict earlier than measured breakthrough.

For test SRS-Avg-Test6 an intermediate flowrate was used. As Figure 9-13 illustrates, significant data scatter was observed in the form of oscillations. On average the data does exhibit a reasonable breakthrough curve and was included in the database for tortuosity factor estimation. The VERSE-LC prediction of breakthrough appears to be sensitive to the dilution factor and pore diffusion coefficient at this flowrate.

For test SRS-Avg-Test7 a fast flowrate was used. As Figure 9-14 illustrates, much smaller data scatter was observed in the form of oscillations. A complete cesium breakthrough curve was achieved and this data set was included in the database for tortuosity factor estimation. The VERSE-LC prediction of breakthrough appears to be less sensitive to the dilution factor and pore diffusion coefficient at this high flowrate.

When comparing the VERSE-LC predictions based on one specific set of input parameter values, we see a systematic shifting of the results from earlier-than to later-than breakthrough 
relative to the three data sets. This suggests that a functional superficial velocity dependence of one or more of the VERSE-LC modeling parameters is not being handled adequately (e.g., axial dispersion or film diffusion coefficient) at this time. However, no attempt to pursue this issue was undertaken.

\subsubsection{SRS High OH Simulant Tests}

Walker et al. (1999) also performed batch contact and column tests using CST IE-911 (Lot number 98-05) with SRS high OH simulant. The column test is basically a repeat of one of the earlier SRS Tank44 studies performed at $\sim 31 \mathrm{C}$ and the key features of this test is listed in Table 9-1 (i.e., test labeled SRS-High-OH-Test1). The size of each column is approximately the same, while the CST packing was based on Lot 98-05 material. Comparisons of the data to VERSELC simulations are shown in Figure 9-15. Several VERSE-LC simulations are shown for this test. The beta parameter used for the cesium isotherm is $2.0987 \times 10^{-4} \mathrm{M}$ based on a fit of data generated using the ZAM code at $31 \mathrm{C}$. No appreciable cesium breakthrough is observed at the $85 \mathrm{~cm}$ axial location. This data set was included in the database for tortuosity factor estimation.

\subsubsection{PNNL Hanford Sample Tests}

Hendrickson (1997) performed a column test using CST IE-911 (Lot number 96-01) with a diluted AW-101 sample. Lee et al. (1997a) also contains a brief description of this test alone with several other CST packed column tests. The column test was performed at $\sim 25 \mathrm{C}$ and the key features of these tests are listed in Table 9-1 (i.e., test labeled PNNL-AW101-Test1). Comparisons of the data to VERSE-LC simulations are shown in Figures 9-16. Several VERSELC simulations are shown. The beta parameter used for the cesium isotherm is $4.7414 \times 10^{-4} \mathrm{M}$ based on a fit of data generated using the ZAM code at $25 \mathrm{C}$. Unfortunately, the report was somewhat unclear as to the composition of the AW-101 simulant actually used in the column test. Therefore, this data set was not included in the tortuosity factor estimation.

\subsubsection{ORNL MVST Sample Tests}

During the mid-90s ORNL performed a "Cesium Removal Demonstration" (CsRD) project where cesium was removed from Melton Valley Storage Tank (MVST) waste using CST packed ion-exchange technology. Approximately 114,000 L (30,000 gal) of supernate was successfully processed. The final report for the CsRD project was issued by Walker, Jr., et al. (1998). Prior to testing the actual pilot-scale facility, various small-scale columns were tested. Below we will look at one of the small-scale tests and then take a look at the performance of the pilot-scale CsRD facility. In some cases due to the older CST batches used or the uncertainties we had in defining feed composition, none of the data sets discussed below were included in the tortuosity factor estimation. This data is primarily shown for completeness and for assessment purposes.

Lee et al. (1997b) performed column tests using CST IE-911 (Lot number -38b) with a MVST W-27 simulant (stored at ORNL). CST Lot number $-38 \mathrm{~b}$ was a developmental sample of CST supplied by UOP. In a summary report Davidson et al. (1998) also discusses these tests along with batch contact testing. The two column tests were performed at $\sim 25 \mathrm{C}$ and the key features 
of these tests are listed in Table 9-1 (i.e., tests labeled ORNL-W27-Test1 and ORNL-W27Test2). Test data and details are provided in Appendix H. These two tests use the same column being operated at different flowrates (i.e., $3 \mathrm{CV} / \mathrm{hr}$ and $6 \mathrm{CV} / \mathrm{hr}$ ). Comparisons of the data to VERSE-LC simulations are shown in Figures 9-17 and 9-18, respectively. Several VERSE-LC simulations are shown. The beta parameter used for the cesium isotherm is $9.3232 \times 10^{-4} \mathrm{M}$ based on a fit of data generated using the ZAM code at $25 \mathrm{C}$ and a measured bed density of $1.15 \mathrm{~g} / \mathrm{ml}$.

For the CsRD facility CST Lot number -38 b (note that this might also be called 96-01) was used. A two column carousel arrangement was used where both the lead and lag columns were always the same size (i.e., $30.6 \mathrm{~cm}$ in diameter and $51.67 \mathrm{~cm}$ in length). The breakthrough data and test details are provided by Walker, Jr., et al. (1998) and are also provided in Appendix H. Four basic processing operations were performed (i.e., referred to as runs). The first run (Run1) was limited in terms of the amount of cesium loading allowed and was performed primarily for assessing the radiation exposure risks. Here Run1 (i.e., constituted only a single lead column) is not considered. The feed compositions for the four CsRD runs were not well defined; therefore, cesium isotherms were generated based on the limited $\mathrm{K}_{\mathrm{d}}$ values for the feeds given by Walker. Jr., et al. (1998).

For Run2 a MVST W-29 supernate flowing at $\sim 3 \mathrm{CV} / \mathrm{hr}$ was processed where again only one freshly packed lead column was used. A total of $\sim 18,000 \mathrm{~L}$ were processed (i.e., $\sim 480 \mathrm{CVs}$ ). A comparison of VERSE-LC predictions to the data is shown in Figure 9-19.

For Run3 both freshly packed lead and lag columns were used. The flowrate was doubled over Run2's value (i.e., 6 CV/hr). Approximately 11,600 L were processed (i.e., $\sim 1040 \mathrm{CVs}$ ) where significant cesium breakthrough was achieved in both the lead and lag columns as shown in Figure 9-20. VERSE-LC predictions are also shown in Figure 9-20.

The fourth and final CsRD run (i.e., Run4) involved one carousel cycle where a total of 3 columns were utilized. During the first cycle $\sim 28,500$ L (i.e., $\sim 750 \mathrm{CVs}$ ) of feed was processed and approximately the same amount was processed during the second cycle. During the process run the flowrate was increased from its starting value of $3 \mathrm{CV} / \mathrm{hr}$ up to $6 \mathrm{CV} / \mathrm{hr}$. For simplicity the VERSE-LC simulations (i.e., cycle 1 named Run4a and cycle 2 Run $4 b$ ) were generated using a constant flowrate of $6 \mathrm{CV} / \mathrm{hr}$. The results based on varying the flowrate would only be slightly different. For cycle 1 a comparison of VERSE-LC predictions to the data is shown in Figure 921 and for cycle 2 is shown in Figure 9-22. The lag column in cycle 1 becomes the lead column during cycle 2. Reasonably accurate predictions for cycle 1 are seen in Figure 9-21 for both columns. However, as shown in Figure 9-22, the third column sitting in the lag position during cycle 2 experienced much earlier breakthrough than predicted. Given the acceptable predictions made for the first two columns, the discrepancies for the third column suggests that this column were either poorly packed or contained low capacity CST material. 
Table 9-1. Key features of Cesium-CST IE-911 fixed bed small-scale columns considered.

\begin{tabular}{|c|c|c|c|c|c|c|c|c|c|c|}
\hline $\begin{array}{l}\text { Column } \\
\text { ID }^{\mathrm{a}}\end{array}$ & $\begin{array}{l}\text { CST IE- } \\
911 \text { Lot } \\
\text { Number }\end{array}$ & $\begin{array}{l}\text { Liquid feed } \\
\text { type }\end{array}$ & $\begin{array}{c}\text { Active } \\
\text { bed } \\
\text { volume } \\
(\mathrm{ml})\end{array}$ & $\begin{array}{l}\text { Diameter } \\
\text { (cm) }\end{array}$ & $\begin{array}{l}\text { Length } \\
\text { (cm) }\end{array}$ & $\begin{array}{l}\text { Column-to- } \\
\text { particle } \\
\text { diameter } \\
\text { ratio }\end{array}$ & $\begin{array}{l}\text { Superficial } \\
\text { velocity } \\
\text { (cm/min) }\end{array}$ & $\mathrm{CV} / \mathrm{hr}$ & $\begin{array}{l}\text { Feed conc.: } \\
\text { total Cs } \\
{[\mathrm{M}]}\end{array}$ & Comments \\
\hline $\begin{array}{c}\text { SRS-Avg-Test1 } \\
\text { (Wilmarth et al., 1999) }\end{array}$ & $98-05$ & $\begin{array}{l}\text { SRS average } \\
\text { simulant }\end{array}$ & 17.7 & 1.5 & 10.0 & 43.6 & 5.5 & 33.0 & $1.30 \times 10^{-4} \mathrm{M}$ & $\begin{array}{l}\text { Test designed to study increased } \\
\text { flowrate. Data used in tortuosity } \\
\text { factor estimation. }\end{array}$ \\
\hline $\begin{array}{c}\text { SRS-Avg-Test2 } \\
\text { (Wilmarth et al., 1999) }\end{array}$ & $98-05$ & $\begin{array}{l}\text { SRS average } \\
\text { simulant }\end{array}$ & 17.7 & 1.5 & 10.0 & 43.6 & 7.0 & 42.0 & $1.24 \times 10^{-4} \mathrm{M}$ & $\begin{array}{l}\text { Test designed to study increased } \\
\text { flowrate. Data used in tortuosity } \\
\text { factor estimation. }\end{array}$ \\
\hline $\begin{array}{c}\text { SRS-Avg-Test3 } \\
\text { (Wilmarth et al., 1999) }\end{array}$ & $98-05$ & $\begin{array}{l}\text { SRS average } \\
\text { simulant }\end{array}$ & 17.7 & 1.5 & 10.0 & 43.6 & 4.1 & 24.6 & $1.43 \times 10^{-4} \mathrm{M}$ & $\begin{array}{l}\text { Two tests were run, Test3a used } \\
\text { CST material with prior exposure } \\
\text { to humid air, while Test3b did not. } \\
\text { Data used in tortuosity factor } \\
\text { estimation. }\end{array}$ \\
\hline $\begin{array}{c}\text { SRS-Avg-Test4 } \\
\text { (Wilmarth et al., 1999) }\end{array}$ & $98-05$ & $\begin{array}{l}\text { SRS average } \\
\text { simulant }\end{array}$ & 49.1 & 2.5 & 10.0 & 72.7 & 4.1 & 24.6 & $1.366 \times 10^{-4} \mathrm{M}$ & $\begin{array}{l}\text { Same conditions as for Test3 but } \\
\text { using a larger diameter column. } \\
\text { Data used in tortuosity factor } \\
\text { estimation. }\end{array}$ \\
\hline $\begin{array}{c}\text { SRS-Avg-Test5 } \\
\text { (Walker et al., 1998) }\end{array}$ & 96-02 & $\begin{array}{l}\text { SRS average } \\
\text { simulant }\end{array}$ & 17.7 & 1.5 & 10.0 & 43.6 & 0.27 & 1.59 & $1.40 \times 10^{-4} \mathrm{M}$ & $\begin{array}{c}\text { Only small portion of } \\
\text { breakthrough curve seen due to } \\
\text { low flowrate. Not used in } \\
\text { tortuosity factor estimation. }\end{array}$ \\
\hline $\begin{array}{c}\text { SRS-Avg-Test6 } \\
\text { (Walker et al., 1998) }\end{array}$ & $96-02$ & $\begin{array}{l}\text { SRS average } \\
\text { simulant }\end{array}$ & 17.7 & 1.5 & 10.0 & 43.6 & 0.98 & 5.88 & $1.40 \times 10^{-4} \mathrm{M}$ & $\begin{array}{l}\text { Significant oscillation seen in } \\
\text { breakthrough data, but still used in } \\
\text { tortuosity factor estimation. }\end{array}$ \\
\hline $\begin{array}{c}\text { SRS-Avg-Test7 } \\
\text { (Walker et al., 1998) }\end{array}$ & 96-04 & $\begin{array}{l}\text { SRS average } \\
\text { simulant }\end{array}$ & 17.7 & 1.43 & 11.0 & 41.6 & 4.1 & 22.1 & $1.40 \times 10^{-4} \mathrm{M}$ & $\begin{array}{c}\text { Complete breakthrough achieved. } \\
\text { Data used in tortuosity factor } \\
\text { estimation. }\end{array}$ \\
\hline $\begin{array}{l}\text { SRS-High-OH-Test1 } \\
\text { (Walker et al., 1999) }\end{array}$ & $98-05$ & $\begin{array}{l}\text { SRS high OH } \\
\text { simulant }\end{array}$ & $\begin{array}{c}17.7 \\
\& \\
150.2\end{array}$ & 1.5 & $\begin{array}{c}10.0 \\
\& \\
85.0\end{array}$ & 43.6 & 5.43 & $\begin{array}{c}32.6 \\
\& \\
3.8 \\
\end{array}$ & $3.70 \times 10^{-4} \mathrm{M}$ & $\begin{array}{c}\text { Two breakthrough locations } \\
\text { sampled. Data used in tortuosity } \\
\text { factor estimation. }\end{array}$ \\
\hline $\begin{array}{l}\text { SRS-Tank44-Test1 } \\
\text { (Walker et al., 1999) }\end{array}$ & $98-05$ & $\begin{array}{c}\text { Diluted SRS } \\
\text { Tank } 44 \\
\text { supernate } \\
\end{array}$ & $\begin{array}{c}17.7 \\
\& \\
150.2 \\
\end{array}$ & 1.5 & $\begin{array}{c}10.0 \\
\& \\
85.0\end{array}$ & 43.6 & 5.32 & $\begin{array}{c}32.6 \\
\& \\
3.8 \\
\end{array}$ & $3.51 \times 10^{-4} \mathrm{M}$ & $\begin{array}{c}\text { Two breakthrough locations } \\
\text { sampled. Data used in tortuosity } \\
\text { factor estimation. }\end{array}$ \\
\hline $\begin{array}{c}\text { PNNL-AW101-Test1 } \\
\text { (Hendrickson, 1997) }\end{array}$ & $96-01$ & $\begin{array}{c}\text { Envelope A } \\
\text { diluted AW-101 } \\
\text { sample }\end{array}$ & 7.9 & 1.0 & 10.0 & 29.1 & 1.06 & 6.3 & $7.26 \times 10^{-5} \mathrm{M}$ & $\begin{array}{l}\text { Actual feed composition unclear } \\
\text { within report. Data not used in } \\
\text { tortuosity factor estimation. }\end{array}$ \\
\hline $\begin{array}{l}\text { ORNL-W27-Test1 } \\
\text { (Lee et al., 1997b) }\end{array}$ & $-38 b$ & $\begin{array}{l}\text { MVST W-27 } \\
\text { sample }\end{array}$ & 10.0 & 1.5 & 5.659 & 43.6 & 0.283 & 3.0 & $7.04 \times 10^{-6} \mathrm{M}$ & $\begin{array}{l}\text { Preliminary study for Cs removal } \\
\text { demonstration facility. Data for } \\
\text { assessment purposes only. }\end{array}$ \\
\hline
\end{tabular}




\begin{tabular}{|c|c|c|c|c|c|c|c|c|c|c|}
\hline $\begin{array}{l}\text { Column } \\
\text { ID }^{\mathrm{a}}\end{array}$ & $\begin{array}{l}\text { CST IE- } \\
911 \text { Lot } \\
\text { Number }\end{array}$ & $\begin{array}{l}\text { Liquid feed } \\
\text { type }\end{array}$ & $\begin{array}{c}\text { Active } \\
\text { bed } \\
\text { volume } \\
(\mathrm{ml})\end{array}$ & $\begin{array}{l}\text { Diameter } \\
\text { (cm) }\end{array}$ & $\begin{array}{c}\text { Length } \\
\text { (cm) }\end{array}$ & $\begin{array}{l}\text { Column-to- } \\
\text { particle } \\
\text { diameter } \\
\text { ratio }\end{array}$ & $\begin{array}{l}\text { Superficial } \\
\text { velocity } \\
\text { (cm/min) }\end{array}$ & $\mathrm{CV} / \mathrm{hr}$ & $\begin{array}{l}\text { Feed conc.: } \\
\text { total Cs } \\
{[\mathrm{M}]}\end{array}$ & Comments \\
\hline $\begin{array}{l}\text { ORNL-W27-Test2 } \\
\text { (Lee et al., 1997b) }\end{array}$ & $-38 b$ & $\begin{array}{l}\text { MVST W-27 } \\
\text { sample }\end{array}$ & 10.0 & 1.5 & 5.659 & 43.6 & 0.566 & 6.0 & $7.04 \times 10^{-6} \mathrm{M}$ & $\begin{array}{l}\text { Preliminary study for Cs removal } \\
\text { demonstration facility. Data for } \\
\text { assessment purposes only. }\end{array}$ \\
\hline $\begin{array}{c}\text { ORNL-CsRD-Run2 } \\
\text { (Walker, Jr., et al., 1998) }\end{array}$ & $-38 b$ & $\begin{array}{l}\text { MVST W-29 } \\
\text { sample }\end{array}$ & $1,581.2$ & 30.6 & 51.672 & 889.5 & 2.58 & 3.0 & $1.35 \times 10^{-5} \mathrm{M}$ & $\begin{array}{l}\text { Part of a Cs removal } \\
\text { demonstration facility. Data for } \\
\text { assessment purposes only. }\end{array}$ \\
\hline \begin{tabular}{|c|} 
ORNL-CsRD-Run3 \\
(Walker, Jr., et al., 1998)
\end{tabular} & $-38 b$ & $\begin{array}{l}\text { MVST W-29 } \\
\text { sample }\end{array}$ & $1,581.2$ & 30.6 & 51.672 & 889.5 & 5.17 & 6.0 & $1.35 \times 10^{-5} \mathrm{M}$ & $\begin{array}{c}\text { Part of a Cs removal } \\
\text { demonstration facility. Data for } \\
\text { assessment purposes only. }\end{array}$ \\
\hline \begin{tabular}{|l|} 
ORNL-CsRD-Run4a,b \\
(Walker, Jr., et al., 1998)
\end{tabular} & $-38 b$ & $\begin{array}{l}\text { MVST W-29 } \\
\text { sample }\end{array}$ & $1,581.2$ & 30.6 & 51.672 & 889.5 & 5.17 & 6.0 & $5.10 \times 10^{-6} \mathrm{M}$ & $\begin{array}{l}\text { Part of a Cs removal } \\
\text { demonstration facility. Data for } \\
\text { assessment purposes only. }\end{array}$ \\
\hline
\end{tabular}

${ }^{\mathrm{a}}$ In some cases more than one column was used in a carousel arrangement. 
Table 9-2. Summary of recommended nominal parameter settings for Cesium-IONSIV ${ }^{\circledR}$ IE-911 CST system column modeling.

\begin{tabular}{|c|c|c|c|c|}
\hline $\begin{array}{c}\text { Key } \\
\text { parameter }\end{array}$ & Definition & $\begin{array}{c}\text { Priority for } \\
\text { predicting } \\
\text { break-through } \\
\text { curve }\end{array}$ & Recommended B. E. settings & Comments \\
\hline $\mathrm{m}_{\mathrm{resin}}$ & mass of resin in column & 1 & column specific & $\begin{array}{l}\text { Key quantity generally assumed to be a } \\
\text { constant during a series of column runs. }\end{array}$ \\
\hline$\eta_{\text {df }}$ & $\begin{array}{l}\text { Dilution factor of CST } \\
\text { when placed in its } \\
\text { engineered-form }\end{array}$ & 1 & $\begin{array}{c}0.68 \\
\text { (Baseline material value while } \\
\text { conservative for most batches) }\end{array}$ & $\begin{array}{l}\text { Batch specific, and based on comparisons of } \\
\text { available equilibrium contact } K_{d} \text { data powder } \\
\text { versus engineered forms. }\end{array}$ \\
\hline$\overline{\mathrm{C}}_{\mathrm{T}}$ & $\begin{array}{l}\text { Cesium total ionic } \\
\text { capacity of resin }\end{array}$ & 1 & $\begin{array}{c}0.58 \\
\left(\mathrm{mmole} / \mathrm{g}_{\text {resin }}\right)\end{array}$ & $\begin{array}{l}\text { Batch and feed specific, and based on available } \\
\text { equilibrium contact } K_{d} \text { data when available. }\end{array}$ \\
\hline$\rho_{\mathrm{b}}$ & bed density & 1 & $1.0\left(\mathrm{~g}_{\text {resin }} / \mathrm{ml}_{\mathrm{bed}}\right)$ & $\begin{array}{l}\text { Ideally measured for each column. Batch } \\
\text { average generic value used otherwise. }\end{array}$ \\
\hline$\beta$ & $\begin{array}{c}\mathrm{XO}_{4}^{-} \text {to } \mathrm{NO}_{3}^{-} \\
\text {selectivity coefficient }\end{array}$ & 1 & $\begin{array}{l}\text { composition dependent } \\
\text { (fit to ZAM generated database) }\end{array}$ & $\begin{array}{l}\text { Based on conc's not activities and total ionic } \\
\text { strength and composition dependent }\end{array}$ \\
\hline $\mathrm{L}, \mathrm{D}, \mathrm{V}_{\mathrm{b}}$ & $\begin{array}{l}\text { Length, diameter, \& } \\
\text { volume of column }\end{array}$ & 1 & column specific & $\begin{array}{l}\text { Does not vary significantly with total ionic } \\
\text { strength. }\end{array}$ \\
\hline$<\mathrm{R}_{\mathrm{p}}>$ & $\begin{array}{l}\text { avg. effective radius of } \\
\text { resin }\end{array}$ & 2 & $\begin{array}{c}172 \mu \mathrm{m} \\
\text { (mean value) }\end{array}$ & $\begin{array}{l}\text { Impacts both film and pore diffusion. } \\
\text { Averaging process remains in question. }\end{array}$ \\
\hline $\mathrm{U}, \mathrm{Q}$ & $\begin{array}{l}\text { superficial velocity \& } \\
\text { volumetric flow rate }\end{array}$ & 2 & column specific & $\begin{array}{l}\text { For mass transfer limited columns flow } \\
\text { perturbation dependent. }\end{array}$ \\
\hline $\mathrm{C}_{\mathrm{o}}$ & $\mathrm{Cs}^{+}$inlet conc & 2 & column specific & $\begin{array}{l}\text { For favorable isotherms higher inlet conc's } \\
\text { sharpen breakthru curves. Only a modest } \\
\text { favorable isotherm over expected feed ranges. }\end{array}$ \\
\hline
\end{tabular}


Table 9-2. Summary of recommended nominal parameter settings for Cesium-IONSIV ${ }^{\circledR}$ IE-911 CST system column modeling (continued).

\begin{tabular}{|c|c|c|c|c|}
\hline $\begin{array}{c}\text { Key } \\
\text { parameter }\end{array}$ & Definition & $\begin{array}{c}\text { Priority for } \\
\text { predicting } \\
\text { break-through } \\
\text { curve }\end{array}$ & Recommended B. E. settings & Comments \\
\hline$\varepsilon_{\mathrm{b}}, \varepsilon_{\mathrm{p}}$ & $\begin{array}{l}\text { bed \& particle } \\
\text { porosities }\end{array}$ & 3 & $\begin{array}{c}0.50 \& 0.24 \\
\text { (average values) }\end{array}$ & $\begin{array}{l}\text { Porosity data very limited and these numbers at } \\
\text { Texas A\&M recommended values. }\end{array}$ \\
\hline $\mathrm{D}_{\mathrm{p}}$ & $\begin{array}{l}\text { pore diffusion } \\
\text { coefficient }\end{array}$ & 3 & $\begin{array}{l}20 \% \text { of Brownian diffusion } \\
\text { value }\end{array}$ & $\begin{array}{l}\text { Based on tortuosity factor optimization using } \\
\text { several more recent column studies. }\end{array}$ \\
\hline $\mathrm{k}_{\mathrm{f}}$ & $\begin{array}{l}\text { liquid film mass } \\
\text { transfer coefficient }\end{array}$ & 3 & $\begin{array}{l}\text { Wilson and Geankoplis (1966) } \\
\text { correlation }\end{array}$ & $\begin{array}{l}\text { This correlation spans the velocity \& particle } \\
\text { sizes of the CST material. }\end{array}$ \\
\hline $\mathrm{D}_{\infty}$ & $\begin{array}{l}\text { Brownian motion } \\
\text { diffusion coefficient }\end{array}$ & 3 & $\begin{array}{l}\text { Computed based on feed ionic } \\
\text { composition }\end{array}$ & $\begin{array}{l}\text { Based on Nernst-Haskell eqn. \& published } \\
\text { ionic conductances at } 25^{\circ} \mathrm{C} \text {. }\end{array}$ \\
\hline $\mathrm{E}_{\mathrm{b}}$ & $\begin{array}{l}\text { axial longitudinal } \\
\text { dispersion coefficient }\end{array}$ & 4 & $\begin{array}{l}\text { Chung \& Wen (1968) } \\
\text { correlation }\end{array}$ & $\begin{array}{l}\text { This correlation based on long column data for } \\
\text { wide range of column conditions. }\end{array}$ \\
\hline $\mathrm{E}_{\mathrm{b}}(\mathrm{L}) / \mathrm{E}_{\mathrm{b}}(\infty)$ & $\begin{array}{l}\text { short column impact } \\
\text { ratio }\end{array}$ & 4 & Liles \& Geankoplis (1960) data & $\begin{array}{l}\text { Power law fit to limited short column tests } \\
\text { with end effects. }\end{array}$ \\
\hline $\mathrm{V}_{\mathrm{CSTR}}$ & $\begin{array}{l}\text { CSTR volumes at } \\
\text { entrance/exit of } \\
\text { columns }\end{array}$ & 4 & column specific & $\begin{array}{l}\text { Estimated based on specified volumes for each } \\
\text { test series or assumed } 25 \% \text { if not available. }\end{array}$ \\
\hline
\end{tabular}


Table 9-3. Root-mean-square residuals for each column study as a function of the ratio of pore to free diffusion coefficients $(\eta)$.

\begin{tabular}{|c|c|c|c|c|c|}
\hline Test ID & $\boldsymbol{\eta}=\mathbf{1 0} \%$ & $\boldsymbol{\eta}=\mathbf{2 0} \%$ & $\boldsymbol{\eta}=\mathbf{2 6} \%$ & $\boldsymbol{\eta}=\mathbf{3 0} \%$ & $\boldsymbol{\eta}=\mathbf{3 5 \%}$ \\
\hline SRS-Avg-Test1 & $8.777 \mathrm{E}-02$ & $6.083 \mathrm{E}-02$ & $5.623 \mathrm{E}-02$ & $5.612 \mathrm{E}-02$ & $5.839 \mathrm{E}-02$ \\
\hline SRS-Avg-Test2 & $6.026 \mathrm{E}-02$ & $2.640 \mathrm{E}-02$ & $3.088 \mathrm{E}-02$ & $3.894 \mathrm{E}-02$ & $4.895 \mathrm{E}-02$ \\
\hline SRS-Avg-Test3 & $1.933 \mathrm{E}-01$ & $1.677 \mathrm{E}-01$ & $1.663 \mathrm{E}-01$ & $1.675 \mathrm{E}-01$ & $1.700 \mathrm{E}-01$ \\
\hline SRS-Avg-Test4 & $1.415 \mathrm{E}-01$ & $1.309 \mathrm{E}-01$ & $1.368 \mathrm{E}-01$ & $1.418 \mathrm{E}-01$ & $1.475 \mathrm{E}-01$ \\
\hline SRS-Avg-Test5 & $1.505 \mathrm{E}-01$ & $7.063 \mathrm{E}-02$ & $5.474 \mathrm{E}-02$ & $4.634 \mathrm{E}-02$ & $3.871 \mathrm{E}-02$ \\
\hline SRS-Avg-Test6 & $1.768 \mathrm{E}-01$ & $1.746 \mathrm{E}-01$ & $1.795 \mathrm{E}-01$ & $1.836 \mathrm{E}-01$ & $1.882 \mathrm{E}-01$ \\
\hline SRS-Avg-Test7 & $6.457 \mathrm{E}-02$ & $4.343 \mathrm{E}-02$ & $5.865 \mathrm{E}-02$ & $7.102 \mathrm{E}-02$ & $8.435 \mathrm{E}-02$ \\
\hline SRS-High-OH-Test1 & $1.758 \mathrm{E}-01$ & $1.311 \mathrm{E}-01$ & $1.289 \mathrm{E}-01$ & $1.319 \mathrm{E}-01$ & $1.376 \mathrm{E}-01$ \\
\hline SRS-Tank44-Test1 & $1.095 \mathrm{E}-01$ & $6.564 \mathrm{E}-02$ & $6.050 \mathrm{E}-02$ & $6.191 \mathrm{E}-02$ & $6.638 \mathrm{E}-02$ \\
\hline PNNL-AW101-Test1 $^{2}$ & $1.924 \mathrm{E}-01$ & $1.055 \mathrm{E}-01$ & $7.756 \mathrm{E}-02$ & $6.366 \mathrm{E}-02$ & $4.985 \mathrm{E}-02$ \\
\hline Overall rms $^{\mathbf{a}}=$ & $1.357 \mathrm{E}-01$ & $1.094 \mathrm{E}-01$ & $1.075 \mathrm{E}-01$ & $1.091 \mathrm{E}-01$ & $1.124 \mathrm{E}-01$ \\
\hline
\end{tabular}

${ }^{a}$ The overall rms value is based on only 8 out of the 10 column tests considered. Tests SRS-Avg-Test5 and PNNLAW101-Test1 were omitted in the estimation of a "best estimate" tortuosity factor.

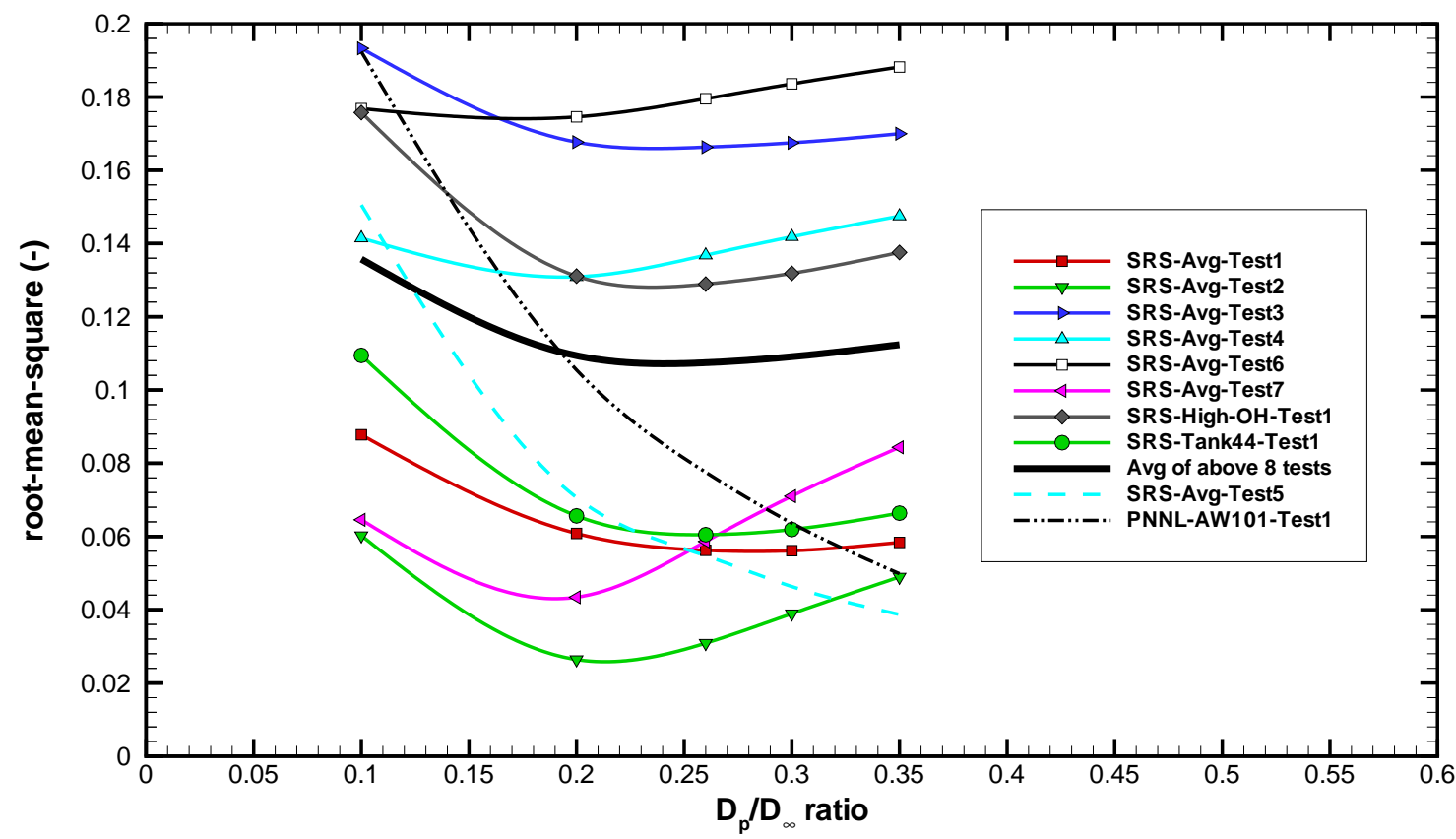

Figure 9-1. Measure of error for individual tests in predicting column exit cesium breakthrough as a function of the ratio of pore to free stream diffusion coefficients. Average behavior of the top eight tests is also plotted. 


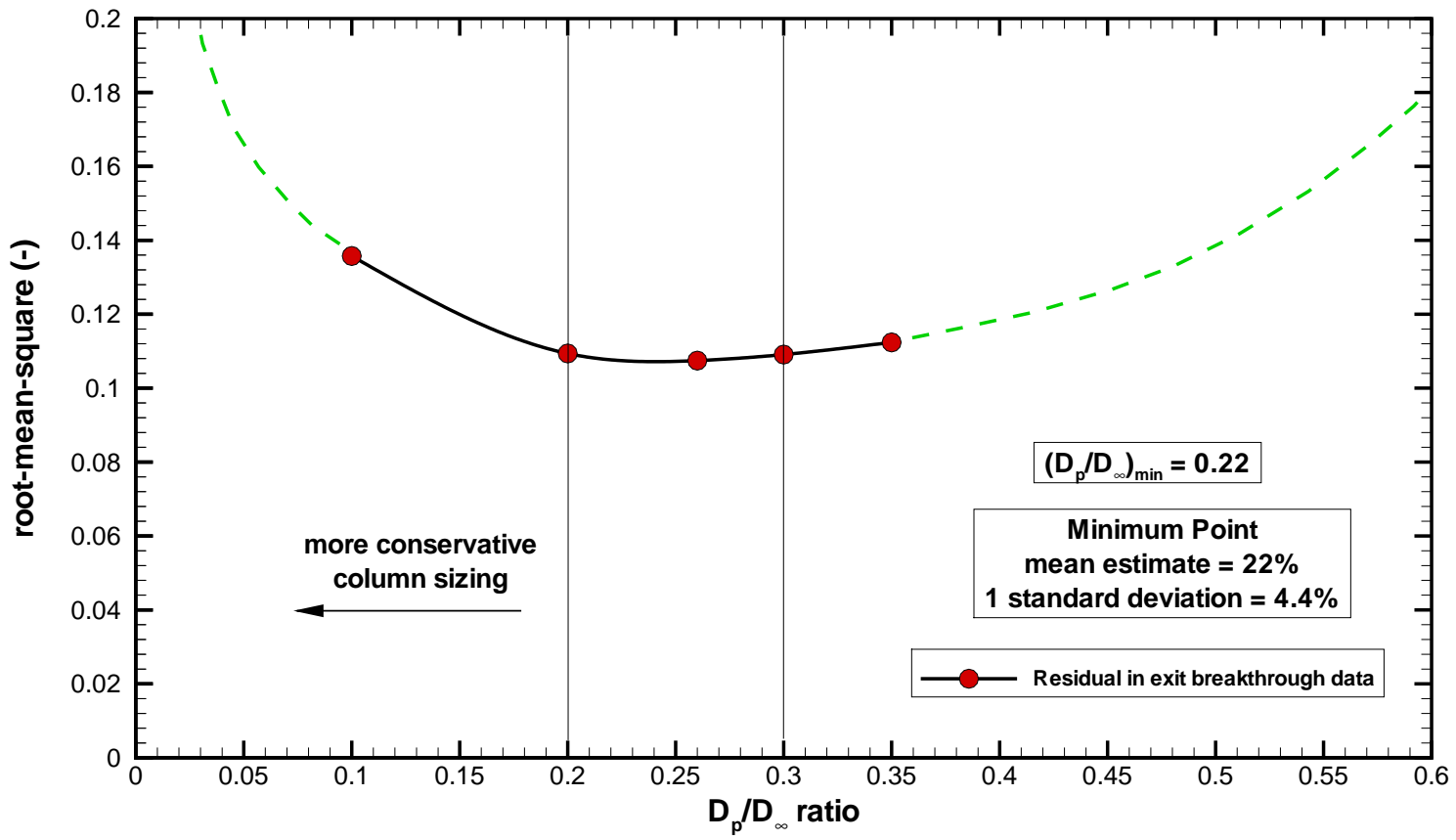

Figure 9-2. Overall measure of error in predicting column exit cesium breakthrough as a function of the pore to free stream diffusion coefficient ratio ( 8 column tests considered in summation).

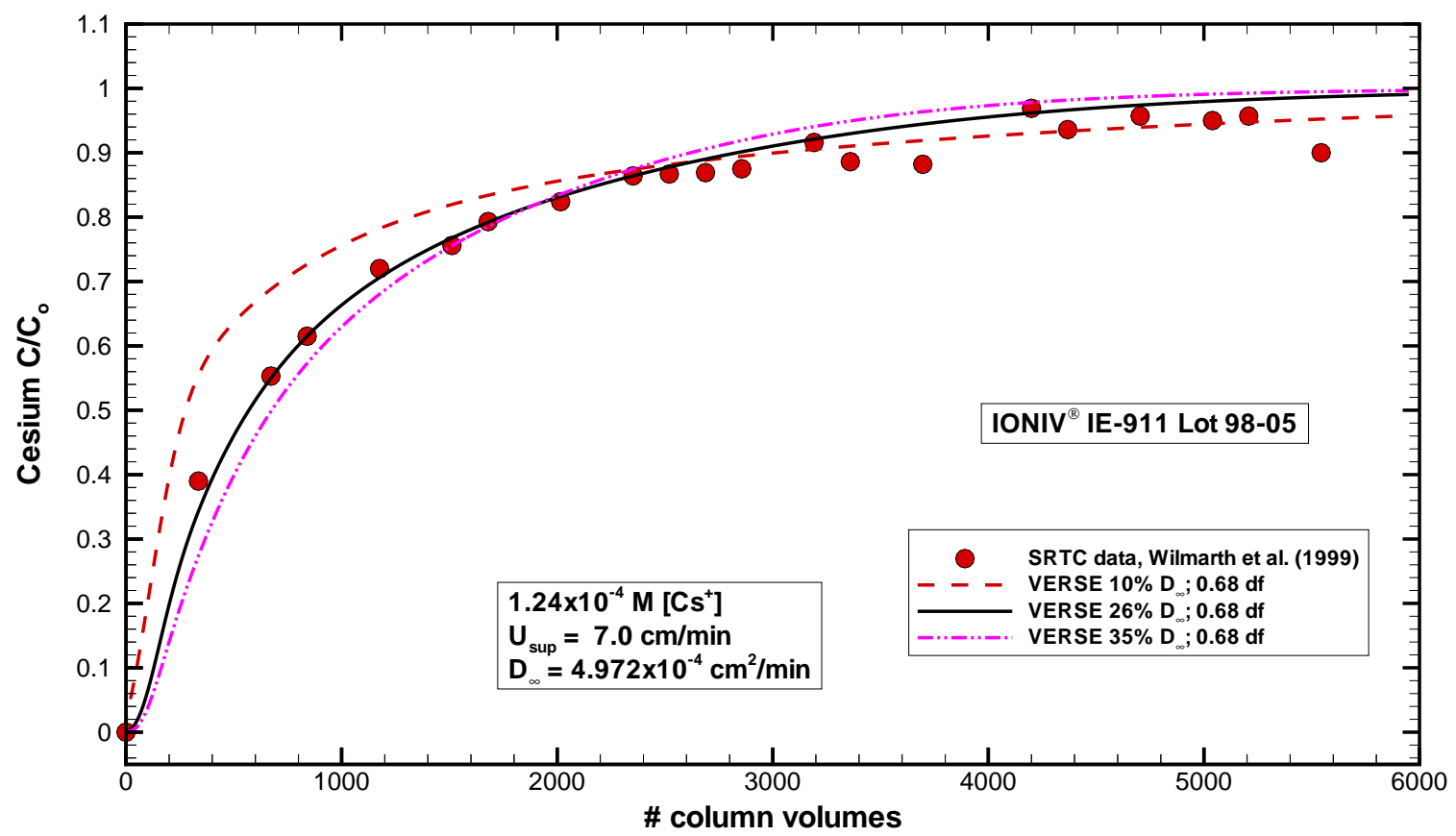

Figure 9-3. Measured versus predicted cesium column exit breakthrough for three assumed pore diffusion coefficient values (test SRS-Avg-Test2 performed by Wilmarth et al. (1999) in a SRS Average simulant liquid at $1.24 \times 10^{-4} \mathrm{M} \mathrm{Cs}$ and at $25 \mathrm{C}$ ). 


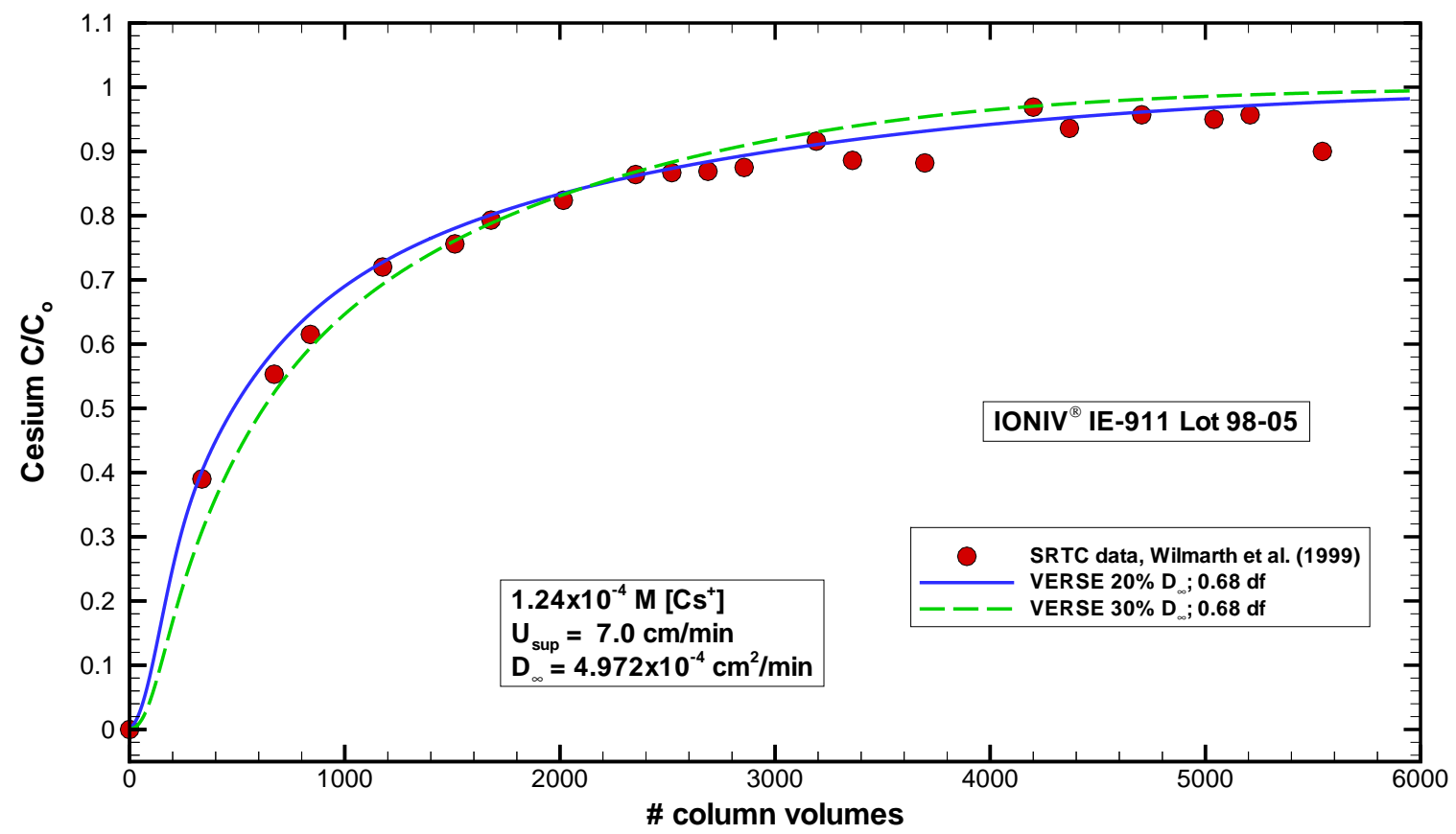

Figure 9-4. Measured versus predicted cesium column exit breakthrough for two assumed pore diffusion coefficient values (test SRS-Avg-Test2 performed by Wilmarth et al. (1999) in a SRS Average simulant liquid at $1.24 \times 10^{-4} \mathrm{M} \mathrm{Cs}$ and at $25 \mathrm{C}$ ).

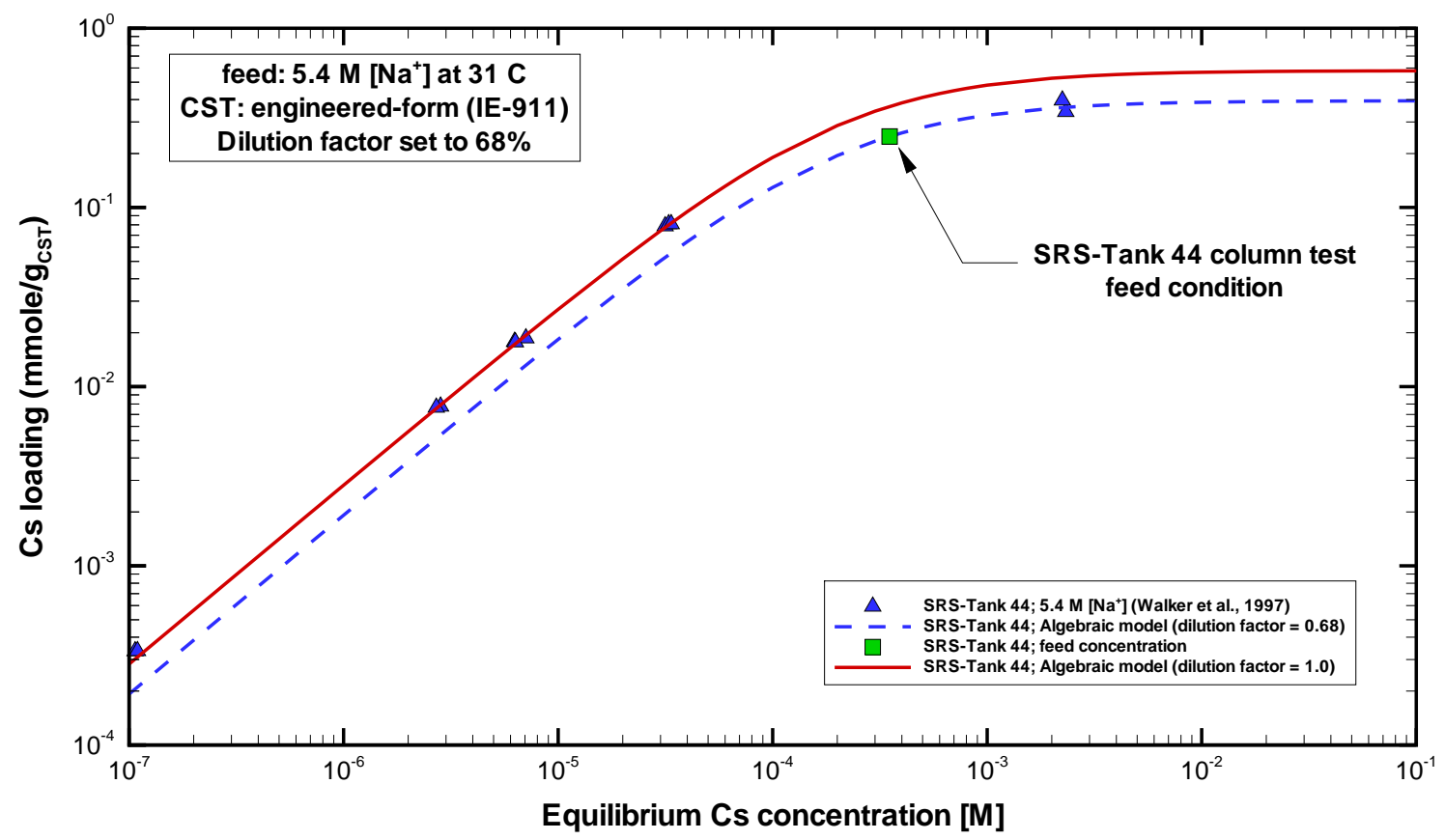

Figure 9-5. A direct comparison of predicted versus measured cesium loadings on IONSIV ${ }^{\circledR}$ IE-911 CST material for diluted SRS Tank 44 waste (Walker et al., 1999). The algebraic model is plotted for both the powder (solid-curve) and engineered (dashed-curve) forms. 


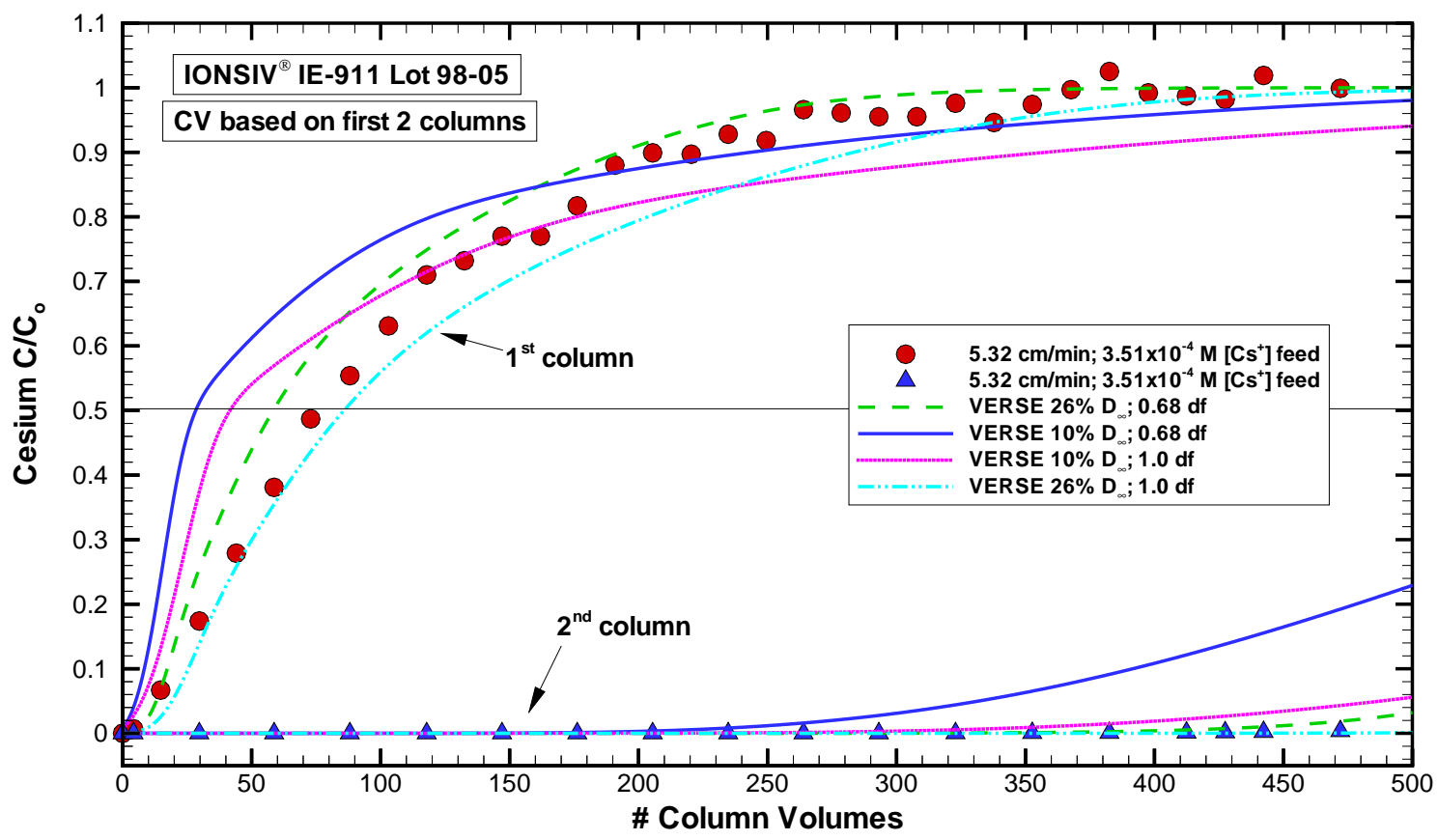

Figure 9-6. VERSE-LC cesium exit breakthrough curves compared to data from Walker et al. (1999) for SRS Tank 44 waste: $\mathrm{D}=1.59 \mathrm{~cm}, \mathrm{~L}=10 \mathrm{~cm}$ and $85 \mathrm{~cm}, \mathrm{U}=5.319 \mathrm{~cm} / \mathrm{min}, \mathrm{T}=31^{\circ} \mathrm{C}$.

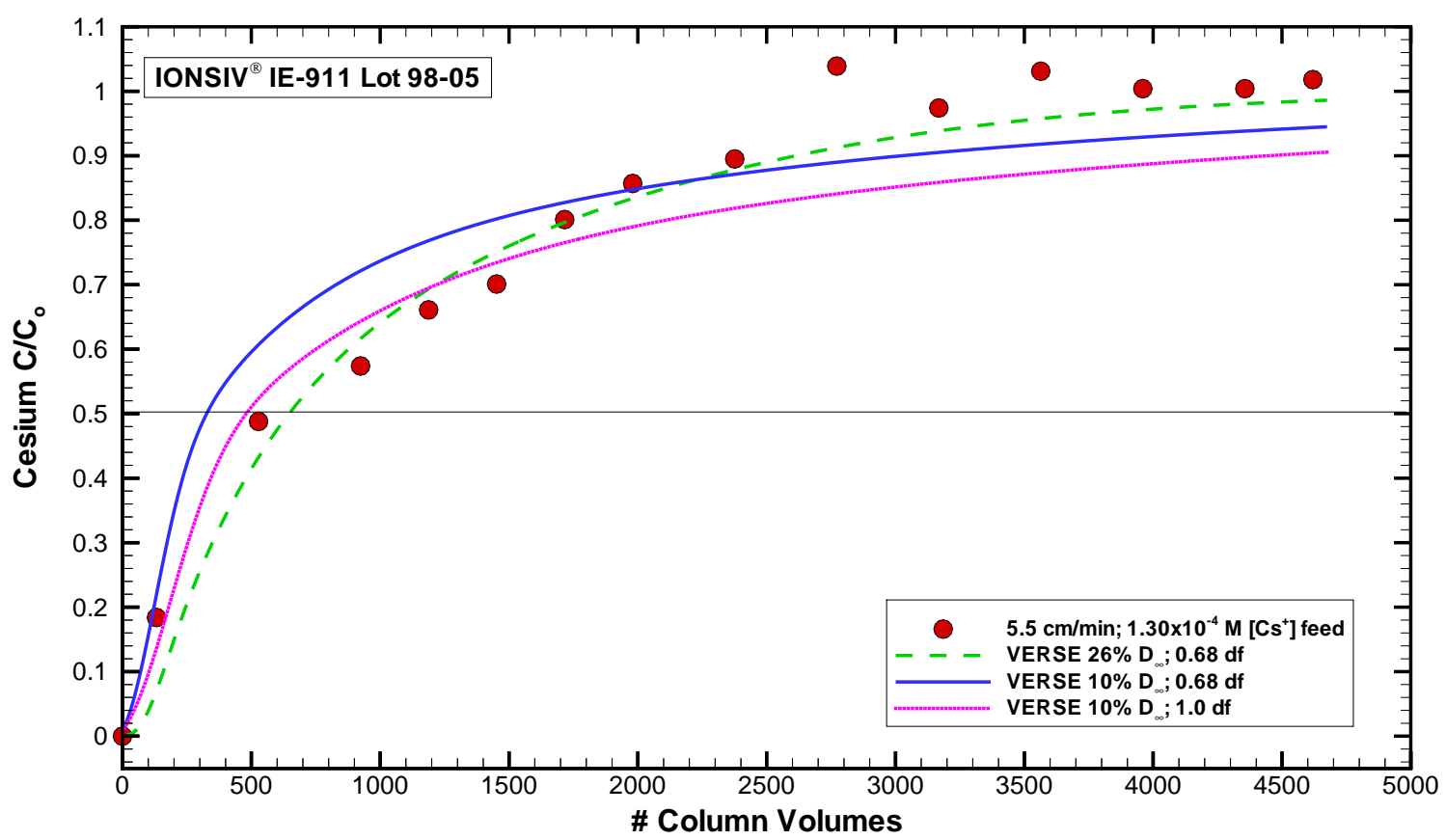

Figure 9-7. VERSE-LC cesium exit breakthrough curve compared to data from SRS-Avg-Test1 (Wilmarth et al., 1999): $\mathrm{D}=1.5 \mathrm{~cm}, \mathrm{~L}=10 \mathrm{~cm}, \mathrm{U}=5.5 \mathrm{~cm} / \mathrm{min}, \mathrm{T}=\sim 25^{\circ} \mathrm{C}$. 


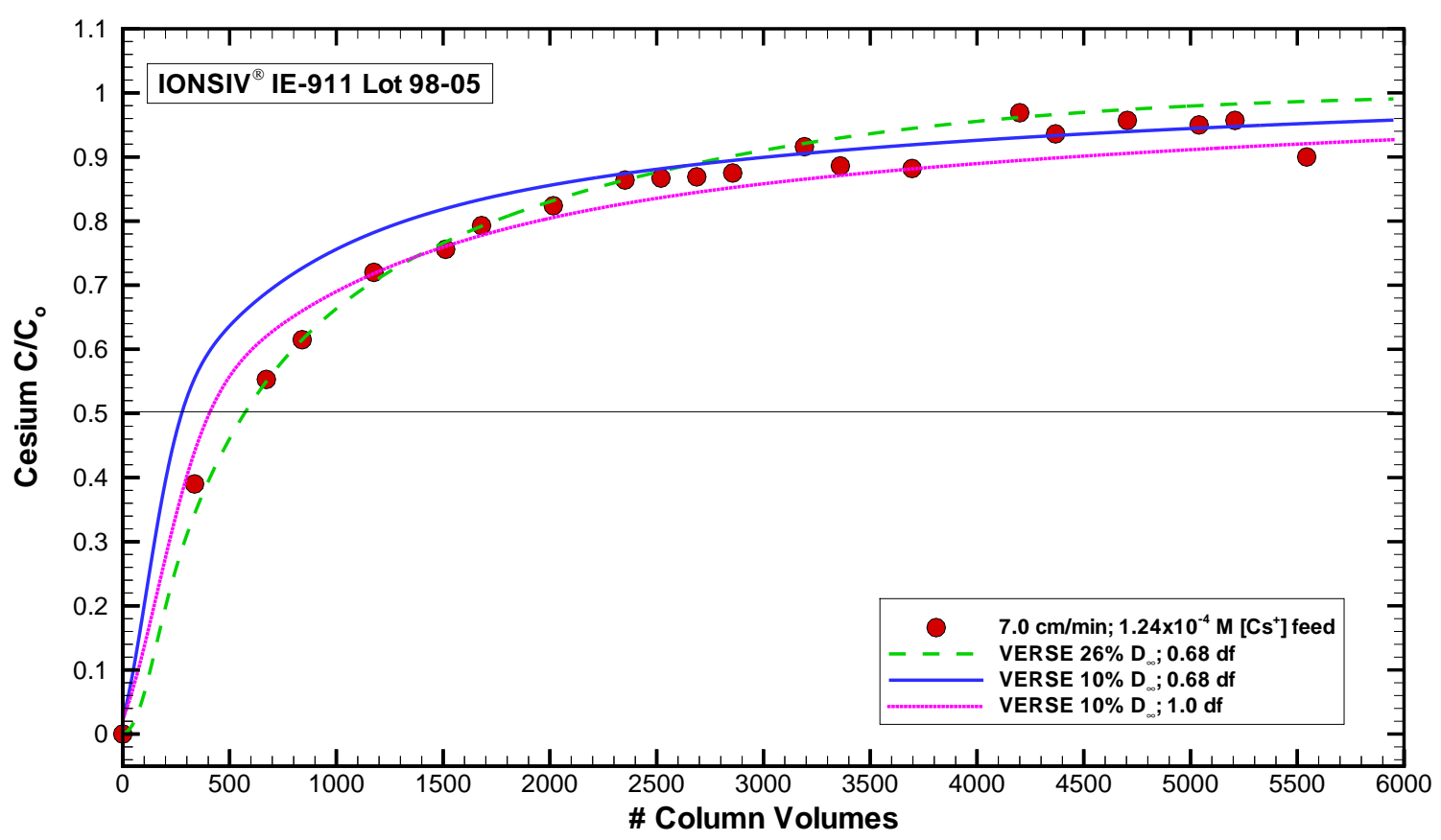

Figure 9-8. VERSE-LC cesium exit breakthrough curve compared to data from SRS-Avg-Test2 (Wilmarth et al., 1999): $\mathrm{D}=1.5 \mathrm{~cm}, \mathrm{~L}=10 \mathrm{~cm}, \mathrm{U}=7.0 \mathrm{~cm} / \mathrm{min}, \mathrm{T}=\sim 25^{\circ} \mathrm{C}$.

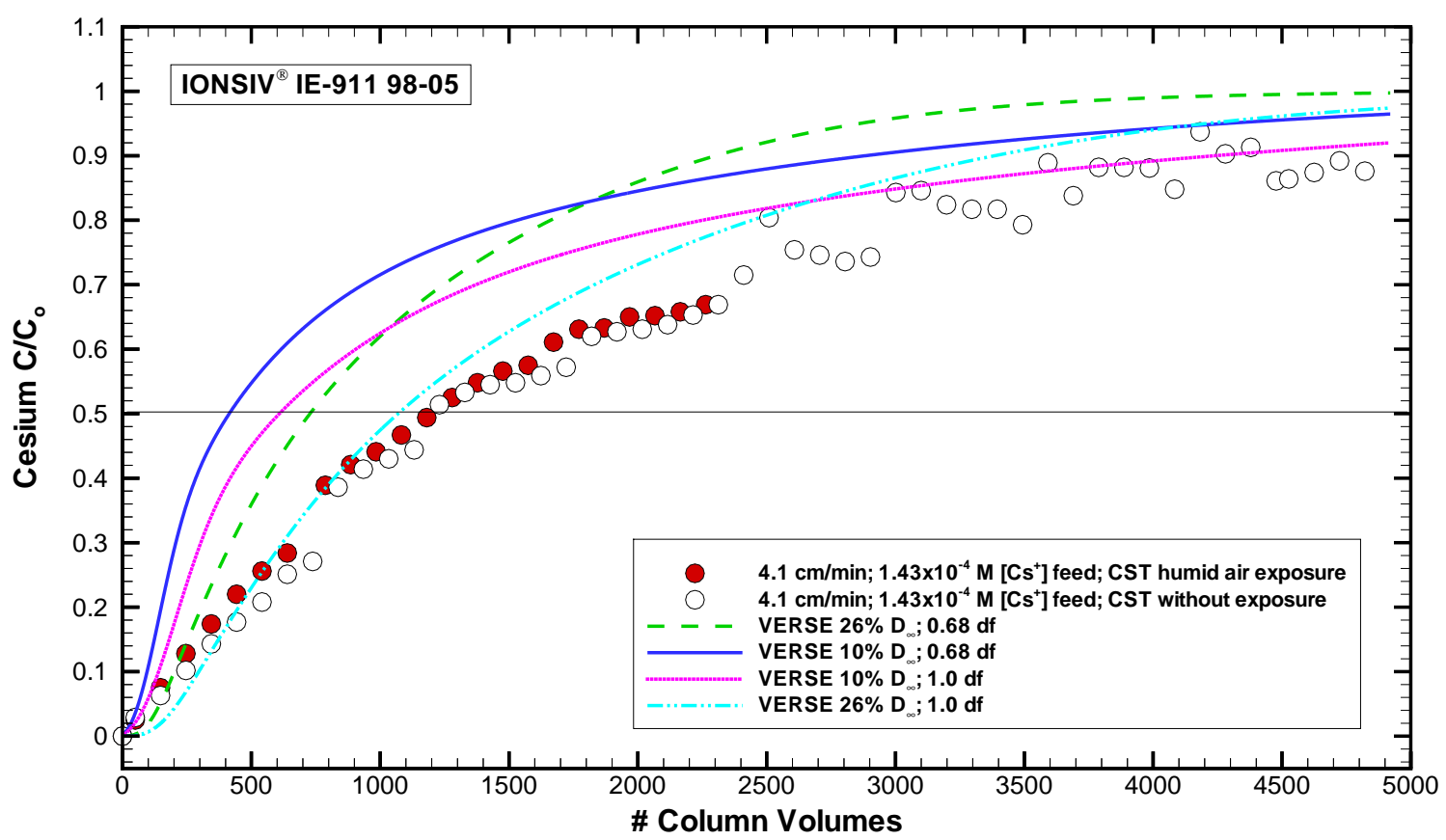

Figure 9-9. VERSE-LC cesium exit breakthrough curve compared to data from SRS-Avg-Test3a and Test3b (Wilmarth et al., 1999): $\mathrm{D}=1.5 \mathrm{~cm}, \mathrm{~L}=10 \mathrm{~cm}, \mathrm{U}=4.1 \mathrm{~cm} / \mathrm{min}, \mathrm{T}=\sim 25^{\circ} \mathrm{C}$. 


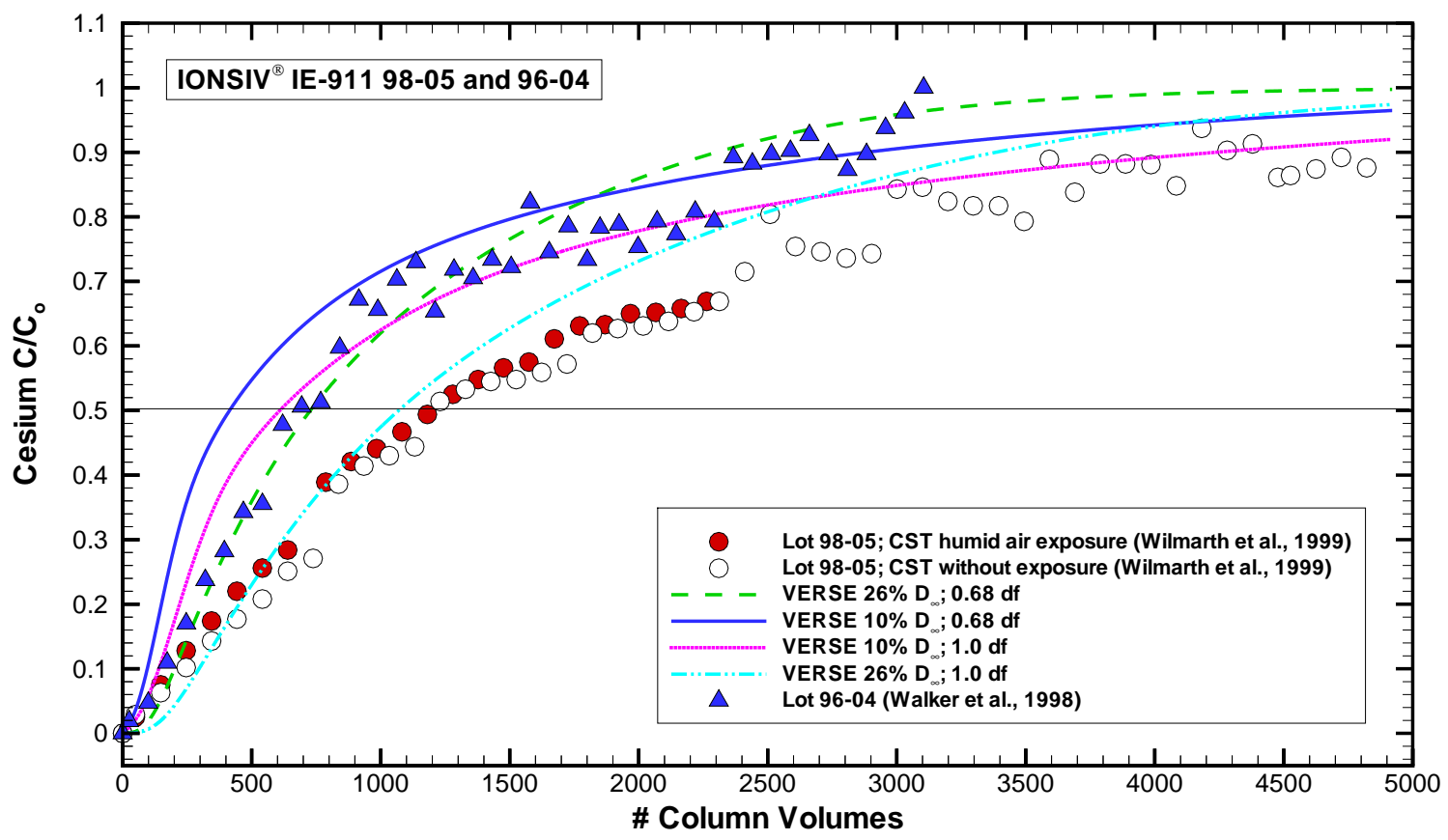

Figure 9-10. VERSE-LC cesium exit breakthrough curve compared to data from Wilmarth et al. (1999) for 98-05 CST material and Walker et al. (1998) for 96-04 CST material: $\mathrm{D}=1.5 \mathrm{~cm}, \mathrm{~L}$ $=10 \mathrm{~cm}, \mathrm{U}=\sim 4.1 \mathrm{~cm} / \mathrm{min}, \mathrm{T}=\sim 25^{\circ} \mathrm{C}$.

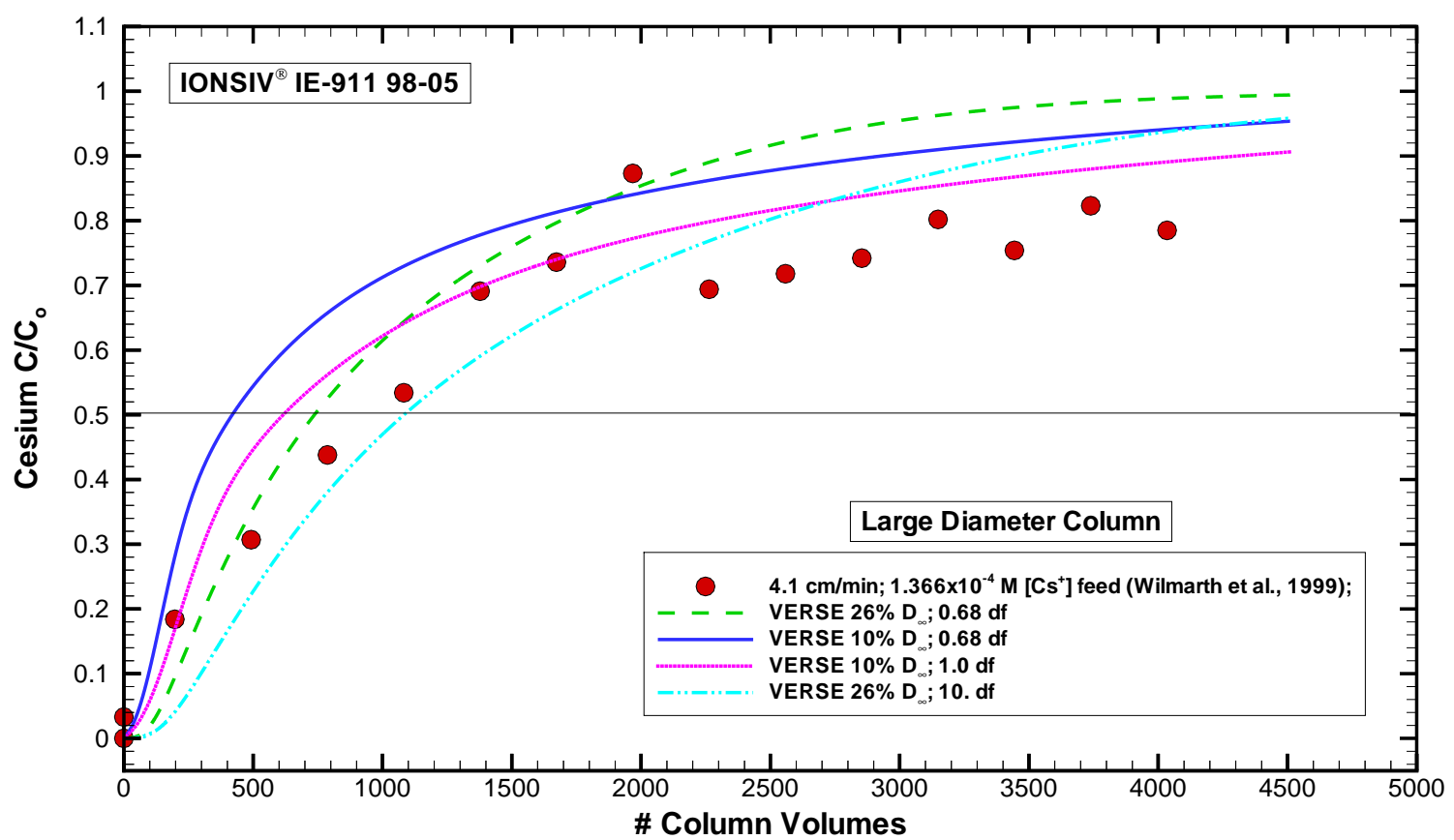

Figure 9-11. VERSE-LC cesium exit breakthrough curve compared to data from SRS-Avg-Test4 (Wilmarth et al., 1999): $\mathrm{D}=2.5 \mathrm{~cm}, \mathrm{~L}=10 \mathrm{~cm}, \mathrm{U}=4.1 \mathrm{~cm} / \mathrm{min}, \mathrm{T}=\sim 25^{\circ} \mathrm{C}$. 


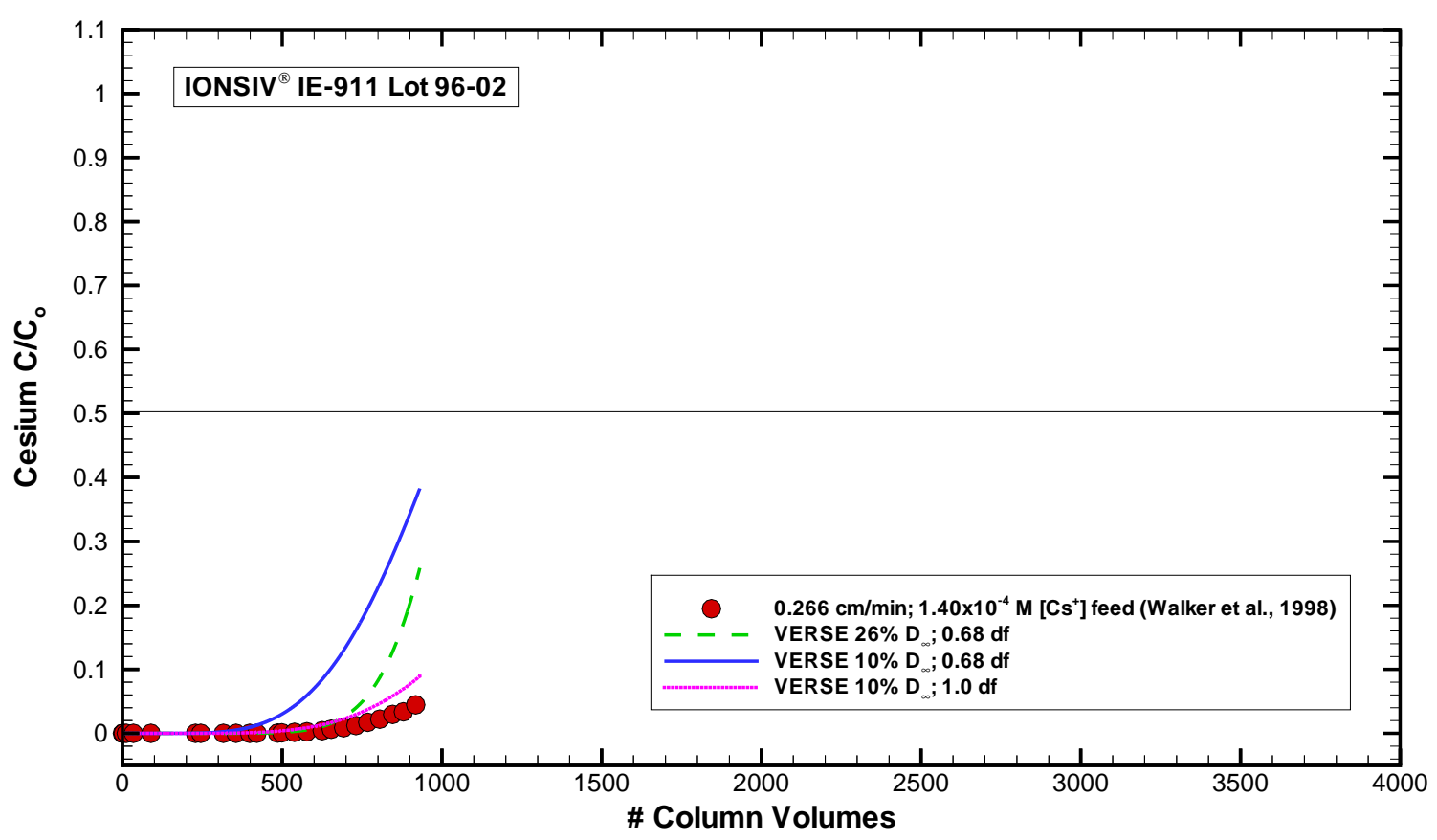

Figure 9-12. VERSE-LC cesium exit breakthrough curve compared to data from SRS-Avg-Test5 (Walker et al., 1998): $\mathrm{D}=1.5 \mathrm{~cm}, \mathrm{~L}=10 \mathrm{~cm}, \mathrm{U}=0.47 \mathrm{~cm} / \mathrm{min}, \mathrm{T}=\sim 22{ }^{\circ} \mathrm{C}$.

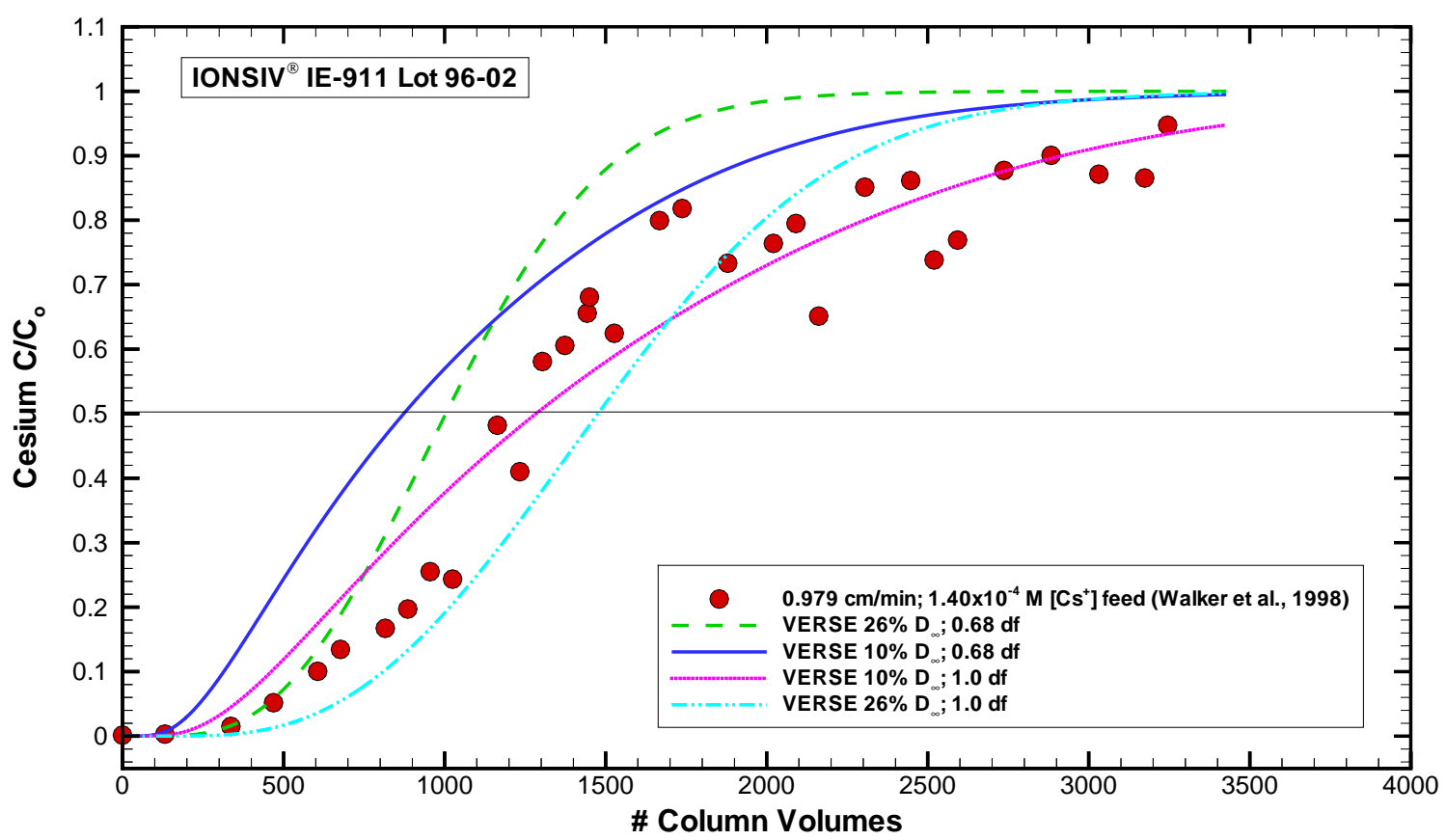

Figure 9-13. VERSE-LC cesium exit breakthrough curve compared to data from SRS-Avg-Test6 (Walker et al., 1998): $\mathrm{D}=1.5 \mathrm{~cm}, \mathrm{~L}=10 \mathrm{~cm}, \mathrm{U}=0.98 \mathrm{~cm} / \mathrm{min}, \mathrm{T}=\sim 22{ }^{\circ} \mathrm{C}$. 


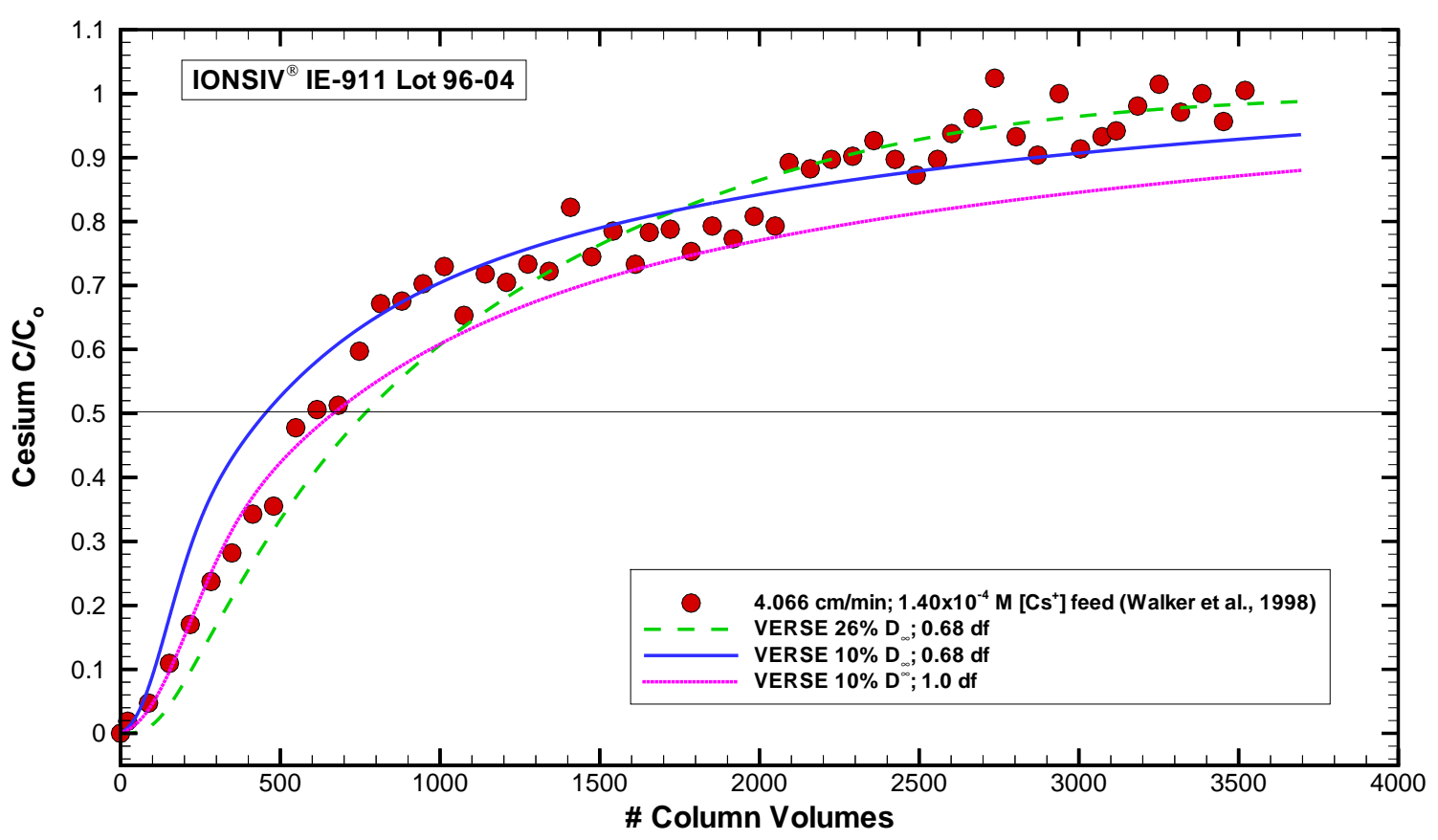

Figure 9-14. VERSE-LC cesium exit breakthrough curve compared to data from SRS-Avg-Test7 (Walker et al., 1998): $\mathrm{D}=1.43 \mathrm{~cm}, \mathrm{~L}=11 \mathrm{~cm}, \mathrm{U}=4.1 \mathrm{~cm} / \mathrm{min}, \mathrm{T}=\sim 22{ }^{\circ} \mathrm{C}$.

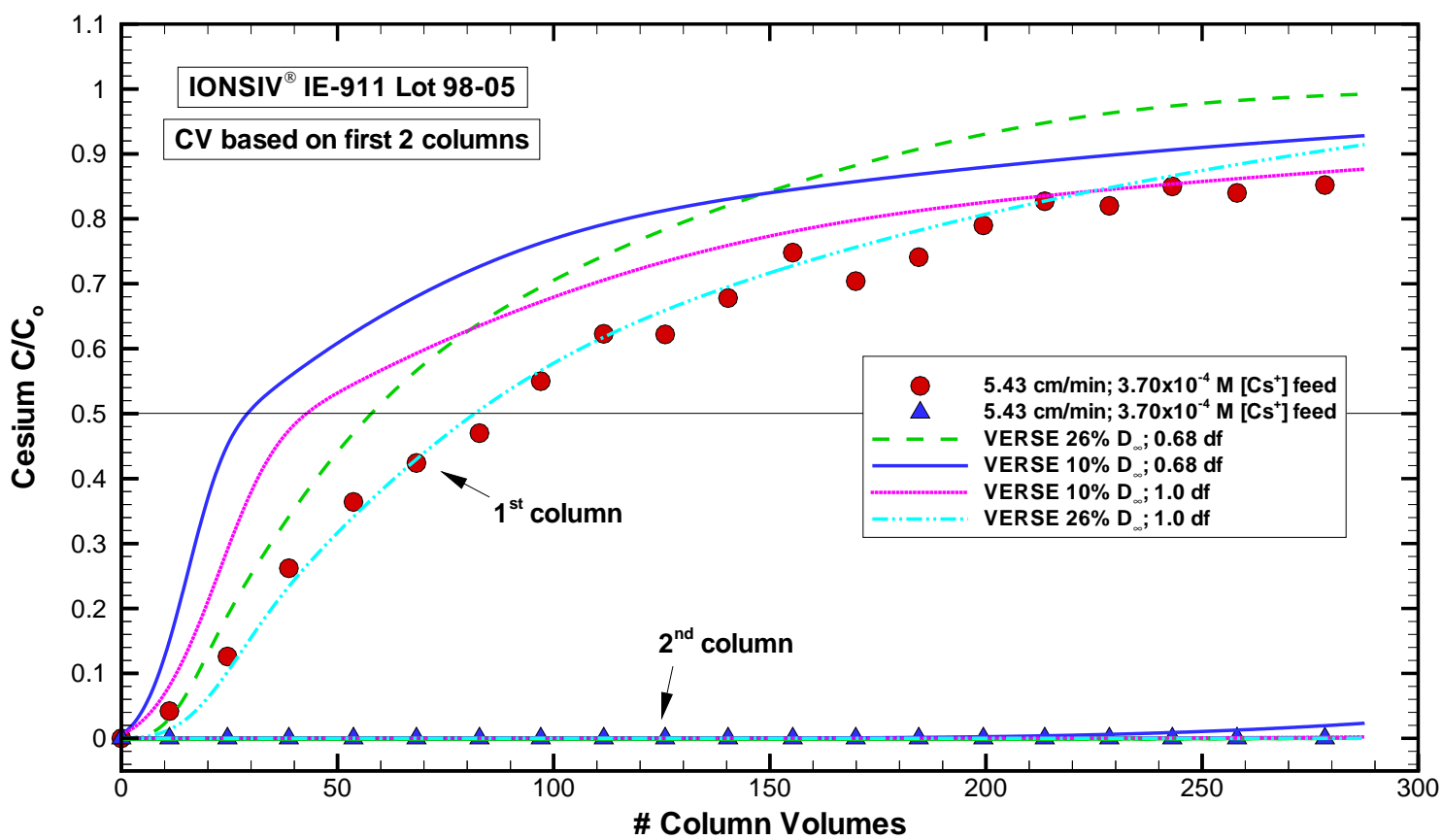

Figure 9-15. VERSE-LC cesium exit breakthrough curve compared to data from SRS-High-OH-Test1 (Walker et al., 1999): $\mathrm{D}=1.5 \mathrm{~cm}, \mathrm{~L}=10 \mathrm{~cm}$ and $85 \mathrm{~cm}, \mathrm{U}=5.43 \mathrm{~cm} / \mathrm{min}, \mathrm{T}=\sim 31^{\circ} \mathrm{C}$. 
Preliminary Ion Exchange Modeling for Removal of Cesium from Hanford Waste Using Hydrous Crystalline Silicotitanate Material

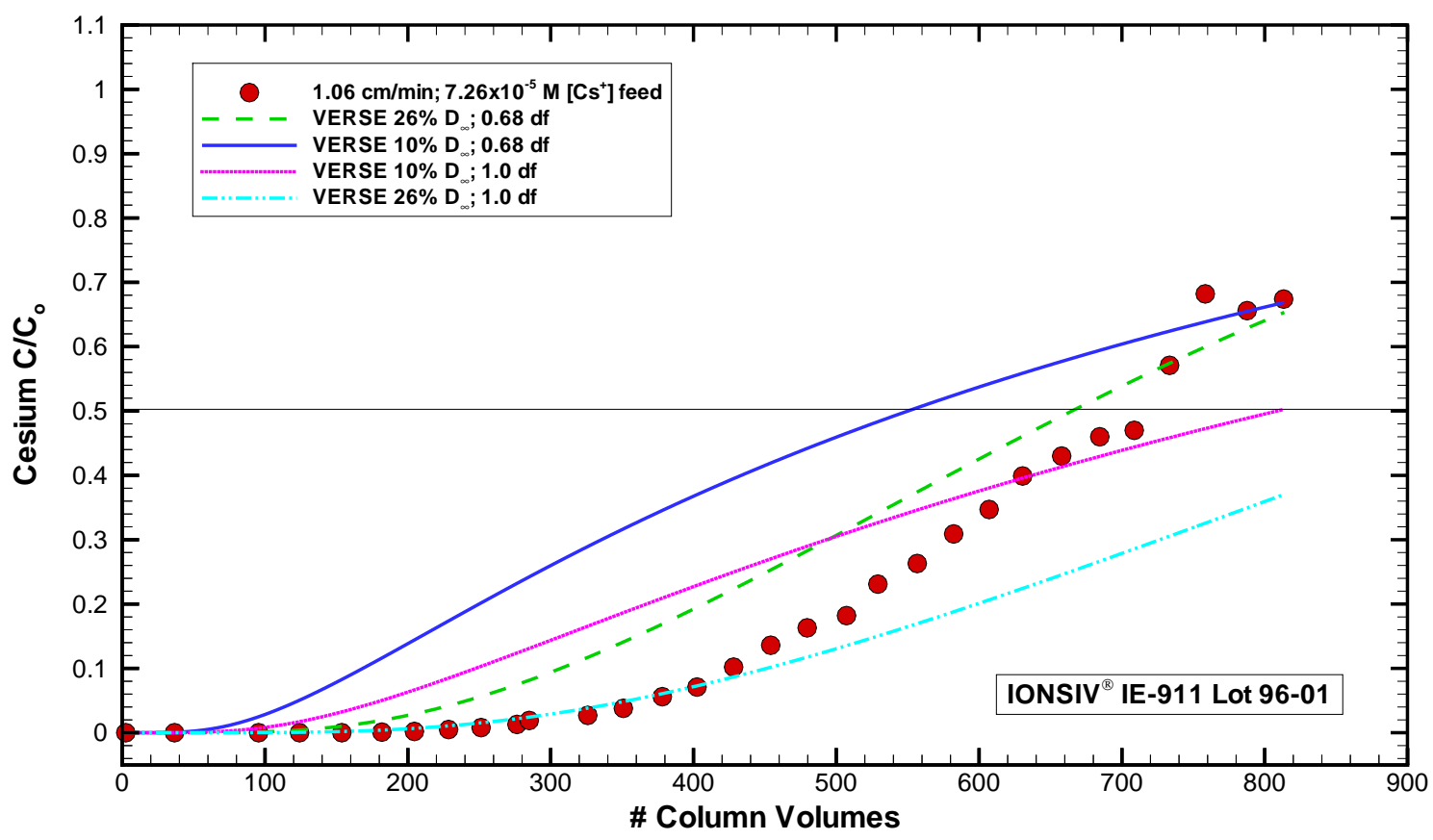

Figure 9-16. VERSE-LC cesium exit breakthrough curve compared to data from PNNL-AW101-Test1 (Hendrickson, 1997): $\mathrm{D}=1.5 \mathrm{~cm}, \mathrm{~L}=10 \mathrm{~cm}$ and $85 \mathrm{~cm}, \mathrm{U}=5.43 \mathrm{~cm} / \mathrm{min}, \mathrm{T}=\sim 31^{\circ} \mathrm{C}$.

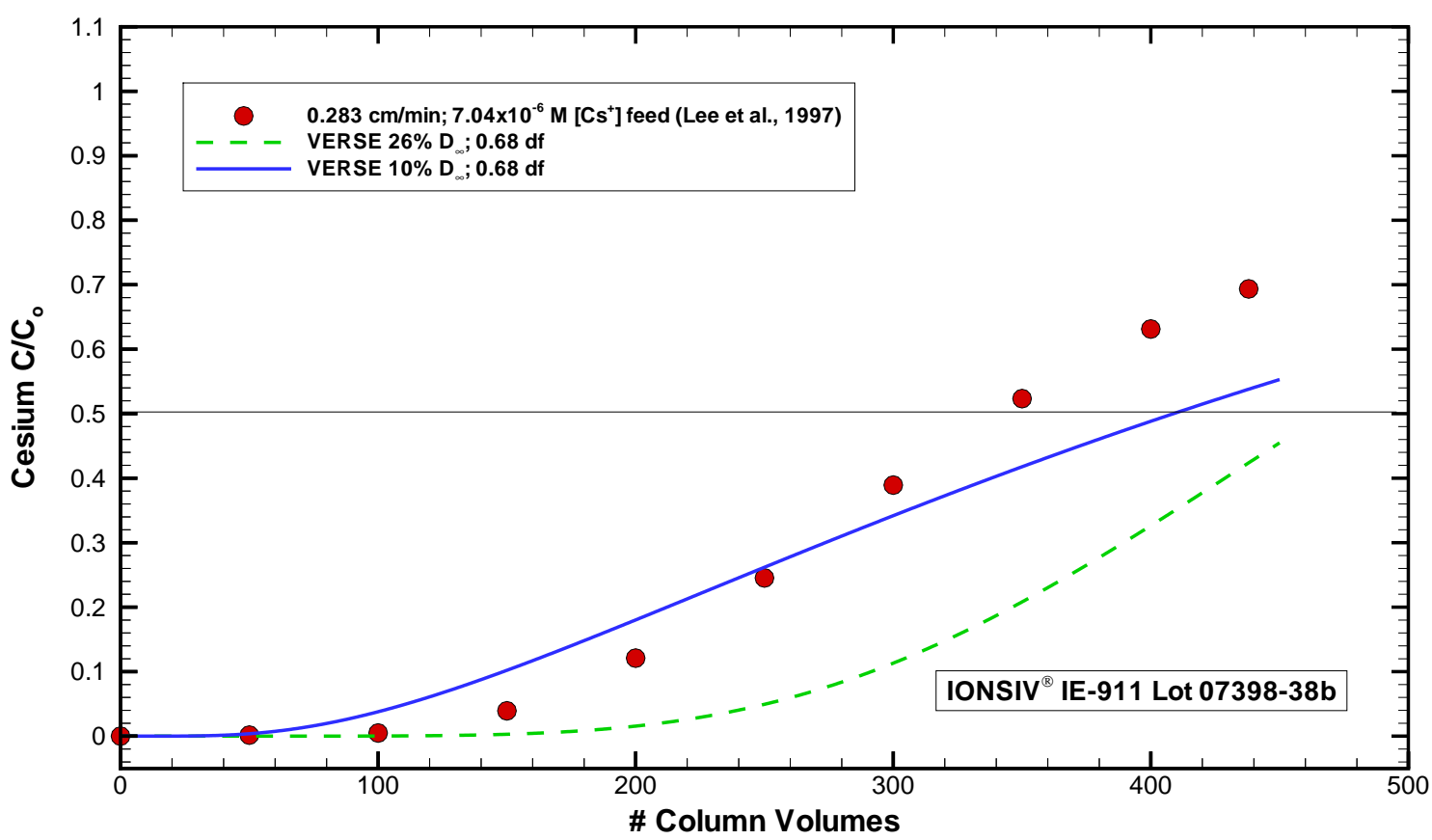

Figure 9-17. VERSE-LC cesium exit breakthrough curve compared to data from ORNL-W27-Test1 (Lee et al., 1997b): $\mathrm{D}=1.5 \mathrm{~cm}, \mathrm{~L}=5.659 \mathrm{~cm}, \mathrm{U}=0.283 \mathrm{~cm} / \mathrm{min}, \mathrm{T}=\sim 25^{\circ} \mathrm{C}$. 
Preliminary Ion Exchange Modeling for Removal of Cesium from Hanford Waste Using Hydrous Crystalline Silicotitanate Material

Revision (Date):

Page:

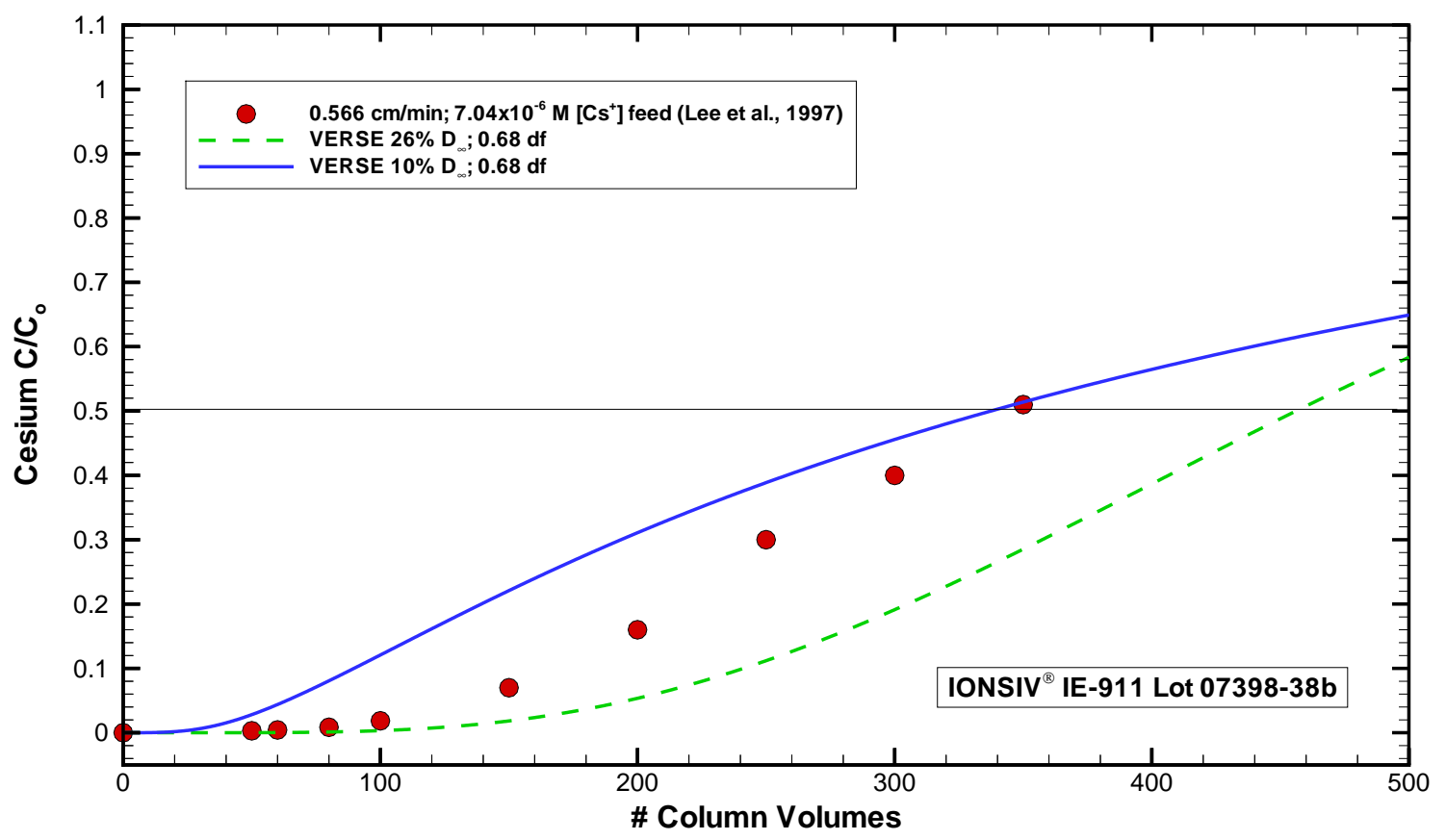

Figure 9-18. VERSE-LC cesium exit breakthrough curve compared to data from ORNL-W27-Test2 (Lee et al., 1997b): $\mathrm{D}=1.5 \mathrm{~cm}, \mathrm{~L}=5.659 \mathrm{~cm}, \mathrm{U}=0.566 \mathrm{~cm} / \mathrm{min}, \mathrm{T}=\sim 25^{\circ} \mathrm{C}$.

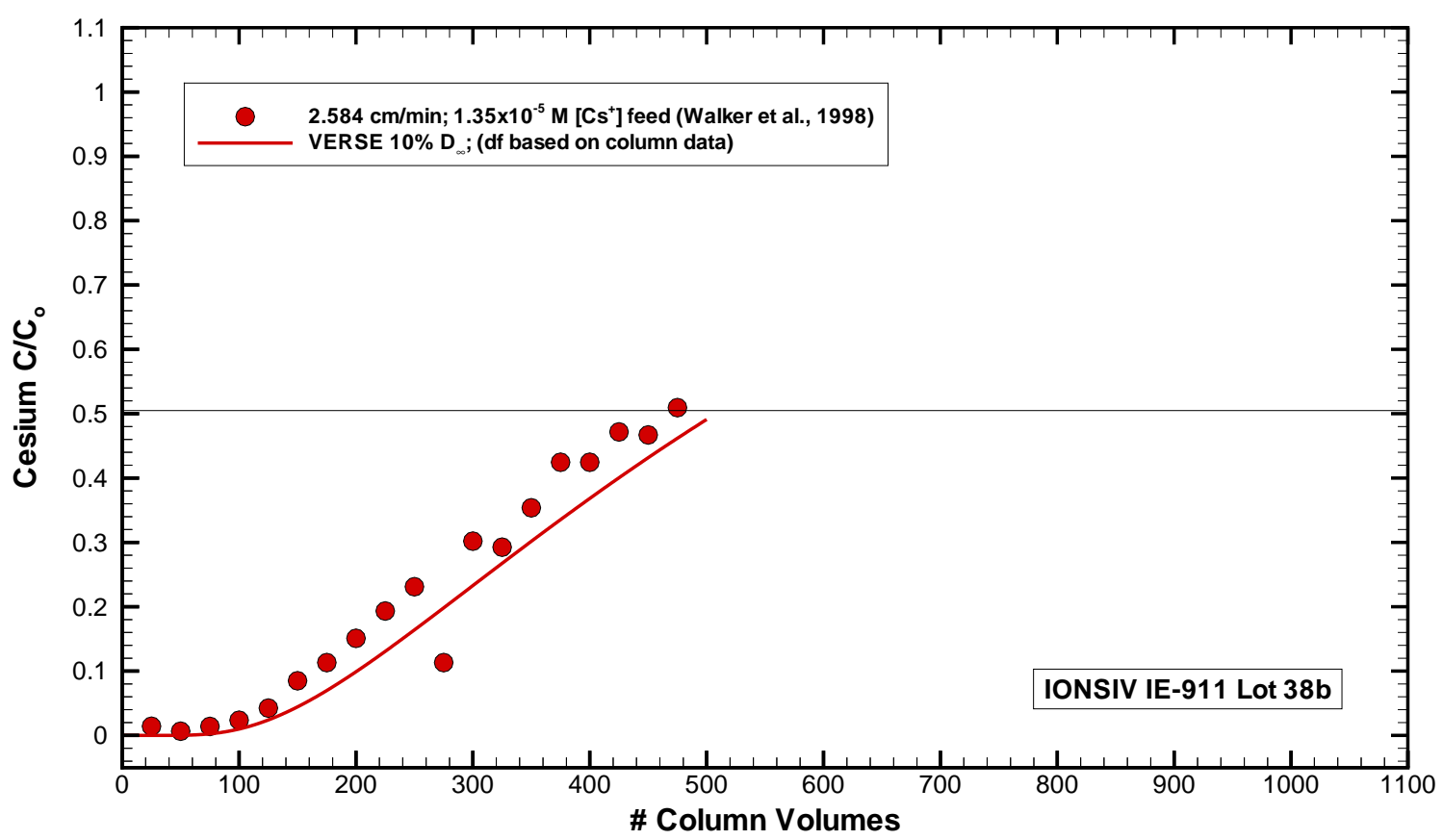

Figure 9-19. VERSE-LC cesium exit breakthrough curve compared to data from ORNL-CsRD-Run2 (Walker, Jr., et al., 1998): $\mathrm{D}=30.6 \mathrm{~cm}, \mathrm{~L}=51.67 \mathrm{~cm}$ per column, $\mathrm{U}=2.584 \mathrm{~cm} / \mathrm{min}$, $\mathrm{T}$ $=\sim 25^{\circ} \mathrm{C}$. 
Preliminary Ion Exchange Modeling for Removal of Cesium from

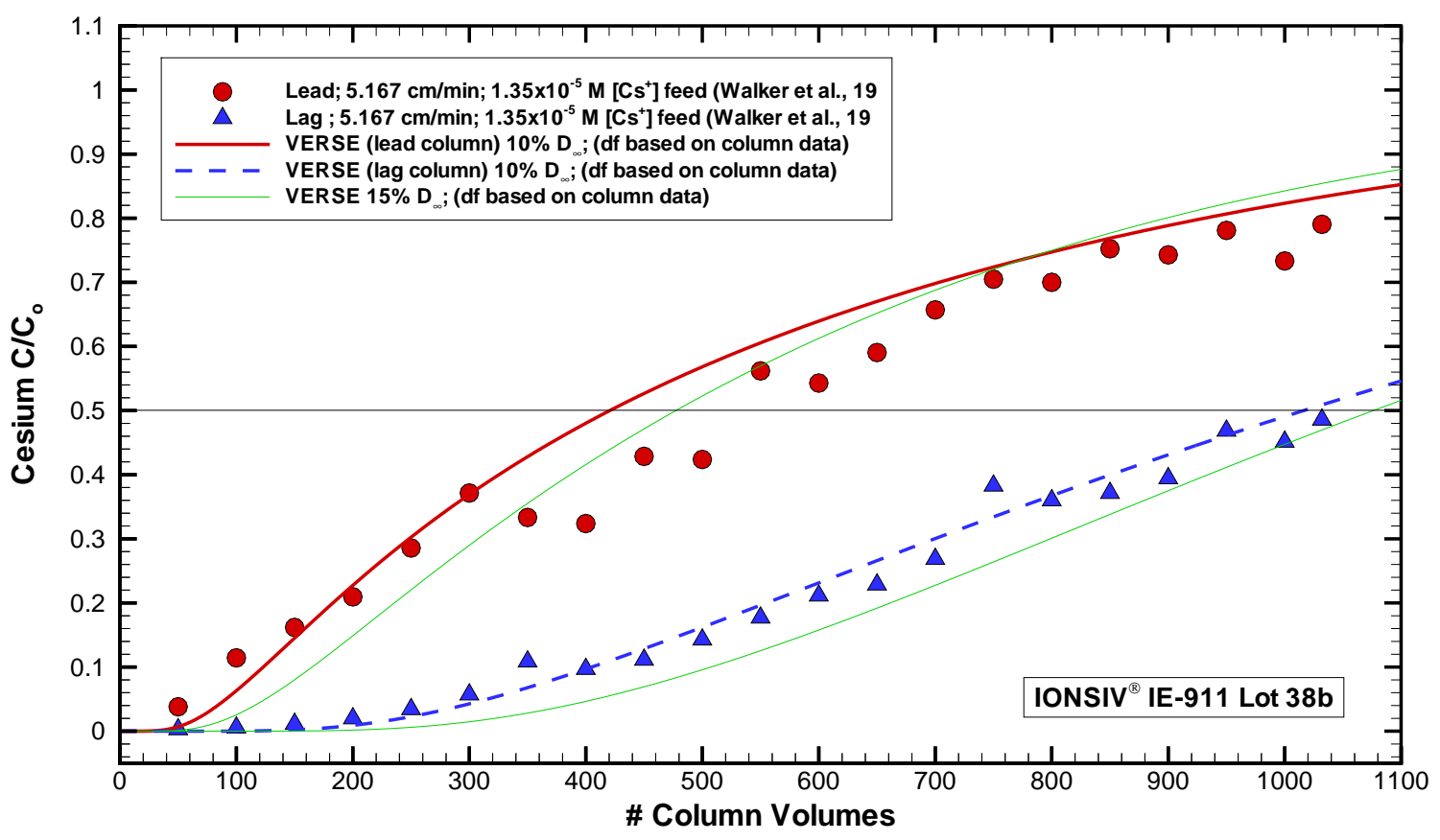

Figure 9-20. VERSE-LC cesium exit breakthrough curve compared to data from ORNL-CsRD-Run3 (Walker, Jr., et al., 1998): $\mathrm{D}=30.6 \mathrm{~cm}, \mathrm{~L}=51.67 \mathrm{~cm}$ per column, $\mathrm{U}=5.167 \mathrm{~cm} / \mathrm{min}, \mathrm{T}$ $=\sim 25^{\circ} \mathrm{C}$.

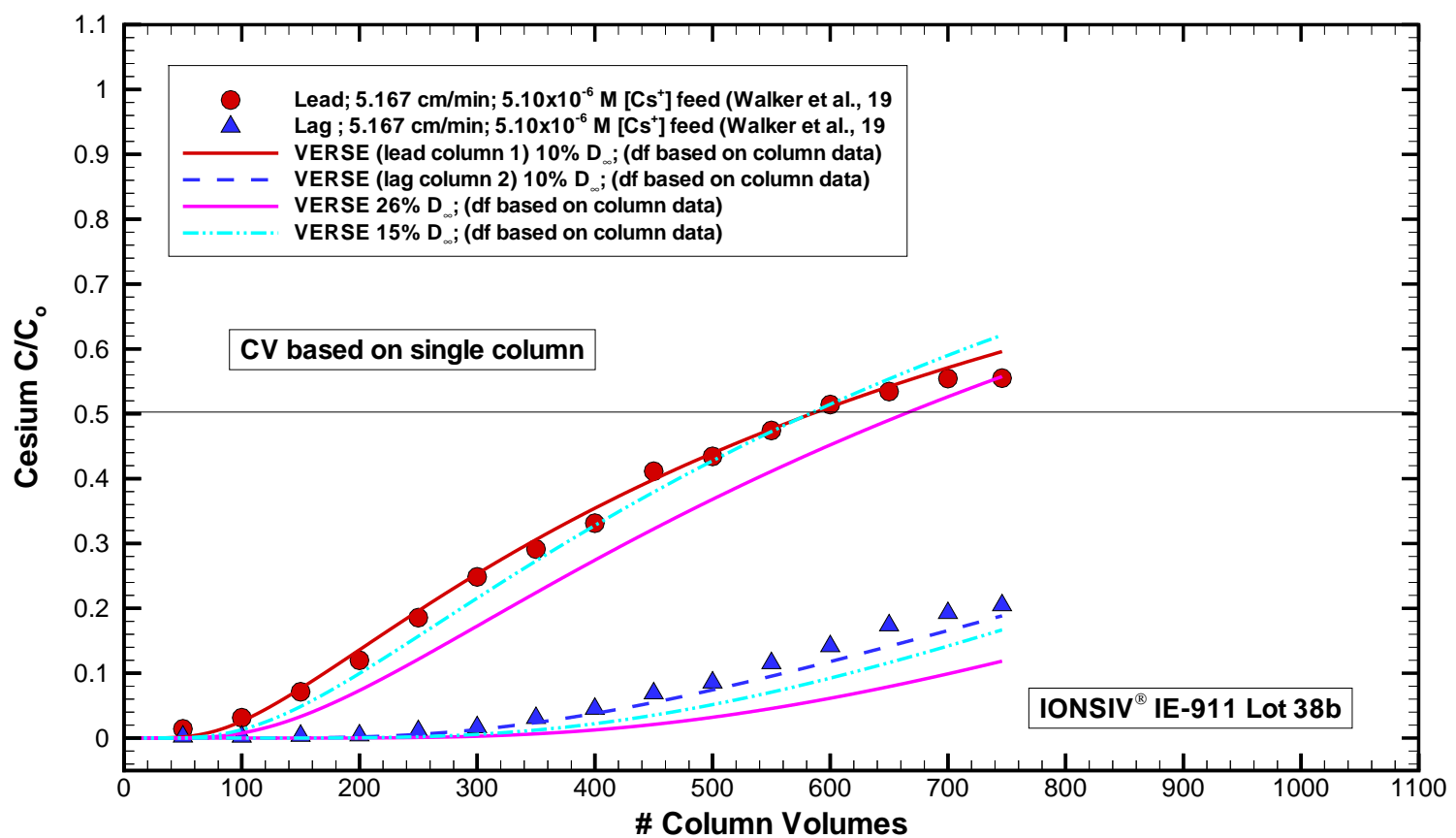

Figure 9-21. VERSE-LC cesium exit breakthrough curve compared to data from ORNL-CsRD-Run4a (Walker, Jr., et al., 1998): $\mathrm{D}=30.6 \mathrm{~cm}, \mathrm{~L}=51.67 \mathrm{~cm}$ per column, $\mathrm{U}=5.167 \mathrm{~cm} / \mathrm{min}, \mathrm{T}$ $=\sim 25^{\circ} \mathrm{C}$. 
Preliminary Ion Exchange Modeling for Removal of Cesium from

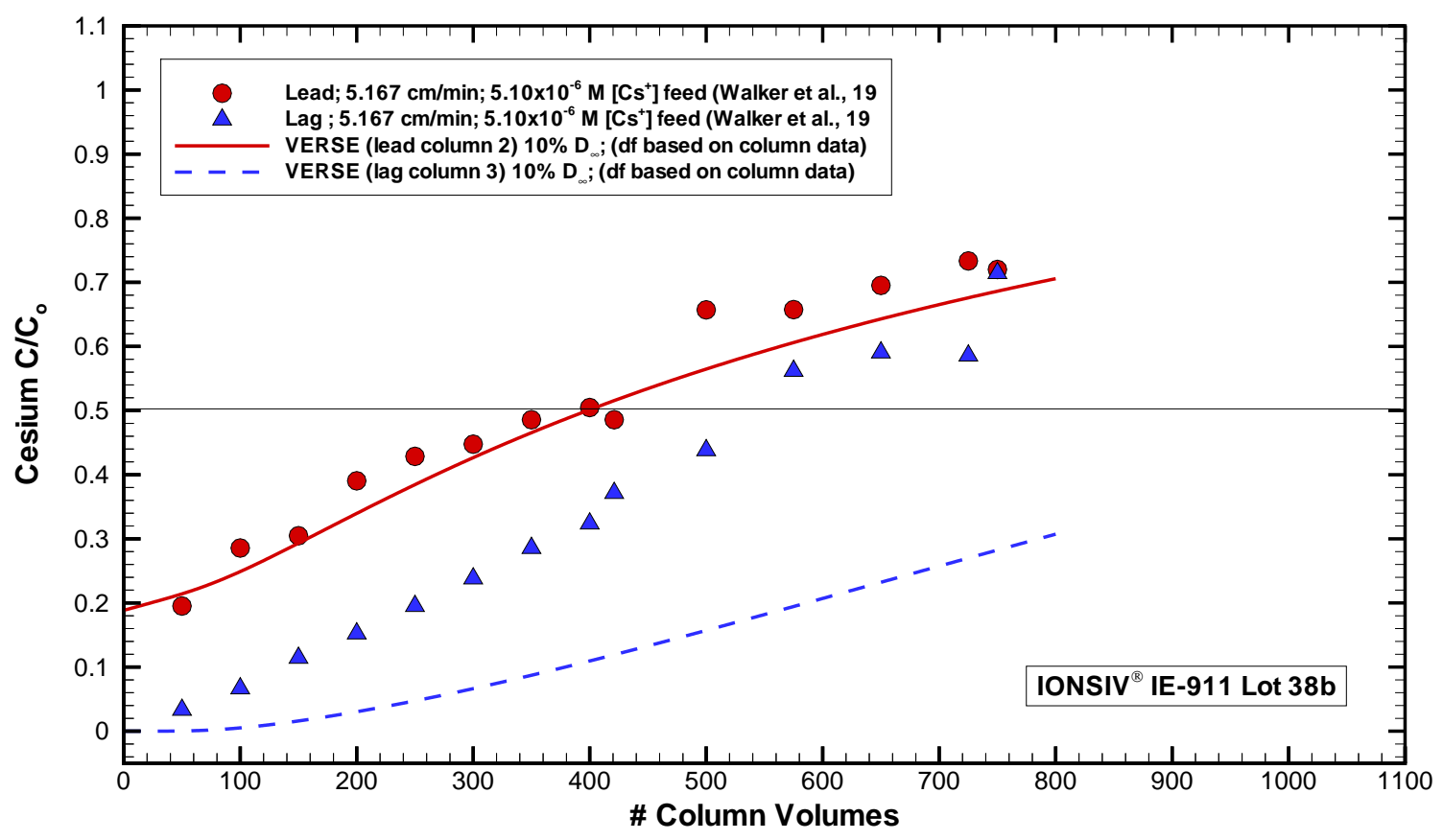

Figure 9-22. VERSE-LC cesium exit breakthrough curve compared to data from ORNL-CsRD-Run4b (Walker, Jr., et al., 1998): $\mathrm{D}=30.6 \mathrm{~cm}, \mathrm{~L}=51.67 \mathrm{~cm}$ per column, $\mathrm{U}=5.167 \mathrm{~cm} / \mathrm{min}, \mathrm{T}$ $=\sim 25^{\circ} \mathrm{C}$. 


\subsection{Full-Scale Column Predictions and Design}

Under "nominal" conditions, the predicted performance of a proposed full-scale ion-exchange facility using CST material is discussed in this section. Nominal conditions imply that the majority of parameter settings chosen are our "best estimate" of their values. It is assumed that the majority of properties associated with CST material (e.g., porosities, bed density, and diffusion coefficients) does not vary significantly between batches and can be adequately defined by their averaged behavior. However, due to batch variability observed in the manufacturing of CST material in its engineered-form, a statistically conservative cesium isotherm is used to accommodate the production range of expected CST material. This is accomplished by using a $68 \%$ dilution factor.

For column sizing purposes a global optimization strategy is employed where the total amount of spent CST material required to process the entire Phase 1 LAW inventory is minimized. Both 2column and 3-column carousel configurations are considered, along with a range of possible L/D geometries. All simulations were performed at an operating temperature of $25 \mathrm{C}$ where best estimate feed conditions for the 16 Phase 1 batch feeds in their scheduled order of processing were applied. Based on very limited analyses, variations in the order of batch feed processing had only small impacts on the total computed spent CST required.

The simulations are based on a VERSE-LC model of the lead and lag columns (and guard column for the 3-column configuration) where the parameter settings are consistent with the values used during the assessment of the available laboratory-scale column experiments. The feed concentrations and flowrates chosen are based on the current best estimate of their values consistent with available data and design objectives.

\subsection{IONSIV ${ }^{\circledR}$ IE-911 CST versus SuperLig ${ }^{\circledR} 644$ Ion-Exchanger Performance}

The CST material is being considered as a potential backup to SuperLig ${ }^{\circledR} 644$ resin for removal of cesium from the Phase 1 LAW inventory. The two main objectives within this chapter are to (1) determine an optimum column design based on CST that meets the facility's exit criteria given its input feeds and (2) determine the performance of CST when used in the current facility's column design. These performance objectives focus only on the loading phase of operation. The broader issues associated with changing over to CST in the overall waste treatment plant (WTP) is not addressed within this report. Since CST is considered to be a nonelutable ion exchange material, the operation within the ion-exchange facility would be impacted, as well as impacts to many other WTP unit operations. Some of the other potential impact issues not addressed are:

- Shielding and heat removal;

- Loaded CST storage;

- Handling and transport;

- HLW glass chemistry;

- Flowsheet compatibility; 
- Gas generation and removal; and

- Schedule impact.

The facility's column design as of when these analyses were being performed had an active bed diameter of $77 \mathrm{~cm}$ and bed length of $225 \mathrm{~cm}$. This design is $\sim 1000 \mathrm{~L}$ in bed volume per column, with a bed L/D of $\sim 2.92$. The available headspace above the active bed was designed such that an increased bed height of $\sim 50 \%$ was acceptable. This is the design specifications used within this effort for comparison purposes. However, note that more recent design changes now have the bed diameter at $107 \mathrm{~cm}$ and the bed height at $119 \mathrm{~cm}$ (i.e., an L/D of 1.1), while the total bed volume remains the same. The shorter columns (i.e., $\sim 50 \%$ shorter) help to reduce overall pressure drop concerns, as well as other operational items.

The impact on loading performance (i.e., removal of cesium from LAW streams) of replacing the SuperLig ${ }^{\circledR} 644$ resin with CST material can be qualitatively summarized by comparing some of the key properties of each ion exchanger. A list of key properties for both ion exchangers is provided in Table 10-1. Geometrically, we see that bed porosities are similar which lead to similar superficial velocities within the columns. Since the average particle size of CST IE-911 is about half that of SuperLig ${ }^{\circledR} 644$ and given similar superficial velocities, we would expect the pressure drop across the beds to be approximately double for CST IE-911 versus SuperLig ${ }^{\circledR} 644$. However, experimentally we see that CST IE-911 particles are much more rigid than SuperLig ${ }^{\circledR}$ 644 particles. The softer SuperLig ${ }^{\circledR} 644$ particles deform more under applied stresses and can result in significant pressure drops across the beds.

The smaller particle sizes also result in higher bed densities for CST IE-911 (i.e., on average a factor of 5 increase is bed density). This density difference becomes significant when considering the cesium loading capacity of a column. As listed in Table 10-1, the total ionic capacity of CST IE-911 versus SuperLig ${ }^{\circledR} 644$ on a mass basis is very similar. However, when comparing the same amount of exchanger on a bed volume basis, CST IE-911's total capacity is $\sim 6$ times that of SuperLig ${ }^{\circledR}$ 644. A comparison of cesium loading curves for LAW-12 (AN-103) in contact with CST IE-911 material and SuperLig ${ }^{\circledR} 644$ resin at 25 C is shown in Figure 10-1. The CST IE-911 material curves are based the algebraic models discussed in section 4, while the SuperLig ${ }^{\circledR} 644$ resin curves were computed using the algebraic model presented by Hamm et al. (2000a). Cesium loading curves on a mass of exchanger and bed volume basis are plotted. The cesium feed concentration for the LAW-12 batch feed is also shown. Over the operating range of interest, the increased cesium loading capacity for CST columns when compared to SuperLig ${ }^{\circledR}$ 644 columns is $\sim 30 \%$ by mass and $\sim 640 \%$ be volume. Basically, CST columns can hold a significantly larger amount of cesium.

The selectivity for $\mathrm{Cs}^{+}$cations versus either $\mathrm{K}^{+}$or $\mathrm{Na}^{+}$cations is very similar between CST IE911 material and SuperLig ${ }^{\circledR} 644$ resin, as shown in Table 10-1. Again, the ionic capacity of CST IE-911 material is much larger when compared on a bed volume basis.

With respect to column "kinetics" (i.e., mass transfer rates), pore diffusion within the CST IE911 particles is $\sim 3.5$ times slower than for SuperLig ${ }^{\circledR} 644$ particles. The slower kinetics of CST 
IE-911 material increases its sensitivity to changes in feed flowrate. This in turn impacts the "Mass Transfer Zone (MTZ)" length, which will be further discussed in a subsection below.

To see the overall impact in loading performance of replacing SuperLig ${ }^{\circledR} 644$ resin with CST IE911 material, two VERSE-LC simulations were performed where the only difference between the two simulations was the ion-exchange material chosen. The two simulations are based on the current ion-exchange facility column design and under its 2-column carousel configuration operating at $25 \mathrm{C}$ (i.e., $1000 \mathrm{~L}$ column beds, bed L/D of $\sim 3$ ). The cesium isotherms shown in Figure 10-1 were used that represent the isotherms for LAW-12 feed (AN-103). The same feed cesium concentration, flowrate, and exit criteria were employed. A comparison of the lead columns exit breakthrough curves is plotted in Figure 10-2 (i.e., the plain solid-curve representing CST IE-911 packed columns and the solid-curve with solid circles representing SuperLig ${ }^{\circledR} 644$ packed columns). The number of column (i.e., "bed") volumes (CV) of feed that can be processed prior to reaching the cesium exit criterion is $\sim 270 \mathrm{CV}$ for SuperLig ${ }^{\circledR} 644$ columns and $\sim 720 \mathrm{CV}$ for CST IE-911 columns. The degree of cesium loading on the lead columns (i.e., percent of saturation) is $\sim 40 \%$ for SuperLig ${ }^{\circledR} 644$ columns and $\sim 20 \%$ for CST IE911 columns. Some of the key properties associated with each exchanger, as discussed above and listed in Table 10-1, can be seen in Figure 10-2. The greater capacity of the CST IE-911 packed columns allow them to process more feed solution, while reduced kinetics of the CST IE911 packed columns limit the level of cesium loading achieved prior to cycling (i.e., reaching the exit cesium criterion).

\subsection{Basic Flowsheet}

The basic flowsheet for the full-scale ion-exchange columns is shown in Figure 10-1 for a 2column carousel configuration. The key parameters defining the full-scale facility are provided in Tables D-2 and D-3 for the Phase 1 LAW inventory. Two identically sized columns in series (i.e., a lead and a lag column) are used where an exit cesium concentration criterion (i.e., an envelope dependent value) is imposed on the lag column. Each column has a void headspace that is approximately the same size as each column. Prior to loading both columns are pretreated and contain an aqueous native solution with an ionic strength of approximately $0.25 \mathrm{M}$ sodium (i.e., essentially zero cesium and potassium are present). During the loading phase the feed solution's ionic strength increases significantly to approximately $5 \mathrm{M}$ sodium and 0 to $1 \mathrm{M}$ potassium. Inlet feed concentration of cesium can vary from 0 to $5 \times 10^{-4} \mathrm{M}$. A design flowrate of $\sim 3 \mathrm{CV} / \mathrm{hr}$ is used during the cesium loading phase. Upon reaching the lag column exit concentration criterion, the cesium loading phase is terminated and for subsequent loading cycles the lag column is placed in the lead column position with a fresh column being placed in the lag position. For SuperLig ${ }^{\circledR} 644$ packed columns the loaded lead column is eluted and pretreated for future use, while for CST IE-911 packed columns the loaded lead column is stored for future disposal. Fresh column implies a cesium free bed. It is assumed that the isotopic feed concentrations of total cesium is made up of ${ }^{137} \mathrm{Cs}$ and ${ }^{133} \mathrm{Cs}$, where their isotopic fractions are envelope dependent.

The cesium, potassium, and sodium concentration profiles along the column train varies with time. Due to the significant selectivity for cesium by SuperLig ${ }^{\circledR} 644$ resin or by CST IE-911 
material, the column exit breakthrough for potassium and sodium occurs very early (i.e., within 5-10 column volumes) with cesium's breakthrough significantly retarded. Key concentration points along the facility are numbered from 0 through 4 as shown in Figure 10-1. The inlet conditions correspond to point 0 while the exit product criterion is imposed at point 4 . Points 1 and 3 represent the locations where the headspace ends and the active bed begins.

A similar discuss applies for 3-column carousel operation where the first column in series is referred to as the lead column, the second column as the middle column, and the third column as the lag column.

\subsection{VERSE-LC Modeling of the Full-Scale Facility}

The VERSE-LC model representing the full-scale flowsheet is shown in Figure 10-2 for both the 2-column and 3-column carousel configurations. To model the full-scale facility using VERSELC the headspaces are considered to be continuous stirred tank reactors (CSTRs). In these CSTRs perfect mixing is assumed where no chemical reactions are taking place. During a VERSE-LC 2-column configuration simulation exit breakthrough curves at points 2 and 4 are generated (and point 6 for 3 -column configuration simulations) along with concentration profiles over both columns at key points in time.

Since the selectivity for $\mathrm{Cs}^{+}$is two orders in magnitude greater than either competitor of interest (i.e., $\mathrm{K}^{+}$or $\mathrm{Na}^{+}$, see Table 10-1), the "effective" single component cesium system is acceptable for transport analysis. The VERSE-LC input parameters are listed for the 11 Envelope A batch feeds, 2 Envelope B batch feeds, and 3 Envelope C batch feeds (i.e., nominal case studies) in Tables D-2 and D-3 of Appendix D. The VERSE-LC input files for typical (nominal) runs are also provided in Appendix D.

During the loading phase for modeling purposes time invariant flowrate and inlet feed conditions are assumed for each LAW batch feed. For one specified carousel configuration and input parameter settings, to compute the total amount of spent CST requires running VERSE-LC 16 times (i.e., once for each batch feed) in sequence. The cesium concentration profiles within the columns (i.e., in the fluid and on the solid) at the end of processing each batch feed are stored and are used as the initial state of the carousel for processing the next batch feed. To automate this process of handling many VERSE-LC runs and I/O files in series, a make utility called "NMAKE" was used. See Appendix D for further discussion on the use of NMAKE for this application.

\subsubsection{Volumetric Flowrate on an Envelope Basis}

The volumetric flowrate of the LAW stream passing through the ion-exchange facility during the loading phase is envelope dependent. The maximum loadings for waste sodium oxide currently planned for ILAW glass forms are provided in Table 10-2. The flowrates used in the VERSELC design calculations are listed in Table 10-3 for the 16 batch feeds. The basis for the flowrates centers on the production goals of glass and the allowable limits of waste sodium oxide $\left(\mathrm{Na}_{2} \mathrm{O}\right)$ loading within the glass matrix. Below the basis for each envelope is discussed. 


\section{Envelope A:}

The maximum waste sodium oxide loading currently planned for LAW Envelope A waste solutions is $\sim 19.6 \mathrm{wt} \%$. In order to support production of $60 \mathrm{MT} /$ day of ILAW glass that contains $19.56 \mathrm{wt} \%$ sodium oxide, $8.71 \mathrm{MT} /$ day of sodium must be feed to the ILAW melters. Assuming the LAW feed solution to the cesium ion exchange process is a $5 \mathrm{M}$ sodium solution, the volume of LAW processed through the Cs ion exchange process must be $\sim 75,773 \mathrm{~L} /$ day $(52.62 \mathrm{~L} / \mathrm{min})$ to support the ILAW glass production rate.

\section{Envelope B:}

The LAW Envelope B wastes contained in tanks 241-AZ-101 and 241-AZ-102 will be processed during the first ten years of operation at the RPP-WTP. During the first ten years of operation, the ILAW glass production rate is nominally $30 \mathrm{MT} /$ day. The maximum waste sodium oxide loading currently planned for LAW Envelope B waste solutions is $7.0 \mathrm{wt} \%$. In order to support production of $30 \mathrm{MT} /$ day of ILAW glass that contains $7.0 \mathrm{wt} \%$ sodium oxide, $1.56 \mathrm{MT} /$ day of sodium must be feed to the ILAW melters. Assuming the LAW feed solution to the cesium ion exchange process is a $5 \mathrm{M}$ sodium solution, the volume of LAW processed through the Cs ion exchange process must be $\sim 13,536 \mathrm{~L} /$ day $(9.40 \mathrm{~L} / \mathrm{min})$ to support the ILAW glass production rate.

After processing the AZ-101 and AZ-102 supernates, the RPP-WTP will receive other Envelope $\mathrm{B}$ solutions that will need to be processed at a rate to support production of $60 \mathrm{MT} /$ day ILAW glass. However, these other Envelope B solutions will not contain the relatively high concentration of cesium that is present in the AZ-101 and AZ-102 supernates. Future Envelope B solutions will have cesium, sodium, and potassium concentrations similar to Envelope A solutions.

\section{Envelope C:}

The LAW Envelope $\mathrm{C}$ wastes contained in tanks 241-AN-102 and 241-AN-107 will be processed during the first ten years of operation at the RPP-WTP. During the first ten years of operation, the ILAW glass production rate is nominally $30 \mathrm{MT} /$ day. The maximum waste sodium oxide loading currently planned for LAW Envelope C waste solutions is $12.0 \mathrm{wt} \%$. In order to support production of $30 \mathrm{MT} / \mathrm{day}$ of ILAW glass that contains $12.0 \mathrm{wt} \%$ sodium oxide, $2.68 \mathrm{MT} /$ day of sodium must be feed to the ILAW melters. Assuming the LAW feed solution to the cesium ion exchange process is a $5 \mathrm{M}$ sodium solution, the volume of LAW processed through the Cs ion exchange process must be $\sim 23,328 \mathrm{~L} /$ day $(16.2 \mathrm{~L} / \mathrm{min})$ to support the ILAW glass production rate.

\subsubsection{Cesium-137 Exit Concentration Criterion on an Envelope Basis}

The LAW stream exiting the ion-exchange facility during the loading phase has a ${ }^{137} \mathrm{Cs}$ concentration criterion that is envelope dependent. Based on a ${ }^{137} \mathrm{Cs}$ content not to exceed 0.3 $\mathrm{Ci} / \mathrm{m}^{3}$ for the glass product made from the ILAW melters, overall (i.e. bucket) average cesium concentrations in the LAW exiting stream can be computed for each envelope. Based on the maximum allowable waste sodium oxide content of the glass and the $0.3 \mathrm{Ci} / \mathrm{m}^{3}$ limit of ${ }^{137} \mathrm{Cs}$ within the glass product, ${ }^{137} \mathrm{Cs}$ exit criterion can be computed on a $\mathrm{Ci} / \mathrm{gmole}$ of sodium basis. Table 10-2 lists these values, along with the values based on a $5 \mathrm{M}$ sodium feed solution in terms 
of $\mu \mathrm{Ci} / \mathrm{ml}$. To convert these ${ }^{137} \mathrm{Cs}$ exit criteria values into total Cs concentration limits, assumed isotopic fractions of ${ }^{137} \mathrm{Cs}$ to total cesium are used (i.e., for calculational purposes the total Cs is assumed to be made up of ${ }^{133} \mathrm{Cs}$ and ${ }^{137} \mathrm{Cs}$ isotopes only). The isotopic fractions assumed for each envelope waste stream is listed in Table 10-2. For each envelope the total cesium exit concentration criterion computed is listed in Table 10-4, where the normalized to the inlet feed concentration is also provided on a batch feed basis.

These exit concentration criteria place limits on the cumulative cesium concentration of the effluent exiting the ion-exchange facility (i.e., the "bucket" average value), not on the instantaneous values during its operation. To perform the carousel operation in an automatic fashion within the VERSE-LC simulations, we impose these cumulative concentration criteria on the VERSE-LC computed instantaneous exit concentrations. No accounting for integration of the breakthrough curve is made. This represents an embedded conservatism within the analysis methodology used.

\subsubsection{Input Concentration and Flowrate Boundary Conditions}

The scheduled sequence of batch feeds is listed in Table 10-3 along with the source tank that it originated from. The volumetric flowrates chosen are envelope dependent. The volumetric flowrates are based on the Phase $130 \mathrm{MT} /$ day operation schedule for Envelopes B and C and on the expanded capability (beyond Phase 1) of $60 \mathrm{MT} /$ day operation for Envelope A, as discussed above. Using these flowrates and the estimated solution volumes of each batch feed, batchprocessing times were computed and are also provided in Table 10-3. With these batch processing times, time dependent inlet cesium concentration and flowrate boundary conditions for use in VERSE-LC simulations were generated (i.e., note that these boundary conditions are stationary values within a given batch feed, but step change between the batch feeds). These boundary conditions are shown in the barchart plotted in Figure 10-5. The total process time required to process the entire Phase 1 inventory is $\sim 4.2$ years of operation. If the Envelope A feeds are processed at the lower $30 \mathrm{MT} /$ day glass production rate, then the total process time becomes $\sim 5.8$ years.

\subsection{Mass Transfer Zone Concept for Column Design}

One approach typically used to size ion-exchange columns is based on the mass transfer zone (MTZ) concept. As discussed in Helfferich and Carr (1993), wave theory indicates that for ionexchange processes under favorable isotherm conditions the shape of the axial concentration profile becomes stationary at long distances down the column. Axial dispersion tends to spread the profile out, while a favorable isotherm tends to sharpen the profile. These two forces become balanced at sufficiently long distances down a column. The stationary shape is an asymptote that is only approached for finite size columns, where the practical distance required is a function of how nonlinear the isotherm is over its operating range.

The approach is based on the following key assumptions:

- Constant inlet feed conditions (concentration $\mathrm{c}_{0}$, flowrate $\mathrm{Q}_{0}$, and temperature $\mathrm{T}_{\mathrm{o}}$ ); 
- Stationary profile achieved within practical lengths;

- Length of MTZ based on stationary conc. profile; and

- Insensitive to carousel process (i.e., cycle number).

For application to a specific carousel configuration (e.g., 2-column case), a lag column exit concentration criterion and a lead column loading criterion must be established. For a conservative design $t$ worst case isotherm must also be chosen where the inlet flowrate and concentration become time invariant. Given these items the length of the MTZ can be estimated as shown by the cartoon illustrated in Figure 10-6. The breakthrough curve shown in Figure 106 represents the stationary concentration profile obtained at long distances down a single virtual column. The downstream half of the MTZ length is established by the criteria chosen, where it is assumed that finite profile development length and carousel cycling have a negligible impact.

To estimate the MTZ length for CST packed columns processing Phase 1 LAW solutions, three VERSE-LC column transport simulations were run based on a long single column (i.e., actual runs were performed using a 2000L 2-column carousel arrangement with cycling turned off). The three feed solutions chosen are LAW-1 (Envelope A, AP-101), LAW-2a (Envelope B, AZ101), and LAW-3 (Envelope C, AN-102). All three LAW feeds have potential worst case isotherms for their respective envelopes.

The normalized axial cesium concentration profile at a point in time prior to when the exit criterion would have been exceeded is plotted in Figure 10-7 for each feed solution. As Figure 10-7 indicates, the MTZ length for each envelope is quite different. Based on this design strategy, the longest MTZ length would be specified (i.e., value corresponding to Envelope A feed). The large differences in MTZ length observed are a direct result of (1) higher flowrates tend to spread out the axial profile, while (2) higher inlet concentrations tend to sharpen the axial profile (i.e., more nonlinear behavior operative).

Based on the envelope dependent results shown in Figure 10-7 and variability seen in isotherms within a given envelope, the MTZ design approach was abandoned. In its place a global optimization strategy was chosen which can directly account for variability of flowrate, feed concentration, and isotherm behavior among the 16 Phase 1 LAW batch feeds. This strategy is recommended for future SuperLig ${ }^{\circledR} 644$ and 639 modeling efforts, too.

\subsection{The Global Optimization Strategy}

To design a CST based ion-exchange facility operating in a carousel manner, the traditional MTZ approach discussed above appeared to be inadequate. The inadequacies were primarily:

- A fairly long virtual column was required to establish a stationary profile, which weakens the assumptions of negligible impact due to finite length columns and carousel cycling; and

- Significant flowrate, feed concentration, exit criterion, and isotherm variability exists between the 16 LAW batch feeds, which make it difficult to obtain a unique worst case scenario for designing to. 
To eliminate these difficulties, a global optimization strategy based on simulating the entire processing of the Phase 1 inventory in its schedule sequence was undertaken. Here, the analysis approach chosen is primarily based on "best estimate" parameter values; however, the chosen isotherms for each batch feed are based on a dilution factor of $68 \%$ to account of the variability observed in the production of CST in its engineered-form.

The assumption is made that once a batch feed has been completely processed, the next batch feed scheduled will begin without starting with fresh columns (i.e., the batch feeds are stacked inline where the carousel cycling only occurs when the exit lag column criterion is reached). The optimization parameter that was chosen to be tracked is the amount of spent CST material used to process each batch feed. Since the original basic concern was over the amount of CST ultimately having to be placed in the glass product, this appeared to be the obvious choice for our optimization parameter.

The carousel operations are discrete events that take place when the exit cesium concentration reaches its criterion at the exit of a lag column. Initially, both the first lead and lag columns are cesium free. Upon reaching the point where the last drop of LAW feed within the inventory is processed, the process is stopped. At this point both the lead and lag columns present are partially loaded, but contributed as full columns to the total spent CST required. In this way, the computed total spent CST becomes a discrete value based on the number of cycles required.

Based on this concept, the total amount of spent CST material required to process the entire Phase 1 LAW inventory can be computed by:

$$
\mathrm{m}_{\mathrm{CST}}=\rho_{\text {bed }} \mathrm{V}_{\text {bed }}\left(\mathrm{n}_{\text {carousel }}+\mathrm{n}_{\text {cycles }}\right) \text {, }
$$

where the bed density is in terms of $\mathrm{g} / \mathrm{ml}$ (i.e., an average value of $1.0 \mathrm{~g} / \mathrm{ml}$ is used), the bed volume per column is given in liters (i.e., this is the key variable for optimization), $\mathrm{n}_{\text {carousel }}$ is the number of columns within a carousel (i.e., 2-column or 3-column arrangements), and $n_{\text {cycles }}$ is the total number of carousel cycles required. The total number of columns given in Eq. (10-1) has two terms since the carousel initially starts out with $\mathrm{n}_{\text {carousel }}$ fresh columns and then adds one new column to the carousel every cycle.

As demonstrated in a following subsection for CST packed columns, variation in the bed L/D geometry, while holding the bed volume of a column constant, has very little impact on spent CST material based on its computed value using Eq. (10-1). Therefore, the spent CST value is a discrete variable that depends primarily on the bed volume of a column. In this way, we are addressing approximately a single-variable optimization problem.

\subsection{Theoretical Minimum Spent CST Material}

A theoretical minimum in spent CST material can be computed based on the assumption that for each batch feed variably sized columns are available that become fully loaded (i.e., saturated) with respect to the feed's cesium concentration. For the $i^{\text {th }}$ batch the maximum CST loading for cesium (mmole $\left.\mathrm{Cs}_{\mathrm{CS}} / \mathrm{g}_{\mathrm{CST}}\right)$ can be computed based on the cesium isotherm and its cesium inlet concentration using: 


$$
\mathrm{Q}_{\mathrm{Cs}, \mathrm{i}}=\frac{\eta_{\mathrm{df}} \overline{\mathrm{C}}_{\mathrm{T}} \mathrm{c}_{\mathrm{Cs}, \mathrm{i}}}{\mathrm{c}_{\mathrm{Cs}, \mathrm{i}}+\beta_{\mathrm{i}}} .
$$

The amount of total cesium present within a given batch feed can be expressed in units of mmole $_{\mathrm{Cs}}$ as:

$$
\mathrm{n}_{\mathrm{Cs}, \mathrm{i}}=1000 \mathrm{c}_{\mathrm{Cs}, \mathrm{i}} \mathrm{V}_{\mathrm{i}} .
$$

Using Eqs. (10-2) and (10-3), the theoretical minimum in spent CST material $(\mathrm{kg})$ for each batch feed becomes:

$$
\mathrm{m}_{\mathrm{CST}, \mathrm{i}}=\frac{\mathrm{c}_{\mathrm{Cs}, \mathrm{i}} \mathrm{V}_{\mathrm{i}}}{\mathrm{Q}_{\mathrm{Cs}, \mathrm{i}}}
$$

where the total amount of spent CST material then becomes:

$$
\mathrm{m}_{\mathrm{CST}}=\sum_{\mathrm{i}=1}^{16}\left[\frac{\mathrm{c}_{\mathrm{Cs}, \mathrm{i}} \mathrm{V}_{\mathrm{i}}}{\mathrm{Q}_{\mathrm{Cs}, \mathrm{i}}}\right] \text {. }
$$

For the 16 batch feeds contained within Phase 1, the theoretical minimum amount of spent CST was computed to be $55,360 \mathrm{~kg}$ and is tabulated in Table 10-5. This value is useful for seeing how efficient a given actual column design might be and is plotted as a dashed-horizontal line in the various spent CST figures to be addressed below.

\subsection{Spent CST Material Dependency on Geometry}

Within a given carousel design, each column is geometrically the same (i.e., the same volume and shape). The two key geometric parameters that define a unique column design are its bed volume and length-to-diameter ratio (L/D). For loading phase considerations, to a much lesser extent the headspace volume above the bed is also needed. The length, L, referred to above represents the height of the active resin bed during the $5 \mathrm{M}$ sodium loading phase.

From a theoretical perspective for fixed boundary conditions (i.e., feed composition and volumetric flowrate), we find that the performance of a column during the loading phase depends upon the overall mass transfer in a unique way. The overall mass transfer limitations result from two components: (1) mass transfer resistance across the liquid film present at each particle's outer surface and (2) mass transfer resistance due to diffusion through each particle's pores. For a fixed volumetric flowrate and bed volume design, when varying the cross-sectional area of the column (i.e., its diameter) varies the bed's liquid superficial velocity. The superficial velocity only impacts the mass transfer film coefficient, not the pore diffusivity coefficient.

For CST IE-911 material we find that the overall mass transfer resistance is dominated by pore diffusion, while the resistance due to film diffusion is relatively negligible under the range of flowing conditions of importance in CST column design here. Given this information, we would 
expect that the estimated amount of spent CST required to process the entire Phase $1 \mathrm{LAW}$ inventory would be insensitive to geometric variations such as L/D.

To confirm this expected insensitivity to L/D, VERSE-LC simulations were run for L/D values of $1,2,3,4$, and 5 . The entire Phase 1 LAW inventory was processed in these simulations. Other than the changes in geometry, all other input parameters were kept at their nominal settings (e.g., $25 \mathrm{C}$ operation, 2-column carousel configuration, $2000 \mathrm{~L}$ bed column size, $500 \mathrm{~L}$ head-space). The results of these five simulation runs are tabulated in Table 10-6. For each L/D test case Table 10-6 provides the column geometry considered, the number of process cycles occurring during each batch feed, the total number of process cycles required, and the total amount of spent CST material required to process the entire Phase 1 LAW inventory. The total amount of spent CST is computed based on the number of cycles required (plus 2 to account for the 2-column configuration and startup), the bed density of the CST material, and the bed volume of a column. These results are also shown in Figure 10-8 where we see that the computed spent CST is very insensitive to $\mathrm{L} / \mathrm{D}$.

The degree of sensitivity resulting from the L/D variations considered, is less than the amount required to cause a change in the total number of cycles required. Therefore, as shown in Table 10-6 and in Figure 10-8 no effect on spent CST is observed.

Given the fact that the estimated amount of spent CST required to process the entire Phase 1 LAW inventory is insensitive to variations in L/D (i.e., under constant bed volume conditions), from a design perspective the $\mathrm{L} / \mathrm{D}$ can be established based on other design considerations (e.g., based on required superficial velocity to sweep out gas bubbles). In the various simulations to be discussed below, typically a $\mathrm{L} / \mathrm{D}=3$ is studied; however, for convenience in some cases the $\mathrm{L} / \mathrm{D}$ is varied (i.e. $\mathrm{L} / \mathrm{D}=2$ for most of the 3 -column carousel cases).

In the various VERSE-LC design simulations performed, the geometry of each column design considered was computed based on the following approach. For the 2-column carousel cases a total bed volume for the column design and a $\mathrm{L} / \mathrm{D}$ are specified. Given these two values the diameter, then length, of the column design can be computed using:

$$
\mathrm{D}_{\text {bed }}=\sqrt[3]{\frac{4 \mathrm{~V}_{\text {bed }}}{\pi\left(\frac{\mathrm{L}}{\mathrm{D}}\right)}},
$$

and

$$
\mathrm{L}_{\text {bed }}=\left(\frac{\mathrm{L}}{\mathrm{D}}\right) \mathrm{D}_{\text {bed }} \text {. }
$$

For the 3-column carousel designs that are used for direct comparison to their 2-column design counterparts, the total bed volume and computed bed diameter based on Eq. (10-6) above is used. The bed length then becomes two-thirds of the 2-column bed length based on Eq. (10-7). In this way, when comparing a 2-column versus a 3-column carousel configuration, we are looking only at how many subdivisions are being made in a total bed (i.e., both have the same 
bed diameter and total bed length). Application of the above approach over a range of column volumes and $\mathrm{L} / \mathrm{D}$ ratios were considered. For the 2-column carousel configurations considered, the resulting geometies are listed in Table 10-7. Table 10-8 contains the geometries considered for the 3-column carousel configurations.

\subsection{CST Column Design and Performance}

Numerous VERSE-LC simulations were run in order to study the behavior of CST packed column operating in a carousel fashion and to provide results for establishing a base design. All of the VERSE-LC simulations were performed at $25 \mathrm{C}$. Both 2-column and 3-column carousel configurations are addressed, where for design purposes a range of potential column volumes was considered. The parameter settings provided in Tables D-2 and D-3 represent our nominal conditions for each batch feed (e.g., at dilution factor of $68 \%$ and a cesium pore diffusivity coefficient of $20 \%$ of its free diffusion value). Sensitivity studies were performed to estimate the impact that potentially "slow kinetics" might have on the performance of a given design. The results of these simulations are discussed below.

\subsubsection{Nominal Case Results for 2-Column Carousels}

Under nominal parameter settings, numerous VERSE-LC simulations were performed where the bed volume of a column was varied from $500 \mathrm{~L}$ up to $9,000 \mathrm{~L}$. The dilution factor was set to $68 \%$ and the cesium pore diffusivity coefficient was set to $20 \%$ of its free diffusion value. Both 2-column and some limited 3-column carousel were considered. These nominal case runs were performed to study the loading behavior of a CST packed column carousel and to estimate a near optimal design size.

The cases where the column volume was set to $1000 \mathrm{~L}$ and with a $\mathrm{L} / \mathrm{D}=3$ represent runs that demonstrate the CST performance when used in the current ion-exchange facility design based on the SuperLig ${ }^{\circledR} 644$ packed columns. For a 2-column carousel configuration the cesium breakthrough curve at history point 2 in Figure 10-4 is plotted in Figure 10-9. The lead column's cesium concentration is plotted versus process time in months for the entire processing period required to process the entire Phase 1 LAW inventory. Also shown in Figure 10-9 are slightly shaded vertical lines that segregate out the individual batch feed processing periods. At the top of the xy frame each batch feed operating period has been labeled. During the processing numerous carousel cycles were performed within each of the 16 batch feed periods. The point in time where a carousel cycle is performed can clearly be seen by the sudden drop to near zero cesium concentration. The actual number of carousel cycles performed per batch feed is tabulated in Table 10-9. The total number of cycles (78) and the total amount of spent CST material required $(80,000 \mathrm{~kg}=80 \mathrm{MT})$ are also listed in Table 10-9.

As Figure 10-9 illustrates, the high cesium feed concentrations of the Envelope B LAW feeds have a profound impact on cesium concentrations throughout the carousel well beyond their process periods. Plotting this breakthrough curve in its normalized form (i.e., exit cesium concentration divided by the current inlet feed value), one can better see the impact. In Figure 10-10 the normalized breakthrough curve is plotted where the percentage of column loading can be quickly approximately determined. For the Envelope A feeds the lead columns are $\sim 20 \%$ 
upon cycling, while for Envelope B feeds the lead columns are $\sim 100 \%$ loaded. For the LAW-3 and LAW-4 Envelope C feeds we see them experiencing loadings greater than $100 \%$ based on their inlet feed concentrations, with final loadings of $\sim 80 \%$.

For example, during the processing of LAW-3 cesium that was originally stored during LAW-2b is being redistributed from the lead column to the lag column for several carousel cycles. This represents loading and unloading of the ion exchange material where in the VERSE-LC model used we are assuming that:

- The ion exchange isotherm is unique and truly represents the solid-liquid equilibrium state regardless of which side it is being approached from; and

- The mass transfer resistances within the CST particles are not directionally dependent, such that incoming and outgoing pore diffusion rates are the same if the same concentration gradient in each direction were applied.

Limited experimental data exist indicated that the isotherms are probably unique, at least for CST material that has not been heated up to significantly. It is suspected that the diffusion rate leaving the CST particles will be much slower than entering. Under these conditions the assumption of similar rates is conservative.

The cesium breakthrough curves at history point 2 in Figure 10-4 are also plotted for three other larger column designs (i.e., $2000 \mathrm{~L}, 3000 \mathrm{~L}$, and $4000 \mathrm{~L}$ ). The breakthrough curve pairs (i.e., concentration and then its normalized value) for the $2000 \mathrm{~L}$ case are shown in Figures 10-11 and 10-12. The breakthrough curves for the $3000 \mathrm{~L}$ case are shown in Figures 10-13 and 10-14. The breakthrough curves for the $4000 \mathrm{~L}$ case are shown in Figures 10-15 and 10-16. The effect of going to increased column volumes beyond the current facility design can clearly be seen in these plots. As one would expect, as the column volume is increased the total number of process cycles drops and lead column loadings rise. For example, when looking at the $4000 \mathrm{~L}$ column volume case shown in Figure 10-16, lead column loadings for Envelope A, B, and C feeds are $\sim 70 \%, \sim 100 \%$, and $+100 \%$, respectively.

The number of carousel cycles and spent CST required for these cases and several other volumes considered are tabulated in Table 10-9. The spent CST results listed in Table 10-9 are plotted in Figure 10-17. Theoretically, the minimum amount of CST required would correspond to the column size where the lag column exit criterion is reached just when the last drop of feed inventory enters the lead column (i.e., just prior to the need to perform the first carousel operation resulting only in a total of two columns used). Note that this minimum point is much greater than the upper limit plotted of $9000 \mathrm{~L}$. For columns larger than this the two columns would be under-loaded. For smaller columns the carousel process occurs at increasing frequency also resulting in reduced loading. The oscillation seen in the data is due to the discrete nature of the computed spent CST required. If numerous data points were to be plotted in Figure 10-17, we would see a shark-tooth pattern forming where a distinct step would occur at the point where the last lead column was nearly full or fresh when the process inventory was consumed. These swings grow in magnitude for larger column volumes. The solid-curve shown in Figure 10-17 is provided only to highlight the general behavior of the data. 
Also shown in Figure 10-17, for the VERSE predictions, we see that the shape of this spent CST curve is rather flat over a large range of column volumes, becoming steep only at small column sizes. The optimal column size over the flat portion of this curve can be based on capital and operational costs for the facility and its carouseling needs. A near optimal column design is achieved at $2000 \mathrm{~L}$ where $\sim 66 \mathrm{MT}$ of spent CST is generated. During Phase 1 the IHLW glass melters will be generating an estimated 2651 MT of glass product based on the Tank Farm COUP report (Kirkbride et al., 2000, see Table 4.1-1). 66 MT of spent CST adds approximately $2.5 \mathrm{wt} \%$ sodium oxide to the IHLW glass melter product. Based on the current column design of a bed volume of $1000 \mathrm{~L}$, the estimated spent CST material increases by $\sim 20 \%$ to $80 \mathrm{MT}$. If the bed volume of CST was to be increased in the current design to 1500 L (i.e., the upper limit of bed volumes in the current design), the estimated spent CST material increases by $\sim 10 \%$ to 72 MT.

\subsubsection{Nominal Case Results for 3-Column Carousels}

Three-column carousel configurations were considered to determine the potential benefit associated with more column stages. Similar results using a 3-column carousel configuration are tabulated in Table 10-10 for a smaller range of column volumes (i.e., $333 \mathrm{~L}, 467 \mathrm{~L}, 667 \mathrm{~L}, 1333$ L, $2000 \mathrm{~L}, 2667 \mathrm{~L}$, and $3333 \mathrm{~L}$ ). These particular column volumes yield total bed volumes of the carousel that are equal to similar total volumes used in the 2-column carousel runs. The spent CST results of these 3-column carousel configurations are tabulated in Table 10-10 and are plotted in Figure 10-18, along with the results of the 2-column carousel runs. To put the 2column and 3-column cases on a common basis for plotting, the computed spent CST is plotted in Figure 10-18 as a function of total carousel bed volume.

As Figure 10-18 shows, only marginal gains can be achieved when a 3-column versus 2-column carousel facility is considered. For example, an $\sim 1 \%$ decrease in spent CST is achieved when going from a 2-column to a 3-column carousel based on a total bed volume of 4000 L (i.e., 2000 $\mathrm{L}$ beds for the 2-column arrangement and $1333 \mathrm{~L}$ for the 3-column arrangement). Theoretically, increased column stages will reduce the amount of spent CST generated; however, the predicted gains are well within the expected accuracy of the methodology and the increased staging is not warranted.

\subsubsection{Impact of Slow Kinetics on Design}

It is well known that CST overall has relatively slow kinetics. From the standpoint of the VERSE-LC model used, the overall kinetics that we are referring to is controlled by the rates of mass transfer across the particle-bed interface (i.e., the film coefficient) followed by mass transfer through the particle pores (i.e., the cesium binary pore diffusion coefficient). In the modeling options chosen we are assuming that the ion exchange process that occurs locally at a CST exchange site is instantaneous. For the range of design conditions of interest to us, we also through sensitivity studies found that pore diffusion is the limiting mass transfer step. One method to increase the CST kinetics is the use of smaller size particles that reduced the diffusion path lengths. Unfortunately, this also increases the pressure drop across the bed. To reduce the increased pressure drop a reduction in the bed L/D could then be considered. 
To assess the overall impact resulting from overall mass transfer resistances (i.e., the "kinetics"), three different column sizes were investigated based on a 2-column carousel configuration where the mass transfer resistances were essentially eliminated. The results of these VERSE-LC simulations are provided in Table 10-11, along with the nominal case runs for comparison. By setting the film and pore diffusivity coefficients to very high values, the impacts due to mass transfer resistances can be eliminated.

To assess the sensitivity pore diffusion has on computed spent CST requirements, additional VERSE-LC simulations were generated based on pore diffusivity coefficients of $10 \%$ and $30 \%$ of its free diffusion value (see Tables 10-12 and 10-13, respectively). The nominal setting for the cesium pore diffusivity coefficient is $20 \%$ of its free diffusion value, whose results were discussed earlier.

The computed spent CST results of these sensitivity runs are all plotted in Figure 10-19 for comparison to the nominal 2-column carousel results. For the $2000 \mathrm{~L}$ bed volume design, the resistances associated with mass transfer only increases the computed spent CST by $\sim 14 \%$ when compared to the nominal result. Impacts of this magnitude would suggest that further reduction in particle size or in bed L/D is not warranted based on improving the "kinetics" alone. However, uncertainty in the actual value of the pore diffusivity coefficient can result in larger impacts.

We see in Figure 10-19, for a fixed column volume design, that as the pore diffusivity coefficient is increased (i.e., infinity, $10 \%, 20 \%, 30 \%, \ldots$ ) its impact on spent CST becomes more aggressive. Future efforts should be focused on obtaining better confidence in the magnitude of this diffusivity coefficient. The impact of pore diffusion also increases for decreasing column volume designs where the more dispersed wave-fronts breakthrough much earlier. In general, as the column volume is increased the impacts associated with "kinetics" diminish and ultimately are lost as shown in Figure 10-19.

Also the theoretical minimum in spent CST required is plotted in Figure 10-19. This value can not be reached using practical designs, but is shown to help gage how efficient or inefficient the current CST material and carousel designs are. For the $2000 \mathrm{~L}$ bed volume design (i.e., $4000 \mathrm{~L}$ total bed volume condition), the actual/practical design only increases the computed spent CST by $\sim 19 \%$.

\subsection{Cesium Column Inventory}

In order to bound the IONSIV ${ }^{\circledR}$ IE-911 CST material's radioactive exposure levels associated with radioactive decay of cesium-137, cesium inventories within the column (i.e., cesium content in the liquid-phase plus adsorbed onto the solid-phase) must be computed during the loading cycle corresponding to the worst case conditions. This bounding inventory calculation is based on the VERSE-LC results described above. This inventory estimate can be used in subsequent analyses (beyond the scope of this report) for estimating conservative exposure levels.

During a loading cycle, cesium is continually being adsorbed onto the IONSIV ${ }^{\circledR}$ IE-911 CST material. At any point in time the total column inventory of cesium is made up of: absorbed 
cesium on the solid resin typically referred to as the cesium loading, cesium contained within the interstitial voids of the bed, and cesium contained within the pores of the resin particles. The sum total of cesium contained within a column is referred to as its column inventory. Due to the high affinity IONSIV ${ }^{\circledR}$ IE-911 CST material has for cesium, after several column volumes of feed has past through the column, the majority of cesium column inventory will reside on the resin.

In order to compute the cumulative cesium inventories along the column, breakthrough curves at various axial locations are used. The molar balance of total cesium for a specified section of the lead column (i.e., from its inlet to a given axial location z) can be expressed as:

$$
\frac{\mathrm{dn}_{\mathrm{oz}}}{\mathrm{dt}}=\dot{\mathrm{n}}_{\mathrm{o}}-\dot{\mathrm{n}}_{\mathrm{z}}(\mathrm{t}) \text {. }
$$

Integration of Eq. (10-8) from an initial condition (i.e., assuming a fresh lead column and a fixed rate of feed input and concentration) up to some specified point in time, $t$, yields:

$$
\mathrm{n}_{\mathrm{oz}}(\mathrm{t})=\dot{\mathrm{n}}_{\mathrm{o}} \mathrm{t}-\int_{0}^{\mathrm{t}} \dot{\mathrm{n}}_{\mathrm{z}}\left(\mathrm{t}^{\prime}\right) \mathrm{dt}^{\prime} .
$$

Given the definition of a breakthrough curve, Eq. (10-9) can be rearranged to:

$$
\mathrm{n}_{\mathrm{oz}}(\mathrm{t})=\mathrm{Qc_{ \textrm {o } }} \mathrm{t}-\mathrm{Q} \int_{0}^{\mathrm{t}} \mathrm{c}_{\mathrm{b}}\left(\mathrm{z}, \mathrm{t}^{\prime}\right) \mathrm{dt^{ \prime }}
$$

Equation (10-10) can be further simplified by making use of the normalized time quantity expressed in terms of column volume (sometimes referred to as bed volume):

$$
\mathrm{n}_{\mathrm{oz}}(\mathrm{t}) \equiv \mathrm{n}_{\mathrm{oz}}(\tau)=\mathrm{c}_{\mathrm{o}} \mathrm{V}_{\mathrm{CV}} \int_{0}^{\tau}\left[1-\frac{\mathrm{c}_{\mathrm{b}}\left(\mathrm{z}, \tau^{\prime}\right)}{\mathrm{c}_{\mathrm{o}}}\right] \mathrm{d} \tau^{\prime}
$$

where

$$
\tau=\frac{\mathrm{tQ}}{\mathrm{V}_{\mathrm{CV}}} .
$$

The integral in Eq. (10-11) represents the area above the normalized breakthrough curve up to the specified point in time. For the case where step changes in flowrate and feed concentration occur (i.e., LAW batch feed changes), Eq. (10-11) applies for each process segment where the cumulative inventory becomes the sum of the computed inventories for all process segments within the given cycle of interest.

Typically, in order to compute the cumulative cesium inventory contained within the lead column, the exit breakthrough curve is used. Envelope B LAW feeds provide the bounding case since their cesium feed concentrations are almost one order larger in magnitude than the other envelopes and due to their lower volumetric flowrate have the longest process time (i.e., 
exposure time). Their lower flowrate also results in much sharper concentration fronts along the column where a plug-flow assumption may be adequate for estimating inventories. As shown in Figure 10-11, which presents the results for the two-column carousel configuration at a $2 \mathrm{~m}^{3}$ column volume design, the $4^{\text {th }}$ and $5^{\text {th }}$ loading cycles experience the largest cesium concentration levels within the lead columns. From an exposure perspective, the $4^{\text {th }}$ loading cycle is larger since its lead column remains at a higher cesium level for a longer duration. It is this $4^{\text {th }}$ loading cycle, where the Envelope B LAW-2a feed is being processed, which represents our bounding case for a $2 \mathrm{~m}^{3}$ column volume design. As shown in Figures 10-9 and 10-13 for $1 \mathrm{~m}^{3}$ and $3 \mathrm{~m}^{3}$ column volume design cases, cycle 9 and cycle 2 are bounding, respectively.

Based on the integration of Eq. (10-11) for a two-column carousel configuration at the column sizes of 1,2 , and $3 \mathrm{~m}^{3}$, the lead column cesium inventories over the process time of their most bounding cycles are shown in Figure 10-20. The cesium inventories plotted in Figure 10-20 are expressed in terms of the total amount of cesium in gmoles contained within the lead column per liter of column (i.e. bed) volume. This cesium inventory concentration increases fairly linearly up to the point where the lead column becomes saturated at its feed value over the entire length of the column (i.e., no additional accumulation within the column can occur). Since the volumetric flowrate for each case shown is identical (i.e., Envelope B's flowrate is 9,400 $\mathrm{ml} / \mathrm{min}$ ), the column saturation point occurs earlier the smaller the column size.

At the end of the process cycle, all three cases shown are processing Envelope B's LAW-2a feed and have the same saturation point. A cesium concentration level of $\sim 0.25$ gmoles $/ \mathrm{L}_{\text {column }}$ is the saturation conditions corresponding to the LAW-2a feed. The initial portion of the larger column's inventory curve is at a different slope than the remainder. This is a result of the fact that the process cycle is initially being fed by Envelope A's LAW-1 feed and then converts over to Envelope B's LAW-2a. 
Table 10-1. Comparison of key parameters ${ }^{\text {a }}$ between IONSIV ${ }^{\circledR}$ IE-911 CST material and SuperLig ${ }^{\circledR} 644$ resin for removal of cesium from LAW by ion exchange.

\begin{tabular}{|c|c|c|}
\hline Parameter & $\begin{array}{l}\text { IONSIV }^{\circledR} \\
\text { IE-911 CST }^{\text {Con }}\end{array}$ & $\begin{array}{c}\text { SuperLig }^{\circledR} \\
644\end{array}$ \\
\hline Bed porosity, (-) & 0.50 & 0.45 \\
\hline Particle porosity, (-) & 0.24 & 0.61 \\
\hline Avg. particle diameter, $(\mu \mathrm{m})$ & 345 & 660 \\
\hline Bed density, $\left(\mathrm{g}_{\text {solid }} / \mathrm{ml}_{\text {bed }}\right)$ & 1.0 & 0.2 \\
\hline $\begin{array}{c}\text { Total Cs capacity, } \\
\text { mass basis }\left(\text { mmole }_{\mathrm{Cs}} \mathbf{g}_{\text {solid }}\right) \\
\text { volume basis }\left(\text { mmole }_{\mathrm{Cs}} / \mathbf{m l}_{\text {bed }}\right)\end{array}$ & $\begin{array}{l}0.3944 \\
0.3944\end{array}$ & $\begin{array}{l}0.3333 \\
0.0667\end{array}$ \\
\hline Pore diffusion coef., $\left(\mathrm{cm}^{2} / \mathrm{min}\right)$ & $0.925 \times 10^{-5}$ & $3.395 \times 10^{-5}$ \\
\hline $\mathrm{Cs}^{+}$to $\mathrm{K}^{+}$selectivity, (-) & $1,400: 1$ & $1,200: 1$ \\
\hline $\mathrm{Cs}^{+}$to $\mathrm{Na}^{+}$selectivity, (-) & $26,000: 1$ & $23,000: 1$ \\
\hline
\end{tabular}

${ }^{\mathrm{a}}$ These are approximate values based on available data and analyses.

Table 10-2. Ion-exchange facility cesium exit concentration criterion on an envelope basis.

\begin{tabular}{|c|c|c|c|c|}
\hline Envelope & $\begin{array}{c}{ }^{137} \mathrm{Cs} \text { exit } \\
\text { criterion }^{\mathrm{a}} \\
(\mathrm{Ci} / \mathrm{gmole} \text { of } \mathrm{Na})\end{array}$ & $\begin{array}{c}{ }^{137} \mathrm{Cs} \text { exit } \\
\text { criterion on a } 5 \\
\mathrm{M} \mathrm{Na}^{+} \text {basis } \\
(\mu \mathrm{Ci} / \mathrm{ml})\end{array}$ & $\begin{array}{c}\text { Isotopic } \\
\text { fraction } \\
\left({ }^{137} \mathrm{Cs} /{ }^{\text {total }} \mathrm{Cs}\right)\end{array}$ & Basis $^{b}$ \\
\hline $\mathbf{A}$ & $1.75 \times 10^{-5}$ & 0.088 & $25 \%$ & $\begin{array}{c}\sim 19.6 \mathrm{wt} \% \text { sodium oxide }\left(\mathrm{Na}_{2} \mathrm{O}\right) \text { at the } \\
60 \mathrm{MT} / \text { day operation }\end{array}$ \\
\hline B & $5.00 \times 10^{-5}$ & 0.250 & $30 \%$ & $\begin{array}{c}\sim 7.0 \mathrm{wt} \% \text { sodium oxide }\left(\mathrm{Na}_{2} \mathrm{O}\right) \text { at the } \\
30 \mathrm{MT} / \text { day operation }\end{array}$ \\
\hline $\mathrm{C}$ & $2.90 \times 10^{-5}$ & 0.150 & $25 \%$ & $\begin{array}{c}\sim 12.0 \mathrm{wt} \% \text { sodium oxide }\left(\mathrm{Na}_{2} \mathrm{O}\right) \text { at the } \\
30 \mathrm{MT} / \text { day operation }\end{array}$ \\
\hline
\end{tabular}

${ }^{\text {a }}$ The actual cesium exit concentration criterion is a bucket average value exiting the lag columns and for VERSE-

LC simulations this criterion is converted into total cesium concentrations in molar units. The values listed limit the $\mathrm{Ci} / \mathrm{m}^{3}$ content in the glass matrix due to ${ }^{137} \mathrm{Cs}$.

${ }^{\mathrm{b}}$ A different operating basis is used for Envelope A (60 MT/d) than for Envelopes B and C (30 MT/d) since Envelopes $\mathrm{B}$ and $\mathrm{C}$ will be completely processed during the Phase 1 campaign. To meet DOE's requirement of an expanded capability beyond Phase 1, the remaining LAWs, all Envelope A wastes, are potentially handled at twice the Phase 1 flowrate. 
Table 10-3. Processing information on the Phase 1 Low activity waste (LAW) feeds listed in their scheduled order to be processed.

\begin{tabular}{|c|c|c|c|c|c|c|c|}
\hline Envelope & $\begin{array}{c}\text { Source } \\
\text { Tank }\end{array}$ & $\begin{array}{c}\text { LAW } \\
\text { batch feed } \\
\text { (id) }\end{array}$ & $\begin{array}{c}\text { Flowrate }^{b} \\
\text { (L/min) }\end{array}$ & $\begin{array}{c}\text { Cesium } \\
\text { feed } \\
\text { conc. } \\
{[\mathrm{M}]}\end{array}$ & $\begin{array}{c}\text { Batch } \\
\text { volume to } \\
\text { be } \\
\text { processed } \\
\left.{ }^{\text {a }}{ }^{3}\right)\end{array}$ & $\begin{array}{c}\text { Batch } \\
\text { process } \\
\text { time } \\
\text { at 30 } \\
\text { MT/d } \\
\text { (day) }\end{array}$ & $\begin{array}{c}\text { Batch } \\
\text { process } \\
\text { time } \\
\text { at } 60 \\
\text { MT/day } \\
\text { (day) }\end{array}$ \\
\hline$\overline{\mathbf{A}}$ & AP-101 & LAW-1 & 52.62 & $3.598 \mathrm{E}-05$ & 4,626 & 122 & 61 \\
\hline B & AZ-101 & LAW-2a & 9.4 & 4.676E-04 & 2,906 & 215 & - \\
\hline B & AZ-102 & LAW-2b & 9.4 & $4.311 \mathrm{E}-04$ & 1,755 & 130 & - \\
\hline $\mathbf{C}$ & AN-102 & LAW-3 & 16.2 & $3.967 \mathrm{E}-05$ & 4,200 & 180 & - \\
\hline $\mathrm{C}$ & AN-102 & LAW-4 & 16.2 & 3.779E-05 & 4,200 & 180 & - \\
\hline $\mathbf{A}$ & AN-104 & LAW-5 & 52.62 & $6.283 \mathrm{E}-05$ & 3,820 & 101 & 50 \\
\hline $\mathbf{A}$ & AN-104 & LAW-6 & 52.62 & $6.328 \mathrm{E}-05$ & 3,540 & 93 & 47 \\
\hline $\mathbf{C}$ & AN-107 & LAW-7 & 16.2 & $4.455 \mathrm{E}-05$ & 5,498 & 236 & - \\
\hline A & AN-105 & LAW-8 & 52.62 & 4.324E-05 & 3,700 & 98 & 49 \\
\hline $\mathbf{A}$ & AN-105 & LAW-9 & 52.62 & 4.444E-05 & 3,600 & 95 & 48 \\
\hline $\mathbf{A}$ & SY-101 & LAW-10 & 52.62 & $3.692 \mathrm{E}-05$ & 2,600 & 69 & 34 \\
\hline $\mathbf{A}$ & SY-101 & LAW-11 & 52.62 & $3.739 \mathrm{E}-05$ & 4,600 & 121 & 61 \\
\hline $\mathbf{A}$ & AN-103 & LAW-12 & 52.62 & $4.831 \mathrm{E}-05$ & 4,720 & 125 & 62 \\
\hline A & AN-103 & LAW-13 & 52.62 & $4.831 \mathrm{E}-05$ & 4,720 & 125 & 62 \\
\hline $\mathbf{A}$ & AW-101 & LAW-14 & 52.62 & $4.569 \mathrm{E}-05$ & 3,940 & 104 & 52 \\
\hline $\mathbf{A}$ & AW-101 & LAW-15 & 52.62 & $4.552 \mathrm{E}-05$ & 5,360 & 141 & 71 \\
\hline
\end{tabular}

${ }^{\mathrm{a}}$ The volume of each batch feed represents the volume of solution entering the ion-exchange facility at a $5 \mathrm{M}$ sodium basis and includes the volume changes that occur upstream to this facility (i.e., pretreatment activities).

b The volumetric flowrates and batch process times are based on the $30 \mathrm{MT} /$ day operation schedule for Envelopes $\mathrm{B}$ and $\mathrm{C}$ and on the expanded capability of $60 \mathrm{MT} /$ day operation for Envelope A.

c The total amount of processing time is $\sim 4.2$ years. If the Envelope A feeds are processed at $30 \mathrm{MT} /$ day, then the total amount of processing time is $\sim 5.8$ years. 
Table 10-4. Ion-exchange facility total cesium exit concentration criterion on a batch feed basis.

\begin{tabular}{|c|c|c|c|c|c|c|}
\hline Envelope & $\begin{array}{c}\text { Isotopic } \\
\text { fraction } \\
\left({ }^{137} \mathrm{Cs} /{ }^{\text {total }} \mathrm{Cs}\right)\end{array}$ & $\begin{array}{l}{ }^{137} \text { Cs exit } \\
\text { criterion }{ }^{a} \\
\text { (Ci/gmole of } \\
\text { Na) }\end{array}$ & $\begin{array}{l}\text { LAW } \\
\text { batch } \\
\text { feed } \\
\text { (id) }\end{array}$ & $\begin{array}{c}\text { Cesium feed } \\
\text { concentration } \\
\left(\mathbf{c}_{\mathbf{o}}\right) \\
{[\mathrm{M}]}\end{array}$ & $\begin{array}{c}\text { Total Cs } \\
\text { exit } \\
\text { criterion }^{b} \\
\text { [M] }\end{array}$ & $\begin{array}{c}\text { Total Cs } \\
\text { exit } \\
\text { criterion } \\
\left(\mathbf{c} / \mathbf{c}_{0}\right)\end{array}$ \\
\hline \multirow[t]{11}{*}{$\mathbf{A}$} & \multirow[t]{11}{*}{$25 \%$} & \multirow[t]{11}{*}{$1.75 \times 10^{-5}$} & "LAW-1 & $3.598 \mathrm{E}-05$ & $2.953 \mathrm{E}-08$ & $8.208 \mathrm{E}-04$ \\
\hline & & & LAW-5 & $6.283 \mathrm{E}-05$ & $2.953 \mathrm{E}-08$ & 4.701E-04 \\
\hline & & & LAW-6 & $6.328 \mathrm{E}-05$ & $2.953 \mathrm{E}-08$ & 4.667E-04 \\
\hline & & & LAW-8 & 4.324E-05 & $2.953 \mathrm{E}-08$ & $6.830 \mathrm{E}-04$ \\
\hline & & & LAW-9 & 4.444E-05 & $2.953 \mathrm{E}-08$ & $6.645 \mathrm{E}-04$ \\
\hline & & & LAW-10 & $3.692 \mathrm{E}-05$ & $2.953 \mathrm{E}-08$ & $7.999 \mathrm{E}-04$ \\
\hline & & & LAW-11 & $3.739 \mathrm{E}-05$ & $2.953 \mathrm{E}-08$ & $7.899 \mathrm{E}-04$ \\
\hline & & & LAW-12 & $4.831 \mathrm{E}-05$ & $2.953 \mathrm{E}-08$ & $6.114 \mathrm{E}-04$ \\
\hline & & & LAW-13 & $4.831 \mathrm{E}-05$ & $2.953 \mathrm{E}-08$ & $6.114 \mathrm{E}-04$ \\
\hline & & & LAW-14 & $4.569 \mathrm{E}-05$ & $2.953 \mathrm{E}-08$ & $6.465 \mathrm{E}-04$ \\
\hline & & & LAW-15 & $4.552 \mathrm{E}-05$ & $2.953 \mathrm{E}-08$ & $6.488 \mathrm{E}-04$ \\
\hline \multirow[t]{2}{*}{ B } & \multirow[t]{2}{*}{$30 \%$} & \multirow[t]{2}{*}{$5.00 \times 10^{-5}$} & LAW-2a & $4.676 \mathrm{E}-04$ & $7.032 \mathrm{E}-08$ & $1.504 \mathrm{E}-04$ \\
\hline & & & LAW-2b & $4.311 \mathrm{E}-04$ & 7.032E-08 & $1.631 \mathrm{E}-04$ \\
\hline \multirow[t]{3}{*}{$\mathrm{C}$} & \multirow[t]{3}{*}{$25 \%$} & \multirow[t]{3}{*}{$2.90 \times 10^{-5}$} & LAW-3 & $3.967 \mathrm{E}-05$ & 4.894E-08 & $1.234 \mathrm{E}-03$ \\
\hline & & & LAW-4 & $3.779 \mathrm{E}-05$ & 4.894E-08 & $1.295 \mathrm{E}-03$ \\
\hline & & & LAW-7 & $4.455 \mathrm{E}-05$ & 4.894E-08 & $1.099 \mathrm{E}-03$ \\
\hline
\end{tabular}

${ }^{a}$ During the loading cycle the solution entering the ion-exchange facility is at a $5 \mathrm{M}$ sodium concentration.

${ }^{\mathrm{b}}$ The ${ }^{\text {total }} \mathrm{Cs}$ criterion is computed based on the ${ }^{137} \mathrm{Cs}$ exit criterion, its isotopic fraction (assumes the total is made up only of the isotopes ${ }^{133} \mathrm{Cs}$ and ${ }^{137} \mathrm{Cs}$ ), and the conversion factor of $1 \mu \mathrm{Ci} / \mathrm{ml}$ of ${ }^{137} \mathrm{Cs}$ equals $8.43820142 \times 10^{-8} \mathrm{M}$ of ${ }^{137} \mathrm{Cs}$. A radioactive half-life of 30.17 years for ${ }^{137} \mathrm{Cs}$ is used taken from GE Nuclear Energy (1996). 
Table 10-5. Estimate of the theoretical minimum amount of spent CST material required to process each Phase 1 LAW batch feed.

\begin{tabular}{|c|c|c|c|c|c|c|}
\hline Envelope & $\begin{array}{c}\text { LAW } \\
\text { batch feed } \\
\text { (id) }\end{array}$ & $\begin{array}{c}\text { Batch } \\
\text { volume to } \\
\text { be } \\
\text { processed }^{\text {a }} \\
\quad\left(\mathbf{m}^{3}\right)\end{array}$ & $\begin{array}{c}\text { Cesium } \\
\text { feed } \\
\text { concentration } \\
\text { [M] }\end{array}$ & $\begin{array}{c}\text { Max cesium } \\
\text { loading }^{b} \\
\left(\text { mmole }_{\mathrm{Cs}} / \mathrm{g}_{\mathrm{CST}}\right)\end{array}$ & $\begin{array}{l}\text { Total Cs } \\
\text { to be } \\
\text { processed } \\
\text { (gmole) }\end{array}$ & $\begin{array}{c}\text { Theoretical } \\
\text { minimum } \\
\text { spent CST } \\
\text { (kg) }\end{array}$ \\
\hline \multirow[t]{11}{*}{$\overline{\mathbf{A}}$} & $\overline{\text { LAW-1 }}$ & $4,4,626$ & 3.598E-05 & 3.375E-02 & 106.4 & 4,931 \\
\hline & LAW-5 & 3,820 & $6.283 \mathrm{E}-05$ & $8.339 \mathrm{E}-02$ & 240.0 & 2,878 \\
\hline & LAW-6 & 3,540 & $6.328 \mathrm{E}-05$ & $8.320 \mathrm{E}-02$ & 224.0 & 2,692 \\
\hline & LAW-8 & 3,700 & $4.324 \mathrm{E}-05$ & $5.787 \mathrm{E}-02$ & 160.0 & 2,765 \\
\hline & LAW-9 & 3,600 & $4.444 \mathrm{E}-05$ & $6.517 \mathrm{E}-02$ & 160.0 & 2,455 \\
\hline & LAW-10 & 2,600 & $3.692 \mathrm{E}-05$ & $5.638 \mathrm{E}-02$ & 96.0 & 1,703 \\
\hline & LAW-11 & 4,600 & $3.739 \mathrm{E}-05$ & $6.073 \mathrm{E}-02$ & 172.0 & 2,832 \\
\hline & LAW-12 & 4,720 & $4.831 \mathrm{E}-05$ & $5.599 \mathrm{E}-02$ & 228.0 & 4,072 \\
\hline & LAW-13 & 4,720 & $4.831 \mathrm{E}-05$ & $5.577 \mathrm{E}-02$ & 228.0 & 4,088 \\
\hline & LAW-14 & 3,940 & $4.569 \mathrm{E}-05$ & $4.411 \mathrm{E}-02$ & 180.0 & 4,081 \\
\hline & LAW-15 & 5,360 & $4.552 \mathrm{E}-05$ & $4.169 \mathrm{E}-02$ & 244.0 & 5,853 \\
\hline \multirow[t]{2}{*}{ B } & LAW-2a & 2,906 & $4.676 \mathrm{E}-04$ & $2.527 \mathrm{E}-01$ & $1,358.5$ & 5,377 \\
\hline & LAW-2b & 1,755 & $4.311 \mathrm{E}-04$ & $2.640 \mathrm{E}-01$ & 756.6 & 2,866 \\
\hline \multirow[t]{3}{*}{$\mathbf{C}$} & LAW-3 & 4,200 & $3.967 \mathrm{E}-05$ & $6.080 \mathrm{E}-02$ & 166.6 & 2,741 \\
\hline & LAW-4 & 4,200 & $3.779 \mathrm{E}-05$ & $5.833 \mathrm{E}-02$ & 158.7 & 2,721 \\
\hline & LAW-7 & 5,498 & $4.455 \mathrm{E}-05$ & $7.409 \mathrm{E}-02$ & 244.9 & 3,306 \\
\hline Total & All LAW's & 63,784 & - & - & - & 55,360 \\
\hline
\end{tabular}

${ }^{\text {a }}$ The volume of each batch feed represents the volume of solution entering the ion-exchange facility at a $5 \mathrm{M}$ sodium basis and includes the volume changes that occur upstream to this facility (i.e., pretreatment activities).

${ }^{b}$ The maximum cesium loading evaluation is based on the feed concentration using the appropriate cesium isotherm (i.e., algebraic model and appropriate beta value). 
Table 10-6. Impact of L/D geometry on estimated amount of total spent CST material generated to process the Phase 1 LAW inventory and the number of cycles required per batch of feed (i.e., based on a $2000 \mathrm{~L}$ 2-column carousel facility operating at $25 \mathrm{C}$ under nominal parameter settings ${ }^{\text {a }}$, except for those parameters explicitly listed in this table).

\begin{tabular}{|c|c|c|c|c|c|}
\hline Column L/D & 1.0 & 2.0 & 3.0 & 4.0 & 5.0 \\
\hline Column length $(\mathrm{cm})$ & 136.6 & 216.8 & 284.0 & 344.1 & 399.3 \\
\hline Column diameter $(\mathrm{cm})$ & 136.6 & 108.4 & 94.7 & 86.0 & 79.9 \\
\hline $\begin{array}{l}\text { Bed volume of one } \\
\text { column (L) }\end{array}$ & $2,000.0$ & $2,000.0$ & $2,000.0$ & $2,000.0$ & $2,000.0$ \\
\hline $\begin{array}{l}\text { Total bed volume of } \\
\text { columns (L) }\end{array}$ & 4,000 & 4,000 & 4,000 & 4,000 & 4,000 \\
\hline LAW-1 & 2 & 2 & 2 & 2 & 2 \\
\hline LAW-2a & 2 & 2 & 2 & 2 & 2 \\
\hline LAW-2b & 1 & 1 & 1 & 1 & 1 \\
\hline LAW-3 & 3 & 3 & 3 & 3 & 3 \\
\hline LAW-4 & 2 & 2 & 2 & 2 & 2 \\
\hline LAW-5 & 2 & 2 & 2 & 2 & 2 \\
\hline LAW-6 & 2 & 2 & 2 & 2 & 2 \\
\hline LAW-7 & 1 & 1 & 1 & 1 & 1 \\
\hline LAW-8 & 1 & 1 & 1 & 1 & 1 \\
\hline LAW-9 & 2 & 2 & 2 & 2 & 2 \\
\hline LAW-10 & 1 & 1 & 1 & 1 & 1 \\
\hline LAW-11 & 1 & 1 & 1 & 1 & 1 \\
\hline LAW-12 & 3 & 3 & 3 & 3 & 3 \\
\hline LAW-13 & 2 & 2 & 2 & 2 & 2 \\
\hline LAW-14 & 3 & 3 & 3 & 3 & 3 \\
\hline LAW-15 & 3 & 3 & 3 & 3 & 3 \\
\hline $\begin{array}{c}\text { Total cycles for all } 16 \\
\text { feeds }\end{array}$ & 31 & 31 & 31 & 31 & 31 \\
\hline Total spent CST (kg) & 66,000 & 66,000 & 66,000 & 66,000 & 66,000 \\
\hline
\end{tabular}

${ }^{a}$ The dilution factor set to $68 \%$ and the cesium pore diffusion coefficient to $20 \%$ of its "free" diffusion value.

b Column volumes here refer to the actual volume containing the CST beds and does not include head space volume. Headspace volume set to $25 \%$ of bed volume (i.e., $500 \mathrm{~L}$ ). 
Table 10-7. Geometric L/D ratios and resulting geometries considered in CST column design simulations using VERSE-LC based on a 2-column carousel configuration.

\begin{tabular}{|c|c|c|c|c|c|c|}
\hline $\begin{array}{c}\text { Individual column } \\
\text { bed volume } \\
(\mathrm{L})\end{array}$ & $\begin{array}{c}\text { Bed } \\
(\mathrm{L} / \mathrm{D}) \\
\text { ratio }\end{array}$ & $\begin{array}{c}\text { Bed } \\
\text { diameter } \\
(\mathrm{cm})\end{array}$ & $\begin{array}{c}\text { Bed column } \\
\text { length } \\
(\mathrm{cm})\end{array}$ & $\begin{array}{c}\text { Head-space } \\
\text { volume } \\
(\mathrm{L})\end{array}$ & $\begin{array}{c}\text { Total bed } \\
\text { length } \\
(\mathrm{L})\end{array}$ & $\begin{array}{c}\text { Total bed } \\
\text { volume } \\
(\mathrm{L})\end{array}$ \\
\hline \hline 500 & 3.0 & 59.6 & 178.9 & 125 & 357.9 & 1000 \\
\hline 700 & 3.0 & 66.7 & 200.2 & 175 & 400.4 & 1400 \\
\hline $1000^{\mathrm{a}}$ & 3.0 & 75.2 & 225.5 & 250 & 450.9 & 2000 \\
\hline 1500 & 3.0 & 86.0 & 258.1 & 375 & 516.2 & 3000 \\
\hline $2000 \mathrm{~b}$ & 3.0 & 94.7 & 284.0 & 500 & 568.1 & 4000 \\
\hline 3000 & 3.0 & 108.4 & 325.2 & 750 & 650.3 & 6000 \\
\hline 4000 & 3.0 & 119.3 & 357.9 & 1000 & 715.8 & 8000 \\
\hline 5000 & 3.0 & 128.5 & 385.5 & 1250 & 771.0 & 10000 \\
\hline 6000 & 3.0 & 136.6 & 409.7 & 1500 & 819.3 & 12000 \\
\hline 7000 & 3.0 & 143.8 & 431.3 & 1750 & 862.5 & 14000 \\
\hline 8000 & 3.0 & 150.3 & 450.9 & 2000 & 901.8 & 16000 \\
\hline 9000 & 3.0 & 156.3 & 469.0 & 2250 & 937.9 & 18000 \\
\hline 2000 & 1.0 & 136.6 & 136.6 & 500 & 273.1 & 4000 \\
\hline 2000 & 2.0 & 108.4 & 216.8 & 500 & 433.5 & 4000 \\
\hline 2000 & 4.0 & 86.0 & 344.1 & 500 & 589.2 & 4000 \\
\hline 2000 & 5.0 & 79.9 & 399.3 & 500 & 4000 \\
\hline
\end{tabular}

${ }^{a}$ At the time these analyses were being performed, this represented the current ion-exchange facility column design for use with SuperLig ${ }^{\circledR} 644$ resin. More recent design efforts have changed the L/D to $\sim 1.1$ (i.e., $119 \mathrm{~cm}$ in length and $107 \mathrm{~cm}$ in diameter).

${ }^{\mathrm{b}}$ This represents the nominal (and near optimum) ion-exchange facility column design for use with IONSIV ${ }^{\circledR}$ IE911 CST material.

Table 10-8. Geometric L/D ratios and resulting geometries considered in CST column design simulations using VERSE-LC based on a 3-column carousel configuration. ${ }^{\mathrm{a}}$

\begin{tabular}{|c|c|c|c|c|c|c|c|}
\hline $\begin{array}{c}\text { Individual column } \\
\text { (in 2-column } \\
\text { carousel) } \\
\text { bed volume } \\
(\mathrm{L})\end{array}$ & $\begin{array}{c}\text { Bed } \\
\text { diameter } \\
(\mathrm{cm})\end{array}$ & $\begin{array}{c}\text { Individual } \\
\text { column } \\
\text { bed volume } \\
(\mathrm{L})\end{array}$ & $\begin{array}{c}\text { Bed column } \\
\text { length } \\
(\mathrm{cm})\end{array}$ & $\begin{array}{c}\text { Bed } \\
(\mathrm{L} / \mathrm{D}) \\
\text { ratio }\end{array}$ & $\begin{array}{c}\text { Head-space } \\
\text { volume } \\
(\mathrm{L})\end{array}$ & $\begin{array}{c}\text { Total bed } \\
\text { length } \\
(\mathrm{L})\end{array}$ & $\begin{array}{c}\text { Total bed } \\
\text { volume } \\
(\mathrm{L})\end{array}$ \\
\hline \hline 500 & 59.6 & 333.3 & 119.3 & 2.0 & 83 & 357.9 & 1000 \\
\hline 700 & 66.7 & 466.7 & 133.5 & 2.0 & 117 & 400.4 & 1400 \\
\hline 1000 & 75.2 & 666.7 & 150.3 & 2.0 & 167 & 450.9 & 2000 \\
\hline 2000 & 94.7 & 1333.3 & 189.4 & 2.0 & 333 & 568.1 & 4000 \\
\hline 3000 & 108.4 & 2000.0 & 216.8 & 2.0 & 500 & 650.3 & 6000 \\
\hline 4000 & 119.3 & 2666.7 & 238.6 & 2.0 & 667 & 715.8 & 8000 \\
\hline 5000 & 128.5 & 3333.3 & 257.0 & 2.0 & 833 & 771.0 & 10000 \\
\hline
\end{tabular}

a The use of a third (i.e., "guard") column in the carousel configuration was not intended to be a normal operating mode, but was a backup column in the original facility design. 
Table 10-9. Estimated amount of total spent CST material generated to process the Phase 1 LAW inventory and the number of cycles required per batch of feed (i.e., based on a 2-column carousel facility operating at $25 \mathrm{C}$ under nominal parameter settings ${ }^{\mathrm{a}}$ ).

\begin{tabular}{|c|c|c|c|c|c|c|c|c|c|c|c|c|}
\hline Column L/D & 3.0 & 3.0 & 3.0 & 3.0 & 3.0 & 3.0 & 3.0 & 3.0 & 3.0 & 3.0 & 3.0 & 3.0 \\
\hline $\begin{array}{l}\text { Column length } \\
(\mathrm{cm})\end{array}$ & 178.9 & 200.2 & 225.5 & 258.1 & 284.0 & 325.2 & 357.9 & 385.5 & 409.7 & 431.3 & 450.9 & 469.0 \\
\hline $\begin{array}{l}\text { Column diameter } \\
(\mathrm{cm})\end{array}$ & 59.6 & 66.7 & 75.2 & 86.0 & 94.7 & 108.4 & 119.3 & 128.5 & 136.6 & 143.8 & 150.3 & 156.3 \\
\hline $\begin{array}{l}\text { Bed volume of one } \\
\text { column (L) }\end{array}$ & 500 & 700 & 1,000 & 1,500 & 2,000 & 3,000 & 4,000 & 5,000 & 6,000 & 7,000 & 8,000 & 9,000 \\
\hline $\begin{array}{l}\text { Total bed volume } \\
\text { of columns (L) }\end{array}$ & 1,000 & 1,400 & 2,000 & 3,000 & 4,000 & 6,000 & 8,000 & 10,000 & 12,000 & 14,000 & 16,000 & 18,000 \\
\hline "LAW-1 cycles & 24 & 14 & 7 & 4 & 2 & 1 & 0 & 0 & 0 & 0 & 0 & 0 \\
\hline LAW-2a cycles & 10 & 6 & 4 & 2 & 2 & 1 & 1 & 0 & 0 & 0 & 0 & 0 \\
\hline LAW-2b cycles & 6 & 5 & 3 & 2 & 1 & 0 & 0 & 1 & 0 & 0 & 0 & 0 \\
\hline LAW-3 cycles & 8 & 5 & 5 & 4 & 3 & 2 & 2 & 1 & 1 & 1 & 1 & 0 \\
\hline LAW-4 cycles & 6 & 5 & 3 & 2 & 2 & 2 & 1 & 1 & 1 & 1 & 0 & 1 \\
\hline LAW-5 cycles & 15 & 8 & 5 & 3 & 2 & 1 & 1 & 1 & 1 & 0 & 1 & 1 \\
\hline LAW-6 cycles & 13 & 7 & 4 & 2 & 2 & 1 & 1 & 0 & 1 & 1 & 0 & 0 \\
\hline LAW-7 cycles & 8 & 5 & 3 & 2 & 1 & 1 & 1 & 1 & 0 & 0 & 1 & 0 \\
\hline LAW-8 cycles & 13 & 8 & 4 & 2 & 1 & 1 & 1 & 1 & 0 & 1 & 1 & 0 \\
\hline LAW-9 cycles & 13 & 7 & 4 & 3 & 2 & 1 & 1 & 1 & 1 & 0 & 0 & 1 \\
\hline LAW-10 cycles & 8 & 5 & 3 & 1 & 1 & 1 & 0 & 0 & 0 & 0 & 1 & 0 \\
\hline LAW-11 cycles & 14 & 8 & 4 & 3 & 1 & 1 & 1 & 1 & 1 & 1 & 0 & 1 \\
\hline LAW-12 cycles & 20 & 11 & 7 & 3 & 3 & 2 & 1 & 1 & 1 & 1 & 1 & 0 \\
\hline LAW-13 cycles & 20 & 11 & 6 & 4 & 2 & 1 & 1 & 0 & 0 & 0 & 0 & 1 \\
\hline LAW-14 cycles & 20 & 11 & 6 & 3 & 3 & 2 & 1 & 1 & 1 & 1 & 1 & 0 \\
\hline LAW-15 cycles & 28 & 17 & 10 & 6 & 3 & 2 & 2 & 2 & 1 & 1 & 1 & 1 \\
\hline $\begin{array}{c}\text { Total cycles for all } \\
16 \text { feeds }\end{array}$ & 226 & 133 & 78 & 46 & 31 & 20 & 15 & 12 & 9 & 8 & 8 & 6 \\
\hline $\begin{array}{l}\text { Total spent CST } \\
(\mathrm{kg})\end{array}$ & 114,000 & 94,500 & 80,000 & 72,000 & 66,000 & 66,000 & 68,000 & 70,000 & 66,000 & 70,000 & 80,000 & 72,000 \\
\hline
\end{tabular}

${ }^{\text {a }}$ The dilution factor set to $68 \%$ and the cesium pore diffusion coefficient to $20 \%$ of its "free" diffusion value.

${ }^{\mathrm{b}}$ Column volumes here refer to the actual volume containing the CST beds and does not include head space volume. Headspace volume set to $25 \%$ of bed volume. 
Table 10-10. Estimated amount of total spent CST material generated to process the Phase 1 LAW inventory and the number of cycles required per batch of feed (i.e., based on a 3-column carousel facility operating at $25 \mathrm{C}$ under nominal parameter settings ${ }^{\mathrm{a}}$ ).

\begin{tabular}{|c|c|c|c|c|c|c|c|}
\hline Column L/D & 2.0 & 2.0 & 2.0 & 2.0 & 2.0 & 2.0 & 2.0 \\
\hline $\begin{array}{l}\text { Column length } \\
(\mathrm{cm})\end{array}$ & 119.3 & 133.5 & 150.3 & 189.4 & 216.8 & 238.6 & 257.0 \\
\hline $\begin{array}{l}\text { Column diameter } \\
(\mathrm{cm})\end{array}$ & 59.6 & 66.7 & 75.2 & 94.7 & 108.4 & 119.3 & 128.5 \\
\hline $\begin{array}{l}\text { Bed volume of one } \\
\text { column (L) }\end{array}$ & 333.3 & 466.7 & 666.7 & $1,333.3$ & $2,000.0$ & $2,666.7$ & $3,333.3$ \\
\hline $\begin{array}{l}\text { Total bed volume } \\
\text { of columns (L) }\end{array}$ & 1,000 & 1,400 & 2,000 & 4,000 & 6,000 & 8,000 & 10,000 \\
\hline LAW-1 cycles & 31 & 18 & 10 & 3 & 1 & 0 & 0 \\
\hline LAW-2a cycles & 14 & 9 & 6 & 2 & 1 & 1 & 0 \\
\hline LAW-2b cycles & 9 & 7 & 4 & 2 & 1 & 0 & 1 \\
\hline LAW-3 cycles & 12 & 9 & 7 & 4 & 3 & 3 & 1 \\
\hline LAW-4 cycles & 10 & 7 & 5 & 3 & 3 & 2 & 2 \\
\hline LAW-5 cycles & 18 & 10 & 7 & 4 & 2 & 1 & 1 \\
\hline LAW-6 cycles & 16 & 9 & 5 & 2 & 2 & 2 & 1 \\
\hline LAW-7 cycles & 11 & 8 & 5 & 2 & 1 & 1 & 1 \\
\hline LAW-8 cycles & 17 & 10 & 6 & 3 & 1 & 1 & 2 \\
\hline LAW-9 cycles & 16 & 9 & 5 & 2 & 2 & 1 & 1 \\
\hline LAW-10 cycles & 10 & 7 & 4 & 1 & 1 & 1 & 0 \\
\hline LAW-11 cycles & 18 & 10 & 6 & 3 & 1 & 1 & 1 \\
\hline LAW-12 cycles & 25 & 14 & 8 & 3 & 3 & 2 & 2 \\
\hline LAW-13 cycles & 25 & 15 & 9 & 3 & 2 & 2 & 1 \\
\hline LAW-14 cycles & 25 & 15 & 9 & 4 & 2 & 2 & 2 \\
\hline LAW-15 cycles & 37 & 21 & 12 & 5 & 3 & 2 & 2 \\
\hline $\begin{array}{l}\text { Total cycles for all } \\
16 \text { feeds }\end{array}$ & 294 & 178 & 108 & 46 & 29 & 22 & 18 \\
\hline $\begin{array}{c}\text { Total spent CST } \\
(\mathrm{kg})\end{array}$ & 99,000 & 84,467 & 74,000 & 65,333 & 64,000 & 66,667 & 70,000 \\
\hline
\end{tabular}

a The dilution factor set to $68 \%$ and the cesium pore diffusion coefficient to $20 \%$ of its "free" diffusion value.

${ }^{\mathrm{b}}$ Column volumes here refer to the actual volume containing the CST beds and does not include head space volume. Headspace volume set to $25 \%$ of bed volume. 
Table 10-11. Impact of mass transfer limitations on estimated amount of total spent CST material generated to process the Phase $1 \mathrm{LAW}$ inventory and the number of cycles required per batch of feed (i.e., based on a 2-column carousel facility operating at $25 \mathrm{C}$ under nominal parameter settings ${ }^{\text {a }}$, except for those parameters explicitly listed in this table).

\begin{tabular}{|c|c|c|c|c|c|c|}
\hline Column L/D & \multicolumn{2}{|c|}{3.0} & \multicolumn{2}{|c|}{3.0} & \multicolumn{2}{|c|}{3.0} \\
\hline Column length $(\mathrm{cm})$ & \multicolumn{2}{|c|}{225.5} & \multicolumn{2}{|c|}{284.0} & \multicolumn{2}{|c|}{325.2} \\
\hline Column diameter $(\mathrm{cm})$ & \multicolumn{2}{|c|}{75.2} & \multicolumn{2}{|c|}{94.7} & \multicolumn{2}{|c|}{108.4} \\
\hline $\begin{array}{l}\text { Bed volume of one } \\
\text { column (L) }\end{array}$ & \multicolumn{2}{|c|}{$1,000.0$} & \multicolumn{2}{|c|}{$2,000.0$} & \multicolumn{2}{|c|}{$3,000.0$} \\
\hline Total bed volume of & \multicolumn{2}{|c|}{2,000} & \multicolumn{2}{|c|}{4,000} & \multicolumn{2}{|c|}{6,000} \\
\hline $\begin{array}{l}\text { State of mass transfer } \\
\text { limits }^{c}\end{array}$ & $\begin{array}{l}\text { Nominal } \\
\text { conditions }\end{array}$ & No MTL & $\begin{array}{l}\text { Nominal } \\
\text { conditions }\end{array}$ & No MTL & $\begin{array}{l}\text { Nominal } \\
\text { conditions }\end{array}$ & No MTL \\
\hline LAW-1 & 7 & 4 & 2 & 1 & 1 & 0 \\
\hline LAW-2a & 4 & 4 & 2 & 2 & 1 & 1 \\
\hline LAW-2b & 3 & 3 & 1 & 1 & 0 & 1 \\
\hline LAW-3 & 5 & 5 & 3 & 3 & 2 & 2 \\
\hline LAW-4 & 3 & 3 & 2 & 2 & 2 & 1 \\
\hline LAW-5 & 5 & 3 & 2 & 1 & 1 & 1 \\
\hline LAW-6 & 4 & 2 & 2 & 2 & 1 & 1 \\
\hline LAW-7 & 3 & 4 & 1 & 1 & 1 & 1 \\
\hline LAW-8 & 4 & 3 & 1 & 1 & 1 & 1 \\
\hline LAW-9 & 4 & 3 & 2 & 1 & 1 & 1 \\
\hline LAW-10 & 3 & 2 & 1 & 1 & 1 & 1 \\
\hline LAW-11 & 4 & 3 & 1 & 2 & 1 & 1 \\
\hline LAW-12 & 7 & 4 & 3 & 2 & 2 & 1 \\
\hline LAW-13 & 6 & 4 & 2 & 2 & 1 & 2 \\
\hline LAW-14 & 6 & 4 & 3 & 2 & 2 & 1 \\
\hline LAW-15 & 10 & 5 & 3 & 3 & 2 & 2 \\
\hline $\begin{array}{c}\text { Total cycles for all } 16 \\
\text { feeds }\end{array}$ & 78 & 56 & 31 & 27 & 20 & 18 \\
\hline Total spent CST (kg) & 80,000 & 58,000 & 66,000 & 58,000 & 66,000 & 60,000 \\
\hline
\end{tabular}

${ }^{\text {a }}$ The dilution factor set to $68 \%$ and the cesium pore diffusion coefficient to $20 \%$ of its "free" diffusion value.

${ }^{\mathrm{b}}$ Column volumes here refer to the actual volume containing the CST beds and does not include head space volume. Head space volume set to $25 \%$ of bed volume.

${ }^{c}$ The mass transfer parameters (i.e., pore diffusivity and film coefficients) are set either to their nominal settings (MTL on) or to very large values to eliminate mass transfer resistance (no MTL). 
Table 10-12. Estimated amount of total spent CST material generated to process the Phase 1 LAW inventory and the number of cycles required per batch of feed (i.e., based on a 2-column carousel facility operating at $25 \mathrm{C}$ under nominal parameter settings ${ }^{\mathrm{a}}$, except for the cesium pore diffusivity coefficient).

\begin{tabular}{|c|c|c|c|c|c|c|c|c|}
\hline Column L/D & 3.0 & 3.0 & 3.0 & 3.0 & 3.0 & 3.0 & 3.0 & 3.0 \\
\hline $\begin{array}{l}\text { Column length } \\
(\mathrm{cm})\end{array}$ & 178.9 & 200.2 & 225.5 & 258.1 & 284.0 & 325.2 & 357.9 & 385.5 \\
\hline $\begin{array}{l}\text { Column diameter } \\
(\mathrm{cm})\end{array}$ & 59.6 & 66.7 & 75.2 & 86.0 & 94.7 & 108.4 & 119.3 & 128.5 \\
\hline $\begin{array}{l}\text { Bed volume of one } \\
\text { column (L) }{ }^{\mathrm{b}}\end{array}$ & 500 & 700 & 1,000 & 1,500 & 2,000 & 3,000 & 4,000 & 5,000 \\
\hline $\begin{array}{l}\text { Total bed volume } \\
\text { of columns (L) }\end{array}$ & 1,000 & 1,400 & 2,000 & 3,000 & 4,000 & 6,000 & 8,000 & 10,000 \\
\hline LAW-1 cycles & 42 & 22 & 12 & 6 & 3 & 2 & 1 & 0 \\
\hline LAW-2a cycles & 10 & 7 & 4 & 2 & 2 & 0 & 0 & 1 \\
\hline LAW-2b cycles & 6 & 4 & 3 & 2 & 1 & 0 & 1 & 0 \\
\hline LAW-3 cycles & 10 & 7 & 5 & 4 & 3 & 2 & 1 & 1 \\
\hline LAW-4 cycles & 9 & 5 & 3 & 2 & 2 & 2 & 1 & 1 \\
\hline LAW-5 cycles & 24 & 13 & 7 & 4 & 2 & 2 & 2 & 1 \\
\hline LAW-6 cycles & 23 & 12 & 7 & 3 & 2 & 1 & 1 & 1 \\
\hline LAW-7 cycles & 11 & 7 & 4 & 2 & 2 & 1 & 1 & 0 \\
\hline LAW-8 cycles & 23 & 12 & 6 & 4 & 2 & 1 & 0 & 1 \\
\hline LAW-9 cycles & 22 & 12 & 6 & 3 & 2 & 1 & 1 & 1 \\
\hline LAW-10 cycles & 14 & 8 & 4 & 2 & 1 & 1 & 1 & 0 \\
\hline LAW-11 cycles & 24 & 13 & 7 & 3 & 3 & 1 & 1 & 1 \\
\hline LAW-12 cycles & 34 & 18 & 10 & 5 & 3 & 2 & 1 & 1 \\
\hline LAW-13 cycles & 34 & 18 & 10 & 5 & 3 & 2 & 1 & 1 \\
\hline LAW-14 cycles & 34 & 19 & 10 & 5 & 3 & 1 & 1 & 1 \\
\hline LAW-15 cycles & 48 & 26 & 13 & 7 & 5 & 3 & 2 & 1 \\
\hline $\begin{array}{c}\text { Total cycles for all } \\
16 \text { feeds }\end{array}$ & 368 & 203 & 111 & 59 & 39 & 22 & 16 & 12 \\
\hline $\begin{array}{c}\text { Total spent CST } \\
(\mathrm{kg})\end{array}$ & 185,000 & 143,500 & 113,000 & 91,500 & 82,000 & 72,000 & 72,000 & 70,000 \\
\hline
\end{tabular}

${ }^{\text {a }}$ The dilution factor set to $68 \%$ and the cesium pore diffusion coefficient to $10 \%$ of its "free" diffusion value.

${ }^{\mathrm{b}}$ Column volumes here refer to the actual volume containing the CST beds and does not include head space volume. Headspace volume set to $25 \%$ of bed volume. 
Table 10-13. Estimated amount of total spent CST material generated to process the Phase 1 LAW inventory and the number of cycles required per batch of feed (i.e., based on a 2-column carousel facility operating at $25 \mathrm{C}$ under nominal parameter settings ${ }^{\mathrm{a}}$, except for the cesium pore diffusivity coefficient).

\begin{tabular}{|c|c|c|c|c|c|c|c|c|}
\hline Column L/D & 3.0 & 3.0 & 3.0 & 3.0 & 3.0 & 3.0 & 3.0 & 3.0 \\
\hline $\begin{array}{l}\text { Column length } \\
(\mathrm{cm})\end{array}$ & 178.9 & 200.2 & 225.5 & 258.1 & 284.0 & 325.2 & 357.9 & 385.5 \\
\hline $\begin{array}{l}\text { Column diameter } \\
(\mathrm{cm})\end{array}$ & 59.6 & 66.7 & 75.2 & 86.0 & 94.7 & 108.4 & 119.3 & 128.5 \\
\hline $\begin{array}{l}\text { Bed volume of one } \\
\text { column (L) }\end{array}$ & 500 & 700 & 1,000 & 1,500 & 2,000 & 3,000 & 4,000 & 5,000 \\
\hline $\begin{array}{l}\text { Total bed volume } \\
\text { of columns (L) }\end{array}$ & 1,000 & 1,400 & 2,000 & 3,000 & 4,000 & 6,000 & 8,000 & 10,000 \\
\hline LAW-1 cycles & 19 & 11 & 6 & 3 & 2 & 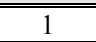 & 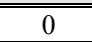 & 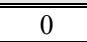 \\
\hline LAW-2a cycles & 10 & 6 & 4 & 2 & 1 & 1 & 1 & 0 \\
\hline LAW-2b cycles & 5 & 4 & 3 & 2 & 2 & 0 & 0 & 1 \\
\hline LAW-3 cycles & 8 & 6 & 5 & 4 & 3 & 2 & 2 & 1 \\
\hline LAW-4 cycles & 6 & 5 & 3 & 2 & 1 & 2 & 1 & 1 \\
\hline LAW-5 cycles & 11 & 6 & 4 & 3 & 3 & 1 & 1 & 0 \\
\hline LAW-6 cycles & 10 & 6 & 3 & 2 & 1 & 1 & 1 & 1 \\
\hline LAW-7 cycles & 7 & 5 & 4 & 2 & 1 & 1 & 1 & 1 \\
\hline LAW-8 cycles & 10 & 6 & 4 & 2 & 2 & 1 & 1 & 1 \\
\hline LAW-9 cycles & 10 & 5 & 3 & 2 & 1 & 1 & 0 & 0 \\
\hline LAW-10 cycles & 6 & 4 & 2 & 1 & 1 & 1 & 1 & 1 \\
\hline LAW-11 cycles & 11 & 7 & 4 & 2 & 1 & 1 & 1 & 1 \\
\hline LAW-12 cycles & 15 & 9 & 5 & 3 & 3 & 1 & 1 & 0 \\
\hline LAW-13 cycles & 15 & 9 & 6 & 4 & 2 & 2 & 1 & 1 \\
\hline LAW-14 cycles & 16 & 9 & 5 & 3 & 2 & 1 & 1 & 1 \\
\hline LAW-15 cycles & 22 & 13 & 8 & 4 & 3 & 2 & 2 & 2 \\
\hline $\begin{array}{c}\text { Total cycles for all } \\
16 \text { feeds }\end{array}$ & 181 & 111 & 69 & 41 & 29 & 19 & 15 & 12 \\
\hline $\begin{array}{c}\text { Total spent CST } \\
(\mathrm{kg})\end{array}$ & 91,500 & 79,100 & 71,000 & 64,500 & 62,000 & 63,000 & 68,000 & 70,000 \\
\hline
\end{tabular}

a The dilution factor set to $68 \%$ and the cesium pore diffusion coefficient to $30 \%$ of its "free" diffusion value.

${ }^{\mathrm{b}}$ Column volumes here refer to the actual volume containing the CST beds and does not include head space volume. Headspace volume set to $25 \%$ of bed volume. 


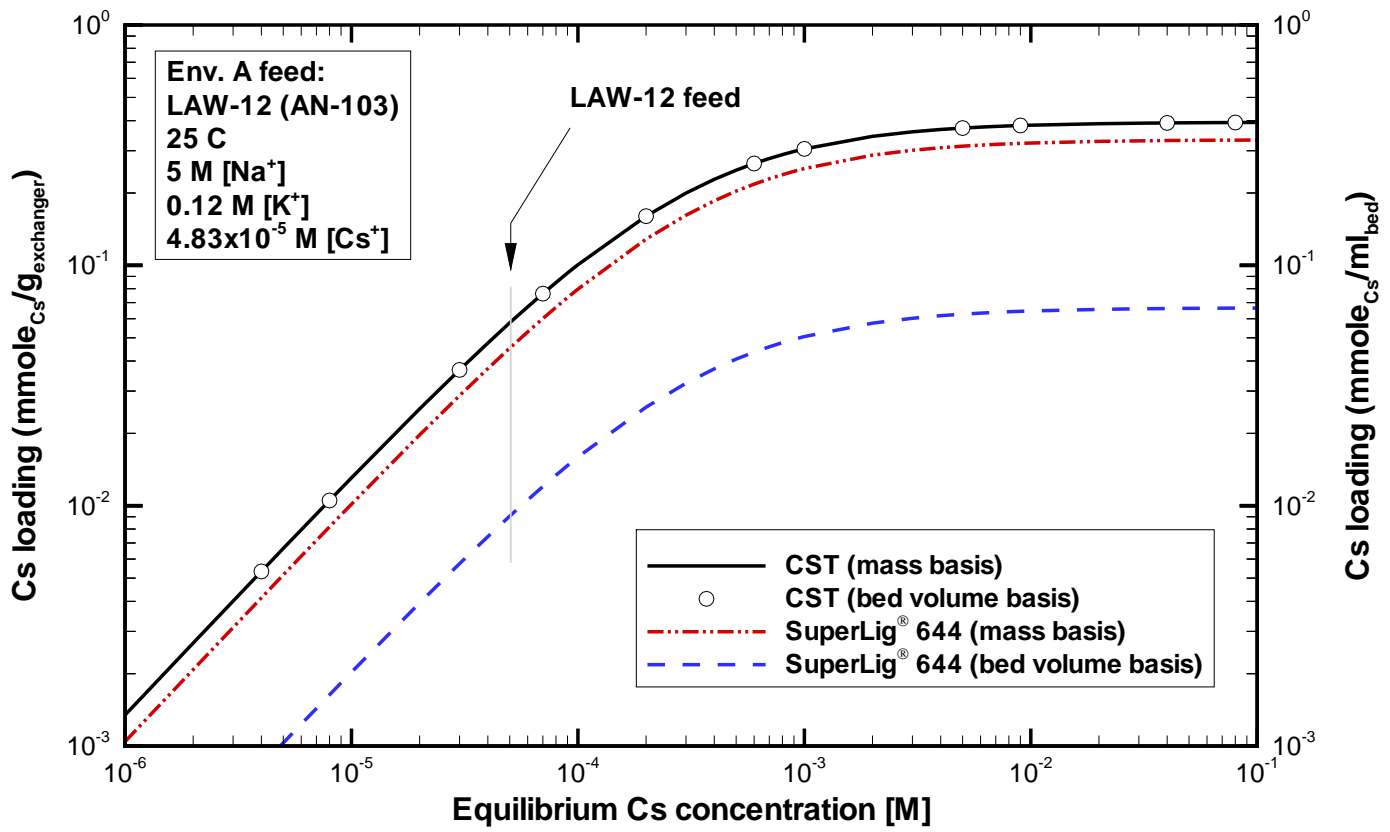

Figure 10-1. Comparison of cesium loading curves for IONSIV ${ }^{\circledR}$ IE-911 CST material and SuperLig ${ }^{\circledR}$ 644 resin in contact with LAW-12 feed solution (241-AN-103) both on a mass and bed volume basis.

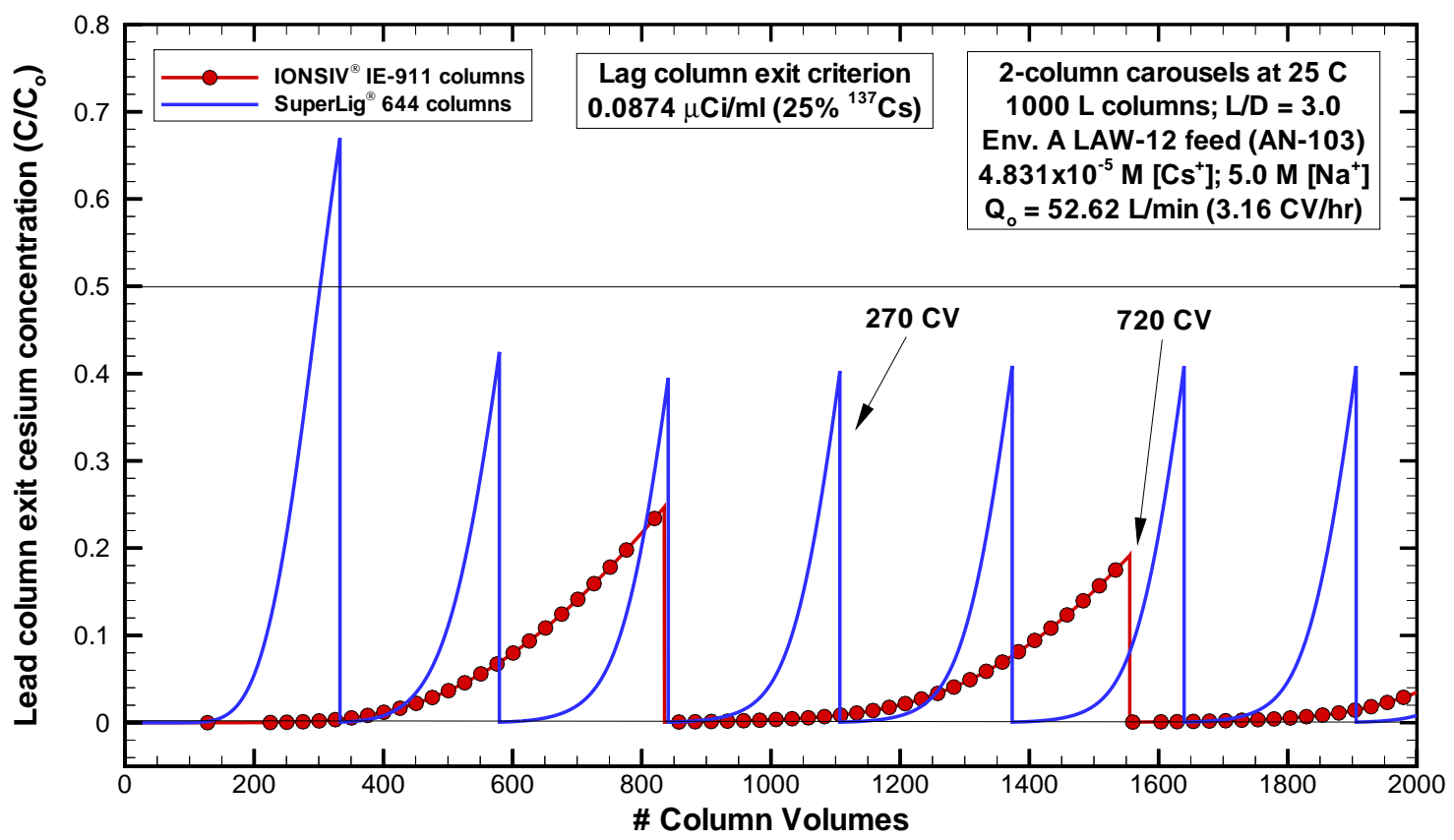

Figure 10-2. Comparison of normalized cesium breakthrough curves for IONSIV ${ }^{\circledR}$ IE-911 CST packed and SuperLig ${ }^{\circledR} 644$ packed columns at $25 \mathrm{C}$ using LAW-12 feed solution (i.e., 241-AN103, identical 2-column carousel configuration, $1000 \mathrm{~L}$ columns, $\mathrm{L} / \mathrm{D}=3$ ). 


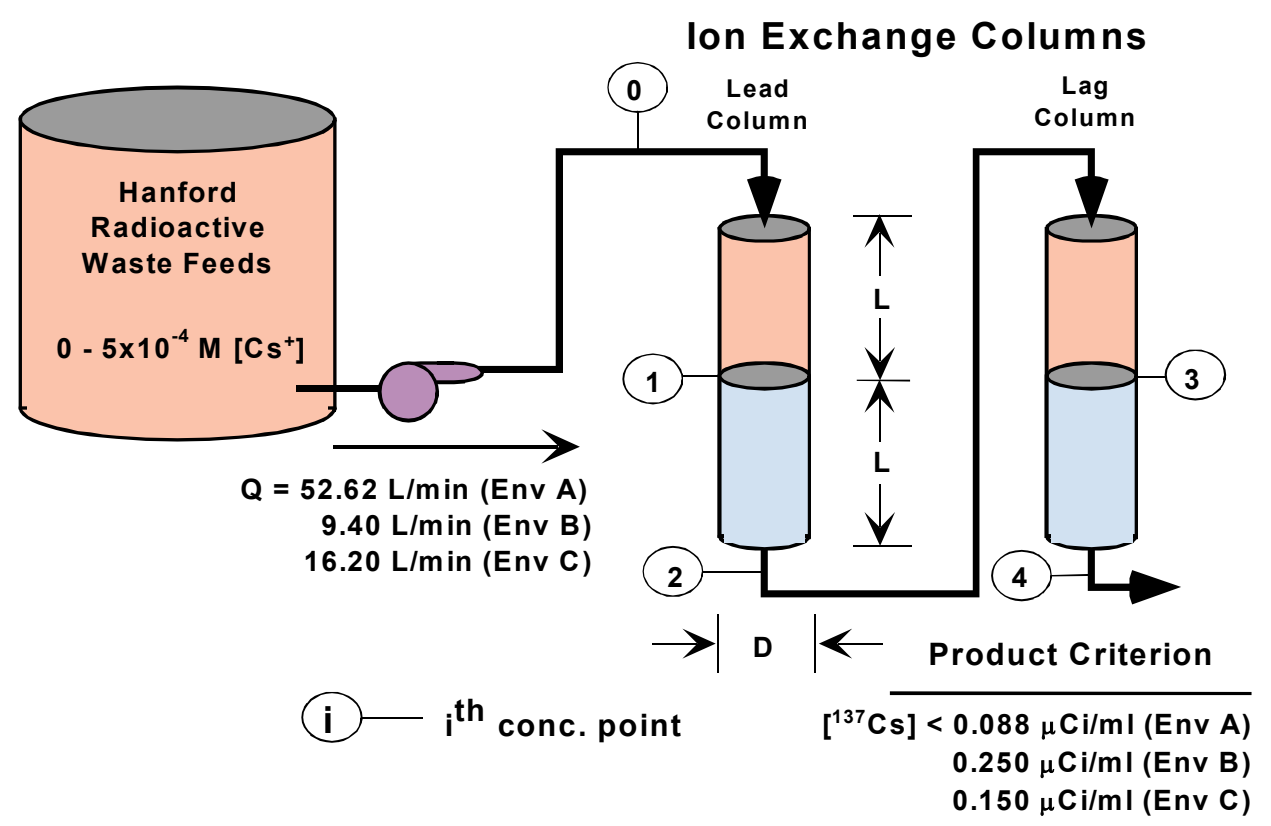

Figure 10-3. Basic flowsheet for a full-scale (two-column carousel configuration) ion-exchange facility for removal of cesium using IONSIV ${ }^{\circledR}$ IE-911 CST material. 


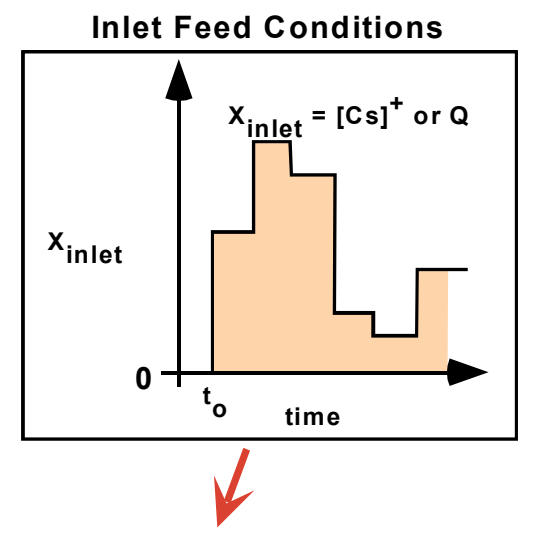

Exit Breakthrough Curves
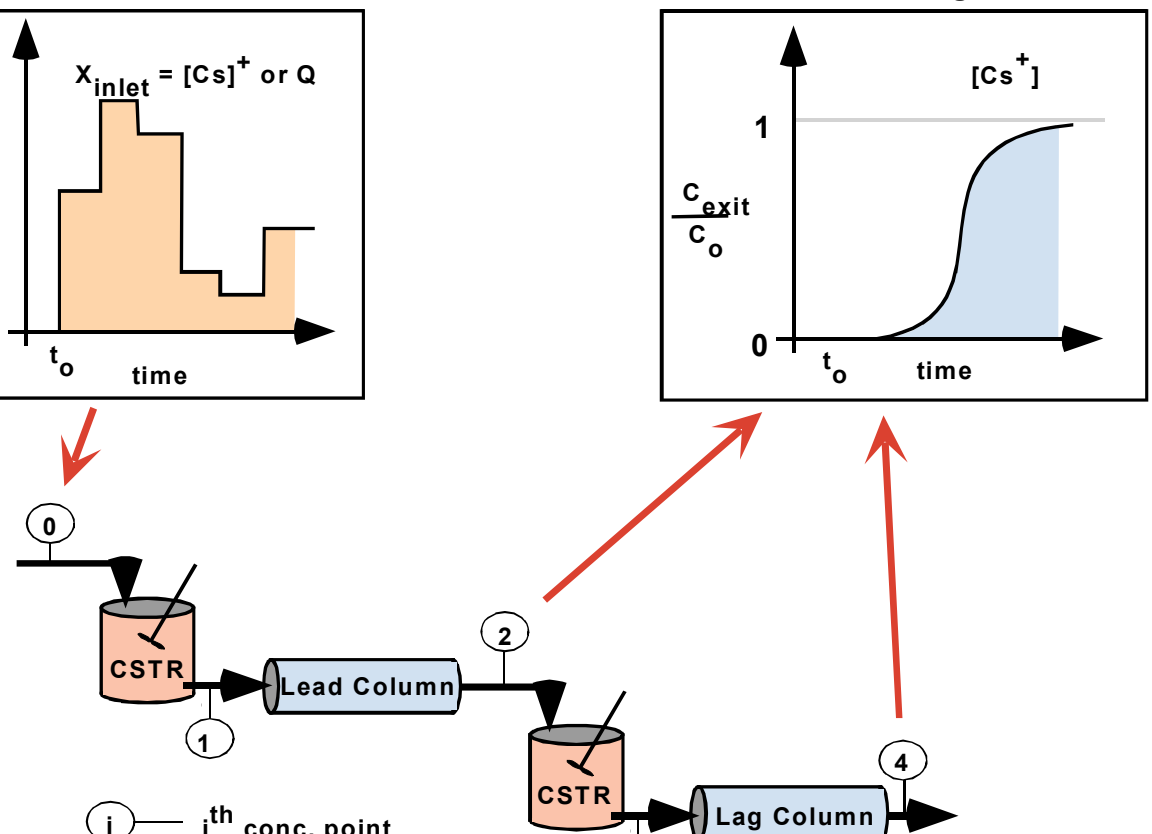

(Two-Column Carousel Configuration)
(4)

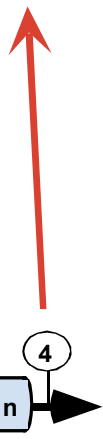

(3)
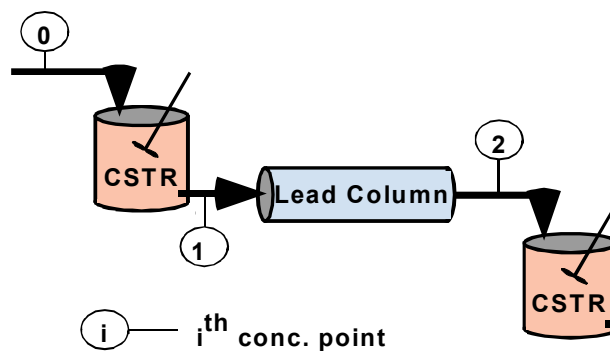

(3)

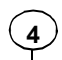

inter Colum n

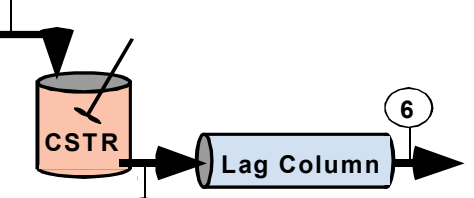

(5) 


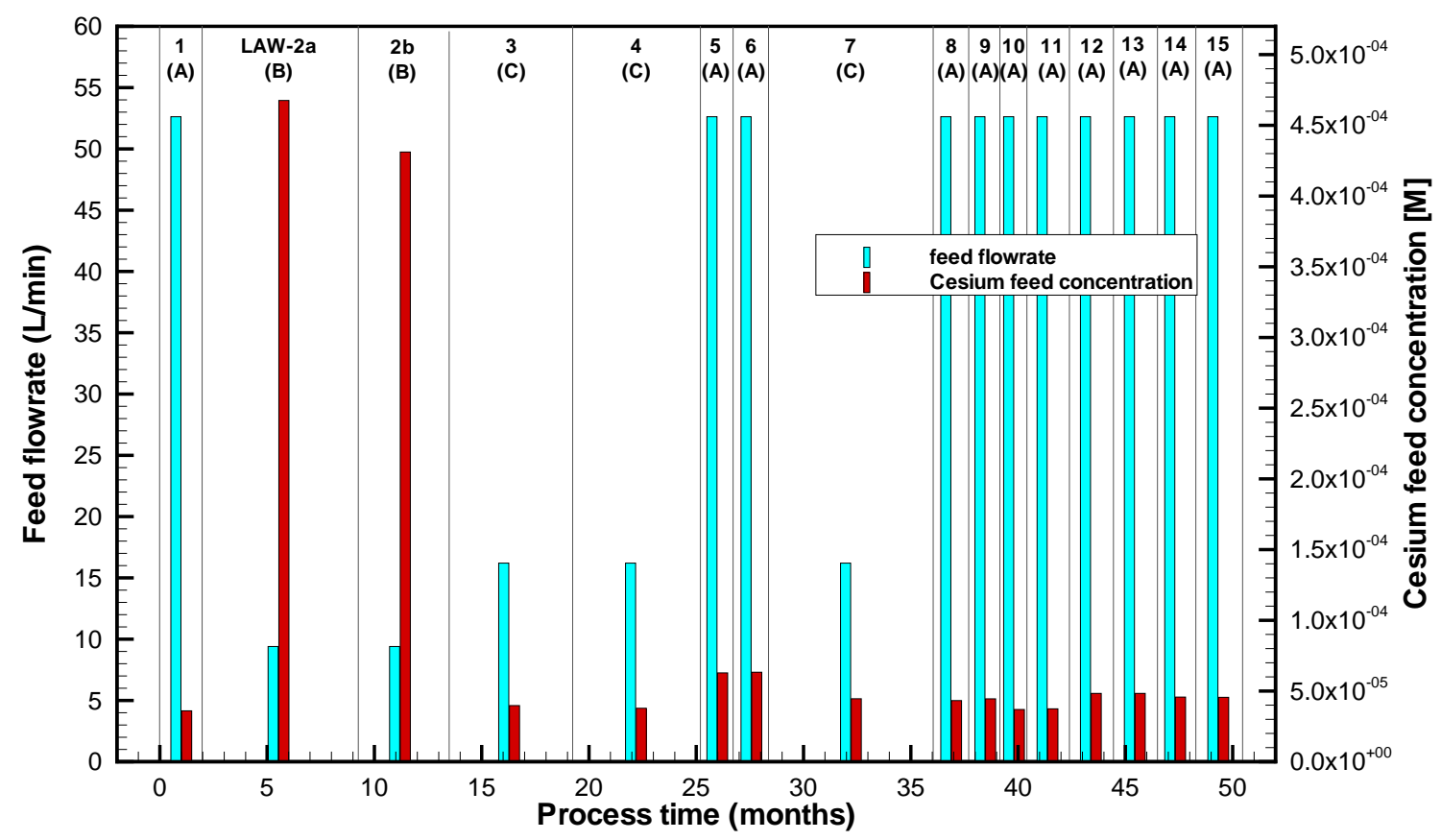

Figure 10-5. Phase 1 LAW batch feed inlet flowrates and cesium concentrations used as input boundary conditions for VERSE-LC CST column design simulations. Constant values are applied over each batch process period.
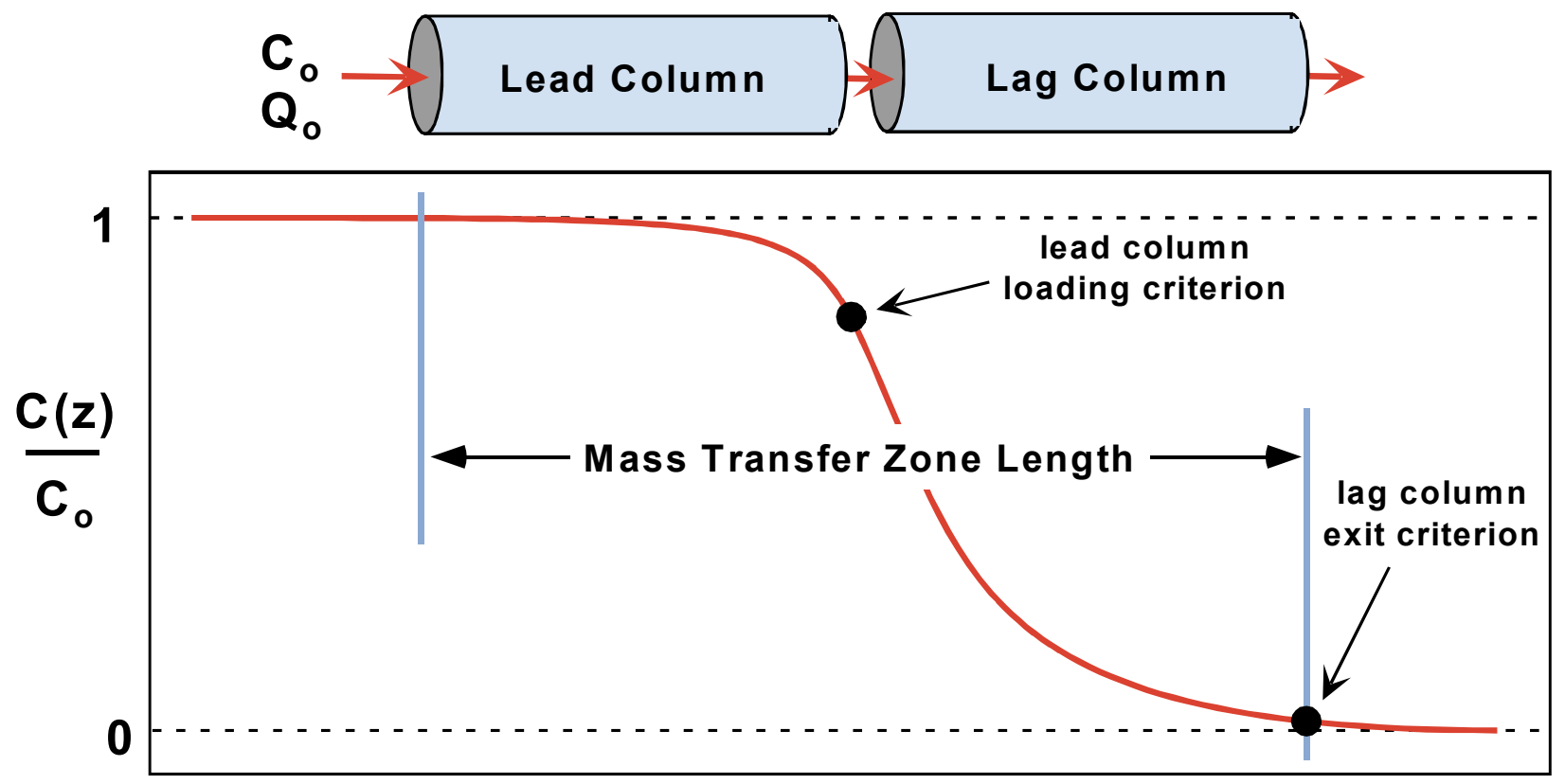

Axial distance down columns

Figure 10-6. The conceptual model defining the length of the mass transfer zone based on a 2-column carousel configuration with specified exit criteria for both columns. 


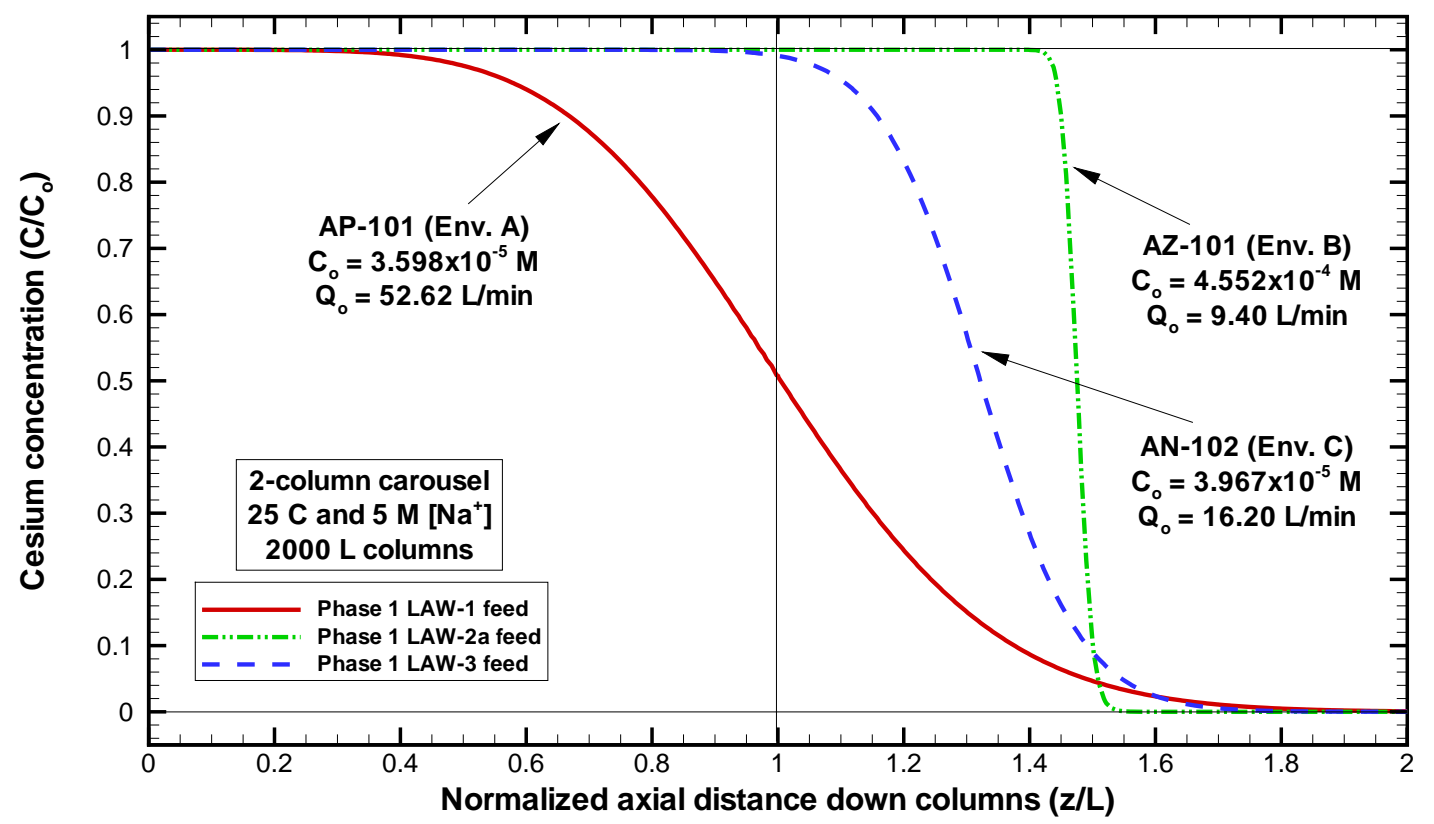

Figure 10-7. Typical impact on mass transfer zone length (i.e., concentration profiles) due to envelope cesium concentration and flowrate differences based on VERSE-LC model predictions when using the IONSIV ${ }^{\circledR}$ IE-911 material.

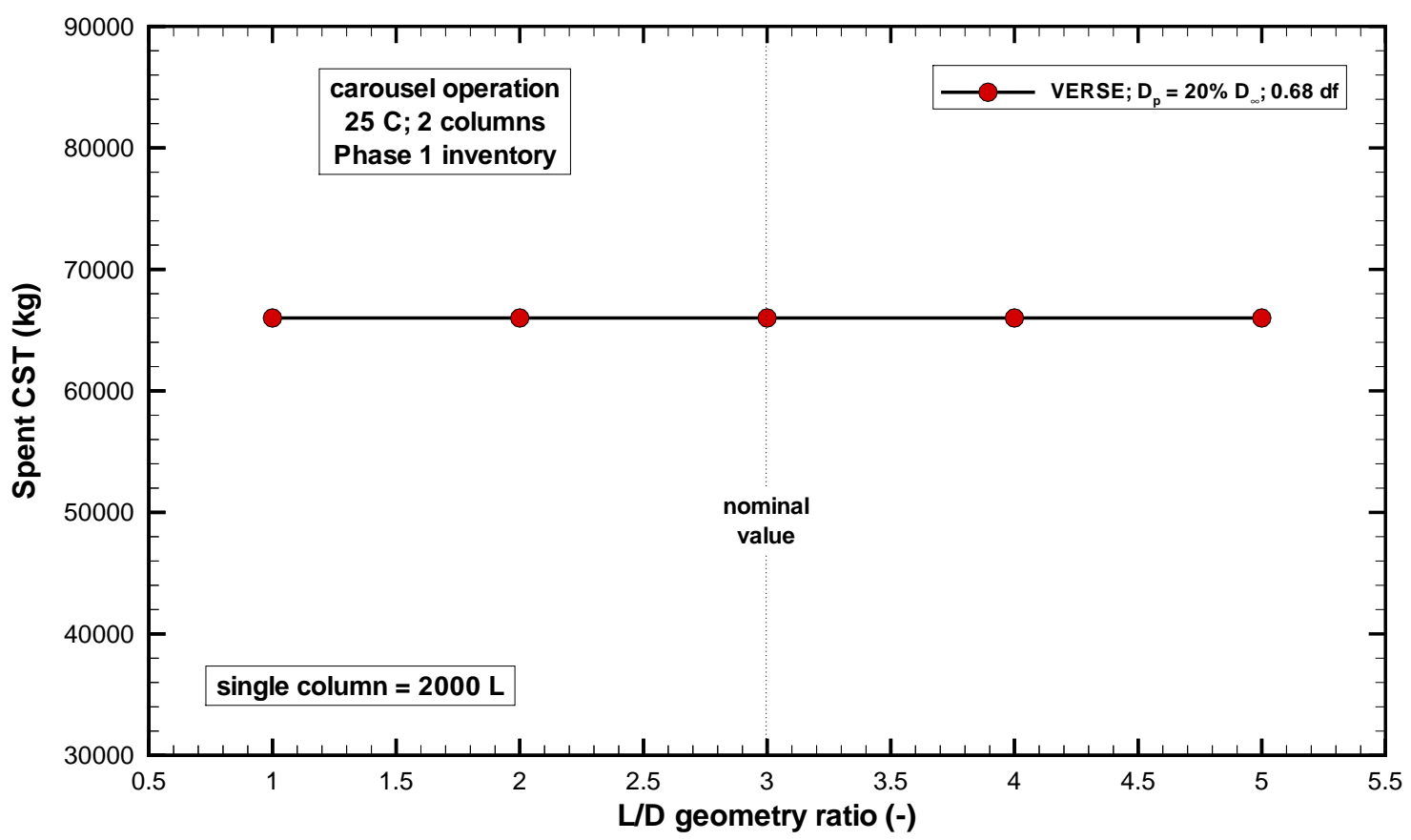

Figure 10-8. Sensitivity of spent CST to the bed L/D geometric ratio for the total processing of the Phase $1 \mathrm{LAW}$ inventory (i.e., a 2-column carousel configuration operating at $25 \mathrm{C}$ with $2000 \mathrm{~L}$ columns of varying L/D geometries). 


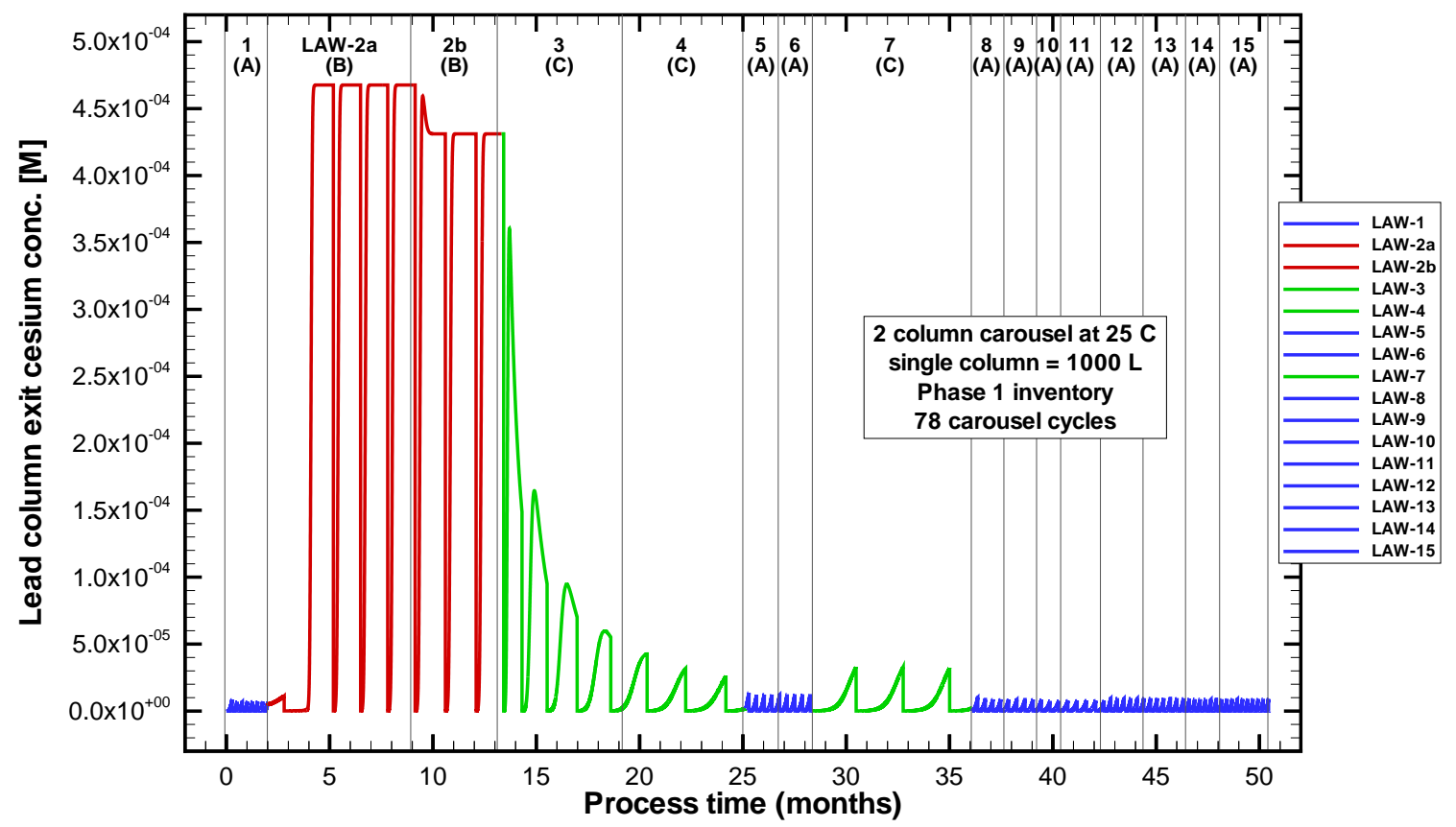

Figure 10-9. VERSE-LC cesium concentration predictions at the exit of the lead column based on CST and the Phase 1 LAW inventory (i.e., a 2-column carousel configuration operating at 25 $\mathrm{C}$ with $1000 \mathrm{~L}$ columns for a total of 78 carousel cycles performed).

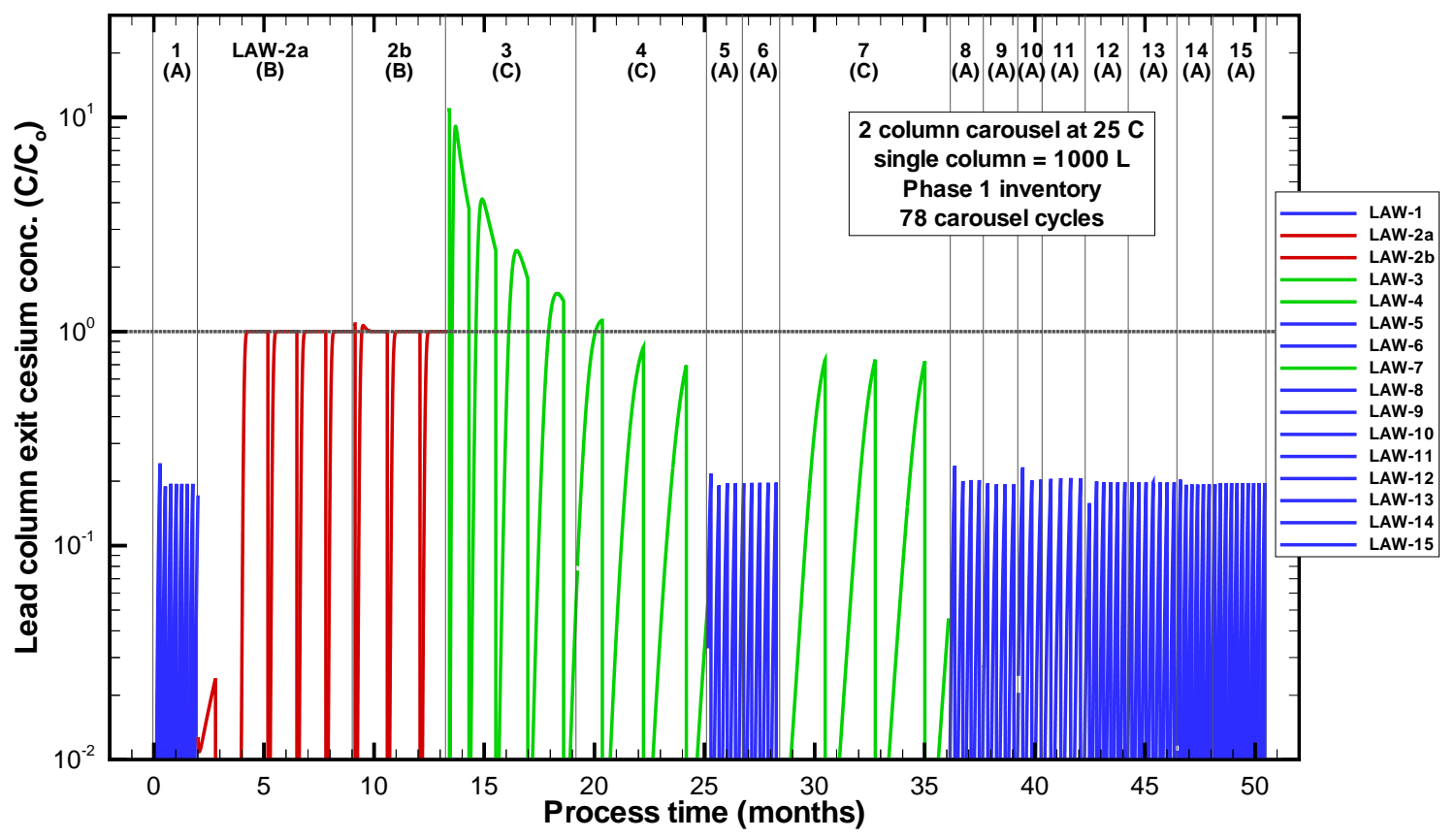

Figure 10-10. VERSE-LC normalized cesium concentration predictions at the exit of the lead column based on CST and the Phase 1 LAW inventory (i.e., a 2-column carousel configuration operating at $25 \mathrm{C}$ with $1000 \mathrm{~L}$ columns for a total of 78 carousel cycles performed). 


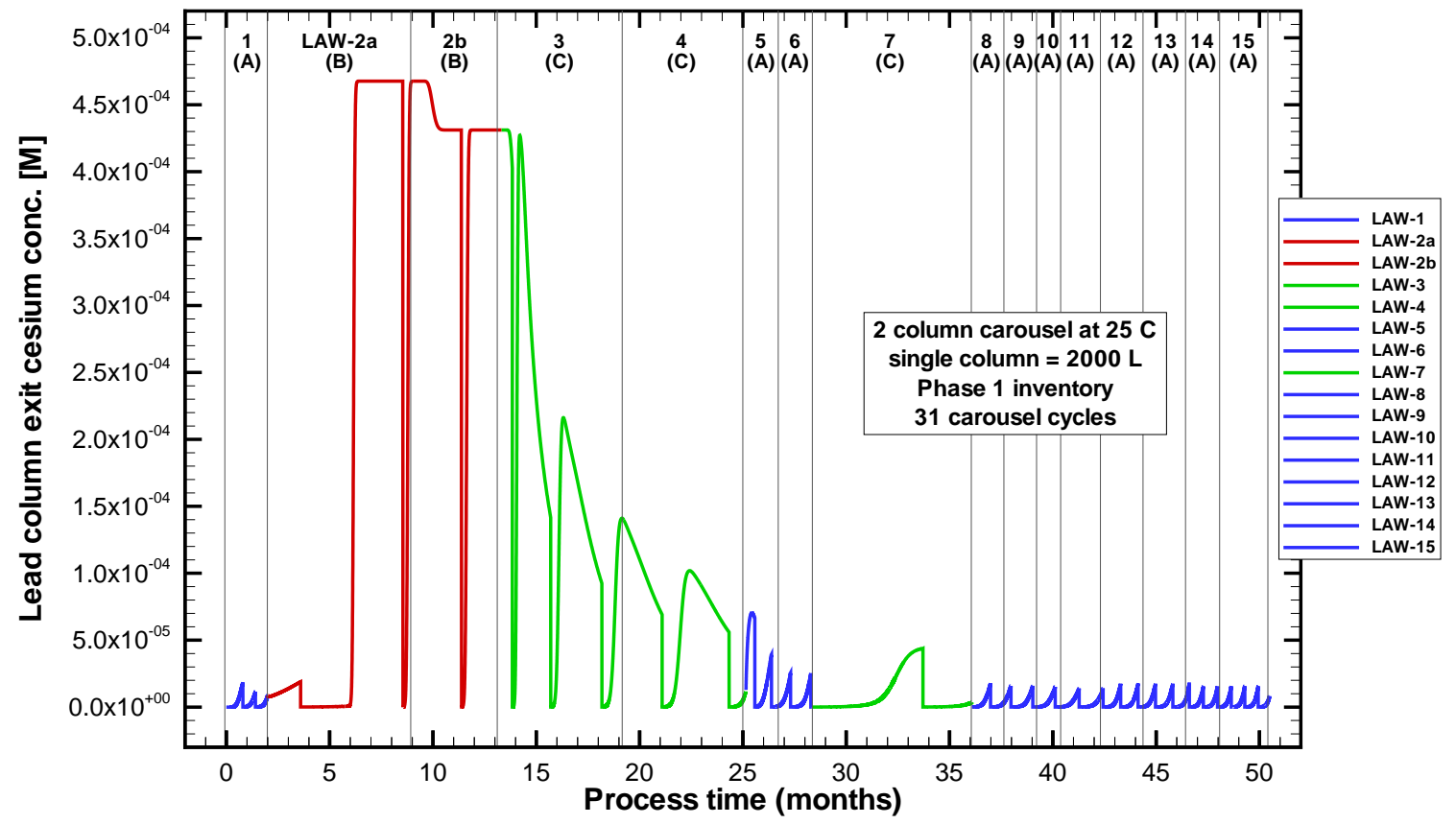

Figure 10-11. VERSE-LC cesium concentration predictions at the exit of the lead column based on CST and the Phase 1 LAW inventory (i.e., a 2-column carousel configuration operating at 25 $\mathrm{C}$ with $2000 \mathrm{~L}$ columns for a total of 31 carousel cycles performed).

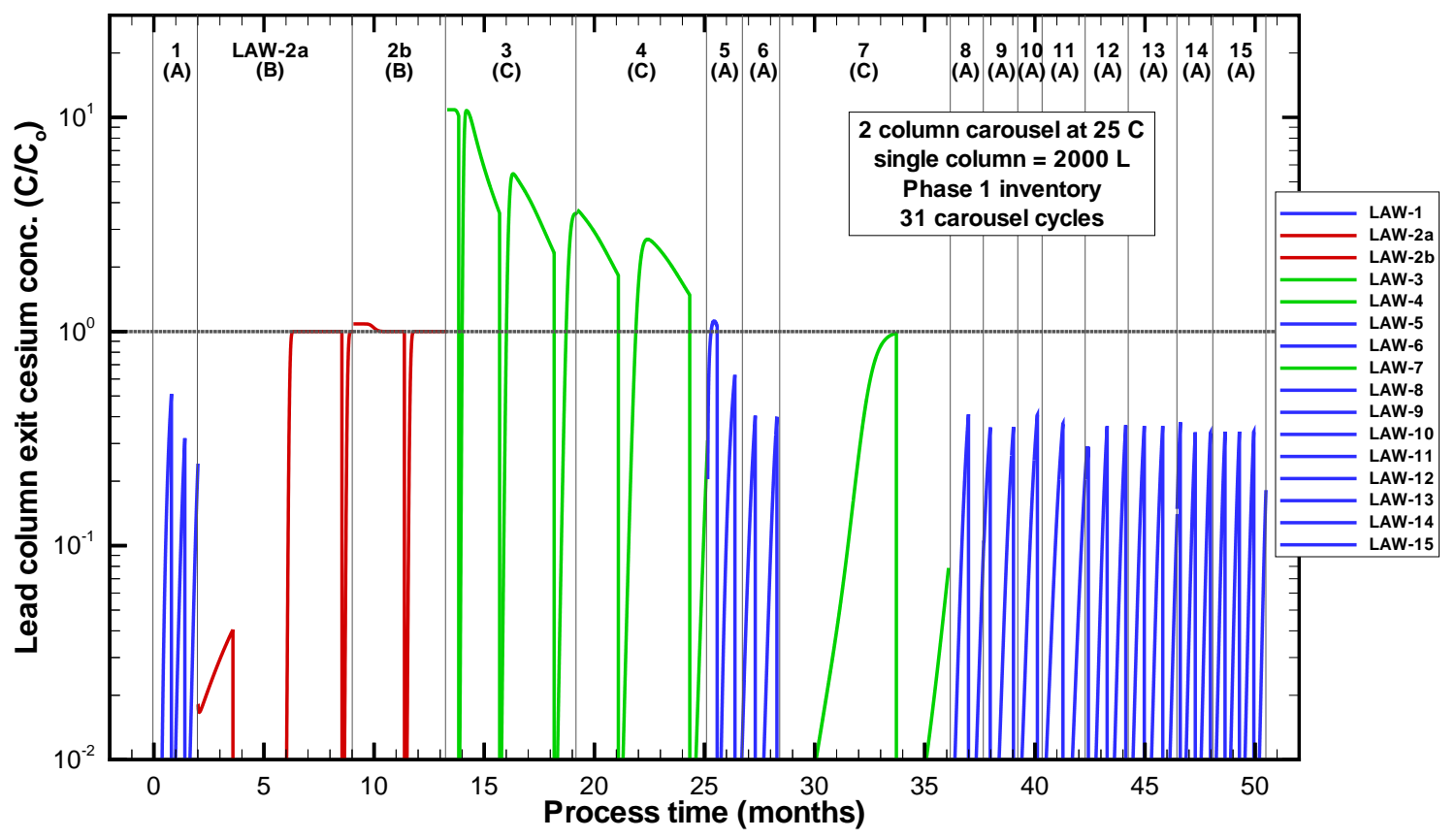

Figure 10-12. VERSE-LC normalized cesium concentration predictions at the exit of the lead column based on CST and the Phase 1 LAW inventory (i.e., a 2-column carousel configuration operating at $25 \mathrm{C}$ with $2000 \mathrm{~L}$ columns for a total of 31 carousel cycles performed). 


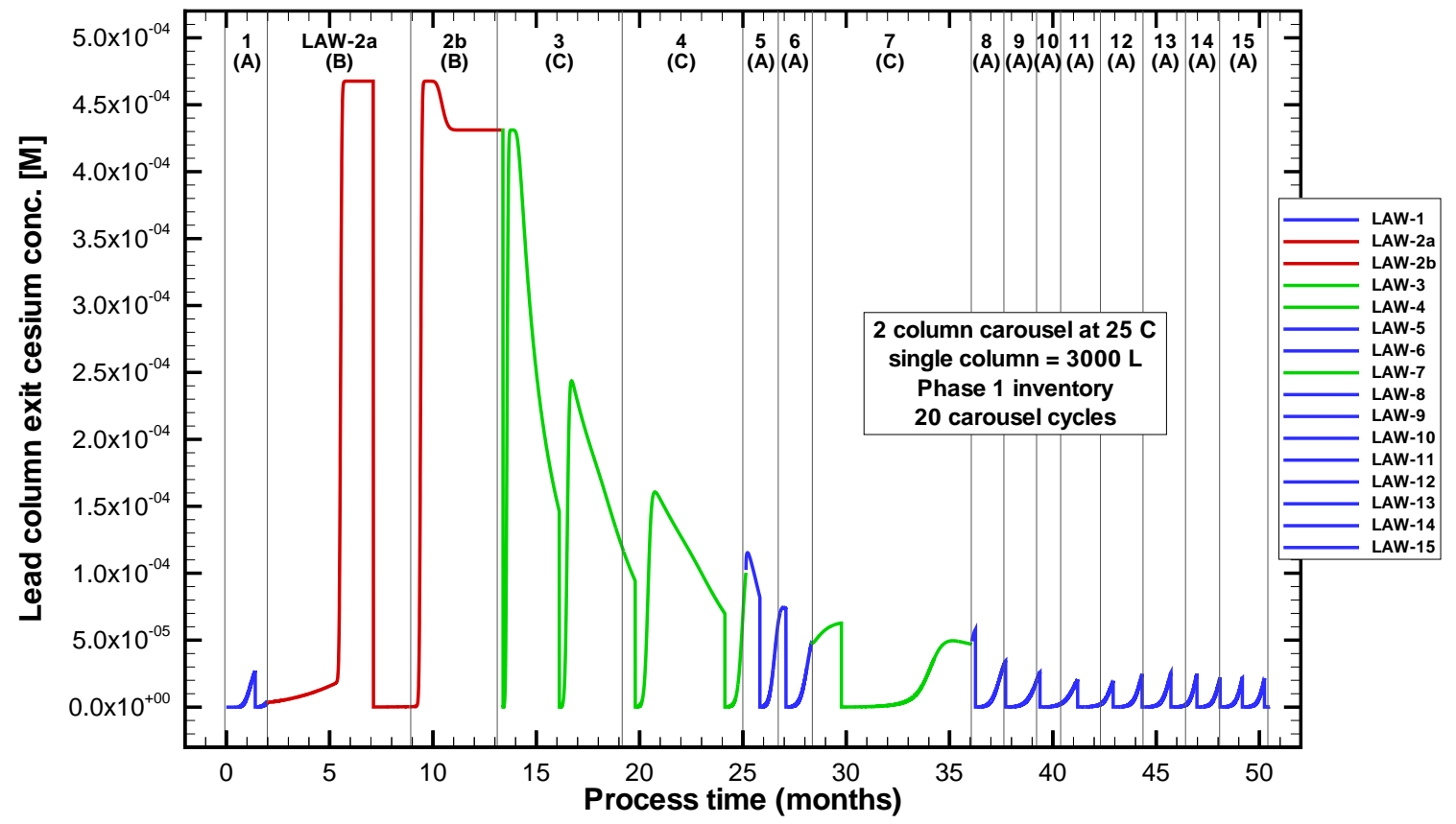

Figure 10-13. VERSE-LC normalized cesium concentration predictions at the exit of the lead column based on CST and the Phase 1 LAW inventory (i.e., a 2-column carousel configuration operating at $25 \mathrm{C}$ with $3000 \mathrm{~L}$ columns for a total of 20 carousel cycles performed).

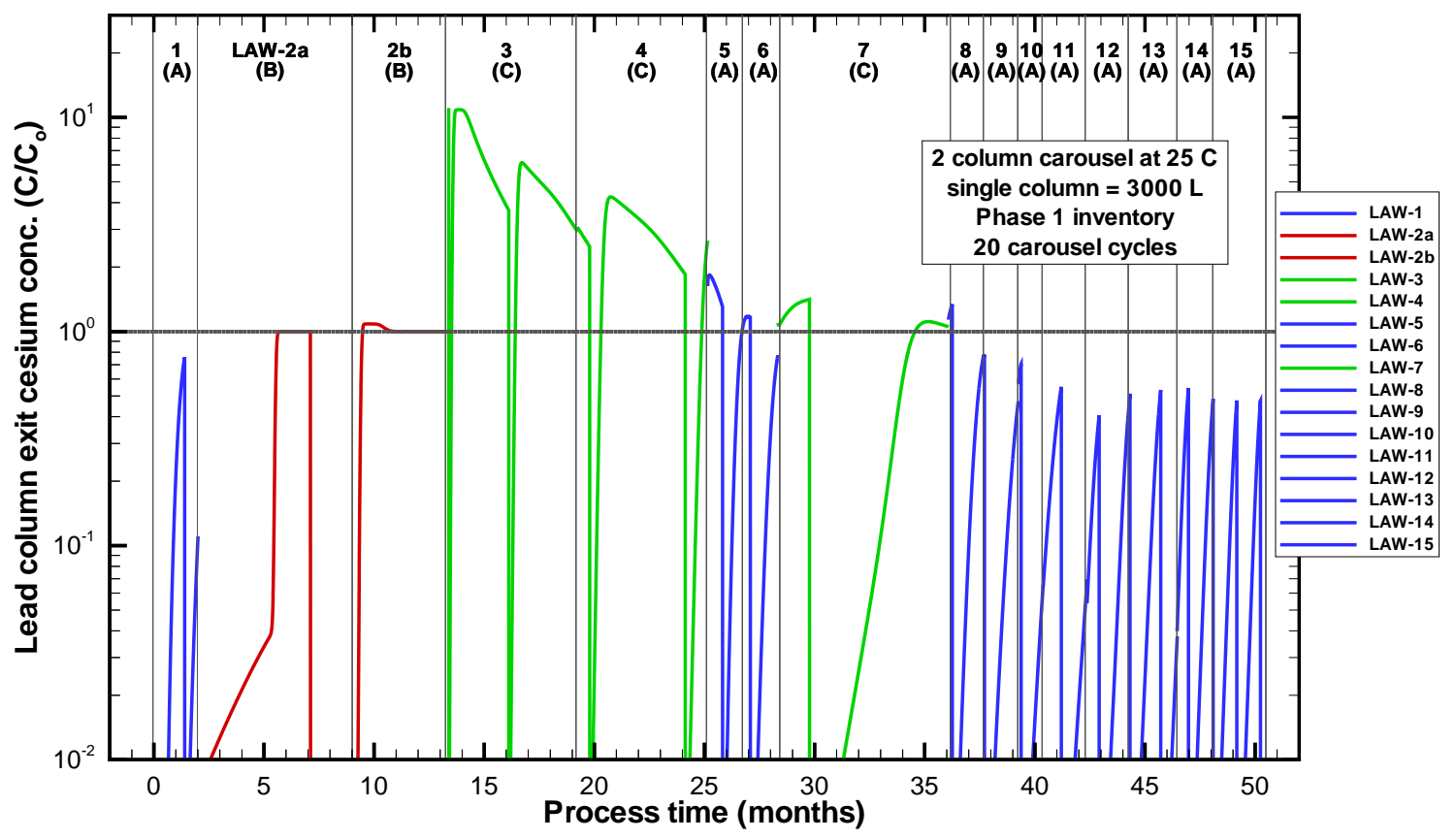

Figure 10-14. VERSE-LC cesium concentration predictions at the exit of the lead column based on CST and the Phase 1 LAW inventory (i.e., a 2-column carousel configuration operating at 25 $\mathrm{C}$ with $3000 \mathrm{~L}$ columns for a total of 20 carousel cycles performed). 


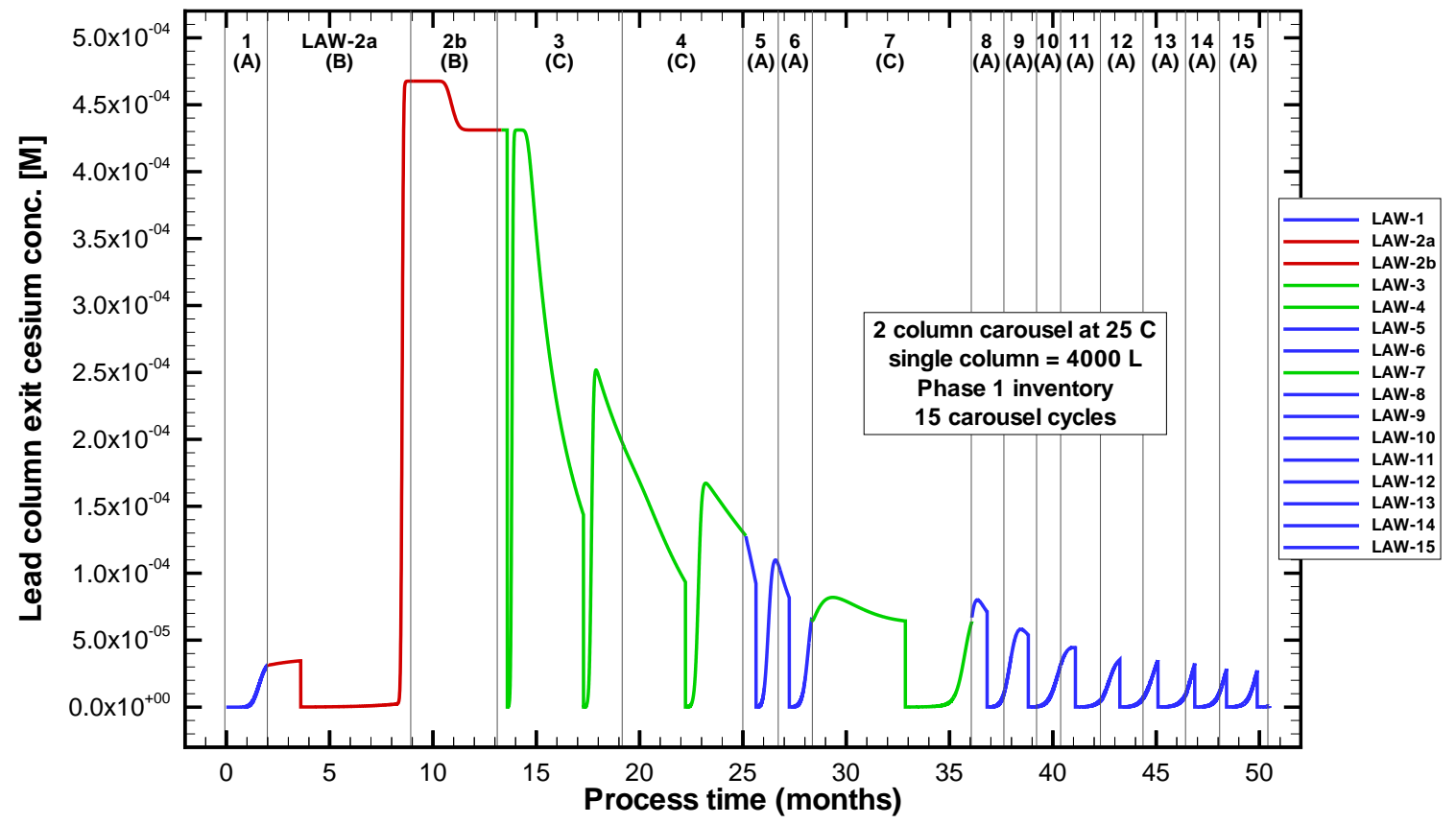

Figure 10-15. VERSE-LC cesium concentration predictions at the exit of the lead column based on CST and the Phase 1 LAW inventory (i.e., a 2-column carousel configuration operating at 25 $\mathrm{C}$ with $4000 \mathrm{~L}$ columns for a total of 15 carousel cycles performed).

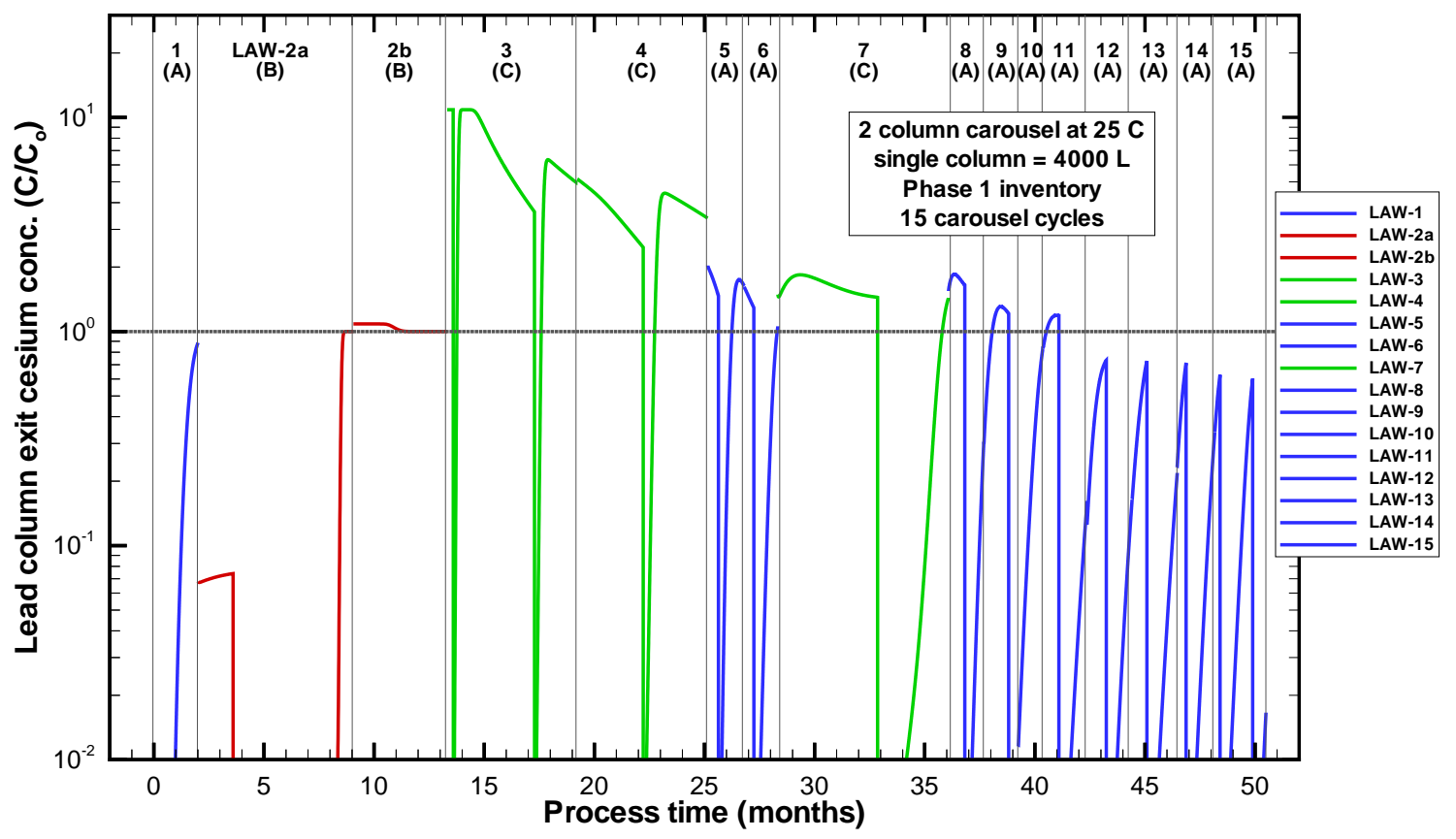

Figure 10-16. VERSE-LC normalized cesium concentration predictions at the exit of the lead column based on CST and the Phase 1 LAW inventory (i.e., a 2-column carousel configuration operating at $25 \mathrm{C}$ with $4000 \mathrm{~L}$ columns for a total of 15 carousel cycles performed). 


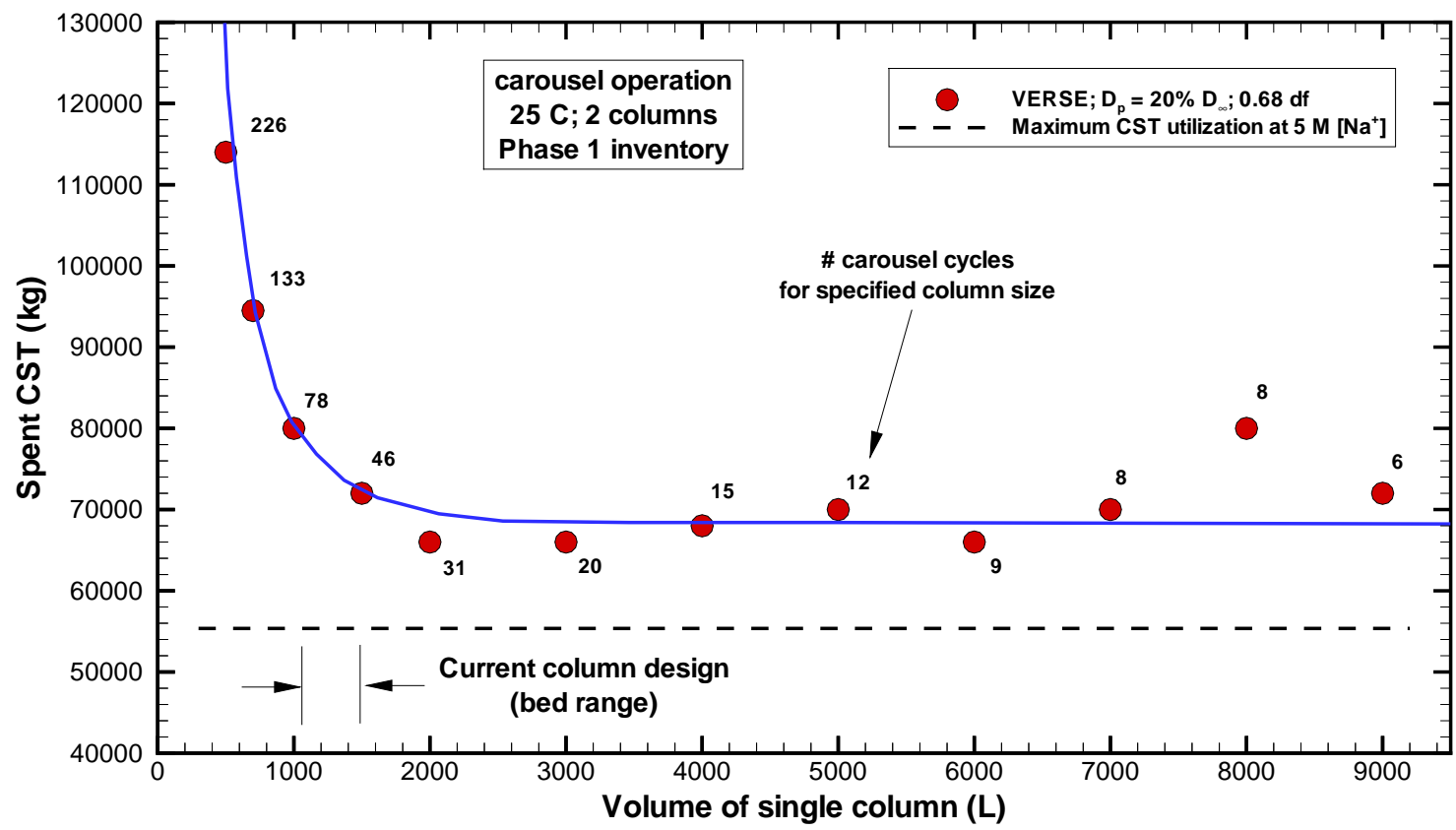

Figure 10-17. Computed total spent CST material required to process the entire Phase 1 LAW inventory based on a two-column carousel configuration at $25 \mathrm{C}$ and nominal parameter settings (solid circles are VERSE-LC results while the solid line represents its average behavior).

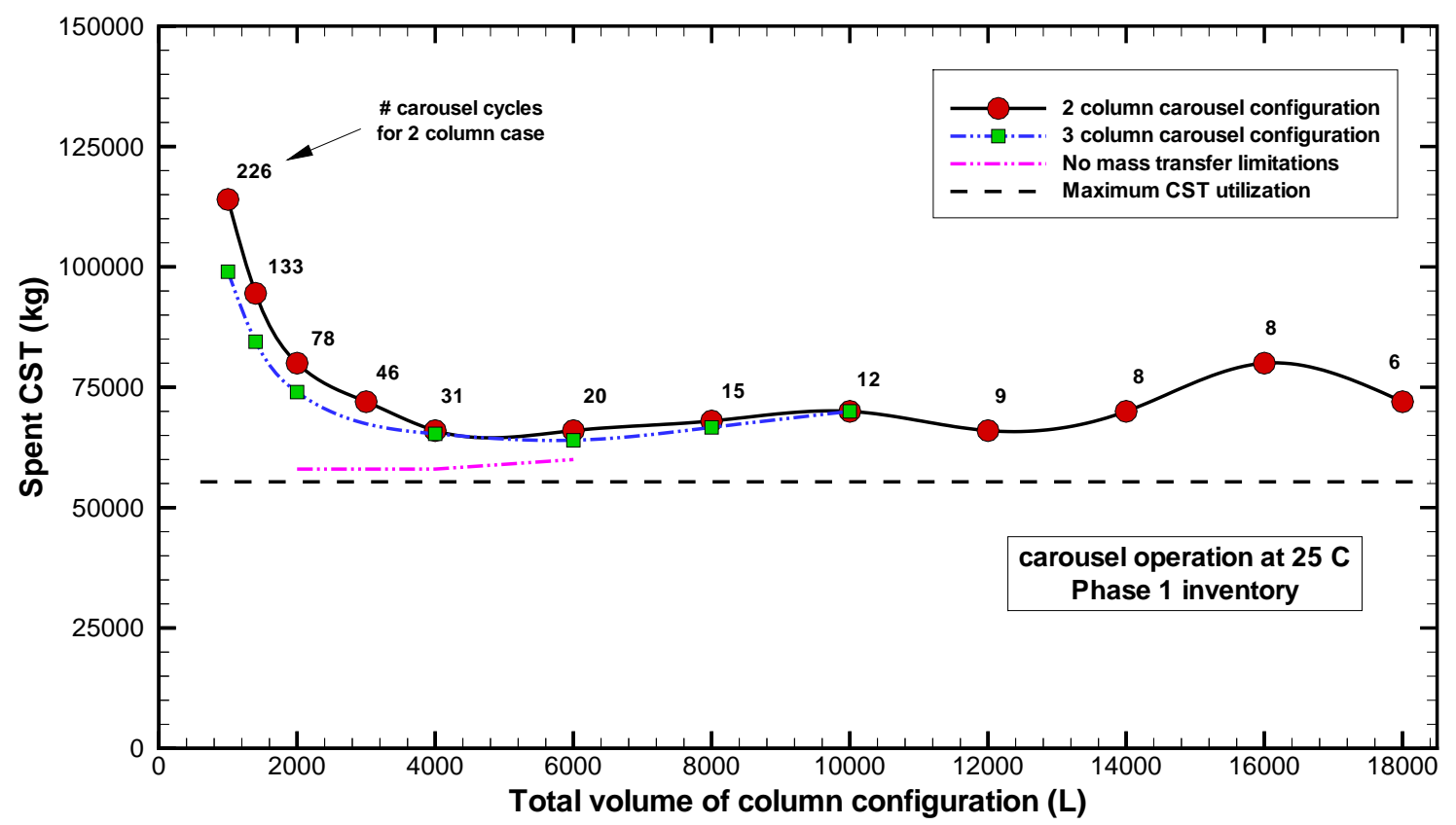

Figure 10-18. Computed total spent CST material required to process the entire Phase 1 LAW inventory at $25 \mathrm{C}$ and nominal parameter settings (VERSE-LC results for 2-column and 3-column carousels and limiting cases). 


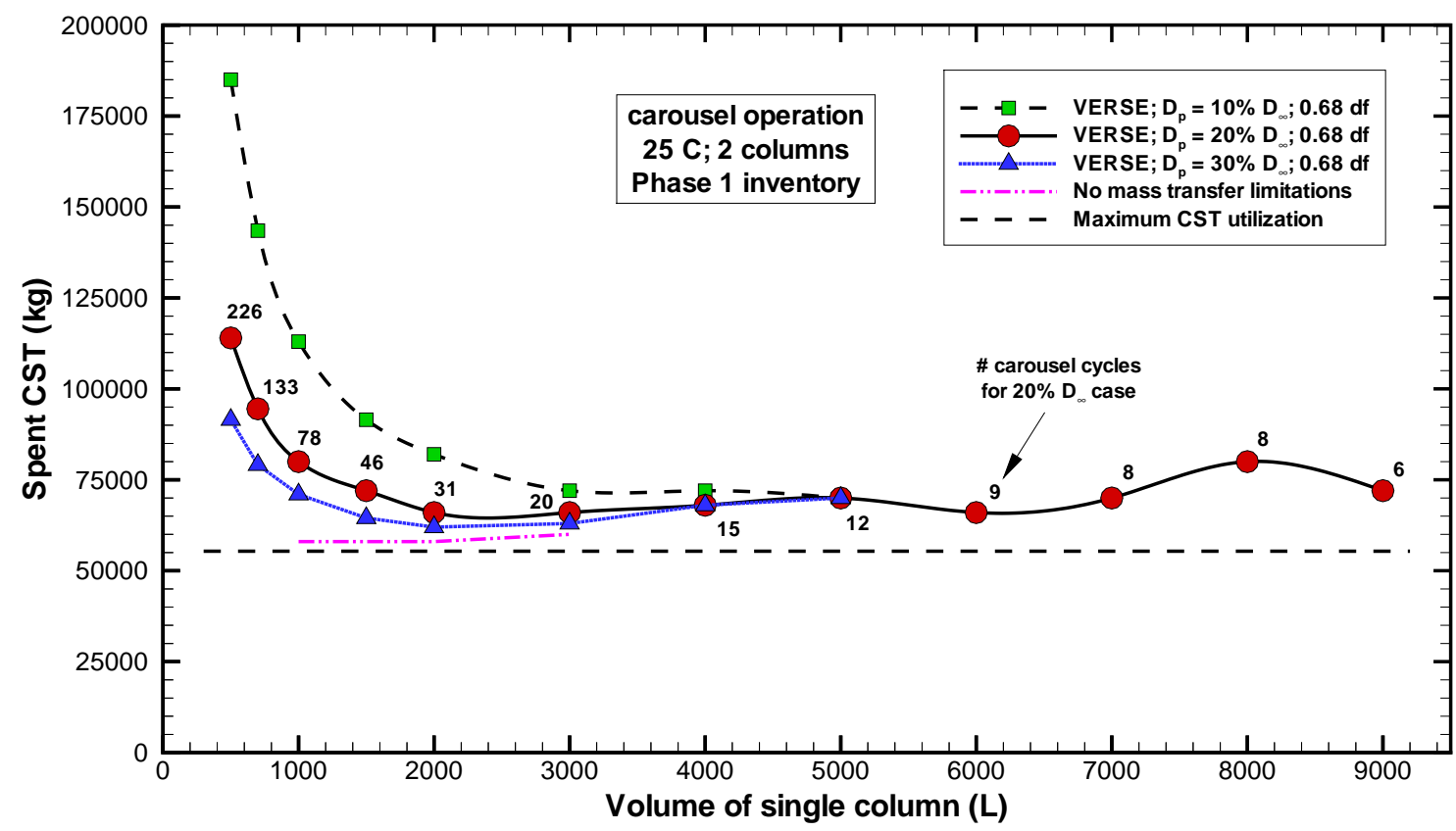

Figure 10-19. Computed total spent CST material required to process the entire Phase 1 LAW inventory based on a two-column carousel configuration at $25 \mathrm{C}$ and nominal parameter settings

(VERSE-LC results for various pore diffusivity coefficients and limiting cases).

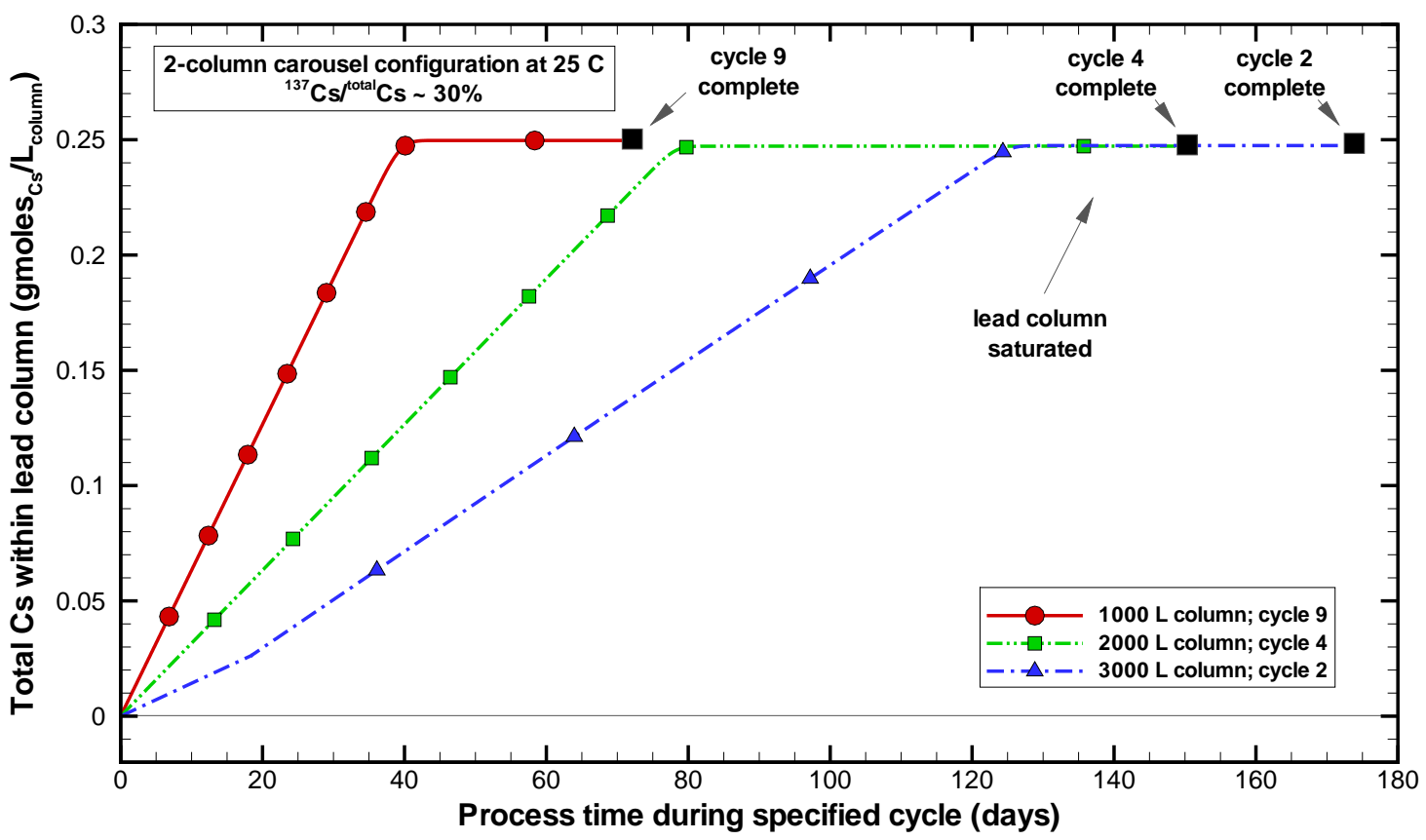

Figure 10-20. Estimated total cesium inventory contained within a lead column during the worst case process cycle for a two-column carousel configuration (results for 3 differing column sizes are shown; worst case cycles occur primarily in the LAW-2a processing period). 


\subsection{References}

Anderson, T. F., D. S. Abrams, and E. A. Grens, II, 1978. "Evaluation of Parameters for Nonlinear Thermodynamic Models," AIChE Journal, Vol. 24, No. 1, pp. 20-29.

Anthony, R. T., C. V. Philip, and S. H. Kim, 2001. "Crystalline Silicotitanate Ion Exchange Support for Salt Alternatives: Final Report," Department of Chemical Engineering, Texas A\&M University, Final report KF-90594-O, August 15.

Berninger, J. A., R. D. Whitley, X. Zhang, and L. N.-H. Wang, 1991. "A Versatile Model for Simulation of Reaction and Nonequilibrium Dynamics in Multicomponent Fixed-Bed Adsorption Processes," Computers Chem. Engr., Vol. 15, No. 11, pp. 749-768.

Bray, L. A., J. E. Amonette, G. N. Brown, T. M. Kafka, and S. F. Yates, 1995. "Efficient Separations and Processing Crosscutting Program: Develop and Test Sorbents," a FY 1995 Annual Progress Report, PNL-10750 (UC-2030), Battelle PNL, September.

Brooks, K. P., 1994. “Cesium Ion Exchange using Actual Waste: Column Size Considerations," TWRSPP-94-091, PNNL, September.

Brown, G. N., L. A. Bray, C. D. Carlson, K. J. Carson, J. R. DesChane, R. J. Elovich, F. V. Hoopes, D. E. Kurath, L. L. Nenninger, and P. K. Tanaka, 1996. "Comparison of Organic and Inorganic Ion Exchangers for Removal of Cesium and Strontium from Simulated and Actual Hanford 241-AW-101 DSSF Tank Waste," PNL-10920 (UC-2030), Battelle PNL, January.

Chung, S. F. and C. Y. Wen, 1968. "Longitudinal Dispersion of Liquid Flowing Through Fixed and Fluidized Beds," AIChE Journal, Vol. 14, No. 6, pp. 857-866.

Clift, R., J. R. Grace, and M. E. Weber, 1978. Bubbles, Drops, and Particles, Academic Press, Inc., Boston.

Collins, J. L., B. Z. Egan, K. K. Anderson, C. W. Chase, J. T. Bell, and G. E. Jernigan, 1998. "Evaluation of Selected Ion Exchangers for the Removal of Cesium and Strontium from MVST W-25 Supernate," Chemical Technology Division, ORNL, (no report number given), August 27.

Cussler, E. L., 1984. Diffusion: Mass Transfer in Fluid Systems, Cambridge University Press, Cambridge.

Davidson. D. J., J. L. Collins, K. K. Anderson, C. W. Chase, and B. Z. Egan, 1998. "Removal of Cesium, Technetium, and Strontium from Tank Waste Supernatant," ORNL/TM-13612, Lockheed Martin ORNL, August.

Ernest, M. V., Jr., R. D. Whitley, Z. Ma., and N.-H. L. Wang, 1997. "Effects of Mass Action Equilibria on Fixed-Bed Multicomponent Ion-Exchange Dynamics," Ind. Eng. Chem. Res., Vol. 36, No. 1, pp. 212-226.

Ernest, M. V., Jr., J. P. Bibler, R. D. Whitley, and N.-H. L. Wang, 1997. "Development of a Carousel Ion-Exchange Process for Removal of Cesium-137 from Alkaline Waste," Ind. Eng. Chem. Res., Vol. 36, No. 7, pp. 2775-2788.

Foo, S. C. and R. G. Rice, 1975. "On the Predication of Ultimate Separation in Parametric Pumps," AIChE Journal, Vol. 21, No. 6, pp.1149-1158.

GE Nuclear Energy, 1996. Nuclides and Isotopes, General Electric Company, Nuclear Energy Operations, $15^{\text {th }}$ Edition, pp.31. 
German, R. M., 1989. Particle Packing Characteristics, Metal Powder Industries Federation, Princeton, New Jersey.

Glasstone, S., and D. Lewis, 1960. Elements of Physical Chemistry, D. Van Nostrand Company, Inc., Princeton, N.J.

Fiskum, S. K., ?, and ??????, 2000. "Inorganic, Radioisotopic, and Organic Analysis of 241-AP101 Tank Waste," PNNL-13354, Battelle PNNL, October.

Fondeur, F. F., T. Hang, M. P. Bussey, W. R. Wilmarth, D. D. Walker, and S. D. Fink, 2000. "The Effect of Carbonate, Oxalate, and Peroxide on the Cesium Loading of IONSIV ${ }^{\circledR}$ IE-910 and IE-911," WSRC-TR-2000-00344, October, 30.

Freund, J. E., 1971. Mathematical Statistics, $2^{\text {nd }}$ Edition, Prentice-Hall, Inc., N.J.

Hamm, L. L., F. G. Smith, III, and A. M. Shadday, 1999. "QA Verification Package for VERSE-LC Version 7.80," WSRC-TR-99-00238, November.

Hamm, L. L., F. G. Smith, III, and D. J. McCabe, 2000a. "Preliminary Ion Exchange Modeling for Removal of Cesium from Hanford Waste Using SuperLig ${ }^{\circledR} 644$ Resin," BNF-003-980220, June 16.

Hamm, L. L., F. G. Smith, III, and D. J. McCabe, 2000b. "Preliminary Ion Exchange Modeling for Removal of Technetium from Hanford Waste Using SuperLig ${ }^{\circledR} 639$ Resin," WSRC-TR2000-00305 (SRT-RPP-2000-00011), August.

Hang, T., L. L. Hamm, and D. J. McCabe, 2001. "Estimating Effects of IONSIV IE-911 (Crystalline Silicotitanate) for Treating Hanford Phase 1 Low-Activity Waste Solutions (U)," WSRC-TR-2001-00033 (SRT-RPP-2001-00016), January 18.

Hay, M. S. and M. G. Bronikowski, 2000. "Chemical Characterization of an Envelope B/D Sample from Hanford Tank 241-AZ-102," BNF-003-98-0249, July 31, revision 0.

Hay, M. S., M. G. Bronikowski, and N. H. Hassan, 2000a. "Chemical Characterization of an Envelope A Sample from Hanford Tank 241-AN-103,” BNF-003-98-0248, July 31, revision 0 .

Hay, M. S., M. G. Bronikowski, C. W. Hsu, and T. L. White, 2000b. "Chemical Characterization of an Envelope C Sample from Hanford Tank 241-AN-102," BNF-003-980250, July 31 , revision 0 .

Hay, M. S., and T. B. Edwards, 1994. "Statistical Analysis of ESP Verification Test Samples," WSRC-RP-94-1224, November 4, revision 0.

Helfferich, 1962. Ion Exchange, McGraw-Hill series in advanced chemistry, McGraw-Hill Book Company, Inc., New York.

Helfferich, F. G. and P. W. Carr, 1993. The review paper "Non-linear Waves in Chromatography , I. Waves, Shocks, and Shapes," Journal of Chromatography, Vol 627, pp. 97-122.

Hendrickson, D. W., 1997. "Hanford Salt Cake Cesium Removal Using Crystalline Silicotitante," SGN Eurisys Services Corporation, SESC-EN-RPT-006. Revision 0, September.

Hritzko, B. J., D. D. Walker, and N. H. L. Wang, 1998. "Design of a Carousel Process for Removing Cesium from SRS Waste Using Crystalline Silicotitanate Ion Exchanger," Progress report from School of Chemical Engineering, Purdue University, October 15. 
Huckman, M. E., I. M. Latheef, and R. G. Anthony, 1998. "Treating Savannah River Waste Using UOP IONSIV IE-911: revised Design Based on Laboratory Column Experiments," Kinetics, Catalysis, and Reaction Engineering Laboratory, Department of Chemical Engineering, Texas A\&M University, October 18.

Huckman, M. E., I. M. Latheef, and R. G. Anthony, 1999. "Ion Exchange of Several Radionuclides on the Hydrous Crystalline Silicotitanate, UOP IONSIV IE-911," Separation Science and Technology, Vol. 34, pp. 1145-1166.

Johnson, M. E., 2000. "Estimating Effects of IONSIV IE-911 (Crystalline Silicotitanate) for Treating Hanford Phase 1 Low-Activity Waste Solutions: Test Specification," TSP-W37500-00029, Revision 0, November 27.

Kirkbride, R. A., G. K. Allen, B. A. Higley, R. M. Orme, R. S. Wittman, J. H. Baldwin, T. W. Crawford, J. Jo, J. N. Strode, T. M. Hohl, S. L. Lambert, D. E. Place, and J. A. Seidl, 2000. "Tank Farm Contractor Operation and Utilization Plan," HNF-SD-WM-SP-012 (Revision 2), April.

Lee, D. D., J. F. Walker, Jr., P. A. Taylor, and D. W. Hendrickson, 1997a. "Cesium-Removal Flow Studies using Ion Exchange," Environmental Progress, Vol. 16, No. 4 pp. 251-262.

Lee, D. D., J. R. Travis, and M. R. Gibson, 1997b. "Hot Demonstration of Proosed Commerical Cesium Removal Technology," ORNL/TM-13169, December.

Liles, A. W. and C. J. Geankoplis, 1960. "Axial Diffusion of Liquids in Packed Beds and End Effects," AIChE Journal, Vol. 6, No. 4, pp. 591-595.

Ma, Z., R. D. Whitley, and N.-H. L. Wang, 1996. "Pore and Surface Diffusion in Multicomponent Adsorption and Liquid Chromatography Systems," AIChE Journal, Vol. 42, No. 5, pp. 1244-1262.

McCabe, D. J., 1995. "Crystalline Silicotitanate Examination Results (U)," WSRC-RP-94-1123, Revision 0, May 18.

McCabe, D. J., 1997. "Examination of Crystalline Silicotitanate Applicability in Removal of Cesium from SRS High Level Waste (U)," WSRC-TR-97-0016, Revision 0, April 25.

Miller, J. E., and N. E. Brown, 1997. "Development and Properties of Crystalline Silicotitanate (CST) Ion Exchangers for Radioactive Waste Applications," Sandia National Laboratories, SAND97-0771, April.

Morales, M., C. W. Spinn, and J. M. Smith, 1951. "Velocities and Effective Thermal Conductivities in Packed Beds," Industrial and Engr. Chemistry, Vol. 43, No. 1, pp. 225-232.

Philip, C. V. and R. G. Anthony, 2000. "Crystalline Silicotitanate Ion Exchange Support for Salt Alternatives," Department of Chemical Engineering, Texas A\&M University, Final report KE-49242-0, September 30.

Qureshi, Z. H., 1999. "Mixing and Sampling of Sludge-Frit-CST Slurries (U)," WSRC-TR-9900309, September.

Reynolds, D. A. and D. L. Herting, 1984. "Solubilities of Sodium Nitrate, Sodium Nitrite, and Sodium Aluminate in Simulated Nuclear Waste," RHO-RE-ST-14P, Rockwell International, Rockwell Hanford Operations, September.

Robinson, S. M., W. D. Arnold, and C. H. Byers, 1994. "Mass-Transfer Mechanisms for Zeolite Ion Exchange in Wastewater Treatment," AIChE Journal, Vol. 40, No. 1, pp. 2045.

Schwartz, C. E. and J. M. Smith, 1953. "Flow Distribution in Packed Beds," Industrial and Engr. Chemistry, Vol. 45, No. 6, pp. 1209-1218. 
Steimke, J. L., M. A. Norato, T. J. Steeper, and D. J. McCabe, 2000. "Summary of Testing of SuperLig ${ }^{\circledR} 639$ at the TFL Ion Exchange Facility," WSRC-TR-2000-00302 (SRT-RPP-200000008), Rev. 0, August 24.

Thibaud-Erkey, C. and R. G. Anthony, 1999. "Mathematical Modeling of an Ion-Exchange Column for Nuclear Waste Remediation," Department of Chemical Engineering, Texas A\&M University, Special report RF-8761-\#2.

Walker, D. D. and C. J. Coleman, 1991. "Densities and Weight \% Solids of Simulated Salt Solutions (U)," WSRC-TR-91-176, April 17.

Walker, D. D., 1998. "Modeling of Crystalline Silicotitanate Ion Exchange Columns," WSRCTR-98-00343, Revision 0, October 12.

Walker, D. D., W. D. King, D. P. Diprete, L. L. Tovo, D. T. Hobbs, and W. L. Wilmarth, 1998a. "Cesium Removal from Simulated SRS High-Level Waste using Crystalline Silicotitanate," WSRC-TR-98-00344, Revision 1, October 16.

Walker, J. F., Jr., P. A. Taylor, R. L. Cummins, B. S. Evans, S. D. Heath, J. D. Hewitt, R. D. Hunt, H. L. Jennings, J. A. Kilby, D. D. Lee, S. Lewis-Lambert, S. A. Richardson, and R. F. Utrera, 1998b. "Cesium-Removal Demonstration Utilizing Crystalline Silicotitanate Sorbent for Processing Melton Valley Storage Tank supernate: Final Report," ORNL/TM-13503, March.

Walker, D. D., 1999a. "Modeling of Crystalline Silicotitanate Ion Exchange Columns using Experimental Data from Simulated SRS Waste," WSRC-TR-98-00396, Revision 0, January 6.

Walker, D. D., 1999b. "Preparation of Simulated Waste Solutions," WSRC-TR-98-00116, Revision 0, April 15.

Walker, D. D., D. J. Adamson, T. D. Allen, R. W. Blessing, W. T. Boyce, B. H. Croy, R. A. ewberry, D. P. Diprete, S. D. Fink, T. Hang, J. C. Hart, M. C. Lee, J. J. Olson, and M. J. Whitaker, 1999. "Cesium Removal from Savannah River Site Radioactive Waste using Crystalline Silicotitanate (IONSIV ${ }^{\mathbb{B}}$ IE-911)," WSRC-TR-99-00308, Revision 0, September 18.

Walker, D. D., W. R. Wilmarth, S. D. Fink, M. Nymar, J. Krumhansel, J. T. Mills, V. H. Dukes, and B. H. Croy, 2001. "Examination of Pre-production Samples of UOP IONSIV ${ }^{\circledR}$ IE-910 and IE-911," WSRC-TR-2001-00221, Revision 0, April 18.

Washburn, E. W., C. J. West, N. E. Dorsey, F. R. Bichowsky, and A. K. Klemenc, 1928. International Critical Tables of Numerical Data, Physics, Chemistry and Technology, Volume IV, by the National Research Council of U.S.A., McGraw-Hill Book Company, Inc., New York.

Wilmarth, W. L., T. Hang, J. T. Mills, V. H. Dukes, and S. D. Fink, 1999. "The Effect of Pretreatment, Superficial Velocity, and Presence of Organic Constituents on IONSIV ${ }^{\circledR}$ IE911 Column Performance," WSRC-TR-99-00313, August 31.

Wilson, E. J. and C. J. Geankoplis, 1966. "Liquid Mass Transfer at Very Low Reynolds Numbers in Packed Beds," Industrial and Engineering Chemistry Fundamentals, Vol. 5, No. 1, pp. 9-14.

UOP, 1996, "IONSIV Ion Exchangers: Crystalline Silicotitanates - Novel Commerical Cesium Ion Exchangers," a product information/specification advertisement brochure.

Zheng, Z., D. Gu, and R. G. Anthony, 1995. "Estimation of Cesium Ion Exchange Distribution Coefficients for Concentrated Electrolytic Solutions When Using Crystalline Silicotitanates," Ind. Eng. Chem. Res., Vol. 34, No. 6, pp. 2142-2147. 
Zheng, Z., C. V. Philip, R. G. Anthony, J. L. Krumhansl, D. E. Trudell, and J. E. Miller, 1996. "Ion Exchange of Group I Metals by Hydrous Crystalline Silicotitanates," Ind. Eng. Chem. Res., Vol. 35, No. 11, pp. 4246-4256.

Zheng, Z., R. G. Anthony, and J. E. Miller, 1997. "Modeling Multicomponent Ion Exchange Equilibrium Utilizing Hydrous Crystalline Silicotitanates by a Multiple Interactive Ion Exchange Site Model,’ Ind. Eng. Chem. Res., Vol. 36, No. 6, pp. 2427-2434. 


\section{Appendix A (BBI Phase 1 LAW Feed Solution Definitions)}

For evaluation and sizing of ion-exchange columns based on CST resin, candidate batch feed compositions for each Envelope (i.e., Envelopes A, B, and C) must be considered. The 16 candidate batch feeds are determined based on available Best Basis Inventory (BBI) Phase 1 LAW feed solution data. Alterations and adjustments were necessary to the raw BBI data in order for it to conform to current pretreatment plans and for overall charge balancing. The alterations and adjustments made focused on only those ionic species who have a direct impact on cesium loading (i.e., those ionic species considered in the cesium isotherm modeling - ZAM code input options). No attempt was made to adjust or correct all species inventories provided in the test specification document; however, for comparative purposes the concentrations of these species are provided.

No recent equilibrium batch contact testing is available for establishing or verifying the batch feed isotherms. Instead, cesium adsorption isotherms are estimated based on the ZAM equilibrium model developed at Texas A\&M for CST powder material. In order to successfully use the ZAM equilibrium model, charge balancing is required for the species chosen as input to the algorithm. Below, the various alterations/adjustments, and the reasoning behind them, are provided. A brief description and user's guide to ZAM is provided in Appendix F where some limited validation of ZAM for Hanford waste feeds is provided (based on earlier AW-101 contact data).

The planned waste treatment processing of the Phase 1 LAW tank inventory consists of processing 16 separate batches of feeds from the ten targeted waste tanks in a sequential fashion (based on a current schedule). The sequence chosen is reflected in the numbering sequence used to label each batch feed (i.e., LAW-1, LAW-2a, LAW-2b, LAW-3, .., LAW-15). Each batch represents a fixed amount of liquid waste volume whose volume includes all planned dilution processes. Decay corrections for the various radioisotopes listed are also considered, based on the scheduled time of processing.

\section{A.1 Altered Tank Solutions}

BBI Phase 1 LAW feed solution data (i.e., 11 Envelope A's, 2 Envelope B's, and 3 Envelope C's) for the following tanks were provided within the test specification document by Johnson (2000):

- Envelope A (AP-101, AN-104, AN-105, SY-101, AN-103, AW-101);

- $\quad$ Envelope B (AZ-101 and AZ-102); and

- Envelope C (AN-102, AN-107).

The following alterations were made to these candidate feed solutions to establish our starting point for performing isotherm modeling: 
- The "bound" $\mathrm{OH}^{-}$inventory for Envelope C's AN-107 (i.e., LAW-7) appeared to be off by one order of magnitude (i.e., $3.48 \times 10^{+7}$ gmoles provided and assumed to be $3.48 \times 10^{+6}$ gmoles). The inventory value was reduced by one order of magnitude based on charge balance considerations.

- Inconsistencies between total cesium and cesium-137 (i.e., in some cases more ${ }^{137} \mathrm{Cs}$ present than total cesium) existed. For Envelope A and C wastes, total cesium was estimated by assuming that ${ }^{137} \mathrm{Cs}$ inventories are correct and that the isotopic fraction of ${ }^{137} \mathrm{Cs}$ to total is $25 \%$. For Envelope B wastes the test specification contains cesium values based on the project baseline assumption that the $\mathrm{AZ}$ tanks are pretreated and waste is then returned to the double-shell tanks (DSTs) for storage and vitrification to occur at a later date. However, a revised baseline assumes that the supernate in the AZ tanks is pretreated and vitrified all at once. Therefore, new values for the original cesium content in the AZ tanks will be based on the Tank Farm COUP report (Kirkbride et al., 2000, see Table 3.1-2) where for tanks AZ-101 and AZ-102 the ${ }^{137} \mathrm{Cs}$ inventories were reported as $1.23 \times 10^{10}$ and $1.33 \times 10^{10} \mathrm{~Bq} / \mathrm{gmole}$ of $\mathrm{Na}$, respectively. This results out to 1.662 and $1.797 \mathrm{Ci} / \mathrm{L}$ at $5.0 \mathrm{M} \mathrm{Na}$. For Envelope B wastes the ${ }^{137} \mathrm{Cs}$ total cesium fraction is set to $30 \%$.

- "Free" $\mathrm{OH}^{-}$inventory was estimated by first summing up the tabulated values for $\mathrm{OH}^{-}$and "bound" $\mathrm{OH}^{-}$. Then, this sum was reduced to account for the amount used to form $\mathrm{Al}(\mathrm{OH})_{4}$ and $\mathrm{Cr}(\mathrm{OH})_{4}^{-}$, where listed inventories for total $\mathrm{Al}$ and total $\mathrm{Cr}$ were assumed to be correct. For one feed solution (i.e., Envelope B LAW-2b) the computed free $\mathrm{OH}^{-}$inventory, based on this logic, would result in a negative value given the total amount of $\mathrm{Al}$ and $\mathrm{Cr}$ reported. Even when total $\mathrm{OH}^{-}$was used (i.e., not reduced by either the $\mathrm{Al}$ or $\mathrm{Cr}$ terms), a negative charge balance would result, indicating that an increase in the cations was needed. The reported tank concentration for $\mathrm{Na}^{+}$was $2.36 \mathrm{M}$, while measurements taken by Hay and Bronikowski (2000) for a sample of Tank AZ-102 gave a concentration for $\mathrm{Na}^{+}$of $2.77 \mathrm{M}$. A negligible difference is seen for the $\mathrm{K}^{+}$concentration between the two data sources. Using this sodium value eliminated the negative $\mathrm{OH}^{-}$concentration upon reaching an ionic charge balance and was considered an acceptable value to use.

- The $\mathrm{H}^{+}$inventory was zero. For modeling purposes the concentration was computed based on the estimated "free" $\mathrm{OH}^{-}$concentration for every feed solution (i.e., this is not a sensitive parameter under these highly alkaline conditions).

- Numerous species listed in the Phase 1 LAW feed solution data provided in the test specification document contained zero inventories for all 16 waste feed solutions. These species were omitted from further calculational processing.

- The species inventories were provided by Johnson (2000) in gmole units along with estimated total liquid volumes. Molar concentrations were computed based on the above alterations to the data set and then dividing by the specified total liquid volume for each candidate feed solution to convert to molarity units. Note that the total liquid volumes provided are the actual tank liquid inventories including estimated increases associated with the necessary dilution processes used during tank retrieval and pipeline transport activities. 
- It is assumed that no liquid-phase or surface reactions are occurring at a kinetic rate of importance where species are being created or destroyed. Potential liquid-phase and precipitation reactions that may occur during a dilution or concentration process have not been considered.

- For waste tank AP-101 (LAW-1) the Tank Farm COUP report (Kirkbride et al., 2000) contains errors in its estimates for inventories of $\mathrm{K}, \mathrm{Na}$, and ${ }^{137} \mathrm{Cs}$ (based on recent information provided by Mike Johnson). As such, more recent analytical analyses performed by Fiskum et al. (2000) on an AP-101 sample are used. The ${ }^{137} \mathrm{Cs}$ inventory provided by Fiskum et al. (2000) was decay adjusted by 8 years to account for the expected time duration between the measurements and LAW-1 pretreatment processing.

- All ${ }^{137}$ Cs inventories are decay corrected from $\sim 1999$ to the date of waste processing in the Waste Treatment Plant (WTP) and all concentrations computed are based on total tank volumes plus any additional liquid processing volumes necessary.

Based on the above stated alterations/processing, the Phase $1 \mathrm{LAW}$ feed solution data in terms of molarity are provided in Tables A-1 and A-2. The assumed valance state, of only those species used in the equilibrium isotherm modeling, is provided.

In computing the "free" $\mathrm{OH}^{-}$concentration levels for each candidate feed batch, a three-step process was used:

- First, "total" $\mathrm{OH}^{-}$concentration levels were computed from the available inventory database;

- Next, "free" $\mathrm{OH}^{-}$concentration levels were estimated by subtracting off from the "total" $\mathrm{OH}^{-}$ four times the amount of total $\mathrm{Al}$ and total $\mathrm{Cr}$ present (i.e., assumed to be in the $\mathrm{Al}^{\mathrm{III}}(\mathrm{OH})_{4}^{-}$ and $\mathrm{Cr}^{\mathrm{III}}(\mathrm{OH})_{4}{ }^{-}$forms);

- Finally, the "free" $\mathrm{OH}^{-}$concentration levels were then adjusted to establish a neutral solution (i.e., to maintain an ionic charge balance based only on those species available in ZAM).

If some fraction of the total $\mathrm{Cr}$ is in the $\mathrm{Cr}{ }^{\mathrm{VI}}$ state, it would probably be in the species form of $\mathrm{Cr}^{\mathrm{VI}} \mathrm{O}_{4}{ }^{-2}$. Under these conditions the second step above should be change to only subtract off the $\mathrm{Al}^{\mathrm{III}}(\mathrm{OH})_{4}{ }^{-}$species. Separate analyses were made where all of the total $\mathrm{Cr}$ was assumed to be in the $\mathrm{Cr}^{\mathrm{VI}}$ state. The final "free" $\mathrm{OH}^{-}$concentration levels were generally within 3\% (for Envelope $\mathrm{A}$ and $\mathrm{B}$ feeds) and $10 \%$ (for Envelope $\mathrm{C}$ feeds) when compared to the above approach, indicating that either method would be acceptable for our purposes within this report.

\section{A.2 Isotopic Dilution Process for Strontium-90}

Strontium is present in high quantities within Envelope $\mathrm{C}$ feed solutions. The current pretreatment plan for the Envelope C waste solutions (i.e., tanks AN-102 and AN-107) is to perform an isotopic dilution process to reduce ${ }^{90} \mathrm{Sr}^{+2}$ within the feed solutions entering the ionexchange facility by adding strontium (in natural abundance) and precipitating out $\mathrm{SrCO}_{3}$. Within the liquid phase an equilibrium reaction involving $\mathrm{Sr}^{+2}, \mathrm{OH}^{-}$, and $\mathrm{SrOH}$ exists: 


$$
\mathrm{Sr}^{+2}+\mathrm{OH}^{-} \longleftrightarrow \mathrm{SrOH}^{+}
$$

The actual amount of $\mathrm{Sr}^{+2}$ present within a liquid solution, that is available for CST ion exchange in the form $\mathrm{SrOH}^{+}$, is currently unknown due to the various reactions occurring and the formation of $\mathrm{Sr}^{+2}$ based complexants (e.g., EDTA). Even though the ZAM equilibrium model can estimate the competitive adsorption rate of $\mathrm{SrOH}^{+}$onto CST, we shall assume that no $\mathrm{SrOH}^{+}$ species are present in the isotherm modeling for Envelope A, B, and C solutions. To remove transuranic (TRU) elements (e.g., $\left.{ }^{241} \mathrm{Am}\right), \mathrm{Na}\left(\mathrm{MnO}_{4}\right)$ is added where TRUs are co-precipitated out of solution along with manganese dioxide.

However, in order to obtain the correct amounts of $\mathrm{Na}^{+}$and $\mathrm{NO}_{3}^{-}$within a feed solution, adjustments are made consistent with the current isotopic dilution process for the Envelope $\mathrm{C}$ solutions (i.e., only done for Envelope C feeds). The processes used to make these adjustments are:

- First, the candidate feed solutions (see batches LAW-3, LAW-4, and LAW-7 in Table A-2) are diluted to $6 \mathrm{M} \mathrm{Na}^{+}$by the addition of water;

- $18 \mathrm{M} \mathrm{NaOH}$ is then added to the above diluted solution until the free $\mathrm{OH}^{-}$concentration reaches $\sim 1 \mathrm{M}$ (i.e., in some cases free $\mathrm{OH}^{-}$concentration already greater than $1 \mathrm{M}$ and no addition was made);

- Assuming zero initial $\mathrm{Sr}^{+2}$ present in the feed solutions, $\operatorname{Sr}\left(\mathrm{NO}_{3}\right)_{2}$ is added until its concentration reaches $\sim 0.075 \mathrm{M}$; and

- Finally, $\mathrm{Na}\left(\mathrm{MnO}_{4}\right)$ is added until its concentration reaches $\sim 0.05 \mathrm{M}$.

- A significant amount of the strontium added forms precipitates that are separated out from the feed solution leaving behind a saturated (at the solubility limits) feed solution isotopically diluted with respect to ${ }^{90} \mathrm{Sr}^{+2}$.

Table A-3 contains the species concentrations for the Envelope $\mathrm{C}$ feed solutions after application of the above pretreatment process steps.

\section{A.3 Adjusted Tank Solutions for $\mathbf{K}_{\mathrm{d}}$ Modeling}

The ZAM equilibrium model for CST (i.e., CSTIEXV\#, version \#, \# = 4 and 5) was chosen to compute the ion exchange characteristics of CST material in the power-form when exposed to various Hanford waste solutions. An estimated correction factor must then be applied to the powder-form results when estimating the performance behavior of the CST engineered-form exchangers. In order to compute the equilibrium cesium loading (and its $\mathrm{K}_{\mathrm{d}}$ value), and final cesium liquid concentration for a specific liquid sample, the liquid sample must be defined such that the important ionic species are specified. Also, the overall charge balance for these specific species must be very close to being neutral (i.e., a net misbalance in charge less than $\sim 5 \times 10^{-4}$ ). The ionic concentrations in the above 16 candidate feed solutions (see Tables A-1 and A-3) were adjusted to meet the restrictions/limitations imposed by ZAM based on the following: 
- Due to pretreatment issues associated with what "free" $\mathrm{Sr}^{2+}$ would be available within the liquid solutions, it was assumed that no $\mathrm{Sr}^{2+}$ would be present. Since a reaction equilibrium between $\mathrm{Sr}^{2+}$ and $\mathrm{SrOH}^{-}$exists within the liquid phase, no $\mathrm{SrOH}^{-}$will be present to compete for exchange sites. Therefore, Cs loadings are over-predicted based on this assumption and its impact should be reconsidered in future efforts.

- Only those cation and anion species available as input to ZAM are considered (see Table A-4 for listing of available ionic species). ZAM does allow the limited input of other special species (i.e., IDs 37-39 for cations and IDs 24-26 for anions, but the current list appeared adequate for our purposes.

- To arrive at a neutral charge balance, based only on those species available to ZAM, the 'free' $\mathrm{OH}^{-}$concentration was adjusted up or down, if necessary.

- In order to determine which feed solution represents a worst case feed for each Envelope, every feed solution was diluted/evaporated to a $5.0 \mathrm{M} \mathrm{Na}^{+}$state. Therefore, the identification of an Envelope's worst feed composition is based on a common $\mathrm{Na}^{+}$basis where sensitivity studies are then performed on each worst case solution where $\mathrm{Na}^{+}$concentrations are varied by evaporation/dilution.

- Since the species $\mathrm{Cr}(\mathrm{OH})_{4}{ }^{-}$, is not available for use in $\mathrm{ZAM}$, species $\mathrm{Al}(\mathrm{OH})_{4}{ }^{-}$represents the total concentration of both species. Sensitivity studies indicated that this would have a small effect on ZAM predicted cesium loadings.

The final "best estimate" concentrations used to model the CST ion-exchange process for each candidate feed solution is listed in Tables A-5 and A-6 where only those input species available as input to ZAM are listed.

ZAM addresses the surface adsorption (i.e., ion-exchange) competition between $\mathrm{Na}^{+}, \mathrm{K}^{+}, \mathrm{Cs}^{+}$, $\mathrm{H}^{+}, \mathrm{Rb}^{+}$, and $\mathrm{SrOH}^{+}$. For this set of analyses it is assumed that $\mathrm{Rb}^{+}$and $\mathrm{SrOH}^{+}$cations are negligible in the liquid feed (i.e., their concentrations are set to zero).

\section{A.4 Feed Solution Densities}

The ZAM equilibrium model for CST computes a point on the cesium adsorption isotherm by performing a simulated batch equilibrium contact experiment. In order to compute the necessary liquid-phase activity coefficients, the ZAM code must have as input the liquid density of the feed sample of interest. The liquid-phase density is used to convert the inputted concentration of ionic species from molarity to molality units. Molality units (gmole/kg-water) are used by the activity coefficient correlation (i.e., Bromley's) and in computing the mixture total ionic strength. Based on several ZAM runs, predicted cesium loadings are not very sensitive to solution density.

Polynomial based correlations exist based on the fitting of available experimentally measured liquid density for a range of LAW samples and simulants. For SRS wastes the Walker correlation (Walker and Coleman, 1991) has been used. For Hanford wastes the Reynolds- 
Herting correlation (Reynolds and Herting, 1984) and more recently the HTWOS Density model (Kirkbride et al., 2000), have been used. Kirkbride et al. (2000) recommends the use of the HTWOS density model due to its flexibility of adding future components. A subset of the available liquid density data is provided in Table A-7.

For Hanford LAWs the functional form of the two correlations (based on molarity concentration and degree $\mathrm{C}$ temperature units) are:

$$
\begin{aligned}
\rho_{\mathrm{RH}}=1.017+ & 0.0587 \mathrm{c}_{\mathrm{AlO}_{2}}-0.01943 \mathrm{c}_{\mathrm{OH}}-0.000883 \mathrm{c}_{\mathrm{NO}_{2}} \mathrm{c}_{\mathrm{NO}_{3}} \\
& +0.0459\left(\mathrm{c}_{\mathrm{AlO}_{2}}+\mathrm{c}_{\mathrm{OH}}+\mathrm{c}_{\mathrm{NO}_{3}}+\mathrm{c}_{\mathrm{NO}_{2}}\right)-0.000505 \mathrm{~T}
\end{aligned}
$$

for the Reynolds-Herting correlation and

$$
\rho_{\mathrm{HTWOS}}=1.0+0.2\left(\begin{array}{l}
-0.0955 \mathrm{c}_{\mathrm{Al}}^{2}+0.383 \mathrm{c}_{\mathrm{Al}}-0.0054 \mathrm{c}_{\mathrm{Na}}^{2}+0.1096 \mathrm{c}_{\mathrm{Na}} \\
-0.073 \mathrm{c}_{\mathrm{NO}_{2}}^{2}+0.373 \mathrm{c}_{\mathrm{NO}_{2}}+0.00046 \mathrm{c}_{\mathrm{NO}_{3}}^{2} \\
+0.201 \mathrm{c}_{\mathrm{NO}_{3}}+0.0197 \mathrm{c}_{\mathrm{OH}}^{2}+0.0077 \mathrm{c}_{\mathrm{OH}}
\end{array}\right)
$$

for the HTWOS density model. Both correlations are limited in what species are accounted for. For waste solutions containing significant quantities of other species it is expected that these correlations will generally under predict liquid density. One simple way to approximate the impact associated with other ionic species is to add their mass to the total mass present assuming that zero volume of mixing occurs as a result of their presence. For example, the HTWOS density model can be modified to account for the added species by the expression:

$$
\rho_{\text {HTWOS }}^{\text {Mod }}=\rho_{\text {HTWOS }}+\frac{\theta}{1000} \sum_{j} c_{j} M_{j}
$$

where the liquid density is in $\mathrm{g} / \mathrm{ml}$, ionic concentrations in molarity, $\mathrm{M}_{\mathrm{j}}$ the ionic molecular weight, the summation is over all ionic species excluding $\mathrm{Al}, \mathrm{Na}, \mathrm{NO}_{2}, \mathrm{NO}_{3}, \mathrm{OH}$, and a correction factor $\theta$. The correction factor would be one if zero volume of mixing occurred upon addition of other species and if the original HTWOS density model was based on samples containing only $\mathrm{Al}, \mathrm{Na}, \mathrm{NO}_{2}, \mathrm{NO}_{3}, \mathrm{OH}$. Based on the data provided in Table A-7, a correction factor $(\theta$ in Eq. (A-3)) of $\sim 0.6$ is required.

Date taken from several sources [i.e., Hay and Bronikowski (2000), Hay et al. (2000a), Hay et al. (2000b), PNNL PNWD-3001, PNNL PNWD-3039, Steimke et al. (2000), and Walker and Coleman (1991)] are provided in Table A-7. A comparison to the Hanford waste data is made between the Reynolds-Herting correlation, the HTWOS density model, and the modified HTWOS density model. The results of the comparison are shown in Table A-8. Based on computed mean bias and root mean square indicators, as expected, the HTWOS density model out performs the Reynolds-Herting correlation. Also, the simple modification to the HTWOS density model provides additional improvements. 
For the creation of cesium adsorption isotherms using the ZAM model, the modified HTWOS density model is chosen (i.e., Eq. (A-3)) where the summation is over all species other than those directly accounted for by the original HTWOS density model. The corresponding "best estimate" liquid densities for the various candidate feed solutions are provided in Tables A-5 and A-6. Liquid density based on both the original HTWOS density model and its modification are listed, along with the percent difference in the estimates. 
Table A-1. Best Basis Inventory (BBI) Phase 1 LAW feed solution data for Envelope A where stated alterations were made to minimize inventory inconsistencies and to establish an ionic charge balance (a value of zero implies that no information was provided on species).

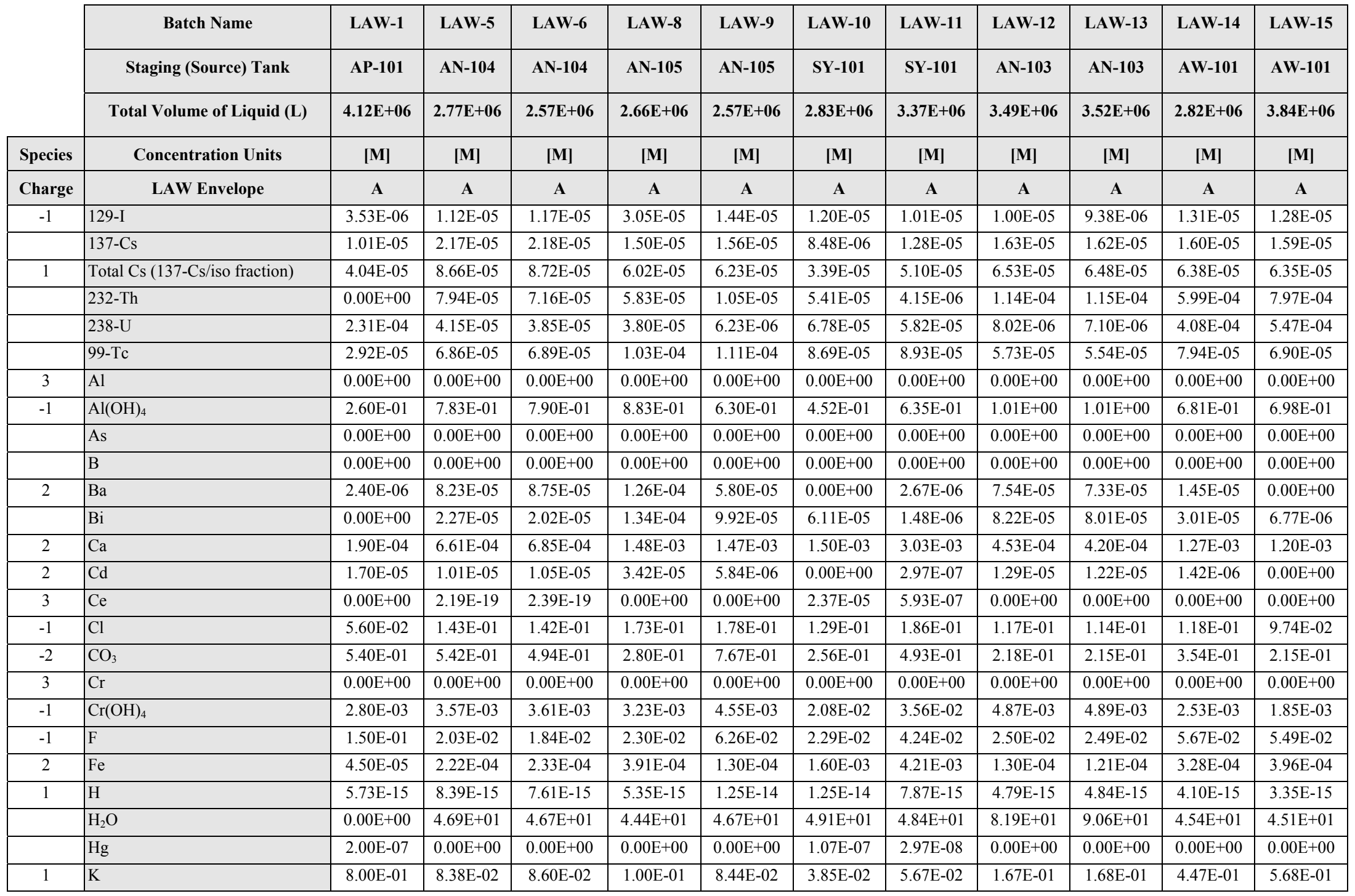




\begin{tabular}{|c|c|c|c|c|c|c|c|c|c|c|c|c|}
\hline & Batch Name & LAW-1 & LAW-5 & LAW-6 & LAW-8 & LAW-9 & LAW-10 & LAW-11 & LAW-12 & LAW-13 & LAW-14 & LAW-15 \\
\hline & Staging (Source) Tank & AP-101 & AN-104 & AN-104 & AN-105 & AN-105 & SY-101 & SY-101 & AN-103 & AN-103 & AW-101 & AW-101 \\
\hline & Total Volume of Liquid (L) & $4.12 E+06$ & $2.77 E+06$ & $2.57 E+06$ & $2.66 \mathrm{E}+06$ & $2.57 E+06$ & $2.83 \mathrm{E}+06$ & $3.37 \mathrm{E}+06$ & $3.49 \mathrm{E}+06$ & $3.52 \mathrm{E}+06$ & $2.82 E+06$ & $3.84 \mathrm{E}+06$ \\
\hline Species & Concentration Units & {$[\mathbf{M}]$} & {$[\mathbf{M}]$} & {$[\mathbf{M}]$} & {$[\mathbf{M}]$} & {$[\mathbf{M}]$} & {$[\mathbf{M}]$} & {$[\mathbf{M}]$} & {$[\mathbf{M}]$} & {$[\mathbf{M}]$} & {$[\mathbf{M}]$} & {$[\mathbf{M}]$} \\
\hline Charge & LAW Envelope & $\mathbf{A}$ & $\mathbf{A}$ & $\mathbf{A}$ & $\mathbf{A}$ & $\mathbf{A}$ & $\mathbf{A}$ & $\mathbf{A}$ & $\mathbf{A}$ & $\mathbf{A}$ & $\mathbf{A}$ & $\mathbf{A}$ \\
\hline 3 & $\mathrm{La}$ & $9.40 \mathrm{E}-06$ & $8.30 \mathrm{E}-06$ & $8.95 \mathrm{E}-06$ & $4.36 \mathrm{E}-13$ & $2.39 \mathrm{E}-12$ & $1.87 \mathrm{E}-05$ & $2.97 \mathrm{E}-07$ & $2.29 \mathrm{E}-06$ & $2.56 \mathrm{E}-06$ & $6.67 \mathrm{E}-12$ & $8.10 \mathrm{E}-12$ \\
\hline 2 & $\mathrm{Mn}$ & $0.00 \mathrm{E}+00$ & $4.73 \mathrm{E}-05$ & 4.94E-05 & $1.11 \mathrm{E}-04$ & $3.07 \mathrm{E}-05$ & $6.15 \mathrm{E}-05$ & $1.15 \mathrm{E}-04$ & 2.72E-05 & $2.47 \mathrm{E}-05$ & $7.06 \mathrm{E}-05$ & $8.41 \mathrm{E}-05$ \\
\hline 1 & $\mathrm{Na}$ & $5.62 \mathrm{E}+00$ & $6.90 \mathrm{E}+00$ & $6.89 \mathrm{E}+00$ & $6.95 \mathrm{E}+00$ & $7.00 \mathrm{E}+00$ & $4.59 \mathrm{E}+00$ & $6.82 \mathrm{E}+00$ & $6.76 \mathrm{E}+00$ & $6.70 \mathrm{E}+00$ & $6.99 \mathrm{E}+00$ & $6.98 \mathrm{E}+00$ \\
\hline & $\mathrm{NH}_{3}$ & $0.00 \mathrm{E}+00$ & $1.35 \mathrm{E}-01$ & $1.44 \mathrm{E}-01$ & $1.12 \mathrm{E}-01$ & $5.33 \mathrm{E}-02$ & $7.74 \mathrm{E}-02$ & $5.91 \mathrm{E}-02$ & $2.31 \mathrm{E}-01$ & $2.33 \mathrm{E}-01$ & $4.18 \mathrm{E}-01$ & $5.42 \mathrm{E}-01$ \\
\hline 2 & $\mathrm{Ni}$ & $1.40 \mathrm{E}-04$ & $8.48 \mathrm{E}-05$ & $8.91 \mathrm{E}-05$ & $3.70 \mathrm{E}-04$ & $1.09 \mathrm{E}-04$ & $7.67 \mathrm{E}-04$ & $2.00 \mathrm{E}-03$ & $5.64 \mathrm{E}-05$ & $4.69 \mathrm{E}-05$ & $1.40 \mathrm{E}-04$ & $1.51 \mathrm{E}-04$ \\
\hline-1 & $\mathrm{NO}_{2}$ & $9.10 \mathrm{E}-01$ & $1.64 \mathrm{E}+00$ & $1.64 \mathrm{E}+00$ & $1.67 \mathrm{E}+00$ & $1.69 \mathrm{E}+00$ & $1.20 \mathrm{E}+00$ & $1.89 \mathrm{E}+00$ & $1.52 \mathrm{E}+00$ & $1.51 \mathrm{E}+00$ & $1.49 \mathrm{E}+00$ & $1.42 \mathrm{E}+00$ \\
\hline-1 & $\mathrm{NO}_{3}$ & $2.10 \mathrm{E}+00$ & $1.90 \mathrm{E}+00$ & $1.87 \mathrm{E}+00$ & $1.78 \mathrm{E}+00$ & $1.97 \mathrm{E}+00$ & $1.31 \mathrm{E}+00$ & $1.64 \mathrm{E}+00$ & $1.65 \mathrm{E}+00$ & $1.64 \mathrm{E}+00$ & $1.85 \mathrm{E}+00$ & $1.81 \mathrm{E}+00$ \\
\hline & $\mathrm{OH}(\mathrm{BOUND})$ & $5.40 \mathrm{E}-01$ & $1.94 \mathrm{E}+00$ & $2.06 \mathrm{E}+00$ & $3.24 \mathrm{E}+00$ & $1.53 \mathrm{E}+00$ & $8.90 \mathrm{E}-01$ & $3.74 \mathrm{E}-01$ & $3.32 \mathrm{E}-01$ & $2.43 \mathrm{E}-01$ & $1.91 \mathrm{E}+00$ & $2.04 \mathrm{E}+00$ \\
\hline & $\mathrm{OH}-$ & $1.86 \mathrm{E}+00$ & $3.32 \mathrm{E}+00$ & $3.24 \mathrm{E}+00$ & $2.08 \mathrm{E}+00$ & $2.05 \mathrm{E}+00$ & $1.66 \mathrm{E}+00$ & $3.62 \mathrm{E}+00$ & $6.22 \mathrm{E}+00$ & $6.31 \mathrm{E}+00$ & $3.34 \mathrm{E}+00$ & $3.78 \mathrm{E}+00$ \\
\hline-1 & OH- (Bound+Free-4Al-4Cr) & $1.75 \mathrm{E}+00$ & $1.19 \mathrm{E}+00$ & $1.31 \mathrm{E}+00$ & $1.87 \mathrm{E}+00$ & $8.03 \mathrm{E}-01$ & 7.99E-01 & $1.27 \mathrm{E}+00$ & $2.09 \mathrm{E}+00$ & $2.07 \mathrm{E}+00$ & $2.44 \mathrm{E}+00$ & $2.99 \mathrm{E}+00$ \\
\hline 2 & $\mathrm{~Pb}$ & $7.20 \mathrm{E}-05$ & $1.29 \mathrm{E}-04$ & $1.35 \mathrm{E}-04$ & $1.84 \mathrm{E}-04$ & $1.01 \mathrm{E}-04$ & $1.39 \mathrm{E}-03$ & $2.37 \mathrm{E}-04$ & $1.58 \mathrm{E}-04$ & $1.56 \mathrm{E}-04$ & $1.93 \mathrm{E}-04$ & $2.24 \mathrm{E}-04$ \\
\hline-3 & $\mathrm{PO}_{4}$ & $1.10 \mathrm{E}-02$ & $2.85 \mathrm{E}-02$ & $2.73 \mathrm{E}-02$ & $1.51 \mathrm{E}-02$ & $3.74 \mathrm{E}-02$ & $3.99 \mathrm{E}-02$ & $4.99 \mathrm{E}-02$ & $1.27 \mathrm{E}-02$ & $1.25 \mathrm{E}-02$ & $1.54 \mathrm{E}-02$ & $7.92 \mathrm{E}-03$ \\
\hline & $\mathrm{Si}$ & $0.00 \mathrm{E}+00$ & $5.67 \mathrm{E}-03$ & $5.64 \mathrm{E}-03$ & $4.55 \mathrm{E}-03$ & $3.02 \mathrm{E}-03$ & $5.30 \mathrm{E}-03$ & 1.19E-02 & $6.56 \mathrm{E}-03$ & $6.59 \mathrm{E}-03$ & 4.79E-03 & $5.39 \mathrm{E}-03$ \\
\hline-2 & $\mathrm{SO}_{4}$ & $4.20 \mathrm{E}-02$ & $6.68 \mathrm{E}-02$ & 6.19E-02 & $3.02 \mathrm{E}-02$ & $5.14 \mathrm{E}-02$ & $3.42 \mathrm{E}-02$ & $3.26 \mathrm{E}-02$ & $1.54 \mathrm{E}-02$ & $1.49 \mathrm{E}-02$ & $2.40 \mathrm{E}-02$ & $1.48 \mathrm{E}-02$ \\
\hline & $\mathrm{Sr}(\mathrm{w} / \mathrm{o} 90 \mathrm{Sr})$ & $0.00 \mathrm{E}+00$ & $1.78 \mathrm{E}-07$ & $1.77 \mathrm{E}-07$ & $3.27 \mathrm{E}-05$ & $2.18 \mathrm{E}-05$ & $1.38 \mathrm{E}-05$ & $1.19 \mathrm{E}-06$ & $2.18 \mathrm{E}-05$ & $2.13 \mathrm{E}-05$ & $6.03 \mathrm{E}-06$ & $5.21 \mathrm{E}-07$ \\
\hline & $\mathrm{Sr}$ (inc. 90Sr) & $0.00 \mathrm{E}+00$ & $1.83 \mathrm{E}-07$ & $1.83 \mathrm{E}-07$ & $3.29 \mathrm{E}-05$ & $2.19 \mathrm{E}-05$ & $1.39 \mathrm{E}-05$ & $1.36 \mathrm{E}-06$ & $2.18 \mathrm{E}-05$ & $2.13 \mathrm{E}-05$ & $6.13 \mathrm{E}-06$ & $6.17 \mathrm{E}-07$ \\
\hline & TOC & $1.60 \mathrm{E}-01$ & $1.68 \mathrm{E}-01$ & $1.67 \mathrm{E}-01$ & $3.91 \mathrm{E}-01$ & $3.17 \mathrm{E}-01$ & $2.84 \mathrm{E}-01$ & $6.02 \mathrm{E}-01$ & $9.17 \mathrm{E}-02$ & $8.24 \mathrm{E}-02$ & $2.06 \mathrm{E}-01$ & $1.69 \mathrm{E}-01$ \\
\hline & $\mathrm{U}(\mathrm{w} / \mathrm{o} 232,3,4,5,6$, and 8$)$ & $0.00 \mathrm{E}+00$ & $1.73 \mathrm{E}-08$ & $1.55 \mathrm{E}-08$ & $3.04 \mathrm{E}-08$ & $3.32 \mathrm{E}-09$ & $8.23 \mathrm{E}-08$ & $4.51 \mathrm{E}-08$ & $8.74 \mathrm{E}-10$ & $0.00 \mathrm{E}+00$ & $3.55 \mathrm{E}-07$ & $5.21 \mathrm{E}-07$ \\
\hline 2 & U (inc. all U isotopes) & $2.60 \mathrm{E}-04$ & 4.19E-05 & $3.88 \mathrm{E}-05$ & $3.83 \mathrm{E}-05$ & $6.26 \mathrm{E}-06$ & $6.82 \mathrm{E}-05$ & $5.85 \mathrm{E}-05$ & $8.08 \mathrm{E}-06$ & $7.16 \mathrm{E}-06$ & $4.11 \mathrm{E}-04$ & $5.52 \mathrm{E}-04$ \\
\hline 2 & $\mathrm{Zn}$ & $0.00 \mathrm{E}+00$ & $0.00 \mathrm{E}+00$ & $0.00 \mathrm{E}+00$ & $0.00 \mathrm{E}+00$ & $0.00 \mathrm{E}+00$ & $0.00 \mathrm{E}+00$ & $0.00 \mathrm{E}+00$ & $0.00 \mathrm{E}+00$ & $0.00 \mathrm{E}+00$ & $0.00 \mathrm{E}+00$ & $0.00 \mathrm{E}+00$ \\
\hline & $\mathrm{Zr}$ & $0.00 \mathrm{E}+00$ & $4.01 \mathrm{E}-05$ & $4.12 \mathrm{E}-05$ & $5.90 \mathrm{E}-05$ & $2.22 \mathrm{E}-05$ & $2.12 \mathrm{E}-05$ & $1.69 \mathrm{E}-05$ & $2.12 \mathrm{E}-05$ & $1.99 \mathrm{E}-05$ & $1.07 \mathrm{E}-04$ & $1.36 \mathrm{E}-04$ \\
\hline & Cations $=$ & 6.4215 & 6.9817 & 6.9759 & 7.0608 & 7.0922 & 4.6431 & 6.9009 & 6.9311 & 6.8742 & 7.4375 & 7.5521 \\
\hline & Anions $=$ & -6.4215 & -6.9817 & -6.9759 & -7.0608 & -7.0922 & -4.6431 & -6.9010 & -6.9311 & -6.8742 & -7.4375 & -7.5521 \\
\hline & Sum $=$ & 0.000 & 0.000 & 0.000 & 0.000 & 0.000 & 0.000 & 0.000 & 0.000 & 0.000 & 0.000 & 0.000 \\
\hline & $\mathrm{Na} / \mathrm{Cs}$ & $1.39 \mathrm{E}+05$ & $7.96 \mathrm{E}+04$ & $7.90 \mathrm{E}+04$ & $1.16 \mathrm{E}+05$ & $1.13 \mathrm{E}+05$ & $1.35 \mathrm{E}+05$ & $1.34 \mathrm{E}+05$ & $1.04 \mathrm{E}+05$ & $1.04 \mathrm{E}+05$ & $1.09 \mathrm{E}+05$ & $1.10 \mathrm{E}+05$ \\
\hline & $\mathrm{K} / \mathrm{Cs}$ & $1.98 \mathrm{E}+04$ & $9.67 \mathrm{E}+02$ & $9.87 \mathrm{E}+02$ & $1.67 \mathrm{E}+03$ & $1.36 \mathrm{E}+03$ & $1.14 \mathrm{E}+03$ & $1.11 \mathrm{E}+03$ & $2.56 \mathrm{E}+03$ & $2.59 \mathrm{E}+03$ & $7.00 \mathrm{E}+03$ & $8.93 \mathrm{E}+03$ \\
\hline
\end{tabular}




\begin{tabular}{|c|c|c|c|c|c|c|c|c|c|c|c|c|}
\hline & Batch Name & LAW-1 & LAW-5 & LAW-6 & LAW-8 & LAW-9 & LAW-10 & LAW-11 & LAW-12 & LAW-13 & LAW-14 & LAW-15 \\
\hline & Staging (Source) Tank & AP-101 & AN-104 & AN-104 & AN-105 & AN-105 & SY-101 & SY-101 & AN-103 & AN-103 & AW-101 & AW-101 \\
\hline & Total Volume of Liquid (L) & $4.12 E+06$ & $2.77 E+06$ & $2.57 \mathrm{E}+06$ & $2.66 \mathrm{E}+06$ & $2.57 E+06$ & $2.83 \mathrm{E}+06$ & $3.37 E+06$ & $3.49 \mathrm{E}+06$ & $3.52 \mathrm{E}+06$ & $2.82 \mathrm{E}+06$ & $3.84 \mathrm{E}+06$ \\
\hline Species & Concentration Units & {$[\mathbf{M}]$} & {$[\mathbf{M}]$} & {$[\mathbf{M}]$} & {$[\mathbf{M}]$} & {$[\mathbf{M}]$} & {$[\mathbf{M}]$} & {$[\mathbf{M}]$} & {$[\mathbf{M}]$} & {$[\mathbf{M}]$} & {$[\mathbf{M}]$} & {$[\mathbf{M}]$} \\
\hline Charge & LAW Envelope & $\mathbf{A}$ & $\mathbf{A}$ & $\mathbf{A}$ & $\mathbf{A}$ & $\mathbf{A}$ & $\mathbf{A}$ & $\mathbf{A}$ & $\mathbf{A}$ & $\mathbf{A}$ & $\mathbf{A}$ & $\mathbf{A}$ \\
\hline & 137-Cs/Total Cs & 0.25 & 0.25 & 0.25 & 0.25 & 0.25 & 0.25 & 0.25 & 0.25 & 0.25 & 0.25 & 0.25 \\
\hline
\end{tabular}

Table A-2. Best Basis Inventory (BBI) Phase 1 LAW feed solution data for Envelopes B and C where stated alterations were made to minimize inventory inconsistencies and to establish an ionic charge balance (a value of zero implies that no information was provided on species).

\begin{tabular}{|c|c|c|c|c|c|c|}
\hline & Batch Name & LAW-2a & LAW-2b & LAW-3 & LAW-4 & LAW-7 \\
\hline & Staging (Source) Tank & AZ-101 & AZ-102 & AN-102 & AN-102 & AN-107 \\
\hline & Total Volume of Liquid (L) & $3.02 \mathrm{E}+06$ & $3.17 \mathrm{E}+06$ & $2.34 E+06$ & $2.34 E+06$ & $3.62 E+06$ \\
\hline Species & Concentration Units & [M] & {$[\mathbf{M}]$} & {$[\mathbf{M}]$} & {$[\mathbf{M}]$} & {$[\mathbf{M}]$} \\
\hline Charge & LAW Envelope & B & B & $\mathrm{C}$ & $\mathrm{C}$ & $\mathrm{C}$ \\
\hline-1 & 129-I & $3.83 \mathrm{E}-02$ & $1.94 \mathrm{E}-02$ & $2.86 \mathrm{E}-05$ & $2.86 \mathrm{E}-05$ & $2.21 \mathrm{E}-05$ \\
\hline & $137-\mathrm{Cs}$ & $1.35 \mathrm{E}-04$ & 7.16E-05 & $1.79 \mathrm{E}-05$ & $1.71 \mathrm{E}-05$ & $1.93 \mathrm{E}-05$ \\
\hline 1 & Total Cs (137-Cs/iso fraction) & $4.50 \mathrm{E}-04$ & $2.39 \mathrm{E}-04$ & $7.18 \mathrm{E}-05$ & $6.84 \mathrm{E}-05$ & 7.73E-05 \\
\hline & 232-Th & $1.00 \mathrm{E}-09$ & $5.31 \mathrm{E}-09$ & $1.17 \mathrm{E}-03$ & $1.17 \mathrm{E}-03$ & $1.85 \mathrm{E}-03$ \\
\hline & $238-U$ & $2.12 \mathrm{E}-02$ & $6.35 \mathrm{E}-01$ & $6.11 \mathrm{E}-04$ & $6.11 \mathrm{E}-04$ & $5.36 \mathrm{E}-04$ \\
\hline & 99-Tc & $1.80 \mathrm{E}-03$ & $1.04 \mathrm{E}-03$ & $6.75 \mathrm{E}-05$ & $6.75 \mathrm{E}-05$ & $4.45 \mathrm{E}-05$ \\
\hline 3 & $\mathrm{Al}$ & $0.00 \mathrm{E}+00$ & $0.00 \mathrm{E}+00$ & $0.00 \mathrm{E}+00$ & $0.00 \mathrm{E}+00$ & $0.00 \mathrm{E}+00$ \\
\hline-1 & $\mathrm{Al}(\mathrm{OH})_{4}$ & $4.05 \mathrm{E}-01$ & $5.52 \mathrm{E}-02$ & 4.66E-01 & 4.66E-01 & $2.36 \mathrm{E}-01$ \\
\hline & As & $0.00 \mathrm{E}+00$ & $0.00 \mathrm{E}+00$ & $0.00 \mathrm{E}+00$ & $0.00 \mathrm{E}+00$ & $0.00 \mathrm{E}+00$ \\
\hline & B & $0.00 \mathrm{E}+00$ & $0.00 \mathrm{E}+00$ & $0.00 \mathrm{E}+00$ & $0.00 \mathrm{E}+00$ & $0.00 \mathrm{E}+00$ \\
\hline 2 & $\mathrm{Ba}$ & $1.97 \mathrm{E}-05$ & $3.10 \mathrm{E}-13$ & $1.81 \mathrm{E}-04$ & $1.81 \mathrm{E}-04$ & $0.00 \mathrm{E}+00$ \\
\hline & $\mathrm{Bi}$ & $0.00 \mathrm{E}+00$ & $0.00 \mathrm{E}+00$ & $0.00 \mathrm{E}+00$ & $0.00 \mathrm{E}+00$ & $2.42 \mathrm{E}-04$ \\
\hline 2 & $\mathrm{Ca}$ & $1.22 \mathrm{E}-05$ & $1.09 \mathrm{E}-04$ & $1.25 \mathrm{E}-02$ & $1.25 \mathrm{E}-02$ & $1.31 \mathrm{E}-02$ \\
\hline 2 & $\mathrm{Cd}$ & $4.19 \mathrm{E}-05$ & $6.60 \mathrm{E}-13$ & $4.70 \mathrm{E}-04$ & $4.66 \mathrm{E}-04$ & $0.00 \mathrm{E}+00$ \\
\hline 3 & $\mathrm{Ce}$ & $0.00 \mathrm{E}+00$ & $0.00 \mathrm{E}+00$ & $0.00 \mathrm{E}+00$ & $0.00 \mathrm{E}+00$ & $0.00 \mathrm{E}+00$ \\
\hline-1 & $\mathrm{Cl}$ & $5.62 \mathrm{E}-03$ & $2.62 \mathrm{E}-04$ & $8.68 \mathrm{E}-02$ & $8.68 \mathrm{E}-02$ & $4.83 \mathrm{E}-02$ \\
\hline
\end{tabular}




\begin{tabular}{|c|c|c|c|c|c|c|}
\hline & Batch Name & LAW-2a & LAW-2b & LAW-3 & LAW-4 & LAW-7 \\
\hline & Staging (Source) Tank & AZ-101 & AZ-102 & AN-102 & AN-102 & AN-107 \\
\hline & Total Volume of Liquid (L) & $3.02 E+06$ & $3.17 E+06$ & $2.34 E+06$ & $2.34 \mathrm{E}+06$ & $3.62 \mathrm{E}+06$ \\
\hline Species & Concentration Units & [M] & [M] & [M] & {$[\mathbf{M}]$} & {$[\mathbf{M}]$} \\
\hline Charge & LAW Envelope & B & B & $\mathbf{C}$ & $\mathbf{C}$ & $\mathbf{C}$ \\
\hline-2 & $\mathrm{CO}_{3}$ & $5.69 \mathrm{E}-01$ & $5.73 \mathrm{E}-01$ & $9.23 \mathrm{E}-01$ & $9.23 \mathrm{E}-01$ & $1.14 \mathrm{E}+00$ \\
\hline 3 & $\mathrm{Cr}$ & $0.00 \mathrm{E}+00$ & $0.00 \mathrm{E}+00$ & $0.00 \mathrm{E}+00$ & $0.00 \mathrm{E}+00$ & $0.00 \mathrm{E}+00$ \\
\hline-1 & $\mathrm{Cr}(\mathrm{OH})_{4}$ & $1.49 \mathrm{E}-02$ & $1.91 \mathrm{E}-02$ & $6.54 \mathrm{E}-03$ & $6.54 \mathrm{E}-03$ & $5.36 \mathrm{E}-03$ \\
\hline-1 & $\mathrm{~F}$ & $9.91 \mathrm{E}-02$ & $5.47 \mathrm{E}-02$ & $7.86 \mathrm{E}-02$ & $7.86 \mathrm{E}-02$ & $1.63 \mathrm{E}-01$ \\
\hline 2 & $\mathrm{Fe}$ & $2.01 \mathrm{E}-04$ & $1.89 \mathrm{E}-04$ & $3.15 \mathrm{E}-03$ & $3.15 \mathrm{E}-03$ & $4.61 \mathrm{E}-02$ \\
\hline \multirow[t]{3}{*}{1} & $\mathrm{H}$ & $6.50 \mathrm{E}-14$ & $6.64 \mathrm{E}-14$ & $5.34 \mathrm{E}-15$ & $5.34 \mathrm{E}-15$ & $8.06 \mathrm{E}-15$ \\
\hline & $\mathrm{H}_{2} \mathrm{O}$ & $4.95 \mathrm{E}+01$ & $5.31 \mathrm{E}+01$ & $2.08 \mathrm{E}+02$ & $2.07 \mathrm{E}+02$ & $1.17 \mathrm{E}+02$ \\
\hline & $\mathrm{Hg}$ & $0.00 \mathrm{E}+00$ & $0.00 \mathrm{E}+00$ & $0.00 \mathrm{E}+00$ & $0.00 \mathrm{E}+00$ & $1.66 \mathrm{E}-06$ \\
\hline 1 & $\mathrm{~K}$ & $1.20 \mathrm{E}-01$ & 7.92E-02 & $7.95 \mathrm{E}-02$ & $7.95 \mathrm{E}-02$ & $3.87 \mathrm{E}-02$ \\
\hline 3 & $\mathrm{La}$ & $2.25 \mathrm{E}-10$ & $1.95 \mathrm{E}-05$ & $0.00 \mathrm{E}+00$ & $0.00 \mathrm{E}+00$ & $2.29 \mathrm{E}-04$ \\
\hline 2 & $\mathrm{Mn}$ & $2.46 \mathrm{E}-07$ & $1.30 \mathrm{E}-05$ & $1.26 \mathrm{E}-03$ & $1.26 \mathrm{E}-03$ & $9.92 \mathrm{E}-03$ \\
\hline \multirow[t]{2}{*}{1} & $\mathrm{Na}$ & $4.82 \mathrm{E}+00$ & $2.77 \mathrm{E}+00$ & $8.97 \mathrm{E}+00$ & $8.97 \mathrm{E}+00$ & $8.45 \mathrm{E}+00$ \\
\hline & $\mathrm{NH}_{3}$ & $1.76 \mathrm{E}-02$ & $7.88 \mathrm{E}-03$ & $7.01 \mathrm{E}-02$ & $7.01 \mathrm{E}-02$ & 4.09E-02 \\
\hline 2 & $\mathrm{Ni}$ & $8.92 \mathrm{E}-07$ & $3.56 \mathrm{E}-05$ & $5.56 \mathrm{E}-03$ & $5.56 \mathrm{E}-03$ & $7.71 \mathrm{E}-03$ \\
\hline-1 & $\mathrm{NO}_{2}$ & $1.43 \mathrm{E}+00$ & $6.55 \mathrm{E}-01$ & $1.44 \mathrm{E}+00$ & $1.44 \mathrm{E}+00$ & $1.21 \mathrm{E}+00$ \\
\hline \multirow[t]{3}{*}{-1} & $\mathrm{NO}_{3}$ & $1.23 \mathrm{E}+00$ & 3.73E-01 & $2.92 \mathrm{E}+00$ & $2.92 \mathrm{E}+00$ & $3.20 \mathrm{E}+00$ \\
\hline & OH(BOUND) & $9.92 \mathrm{E}-01$ & $1.40 \mathrm{E}-02$ & $2.83 \mathrm{E}+00$ & $2.83 \mathrm{E}+00$ & $9.61 \mathrm{E}-01$ \\
\hline & OH- & $1.01 \mathrm{E}+00$ & $1.26 \mathrm{E}-01$ & $3.80 \mathrm{E}-01$ & $3.80 \mathrm{E}-01$ & $1.54 \mathrm{E}+00$ \\
\hline-1 & OH- (Bound+Free-4Al-4Cr) & $1.54 \mathrm{E}-01$ & $1.51 \mathrm{E}-01$ & $1.87 \mathrm{E}+00$ & $1.87 \mathrm{E}+00$ & $1.24 \mathrm{E}+00$ \\
\hline 2 & $\mathrm{~Pb}$ & $0.00 \mathrm{E}+00$ & $0.00 \mathrm{E}+00$ & $7.78 \mathrm{E}-04$ & $7.78 \mathrm{E}-04$ & $1.76 \mathrm{E}-03$ \\
\hline \multirow[t]{2}{*}{-3} & $\mathrm{PO}_{4}$ & $1.55 \mathrm{E}-02$ & $1.41 \mathrm{E}-03$ & 4.14E-02 & $4.14 \mathrm{E}-02$ & $3.54 \mathrm{E}-02$ \\
\hline & $\mathrm{Si}$ & $1.31 \mathrm{E}-02$ & $1.89 \mathrm{E}-02$ & $4.53 \mathrm{E}-03$ & $4.53 \mathrm{E}-03$ & $2.18 \mathrm{E}-03$ \\
\hline \multirow[t]{5}{*}{-2} & $\mathrm{SO}_{4}$ & $1.89 \mathrm{E}-01$ & $1.87 \mathrm{E}-01$ & $1.32 \mathrm{E}-01$ & $1.32 \mathrm{E}-01$ & $8.15 \mathrm{E}-02$ \\
\hline & $\mathrm{Sr}(\mathrm{w} / \mathrm{o} 90 \mathrm{Sr})$ & $1.03 \mathrm{E}-05$ & $2.11 \mathrm{E}-06$ & $3.42 \mathrm{E}-05$ & $3.42 \mathrm{E}-05$ & $6.69 \mathrm{E}-05$ \\
\hline & $\mathrm{Sr}$ (inc. 90Sr) & $1.33 \mathrm{E}-05$ & $2.13 \mathrm{E}-06$ & $3.85 \mathrm{E}-05$ & $3.80 \mathrm{E}-05$ & $7.38 \mathrm{E}-05$ \\
\hline & TOC & $1.20 \mathrm{E}-01$ & $1.31 \mathrm{E}-01$ & $1.78 \mathrm{E}+00$ & $1.78 \mathrm{E}+00$ & $2.93 \mathrm{E}+00$ \\
\hline & $\mathrm{U}(\mathrm{w} / \mathrm{o} 232,3,4,5,6$, and 8$)$ & $2.84 \mathrm{E}-08$ & $2.14 \mathrm{E}-07$ & $4.27 \mathrm{E}-07$ & $4.27 \mathrm{E}-07$ & $0.00 \mathrm{E}+00$ \\
\hline 2 & U (inc. all U isotopes) & $2.14 \mathrm{E}-05$ & $6.42 \mathrm{E}-04$ & $6.15 \mathrm{E}-04$ & $6.15 \mathrm{E}-04$ & 5.39E-04 \\
\hline
\end{tabular}




\begin{tabular}{|c|c|c|c|c|c|c|}
\hline & Batch Name & LAW-2a & LAW-2b & LAW-3 & LAW-4 & LAW-7 \\
\hline & Staging (Source) Tank & AZ-101 & AZ-102 & AN-102 & AN-102 & AN-107 \\
\hline & Total Volume of Liquid (L) & $3.02 E+06$ & $3.17 E+06$ & $2.34 \mathrm{E}+06$ & $2.34 \mathrm{E}+06$ & $3.62 \mathrm{E}+06$ \\
\hline Species & Concentration Units & {$[\mathbf{M}]$} & {$[\mathbf{M}]$} & {$[\mathbf{M}]$} & {$[\mathbf{M}]$} & {$[\mathbf{M}]$} \\
\hline Charge & LAW Envelope & B & B & $\mathbf{C}$ & C & $\mathbf{C}$ \\
\hline \multirow[t]{8}{*}{2} & $\mathrm{Zn}$ & $0.00 \mathrm{E}+00$ & $0.00 \mathrm{E}+00$ & $0.00 \mathrm{E}+00$ & $0.00 \mathrm{E}+00$ & $0.00 \mathrm{E}+00$ \\
\hline & $\mathrm{Zr}$ & $0.00 \mathrm{E}+00$ & $4.53 \mathrm{E}-05$ & $5.77 \mathrm{E}-04$ & $5.77 \mathrm{E}-04$ & $1.00 \mathrm{E}-03$ \\
\hline & cations $=$ & 4.9364 & 2.8513 & 9.1030 & 9.1030 & 8.6508 \\
\hline & anions $=$ & -4.9364 & -2.8513 & -9.1030 & -9.1030 & -8.6508 \\
\hline & sum $=$ & 0.000 & 0.000 & 0.000 & 0.000 & 0.000 \\
\hline & $\mathrm{Na} / \mathrm{Cs}$ & $1.07 \mathrm{E}+04$ & $1.16 \mathrm{E}+04$ & $1.25 \mathrm{E}+05$ & $1.31 \mathrm{E}+05$ & $1.09 \mathrm{E}+05$ \\
\hline & $\mathrm{K} / \mathrm{Cs}$ & $2.67 \mathrm{E}+02$ & $3.32 \mathrm{E}+02$ & $1.11 \mathrm{E}+03$ & $1.16 \mathrm{E}+03$ & $5.00 \mathrm{E}+02$ \\
\hline & 137-Cs/Total Cs & 0.30 & 0.30 & 0.25 & 0.25 & 0.25 \\
\hline
\end{tabular}

Table A-3. Best Basis Inventory (BBI) Phase 1 LAW feed solution data for Envelope C where the impact from the isotopic dilution pretreatment process for Strontium-90 and permanganate addition for TRUs separation have been taken into account.

\begin{tabular}{|c|c|c|c|c|}
\cline { 2 - 5 } \multicolumn{1}{c|}{} & \multicolumn{1}{c|}{ Batch Name } & LAW-3 & LAW-4 & LAW-7 \\
\cline { 2 - 5 } \multicolumn{1}{c|}{ Staging (Source) Tank } & AN-102 & AN-102 & AN-107 \\
\cline { 2 - 5 } \multicolumn{1}{c|}{ Total Volume of Liquid (L) } & $\mathbf{3 . 4 7 E + 0 6}$ & $\mathbf{3 . 4 7 E + 0 6}$ & $\mathbf{4 . 4 6 E + 0 6}$ \\
\hline Species & \multicolumn{1}{|c|}{ Concentration Units } & {$[\mathbf{M}]$} & {$[\mathbf{M}]$} & {$[\mathbf{M}]$} \\
\hline Charge & \multicolumn{1}{|c|}{ LAW Envelope } & $\mathbf{C}$ & $\mathbf{C}$ & $\mathbf{C}$ \\
\hline-1 & $129-\mathrm{I}$ & $1.91 \mathrm{E}-05$ & $1.91 \mathrm{E}-05$ & $1.57 \mathrm{E}-05$ \\
\hline & $137-\mathrm{Cs}$ & $1.20 \mathrm{E}-05$ & $1.14 \mathrm{E}-05$ & $1.37 \mathrm{E}-05$ \\
\hline 1 & Total Cs (137-Cs/iso fract) & $4.80 \mathrm{E}-05$ & $4.57 \mathrm{E}-05$ & $5.49 \mathrm{E}-05$ \\
\hline & $232-T h$ & $7.83 \mathrm{E}-04$ & $7.83 \mathrm{E}-04$ & $1.31 \mathrm{E}-03$ \\
\hline & $238-\mathrm{U}$ & $4.09 \mathrm{E}-04$ & $4.09 \mathrm{E}-04$ & $3.80 \mathrm{E}-04$ \\
\hline & $99-\mathrm{Tc}$ & $4.51 \mathrm{E}-05$ & $4.51 \mathrm{E}-05$ & $3.16 \mathrm{E}-05$ \\
\hline 3 & Al & $0.00 \mathrm{E}+00$ & $0.00 \mathrm{E}+00$ & $0.00 \mathrm{E}+00$ \\
\hline
\end{tabular}




\section{WESTINGHOUSE SAVANNAH RIVER COMPANY}

Preliminary Ion Exchange Modeling for Removal of Cesium from

Hanford Waste Using Hydrous Crystalline Silicotitanate Material
Report:

Revision (Date):

Page:
WSRC-TR-2001-00400 $0(07 / 05 / 02)$ 157 of 338

\begin{tabular}{|c|c|c|c|c|}
\hline & Batch Name & LAW-3 & LAW-4 & LAW-7 \\
\hline & Staging (Source) Tank & AN-102 & AN-102 & AN-107 \\
\hline & Total Volume of Liquid (L) & $3.47 \mathrm{E}+06$ & $3.47 \mathrm{E}+06$ & $4.46 E+06$ \\
\hline Species & Concentration Units & {$[\mathbf{M}]$} & {$[\mathbf{M}]$} & {$[\mathbf{M}]$} \\
\hline Charge & LAW Envelope & $\mathbf{C}$ & $\mathbf{C}$ & $\mathbf{C}$ \\
\hline \multirow[t]{3}{*}{-1} & $\mathrm{Al}\left(\mathrm{OH}_{4}\right.$ & $3.11 \mathrm{E}-01$ & $3.11 \mathrm{E}-01$ & $1.68 \mathrm{E}-01$ \\
\hline & As & $0.00 \mathrm{E}+00$ & $0.00 \mathrm{E}+00$ & $0.00 \mathrm{E}+00$ \\
\hline & B & $0.00 \mathrm{E}+00$ & $0.00 \mathrm{E}+00$ & $0.00 \mathrm{E}+00$ \\
\hline \multirow[t]{2}{*}{2} & $\mathrm{Ba}$ & $1.21 \mathrm{E}-04$ & $1.21 \mathrm{E}-04$ & $0.00 \mathrm{E}+00$ \\
\hline & $\mathrm{Bi}$ & $0.00 \mathrm{E}+00$ & $0.00 \mathrm{E}+00$ & $1.72 \mathrm{E}-04$ \\
\hline 2 & $\mathrm{Ca}$ & $8.37 \mathrm{E}-03$ & $8.37 \mathrm{E}-03$ & $9.31 \mathrm{E}-03$ \\
\hline 2 & $\mathrm{Cd}$ & $3.14 \mathrm{E}-04$ & $3.11 \mathrm{E}-04$ & $0.00 \mathrm{E}+00$ \\
\hline 3 & $\mathrm{Ce}$ & $0.00 \mathrm{E}+00$ & $0.00 \mathrm{E}+00$ & $0.00 \mathrm{E}+00$ \\
\hline-1 & $\mathrm{Cl}$ & $5.80 \mathrm{E}-02$ & $5.80 \mathrm{E}-02$ & $3.43 \mathrm{E}-02$ \\
\hline-2 & $\mathrm{CO}_{3}$ & $6.17 \mathrm{E}-01$ & $6.17 \mathrm{E}-01$ & $8.08 \mathrm{E}-01$ \\
\hline 3 & $\mathrm{Cr}$ & $0.00 \mathrm{E}+00$ & $0.00 \mathrm{E}+00$ & $0.00 \mathrm{E}+00$ \\
\hline-1 & $\mathrm{Cr}(\mathrm{OH})_{4}$ & $4.37 \mathrm{E}-03$ & $4.37 \mathrm{E}-03$ & $3.80 \mathrm{E}-03$ \\
\hline-1 & $\mathrm{~F}$ & $5.26 \mathrm{E}-02$ & $5.26 \mathrm{E}-02$ & $1.16 \mathrm{E}-01$ \\
\hline 2 & $\mathrm{Fe}$ & $2.11 \mathrm{E}-03$ & $2.11 \mathrm{E}-03$ & $3.27 \mathrm{E}-02$ \\
\hline \multirow[t]{3}{*}{1} & $\mathrm{H}$ & $8.00 \mathrm{E}-15$ & $8.00 \mathrm{E}-15$ & $1.02 \mathrm{E}-14$ \\
\hline & $\mathrm{H}_{2} \mathrm{O}$ & $1.39 \mathrm{E}+02$ & $1.38 \mathrm{E}+02$ & $8.27 \mathrm{E}+01$ \\
\hline & $\mathrm{Hg}$ & $0.00 \mathrm{E}+00$ & $0.00 \mathrm{E}+00$ & $1.18 \mathrm{E}-06$ \\
\hline 1 & $\mathrm{~K}$ & $5.31 \mathrm{E}-02$ & $5.31 \mathrm{E}-02$ & $2.75 \mathrm{E}-02$ \\
\hline 3 & $\mathrm{La}$ & $0.00 \mathrm{E}+00$ & $0.00 \mathrm{E}+00$ & $1.62 \mathrm{E}-04$ \\
\hline 2 & $\mathrm{Mn}$ & $5.00 \mathrm{E}-02$ & $5.00 \mathrm{E}-02$ & $5.00 \mathrm{E}-02$ \\
\hline \multirow[t]{2}{*}{1} & $\mathrm{Na}$ & $6.05 \mathrm{E}+00$ & $6.05 \mathrm{E}+00$ & $6.16 \mathrm{E}+00$ \\
\hline & $\mathrm{NH}_{3}$ & $4.69 \mathrm{E}-02$ & $4.69 \mathrm{E}-02$ & $2.90 \mathrm{E}-02$ \\
\hline 2 & $\mathrm{Ni}$ & $3.71 \mathrm{E}-03$ & $3.71 \mathrm{E}-03$ & $5.47 \mathrm{E}-03$ \\
\hline-1 & $\mathrm{NO}_{2}$ & $9.63 \mathrm{E}-01$ & $9.63 \mathrm{E}-01$ & $8.57 \mathrm{E}-01$ \\
\hline \multirow[t]{3}{*}{-1} & $\mathrm{NO}_{3}$ & $2.10 \mathrm{E}+00$ & $2.10 \mathrm{E}+00$ & $2.42 \mathrm{E}+00$ \\
\hline & $\mathrm{OH}(\mathrm{BOUND})$ & $1.89 \mathrm{E}+00$ & $1.89 \mathrm{E}+00$ & $6.82 \mathrm{E}-01$ \\
\hline & $\mathrm{OH}-$ & $2.54 \mathrm{E}-01$ & $2.54 \mathrm{E}-01$ & $1.09 \mathrm{E}+00$ \\
\hline-1 & OH- (Bound+Free-4Al-4Cr) & $1.25 \mathrm{E}+00$ & $1.25 \mathrm{E}+00$ & $9.79 \mathrm{E}-01$ \\
\hline
\end{tabular}




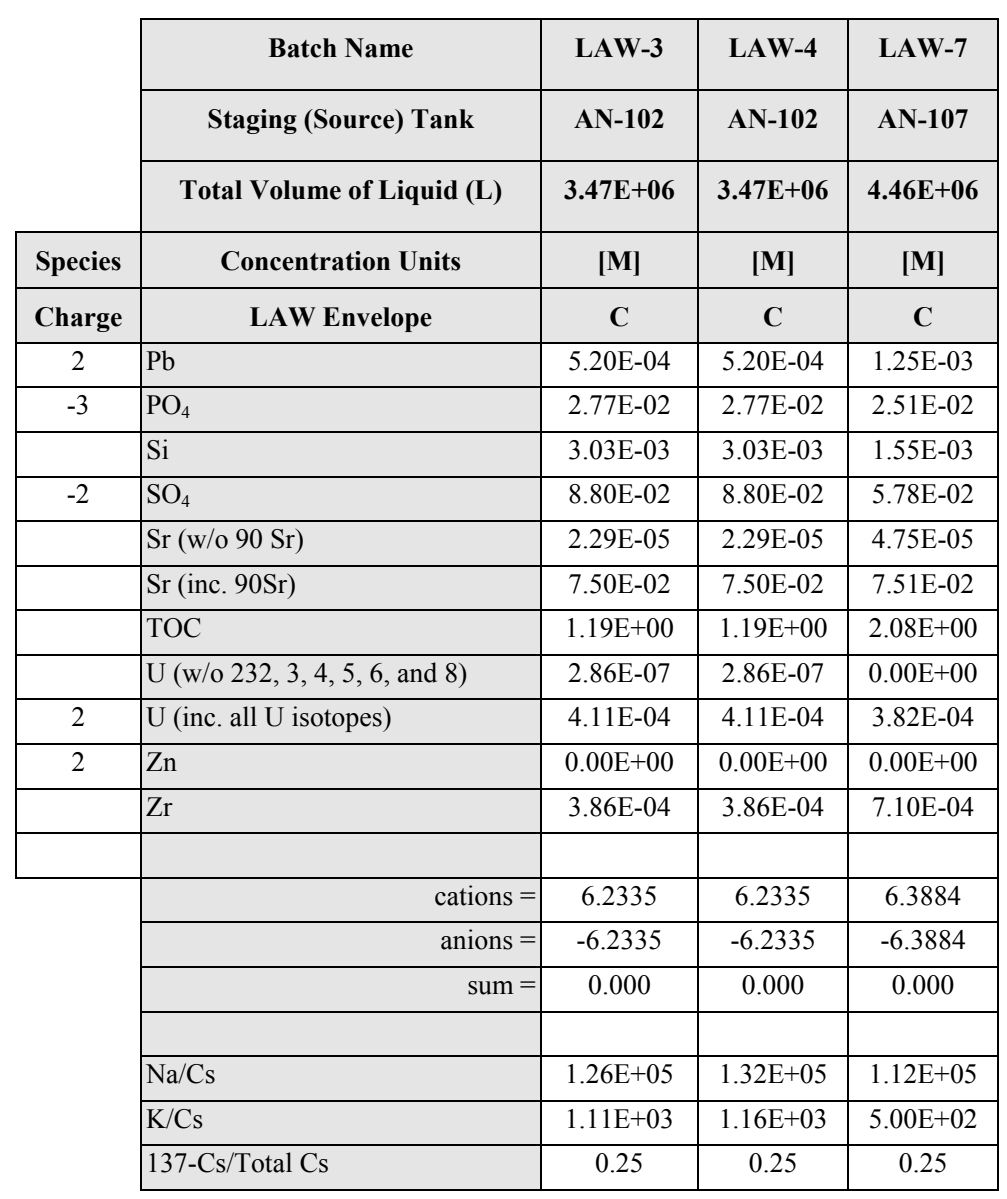


Table A-4. Ionic species available within the ZAM CST ion-exchange equilibrium model.

\begin{tabular}{|c|c|c|}
\hline ID & Cations & Anions \\
\hline 1 & $\mathrm{H}^{+}$ & $\mathrm{F}^{-}$ \\
\hline 2 & $\mathrm{Li}^{+}$ & $\mathrm{Cl}^{-}$ \\
\hline 3 & $\mathrm{Na}^{+}$ & $\mathrm{Br}^{-}$ \\
\hline 4 & $\mathrm{~K}^{+}$ & $\mathrm{I}^{-}$ \\
\hline 5 & $\mathrm{Rb}^{+}$ & $\mathrm{ClO}_{3}{ }^{-}$ \\
\hline 6 & $\mathrm{Cs}^{+}$ & $\mathrm{ClO}^{-}$ \\
\hline 7 & $\mathrm{NH}_{4}{ }^{+}$ & $\mathrm{BrO}_{3}{ }^{-}$ \\
\hline 8 & $\mathrm{Tl}^{+}$ & $\mathrm{IO}_{3}{ }^{-}$ \\
\hline 9 & $\mathrm{Ag}^{+}$ & $\mathrm{NO}_{3}{ }^{-}$ \\
\hline 10 & $\mathrm{Be}^{2+}$ & $\mathrm{H}_{2} \mathrm{PO}_{4}^{-}$ \\
\hline 11 & $\mathrm{Mg}^{2+}$ & $\mathrm{H}_{2} \mathrm{AsO}_{4}{ }^{-}$ \\
\hline 12 & $\mathrm{Ca}^{2+}$ & $\mathrm{CNS}^{-}$ \\
\hline 13 & $\mathrm{Sr}^{2+}$ & $\mathrm{OH}^{-}$ \\
\hline 14 & $\mathrm{Ba}^{2+}$ & $\mathrm{CrO}_{4}{ }^{2-}$ \\
\hline 15 & $\mathrm{Mn}^{2+}$ & $\mathrm{SO}_{4}{ }^{2-}$ \\
\hline 16 & $\mathrm{Fe}^{2+}$ & $\mathrm{S}_{2} \mathrm{O}_{3}{ }^{2-}$ \\
\hline 17 & $\mathrm{Co}^{2+}$ & $\mathrm{HPO}_{4}{ }^{2-}$ \\
\hline 18 & $\mathrm{Ni}^{2+}$ & $\mathrm{HAsO}_{4}{ }^{2-}$ \\
\hline $\mathrm{Cu}^{2+}$ & $\mathrm{CO}_{3}{ }^{2-}$ \\
\hline $\mathrm{Zn}^{2+}$ & $\mathrm{PO}_{4}{ }^{3-}$ \\
\hline
\end{tabular}

\begin{tabular}{|c|c|c|}
\hline ID & Cations & Anions \\
\hline 21 & $\mathrm{Cd}^{2+}$ & $\mathrm{AsO}_{4}{ }^{3-}$ \\
\hline 22 & $\mathrm{~Pb}^{2+}$ & $\mathrm{Fe}(\mathrm{CN})_{6}{ }^{3-}$ \\
\hline 23 & $\mathrm{UO}_{2}{ }^{2+}$ & $\mathrm{Mo}(\mathrm{CN})_{8}^{3-}$ \\
\hline 24 & $\mathrm{Cr}^{3+}$ & User defined \\
\hline 25 & $\mathrm{Al}^{3+}$ & User defined \\
\hline 26 & $\mathrm{Sc}^{3+}$ & User defined \\
\hline 27 & $\mathrm{Y}^{3+}$ & $\mathrm{NO}_{2}^{-}$ \\
\hline 28 & $\mathrm{La}^{3+}$ & $\mathrm{Al}(\mathrm{OH})_{4}^{-}$ \\
\hline 29 & $\mathrm{Ce}^{3+}$ & $\mathrm{na}^{\mathrm{b}}$ \\
\hline 30 & $\mathrm{Pr}^{3+}$ & na \\
\hline 31 & $\mathrm{Nd}^{3+}$ & na \\
\hline 32 & $\mathrm{Sm}^{3+}$ & na \\
\hline 33 & $\mathrm{Eu}^{3+}$ & na \\
\hline 34 & $\mathrm{Ga}^{3+}$ & na \\
\hline 35 & $\mathrm{Co}^{3+}$ & na \\
\hline 36 & $\mathrm{Th}^{4+}$ & na \\
\hline 37 & User defined $^{2}$ & na \\
\hline 38 & User defined & na \\
\hline 39 & User defined & na \\
\hline 40 & $\mathrm{SrOH}^{+}$ & na \\
\hline
\end{tabular}

Array locations in storage that are available for user to specify additional species.

${ }^{\mathrm{b}}$ Array locations in storage that are currently unused. 
Table A-5. Best estimate Phase 1 LAW feed solution data for Envelope A adjusted to $5 \mathrm{M} \mathrm{Na}{ }^{\text {a }}$ and used as input to the CST equilibrium model.

\begin{tabular}{|c|c|c|c|c|c|c|c|c|c|c|c|c|}
\hline & Batch Name & LAW-1 & LAW-5 & LAW-6 & LAW-8 & LAW-9 & LAW-10 & LAW-11 & LAW-12 & LAW-13 & LAW-14 & LAW-15 \\
\hline & Staging (Source) Tank & AP-101 & AN-104 & AN-104 & AN-105 & AN-105 & SY-101 & SY-101 & AN-103 & AN-103 & AW-101 & AW-101 \\
\hline & Total Volume of Liquid (L) & $4.63 E+06$ & $3.82 \mathrm{E}+06$ & $3.54 \mathrm{E}+06$ & $3.70 \mathrm{E}+06$ & $3.60 \mathrm{E}+06$ & $2.60 \mathrm{E}+06$ & $4.60 \mathrm{E}+06$ & $4.72 E+06$ & $4.72 E+06$ & $3.94 \mathrm{E}+06$ & $5.36 \mathrm{E}+06$ \\
\hline Species & Concentration Units & {$[\mathbf{M}]$} & {$[\mathbf{M}]$} & {$[\mathbf{M}]$} & {$[\mathbf{M}]$} & {$[\mathbf{M}]$} & {$[\mathbf{M}]$} & {$[\mathbf{M}]$} & {$[\mathbf{M}]$} & {$[\mathbf{M}]$} & {$[\mathbf{M}]$} & {$[\mathbf{M}]$} \\
\hline Charge & LAW Envelope & $\mathbf{A}$ & $\mathbf{A}$ & $\mathbf{A}$ & $\mathbf{A}$ & $\mathbf{A}$ & $\mathbf{A}$ & $\mathbf{A}$ & $\mathbf{A}$ & $\mathbf{A}$ & $\mathbf{A}$ & $\mathbf{A}$ \\
\hline \multicolumn{13}{|l|}{ Cations } \\
\hline 1 & $\mathrm{Na}$ & $5.00 \mathrm{E}+00$ & $5.00 \mathrm{E}+00$ & $5.00 \mathrm{E}+00$ & $5.00 \mathrm{E}+00$ & $5.00 \mathrm{E}+00$ & $5.00 \mathrm{E}+00$ & $5.00 \mathrm{E}+00$ & $5.00 \mathrm{E}+00$ & $5.00 \mathrm{E}+00$ & $5.00 \mathrm{E}+00$ & $5.00 \mathrm{E}+00$ \\
\hline 1 & Cs (total) & $3.60 \mathrm{E}-05$ & $6.28 \mathrm{E}-05$ & $6.33 \mathrm{E}-05$ & $4.32 \mathrm{E}-05$ & $4.44 \mathrm{E}-05$ & $3.69 \mathrm{E}-05$ & $3.74 \mathrm{E}-05$ & $4.83 \mathrm{E}-05$ & $4.83 \mathrm{E}-05$ & $4.57 \mathrm{E}-05$ & $4.55 \mathrm{E}-05$ \\
\hline 1 & $\mathrm{H}$ & $6.44 \mathrm{E}-15$ & $1.16 \mathrm{E}-14$ & $1.05 \mathrm{E}-14$ & $7.44 \mathrm{E}-15$ & $1.74 \mathrm{E}-14$ & $1.15 \mathrm{E}-14$ & $1.07 \mathrm{E}-14$ & $6.48 \mathrm{E}-15$ & $6.48 \mathrm{E}-15$ & $5.72 \mathrm{E}-15$ & $4.67 \mathrm{E}-15$ \\
\hline 1 & $\mathrm{Rb}$ & $0.00 \mathrm{E}+00$ & $0.00 \mathrm{E}+00$ & $0.00 \mathrm{E}+00$ & $0.00 \mathrm{E}+00$ & $0.00 \mathrm{E}+00$ & $0.00 \mathrm{E}+00$ & $0.00 \mathrm{E}+00$ & $0.00 \mathrm{E}+00$ & $0.00 \mathrm{E}+00$ & $0.00 \mathrm{E}+00$ & $0.00 \mathrm{E}+00$ \\
\hline 1 & $\mathrm{~K}$ & $7.12 \mathrm{E}-01$ & $6.07 \mathrm{E}-02$ & $6.24 \mathrm{E}-02$ & $7.22 \mathrm{E}-02$ & $6.03 \mathrm{E}-02$ & $4.19 \mathrm{E}-02$ & $4.15 \mathrm{E}-02$ & $1.24 \mathrm{E}-01$ & $1.25 \mathrm{E}-01$ & $3.20 \mathrm{E}-01$ & $4.07 \mathrm{E}-01$ \\
\hline 1 & $\mathrm{SrOH}$ & $0.00 \mathrm{E}+00$ & $0.00 \mathrm{E}+00$ & $0.00 \mathrm{E}+00$ & $0.00 \mathrm{E}+00$ & $0.00 \mathrm{E}+00$ & $0.00 \mathrm{E}+00$ & $0.00 \mathrm{E}+00$ & $0.00 \mathrm{E}+00$ & $0.00 \mathrm{E}+00$ & $0.00 \mathrm{E}+00$ & $0.00 \mathrm{E}+00$ \\
\hline 2 & Sr (total) & $0.00 \mathrm{E}+00$ & $0.00 \mathrm{E}+00$ & $0.00 \mathrm{E}+00$ & $0.00 \mathrm{E}+00$ & $0.00 \mathrm{E}+00$ & $0.00 \mathrm{E}+00$ & $0.00 \mathrm{E}+00$ & $0.00 \mathrm{E}+00$ & $0.00 \mathrm{E}+00$ & $0.00 \mathrm{E}+00$ & $0.00 \mathrm{E}+00$ \\
\hline 2 & $\mathrm{Ba}$ & $2.14 \mathrm{E}-06$ & $5.97 \mathrm{E}-05$ & $6.36 \mathrm{E}-05$ & $9.08 \mathrm{E}-05$ & $4.14 \mathrm{E}-05$ & $0.00 \mathrm{E}+00$ & $1.96 \mathrm{E}-06$ & $5.57 \mathrm{E}-05$ & $5.47 \mathrm{E}-05$ & $1.04 \mathrm{E}-05$ & $0.00 \mathrm{E}+00$ \\
\hline 2 & $\mathrm{Ca}$ & $1.69 \mathrm{E}-04$ & $4.79 \mathrm{E}-04$ & $4.97 \mathrm{E}-04$ & $1.06 \mathrm{E}-03$ & $1.05 \mathrm{E}-03$ & $1.63 \mathrm{E}-03$ & $2.22 \mathrm{E}-03$ & $3.35 \mathrm{E}-04$ & $3.14 \mathrm{E}-04$ & $9.09 \mathrm{E}-04$ & $8.60 \mathrm{E}-04$ \\
\hline 2 & $\mathrm{Cd}$ & $1.51 \mathrm{E}-05$ & $7.33 \mathrm{E}-06$ & $7.63 \mathrm{E}-06$ & $2.46 \mathrm{E}-05$ & $4.17 \mathrm{E}-06$ & $0.00 \mathrm{E}+00$ & $2.17 \mathrm{E}-07$ & $9.53 \mathrm{E}-06$ & $9.11 \mathrm{E}-06$ & $1.02 \mathrm{E}-06$ & $0.00 \mathrm{E}+00$ \\
\hline 2 & $\mathrm{Mn}$ & $0.00 \mathrm{E}+00$ & $3.43 \mathrm{E}-05$ & $3.59 \mathrm{E}-05$ & $7.95 \mathrm{E}-05$ & 2.19E-05 & $6.69 \mathrm{E}-05$ & $8.41 \mathrm{E}-05$ & $2.01 \mathrm{E}-05$ & $1.84 \mathrm{E}-05$ & $5.05 \mathrm{E}-05$ & $6.03 \mathrm{E}-05$ \\
\hline 2 & $\mathrm{Ni}$ & $1.25 \mathrm{E}-04$ & $6.15 \mathrm{E}-05$ & $6.47 \mathrm{E}-05$ & $2.66 \mathrm{E}-04$ & $7.78 \mathrm{E}-05$ & $8.35 \mathrm{E}-04$ & $1.46 \mathrm{E}-03$ & $4.17 \mathrm{E}-05$ & $3.50 \mathrm{E}-05$ & $1.00 \mathrm{E}-04$ & $1.08 \mathrm{E}-04$ \\
\hline 2 & $\mathrm{U}$ (total) & $2.31 \mathrm{E}-04$ & $3.04 \mathrm{E}-05$ & $2.82 \mathrm{E}-05$ & $2.76 \mathrm{E}-05$ & $4.47 \mathrm{E}-06$ & $7.42 \mathrm{E}-05$ & $4.28 \mathrm{E}-05$ & $5.97 \mathrm{E}-06$ & $5.34 \mathrm{E}-06$ & $2.94 \mathrm{E}-04$ & $3.96 \mathrm{E}-04$ \\
\hline 2 & $\mathrm{Zn}$ & $0.00 \mathrm{E}+00$ & $0.00 \mathrm{E}+00$ & $0.00 \mathrm{E}+00$ & $0.00 \mathrm{E}+00$ & $0.00 \mathrm{E}+00$ & $0.00 \mathrm{E}+00$ & $0.00 \mathrm{E}+00$ & $0.00 \mathrm{E}+00$ & $0.00 \mathrm{E}+00$ & $0.00 \mathrm{E}+00$ & $0.00 \mathrm{E}+00$ \\
\hline 2 & $\mathrm{Fe}$ & $4.00 \mathrm{E}-05$ & $1.61 \mathrm{E}-04$ & $1.69 \mathrm{E}-04$ & $2.81 \mathrm{E}-04$ & $9.25 \mathrm{E}-05$ & $1.74 \mathrm{E}-03$ & 3.09E-03 & $9.60 \mathrm{E}-05$ & $9.05 \mathrm{E}-05$ & $2.35 \mathrm{E}-04$ & $2.84 \mathrm{E}-04$ \\
\hline 2 & $\mathrm{~Pb}$ & $6.41 \mathrm{E}-05$ & $9.37 \mathrm{E}-05$ & $9.77 \mathrm{E}-05$ & $1.32 \mathrm{E}-04$ & $7.22 \mathrm{E}-05$ & $1.52 \mathrm{E}-03$ & $1.74 \mathrm{E}-04$ & $1.17 \mathrm{E}-04$ & $1.16 \mathrm{E}-04$ & $1.38 \mathrm{E}-04$ & $1.61 \mathrm{E}-04$ \\
\hline 3 & $\mathrm{Al}$ & $0.00 \mathrm{E}+00$ & $0.00 \mathrm{E}+00$ & $0.00 \mathrm{E}+00$ & $0.00 \mathrm{E}+00$ & $0.00 \mathrm{E}+00$ & $0.00 \mathrm{E}+00$ & $0.00 \mathrm{E}+00$ & $0.00 \mathrm{E}+00$ & $0.00 \mathrm{E}+00$ & $0.00 \mathrm{E}+00$ & $0.00 \mathrm{E}+00$ \\
\hline 3 & $\mathrm{Cr}$ & $0.00 \mathrm{E}+00$ & $0.00 \mathrm{E}+00$ & $0.00 \mathrm{E}+00$ & $0.00 \mathrm{E}+00$ & $0.00 \mathrm{E}+00$ & $0.00 \mathrm{E}+00$ & $0.00 \mathrm{E}+00$ & $0.00 \mathrm{E}+00$ & $0.00 \mathrm{E}+00$ & $0.00 \mathrm{E}+00$ & $0.00 \mathrm{E}+00$ \\
\hline 3 & $\mathrm{La}$ & $8.36 \mathrm{E}-06$ & $6.02 \mathrm{E}-06$ & $6.50 \mathrm{E}-06$ & $3.14 \mathrm{E}-13$ & $1.71 \mathrm{E}-12$ & $2.04 \mathrm{E}-05$ & $2.17 \mathrm{E}-07$ & $1.69 \mathrm{E}-06$ & $1.91 \mathrm{E}-06$ & $4.77 \mathrm{E}-12$ & $5.80 \mathrm{E}-12$ \\
\hline 3 & $\mathrm{Ce}$ & $0.00 \mathrm{E}+00$ & $1.59 \mathrm{E}-19$ & $1.73 \mathrm{E}-19$ & $0.00 \mathrm{E}+00$ & $0.00 \mathrm{E}+00$ & $2.58 \mathrm{E}-05$ & $4.35 \mathrm{E}-07$ & $0.00 \mathrm{E}+00$ & $0.00 \mathrm{E}+00$ & $0.00 \mathrm{E}+00$ & $0.00 \mathrm{E}+00$ \\
\hline \multicolumn{13}{|l|}{ Anions } \\
\hline-1 & $\mathrm{OH}$ (free) & $1.55 \mathrm{E}+00$ & $8.64 \mathrm{E}-01$ & $9.54 \mathrm{E}-01$ & $1.34 \mathrm{E}+00$ & $5.73 \mathrm{E}-01$ & $8.70 \mathrm{E}-01$ & $9.31 \mathrm{E}-01$ & $1.54 \mathrm{E}+00$ & $1.54 \mathrm{E}+00$ & $1.75 \mathrm{E}+00$ & $2.14 \mathrm{E}+00$ \\
\hline-1 & $\mathrm{NO}_{3}$ & $1.87 \mathrm{E}+00$ & $1.38 \mathrm{E}+00$ & $1.36 \mathrm{E}+00$ & $1.28 \mathrm{E}+00$ & $1.41 \mathrm{E}+00$ & $1.43 \mathrm{E}+00$ & $1.20 \mathrm{E}+00$ & $1.22 \mathrm{E}+00$ & $1.22 \mathrm{E}+00$ & $1.32 \mathrm{E}+00$ & $1.30 \mathrm{E}+00$ \\
\hline-1 & $\mathrm{NO}_{2}$ & $8.10 \mathrm{E}-01$ & $1.19 \mathrm{E}+00$ & $1.19 \mathrm{E}+00$ & $1.20 \mathrm{E}+00$ & $1.21 \mathrm{E}+00$ & $1.31 \mathrm{E}+00$ & $1.39 \mathrm{E}+00$ & $1.13 \mathrm{E}+00$ & $1.13 \mathrm{E}+00$ & $1.06 \mathrm{E}+00$ & $1.01 \mathrm{E}+00$ \\
\hline-1 & $\mathrm{Cl}$ & $4.98 \mathrm{E}-02$ & $1.03 \mathrm{E}-01$ & $1.03 \mathrm{E}-01$ & $1.25 \mathrm{E}-01$ & $1.27 \mathrm{E}-01$ & $1.40 \mathrm{E}-01$ & $1.37 \mathrm{E}-01$ & $8.62 \mathrm{E}-02$ & $8.52 \mathrm{E}-02$ & $8.43 \mathrm{E}-02$ & $6.98 \mathrm{E}-02$ \\
\hline
\end{tabular}




\begin{tabular}{|c|c|c|c|c|c|c|c|c|c|c|c|c|}
\hline & Batch Name & LAW-1 & LAW-5 & LAW-6 & LAW-8 & LAW-9 & LAW-10 & LAW-11 & LAW-12 & LAW-13 & LAW-14 & LAW-15 \\
\hline & Staging (Source) Tank & AP-101 & AN-104 & AN-104 & AN-105 & AN-105 & SY-101 & SY-101 & AN-103 & AN-103 & AW-101 & AW-101 \\
\hline & Total Volume of Liquid (L) & $4.63 E+06$ & $3.82 \mathrm{E}+06$ & $3.54 \mathrm{E}+06$ & $3.70 \mathrm{E}+06$ & $3.60 \mathrm{E}+06$ & $2.60 \mathrm{E}+06$ & $4.60 \mathrm{E}+06$ & $4.72 E+06$ & $4.72 E+06$ & $3.94 \mathrm{E}+06$ & $5.36 \mathrm{E}+06$ \\
\hline Species & Concentration Units & {$[\mathbf{M}]$} & {$[\mathbf{M}]$} & {$[\mathbf{M}]$} & {$[\mathbf{M}]$} & {$[\mathbf{M}]$} & {$[\mathbf{M}]$} & {$[\mathbf{M}]$} & {$[\mathbf{M}]$} & {$[\mathbf{M}]$} & {$[\mathbf{M}]$} & {$[\mathbf{M}]$} \\
\hline Charge & LAW Envelope & $\mathbf{A}$ & $\mathbf{A}$ & $\mathbf{A}$ & $\mathbf{A}$ & $\mathbf{A}$ & $\mathbf{A}$ & $\mathbf{A}$ & $\mathbf{A}$ & $\mathbf{A}$ & $\mathbf{A}$ & $\mathbf{A}$ \\
\hline-1 & $\mathrm{~F}$ & $1.33 \mathrm{E}-01$ & $1.47 \mathrm{E}-02$ & $1.34 \mathrm{E}-02$ & $1.65 \mathrm{E}-02$ & $4.47 \mathrm{E}-02$ & $2.50 \mathrm{E}-02$ & $3.11 \mathrm{E}-02$ & $1.85 \mathrm{E}-02$ & $1.86 \mathrm{E}-02$ & $4.06 \mathrm{E}-02$ & $3.94 \mathrm{E}-02$ \\
\hline-1 & $\mathrm{Al}(\mathrm{OH})_{4}$ & $2.31 \mathrm{E}-01$ & $5.68 \mathrm{E}-01$ & $5.73 \mathrm{E}-01$ & $6.35 \mathrm{E}-01$ & $4.50 \mathrm{E}-01$ & $4.92 \mathrm{E}-01$ & $4.65 \mathrm{E}-01$ & $7.50 \mathrm{E}-01$ & $7.56 \mathrm{E}-01$ & $4.87 \mathrm{E}-01$ & $5.00 \mathrm{E}-01$ \\
\hline-1 & $\mathrm{Cr}(\mathrm{OH})_{4}$ & $2.49 \mathrm{E}-03$ & $2.59 \mathrm{E}-03$ & $2.62 \mathrm{E}-03$ & $2.32 \mathrm{E}-03$ & $3.25 \mathrm{E}-03$ & $2.27 \mathrm{E}-02$ & $2.61 \mathrm{E}-02$ & $3.60 \mathrm{E}-03$ & $3.64 \mathrm{E}-03$ & $1.81 \mathrm{E}-03$ & $1.32 \mathrm{E}-03$ \\
\hline-1 & $129-\mathrm{I}$ & $3.14 \mathrm{E}-06$ & $8.12 \mathrm{E}-06$ & $8.47 \mathrm{E}-06$ & $2.19 \mathrm{E}-05$ & $1.03 \mathrm{E}-05$ & $1.31 \mathrm{E}-05$ & 7.39E-06 & 7.42E-06 & $6.99 \mathrm{E}-06$ & $9.39 \mathrm{E}-06$ & $9.14 \mathrm{E}-06$ \\
\hline-2 & $\mathrm{CO}_{3}$ & $4.80 \mathrm{E}-01$ & $3.93 \mathrm{E}-01$ & $3.59 \mathrm{E}-01$ & $2.01 \mathrm{E}-01$ & $5.47 \mathrm{E}-01$ & $2.78 \mathrm{E}-01$ & $3.61 \mathrm{E}-01$ & $1.61 \mathrm{E}-01$ & $1.60 \mathrm{E}-01$ & $2.53 \mathrm{E}-01$ & $1.54 \mathrm{E}-01$ \\
\hline-2 & $\mathrm{SO}_{4}$ & $3.74 \mathrm{E}-02$ & $4.84 \mathrm{E}-02$ & $4.49 \mathrm{E}-02$ & $2.17 \mathrm{E}-02$ & $3.67 \mathrm{E}-02$ & $3.73 \mathrm{E}-02$ & $2.39 \mathrm{E}-02$ & $1.14 \mathrm{E}-02$ & $1.11 \mathrm{E}-02$ & $1.72 \mathrm{E}-02$ & $1.06 \mathrm{E}-02$ \\
\hline-3 & $\mathrm{PO}_{4}$ & $9.79 \mathrm{E}-03$ & $2.07 \mathrm{E}-02$ & $1.98 \mathrm{E}-02$ & $1.08 \mathrm{E}-02$ & $2.67 \mathrm{E}-02$ & $4.35 \mathrm{E}-02$ & $3.65 \mathrm{E}-02$ & $9.39 \mathrm{E}-03$ & $9.34 \mathrm{E}-03$ & $1.10 \mathrm{E}-02$ & $5.67 \mathrm{E}-03$ \\
\hline & $\begin{array}{l}\text { Best estimate density based on } \\
\text { HTWOS model }(\mathrm{g} / \mathrm{ml})=\end{array}$ & 1.237 & 1.210 & 1.211 & 1.212 & 1.210 & 1.217 & 1.211 & 1.209 & 1.209 & 1.214 & 1.217 \\
\hline & $\begin{array}{l}\text { Best estimate density based on } \\
\text { modified HTWOS model }(\mathrm{g} / \mathrm{ml})=\end{array}$ & 1.277 & 1.232 & 1.231 & 1.225 & 1.238 & 1.237 & 1.232 & 1.221 & 1.221 & 1.234 & 1.235 \\
\hline & $\%$ difference in density estimate $=$ & 3.2 & 1.8 & 1.7 & 1.1 & 2.3 & 1.6 & 1.7 & 1.0 & 1.0 & 1.7 & 1.5 \\
\hline & Cations $=$ & 5.7131 & 5.0626 & 5.0644 & 5.0761 & 5.0630 & 5.0538 & 5.0557 & 5.1249 & 5.1265 & 5.3233 & 5.4105 \\
\hline & Anions $=$ & -5.7131 & -5.0626 & -5.0644 & -5.0761 & -5.0630 & -5.0538 & -5.0557 & -5.1249 & -5.1265 & -5.3233 & -5.4105 \\
\hline & Sum $=$ & 0.000 & 0.000 & 0.000 & 0.000 & 0.000 & 0.000 & 0.000 & 0.000 & 0.000 & 0.000 & 0.000 \\
\hline & Ionic strength (gmole/kg) & 6.304 & 6.727 & 6.652 & 6.304 & 6.951 & 6.553 & 6.618 & 6.324 & 6.327 & 6.617 & 6.487 \\
\hline & $\mathrm{Na} / \mathrm{Cs}$ & $1.39 \mathrm{E}+05$ & $7.96 \mathrm{E}+04$ & $7.90 \mathrm{E}+04$ & $1.16 \mathrm{E}+05$ & $1.13 \mathrm{E}+05$ & $1.35 \mathrm{E}+05$ & $1.34 \mathrm{E}+05$ & $1.04 \mathrm{E}+05$ & $1.04 \mathrm{E}+05$ & $1.09 \mathrm{E}+05$ & $1.10 \mathrm{E}+05$ \\
\hline & $\mathrm{K} / \mathrm{Cs}$ & $1.98 \mathrm{E}+04$ & $9.67 \mathrm{E}+02$ & $9.87 \mathrm{E}+02$ & $1.67 \mathrm{E}+03$ & $1.36 \mathrm{E}+03$ & $1.14 \mathrm{E}+03$ & $1.11 \mathrm{E}+03$ & $2.56 \mathrm{E}+03$ & $2.59 \mathrm{E}+03$ & $7.00 \mathrm{E}+03$ & $8.93 E+03$ \\
\hline & $\mathrm{Na} / \mathrm{K}$ & 7.0 & 82.3 & 80.1 & 69.3 & 82.9 & 119.3 & 120.4 & 40.5 & 39.9 & 15.6 & 12.3 \\
\hline & 137-Cs/Total Cs & 0.25 & 0.25 & 0.25 & 0.25 & 0.25 & 0.25 & 0.25 & 0.25 & 0.25 & 0.25 & 0.25 \\
\hline
\end{tabular}

${ }^{\mathrm{a}}$ For analysis at other $\mathrm{Na}^{+}$concentration levels, such as $4 \mathrm{M}$ or $6 \mathrm{M}$ feeds, the concentrations of each species provided in this table is computed based on a simple ratio. No shifts in molar ratios due to potential liquid-phase or precipitation reactions are considered. 
Table A-6. Best estimate Phase 1 LAW feed solution data for Envelopes B and C adjusted to $5 \mathrm{M} \mathrm{Na}^{\text {a }}$ and used as input to the CST equilibrium model.

\begin{tabular}{|c|c|c|c|c|c|c|}
\hline & Batch Name & LAW-2a & LAW-2b & LAW-3 & LAW-4 & LAW-7 \\
\hline & Staging (Source) Tank & AZ-101 & AZ-102 & AN-102 & AN-102 & AN-107 \\
\hline & Total Volume of Liquid (L) & $2.91 \mathrm{E}+06$ & $1.76 E+06$ & $4.20 \mathrm{E}+06$ & $4.20 \mathrm{E}+06$ & $5.50 \mathrm{E}+06$ \\
\hline Species & Concentration Units & {$[\mathbf{M}]$} & {$[\mathbf{M}]$} & {$[\mathbf{M}]$} & {$[\mathbf{M}]$} & {$[\mathbf{M}]$} \\
\hline Charge & LAW Envelope & B & B & $\mathbf{C}$ & C & $\mathbf{C}$ \\
\hline \multicolumn{7}{|l|}{ Cations } \\
\hline 1 & $\mathrm{Na}$ & $5.00 \mathrm{E}+00$ & $5.00 \mathrm{E}+00$ & $5.00 \mathrm{E}+00$ & $5.00 \mathrm{E}+00$ & $5.00 \mathrm{E}+00$ \\
\hline 1 & Cs (total) & $4.68 \mathrm{E}-04$ & $4.31 \mathrm{E}-04$ & $3.97 \mathrm{E}-05$ & $3.78 \mathrm{E}-05$ & $4.45 \mathrm{E}-05$ \\
\hline 1 & $\mathrm{H}$ & $6.26 \mathrm{E}-14$ & $3.68 \mathrm{E}-14$ & $9.68 \mathrm{E}-15$ & $9.68 \mathrm{E}-15$ & $1.26 \mathrm{E}-14$ \\
\hline 1 & $\mathrm{Rb}$ & $0.00 \mathrm{E}+00$ & $0.00 \mathrm{E}+00$ & $0.00 \mathrm{E}+00$ & $0.00 \mathrm{E}+00$ & $0.00 \mathrm{E}+00$ \\
\hline 1 & $\mathrm{~K}$ & $1.25 \mathrm{E}-01$ & $1.43 \mathrm{E}-01$ & $4.39 \mathrm{E}-02$ & $4.39 \mathrm{E}-02$ & $2.23 \mathrm{E}-02$ \\
\hline 1 & $\mathrm{SrOH}$ & $0.00 \mathrm{E}+00$ & $0.00 \mathrm{E}+00$ & $0.00 \mathrm{E}+00$ & $0.00 \mathrm{E}+00$ & $0.00 \mathrm{E}+00$ \\
\hline 2 & Sr (total) & $0.00 \mathrm{E}+00$ & $0.00 \mathrm{E}+00$ & $0.00 \mathrm{E}+00$ & $0.00 \mathrm{E}+00$ & $0.00 \mathrm{E}+00$ \\
\hline 2 & $\mathrm{Ba}$ & $2.04 \mathrm{E}-05$ & $5.60 \mathrm{E}-13$ & $1.00 \mathrm{E}-04$ & $1.00 \mathrm{E}-04$ & $0.00 \mathrm{E}+00$ \\
\hline 2 & $\mathrm{Ca}$ & $1.27 \mathrm{E}-05$ & $1.98 \mathrm{E}-04$ & $6.92 \mathrm{E}-03$ & $6.92 \mathrm{E}-03$ & $7.56 \mathrm{E}-03$ \\
\hline 2 & $\mathrm{Cd}$ & $4.35 \mathrm{E}-05$ & $1.19 \mathrm{E}-12$ & $2.60 \mathrm{E}-04$ & $2.57 \mathrm{E}-04$ & $0.00 \mathrm{E}+00$ \\
\hline 2 & $\mathrm{Mn}$ & $2.55 \mathrm{E}-07$ & $2.34 \mathrm{E}-05$ & $4.13 \mathrm{E}-02$ & $4.13 \mathrm{E}-02$ & $4.06 \mathrm{E}-02$ \\
\hline 2 & $\mathrm{Ni}$ & $9.26 \mathrm{E}-07$ & $6.43 \mathrm{E}-05$ & $3.07 \mathrm{E}-03$ & $3.07 \mathrm{E}-03$ & $4.44 \mathrm{E}-03$ \\
\hline 2 & $\mathrm{U}$ (total) & $2.23 \mathrm{E}-05$ & $1.16 \mathrm{E}-03$ & $3.40 \mathrm{E}-04$ & $3.40 \mathrm{E}-04$ & $3.10 \mathrm{E}-04$ \\
\hline 2 & $\mathrm{Zn}$ & $0.00 \mathrm{E}+00$ & $0.00 \mathrm{E}+00$ & $0.00 \mathrm{E}+00$ & $0.00 \mathrm{E}+00$ & $0.00 \mathrm{E}+00$ \\
\hline 2 & $\mathrm{Fe}$ & $2.09 \mathrm{E}-04$ & $3.41 \mathrm{E}-04$ & $1.74 \mathrm{E}-03$ & $1.74 \mathrm{E}-03$ & $2.66 \mathrm{E}-02$ \\
\hline 2 & $\mathrm{~Pb}$ & $0.00 \mathrm{E}+00$ & $0.00 \mathrm{E}+00$ & $4.30 \mathrm{E}-04$ & $4.30 \mathrm{E}-04$ & $1.02 \mathrm{E}-03$ \\
\hline 3 & $\mathrm{Al}$ & $0.00 \mathrm{E}+00$ & $0.00 \mathrm{E}+00$ & $0.00 \mathrm{E}+00$ & $0.00 \mathrm{E}+00$ & $0.00 \mathrm{E}+00$ \\
\hline 3 & $\mathrm{Cr}$ & $0.00 \mathrm{E}+00$ & $0.00 \mathrm{E}+00$ & $0.00 \mathrm{E}+00$ & $0.00 \mathrm{E}+00$ & $0.00 \mathrm{E}+00$ \\
\hline 3 & $\mathrm{La}$ & $2.33 \mathrm{E}-10$ & $3.52 \mathrm{E}-05$ & $0.00 \mathrm{E}+00$ & $0.00 \mathrm{E}+00$ & $1.32 \mathrm{E}-04$ \\
\hline 3 & $\mathrm{Ce}$ & $0.00 \mathrm{E}+00$ & $0.00 \mathrm{E}+00$ & $0.00 \mathrm{E}+00$ & $0.00 \mathrm{E}+00$ & $0.00 \mathrm{E}+00$ \\
\hline \multicolumn{7}{|l|}{ anions } \\
\hline-1 & $\mathrm{OH}$ (free) & $1.60 \mathrm{E}-01$ & 2.72E-01 & $1.03 \mathrm{E}+00$ & $1.03 \mathrm{E}+00$ & 7.94E-01 \\
\hline-1 & $\mathrm{NO}_{3}$ & $1.28 \mathrm{E}+00$ & $6.74 \mathrm{E}-01$ & $1.74 \mathrm{E}+00$ & $1.74 \mathrm{E}+00$ & $1.97 \mathrm{E}+00$ \\
\hline-1 & $\mathrm{NO}_{2}$ & $1.48 \mathrm{E}+00$ & $1.18 \mathrm{E}+00$ & $7.96 \mathrm{E}-01$ & $7.96 \mathrm{E}-01$ & $6.95 \mathrm{E}-01$ \\
\hline
\end{tabular}




\begin{tabular}{|c|c|c|c|c|c|c|}
\hline & Batch Name & LAW-2a & LAW-2b & LAW-3 & LAW-4 & LAW-7 \\
\hline & Staging (Source) Tank & AZ-101 & AZ-102 & AN-102 & AN-102 & AN-107 \\
\hline & Total Volume of Liquid (L) & $2.91 E+06$ & $1.76 E+06$ & $4.20 \mathrm{E}+06$ & $4.20 \mathrm{E}+06$ & $5.50 \mathrm{E}+06$ \\
\hline Species & Concentration Units & [M] & [M] & {$[\mathbf{M}]$} & [M] & [M] \\
\hline Charge & LAW Envelope & B & B & C & C & C \\
\hline-1 & $\mathrm{Cl}$ & $5.83 \mathrm{E}-03$ & $4.72 \mathrm{E}-04$ & $4.79 \mathrm{E}-02$ & 4.79E-02 & $2.78 \mathrm{E}-02$ \\
\hline-1 & $F$ & $1.03 \mathrm{E}-01$ & $9.87 \mathrm{E}-02$ & $4.35 \mathrm{E}-02$ & $4.35 \mathrm{E}-02$ & $9.39 \mathrm{E}-02$ \\
\hline-1 & $\mathrm{Al}(\mathrm{OH})_{4}$ & $4.21 \mathrm{E}-01$ & 9.97E-02 & $2.57 \mathrm{E}-01$ & $2.57 \mathrm{E}-01$ & $1.36 \mathrm{E}-01$ \\
\hline-1 & $\mathrm{Cr}(\mathrm{OH})_{4}$ & $1.55 \mathrm{E}-02$ & $3.45 \mathrm{E}-02$ & $3.61 \mathrm{E}-03$ & $3.61 \mathrm{E}-03$ & $3.09 \mathrm{E}-03$ \\
\hline-1 & $129-\mathrm{I}$ & $3.97 \mathrm{E}-02$ & $3.50 \mathrm{E}-02$ & $1.58 \mathrm{E}-05$ & $1.58 \mathrm{E}-05$ & $1.27 \mathrm{E}-05$ \\
\hline-2 & $\mathrm{CO}_{3}$ & $5.91 \mathrm{E}-01$ & $1.03 \mathrm{E}+00$ & $5.10 \mathrm{E}-01$ & $5.10 \mathrm{E}-01$ & $6.55 \mathrm{E}-01$ \\
\hline-2 & $\mathrm{SO}_{4}$ & $1.97 \mathrm{E}-01$ & 3.37E-01 & 7.27E-02 & 7.27E-02 & 4.69E-02 \\
\hline-3 & $\mathrm{PO}_{4}$ & $1.61 \mathrm{E}-02$ & $2.54 \mathrm{E}-03$ & $2.29 \mathrm{E}-02$ & $2.29 \mathrm{E}-02$ & $2.04 \mathrm{E}-02$ \\
\hline & $\begin{array}{l}\text { Best estimate density based on } \\
\text { HTWOS model }(\mathrm{g} / \mathrm{ml})=\end{array}$ & 1.213 & 1.178 & 1.209 & 1.209 & 1.211 \\
\hline & $\begin{array}{l}\text { Best estimate density based on } \\
\text { modified HTWOS model }(\mathrm{g} / \mathrm{ml})=\end{array}$ & 1.254 & 1.242 & 1.237 & 1.237 & 1.243 \\
\hline & $\%$ difference in density estimate $=$ & 3.4 & 5.5 & 2.3 & 2.3 & 2.7 \\
\hline & cations $=$ & 5.1256 & 5.1467 & 5.1523 & 5.1523 & 5.1836 \\
\hline & anions $=$ & -5.1256 & -5.1467 & -5.1524 & -5.1524 & -5.1836 \\
\hline & sum $=$ & 0.000 & 0.000 & 0.000 & 0.000 & 0.000 \\
\hline & Ionic strength (gmole/kg) & 7.361 & 8.171 & 6.995 & 6.995 & 7.209 \\
\hline & $\mathrm{Na} / \mathrm{Cs}$ & $1.07 \mathrm{E}+04$ & $1.16 \mathrm{E}+04$ & $1.26 \mathrm{E}+05$ & $1.32 \mathrm{E}+05$ & $1.12 \mathrm{E}+05$ \\
\hline & $\mathrm{K} / \mathrm{Cs}$ & $2.67 \mathrm{E}+02$ & $3.32 \mathrm{E}+02$ & $1.11 \mathrm{E}+03$ & $1.16 \mathrm{E}+03$ & $5.00 \mathrm{E}+02$ \\
\hline & $\mathrm{Na} / \mathrm{K}$ & 40.0 & 35.0 & 113.8 & 113.8 & 224.5 \\
\hline & 137-Cs/Total Cs & 0.30 & 0.30 & 0.25 & 0.25 & 0.25 \\
\hline
\end{tabular}

${ }^{\text {a }}$ For analysis at other $\mathrm{Na}^{+}$concentration levels, such as $4 \mathrm{M}$ or $6 \mathrm{M}$ feeds, the concentrations of each species provided in this table is computed based on a simple ratio. No shifts in molar ratios due to potential liquid-phase or precipitation reactions are considered. 
Table A-7. Hanford and SRS measured liquid-phase densities for several LAW liquid samples and simulants considered.

\begin{tabular}{|c|c|c|c|c|c|c|c|c|c|c|c|c|}
\hline LAW Sample & Envelope & $\begin{array}{l}\text { Temperature } \\
\text { (C) }\end{array}$ & $\begin{array}{l}\mathbf{N a} \\
{[\mathrm{M}]}\end{array}$ & $\begin{array}{l}\mathbf{N O}_{2} \\
{[\mathbf{M}]}\end{array}$ & $\begin{array}{l}\mathrm{NO}_{3} \\
{[\mathrm{M}]}\end{array}$ & $\begin{array}{c}\text { Al total } \\
\text { [M] }\end{array}$ & $\begin{array}{c}\text { OH (free) } \\
{[\mathrm{M}]}\end{array}$ & $\begin{array}{c}\mathbf{K} \\
{[\mathbf{M}]}\end{array}$ & $\begin{array}{l}\mathrm{CO}_{3} \\
{[\mathrm{M}]}\end{array}$ & $\begin{array}{l}\mathrm{SO}_{4} \\
{[\mathrm{M}]}\end{array}$ & $\begin{array}{c}\text { Measured } \\
\text { density } \\
(\mathrm{g} / \mathrm{ml})\end{array}$ & Reference \\
\hline $\mathrm{AN}-103$; as received & A sample & $20^{a}$ & 11.700 & 2.318 & 3.499 & 1.556 & 4.836 & 0.270 & 0.568 & 0.017 & 1.490 & $\begin{array}{c}\text { Hay and } \\
\text { Bronikowski, } 2000\end{array}$ \\
\hline AN-103, diluted & A sample & $20^{a}$ & 5.250 & 1.040 & 1.570 & 0.698 & 2.170 & 0.121 & 0.255 & 0.008 & 1.260 & $\begin{array}{c}\text { Hay and } \\
\text { Bronikowski, } 2000\end{array}$ \\
\hline AZ-102, as received & B sample & $20^{\mathrm{a}}$ & 2.770 & 0.659 & 0.273 & 0.0279 & 0.109 & 0.081 & 0.392 & 0.172 & 1.150 & Hay et al., 2000a \\
\hline $\mathrm{AN}-102$, as received & C sample & $20^{a}$ & 10.200 & 1.795 & 3.082 & 0.543 & 1.298 & 0.056 & 0.777 & 0.135 & 1.470 & Hay et al., 2000b \\
\hline AN-102, diluted & C sample & $20^{\mathrm{a}}$ & 6.420 & 1.130 & 1.940 & 0.342 & 0.817 & 0.035 & 0.489 & 0.085 & 1.330 & Hay et al., $2000 \mathrm{~b}$ \\
\hline TFL Simulant & A simulant & $20^{a}$ & 5.000 & 1.132 & 1.247 & 0.690 & 1.627 & 0.089 & 0.069 & 0.004 & 1.225 & Steimke et al., 2000 \\
\hline AW-101; PNNL & A sample & $?$ & 4.590 & 1.010 & 1.500 & 0.410 & 2.170 & 0.390 & $?$ & $?$ & 1.228 & PNWD-3001 \\
\hline AN-107, PNNL & C sample & $?$ & 4.840 & 0.620 & 1.820 & 0.087 & 0.800 & 0.019 & $?$ & $?$ & 1.241 & PNWD-3039 \\
\hline 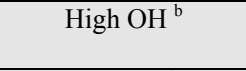 & SRS simulant & 25 & 5.000 & 0.500 & 1.429 & 0.500 & 2.457 & 0.000 & 0.018 & 0.018 & 1.217 & $\begin{array}{c}\text { Walker and } \\
\text { Coleman, } 1991\end{array}$ \\
\hline Average $^{b}$ & SRS simulant & 25 & 5.000 & 0.707 & 1.907 & 0.300 & 1.393 & 0.000 & 0.164 & 0.136 & 1.229 & $\begin{array}{c}\text { Walker and } \\
\text { Coleman, } 1991\end{array}$ \\
\hline High $\mathrm{NO}_{3}{ }^{\mathrm{b}}$ & SRS simulant & 25 & 5.000 & 0.300 & 2.586 & 0.164 & 0.664 & 0.000 & 0.321 & 0.300 & 1.244 & $\begin{array}{c}\text { Walker and } \\
\text { Coleman, } 1991 \\
\end{array}$ \\
\hline
\end{tabular}

a These tests were performed in hot-cells where the ambient temperature of the cells were not controlled or closely measured. Historically, measured temperatures on the order of $20 \mathrm{C}$ is assumed.

${ }^{\mathrm{b}}$ For SRS wastes simulants are typically studied that reflect the average tank compositions and also the upper $\mathrm{OH}_{\text {and }} \mathrm{NO}_{3}$ expected conditions. These specific destinations (i.e., average, high $\mathrm{OH}$, high $\mathrm{NO}_{3}$ ) have been in use for many years and their specific species compositions have varied slightly over the years.

The concentrations listed are consistent with those at the time of the listed measurement. 
Table A-8. Comparison of measured to predicted liquid-phase densities for several Hanford LAW liquid samples and simulants based on available correlations.

\begin{tabular}{|c|c|c|c|c|c|c|c|c|}
\hline LAW Sample & Envelope & $\begin{array}{c}\text { Measured } \\
\text { density } \\
(\mathrm{g} / \mathrm{ml})\end{array}$ & $\begin{array}{l}\text { Reynolds- } \\
\text { Herting calc. } \\
\text { density } \\
(\mathrm{g} / \mathrm{ml})\end{array}$ & $\%$ Error & $\begin{array}{c}\text { HTWOS calc. } \\
\text { density } \\
(\mathrm{g} / \mathrm{ml})\end{array}$ & \% Error & $\begin{array}{c}\text { Modified }^{a} \\
\text { HTWOS calc. } \\
\text { density } \\
(\mathrm{g} / \mathrm{ml})\end{array}$ & \% Error \\
\hline $\mathrm{AN}-103$; as received & A sample & 1.490 & 1.557 & 4.5 & 1.517 & 1.8 & 1.545 & 3.7 \\
\hline AN-103, diluted & A sample & 1.260 & 1.256 & -0.3 & 1.277 & 1.3 & 1.289 & 2.3 \\
\hline AZ-102, as received & B sample & 1.150 & 1.055 & -8.2 & 1.109 & -3.6 & 1.134 & -1.3 \\
\hline $\mathrm{AN}-102$, as received & C sample & 1.470 & 1.317 & -10.4 & 1.367 & -7.0 & 1.405 & -4.5 \\
\hline AN-102, diluted & C sample & 1.330 & 1.203 & -9.5 & 1.268 & -4.7 & 1.291 & -2.9 \\
\hline TFL Simulant & A simulant & 1.225 & 1.230 & 0.4 & 1.255 & 2.5 & 1.260 & 2.9 \\
\hline AW-101; PNNL & A sample & 1.228 & 1.221 & -0.6 & 1.249 & 1.7 & 1.258 & 2.4 \\
\hline AN-107, PNNL & C sample & 1.241 & 1.148 & -7.5 & 1.205 & -2.9 & 1.206 & -2.9 \\
\hline Mean bias (\%) & & & & -3.95 & & -1.35 & & -0.03 \\
\hline Root mean square (\%) & & & & 2.32 & & 1.29 & & 1.06 \\
\hline High $\mathrm{OH}^{\mathrm{b}}$ & SRS simulant & 1.217 & 1.210 & -0.6 & 1.235 & 1.5 & 1.237 & 1.6 \\
\hline Average $^{b}$ & SRS simulant & 1.229 & 1.191 & -3.1 & 1.236 & 0.6 & 1.250 & 1.7 \\
\hline High $\mathrm{NO}_{3}{ }^{\mathrm{b}}$ & SRS simulant & 1.244 & 1.171 & -5.9 & 1.223 & -1.7 & 1.252 & 0.6 \\
\hline
\end{tabular}

a The modification results shown only account for the additional species $\mathrm{K}, \mathrm{CO}_{3}$, and $\mathrm{SO}_{4}$. For the candidate feed solutions all additional species are accounted for based on Eq. (A-3) approach where the correction factor was set to 0.6. 


\section{Appendix B (Cesium Isotherms for LAW Phase 1 Batch Feeds)}

In column sizing one of two possible design strategies are typically considered: (1) bounding analysis where "worst case" feed compositions are used or (2) global optimization where best estimate feed compositions for each individual batch are used. Each approach has its on advantages and disadvantages. For example, the bounding approach requires less analysis overall but it may be difficult to establish a reasonable bound that is not too excessive. Since the amount of waste to be processed, flowrate, and key feed compositions depend significantly on which envelope is being considered, the global optimization strategy is taken. However, in this appendix the isotherms required by either design approach are determined. The least favorable isotherm for each envelope is determined by comparison of all batch feed isotherms within each envelope.

For the CST exchanger material, individual and least favorable isotherms for each batch feed and Envelope (i.e., Envelopes A, B, and C) are determined, respectively. The isotherms corresponding to the 16 batch feeds are determined based on available BBI Phase 1 LAW feed solution data. For each envelope class, worst case here implies the largest amount of CST material necessary to achieve the same level of decontamination factor for operating columns, while least favorable represents a lower cesium loading curve. For linear isotherms this can easily be established based on the lowest $\mathrm{K}_{d}$ value (note: for a linear isotherm its $\mathrm{K}_{d}$ value is a constant). However, for the Cesium-CST system the isotherm is slightly non-linear over the region of interest and in general differing isotherms could cross each other. Therefore, a definitive determination of the worst case isotherm requires consideration over the entire operating range for cesium. One additional complication is the fact that for each candidate feed within a given envelope its cesium concentration varies. The least favorable isotherm is easily determined by inspection of all isotherms within a given envelope. To determine which isotherm is the worst case requires consideration of the feed cesium concentration as well.

Appendix A contains the BBI data set and discusses the various alterations and adjustments that were necessary. In order to successfully use the ZAM equilibrium model, a tight charge balance (i.e., less than $5 \times 10^{-4}$ mismatch) is required for the species chosen as input to the algorithm. Tables A-5 and A-6 contain the best estimate BBI feed solutions (i.e., 16 in total) all adjusted to a common $\mathrm{Na}^{+}$concentration of $5 \mathrm{M}$. This common $\mathrm{Na}^{+}$concentration is chosen for establishing the individual isotherms based on the current feed design to the ion-exchange facilities for cesium then technetium removal.

In order to generate an isotherm using ZAM, the input $\mathrm{Cs}^{+}$concentration must be varied over the region of interest (i.e., zero up to the maximum expected $\mathrm{Cs}^{+}$feed concentration). To maintain a tight charge balance, the best estimate feed compositions provided in Tables A-5 and A-6 were adjusted by adding/subtracting $\mathrm{CsCl}$ to the solutions. All other species were kept at their tabulated values. For fitting an appropriate algebraic isotherm model to this data, alterations such as concentrating or diluting were also considered. For example, feed solutions having a 4 $\mathrm{M} \mathrm{Na}^{+}$concentration were generated by dilution of the $5 \mathrm{M}$ feeds (i.e., an $80 \%$ dilution was applied to each constituent). Also, to obtain a higher degree of confidence in the estimated 
parameters to the algebraic models several additional equilibrium data points were generated (e.g., variations in the $\mathrm{Na} / \mathrm{K}$ and $\mathrm{Na} / \mathrm{SrOH}$ ratios were considered).

Table B-1 contains some of the key parameter values for CST in its powder-form and three of its engineered-forms. For comparison purposes, SuperLig ${ }^{\circledR} 644$ properties are also provided.

\section{B.1 Molar Ratios of $\mathrm{Na} / \mathrm{Cs}, \mathrm{K} / \mathrm{Cs}$, and $\mathrm{SrOH} / \mathrm{Cs}$}

As stated earlier, ZAM addresses the surface ion-exchange competition between $\mathrm{Na}^{+}, \mathrm{K}^{+}, \mathrm{Cs}^{+}$, $\mathrm{H}^{+}, \mathrm{Rb}^{+}$, and $\mathrm{SrOH}^{+}$. For this set of analyses it is assumed that $\mathrm{Rb}^{+}$cations are not present in the liquid feed (i.e., their concentrations are set to zero since only trace amounts are typically present) and under loading conditions the $\mathrm{H}^{+}$concentration levels are extremely small (i.e., $<1 \times 10^{-14} \mathrm{M}$ ). Under these assumptions, only the species $\mathrm{Na}^{+}, \mathrm{K}^{+}, \mathrm{SrOH}^{+}$, and $\mathrm{Cs}^{+}$are competing for surface sites. The molar feed ratios of $\left[\mathrm{Na}^{+}, / \mathrm{Cs}^{+}\right],\left[\mathrm{K}^{+} / \mathrm{Cs}^{+}\right]$, and $\left[\mathrm{SrOH}^{+} / \mathrm{Cs}^{+}\right]$provide us with some initial indication of which candidate feed solutions will be less favorable. For the nominal set of analyses performed within this report, $\mathrm{SrOH}^{+}$is assumed to be zero within the feed solutions; therefore, only the other two molar ratios are considered. The impact of $\mathrm{SrOH}^{+}$within the feed is addressed as a sensitivity parameter. For each envelope class the molar ratios provided in Tables A-5 and A-6 are plotted in Figures B-1, B-2, and B-3 for Envelopes A, B, and $\mathrm{C}$, respectively. The higher a ratio becomes the more competition for surface sites will occur. Based on this simple indicator (i.e., maximum number of $\mathrm{Na}^{+}$plus $\mathrm{K}^{+}$competitors versus $\mathrm{Cs}^{+}$) the following feed solutions should ultimately result in the least favorable isotherms (see Table B-2):

- For Envelope A Figure B-1 indicates feed solution LAW-1 is the least favorable, but the worst case isotherm may be one with a higher cesium feed concentration such as LAW-15;

- For Envelope B Figure B-2 indicates feed solution LAW-2b is both the least favorable and worst case isotherm; and

- For Envelope C Figure B-3 indicates feed solutions LAW-3 and LAW-4 are the least favorable isotherms, but the worst case isotherm may be LAW-7 who has the highest cesium feed concentration.

For comparison purposes, the molar ratios for all feed solutions are plotted together in Figure B4. Also in Figure B-4, for each envelope the least favorable isotherm is highlighted. To see how representative the Phase 1 LAW batch feeds are when compared to the entire LAW inventory, Figure B-5 contains the molar ratios for all 177 Hanford waste tank inventories (10 of which define the Phase 1 campaign).

\section{B.2 Isotherm Predictions using the ZAM Model}

The ZAM code performs a simulated batch contact test producing one point on a given isotherm curve. To establish an entire isotherm curve, for each feed solution of interest the ZAM equilibrium isotherm model was run for a range of 14 initial total $\mathrm{Cs}^{+}$concentrations (i.e., 0.0 , $1.0 \times 10^{-6}, 1.0 \times 10^{-5}, 5.0 \times 10^{-5}, 1.0 \times 10^{-4}, 5.0 \times 10^{-4}, 1.0 \times 10^{-3}, 2.0 \times 10^{-3}, 3.0 \times 10^{-3}, 5.0 \times 10^{-3}, 1.0 \times 10^{-2}$, $3.0 \times 10^{-2}, 5.0 \times 10^{-2}, 1.0 \times 10^{-1}$ in units of M). In order to maintain liquid concentrations nearly 
constant a high value for the phase ratio (i.e., liquid volume to solid mass) of $1.0 \times 10^{5} \mathrm{ml} / \mathrm{g}$ was chosen. All calculations were performed at $25{ }^{\circ} \mathrm{C}$ with a liquid solution density set to the modified HTWOS model estimates provided in Tables A-5 and A-6. The initial form of the resin was set to the Na-form. Cesium and potassium loadings, along with their $\mathrm{K}_{\mathrm{d}}$ and final equilibrium liquid concentration values, were recorded. Note that the isotherm curve created is not dependent upon the choice of phase ratio or initial CST form, but does depend upon temperature and solution density.

The total cation exchange capacity of the CST material in its powder-form (batch IE-910) is species dependent. Two types of exchange sites exist on the CST solid. The total ion exchange capacity is stated to be $\sim 4.6 \mathrm{mmole} / \mathrm{g}_{\mathrm{CST}}$, but the cesium exchange capacity is much less indicating that not all sites are available for cesium exchange (see, Zheng et al., 1996). In the ideal solid region (i.e., prior to the first step of the isotherm), the apparent total capacities are $\sim 0.58 \mathrm{mmole} / \mathrm{g}_{\text {CST }}$ for $\mathrm{Cs}^{+}, \sim 1.2 \mathrm{mmole} / \mathrm{g}_{\text {CST }}$ for $\mathrm{K}^{+}, \sim 1.18 \mathrm{mmole} / \mathrm{g}_{\text {CST }}$ for $\mathrm{Rb}^{+}$, and $\sim 1.0$ mmole $/ \mathrm{g}_{\mathrm{CST}}$ for $\mathrm{SrOH}^{+}$. For the expected feed concentrations it is anticipated that the entire columns will be operating within this ideal solid region. The ZAM algorithm employs the above stated total exchange capacities that apply only to the CST in its powder-form (IE-910). A brief discussion about ZAM, along with limited verification/validation assessments, is provided in Appendix F.

For each of the 16 batch feeds defined in Table A-5 and Table A-6, and for each of the 14 cesium concentration values chosen, a simulated batch contact test was performed using the ZAM code. The results of these ZAM runs are tabulated in Tables B-3 through B-18 where:

- for Envelope A feeds Table B-3 through Table B-13 correspond to batch feeds LAW-1, LAW-5, LAW-6, LAW-8, LAW-9, LAW-10, LAW-11, LAW-12, LAW-13, LAW-14, and LAW-15, respectively;

- for Envelope B feeds Table B-14 and Table B-15 correspond to batch feeds LAW-2a and LAW-2b, respectively; and

- for Envelope C feeds Table B-16 through Table B-18 correspond to batch feeds LAW-3, LAW-4, and LAW-7, respectively.

In each table the equilibrium cesium concentration, cesium $\mathrm{K}_{\mathrm{d}}$, and cesium loading are given for each ZAM simulated batch contact test, along with similar information for potassium. The ionic strength of each solution is also provided. The ZAM input files used to generate the data corresponding to the cesium concentration of the 16 batch feeds are provided in Appendix G (along with some of the sensitivity input files). For each batch feed condition, the remaining 13 input files can be obtained by adding or removing $\mathrm{CsCl}$ from the concentration levels given in input files listed in Appendix G. The database provided in Tables B-4 through B-18 constitutes our cesium isotherm database for $5 \mathrm{M}$ sodium adjusted feeds.

\section{B.3 Isotherm Model for VERSE-LC Application}

In order to perform column transport simulations, the ZAM generated batch contact test data, presented above, must be correlated using one of the VERSE-LC isotherm modeling options. As 
demonstrated by Hamm et al. (2000a, Figure 3-3), for ion exchange competitors with affinities significantly less than the value for cesium, a single-component transport modeling approach is adequate for the cesium loading phase. To perform single-component transport simulations, an "effective" binary isotherm model in an algebraic form must be available for use in the VERSELC code. Based on our previous experience using VERSE-LC for modeling SuperLig ${ }^{\circledR} 644$ and SuperLig $^{\circledR} 639$ resins (Hamm et al., 2000a and 2000b), the VERSE-LC Freundlich/Langmuir Hybrid isotherm model was chosen. As described by Hamm et al. (2000a, see Chapter 4), the cations cesium, potassium, sodium, and strontium hydroxide form a 4-component homovalent system where the surface loading for cesium on the CST material can be expressed as:

$$
\begin{aligned}
& \mathrm{Q}_{\mathrm{Cs}^{+}}=\frac{\eta_{\mathrm{df}} \overline{\mathrm{C}}_{\mathrm{T}_{\mathrm{pCs}}} \mathrm{c}_{\mathrm{pCs}^{+}}+\widetilde{\mathrm{K}}_{21} \mathrm{c}_{\mathrm{pK}^{+}}+\widetilde{\mathrm{K}}_{31} \mathrm{c}_{\mathrm{pNa}^{+}}+\widetilde{\mathrm{K}}_{41} \mathrm{c}_{\mathrm{pSrOH}^{+}}}{\mathrm{c}}, \\
& \mathrm{Q}_{\mathrm{Cs}^{+}}=\frac{\eta_{\mathrm{df}} \overline{\mathrm{C}}_{\mathrm{T}_{\mathrm{pCs}}}}{\mathrm{c}_{\mathrm{pCs}^{+}}+\beta}
\end{aligned}
$$

or as

where

$$
\beta=\widetilde{\mathrm{K}}_{21} \mathrm{c}_{\mathrm{pK}^{+}}+\widetilde{\mathrm{K}}_{31} \mathrm{c}_{\mathrm{pNa}^{+}}+\widetilde{\mathrm{K}}_{41} \mathrm{c}_{\mathrm{pSrOH}^{+}} .
$$

It has been assumed that the binary selectivity coefficients are not composition dependent, but are true constants (i.e., note that the selectivity coefficients actually contain the true equilibrium constants and liquid/solid phase activity coefficients). This assumption appears to be somewhat adequate when considering different feeds within the same envelope (i.e., generally only small variations are observed in selectivity coefficients within a given envelope), but should not be used between envelopes. The composite impact on cesium loading from the other cation competitors is summed up in the beta parameter shown. Equation (B-1a) can be rearranged into a more convenient form where liquid concentrations are expressed as molar ratios by:

$$
\mathrm{Q}_{\mathrm{Cs}^{+}}=\frac{\eta_{\mathrm{df}} \overline{\mathrm{C}}_{\mathrm{T}}}{1+\widetilde{\mathrm{K}}_{21}\left[\frac{\mathrm{c}_{\mathrm{pK}}}{\mathrm{c}_{\mathrm{pCs}}}\right]+\widetilde{\mathrm{K}}_{31}\left[\frac{\mathrm{c}_{\mathrm{pNa}^{+}}}{\mathrm{c}_{\mathrm{pCs}}}\right]+\widetilde{\mathrm{K}}_{41}\left[\frac{\mathrm{c}_{\mathrm{pSrOH}^{+}}}{\mathrm{c}_{\mathrm{pCs}^{+}}}\right]}=\frac{\eta_{\mathrm{df}} \overline{\mathrm{C}}_{\mathrm{T}}}{1+\frac{\beta}{\mathrm{c}_{\mathrm{pCs}}}} \text {. }
$$

Under the simple assumption of constant binary selectivity coefficients, we see that the cesium loading isotherm is only a function of the molar ratios $\left[\mathrm{K}^{+}, / \mathrm{Cs}^{+}\right],\left[\mathrm{Na}^{+} / \mathrm{Cs}^{+}\right]$, and $\left[\mathrm{SrOH}^{+} / \mathrm{Cs}^{+}\right]$ where the total cesium exchange capacity is a constant value within the ideal solid region (i.e., depends only on the exchange material properties and for the powder-form its value has been determined to be $\overline{\mathrm{C}}_{\mathrm{T}}=0.58 \mathrm{mmole} / \mathrm{g}_{\mathrm{CST}}$ ). Equation (B-2) also indicates that the larger the beta value the less favorable the cesium isotherm.

The VERSE-LC Freundlich/Langmuir Hybrid model is expressed as:

$$
\overline{\mathrm{C}}_{\mathrm{pi}}=\frac{\mathrm{a}_{\mathrm{i}} \mathrm{c}_{\mathrm{pi}}^{\mathrm{M}_{\mathrm{a}}}}{\beta_{\mathrm{i}}+\mathrm{b}_{1} \mathrm{c}_{\mathrm{p} 1}^{\mathrm{M}_{\mathrm{b} 1}}+\mathrm{b}_{2} \mathrm{c}_{\mathrm{p} 2}^{\mathrm{M}_{\mathrm{b}}}+\mathrm{b}_{3} \mathrm{c}_{\mathrm{p} 3}^{\mathrm{M}_{\mathrm{b} 3}}+\mathrm{b}_{4} \mathrm{c}_{\mathrm{p} 4}^{\mathrm{M}_{\mathrm{b} 4}}} \quad \text { for } \quad \mathrm{i}=1,4,
$$


where the model parameters $\left(a_{i}, b_{i}, M_{a i}, M_{b i}\right.$, and $\beta_{i}$ for $\left.i=1,4\right)$ can be determined from the parameter values associated with the 4-component homovalent model.

The Freundlich/Langmuir Hybrid model can also be used for an "effective" single-component case as well. Here the potassium, sodium, and strontium hydroxide concentrations throughout the column are assumed to be at their feed concentration levels. For an "effective" singlecomponent total cesium isotherm, Eq. (B-3) under these conditions reduces to:

$$
\overline{\mathrm{C}}_{\mathrm{p} 1}=\frac{\mathrm{a}_{1} \mathrm{c}_{\mathrm{p} 1}^{\mathrm{M}_{\mathrm{a}}}}{\left[\beta_{\mathrm{i}}+\mathrm{b}_{2} \mathrm{c}_{\mathrm{p} 2}^{\mathrm{M}_{\mathrm{b} 2}}+\mathrm{b}_{3} \mathrm{c}_{\mathrm{p} 3}^{\mathrm{M}_{\mathrm{b} 3}}+\mathrm{b}_{4} \mathrm{c}_{\mathrm{p} 4}^{\mathrm{M}_{\mathrm{b}}}\right]+\mathrm{b}_{1} \mathrm{c}_{\mathrm{p} 1}^{\mathrm{M}_{\mathrm{b} 1}}} \Rightarrow \frac{\mathrm{a}_{1} \mathrm{c}_{\mathrm{pl}}^{\mathrm{M}_{\mathrm{a}}}}{\hat{\beta}_{\mathrm{i}}+\mathrm{b}_{1} \mathrm{c}_{\mathrm{pl}}^{\mathrm{M}_{\mathrm{b} 1}}},
$$

where the beta parameter for cesium becomes dependent upon the potassium, sodium, and strontium hydroxide feed concentrations. The relationship between the two models expressed by Eqs. (B-3) and (B-4) (i.e., 4-component homovalent and "effective" single-component isotherm models, respectively) is provided in Table B-19. The dilution factor, $\eta_{\mathrm{df}}$, is set to unity when CST in its powder-form is being considered.

Since ZAM can be used directly to generate cesium isotherms for each candidate feed, we shall develop cesium isotherms based on the "effective" single-component model and not explicitly rely on the selectivity coefficients $\left(\mathrm{K}_{\mathrm{i}}, \mathrm{i}=2,4\right)$ required by the 4-component homovalent model. In this way, the propagation of errors will be minimized.

\section{B.4 Isotherm Parameter Estimation Technique}

To establish a cesium isotherm, three independent binary selectivity coefficient parameters (or depending upon the model chosen a single beta parameter directly) must be determined based on equilibrium contact test data or ZAM generated data. As seen by Eq. (B-2), based on the functional form chosen the fitting parameter(s) can be obtained from equilibrium contact tests where only one of the cations solid-phase concentration (or surface loading) is known.

A non-linear regression analysis based on the maximum likelihood principle (see Anderson et al., 1978) was chosen consistent with earlier work (Hamm et al., 2000a and 2000b). The maximum likelihood approach minimizes a cost function based on the sum of squares of weighted residuals. Special attention must be given to the choice of weights. Due to the broad range of cesium concentrations considered (i.e.,), a logarithmic weighting criterion for parameter estimation was found to be superior (i.e., more uniform weighting over several orders of magnitude in cesium concentration was achieved). The cost function being minimized is

$$
\Phi\left(\widetilde{\mathrm{K}}_{21}, \widetilde{\mathrm{K}}_{31}, \widetilde{\mathrm{K}}_{41}\right)=\sum_{\mathrm{i}=1}^{\mathrm{n}}\left[\mathrm{R}_{\mathrm{i}}^{2}\right],
$$

where the chosen weighted residual function is

$$
\mathrm{R}_{\mathrm{i}}=\ln \left(\mathrm{Q}_{\mathrm{Cs}^{+}}^{\mathrm{ZAM}}\right)-\ln \left(\mathrm{Q}_{\mathrm{Cs}^{+}}^{\mathrm{a} \text { lg ebraic }}\right) .
$$


Equation (B-5) results in uniform weighting among all of the data points. Application of the maximum likelihood algorithm (using logarithmetric weighting) to the algebraic model (Eq. B-2) results in "best estimate" values for the three selectivity coefficients (or beta parameter directly), as discussed below for each envelope. The various other binary selectivity coefficients (i.e., combinations of the three primary ones) can be computed based on reciprocity identities. The confidence levels, covariance matrix, and correlation coefficient matrix for the fitted parameters are also computed during the optimization process.

\section{B.5 The Least Favorable Isotherm}

As Eq. (B-2) indicates, along with the excellent agreement achieved in fitting this algebraic model to the various isotherms created using the ZAM model, the dominant factors affecting an isotherm are the molar ratios. Over the range of candidate feed solutions considered, their compositional variations within the liquid-phase play only a secondary role. Therefore, the determination of a least favorable isotherm for each set of candidate feed solutions within a given envelope can be directly determined by Eq. (B-2) once the three binary selectivity coefficients are known. Using Eq. (B-2), the following criterion can be derived that establishes conditions under which the least favorable isotherm can be determined:

$$
\begin{aligned}
\left(\widetilde{\mathrm{K}}_{21}\left[\frac{\mathrm{c}_{\mathrm{pK}^{+}}}{\mathrm{c}_{\mathrm{pCs}^{+}}}\right]+\widetilde{\mathrm{K}}_{31}\left[\frac{\mathrm{c}_{\mathrm{pNa}^{+}}}{\mathrm{c}_{\mathrm{pCs}^{+}}}\right]+\widetilde{\mathrm{K}}_{41}\left[\frac{\mathrm{c}_{\mathrm{pSrOH}^{+}}}{\mathrm{c}_{\mathrm{pCs}^{+}}}\right]\right)_{\text {least favorable }} \\
\geq\left(\widetilde{\mathrm{K}}_{21}\left[\frac{\mathrm{c}_{\mathrm{pK}^{+}}}{\mathrm{c}_{\mathrm{pCs}^{+}}}\right]+\widetilde{\mathrm{K}}_{31}\left[\frac{\mathrm{c}_{\mathrm{pNa}^{+}}}{\mathrm{c}_{\mathrm{pCs}^{+}}}\right]+\widetilde{\mathrm{K}}_{41}\left[\frac{\mathrm{c}_{\mathrm{pSrOH}^{+}}}{\mathrm{c}_{\mathrm{pCs}^{+}}}\right]\right)_{\text {candidate feed solution }}
\end{aligned}
$$

Table B-1 lists the numerical value of Eq. (B-6) for each candidate feed solution where it is assumed that the $[\mathrm{SrOH} / \mathrm{Cs}]$ ratio is zero. For example, as shown in Table B-1 for Envelope A, the worst case isotherm results from the feed solution corresponding to LAW-1. Overall estimates (Envelope A only) for the three binary selectivity coefficients were obtained using the non-linear regression analysis scheme described above. The overall regression results for the 11 Envelope A feeds is provided in Tables B-20 and B-21. The estimated binary selectivity coefficients obtained are (see Table B-20):

- for $\left(\mathrm{SrOH}^{+}\right.$versus $\left.\mathrm{Cs}^{+}\right) \mathrm{K}_{14} \approx 6.9 \rightarrow \mathrm{K}_{41} \approx 1.443 \times 10^{-1}$;

- for $\left(\mathrm{K}^{+}\right.$versus $\left.\mathrm{Cs}^{+}\right) \mathrm{K}_{12} \approx 1,400 \rightarrow \mathrm{K}_{21} \approx 7.143 \times 10^{-4}$; and

- for $\left(\mathrm{Na}^{+}\right.$versus $\left.\mathrm{Cs}^{+}\right) \mathrm{K}_{13} \approx 26,000 \rightarrow \mathrm{K}_{31} \approx 3.846 \times 10^{-5}$.

The parameter estimation results for Envelope A (i.e., 11 batch fees) are provided in Table B-20 where the various possible binary selectivity coefficients for this 4-component isotherm are provided. Also provided are the approximate standard errors for each coefficient. The correlation coefficient matrix for the fitting process is given in Table B-21. As Table B-21 indicates, the degree of cross-correlation between $\mathrm{SrOH}^{+}$versus $\mathrm{K}^{+}$and $\mathrm{SrOH}^{+}$versus $\mathrm{Na}^{+}$is 
high (i.e., 0.1118 and -0.2493 ; where a good indication would have been near 1.0 or -1.0 ). Even though the $\mathrm{SrOH}^{+}$selectivity coefficient, $\mathrm{K}_{14}$, is not uniquely defined, comparison of predicted versus ZAM generated data remains excellent. These results support the need to handle each batch feed separately, in order in increase our predictive capability.

Note that Eq. (B-2) is based on the assumption of constant binary selectivity coefficients which implies that for the range of feed solutions considered, compositional variations have only a minor impact on the isotherm. However, compositional variations between envelopes are significant enough to warrant, in general, different estimates for the three binary selectivity coefficients. However, due to the poor parameter confidence levels achieved, the binary selectivity coefficients for Envelopes B and C are not presented here.

\section{B.6 "Effective" Single-Component Isotherm Models}

As discussed above, an effective single-component total cesium isotherm can be expressed as:

$$
\mathrm{Q}_{\mathrm{Cs}}=\frac{\eta_{\mathrm{df}} \overline{\mathrm{C}}_{\mathrm{T}} \mathrm{c}_{\mathrm{Cs}}}{\mathrm{c}_{\mathrm{Cs}}+\left[\widetilde{\mathrm{K}}_{21} \mathrm{c}_{\mathrm{K}}+\widetilde{\mathrm{K}}_{31} \mathrm{c}_{\mathrm{Na}}+\widetilde{\mathrm{K}}_{41} \mathrm{c}_{\mathrm{SrOH}}+\cdots\right]} \Rightarrow \frac{\eta_{\mathrm{df}} \overline{\mathrm{C}}_{\mathrm{T}} \mathrm{c}_{\mathrm{Cs}}}{\mathrm{c}_{\mathrm{Cs}}+\beta},
$$

where the beta parameter for cesium becomes dependent upon the other ionic competitors for CST adsorption (i.e., $\mathrm{K}^{+}, \mathrm{Na}^{+}, \mathrm{SrOH}^{+}$, and $\mathrm{Rb}^{+}$). The beta parameter contains the selectivity coefficients making it dependent upon temperature and liquid composition of all of the ionic species in solution. The dilution factor $\left(\eta_{\mathrm{df}}\right)$ is unity when considering a specific power-form and is less than one upon addition of an inert binder. Based on analyses discussed in Appendix $\mathrm{C}$ the best estimate dilution factor for the engineered-form is set to 0.68 . The total cesium capacity term is only a function of which batch of powder-form material is being considered and is set to $0.58 \mathrm{mmole}_{\mathrm{CS}} / \mathrm{g}_{\mathrm{CST}}$.

For each candidate feed solution the ZAM model was run to obtain a database for determining a beta value for that particular feed. This database is generated by varying the cesium and chloride concentrations, while holding all other ionic concentrations at their best estimate values. Using the database generated for each batch feed and Eq. (B-7) above, a "best estimate" of the beta parameter for each batch feed was computed. The summary results from the non-linear regression analyses are listed in Table B-22 for all 16 batch feeds, along with the overall average for each envelope. As shown in Table B-22, the root-mean-square error in predicting the cesium loading over the entire cesium concentration range is excellent. Based on the results shown in Table B-22, the Freundlich/Langmuir Hybrid modeling parameters for the 16 batch feeds are tabulated in Table B-23. The leading coefficient, $\mathrm{a}_{\mathrm{i}}$, varies depending upon which form of the CST material is to be considered.

Using the algebraic model, predictions for comparison to the ZAM model were generated for each batch feed and are tabulated in Tables B-24 through B-39. Tables B-24 through B-39 contain the algebraic model predictions for both the powder-form and the engineered-form. Residual plots of the algebraic model predictions of cesium loading versus the ZAM model predictions of cesium loading are provided in Figures B-6 through B-8 for the Envelope A, B, 
and $\mathrm{C}$ feeds, respectively. In all cases the algebraic model provides an excellent fit to the ZAM generated databases. A bar-chart of the beta values for each batch feed is illustrated in Figure B9 where the various beta values have been grouped according to envelope. As Eq. (B-7) indicates, the higher the beta value the less favorable the isotherm.

\section{B.6.1 PHASE 1 Envelope A LAW Isotherms}

As Figure B-9 illustrates, a wide variation in isotherms is seen for the Envelope A batch feeds. In Figure B-10 a log-log plot of the cesium loading isotherms for all 11 Envelope A batch feeds is shown. Also shown, is the location where the column inlet cesium concentration of each feed would be. The least favorable isotherm can be easily seen in Figure B-10 to be LAW-1. However, since LAW-1's cesium concentration is not also the highest value, LAW-1 may not be the worst case isotherm, as well. Column transport analyses would be required to determine which of these isotherms would generate more total spent CST material to process the LAW solutions.

\section{B.6.2 PHASE 1 Envelope B LAW Isotherms}

As Figure B-9 illustrates, a modest variation in isotherms is seen for the Envelope B batch feeds. In Figure B-11 a log-log plot of the cesium loading isotherms for both Envelope B batch feeds is shown. Also shown, is the location where the column inlet cesium concentration of each feed would be. The least favorable isotherm can be easily seen in Figure B-11 to be LAW-2a. Since LAW-2a also contains the higher cesium feed concentration, it is also the worst case isotherm.

\section{B.6.3 PHASE 1 Envelope C LAW Isotherms}

As Figure B-9 illustrates, a small variation in isotherms is seen for the Envelope C batch feeds. In Figure B-12 a log-log plot of the cesium loading isotherms for all 3 Envelope $\mathrm{C}$ batch feeds is shown. Also shown, is the location where the column inlet cesium concentration of each feed would be. The least favorable isotherm can be easily seen in Figure B-12 to be LAW-3 and LAW-4. However, since neither LAW-3's nor LAW-4's cesium concentration is also the highest value, LAW-7 may be the worst case isotherm. Column transport analyses would be required to determine which of these isotherms would generate more total spent CST material to process the LAW solutions.

\section{B.6.4 Comparison of Least Favorable Isotherms}

In Figures B-10, B-11, and B-12, the least favorable isotherms were seen to be LAW-1 for Envelope A, LAW-2a for Envelope B, and LAW-3 (or LAW-4) for Envelope C. A direct comparison of these three isotherms is given in Figure B-13. The least favorable isotherm among these is LAW-1; however, LAW-1 also has the lowest cesium feed concentration. To determine which isotherm would be the worst case isotherm again requires separate column transport analyses. Based on issues such as these and others (i.e., envelope dependent cesium exit criteria, waste volumes, flowrates, and cesium feed concentrations), a global strategy for 
CST column design was chosen where the overall worst case isotherm is not considered; instead, all 16 best estimate isotherms are employed directly.

\section{B.6.5 LAW-1 Feed ( $\mathrm{KNO}_{3}$ precipitation Issue)}

The best estimate feed composition for LAW-1 (i.e., Tank AP-101) presented a unique problem when the $5.0 \mathrm{M}$ sodium feed was used in developing its isotherm using the ZAM model. For this feed composition ZAM predicts that some $\mathrm{KNO}_{3}$ will precipitate out indicating that the $\mathrm{KNO}_{3}$ solubility limit had been exceeded. Based on experience with making Hanford and SRS simulants, $\mathrm{KNO}_{3}$ precipitation at these concentrations (i.e., for LAW-1 at $5.0 \mathrm{M} \mathrm{Na}^{+}$and $25 \mathrm{C}$, $\mathrm{K}^{+}$is at $0.712 \mathrm{M}$ and $\mathrm{NO}_{3}{ }^{-}$is at $1.87 \mathrm{M}$ ) had not been observed.

For a simple solution, a comparison of the ZAM prediction versus measured data (Washburn et al., 1928) for the solubility limit of $\mathrm{KNO}_{3}$ is shown in Figure B-14. The results shown are for an aqueous solution of $\mathrm{Na}^{+}, \mathrm{K}^{+}, \mathrm{OH}^{-}$, and $\mathrm{NO}_{3}{ }^{-}$where a fixed amount of $\mathrm{K}^{+}$and $\mathrm{NO}_{3}{ }^{-}$(i.e., at two different molar ratios) were considered over a wide range of varying $\mathrm{NaOH}$. The decreasing solubility upon increasing ionic strength clearly shows the expected "salt-effect". Unfortunately, for the case of pure $\mathrm{K}^{+}$and $\mathrm{NO}_{3}{ }^{-}$(i.e., no $\mathrm{NAOH}$ present) the ZAM prediction of $\sim 1.1 \mathrm{M}$ is significantly lower than the measured value of $\sim 3.3 \mathrm{M}$. It would appear that either the $\mathrm{KNO}_{3}$ Bromley activity coefficient or the $\mathrm{KNO}_{3}$ solubility product being used is incorrect.

Once $\mathrm{KNO}_{3}$ precipitation is encountered within the ZAM model, ZAM performs mass balance calculations to appropriately reduce the initial feed concentrations of $\mathrm{K}^{+}$and $\mathrm{NO}_{3}{ }^{-}$to account for the level of $\mathrm{KNO}_{3}$ precipitation predicted to occur and then performs the solid-liquid equilibrium calculation using the new adjusted feed composition. Unfortunately, we are unable to turn off this feature directly within ZAM through input and must through the following extrapolation procedure to estimate the cesium loadings consistent with the initial feed compositions.

For the Phase 1 LAW-1 feed the nominal nitrate to nitrite concentration ratio is lowered such that the point where $\mathrm{KNO}_{3}$ precipitation starts to occur is located (i.e., an equal amount of nitrite is added in place of nitrate). The cesium loading curve resulting from this analysis is shown in Figure B-15, where the input nitrate to nitrite concentration ratio into ZAM is varied on both sides of this solubility point. The solid line represents the ZAM results where a clear discontinuity in slope is experienced at the point of reaching the predicted $\mathrm{KNO}_{3}$ solubility limit. Prior to reaching this solubility limit, increased nitrate to nitrite concentration ratio results in a decreased cesium loading primarily due to the increased ionic strength. Without $\mathrm{KNO}_{3}$ precipitation occurring, further increase in the nitrate to nitrite concentration ratio would follow the linear extrapolation shown as a dashed line in Figure B-15. With $\mathrm{KNO}_{3}$ precipitation occurring, the decreased quantity of liquid-phase $\mathrm{K}^{+}$and $\mathrm{NO}_{3}{ }^{-}$present $\left(\mathrm{K}^{+}\right.$as a competitor and both as contributors to ionic strength) reduces this trend. In generating the entire cesium isotherm for the Phase $1 \mathrm{LAW}-1$ feed, the above extrapolation technique is used at every cesium concentration point considered. The database generated is tabulated in Table B-3.

After writing this chapter the work of Anthony et al. (2001) was provided to the authors. Anthony et al. (2001) updated the ZAM code where the solubility product for $\mathrm{KNO}_{3}$ would be inputted through the input. As stated within their report, a solubility product fixed to $0.19 \mathrm{M}$ 
was in the earlier version of ZAM. This value was based on the presence of salt formation induced by TAM5 during a $1.6 \mathrm{M} \mathrm{NaKNO}_{3}$ step change experiment and looking at the potassium isotherms. For solutions without TAM5 no such low solubility product is observed (even in the presence of CST in its engineered-forms).

\section{B.6.6 Impact of Strontium on Envelope C Isotherms}

There are three candidate feed solutions to be considered for Envelope C. The total strontium concentration estimates available were:

- $<2.28 \times 10^{-6} \mathrm{M}$ total $\mathrm{Sr}^{2+}$ for tank AN-103

- $<2.85 \times 10^{-6} \mathrm{M}^{-}$total $\mathrm{Sr}^{2+}$ for tank AZ-102

- $2.04 \times 10^{-5} \mathrm{M}$ total $\mathrm{Sr}^{2+}$ for tank AN-102

- $\quad 1.633 \times 10^{-3} \mathrm{M}_{\text {total }} \mathrm{Sr}^{2+}$ for tank AN-102 post Sr/TRU

- $\quad 5.0 \times 10^{-4} \mathrm{M}$ total $\mathrm{Sr}^{2+}$ for tank $\mathrm{AN}-107$ post $\mathrm{Sr} / \mathrm{TRU}$

The value of $1.633 \times 10^{-3} \mathrm{M}$ total strontium in solution was chosen as an upper bound value for free strontium in solution based on the currently available analytical analyses listed above. Based on complexants (e.g., EDTA) the free strontium available to form strontium hydroxide should be much smaller. The following liquid-phase equilibrium reaction is being modeled within the ZAM code:

$$
\mathrm{Sr}^{+2}(1)+\mathrm{OH}^{-}(1) \stackrel{\mathrm{K}_{\mathrm{eq}}}{\longleftrightarrow} \mathrm{SrOH}^{+}(1)
$$

where the equilibrium constant is $\sim 197$. Based on ZAM equilibrium calculations using this mass-action equation and equilibrium constant, the majority of free $\mathrm{Sr}^{2+}$ initially assumed in solution (i.e., $1.633 \times 10^{-5} \mathrm{M}$ ) is converted into $\mathrm{SrOH}^{+}$, approximately $1.625 \times 10^{-5} \mathrm{M}$, which competes for CST surface sites.

In fitting the algebraic isotherm model the following data sets were considered:

- LAW-3 candidate feed at $5 \mathrm{M} \mathrm{Na}^{+}$whose species concentrations are specified in Table A-6 where free $\mathrm{Sr}^{2+}$ concentration was set to zero (i.e., the nominal case); and

- LAW-3 candidate feed at $5 \mathrm{M} \mathrm{Na}^{+}$whose species concentrations are specified in Table A-6, except for free strontium which was set to $1.633 \times 10^{-5} \mathrm{M} \mathrm{Sr}^{2+}$.

As shown in Table B-22, the beta value for the nominal LAW-3 batch feed is $2.1769 \times 10^{-4} \mathrm{M}$. When the free strontium concentration is increased from zero to $1.633 \times 10^{-5} \mathrm{M} \mathrm{Sr}^{2+}$ (i.e., zero to $1.625 \times 10^{-5} \mathrm{M} \mathrm{SrOH}^{+}$) the computed beta value increases to $3.8445 \times 10^{-4} \mathrm{M}$ (i.e., an increase of $\sim 77 \%$ ). A comparison of the two LAW-3 isotherms is shown in Figure B-16.

As Figure B-16 suggests, the presence of free strontium in solution can have a significant impact on the cesium isotherm. In this bounding case approximately $50 \%$ reduction in cesium loading is observed over the operating range of the isotherm. Prior to final CST column design, 
additional investigations are warranted to determine the expected levels of free strontium in solution and its ultimate impact on the cesium isotherms.

\section{B.7 Cesium Isotherm Sensitivity Study and Error Analysis}

Our cesium isotherm is based on the ZAM model multiplied by a bias term (referred to in this report as a dilution factor). The ZAM model solves the appropriate liquid-solid equilibrium equations for the Cesium-CST system where its modeling parameters were determined based on CST powder batch and kinetic contact tests. The dilution factor is based on the measured deviations of engineered contact test data when compared to ZAM predicted powder behavior. In this section we discuss the uncertainties associated with our cesium isotherm model.

To evaluate the impact on the cesium isotherms associated with uncertainties in the composition of the various batch feeds, a sensitivity analysis was performed. The Envelope A LAW-15 batch feed was chosen where various species composition values, along with temperature and feed density, were individually altered from there "nominal" settings. The results of this sensitivity study are provided in Tables B-40 and B-41. These tables list the sensitivity variables considered and their impact on cesium loading (i.e., Table B-40 for the nominal state set to $100 \%$ of the cesium feed concentration and Table B-41 for $50 \%$ of the cesium feed concentration). A simple star-pattern approach to the sensitivity analysis was considered to be adequate (i.e., for each parameter considered its value was varied while all other parameters were kept at their nominal settings).

Using the sensitivity results, a "simplified" error analysis is performed where an estimate of the overall uncertainty in the cesium isotherm is computed. Much of this effort is based on a good engineering judgement approach where errors in various variables are stated based on an assumed/implied confidence level of approximately 2-sigma. Where available, supporting data are used in establishing these error estimates.

\section{B.7.1 The Uncertainty Approach Taken}

For estimating our level of confidence in the predicted cesium isotherms, a best estimate plus quantified uncertainty approach is being attempted. A rough measure of uncertainty in a cesium isotherm can be estimated based on the following approach.

The ZAM model solves a set of equations describing the liquid-solid equilibrium behavior for the Cesium-CST system. At an equilibrium state the cesium loading can be functionally expressed as:

$$
\mathrm{Q}=\eta_{\mathrm{df}} \mathrm{F}\left(\overrightarrow{\mathrm{c}}, \mathrm{T}, \rho_{\mathrm{soln}}, \mathrm{c}_{\mathrm{Cs}}\right),
$$

where the concentration vector represents all cations and anions (excluding cesium) within the candidate feed that is considered within the ZAM model. Equation (B-9) represents a nonlinear cesium isotherm that can be linearized about some nominal cesium concentration (e.g., its feed value) yielding: 


$$
\begin{aligned}
\mathrm{Q}-\mathrm{Q}_{\mathrm{o}}=( & \left.\frac{\partial \mathrm{Q}}{\partial \eta_{\mathrm{df}}}\right)_{\mathrm{o}}\left[\eta_{\mathrm{df}}-\eta_{\mathrm{df}, \mathrm{o}}\right]+\left(\frac{\partial \mathrm{Q}}{\partial \mathrm{T}}\right)_{\mathrm{o}}\left[\mathrm{T}-\mathrm{T}_{\mathrm{o}}\right]+\left(\frac{\partial \mathrm{Q}}{\partial \rho_{\mathrm{so}} \ln }\right)_{\mathrm{o}}\left[\rho_{\mathrm{soln}}-\rho_{\mathrm{soln}, \mathrm{o}}\right] \\
& +\sum_{\mathrm{i} \neq \mathrm{Cs}}\left(\frac{\partial \mathrm{Q}}{\partial \mathrm{c}_{\mathrm{i}}}\right)_{\mathrm{o}}\left[\mathrm{c}_{\mathrm{i}}-\mathrm{c}_{\mathrm{i}, \mathrm{o}}\right]
\end{aligned}
$$

where the subscript o implies that the variable is evaluated at its nominal value. If we consider these variables to be random variables that are independent, then the propagation of errors approach (Freund, 1971) provides us with a simple method of combining in a statistical way the uncertainty associated with each of these variables to produce a composite impact.

To make use of the propagation of errors approach the linear equation Eq. (B-10) must be used, where an overall variance results in:

$$
\sigma_{\mathrm{Q}}^{2}=\left(\frac{\partial \mathrm{Q}}{\partial \eta_{\mathrm{df}}}\right)_{0}^{2} \sigma_{\eta_{\mathrm{df}}}^{2}+\left(\frac{\partial \mathrm{Q}}{\partial \mathrm{T}}\right)_{\mathrm{o}}^{2} \sigma_{\mathrm{T}}^{2}+\left(\frac{\partial \mathrm{Q}}{\partial \rho_{\mathrm{so}} \ln }\right)_{\mathrm{o}}^{2} \sigma_{\rho_{\mathrm{soln}}}^{2}+\sum_{\mathrm{i} \neq \mathrm{Cs}}\left(\frac{\partial \mathrm{Q}}{\partial \mathrm{c}_{\mathrm{i}}}\right)_{\mathrm{o}}^{2} \sigma_{\mathrm{c}_{\mathrm{i}}}^{2},
$$

and due to the actual nonlinear behavior of the isotherm, the overall variance becomes dependent upon the choice of the nominal state (i.e., the variance varies with respect to cesium concentration). By defining an error estimate in terms of percentage and dividing through by $\mathrm{Q}$, Eq. (B-11) can be expressed as:

$$
\mathrm{E}_{\mathrm{Q}}^{2}=\mathrm{E}_{\eta_{\mathrm{df}}}^{2}+\mathrm{E}_{\mathrm{T}}^{2}+\mathrm{E}_{\rho_{\mathrm{soln}}}^{2}+\sum_{\mathrm{i} \neq \mathrm{Cs}} \mathrm{E}_{\mathrm{c}_{\mathrm{i}}}^{2},
$$

where the error estimates in terms of percentages are:

$$
\mathrm{E}_{\mathrm{x}}=100\left(\frac{\partial \mathrm{Q}}{\partial \mathrm{x}}\right)_{\mathrm{o}} \sigma_{\mathrm{x}}^{2} .
$$

\section{B.7.2 Feed Composition Uncertainties}

In order to estimate uncertainty levels in the feed composition a comparison must be made between the candidate feed batches provided in Tables A-1 and A-2 and actual measured samples. Recent characterization data of three waste tank samples have been performed by Hay et al. (2000a) for Envelope A's AN-103 (sample size of 1.2 L), by Hay and Bronikowski (2000) for Envelope B's AZ-102 (sample size of 14.25 L), and by Hay et al. (2000b) for Envelope C's AN-102 (sample size of $3.75 \mathrm{~L}$ ). In their reports they state that "recent experience at SRS indicates the combined sampling and analytical error associated with obtaining small samples from a well mixed waste tank is on the order of 15-20\%" (Hay and Edwards, 1994). In Table B42 a comparison is made between the measured feed compositions and the candidate feeds listed in Table A-1 and A-2. The percent difference in measured versus LAW candidate feed compositions are listed for each envelope, alone with a root-mean-square value for the key species of interest. The majority of error values listed are greater than $20 \%$. These tanks are probably not well mixed. The degree of uncertainty in tank variability due to lack of 
homogeneity will be highly tank and time dependent. We shall implicitly account for tank nonhomogeneity, and our lack of knowledge, by setting our error estimates for each species to $50 \%$ (except for sodium). Since the sodium level of the pretreatment feed will be adjusted to a target concentration of $\sim 5.0 \mathrm{M}$, for sodium we shall set our error estimate to back $20 \%$.

Free $\mathrm{OH}^{-}$concentrations appear to be difficult to pin down since analytical methods typically used to determine this quantity actually measure total $\mathrm{OH}^{-}$and then compute the free $\mathrm{OH}^{-}$by assuming certain chemical reactions (e.g., aluminate or carbonate formations) have consumed (bound) $\mathrm{OH}^{-}$. In these analyses presented here we allow the free $\mathrm{OH}^{-}$to vary in order to achieve a charge balance for all candidate feed solutions (i.e., both nominal compositions, as well as compositions used in the sensitivity studies). For sensitivity studies species concentration variations can not be arbitrarily chosen, but must adhere to the overall ionic charge balance. For each species variation considered, the $\mathrm{OH}^{-}$concentration is adjusted to maintain the charge balance. Table B-43 provides the species concentrations for each sensitivity study performed. In reality a more complex variation of several species concentrations would be occurring. However, for our purposes this more limited star-pattern approach was considered to be adequate.

As stated elsewhere within this report, no account is taken both nominally or statistically for the loading of $\mathrm{SrOH}^{+}$onto the CST material. It is assumed that the total $\mathrm{Sr}$ present is not available to form the $\mathrm{SrOH}^{+}$species (i.e., zero free strontium). This assumption should be addressed in any future considerations of CST columns since the loading capacity for $\mathrm{SrOH}^{+}$is on the same order as or greater than for $\mathrm{Cs}^{+}$.

\section{B.7.3 Feed Density and Process Temperature Uncertainties}

As discussed in Appendix A, a modified HTWOS density model was chosen for estimating the liquid-phase density of the various candidate feed solutions. A comparison of predicted versus measure densities are shown in Table A-8 where the overall root-mean-square is $\sim 1 \%$ for the available Hanford data and $\sim 1.4 \%$ for available SRS waste. For our purposes will shall assume an error estimate of $\sim 3 \%$ to be acceptable (i.e., two times $1.5 \%$ ). Note that a decreased solution density only marginally decreases the cesium loadings as can be seen in Figure B-17.

Current column designs assume an operating temperature of $\sim 25 \mathrm{C}$. To take into account potential non-uniform temperatures within the columns and CST material itself, due to ${ }^{137} \mathrm{Cs}$ decay and possible operational upsets, a $5 \mathrm{C}$ variation (i.e., 1.68\%) is considered. Note that increased temperature decreases the cesium loadings.

\section{B.7.4 Dilution Factor Uncertainty}

In Appendix $\mathrm{C}$ the dilution factor (i.e., ratio of total cesium capacity for an engineered-form versus total cesium capacity for its powder-form) is discussed. Based on the various engineered forms tested a range of dilution factors were observed. A mean value of 0.76 was computed; however, for more recent engineered forms a mean value of 0.68 appeared to be more appropriate. Based on this lower value for dilution factor an $\sim 15 \%$ error estimate seems reasonable. 


\section{B.7.5 Sensitivity Results and Error Estimates}

A sensitivity study was performed using the candidate feed LAW-15. Batch LAW-15 was chosen since Envelope A constitutes the major amount of LAW tank waste to be process (i.e., $71 \%$ by volume Envelope A, 7\% Envelope B, and 22\% Envelope C when adjusted to a common 5.0 M sodium basis) and among the Envelope A feed candidates it demonstrates a potentially worst case isotherm.

To determine the impact of each variable listed in Table B-40 or Table B-41, a ZAM model run was made. The results of the ZAM model runs are listed in Tables B-40 and B-41 for the nominal cesium feed concentration value of $4.552 \times 10^{-5} \mathrm{M}$ and for $50 \%$ of this value, respectively. The input concentrations used for each sensitivity case are provided in Table B-43.

During the sensitivity runs, the ZAM code predicted that $\mathrm{KNO}_{3}$ would start to precipitate out of solution, reducing the amount of $\mathrm{K}^{+}$in solution for competition with $\mathrm{Cs}^{+}$for CST sites. At approximately $41.5 \%$ increase in $\mathrm{K}^{+}$above its nominal value, precipitation started. To estimate the cesium loading impact associated with a $50 \%$ increase in $\mathrm{K}^{+}$, an extrapolation of results were made. The extrapolation performed is shown in Figure B-18 as the dashed line, while the solid curve shown represents the unaltered results from ZAM over the $0 \%$ to $60 \%$ increase in $\mathrm{K}^{+}$ concentration inputs. At a $50 \%$ increase in $\mathrm{K}^{+}$the extrapolation yields a $5.8 \%$ decrease in cesium loading.

As the entries in Tables B-40 and B-41 indicate, an overall error for the cesium isotherm is $\sim 28 \%$ and is not sensitive to the chosen nominal value for cesium concentration. Except for the dilution factor, which exhibits a one-for-one impact as expected, all the other variables have a reduced impact (i.e., a smaller $\%$ change in loading than the $\%$ change in the variable itself). 
Table B-1. Key CST exchange properties taken from literature.

\begin{tabular}{|c|c|c|c|c|c|c|}
\hline Resin Form & Batch Name & $\underset{(-)}{\text { F Factor }}{ }^{a}$ & $\begin{array}{l}\text { Bulk Dry } \\
\text { Density } \\
\left(\mathrm{g} / \mathrm{ml}_{\text {bed }}\right)\end{array}$ & $\begin{array}{c}\text { Dilution Factor } \\
(-)\end{array}$ & $\begin{array}{c}\text { Cesium Total Ion- } \\
\text { Exchange Capacity } \\
\left.\text { (mmole } / \mathrm{g}_{\text {solid }}\right)\end{array}$ & $\begin{array}{l}\text { Cesium Total Ion- } \\
\text { Exchange Capacity } \\
\left.\text { (mmole } / \mathrm{ml}_{\text {bed }}\right)\end{array}$ \\
\hline SuperLig $^{(} 644$ & 10-SM-171 & 0.9751 & 0.2238 & 1.0 & 0.3333 & 0.0746 \\
\hline Powder & IE-910 & 0.9680 & 0.7738 & 1.0 & 0.580 & 0.4488 \\
\hline Engineered & IE-911 (38b) & 0.8870 & 1.1300 & not reported & not reported & not reported \\
\hline Engineered & IE-911 (08) & 0.8990 & 0.8999 & not reported & not reported & not reported \\
\hline Engineered & Nominal ${ }^{d}$ & 0.80 & 1.00 & 0.68 (estimated) & 0.3944 (estimated) & 0.3944 (estimated) \\
\hline
\end{tabular}

${ }^{\mathrm{a}}$ Data obtained from Brown et al. (1996, Table 3.1).

${ }^{\mathrm{b}}$ Data obtained from Zheng et al. (1996) and confirmed by running ZAM at very large liquid cesium concentrations.

${ }^{c}$ During the manufacturing production process of IONSIV ${ }^{\circledR}$ IE-911 resin, an inert binder is added to CST powder to create an engineered form that is useable in ion-exchange columns. The additional inert binding material reduces the total Cs ion-exchange capacity and this reduction factor is referred to as its "dilution factor".

${ }^{d}$ The average properties of IONSIV ${ }^{\circledR}$ IE-911 exchanger as stated by UOP (1996).

Table B-2. Key molar ratios for the various LAW batch feed solutions.

\begin{tabular}{|c|c|c|c|c|c|c|c|c|}
\hline Envelope & $\begin{array}{c}\text { Batch } \\
\text { Name }\end{array}$ & $\begin{array}{c}\text { Staging } \\
\text { (Source) Tank }\end{array}$ & $\mathbf{N a / C s}$ & $\mathbf{K} / \mathbf{C s}$ & $\mathbf{( N a + K ) / C s}$ & $\mathbf{N a} / \mathbf{K}$ & $\begin{array}{c}\text { High (Na+K) } \\
\text { Level Ranking }\end{array}$ & $\begin{array}{c}\text { Worst Case } \\
\mathbf{b} \\
\text { Indicator }\end{array}$ \\
\hline A & LAW-1 & AP-101 & $1.39 \mathrm{E}+05$ & $1.98 \mathrm{E}+04$ & $1.59 \mathrm{E}+05$ & 7.0 & 1 & 19.5 \\
\hline A & LAW-5 & AN-104 & $7.96 \mathrm{E}+04$ & $9.67 \mathrm{E}+02$ & $8.06 \mathrm{E}+04$ & 82.3 & 9 & 3.8 \\
\hline A & LAW-6 & AN-104 & $7.90 \mathrm{E}+04$ & $9.87 \mathrm{E}+02$ & $8.00 \mathrm{E}+04$ & 80.1 & 10 & 3.7 \\
\hline A & LAW-8 & AN-105 & $1.16 \mathrm{E}+05$ & $1.67 \mathrm{E}+03$ & $1.17 \mathrm{E}+05$ & 69.3 & 5 & 5.6 \\
\hline A & LAW-9 & AN-105 & $1.13 \mathrm{E}+05$ & $1.36 \mathrm{E}+03$ & $1.14 \mathrm{E}+05$ & 82.9 & 7 & 5.3 \\
\hline A & LAW-10 & SY-101 & $1.35 \mathrm{E}+05$ & $1.14 \mathrm{E}+03$ & $1.37 \mathrm{E}+05$ & 119.3 & 2 & 6.0 \\
\hline A & LAW-11 & SY-101 & $1.34 \mathrm{E}+05$ & $1.11 \mathrm{E}+03$ & $1.35 \mathrm{E}+05$ & 120.4 & 3 & 5.9 \\
\hline A & LAW-12 & AN-103 & $1.04 \mathrm{E}+05$ & $2.56 \mathrm{E}+03$ & $1.06 \mathrm{E}+05$ & 40.5 & 8 & 5.8 \\
\hline A & LAW-13 & AN-103 & $1.04 \mathrm{E}+05$ & $2.59 \mathrm{E}+03$ & $1.06 \mathrm{E}+05$ & 39.9 & 8 & 5.8 \\
\hline A & LAW-14 & AW-101 & $1.09 \mathrm{E}+05$ & $7.00 \mathrm{E}+03$ & $1.16 \mathrm{E}+05$ & 15.6 & 6 & 9.2 \\
\hline A & LAW-15 & AW-101 & $1.10 \mathrm{E}+05$ & $8.93 \mathrm{E}+03$ & $1.19 \mathrm{E}+05$ & 12.3 & 4 & 10.6 \\
\hline \hline B & LAW-2a & $\begin{array}{c}\text { Pretreated } \\
\text { A-101/AZ-102 }\end{array}$ & $1.07 \mathrm{E}+04$ & $2.67 \mathrm{E}+02$ & $1.10 \mathrm{E}+04$ & 40.0 & 2 & - \\
\hline B & LAW-2b & Pretreated & $1.16 \mathrm{E}+04$ & $3.32 \mathrm{E}+02$ & $1.19 \mathrm{E}+04$ & 35.0 & 1 & - \\
\hline C & LAW-3 & AN-102 & $1.26 \mathrm{E}+05$ & $1.11 \mathrm{E}+03$ & $1.27 \mathrm{E}+05$ & 113.8 & 2 & - \\
\hline C & LAW-4 & AN-102 & $1.32 \mathrm{E}+05$ & $1.16 \mathrm{E}+03$ & $1.33 \mathrm{E}+05$ & 113.8 & 1 & - \\
\hline C & LAW-7 & AN-107 & $1.12 \mathrm{E}+05$ & $5.00 \mathrm{E}+02$ & $1.13 \mathrm{E}+05$ & 224.5 & 3 & - \\
\hline
\end{tabular}

${ }^{\text {a }}$ Ranking was performed over each envelope where the highest $\left(\mathrm{Na}^{+}+\mathrm{K}^{+}\right)$level is ranked number 1 . However, the higher the concentration of $\mathrm{K}^{+}$relative to $\mathrm{Na}^{+}$in a feed solution, the more competition for surface sites experienced since the CST material has a much stronger affinity for $\mathrm{K}^{+}$than for $\mathrm{Na}^{+}$.

${ }^{b}$ The worst case indicator is based on Eq. (B-2) and the appropriate binary selectivity coefficients. The higher the number the worst (i.e., lower cesium adsorption) the isotherm. Here, appropriate Envelope A weighting between $\mathrm{K}^{+}\left(\mathrm{K}_{12}=1,400\right)$ and $\mathrm{Na}^{+}\left(\mathrm{K}_{13}=26,000\right.$, ) levels due to affinity differences is achieved. 
WESTINGHOUSE SAVANNAH RIVER COMPANY

Report:

WSRC-TR-2000-00???

Preliminary Ion Exchange Modeling for Removal of Cesium from

Revision (Date):

$0(07 / 05 / 02)$

Hanford Waste Using Crystalline Silicotitanate Material

Page:

182 of 338

Table B-3. "Extrapolated" ZAM equilibrium isotherm model predictions for CST material in the powder form (IE-910) in contact with Envelope A's LAW-1 feed solution at $5 \mathrm{M}\left[\mathrm{Na}^{+}\right]{ }^{\mathrm{a}}$

\begin{tabular}{|c|c|c|c|c|c|c|c|}
\hline Cs (initial) & Cs (final) ${ }^{b}$ & $\mathrm{Na} / \mathrm{Cs}$ & Ionic strength $^{c}$ & $\operatorname{Cs} K_{d}$ & Cs loading & $\mathbf{K ~ K}_{\mathrm{d}}{ }^{\mathrm{c}}$ & K loading ${ }^{c}$ \\
\hline$[\mathbf{M}]$ & {$[\mathbf{M}]$} & [molar ratio] & [gmole/kg] & {$[\mathrm{ml} / \mathrm{g}]$} & [mmole/g] & {$[\mathrm{ml} / \mathrm{g}]$} & [mmole/g] \\
\hline 0 & same as initial & infinity & - & 0.0 & $0.000 \mathrm{E}+00$ & - & - \\
\hline 0.000001 & same as initial & $5.000 \mathrm{E}+06$ & - & 1326.5 & $1.326 \mathrm{E}-03$ & - & - \\
\hline 0.00001 & same as initial & $5.000 \mathrm{E}+05$ & - & 1300.3 & $1.300 \mathrm{E}-02$ & - & - \\
\hline 0.00005 & same as initial & $1.000 \mathrm{E}+05$ & - & 1197.8 & 5.989E-02 & - & - \\
\hline 0.0001 & same as initial & $5.000 \mathrm{E}+04$ & - & 1089.6 & $1.090 \mathrm{E}-01$ & - & - \\
\hline 0.0005 & same as initial & $1.000 \mathrm{E}+04$ & - & 625.8 & $3.129 \mathrm{E}-01$ & - & - \\
\hline 0.001 & same as initial & $5.000 \mathrm{E}+03$ & - & 407.3 & $4.073 \mathrm{E}-01$ & - & - \\
\hline 0.002 & same as initial & $2.500 \mathrm{E}+03$ & - & 239.4 & $4.788 \mathrm{E}-01$ & - & - \\
\hline 0.003 & same as initial & $1.667 \mathrm{E}+03$ & - & 169.5 & $5.084 \mathrm{E}-01$ & - & - \\
\hline 0.005 & same as initial & $1.000 \mathrm{E}+03$ & - & 107.0 & $5.348 \mathrm{E}-01$ & - & - \\
\hline 0.01 & same as initial & $5.000 \mathrm{E}+02$ & - & 55.7 & $5.565 \mathrm{E}-01$ & - & - \\
\hline 0.03 & same as initial & $1.667 \mathrm{E}+02$ & - & 19.1 & 5.719E-01 & - & - \\
\hline 0.05 & same as initial & $1.000 \mathrm{E}+02$ & - & 11.5 & $5.751 \mathrm{E}-01$ & - & - \\
\hline 0.1 & same as initial & $5.000 \mathrm{E}+01$ & - & 5.8 & $5.775 \mathrm{E}-01$ & - & - \\
\hline $\begin{array}{c}3.598 \mathrm{E}-05 \\
\text { (feed conc.) }\end{array}$ & same as initial & $1.1 .390 \mathrm{E}+05$ & - & 1238.0 & "4.455E-02 & - & - \\
\hline
\end{tabular}

${ }^{\text {a }}$ At a $5.0 \mathrm{M}$ sodium concentration level the solution density is estimated to be $1.277 \mathrm{~g} / \mathrm{ml}$ and at $0.712 \mathrm{M}\left[\mathrm{K}^{+}\right]$.

${ }^{b}$ Final cesium equilibrium concentrations are approx. equal to initial values due to extrapolation scheme used.

${ }^{c}$ These quantities were not computed during the application of the extrapolation scheme chosen to correct ZAM model output due to its unwarranted prediction of $\mathrm{KNO}_{3}$ precipitation.

Table B-4. ZAM equilibrium isotherm model predictions for CST material in the powder form (IE-910) in contact with Envelope A's LAW-5 feed solution at $5 \mathrm{M}\left[\mathrm{Na}^{+}\right]^{\text {a }}$

\begin{tabular}{|c|c|c|c|c|c|c|c|}
\hline Cs (initial) & Cs (final) ${ }^{b}$ & $\mathrm{Na} / \mathrm{Cs}$ & Ionic strength & $\operatorname{Cs} K_{d}$ & Cs loading & $\mathbf{K} \mathbf{K}_{\mathrm{d}}$ & K loading \\
\hline$[\mathbf{M}]$ & {$[\mathbf{M}]$} & [molar ratio] & [gmole/kg] & {$[\mathrm{ml} / \mathrm{g}]$} & {$[\mathrm{mmole} / \mathrm{g}]$} & {$[\mathrm{ml} / \mathrm{g}]$} & [mmole/g] \\
\hline 0 & $0.000 \mathrm{E}+00$ & infinity & 6.727 & 0.0 & $0.000 \mathrm{E}+00$ & 5.548 & $3.369 \mathrm{E}-01$ \\
\hline 0.000001 & 9.759E-07 & $5.123 \mathrm{E}+06$ & 6.727 & 2465.0 & $2.406 \mathrm{E}-03$ & 5.539 & $3.364 \mathrm{E}-01$ \\
\hline 0.00001 & $9.768 \mathrm{E}-06$ & $5.119 \mathrm{E}+05$ & 6.727 & 2376.0 & $2.321 \mathrm{E}-02$ & 5.455 & $3.313 \mathrm{E}-01$ \\
\hline 0.00005 & $4.900 \mathrm{E}-05$ & $1.020 \mathrm{E}+05$ & 6.727 & 2047.0 & $1.003 \mathrm{E}-01$ & 5.146 & $3.125 \mathrm{E}-01$ \\
\hline 0.0001 & $9.829 \mathrm{E}-05$ & $5.087 \mathrm{E}+04$ & 6.727 & 1744.0 & $1.714 \mathrm{E}-01$ & 4.861 & $2.952 \mathrm{E}-01$ \\
\hline 0.0005 & $4.961 \mathrm{E}-04$ & $1.008 \mathrm{E}+04$ & 6.728 & 794.0 & $3.939 \mathrm{E}-01$ & 3.969 & $2.410 \mathrm{E}-01$ \\
\hline 0.001 & $9.953 \mathrm{E}-04$ & $5.024 \mathrm{E}+03$ & 6.729 & 471.6 & 4.694E-01 & 3.666 & $2.226 \mathrm{E}-01$ \\
\hline 0.002 & $1.995 \mathrm{E}-03$ & $2.506 \mathrm{E}+03$ & 6.732 & 260.2 & 5.191E-01 & 3.467 & $2.106 \mathrm{E}-01$ \\
\hline 0.003 & $2.995 \mathrm{E}-03$ & $1.669 \mathrm{E}+03$ & 6.734 & 179.6 & $5.379 \mathrm{E}-01$ & 3.391 & $2.059 \mathrm{E}-01$ \\
\hline 0.005 & 4.994E-03 & $1.001 \mathrm{E}+03$ & 6.739 & 110.9 & $5.538 \mathrm{E}-01$ & 3.326 & $2.020 \mathrm{E}-01$ \\
\hline 0.01 & $9.994 \mathrm{E}-03$ & $5.003 \mathrm{E}+02$ & 6.751 & 56.7 & $5.667 \mathrm{E}-01$ & 3.273 & $1.988 \mathrm{E}-01$ \\
\hline 0.03 & $2.999 \mathrm{E}-02$ & $1.667 \mathrm{E}+02$ & 6.801 & 19.2 & $5.755 \mathrm{E}-01$ & 3.229 & $1.961 \mathrm{E}-01$ \\
\hline 0.05 & 4.999E-02 & $1.000 \mathrm{E}+02$ & 6.851 & 11.6 & $5.774 \mathrm{E}-01$ & 3.213 & $1.951 \mathrm{E}-01$ \\
\hline 0.1 & $9.999 \mathrm{E}-02$ & $5.001 \mathrm{E}+01$ & 6.979 & 5.8 & $5.785 \mathrm{E}-01$ & 3.185 & $1.934 \mathrm{E}-01$ \\
\hline $\begin{array}{c}6.283 \mathrm{E}-05 \\
\text { (feed conc.) }\end{array}$ & 6.162E-05 & $8.114 \mathrm{E}+04$ & 6.727 & 191960.0 & $1.208 \mathrm{E}-01$ & 5.064 & 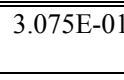 \\
\hline
\end{tabular}

${ }^{\mathrm{a}}$ At a $5.0 \mathrm{M}$ sodium concentration level the solution density is estimated to be $1.232 \mathrm{~g} / \mathrm{ml}$ and at $0.06073 \mathrm{M}\left[\mathrm{K}^{+}\right]$.

b Final cesium equilibrium concentrations are only slightly less than initial values due to large phase ratio used. 
WESTINGHOUSE SAVANNAH RIVER COMPANY

Report:

WSRC-TR-2000-00???

Preliminary Ion Exchange Modeling for Removal of Cesium from

Revision (Date):

$0(07 / 05 / 02)$

Hanford Waste Using Crystalline Silicotitanate Material

Page:

183 of 338

Table B-5. ZAM equilibrium isotherm model predictions for CST material in the powder form (IE-910) in contact with Envelope A's LAW-6 feed solution at $5 \mathrm{M}\left[\mathrm{Na}^{+}\right]^{\mathrm{a}}{ }^{\mathrm{C}}$

\begin{tabular}{|c|c|c|c|c|c|c|c|}
\hline Cs (initial) & Cs (final) $^{\mathbf{b}}$ & $\mathbf{N a / C s}$ & Ionic strength & $\mathbf{C s ~}_{\mathbf{d}}$ & Cs loading & $\mathbf{K}_{\mathbf{d}}$ & K loading \\
\hline$[\mathbf{M}]$ & {$[\mathbf{M}]$} & {$[\mathbf{m o l a r}$ ratio] } & {$[\mathbf{g m o l e} / \mathbf{k g}]$} & {$[\mathbf{m l} / \mathbf{g}]$} & {$[\mathbf{m m o l e} / \mathbf{g}]$} & {$[\mathbf{m l} / \mathbf{g}]$} & {$[\mathbf{m m o l e} / \mathbf{g}]$} \\
\hline 0 & $0.000 \mathrm{E}+00$ & infinity & 6.652 & 0.0 & $0.000 \mathrm{E}+00$ & 5.505 & $3.437 \mathrm{E}-01$ \\
\hline 0.000001 & $9.762 \mathrm{E}-07$ & $5.122 \mathrm{E}+06$ & 6.652 & 2441.0 & $2.383 \mathrm{E}-03$ & 5.496 & $3.431 \mathrm{E}-01$ \\
\hline 0.00001 & $9.770 \mathrm{E}-06$ & $5.118 \mathrm{E}+05$ & 6.652 & 2353.0 & $2.299 \mathrm{E}-02$ & 5.414 & $3.380 \mathrm{E}-01$ \\
\hline 0.00005 & $4.901 \mathrm{E}-05$ & $1.020 \mathrm{E}+05$ & 6.652 & 2030.0 & $9.949 \mathrm{E}-02$ & 5.113 & $3.192 \mathrm{E}-01$ \\
\hline 0.0001 & $9.830 \mathrm{E}-05$ & $5.086 \mathrm{E}+04$ & 6.652 & 1731.0 & $1.702 \mathrm{E}-01$ & 4.834 & $3.018 \mathrm{E}-01$ \\
\hline 0.0005 & $4.961 \mathrm{E}-04$ & $1.008 \mathrm{E}+04$ & 6.653 & 791.5 & $3.927 \mathrm{E}-01$ & 3.957 & $2.470 \mathrm{E}-01$ \\
\hline 0.001 & $9.953 \mathrm{E}-04$ & $5.024 \mathrm{E}+03$ & 6.654 & 470.7 & $4.685 \mathrm{E}-01$ & 3.657 & $2.283 \mathrm{E}-01$ \\
\hline 0.002 & $1.995 \mathrm{E}-03$ & $2.506 \mathrm{E}+03$ & 6.657 & 259.9 & $5.185 \mathrm{E}-01$ & 3.460 & $2.160 \mathrm{E}-01$ \\
\hline 0.003 & $2.995 \mathrm{E}-03$ & $1.669 \mathrm{E}+03$ & 6.659 & 179.5 & $5.376 \mathrm{E}-01$ & 3.385 & $2.113 \mathrm{E}-01$ \\
\hline 0.005 & $4.994 \mathrm{E}-03$ & $1.001 \mathrm{E}+03$ & 6.664 & 110.9 & $5.538 \mathrm{E}-01$ & 3.320 & $2.073 \mathrm{E}-01$ \\
\hline 0.01 & $9.994 \mathrm{E}-03$ & $5.003 \mathrm{E}+02$ & 6.677 & 56.7 & $5.665 \mathrm{E}-01$ & 3.267 & $2.040 \mathrm{E}-01$ \\
\hline 0.03 & $2.999 \mathrm{E}-02$ & $1.667 \mathrm{E}+02$ & 6.726 & 19.2 & $5.752 \mathrm{E}-01$ & 3.223 & $2.012 \mathrm{E}-01$ \\
\hline 0.05 & $4.999 \mathrm{E}-02$ & $1.000 \mathrm{E}+02$ & 6.776 & 11.6 & $5.774 \mathrm{E}-01$ & 3.207 & $2.002 \mathrm{E}-01$ \\
\hline 0.1 & $9.999 \mathrm{E}-02$ & $5.001 \mathrm{E}+01$ & 6.902 & 5.8 & $5.785 \mathrm{E}-01$ & 3.180 & $1.985 \mathrm{E}-01$ \\
\hline \hline $\begin{array}{c}6.328 \mathrm{E}-05 \\
\text { (feed conc.) }\end{array}$ & $6.207 \mathrm{E}-05$ & $8.055 \mathrm{E}+04$ & 6.652 & 1941.0 & $1.205 \mathrm{E}-01$ & 5.030 & $3.140 \mathrm{E}-01$ \\
\hline
\end{tabular}

${ }^{a}$ At a $5.0 \mathrm{M}$ sodium concentration level the solution density is estimated to be $1.231 \mathrm{~g} / \mathrm{ml}$ and at $0.06243 \mathrm{M}\left[\mathrm{K}^{+}\right]$.

${ }^{\mathrm{b}}$ Final cesium equilibrium concentrations are only slightly less than initial values due to large phase ratio used.

Table B-6. ZAM equilibrium isotherm model predictions for CST material in the powder form (IE-910) in contact with Envelope A's LAW-8 feed solution at $5 \mathrm{M}\left[\mathrm{Na}^{+}\right]^{\mathrm{a}}{ }^{\mathrm{C}}$

\begin{tabular}{|c|c|c|c|c|c|c|c|}
\hline Cs (initial) & Cs (final) ${ }^{b}$ & $\mathrm{Na} / \mathrm{Cs}$ & Ionic strength & $\operatorname{Cs} K_{d}$ & Cs loading & $K_{\mathrm{d}}$ & K loading \\
\hline$[\mathbf{M}]$ & {$[\mathbf{M}]$} & [molar ratio] & [gmole/kg] & {$[\mathrm{ml} / \mathbf{g}]$} & [mmole/g] & {$[\mathrm{ml} / \mathbf{g}]$} & [mmole/g] \\
\hline 0 & $0.000 \mathrm{E}+00$ & infinity & 6.304 & 0.0 & $0.000 \mathrm{E}+00$ & 5.231 & $3.775 \mathrm{E}-01$ \\
\hline 0.000001 & $9.775 \mathrm{E}-07$ & $5.115 \mathrm{E}+06$ & 6.304 & 2297.0 & $2.245 \mathrm{E}-03$ & 5.223 & $3.769 \mathrm{E}-01$ \\
\hline 0.00001 & $9.783 \mathrm{E}-06$ & $5.111 \mathrm{E}+05$ & 6.304 & 2220.0 & $2.172 \mathrm{E}-02$ & 5.153 & $3.718 \mathrm{E}-01$ \\
\hline 0.00005 & $4.905 \mathrm{E}-05$ & $1.019 \mathrm{E}+05$ & 6.304 & 1930.0 & $9.467 \mathrm{E}-02$ & 4.891 & $3.529 \mathrm{E}-01$ \\
\hline 0.0001 & $9.837 \mathrm{E}-05$ & $5.083 \mathrm{E}+04$ & 6.304 & 1658.0 & $1.631 \mathrm{E}-01$ & 4.646 & $3.353 \mathrm{E}-01$ \\
\hline 0.0005 & $4.962 \mathrm{E}-04$ & $1.008 \mathrm{E}+04$ & 6.305 & 775.7 & $3.849 \mathrm{E}-01$ & 3.851 & $2.779 \mathrm{E}-01$ \\
\hline 0.001 & $9.954 \mathrm{E}-04$ & $5.023 \mathrm{E}+03$ & 6.307 & 465.1 & $4.630 \mathrm{E}-01$ & 3.571 & $2.577 \mathrm{E}-01$ \\
\hline 0.002 & $1.995 \mathrm{E}-03$ & $2.506 \mathrm{E}+03$ & 6.309 & 258.2 & $5.151 \mathrm{E}-01$ & 3.384 & $2.442 \mathrm{E}-01$ \\
\hline 0.003 & $2.995 \mathrm{E}-03$ & $1.669 \mathrm{E}+03$ & 6.311 & 178.7 & $5.352 \mathrm{E}-01$ & 3.312 & $2.390 \mathrm{E}-01$ \\
\hline 0.005 & 4.994E-03 & $1.001 \mathrm{E}+03$ & 6.316 & 110.5 & $5.518 \mathrm{E}-01$ & 3.250 & $2.345 \mathrm{E}-01$ \\
\hline 0.01 & $9.994 \mathrm{E}-03$ & $5.003 \mathrm{E}+02$ & 6.328 & 56.6 & 5.657E-01 & 3.199 & $2.308 \mathrm{E}-01$ \\
\hline 0.03 & 2.999E-02 & $1.667 \mathrm{E}+02$ & 6.376 & 19.2 & 5.749E-01 & 3.157 & $2.278 \mathrm{E}-01$ \\
\hline 0.05 & 4.999E-02 & $1.000 \mathrm{E}+02$ & 6.424 & 11.5 & $5.769 \mathrm{E}-01$ & 3.141 & $2.267 \mathrm{E}-01$ \\
\hline 0.1 & 9.999E-02 & $5.001 \mathrm{E}+01$ & 6.547 & 5.8 & $5.784 \mathrm{E}-01$ & 3.115 & $2.248 \mathrm{E}-01$ \\
\hline $\begin{array}{l}4.324 \mathrm{E}-05 \\
\text { (feed conc.) }\end{array}$ & 4.241E-05 & $1.179 \mathrm{E}+05$ & 6.304 & 1974.0 & 8.372E-02 & 4.931 & $3.558 \mathrm{E}-01$ \\
\hline
\end{tabular}


WESTINGHOUSE SAVANNAH RIVER COMPANY

Report:

WSRC-TR-2000-00???

Preliminary Ion Exchange Modeling for Removal of Cesium from

Revision (Date):

$0(07 / 05 / 02)$

Hanford Waste Using Crystalline Silicotitanate Material

Page:

184 of 338

Table B-7. ZAM equilibrium isotherm model predictions for CST material in the powder form (IE-910) in contact with Envelope A's LAW-9 feed solution at $5 \mathrm{M}\left[\mathrm{Na}^{+}\right]^{\mathrm{a}}{ }^{\mathrm{C}}$

\begin{tabular}{|c|c|c|c|c|c|c|c|}
\hline Cs (initial) & Cs (final) $^{\mathbf{b}}$ & $\mathbf{N a / C s}$ & Ionic strength & $\mathbf{C s ~}_{\mathbf{d}}$ & Cs loading & $\mathbf{K}_{\mathbf{d}}$ & K loading \\
\hline$[\mathbf{M}]$ & {$[\mathbf{M}]$} & {$[\mathbf{m o l a r}$ ratio] } & {$[\mathbf{g m o l e} / \mathbf{k g}]$} & {$[\mathbf{m l} / \mathbf{g}]$} & {$[\mathbf{m m o l e} / \mathbf{g}]$} & {$[\mathbf{m l} / \mathbf{g}]$} & {$[\mathbf{m m o l e} / \mathbf{g}]$} \\
\hline 0 & $0.000 \mathrm{E}+00$ & infinity & 6.951 & 0.0 & $0.000 \mathrm{E}+00$ & 5.626 & $3.391 \mathrm{E}-01$ \\
\hline 0.000001 & $9.749 \mathrm{E}-07$ & $5.129 \mathrm{E}+06$ & 6.951 & 2573.0 & $2.508 \mathrm{E}-03$ & 5.616 & $3.385 \mathrm{E}-01$ \\
\hline 0.00001 & $9.758 \mathrm{E}-06$ & $5.124 \mathrm{E}+05$ & 6.951 & 2476.0 & $2.416 \mathrm{E}-02$ & 5.528 & $3.332 \mathrm{E}-01$ \\
\hline 0.00005 & $4.896 \mathrm{E}-05$ & $1.021 \mathrm{E}+05$ & 6.951 & 2121.0 & $1.038 \mathrm{E}-01$ & 5.205 & $3.137 \mathrm{E}-01$ \\
\hline 0.0001 & $9.823 \mathrm{E}-05$ & $5.090 \mathrm{E}+04$ & 6.951 & 1797.0 & $1.765 \mathrm{E}-01$ & 4.910 & $2.960 \mathrm{E}-01$ \\
\hline 0.0005 & $4.960 \mathrm{E}-04$ & $1.008 \mathrm{E}+04$ & 6.952 & 805.0 & $3.993 \mathrm{E}-01$ & 4.008 & $2.416 \mathrm{E}-01$ \\
\hline 0.001 & $9.953 \mathrm{E}-04$ & $5.024 \mathrm{E}+03$ & 6.954 & 475.5 & $4.733 \mathrm{E}-01$ & 3.708 & $2.235 \mathrm{E}-01$ \\
\hline 0.002 & $1.995 \mathrm{E}-03$ & $2.506 \mathrm{E}+03$ & 6.956 & 261.3 & $5.213 \mathrm{E}-01$ & 3.512 & $2.117 \mathrm{E}-01$ \\
\hline 0.003 & $2.995 \mathrm{E}-03$ & $1.669 \mathrm{E}+03$ & 6.959 & 180.2 & $5.397 \mathrm{E}-01$ & 3.438 & $2.072 \mathrm{E}-01$ \\
\hline 0.005 & $4.994 \mathrm{E}-03$ & $1.001 \mathrm{E}+03$ & 6.964 & 111.1 & $5.548 \mathrm{E}-01$ & 3.375 & $2.034 \mathrm{E}-01$ \\
\hline 0.01 & $4.994 \mathrm{E}-03$ & $1.001 \mathrm{E}+03$ & 6.964 & 111.1 & $5.548 \mathrm{E}-01$ & 3.375 & $2.034 \mathrm{E}-01$ \\
\hline 0.03 & $2.999 \mathrm{E}-02$ & $1.667 \mathrm{E}+02$ & 7.026 & 19.2 & $5.755 \mathrm{E}-01$ & 3.280 & $1.977 \mathrm{E}-01$ \\
\hline 0.05 & $4.999 \mathrm{E}-02$ & $1.000 \mathrm{E}+02$ & 7.077 & 11.6 & $5.774 \mathrm{E}-01$ & 3.264 & $1.968 \mathrm{E}-01$ \\
\hline 0.1 & $9.999 \mathrm{E}-02$ & $5.001 \mathrm{E}+01$ & 7.206 & 5.8 & $5.786 \mathrm{E}-01$ & 3.237 & $1.951 \mathrm{E}-01$ \\
\hline \hline $\begin{array}{c}4.444 \mathrm{E}-05 \\
\text { (feed conc.) }\end{array}$ & $4.350 \mathrm{E}-05$ & $1.149 \mathrm{E}+05$ & 6.951 & 2164.0 & $9.413 \mathrm{E}-02$ & 5.244 & $3.161 \mathrm{E}-01$ \\
\hline
\end{tabular}

${ }^{a}$ At a $5.0 \mathrm{M}$ sodium concentration level the solution density is estimated to be $1.238 \mathrm{~g} / \mathrm{ml}$ and at $0.06027 \mathrm{M}\left[\mathrm{K}^{+}\right]$.

${ }^{\mathrm{b}}$ Final cesium equilibrium concentrations are only slightly less than initial values due to large phase ratio used.

Table B-8. ZAM equilibrium isotherm model predictions for CST material in the powder form (IE-910) in contact with Envelope A's LAW-10 feed solution at $5 \mathrm{M}\left[\mathrm{Na}^{+}\right]$. $^{\mathrm{a}}$

\begin{tabular}{|c|c|c|c|c|c|c|c|}
\hline Cs (initial) & Cs (final) ${ }^{b}$ & $\mathrm{Na} / \mathrm{Cs}$ & Ionic strength & $\operatorname{Cs} K_{d}$ & Cs loading & $K_{\mathrm{d}}$ & K loading \\
\hline$[\mathbf{M}]$ & {$[\mathbf{M}]$} & [molar ratio] & [gmole/kg] & {$[\mathrm{ml} / \mathbf{g}]$} & [mmole/g] & {$[\mathrm{ml} / \mathbf{g}]$} & [mmole/g] \\
\hline 0 & $0.000 \mathrm{E}+00$ & infinity & 6.553 & 0.0 & $0.000 \mathrm{E}+00$ & 6.011 & $2.520 \mathrm{E}-01$ \\
\hline 0.000001 & $9.746 \mathrm{E}-07$ & $5.130 \mathrm{E}+06$ & 6.553 & 2609.0 & $2.543 \mathrm{E}-03$ & 5.999 & $2.515 \mathrm{E}-01$ \\
\hline 0.00001 & $9.755 \mathrm{E}-06$ & $5.126 \mathrm{E}+05$ & 6.553 & 2509.0 & $2.448 \mathrm{E}-02$ & 5.894 & $2.471 \mathrm{E}-01$ \\
\hline 0.00005 & $4.895 \mathrm{E}-05$ & $1.021 \mathrm{E}+05$ & 6.553 & 2146.0 & $1.050 \mathrm{E}-01$ & 5.508 & $2.309 \mathrm{E}-01$ \\
\hline 0.0001 & $9.822 \mathrm{E}-05$ & $5.091 \mathrm{E}+04$ & 6.553 & 1815.0 & $1.783 \mathrm{E}-01$ & 5.158 & $2.162 \mathrm{E}-01$ \\
\hline 0.0005 & $4.960 \mathrm{E}-04$ & $1.008 \mathrm{E}+04$ & 6.554 & 808.5 & $4.010 \mathrm{E}-01$ & 4.092 & $1.715 \mathrm{E}-01$ \\
\hline 0.001 & $9.953 \mathrm{E}-04$ & $5.024 \mathrm{E}+03$ & 6.555 & 476.7 & $4.745 \mathrm{E}-01$ & 3.741 & $1.568 \mathrm{E}-01$ \\
\hline 0.002 & $1.995 \mathrm{E}-03$ & $2.506 \mathrm{E}+03$ & 6.558 & 261.7 & $5.221 \mathrm{E}-01$ & 3.512 & $1.472 \mathrm{E}-01$ \\
\hline 0.003 & $2.995 \mathrm{E}-03$ & $1.669 \mathrm{E}+03$ & 6.560 & 180.3 & $5.400 \mathrm{E}-01$ & 3.426 & $1.436 \mathrm{E}-01$ \\
\hline 0.005 & 4.994E-03 & $1.001 \mathrm{E}+03$ & 6.565 & 111.2 & $5.553 \mathrm{E}-01$ & 3.352 & $1.405 \mathrm{E}-01$ \\
\hline 0.01 & $9.994 \mathrm{E}-03$ & $5.003 \mathrm{E}+02$ & 6.577 & 56.8 & 5.674E-01 & 3.291 & $1.380 \mathrm{E}-01$ \\
\hline 0.03 & 2.999E-02 & $1.667 \mathrm{E}+02$ & 6.626 & 19.2 & $5.755 \mathrm{E}-01$ & 3.242 & $1.359 \mathrm{E}-01$ \\
\hline 0.05 & 4.999E-02 & $1.000 \mathrm{E}+02$ & 6.675 & 11.6 & $5.774 \mathrm{E}-01$ & 3.224 & $1.352 \mathrm{E}-01$ \\
\hline 0.1 & 9.999E-02 & $5.001 \mathrm{E}+01$ & 6.799 & 5.8 & $5.786 \mathrm{E}-01$ & 3.193 & $1.339 \mathrm{E}-01$ \\
\hline $\begin{array}{c}3.692 \mathrm{E}-05 \\
\text { (feed conc.) }\end{array}$ & 3.611E-05 & $1.385 \mathrm{E}+05$ & 6.553 & 2253.0 & $8.136 \mathrm{E}-02$ & (5.622 & $2.357 \mathrm{E}-01$ \\
\hline
\end{tabular}


WESTINGHOUSE SAVANNAH RIVER COMPANY

Report:

WSRC-TR-2000-00???

Preliminary Ion Exchange Modeling for Removal of Cesium from

Revision (Date):

$0(07 / 05 / 02)$

Hanford Waste Using Crystalline Silicotitanate Material

Page:

185 of 338

Table B-9. ZAM equilibrium isotherm model predictions for CST material in the powder form (IE-910) in contact with Envelope A's LAW-11 feed solution at $5 \mathrm{M}\left[\mathrm{Na}^{+}\right]^{\mathrm{a}}$

\begin{tabular}{|c|c|c|c|c|c|c|c|}
\hline Cs (initial) & Cs (final) $^{\mathbf{b}}$ & $\mathbf{N a / C s}$ & Ionic strength & $\mathbf{C s ~}_{\mathbf{d}}$ & Cs loading & $\mathbf{K}_{\mathbf{d}}$ & K loading \\
\hline$[\mathbf{M}]$ & {$[\mathbf{M}]$} & {$[\mathbf{m o l a r}$ ratio] } & {$[\mathbf{g m o l e} / \mathbf{k g}]$} & {$[\mathbf{m l} / \mathbf{g}]$} & {$[\mathbf{m m o l e} / \mathbf{g}]$} & {$[\mathbf{m l} / \mathbf{g}]$} & {$[\mathbf{m m o l e} / \mathbf{g}]$} \\
\hline 0 & $0.000 \mathrm{E}+00$ & infinity & 6.618 & 0.0 & $0.000 \mathrm{E}+00$ & 6.323 & $2.625 \mathrm{E}-01$ \\
\hline 0.000001 & $9.727 \mathrm{E}-07$ & $5.140 \mathrm{E}+06$ & 6.618 & 2810.0 & $2.733 \mathrm{E}-03$ & 6.309 & $2.619 \mathrm{E}-01$ \\
\hline 0.00001 & $9.737 \mathrm{E}-06$ & $5.135 \mathrm{E}+05$ & 6.618 & 2696.0 & $2.625 \mathrm{E}-02$ & 6.192 & $2.571 \mathrm{E}-01$ \\
\hline 0.00005 & $4.889 \mathrm{E}-05$ & $1.023 \mathrm{E}+05$ & 6.618 & 2281.0 & $1.115 \mathrm{E}-01$ & 5.768 & $2.395 \mathrm{E}-01$ \\
\hline 0.0001 & $9.813 \mathrm{E}-05$ & $5.095 \mathrm{E}+04$ & 6.618 & 1911.0 & $1.875 \mathrm{E}-01$ & 5.389 & $2.238 \mathrm{E}-01$ \\
\hline 0.0005 & $4.959 \mathrm{E}-04$ & $1.008 \mathrm{E}+04$ & 6.619 & 827.0 & $4.101 \mathrm{E}-01$ & 4.281 & $1.777 \mathrm{E}-01$ \\
\hline 0.001 & $9.952 \mathrm{E}-04$ & $5.024 \mathrm{E}+03$ & 6.620 & 483.0 & $4.807 \mathrm{E}-01$ & 3.930 & $1.632 \mathrm{E}-01$ \\
\hline 0.002 & $1.995 \mathrm{E}-03$ & $2.506 \mathrm{E}+03$ & 6.622 & 263.6 & $5.259 \mathrm{E}-01$ & 3.705 & $1.538 \mathrm{E}-01$ \\
\hline 0.003 & $2.995 \mathrm{E}-03$ & $1.669 \mathrm{E}+03$ & 6.625 & 181.2 & $5.427 \mathrm{E}-01$ & 3.620 & $1.503 \mathrm{E}-01$ \\
\hline 0.005 & $4.994 \mathrm{E}-03$ & $1.001 \mathrm{E}+03$ & 6.630 & 111.5 & $5.568 \mathrm{E}-01$ & 3.548 & $1.473 \mathrm{E}-01$ \\
\hline 0.01 & $9.994 \mathrm{E}-03$ & $5.003 \mathrm{E}+02$ & 6.642 & 56.9 & $5.683 \mathrm{E}-01$ & 3.490 & $1.449 \mathrm{E}-01$ \\
\hline 0.03 & $2.999 \mathrm{E}-02$ & $1.667 \mathrm{E}+02$ & 6.691 & 19.2 & $5.758 \mathrm{E}-01$ & 3.442 & $1.429 \mathrm{E}-01$ \\
\hline 0.05 & $4.999 \mathrm{E}-02$ & $1.000 \mathrm{E}+02$ & 6.740 & 11.6 & $5.774 \mathrm{E}-01$ & 3.424 & $1.422 \mathrm{E}-01$ \\
\hline 0.1 & $9.999 \mathrm{E}-02$ & $5.001 \mathrm{E}+01$ & 6.864 & 5.8 & $5.787 \mathrm{E}-01$ & 3.395 & $1.410 \mathrm{E}-01$ \\
\hline \hline $\begin{array}{c}3.739 \mathrm{E}-05 \\
\text { (feed conc.) }\end{array}$ & $3.652 \mathrm{E}-05$ & $1.369 \mathrm{E}+05$ & 6.618 & 2397.0 & $8.754 \mathrm{E}-02$ & 5.887 & $2.444 \mathrm{E}-01$ \\
\hline
\end{tabular}

${ }^{a}$ At a $5.0 \mathrm{M}$ sodium concentration level the solution density is estimated to be $1.232 \mathrm{~g} / \mathrm{ml}$ and at $0.04152 \mathrm{M}\left[\mathrm{K}^{+}\right]$.

${ }^{\mathrm{b}}$ Final cesium equilibrium concentrations are only slightly less than initial values due to large phase ratio used.

Table B-10. ZAM equilibrium isotherm model predictions for CST material in the powder form (IE-910) in contact with Envelope A's LAW-12 feed solution at $5 \mathrm{M}\left[\mathrm{Na}^{+}\right]^{\text {a }}$

\begin{tabular}{|c|c|c|c|c|c|c|c|}
\hline Cs (initial) & Cs (final) ${ }^{\mathbf{b}}$ & $\mathbf{N a} / \mathbf{C s}$ & Ionic strength & $\mathbf{C s} \mathbf{K}_{\mathbf{d}}$ & $\mathbf{C s}$ loading & $\mathbf{K ~ K ~}_{\mathbf{d}}$ & $\mathbf{K}$ loading \\
\hline$[\mathbf{M}]$ & {$[\mathbf{M}]$} & {$[\mathbf{m o l a r}$ ratio] } & {$[\mathbf{g m o l e} / \mathbf{k g}]$} & {$[\mathbf{m l} / \mathbf{g}]$} & {$[\mathbf{m m o l e} / \mathbf{g}]$} & {$[\mathbf{m l} / \mathbf{g}]$} & {$[\mathbf{m m o l e} / \mathbf{g}]$} \\
\hline 0 & $0.000 \mathrm{E}+00$ & infinity & 6.324 & 0.0 & $0.000 \mathrm{E}+00$ & 4.207 & $5.196 \mathrm{E}-01$ \\
\hline 0.000001 & $9.806 \mathrm{E}-07$ & $5.099 \mathrm{E}+06$ & 6.324 & 1980.0 & $1.942 \mathrm{E}-03$ & 4.203 & $5.191 \mathrm{E}-01$ \\
\hline 0.00001 & $9.811 \mathrm{E}-06$ & $5.096 \mathrm{E}+05$ & 6.324 & 1922.0 & $1.886 \mathrm{E}-02$ & 4.164 & $5.143 \mathrm{E}-01$ \\
\hline 0.00005 & $4.916 \mathrm{E}-05$ & $1.017 \mathrm{E}+05$ & 6.324 & 1700.0 & $8.357 \mathrm{E}-02$ & 4.015 & $4.959 \mathrm{E}-01$ \\
\hline 0.0001 & $9.854 \mathrm{E}-05$ & $5.074 \mathrm{E}+04$ & 6.324 & 1485.0 & $1.463 \mathrm{E}-01$ & 3.871 & $4.781 \mathrm{E}-01$ \\
\hline 0.0005 & $4.963 \mathrm{E}-04$ & $1.007 \mathrm{E}+04$ & 6.325 & 735.7 & $3.651 \mathrm{E}-01$ & 3.367 & $4.158 \mathrm{E}-01$ \\
\hline 0.001 & $9.955 \mathrm{E}-04$ & $5.023 \mathrm{E}+03$ & 6.327 & 450.5 & $4.485 \mathrm{E}-01$ & 3.176 & $3.922 \mathrm{E}-01$ \\
\hline 0.002 & $1.995 \mathrm{E}-03$ & $2.506 \mathrm{E}+03$ & 6.329 & 253.6 & $5.059 \mathrm{E}-01$ & 3.043 & $3.758 \mathrm{E}-01$ \\
\hline 0.003 & $2.995 \mathrm{E}-03$ & $1.669 \mathrm{E}+03$ & 6.331 & 176.5 & $5.286 \mathrm{E}-01$ & 2.991 & $3.694 \mathrm{E}-01$ \\
\hline 0.005 & $4.995 \mathrm{E}-03$ & $1.001 \mathrm{E}+03$ & 6.336 & 109.7 & $5.480 \mathrm{E}-01$ & 2.945 & $3.637 \mathrm{E}-01$ \\
\hline 0.01 & $9.994 \mathrm{E}-03$ & $5.003 \mathrm{E}+02$ & 6.348 & 56.4 & $5.635 \mathrm{E}-01$ & 2.908 & $3.591 \mathrm{E}-01$ \\
\hline 0.03 & $2.999 \mathrm{E}-02$ & $1.667 \mathrm{E}+02$ & 6.397 & 19.2 & $5.743 \mathrm{E}-01$ & 2.876 & $3.552 \mathrm{E}-01$ \\
\hline 0.05 & $4.999 \mathrm{E}-02$ & $1.000 \mathrm{E}+02$ & 6.446 & 11.5 & $5.764 \mathrm{E}-01$ & 2.865 & $3.538 \mathrm{E}-01$ \\
\hline 0.1 & $9.999 \mathrm{E}-02$ & $5.001 \mathrm{E}+01$ & 6.570 & 5.8 & $5.782 \mathrm{E}-01$ & 2.844 & $3.512 \mathrm{E}-01$ \\
\hline \hline $\begin{array}{c}4.831 \mathrm{E}-05 \\
\text { feed conc.) }\end{array}$ & $4.749 \mathrm{E}-05$ & $1.053 \mathrm{E}+05$ & 6.324 & 1709.0 & $8.116 \mathrm{E}-02$ & 4.021 & $4.966 \mathrm{E}-01$ \\
\hline
\end{tabular}

${ }^{a}$ At a $5.0 \mathrm{M}$ sodium concentration level the solution density is estimated to be $1.221 \mathrm{~g} / \mathrm{ml}$ and at $0.1235 \mathrm{M}\left[\mathrm{K}^{+}\right]$.

${ }^{b}$ Final cesium equilibrium concentrations are only slightly less than initial values due to large phase ratio used. 
WESTINGHOUSE SAVANNAH RIVER COMPANY

Report:

WSRC-TR-2000-00???

Preliminary Ion Exchange Modeling for Removal of Cesium from

Revision (Date):

$0(07 / 05 / 02)$

Hanford Waste Using Crystalline Silicotitanate Material

Page:

186 of 338

Table B-11. ZAM equilibrium isotherm model predictions for CST material in the powder form (IE-910) in contact with Envelope A's LAW-13 feed solution at $5 \mathrm{M}\left[\mathrm{Na}^{+}\right]^{\text {a }}$

\begin{tabular}{|c|c|c|c|c|c|c|c|}
\hline Cs (initial) & Cs (final) $^{\mathbf{b}}$ & $\mathbf{N a / C s}$ & Ionic strength & $\mathbf{C s}_{\mathbf{d}}$ & Cs loading & $\mathbf{K}_{\mathbf{d}}$ & K loading \\
\hline$[\mathbf{M}]$ & {$[\mathbf{M}]$} & {$[\mathbf{m o l a r}$ ratio] } & {$[\mathbf{g m o l e} / \mathbf{k g}]$} & {$[\mathbf{m l} / \mathbf{g}]$} & {$[\mathbf{m m o l e} / \mathbf{g}]$} & {$[\mathbf{m l} / \mathbf{g}]$} & {$[\mathbf{m m o l e} / \mathbf{g}]$} \\
\hline 0 & $0.000 \mathrm{E}+00$ & infinity & 6.327 & 0.0 & $0.000 \mathrm{E}+00$ & 4.177 & $5.230 \mathrm{E}-01$ \\
\hline 0.000001 & $9.807 \mathrm{E}-07$ & $5.098 \mathrm{E}+06$ & 6.327 & 1971.0 & $1.933 \mathrm{E}-03$ & 4.173 & $5.225 \mathrm{E}-01$ \\
\hline 0.00001 & $9.812 \mathrm{E}-06$ & $5.096 \mathrm{E}+05$ & 6.327 & 1914.0 & $1.878 \mathrm{E}-02$ & 4.135 & $5.177 \mathrm{E}-01$ \\
\hline 0.00005 & $4.917 \mathrm{E}-05$ & $1.017 \mathrm{E}+05$ & 6.327 & 1694.0 & $8.329 \mathrm{E}-02$ & 3.988 & $4.993 \mathrm{E}-01$ \\
\hline 0.0001 & $9.854 \mathrm{E}-05$ & $5.074 \mathrm{E}+04$ & 6.327 & 1480.0 & $1.458 \mathrm{E}-01$ & 3.846 & $4.815 \mathrm{E}-01$ \\
\hline 0.0005 & $4.964 \mathrm{E}-04$ & $1.007 \mathrm{E}+04$ & 6.328 & 734.4 & $3.646 \mathrm{E}-01$ & 3.350 & $4.194 \mathrm{E}-01$ \\
\hline 0.001 & $9.955 \mathrm{E}-04$ & $5.023 \mathrm{E}+03$ & 6.330 & 450.0 & $4.480 \mathrm{E}-01$ & 3.161 & $3.958 \mathrm{E}-01$ \\
\hline 0.002 & $1.995 \mathrm{E}-03$ & $2.506 \mathrm{E}+03$ & 6.332 & 253.4 & $5.055 \mathrm{E}-01$ & 3.029 & $3.792 \mathrm{E}-01$ \\
\hline 0.003 & $2.995 \mathrm{E}-03$ & $1.669 \mathrm{E}+03$ & 6.334 & 176.4 & $5.283 \mathrm{E}-01$ & 2.978 & $3.728 \mathrm{E}-01$ \\
\hline 0.005 & $4.995 \mathrm{E}-03$ & $1.001 \mathrm{E}+03$ & 6.339 & 109.7 & $5.480 \mathrm{E}-01$ & 2.933 & $3.672 \mathrm{E}-01$ \\
\hline 0.01 & $9.994 \mathrm{E}-03$ & $5.003 \mathrm{E}+02$ & 6.351 & 56.4 & $5.634 \mathrm{E}-01$ & 2.896 & $3.626 \mathrm{E}-01$ \\
\hline 0.03 & $2.999 \mathrm{E}-02$ & $1.667 \mathrm{E}+02$ & 6.400 & 19.2 & $5.743 \mathrm{E}-01$ & 2.864 & $3.586 \mathrm{E}-01$ \\
\hline 0.05 & $4.999 \mathrm{E}-02$ & $1.000 \mathrm{E}+02$ & 6.449 & 11.5 & $5.764 \mathrm{E}-01$ & 2.853 & $3.572 \mathrm{E}-01$ \\
\hline 0.1 & $9.999 \mathrm{E}-02$ & $5.001 \mathrm{E}+01$ & 6.573 & 5.8 & $5.782 \mathrm{E}-01$ & 2.832 & $3.546 \mathrm{E}-01$ \\
\hline \hline $\begin{array}{c}4.831 \mathrm{E}-05 \\
\text { (feed conc.) }\end{array}$ & $4.750 \mathrm{E}-05$ & $1.053 \mathrm{E}+05$ & 6.327 & 1702.0 & $8.085 \mathrm{E}-02$ & 3.994 & $5.000 \mathrm{E}-01$ \\
\hline
\end{tabular}

${ }^{a}$ At a $5.0 \mathrm{M}$ sodium concentration level the solution density is estimated to be $1.221 \mathrm{~g} / \mathrm{ml}$ and at $0.1252 \mathrm{M}\left[\mathrm{K}^{+}\right]$.

${ }^{\mathrm{b}}$ Final cesium equilibrium concentrations are only slightly less than initial values due to large phase ratio used.

Table B-12. ZAM equilibrium isotherm model predictions for CST material in the powder form (IE-910) in contact with Envelope A's LAW-14 feed solution at $5 \mathrm{M}\left[\mathrm{Na}^{+}\right]^{\text {a }}$

\begin{tabular}{|c|c|c|c|c|c|c|c|}
\hline Cs (initial) & Cs (final) $^{\mathbf{b}}$ & $\mathbf{N a} / \mathbf{C s}$ & Ionic strength & $\mathbf{C s ~ K}_{\mathbf{d}}$ & Cs loading & $\mathbf{K ~ K}_{\mathbf{d}}$ & $\mathbf{K}$ loading \\
\hline$[\mathbf{M}]$ & {$[\mathbf{M}]$} & {$[\mathbf{m o l a r}$ ratio] } & {$[\mathbf{g m o l e} / \mathbf{k g}]$} & {$[\mathbf{m l} / \mathbf{g}]$} & {$[\mathbf{m m o l e} / \mathbf{g}]$} & {$[\mathbf{m l} / \mathbf{g}]$} & {$[\mathbf{m m o l e} / \mathbf{g}]$} \\
\hline 0 & $0.000 \mathrm{E}+00$ & infinity & 6.617 & 0.0 & $0.000 \mathrm{E}+00$ & 2.444 & $7.816 \mathrm{E}-01$ \\
\hline 0.000001 & $9.843 \mathrm{E}-07$ & $5.080 \mathrm{E}+06$ & 6.617 & 1594.0 & $1.569 \mathrm{E}-03$ & 2.443 & $7.813 \mathrm{E}-01$ \\
\hline 0.00001 & $9.847 \mathrm{E}-06$ & $5.078 \mathrm{E}+05$ & 6.617 & 1557.0 & $1.533 \mathrm{E}-02$ & 2.433 & $7.781 \mathrm{E}-01$ \\
\hline 0.00005 & $4.931 \mathrm{E}-05$ & $1.014 \mathrm{E}+05$ & 6.617 & 1407.0 & $6.938 \mathrm{E}-02$ & 2.396 & $7.662 \mathrm{E}-01$ \\
\hline 0.0001 & $9.876 \mathrm{E}-05$ & $5.063 \mathrm{E}+04$ & 6.617 & 1257.0 & $1.241 \mathrm{E}-01$ & 2.359 & $7.544 \mathrm{E}-01$ \\
\hline 0.0005 & $4.966 \mathrm{E}-04$ & $1.007 \mathrm{E}+04$ & 6.618 & 674.8 & $3.351 \mathrm{E}-01$ & 2.215 & $7.084 \mathrm{E}-01$ \\
\hline 0.001 & $9.957 \mathrm{E}-04$ & $5.022 \mathrm{E}+03$ & 6.619 & 426.9 & $4.251 \mathrm{E}-01$ & 2.154 & $6.888 \mathrm{E}-01$ \\
\hline 0.002 & $1.995 \mathrm{E}-03$ & $2.506 \mathrm{E}+03$ & 6.622 & 246.0 & $4.908 \mathrm{E}-01$ & 2.109 & $6.745 \mathrm{E}-01$ \\
\hline 0.003 & $2.995 \mathrm{E}-03$ & $1.669 \mathrm{E}+03$ & 6.624 & 172.7 & $5.172 \mathrm{E}-01$ & 2.091 & $6.687 \mathrm{E}-01$ \\
\hline 0.005 & $4.995 \mathrm{E}-03$ & $1.001 \mathrm{E}+03$ & 6.629 & 108.2 & $5.405 \mathrm{E}-01$ & 2.075 & $6.636 \mathrm{E}-01$ \\
\hline 0.01 & $9.994 \mathrm{E}-03$ & $5.003 \mathrm{E}+02$ & 6.641 & 56.0 & $5.596 \mathrm{E}-01$ & 2.061 & $6.591 \mathrm{E}-01$ \\
\hline 0.03 & $2.999 \mathrm{E}-02$ & $1.667 \mathrm{E}+02$ & 6.690 & 19.1 & $5.728 \mathrm{E}-01$ & 2.049 & $6.553 \mathrm{E}-01$ \\
\hline 0.05 & $4.999 \mathrm{E}-02$ & $1.000 \mathrm{E}+02$ & 6.739 & 11.5 & $5.759 \mathrm{E}-01$ & 2.045 & $6.540 \mathrm{E}-01$ \\
\hline 0.1 & $4.999 \mathrm{E}-02$ & $1.000 \mathrm{E}+02$ & 6.739 & 11.5 & $5.759 \mathrm{E}-01$ & 2.045 & $6.540 \mathrm{E}-01$ \\
\hline \hline $\begin{array}{c}4.569 \mathrm{E}-05 \\
\text { feed conc.) }\end{array}$ & $4.504 \mathrm{E}-05$ & $1.110 \mathrm{E}+05$ & 6.617 & 1422.0 & $6.405 \mathrm{E}-02$ & 2.400 & $7.675 \mathrm{E}-01$ \\
\hline
\end{tabular}

${ }^{\text {a }}$ At a $5.0 \mathrm{M}$ sodium concentration level the solution density is estimated to be $1.234 \mathrm{~g} / \mathrm{ml}$ and at $0.3198 \mathrm{M}\left[\mathrm{K}^{+}\right]$.

${ }^{b}$ Final cesium equilibrium concentrations are only slightly less than initial values due to large phase ratio used. 
WESTINGHOUSE SAVANNAH RIVER COMPANY

Report:

WSRC-TR-2000-00???

Preliminary Ion Exchange Modeling for Removal of Cesium from

Revision (Date):

$0(07 / 05 / 02)$

Hanford Waste Using Crystalline Silicotitanate Material

Page:

187 of 338

Table B-13. ZAM equilibrium isotherm model predictions for CST material in the powder form (IE-910) in contact with Envelope A's LAW-15 feed solution at $5 \mathrm{M}\left[\mathrm{Na}^{+}\right]^{\mathrm{a}}$

\begin{tabular}{|c|c|c|c|c|c|c|c|}
\hline Cs (initial) & Cs (final) ${ }^{b}$ & $\mathrm{Na} / \mathrm{Cs}$ & Ionic strength & $\operatorname{Cs} K_{d}$ & Cs loading & $\mathbf{K} \mathbf{K}_{\mathrm{d}}$ & K loading \\
\hline [M] & {$[\mathbf{M}]$} & [molar ratio] & [gmole/kg] & {$[\mathrm{ml} / \mathrm{g}]$} & [mmole/g] & {$[\mathrm{ml} / \mathrm{g}]$} & [mmole/g] \\
\hline 0 & $0.000 \mathrm{E}+00$ & infinity & 6.487 & 0.0 & $0.000 \mathrm{E}+00$ & 2.068 & $8.411 \mathrm{E}-01$ \\
\hline 0.000001 & 9.852E-07 & $5.075 \mathrm{E}+06$ & 6.487 & 1502.0 & $1.480 \mathrm{E}-03$ & 2.067 & $8.406 \mathrm{E}-01$ \\
\hline 0.00001 & $9.855 \mathrm{E}-06$ & $5.074 \mathrm{E}+05$ & 6.487 & 1468.0 & $1.447 \mathrm{E}-02$ & 2.061 & $8.382 \mathrm{E}-01$ \\
\hline 0.00005 & $4.934 \mathrm{E}-05$ & $1.013 \mathrm{E}+05$ & 6.487 & 1335.0 & $6.587 \mathrm{E}-02$ & 2.038 & 8.289E-01 \\
\hline 0.0001 & $9.882 \mathrm{E}-05$ & $5.060 \mathrm{E}+04$ & 6.487 & 1198.0 & $1.184 \mathrm{E}-01$ & 2.014 & $8.191 \mathrm{E}-01$ \\
\hline 0.0005 & $4.967 \mathrm{E}-04$ & $1.007 \mathrm{E}+04$ & 6.488 & 657.7 & $3.267 \mathrm{E}-01$ & 1.918 & $7.801 \mathrm{E}-01$ \\
\hline 0.001 & $9.958 \mathrm{E}-04$ & $5.021 \mathrm{E}+03$ & 6.489 & 420.0 & $4.182 \mathrm{E}-01$ & 1.876 & $7.630 \mathrm{E}-01$ \\
\hline 0.002 & $1.995 \mathrm{E}-03$ & $2.506 \mathrm{E}+03$ & 6.491 & 243.6 & $4.860 \mathrm{E}-01$ & 1.845 & 7.504E-01 \\
\hline 0.003 & $2.995 \mathrm{E}-03$ & $1.669 \mathrm{E}+03$ & 6.494 & 171.6 & 5.139E-01 & 1.832 & $7.451 \mathrm{E}-01$ \\
\hline 0.005 & $4.995 \mathrm{E}-03$ & $1.001 \mathrm{E}+03$ & 6.498 & 107.8 & $5.385 \mathrm{E}-01$ & 1.821 & $7.406 \mathrm{E}-01$ \\
\hline 0.01 & $9.994 \mathrm{E}-03$ & $5.003 \mathrm{E}+02$ & 6.510 & 55.9 & $5.584 \mathrm{E}-01$ & 1.811 & $7.365 \mathrm{E}-01$ \\
\hline 0.03 & 2.999E-02 & $1.667 \mathrm{E}+02$ & 6.559 & 19.1 & $5.725 \mathrm{E}-01$ & 1.803 & 7.333E-01 \\
\hline 0.05 & 4.999E-02 & $1.000 \mathrm{E}+02$ & 6.607 & 11.5 & $5.754 \mathrm{E}-01$ & 1.799 & $7.317 \mathrm{E}-01$ \\
\hline 0.1 & 9.999E-02 & $5.001 \mathrm{E}+01$ & 6.730 & 5.8 & $5.776 \mathrm{E}-01$ & 1.794 & $7.296 \mathrm{E}-01$ \\
\hline $\begin{array}{l}4.552 \mathrm{E}-05 \\
\text { (feed conc.) }\end{array}$ & 4.492E-05 & $1.113 \mathrm{E}+05$ & 6.487 & 1349.0 & $6.060 \mathrm{E}-02$ & 2.040 & $8.297 \mathrm{E}-01$ \\
\hline
\end{tabular}

${ }^{a}$ At a $5.0 \mathrm{M}$ sodium concentration level the solution density is estimated to be $1.235 \mathrm{~g} / \mathrm{ml}$ and at $0.4067 \mathrm{M}\left[\mathrm{K}^{+}\right]$.

${ }^{\mathrm{b}}$ Final cesium equilibrium concentrations are only slightly less than initial values due to large phase ratio used.

Table B-14. ZAM equilibrium isotherm model predictions for CST material in the powder form (IE-910) in contact with Envelope B's LAW-2a feed solution at $5 \mathrm{M}\left[\mathrm{Na}^{+}\right]^{\text {a }}$

\begin{tabular}{|c|c|c|c|c|c|c|c|}
\hline Cs (initial) & Cs (final) ${ }^{b}$ & $\mathrm{Na} / \mathrm{Cs}$ & Ionic strength & $\operatorname{Cs} K_{d}$ & Cs loading & $\mathbf{K} \mathbf{K}_{\mathrm{d}}$ & K loading \\
\hline$[\mathbf{M}]$ & {$[\mathbf{M}]$} & [molar ratio] & [gmole/kg] & {$[\mathrm{ml} / \mathrm{g}]$} & [mmole/g] & {$[\mathrm{ml} / \mathrm{g}]$} & [mmole/g] \\
\hline 0 & $0.000 \mathrm{E}+00$ & infinity & 7.360 & 0.0 & $0.000 \mathrm{E}+00$ & 4.143 & 5.179E-01 \\
\hline 0.000001 & $9.784 \mathrm{E}-07$ & $5.110 \mathrm{E}+06$ & 7.360 & 2203.0 & $2.155 \mathrm{E}-03$ & 4.138 & 5.173E-01 \\
\hline 0.00001 & $9.791 \mathrm{E}-06$ & $5.107 \mathrm{E}+05$ & 7.360 & 2132.0 & $2.087 \mathrm{E}-02$ & 4.095 & 5.119E-01 \\
\hline 0.00005 & 4.909E-05 & $1.019 \mathrm{E}+05$ & 7.360 & 1863.0 & $9.145 \mathrm{E}-02$ & 3.935 & 4.919E-01 \\
\hline 0.0001 & $9.842 \mathrm{E}-05$ & $5.080 \mathrm{E}+04$ & 7.360 & 1608.0 & $1.583 \mathrm{E}-01$ & 3.783 & 4.729E-01 \\
\hline 0.0005 & $4.962 \mathrm{E}-04$ & $1.008 \mathrm{E}+04$ & 7.361 & 764.6 & $3.794 \mathrm{E}-01$ & 3.280 & 4.100E-01 \\
\hline 0.001 & $9.954 \mathrm{E}-04$ & $5.023 \mathrm{E}+03$ & 7.362 & 461.1 & $4.590 \mathrm{E}-01$ & 3.099 & $3.874 \mathrm{E}-01$ \\
\hline 0.002 & $1.995 \mathrm{E}-03$ & $2.506 \mathrm{E}+03$ & 7.365 & 256.9 & $5.125 \mathrm{E}-01$ & 2.977 & $3.721 \mathrm{E}-01$ \\
\hline 0.003 & $2.995 \mathrm{E}-03$ & $1.669 \mathrm{E}+03$ & 7.368 & 178.1 & $5.334 \mathrm{E}-01$ & 2.930 & $3.663 \mathrm{E}-01$ \\
\hline 0.005 & 4.994E-03 & $1.001 \mathrm{E}+03$ & 7.373 & 110.3 & $5.508 \mathrm{E}-01$ & 2.889 & 3.611E-01 \\
\hline 0.01 & $9.994 \mathrm{E}-03$ & $5.003 \mathrm{E}+02$ & 7.386 & 56.5 & $5.651 \mathrm{E}-01$ & 2.855 & $3.569 \mathrm{E}-01$ \\
\hline 0.03 & $2.999 \mathrm{E}-02$ & $1.667 \mathrm{E}+02$ & 7.438 & 19.2 & 5.749E-01 & 2.826 & $3.533 \mathrm{E}-01$ \\
\hline 0.05 & 4.999E-02 & $1.000 \mathrm{E}+02$ & 7.490 & 11.5 & $5.769 \mathrm{E}-01$ & 2.815 & $3.519 \mathrm{E}-01$ \\
\hline 0.1 & $9.999 \mathrm{E}-02$ & $5.001 \mathrm{E}+01$ & 7.624 & 5.8 & $5.784 \mathrm{E}-01$ & 2.795 & 3.494E-01 \\
\hline $\begin{array}{c}4.676 \mathrm{E}-04 \\
\text { (feed conc.) }\end{array}$ & $4 \quad 4.638 \mathrm{E}-04$ & $1.078 \mathrm{E}+04$ & 7.361 & $\begin{array}{l}798.7 \\
\end{array}$ & 3.704E-01 & 3.301 & 4.126E-01 \\
\hline
\end{tabular}

${ }^{\text {a }}$ At a $5.0 \mathrm{M}$ sodium concentration level the solution density is estimated to be $1.254 \mathrm{~g} / \mathrm{ml}$ and at $0.1250 \mathrm{M}\left[\mathrm{K}^{+}\right]$.

${ }^{b}$ Final cesium equilibrium concentrations are only slightly less than initial values due to large phase ratio used. 
WESTINGHOUSE SAVANNAH RIVER COMPANY

Report:

WSRC-TR-2000-00???

Preliminary Ion Exchange Modeling for Removal of Cesium from

Revision (Date):

$0(07 / 05 / 02)$

Hanford Waste Using Crystalline Silicotitanate Material

Page:

188 of 338

Table B-15. ZAM equilibrium isotherm model predictions for CST material in the powder form (IE-910) in contact with Envelope B's LAW-2b feed solution at $5 \mathrm{M}\left[\mathrm{Na}^{+}\right]^{\mathrm{a}}$

\begin{tabular}{|c|c|c|c|c|c|c|c|}
\hline Cs (initial) & Cs (final) ${ }^{b}$ & $\mathrm{Na} / \mathrm{Cs}$ & Ionic strength & $\operatorname{Cs} K_{d}$ & Cs loading & $\mathbf{K} \mathbf{K}_{\mathrm{d}}$ & K loading \\
\hline [M] & {$[\mathbf{M}]$} & [molar ratio] & [gmole/kg] & {$[\mathrm{ml} / \mathrm{g}]$} & [mmole/g] & {$[\mathrm{ml} / \mathrm{g}]$} & [mmole/g] \\
\hline 0 & $0.000 \mathrm{E}+00$ & infinity & 8.170 & 0.0 & $0.000 \mathrm{E}+00$ & 4.453 & $6.363 \mathrm{E}-01$ \\
\hline 0.000001 & $9.736 \mathrm{E}-07$ & $5.136 \mathrm{E}+06$ & 8.170 & 2711.0 & $2.639 \mathrm{E}-03$ & 4.448 & $6.356 \mathrm{E}-01$ \\
\hline 0.00001 & $9.746 \mathrm{E}-06$ & $5.130 \mathrm{E}+05$ & 8.170 & 2604.0 & $2.538 \mathrm{E}-02$ & 4.405 & $6.295 \mathrm{E}-01$ \\
\hline 0.00005 & $4.892 \mathrm{E}-05$ & $1.022 \mathrm{E}+05$ & 8.170 & 2215.0 & $1.084 \mathrm{E}-01$ & 4.247 & $6.069 \mathrm{E}-01$ \\
\hline 0.0001 & $9.817 \mathrm{E}-05$ & $5.093 \mathrm{E}+04$ & 8.170 & 1864.0 & $1.830 \mathrm{E}-01$ & 4.106 & $5.867 \mathrm{E}-01$ \\
\hline 0.0005 & 4.959E-04 & $1.008 \mathrm{E}+04$ & 8.171 & 818.1 & $4.057 \mathrm{E}-01$ & 3.683 & $5.263 \mathrm{E}-01$ \\
\hline 0.001 & $9.952 \mathrm{E}-04$ & $5.024 \mathrm{E}+03$ & 8.172 & 480.0 & 4.777E-01 & 3.546 & $5.067 \mathrm{E}-01$ \\
\hline 0.002 & $1.995 \mathrm{E}-03$ & $2.506 \mathrm{E}+03$ & 8.175 & 262.7 & 5.241E-01 & 3.458 & $4.941 \mathrm{E}-01$ \\
\hline 0.003 & $2.995 \mathrm{E}-03$ & $1.669 \mathrm{E}+03$ & 8.178 & 180.8 & $5.415 \mathrm{E}-01$ & 3.424 & 4.893E-01 \\
\hline 0.005 & 4.994E-03 & $1.001 \mathrm{E}+03$ & 8.183 & 111.4 & 5.563E-01 & 3.396 & $4.853 \mathrm{E}-01$ \\
\hline 0.01 & $9.994 \mathrm{E}-03$ & $5.003 \mathrm{E}+02$ & 8.197 & 56.8 & 5.679E-01 & 3.373 & $4.820 \mathrm{E}-01$ \\
\hline 0.03 & 2.999E-02 & $1.667 \mathrm{E}+02$ & 8.251 & 19.2 & $5.758 \mathrm{E}-01$ & 3.354 & 4.793E-01 \\
\hline 0.05 & 4.999E-02 & $1.000 \mathrm{E}+02$ & 8.305 & 11.6 & $5.774 \mathrm{E}-01$ & 3.346 & $4.781 \mathrm{E}-01$ \\
\hline 0.1 & 9.999E-02 & $5.001 \mathrm{E}+01$ & 8.444 & 5.8 & $5.786 \mathrm{E}-01$ & 3.334 & $4.764 \mathrm{E}-01$ \\
\hline $\begin{array}{l}4.311 \mathrm{E}-04 \\
\text { (feed conc.) }\end{array}$ & $4.272 \mathrm{E}-04$ & $1.170 \mathrm{E}+04$ & 8.171 & 905.9 & $3.870 \mathrm{E}-01$ & 3.718 & 5.313E-01 \\
\hline
\end{tabular}

${ }^{a}$ At a $5.0 \mathrm{M}$ sodium concentration level the solution density is estimated to be $1.242 \mathrm{~g} / \mathrm{ml}$ and at $0.1429 \mathrm{M}\left[\mathrm{K}^{+}\right]$.

${ }^{\mathrm{b}}$ Final cesium equilibrium concentrations are only slightly less than initial values due to large phase ratio used.

Table B-16. ZAM equilibrium isotherm model predictions for CST material in the powder form (IE-910) in contact with Envelope C's LAW-3 feed solution at $5 \mathrm{M}\left[\mathrm{Na}^{+}\right]^{\mathrm{a}}$

\begin{tabular}{|c|c|c|c|c|c|c|c|}
\hline Cs (initial) & Cs (final) ${ }^{b}$ & $\mathrm{Na} / \mathrm{Cs}$ & Ionic strength & $\operatorname{Cs} K_{d}$ & Cs loading & $\mathbf{K} \mathbf{K}_{\mathrm{d}}$ & K loading \\
\hline$[\mathbf{M}]$ & {$[\mathbf{M}]$} & [molar ratio] & [gmole/kg] & {$[\mathrm{ml} / \mathrm{g}]$} & [mmole/g] & {$[\mathrm{ml} / \mathrm{g}]$} & [mmole/g] \\
\hline 0 & $0.000 \mathrm{E}+00$ & infinity & 6.995 & 0.0 & $0.000 \mathrm{E}+00$ & 6.339 & $2.784 \mathrm{E}-01$ \\
\hline 0.000001 & $9.742 \mathrm{E}-07$ & $5.132 \mathrm{E}+06$ & 6.995 & 2653.0 & $2.585 \mathrm{E}-03$ & 6.326 & $2.778 \mathrm{E}-01$ \\
\hline 0.00001 & $9.751 \mathrm{E}-06$ & $5.128 \mathrm{E}+05$ & 6.995 & 2550.0 & $2.487 \mathrm{E}-02$ & 6.217 & $2.731 \mathrm{E}-01$ \\
\hline 0.00005 & $4.894 \mathrm{E}-05$ & $1.022 \mathrm{E}+05$ & 6.995 & 2175.0 & $1.064 \mathrm{E}-01$ & 5.817 & $2.555 \mathrm{E}-01$ \\
\hline 0.0001 & $9.820 \mathrm{E}-05$ & $5.092 \mathrm{E}+04$ & 6.995 & 1836.0 & $1.803 \mathrm{E}-01$ & 5.455 & $2.396 \mathrm{E}-01$ \\
\hline 0.0005 & $4.960 \mathrm{E}-04$ & $1.008 \mathrm{E}+04$ & 6.996 & 812.7 & $4.031 \mathrm{E}-01$ & 4.362 & $1.916 \mathrm{E}-01$ \\
\hline 0.001 & $9.952 \mathrm{E}-04$ & $5.024 \mathrm{E}+03$ & 6.997 & 478.1 & $4.758 \mathrm{E}-01$ & 4.005 & $1.759 \mathrm{E}-01$ \\
\hline 0.002 & $1.995 \mathrm{E}-03$ & $2.506 \mathrm{E}+03$ & 7.000 & 262.1 & $5.229 \mathrm{E}-01$ & 3.774 & $1.658 \mathrm{E}-01$ \\
\hline 0.003 & $2.995 \mathrm{E}-03$ & $1.669 \mathrm{E}+03$ & 7.002 & 180.5 & $5.406 \mathrm{E}-01$ & 3.686 & $1.619 \mathrm{E}-01$ \\
\hline 0.005 & 4.994E-03 & $1.001 \mathrm{E}+03$ & 7.007 & 111.3 & $5.558 \mathrm{E}-01$ & 3.611 & $1.586 \mathrm{E}-01$ \\
\hline 0.01 & $9.994 \mathrm{E}-03$ & $5.003 \mathrm{E}+02$ & 7.019 & 56.8 & $5.676 \mathrm{E}-01$ & 3.551 & $1.560 \mathrm{E}-01$ \\
\hline 0.03 & $2.999 \mathrm{E}-02$ & $1.667 \mathrm{E}+02$ & 7.069 & 19.2 & $5.758 \mathrm{E}-01$ & 3.502 & $1.538 \mathrm{E}-01$ \\
\hline 0.05 & $4.999 \mathrm{E}-02$ & $1.000 \mathrm{E}+02$ & 7.120 & 11.6 & $5.774 \mathrm{E}-01$ & 3.484 & $1.530 \mathrm{E}-01$ \\
\hline 0.1 & $9.999 \mathrm{E}-02$ & $5.001 \mathrm{E}+01$ & 7.247 & 5.8 & $5.786 \mathrm{E}-01$ & 3.455 & $1.517 \mathrm{E}-01$ \\
\hline $\begin{array}{c}3.967 \mathrm{E}-05 \\
\text { (feed conc.) }\end{array}$ & 3.880E-05 & $1.289 \mathrm{E}+05$ & 6.995 & 2261.0 & "8.773E-02 & 5.909 & $2.595 \mathrm{E}-01$ \\
\hline
\end{tabular}


WESTINGHOUSE SAVANNAH RIVER COMPANY

Report:

WSRC-TR-2000-00???

Preliminary Ion Exchange Modeling for Removal of Cesium from

Revision (Date):

$0(07 / 05 / 02)$

Hanford Waste Using Crystalline Silicotitanate Material

Page:

189 of 338

Table B-17. ZAM equilibrium isotherm model predictions for CST material in the powder form (IE-910) in contact with Envelope C's LAW-4 feed solution at $5 \mathrm{M}\left[\mathrm{Na}^{+}{ }^{+}{ }^{\mathrm{a}}\right.$

\begin{tabular}{|c|c|c|c|c|c|c|c|}
\hline Cs (initial) & Cs (final) $^{\mathbf{b}}$ & $\mathbf{N a / C s}$ & Ionic strength & $\mathbf{C s ~}_{\mathbf{d}}$ & Cs loading & $\mathbf{K}_{\mathbf{d}}$ & K loading \\
\hline$[\mathbf{M}]$ & {$[\mathbf{M}]$} & {$[\mathbf{m o l a r}$ ratio] } & {$[\mathbf{g m o l e} / \mathbf{k g}]$} & {$[\mathbf{m l} / \mathbf{g}]$} & {$[\mathbf{m m o l e} / \mathbf{g}]$} & {$[\mathbf{m l} / \mathbf{g}]$} & {$[\mathbf{m m o l e} / \mathbf{g}]$} \\
\hline 0 & $0.000 \mathrm{E}+00$ & infinity & 6.995 & 0.0 & $0.000 \mathrm{E}+00$ & 6.339 & $2.784 \mathrm{E}-01$ \\
\hline 0.000001 & $9.742 \mathrm{E}-07$ & $5.132 \mathrm{E}+06$ & 6.995 & 2653.0 & $2.585 \mathrm{E}-03$ & 6.326 & $2.778 \mathrm{E}-01$ \\
\hline 0.00001 & $9.751 \mathrm{E}-06$ & $5.128 \mathrm{E}+05$ & 6.995 & 2550.0 & $2.487 \mathrm{E}-02$ & 6.217 & $2.731 \mathrm{E}-01$ \\
\hline 0.00005 & $4.894 \mathrm{E}-05$ & $1.022 \mathrm{E}+05$ & 6.995 & 2175.0 & $1.064 \mathrm{E}-01$ & 5.817 & $2.555 \mathrm{E}-01$ \\
\hline 0.0001 & $9.820 \mathrm{E}-05$ & $5.092 \mathrm{E}+04$ & 6.995 & 1836.0 & $1.803 \mathrm{E}-01$ & 5.455 & $2.396 \mathrm{E}-01$ \\
\hline 0.0005 & $4.960 \mathrm{E}-04$ & $1.008 \mathrm{E}+04$ & 6.996 & 812.7 & $4.031 \mathrm{E}-01$ & 4.362 & $1.916 \mathrm{E}-01$ \\
\hline 0.001 & $9.952 \mathrm{E}-04$ & $5.024 \mathrm{E}+03$ & 6.997 & 478.1 & $4.758 \mathrm{E}-01$ & 4.005 & $1.759 \mathrm{E}-01$ \\
\hline 0.002 & $1.995 \mathrm{E}-03$ & $2.506 \mathrm{E}+03$ & 7.000 & 262.1 & $5.229 \mathrm{E}-01$ & 3.774 & $1.658 \mathrm{E}-01$ \\
\hline 0.003 & $2.995 \mathrm{E}-03$ & $1.669 \mathrm{E}+03$ & 7.002 & 180.5 & $5.406 \mathrm{E}-01$ & 3.686 & $1.619 \mathrm{E}-01$ \\
\hline 0.005 & $4.994 \mathrm{E}-03$ & $1.001 \mathrm{E}+03$ & 7.007 & 111.3 & $5.558 \mathrm{E}-01$ & 3.611 & $1.586 \mathrm{E}-01$ \\
\hline 0.01 & $9.994 \mathrm{E}-03$ & $5.003 \mathrm{E}+02$ & 7.019 & 56.8 & $5.676 \mathrm{E}-01$ & 3.551 & $1.560 \mathrm{E}-01$ \\
\hline 0.03 & $2.999 \mathrm{E}-02$ & $1.667 \mathrm{E}+02$ & 7.069 & 19.2 & $5.758 \mathrm{E}-01$ & 3.502 & $1.538 \mathrm{E}-01$ \\
\hline 0.05 & $4.999 \mathrm{E}-02$ & $1.000 \mathrm{E}+02$ & 7.120 & 11.6 & $5.774 \mathrm{E}-01$ & 3.484 & $1.530 \mathrm{E}-01$ \\
\hline 0.1 & $9.999 \mathrm{E}-02$ & $5.001 \mathrm{E}+01$ & 7.247 & 5.8 & $5.786 \mathrm{E}-01$ & 3.455 & $1.517 \mathrm{E}-01$ \\
\hline \hline $\begin{array}{c}3.779 \mathrm{E}-05 \\
\text { (feed conc.) }\end{array}$ & $3.694 \mathrm{E}-05$ & $1.354 \mathrm{E}+05$ & 6.995 & 2278.0 & $8.415 \mathrm{E}-02$ & 5.926 & $2.603 \mathrm{E}-01$ \\
\hline
\end{tabular}

${ }^{a}$ At a $5.0 \mathrm{M}$ sodium concentration level the solution density is estimated to be $1.237 \mathrm{~g} / \mathrm{ml}$ and at $0.04393 \mathrm{M}\left[\mathrm{K}^{+}\right]$.

${ }^{\mathrm{b}}$ Final cesium equilibrium concentrations are only slightly less than initial values due to large phase ratio used.

Table B-18. ZAM equilibrium isotherm model predictions for CST material in the powder form (IE-910) in contact with Envelope C's LAW-7 feed solution at $5 \mathrm{M}\left[\mathrm{Na}^{+}\right]^{\mathrm{a}}$

\begin{tabular}{|c|c|c|c|c|c|c|c|}
\hline Cs (initial) & Cs (final) ${ }^{b}$ & $\mathrm{Na} / \mathrm{Cs}$ & Ionic strength & $\operatorname{Cs} K_{d}$ & Cs loading & $\mathbf{K} \mathbf{K}_{\mathrm{d}}$ & K loading \\
\hline$[\mathbf{M}]$ & {$[\mathbf{M}]$} & [molar ratio] & [gmole/kg] & {$[\mathrm{ml} / \mathrm{g}]$} & [mmole/g] & {$[\mathrm{ml} / \mathrm{g}]$} & [mmole/g] \\
\hline 0 & $0.000 \mathrm{E}+00$ & infinity & 7.209 & 0.0 & $0.000 \mathrm{E}+00$ & 7.316 & $1.629 \mathrm{E}-01$ \\
\hline 0.000001 & $9.709 \mathrm{E}-07$ & $5.150 \mathrm{E}+06$ & 7.209 & 2997.0 & $2.910 \mathrm{E}-03$ & 7.297 & $1.625 \mathrm{E}-01$ \\
\hline 0.00001 & $9.721 \mathrm{E}-06$ & $5.144 \mathrm{E}+05$ & 7.209 & 2867.0 & $2.787 \mathrm{E}-02$ & 7.139 & $1.590 \mathrm{E}-01$ \\
\hline 0.00005 & $4.883 \mathrm{E}-05$ & $1.024 \mathrm{E}+05$ & 7.209 & 2403.0 & $1.173 \mathrm{E}-01$ & 6.572 & $1.464 \mathrm{E}-01$ \\
\hline 0.0001 & $9.804 \mathrm{E}-05$ & $5.100 \mathrm{E}+04$ & 7.210 & 1996.0 & $1.957 \mathrm{E}-01$ & 6.075 & $1.353 \mathrm{E}-01$ \\
\hline 0.0005 & $4.958 \mathrm{E}-04$ & $1.008 \mathrm{E}+04$ & 7.211 & 842.5 & $4.177 \mathrm{E}-01$ & 4.666 & $1.039 \mathrm{E}-01$ \\
\hline 0.001 & $9.951 \mathrm{E}-04$ & $5.025 \mathrm{E}+03$ & 7.212 & 488.3 & $4.859 \mathrm{E}-01$ & 4.234 & $9.429 \mathrm{E}-02$ \\
\hline 0.002 & $1.995 \mathrm{E}-03$ & $2.506 \mathrm{E}+03$ & 7.214 & 265.1 & $5.289 \mathrm{E}-01$ & 3.961 & $8.821 \mathrm{E}-02$ \\
\hline 0.003 & $2.995 \mathrm{E}-03$ & $1.669 \mathrm{E}+03$ & 7.217 & 182.0 & $5.451 \mathrm{E}-01$ & 3.859 & $8.594 \mathrm{E}-02$ \\
\hline 0.005 & 4.994E-03 & $1.001 \mathrm{E}+03$ & 7.222 & 111.8 & $5.583 \mathrm{E}-01$ & 3.772 & $8.400 \mathrm{E}-02$ \\
\hline 0.01 & $9.994 \mathrm{E}-03$ & $5.003 \mathrm{E}+02$ & 7.234 & 56.9 & $5.690 \mathrm{E}-01$ & 3.703 & $8.247 \mathrm{E}-02$ \\
\hline 0.03 & $2.999 \mathrm{E}-02$ & $1.667 \mathrm{E}+02$ & 7.285 & 19.2 & $5.761 \mathrm{E}-01$ & 3.646 & $8.120 \mathrm{E}-02$ \\
\hline 0.05 & $4.999 \mathrm{E}-02$ & $1.000 \mathrm{E}+02$ & 7.336 & 11.6 & 5.779E-01 & 3.627 & $8.077 \mathrm{E}-02$ \\
\hline 0.1 & $9.999 \mathrm{E}-02$ & $5.001 \mathrm{E}+01$ & 7.465 & 5.8 & $5.788 \mathrm{E}-01$ & 3.594 & $8.004 \mathrm{E}-02$ \\
\hline $\begin{array}{l}4.455 \mathrm{E}-05 \\
\text { (feed conc.) }\end{array}$ & $\begin{array}{l}4.348 \mathrm{E}-05 \\
\end{array}$ & $1.150 \mathrm{E}+05$ & 7.209 & 2457.0 & "1.068E-01 & 6.638 & $\begin{array}{l}1.478 \mathrm{E}-01 \\
\end{array}$ \\
\hline
\end{tabular}


Preliminary Ion Exchange Modeling for Removal of Cesium from Revision (Date):

Table B-19. Parameter identities between a 4-component VERSE-LC Freundlich/Langmuir Hybrid equilibrium isotherm model and the "effective" single-component homovalent isotherm model.

\begin{tabular}{|c|c|c|c|c||c|}
\hline Parameter & $\begin{array}{c}\text { Total Cesium } \\
\text { (component 1) }\end{array}$ & $\begin{array}{c}\text { Potassium } \\
\text { (component 2) }\end{array}$ & $\begin{array}{c}\text { Sodium } \\
\text { (component 3) }\end{array}$ & $\begin{array}{c}\text { Strontium } \\
\text { hydroxide } \\
\text { (component 4) }\end{array}$ & $\begin{array}{c}\text { Total Cesium } \\
\text { (effective single } \\
\text { component) }\end{array}$ \\
\hline $\begin{array}{c}\mathbf{a}_{\mathbf{I}} \\
(\text { gmoles/L }\end{array}$ & $\eta_{\mathrm{df}} \rho_{\mathrm{b}} \overline{\mathrm{C}}_{\mathrm{T}}$ & $\eta_{\mathrm{df}} \rho_{\mathrm{b}} \overline{\mathrm{C}}_{\mathrm{T}} \widetilde{\mathrm{K}}_{21}$ & $\eta_{\mathrm{df}} \rho_{\mathrm{b}} \overline{\mathrm{C}}_{\mathrm{T}} \widetilde{\mathrm{K}}_{31}$ & $\eta_{\mathrm{df}} \rho_{\mathrm{b}} \overline{\mathrm{C}}_{\mathrm{T}} \widetilde{\mathrm{K}}_{41}$ & $\eta_{\mathrm{df}} \rho_{\mathrm{b}} \overline{\mathrm{C}}_{\mathrm{T}}$ \\
\hline $\mathbf{b}_{\mathbf{i}}\left(\mathbf{M}^{-1}\right)$ & 1.0 & $\widetilde{\mathrm{K}}_{21}$ & $\widetilde{\mathrm{K}}_{31}$ & $\widetilde{\mathrm{K}}_{41}$ & 1.0 \\
\hline $\mathbf{M}_{\mathrm{ai}}(-)$ & 1.0 & 1.0 & 1.0 & 1.0 & 1.0 \\
\hline $\mathbf{M}_{\mathrm{bi}}(-)$ & 1.0 & 1.0 & 1.0 & 1.0 & 1.0 \\
\hline$\beta_{\mathbf{i}}(-)$ & 0.0 & 0.0 & 0.0 & 0.0 & $\widetilde{\mathrm{K}}_{21} \mathrm{c}_{\mathrm{p} 2}^{\mathrm{M}_{\mathrm{b} 2}}+\widetilde{\mathrm{K}}_{31} \mathrm{c}_{\mathrm{p} 3}^{\mathrm{M}_{\mathrm{b} 3}}$ \\
$+\widetilde{\mathrm{K}}_{41} \mathrm{c}_{\mathrm{p} 4}^{\mathrm{M}_{\mathrm{b} 4}}$ \\
\hline
\end{tabular}

${ }^{a}$ The parameters for components 1,2,3, and 4 refer to total cesium, potassium, sodium, and strontium hydroxide, respectively, when the ion-exchange system of interest is the Cesium-CST system. 
Table B-20. Estimated selectivity coefficients for the Cesium-CST system based on ZAM predictions and the 4-component homovalent isotherm model for the Envelope A feed solutions.

\begin{tabular}{|c|c|c|c|c|}
\hline $\begin{array}{c}\text { Selectivity } \\
\text { Coefficient }\end{array}$ & Definition & $\begin{array}{c}\text { Parameter } \\
\text { Estimate }\end{array}$ & $\begin{array}{c}\text { Approx. } \\
\text { Standard } \\
\text { Error }\end{array}$ & $\begin{array}{c}\text { Relative } \\
\text { Error } \\
\text { (\%) }\end{array}$ \\
\hline \hline$\widetilde{\mathrm{K}}_{21}$ & $\begin{array}{c}\mathrm{K}^{+} \text {to Cs } \\
\text { selectivity }\end{array}$ & $7.0665 \times 10^{-4}$ & $3.9835 \times 10^{-5}$ & $5.6 \%$ \\
\hline$\widetilde{\mathrm{K}}_{31}$ & $\begin{array}{c}\mathrm{Na}^{+} \text {to Cs } \\
\text { selectivity }\end{array}$ & $3.7897 \times 10^{-5}$ & $6.4175 \times 10^{-7}$ & $1.7 \%$ \\
\hline$\widetilde{\mathrm{K}}_{41}$ & $\begin{array}{c}\text { SrOH} \\
\text { selectivity }^{+}\end{array}$ & $1.4432 \times 10^{-1}$ & $4.3031 \times 10^{-2}$ & $29.8 \%$ \\
\hline$\widetilde{\mathrm{K}}_{12}$ & $\begin{array}{c}\mathrm{Cs}^{+} \text {to } \mathrm{K}^{+} \\
\text {selectivity }\end{array}$ & $1,415.1$ & 79.2 & $5.6 \%$ \\
\hline$\widetilde{\mathrm{K}}_{13}$ & $\begin{array}{c}\mathrm{Cs}^{+} \text {to Na } \\
\text { selectivity }\end{array}$ & $26,387.3$ & 448.6 & $1.7 \%$ \\
\hline$\widetilde{\mathrm{K}}_{14}$ & $\begin{array}{c}\mathrm{Cs}^{+} \text {to SrOH } \\
\text { selectivity }\end{array}$ & 6.9 & 2.1 & $29.8 \%$ \\
\hline \hline$\widetilde{\mathrm{K}}_{23}$ & $\begin{array}{c}\mathrm{K}^{+} \text {to Na } \\
\text { selectivity }\end{array}$ & 18.6 & - & - \\
\hline$\widetilde{\mathrm{K}}_{42}$ & $\begin{array}{c}\text { SrOH } \\
\text { selectivity }\end{array}$ & 204.2 & - & - \\
\hline$\widetilde{\mathrm{K}}_{43}$ & $\begin{array}{c}\text { SrOH} \\
\text { selectivity }\end{array}$ & $3,808.2$ & - & \\
\hline
\end{tabular}


Preliminary Ion Exchange Modeling for Removal of Cesium from

Table B-21. The resulting correlation coefficient matrix for the fit of the selectivity coefficients for the Cesium-CST system, ZAM produced data, and the 4-component homovalent isotherm model for the Envelope A feed solutions.

\begin{tabular}{|c|c|c|c|}
\hline $\begin{array}{c}\text { Selectivity } \\
\text { Coefficient } \\
\text { (species) }\end{array}$ & $\begin{array}{c}\widetilde{\mathrm{K}}_{21} \\
\left(\mathrm{~K}^{+}\right)\end{array}$ & $\begin{array}{c}\widetilde{\mathrm{K}}_{31} \\
\left(\mathrm{Na}^{+}\right)\end{array}$ & $\begin{array}{c}\widetilde{\mathrm{K}}_{41} \\
\left(\mathrm{SrOH}^{+}\right)\end{array}$ \\
\hline \hline $\begin{array}{c}\widetilde{\mathrm{K}}_{21} \\
\left(\mathrm{~K}^{+}\right)\end{array}$ & 1.0000 & -0.7984 & 0.1118 \\
\hline$\widetilde{\mathrm{K}}_{31}$ & -0.7984 & 1.0000 & -0.2493 \\
\hline$\left(\mathrm{Na}^{+}\right)$ & 0.1118 & -0.2493 & 1.0000 \\
\hline$\widetilde{\mathrm{K}}_{41}$ & & & \\
$\left(\mathrm{SrOH}^{+}\right)$ & & & \\
\hline
\end{tabular}

Table B-22. Estimated beta parameter values and error estimate for a one-component (total cesium) homovalent algebraic isotherm model based on CST in its powder-form (IE-910) where the data sets used were created using the ZAM code.

\begin{tabular}{|c|c|c|c|}
\hline Feed Solution & $\begin{array}{c}\text { "Best Estimate" } \\
\beta_{\mathbf{i}} \\
{[\mathbf{M}]}\end{array}$ & $\begin{array}{c}\text { Standard Error in } \\
\beta_{\mathbf{i}} \\
{[\mathbf{M}]}\end{array}$ & $\begin{array}{c}\text { Root Mean Square } \\
\text { in Cs loading } \\
\text { (\% difference) }\end{array}$ \\
\hline \hline Envelope A & \multicolumn{3}{|c|}{} \\
\hline LAW-1 & $4.3376 \mathrm{E}-04$ & $\pm 8.3713 \mathrm{E}-07$ & 0.3912 \\
\hline LAW-5 & $2.3431 \mathrm{E}-04$ & $\pm 2.0648 \mathrm{E}-08$ & 0.0137 \\
\hline LAW-6 & $2.3668 \mathrm{E}-04$ & $\pm 2.2931 \mathrm{E}-08$ & 0.0202 \\
\hline LAW-8 & $2.5147 \mathrm{E}-04$ & $\pm 3.8060 \mathrm{E}-08$ & 0.0270 \\
\hline LAW-9 & $2.2453 \mathrm{E}-04$ & $\pm 2.8251 \mathrm{E}-08$ & 0.0220 \\
\hline LAW-10 & $2.2135 \mathrm{E}-04$ & $\pm 2.2209 \mathrm{E}-08$ & 0.0161 \\
\hline LAW-11 & $2.0543 \mathrm{E}-04$ & $\pm 2.4697 \mathrm{E}-08$ & 0.0229 \\
\hline LAW-12 & $2.9196 \mathrm{E}-04$ & $\pm 3.2849 \mathrm{E}-08$ & 0.0177 \\
\hline LAW-13 & $2.9328 \mathrm{E}-04$ & $\pm 3.2820 \mathrm{E}-08$ & 0.0184 \\
\hline LAW-14 & $3.6283 \mathrm{E}-04$ & $\pm 4.0017 \mathrm{E}-08$ & 0.0262 \\
\hline LAW-15 & $3.8513 \mathrm{E}-04$ & $\pm 3.7129 \mathrm{E}-08$ & 0.0219 \\
\hline Overall avg. & $2.8552 \mathrm{E}-04$ & $\pm 1.0334 \mathrm{E}-07$ & 0.0543 \\
\hline \hline Envelope B & \multicolumn{3}{|l}{} \\
\hline LAW-2a & $2.6230 \mathrm{E}-04$ & $\pm 2.9902 \mathrm{E}-08$ & 0.0173 \\
\hline LAW-2b & $2.1296 \mathrm{E}-04$ & $\pm 2.3999 \mathrm{E}-08$ & 0.0138 \\
\hline Overall avg. & $2.3763 \mathrm{E}-04$ & $\pm 2.6951 \mathrm{E}-08$ & 0.0156 \\
\hline \hline Envelope C & \multicolumn{3}{|l}{} \\
\hline LAW-3 & $2.1769 \mathrm{E}-04$ & $\pm 2.4699 \mathrm{E}-08$ & 0.0135 \\
\hline LAW-4 & $2.1769 \mathrm{E}-04$ & $\pm 2.4646 \mathrm{E}-08$ & 0.0132 \\
\hline LAW-7 & $1.9258 \mathrm{E}-04$ & $\pm 2.0511 \mathrm{E}-08$ & 0.0166 \\
\hline Overall avg. & $2.0932 \mathrm{E}-04$ & $\pm 2.3285 \mathrm{E}-08$ & 0.0144 \\
\hline
\end{tabular}

${ }^{a}$ Based on a percent difference in Cs loading [i.e., 100(algebraic-ZAM)/ZAM]. 
WESTINGHOUSE SAVANNAH RIVER COMPANY

Report:

WSRC-TR-2000-00???

Preliminary Ion Exchange Modeling for Removal of Cesium from

Revision (Date):

$0(07 / 05 / 02)$

Hanford Waste Using Crystalline Silicotitanate Material

Page:

193 of 338

Table B-23. Parameter settings for an "effective" single component Freundlich/Langmuir Hybrid equilibrium isotherm model for total cesium on CST based on the 1-component homovalent model.

\begin{tabular}{|c|c|c|c|c|c|c|}
\hline Feed Solution & $\begin{array}{c}\text { "Powder-form" } \\
\text { (IE-910) } \\
\mathbf{a}_{\mathrm{i}} \\
\left.\text { (gmoles/L } \mathbf{L}_{\mathrm{CV}}\right)\end{array}$ & $\begin{array}{c}\text { "Engineered-form" } \\
\left(\text { IE-911) }{ }^{\mathrm{a}}\right. \\
\mathbf{a}_{\mathrm{i}} \\
\left(\text { gmoles } / \mathbf{L}_{\mathrm{CV}}\right)\end{array}$ & $\begin{array}{c}\mathbf{b}_{\mathbf{i}} \\
\left(\mathbf{M}^{-1}\right)\end{array}$ & $\begin{array}{c}\mathbf{M}_{\mathrm{ai}} \\
(-)\end{array}$ & $\begin{array}{c}\mathbf{M}_{\mathbf{b i}} \\
(-)\end{array}$ & $\begin{array}{c}\text { "Effective" b } \\
\beta_{I} \\
(-)\end{array}$ \\
\hline \multicolumn{7}{|l|}{ Envelope A } \\
\hline LAW-1 & 0.580 & 0.3944 & 1.0 & 1.0 & 1.0 & 4.3376E-04 \\
\hline LAW-5 & 0.580 & 0.3944 & 1.0 & 1.0 & 1.0 & $2.3431 \mathrm{E}-04$ \\
\hline LAW-6 & 0.580 & 0.3944 & 1.0 & 1.0 & 1.0 & $2.3668 \mathrm{E}-04$ \\
\hline LAW-8 & 0.580 & 0.3944 & 1.0 & 1.0 & 1.0 & $2.5147 \mathrm{E}-04$ \\
\hline LAW-9 & 0.580 & 0.3944 & 1.0 & 1.0 & 1.0 & $2.2453 \mathrm{E}-04$ \\
\hline LAW-10 & 0.580 & 0.3944 & 1.0 & 1.0 & 1.0 & $2.2135 \mathrm{E}-04$ \\
\hline LAW-11 & 0.580 & 0.3944 & 1.0 & 1.0 & 1.0 & $2.0543 \mathrm{E}-04$ \\
\hline LAW-12 & 0.580 & 0.3944 & 1.0 & 1.0 & 1.0 & $2.9196 \mathrm{E}-04$ \\
\hline LAW-13 & 0.580 & 0.3944 & 1.0 & 1.0 & 1.0 & $2.9328 \mathrm{E}-04$ \\
\hline LAW-14 & 0.580 & 0.3944 & 1.0 & 1.0 & 1.0 & $3.6283 \mathrm{E}-04$ \\
\hline LAW-15 & 0.580 & 0.3944 & 1.0 & 1.0 & 1.0 & 3.8513E-04 \\
\hline \multicolumn{7}{|l|}{ Envelope B } \\
\hline LAW-2a & 0.580 & 0.3944 & 1.0 & 1.0 & 1.0 & $2.6230 \mathrm{E}-04$ \\
\hline LAW-2b & 0.580 & 0.3944 & 1.0 & 1.0 & 1.0 & $2.1296 \mathrm{E}-04$ \\
\hline \multicolumn{7}{|l|}{ Envelope C } \\
\hline LAW-3 & 0.580 & 0.3944 & 1.0 & 1.0 & 1.0 & $2.1769 \mathrm{E}-04$ \\
\hline LAW-4 & 0.580 & 0.3944 & 1.0 & 1.0 & 1.0 & $2.1769 \mathrm{E}-04$ \\
\hline LAW-7 & 0.580 & 0.3944 & 1.0 & 1.0 & 1.0 & $1.9258 \mathrm{E}-04$ \\
\hline
\end{tabular}

${ }^{\text {a }}$ A dilution factor of $68 \%$ is assumed when going from the powder-form to the engineered-form and a bed density of $1.0 \mathrm{~g} / \mathrm{ml}$ assumed.

${ }^{\mathrm{b}}$ These are "best estimate" values based on the maximum likelihood algorithm.

Table B-24. "Extrapolated" ZAM equilibrium model versus algebraic model cesium loading predictions for CST material in contact with Envelope A's LAW-1 feed solution at $5 \mathrm{M}^{\mathrm{N}} \mathrm{Na}^{+}{ }^{+}{ }^{a}$

\begin{tabular}{|c|c|c|c|c|c|}
\hline \multirow[b]{2}{*}{ Cs (initial) } & \multirow[b]{2}{*}{ Cs (final) ${ }^{b}$} & \multicolumn{3}{|c|}{ Powder form } & \multirow{2}{*}{$\begin{array}{c}\text { Engineered form } \\
\text { Cs loading } \\
\text { (Algebraic model) }\end{array}$} \\
\hline & & $\begin{array}{c}\text { Cs loading } \\
\text { (ZAM model) }\end{array}$ & $\begin{array}{c}\text { Cs loading } \\
\text { (Algebraic model) }\end{array}$ & Loading difference & \\
\hline [M] & {$[\mathbf{M}]$} & [mmole/g] & [mmole/g] & {$[\%]$} & [mmole/g] \\
\hline 0 & same as initial & $0.000 \mathrm{E}+00$ & $0.000 \mathrm{E}+00$ & na & $0.000 \mathrm{E}+00$ \\
\hline 0.000001 & same as initial & $1.326 \mathrm{E}-03$ & $1.334 \mathrm{E}-03$ & 0.5744 & $9.072 \mathrm{E}-04$ \\
\hline 0.00001 & same as initial & $1.300 \mathrm{E}-02$ & $1.307 \mathrm{E}-02$ & 0.5132 & $8.888 \mathrm{E}-03$ \\
\hline 0.00005 & same as initial & $5.989 \mathrm{E}-02$ & $5.995 \mathrm{E}-02$ & 0.0975 & $4.076 \mathrm{E}-02$ \\
\hline 0.0001 & same as initial & $1.090 \mathrm{E}-01$ & $1.087 \mathrm{E}-01$ & -0.2698 & $7.389 \mathrm{E}-02$ \\
\hline 0.0005 & same as initial & $3.129 \mathrm{E}-01$ & $3.106 \mathrm{E}-01$ & -0.7442 & $2.112 \mathrm{E}-01$ \\
\hline 0.001 & same as initial & $4.073 \mathrm{E}-01$ & $4.045 \mathrm{E}-01$ & -0.6848 & $2.751 \mathrm{E}-01$ \\
\hline 0.002 & same as initial & $4.788 \mathrm{E}-01$ & $4.766 \mathrm{E}-01$ & -0.4595 & $3.241 \mathrm{E}-01$ \\
\hline 0.003 & same as initial & $5.084 \mathrm{E}-01$ & $5.067 \mathrm{E}-01$ & -0.3200 & $3.446 \mathrm{E}-01$ \\
\hline 0.005 & same as initial & $5.348 \mathrm{E}-01$ & $5.337 \mathrm{E}-01$ & -0.2110 & $3.629 \mathrm{E}-01$ \\
\hline 0.01 & same as initial & $5.565 \mathrm{E}-01$ & $5.559 \mathrm{E}-01$ & -0.1156 & $3.780 \mathrm{E}-01$ \\
\hline 0.03 & same as initial & $5.719 \mathrm{E}-01$ & $5.717 \mathrm{E}-01$ & -0.0356 & $3.888 \mathrm{E}-01$ \\
\hline 0.05 & same as initial & $5.751 \mathrm{E}-01$ & $5.750 \mathrm{E}-01$ & -0.0218 & $3.910 \mathrm{E}-01$ \\
\hline
\end{tabular}


Preliminary Ion Exchange Modeling for Removal of Cesium from

\begin{tabular}{|c|c|c|c|c||c|}
\cline { 3 - 6 } \multicolumn{2}{c|}{} & \multicolumn{3}{c||}{} & Engineered form $^{\mathbf{c}}$ \\
\hline Cs (initial) & Cs (final) & $\begin{array}{c}\text { Powder form } \\
\text { Cs loading } \\
\text { (ZAM model) }\end{array}$ & $\begin{array}{c}\text { Cs loading } \\
\text { (Algebraic model) }\end{array}$ & Loading difference & $\begin{array}{c}\text { Cs loading } \\
\text { (Algebraic model) }\end{array}$ \\
\hline$[\mathbf{M}]$ & {$[\mathbf{M}]$} & {$[\mathbf{m m o l e} / \mathbf{g}]$} & {$[\mathbf{m m o l e} / \mathbf{g}]$} & {$[\%]$} & {$[\mathbf{m m o l e} / \mathbf{g}]$} \\
\hline 0.1 & same as initial & $5.775 \mathrm{E}-01$ & $5.775 \mathrm{E}-01$ & 0.0050 & $3.927 \mathrm{E}-01$ \\
\hline \hline $\begin{array}{c}3.598 \mathrm{E}-05 \\
\text { (feed conc.) }\end{array}$ & same as initial & $4.455 \mathrm{E}-02$ & $4.443 \mathrm{E}-02$ & -0.2663 & $3.021 \mathrm{E}-02$ \\
\hline
\end{tabular}

a At a $5.0 \mathrm{M}$ sodium concentration level the solution density is estimated to be $1.277 \mathrm{~g} / \mathrm{ml}$ and at $0.712 \mathrm{M}\left[\mathrm{K}^{+}\right]$.

${ }^{\mathrm{b}}$ Final cesium equilibrium concentrations are approx. equal to initial values due to extrapolation scheme used.

c To estimate isotherm for engineered form a dilution factor of 0.68 is employed.

Table B-25. ZAM equilibrium model versus algebraic model cesium loading predictions for CST material in contact with Envelope A's LAW-5 feed solution at $5 \mathrm{M}\left[\mathrm{Na}^{+}\right]^{\mathrm{a}}{ }^{\mathrm{a}}$

\begin{tabular}{|c|c|c|c|c|c|}
\hline & \multirow{2}{*}{\multicolumn{3}{|c|}{ Powder form }} & \multirow{3}{*}{$\begin{array}{c}\text { Engineered form } \\
\text { Cs loading } \\
\text { (Algebraic model) }\end{array}$} \\
\hline & & & & & \\
\hline Cs (initial) & Cs (final) ${ }^{b}$ & $\begin{array}{l}\text { Cs loading } \\
\text { (ZAM model) }\end{array}$ & $\begin{array}{c}\text { Cs loading } \\
\text { (Algebraic model) }\end{array}$ & Loading difference & \\
\hline$[\mathbf{M}]$ & {$[\mathbf{M}]$} & [mmole/g] & [mmole/g] & [\%] & [mmole/g] \\
\hline 0 & $0.000 \mathrm{E}+00$ & $0.000 \mathrm{E}+00$ & $0.000 \mathrm{E}+00$ & na & $0.000 \mathrm{E}+00$ \\
\hline 0.000001 & $9.759 \mathrm{E}-07$ & $2.406 \mathrm{E}-03$ & $2.406 \mathrm{E}-03$ & 0.0035 & $1.636 \mathrm{E}-03$ \\
\hline 0.00001 & $9.768 \mathrm{E}-06$ & $2.321 \mathrm{E}-02$ & $2.321 \mathrm{E}-02$ & 0.0122 & $1.578 \mathrm{E}-02$ \\
\hline 0.00005 & $4.900 \mathrm{E}-05$ & $1.003 \mathrm{E}-01$ & $1.003 \mathrm{E}-01$ & 0.0111 & $6.821 \mathrm{E}-02$ \\
\hline 0.0001 & $9.829 \mathrm{E}-05$ & $1.714 \mathrm{E}-01$ & $1.714 \mathrm{E}-01$ & -0.0094 & $1.166 \mathrm{E}-01$ \\
\hline 0.0005 & $4.961 \mathrm{E}-04$ & 3.939E-01 & 3.939E-01 & 0.0094 & $2.679 \mathrm{E}-01$ \\
\hline 0.001 & $9.953 \mathrm{E}-04$ & 4.694E-01 & $4.695 \mathrm{E}-01$ & 0.0200 & $3.192 \mathrm{E}-01$ \\
\hline 0.002 & $1.995 \mathrm{E}-03$ & 5.191E-01 & $5.190 \mathrm{E}-01$ & -0.0115 & $3.529 \mathrm{E}-01$ \\
\hline 0.003 & $2.995 \mathrm{E}-03$ & $5.379 \mathrm{E}-01$ & $5.379 \mathrm{E}-01$ & 0.0027 & $3.658 \mathrm{E}-01$ \\
\hline 0.005 & 4.994E-03 & $5.538 \mathrm{E}-01$ & $5.540 \mathrm{E}-01$ & 0.0311 & $3.767 \mathrm{E}-01$ \\
\hline 0.01 & $9.994 \mathrm{E}-03$ & $5.667 \mathrm{E}-01$ & $5.667 \mathrm{E}-01$ & 0.0095 & $3.854 \mathrm{E}-01$ \\
\hline 0.03 & $2.999 \mathrm{E}-02$ & $5.755 \mathrm{E}-01$ & $5.755 \mathrm{E}-01$ & -0.0008 & $3.913 \mathrm{E}-01$ \\
\hline 0.05 & $4.999 \mathrm{E}-02$ & $5.774 \mathrm{E}-01$ & $5.773 \mathrm{E}-01$ & -0.0156 & $3.926 \mathrm{E}-01$ \\
\hline 0.1 & $9.999 \mathrm{E}-02$ & $5.785 \mathrm{E}-01$ & $5.786 \mathrm{E}-01$ & 0.0176 & $3.935 \mathrm{E}-01$ \\
\hline $\begin{array}{l}6.283 \mathrm{E}-05 \\
\text { (feed conc.) }\end{array}$ & $6.162 \mathrm{E}-05$ & $1.208 \mathrm{E}-01$ & $1.208 \mathrm{E}-01$ & -0.0039 & $8.212 \mathrm{E}-02$ \\
\hline & & & root mean square $=$ & 0.0137 & \\
\hline
\end{tabular}

${ }^{a}$ At a $5.0 \mathrm{M}$ sodium concentration level the solution density is estimated to be $1.232 \mathrm{~g} / \mathrm{ml}$ and at $0.06073 \mathrm{M}\left[\mathrm{K}^{+}\right]$.

${ }^{\mathrm{b}}$ Final cesium equilibrium concentrations are only slightly less than initial values due to large phase ratio used.

c To estimate isotherm for engineered form a dilution factor of 0.68 is employed.

Table B-26. ZAM equilibrium model versus algebraic model cesium loading predictions for CST material in contact with Envelope A's LAW-6 feed solution at $5 \mathrm{M}\left[\mathrm{Na}^{+}\right]^{\mathrm{a}}{ }^{\mathrm{a}}$

\begin{tabular}{|c|c|c|c|c||c|}
\cline { 3 - 6 } \multicolumn{2}{c|}{} & \multicolumn{3}{c||}{} & Engineered form $^{\mathbf{c}}$ \\
\hline Cs (initial) & Cs (final) & $\begin{array}{c}\text { Powder form } \\
\text { Cs loading } \\
\text { (ZAM model) }\end{array}$ & $\begin{array}{c}\text { Cs loading } \\
\text { (Algebraic model) }\end{array}$ & Loading difference & $\begin{array}{c}\text { Cs loading } \\
\text { (Algebraic model) }\end{array}$ \\
\hline$[\mathbf{M}]$ & {$[\mathbf{M}]$} & {$[\mathbf{m m o l e} / \mathbf{g}]$} & {$[\mathbf{m m o l e} / \mathbf{g}]$} & {$[\%]$} & [mmole/g] \\
\hline 0 & $0.000 \mathrm{E}+00$ & $0.000 \mathrm{E}+00$ & $0.000 \mathrm{E}+00$ & na & $0.000 \mathrm{E}+00$ \\
\hline 0.000001 & $9.762 \mathrm{E}-07$ & $2.383 \mathrm{E}-03$ & $2.382 \mathrm{E}-03$ & -0.0205 & $1.620 \mathrm{E}-03$ \\
\hline 0.00001 & $9.770 \mathrm{E}-06$ & $2.299 \mathrm{E}-02$ & $2.299 \mathrm{E}-02$ & 0.0178 & $1.564 \mathrm{E}-02$ \\
\hline 0.00005 & $4.901 \mathrm{E}-05$ & $9.949 \mathrm{E}-02$ & $9.950 \mathrm{E}-02$ & 0.0085 & $6.766 \mathrm{E}-02$ \\
\hline
\end{tabular}


WESTINGHOUSE SAVANNAH RIVER COMPANY

Report:

WSRC-TR-2000-00???

Preliminary Ion Exchange Modeling for Removal of Cesium from

Revision (Date):

$0(07 / 05 / 02)$

Hanford Waste Using Crystalline Silicotitanate Material

Page:

195 of 338

\begin{tabular}{|c|c|c|c|c|c|}
\hline \multirow[b]{2}{*}{ Cs (initial) } & \multirow[b]{2}{*}{ Cs (final) ${ }^{b}$} & \multicolumn{3}{|c|}{ Powder form } & \multirow{2}{*}{$\begin{array}{c}\text { Engineered form } \\
\begin{array}{c}\text { Cs loading } \\
\text { (Algebraic model) }\end{array}\end{array}$} \\
\hline & & $\begin{array}{c}\text { Cs loading } \\
\text { (ZAM model) }\end{array}$ & $\begin{array}{c}\text { Cs loading } \\
\text { (Algebraic model) }\end{array}$ & Loading difference & \\
\hline$[\mathbf{M}]$ & {$[\mathbf{M}]$} & [mmole/g] & [mmole/g] & {$[\%]$} & [mmole/g] \\
\hline 0.0001 & $9.830 \mathrm{E}-05$ & $1.702 \mathrm{E}-01$ & $1.702 \mathrm{E}-01$ & 0.0258 & $1.157 \mathrm{E}-01$ \\
\hline 0.0005 & $4.961 \mathrm{E}-04$ & $3.927 \mathrm{E}-01$ & $3.927 \mathrm{E}-01$ & 0.0008 & $2.670 \mathrm{E}-01$ \\
\hline 0.001 & $9.953 \mathrm{E}-04$ & $4.685 \mathrm{E}-01$ & $4.686 \mathrm{E}-01$ & 0.0185 & $3.186 \mathrm{E}-01$ \\
\hline 0.002 & $1.995 \mathrm{E}-03$ & $5.185 \mathrm{E}-01$ & $5.185 \mathrm{E}-01$ & -0.0024 & $3.526 \mathrm{E}-01$ \\
\hline 0.003 & $2.995 \mathrm{E}-03$ & $5.376 \mathrm{E}-01$ & $5.375 \mathrm{E}-01$ & -0.0149 & $3.655 \mathrm{E}-01$ \\
\hline 0.005 & 4.994E-03 & $5.538 \mathrm{E}-01$ & $5.538 \mathrm{E}-01$ & -0.0142 & $3.766 \mathrm{E}-01$ \\
\hline 0.01 & 9.994E-03 & $5.665 \mathrm{E}-01$ & $5.666 \mathrm{E}-01$ & 0.0216 & $3.853 \mathrm{E}-01$ \\
\hline 0.03 & $2.999 \mathrm{E}-02$ & $5.752 \mathrm{E}-01$ & $5.755 \mathrm{E}-01$ & 0.0435 & $3.913 \mathrm{E}-01$ \\
\hline 0.05 & 4.999E-02 & 5.774E-01 & 5.773E-01 & -0.0204 & $3.925 \mathrm{E}-01$ \\
\hline 0.1 & 9.999E-02 & $5.785 \mathrm{E}-01$ & 5.786E-01 & 0.0152 & $3.935 \mathrm{E}-01$ \\
\hline $\begin{array}{l}6.328 \mathrm{E}-05 \\
\text { (feed conc.) }\end{array}$ & 6.207E-05 & $1.205 \mathrm{E}-01$ & (1.205E-01 & 0.0218 & "8.194E-02 \\
\hline & & & root mean square $=$ & 0.0202 & \\
\hline
\end{tabular}

${ }^{a}$ At a $5.0 \mathrm{M}$ sodium concentration level the solution density is estimated to be $1.231 \mathrm{~g} / \mathrm{ml}$ and at $0.06243 \mathrm{M}\left[\mathrm{K}^{+}\right]$.

${ }^{\mathrm{b}}$ Final cesium equilibrium concentrations are only slightly less than initial values due to large phase ratio used.

c To estimate isotherm for engineered form a dilution factor of 0.68 is employed.

Table B-27. ZAM equilibrium model versus algebraic model cesium loading predictions for CST material in contact with Envelope A's LAW-8 feed solution at $5 \mathrm{M}\left[\mathrm{Na}^{+}\right]^{\mathrm{a}}{ }^{\mathrm{a}}$

\begin{tabular}{|c|c|c|c|c|c|}
\hline \multirow[b]{2}{*}{ Cs (initial) } & \multirow[b]{2}{*}{ Cs (final) ${ }^{b}$} & \multicolumn{3}{|c|}{ Powder form } & \multirow{2}{*}{$\begin{array}{c}\text { Engineered form } \\
\text { Cs loading } \\
\text { (Algebraic model) }\end{array}$} \\
\hline & & $\begin{array}{c}\text { Cs loading } \\
\text { (ZAM model) }\end{array}$ & $\begin{array}{c}\text { Cs loading } \\
\text { (Algebraic model) }\end{array}$ & Loading difference & \\
\hline$[\mathbf{M}]$ & {$[\mathbf{M}]$} & [mmole/g] & [mmole/g] & {$[\%]$} & [mmole/g] \\
\hline 0 & $0.000 \mathrm{E}+00$ & $0.000 \mathrm{E}+00$ & $0.000 \mathrm{E}+00$ & na & $0.000 \mathrm{E}+00$ \\
\hline 0.000001 & $9.775 \mathrm{E}-07$ & $2.245 \mathrm{E}-03$ & $2.246 \mathrm{E}-03$ & 0.0221 & $1.527 \mathrm{E}-03$ \\
\hline 0.00001 & $9.783 \mathrm{E}-06$ & $2.172 \mathrm{E}-02$ & $2.172 \mathrm{E}-02$ & 0.0032 & $1.477 \mathrm{E}-02$ \\
\hline 0.00005 & $4.905 \mathrm{E}-05$ & $9.467 \mathrm{E}-02$ & $9.467 \mathrm{E}-02$ & -0.0006 & $6.437 \mathrm{E}-02$ \\
\hline 0.0001 & $9.837 \mathrm{E}-05$ & $1.631 \mathrm{E}-01$ & $1.631 \mathrm{E}-01$ & -0.0060 & $1.109 \mathrm{E}-01$ \\
\hline 0.0005 & $4.962 \mathrm{E}-04$ & $3.849 \mathrm{E}-01$ & $3.849 \mathrm{E}-01$ & 0.0056 & $2.617 \mathrm{E}-01$ \\
\hline 0.001 & $9.954 \mathrm{E}-04$ & $4.630 \mathrm{E}-01$ & $4.630 \mathrm{E}-01$ & 0.0139 & $3.149 \mathrm{E}-01$ \\
\hline 0.002 & $1.995 \mathrm{E}-03$ & $5.151 \mathrm{E}-01$ & $5.151 \mathrm{E}-01$ & -0.0066 & $3.503 \mathrm{E}-01$ \\
\hline 0.003 & $2.995 \mathrm{E}-03$ & 5.352E-01 & $5.351 \mathrm{E}-01$ & -0.0249 & $3.638 \mathrm{E}-01$ \\
\hline 0.005 & 4.994E-03 & $5.518 \mathrm{E}-01$ & $5.522 \mathrm{E}-01$ & 0.0648 & $3.755 \mathrm{E}-01$ \\
\hline 0.01 & 9.994E-03 & $5.657 \mathrm{E}-01$ & $5.658 \mathrm{E}-01$ & 0.0183 & $3.847 \mathrm{E}-01$ \\
\hline 0.03 & $2.999 \mathrm{E}-02$ & 5.749E-01 & $5.752 \mathrm{E}-01$ & 0.0467 & $3.911 \mathrm{E}-01$ \\
\hline 0.05 & 4.999E-02 & $5.769 \mathrm{E}-01$ & $5.771 \mathrm{E}-01$ & 0.0368 & $3.924 \mathrm{E}-01$ \\
\hline 0.1 & $9.999 \mathrm{E}-02$ & $5.784 \mathrm{E}-01$ & $5.785 \mathrm{E}-01$ & 0.0178 & $3.934 \mathrm{E}-01$ \\
\hline $\begin{array}{l}4.324 \mathrm{E}-05 \\
\text { (feed conc.) }\end{array}$ & $4.241 \mathrm{E}-05$ & $8.372 \mathrm{E}-02$ & $8.370 \mathrm{E}-02$ & -0.0205 & $5.692 \mathrm{E}-02$ \\
\hline & & & root mean square $=$ & 0.0270 & \\
\hline
\end{tabular}

${ }^{\text {a }}$ At a $5.0 \mathrm{M}$ sodium concentration level the solution density is estimated to be $1.225 \mathrm{~g} / \mathrm{ml}$ and at $0.07216 \mathrm{M}\left[\mathrm{K}^{+}\right]$.

${ }^{\mathrm{b}}$ Final cesium equilibrium concentrations are only slightly less than initial values due to large phase ratio used. c To estimate isotherm for engineered form a dilution factor of 0.68 is employed. 
WESTINGHOUSE SAVANNAH RIVER COMPANY

Preliminary Ion Exchange Modeling for Removal of Cesium from Hanford Waste Using Crystalline Silicotitanate Material
Report:

WSRC-TR-2000-00???

Revision (Date):

Page:
$0(07 / 05 / 02)$

196 of 338

Table B-28. ZAM equilibrium model versus algebraic model cesium loading predictions for CST material in contact with Envelope A's LAW-9 feed solution at $5 \mathrm{M}\left[\mathrm{Na}^{+}\right]^{\mathrm{a}}{ }^{\mathrm{a}}$

\begin{tabular}{|c|c|c|c|c|c|}
\hline \multirow[b]{2}{*}{ Cs (initial) } & \multirow[b]{2}{*}{ Cs (final) ${ }^{b}$} & \multicolumn{3}{|c|}{ Powder form } & \multirow{2}{*}{$\begin{array}{c}\text { Engineered form } \\
\text { Cs loading } \\
\text { (Algebraic model) }\end{array}$} \\
\hline & & $\begin{array}{l}\text { Cs loading } \\
\text { (ZAM model) }\end{array}$ & $\begin{array}{c}\text { Cs loading } \\
\text { (Algebraic model) }\end{array}$ & Loading difference & \\
\hline [M] & [M] & [mmole/g] & [mmole/g] & [\%] & [mmole/g] \\
\hline 0 & $0.000 \mathrm{E}+00$ & $0.000 \mathrm{E}+00$ & $0.000 \mathrm{E}+00$ & na & $0.000 \mathrm{E}+00$ \\
\hline 0.000001 & $9.749 \mathrm{E}-07$ & $2.508 \mathrm{E}-03$ & $2.507 \mathrm{E}-03$ & -0.0386 & $1.705 \mathrm{E}-03$ \\
\hline 0.00001 & $9.758 \mathrm{E}-06$ & $2.416 \mathrm{E}-02$ & $2.416 \mathrm{E}-02$ & -0.0167 & $1.643 \mathrm{E}-02$ \\
\hline 0.00005 & $4.896 \mathrm{E}-05$ & $1.038 \mathrm{E}-01$ & $1.038 \mathrm{E}-01$ & -0.0125 & $7.061 \mathrm{E}-02$ \\
\hline 0.0001 & $9.823 \mathrm{E}-05$ & $1.765 \mathrm{E}-01$ & $1.765 \mathrm{E}-01$ & 0.0000 & $1.200 \mathrm{E}-01$ \\
\hline 0.0005 & $4.960 \mathrm{E}-04$ & $3.993 \mathrm{E}-01$ & $3.993 \mathrm{E}-01$ & -0.0046 & $2.715 \mathrm{E}-01$ \\
\hline 0.001 & $9.953 \mathrm{E}-04$ & $4.733 \mathrm{E}-01$ & $4.732 \mathrm{E}-01$ & -0.0050 & $3.218 \mathrm{E}-01$ \\
\hline 0.002 & $1.995 \mathrm{E}-03$ & $5.213 \mathrm{E}-01$ & $5.213 \mathrm{E}-01$ & 0.0063 & $3.545 \mathrm{E}-01$ \\
\hline 0.003 & $2.995 \mathrm{E}-03$ & $5.397 \mathrm{E}-01$ & $5.396 \mathrm{E}-01$ & -0.0275 & $3.669 \mathrm{E}-01$ \\
\hline 0.005 & 4.994E-03 & $5.548 \mathrm{E}-01$ & $5.550 \mathrm{E}-01$ & 0.0382 & $3.774 \mathrm{E}-01$ \\
\hline 0.01 & $4.994 \mathrm{E}-03$ & $5.548 \mathrm{E}-01$ & $5.550 \mathrm{E}-01$ & 0.0382 & $3.774 \mathrm{E}-01$ \\
\hline 0.03 & $2.999 \mathrm{E}-02$ & $5.755 \mathrm{E}-01$ & $5.757 \mathrm{E}-01$ & 0.0316 & $3.915 \mathrm{E}-01$ \\
\hline 0.05 & 4.999E-02 & $5.774 \mathrm{E}-01$ & $5.774 \mathrm{E}-01$ & 0.0038 & $3.926 \mathrm{E}-01$ \\
\hline 0.1 & $9.999 \mathrm{E}-02$ & $5.786 \mathrm{E}-01$ & $5.787 \mathrm{E}-01$ & 0.0101 & $3.935 \mathrm{E}-01$ \\
\hline $\begin{array}{l}4.444 \mathrm{E}-05 \\
\text { (feed conc.) }\end{array}$ & $4.350 \mathrm{E}-05$ & $9.413 \mathrm{E}-02$ & $9.413 \mathrm{E}-02$ & -0.0029 & $6.401 \mathrm{E}-02$ \\
\hline & & & root mean square $=$ & 0.0220 & \\
\hline
\end{tabular}

${ }^{a}$ At a $5.0 \mathrm{M}$ sodium concentration level the solution density is estimated to be $1.238 \mathrm{~g} / \mathrm{ml}$ and at $0.06027 \mathrm{M}\left[\mathrm{K}^{+}\right]$.

${ }^{\mathrm{b}}$ Final cesium equilibrium concentrations are only slightly less than initial values due to large phase ratio used.

c To estimate isotherm for engineered form a dilution factor of 0.68 is employed.

Table B-29. ZAM equilibrium model versus algebraic model cesium loading predictions for CST material in contact with Envelope A's LAW-10 feed solution at $5 \mathrm{M}\left[\mathrm{Na}^{+}\right]$. ${ }^{\mathrm{a}}$

\begin{tabular}{|c|c|c|c|c||c|}
\cline { 3 - 6 } \multicolumn{2}{c|}{} & \multicolumn{3}{c|}{ Powder form } & Engineered form $^{\mathbf{c}}$ \\
\hline Cs (initial) & Cs (final) & $\begin{array}{c}\text { b } \\
\text { Cs loading } \\
\text { (ZAM model) }\end{array}$ & $\begin{array}{c}\text { Cs loading } \\
\text { (Algebraic model) }\end{array}$ & Loading difference & $\begin{array}{c}\text { Cs loading } \\
\text { (Algebraic model) }\end{array}$ \\
\hline$[\mathbf{M}]$ & {$[\mathbf{M}]$} & {$[\mathbf{m m o l e / g}]$} & {$[\mathbf{m m o l e / g}]$} & [\%] & [mmole/g] \\
\hline 0 & $0.000 \mathrm{E}+00$ & $0.000 \mathrm{E}+00$ & $0.000 \mathrm{E}+00$ & -0.0077 & $0.000 \mathrm{E}+00$ \\
\hline 0.000001 & $9.746 \mathrm{E}-07$ & $2.543 \mathrm{E}-03$ & $2.543 \mathrm{E}-03$ & 0.0272 & $1.729 \mathrm{E}-03$ \\
\hline 0.00001 & $9.755 \mathrm{E}-06$ & $2.448 \mathrm{E}-02$ & $2.448 \mathrm{E}-02$ & -0.0110 & $7.665 \mathrm{E}-02$ \\
\hline 0.00005 & $4.895 \mathrm{E}-05$ & $1.050 \mathrm{E}-01$ & $1.050 \mathrm{E}-01$ & -0.0034 & $1.212 \mathrm{E}-01$ \\
\hline 0.0001 & $9.822 \mathrm{E}-05$ & $1.783 \mathrm{E}-01$ & $1.783 \mathrm{E}-01$ & 0.0039 & $2.727 \mathrm{E}-01$ \\
\hline 0.0005 & $4.960 \mathrm{E}-04$ & $4.010 \mathrm{E}-01$ & $4.010 \mathrm{E}-01$ & 0.0040 & $3.226 \mathrm{E}-01$ \\
\hline 0.001 & $9.953 \mathrm{E}-04$ & $4.745 \mathrm{E}-01$ & $4.745 \mathrm{E}-01$ & -0.0032 & $3.550 \mathrm{E}-01$ \\
\hline 0.002 & $1.995 \mathrm{E}-03$ & $5.221 \mathrm{E}-01$ & $5.221 \mathrm{E}-01$ & 0.0159 & $3.673 \mathrm{E}-01$ \\
\hline 0.003 & $2.995 \mathrm{E}-03$ & $5.400 \mathrm{E}-01$ & $5.401 \mathrm{E}-01$ & 0.0092 & $3.777 \mathrm{E}-01$ \\
\hline 0.005 & $4.994 \mathrm{E}-03$ & $5.553 \mathrm{E}-01$ & $5.554 \mathrm{E}-01$ & 0.0129 & $3.859 \mathrm{E}-01$ \\
\hline 0.01 & $9.994 \mathrm{E}-03$ & $5.674 \mathrm{E}-01$ & $5.674 \mathrm{E}-01$ & 0.0421 & $3.915 \mathrm{E}-01$ \\
\hline 0.03 & $2.999 \mathrm{E}-02$ & $5.755 \mathrm{E}-01$ & $5.758 \mathrm{E}-01$ & 0.0102 & $3.927 \mathrm{E}-01$ \\
\hline 0.05 & $4.999 \mathrm{E}-02$ & $5.774 \mathrm{E}-01$ & $5.774 \mathrm{E}-01$ & 0.0133 & $3.935 \mathrm{E}-01$ \\
\hline 0.1 & $9.999 \mathrm{E}-02$ & $5.786 \mathrm{E}-01$ & $5.787 \mathrm{E}-01$ & -0.0099 & $5.532 \mathrm{E}-02$ \\
\hline \hline $3.692 \mathrm{E}-05$ & $3.611 \mathrm{E}-05$ & $8.136 \mathrm{E}-02$ & $8.135 \mathrm{E}-02$ & & \\
\hline feed conc.) & & & & & \\
\hline
\end{tabular}


WESTINGHOUSE SAVANNAH RIVER COMPANY

Report:

WSRC-TR-2000-00???

Preliminary Ion Exchange Modeling for Removal of Cesium from

Revision (Date):

$0(07 / 05 / 02)$

Hanford Waste Using Crystalline Silicotitanate Material

Page:

197 of 338

\begin{tabular}{|c|c|c|c|c||c|}
\cline { 3 - 6 } \multicolumn{2}{c|}{} & \multicolumn{3}{c||}{ Engineered form $^{\mathrm{c}}$} \\
\hline \multirow{2}{*}{ Cs (initial) } & Cs (final) ${ }^{\mathrm{b}}$ & $\begin{array}{c}\text { Cs loading } \\
\text { (ZAM model) }\end{array}$ & $\begin{array}{c}\text { Cs loading } \\
\text { (Algebraic model) }\end{array}$ & Loading difference & $\begin{array}{c}\text { Cs loading } \\
\text { (Algebraic model) }\end{array}$ \\
\hline \multirow{2}{*}[\mathrm{M}]{} & {$[\mathrm{M}]$} & {$[\mathrm{mmole} / \mathrm{g}]$} & {$[\mathrm{mmole} / \mathrm{g}]$} & {$[\%]$} & {$[\mathrm{mmole} / \mathrm{g}]$} \\
\hline
\end{tabular}

${ }^{a}$ At a $5.0 \mathrm{M}$ sodium concentration level the solution density is estimated to be $1.237 \mathrm{~g} / \mathrm{ml}$ and at $0.04192 \mathrm{M}\left[\mathrm{K}^{+}\right]$.

${ }^{\mathrm{b}}$ Final cesium equilibrium concentrations are only slightly less than initial values due to large phase ratio used.

c To estimate isotherm for engineered form a dilution factor of 0.68 is employed.

Table B-30. ZAM equilibrium model versus algebraic model cesium loading predictions for CST material in contact with Envelope A's LAW-11 feed solution at $5 \mathrm{M}\left[\mathrm{Na}^{+}\right]^{\mathrm{a}}$.

\begin{tabular}{|c|c|c|c|c|c|}
\hline \multirow[b]{2}{*}{ Cs (initial) } & \multirow[b]{2}{*}{ Cs (final) ${ }^{b}$} & \multicolumn{3}{|c|}{ Powder form } & \multirow{2}{*}{\begin{tabular}{|c|} 
Engineered form \\
Cs loading \\
(Algebraic model)
\end{tabular}} \\
\hline & & $\begin{array}{c}\text { Cs loading } \\
\text { (ZAM model) }\end{array}$ & $\begin{array}{c}\text { Cs loading } \\
\text { (Algebraic model) }\end{array}$ & Loading difference & \\
\hline$[\mathbf{M}]$ & {$[\mathbf{M}]$} & {$[\mathrm{mmole} / \mathrm{g}]$} & {$[\mathrm{mmole} / \mathrm{g}]$} & {$[\%]$} & {$[\mathrm{mmole} / \mathrm{g}]$} \\
\hline 0 & $0.000 \mathrm{E}+00$ & $0.000 \mathrm{E}+00$ & $0.000 \mathrm{E}+00$ & na & $0.000 \mathrm{E}+00$ \\
\hline 0.000001 & $9.727 \mathrm{E}-07$ & $2.733 \mathrm{E}-03$ & $2.733 \mathrm{E}-03$ & 0.0015 & $1.859 \mathrm{E}-03$ \\
\hline 0.00001 & $9.737 \mathrm{E}-06$ & $2.625 \mathrm{E}-02$ & $2.625 \mathrm{E}-02$ & -0.0156 & $1.785 \mathrm{E}-02$ \\
\hline 0.00005 & 4.889E-05 & $1.115 \mathrm{E}-01$ & $1.115 \mathrm{E}-01$ & -0.0179 & $7.582 \mathrm{E}-02$ \\
\hline 0.0001 & 9.813E-05 & $1.875 \mathrm{E}-01$ & $1.875 \mathrm{E}-01$ & -0.0178 & $1.275 \mathrm{E}-01$ \\
\hline 0.0005 & 4.959E-04 & 4.101E-01 & 4.101E-01 & 0.0000 & $2.789 \mathrm{E}-01$ \\
\hline 0.001 & 9.952E-04 & $4.807 \mathrm{E}-01$ & $4.808 \mathrm{E}-01$ & 0.0165 & $3.269 \mathrm{E}-01$ \\
\hline 0.002 & $1.995 \mathrm{E}-03$ & $5.259 \mathrm{E}-01$ & $5.259 \mathrm{E}-01$ & -0.0057 & $3.576 \mathrm{E}-01$ \\
\hline 0.003 & $2.995 \mathrm{E}-03$ & $5.427 \mathrm{E}-01$ & $5.428 \mathrm{E}-01$ & 0.0142 & $3.691 \mathrm{E}-01$ \\
\hline 0.005 & 4.994E-03 & $5.568 \mathrm{E}-01$ & $5.571 \mathrm{E}-01$ & 0.0455 & $3.788 \mathrm{E}-01$ \\
\hline 0.01 & $9.994 \mathrm{E}-03$ & $5.683 \mathrm{E}-01$ & $5.683 \mathrm{E}-01$ & 0.0104 & $3.865 \mathrm{E}-01$ \\
\hline 0.03 & $2.999 \mathrm{E}-02$ & $5.758 \mathrm{E}-01$ & $5.761 \mathrm{E}-01$ & 0.0427 & $3.917 \mathrm{E}-01$ \\
\hline 0.05 & 4.999E-02 & 5.774E-01 & $5.776 \mathrm{E}-01$ & 0.0419 & $3.928 \mathrm{E}-01$ \\
\hline 0.1 & $9.999 \mathrm{E}-02$ & $5.787 \mathrm{E}-01$ & $5.788 \mathrm{E}-01$ & 0.0119 & $3.936 \mathrm{E}-01$ \\
\hline $\begin{array}{l}3.739 \mathrm{E}-05 \\
\text { (feed conc.) }\end{array}$ & 3.652E-05 & 8.754E-02 & $8.755 \mathrm{E}-02$ & 0.0079 & (5.953E-02 \\
\hline & & & root mean square $=$ & 0.0229 & \\
\hline
\end{tabular}

${ }^{a}$ At a $5.0 \mathrm{M}$ sodium concentration level the solution density is estimated to be $1.232 \mathrm{~g} / \mathrm{ml}$ and at $0.04152 \mathrm{M}\left[\mathrm{K}^{+}\right]$.

${ }^{\mathrm{b}}$ Final cesium equilibrium concentrations are only slightly less than initial values due to large phase ratio used.

c To estimate isotherm for engineered form a dilution factor of 0.68 is employed.

Table B-31. ZAM equilibrium model versus algebraic model cesium loading predictions for CST material in contact with Envelope A's LAW-12 feed solution at $5 \mathrm{M}\left[\mathrm{Na}^{+}\right]^{\mathrm{a}}{ }^{\mathrm{a}}$

\begin{tabular}{|c|c|c|c|c|c|}
\hline \multirow[b]{2}{*}{ Cs (initial) } & \multirow[b]{2}{*}{ Cs (final) ${ }^{b}$} & \multicolumn{3}{|c|}{ Powder form } & \multirow{2}{*}{$\begin{array}{c}\text { Engineered form } \\
\begin{array}{c}\text { Cs loading } \\
\text { (Algebraic model) }\end{array}\end{array}$} \\
\hline & & $\begin{array}{l}\text { Cs loading } \\
\text { (ZAM model) }\end{array}$ & $\begin{array}{c}\text { Cs loading } \\
\text { (Algebraic model) }\end{array}$ & Loading difference & \\
\hline$[\mathbf{M}]$ & {$[\mathbf{M}]$} & [mmole/g] & [mmole/g] & [\%] & [mmole/g] \\
\hline 0 & $0.000 \mathrm{E}+00$ & $0.000 \mathrm{E}+00$ & $0.000 \mathrm{E}+00$ & na & $0.000 \mathrm{E}+00$ \\
\hline 0.000001 & $9.806 \mathrm{E}-07$ & $1.942 \mathrm{E}-03$ & $1.942 \mathrm{E}-03$ & -0.0039 & $1.320 \mathrm{E}-03$ \\
\hline 0.00001 & $9.811 \mathrm{E}-06$ & $1.886 \mathrm{E}-02$ & $1.886 \mathrm{E}-02$ & -0.0007 & $1.282 \mathrm{E}-02$ \\
\hline 0.00005 & $4.916 \mathrm{E}-05$ & $8.357 \mathrm{E}-02$ & $8.359 \mathrm{E}-02$ & 0.0166 & $5.684 \mathrm{E}-02$ \\
\hline 0.0001 & $9.854 \mathrm{E}-05$ & $1.463 \mathrm{E}-01$ & $1.464 \mathrm{E}-01$ & 0.0185 & $9.952 \mathrm{E}-02$ \\
\hline 0.0005 & $4.963 \mathrm{E}-04$ & $3.651 \mathrm{E}-01$ & $3.652 \mathrm{E}-01$ & 0.0133 & $2.483 \mathrm{E}-01$ \\
\hline
\end{tabular}


WESTINGHOUSE SAVANNAH RIVER COMPANY

Report:

WSRC-TR-2000-00???

Preliminary Ion Exchange Modeling for Removal of Cesium from

Revision (Date):

$0(07 / 05 / 02)$

Hanford Waste Using Crystalline Silicotitanate Material

Page:

198 of 338

\begin{tabular}{|c|c|c|c|c|c|}
\hline \multirow[b]{2}{*}{ Cs (initial) } & \multirow[b]{2}{*}{ Cs (final) ${ }^{b}$} & \multicolumn{3}{|c|}{ Powder form } & \multirow{2}{*}{$\begin{array}{c}\text { Engineered form } \\
\text { Cs loading } \\
\text { (Algebraic model) }\end{array}$} \\
\hline & & $\begin{array}{c}\text { Cs loading } \\
\text { (ZAM model) }\end{array}$ & $\begin{array}{c}\text { Cs loading } \\
\text { (Algebraic model) }\end{array}$ & Loading difference & \\
\hline$[\mathbf{M}]$ & {$[\mathbf{M}]$} & [mmole/g] & [mmole/g] & [\%] & [mmole/g] \\
\hline 0.001 & $9.955 \mathrm{E}-04$ & $4.485 \mathrm{E}-01$ & $4.485 \mathrm{E}-01$ & -0.0001 & $3.050 \mathrm{E}-01$ \\
\hline 0.002 & $1.995 \mathrm{E}-03$ & $5.059 \mathrm{E}-01$ & $5.060 \mathrm{E}-01$ & 0.0046 & $3.440 \mathrm{E}-01$ \\
\hline 0.003 & $2.995 \mathrm{E}-03$ & $5.286 \mathrm{E}-01$ & $5.285 \mathrm{E}-01$ & -0.0256 & $3.594 \mathrm{E}-01$ \\
\hline 0.005 & $4.995 \mathrm{E}-03$ & $5.480 \mathrm{E}-01$ & $5.480 \mathrm{E}-01$ & 0.0035 & $3.726 \mathrm{E}-01$ \\
\hline 0.01 & $9.994 \mathrm{E}-03$ & $5.635 \mathrm{E}-01$ & $5.635 \mathrm{E}-01$ & 0.0134 & $3.832 \mathrm{E}-01$ \\
\hline 0.03 & $2.999 \mathrm{E}-02$ & 5.743E-01 & 5.744E-01 & 0.0173 & $3.906 \mathrm{E}-01$ \\
\hline 0.05 & 4.999E-02 & $5.764 \mathrm{E}-01$ & $5.766 \mathrm{E}-01$ & 0.0429 & $3.921 \mathrm{E}-01$ \\
\hline 0.1 & $9.999 \mathrm{E}-02$ & $5.782 \mathrm{E}-01$ & $5.783 \mathrm{E}-01$ & 0.0120 & $3.933 \mathrm{E}-01$ \\
\hline $\begin{array}{l}4.831 \mathrm{E}-05 \\
\text { (feed conc.) }\end{array}$ & 4.749E-05 & $8.116 \mathrm{E}-02$ & $8.114 \mathrm{E}-02$ & -0.0207 & $5.518 \mathrm{E}-02$ \\
\hline & & & root mean square $=$ & 0.0177 & \\
\hline
\end{tabular}

${ }^{a}$ At a $5.0 \mathrm{M}$ sodium concentration level the solution density is estimated to be $1.221 \mathrm{~g} / \mathrm{ml}$ and at $0.1235 \mathrm{M}\left[\mathrm{K}^{+}\right]$

${ }^{\mathrm{b}}$ Final cesium equilibrium concentrations are only slightly less than initial values due to large phase ratio used.

c To estimate isotherm for engineered form a dilution factor of 0.68 is employed.

Table B-32. ZAM equilibrium model versus algebraic model cesium loading predictions for CST material in contact with Envelope A's LAW-13 feed solution at $5 \mathrm{M}\left[\mathrm{Na}^{+}\right]$.

\begin{tabular}{|c|c|c|c|c||c|}
\cline { 3 - 6 } \multicolumn{2}{c|}{} & \multicolumn{3}{c|}{} & Engineered form $^{\text {c }}$ \\
\hline Cs (initial) & Cs (final) ${ }^{\mathbf{b}}$ & $\begin{array}{c}\text { Powder form } \\
\text { (ZAM model) }\end{array}$ & $\begin{array}{c}\text { Cs loading } \\
\text { (Algebraic model) }\end{array}$ & Loading difference & $\begin{array}{c}\text { Cs loading } \\
\text { (Algebraic model) }\end{array}$ \\
\hline$[\mathbf{M}]$ & {$[\mathbf{M}]$} & [mmole/g] & [mmole/g] & [\%] & [mmole/g] \\
\hline 0 & $0.000 \mathrm{E}+00$ & $0.000 \mathrm{E}+00$ & $0.000 \mathrm{E}+00$ & na & $0.000 \mathrm{E}+00$ \\
\hline 0.000001 & $9.807 \mathrm{E}-07$ & $1.933 \mathrm{E}-03$ & $1.933 \mathrm{E}-03$ & 0.0021 & $1.314 \mathrm{E}-03$ \\
\hline 0.00001 & $9.812 \mathrm{E}-06$ & $1.878 \mathrm{E}-02$ & $1.878 \mathrm{E}-02$ & -0.0204 & $1.277 \mathrm{E}-02$ \\
\hline 0.00005 & $4.917 \mathrm{E}-05$ & $8.329 \mathrm{E}-02$ & $8.328 \mathrm{E}-02$ & -0.0190 & $5.663 \mathrm{E}-02$ \\
\hline 0.0001 & $9.854 \mathrm{E}-05$ & $1.458 \mathrm{E}-01$ & $1.459 \mathrm{E}-01$ & 0.0183 & $9.919 \mathrm{E}-02$ \\
\hline 0.0005 & $4.964 \mathrm{E}-04$ & $3.646 \mathrm{E}-01$ & $3.646 \mathrm{E}-01$ & 0.0102 & $2.479 \mathrm{E}-01$ \\
\hline 0.001 & $9.955 \mathrm{E}-04$ & $4.480 \mathrm{E}-01$ & $4.480 \mathrm{E}-01$ & 0.0084 & $3.046 \mathrm{E}-01$ \\
\hline 0.002 & $1.995 \mathrm{E}-03$ & $5.055 \mathrm{E}-01$ & $5.057 \mathrm{E}-01$ & 0.0258 & $3.439 \mathrm{E}-01$ \\
\hline 0.003 & $2.995 \mathrm{E}-03$ & $5.283 \mathrm{E}-01$ & $5.283 \mathrm{E}-01$ & -0.0091 & $3.592 \mathrm{E}-01$ \\
\hline 0.005 & $4.995 \mathrm{E}-03$ & $5.480 \mathrm{E}-01$ & $5.478 \mathrm{E}-01$ & -0.0214 & $3.725 \mathrm{E}-01$ \\
\hline 0.01 & $9.994 \mathrm{E}-03$ & $5.634 \mathrm{E}-01$ & $5.635 \mathrm{E}-01$ & 0.0183 & $3.832 \mathrm{E}-01$ \\
\hline 0.03 & $2.999 \mathrm{E}-02$ & $5.743 \mathrm{E}-01$ & $5.744 \mathrm{E}-01$ & 0.0130 & $3.906 \mathrm{E}-01$ \\
\hline 0.05 & $4.999 \mathrm{E}-02$ & $5.764 \mathrm{E}-01$ & $5.766 \mathrm{E}-01$ & 0.0403 & $3.921 \mathrm{E}-01$ \\
\hline 0.1 & $9.999 \mathrm{E}-02$ & $5.782 \mathrm{E}-01$ & $5.783 \mathrm{E}-01$ & 0.0107 & $3.932 \mathrm{E}-01$ \\
\hline \hline $4.831 \mathrm{E}-05$ & $4.750 \mathrm{E}-05$ & $8.085 \mathrm{E}-02$ & $8.084 \mathrm{E}-02$ & -0.0013 & $5.497 \mathrm{E}-02$ \\
(feed conc.) & & & & & \\
\hline & & & root mean square & 0.0184 & \\
\hline
\end{tabular}

${ }^{a}$ At a $5.0 \mathrm{M}$ sodium concentration level the solution density is estimated to be $1.221 \mathrm{~g} / \mathrm{ml}$ and at $0.1252 \mathrm{M}\left[\mathrm{K}^{+}\right]$

${ }^{\mathrm{b}}$ Final cesium equilibrium concentrations are only slightly less than initial values due to large phase ratio used.

c To estimate isotherm for engineered form a dilution factor of 0.68 is employed.

Table B-33. ZAM equilibrium model versus algebraic model cesium loading predictions for CST material in contact with Envelope A's LAW-14 feed solution at $5 \mathrm{M}\left[\mathrm{Na}^{+}\right]^{\text {a }}$ 
WESTINGHOUSE SAVANNAH RIVER COMPANY

Report:

WSRC-TR-2000-00???

Preliminary Ion Exchange Modeling for Removal of Cesium from

Revision (Date):

$0(07 / 05 / 02)$

Hanford Waste Using Crystalline Silicotitanate Material

Page:

199 of 338

\begin{tabular}{|c|c|c|c|c||c|}
\hline Cs (initial) & Cs (final) & $\begin{array}{c}\text { Cs loading } \\
\text { (ZAM model) }\end{array}$ & $\begin{array}{c}\text { Cs loading } \\
\text { (Algebraic model) }\end{array}$ & Loading difference & $\begin{array}{c}\text { Cs loading } \\
\text { (Algebraic model) }\end{array}$ \\
\hline$[\mathbf{M}]$ & {$[\mathbf{M}]$} & {$[\mathbf{m m o l e} / \mathbf{g}]$} & {$[\mathbf{m m o l e} / \mathbf{g}]$} & {$[\%]$} & {$[\mathbf{m m o l e} / \mathbf{g}]$} \\
\hline 0 & $0.000 \mathrm{E}+00$ & $0.000 \mathrm{E}+00$ & $0.000 \mathrm{E}+00$ & na & $0.000 \mathrm{E}+00$ \\
\hline 0.000001 & $9.843 \mathrm{E}-07$ & $1.569 \mathrm{E}-03$ & $1.569 \mathrm{E}-03$ & 0.0138 & $1.067 \mathrm{E}-03$ \\
\hline 0.00001 & $9.847 \mathrm{E}-06$ & $1.533 \mathrm{E}-02$ & $1.532 \mathrm{E}-02$ & -0.0445 & $1.042 \mathrm{E}-02$ \\
\hline 0.00005 & $4.931 \mathrm{E}-05$ & $6.938 \mathrm{E}-02$ & $6.939 \mathrm{E}-02$ & 0.0205 & $4.719 \mathrm{E}-02$ \\
\hline 0.0001 & $9.876 \mathrm{E}-05$ & $1.241 \mathrm{E}-01$ & $1.241 \mathrm{E}-01$ & -0.0377 & $8.438 \mathrm{E}-02$ \\
\hline 0.0005 & $4.966 \mathrm{E}-04$ & $3.351 \mathrm{E}-01$ & $3.351 \mathrm{E}-01$ & 0.0098 & $2.279 \mathrm{E}-01$ \\
\hline 0.001 & $9.957 \mathrm{E}-04$ & $4.251 \mathrm{E}-01$ & $4.251 \mathrm{E}-01$ & 0.0075 & $2.891 \mathrm{E}-01$ \\
\hline 0.002 & $1.995 \mathrm{E}-03$ & $4.908 \mathrm{E}-01$ & $4.907 \mathrm{E}-01$ & -0.0045 & $3.337 \mathrm{E}-01$ \\
\hline 0.003 & $2.995 \mathrm{E}-03$ & $5.172 \mathrm{E}-01$ & $5.173 \mathrm{E}-01$ & 0.0177 & $3.518 \mathrm{E}-01$ \\
\hline 0.005 & $4.995 \mathrm{E}-03$ & $5.405 \mathrm{E}-01$ & $5.407 \mathrm{E}-01$ & 0.0488 & $3.677 \mathrm{E}-01$ \\
\hline 0.01 & $9.994 \mathrm{E}-03$ & $5.596 \mathrm{E}-01$ & $5.597 \mathrm{E}-01$ & 0.0209 & $3.806 \mathrm{E}-01$ \\
\hline 0.03 & $2.999 \mathrm{E}-02$ & $5.728 \mathrm{E}-01$ & $5.731 \mathrm{E}-01$ & 0.0450 & $3.897 \mathrm{E}-01$ \\
\hline 0.05 & $4.999 \mathrm{E}-02$ & $5.759 \mathrm{E}-01$ & $5.758 \mathrm{E}-01$ & -0.0111 & $3.916 \mathrm{E}-01$ \\
\hline 0.1 & $4.999 \mathrm{E}-02$ & $5.759 \mathrm{E}-01$ & $5.758 \mathrm{E}-01$ & -0.0111 & $3.916 \mathrm{E}-01$ \\
\hline \hline $\begin{array}{c}4.569 \mathrm{E}-05 \\
\text { feed conc.) }\end{array}$ & $4.504 \mathrm{E}-05$ & $6.405 \mathrm{E}-02$ & $6.405 \mathrm{E}-02$ & 0.0015 & $4.355 \mathrm{E}-02$ \\
\hline & & & & & \\
\hline
\end{tabular}

${ }^{\text {a }}$ At a $5.0 \mathrm{M}$ sodium concentration level the solution density is estimated to be $1.234 \mathrm{~g} / \mathrm{ml}$ and at $0.3198 \mathrm{M}\left[\mathrm{K}^{+}\right]$.

${ }^{\mathrm{b}}$ Final cesium equilibrium concentrations are only slightly less than initial values due to large phase ratio used.

c To estimate isotherm for engineered form a dilution factor of 0.68 is employed.

Table B-34. ZAM equilibrium model versus algebraic model cesium loading predictions for CST material in contact with Envelope A's LAW-15 feed solution at $5 \mathrm{M}\left[\mathrm{Na}^{+}\right] .{ }^{\mathrm{a}}$

\begin{tabular}{|c|c|c|c|c|c|}
\hline \multirow[b]{2}{*}{ Cs (initial) } & \multirow[b]{2}{*}{ Cs (final) ${ }^{b}$} & \multicolumn{3}{|c|}{ Powder form } & \multirow{2}{*}{$\begin{array}{c}\text { Engineered form } \\
\text { Cs loading } \\
\text { (Algebraic model) }\end{array}$} \\
\hline & & $\begin{array}{l}\text { Cs loading } \\
\text { (ZAM model) }\end{array}$ & $\begin{array}{c}\text { Cs loading } \\
\text { (Algebraic model) }\end{array}$ & Loading difference & \\
\hline [M] & [M] & [mmole/g] & [mmole/g] & [\%] & [mmole/g] \\
\hline 0 & $0.000 \mathrm{E}+00$ & $0.000 \mathrm{E}+00$ & $0.000 \mathrm{E}+00$ & na & $0.000 \mathrm{E}+00$ \\
\hline 0.000001 & $9.852 \mathrm{E}-07$ & $1.480 \mathrm{E}-03$ & $1.480 \mathrm{E}-03$ & 0.0095 & $1.006 \mathrm{E}-03$ \\
\hline 0.00001 & $9.855 \mathrm{E}-06$ & $1.447 \mathrm{E}-02$ & $1.447 \mathrm{E}-02$ & 0.0279 & $9.840 \mathrm{E}-03$ \\
\hline 0.00005 & $4.934 \mathrm{E}-05$ & $6.587 \mathrm{E}-02$ & $6.587 \mathrm{E}-02$ & -0.0030 & $4.479 \mathrm{E}-02$ \\
\hline 0.0001 & $9.882 \mathrm{E}-05$ & $1.184 \mathrm{E}-01$ & $1.184 \mathrm{E}-01$ & 0.0393 & $8.053 \mathrm{E}-02$ \\
\hline 0.0005 & $4.967 \mathrm{E}-04$ & $3.267 \mathrm{E}-01$ & $3.267 \mathrm{E}-01$ & 0.0035 & $2.221 \mathrm{E}-01$ \\
\hline 0.001 & $9.958 \mathrm{E}-04$ & $4.182 \mathrm{E}-01$ & $4.182 \mathrm{E}-01$ & 0.0016 & $2.844 \mathrm{E}-01$ \\
\hline 0.002 & $1.995 \mathrm{E}-03$ & $4.860 \mathrm{E}-01$ & $4.861 \mathrm{E}-01$ & 0.0346 & $3.306 \mathrm{E}-01$ \\
\hline 0.003 & $2.995 \mathrm{E}-03$ & $5.139 \mathrm{E}-01$ & $5.139 \mathrm{E}-01$ & -0.0052 & $3.495 \mathrm{E}-01$ \\
\hline 0.005 & $4.995 \mathrm{E}-03$ & $5.385 \mathrm{E}-01$ & $5.385 \mathrm{E}-01$ & 0.0038 & $3.662 \mathrm{E}-01$ \\
\hline 0.01 & 9.994E-03 & $5.584 \mathrm{E}-01$ & $5.585 \mathrm{E}-01$ & 0.0204 & $3.798 \mathrm{E}-01$ \\
\hline 0.03 & $2.999 \mathrm{E}-02$ & $5.725 \mathrm{E}-01$ & $5.726 \mathrm{E}-01$ & 0.0239 & $3.894 \mathrm{E}-01$ \\
\hline 0.05 & 4.999E-02 & $5.754 \mathrm{E}-01$ & $5.756 \mathrm{E}-01$ & 0.0314 & $3.914 \mathrm{E}-01$ \\
\hline 0.1 & $9.999 \mathrm{E}-02$ & $5.776 \mathrm{E}-01$ & $5.778 \mathrm{E}-01$ & 0.0229 & $3.929 \mathrm{E}-01$ \\
\hline $\begin{array}{l}4.552 \mathrm{E}-05 \\
\text { (feed conc.) }\end{array}$ & 4.492E-05 & $6.060 \mathrm{E}-02$ & $6.058 \mathrm{E}-02$ & -0.0237 & 4.120E-02 \\
\hline & & & root mean square $=$ & 0.0219 & \\
\hline
\end{tabular}

${ }^{\text {a }}$ At a $5.0 \mathrm{M}$ sodium concentration level the solution density is estimated to be $1.235 \mathrm{~g} / \mathrm{ml}$ and at $0.4067 \mathrm{M}\left[\mathrm{K}^{+}\right]$.

${ }^{b}$ Final cesium equilibrium concentrations are only slightly less than initial values due to large phase ratio used. c To estimate isotherm for engineered form a dilution factor of 0.68 is employed. 
WESTINGHOUSE SAVANNAH RIVER COMPANY

Preliminary Ion Exchange Modeling for Removal of Cesium from Hanford Waste Using Crystalline Silicotitanate Material
Report:

WSRC-TR-2000-00???

Revision (Date):

Page:
$0(07 / 05 / 02)$ 200 of 338

Table B-35. ZAM equilibrium model versus algebraic model cesium loading predictions for CST

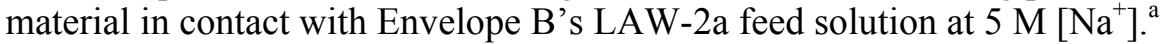

\begin{tabular}{|c|c|c|c|c|c|}
\hline \multirow[b]{2}{*}{ Cs (initial) } & \multirow[b]{2}{*}{ Cs (final) ${ }^{b}$} & \multicolumn{3}{|c|}{ Powder form } & \multirow{2}{*}{$\begin{array}{c}\text { Engineered form } \\
\text { Cs loading } \\
\text { (Algebraic model) }\end{array}$} \\
\hline & & $\begin{array}{l}\text { Cs loading } \\
\text { (ZAM model) }\end{array}$ & $\begin{array}{c}\text { Cs loading } \\
\text { (Algebraic model) }\end{array}$ & Loading difference & \\
\hline [M] & {$[\mathbf{M}]$} & [mmole/g] & [mmole/g] & [\%] & [mmole/g] \\
\hline 0 & $0.000 \mathrm{E}+00$ & $0.000 \mathrm{E}+00$ & $0.000 \mathrm{E}+00$ & na & $0.000 \mathrm{E}+00$ \\
\hline 0.000001 & $9.784 \mathrm{E}-07$ & $2.155 \mathrm{E}-03$ & $2.155 \mathrm{E}-03$ & -0.0004 & $1.466 \mathrm{E}-03$ \\
\hline 0.00001 & $9.791 \mathrm{E}-06$ & $2.087 \mathrm{E}-02$ & $2.087 \mathrm{E}-02$ & -0.0169 & $1.419 \mathrm{E}-02$ \\
\hline 0.00005 & 4.909E-05 & $9.145 \mathrm{E}-02$ & $9.144 \mathrm{E}-02$ & -0.0206 & $6.218 \mathrm{E}-02$ \\
\hline 0.0001 & $9.842 \mathrm{E}-05$ & $1.583 \mathrm{E}-01$ & $1.582 \mathrm{E}-01$ & -0.0065 & $1.076 \mathrm{E}-01$ \\
\hline 0.0005 & $4.962 \mathrm{E}-04$ & $3.794 \mathrm{E}-01$ & $3.794 \mathrm{E}-01$ & 0.0088 & $2.580 \mathrm{E}-01$ \\
\hline 0.001 & $9.954 \mathrm{E}-04$ & $4.590 \mathrm{E}-01$ & $4.590 \mathrm{E}-01$ & 0.0129 & $3.121 \mathrm{E}-01$ \\
\hline 0.002 & $1.995 \mathrm{E}-03$ & $5.125 \mathrm{E}-01$ & $5.126 \mathrm{E}-01$ & 0.0172 & $3.486 \mathrm{E}-01$ \\
\hline 0.003 & $2.995 \mathrm{E}-03$ & $5.334 \mathrm{E}-01$ & $5.333 \mathrm{E}-01$ & -0.0216 & $3.626 \mathrm{E}-01$ \\
\hline 0.005 & 4.994E-03 & $5.508 \mathrm{E}-01$ & $5.511 \mathrm{E}-01$ & 0.0397 & $3.747 \mathrm{E}-01$ \\
\hline 0.01 & $9.994 \mathrm{E}-03$ & $5.651 \mathrm{E}-01$ & $5.652 \mathrm{E}-01$ & 0.0188 & $3.843 \mathrm{E}-01$ \\
\hline 0.03 & $2.999 \mathrm{E}-02$ & $5.749 \mathrm{E}-01$ & $5.750 \mathrm{E}-01$ & 0.0109 & $3.910 \mathrm{E}-01$ \\
\hline 0.05 & 4.999E-02 & $5.769 \mathrm{E}-01$ & $5.770 \mathrm{E}-01$ & 0.0153 & $3.923 \mathrm{E}-01$ \\
\hline 0.1 & 9.999E-02 & $5.784 \mathrm{E}-01$ & $5.785 \mathrm{E}-01$ & 0.0070 & $3.934 \mathrm{E}-01$ \\
\hline $\begin{array}{l}4.676 \mathrm{E}-04 \\
\text { (feed conc.) }\end{array}$ & 4.638E-04 & 3.704E-01 & $3.705 \mathrm{E}-01$ & 0.0110 & $2.519 \mathrm{E}-01$ \\
\hline & & & root mean square $=$ & 0.0173 & \\
\hline
\end{tabular}

${ }^{\text {a }}$ At a $5.0 \mathrm{M}$ sodium concentration level the solution density is estimated to be $1.254 \mathrm{~g} / \mathrm{ml}$ and at $0.1250 \mathrm{M}\left[\mathrm{K}^{+}\right]$.

${ }^{b}$ Final cesium equilibrium concentrations are only slightly less than initial values due to large phase ratio used. c To estimate isotherm for engineered form a dilution factor of 0.68 is employed.

Table B-36. ZAM equilibrium model versus algebraic model cesium loading predictions for CST material in contact with Envelope B's LAW-2b feed solution at $5 \mathrm{M}\left[\mathrm{Na}^{+}\right]^{\mathrm{a}}$

\begin{tabular}{|c|c|c|c|c|c|}
\hline \multirow[b]{2}{*}{ Cs (initial) } & \multirow[b]{2}{*}{ Cs (final) ${ }^{b}$} & \multicolumn{3}{|c|}{ Powder form } & \multirow{2}{*}{$\begin{array}{c}\text { Engineered form } \\
\text { Cs loading } \\
\text { (Algebraic model) }\end{array}$} \\
\hline & & $\begin{array}{c}\text { Cs loading } \\
\text { (ZAM model) }\end{array}$ & $\begin{array}{c}\text { Cs loading } \\
\text { (Algebraic model) }\end{array}$ & Loading difference & \\
\hline$[\mathbf{M}]$ & {$[\mathbf{M}]$} & [mmole/g] & [mmole/g] & {$[\%]$} & [mmole/g] \\
\hline 0 & $0.000 \mathrm{E}+00$ & $0.000 \mathrm{E}+00$ & $0.000 \mathrm{E}+00$ & na & $0.000 \mathrm{E}+00$ \\
\hline 0.000001 & $9.736 \mathrm{E}-07$ & $2.639 \mathrm{E}-03$ & $2.640 \mathrm{E}-03$ & 0.0045 & $1.795 \mathrm{E}-03$ \\
\hline 0.00001 & $9.746 \mathrm{E}-06$ & $2.538 \mathrm{E}-02$ & $2.538 \mathrm{E}-02$ & 0.0127 & $1.726 \mathrm{E}-02$ \\
\hline 0.00005 & 4.892E-05 & $1.084 \mathrm{E}-01$ & $1.083 \mathrm{E}-01$ & -0.0111 & $7.368 \mathrm{E}-02$ \\
\hline 0.0001 & $9.817 \mathrm{E}-05$ & $1.830 \mathrm{E}-01$ & $1.830 \mathrm{E}-01$ & 0.0093 & $1.244 \mathrm{E}-01$ \\
\hline 0.0005 & 4.959E-04 & $4.057 \mathrm{E}-01$ & $4.058 \mathrm{E}-01$ & 0.0141 & $2.759 \mathrm{E}-01$ \\
\hline 0.001 & $9.952 \mathrm{E}-04$ & 4.777E-01 & 4.778E-01 & 0.0143 & $3.249 \mathrm{E}-01$ \\
\hline 0.002 & $1.995 \mathrm{E}-03$ & $5.241 \mathrm{E}-01$ & $5.241 \mathrm{E}-01$ & -0.0054 & $3.564 \mathrm{E}-01$ \\
\hline 0.003 & $2.995 \mathrm{E}-03$ & $5.415 \mathrm{E}-01$ & $5.415 \mathrm{E}-01$ & 0.0001 & $3.682 \mathrm{E}-01$ \\
\hline 0.005 & 4.994E-03 & $5.563 \mathrm{E}-01$ & $5.563 \mathrm{E}-01$ & -0.0095 & $3.783 \mathrm{E}-01$ \\
\hline 0.01 & 9.994E-03 & $5.679 \mathrm{E}-01$ & 5.679E-01 & 0.0070 & $3.862 \mathrm{E}-01$ \\
\hline 0.03 & 2.999E-02 & $5.758 \mathrm{E}-01$ & $5.759 \mathrm{E}-01$ & 0.0178 & $3.916 \mathrm{E}-01$ \\
\hline 0.05 & 4.999E-02 & $5.774 \mathrm{E}-01$ & $5.775 \mathrm{E}-01$ & 0.0269 & $3.927 \mathrm{E}-01$ \\
\hline 0.1 & 9.999E-02 & $5.786 \mathrm{E}-01$ & $5.788 \mathrm{E}-01$ & 0.0216 & $3.936 \mathrm{E}-01$ \\
\hline $\begin{array}{l}4.311 \mathrm{E}-04 \\
\text { (feed conc.) }\end{array}$ & 4.272E-04 & 3.870E-01 & 3.871E-01 & 0.0136 & 2.632E-01 \\
\hline
\end{tabular}


Preliminary Ion Exchange Modeling for Removal of Cesium from

\begin{tabular}{|c|c|c|c|c||c|}
\cline { 3 - 6 } \multicolumn{2}{c|}{} & \multicolumn{3}{c||}{ Engineered form $^{\mathrm{c}}$} \\
\hline \multirow{2}{*}{ Cs (initial) } & Cs (final) ${ }^{\mathrm{b}}$ & $\begin{array}{c}\text { Cs loading } \\
\text { (ZAM model) }\end{array}$ & $\begin{array}{c}\text { Cs loading } \\
\text { (Algebraic model) }\end{array}$ & Loading difference & $\begin{array}{c}\text { Cs loading } \\
\text { (Algebraic model) }\end{array}$ \\
\hline \multirow{2}{*}[\mathrm{M}]{} & {$[\mathrm{M}]$} & {$[\mathrm{mmole} / \mathrm{g}]$} & {$[\mathrm{mmole} / \mathrm{g}]$} & {$[\%]$} & {$[\mathrm{mmole} / \mathrm{g}]$} \\
\hline
\end{tabular}

${ }^{\text {a }}$ At a $5.0 \mathrm{M}$ sodium concentration level the solution density is estimated to be $1.242 \mathrm{~g} / \mathrm{ml}$ and at $0.1429 \mathrm{M}\left[\mathrm{K}^{+}\right]$.

${ }^{b}$ Final cesium equilibrium concentrations are only slightly less than initial values due to large phase ratio used.

c To estimate isotherm for engineered form a dilution factor of 0.68 is employed.

Table B-37. ZAM equilibrium model versus algebraic model cesium loading predictions for CST material in contact with Envelope C's LAW-3 feed solution at $5 \mathrm{M}\left[\mathrm{Na}^{+}\right]^{\mathrm{a}}$

\begin{tabular}{|c|c|c|c|c|c|}
\hline \multirow[b]{2}{*}{ Cs (initial) } & \multirow[b]{2}{*}{ Cs (final) ${ }^{b}$} & \multicolumn{3}{|c|}{ Powder form } & \multirow{2}{*}{$\begin{array}{c}\text { Engineered form } \\
\begin{array}{c}\text { Cs loading } \\
\text { (Algebraic model) }\end{array}\end{array}$} \\
\hline & & $\begin{array}{l}\text { Cs loading } \\
\text { (ZAM model) }\end{array}$ & $\begin{array}{c}\text { Cs loading } \\
\text { (Algebraic model) }\end{array}$ & Loading difference & \\
\hline [M] & [M] & [mmole/g] & {$[\mathrm{mmole} / \mathrm{g}]$} & [\%] & [mmole/g] \\
\hline 0 & $0.000 \mathrm{E}+00$ & $0.000 \mathrm{E}+00$ & $0.000 \mathrm{E}+00$ & na & $0.000 \mathrm{E}+00$ \\
\hline 0.000001 & $9.742 \mathrm{E}-07$ & $2.585 \mathrm{E}-03$ & $2.584 \mathrm{E}-03$ & -0.0200 & $1.757 \mathrm{E}-03$ \\
\hline 0.00001 & $9.751 \mathrm{E}-06$ & $2.487 \mathrm{E}-02$ & $2.487 \mathrm{E}-02$ & 0.0044 & $1.691 \mathrm{E}-02$ \\
\hline 0.00005 & 4.894E-05 & $1.064 \mathrm{E}-01$ & $1.065 \mathrm{E}-01$ & 0.0138 & $7.239 \mathrm{E}-02$ \\
\hline 0.0001 & $9.820 \mathrm{E}-05$ & $1.803 \mathrm{E}-01$ & $1.803 \mathrm{E}-01$ & 0.0045 & $1.226 \mathrm{E}-01$ \\
\hline 0.0005 & $4.960 \mathrm{E}-04$ & $4.031 \mathrm{E}-01$ & $4.031 \mathrm{E}-01$ & -0.0027 & $2.741 \mathrm{E}-01$ \\
\hline 0.001 & 9.952E-04 & $4.758 \mathrm{E}-01$ & $4.759 \mathrm{E}-01$ & 0.0202 & $3.236 \mathrm{E}-01$ \\
\hline 0.002 & $1.995 \mathrm{E}-03$ & 5.229E-01 & $5.229 \mathrm{E}-01$ & 0.0093 & $3.556 \mathrm{E}-01$ \\
\hline 0.003 & $2.995 \mathrm{E}-03$ & 5.406E-01 & 5.407E-01 & 0.0189 & $3.677 \mathrm{E}-01$ \\
\hline 0.005 & 4.994E-03 & $5.558 \mathrm{E}-01$ & $5.558 \mathrm{E}-01$ & -0.0105 & 3.779E-01 \\
\hline 0.01 & $9.994 \mathrm{E}-03$ & $5.676 \mathrm{E}-01$ & $5.676 \mathrm{E}-01$ & 0.0135 & $3.860 \mathrm{E}-01$ \\
\hline 0.03 & $2.999 \mathrm{E}-02$ & $5.758 \mathrm{E}-01$ & $5.758 \mathrm{E}-01$ & 0.0021 & $3.916 \mathrm{E}-01$ \\
\hline 0.05 & 4.999E-02 & $5.774 \mathrm{E}-01$ & $5.775 \mathrm{E}-01$ & 0.0174 & $3.927 \mathrm{E}-01$ \\
\hline 0.1 & $9.999 \mathrm{E}-02$ & $5.786 \mathrm{E}-01$ & $5.787 \mathrm{E}-01$ & 0.0169 & $3.935 \mathrm{E}-01$ \\
\hline $\begin{array}{l}3.967 \mathrm{E}-05 \\
\text { (feed conc.) }\end{array}$ & $3.880 \mathrm{E}-05$ & $8.773 \mathrm{E}-02$ & $8.774 \mathrm{E}-02$ & 0.0131 & $5.966 \mathrm{E}-02$ \\
\hline & & & root mean square $=$ & 0.0135 & \\
\hline
\end{tabular}

${ }^{a}$ At a $5.0 \mathrm{M}$ sodium concentration level the solution density is estimated to be $1.237 \mathrm{~g} / \mathrm{ml}$ and at $0.04393 \mathrm{M}\left[\mathrm{K}^{+}\right]$.

${ }^{\mathrm{b}}$ Final cesium equilibrium concentrations are only slightly less than initial values due to large phase ratio used.

c To estimate isotherm for engineered form a dilution factor of 0.68 is employed.

Table B-38. ZAM equilibrium model versus algebraic model cesium loading predictions for CST material in contact with Envelope C's LAW-4 feed solution at $5 \mathrm{M}\left[\mathrm{Na}^{+}\right]^{\mathrm{a}}$

\begin{tabular}{|c|c|c|c|c|c|}
\hline \multirow[b]{2}{*}{ Cs (initial) } & \multirow[b]{2}{*}{ Cs (final) ${ }^{b}$} & \multicolumn{3}{|c|}{ Powder form } & \multirow{2}{*}{$\begin{array}{c}\text { Engineered form } \\
\text { Cs loading } \\
\text { (Algebraic model) }\end{array}$} \\
\hline & & $\begin{array}{l}\text { Cs loading } \\
\text { (ZAM model) }\end{array}$ & $\begin{array}{c}\text { Cs loading } \\
\text { (Algebraic model) }\end{array}$ & Loading difference & \\
\hline$[\mathbf{M}]$ & {$[\mathbf{M}]$} & [mmole/g] & {$[$ mmole/g] } & {$[\%]$} & [mmole/g] \\
\hline 0 & $0.000 \mathrm{E}+00$ & $0.000 \mathrm{E}+00$ & $0.000 \mathrm{E}+00$ & na & $0.000 \mathrm{E}+00$ \\
\hline 0.000001 & $9.742 \mathrm{E}-07$ & $2.585 \mathrm{E}-03$ & $2.584 \mathrm{E}-03$ & -0.0200 & $1.757 \mathrm{E}-03$ \\
\hline 0.00001 & $9.751 \mathrm{E}-06$ & $2.487 \mathrm{E}-02$ & $2.487 \mathrm{E}-02$ & 0.0044 & $1.691 \mathrm{E}-02$ \\
\hline 0.00005 & $4.894 \mathrm{E}-05$ & $1.064 \mathrm{E}-01$ & $1.065 \mathrm{E}-01$ & 0.0138 & $7.239 \mathrm{E}-02$ \\
\hline 0.0001 & $9.820 \mathrm{E}-05$ & $1.803 \mathrm{E}-01$ & $1.803 \mathrm{E}-01$ & 0.0045 & $1.226 \mathrm{E}-01$ \\
\hline 0.0005 & $4.960 \mathrm{E}-04$ & $4.031 \mathrm{E}-01$ & $4.031 \mathrm{E}-01$ & -0.0027 & $2.741 \mathrm{E}-01$ \\
\hline
\end{tabular}


WESTINGHOUSE SAVANNAH RIVER COMPANY

Report:

WSRC-TR-2000-00???

Preliminary Ion Exchange Modeling for Removal of Cesium from

Revision (Date):

$0(07 / 05 / 02)$

Hanford Waste Using Crystalline Silicotitanate Material

Page:

202 of 338

\begin{tabular}{|c|c|c|c|c|c|}
\hline \multirow[b]{2}{*}{ Cs (initial) } & \multirow[b]{2}{*}{ Cs (final) ${ }^{b}$} & \multicolumn{3}{|c|}{ Powder form } & \multirow{2}{*}{$\begin{array}{c}\text { Engineered form } \\
\text { Cs loading } \\
\text { (Algebraic model) }\end{array}$} \\
\hline & & $\begin{array}{c}\text { Cs loading } \\
\text { (ZAM model) }\end{array}$ & $\begin{array}{c}\text { Cs loading } \\
\text { (Algebraic model) }\end{array}$ & Loading difference & \\
\hline$[\mathbf{M}]$ & {$[\mathbf{M}]$} & [mmole/g] & [mmole/g] & [\%] & [mmole/g] \\
\hline 0.001 & $9.952 \mathrm{E}-04$ & $4.758 \mathrm{E}-01$ & $4.759 \mathrm{E}-01$ & 0.0202 & $3.236 \mathrm{E}-01$ \\
\hline 0.002 & $1.995 \mathrm{E}-03$ & $5.229 \mathrm{E}-01$ & $5.229 \mathrm{E}-01$ & 0.0093 & $3.556 \mathrm{E}-01$ \\
\hline 0.003 & $2.995 \mathrm{E}-03$ & $5.406 \mathrm{E}-01$ & $5.407 \mathrm{E}-01$ & 0.0189 & $3.677 \mathrm{E}-01$ \\
\hline 0.005 & 4.994E-03 & $5.558 \mathrm{E}-01$ & $5.558 \mathrm{E}-01$ & -0.0105 & $3.779 \mathrm{E}-01$ \\
\hline 0.01 & $9.994 \mathrm{E}-03$ & $5.676 \mathrm{E}-01$ & $5.676 \mathrm{E}-01$ & 0.0135 & $3.860 \mathrm{E}-01$ \\
\hline 0.03 & $2.999 \mathrm{E}-02$ & $5.758 \mathrm{E}-01$ & $5.758 \mathrm{E}-01$ & 0.0021 & $3.916 \mathrm{E}-01$ \\
\hline 0.05 & 4.999E-02 & $5.774 \mathrm{E}-01$ & $5.775 \mathrm{E}-01$ & 0.0174 & $3.927 \mathrm{E}-01$ \\
\hline 0.1 & $9.999 \mathrm{E}-02$ & $5.786 \mathrm{E}-01$ & $5.787 \mathrm{E}-01$ & 0.0169 & $3.935 \mathrm{E}-01$ \\
\hline $\begin{array}{l}3.779 \mathrm{E}-05 \\
\text { (feed conc.) }\end{array}$ & $3.694 \mathrm{E}-05$ & $8.415 \mathrm{E}-02$ & $8.414 \mathrm{E}-02$ & -0.0081 & $5.722 \mathrm{E}-02$ \\
\hline & & & root mean square $=$ & 0.0132 & \\
\hline
\end{tabular}

${ }^{\text {a }}$ At a $5.0 \mathrm{M}$ sodium concentration level the solution density is estimated to be $1.237 \mathrm{~g} / \mathrm{ml}$ and at $0.04393 \mathrm{M}\left[\mathrm{K}^{+}\right]$.

${ }^{\mathrm{b}}$ Final cesium equilibrium concentrations are only slightly less than initial values due to large phase ratio used.

c To estimate isotherm for engineered form a dilution factor of 0.68 is employed.

Table B-39. ZAM equilibrium model versus algebraic model cesium loading predictions for CST material in contact with Envelope C's LAW-7 feed solution at $5 \mathrm{M}\left[\mathrm{Na}^{+}\right]^{\mathrm{a}}{ }^{\mathrm{a}}$

\begin{tabular}{|c|c|c|c|c|c|}
\hline \multirow[b]{2}{*}{ Cs (initial) } & \multirow[b]{2}{*}{ Cs (final) ${ }^{b}$} & \multicolumn{3}{|c|}{ Powder form } & \multirow{2}{*}{$\begin{array}{c}\text { Engineered form } \\
\text { Cs loading } \\
\text { (Algebraic model) }\end{array}$} \\
\hline & & $\begin{array}{l}\text { Cs loading } \\
\text { (ZAM model) }\end{array}$ & $\begin{array}{c}\text { Cs loading } \\
\text { (Algebraic model) }\end{array}$ & Loading difference & \\
\hline$[\mathbf{M}]$ & {$[\mathbf{M}]$} & [mmole/g] & [mmole/g] & {$[\%]$} & [mmole/g] \\
\hline 0 & $0.000 \mathrm{E}+00$ & $0.000 \mathrm{E}+00$ & $0.000 \mathrm{E}+00$ & $\mathrm{Na}$ & $0.000 \mathrm{E}+00$ \\
\hline 0.000001 & $9.709 \mathrm{E}-07$ & $2.910 \mathrm{E}-03$ & $2.909 \mathrm{E}-03$ & -0.0124 & $1.978 \mathrm{E}-03$ \\
\hline 0.00001 & $9.721 \mathrm{E}-06$ & $2.787 \mathrm{E}-02$ & $2.787 \mathrm{E}-02$ & 0.0005 & $1.895 \mathrm{E}-02$ \\
\hline 0.00005 & $4.883 \mathrm{E}-05$ & $1.173 \mathrm{E}-01$ & $1.173 \mathrm{E}-01$ & -0.0187 & $7.978 \mathrm{E}-02$ \\
\hline 0.0001 & $9.804 \mathrm{E}-05$ & $1.957 \mathrm{E}-01$ & $1.957 \mathrm{E}-01$ & -0.0134 & $1.330 \mathrm{E}-01$ \\
\hline 0.0005 & $4.958 \mathrm{E}-04$ & $4.177 \mathrm{E}-01$ & $4.177 \mathrm{E}-01$ & 0.0069 & $2.841 \mathrm{E}-01$ \\
\hline 0.001 & $9.951 \mathrm{E}-04$ & $4.859 \mathrm{E}-01$ & $4.860 \mathrm{E}-01$ & 0.0096 & $3.304 \mathrm{E}-01$ \\
\hline 0.002 & $1.995 \mathrm{E}-03$ & $5.289 \mathrm{E}-01$ & $5.289 \mathrm{E}-01$ & 0.0125 & $3.597 \mathrm{E}-01$ \\
\hline 0.003 & $2.995 \mathrm{E}-03$ & $5.451 \mathrm{E}-01$ & $5.450 \mathrm{E}-01$ & -0.0241 & $3.706 \mathrm{E}-01$ \\
\hline 0.005 & $4.994 \mathrm{E}-03$ & $5.583 \mathrm{E}-01$ & $5.585 \mathrm{E}-01$ & 0.0242 & $3.798 \mathrm{E}-01$ \\
\hline 0.01 & 9.994E-03 & $5.690 \mathrm{E}-01$ & $5.690 \mathrm{E}-01$ & 0.0135 & $3.869 \mathrm{E}-01$ \\
\hline 0.03 & $2.999 \mathrm{E}-02$ & $5.761 \mathrm{E}-01$ & $5.763 \mathrm{E}-01$ & 0.0332 & $3.919 \mathrm{E}-01$ \\
\hline 0.05 & $4.999 \mathrm{E}-02$ & $5.779 \mathrm{E}-01$ & $5.778 \mathrm{E}-01$ & -0.0191 & $3.929 \mathrm{E}-01$ \\
\hline 0.1 & $9.999 \mathrm{E}-02$ & $5.788 \mathrm{E}-01$ & $5.789 \mathrm{E}-01$ & 0.0074 & $3.936 \mathrm{E}-01$ \\
\hline $\begin{array}{l}4.455 \mathrm{E}-05 \\
\text { (feed conc.) }\end{array}$ & 4.348E-05 & $1.068 \mathrm{E}-01$ & $1.068 \mathrm{E}-01$ & 0.0001 & $7.264 \mathrm{E}-02$ \\
\hline & & & root mean square $=$ & 0.0166 & \\
\hline
\end{tabular}

${ }^{a}$ At a $5.0 \mathrm{M}$ sodium concentration level the solution density is estimated to be $1.243 \mathrm{~g} / \mathrm{ml}$ and at $0.02227 \mathrm{M}\left[\mathrm{K}^{+}\right]$.

${ }^{\mathrm{b}}$ Final cesium equilibrium concentrations are only slightly less than initial values due to large phase ratio used.

c To estimate isotherm for engineered form a dilution factor of 0.68 is employed. 
Table B-40. Cesium isotherm (engineered-form) sensitivity results based on the ZAM model for deviations about the nominal settings for the Phase $1 \mathrm{LAW}-15$ feed solution at $5.0 \mathrm{M} \mathrm{Na}^{+}, 25 \mathrm{C}$, and $4.552 \times 10^{-5} \mathrm{M} \mathrm{Cs}^{+}(100 \%$ of feed value $)$.

\begin{tabular}{|c|c|c|c|c|c|c|}
\hline $\begin{array}{l}\text { Sensitivity } \\
\text { Case }\end{array}$ & $\begin{array}{l}\text { Variable } \\
\text { change } \\
(\%)\end{array}$ & $\begin{array}{c}\text { Ionic } \\
\text { Strength } \\
\text { (gmole/kg) }\end{array}$ & $\begin{array}{l}\text { Cs Kd } \\
(\mathrm{ml} / \mathrm{g})\end{array}$ & $\begin{array}{l}\text { Cs loading } \\
(\mathrm{mmole} / \mathrm{g})\end{array}$ & $\begin{array}{l}\text { Cs loading } \\
\text { change } \\
(\%)\end{array}$ & Comments \\
\hline Nominal & $0 \%$ & 6.49 & 917 & $4.121 \mathrm{E}-02$ & 0.0 & Nominal settings at $5.0 \mathrm{M} \mathrm{Na}^{+}$and $25 \mathrm{C}$. \\
\hline $\begin{array}{c}\text { Dilution } \\
\text { factor }\end{array}$ & $-15 \%$ & 6.49 & 780 & $3.503 \mathrm{E}-02$ & -15.0 & $\begin{array}{l}\text { Dilution factor (engineered to powder capacity ratio) } \\
\text { was reduced to } 0.578 \text {. }\end{array}$ \\
\hline $\mathbf{H}^{+}$ & no impact & 6.49 & 917 & 4.121E-02 & 0.0 & $\begin{array}{l}\text { Has no impact since hydroxyl ion is used to set solution } \\
\mathrm{pH} \text { level in ZAM model directly. }\end{array}$ \\
\hline Density & $-3.0 \%$ & 6.77 & 906 & $4.069 \mathrm{E}-02$ & -1.3 & Liquid-phase density was decreased by $3 \%$. \\
\hline Temperature & $+1.7 \%$ & 6.49 & 838 & $3.770 \mathrm{E}-02$ & -8.5 & Temperature was increased by $5 \mathrm{C}$. \\
\hline $\mathbf{K}^{+}$ & $+50 \%$ & 6.74 & 874 & $3.927 \mathrm{E}-02$ & -5.8 & $\begin{array}{l}\text { Potassium was increased by } 50 \% \text { and } \mathrm{OH}^{-} \text {adjusted } \\
\text { appropriately. Compensating effects minimize its } \\
\text { overall impact. Extrapolated value where no } \mathrm{KNO}_{3} \text { is } \\
\text { allowed to precipitate out of solution. }\end{array}$ \\
\hline $\mathbf{N a}^{+}$ & $+20.0 \%$ & 7.99 & 800 & $3.601 \mathrm{E}-02$ & -12.6 & $\begin{array}{l}\text { Sodium was increased by } 20 \% \text { and } \mathrm{OH}^{-} \text {adjusted } \\
\text { appropriately.. }\end{array}$ \\
\hline $\mathrm{NO}_{2}^{-}$ & $+50 \%$ & 6.60 & 896 & $4.024 \mathrm{E}-02$ & -2.4 & $\begin{array}{l}\text { Nitrite was increased by } 50 \% \text { and } \mathrm{OH}^{-} \text {adjusted } \\
\text { appropriately. }\end{array}$ \\
\hline $\mathrm{NO}_{3}^{-}$ & $+50 \%$ & 6.71 & 782 & $3.519 \mathrm{E}-02$ & -14.6 & $\begin{array}{l}\text { Nitrate was increased by } 50 \% \text { and } \mathrm{OH}^{-} \text {adjusted } \\
\text { appropriately. }\end{array}$ \\
\hline $\mathrm{Al}(\mathrm{OH})_{4}^{-}$ & $+50 \%$ & 6.63 & 890 & $3.999 \mathrm{E}-02$ & -2.9 & $\begin{array}{l}\text { Aluminate was increased by } 50 \% \text { and } \mathrm{OH}^{-} \text {adjusted } \\
\text { appropriately. All Al within the feed inventory is } \\
\text { assumed to be converted into the } \mathrm{Al}(\mathrm{OH})_{4}^{-} \text {species. The } \\
\text { total amount of } \mathrm{OH}^{-} \text {inventory is reduced by the } \\
\text { appropriate amount for this reaction. }\end{array}$ \\
\hline $\mathrm{Cl}^{-}$ & $+50 \%$ & 6.49 & 913 & 4.099E-02 & -0.5 & $\begin{array}{l}\text { Chloride was increased by } 50 \% \text { and } \mathrm{OH}^{-} \text {adjusted } \\
\text { appropriately. }\end{array}$ \\
\hline $\mathrm{CO}_{3}{ }^{-2}$ & $-50 \%$ & 6.33 & 905 & $4.066 \mathrm{E}-02$ & -1.3 & $\begin{array}{l}\text { Carbonate was decreased by } 50 \% \text { and } \mathrm{OH}^{-} \text {adjusted } \\
\text { appropriately. Carbonate levels increase over time } \\
\text { within a waste tank due to a liquid-phase reaction } \\
\text { between } \mathrm{OH}^{-} \text {and } \mathrm{CO}_{2} \text { (present in the vent gas space). }\end{array}$ \\
\hline $\begin{array}{c}\mathrm{CrO}_{4}^{-2} \\
\quad \text { or } \\
\mathrm{Cr}(\mathrm{OH})_{4}^{-}\end{array}$ & $+50 \%$ & 8.10 & 886 & $3.982 \mathrm{E}-02$ & -3.4 & $\begin{array}{l}\text { Chromate was increased by } 50 \% \text { and } \mathrm{OH}^{-} \text {adjusted } \\
\text { appropriately. The chromate } \mathrm{CrO}_{4}^{-2} \text { anion has } \\
\text { chromium(VI), while for the } \mathrm{Cr}(\mathrm{OH})_{4}^{-} \text {anion has } \\
\text { chromium(III). The impact of total } \mathrm{Cr} \text { using ZAM can } \\
\text { only be checked using the chromate anion since ZAM } \\
\text { currently only handles the chromate species in the } \\
\text { aqueous solution. The total amount of } \mathrm{OH}^{-} \text {inventory is } \\
\text { reduced by the appropriate amount for these reactions. }\end{array}$ \\
\hline $\mathbf{O H}^{-}$ & varies & - & - & - & - & $\begin{array}{l}\text { No explicit sensitivity results are listed for the hydroxyl } \\
\text { ion since its impact is accounted for automatically for } \\
\text { in the other results (i.e., } \mathrm{OH}^{-} \text {is varied to maintain } \\
\text { charge balance). }\end{array}$ \\
\hline \multicolumn{5}{|c|}{$\begin{array}{l}\text { Overall error estimate at } 100 \% \text { cesium feed concentration } \\
\qquad(\%)\end{array}$} & $27 \%$ & $\begin{array}{l}\text { Represents an overall error estimate in the cesium } \\
\text { loading curve due to the various uncertainties listed } \\
\text { above. }\end{array}$ \\
\hline
\end{tabular}


WESTINGHOUSE SAVANNAH RIVER COMPANY

Report:

WSRC-TR-2000-00???

Preliminary Ion Exchange Modeling for Removal of Cesium from

Revision (Date):

$0(07 / 05 / 02)$

Hanford Waste Using Crystalline Silicotitanate Material

Page:

204 of 338

Table B-41. Cesium isotherm (engineered-form) sensitivity results based on the ZAM model for deviations about the nominal settings for the Phase $1 \mathrm{LAW}-15$ feed solution at $5.0 \mathrm{M} \mathrm{Na}^{+}, 25 \mathrm{C}$, and $2.276 \times 10^{-5} \mathrm{M} \mathrm{Cs}^{+}(50 \%$ of feed value).

\begin{tabular}{|c|c|c|c|c|c|c|}
\hline $\begin{array}{l}\text { Sensitivity } \\
\text { Case }\end{array}$ & $\begin{array}{l}\text { Variable } \\
\text { change } \\
(\%)\end{array}$ & $\begin{array}{c}\text { Ionic } \\
\text { Strength } \\
\text { (gmole/kg) }\end{array}$ & $\begin{array}{l}\text { Cs Kd } \\
(\mathrm{ml} / \mathrm{g})\end{array}$ & $\begin{array}{l}\text { Cs loading } \\
(\mathrm{mmole} / \mathrm{g})\end{array}$ & $\begin{array}{c}\text { Cs loading } \\
\text { change } \\
(\%)\end{array}$ & Comments \\
\hline Nominal & $0 \%$ & 6.49 & 968 & $2.171 \mathrm{E}-02$ & 0.0 & Nominal settings at $5.0 \mathrm{M} \mathrm{Na}^{+}$and $25 \mathrm{C}$. \\
\hline $\begin{array}{l}\text { Dilution } \\
\text { factor }\end{array}$ & $-15 \%$ & 6.49 & 822 & $1.846 \mathrm{E}-02$ & -15.0 & $\begin{array}{l}\text { Dilution factor (engineered to powder capacity ratio) } \\
\text { was reduced to } 0.578 \text {. }\end{array}$ \\
\hline $\mathbf{H}^{+}$ & no impact & 6.49 & 968 & $2.171 \mathrm{E}-02$ & 0.0 & $\begin{array}{l}\text { Has no impact since hydroxyl ion is used to set solution } \\
\mathrm{pH} \text { level in ZAM model directly. }\end{array}$ \\
\hline Density & $-3.0 \%$ & 6.77 & 955 & $2.143 \mathrm{E}-02$ & -1.3 & Liquid-phase density was decreased by $3 \%$. \\
\hline Temperature & $+1.7 \%$ & 6.49 & 881 & $1.979 \mathrm{E}-02$ & -8.9 & Temperature was increased by $5 \mathrm{C}$. \\
\hline $\mathbf{K}^{+}$ & $+50 \%$ & 6.74 & 920 & $2.066 \mathrm{E}-02$ & -6.0 & $\begin{array}{l}\text { Potassium was increased by } 50 \% \text { and } \mathrm{OH}^{-} \text {adjusted } \\
\text { appropriately. Compensating effects minimize its } \\
\text { overall impact. Extrapolated value where no } \mathrm{KNO}_{3} \text { is } \\
\text { allowed to precipitate out of solution. }\end{array}$ \\
\hline $\mathrm{Na}^{+}$ & $+20.0 \%$ & 7.99 & 839 & $1.886 \mathrm{E}-02$ & -13.1 & $\begin{array}{l}\text { Sodium was increased by } 20 \% \text { and } \mathrm{OH}^{-} \text {adjusted } \\
\text { appropriately.. }\end{array}$ \\
\hline $\mathrm{NO}_{2}^{-}$ & $+50 \%$ & 6.60 & 944 & $2.119 \mathrm{E}-02$ & -2.4 & $\begin{array}{l}\text { Nitrite was increased by } 50 \% \text { and } \mathrm{OH}^{-} \text {adjusted } \\
\text { appropriately. }\end{array}$ \\
\hline $\mathrm{NO}_{3}^{-}$ & $+50 \%$ & 6.71 & 819 & $1.841 \mathrm{E}-02$ & -15.2 & $\begin{array}{l}\text { Nitrate was increased by } 50 \% \text { and } \mathrm{OH}^{-} \text {adjusted } \\
\text { appropriately. }\end{array}$ \\
\hline $\mathrm{Al}(\mathrm{OH})_{4}{ }^{-}$ & $+50 \%$ & 6.63 & 938 & $2.105 \mathrm{E}-02$ & -3.0 & $\begin{array}{l}\text { Aluminate was increased by } 50 \% \text { and } \mathrm{OH}^{-} \text {adjusted } \\
\text { appropriately. All } \mathrm{Al} \text { within the feed inventory is } \\
\text { assumed to be converted into the } \mathrm{Al}(\mathrm{OH})_{4}^{-} \text {species. The } \\
\text { total amount of } \mathrm{OH}^{-} \text {inventory is reduced by the } \\
\text { appropriate amount for this reaction. }\end{array}$ \\
\hline $\mathrm{Cl}^{-}$ & $+50 \%$ & 6.49 & 963 & $2.161 \mathrm{E}-02$ & -0.5 & $\begin{array}{l}\text { Chloride was increased by } 50 \% \text { and } \mathrm{OH}^{-} \text {adjusted } \\
\text { appropriately. }\end{array}$ \\
\hline $\mathrm{CO}_{3}^{-2}$ & $-50 \%$ & 6.33 & 955 & $2.143 \mathrm{E}-02$ & -1.3 & $\begin{array}{l}\text { Carbonate was decreased by } 50 \% \text { and } \mathrm{OH}^{-} \text {adjusted } \\
\text { appropriately. Carbonate levels increase over time } \\
\text { within a waste tank due to a liquid-phase reaction } \\
\text { between } \mathrm{OH}^{-} \text {and } \mathrm{CO}_{2} \text { (present in the vent gas space). }\end{array}$ \\
\hline $\begin{array}{l}\mathrm{CrO}_{4}^{-2} \\
\quad \text { or } \\
\mathrm{Cr}(\mathrm{OH})_{4}^{-}\end{array}$ & $+50 \%$ & 8.10 & 933 & $2.094 \mathrm{E}-02$ & -3.5 & $\begin{array}{l}\text { Chromate was increased by } 50 \% \text { and } \mathrm{OH}^{-} \text {adjusted } \\
\text { appropriately. The chromate } \mathrm{CrO}_{4}^{-2} \text { anion has } \\
\text { chromium(VI), while for the } \mathrm{Cr}(\mathrm{OH})_{4}^{-} \text {anion has } \\
\text { chromium(III). The impact of total } \mathrm{Cr} \text { using ZAM can } \\
\text { only be checked using the chromate anion since ZAM } \\
\text { currently only handles the chromate species in the } \\
\text { aqueous solution. The total amount of } \mathrm{OH}^{-} \text {inventory is } \\
\text { reduced by the appropriate amount for these reactions. }\end{array}$ \\
\hline $\mathrm{OH}^{-}$ & varies & - & - & - & - & $\begin{array}{l}\text { No explicit sensitivity results are listed for the hydroxyl } \\
\text { ion since its impact is accounted for automatically for } \\
\text { in the other results (i.e., } \mathrm{OH}^{-} \text {is varied to maintain } \\
\text { charge balance). }\end{array}$ \\
\hline \multicolumn{5}{|c|}{$\begin{array}{l}\text { Overall error estimate at } 50 \% \text { of cesium feed concentration } \\
\qquad(\%)\end{array}$} & $28 \%$ & $\begin{array}{l}\text { Represents an overall error estimate in the cesium } \\
\text { loading curve due to the various uncertainties listed } \\
\text { above. }\end{array}$ \\
\hline
\end{tabular}


Table B-42. Error estimates associated with feed compositions for the key species of interest.

\begin{tabular}{|c|c|c|c|c|c|c|c|c|c|c|c|c|c|}
\hline \multicolumn{5}{|c|}{ AN-103 (Env-A [Hay et al., 2000a]; LAW-12 and 13) } & \multicolumn{4}{|c|}{$\begin{array}{c}\text { AZ-102 (Env-B [Hay and Bronikowski, 2000]; } \\
\text { LAW-2b) }\end{array}$} & \multicolumn{4}{|c|}{$\begin{array}{c}\text { AN-102 (Env-A [Hay et al., 2000b]; LAW-3 and } \\
\text { 4) }\end{array}$} & \multirow{2}{*}{$\begin{array}{c}\text { Summary } \\
\text { rms } \\
\text { difference }\end{array}$} \\
\hline & data & $\begin{array}{c}\text { LAW-12 } \\
\text { feed }\end{array}$ & $\begin{array}{l}\text { Adjusted } \\
\text { data }\end{array}$ & Difference & Data & $\begin{array}{l}\text { LAW-2b } \\
\text { feed }\end{array}$ & $\begin{array}{l}\text { Adjusted } \\
\text { data }\end{array}$ & Difference & data & $\begin{array}{c}\text { LAW-3 } \\
\text { feed }\end{array}$ & $\begin{array}{l}\text { Adjusted } \\
\text { data }\end{array}$ & Difference & \\
\hline \multirow[t]{2}{*}{ Species } & {$[\mathbf{M}]$} & {$[\mathbf{M}]$} & {$[\mathbf{M}]$} & $(\%)$ & {$[\mathbf{M}]$} & {$[\mathbf{M}]$} & [M] & $(\%)$ & {$[\mathbf{M}]$} & {$[\mathbf{M}]$} & {$[\mathbf{M}]$} & $(\%)$ & $(\%)$ \\
\hline & - & factor $=$ & 1.29 & - & - & factor $=$ & 8.53 & - & - & factor $=$ & 1.40 & - & - \\
\hline $\mathrm{Na}^{+}$ & 5.25 & $6.76 \mathrm{E}+00$ & $6.76 \mathrm{E}+00$ & 0.0 & 2.77 & $2.36 \mathrm{E}+00$ & $2.36 \mathrm{E}+00$ & 0.0 & 6.42 & $8.97 \mathrm{E}+00$ & $8.97 \mathrm{E}+00$ & 0.0 & 0.0 \\
\hline $\mathrm{K}^{+}$ & $1.21 \mathrm{E}-01$ & $1.67 \mathrm{E}-01$ & $1.56 \mathrm{E}-01$ & 7.2 & 8.07E-02 & 7.92E-02 & $6.88 \mathrm{E}-02$ & 15.1 & $3.52 \mathrm{E}-02$ & 7.95E-02 & 4.92E-02 & 61.5 & 36.8 \\
\hline Total Cr & $1.41 \mathrm{E}-03$ & 4.87E-03 & $1.82 \mathrm{E}-03$ & 168.2 & $1.48 \mathrm{E}-02$ & $2.17 \mathrm{E}-05$ & $1.26 \mathrm{E}-02$ & -99.8 & $3.06 \mathrm{E}-03$ & $6.54 \mathrm{E}-03$ & $4.28 \mathrm{E}-03$ & 52.9 & 117.0 \\
\hline Total Al & $6.98 \mathrm{E}-01$ & $1.01 \mathrm{E}+00$ & 8.99E-01 & 12.8 & $2.79 \mathrm{E}-02$ & $5.52 \mathrm{E}-02$ & $2.38 \mathrm{E}-02$ & 132.1 & $3.42 \mathrm{E}-01$ & 4.66E-01 & $4.78 \mathrm{E}-01$ & -2.6 & 76.7 \\
\hline $\mathrm{NO}_{3}^{-}$ & $1.57 \mathrm{E}+00$ & $1.65 \mathrm{E}+00$ & $2.02 \mathrm{E}+00$ & -18.2 & $2.73 \mathrm{E}-01$ & $3.73 \mathrm{E}-01$ & $2.33 \mathrm{E}-01$ & 60.3 & $1.94 \mathrm{E}+00$ & $2.92 \mathrm{E}+00$ & $2.71 \mathrm{E}+00$ & 7.6 & 36.7 \\
\hline $\mathrm{NO}_{2}^{-}$ & $1.04 \mathrm{E}+00$ & $1.52 \mathrm{E}+00$ & $1.34 \mathrm{E}+00$ & 13.8 & $6.59 \mathrm{E}-01$ & $6.55 \mathrm{E}-01$ & $5.62 \mathrm{E}-01$ & 16.5 & $1.13 \mathrm{E}+00$ & $1.44 \mathrm{E}+00$ & $1.58 \mathrm{E}+00$ & -8.8 & 13.4 \\
\hline $\mathrm{CO}_{3}^{-}$ & $2.55 \mathrm{E}-01$ & $2.18 \mathrm{E}-01$ & $3.28 \mathrm{E}-01$ & -33.5 & 3.92E-01 & 5.73E-01 & 3.34E-01 & 71.6 & 4.89E-01 & $9.23 \mathrm{E}-01$ & $6.84 \mathrm{E}-01$ & 35.0 & 49.9 \\
\hline $\mathrm{Cl}^{-}$ & $6.15 \mathrm{E}-02$ & $1.17 \mathrm{E}-01$ & 7.92E-02 & 47.7 & $1.41 \mathrm{E}-03$ & 2.62E-04 & $1.20 \mathrm{E}-03$ & -78.2 & $6.76 \mathrm{E}-02$ & $8.68 \mathrm{E}-02$ & $9.45 \mathrm{E}-02$ & -8.1 & 53.1 \\
\hline $\mathrm{SO}_{4}^{-2}$ & 7.73E-03 & $1.54 \mathrm{E}-02$ & $9.96 \mathrm{E}-03$ & 54.5 & $1.72 \mathrm{E}-01$ & $1.87 \mathrm{E}-01$ & $1.47 \mathrm{E}-01$ & 27.3 & $8.48 \mathrm{E}-02$ & $1.32 \mathrm{E}-01$ & $1.19 \mathrm{E}-01$ & 11.0 & 35.8 \\
\hline Total $\mathrm{Sr}^{\mathrm{b}}$ & $2.33 \mathrm{E}-06$ & $2.18 \mathrm{E}-05$ & $3.00 \mathrm{E}-06$ & 626.4 & $2.85 \mathrm{E}-06$ & $2.13 \mathrm{E}-06$ & $2.43 \mathrm{E}-06$ & -12.3 & $2.04 \mathrm{E}-05$ & $3.85 \mathrm{E}-05$ & $2.85 \mathrm{E}-05$ & 35.0 & 362.3 \\
\hline $\mathrm{OH}^{-}$(free) & $2.81 \mathrm{E}+00^{\mathrm{a}}$ & $6.22 \mathrm{E}+00$ & $3.62 \mathrm{E}+00$ & 71.8 & $1.09 \mathrm{E}-01$ & $1.26 \mathrm{E}-01$ & $9.29 \mathrm{E}-02$ & 35.2 & $8.17 \mathrm{E}-01$ & $2.83 \mathrm{E}+00$ & $1.14 \mathrm{E}+00$ & 147.7 & 97.0 \\
\hline $\mathrm{OH}^{-}$(bound) & - & 3.32E-01 & $3.60 \mathrm{E}+00$ & -90.8 & - & $1.40 \mathrm{E}-02$ & $1.46 \mathrm{E}-01$ & -90.4 & - & $3.80 \mathrm{E}-01$ & $1.93 \mathrm{E}+00$ & -80.3 & 87.3 \\
\hline $\mathrm{OH}^{-}$(total) & - & $6.55 \mathrm{E}+00$ & $7.22 \mathrm{E}+00$ & -9.3 & - & $1.40 \mathrm{E}-01$ & 2.39E-01 & -41.5 & - & $3.21 \mathrm{E}+00$ & $3.07 \mathrm{E}+00$ & 4.5 & 24.7 \\
\hline
\end{tabular}

a For $\mathrm{AN}-103$ data the $\mathrm{OH}^{-}$(free) is based on a simple dilution factor and not the data since significant dissolution of solids occurred during dilution process.

${ }^{\mathrm{b}} \mathrm{CST}$ has a strong affinity for $\mathrm{SrOH}^{+}$; however, the actual amount of total $\mathrm{Sr}$ available in solution to form $\mathrm{SrOH}^{+}$is currently believed to be small but is unknown at this time. In this report the Sr concentration has been set to zero. Future work will need to address this issue.

Table B-43. Phase 1 LAW-15 feed solution data used as input to the CST equilibrium model sensitivity study.

\begin{tabular}{|c|c|c|c|c|c|c|c|c|c|c|c|c|}
\hline Species & Sensitivity Case & Nominal & $\mathrm{Al}(\mathrm{OH})_{4}^{-}$ & $\mathrm{CrO}_{4}^{-2}$ & pH & $\mathrm{NO}_{3}^{-}$ & $\mathrm{NO}_{2}^{-}$ & $\mathrm{CO}_{3}^{-2}$ & $\mathrm{Cl}^{-}$ & $\mathbf{K}^{+}$ & $\mathrm{SrOH}^{-}$ & $\mathrm{Na}^{+}$ \\
\hline Charge & Concentration Units & {$[\mathbf{M}]$} & {$[\mathbf{M}]$} & {$[\mathbf{M}]$} & {$[\mathbf{M}]$} & {$[\mathbf{M}]$} & {$[\mathbf{M}]$} & {$[\mathbf{M}]$} & [M] & {$[\mathbf{M}]$} & {$[\mathbf{M}]$} & {$[\mathbf{M}]$} \\
\hline \multicolumn{13}{|l|}{ Cations } \\
\hline 1 & $\mathrm{Na}$ & $5.00 \mathrm{E}+00$ & $5.00 \mathrm{E}+00$ & $5.00 \mathrm{E}+00$ & $5.00 \mathrm{E}+00$ & $5.00 \mathrm{E}+00$ & $5.00 \mathrm{E}+00$ & $5.00 \mathrm{E}+00$ & $5.00 \mathrm{E}+00$ & $5.00 \mathrm{E}+00$ & $5.00 \mathrm{E}+00$ & $6.00 \mathrm{E}+00$ \\
\hline 1 & Cs (total) & $4.55 \mathrm{E}-05$ & $4.55 \mathrm{E}-05$ & $4.55 \mathrm{E}-05$ & $4.55 \mathrm{E}-05$ & $4.55 \mathrm{E}-05$ & $4.55 \mathrm{E}-05$ & $4.55 \mathrm{E}-05$ & $4.55 \mathrm{E}-05$ & $4.55 \mathrm{E}-05$ & $4.55 \mathrm{E}-05$ & $4.55 \mathrm{E}-05$ \\
\hline
\end{tabular}




\begin{tabular}{|c|c|c|c|c|c|c|c|c|c|c|c|c|}
\hline Species & Sensitivity Case & Nominal & $\mathrm{Al}(\mathrm{OH})_{4}^{-}$ & $\mathrm{CrO}_{4}^{-2}$ & pH & $\mathrm{NO}_{3}^{-}$ & $\mathrm{NO}_{2}^{-}$ & $\mathrm{CO}_{3}^{-2}$ & $\mathrm{Cl}^{-}$ & $\mathbf{K}^{+}$ & $\mathrm{SrOH}^{-}$ & $\mathrm{Na}^{+}$ \\
\hline Charge & Concentration Units & {$[\mathbf{M}]$} & {$[\mathbf{M}]$} & {$[\mathbf{M}]$} & {$[\mathbf{M}]$} & [M] & {$[\mathbf{M}]$} & {$[\mathbf{M}]$} & [M] & [M] & [M] & {$[\mathbf{M}]$} \\
\hline 1 & $\mathrm{H}^{+}$ & $1.07 \mathrm{E}-15$ & $1.07 \mathrm{E}-15$ & $1.07 \mathrm{E}-15$ & $4.67 \mathrm{E}-15$ & $1.07 \mathrm{E}-15$ & $1.07 \mathrm{E}-15$ & $1.07 \mathrm{E}-15$ & $1.07 \mathrm{E}-15$ & $1.07 \mathrm{E}-15$ & $1.07 \mathrm{E}-15$ & $1.07 \mathrm{E}-15$ \\
\hline 1 & $\mathrm{Rb}$ & $0.00 \mathrm{E}+00$ & $0.00 \mathrm{E}+00$ & $0.00 \mathrm{E}+00$ & $0.00 \mathrm{E}+00$ & $0.00 \mathrm{E}+00$ & $0.00 \mathrm{E}+00$ & $0.00 \mathrm{E}+00$ & $0.00 \mathrm{E}+00$ & $0.00 \mathrm{E}+00$ & $0.00 \mathrm{E}+00$ & $0.00 \mathrm{E}+00$ \\
\hline 1 & $\mathrm{~K}$ & $4.07 \mathrm{E}-01$ & 4.07E-01 & $4.07 \mathrm{E}-01$ & $4.07 \mathrm{E}-01$ & $4.07 \mathrm{E}-01$ & $4.07 \mathrm{E}-01$ & $4.07 \mathrm{E}-01$ & $4.07 \mathrm{E}-01$ & $6.10 \mathrm{E}-01$ & $4.07 \mathrm{E}-01$ & $4.07 \mathrm{E}-01$ \\
\hline 1 & $\mathrm{SrOH}$ & $0.00 \mathrm{E}+00$ & $0.00 \mathrm{E}+00$ & $0.00 \mathrm{E}+00$ & $0.00 \mathrm{E}+00$ & $0.00 \mathrm{E}+00$ & $0.00 \mathrm{E}+00$ & $0.00 \mathrm{E}+00$ & $0.00 \mathrm{E}+00$ & $0.00 \mathrm{E}+00$ & $0.00 \mathrm{E}+00$ & $0.00 \mathrm{E}+00$ \\
\hline 2 & $\mathrm{Sr}$ & $0.00 \mathrm{E}+00$ & $0.00 \mathrm{E}+00$ & $0.00 \mathrm{E}+00$ & $0.00 \mathrm{E}+00$ & $0.00 \mathrm{E}+00$ & $0.00 \mathrm{E}+00$ & $0.00 \mathrm{E}+00$ & $0.00 \mathrm{E}+00$ & $0.00 \mathrm{E}+00$ & $2.28 \mathrm{E}-05$ & $0.00 \mathrm{E}+00$ \\
\hline 2 & $\mathrm{Ba}$ & $0.00 \mathrm{E}+00$ & $0.00 \mathrm{E}+00$ & $0.00 \mathrm{E}+00$ & $0.00 \mathrm{E}+00$ & $0.00 \mathrm{E}+00$ & $0.00 \mathrm{E}+00$ & $0.00 \mathrm{E}+00$ & $0.00 \mathrm{E}+00$ & $0.00 \mathrm{E}+00$ & $0.00 \mathrm{E}+00$ & $0.00 \mathrm{E}+00$ \\
\hline 2 & $\mathrm{Ca}$ & $8.60 \mathrm{E}-04$ & $8.60 \mathrm{E}-04$ & $8.60 \mathrm{E}-04$ & 8.60E-04 & $8.60 \mathrm{E}-04$ & $8.60 \mathrm{E}-04$ & $8.60 \mathrm{E}-04$ & $8.60 \mathrm{E}-04$ & $8.60 \mathrm{E}-04$ & $8.60 \mathrm{E}-04$ & $8.60 \mathrm{E}-04$ \\
\hline 2 & $\mathrm{Cd}$ & $0.00 \mathrm{E}+00$ & $0.00 \mathrm{E}+00$ & $0.00 \mathrm{E}+00$ & $0.00 \mathrm{E}+00$ & $0.00 \mathrm{E}+00$ & $0.00 \mathrm{E}+00$ & $0.00 \mathrm{E}+00$ & $0.00 \mathrm{E}+00$ & $0.00 \mathrm{E}+00$ & $0.00 \mathrm{E}+00$ & $0.00 \mathrm{E}+00$ \\
\hline 2 & Mn & $6.03 \mathrm{E}-05$ & $6.03 \mathrm{E}-05$ & $6.03 \mathrm{E}-05$ & $6.03 \mathrm{E}-05$ & $6.03 \mathrm{E}-05$ & $6.03 \mathrm{E}-05$ & $6.03 \mathrm{E}-05$ & $6.03 \mathrm{E}-05$ & $6.03 \mathrm{E}-05$ & $6.03 \mathrm{E}-05$ & $6.03 \mathrm{E}-05$ \\
\hline 2 & $\mathrm{Ni}$ & $1.08 \mathrm{E}-04$ & $1.08 \mathrm{E}-04$ & $1.08 \mathrm{E}-04$ & $1.08 \mathrm{E}-04$ & $1.08 \mathrm{E}-04$ & $1.08 \mathrm{E}-04$ & $1.08 \mathrm{E}-04$ & $1.08 \mathrm{E}-04$ & $1.08 \mathrm{E}-04$ & $1.08 \mathrm{E}-04$ & $1.08 \mathrm{E}-04$ \\
\hline 2 & $\mathrm{U}$ (total) & $3.96 \mathrm{E}-04$ & $3.96 \mathrm{E}-04$ & $3.96 \mathrm{E}-04$ & $3.96 \mathrm{E}-04$ & $3.96 \mathrm{E}-04$ & $3.96 \mathrm{E}-04$ & $3.96 \mathrm{E}-04$ & $3.96 \mathrm{E}-04$ & $3.96 \mathrm{E}-04$ & $3.96 \mathrm{E}-04$ & $3.96 \mathrm{E}-04$ \\
\hline 2 & $\mathrm{Zn}$ & $0.00 \mathrm{E}+00$ & $0.00 \mathrm{E}+00$ & $0.00 \mathrm{E}+00$ & $0.00 \mathrm{E}+00$ & $0.00 \mathrm{E}+00$ & $0.00 \mathrm{E}+00$ & $0.00 \mathrm{E}+00$ & $0.00 \mathrm{E}+00$ & $0.00 \mathrm{E}+00$ & $0.00 \mathrm{E}+00$ & $0.00 \mathrm{E}+00$ \\
\hline 2 & $\mathrm{Fe}$ & $2.84 \mathrm{E}-04$ & $2.84 \mathrm{E}-04$ & $2.84 \mathrm{E}-04$ & $2.84 \mathrm{E}-04$ & $2.84 \mathrm{E}-04$ & $2.84 \mathrm{E}-04$ & $2.84 \mathrm{E}-04$ & $2.84 \mathrm{E}-04$ & $2.84 \mathrm{E}-04$ & $2.84 \mathrm{E}-04$ & $2.84 \mathrm{E}-04$ \\
\hline 2 & $\mathrm{~Pb}$ & $1.61 \mathrm{E}-04$ & $1.61 \mathrm{E}-04$ & $1.61 \mathrm{E}-04$ & $1.61 \mathrm{E}-04$ & $1.61 \mathrm{E}-04$ & $1.61 \mathrm{E}-04$ & $1.61 \mathrm{E}-04$ & $1.61 \mathrm{E}-04$ & $1.61 \mathrm{E}-04$ & $1.61 \mathrm{E}-04$ & $1.61 \mathrm{E}-04$ \\
\hline 3 & $\mathrm{Cr}$ & $0.00 \mathrm{E}+00$ & $0.00 \mathrm{E}+00$ & $0.00 \mathrm{E}+00$ & $0.00 \mathrm{E}+00$ & $0.00 \mathrm{E}+00$ & $0.00 \mathrm{E}+00$ & $0.00 \mathrm{E}+00$ & $0.00 \mathrm{E}+00$ & $0.00 \mathrm{E}+00$ & $0.00 \mathrm{E}+00$ & $0.00 \mathrm{E}+00$ \\
\hline 3 & $\mathrm{La}$ & $5.80 \mathrm{E}-12$ & $5.80 \mathrm{E}-12$ & $5.80 \mathrm{E}-12$ & $5.80 \mathrm{E}-12$ & $5.80 \mathrm{E}-12$ & $5.80 \mathrm{E}-12$ & $5.80 \mathrm{E}-12$ & $5.80 \mathrm{E}-12$ & $5.80 \mathrm{E}-12$ & $5.80 \mathrm{E}-12$ & $5.80 \mathrm{E}-12$ \\
\hline 3 & $\mathrm{Ce}$ & $0.00 \mathrm{E}+00$ & $0.00 \mathrm{E}+00$ & $0.00 \mathrm{E}+00$ & $0.00 \mathrm{E}+00$ & $0.00 \mathrm{E}+00$ & $0.00 \mathrm{E}+00$ & $0.00 \mathrm{E}+00$ & $0.00 \mathrm{E}+00$ & $0.00 \mathrm{E}+00$ & $0.00 \mathrm{E}+00$ & $0.00 \mathrm{E}+00$ \\
\hline \multicolumn{13}{|l|}{ Anions } \\
\hline-1 & OH- (free) & $2.14 \mathrm{E}+00$ & $1.89 \mathrm{E}+00$ & $1.14 \mathrm{E}+00$ & $2.14 \mathrm{E}+00$ & $1.49 \mathrm{E}+00$ & $1.63 \mathrm{E}+00$ & $2.29 \mathrm{E}+00$ & $2.11 \mathrm{E}+00$ & $2.34 \mathrm{E}+00$ & $2.14 \mathrm{E}+00$ & $3.14 \mathrm{E}+00$ \\
\hline-1 & NO3 & $1.30 \mathrm{E}+00$ & $1.30 \mathrm{E}+00$ & $1.30 \mathrm{E}+00$ & $1.30 \mathrm{E}+00$ & $1.95 \mathrm{E}+00$ & $1.30 \mathrm{E}+00$ & $1.30 \mathrm{E}+00$ & $1.30 \mathrm{E}+00$ & $1.30 \mathrm{E}+00$ & $1.30 \mathrm{E}+00$ & $1.30 \mathrm{E}+00$ \\
\hline-1 & $\mathrm{NO} 2$ & $1.01 \mathrm{E}+00$ & $1.01 \mathrm{E}+00$ & $1.01 \mathrm{E}+00$ & $1.01 \mathrm{E}+00$ & $1.01 \mathrm{E}+00$ & $1.52 \mathrm{E}+00$ & $1.01 \mathrm{E}+00$ & $1.01 \mathrm{E}+00$ & $1.01 \mathrm{E}+00$ & $1.01 \mathrm{E}+00$ & $1.01 \mathrm{E}+00$ \\
\hline-1 & $\mathrm{Cl}$ & $6.98 \mathrm{E}-02$ & $6.98 \mathrm{E}-02$ & $6.98 \mathrm{E}-02$ & $6.98 \mathrm{E}-02$ & $6.98 \mathrm{E}-02$ & $6.98 \mathrm{E}-02$ & $6.98 \mathrm{E}-02$ & $1.05 \mathrm{E}-01$ & $6.98 \mathrm{E}-02$ & $6.98 \mathrm{E}-02$ & $6.98 \mathrm{E}-02$ \\
\hline-1 & F & $3.94 \mathrm{E}-02$ & $3.94 \mathrm{E}-02$ & $3.94 \mathrm{E}-02$ & $3.94 \mathrm{E}-02$ & $3.94 \mathrm{E}-02$ & $3.94 \mathrm{E}-02$ & $3.94 \mathrm{E}-02$ & $3.94 \mathrm{E}-02$ & $3.94 \mathrm{E}-02$ & $3.94 \mathrm{E}-02$ & $3.94 \mathrm{E}-02$ \\
\hline-1 & $\mathrm{Al}(\mathrm{OH}) 4$ & $5.01 \mathrm{E}-01$ & $7.52 \mathrm{E}-01$ & $0.00 \mathrm{E}+00$ & $5.01 \mathrm{E}-01$ & $5.01 \mathrm{E}-01$ & $5.01 \mathrm{E}-01$ & $5.01 \mathrm{E}-01$ & $5.01 \mathrm{E}-01$ & $5.01 \mathrm{E}-01$ & $5.01 \mathrm{E}-01$ & $5.01 \mathrm{E}-01$ \\
\hline-1 & 129-I & $9.14 \mathrm{E}-06$ & $9.14 \mathrm{E}-06$ & $9.14 \mathrm{E}-06$ & $9.14 \mathrm{E}-06$ & $9.14 \mathrm{E}-06$ & $9.14 \mathrm{E}-06$ & $9.14 \mathrm{E}-06$ & $9.14 \mathrm{E}-06$ & $9.14 \mathrm{E}-06$ & $9.14 \mathrm{E}-06$ & $9.14 \mathrm{E}-06$ \\
\hline-2 & $\mathrm{CrO} 4$ & $0.00 \mathrm{E}+00$ & $0.00 \mathrm{E}+00$ & $7.52 \mathrm{E}-01$ & $0.00 \mathrm{E}+00$ & $0.00 \mathrm{E}+00$ & $0.00 \mathrm{E}+00$ & $0.00 \mathrm{E}+00$ & $0.00 \mathrm{E}+00$ & $0.00 \mathrm{E}+00$ & $0.00 \mathrm{E}+00$ & $0.00 \mathrm{E}+00$ \\
\hline-2 & $\mathrm{CO} 3$ & $1.54 \mathrm{E}-01$ & $1.54 \mathrm{E}-01$ & $1.54 \mathrm{E}-01$ & $1.54 \mathrm{E}-01$ & $1.54 \mathrm{E}-01$ & $1.54 \mathrm{E}-01$ & 7.69E-02 & $1.54 \mathrm{E}-01$ & $1.54 \mathrm{E}-01$ & $1.54 \mathrm{E}-01$ & $1.54 \mathrm{E}-01$ \\
\hline-2 & $\mathrm{SO} 4$ & $1.06 \mathrm{E}-02$ & $1.06 \mathrm{E}-02$ & $1.06 \mathrm{E}-02$ & $1.06 \mathrm{E}-02$ & $1.06 \mathrm{E}-02$ & $1.06 \mathrm{E}-02$ & $1.06 \mathrm{E}-02$ & $1.06 \mathrm{E}-02$ & $1.06 \mathrm{E}-02$ & $1.06 \mathrm{E}-02$ & $1.06 \mathrm{E}-02$ \\
\hline \multirow{2}{*}{-3} & cations $=$ & 5.4105 & 5.4105 & 5.4105 & 5.4105 & 5.4105 & 5.4105 & 5.4105 & 5.4105 & 5.6139 & 5.4105 & 6.4105 \\
\hline & anions $=$ & -5.4105 & -5.4105 & -5.4105 & -5.4105 & -5.4105 & -5.4105 & -5.4105 & -5.4105 & -5.6138 & -5.4105 & -6.4105 \\
\hline
\end{tabular}


Hanford Waste Using Crystalline Silicotitanate Material

Page: 


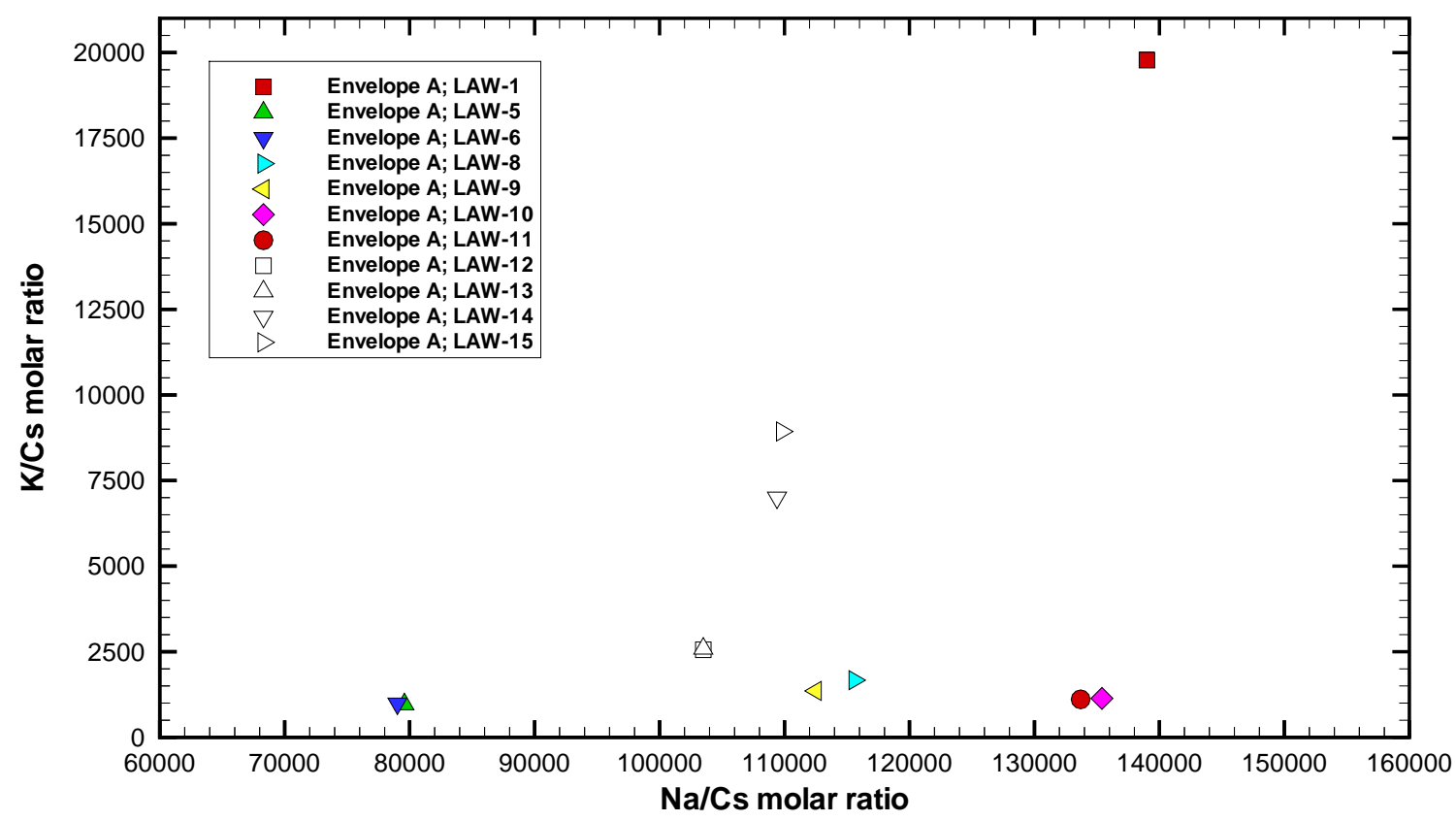

Figure B-1. Molar ratios of $\mathrm{Na} / \mathrm{Cs}$ versus $\mathrm{K} / \mathrm{Cs}$ for the Envelope A candidate feed solutions.

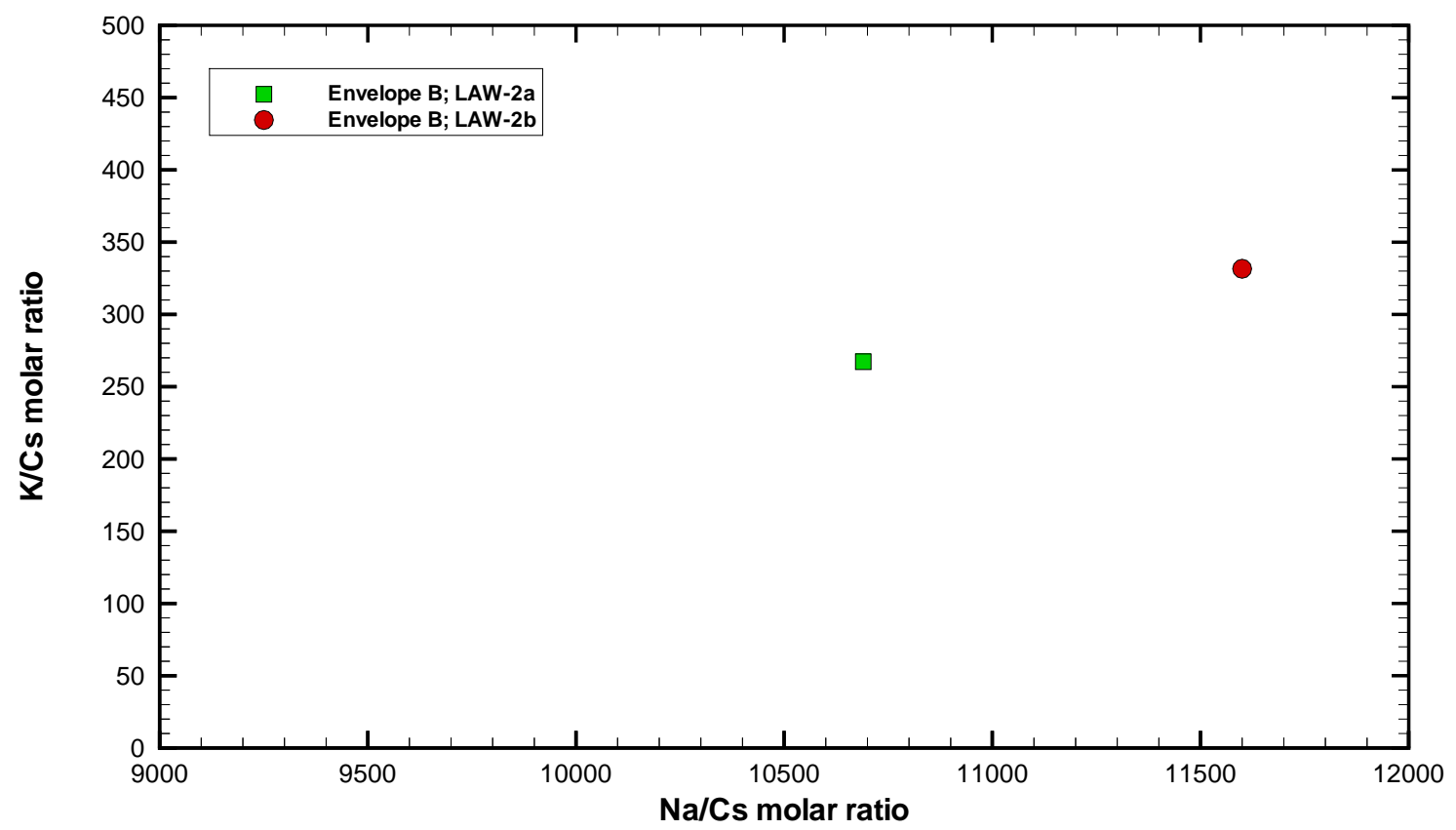

Figure B-2. Molar ratios of $\mathrm{Na} / \mathrm{Cs}$ versus $\mathrm{K} / \mathrm{Cs}$ for the Envelope B candidate feed solutions. 
Preliminary Ion Exchange Modeling for Removal of Cesium from

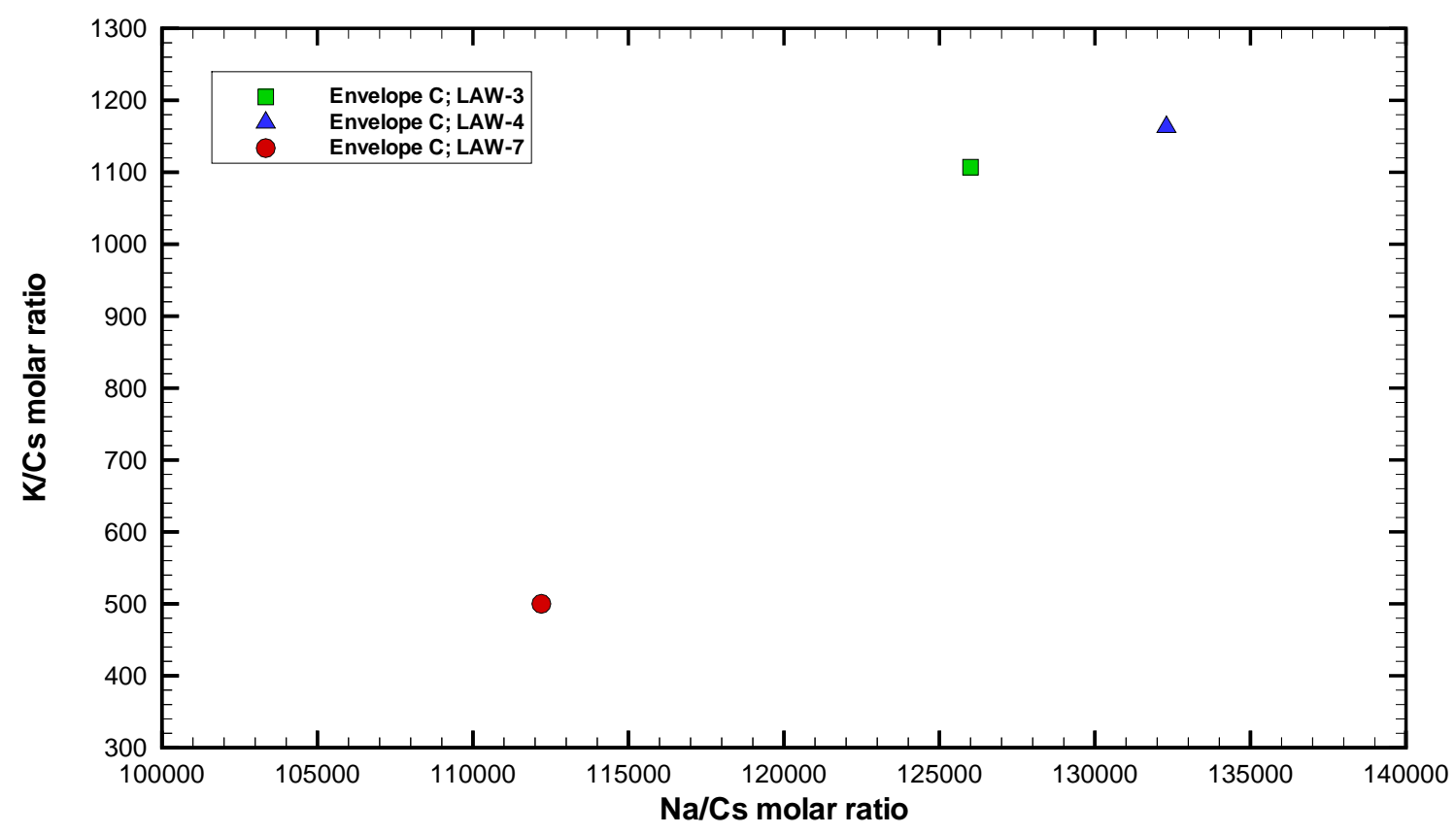

Figure B-3. Molar ratios of $\mathrm{Na} / \mathrm{Cs}$ versus $\mathrm{K} / \mathrm{Cs}$ for the Envelope $\mathrm{C}$ candidate feed solutions.

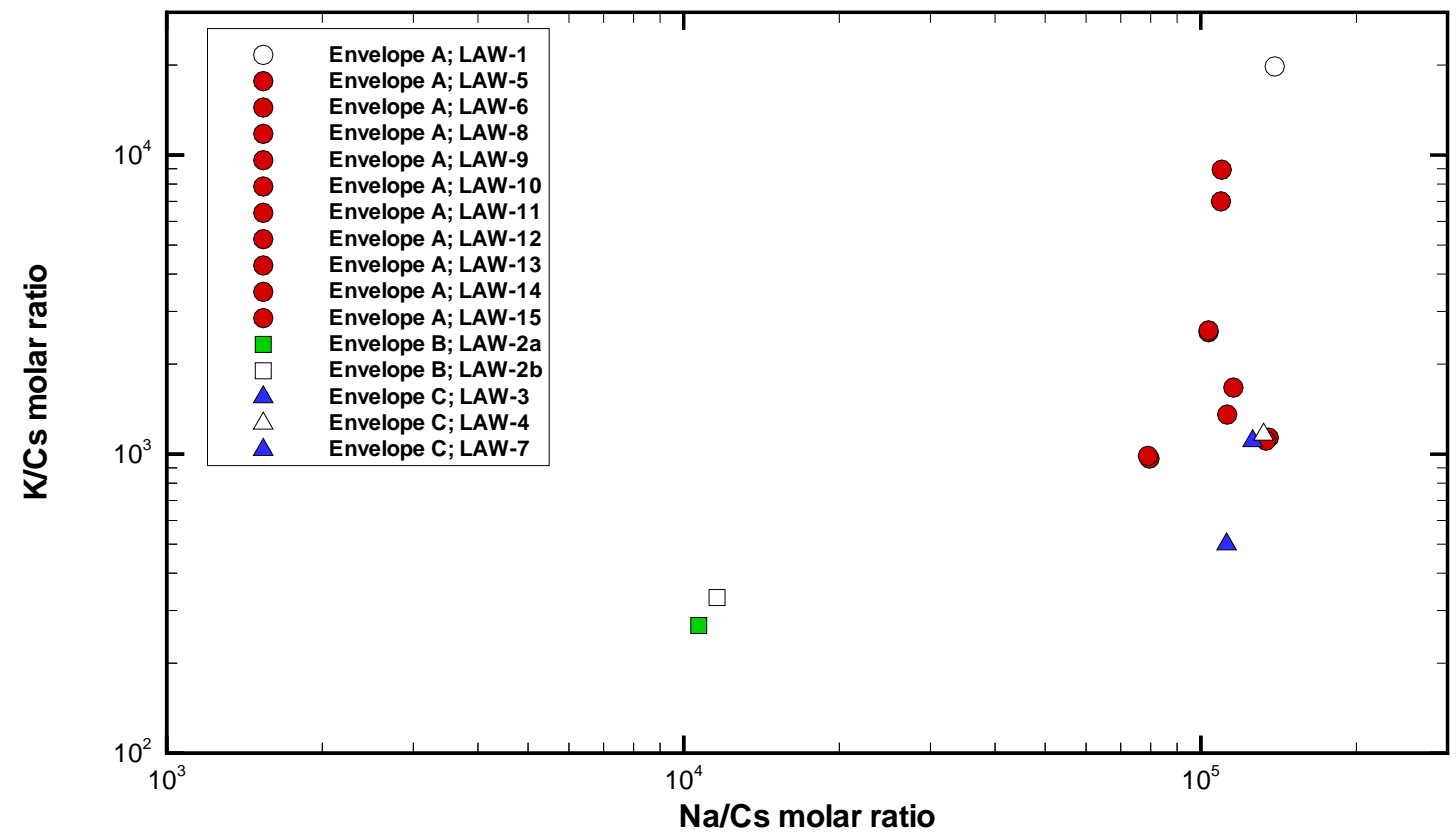

Figure B-4. Molar ratios of $\mathrm{Na} / \mathrm{Cs}$ versus $\mathrm{K} / \mathrm{Cs}$ for all Envelope $\mathrm{A}, \mathrm{B}$, and $\mathrm{C}$ candidate feed solutions. The least favorable feed solutions for each envelope are highlighted by open symbols. 


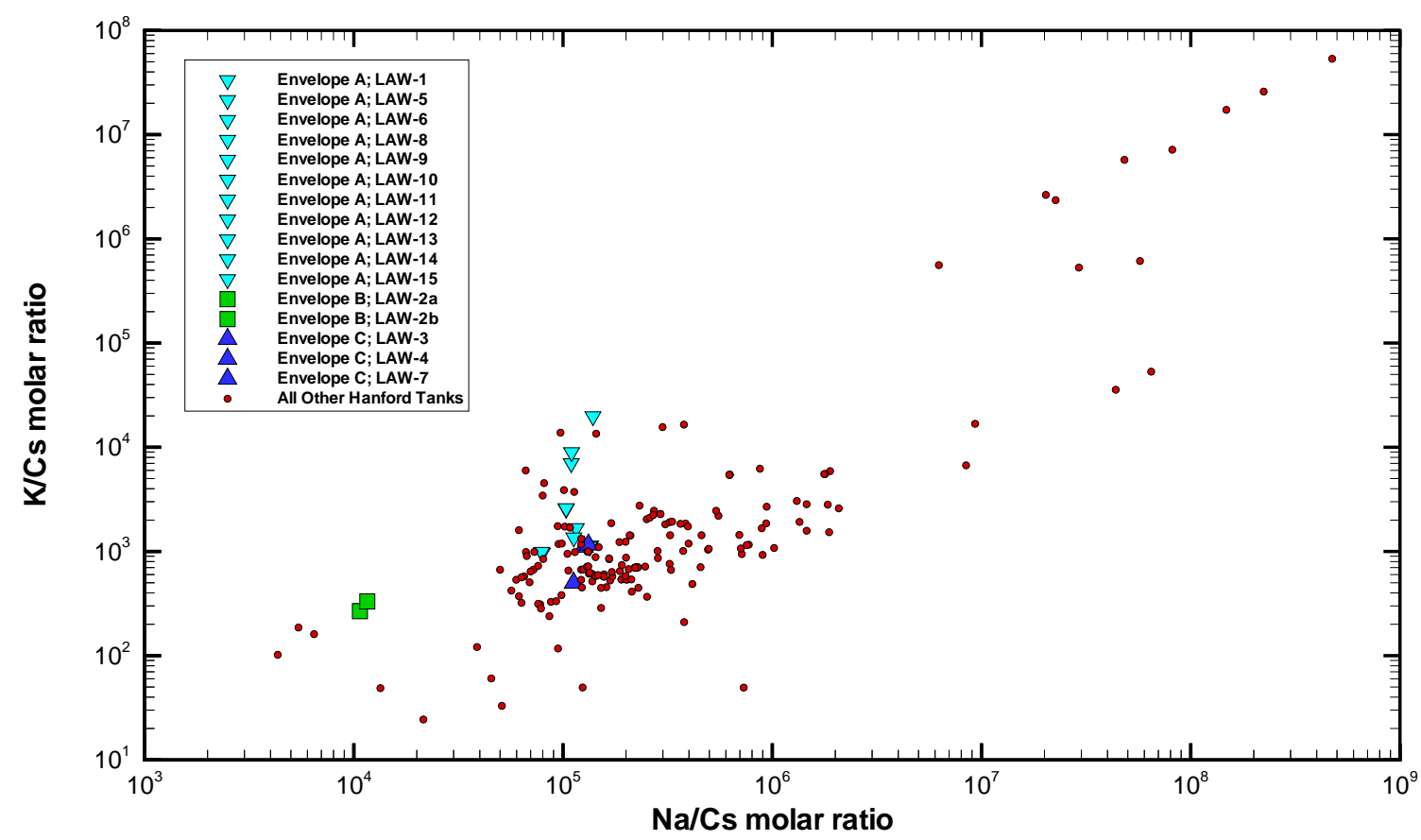

Figure B-5. LAW molar ratios of $\mathrm{Na} / \mathrm{Cs}$ versus $\mathrm{K} / \mathrm{Cs}$ for all 177 Hanford waste tanks. The Phase $1 \mathrm{LAW}$ batch feeds are highlighted using larger symbols.

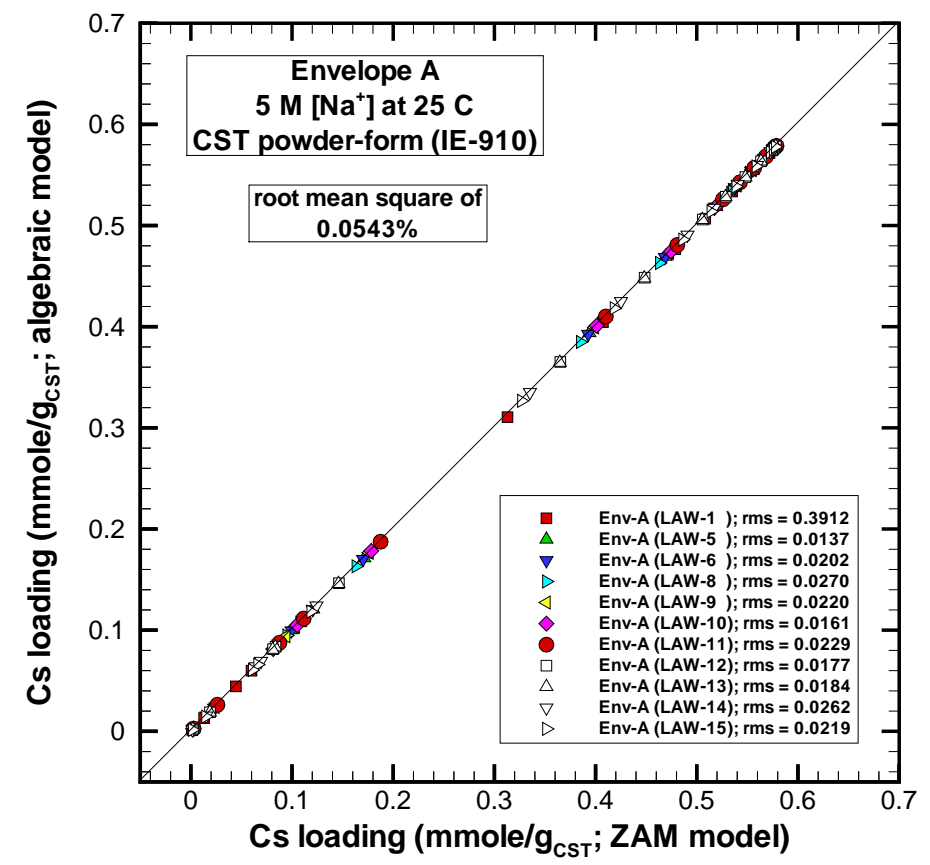

Figure B-6. Comparison of ZAM model versus "effective" single-component Freundlich/Langmuir Hybrid isotherm model predictions for cesium loadings on CST material in the powderform (IE-910) for the eleven Envelope A candidate feed solutions. 


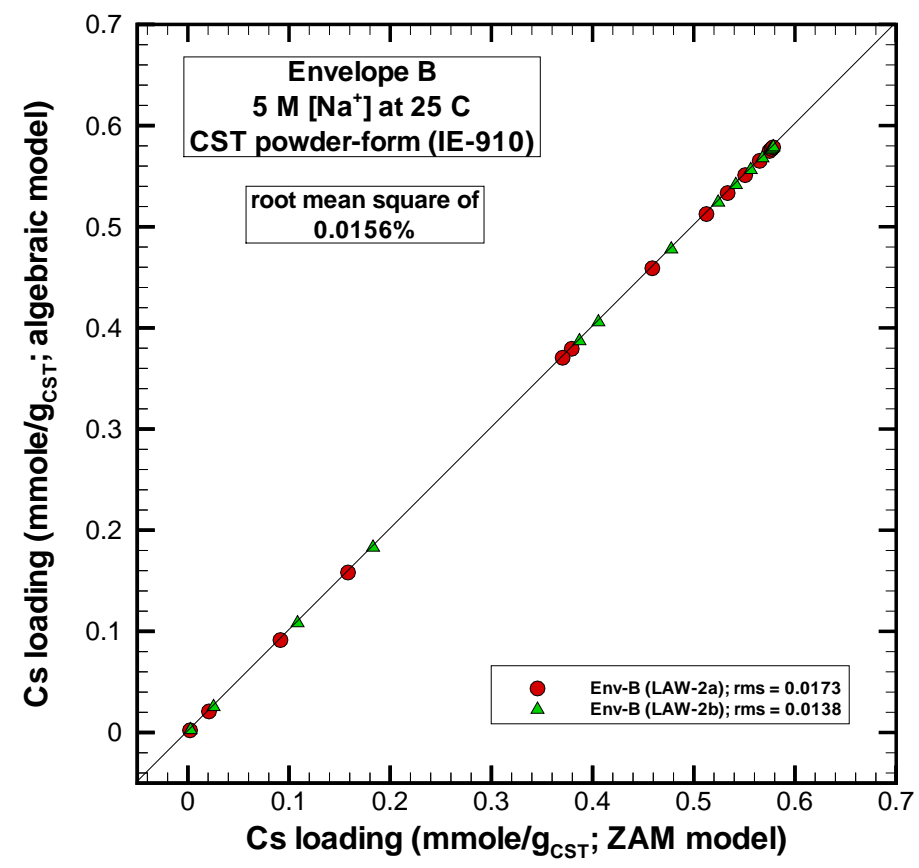

Figure B-7. Comparison of ZAM model versus "effective" single-component Freundlich/Langmuir Hybrid isotherm model predictions for cesium loadings on CST material in the powderform (IE-910) for the two Envelope B candidate feed solutions.

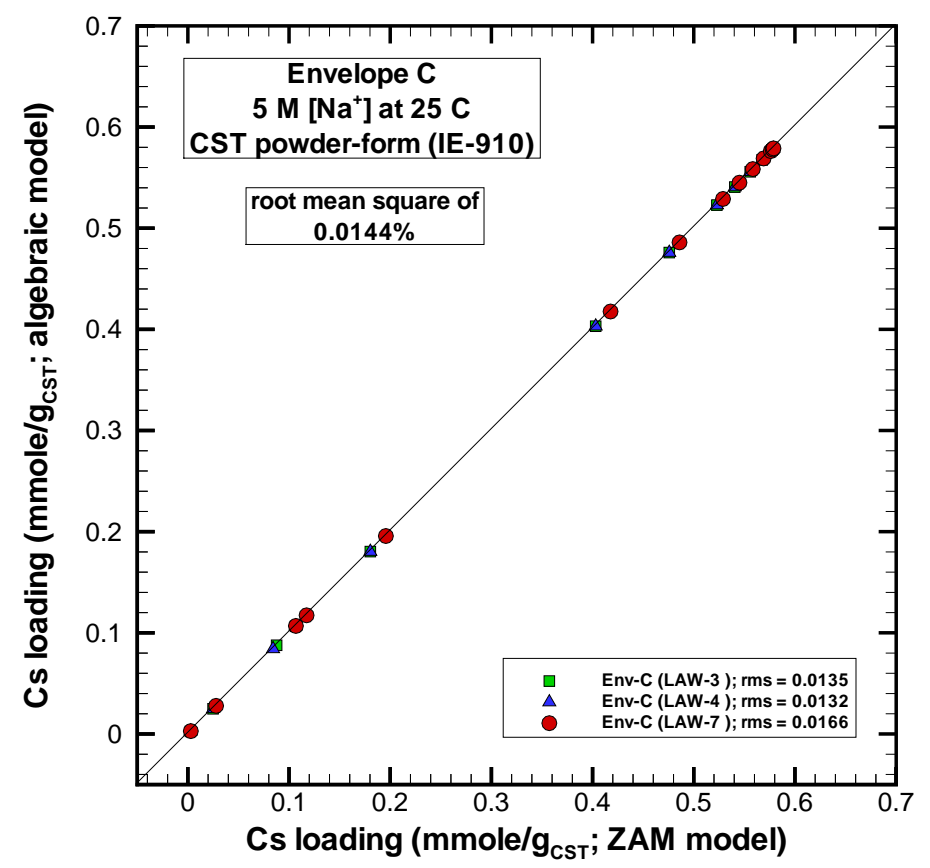

Figure B-8. Comparison of ZAM model versus "effective" single-component Freundlich /Langmuir Hybrid isotherm model predictions for cesium loadings on CST material in the powderform (IE-910) for the three Envelope C candidate feed solutions. 


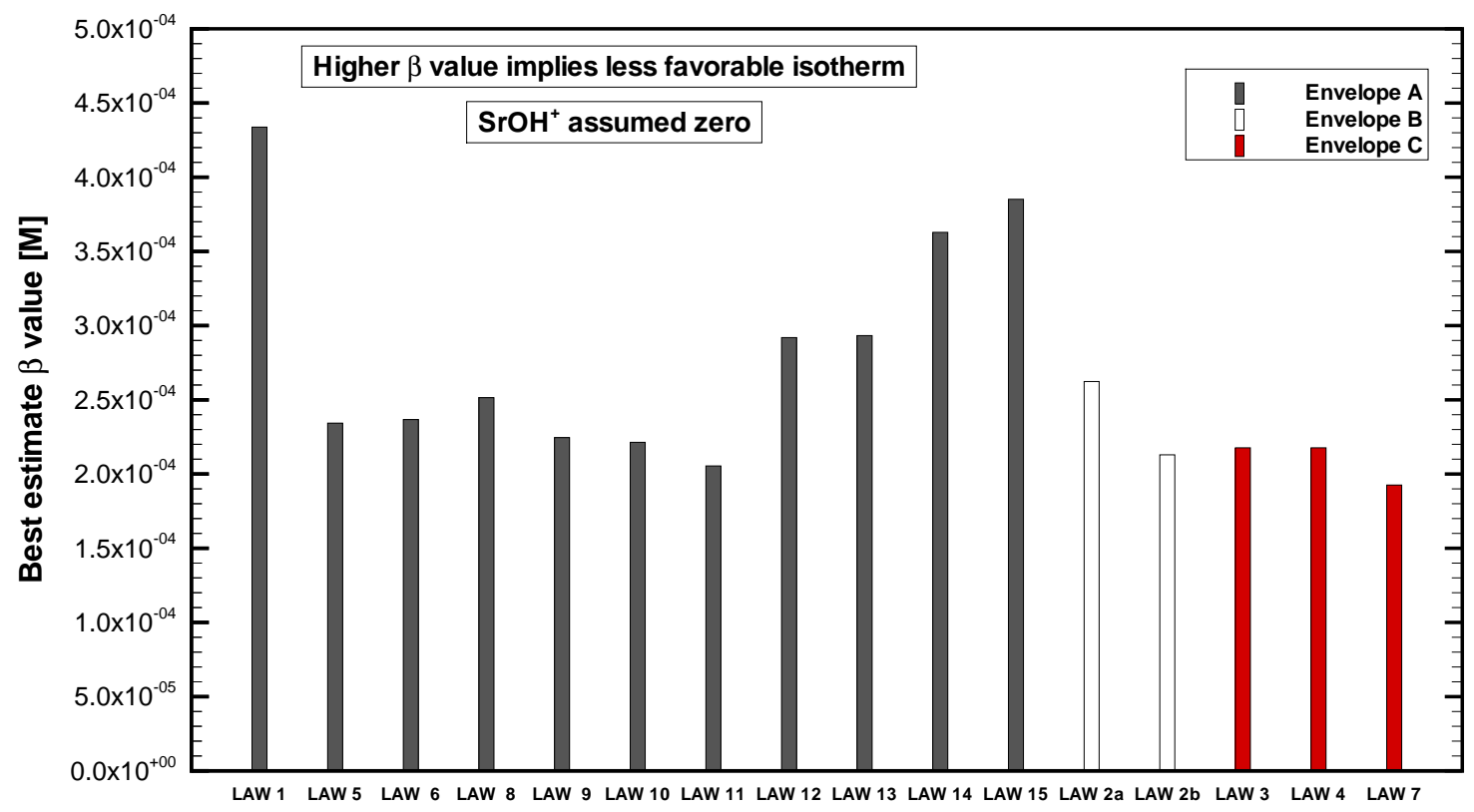

Candidate feed solutions

Figure B-9. The estimated Phase 1 feed beta values used in the cesium effective single-component isotherm model for CST powder-form and engineered-form materials. The beta values are grouped by envelope and are based on feeds assuming zero $\mathrm{Rb}^{+}$and $\mathrm{SrOH}^{+}$present.

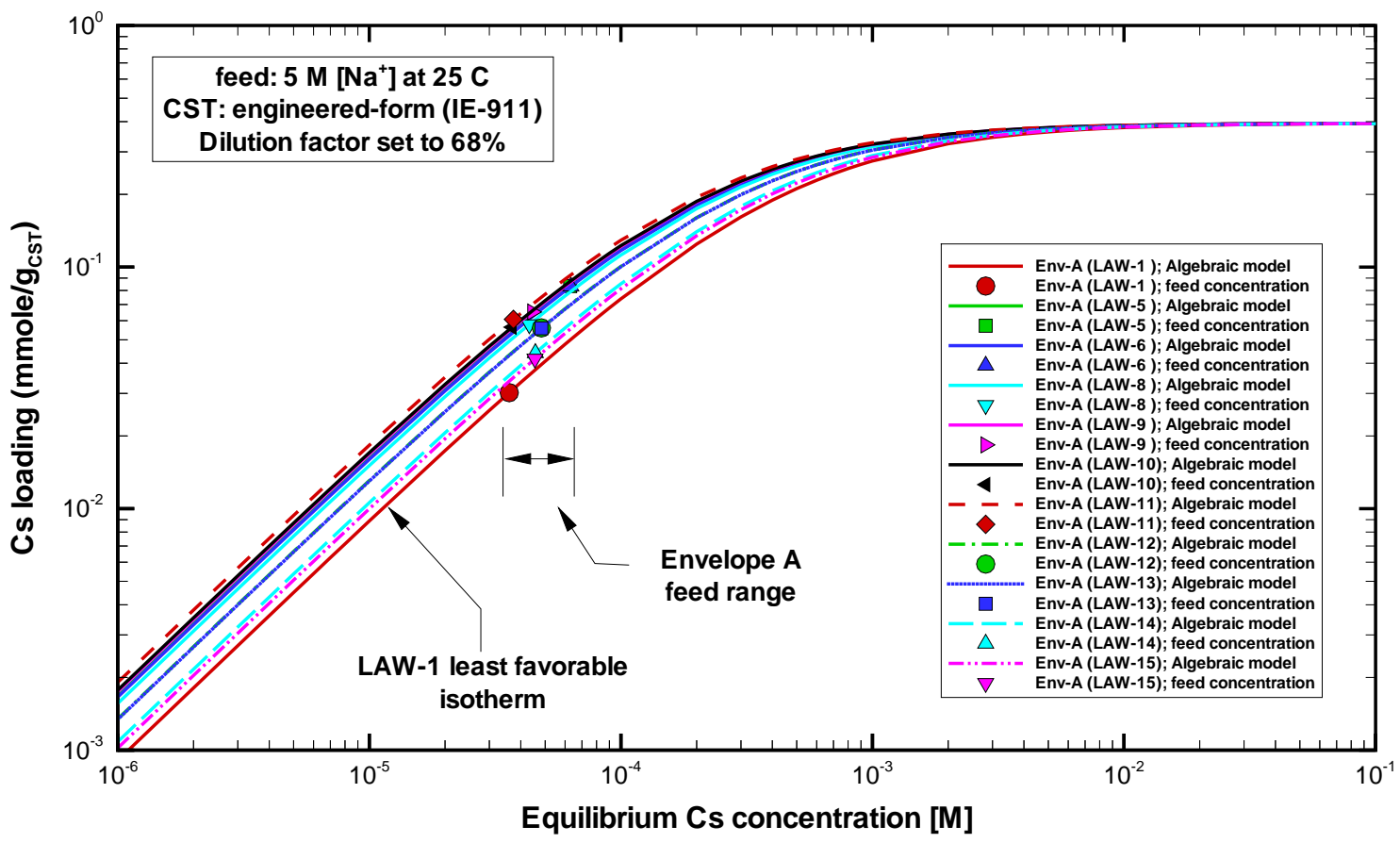

Figure B-10. Comparison of Envelope A isotherms for the CST material in its engineered-form (IE-911). The lines represent predictions based on the "effective" single-component Freundlich/ Langmuir Hybrid isotherm model and symbols indicate the feed concentrations of cesium. 


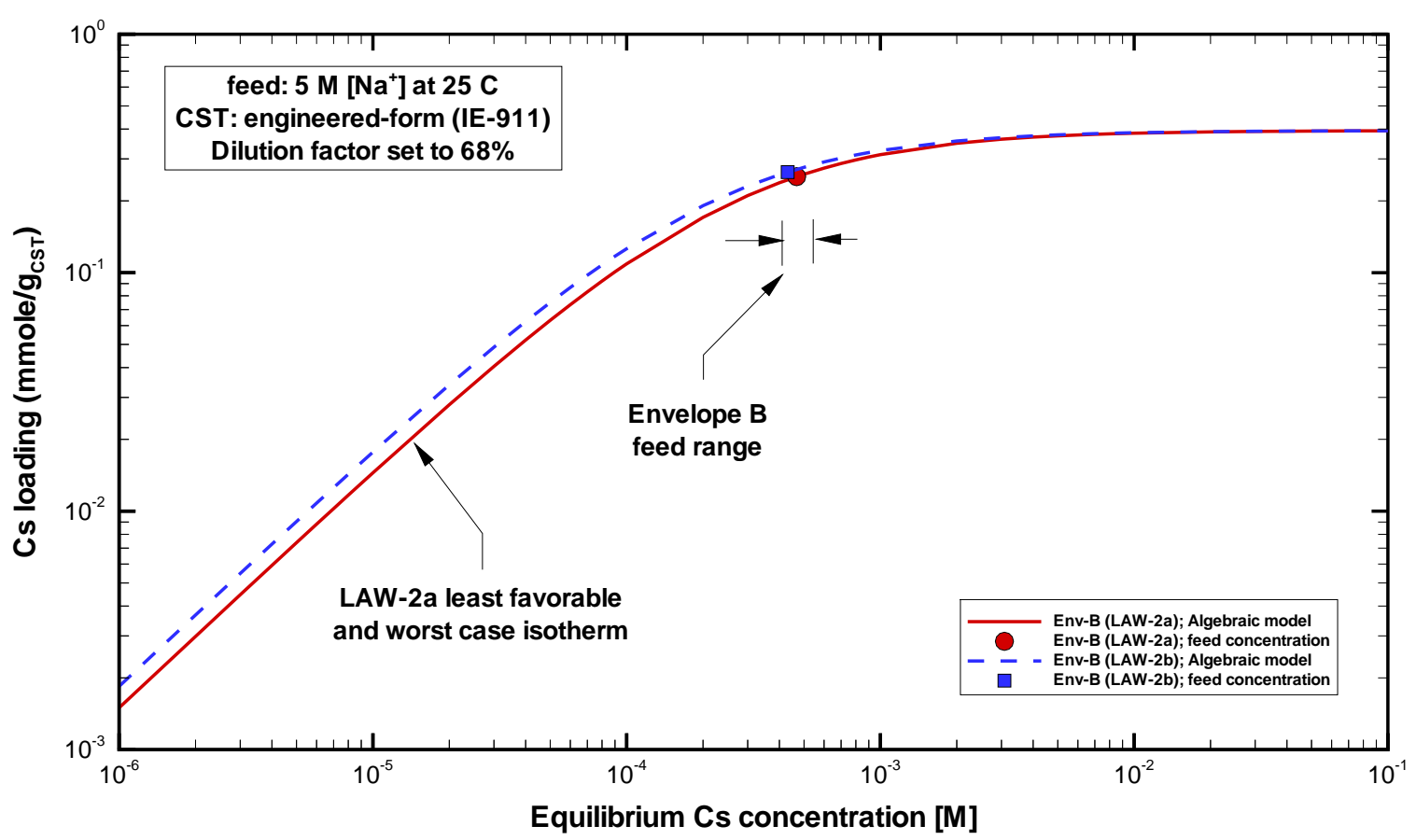

Figure B-11. Comparison of Envelope B isotherms for the CST material in its engineered-form (IE-911). The lines represent predictions based on the "effective" single-component Freundlich/ Langmuir Hybrid isotherm model and symbols indicate cesium feed concentrations.

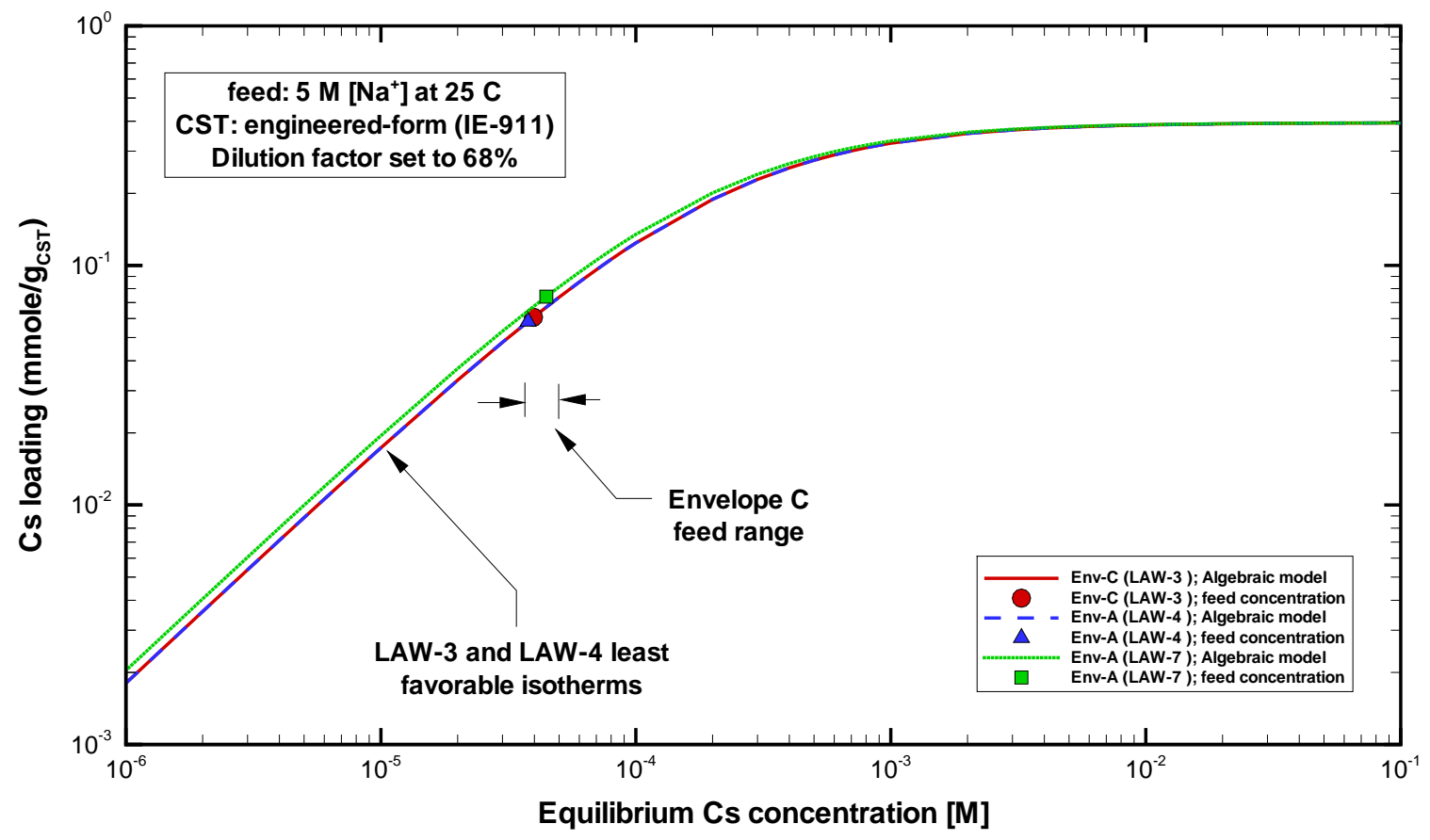

Figure B-12. Comparison of Envelope C isotherms for the CST material in its engineered-form (IE-911). The lines represent predictions based on the "effective" single-component Freundlich/ Langmuir Hybrid isotherm model and symbols indicate cesium feed concentrations. 


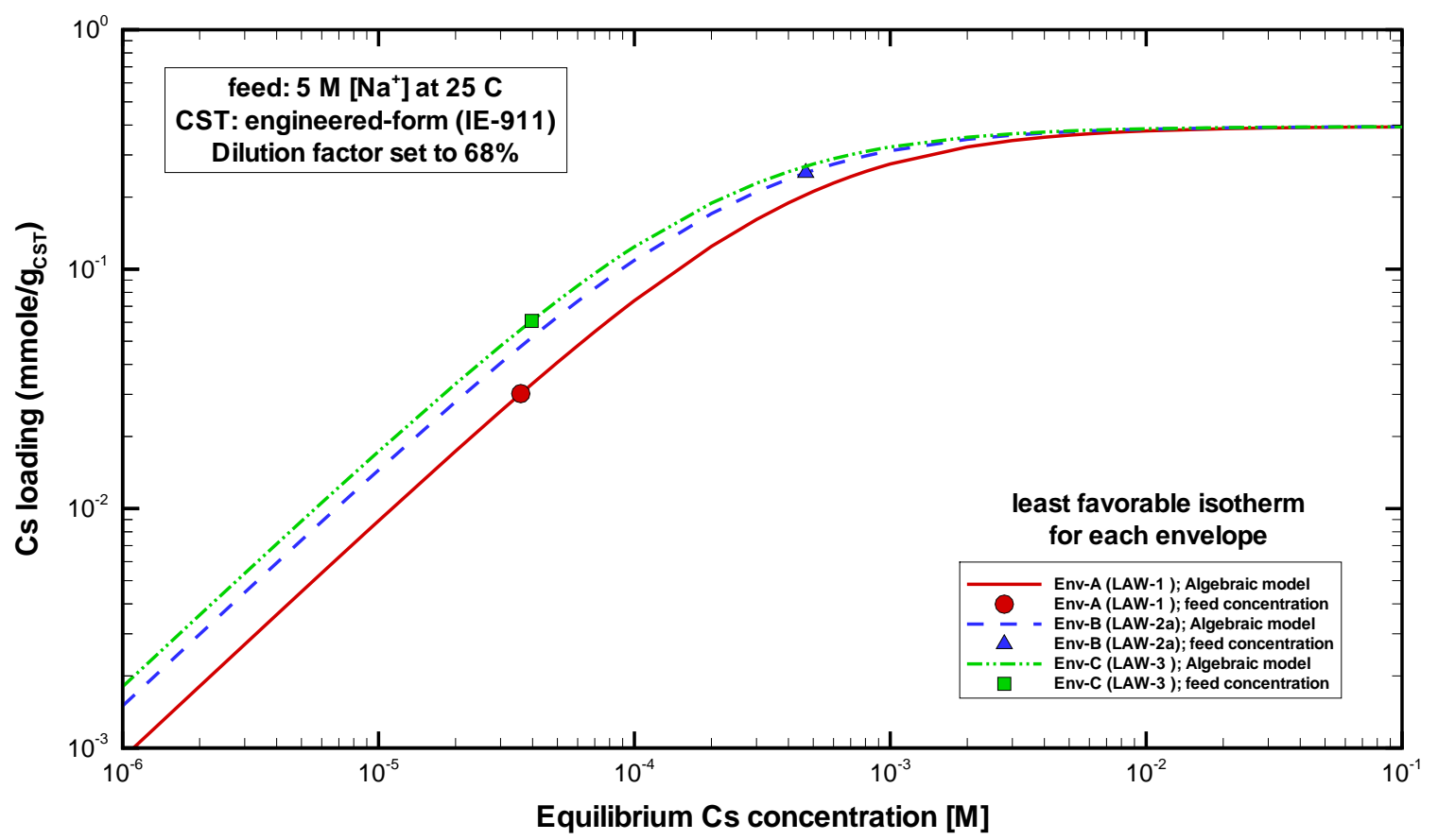

Figure B-13. Comparison of the least favorable isotherms for Envelope A, B, and C feeds for the CST material in its engineered-form (IE-911). The lines represent predictions based on the "effective" single-component Freundlich/Langmuir Hybrid isotherm model.

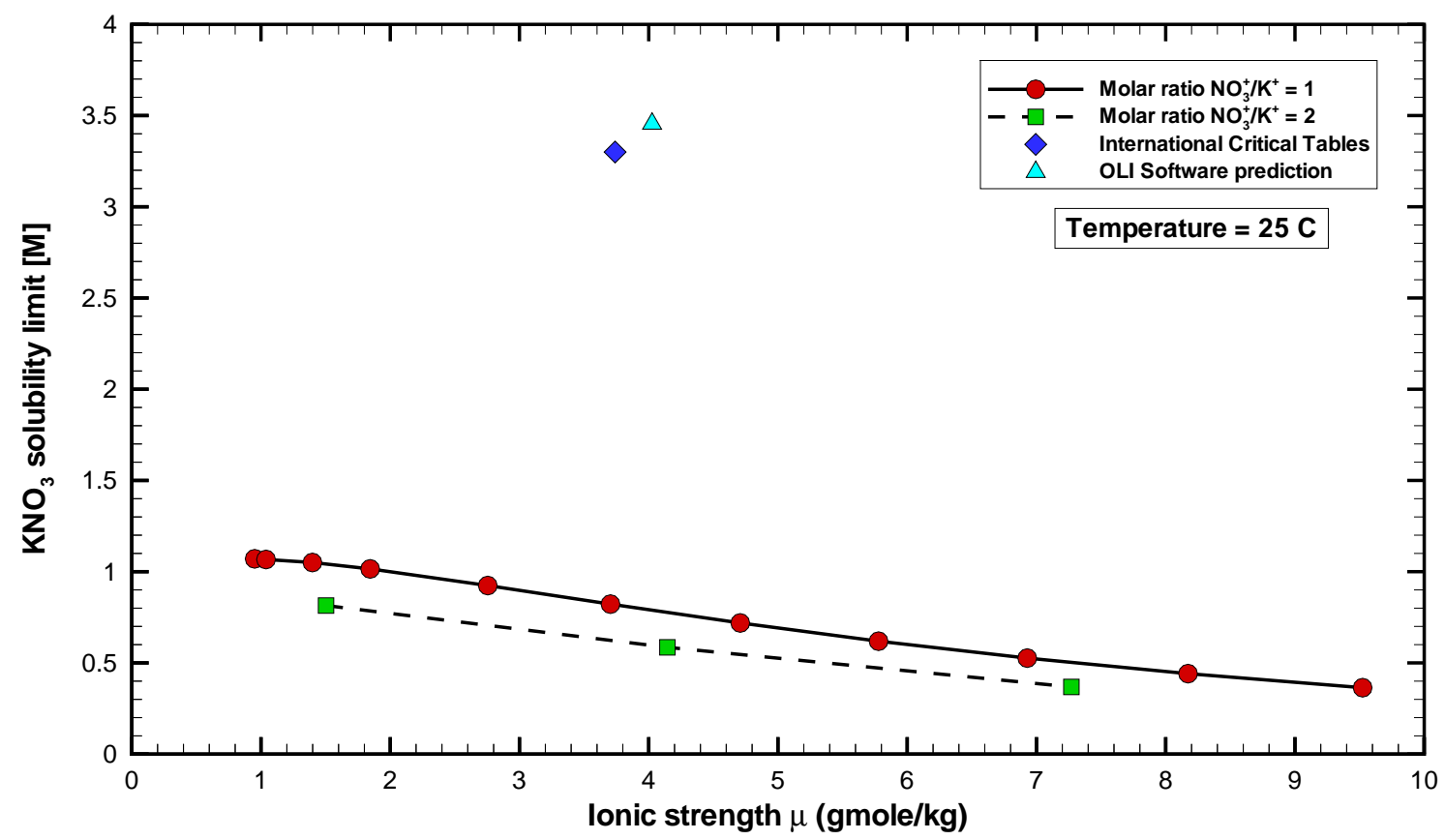

Figure B-14. Comparison of ZAM model predicted versus experimental (Washburn et al., 1928) $\mathrm{KNO}_{3}$ solubility limit at $25 \mathrm{C}$ (also shown is OLI version 6.5 prediction). For ZAM predictions $\mathrm{NaOH}$ was added to see the ionic strength effect. 


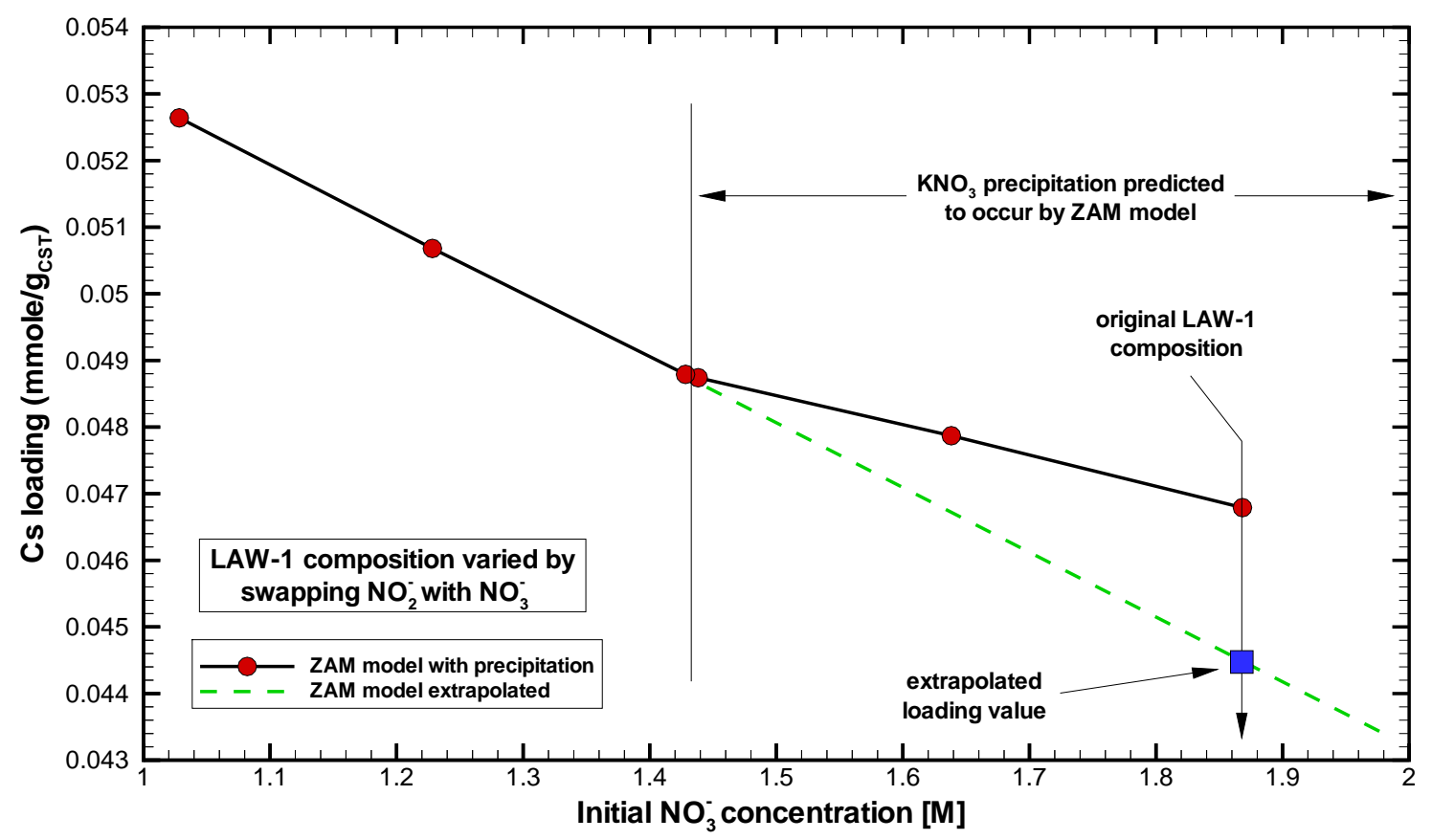

Figure B-15. Predicted impact for cesium loading on CST engineered-form material upon a swapping of nitrate with nitrite starting with a nominal solution of LAW-1 feed at $5.0 \mathrm{M} \mathrm{Na}^{+}$and $25 \mathrm{C}$ (the effect with [solid line] and without [dashed line] $\mathrm{KNO}_{3}$ precipitation is shown).

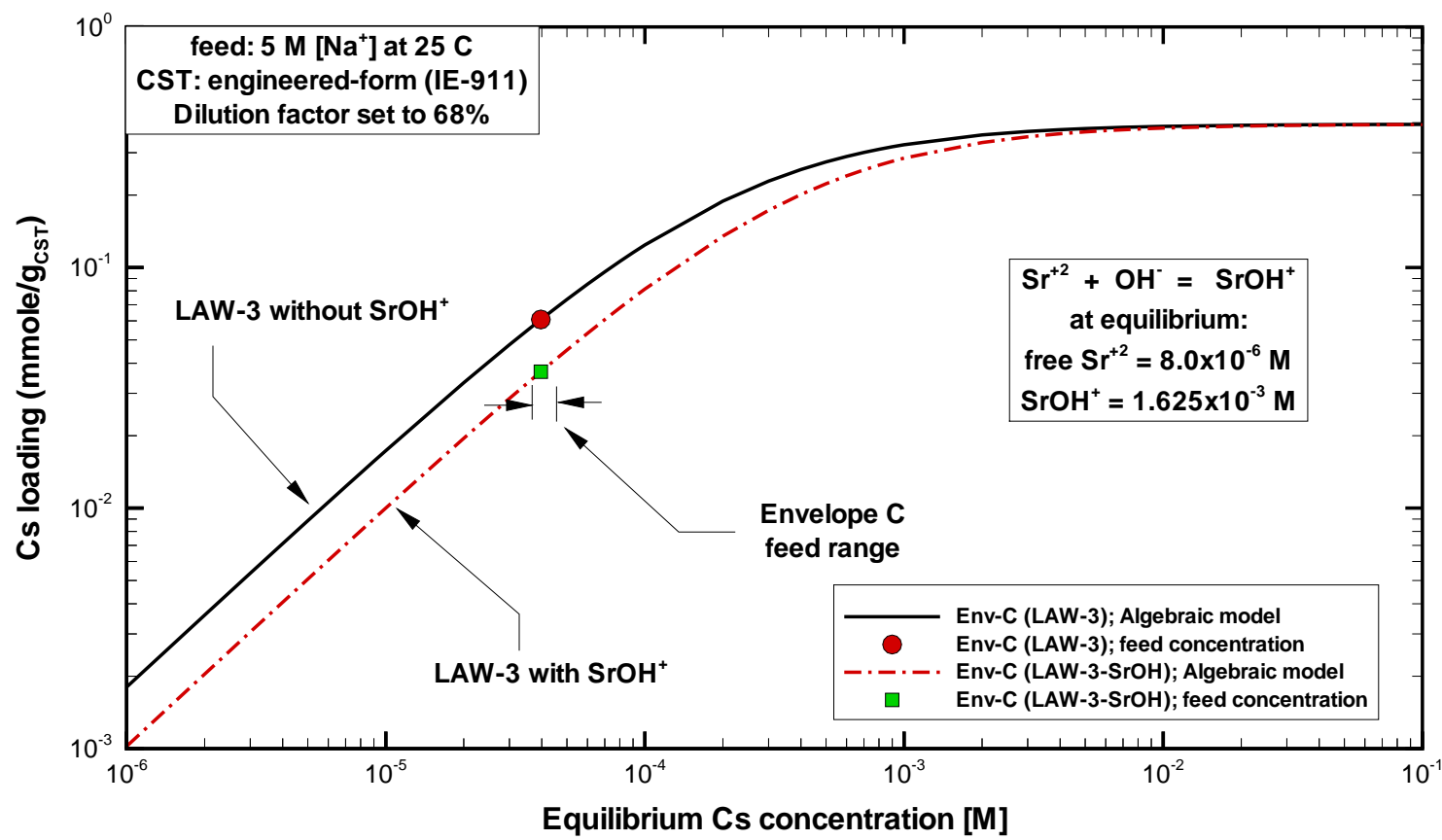

Figure B-16. Estimated impact of aqueous strontium hydroxide on cesium loadings for CST engineeredform material starting with a nominal solution of LAW-3 feed at $5.0 \mathrm{M} \mathrm{Na}^{+}$and $25 \mathrm{C}$ (zero $\mathrm{SrOH}^{+}$present [solid line] and upper bound of $\mathrm{SrOH}^{+}$present [dashed line]). 


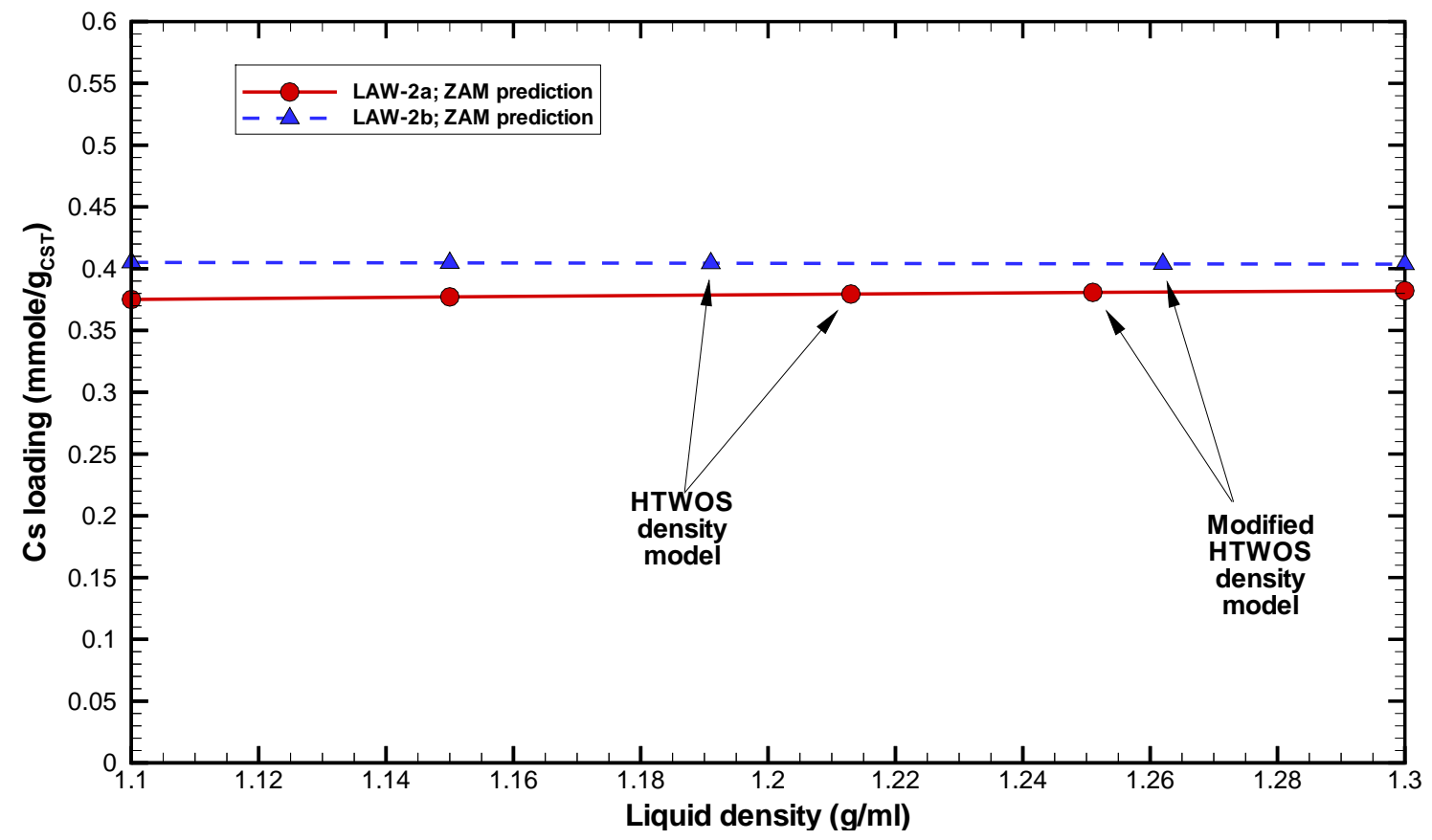

Figure B-17. Predicted impact of solution density on ZAM prediction of cesium loading on CST material based on LAW-2a and LAW-2b feeds at $5.0 \mathrm{M} \mathrm{Na}^{+}$and $25 \mathrm{C}$ (the modified HTWOS density model values represent nominal conditions).

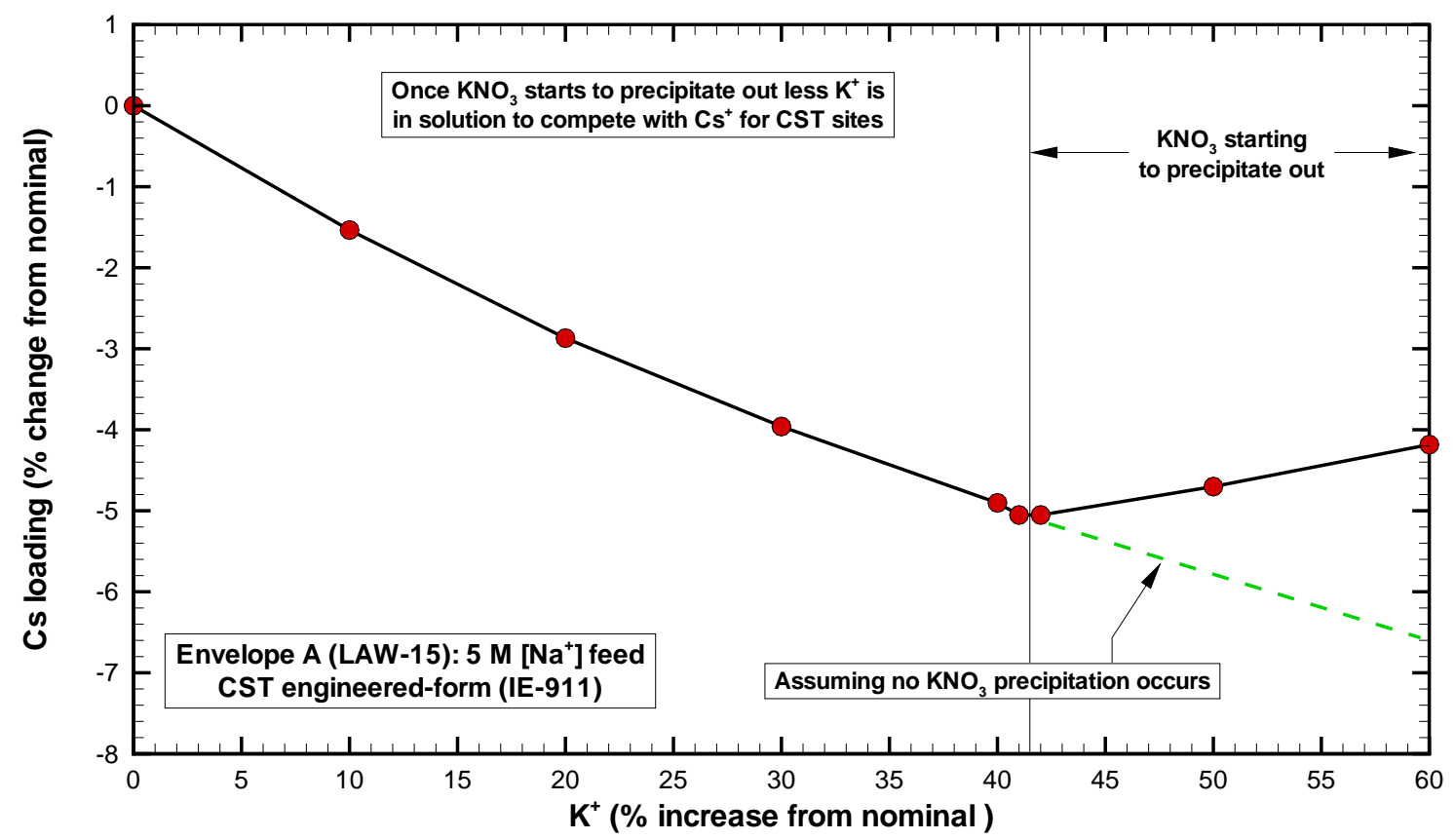

Figure B-18. Predicted impact for cesium loading on CST engineered-form material upon an increase in potassium concentration starting with a nominal solution of LAW-15 feed at $5.0 \mathrm{M} \mathrm{Na}^{+}$ and $25 \mathrm{C}$ (the effect with [solid line] and without [dashed line] $\mathrm{KNO}_{3}$ precipitation is shown). 


\section{Appendix C (Dilution Factor for IONSIV ${ }^{\circledR}$ IE-911) $^{2}$}

The basic ion exchange material is originally in a powder-form (i.e., very fine particles) and is referred to by UOP as IE-910 (and earlier on in its development phase by Texas A\&M University as TAM-5). In order to make use of this ion exchanger an "engineered-form" must be generated and ultimately processed at a production level. The selectivity and capacity characteristics of the ion exchanger are addressed primarily from the powder-form perspective. The smaller the particles the more improved the mass transfer rates will be, while in contrast, the larger the flow resistance (pressure drop) through a packed bed. Therefore, an optimal size particle potentially exists for the engineered-forms.

The IE-910 material is too fine for optimal use in large-scale ion-exchange columns. Commercial development of an engineered-form referred to by UOP as IE-911 has been underway during the later part of the 90's focusing on achieving optimal mass transfer efficiency within optimally sized particles. During the manufacturing production process of IONSIV ${ }^{\circledR}$ IE- $^{-}$ 911 material, an inert binder is added to IE-910 powder to create an engineered form that consists of larger sized particles made up from binding together numerous fine particles of IE910. The additional inert binding material reduces the total "effective" Cs ion-exchange capacity on a per weight basis and this reduction factor is referred to as its "dilution factor".

Typically, the engineered forms of IE-911 contain approximately 25 -to-30\% by mass inert binder. If during the production process, the inert binder does not chemically alter the CST or block off assessable surface adsorption sites; then, the resulting total cesium capacity of the IE911 resin would simply be 70 -to-75\% of its original IE-910 powder value. For an engineeredform this is probably its upper limit. For example, for an engineered-form containing 30\% binder this would result in a total $\mathrm{Cs}^{+}$exchange capacity of:

$$
\left[\overline{\mathrm{C}}_{\mathrm{T}}\right]_{\mathrm{Cs}}^{\text {Engineered-Form }}=\left(0.58 \frac{\text { mmole }_{\mathrm{Cs}}}{\mathrm{g}_{\text {powder }}}\right)\left(0.70 \frac{\mathrm{g}_{\text {powder }}}{\mathrm{g}_{\mathrm{CST}}}\right) \Rightarrow 0.406 \frac{\mathrm{mmole}_{\mathrm{Cs}}}{\mathrm{g}_{\mathrm{CST}}},
$$

and for $\mathrm{SrOH}^{+}$:

$$
\left[\overline{\mathrm{C}}_{\mathrm{T}}\right]_{\text {SrOH }}^{\text {Engineered-Form }}=\left(1.00 \frac{\text { mmole }_{\mathrm{SrOH}}}{\mathrm{g}_{\text {powder }}}\right)\left(0.70 \frac{\mathrm{g}_{\text {powder }}}{\mathrm{g}_{\mathrm{CST}}}\right) \Rightarrow 0.700 \frac{\mathrm{mmole}_{\mathrm{SrOH}}}{\mathrm{g}_{\mathrm{CST}}},
$$

where it is assumed that the dilution factor is not species dependent.

The powder-form total ionic capacities used in Eqs. (C-1) are those based on the original development of TAM-5 and reported by Zheng et al. (1996). During the crystal growth process in making CST powder several solid-phases can result. One particular solid-phase produces a material with superior Cs selectivity. The current manufacturer (i.e., UOP) states that their process improvements result in CST powder that is "better" than earlier batches by optimizing the growth of this particular solid-phase. This implies that the newer powder-form batches should have higher total ionic capacities than the values stated above. 
To obtain a better estimate and basis for the dilution factor, comparisons are made between ionexchange data for the more recent engineered-forms versus its original powder-forms. During the mid-90's the CST materials were tested using Hanford waste solutions. More recently, the SRS has been testing the CST materials using SRS waste solutions and newer engineered-forms. Below, analyses are presented that establish our "best estimate" of this dilution factor. The value ultimately chosen, $68 \%$, represents our best estimate of its value for the Baseline engineeredform and based on the various other engineered-forms this value appears to be conservative (i.e., $\sim 85 \%$ confidence level, mean minus one sigma). The use of $68 \%$ for the dilution factor sets a CST material acceptance criterion that should be reasonably achieved by the manufacturer of the CST engineered-forms.

After writing this appendix the work of Anthony et al. (2001) was provided to the authors. Anthony et al. (2001) upgraded the ZAM model to take into account the effect of the binder used to make CST in its engineered-forms. Based on SRS average simulant at $25 \mathrm{C}$, the improved ZAM predictions for IE-911 granules when compared to the ZAM predictions for CST powder yield a dilution factor of $\sim 73 \%$. This value is very consistent with the data provided in this report where the conservative value of $68 \%$ is used.

\section{C.1 Cesium loading on IONSIV ${ }^{\circledR}$ IE-911 versus IONSIV $^{\circledR}$ IE-910}

Recently, using SRS-average simulant, $\mathrm{K}_{\mathrm{d}}$ equilibrium contact tests were performed by Walker et al. (2001) at SRTC. The composition of the SRS-average simulant is given in Table C-1, along with the composition of various other simulants studied. A comparison of the cesium $\mathrm{K}_{\mathrm{d}}$ data at $36.2 \mathrm{C}$ is shown in Figure $\mathrm{C}-1$ and the data is tabulated in Table C-2. This data set contains one set of data using the powder-form and three using engineered-forms. Similar data by Walker et al. (2001) taken at other temperatures are provided in Table C-3 and by Fondeur et al. (2000) in Table C-4. The powder-form data provided corresponds to the same batch of powder-form used in the creation of the Baseline and New IE-911 batches. The engineered-form Old IE-911 was made using an older powder-form not listed here. Figure C-2 presents the same data sets in terms of the cesium loading.

Figures $\mathrm{C}-1$ and $\mathrm{C}-2$ indicate that the engineered-forms have consistently less selectivity than the powder-form when viewed on a per mass basis. The engineered-forms Baseline IE-911 and Old IE-911 are production level batches, while New IE-911 is a smaller lab-scale batch. The variability between the three engineered-forms result from:

- Two different powder-forms were used in creating the three batches;

- The fraction of inert binder added may have been different for the three batches; and

- The production techniques were different (e.g., lab-scale versus production-scale).

For determining an appropriate "best estimate" dilution factor we restrict our analysis to comparing the Baseline IE-911 to its New IE-910 powder. In this way, we are comparing only the impact of adding inert binder at the production-scale level to a specific powder-form. To estimate the level of variability observed between differing batches of engineered-form CST material, dilution factors are computed and compared for the more recent data sets available. 


\section{C.2 Averaged Dilution Factor for Baseline IE-911 CST Material}

In order to establish cesium isotherms for Envelopes $\mathrm{A}, \mathrm{B}$, and $\mathrm{C}$, the Texas A\&M equilibrium isotherm model (i.e., ZAM) is being used. The ZAM model is based on CST in its powder-form (i.e., actually TAM-5 powder-form from an earlier state around 1995). To make use of the ZAM model, adjustments must be made to its predictions when being applied to CST in its engineeredforms. Below we discuss a proposed way in which to make such adjustments. Typically, the engineered forms of IE-911 contain inert binder at approximately 25 -to-30\% by mass. If during the production process, the inert binder does not chemically alter the CST or block off assessable surface adsorption sites; then, the resulting total cesium capacity of the IE-911 resin would simply be 70 -to- $75 \%$ of its original IE-910 powder value. For an engineered-form this is probably its upper limit.

At any given equilibrium cesium concentration a dilution factor between IE-911 and IE-910 can be computed based on:

$$
\eta_{\mathrm{df}}\left(\mathrm{c}_{\mathrm{Cs}}\right) \equiv\left(\frac{\mathrm{Q}_{\mathrm{Cs}}^{\mathrm{IE}-911}}{\mathrm{Q}_{\mathrm{Cs}}^{\mathrm{IE}-910}}\right)
$$

Over the range of the data being considered the cesium loading curve can be expressed as:

$$
\mathrm{Q}_{\mathrm{Cs}}^{\mathrm{IE}-91 \mathrm{x}}=\mathrm{a}^{\mathrm{IE}-91 \mathrm{x}}\left[\mathrm{c}_{\mathrm{Cs}}\right]^{\mathrm{b}^{\mathrm{IE}-91 \mathrm{x}}},
$$

which represents a linear line on a log-log plot (i.e., linearity is observed at lower cesium concentrations with nonlinear behavior beginning to occur at higher concentrations). Substitution of Eq. (C-3) into Eq. (C-2) results in an expression for the dilution factor given by:

$$
\eta_{\mathrm{df}}\left(\mathrm{c}_{\mathrm{Cs}}\right)=\left[\frac{\mathrm{a}^{\mathrm{IE}-911}}{\mathrm{a}^{\mathrm{IE}-910}}\right]\left[\mathrm{c}_{\mathrm{Cs}}\right]^{\left(\mathrm{b}^{\mathrm{IE}-911}-\mathrm{b}^{\mathrm{IE}-910}\right)} .
$$

In principle the dilution factor should be a true constant; however, due to analytical uncertainties the value computed using Eq. (C-4) will vary some. To eliminate the variation an algebraically averaged value over the entire cesium data range is computed using:

$$
\left\langle\eta_{\mathrm{df}}\right\rangle=\left[\frac{1}{\mathrm{c}_{\mathrm{Cs}}^{\max }-\mathrm{c}_{\mathrm{Cs}}^{\min }}\right]\left[\int_{\mathrm{c}_{\mathrm{Cs}}^{\min }}^{\mathrm{c}_{\mathrm{Cs}}^{\max } \eta_{\mathrm{df}}}\left(\mathrm{c}_{\mathrm{Cs}}^{\prime}\right) \mathrm{dc} \mathrm{c}_{\mathrm{Cs}}^{\prime}\right],
$$

where upon substitution of Eq. (C-4) into Eq. (C-5a) and integrating yields

$$
\left\langle\eta_{\mathrm{df}}\right\rangle=\frac{\mathrm{a}^{\mathrm{IE}-911}}{\mathrm{a}^{\mathrm{IE}-910}\left(\mathrm{c}_{\mathrm{Cs}}^{\max }-\mathrm{c}_{\mathrm{Cs}}^{\min }\right)\left(\mathrm{b}^{\mathrm{IE}-911}-\mathrm{b}^{\mathrm{IE}-910}+1\right)} .
$$

The cesium loading data at $36.2 \mathrm{C}$ by Walker et al. (2001) for the Baseline IE-911 and its New IE-910 powder are shown in Figure C-3. The data correspond to newer powder-form CST (i.e., 
solid circles), along with a production version baseline of engineered-form CST made using the new powder-form (i.e., solid triangles). As shown in Figure C-3, the equilibrium cesium concentration ranges from $\sim 3 \times 10^{-7}$ to $\sim 7 \times 10^{-5} \mathrm{M}$. Also shown in Figure C-3 are the power-law (i.e., Eq. (C-3)) fits to the cesium loading data (i.e., solid-line representing the powder-form fit and dashed-line representing the engineered-form fit). The power-law fits represent the data well over the range being considered. For comparison purposes the power-law Eq. (C-3) was fitted to all of the CST material presented in Table C-2. The a and b coefficients to the powerlaw equation for each material is listed in Table $\mathrm{C}-5$, along with their integral average dilution factor estimate.

Making use of Eq. (C-4), point values of the dilution factor can be computed as tabulated in Table C-5. For example, for the Baseline IE-911 material we see the dilution factor varying from $75.5 \%$ at its lowest cesium concentration to $61.0 \%$ at its highest value. Using the powerlaw fits, the average deviation over the entire cesium concentration range was computed to be $\sim 68 \%$ based on Eq. (C-6). Also shown in Figure C-3, are the ranges of inlet cesium feed concentrations for each Hanford LAW envelope and the lag column exit cesium criterion.

Conservatively, for our current column transport efforts we shall set the total $\mathrm{Cs}^{+}$exchange capacity to:

$$
\left[\overline{\mathrm{C}}_{\mathrm{T}}\right]_{\mathrm{Cs}}^{\text {Engineered-Form }}=\left(0.58 \frac{\mathrm{mmole}_{\mathrm{Cs}}}{\mathrm{g}_{\text {powder }}}\right)\left(0.68 \frac{\mathrm{g}_{\text {powder }}}{\mathrm{g}_{\mathrm{CST}}}\right) \Rightarrow 0.3944 \frac{\mathrm{mmole}_{\mathrm{Cs}}}{\mathrm{g}_{\mathrm{CST}}},
$$

and for $\mathrm{SrOH}^{+}$to:

$$
\left[\overline{\mathrm{C}}_{\mathrm{T}} \text { J }_{\text {SrOH }}^{\text {Engineered-Form }}=\left(1.00 \frac{\mathrm{mmole}_{\mathrm{SrOH}}}{\mathrm{g}_{\text {powder }}}\right)\left(0.68 \frac{\mathrm{g}_{\text {powder }}}{\mathrm{g}_{\mathrm{CST}}}\right) \Rightarrow 0.680 \frac{\mathrm{mmole}_{\mathrm{SrOH}}}{\mathrm{g}_{\mathrm{CST}}} .\right.
$$

In Figure C-4 the same set of data as shown in Figure C-3 is re-plotted. However, the power-law fits as shown in Figure C-3 are now replaced with ZAM predictions. The solid-curve represents the ZAM predictions for the powder-form material. Using as a constant dilution factor, $68 \%$, the adjusted ZAM predictions are shown as a dashed-curve. Also shown in Figure C-4, are the ranges of inlet cesium feed concentrations for each envelope and the lag column exit criterion.

In the log-log plot shown in Figure C-4, both sets of data exhibit approximately linear behavior, while the ZAM model shows slight curvature at increasing cesium concentrations due to the finite/fixed amount of exchange sites available within the model. The current manufacturer (i.e., UOP) states that their process improvements result in CST powder that is "better" than earlier batches. The data sets at the high cesium concentration test conditions, show slight up-turns in loading. This might indicate that a slightly increased total cesium exchange capacity may exist or a varying capacity exists at higher cesium concentration levels; however, no explicit attempts to take credit for this is proposed. From an overall perspective the two predictions appear reasonable and the estimated dilution factor falls below its expected maximum bound of 70-to$75 \%$. 
Based on these simple analyses we propose to use this dilution factor for the CST column simulations. Above an average dilution factor was computed based on equilibrium contact tests performed at $36.2 \mathrm{C}$. Below we shall address the impact on the isotherm when at lower operating temperatures and its variation dependence on cesium concentration.

\section{C.3 Dilution Factor Variability}

As stated above, the expectation is that for a specific batch of the engineered-form the dilution factor is a true constant and not a function of temperature or liquid composition. Unfortunately, the currently available batch contact data produces dilution factors with a modest variation in magnitude. Below we shall show the computed variation and compare it to the earlier average value of 0.68 for the Baseline IE-911 material (i.e., estimated using a power-law/integration method).

\section{C.3.1 Impact of Cesium Concentration}

As discussed in Appendix B, an effective single-component total cesium isotherm can be expressed as:

$$
\mathrm{Q}_{\mathrm{Cs}}=\frac{\eta_{\mathrm{DF}} \overline{\mathrm{C}}_{\mathrm{T}} \mathrm{c}_{\mathrm{Cs}}}{\mathrm{c}_{\mathrm{Cs}}+\left[\widetilde{\mathrm{K}}_{21} \mathrm{c}_{\mathrm{K}}+\widetilde{\mathrm{K}}_{31} \mathrm{c}_{\mathrm{Na}}+\widetilde{\mathrm{K}}_{41} \mathrm{c}_{\mathrm{SrOH}}+\cdots\right]} \Rightarrow \frac{\eta_{\mathrm{DF}} \overline{\mathrm{C}}_{\mathrm{T}} \mathrm{c}_{\mathrm{Cs}}}{\mathrm{c}_{\mathrm{Cs}}+\beta}
$$

where the beta parameter for cesium becomes dependent upon the other ionic competitors for CST exchange (i.e., $\mathrm{K}^{+}, \mathrm{Na}^{+}, \mathrm{SrOH}^{+}$, and $\mathrm{Rb}^{+}$). The beta parameter contains the selectivity coefficients making it dependent upon temperature and liquid composition. The dilution factor $\left(\eta_{\mathrm{DF}}\right)$ is unity when considering a specific powder-form and is less than one upon addition of an inert binder. The total cesium capacity term is only a function of which batch of powder-form material is being considered.

Assuming that the New IE-910 powder-form material has the total cesium capacity of 0.58 mmole/g $\mathrm{g}_{\text {CST }}$, Eq. (C-8) was fitted to various available batch contact data to estimate beta values for different liquid compositions and temperatures. The results of the fitting process are listed in Table C-6. For each batch contact data point obtained for each engineered-form, the appropriate beta value was used and a dilution factor was estimated. Averaged values (i.e., original plus duplicate measurement values) for the equilibrium cesium concentration and cesium loading were used in computing these parameters. These results are also listed in Table C-6. Here we are making the reasonable assumption that the beta value for the engineered-form is the same as the value for its powder-form when considering data at the same temperature and nearly the same liquid composition.

The calculated variation observed in dilution factor is shown in Figure C-5 with respect to equilibrium cesium concentration. Three different batches of engineered-forms are provided, with each batch represented by a different symbol. No apparent explanation can be provided at this time for the level of variation observed. Also provided is the sample mean and one standard deviation values for the dilution factor estimates shown as horizontal lines (i.e., mean the solid- 
line and the mean minus one standard deviation the dashed-line). The mean minus one standard deviation value of $\sim 0.66$ is very close to the computed average value of 0.68 for the Baseline material.

Given the variability within the estimated dilution factors, partly stemming from data uncertainties and actual batch variability, the average value of 0.68 still appears acceptable.

\section{C.3.2 Impact of Temperature}

During the loading phase of column operation cesium ions are replacing sodium ions at surface sites. This is the dominant ion-exchange reaction occurring when the more selective cesium ion takes a surface site as expressed by the mass-action relation:

$$
\left[\overline{\mathrm{Na}^{+}}\right]+\left[\mathrm{Cs}^{+}\right] \stackrel{\mathrm{Na}-\mathrm{Cs}_{\mathrm{eq}}}{\longleftrightarrow}\left[\overline{\mathrm{Cs}^{+}}\right]+\left[\mathrm{Na}^{+}\right],
$$

where the bar over a species implies that it resides on a surface site. Ion exchange is not a chemical reaction and generally occurs with little evolution or uptake of heat (see Helfferich, 1962). Therefore, the temperature dependence of ion-exchange equilibria is usually minor. For the mass-action relationship expressed by Eq. (C-9) when using CST material we have an exothermic heat of ion-exchange (i.e., $\Delta \mathrm{H}_{\mathrm{IX}}<0$ ). The temperature dependence of ion-exchange equilibria is given by the thermodynamic relation:

$$
\left(\frac{\mathrm{d} \ln \mathrm{K}_{\mathrm{eq}}}{\mathrm{dT}}\right)_{\mathrm{P}}=\frac{\Delta \mathrm{H}_{\mathrm{IX}}}{\mathrm{RT}^{2}} .
$$

Assuming that the heat of ion-exchange is constant, Eq. (C-10) can be integrated resulting in:

$$
\ln \left(\frac{\mathrm{K}_{\mathrm{eq}, 2}}{\mathrm{~K}_{\mathrm{eq}, 1}}\right)=-\frac{\Delta \mathrm{H}_{\mathrm{IX}}}{\mathrm{R}}\left[\frac{1}{\mathrm{~T}_{2}}-\frac{1}{\mathrm{~T}_{1}}\right] .
$$

When considering an exothermic reaction, Eq. (C-11) states that a rise in temperature will reduce the thermodynamic equilibrium constant. For our ion-exchange process, this results in reduced cesium adsorption at higher temperatures.

In the ZAM model, Zheng et al. (1997) are using a triple-site (i.e., supper-site) concept that can be justified based on the differences measured in total ionic capacities for the various competitors. Ion exchange of cesium with a super-site on the CST material is represented by the set of mass-action relations:

$$
\begin{gathered}
{\left[\overline{\mathrm{Na}_{3}^{+}}\right]+\left[\mathrm{Cs}^{+}\right] \stackrel{\mathrm{K}_{\mathrm{eq}, 1}}{\longleftrightarrow}\left[\overline{\mathrm{Na}_{2}^{+} \mathrm{Cs}^{+}}\right]+\left[\mathrm{Na}^{+}\right],} \\
{\left[\overline{\mathrm{H}^{+} \mathrm{Na}_{2}^{+}}\right]+\left[\mathrm{Cs}^{+}\right] \stackrel{\mathrm{K}_{\mathrm{eq}, 2}}{\longleftrightarrow}\left[\overline{\mathrm{H}^{+} \mathrm{Na}^{+} \mathrm{Cs}^{+}}\right]+\left[\mathrm{Na}^{+}\right] \text {, and }}
\end{gathered}
$$




$$
\left[\overline{\mathrm{H}_{2}^{+} \mathrm{Na}^{+}}\right]+\left[\mathrm{Cs}^{+}\right] \stackrel{\mathrm{K}_{\mathrm{eq}, 3}}{\longleftrightarrow}\left[\overline{\mathrm{H}_{2}^{+} \mathrm{Cs}^{+}}\right]+\left[\mathrm{Na}^{+}\right] .
$$

The estimated values of the heat of ion-exchange for Eqs. (C-12) are reported by Zheng et al. (1997) to be:

- Eq. (C-12a) $\Delta \mathrm{H}_{\mathrm{IX}}=-2.18 \times 10^{4} \mathrm{~J} / \mathrm{gmole}$;

- Eq. (C-12b) $\Delta \mathrm{H}_{\mathrm{IX}}=1.46 \times 10^{4} \mathrm{~J} / \mathrm{gmole}$; and

- Eq. (C-12c) $\Delta \mathrm{H}_{\mathrm{IX}}=$ not recorded (probably small).

For cesium removal during the loading cycle the feed will be under very high $\mathrm{pH}$ conditions where the CST material will be predominately in its sodium form. Therefore, under these conditions the dominant mass-action reaction expected to be occurring is expressed by Eq. (C12a) resulting in an overall exothermic reaction. The above ZAM modeling parameters are based on batch contact data over a broad $\mathrm{pH}$ range covering a temperature range of 25 to $44 \mathrm{C}$ (see Zheng et al. $(1996,1997)$ ). In the ZAM model the total ionic capacity values are constants, not a direct function of temperature.

Walker et al. (2001) have recently measured the temperature dependence of several CST materials for cesium loading. Their data are tabulated in Table C-3. For the new powder-form IE-910 material, a comparison of the measured to ZAM predicted cesium loadings is provided in Figure C-6 (also showing the data point by Fondeur et al. (2000)). As can be seen in Figure C-6, both the measured and the predicted loading curves show reduced cesium loadings at higher temperatures, consistent with the above arguments. As observed earlier the ZAM model underpredicts the cesium loading measured for the new powder-form material at these liquid cesium concentrations. However, the ZAM model shifts the isotherms approximately the same amount as indicated by the data.

The temperature dependence of the computed point values for the dilution factor is shown in Figure C-7 based on the data provided in Table C-6. Similar to the comments made for the cesium concentration dependence observed, no apparent explanation can be provided at this time for the level of variation observed.

\section{C.4 Cesium Loading Curve Comparisons}

Using the ZAM code to predict CST powder-form behavior and the average dilution factor value of $68 \%$ to adjust the ZAM results to predict CST engineered-form behavior, several other data sets are compared. Walker et al. (2001) also measured cesium loadings using same standard simulant composition typical used by UOP for their performance testing (i.e., see Table C-1 for UOP simulant composition). Their data is tabulated in Table C-6 and is plotted in Figures C-8 and C-9. Figure C-8 contains the only the Baseline data and its powder-form data similar to the plot shown in Figure C-4 for direct comparison. The shift in the data (i.e., powder-form to engineered-form) is consistent with the shift computed with ZAM. However, as seen in Figure C-4, ZAM under-predicts the cesium loadings at these higher cesium concentration levels. To 
see the variability in cesium loading due to batch differences among engineered-forms, Figure C9 is a close-up of Figure C-8 where the data for the other two engineered-forms has been added.

Similar data was taken by Fondeur et al. (2000) at $25 \mathrm{C}$ using SRS-average simulant. This data is listed in Table C-4 and is plotted in Figure C-10. Excellent agreement is seen between the powder-form and Baseline engineered-form data and their corresponding ZAM predictions.

Cesium loadings were also measured for the Baseline CST material (IE-911) using actual waste samples taken from SRS Tank 44 by Walker et al. (1997). The composition of the SRS Tank 44 samples is given in Table $\mathrm{C}-1$ and the data obtained from the batch contact testing is listed in Table C-7. A comparison of the data to the ZAM predictions is shown in Figure C-11. The data is for the Baseline engineered-form of CST, while the dashed-curve represents the ZAM prediction of the engineered-form of CST. Unfortunately, the data follows the powder-form predictions of ZAM at the lower tested cesium concentrations and then falls on the engineeredform predictions of ZAM at the highest tested cesium concentration. This behavior can not be explained at this time, but does indicate to some extent why there has been a lot of confusion historically over the issue of whether or not a dilution factor is required. The low concentration data would suggest that no dilution effects occur (i.e., a dilution factor of unity), while the highest concentration indicates a dilution effect of $68 \%$ occurs.

Batch contact tests based on some of the earlier CST forms was performed by McCabe (1995) for powder-form CST (DG-112) and by McCabe (1997) for engineered-form CST (38b). Both sets of tests were done at $\sim 25 \mathrm{C}$ using the same SRS simulant. The composition of the SRS simulant used in given in Table $\mathrm{C}-1$ and the test data is listed in Table $\mathrm{C}-8$. A comparison of the data to ZAM predictions is shown in Figure C-12. The ZAM predictions compare favorably to the data, where the data supports the use of a constant dilution factor of $\sim 68 \%$.

Some of the key properties for the various CST materials considered above is provided in Table C-9.

\section{C.5 Strontium Loading Curve Comparisons}

Walker et al. (2001) also performed a series of batch contact tests at $36.2 \mathrm{C}$ using the SRSaverage simulant spiked with $\mathrm{Sr}^{+2}$ at an initial concentration of $1.141 \times 10^{-5} \mathrm{M}$. Measured strontium $\mathrm{K}_{\mathrm{d}}$ and loading values are listed in Table C-10. ZAM predictions for both the powerform and engineered-form were also computed and a comparison to the experimental data is provided in Figure C-13. As shown in Figure C-13, the three different engineered-forms have similar strontium loading values; however, the powder-form values appear to be inconsistent when compared to the engineered-form values.

In general the ZAM predictions have similar behavior when viewed with the engineered-form data (i.e., loading values are somewhat in the same ballpark). However, overall the comparisons between the data and/or predictions are poor. 
Preliminary Ion Exchange Modeling for Removal of Cesium from

Revision (Date):

Table C-1. Ionic species molar concentrations for simulated waste solutions used in ZAM batch contact simulations for estimating the dilution factor.

\begin{tabular}{|c|c|c|c|c|c|c|}
\hline $\begin{array}{l}\text { Species } \\
\text { ID }\end{array}$ & $\begin{array}{c}\text { SRS } \\
\text { Average } \\
\text { simulant } \\
{[\mathrm{M}]}\end{array}$ & $\begin{array}{c}\text { SRS High } \\
\text { OH }^{-} \\
\text {simulant } \\
{[\mathrm{M}]}\end{array}$ & $\begin{array}{c}\text { SRS Tank } \\
44 \text { sample } \\
{[\mathrm{M}]}\end{array}$ & $\begin{array}{c}\text { UOP } \\
\text { simulant } \\
{[\mathrm{M}]}\end{array}$ & $\begin{array}{c}\text { McCabe } \\
\text { simulant } \\
{[\mathrm{M}]}\end{array}$ & $\begin{array}{c}\text { TAM } \\
\text { Exp1 } \\
\text { [M] }\end{array}$ \\
\hline \multicolumn{7}{|l|}{ Cations } \\
\hline $\mathrm{Na}^{+}$ & 5.6 & 5.6 & 5.4 & 5.7 & 5.55 & 5.7 \\
\hline $\mathrm{Cs}^{+\mathrm{a}}$ & $1.4 \times 10^{-4}$ & $3.7 \times 10^{-4}$ & $3.51 \times 10^{-4}$ & $7.4403 \times 10^{-4}$ & $2.4 \times 10^{-4}$ & $1.0 \times 10^{-4}$ \\
\hline $\mathrm{K}^{+}$ & 0.015 & 0.03 & 0.051 & - & 0.015 & - \\
\hline $\mathrm{H}^{+}$ & $5.2 \times 10^{-15}$ & $3.3 \times 10^{-15}$ & $2.3 \times 10^{-15}$ & $1.7 \times 10^{-15}$ & $3.4 \times 10^{-15}$ & $1.7 \times 10^{-14}$ \\
\hline \multicolumn{7}{|l|}{ Anions } \\
\hline $\mathrm{NO}_{3}^{-}$ & 2.14 & 1.1 & 0.37 & 5.1 & 1.2152 & 5.1 \\
\hline $\mathrm{NO}_{2}^{-}$ & 0.52 & 0.74 & 0.35 & - & 0.71 & - \\
\hline $\mathrm{Cl}^{-}$ & 0.025 & $3.637 \times 10^{-2}$ & 0.009 & $7.4403 \times 10^{-4}$ & - & - \\
\hline $\mathrm{F}^{-}$ & 0.032 & 0.01 & - & - & - & - \\
\hline $\mathrm{OH}^{-}$(free) & 1.938 & 3.05 & 4.3 & 0.6 & 2.9 & 0.6 \\
\hline $\mathrm{Al}(\mathrm{OH})_{4}^{-}$ & 0.31 & 0.27 & 0.126 & - & - & - \\
\hline $\mathrm{CO}_{3}{ }^{2-}$ & 0.16 & 0.17 & 0.1412 & - & 0.2 & - \\
\hline $\mathrm{SO}_{4}{ }^{2-}$ & 0.15 & 0.03 & 0.001 & - & 0.17 & - \\
\hline $\mathrm{PO}_{4}{ }^{3-}$ & 0.01 & 0.008 & 0.0001 & - & - & - \\
\hline Cations $=$ & 5.615 & 5.630 & 5.451 & 5.701 & 5.565 & 5.700 \\
\hline Anions $=$ & -5.615 & -5.630 & -5.451 & -5.701 & $\frac{.005}{-5.565}$ & $\frac{-7.700}{-5.700}$ \\
\hline Sum $=$ & 0.000 & 0.000 & 0.000 & 0.000 & 0.000 & 0.000 \\
\hline
\end{tabular}

${ }^{\text {a }}$ For varying Cs the nominal SRS-average value for $1 \times 10^{-4} \mathrm{M}$ is adjusted where the total concentration of Cs plus $\mathrm{Na}$ is maintained constant. No variation in liquid sample composition occurred for the UOP experiments. 
Table C-2. Cesium batch contact test data taken by Walker et al. (2001) for various batches of CST material in contact with SRS-average simulated waste samples at $36.2 \mathrm{C}$.

\begin{tabular}{|c|c|c|c|c|c|}
\hline CST Form & Batch Name ${ }^{a}$ & $\begin{array}{c}\text { Na Concentration } \\
{[\mathrm{M}]}\end{array}$ & $\begin{array}{c}\text { Cs Equilibrium } \\
\text { Concentration } \\
{[\mathrm{M}]}\end{array}$ & $\begin{array}{c}\text { Cs K } K_{d} \\
\left(\mathrm{ml} / \mathrm{g}_{\mathrm{CST}}\right)\end{array}$ & $\begin{array}{c}\text { Cs loading } \\
\left.\text { (mmole } / \mathrm{g}_{\mathrm{CST}}\right)\end{array}$ \\
\hline \multirow[t]{8}{*}{ powder } & \multirow{8}{*}{$\begin{array}{c}\text { New IE-910 } \\
(?)\end{array}$} & 5.59939 & $3.8557 \mathrm{E}-05$ & 2040.98 & $7.8695 \mathrm{E}-02$ \\
\hline & & 5.59939 & $4.0307 \mathrm{E}-05$ & 2038.93 & $8.2183 \mathrm{E}-02$ \\
\hline & & 5.59999 & $9.0107 \mathrm{E}-06$ & 1747.32 & $1.5745 \mathrm{E}-02$ \\
\hline & & 5.59999 & 8.9799E-06 & 1762.20 & $1.5824 \mathrm{E}-02$ \\
\hline & & 5.60011 & $1.8895 \mathrm{E}-06$ & 1682.41 & $3.1789 \mathrm{E}-03$ \\
\hline & & 5.60011 & $1.6621 \mathrm{E}-06$ & 1937.59 & $3.2204 \mathrm{E}-03$ \\
\hline & & 5.60013 & 3.7494E-07 & 1689.98 & $6.3365 \mathrm{E}-04$ \\
\hline & & 5.60013 & $3.5027 \mathrm{E}-07$ & 1758.94 & $6.1610 \mathrm{E}-04$ \\
\hline \multirow[t]{8}{*}{ Engineered } & \multirow{8}{*}{$\begin{array}{c}\text { Baseline } \\
(9090-76)\end{array}$} & 5.59939 & $\begin{array}{c}5.7745 \mathrm{E}-05 \\
\end{array}$ & 1508.1 & $\begin{array}{c}8.7083 \mathrm{E}-02 \\
\end{array}$ \\
\hline & & 5.59939 & $6.2271 \mathrm{E}-05$ & 1370.2 & $8.5321 \mathrm{E}-02$ \\
\hline & & 5.59999 & $1.3578 \mathrm{E}-05$ & 1231.9 & $1.6727 \mathrm{E}-02$ \\
\hline & & 5.59999 & $1.5117 \mathrm{E}-05$ & 1098.2 & $1.6602 \mathrm{E}-02$ \\
\hline & & 5.60011 & $2.6678 \mathrm{E}-06$ & 1233.5 & $3.2906 \mathrm{E}-03$ \\
\hline & & 5.60011 & $2.6514 \mathrm{E}-06$ & 1207.3 & $3.2010 \mathrm{E}-03$ \\
\hline & & 5.60013 & $6.5170 \mathrm{E}-07$ & 1000.3 & $6.5189 \mathrm{E}-04$ \\
\hline & & 5.60013 & 5.8351E-07 & 1105.9 & $6.4530 \mathrm{E}-04$ \\
\hline \multirow[t]{8}{*}{ Engineered } & \multirow{8}{*}{$\begin{array}{l}\text { New IE-911 } \\
(30950-49)\end{array}$} & 5.59939 & 5.6684E-05 & 1373.3 & 7.7847E-02 \\
\hline & & 5.59939 & $6.3489 \mathrm{E}-05$ & 1248.8 & $7.9283 \mathrm{E}-02$ \\
\hline & & 5.59999 & $1.4273 \mathrm{E}-05$ & 1072.8 & $1.5311 \mathrm{E}-02$ \\
\hline & & 5.59999 & $1.3657 \mathrm{E}-05$ & 1131.7 & $1.5456 \mathrm{E}-02$ \\
\hline & & 5.60011 & $2.6940 \mathrm{E}-06$ & 1133.6 & $3.0540 \mathrm{E}-03$ \\
\hline & & 5.60011 & $2.7748 \mathrm{E}-06$ & 1109.0 & $3.0773 \mathrm{E}-03$ \\
\hline & & 5.60013 & $6.1033 \mathrm{E}-07$ & 980.1 & 5.9819E-04 \\
\hline & & 5.60013 & $5.8064 \mathrm{E}-07$ & 1045.8 & $6.0726 \mathrm{E}-04$ \\
\hline \multirow[t]{8}{*}{ Engineered } & \multirow{8}{*}{$\begin{array}{l}\text { Old IE-911 } \\
(99-9)\end{array}$} & 5.59939 & 5.3253E-05 & 1539.0 & $8.1956 \mathrm{E}-02$ \\
\hline & & 5.59939 & $5.3145 \mathrm{E}-05$ & 1514.0 & $8.0462 \mathrm{E}-02$ \\
\hline & & 5.59999 & $1.1487 \mathrm{E}-05$ & 1424.0 & $1.6357 \mathrm{E}-02$ \\
\hline & & 5.59999 & $1.1315 \mathrm{E}-05$ & 1416.6 & $1.6028 \mathrm{E}-02$ \\
\hline & & 5.60011 & $2.1228 \mathrm{E}-06$ & 1523.7 & $3.2345 \mathrm{E}-03$ \\
\hline & & 5.60011 & $2.0731 \mathrm{E}-06$ & 1555.5 & $3.2246 \mathrm{E}-03$ \\
\hline & & 5.60013 & $4.3556 \mathrm{E}-07$ & 1486.6 & $6.4750 \mathrm{E}-04$ \\
\hline & & 5.60013 & $4.3003 \mathrm{E}-07$ & 1465.8 & $6.3033 \mathrm{E}-04$ \\
\hline
\end{tabular}

${ }^{a}$ The engineered-forms Baseline IE-911 and New IE-911 were made using the power-form New IE-910 material. The Old IE-911 engineered-form material was made using an earlier batch of power-form IE-910. 
Table C-3. Cesium batch contact test data taken by Walker et al. (2001) for various batches of CST material in contact with SRS-average simulated waste samples at different temperatures.

\begin{tabular}{|c|c|c|c|c|c|c|}
\hline CST Form & Batch Name ${ }^{a}$ & $\begin{array}{c}\text { Temperature } \\
\text { (C) }\end{array}$ & $\begin{array}{c}\text { Na Equilibrium } \\
\text { Concentration } \\
{[\mathrm{M}]}\end{array}$ & $\begin{array}{c}\text { Cs Equilibrium } \\
\text { Concentration } \\
{[\mathrm{M}]}\end{array}$ & $\begin{array}{c}\text { Cs K } K_{d} \\
\left(\mathrm{ml} / \mathrm{g}_{\mathrm{CST}}\right)\end{array}$ & $\begin{array}{l}\text { Cs loading } \\
\left.\text { (mmole/g } / \mathrm{g}_{\mathrm{CST}}\right)\end{array}$ \\
\hline \multirow[t]{6}{*}{ powder } & \multirow{6}{*}{$\begin{array}{c}\text { New IE-910 } \\
(?)\end{array}$} & 36.2 & 5.59939 & $3.8557 \mathrm{E}-05$ & 2040.98 & $7.8695 \mathrm{E}-02$ \\
\hline & & 36.2 & 5.59939 & 4.0307E-05 & 2038.93 & $8.2183 \mathrm{E}-02$ \\
\hline & & 30.2 & 5.59939 & $4.0075 \mathrm{E}-05$ & 2056.68 & $8.2420 \mathrm{E}-02$ \\
\hline & & 30.2 & 5.59939 & $3.8060 \mathrm{E}-05$ & 2175.98 & $8.2817 \mathrm{E}-02$ \\
\hline & & 26.7 & 5.59940 & $3.4254 \mathrm{E}-05$ & 2246.30 & 7.6944E-02 \\
\hline & & 26.7 & 5.59940 & $3.4254 \mathrm{E}-05$ & 2369.63 & $8.1169 \mathrm{E}-02$ \\
\hline \multirow[t]{6}{*}{ Engineered } & \multirow{6}{*}{$\begin{array}{l}\text { Baseline } \\
(9090-76)\end{array}$} & 36.2 & 5.59939 & $\begin{array}{l}5.7745 \mathrm{E}-05 \\
\end{array}$ & 1508.07 & 8.7083E-02 \\
\hline & & 36.2 & 5.59939 & $6.2271 \mathrm{E}-05$ & 1370.16 & $8.5321 \mathrm{E}-02$ \\
\hline & & 30.2 & 5.59939 & $4.9254 \mathrm{E}-05$ & 1807.22 & $8.9012 \mathrm{E}-02$ \\
\hline & & 30.2 & 5.59939 & $5.0075 \mathrm{E}-05$ & 1773.76 & $8.8820 \mathrm{E}-02$ \\
\hline & & 26.7 & 5.59940 & 4.7239E-05 & 1839.95 & $8.6917 \mathrm{E}-02$ \\
\hline & & 26.7 & 5.59940 & $4.3881 \mathrm{E}-05$ & 1933.33 & $8.4836 \mathrm{E}-02$ \\
\hline \multirow[t]{6}{*}{ Engineered } & \multirow{6}{*}{$\begin{array}{l}\text { New IE-911 } \\
(30950-49)\end{array}$} & 36.2 & $\begin{array}{l}5.59939 \\
\end{array}$ & 5.6684E-05 & 1373.34 & 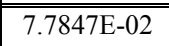 \\
\hline & & 36.2 & 5.59939 & $6.3489 \mathrm{E}-05$ & 1248.78 & $7.9283 \mathrm{E}-02$ \\
\hline & & 30.2 & 5.59939 & $5.0821 \mathrm{E}-05$ & 1576.51 & $8.0120 \mathrm{E}-02$ \\
\hline & & 30.2 & 5.59939 & 5.1493E-05 & 1571.19 & $8.0905 \mathrm{E}-02$ \\
\hline & & 26.7 & 5.59940 & 4.6343E-05 & 1748.13 & $8.1014 \mathrm{E}-02$ \\
\hline & & 26.7 & 5.59940 & $4.6791 \mathrm{E}-05$ & 1698.47 & 7.9473E-02 \\
\hline \multirow[t]{6}{*}{ Engineered } & \multirow{6}{*}{$\begin{array}{l}\text { Old IE-911 } \\
(99-9)\end{array}$} & 36.2 & 5.59939 & $\begin{array}{l}5.3253 \mathrm{E}-05 \\
\end{array}$ & 1538.99 & $8.1956 \mathrm{E}-02$ \\
\hline & & 36.2 & 5.59939 & $5.3145 \mathrm{E}-05$ & 1514.02 & $8.0462 \mathrm{E}-02$ \\
\hline & & 30.2 & 5.59939 & $4.2985 \mathrm{E}-05$ & 1875.92 & $8.0637 \mathrm{E}-02$ \\
\hline & & 30.2 & 5.59939 & $4.1418 \mathrm{E}-05$ & 1945.68 & $8.0586 \mathrm{E}-02$ \\
\hline & & 26.7 & 5.59940 & $4.1418 \mathrm{E}-05$ & 1982.71 & $8.2120 \mathrm{E}-02$ \\
\hline & & 26.7 & 5.59940 & $3.8955 \mathrm{E}-05$ & 2101.12 & $8.1850 \mathrm{E}-02$ \\
\hline
\end{tabular}

a The engineered-forms Baseline IE-911 and New IE-911 were made using the power-form New IE-910 material. The Old IE-911 engineered-form material was made using an earlier batch of power-form IE-910.

Table C-4. Cesium batch contact test data taken by Fondeur et al. (2000) for two batches of CST material in contact with SRS-average simulated waste samples at $25 \mathrm{C}$.

\begin{tabular}{|c|c|c|c|c|c|c|}
\hline CST Form & Batch Name & $\begin{array}{c}\text { Temperature } \\
\text { (C) }\end{array}$ & $\begin{array}{c}\text { Na Equilibrium } \\
\text { Concentration } \\
{[\mathbf{M}]}\end{array}$ & $\begin{array}{c}\text { Cs Equilibrium } \\
\text { Concentration } \\
{[\mathbf{M}]}\end{array}$ & $\begin{array}{c}\mathbf{C s ~}_{\mathbf{d}} \\
\left(\mathbf{m l} / \mathbf{g}_{\mathbf{c S T}}\right)\end{array}$ & $\begin{array}{c}\text { Cs loading } \\
\left(\mathbf{m m o l e} / \mathbf{g}_{\text {CST }}\right)\end{array}$ \\
\hline powder & $\begin{array}{c}\text { New IE-910 } \\
(?)\end{array}$ & 25.0 & 5.6 & $1.2243 \mathrm{E}-05$ & 2087.0 & $2.5551 \mathrm{E}-02$ \\
\hline \hline Engineered & $\begin{array}{c}\text { Baseline } \\
(9090-76)\end{array}$ & 25.0 & 5.6 & $1.5909 \mathrm{E}-05$ & 1560.0 & $2.4818 \mathrm{E}-02$ \\
\hline
\end{tabular}

${ }^{a}$ The engineered-form Baseline IE-911 was made using the power-form New IE-910 material. 
Table C-5. Power-law coefficients based on cesium loading data taken by Walker et al. (2001) for various batches of CST material in contact with SRS-average simulated waste samples at $36.2 \mathrm{C}$.

\begin{tabular}{|c|c|c|c|c|}
\hline CST Form & Batch Name & $\begin{array}{c}\text { Power-law } \\
\text { coefficient } \\
\text { a }\end{array}$ & $\begin{array}{c}\text { Power-law } \\
\text { coefficient } \\
\text { b }\end{array}$ & $\begin{array}{l}\text { Integral average } \\
\text { dilution factor }\end{array}$ \\
\hline powder & $\begin{array}{c}\text { New IE-910 } \\
(?)\end{array}$ & 2631.6 & 1.0293 & $\begin{array}{c}1.0 \\
\text { (reference) }\end{array}$ \\
\hline Engineered & $\begin{array}{l}\text { Baseline } \\
(9090-76)\end{array}$ & 2370.5 & 1.0560 & 0.680 \\
\hline Engineered & $\begin{array}{c}\text { New IE-911 } \\
(30950-49)\end{array}$ & 2011.7 & 1.0479 & 0.628 \\
\hline Engineered & $\begin{array}{c}\text { Old IE-911 } \\
(99-9)\end{array}$ & 1506.8 & 1.0009 & 0.772 \\
\hline
\end{tabular}

${ }^{\text {a }}$ The integrated average dilution factor, based on Eq. (C-5b), was obtained using as the lower and upper cesium concentration integration limits, $3 \times 10^{-7} \mathrm{M}$ and $7 \times 10^{-5} \mathrm{M}$, respectively. The power-law fit for the New IE-910 powder is used for each dilution factor estimate.

Table C-6. Estimated dilution factors based on an "effective" single component homovalent isotherm model of the cesium loading curve computed for selected SRS batch contact data ${ }^{\text {a }}$.

\begin{tabular}{|c|c|c|c|c|c|c|c|c|}
\hline $\begin{array}{l}\text { CST } \\
\text { Form }\end{array}$ & $\begin{array}{l}\text { Batch } \\
\text { Name }\end{array}$ & $\begin{array}{l}\text { Liquid } \\
\text { sample }\end{array}$ & $\begin{array}{c}T \\
(C)\end{array}$ & $\begin{array}{c}\text { Equilibrium } \\
\mathrm{Cs}^{\mathrm{c}} \\
{[\mathrm{M}]}\end{array}$ & $\begin{array}{c}\text { Cs loading } \\
\left.\text { (mmole } / \mathrm{g}_{\mathrm{CST}}\right)\end{array}$ & $\begin{array}{c}\text { Beta } \\
\text { parameter }^{d}\end{array}$ & $\begin{array}{c}\text { Total } \\
\text { capacity } \\
\text { parameter }\end{array}$ & $\begin{array}{l}\text { Dilution } \\
\text { Factor }\end{array}$ \\
\hline Powder & $\begin{array}{c}\text { New-IE-910 } \\
(?)\end{array}$ & SRS-Avg & $\begin{array}{l}36.2 \\
30.2 \\
26.7 \\
25.0 \\
36.2\end{array}$ & - & - & $\begin{array}{c}3.057 \mathrm{E}-04^{\mathbf{b}} \\
2.352 \mathrm{E}-04 \\
2.170 \mathrm{E}-04 \\
2.657 \mathrm{E}-04 \\
5.3290 \mathrm{E}-04\end{array}$ & $\begin{array}{c}0.580 \\
\text { (specified) }\end{array}$ & $\begin{array}{c}1.000 \\
\text { (reference) }\end{array}$ \\
\hline \multirow[t]{8}{*}{ Engineered } & $\begin{array}{c}\text { Baseline-IE-911 } \\
(9090-76)\end{array}$ & SRS-Avg & 36.2 & "6.0008E-05 & "8.6202E-02 & 2.4489E-04 & 0.438 & 0.755 \\
\hline & & SRS-Avg & 36.2 & $1.4347 \mathrm{E}-05$ & $1.6664 \mathrm{E}-02$ & $3.2154 \mathrm{E}-04$ & 0.390 & 0.673 \\
\hline & & SRS-Avg & 36.2 & $2.6596 \mathrm{E}-06$ & $3.2458 \mathrm{E}-03$ & $3.2012 \mathrm{E}-04$ & 0.394 & 0.679 \\
\hline & & SRS-Avg & 36.2 & $6.1761 \mathrm{E}-07$ & $6.4860 \mathrm{E}-04$ & $3.3620 \mathrm{E}-04$ & 0.354 & 0.610 \\
\hline & & UOP & 36.2 & $1.1555 \mathrm{E}-04$ & $7.7318 \mathrm{E}-02$ & $5.3290 \mathrm{E}-04$ & 0.434 & 0.748 \\
\hline & & SRS-Avg & 30.2 & $4.9664 \mathrm{E}-05$ & $8.8916 \mathrm{E}-02$ & $2.3519 \mathrm{E}-04$ & 0.510 & 0.879 \\
\hline & & SRS-Avg & 26.7 & $4.5560 \mathrm{E}-05$ & $8.5876 \mathrm{E}-02$ & $2.1705 \mathrm{E}-04$ & 0.495 & 0.853 \\
\hline & & SRS-Avg & 25.0 & $1.5909 \mathrm{E}-05$ & $2.4818 \mathrm{E}-02$ & $2.6567 \mathrm{E}-04$ & 0.439 & 0.757 \\
\hline \multirow[t]{7}{*}{ Engineered } & $\begin{array}{l}\text { New-IE-911 } \\
(30950-49)\end{array}$ & SRS-Avg & 36.2 & "6.0087E-05 & $7.8565 \mathrm{E}-02$ & 2.4489E-04 & 0.399 & 0.688 \\
\hline & & SRS-Avg & 36.2 & $1.3965 \mathrm{E}-05$ & $1.5383 \mathrm{E}-02$ & $3.2154 \mathrm{E}-04$ & 0.370 & 0.637 \\
\hline & & SRS-Avg & 36.2 & $2.7344 \mathrm{E}-06$ & $3.0656 \mathrm{E}-03$ & $3.2012 \mathrm{E}-04$ & 0.362 & 0.624 \\
\hline & & SRS-Avg & 36.2 & 5.9549E-07 & $6.0272 \mathrm{E}-04$ & $3.3620 \mathrm{E}-04$ & 0.341 & 0.588 \\
\hline & & UOP & 36.2 & $1.2488 \mathrm{E}-04$ & 7.1069E-02 & $5.3290 \mathrm{E}-04$ & 0.374 & 0.645 \\
\hline & & SRS-Avg & 30.2 & $5.1157 \mathrm{E}-05$ & $8.0512 \mathrm{E}-02$ & $2.3519 \mathrm{E}-04$ & 0.451 & 0.777 \\
\hline & & SRS-Avg & 26.7 & $4.6567 \mathrm{E}-05$ & $8.0244 \mathrm{E}-02$ & $2.1705 \mathrm{E}-04$ & 0.454 & 0.783 \\
\hline \multirow[t]{6}{*}{ Engineered } & $\begin{array}{l}\text { Old-IE-911 } \\
(99-9)\end{array}$ & SRS-Avg & 36.2 & 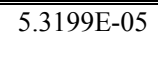 & "8.1209E-02 & "2.4489E-04 & 0.455 & 0.785 \\
\hline & & SRS-Avg & 36.2 & $1.1401 \mathrm{E}-05$ & $1.6193 \mathrm{E}-02$ & $3.2154 \mathrm{E}-04$ & 0.473 & 0.815 \\
\hline & & SRS-Avg & 36.2 & $2.0979 \mathrm{E}-06$ & $3.2296 \mathrm{E}-03$ & $3.2012 \mathrm{E}-04$ & 0.496 & 0.855 \\
\hline & & SRS-Avg & 36.2 & $4.3279 \mathrm{E}-07$ & $6.3892 \mathrm{E}-04$ & $3.3620 \mathrm{E}-04$ & 0.497 & 0.857 \\
\hline & & UOP & 36.2 & $9.6517 \mathrm{E}-05$ & 7.4439E-02 & $5.3290 \mathrm{E}-04$ & 0.485 & 0.837 \\
\hline & & SRS-Avg & 30.2 & $4.2201 \mathrm{E}-05$ & $8.0611 \mathrm{E}-02$ & $2.3519 \mathrm{E}-04$ & 0.530 & 0.914 \\
\hline
\end{tabular}


Preliminary Ion Exchange Modeling for Removal of Cesium from

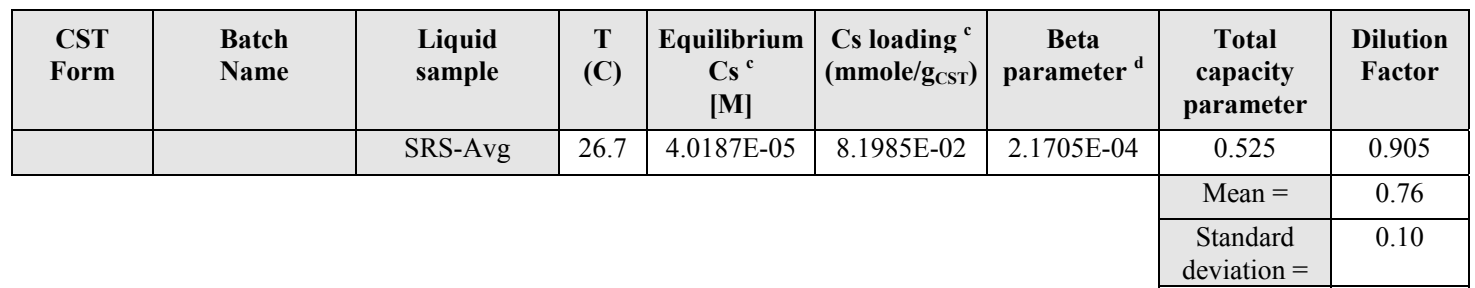

${ }^{a}$ Data obtained from Walker et al. (2001) and Fondeur et al. (2000).

${ }^{\mathrm{b}}$ Beta parameter value averaged over the cesium concentration range of $3 \times 10^{-7}$ to $7 \times 10^{-5} \mathrm{M}$, but is not used in calculating point dilution factor estimates.

${ }^{c}$ The cesium concentration and loading values are averages of the two tests performed for each data point (i.e., original sample plus its duplicate).

${ }^{\mathrm{c}}$ The beta parameter value used for each dilution factor estimate is based on powder-form data at the temperature of interest and the nearest cesium concentration available.

Table C-7. Cesium batch contact test data taken by Walker et al. (1997) for the Baseline CST material in contact with SRS Tank 44 actual waste samples at $31 \mathrm{C}^{\text {a }}$

\begin{tabular}{|c|c|c|c|c|c|}
\hline CST Form & Batch Name & $\begin{array}{c}\text { Na Concentration } \\
{[\mathrm{M}]}\end{array}$ & $\begin{array}{c}\text { Cs Equilibrium } \\
\text { Concentration } \\
{[\mathrm{M}]}\end{array}$ & $\begin{array}{c}\mathrm{Cs} \mathrm{K_{d }} \\
\left(\mathrm{ml} / \mathrm{g}_{\mathrm{CST}}\right)\end{array}$ & $\begin{array}{l}\text { Cs loading } \\
\left(\text { mmole } / \mathbf{g}_{\text {CST }}\right)\end{array}$ \\
\hline \multirow[t]{12}{*}{ Engineered } & \multirow{12}{*}{$\begin{array}{c}\text { Baseline } \\
(9090-76)\end{array}$} & 5.40 & $2.3239 \mathrm{E}-03$ & 147.1 & $3.4187 \mathrm{E}-01$ \\
\hline & & 5.40 & $2.2409 \mathrm{E}-03$ & 176.9 & $3.9642 \mathrm{E}-01$ \\
\hline & & 5.40 & $3.1525 \mathrm{E}-05$ & 2497.3 & $7.8727 \mathrm{E}-02$ \\
\hline & & 5.40 & $3.2742 \mathrm{E}-05$ & 2470.0 & $8.0873 \mathrm{E}-02$ \\
\hline & & 5.40 & $3.3638 \mathrm{E}-05$ & 2404.4 & $8.0879 \mathrm{E}-02$ \\
\hline & & 5.40 & $6.2606 \mathrm{E}-06$ & 2848.2 & $1.7831 \mathrm{E}-02$ \\
\hline & & 5.40 & 7.0744E-06 & 2637.1 & $1.8656 \mathrm{E}-02$ \\
\hline & & 5.40 & $6.3195 \mathrm{E}-06$ & 2805.8 & $1.7731 \mathrm{E}-02$ \\
\hline & & 5.40 & $2.8309 \mathrm{E}-06$ & 2743.0 & $7.7653 \mathrm{E}-03$ \\
\hline & & 5.40 & $2.7029 \mathrm{E}-06$ & 2831.9 & $7.6545 \mathrm{E}-03$ \\
\hline & & 5.40 & $1.0667 \mathrm{E}-07$ & 3135.1 & 3.3444E-04 \\
\hline & & 5.40 & $1.0940 \mathrm{E}-07$ & 3066.2 & $3.3543 \mathrm{E}-04$ \\
\hline
\end{tabular}

${ }^{\mathrm{a}}$ The solution density was $1.2015 \mathrm{~g} / \mathrm{ml}$ and its viscosity was $2.6 \mathrm{cP}$ at $31 \mathrm{C}$. 
Table C-8. Cesium batch contact test data taken by McCabe (1995 \& 1997) for two CST materials in contact with a SRS simulant at $25 \mathrm{C}$. $^{\text {a }}$

\begin{tabular}{|c|c|c|c|c|c|c|}
\hline CST Form & Batch Name ${ }^{a}$ & $\begin{array}{c}\text { Temperature } \\
\text { (C) }\end{array}$ & $\begin{array}{c}\text { Na Equilibrium } \\
\text { Concentration } \\
{[\mathrm{M}]}\end{array}$ & $\begin{array}{c}\text { Cs Equilibrium } \\
\text { Concentration } \\
{[\mathrm{M}]}\end{array}$ & $\underset{\left(\mathbf{m l} / \mathrm{g}_{\mathrm{CST}}\right)}{\operatorname{Cs~K_{d}}}$ & $\begin{array}{l}\text { Cs loading } \\
\left.\text { (mmole/g } \text { gST }_{\text {CST }}\right)\end{array}$ \\
\hline \multirow[t]{8}{*}{ Powder } & \multirow{8}{*}{$\begin{array}{c}\text { TAM-5 } \\
\text { (DG-112) }\end{array}$} & 25.0 & 5.599 & $3.8557 \mathrm{E}-05$ & 2041.0 & $7.8695 \mathrm{E}-02$ \\
\hline & & 25.0 & 5.599 & $4.0307 \mathrm{E}-05$ & 2038.9 & $8.2183 \mathrm{E}-02$ \\
\hline & & 25.0 & 5.600 & $9.0107 \mathrm{E}-06$ & 1747.3 & $1.5745 \mathrm{E}-02$ \\
\hline & & 25.0 & 5.600 & $8.9799 \mathrm{E}-06$ & 1762.2 & $1.5824 \mathrm{E}-02$ \\
\hline & & 25.0 & 5.600 & $1.8895 \mathrm{E}-06$ & 1682.4 & $3.1789 \mathrm{E}-03$ \\
\hline & & 25.0 & 5.600 & $1.6621 \mathrm{E}-06$ & 1937.6 & $3.2204 \mathrm{E}-03$ \\
\hline & & 25.0 & 5.600 & $3.7494 \mathrm{E}-07$ & 1690.0 & $6.3365 \mathrm{E}-04$ \\
\hline & & 25.0 & 5.600 & $3.5027 \mathrm{E}-07$ & 1758.9 & $6.1610 \mathrm{E}-04$ \\
\hline \multirow[t]{7}{*}{ Engineered } & \multirow{7}{*}{$\begin{array}{c}\text { Early batch } \\
(38 \mathrm{~B})\end{array}$} & 25.0 & 5.5500 & $3.6343 \mathrm{E}-05$ & 1503.6 & $5.4646 \mathrm{E}-02$ \\
\hline & & 25.0 & 5.5497 & $7.2761 \mathrm{E}-05$ & 1770.0 & $1.2879 \mathrm{E}-01$ \\
\hline & & 25.0 & 5.5495 & $1.1358 \mathrm{E}-04$ & 1281.9 & $1.4560 \mathrm{E}-01$ \\
\hline & & 25.0 & 5.5492 & $2.2881 \mathrm{E}-04$ & 850.9 & $1.9470 \mathrm{E}-01$ \\
\hline & & 25.0 & 5.5490 & $3.5940 \mathrm{E}-04$ & 641.7 & $2.3062 \mathrm{E}-01$ \\
\hline & & 25.0 & 5.5488 & $4.9321 \mathrm{E}-04$ & 491.4 & $2.4235 \mathrm{E}-01$ \\
\hline & & 25.0 & 5.5486 & $6.7448 \mathrm{E}-04$ & 367.6 & $2.4793 \mathrm{E}-01$ \\
\hline
\end{tabular}

${ }^{\mathrm{a}}$ These are CST materials early on in their development history.

Table C-9. Key CST exchange properties taken from literature.

\begin{tabular}{|c|c|c|c|c|c|}
\hline CST Form & Batch Name & $\begin{array}{c}\text { F Factor } \\
\mathbf{( - )}\end{array}$ & $\begin{array}{c}\text { Bulk Dry } \\
\text { Density } \\
\mathbf{( g / m l )}\end{array}$ & $\begin{array}{c}\text { Dilution Factor } \\
(-)\end{array}$ & $\begin{array}{c}\text { Cesium Total Ion- } \\
\text { Exchange } \\
\text { Capacity } \\
\text { bole/g) }\end{array}$ \\
\hline SuperLig $^{\circledast}$ 644 & $10-$-SM-171 & 0.9751 & 0.2238 & $\mathrm{Na}$ & 0.3333 \\
\hline powder & IE-910 & 0.9680 & 0.7738 & 1.0 & 0.580 \\
\hline powder & New-IE-910 & 0.8573 & - & 1.0 & 0.580 \\
\hline Engineered & IE-911 (38b) & 0.8870 & 1.1300 & not reported & not reported \\
\hline Engineered & IE-911 (08) & 0.8990 & 0.8999 & not reported & not reported \\
\hline Engineered & Baseline & 0.7875 & 1.00 & 0.68 & 0.3944 \\
\hline Engineered & New-IE-911 & 0.8493 & 1.00 & 0.63 & 0.3654 \\
\hline Engineered & Old-IE-911 & 0.8331 & 1.00 & 0.77 & 0.4466 \\
\hline
\end{tabular}

${ }^{a}$ Data obtained from Brown et al. (1996, Table 3.1) or Walker et al. (2001).

${ }^{\mathrm{b}}$ Data obtained from Zheng et al. (1996) and confirmed by running ZAM at a very large liquid cesium concentration for the powder-form and estimated for the engineered-forms.

${ }^{\mathrm{c}}$ During the manufacturing production process of IONSIV ${ }^{\circledR}$ IE-911 resin, an inert binder is added to CST powder to create an engineered form that is useable in ion-exchange columns. The additional inert binding material reduces the total Cs ion-exchange capacity and this reduction factor is referred to as its "dilution factor". 
Table C-10. Strontium batch contact test data taken by Walker et al. (2001) for various batches of CST material in contact with SRS-average simulated waste samples at $36.2 \mathrm{C}$.

\begin{tabular}{|c|c|c|c|c|c|}
\hline CST Form & Batch Name ${ }^{a}$ & $\begin{array}{c}\text { Na Concentration } \\
{[\mathrm{M}]}\end{array}$ & $\begin{array}{c}\text { Sr Equilibrium } \\
\text { Concentration } \\
\text { [M] }\end{array}$ & $\begin{array}{c}\mathrm{Sr} \mathrm{K}_{\mathrm{d}} \\
\left(\mathrm{ml} / \mathrm{g}_{\mathrm{CST}}\right)\end{array}$ & $\begin{array}{l}\text { Sr loading } \\
\text { (mmole/gCsT) }\end{array}$ \\
\hline \multirow[t]{6}{*}{ powder } & \multirow{6}{*}{$\begin{array}{c}\text { New IE-910 } \\
(?)\end{array}$} & 5.60 & $1.297 \mathrm{E}-07$ & 20096.5 & $2.606 \mathrm{E}-03$ \\
\hline & & 5.60 & $1.235 \mathrm{E}-07$ & 20545.1 & $2.537 \mathrm{E}-03$ \\
\hline & & 5.60 & $7.608 \mathrm{E}-08$ & 8682.3 & $6.605 \mathrm{E}-04$ \\
\hline & & 5.60 & $6.577 \mathrm{E}-08$ & 9952.4 & $6.546 \mathrm{E}-04$ \\
\hline & & 5.60 & $5.527 \mathrm{E}-08$ & 4781.3 & 2.643E-04 \\
\hline & & 5.60 & 5.694E-08 & 4629.6 & $2.636 \mathrm{E}-04$ \\
\hline \multirow[t]{6}{*}{ Engineered } & \multirow{6}{*}{$\begin{array}{l}\text { Baseline } \\
(9090-76)\end{array}$} & 5.60 & $2.519 \mathrm{E}-07$ & 11074.7 & $2.790 \mathrm{E}-03$ \\
\hline & & 5.60 & 2.499E-07 & 11124.0 & 2.779E-03 \\
\hline & & 5.60 & $1.241 \mathrm{E}-07$ & 5626.8 & $6.982 \mathrm{E}-04$ \\
\hline & & 5.60 & $1.085 \mathrm{E}-07$ & 6554.1 & $7.110 \mathrm{E}-04$ \\
\hline & & 5.60 & 4.781E-08 & 5991.8 & $2.865 \mathrm{E}-04$ \\
\hline & & 5.60 & 4.849E-08 & 5933.9 & $2.878 \mathrm{E}-04$ \\
\hline \multirow[t]{6}{*}{ Engineered } & \multirow{6}{*}{$\begin{array}{l}\text { New IE-911 } \\
(30950-49)\end{array}$} & 5.60 & $2.261 \mathrm{E}-07$ & 11445.2 & $2.588 \mathrm{E}-03$ \\
\hline & & 5.60 & $2.419 \mathrm{E}-07$ & 10144.2 & $2.454 \mathrm{E}-03$ \\
\hline & & 5.60 & $1.057 \mathrm{E}-07$ & 6215.3 & $6.571 \mathrm{E}-04$ \\
\hline & & 5.60 & $1.052 \mathrm{E}-07$ & 6266.2 & $6.594 \mathrm{E}-04$ \\
\hline & & 5.60 & $4.840 \mathrm{E}-08$ & 5520.3 & 2.672E-04 \\
\hline & & 5.60 & 5.272E-08 & 5062.5 & $2.669 \mathrm{E}-04$ \\
\hline \multirow[t]{6}{*}{ Engineered } & \multirow{6}{*}{$\begin{array}{l}\text { Old IE-911 } \\
\qquad(99-9)\end{array}$} & 5.60 & $2.659 \mathrm{E}-07$ & 9337.2 & $2.482 \mathrm{E}-03$ \\
\hline & & 5.60 & $2.560 \mathrm{E}-07$ & 10000.9 & $2.561 \mathrm{E}-03$ \\
\hline & & 5.60 & $1.222 \mathrm{E}-07$ & 5448.4 & $6.660 \mathrm{E}-04$ \\
\hline & & 5.60 & $1.094 \mathrm{E}-07$ & 6114.2 & 6.687E-04 \\
\hline & & 5.60 & 4.663E-08 & 5794.6 & 2.702E-04 \\
\hline & & 5.60 & 4.791E-08 & 5674.2 & 2.719E-04 \\
\hline
\end{tabular}

a The engineered-forms Baseline IE-911 and New IE-911 were made using the power-form New IE-910 material.

The Old IE-911 engineered-form material was made using an earlier batch of power-form IE-910.

${ }^{\mathrm{b}}$ The initial $\mathrm{Sr}^{+2}$ concentrations were all experimentally set to $1.141 \times 10^{-5} \mathrm{M}$. The actual free $\mathrm{Sr}^{+2}$ concentrations available for forming aqueous $\mathrm{SrOH}^{+}$are unknown, but were assumed to be equal to the total $\mathrm{Sr}^{+2}$ present. 


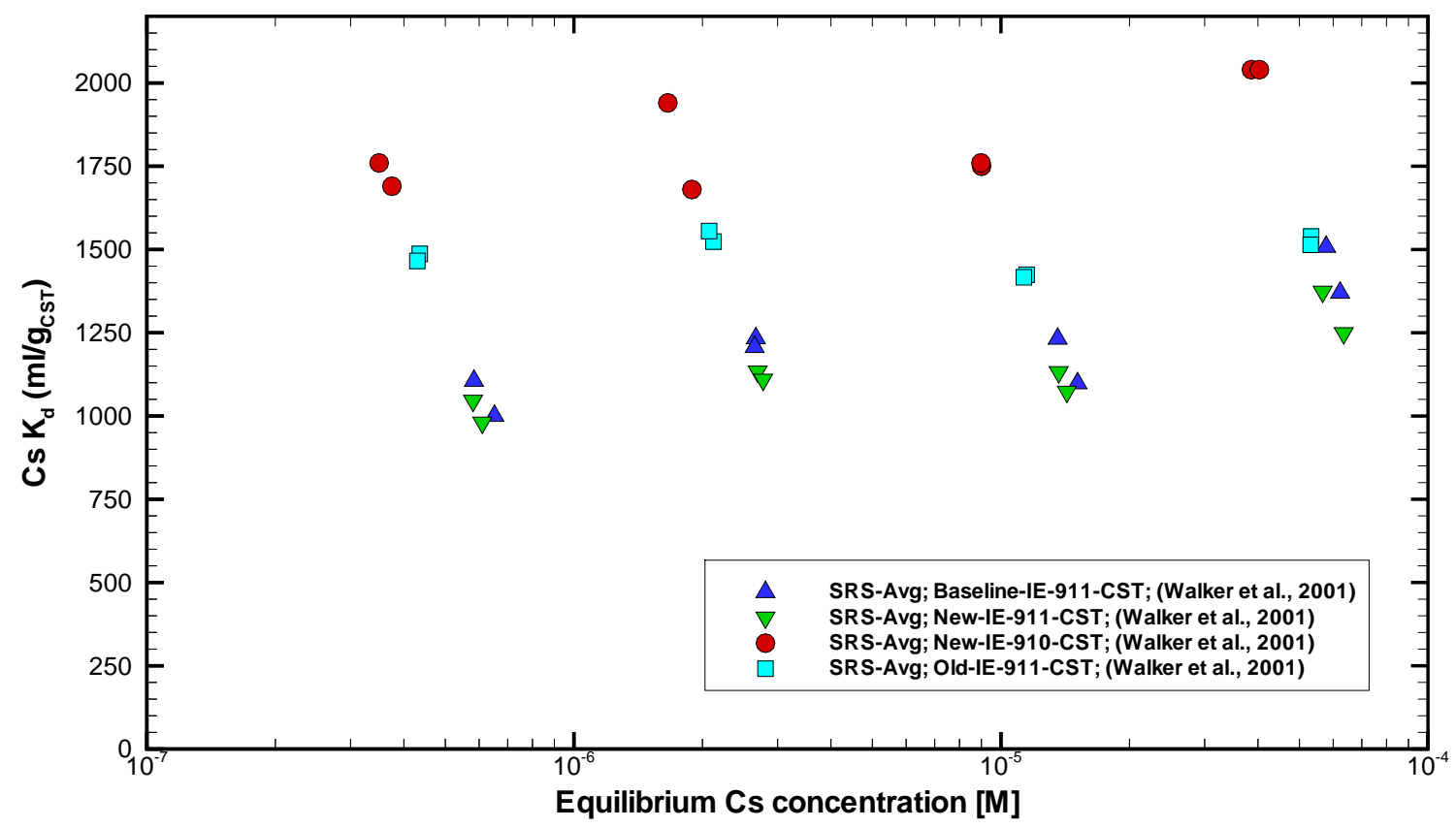

Figure C-1. Comparison of measured cesium $\mathrm{K}_{\mathrm{d}}$ values for several CST batches taken by Walker et al. (2001) in contact with a SRS-average simulated waste sample at $36.2 \mathrm{C}$.

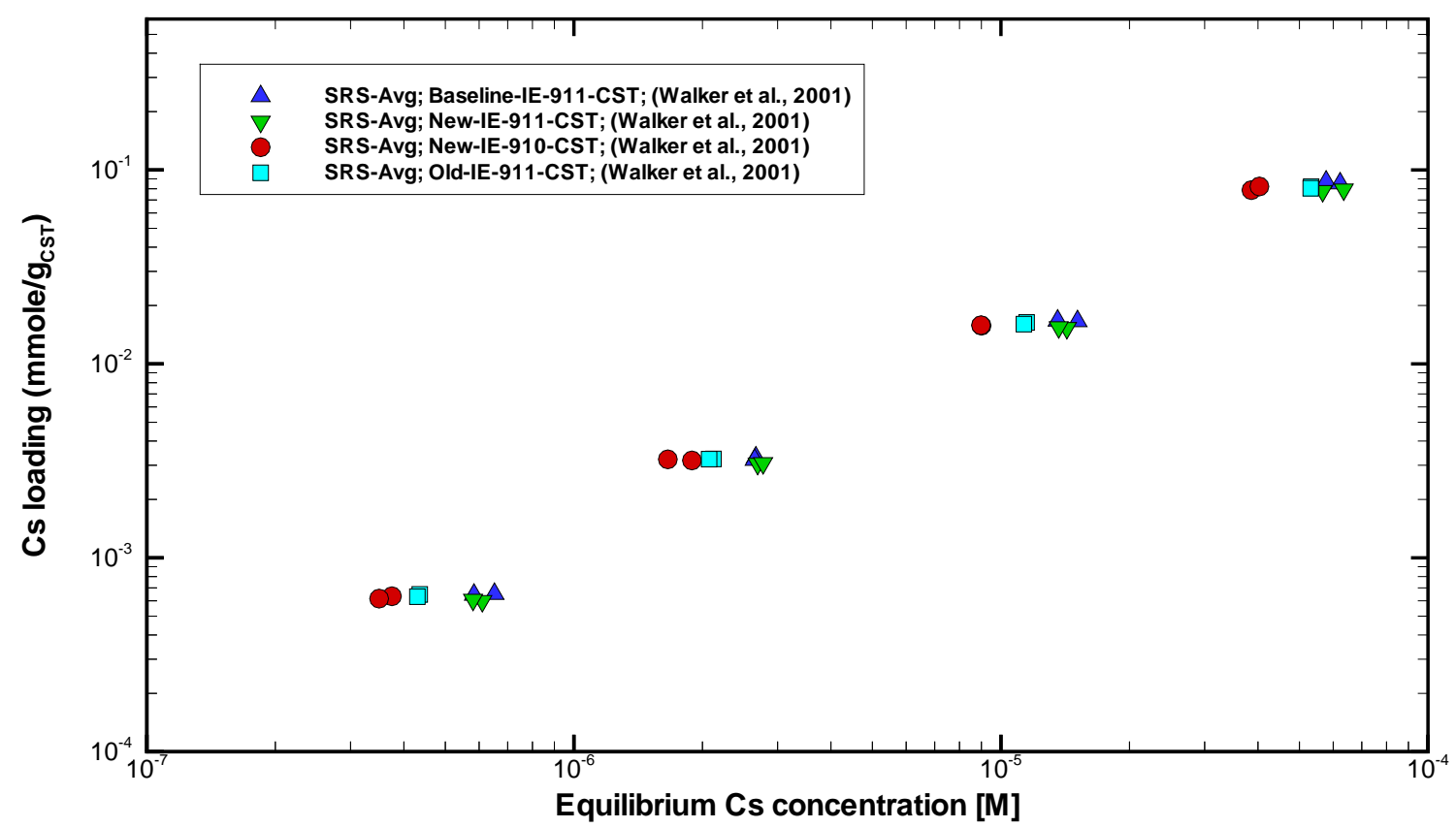

Figure C-2. Comparison of measured cesium loading values for several CST batches taken by Walker et al. (2001) in contact with a SRS-average simulated waste sample at 36.2 C. 


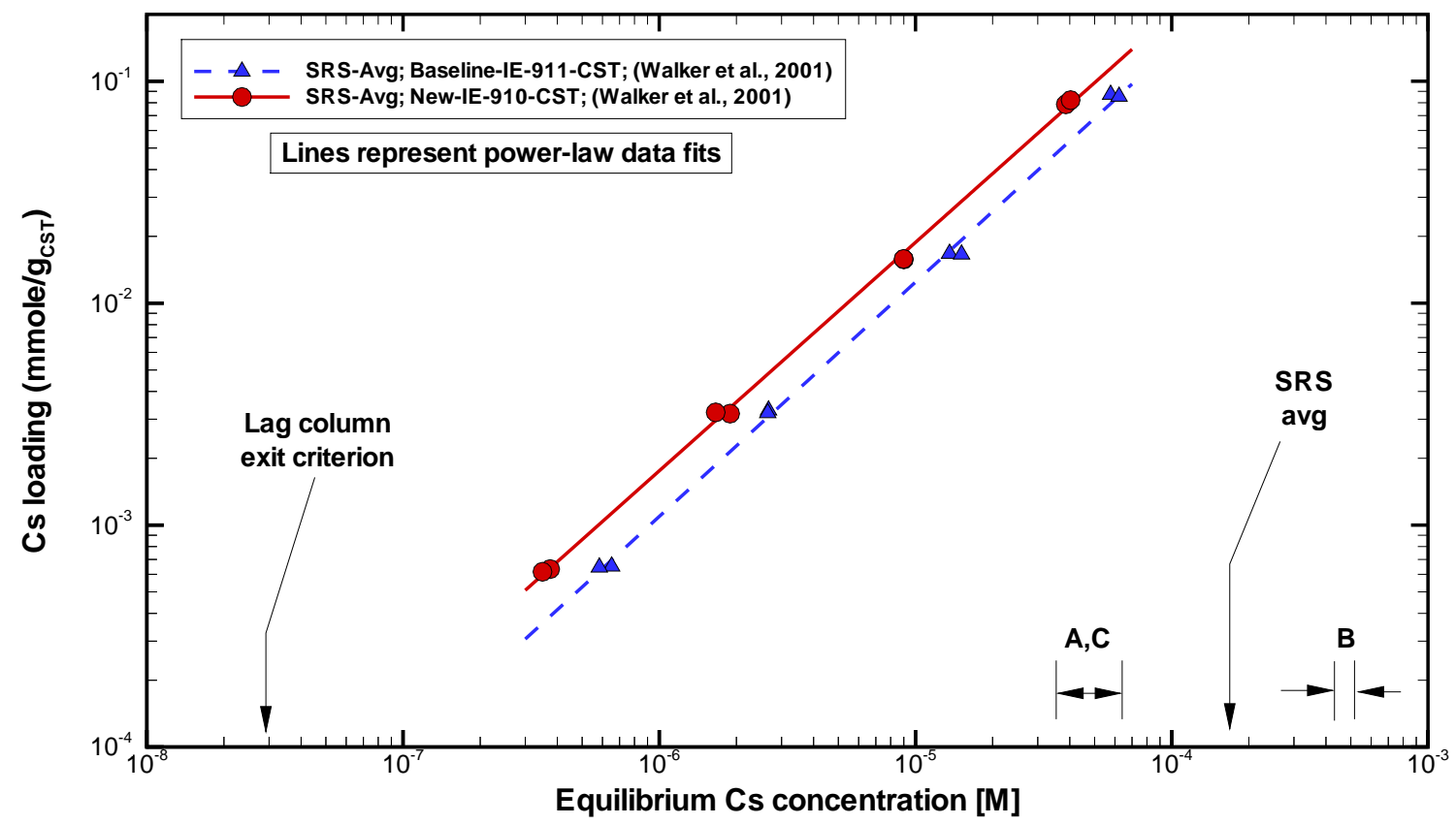

Figure C-3. Comparison of SRS-Avg simulant cesium CST loading data recently taken by Walker et al. (2001) to power-law fits through the data sets at $36.2 \mathrm{C}$.

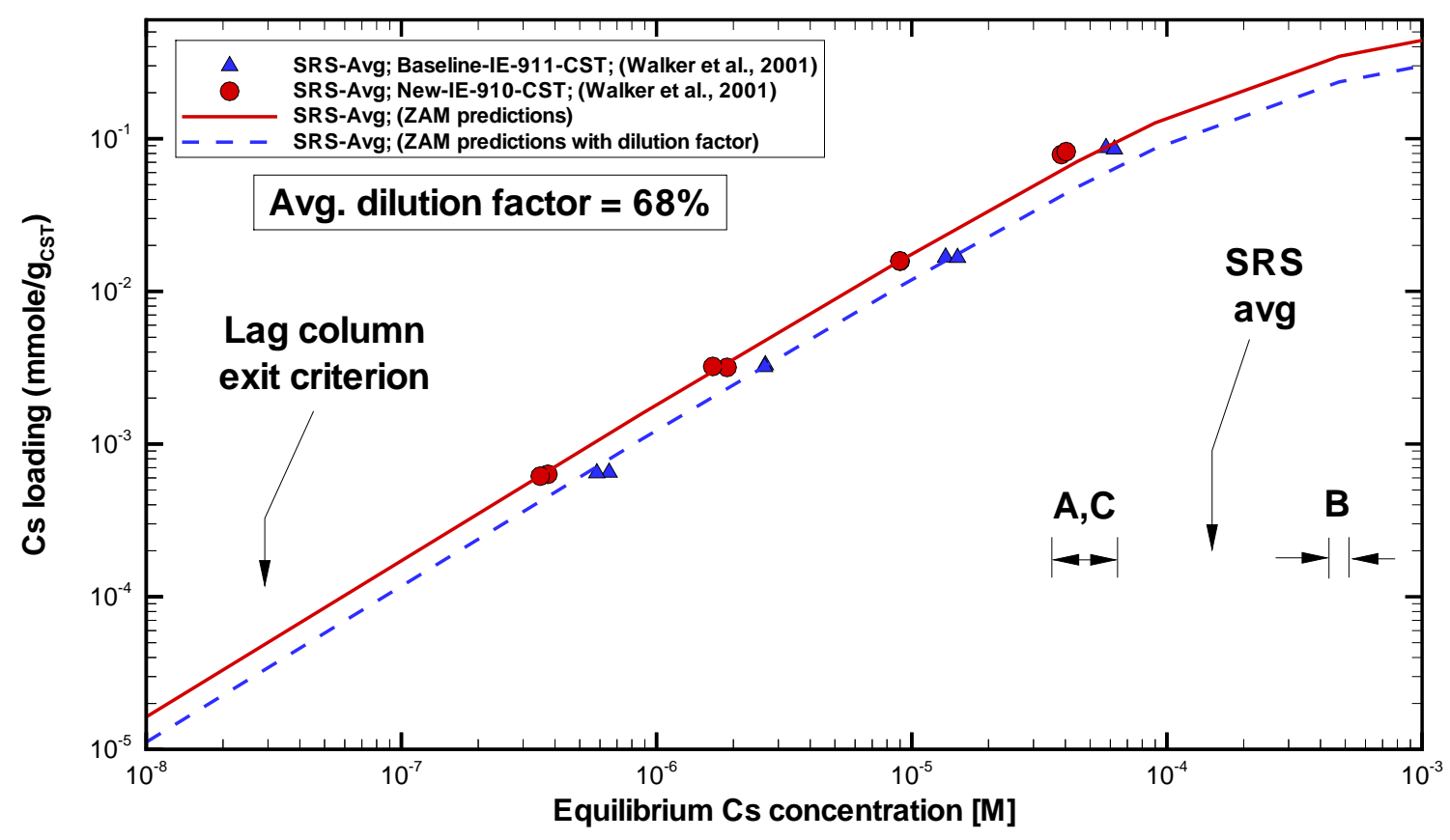

Figure C-4. Comparison of SRS-Avg simulant cesium CST loading data recently taken by Walker et al. (2001) to both best estimate and adjusted ZAM predictions at $36.2 \mathrm{C}$. 


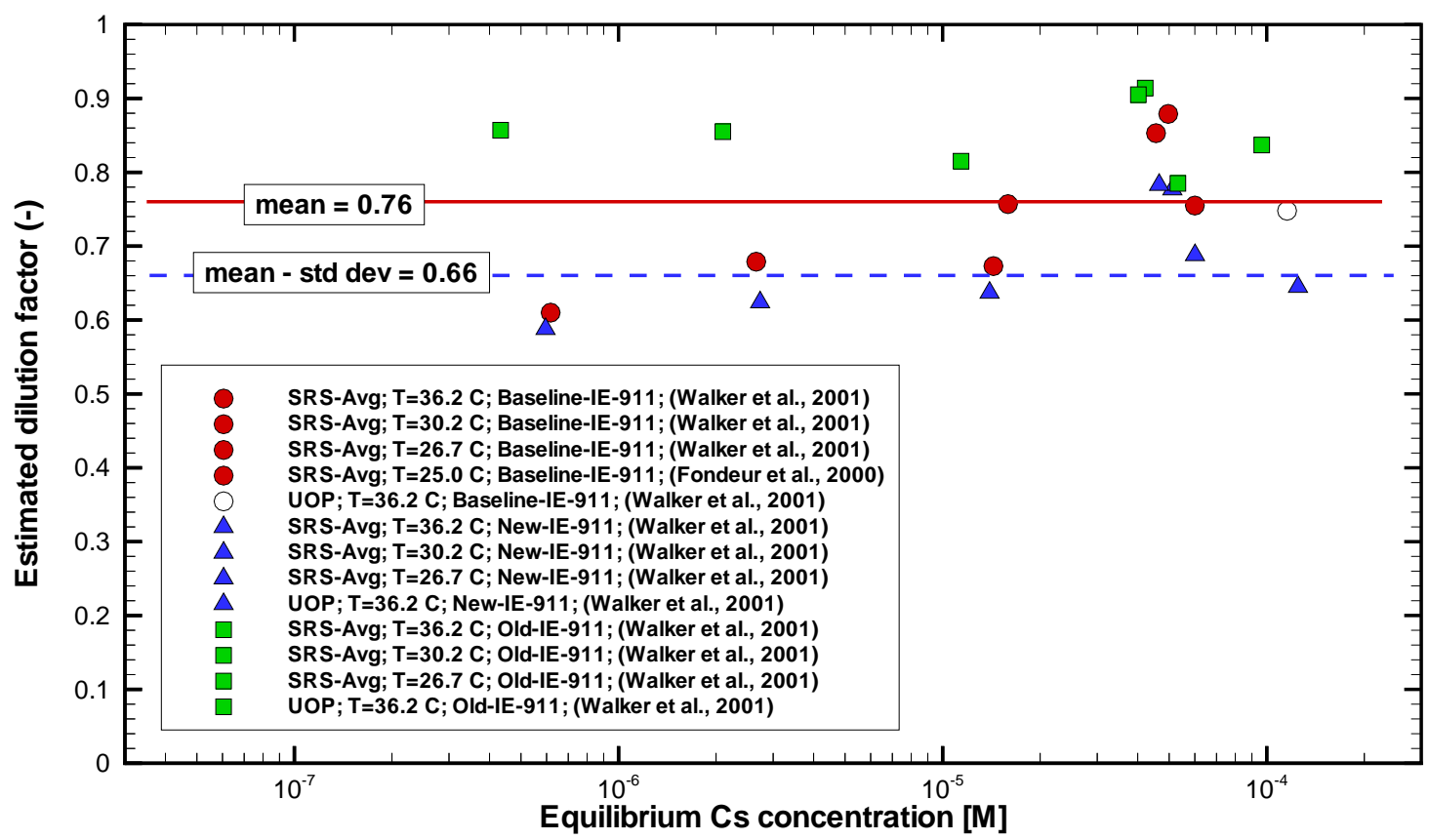

Figure C-5. Comparison of dilution factor for several CST batch contact data sets with respect to equilibrium cesium concentration.

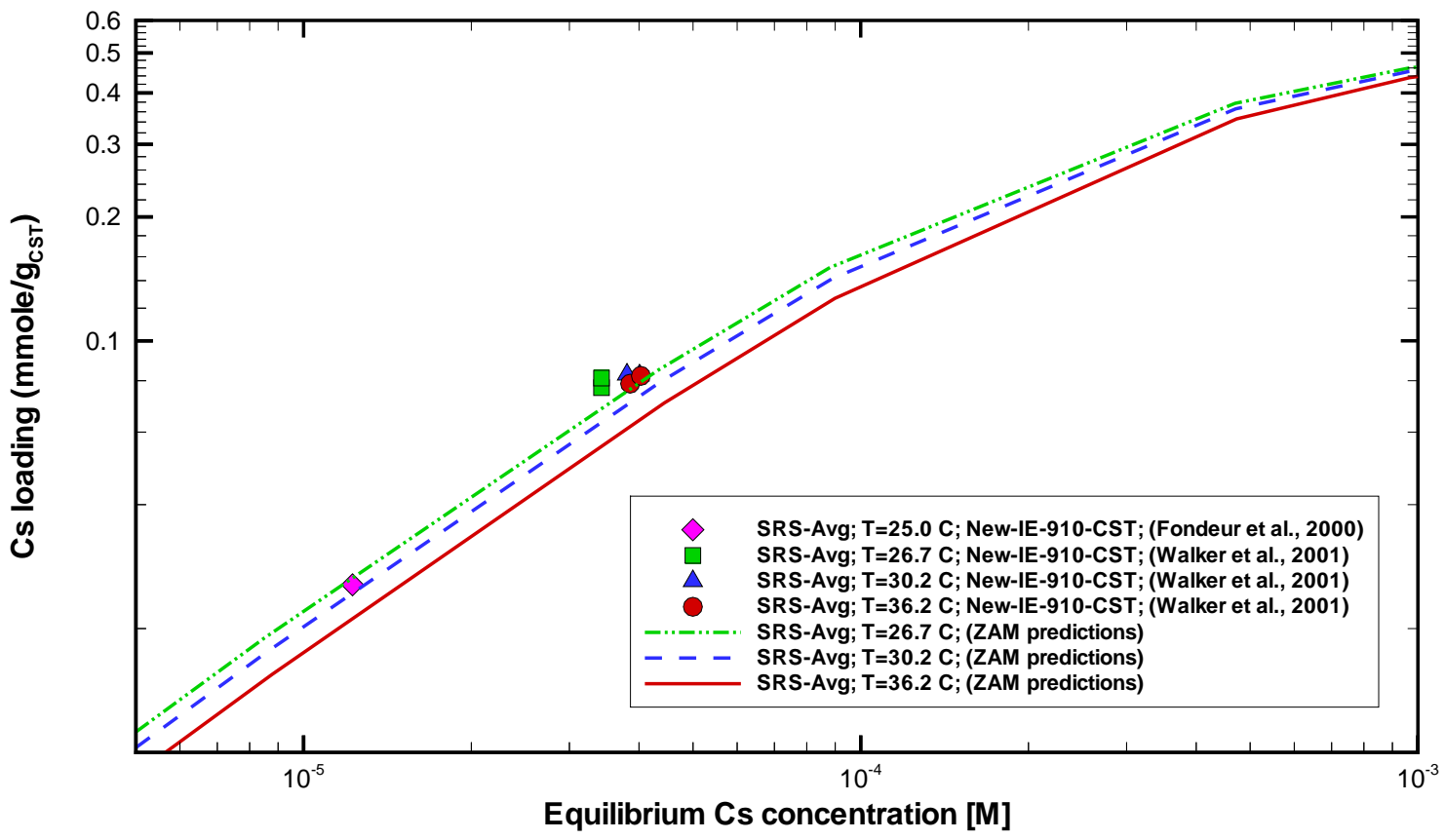

Figure C-6. Comparison of SRS-Avg simulant cesium CST (new powder-form) loading data taken by Fondeur et al. (2000) and Walker et al. (2001) to best estimate ZAM predictions at 26.7, 30.2 , and $36.2 \mathrm{C}$. 


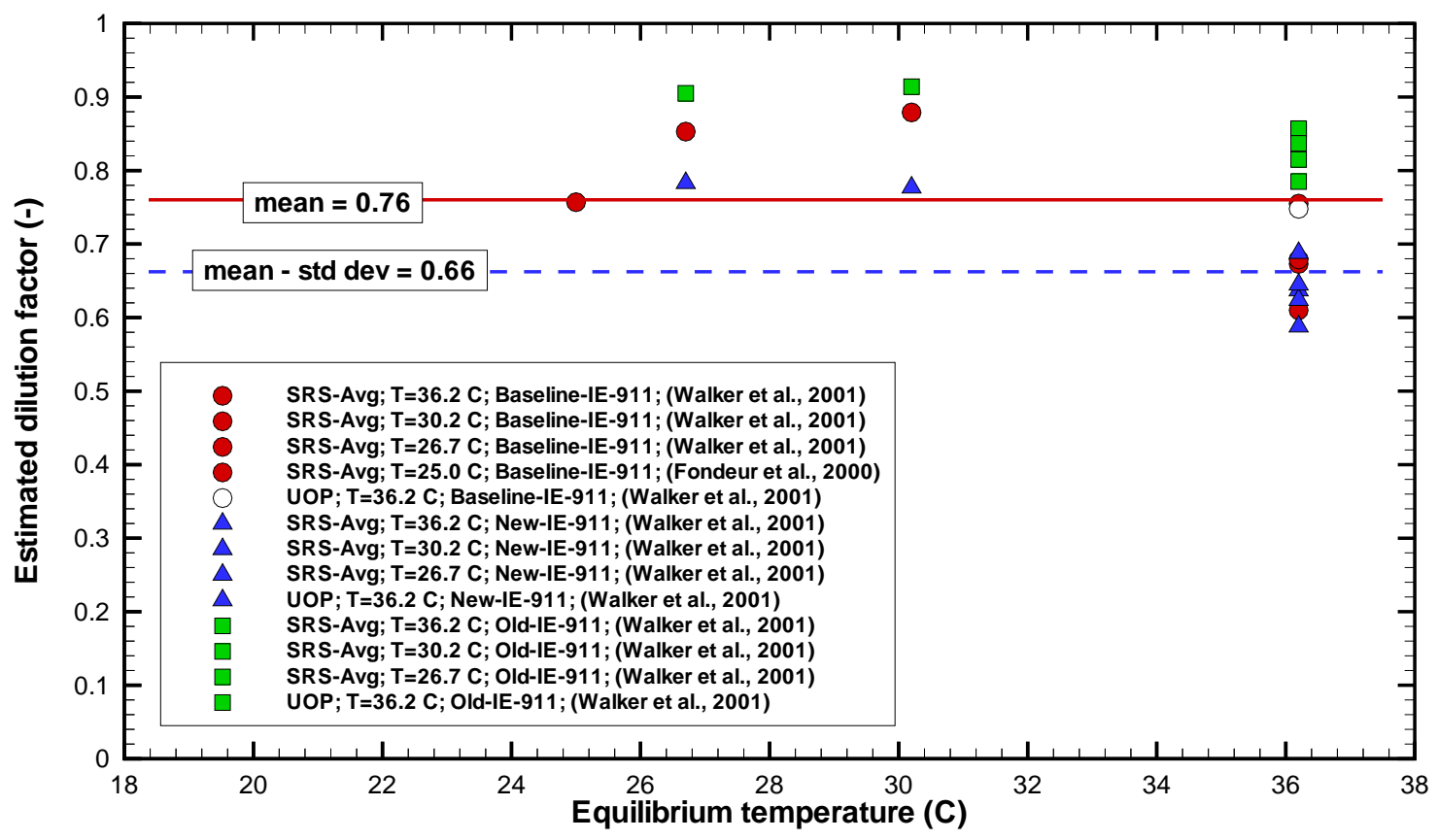

Figure C-7. Comparison of dilution factor for several CST batch contact data sets with respect to equilibrium contact temperature.

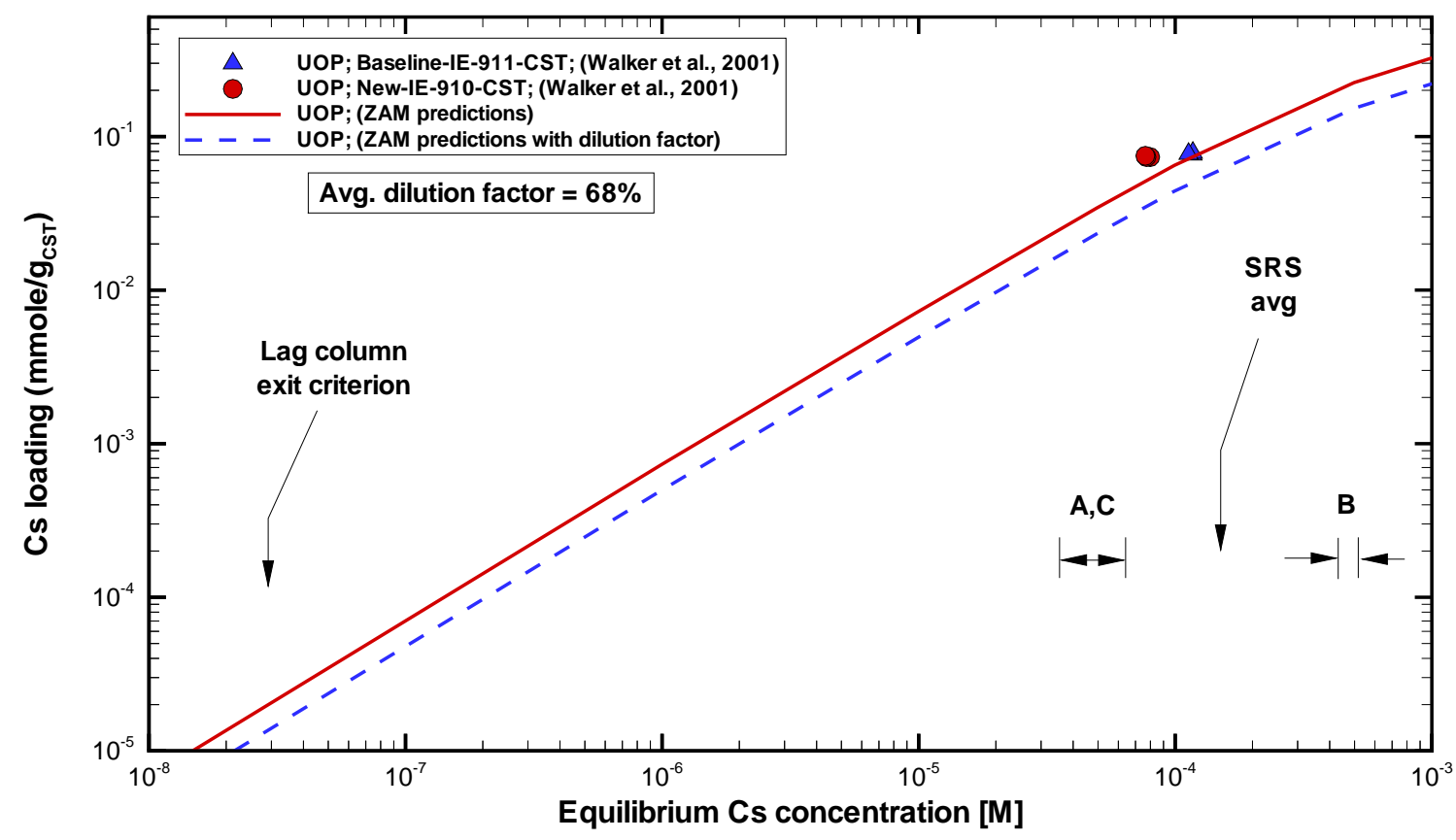

Figure C-8. Comparison of UOP simulant cesium CST loading data recently taken by Walker et al. (2001) to both best estimate and adjusted ZAM predictions at $36.2 \mathrm{C}$. 


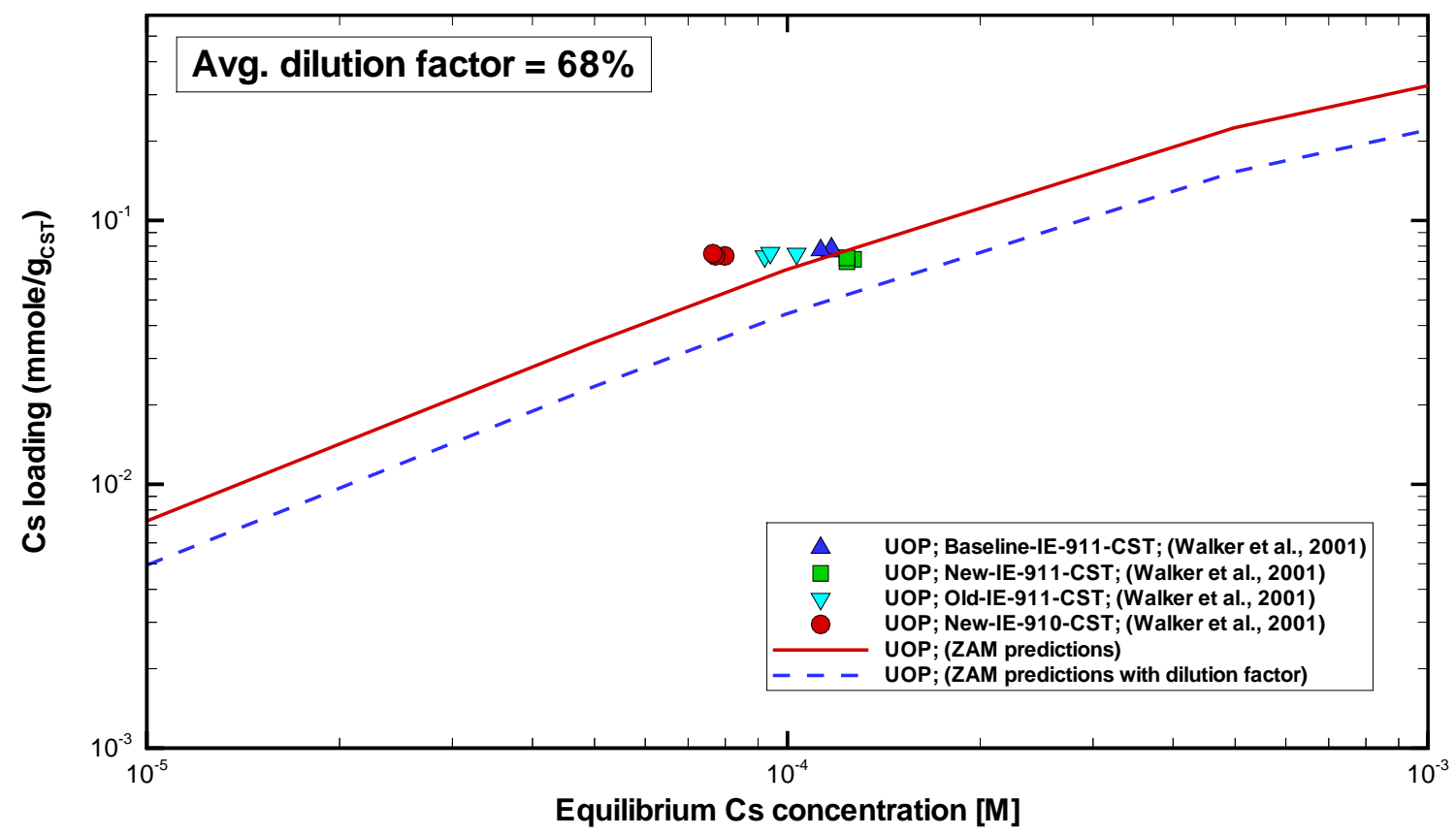

Figure C-9. A close-up comparison of UOP simulant cesium CST loading data recently taken by Walker et al. (2001) to both best estimate and adjusted ZAM predictions at 36.2 C.

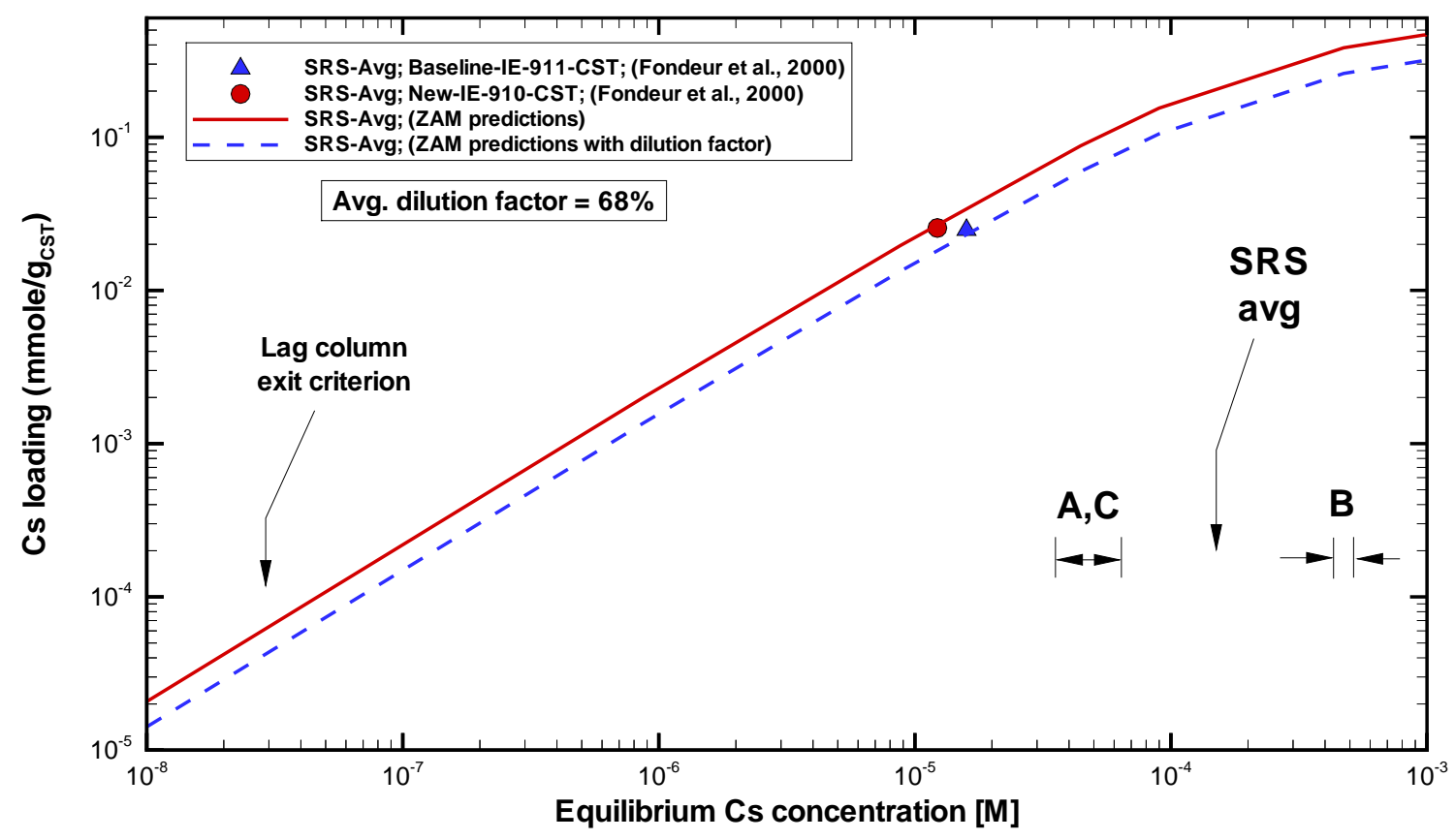

Figure C-10. Comparison of SRS-Avg simulant cesium CST loading data taken by Fondeur et al. (2000) to both best estimate and adjusted ZAM predictions at $\sim 25 \mathrm{C}$. 


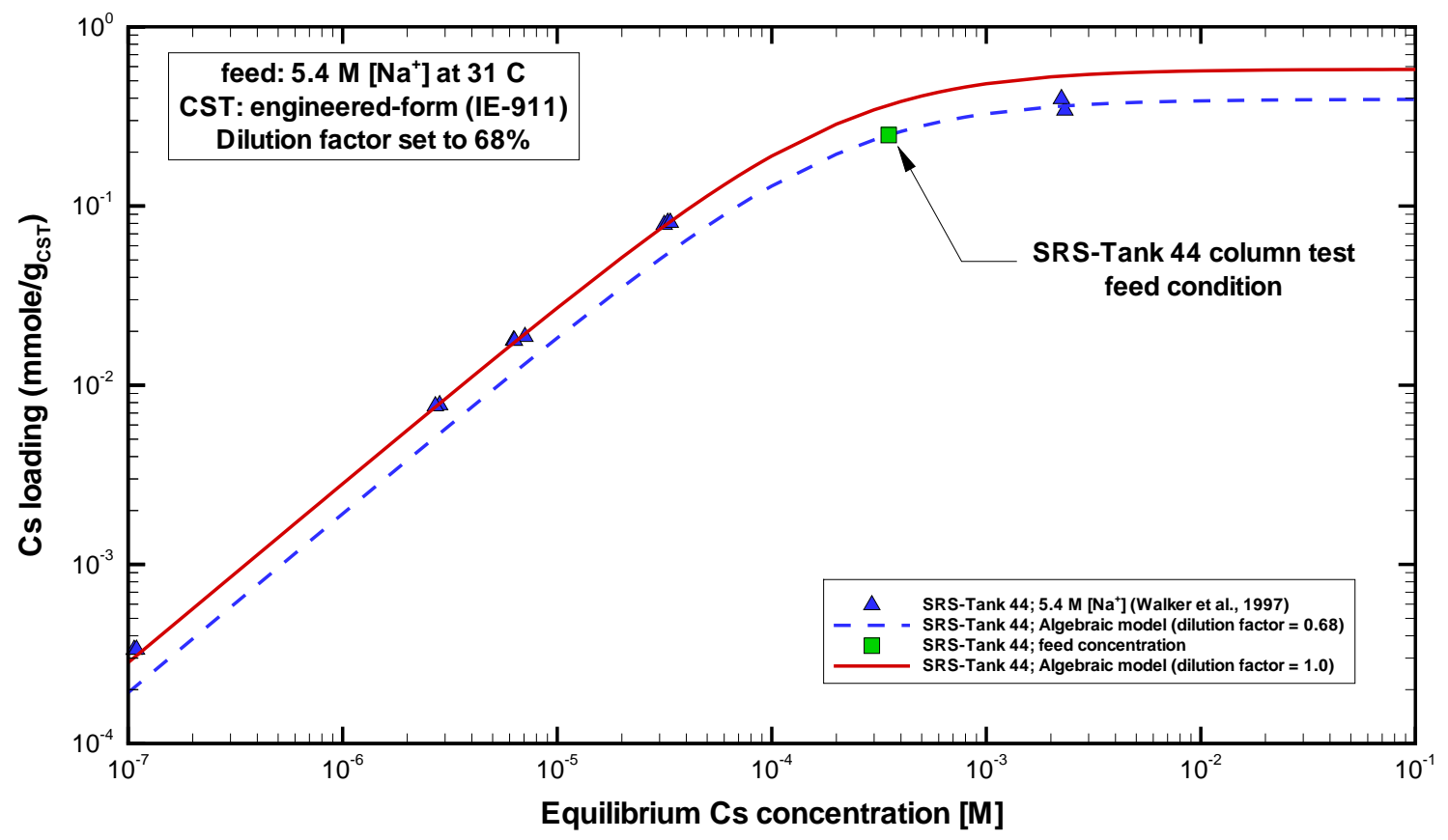

Figure C-11. Comparison of SRS-Tank 44 sample cesium CST loading data taken by Walker et al. (1997) to both best estimate and adjusted ZAM predictions at $31 \mathrm{C}$.

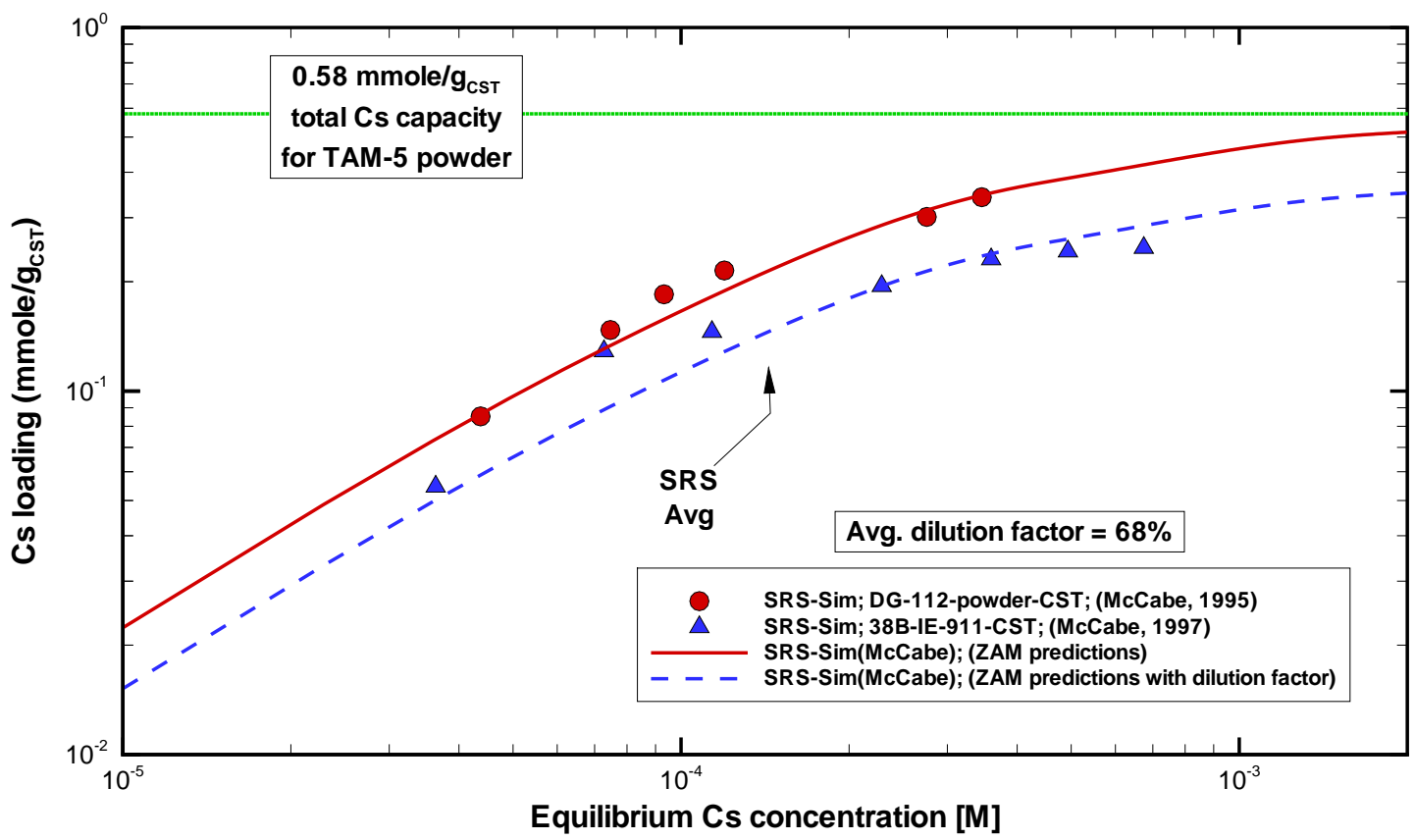

Figure C-12. Comparison of SRS simulant cesium loading data on CST powder and an early on engineered-form material taken by McCabe (1995 [powder data] and 1997 [engineeredform data]) to both best estimate and adjusted ZAM predictions at $\sim 25 \mathrm{C}$. 
Preliminary Ion Exchange Modeling for Removal of Cesium from

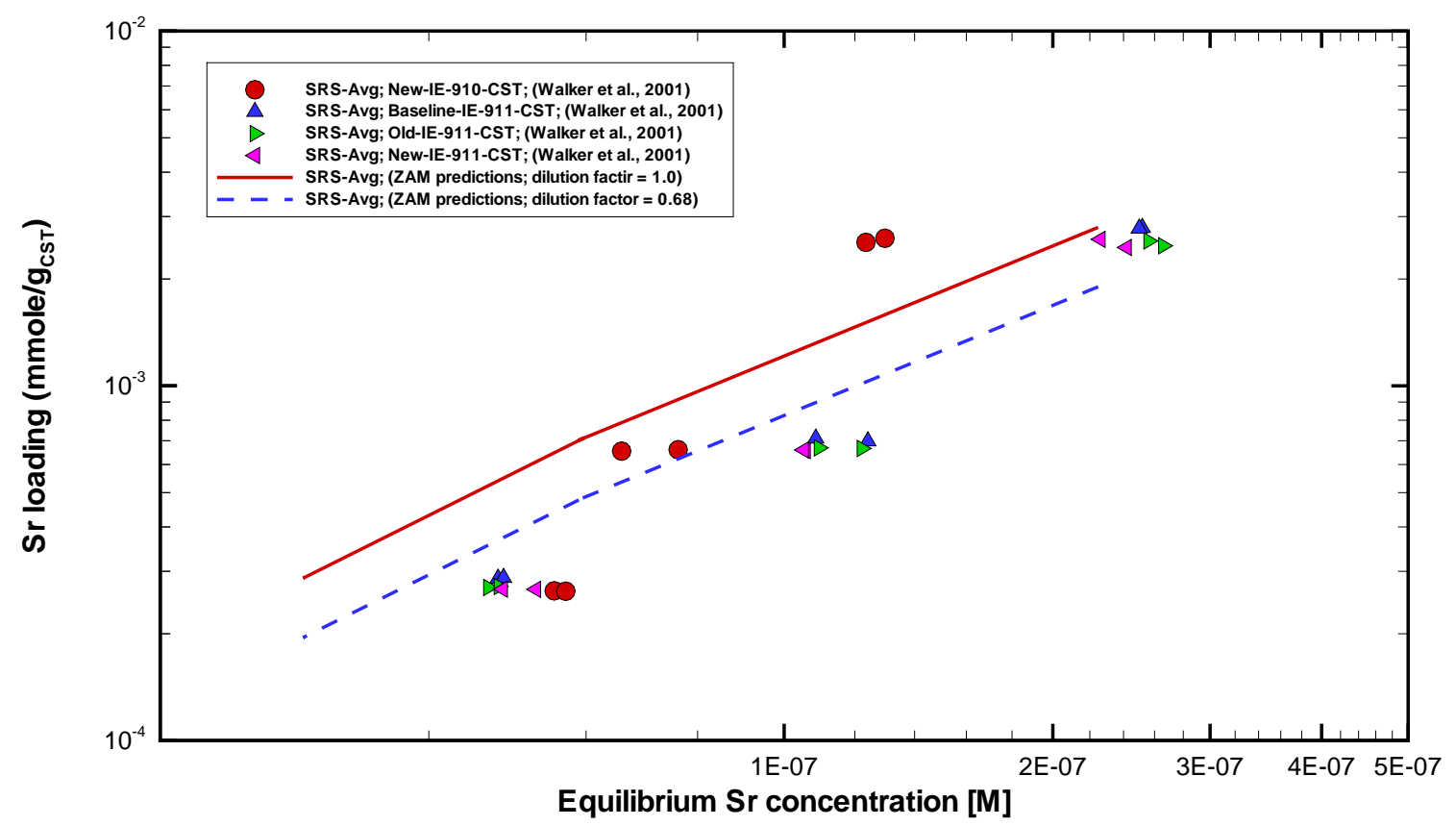

Figure C-13. Comparison of SRS-Avg simulant strontium CST loading data recently taken by Walker et al. (2001) to both best estimate and adjusted ZAM predictions at 36.2 C. 


\section{Appendix D (VERSE-LC Input Files for Phase 1 Batch Feeds)}

VERSE-LC column transport simulations were performed to estimate the total amount of spent CST material required to process the entire Phase 1 inventory. Various column sizes and carousel configurations (i.e., 2-column and 3-column cases) were considered. For reference the VERSE-LC input files for 16 Phase 1 batch feeds are provided in this appendix. Only the input files for the nominal VERSE-LC runs are provided (i.e., 2-column carousel configuration, 2000 $\mathrm{L}$ column volume, $\mathrm{L} / \mathrm{D}=3$ geometry, cesium pore diffusivity equal to $20 \%$ of its free diffusion value). The other input files can be obtained by changing those specific parameter settings as discussed in Chapter 10. One representative VERSE-LC main output file is provided for each of the envelopes (i.e., LAW-1 for Envelope A, LAW-2b for Envelope B, and LAW-3 for Envelope C).

For one specified carousel configuration and input parameter settings, to compute the total amount of spent CST requires running VERSE-LC 16 times (i.e., once for each batch feed) in sequence. Table D-1 provides the run sequence used and the necessary I/O options used in VERSE-LC. Basically, once the total volume of a given batch feed was processed the cesium concentration profiles contained within the lead and lag columns (and intermediate column if present) were stored on an output file (i.e., bed and pore concentrations throughout the two or three columns). On the subsequent batch feed, the previous batch feed output file was read into the current run as its initial cesium concentration profiles. The VERSE-LC simulations were performed on a PC-based Windows-95 machine.

To automate this process of handling many VERSE-LC runs and I/O files in series, a make utility called "NMAKE" was used. A sample input file to NMAKE (i.e., Makefile) is also provided below within this appendix where two cases (vol_a and vol_b) are being executed. In this example, directories were created to hold the files for each of the two case studies (Dir_a and Dir_b). Within each directory subdirectories containing the VERSE-LC input file for each of the 16 batch feeds was created (e.g., ... Dir_a $\backslash L A W \_1, \ldots$. Dir_a $\backslash L A W-2 a$, etc.). Using copy and directory change commands, the above file sequencing was achieved.

The nominal settings for key VERSE-LC input parameters are tabulated in Table D-2 for the Envelope A batch feeds and in Table D-3 for the Envelope B and C batch feeds. At the bottom of the three example VERSE-LC main output files, the process time(s) when a carousel cycle occurred is written out. This provides the number of cycles required on a batch by batch basis as tabulated in Chapter 10. 
Table D-1. Listing of batch feed sequence and VERSE-LC I/O transferring.

\begin{tabular}{|c|c|c|c|}
\hline Envelope & $\begin{array}{c}\text { Phase 1 LAW } \\
\text { batch feed } \\
\text { sequence }\end{array}$ & VERSE-LC input & VERSE-LC output \\
\hline A & LAW-1 & Initially fresh columns & yio file of LAW-1 \\
\hline B & LAW-2a & yio file of LAW-1 & yio file of LAW-2a \\
\hline B & LAW-2b & yio file of LAW-2a & yio file of LAW-2b \\
\hline C & LAW-3 & yio file of LAW-2b & yio file of LAW-3 \\
\hline C & LAW-4 & yio file of LAW-3 & yio file of LAW-4 \\
\hline A & LAW-5 & yio file of LAW-4 & yio file of LAW-5 \\
\hline A & LAW-6 & yio file of LAW-5 & yio file of LAW-6 \\
\hline A & LAW-7 & yio file of LAW-6 & yio file of LAW-7 \\
\hline A & LAW-8 & yio file of LAW-7 & yio file of LAW-8 \\
\hline A & LAW-9 & yio file of LAW-8 & yio file of LAW-9 \\
\hline A & LAW-10 & yio file of LAW-9 & yio file of LAW-10 \\
\hline A & LAW-11 & yio file of LAW-10 & yio file of LAW-11 \\
\hline A & LAW-12 & yio file of LAW-11 & yio file of LAW-12 \\
\hline A & LAW-13 & yio file of LAW-12 & yio file of LAW-13 \\
\hline A & LAW-14 & yio file of LAW-13 & yio file of LAW-14 \\
\hline A & LAW-15 & yio file of LAW-14 & \\
\hline & & & \\
\hline
\end{tabular}


Table D-2. VERSE-LC nominal input parameters settings used in the Envelope A batch feed simulations at $25 \mathrm{C}$.

\begin{tabular}{|c|c|c|c|c|c|c|c|c|c|c|c|}
\hline Feed Batch Name & LAW-1 & LAW-5 & LAW-6 & LAW-8 & LAW-9 & LAW-10 & LAW-11 & LAW-12 & LAW-13 & LAW-14 & LAW-15 \\
\hline Tank source & AP-101 & AN-104 & AN-104 & AN-105 & AN-105 & SY-101 & SY-101 & AN-103 & AN-103 & AW-101 & AW-101 \\
\hline \multicolumn{12}{|l|}{ Input Parameters } \\
\hline Flow rate $(\mathrm{L} / \mathrm{min})$ & 52.62 & 52.62 & 52.62 & 52.62 & 52.62 & 52.62 & 52.62 & 52.62 & 52.62 & 52.62 & 52.62 \\
\hline Feed ${ }^{\text {total }}$ Cs conc. (M) & $3.598 \mathrm{E}-05$ & $6.283 \mathrm{E}-05$ & $6.328 \mathrm{E}-05$ & 4.324E-05 & 4.444E-05 & 3.692E-05 & $3.739 \mathrm{E}-05$ & 4.831E-05 & $4.831 \mathrm{E}-05$ & $4.569 \mathrm{E}-05$ & $4.552 \mathrm{E}-05$ \\
\hline Fluid viscosity (Poise) & 2.6125 & 2.6125 & 2.6125 & 2.6125 & 2.6125 & 2.6125 & 2.6125 & 2.6125 & 2.6125 & 2.6125 & 2.6125 \\
\hline Fluid density (g/ml) a & 1.277 & 1.232 & 1.231 & 1.225 & 1.238 & 1.237 & 1.232 & 1.221 & 1.221 & 1.234 & 1.235 \\
\hline Particle radius $(\mu \mathrm{m})$ & 172.0 & 172.0 & 172.0 & 172.0 & 172.0 & 172.0 & 172.0 & 172.0 & 172.0 & 172.0 & 172.0 \\
\hline Bed porosity & 0.50 & 0.50 & 0.50 & 0.50 & 0.50 & 0.50 & 0.50 & 0.50 & 0.50 & 0.50 & 0.50 \\
\hline Particle porosity & 0.24 & 0.24 & 0.24 & 0.24 & 0.24 & 0.24 & 0.24 & 0.24 & 0.24 & 0.24 & 0.24 \\
\hline Pore diffusivity $\left(\mathrm{cm}^{2} / \mathrm{min}\right)^{b}$ & $9.044 \mathrm{E}-05$ & 8.624E-05 & $8.715 \mathrm{E}-05$ & $9.090 \mathrm{E}-05$ & 8.303E-05 & $8.669 \mathrm{E}-05$ & $8.698 \mathrm{E}-05$ & $9.248 \mathrm{E}-05$ & $9.247 \mathrm{E}-05$ & $9.347 \mathrm{E}-05$ & $9.643 \mathrm{E}-05$ \\
\hline Brownian diffusivity $\left(\mathrm{cm}^{2} / \mathrm{min}\right)$ & $4.522 \mathrm{E}-04$ & $4.312 \mathrm{E}-04$ & $4.357 \mathrm{E}-04$ & $4.545 \mathrm{E}-04$ & 4.152E-04 & 4.334E-04 & $4.349 \mathrm{E}-04$ & 4.624E-04 & 4.624E-04 & 4.674E-04 & 4.822E-04 \\
\hline Fr/La Hybrid a (moles/L B.V.) ${ }^{\text {b }}$ & 0.3944 & 0.3944 & 0.3944 & 0.3944 & 0.3944 & 0.3944 & 0.3944 & 0.3944 & 0.3944 & 0.3944 & 0.3944 \\
\hline Fr/La Hybrid b (1/M) & 1.0 & 1.0 & 1.0 & 1.0 & 1.0 & 1.0 & 1.0 & 1.0 & 1.0 & 1.0 & 1.0 \\
\hline Fr/La Hybrid $M_{a}$ & 1.0 & 1.0 & 1.0 & 1.0 & 1.0 & 1.0 & 1.0 & 1.0 & 1.0 & 1.0 & 1.0 \\
\hline Fr/La Hybrid $M_{b}$ & 1.0 & 1.0 & 1.0 & 1.0 & 1.0 & 1.0 & 1.0 & 1.0 & 1.0 & 1.0 & 1.0 \\
\hline Fr/La Hybrid beta & $3.8445 \mathrm{E}-04$ & $2.3431 \mathrm{E}-04$ & $2.3668 \mathrm{E}-04$ & $2.5147 \mathrm{E}-04$ & $2.2453 \mathrm{E}-04$ & $2.2135 \mathrm{E}-04$ & $2.0543 \mathrm{E}-04$ & $2.9196 \mathrm{E}-04$ & $2.9328 \mathrm{E}-04$ & $3.6283 \mathrm{E}-04$ & 3.8513E-04 \\
\hline Total Cs exit criterion [M] & $2.9534 \mathrm{E}-08$ & $2.9534 \mathrm{E}-08$ & 2.9534E-08 & $2.9534 \mathrm{E}-08$ & $2.9534 \mathrm{E}-08$ & $2.9534 \mathrm{E}-08$ & $2.9534 \mathrm{E}-08$ & $2.9534 \mathrm{E}-08$ & $2.9534 \mathrm{E}-08$ & $2.9534 \mathrm{E}-08$ & 2.9534E-08 \\
\hline
\end{tabular}

${ }^{a}$ The bed density used represents a typical average value of measured engineered-form CST material where swelling due to feed ionic strength variations have negligible effects.

a The dilution factor is nominally set to $68 \%$ and the cesium pore diffusion coefficient to $20 \%$ of its "free" diffusion value. 
Table D-3. VERSE-LC nominal input parameters settings used in the Envelopes B and C batch feed simulations at 25 C.

\begin{tabular}{|c|c|c|c|c|c|}
\hline Envelope & B & B & C & $\mathrm{C}$ & C \\
\hline Feed Batch Name & LAW-2a & LAW-2b & LAW-3 & LAW-4 & LAW-7 \\
\hline Tank source & AZ-101 & AZ-102 & AN-102 & AN-102 & AN-107 \\
\hline \multicolumn{6}{|l|}{ Input Parameters } \\
\hline Flow rate (L/min) & 9.4 & 9.4 & 16.2 & 16.2 & 16.2 \\
\hline Feed ${ }^{\text {total }}$ Cs conc. $(M)$ & 4.676E-04 & $4.311 \mathrm{E}-04$ & $3.967 \mathrm{E}-05$ & $3.779 \mathrm{E}-05$ & 4.455E-05 \\
\hline Fluid viscosity (Poise) & 2.6125 & 2.6125 & 2.6125 & 2.6125 & 2.6125 \\
\hline Fluid density $(\mathrm{g} / \mathrm{ml})^{a}$ & 1.254 & 1.242 & 1.237 & 1.237 & 1.243 \\
\hline Particle radius $(\mu \mathrm{m})$ & 172.0 & 172.0 & 172.0 & 172.0 & 172.0 \\
\hline Bed porosity & 0.50 & 0.50 & 0.50 & 0.50 & 0.50 \\
\hline Particle porosity & 0.24 & 0.24 & 0.24 & 0.24 & 0.24 \\
\hline Pore diffusivity $\left(\mathrm{cm}^{2} / \mathrm{min}\right)^{b}$ & $7.828 \mathrm{E}-05$ & $7.614 \mathrm{E}-05$ & $8.709 \mathrm{E}-05$ & $8.709 \mathrm{E}-05$ & $8.435 \mathrm{E}-05$ \\
\hline Brownian diffusivity $\left(\mathrm{cm}^{2} / \mathrm{min}\right)$ & $3.914 \mathrm{E}-04$ & $3.807 \mathrm{E}-04$ & $4.354 \mathrm{E}-04$ & 4.354E-04 & $4.217 \mathrm{E}-04$ \\
\hline Fr/La Hybrid a (moles/L B.V.) ${ }^{\text {b }}$ & 0.3944 & 0.3944 & 0.3944 & 0.3944 & 0.3944 \\
\hline Fr/La Hybrid b (1/M) & 1.0 & 1.0 & 1.0 & 1.0 & 1.0 \\
\hline $\mathrm{Fr} /$ La Hybrid $\mathrm{M}_{\mathrm{a}}$ & 1.0 & 1.0 & 1.0 & 1.0 & 1.0 \\
\hline Fr/La Hybrid $M_{b}$ & 1.0 & 1.0 & 1.0 & 1.0 & 1.0 \\
\hline Fr/La Hybrid beta & $2.6230 \mathrm{E}-04$ & $2.1296 \mathrm{E}-04$ & $2.1769 \mathrm{E}-04$ & $2.1769 \mathrm{E}-04$ & $1.9258 \mathrm{E}-04$ \\
\hline Total Cs exit criterion [M] & 7.0318E-08 & $7.0318 \mathrm{E}-08$ & $4.8942 \mathrm{E}-08$ & $4.8942 \mathrm{E}-08$ & 4.8942E-08 \\
\hline
\end{tabular}

${ }^{\text {a }}$ The bed density used represents a typical average value of measured engineered-form CST material where swelling due to feed ionic strength variations have negligible effects.

a The dilution factor is nominally set to $68 \%$ and the cesium pore diffusion coefficient to $20 \%$ of its "free" diffusion value. 


\section{Makefile for NMAKE Utility (2 nominal case runs of VERSE-LC)}

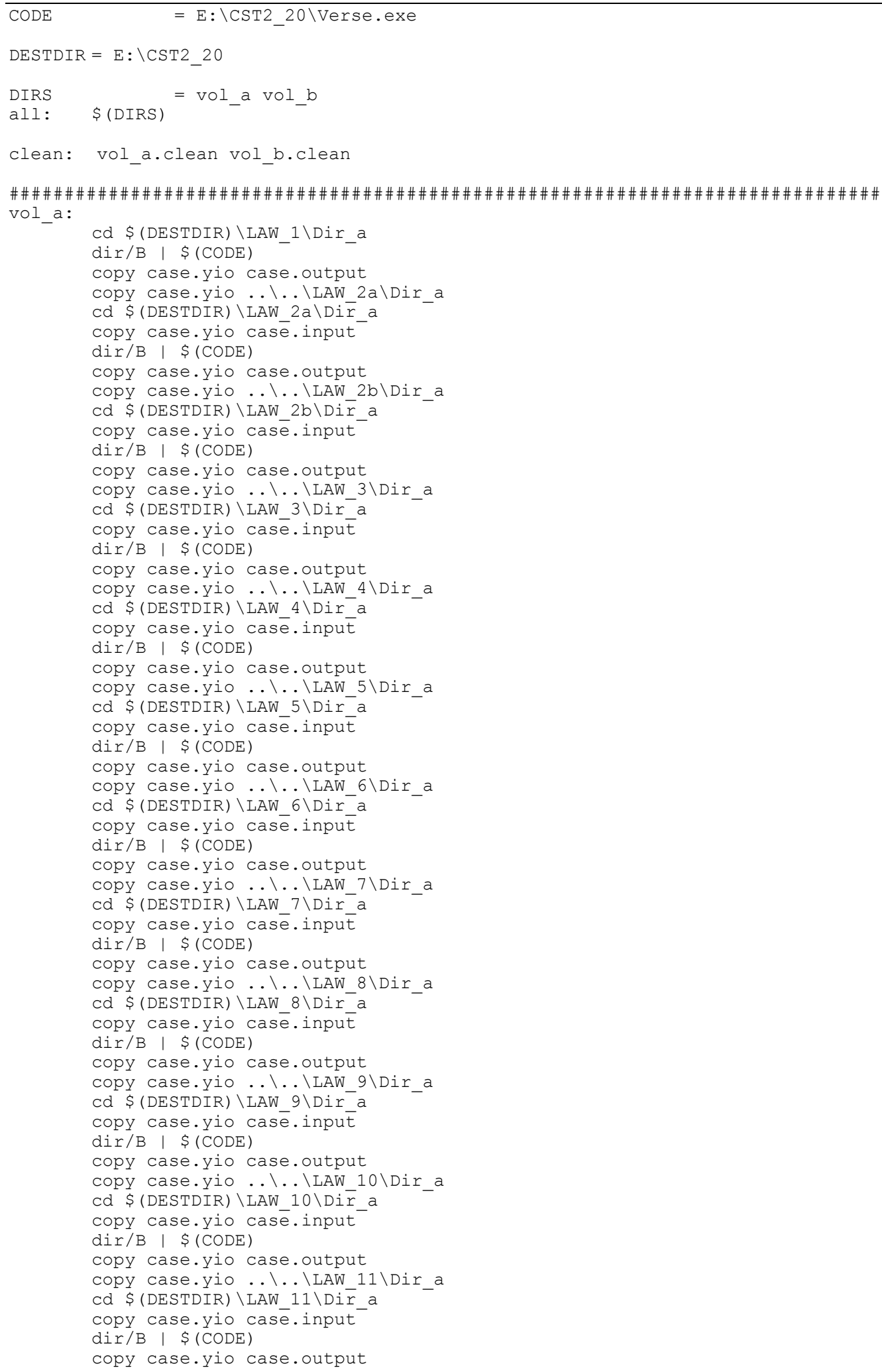


copy case.yio... . \LAW_12\Dir_a

cd $\$($ DESTDIR) $\backslash$ LAW_ $12 \backslash$ Dir_a

copy case.yio case.input

dir/B | \$ (CODE)

copy case.yio case.output

copy case.yio..... \LAW_13\Dir_a

cd $\$(D E S T D I R) \backslash L A W \_13 \backslash D i \bar{r} \_a$

copy case.yio casēe.input

$\operatorname{dir/B~|~\$ (CODE)~}$

copy case.yio case.output

copy case.yio ... .. \LAW 14\Dir a

cd $\$($ DESTDIR) $\backslash$ LAW_14 $\backslash$ Dir_a

copy case.yio casè. input

dir/B | \$(CODE)

copy case.yio case.output

copy case.yio ..... \LAW 15\Dir_a

cd $\$(D E S T D I R) \backslash L A W \_15 \backslash D i \bar{r} \_a$

copy case.yio casē.input

$\operatorname{dir/B~|~\$ (CODE)~}$

vol_b:

$\mathrm{cd} \$(\mathrm{DESTDIR}) \backslash \mathrm{LAW} \_1 \backslash \mathrm{Dir} \_\mathrm{b}$

$\operatorname{dir} / \mathrm{B} \mid$ | (CODE)

copy case.yio case.output

copy case.yio ... .. \LAW_2a\Dir_b

cd $\$$ (DESTDIR) \LAW $2 a \backslash D i \bar{r}$ b

copy case.yio case.input

$\mathrm{dir} / \mathrm{B} \mid$ | (CODE)

copy case.yio case.output

copy case.yio ... .. \LAW_2b\Dir_b

$\mathrm{cd} \$($ DESTDIR) $\backslash$ LAW_2b $\backslash$ Dir_b

copy case.yio casēe.input

$\operatorname{dir/B~|~\$ (CODE)~}$

copy case.yio case.output

copy case.yio ... .. \LAW 3\Dir b

$\mathrm{cd} \$(\mathrm{DESTDIR}) \backslash \mathrm{LAW} 3 \backslash \mathrm{Dir}^{-} \mathrm{b}$

copy case.yio casē.input

$\operatorname{dir} / \mathrm{B} \mid$ | (CODE)

copy case.yio case.output

copy case.yio... .. \LAW 4\Dir b

cd $\$(D E S T D I R) \backslash L A W \quad 4 \backslash$ Dir b

copy case.yio casēe.input

$\operatorname{dir/B~|~\$ (CODE)~}$

copy case.yio case.output

copy case.yio .. \.. \LAW_5 \Dir b

$\mathrm{cd} \$(\mathrm{DESTDIR}) \backslash$ LAW $5 \backslash$ Dir b

copy case.yio casē.input

dir/B | \$ (CODE)

copy case.yio case.output

copy case.yio ... . \LAW_6\Dir b

$\mathrm{cd} \$(\mathrm{DESTDIR}) \backslash$ LAW $6 \backslash$ Dir b

copy case.yio casē.input

dir/B | \$(CODE)

copy case.yio case.output

copy case.yio ... . . LAW_7 Dir_b

cd $\$(D E S T D I R) \backslash L A W \quad 7 \backslash D i r b$

copy case.yio casēe.input

$\mathrm{dir} / \mathrm{B} \mid$ | (CODE)

copy case.yio case.output

copy case.yio ... ... \LAW_8\Dir_b

$\mathrm{cd} \$(\mathrm{DESTDIR}) \backslash \mathrm{LAW}^{\mathrm{B}} 8 \backslash \mathrm{Dir}^{-} \mathrm{b}$

copy case.yio casēe.input

$\mathrm{dir} / \mathrm{B} \mid$ | (CODE)

copy case.yio case.output

copy case.yio ... . . \LAW_9 \Dir_b

$\mathrm{cd} \$($ DESTDIR) \LAW_9 $\backslash$ Dir_b

copy case.yio casē.input

dir/B | \$ (CODE)

copy case.yio case.output

copy case.yio ..... \LAW 10\Dir b

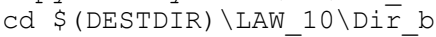

copy case.yio casēe.input

dir/B | \$(CODE)

copy case.yio case.output 
copy case.yio ... . \LAW_11\Dir_b

cd $\$(D E S T D I R) \backslash$ LAW_11 1 Dir_b

copy case.yio case..input

dir/B | \$ (CODE)

copy case.yio case.output

copy case.yio ... . \LAW_12\Dir_b

$\mathrm{cd} \$($ DESTDIR) \LAW_12\Dir_b

copy case.yio case. input

dir/B | \$(CODE)

copy case.yio case.output

copy case.yio ... .. \LAW_13\Dir_b

cd $\$($ DESTDIR) $\backslash$ LAW_13\Dir_b

copy case.yio case. input

dir/B | \$(CODE)

copy case.yio case.output

copy case.yio ... .. \LAW 14\Dir b

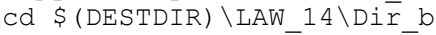

copy case.yio case. input

$\operatorname{dir/B~|~\$ (CODE)~}$

copy case.yio case.output

copy case.yio .. .. \LAW_15\Dir_b

cd \$ (DESTDIR) \LAW_15\Dir_b

copy case.yio casē.input

$\operatorname{dir} / \mathrm{B} \mid$ | (CODE)

vol_a.clean:

cd \$ (DESTDIR) \LAW_1 \Dir_a

- Qerase *.run

- Qerase *.h*

- Qerase *.p*

- Qerase *.yio*

- Qerase *.output

cd $\$(D E S T D I R) \backslash L A W \_5 \backslash D i r \_a$

- Qerase*.run

- Qerase *.h*

- Qerase *.p*

- Qerase *.yio*

- Cerase *.input

- derase *.output

cd \$ (DESTDIR) \LAW 6\Dir a

- Qerase *.run

- Qerase *.h*

- Qerase *.p*

- Qerase *.yio*

- derase *.input

- Qerase *.output

cd $\$(D E S T D I R) \backslash L A W \_8 \backslash D i r \_a$

- Qerase *.run

- derase *.h*

- Qerase *.p*

- Qerase *.yio*

- Qerase *.input

- derase *.output

cd \$(DESTDIR) \LAW_9 $\backslash$ Dir_a

- Qerase *.run

- Qerase *. $h *$

- Qerase *.p*

- Qerase *.yio*

- Qerase *.input

- derase *.output

cd $\$(D E S T D I R) \backslash L A W \_10 \backslash D i r \_a$

- Qerase *.run

- Qerase *.h*

- Qerase *.p*

- Qerase *.yio*

- Qerase *.input

- derase *.output

cd $\$(D E S T D I R) \backslash L A W \_11 \backslash$ Dir_a

- derase *.run

- Qerase *.h*

- Qerase *.p*

- Qerase *.yio*

- Qerase *.input

- derase *. output 


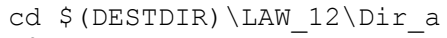

- Qerase *.run

- derase *.h*

- derase *.p*

- Qerase *.yio*

- Qerase *.input

- derase *.output

cd $\$($ DESTDIR) \LAW 13\Dir a

- Qerase *.run

- Qerase *.h*

- Qerase *. $\mathrm{p}^{*}$

- Qerase *.yio*

- Qerase *.input

- derase *.output

cd $\$(D E S T D I R) \backslash L A W \_14 \backslash$ Dir_a

- derase *.run

- Qerase *.h*

- Qerase*.p*

- Qerase *.yio*

- Qerase *.input

- derase *.output

cd \$ (DESTDIR) \LAW_15\Dir_a

- Qerase *.run

- Qerase *.h*

- Qerase *.p*

- Qerase *.yio*

- Cerase *.input

- derase *.output

cd $\$($ DESTDIR) \LAW_2a \Dir_a

- Qerase *.run

- Qerase *.h*

- Qerase *.p*

- Qerase *.yio*

- Qerase *.input

- Qerase *.output

cd $\$($ DESTDIR) \LAW_2b\Dir_a

- Qerase *.run

- Qerase *.h*

- Qerase *.p*

- Qerase *.yio*

- Qerase *.input

- Cerase *.output

cd \$(DESTDIR) \LAW 3\Dir a

- Qerase*.run

- Qerase *.h*

- Qerase *.p*

- Qerase *.yio*

- Qerase *.input

- Qerase *.output

cd $\$(D E S T D I R) \backslash L A W \_4 \backslash D i r \_a$

- Qerase *.run

- Qerase *.h*

- Qerase *.p*

- Cerase *.yio*

- Qerase *.input

- Qerase *.output

cd \$ (DESTDIR) \LAW_7\Dir_a

- Cerase *.run

- Qerase *.h*

- derase *.p*

- Qerase *.yio*

- Qerase *.input

vol_b.clean:

cd \$(DESTDIR) \LAW_1 \Dir_b

- Qerase *.run

- @erase *.h*

- Qerase *.p*

- Qerase *.yio*

- Qerase *.output

cd \$ (DESTDIR) \LAW_5 \Dir_b

- Qerase *.run

- Qerase *.h*

- derase *.p* 


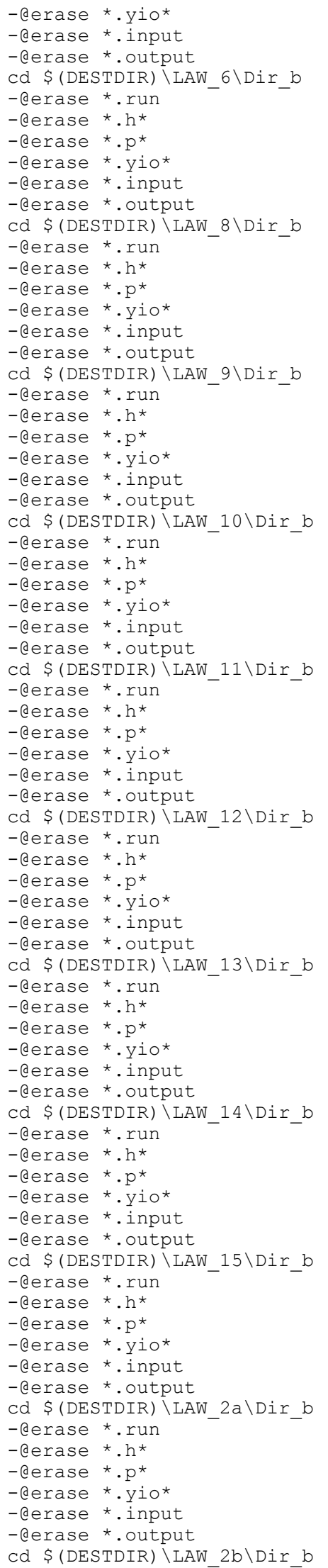


Preliminary Ion Exchange Modeling for Removal of Cesium from

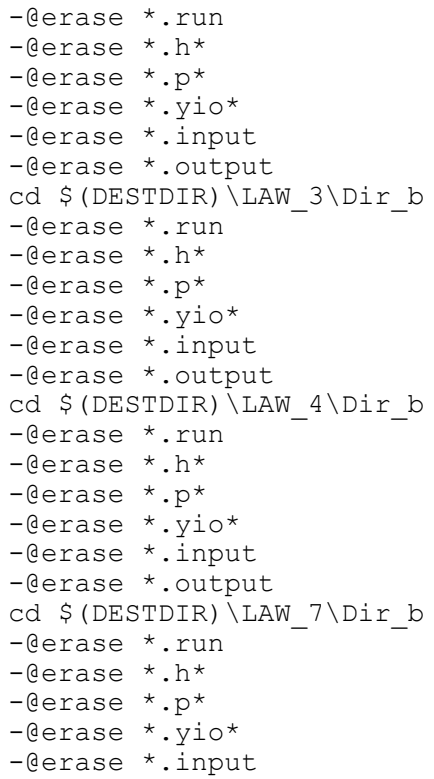




\section{VERSE-LC Input for Phase 1 LAW-1 Batch Feed (nominal case; 2000 L)}

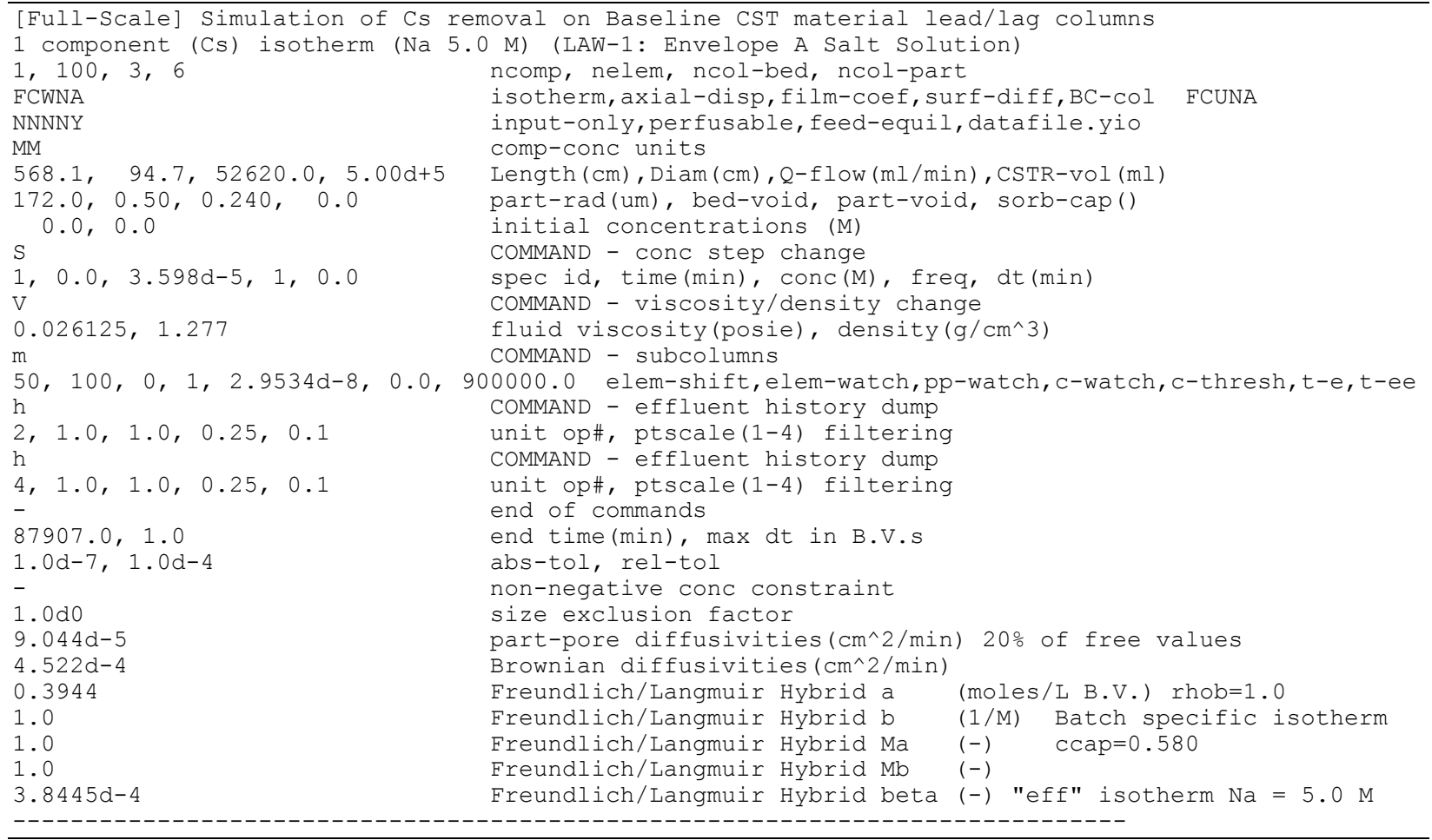

\section{VERSE-LC Input for Phase 1 LAW-2a Batch Feed (nominal case; 2000 L)}

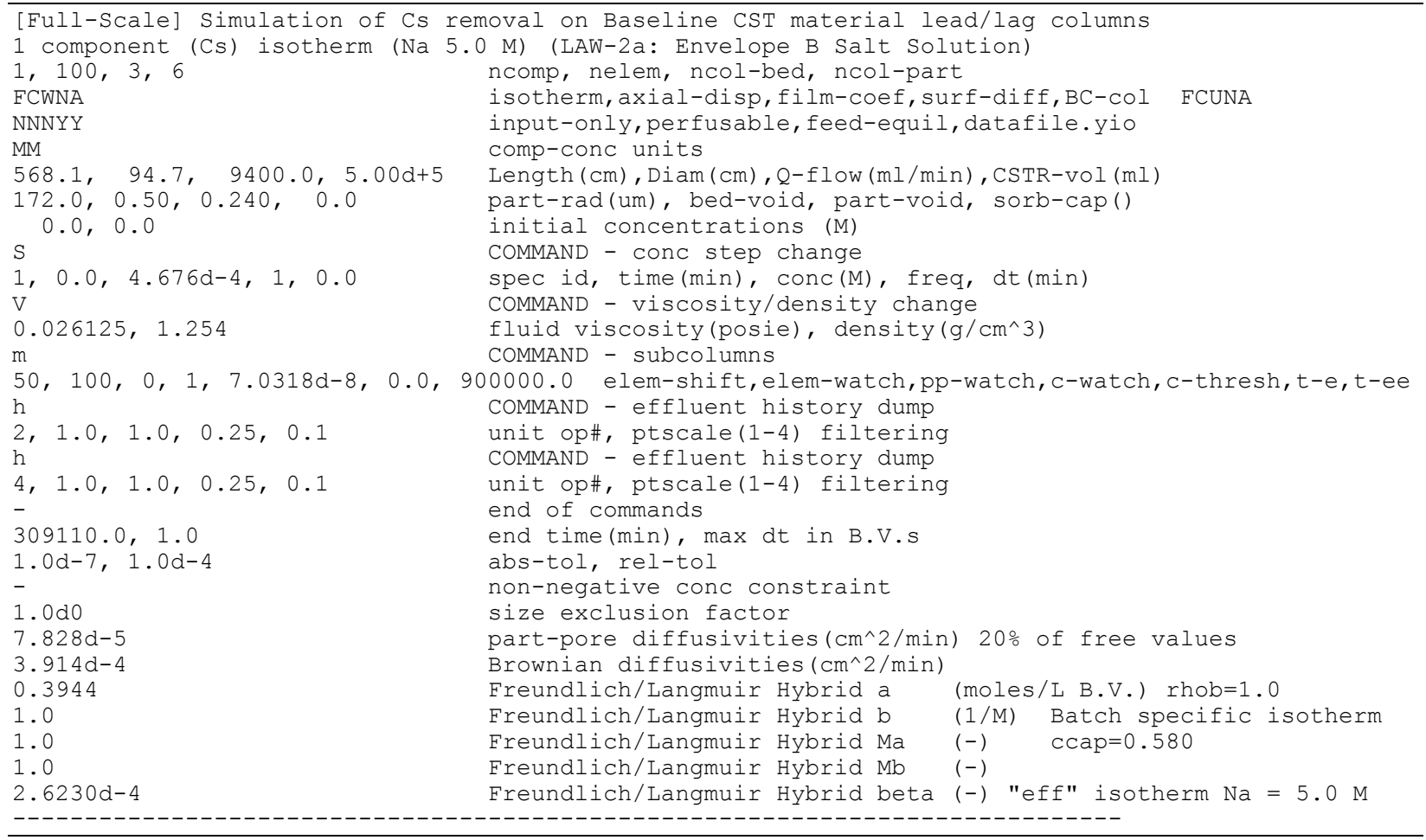




\section{VERSE-LC Input for Phase 1 LAW-2b Batch Feed (nominal case; 2000 L)}

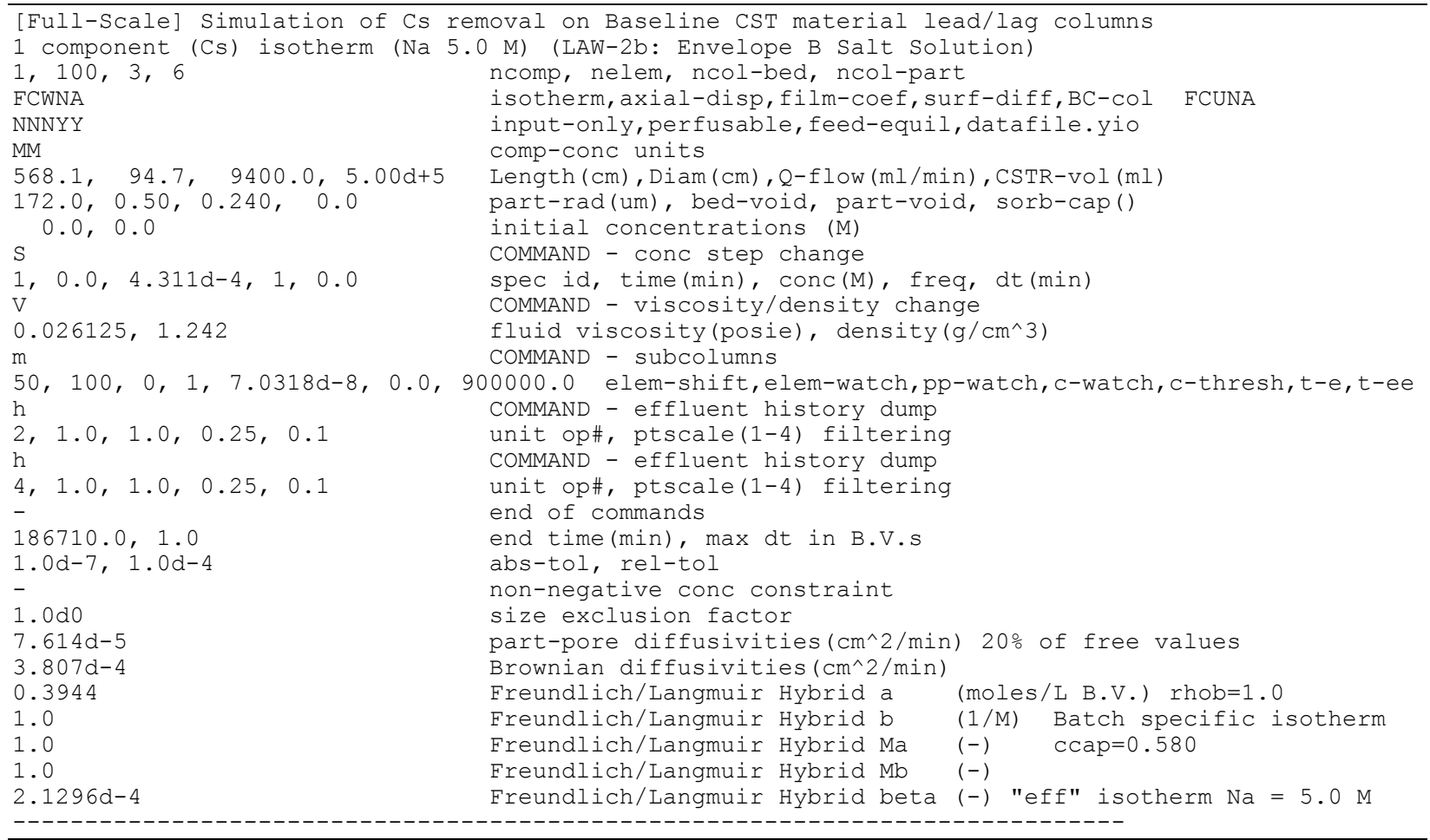

\section{VERSE-LC Input for Phase 1 LAW-3 Batch Feed (nominal case; 2000 L)}

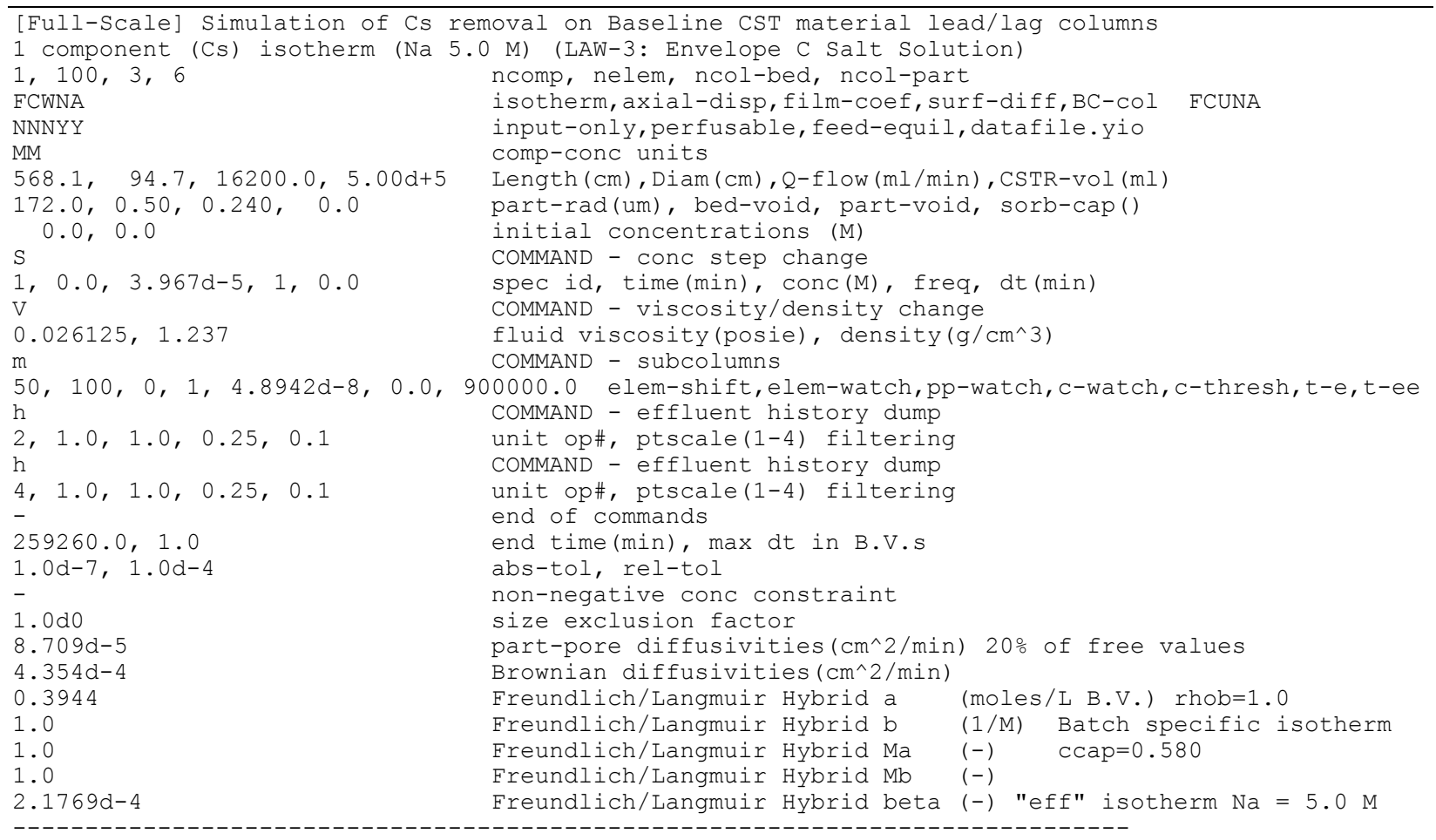




\section{VERSE-LC Input for Phase 1 LAW-4 Batch Feed (nominal case; 2000 L)}

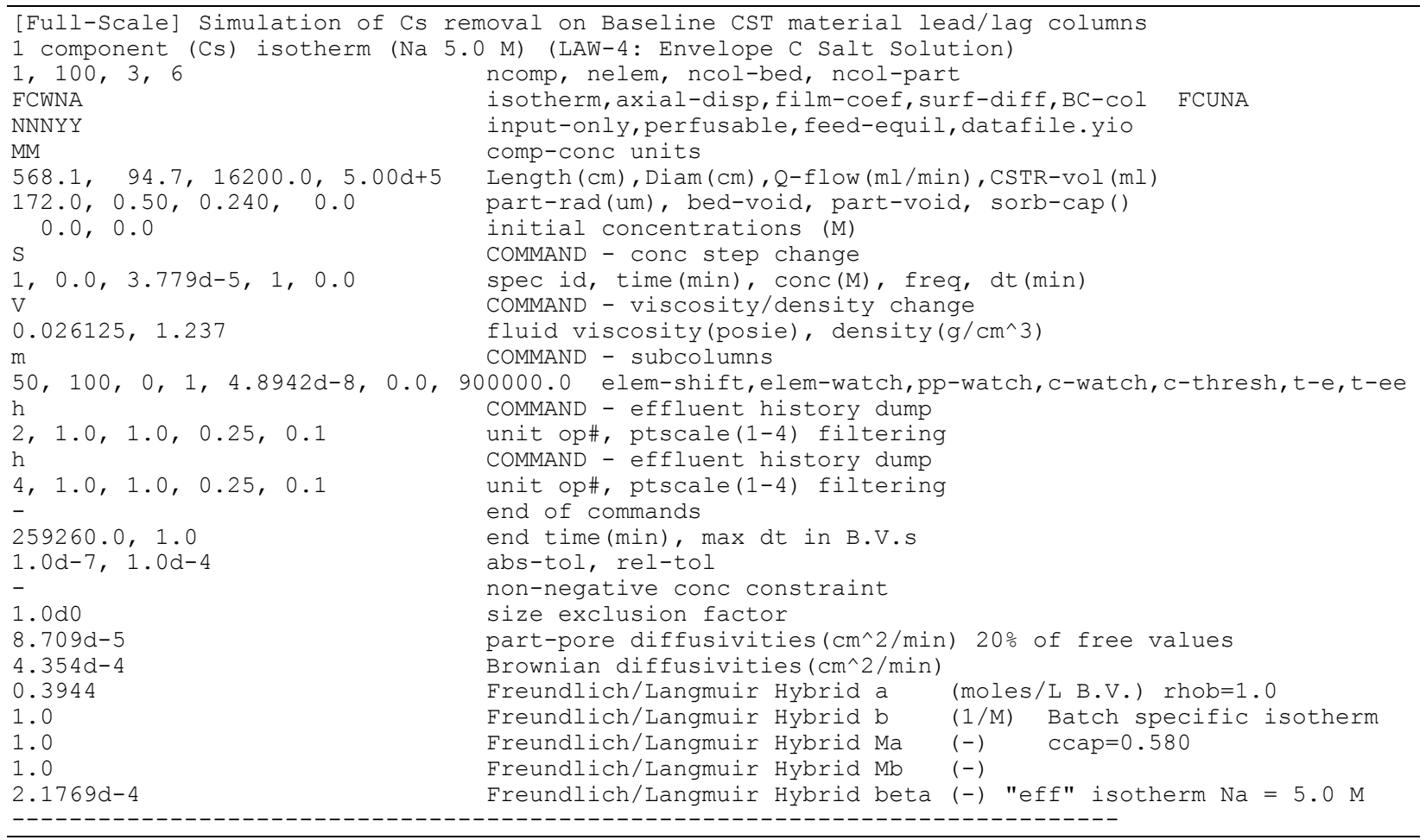

\section{VERSE-LC Input for Phase 1 LAW-5 Batch Feed (nominal case; 2000 L)}

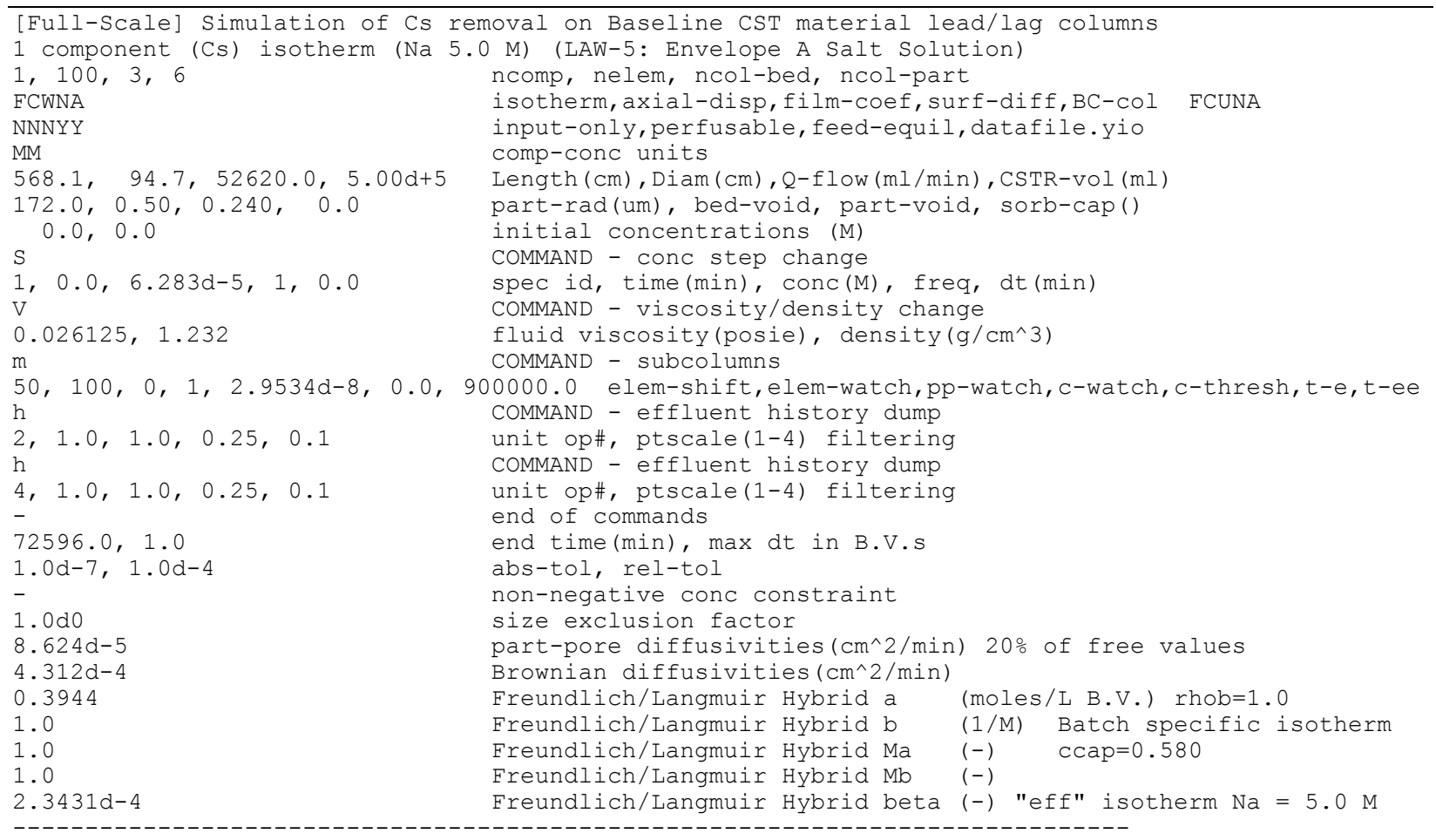




\section{VERSE-LC Input for Phase 1 LAW-6 Batch Feed (nominal case; 2000 L)}

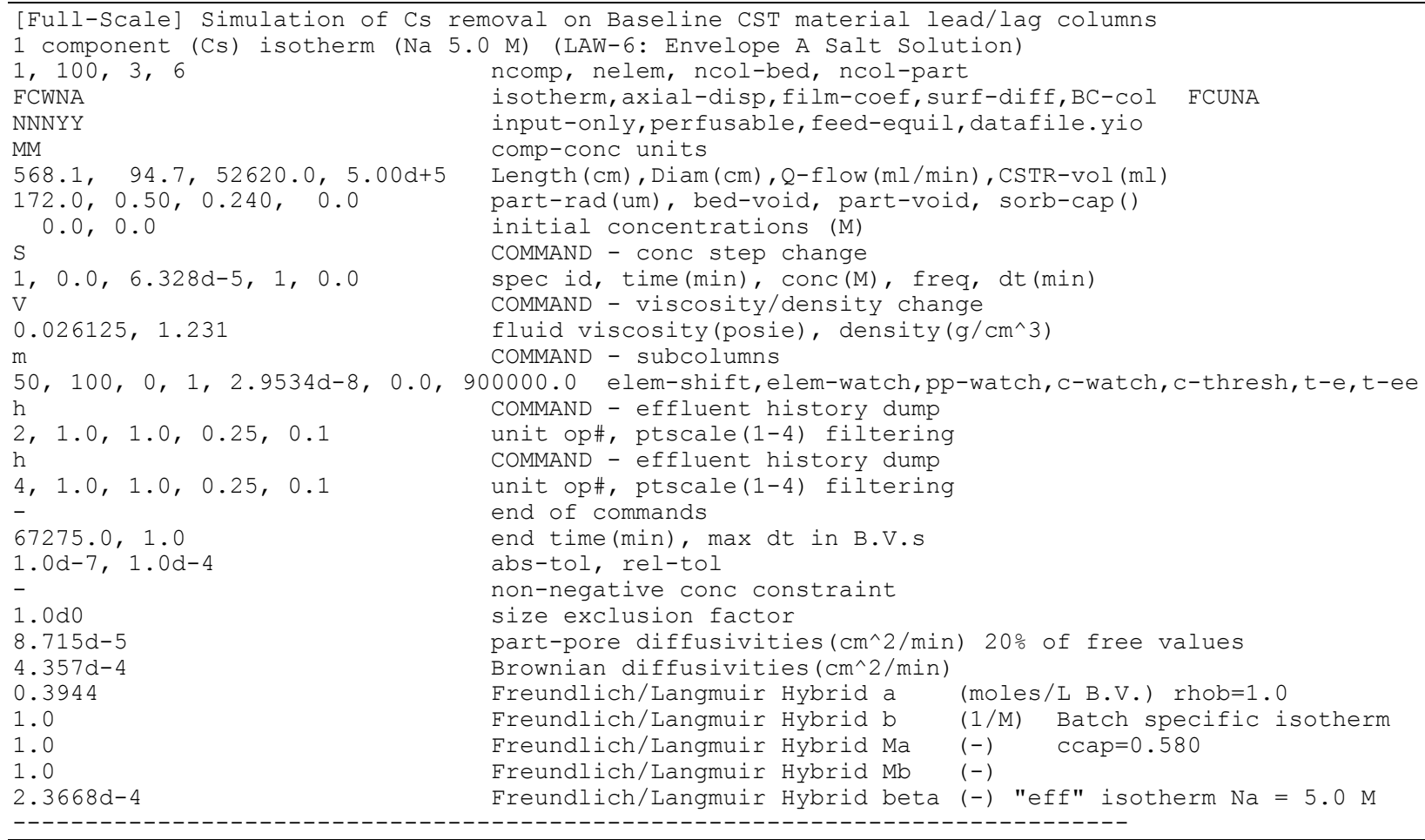

\section{VERSE-LC Input for Phase 1 LAW-7 Batch Feed (nominal case; 2000 L)}

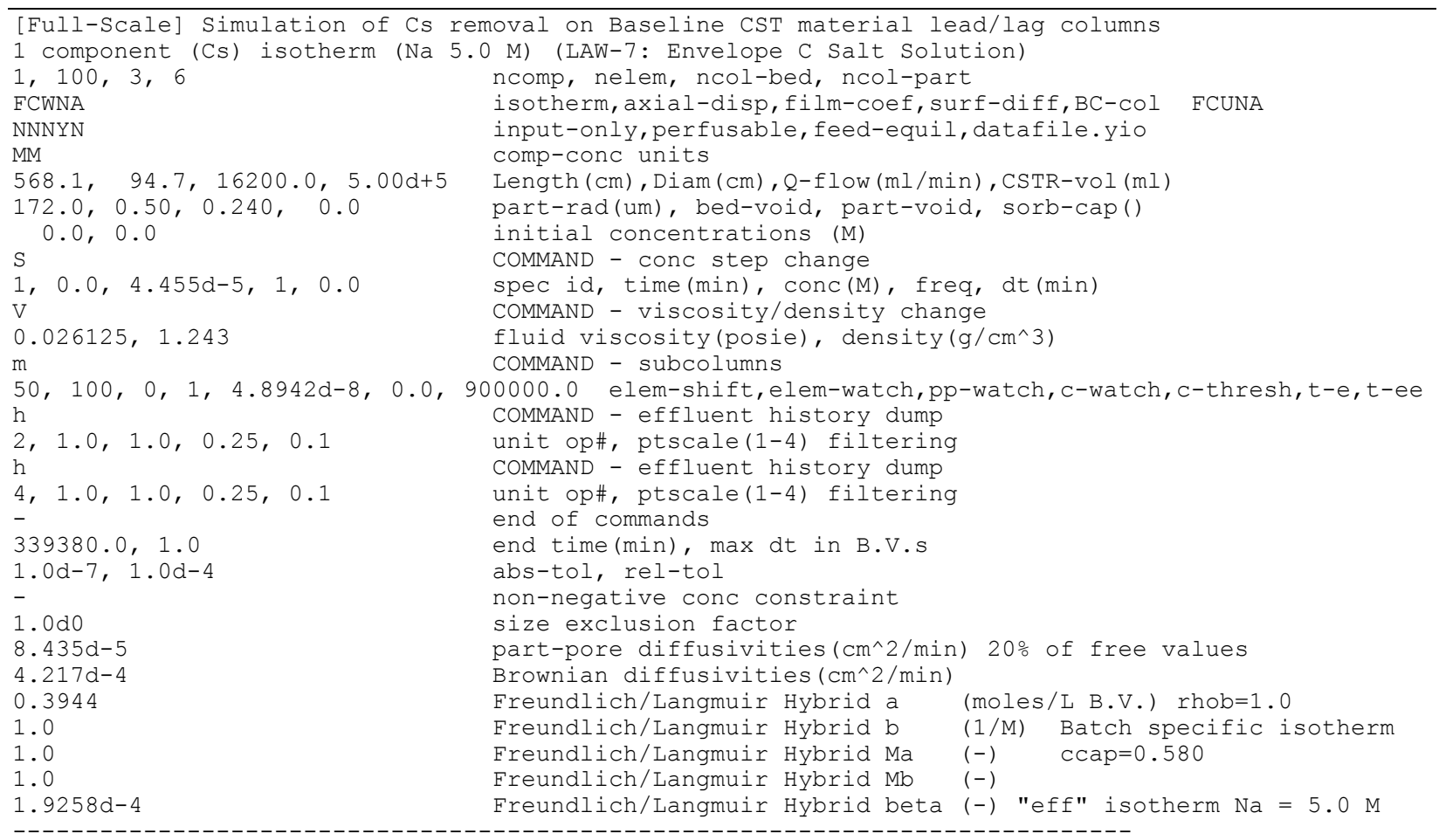




\section{VERSE-LC Input for Phase 1 LAW-8 Batch Feed (nominal case; 2000 L)}

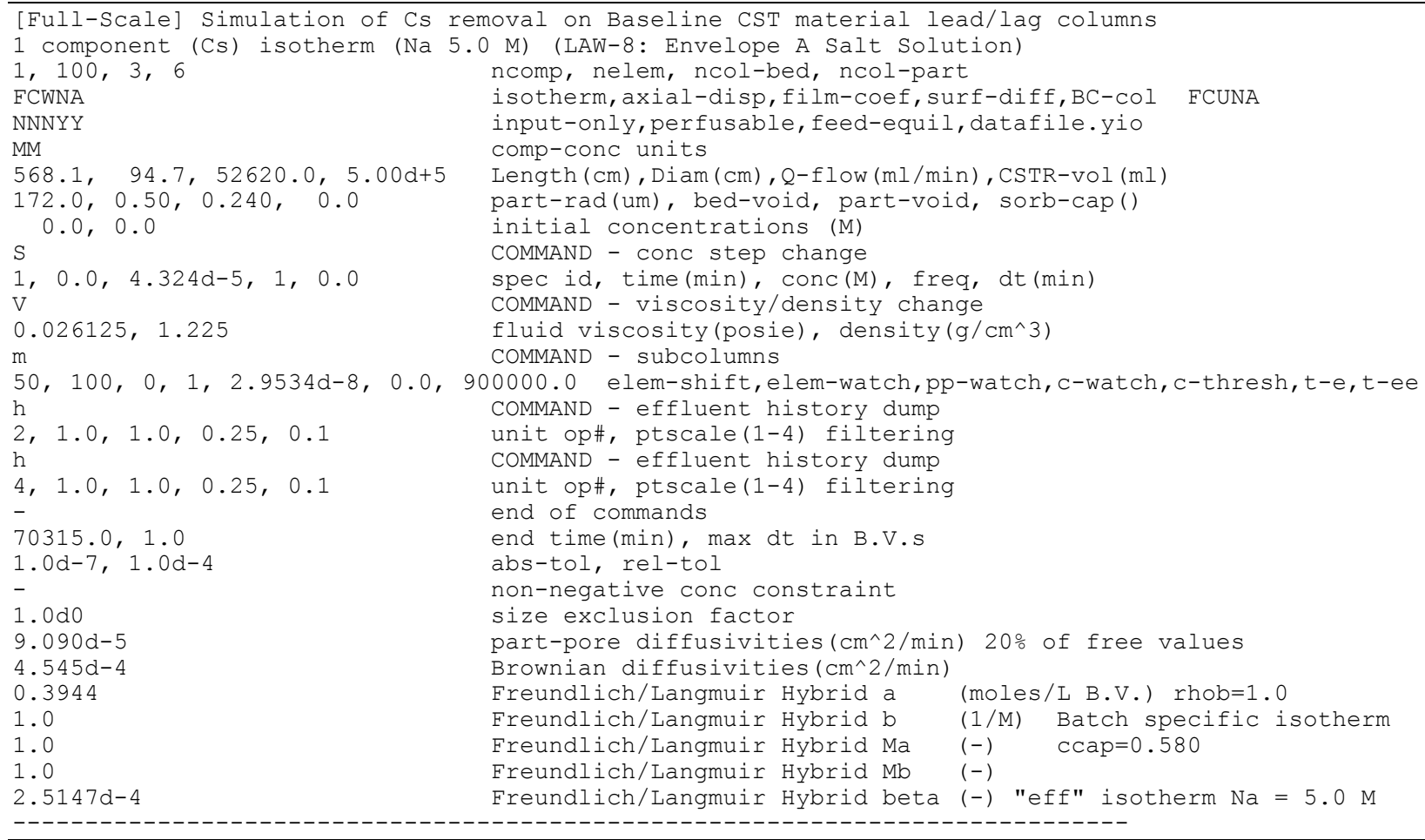

\section{VERSE-LC Input for Phase 1 LAW-9 Batch Feed (nominal case; 2000 L)}

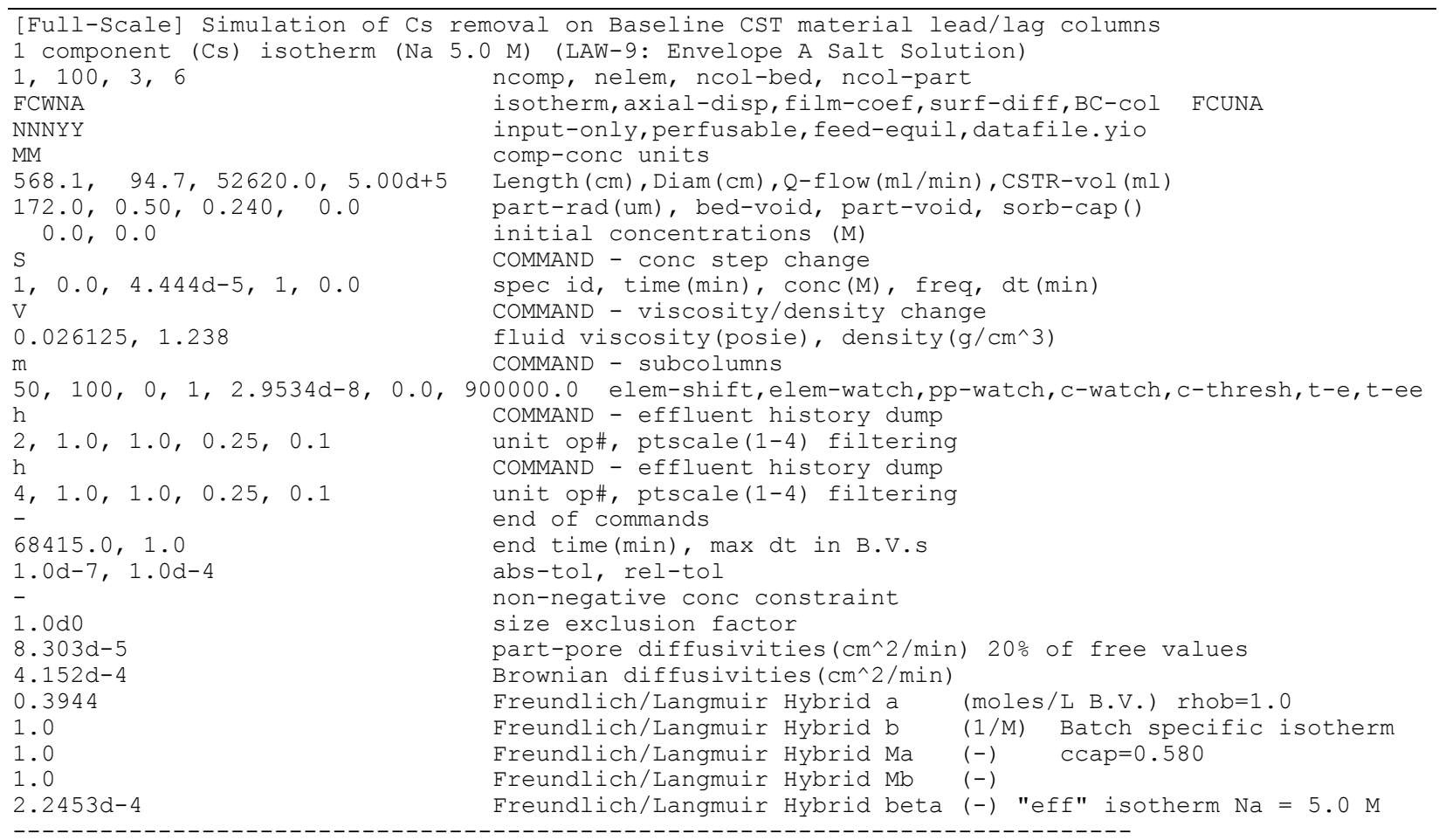




\section{VERSE-LC Input for Phase 1 LAW-10 Batch Feed (nominal case; 2000 L)}

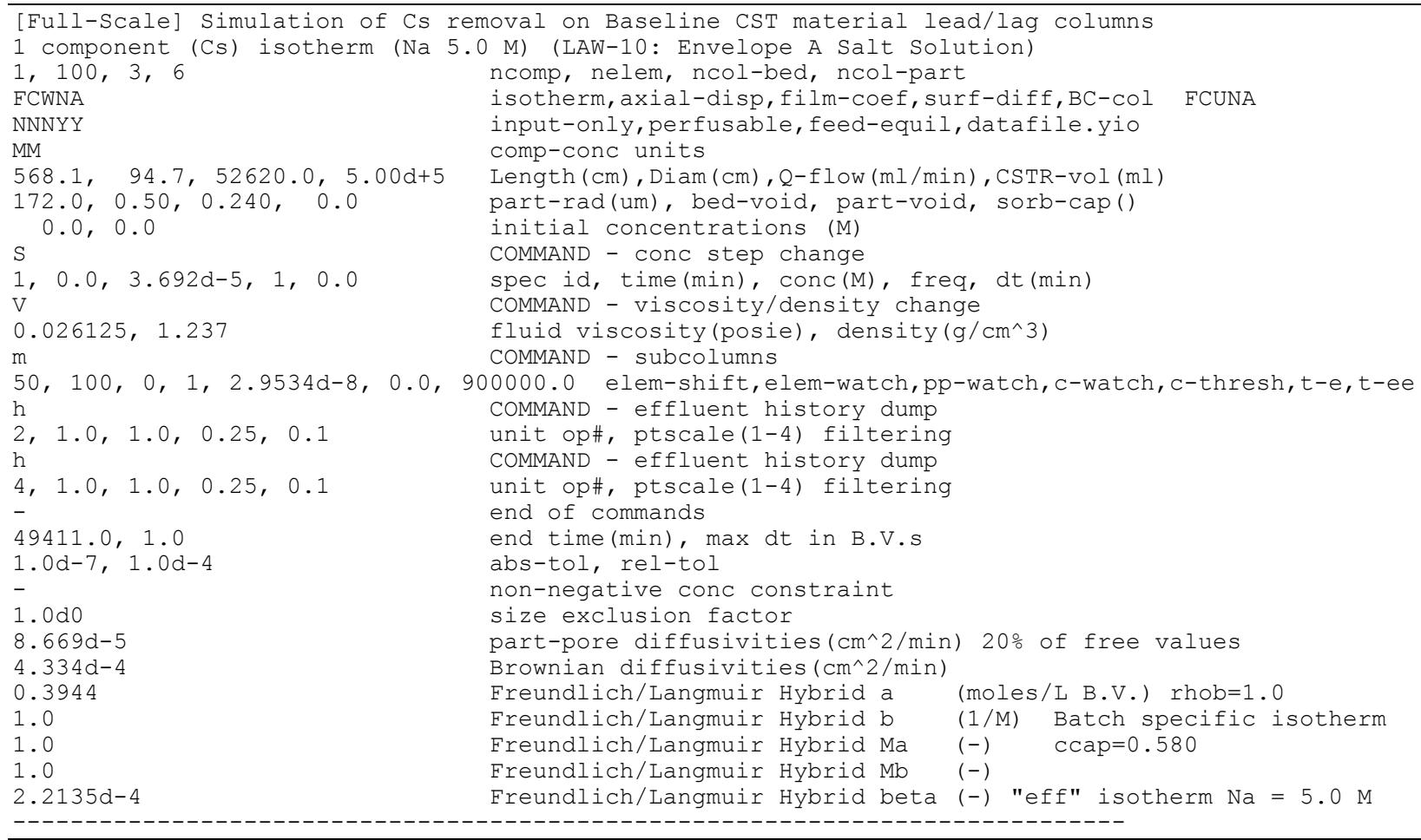

\section{VERSE-LC Input for Phase 1 LAW-11 Batch Feed (nominal case; 2000 L)}

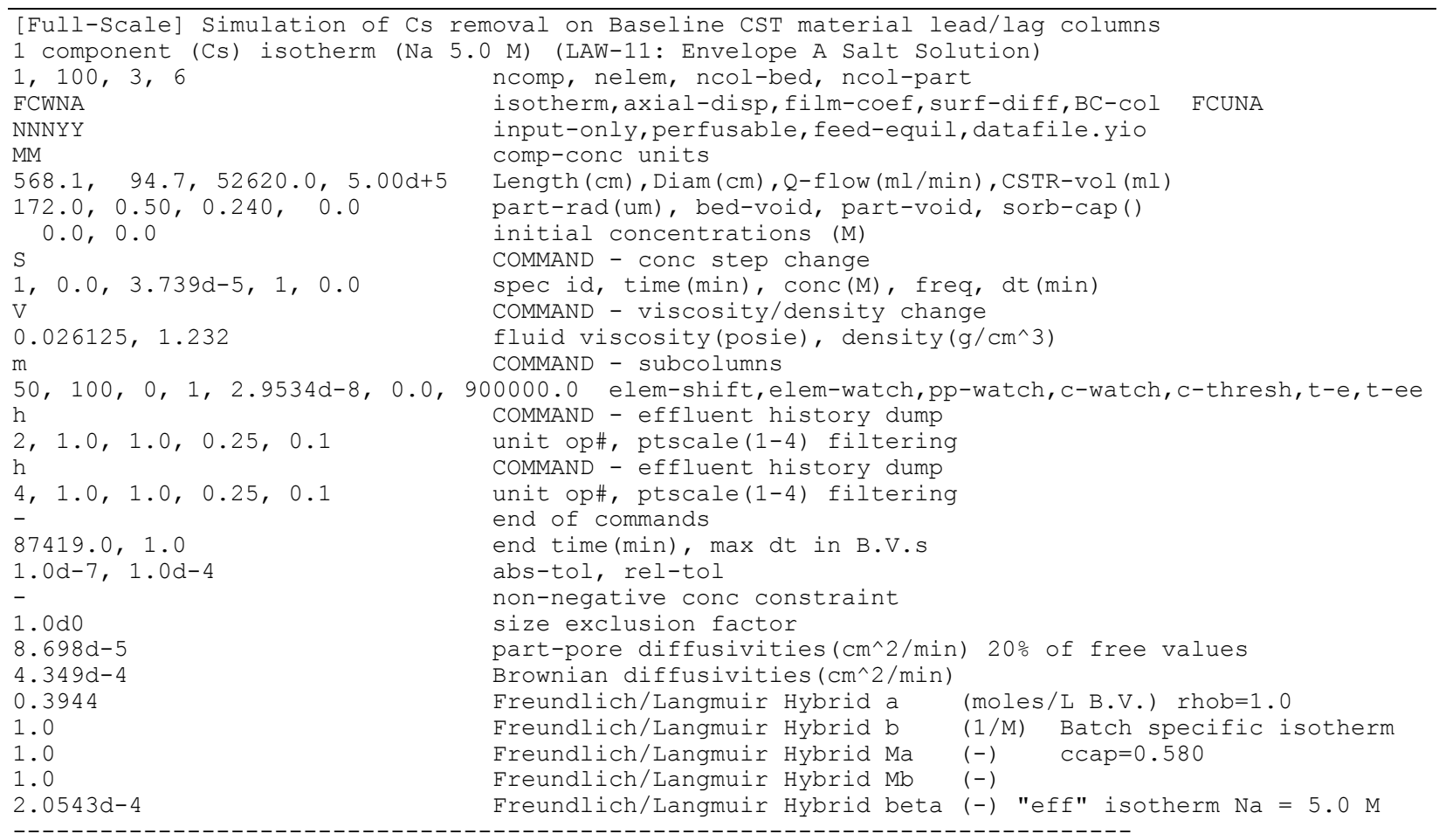




\section{VERSE-LC Input for Phase 1 LAW-12 Batch Feed (nominal case; 2000 L)}

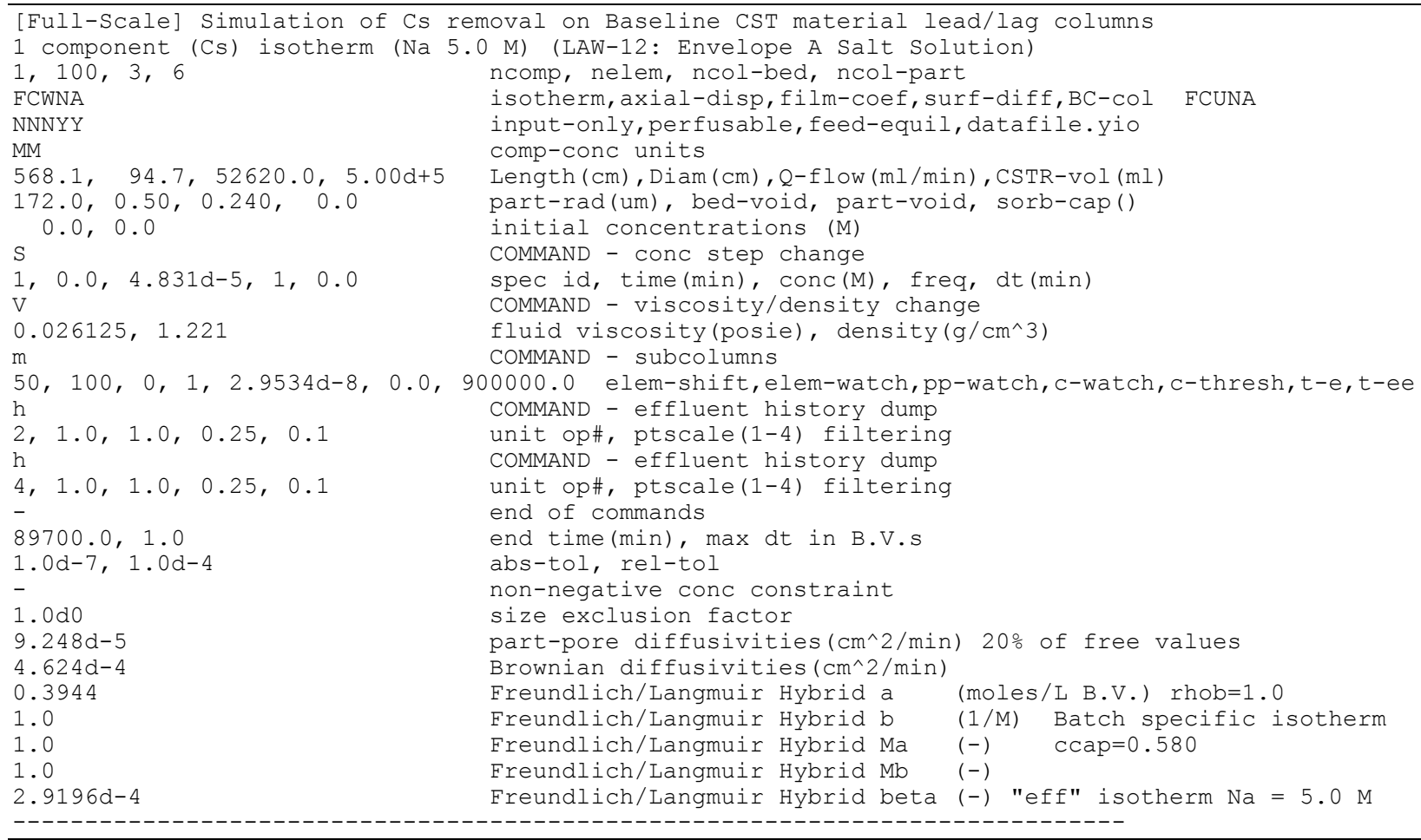

\section{VERSE-LC Input for Phase 1 LAW-13 Batch Feed (nominal case; 2000 L)}

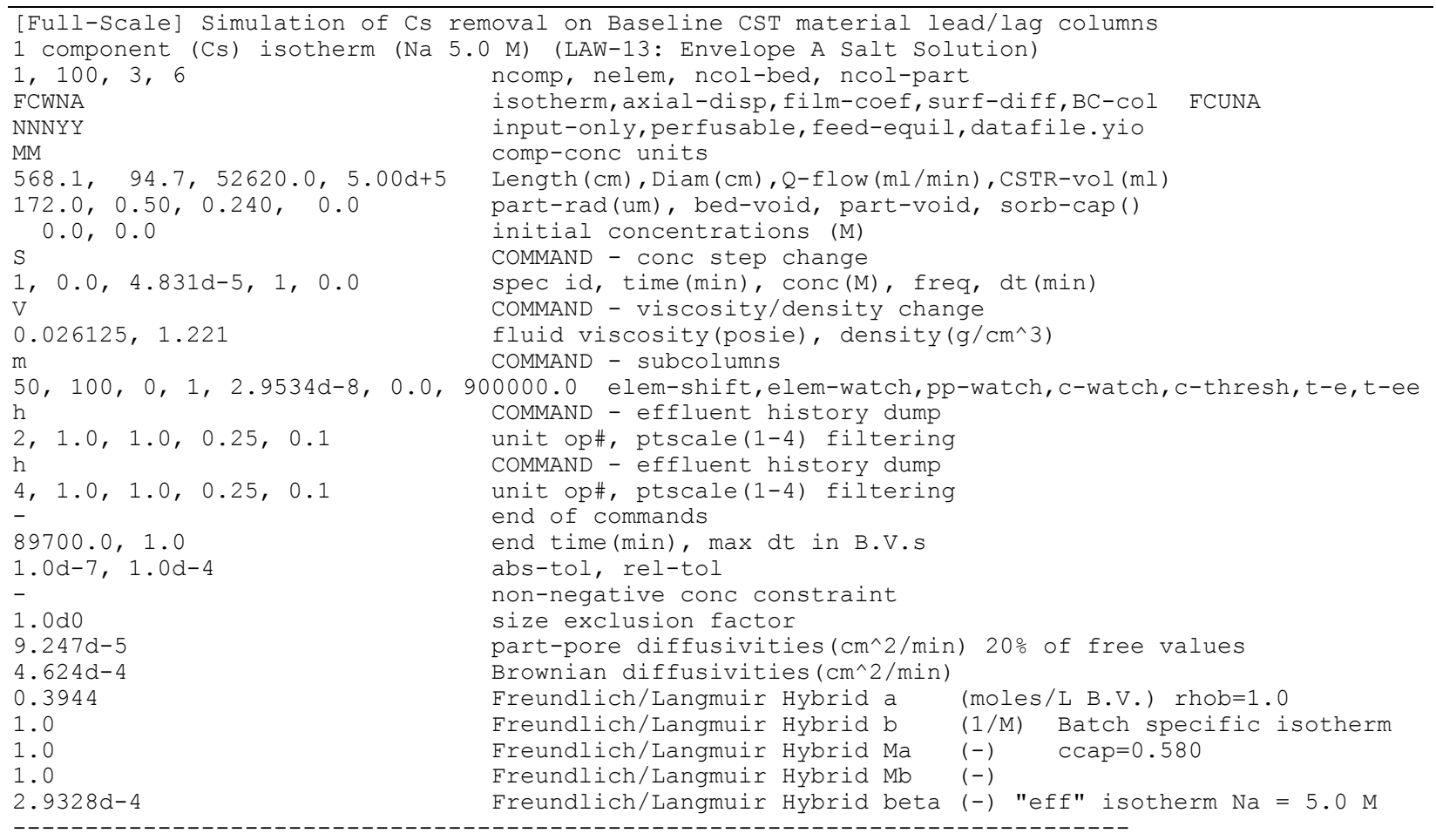




\section{VERSE-LC Input for Phase 1 LAW-14 Batch Feed (nominal case; 2000 L)}

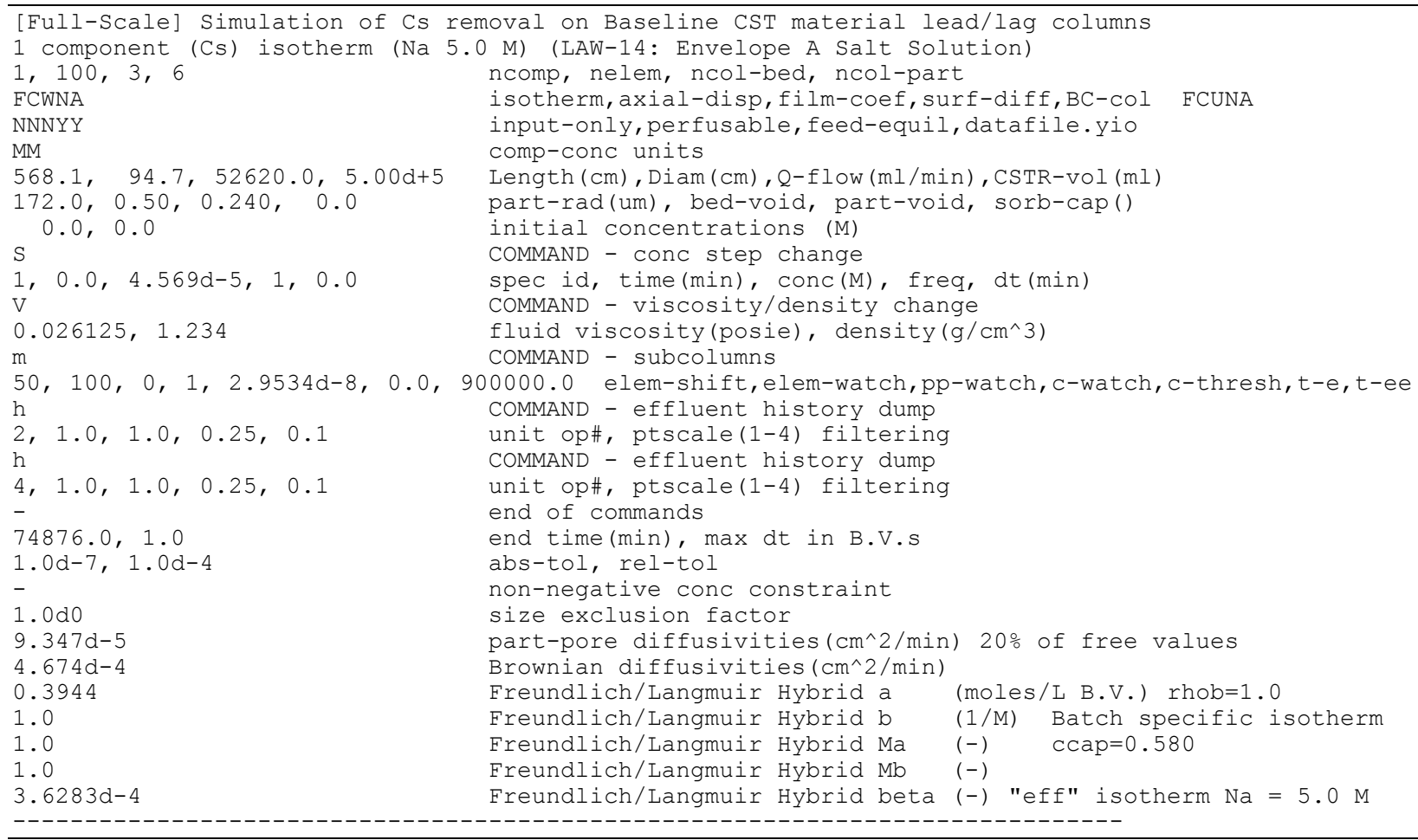

\section{VERSE-LC Input for Phase 1 LAW-15 Batch Feed (nominal case; 2000 L)}

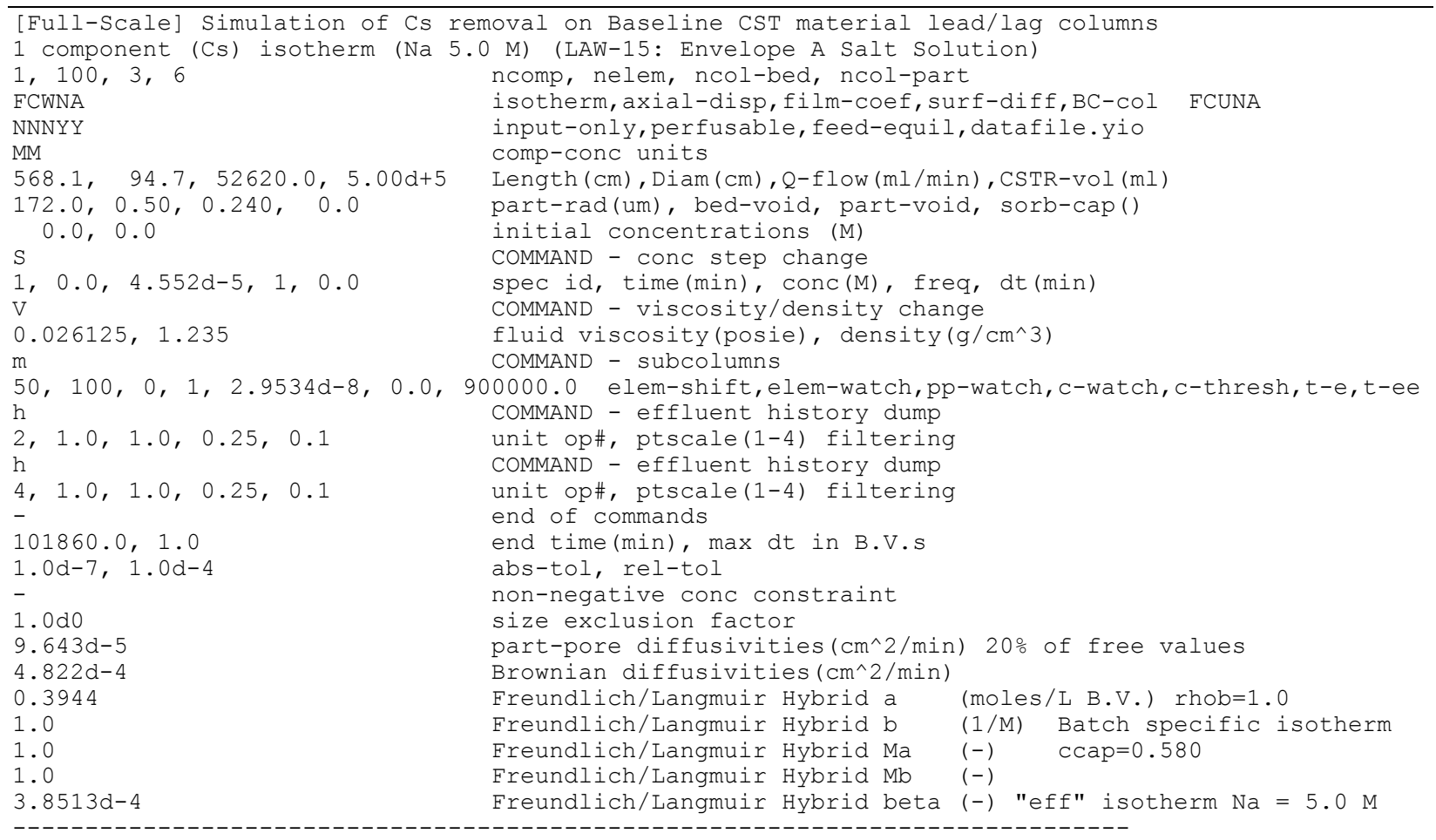




\section{VERSE-LC Output for Phase 1 LAW-1 Batch Feed (nominal case; 2000 L)}

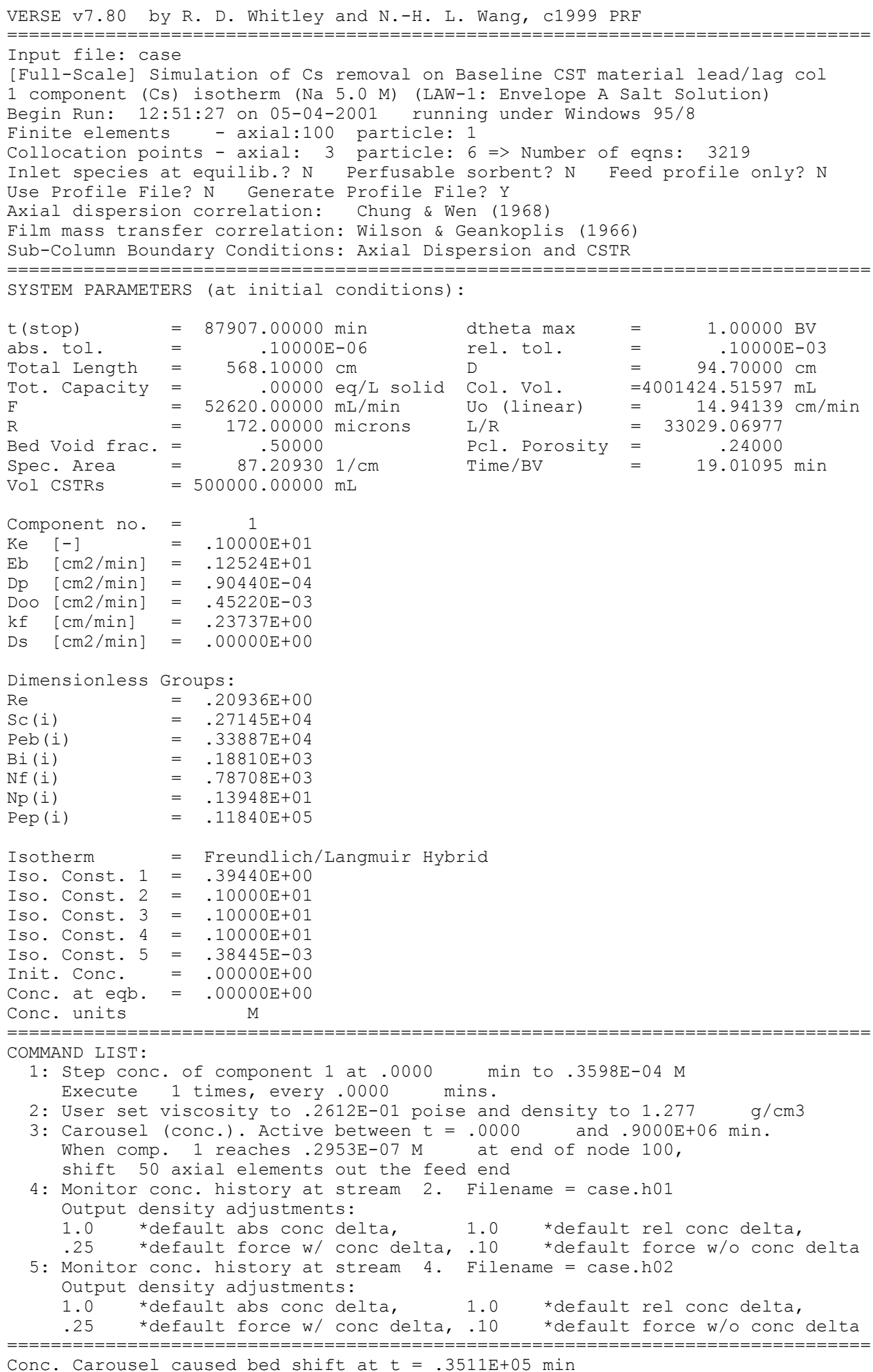


Conc. Carousel caused bed shift at $t=.6174 \mathrm{E}+05 \mathrm{~min}$

VERSE-LC finished in 5032 steps. Average step size 17.47 minutes

End run: 12:58:15 on 05-04-2001

Integrated Areas in History Files:

case.h01 .253632

case.h02 .206094E-03

\section{VERSE-LC Output for Phase 1 LAW-2b Batch Feed (nominal case; 2000 L)}

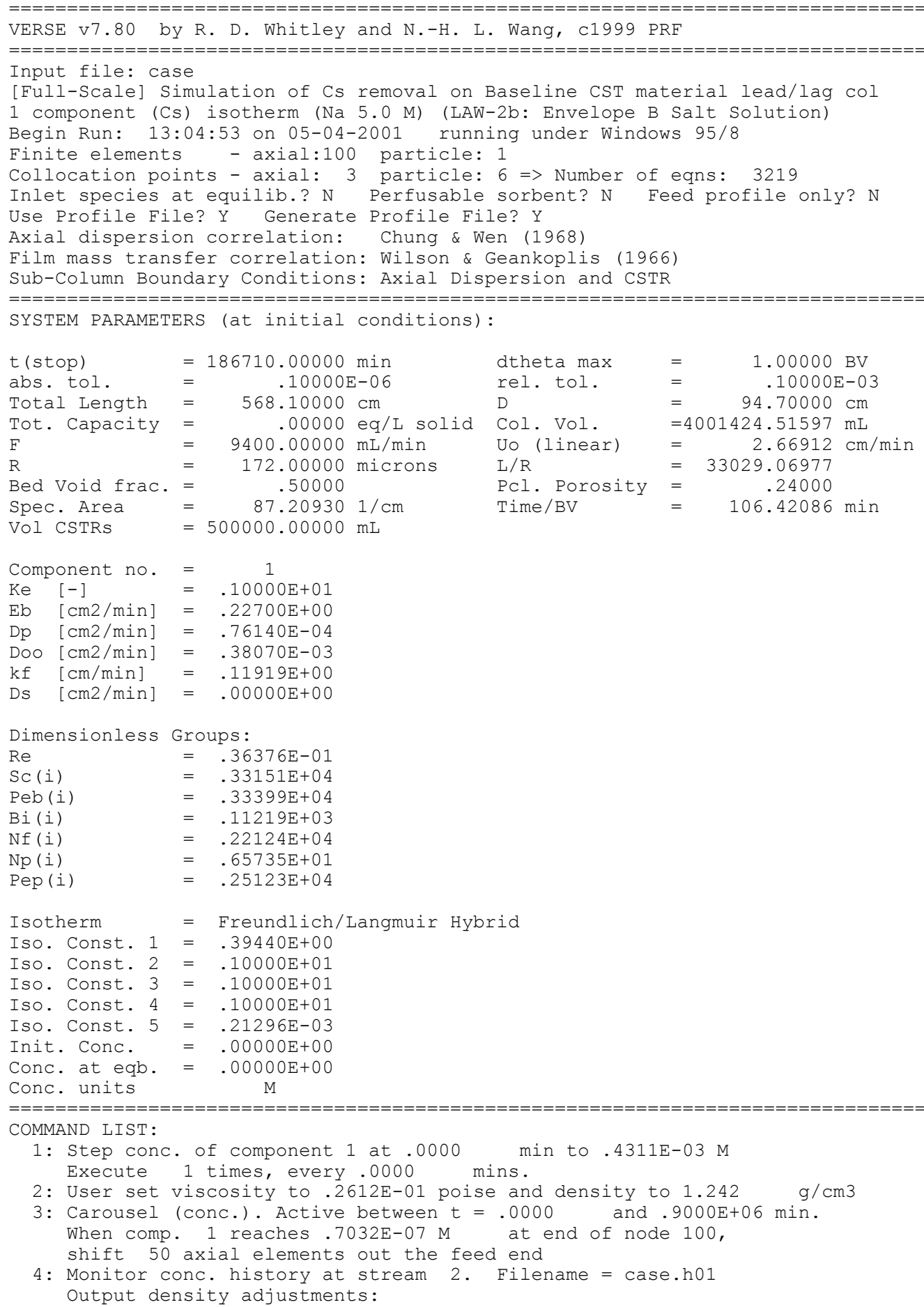


1.0 *default abs conc delta, 1.0 *default rel conc delta,

.25 *default force w/ conc delta, .10 *default force w/o conc delta

5: Monitor conc. history at stream 4. Filename = case.h02

Output density adjustments:

1.0 *default abs conc delta, 1.0 *default rel conc delta,

.25 *default force w/ conc delta, .10 *default force w/o conc delta

Conc. Carousel caused bed shift at $t=.1018 \mathrm{E}+06 \mathrm{~min}$

VERSE-LC finished in 1798 steps. Average step size 103.8 minutes

End run: 13:06:47 on 05-04-2001

Integrated Areas in History Files:

case.h01 77.2638

case.h02 .782044E-04

\section{VERSE-LC Output for Phase 1 LAW-3 Batch Feed (nominal case; 2000 L)}

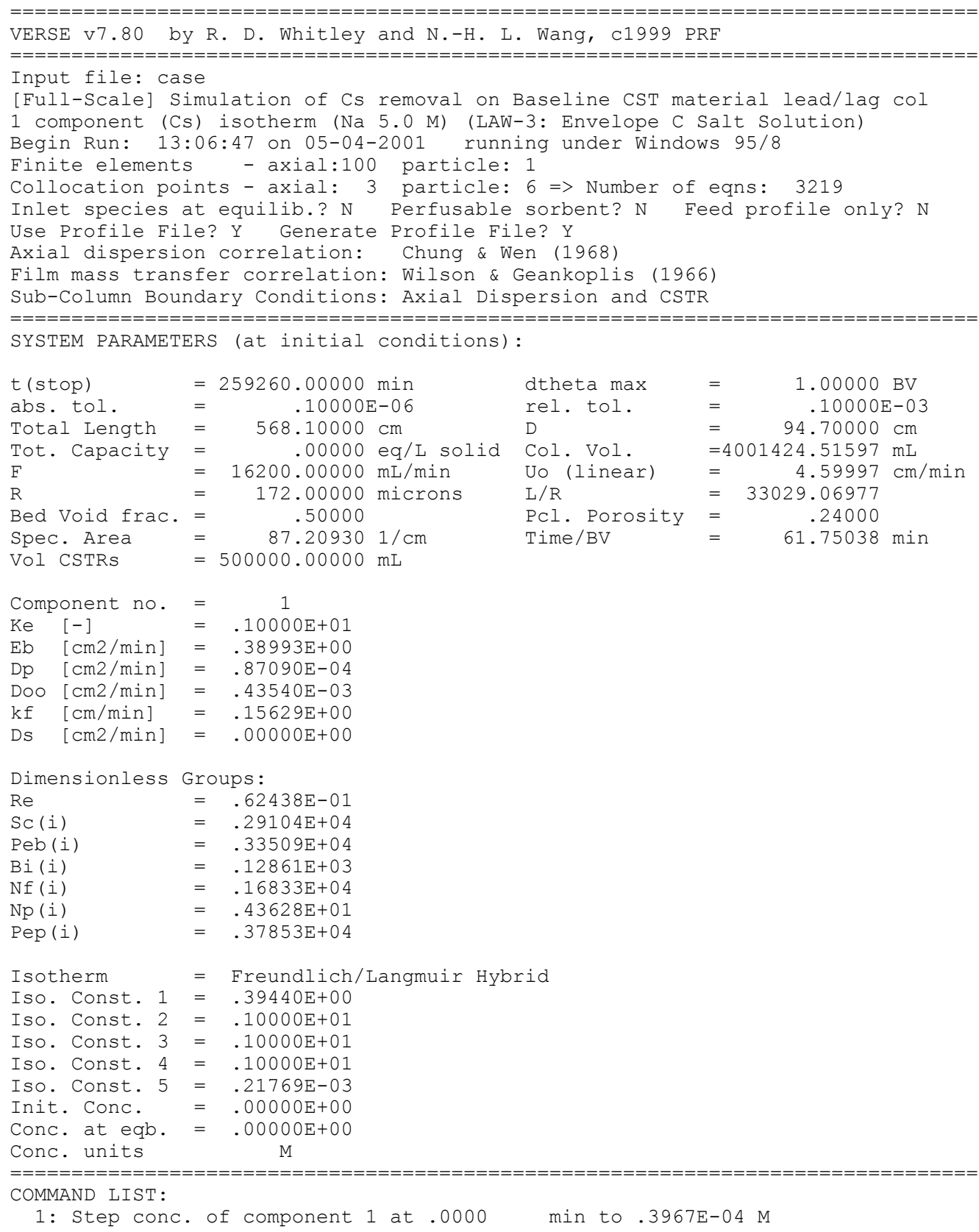


Execute 1 times, every .0000 mins.

2: User set viscosity to.2612E-01 poise and density to $1.237 \mathrm{~g} / \mathrm{cm} 3$

3: Carousel (conc.). Active between $t=.0000$ and .9000E+06 min.

When comp. 1 reaches .4894E-07 M at end of node 100,

shift 50 axial elements out the feed end

4: Monitor conc. history at stream 2. Filename = case.h01

Output density adjustments:

1.0 *default abs conc delta, 1.0 *default rel conc delta,

.25 *default force w/ conc delta, .10 *default force w/o conc delta

5: Monitor conc. history at stream 4. Filename = case.h02

Output density adjustments:

1.0 *default abs conc delta, 1.0 *default rel conc delta,

.25 *default force w/ conc delta, .10 *default force w/o conc delta

.

Conc. Carousel caused bed shift at $t=.2286 \mathrm{E}+05 \mathrm{~min}$

Conc. Carousel caused bed shift at $t=.1043 \mathrm{E}+06 \mathrm{~min}$

Conc. Carousel caused bed shift at $t=.2129 \mathrm{E}+06 \mathrm{~min}$

VERSE-LC finished in 4561 steps. Average step size 56.84 minutes

End run: 13:13:25 on 05-04-2001

Integrated Areas in History Files:

$\begin{array}{ll}\text { case.h01 } & 46.7109\end{array}$

case.h02 .257035E-03 


\section{Appendix E (Batch Kinetics Test Input and Output Files)}

For reference the VERSE-LC input and output files for the batch kinetics test simulations are provided in this appendix. Several simulations were run. This appendix contains the measured transient liquid-phase cesium response corresponding to several batch kinetics tests performed using CST powder and engineered forms. For each CST material a cesium isotherm was created based on the batch $\mathrm{K}_{\mathrm{d}}$ test with the longest contact time. A beta factor was computed where it is assumed that $100 \%$ equilibrium is reached once this contact time has occurred. Based on the amounts of CST material and solution used for each batch contact test, material balance calculations were used to estimate the test's bed density and porosity. The various values computed and used in the VERSE-LC simulations are also tabulated in this appendix.

\section{E.1 PNNL Kinetics Studies}

The batch kinetics test data taken by Brown et al. (1996) are listed in Table C-1. Table C-2 provides a listing of the key test parameters. Three CST materials were tested where timed $\mathrm{K}_{\mathrm{d}}$ tests were performed. A listing of input VERSE-LC files is also provided in this appendix for all three materials, while only a VERSE-LC output file for the powder form is given.

\section{E.2 SRS Kinetics Studies}

The batch kinetics test data taken by Fondeur et al. (2000) are listed in Table C-3. Table C-4 provides a listing of the key test parameters. One CST material was tested (i.e., the Baseline engineered-form of CST) where timed $\mathrm{K}_{\mathrm{d}}$ tests were performed. A listing of an input and its output VERSE-LC files are also provided in this appendix.

\section{E.3 Particle Size Impact on Kinetics}

A series of transient cesium uptake tests were performed to investigate the impact that CST particle size has on its kinetics. This was part of an earlier effort to determining an optimum sized engineered-form of CST. The batch kinetics test data taken by Miller and Brown (1997) and by Anthony et al. (1996) are listed in Table C-5. Table C-6 provides a listing of the key test parameters. Three CST materials were tested (i.e., TAM5 powder and two TAM5 generated engineered-forms of CST with average particle diameters of $\sim 112 \mu \mathrm{m}$ and $\sim 334 \mu \mathrm{m}$ ) where transient cesium uptake tests were performed. A listing of input VERSE-LC files is also provided in this appendix.

\section{E.4 ORNL Kinetics Studies}

The batch kinetics test data taken by Davidson et al. (1998) are listed in Table C-7. Table C-8 provides a listing of the key test parameters. One CST material was tested (i.e., a powder-form of CST) where timed $\mathrm{K}_{\mathrm{d}}$ tests were performed. 
WESTINGHOUSE SAVANNAH RIVER COMPANY

Preliminary Ion Exchange Modeling for Removal of Cesium from Hanford Waste Using Hydrous Crystalline Silicotitanate Material
Report: WSRC-TR-2001-00400

Revision (Date): $\quad 0(07 / 05 / 02)$

Page:
262 of 338

Table E-1. Cesium uptake measurements made at $25 \mathrm{C}$ during the batch kinetics tests of Brown et al., 1996 (initial cesium concentration of $1.0 \times 10^{-4} \mathrm{M}$ ).

\begin{tabular}{|c|c|c|c|c|c|c|}
\hline & \multicolumn{3}{|c|}{$\begin{array}{c}\text { Cs K value, } \\
\text { (ml/g) }\end{array}$} & \multicolumn{2}{c|}{ Final Liquid Cs conc., } \\
[M]
\end{tabular}


WESTINGHOUSE SAVANNAH RIVER COMPANY

Preliminary Ion Exchange Modeling for Removal of Cesium from Hanford Waste Using Hydrous Crystalline Silicotitanate Material
Report:

WSRC-TR-2001-00400

Revision (Date):

Page:
$0(07 / 05 / 02)$ 263 of 338

Table E-2. Key parameters measured or specified during the batch kinetics tests of Brown et al. (1996) and used to establish the cesium isotherms used in VERSE-LC kinetic modeling.

\begin{tabular}{|c|c|c|c|}
\hline Parameter & $\begin{array}{l}\text { Parameter setting } \\
\text { for powder form }\end{array}$ & $\begin{array}{c}\text { Parameter setting } \\
\text { for engineered- } \\
\text { form (08) }\end{array}$ & $\begin{array}{l}\text { Parameter setting } \\
\text { for engineered- } \\
\text { form }(\mathbf{3 8 b})\end{array}$ \\
\hline CST material & IE-910 & IE-911 & IE-911 \\
\hline Temperature & $25^{\circ} \mathrm{C}$ & $25^{\circ} \mathrm{C}$ & $25^{\circ} \mathrm{C}$ \\
\hline Initial liquid $\mathrm{Na}^{+}$conc. & $5.0 \mathrm{M}$ & $5.0 \mathrm{M}$ & $5.0 \mathrm{M}$ \\
\hline Initial liquid $\mathrm{Cs}^{+}$conc. & $1.0 \times 10^{-4} \mathrm{M}$ & $1.0 \times 10^{-4} \mathrm{M}$ & $1.0 \times 10^{-4} \mathrm{M}$ \\
\hline $\begin{array}{l}\text { Final liquid } \mathrm{Cs}^{+} \text {conc. } \\
\text { (actually at } 120 \text { hours) }\end{array}$ & $5.060 \times 10^{-6} \mathrm{M}$ & $9.295 \times 10^{-6} \mathrm{M}$ & $1.026 \times 10^{-5} \mathrm{M}$ \\
\hline Initial liquid sample volume & $10.0 \mathrm{ml}$ & $10.0 \mathrm{ml}$ & $10.0 \mathrm{ml}$ \\
\hline Initial resin mass & $0.1 \mathrm{~g}$ & $0.1 \mathrm{~g}$ & $0.1 \mathrm{~g}$ \\
\hline Batch ID & na & Lot 08 & Lot $38 \mathrm{~b}$ \\
\hline Solution composition & $70 \% \mathrm{AW}-101 \mathrm{DSSF}$ & $70 \%$ AW-101 DSSF & $70 \%$ AW-101 DSSF \\
\hline Particle porosity & 0.1 & 0.24 & 0.24 \\
\hline solid density & $1.176 \mathrm{~g} / \mathrm{ml}$ & $1.717 \mathrm{~g} / \mathrm{ml}$ & $1.368 \mathrm{~g} / \mathrm{ml}$ \\
\hline Solution density & $1.409 \mathrm{~g} / \mathrm{ml}$ & $1.409 \mathrm{~g} / \mathrm{ml}$ & $1.409 \mathrm{~g} / \mathrm{ml}$ \\
\hline Solution viscosity & na & na & na \\
\hline $\mathrm{F}$ factor & 0.968 & 0.887 & 0.879 \\
\hline Phase ratio & 103.31 & 112.74 & 111.23 \\
\hline Bed density & $0.009601 \mathrm{~g} / \mathrm{ml}$ & $0.008824 \mathrm{~g} / \mathrm{ml}$ & $0.008931 \mathrm{~g} / \mathrm{ml}$ \\
\hline Bed porosity & 0.989259 & 0.993240 & 0.991409 \\
\hline $\begin{array}{l}\text { Estimated beta value for cesium } \\
\text { isotherm }\end{array}$ & $2.94143 \times 10^{-4} \mathrm{M}$ & $3.49210 \times 10^{-4} \mathrm{M}$ & $3.94901 \times 10^{-4} \mathrm{M}$ \\
\hline
\end{tabular}

Table E-3. Cesium uptake measurements made at $25 \mathrm{C}$ during the batch kinetics tests of Fondeur et al., 2000. Also included are estimated conditions at earlier contact times.

\begin{tabular}{|c|c|c|c|c|c|}
\hline $\begin{array}{c}\text { Batch Kinetics } \\
\text { Contact Test } \\
\text { ID }\end{array}$ & $\begin{array}{l}\text { Contact } \\
\text { time } \\
\text { (hrs) }\end{array}$ & $\begin{array}{c}\text { Initial Liquid } \\
\mathrm{Cs}^{+} \text {conc. } \\
{[\mathrm{M}]}\end{array}$ & $\begin{array}{c}K_{d} \text { value } \\
(\mathrm{ml} / \mathrm{g})\end{array}$ & $\begin{array}{c}\text { Final Liquid } \\
\mathrm{Cs}^{+} \text {conc. } \\
{[\mathrm{M}]}\end{array}$ & $\begin{array}{c}\text { Approach to } \\
\text { equilibrium } \\
(\%)\end{array}$ \\
\hline Fondeur & 0.0 & $1.40 \mathrm{E}-04$ & - & $1.400 \mathrm{E}-04$ & 0.00 \\
\hline estimated & 0.03333 & $1.40 \mathrm{E}-04$ & 2.0 & $1.388 \mathrm{E}-04$ & 0.98 \\
\hline estimated & 0.1 & $1.40 \mathrm{E}-04$ & 7.0 & $1.359 \mathrm{E}-04$ & 3.36 \\
\hline estimated & 0.5 & $1.40 \mathrm{E}-04$ & 25.0 & $1.264 \mathrm{E}-04$ & 11.15 \\
\hline estimated & 1.0 & $1.40 \mathrm{E}-04$ & 51.0 & $1.148 \mathrm{E}-04$ & 20.67 \\
\hline estimated & 3.0 & $1.40 \mathrm{E}-04$ & 147.0 & $8.578 \mathrm{E}-05$ & 44.50 \\
\hline estimated & 6.0 & $1.40 \mathrm{E}-04$ & 265.0 & $6.544 \mathrm{E}-05$ & 61.20 \\
\hline estimated & 12.0 & $1.40 \mathrm{E}-04$ & 500.0 & 4.444E-05 & 78.43 \\
\hline
\end{tabular}


WESTINGHOUSE SAVANNAH RIVER COMPANY

Report: $\quad$ WSRC-TR-2001-00400

Preliminary Ion Exchange Modeling for Removal of Cesium from Hanford Waste Using Hydrous Crystalline Silicotitanate Material

Revision (Date): $\quad 0(07 / 05 / 02)$

Page:

264 of 338

\begin{tabular}{|l|c|c|c|c|c|}
\hline Fondeur & 24.0 & $1.40 \mathrm{E}-04$ & 758.13 & $3.286 \mathrm{E}-05$ & 87.93 \\
\hline Fondeur & 48.0 & $1.40 \mathrm{E}-04$ & 1002.34 & $2.637 \mathrm{E}-05$ & 93.27 \\
\hline Fondeur & 72.0 & $1.40 \mathrm{E}-04$ & 1099.57 & $2.444 \mathrm{E}-05$ & 94.85 \\
\hline Fondeur & 96.0 & $1.40 \mathrm{E}-04$ & 1227.15 & $2.230 \mathrm{E}-05$ & 96.60 \\
\hline Fondeur & 120.0 & $1.40 \mathrm{E}-04$ & 1389 & $2.008 \mathrm{E}-05$ & 98.43 \\
\hline Fondeur & 144.0 & $1.40 \mathrm{E}-04$ & 1496 & $1.884 \mathrm{E}-05$ & 99.45 \\
\hline Fondeur & 168.0 & $1.40 \mathrm{E}-04$ & 1523 & $1.855 \mathrm{E}-05$ & 99.69 \\
\hline Fondeur & 192.0 & $1.40 \mathrm{E}-04$ & 1560 & $1.816 \mathrm{E}-05$ & 100.00 \\
\hline
\end{tabular}

Table E-4. Key parameters measured or specified during the batch kinetics tests of Fondeur et al. (2000) and used to establish the cesium isotherm used in VERSE-LC kinetic modeling.

\begin{tabular}{|c|c|}
\hline Parameter & Parameter setting \\
\hline CST material & IE-911 \\
\hline Temperature & $25^{\circ} \mathrm{C}$ \\
\hline Initial liquid $\mathrm{Na}^{+}$conc. & $5.6 \mathrm{M}$ \\
\hline Initial liquid $\mathrm{Cs}^{+}$conc. & $1.40 \times 10^{-4} \mathrm{M}$ \\
\hline $\begin{array}{l}\text { Final liquid } \mathrm{Cs}^{+} \text {conc. } \\
\text { (actually at } 192 \text { hours) }\end{array}$ & $1.816 \times 10^{-5} \mathrm{M}$ \\
\hline Initial liquid sample volume & $20.0 \mathrm{ml}$ \\
\hline Initial resin mass & $0.1 \mathrm{~g}$ \\
\hline Batch ID & Lot 9090-76 \\
\hline Solution composition & SRS Avg. \\
\hline Particle porosity & 0.24 \\
\hline solid density & $1.520 \mathrm{~g} / \mathrm{ml}$ \\
\hline solution density & $1.253 \mathrm{~g} / \mathrm{ml}$ \\
\hline solution viscosity & $2.78 \mathrm{cP}$ \\
\hline $\mathrm{F}$ factor & 0.86 \\
\hline Phase ratio & 232.56 \\
\hline Bed density & $0.004288 \mathrm{~g} / \mathrm{ml}$ \\
\hline Bed porosity & 0.996288 \\
\hline $\begin{array}{c}\text { Estimated beta value for cesium } \\
\text { isotherm }\end{array}$ & $3.53632 \times 10^{-4} \mathrm{M}$ \\
\hline
\end{tabular}


Table E-5. Cesium uptake measurements made at $25 \mathrm{C}$ during the transient cesium uptake tests of Miller and Brown (1997) and Anthony et al., 1996.

\begin{tabular}{|c|c|c|c|c|c|}
\hline \multicolumn{2}{|c|}{$\begin{array}{c}\text { Miller and Brown (1997) } \\
\text { TAM5 powder } \\
\text { avg diameter of 0.8 } \mu \mathrm{m}\end{array}$} & \multicolumn{2}{c|}{$\begin{array}{c}\text { Anthony et al. (1996) } \\
\text { Engineered-form } \\
\text { avg diameter of 112 } \mu \mathrm{m}\end{array}$} & \multicolumn{2}{c|}{$\begin{array}{c}\text { Anthony et al. (1996) } \\
\text { Engineered-form } \\
\text { avg diameter of 334 } \mu \text { m }\end{array}$} \\
\hline $\begin{array}{c}\text { Contact } \\
\text { time } \\
\text { (hrs) }\end{array}$ & $\begin{array}{c}\text { Liquid Cs } \\
\text { conc. } \\
{[\mathbf{M}]}\end{array}$ & $\begin{array}{c}\text { Contact } \\
\text { time } \\
\text { (hrs) }\end{array}$ & $\begin{array}{c}\text { Liquid Cs } \\
\text { conc. } \\
{[\mathbf{M}]}\end{array}$ & $\begin{array}{c}\text { Contact } \\
\text { time } \\
\text { (hrs) }\end{array}$ & $\begin{array}{c}\text { Liquid Cs } \\
\text { conc. } \\
\text { [M] }\end{array}$ \\
\hline \hline 0.0 & $1.0 \mathrm{E}-04$ & 0.0 & $1.0 \mathrm{E}-04$ & 0.0 & $1.0 \mathrm{E}-04$ \\
\hline 0.030 & $6.154 \mathrm{E}-05$ & 0.0405 & $7.000 \mathrm{E}-05$ & 0.0405 & $8.909 \mathrm{E}-05$ \\
\hline 0.070 & $5.385 \mathrm{E}-05$ & 0.0811 & $5.500 \mathrm{E}-05$ & 0.0811 & $8.136 \mathrm{E}-05$ \\
\hline 0.080 & $5.055 \mathrm{E}-05$ & 0.1216 & $4.818 \mathrm{E}-05$ & 0.1216 & $7.773 \mathrm{E}-05$ \\
\hline 0.130 & $4.286 \mathrm{E}-05$ & 0.1622 & $4.364 \mathrm{E}-05$ & 0.1622 & $7.455 \mathrm{E}-05$ \\
\hline 0.160 & $4.011 \mathrm{E}-05$ & 0.2595 & $3.727 \mathrm{E}-05$ & 0.2595 & $6.864 \mathrm{E}-05$ \\
\hline 0.245 & $3.516 \mathrm{E}-05$ & 0.3243 & $3.364 \mathrm{E}-05$ & 0.3243 & $6.500 \mathrm{E}+05$ \\
\hline 0.330 & $3.187 \mathrm{E}-05$ & 0.4865 & $2.864 \mathrm{E}-05$ & 0.4865 & $5.864 \mathrm{E}-05$ \\
\hline 0.500 & $2.692 \mathrm{E}-05$ & 1.0135 & $2.000 \mathrm{E}-05$ & 1.0135 & $4.591 \mathrm{E}-05$ \\
\hline 1.000 & $1.758 \mathrm{E}-05$ & 1.9865 & $1.864 \mathrm{E}-05$ & 1.9865 & $3.455 \mathrm{E}-05$ \\
\hline- & - & 6.0000 & $1.682 \mathrm{E}-05$ & 6.0000 & $2.046 \mathrm{E}-05$ \\
\hline- & - & 12.0000 & $1.546 \mathrm{E}-05$ & 12.0000 & $1.864 \mathrm{E}-05$ \\
\hline
\end{tabular}


Preliminary Ion Exchange Modeling for Removal of Cesium from

Revision (Date):

Table E-6. Key parameters measured or specified during the transient cesium uptake tests of Miller and Brown (1997) and Anthony et al. (1996) and used to establish the cesium isotherm used in VERSE-LC kinetic modeling.

\begin{tabular}{|c|c|c|c|}
\hline Parameter & Parameter setting & Parameter setting & Parameter setting \\
\hline CST material & Avg diameter $0.8 \mathrm{~mm}$ & Avg diameter $112 \mathrm{~mm}$ & Avg diameter $334 \mathrm{~mm}$ \\
\hline Temperature & $25^{\circ} \mathrm{C}$ & $25^{\circ} \mathrm{C}$ & $25^{\circ} \mathrm{C}$ \\
\hline Initial liquid $\mathrm{Na}^{+}$conc. & $5.0 \mathrm{M}$ & $5.0 \mathrm{M}$ & $5.0 \mathrm{M}$ \\
\hline Initial liquid $\mathrm{Cs}^{+}$conc. & $1.0 \times 10^{-4} \mathrm{M}$ & $1.0 \times 10^{-4} \mathrm{M}$ & $1.0 \times 10^{-4} \mathrm{M}$ \\
\hline $\begin{array}{l}\text { Final liquid } \mathrm{Cs}^{+} \text {conc. } \\
\text { (actually at } 120 \text { hours) }\end{array}$ & $1.27 \times 10^{-5} \mathrm{M}$ & $1.27 \times 10^{-5} \mathrm{M}$ & $1.27 \times 10^{-5} \mathrm{M}$ \\
\hline Initial liquid sample volume & $19.0 \mathrm{ml}$ & $19.0 \mathrm{ml}$ & $19.0 \mathrm{ml}$ \\
\hline Initial resin mass & $0.1 \mathrm{~g}$ & $0.1 \mathrm{~g}$ & $0.1 \mathrm{~g}$ \\
\hline Batch ID & TAM5 powder & TAM5 generated & TAM5 generated \\
\hline Solution composition & DSSF5 simulant & DSSF5 simulant & DSSF5 simulant \\
\hline Particle porosity & .1 & 0.24 & 0.24 \\
\hline solid density & $1.520 \mathrm{~g} / \mathrm{ml}$ & $1.520 \mathrm{~g} / \mathrm{ml}$ & $1.520 \mathrm{~g} / \mathrm{ml}$ \\
\hline solution density & $1.26 \mathrm{~g} / \mathrm{ml}$ & $1.26 \mathrm{~g} / \mathrm{ml}$ & $1.26 \mathrm{~g} / \mathrm{ml}$ \\
\hline solution viscosity & na & na & na \\
\hline $\mathrm{F}$ factor & 1.0 & 1.0 & 1.0 \\
\hline Phase ratio & 190.00 & 190.00 & 190.00 \\
\hline Bed density & $0.005245 \mathrm{~g} / \mathrm{ml}$ & $0.005245 \mathrm{~g} / \mathrm{ml}$ & $0.005245 \mathrm{~g} / \mathrm{ml}$ \\
\hline Bed porosity & 0.996166 & 0.995460 & 0.995460 \\
\hline $\begin{array}{l}\text { Estimated beta value for cesium } \\
\text { isotherm }\end{array}$ & $2.89276 \times 10^{-4} \mathrm{M}$ & $2.89276 \times 10^{-4} \mathrm{M}$ & $2.89276 \times 10^{-4} \mathrm{M}$ \\
\hline
\end{tabular}


WESTINGHOUSE SAVANNAH RIVER COMPANY

Preliminary Ion Exchange Modeling for Removal of Cesium from Hanford Waste Using Hydrous Crystalline Silicotitanate Material
Report: $\quad$ WSRC-TR-2001-00400

Revision (Date): $\quad 0(07 / 05 / 02)$

Page:

Table E-7. Cesium uptake measurements made at $25 \mathrm{C}$ during the batch kinetics tests of Davidson et al., 1998.

\begin{tabular}{|c|c|c|c|c|c|}
\hline Phase ratio & $\begin{array}{c}\text { Contact } \\
\text { time } \\
\text { (hrs) }\end{array}$ & $\begin{array}{c}\text { Initial Liquid } \\
\mathrm{Cs}^{+} \text {conc. } \\
{[\mathrm{M}]}\end{array}$ & $\begin{array}{c}\mathrm{K}_{\mathrm{d}} \text { value } \\
(\mathrm{ml} / \mathrm{g})\end{array}$ & $\begin{array}{c}\text { Final Liquid } \\
\mathrm{Cs}^{+} \text {conc. } \\
{[\mathrm{M}]}\end{array}$ & $\begin{array}{c}\text { Approach to } \\
\text { equilibrium } \\
(\%)\end{array}$ \\
\hline 100 & 0.00 & $1.418 \mathrm{E}-06$ & - & $1.418 \mathrm{E}-06$ & 0.00 \\
\hline 100 & 0.25 & $1.418 \mathrm{E}-06$ & 297 & $3.572 \mathrm{E}-07$ & 83.69 \\
\hline 100 & 2.00 & $1.418 \mathrm{E}-06$ & 321 & $3.368 \mathrm{E}-07$ & 85.29 \\
\hline 100 & 24.00 & $1.418 \mathrm{E}-06$ & 686 & $1.804 \mathrm{E}-07$ & 97.63 \\
\hline 100 & 72.00 & $1.418 \mathrm{E}-06$ & 976 & $1.318 \mathrm{E}-07$ & 101.47 \\
\hline 100 & 144.00 & $1.418 \mathrm{E}-06$ & 843 & $1.504 \mathrm{E}-07$ & 100.00 \\
\hline 200 & 0.00 & $1.418 \mathrm{E}-06$ & - & $1.418 \mathrm{E}-06$ & 0.00 \\
\hline 200 & 0.25 & $1.418 \mathrm{E}-06$ & 451 & $4.356 \mathrm{E}-07$ & 83.74 \\
\hline 200 & 2.00 & $1.418 \mathrm{E}-06$ & 662 & $3.290 \mathrm{E}-07$ & 92.83 \\
\hline 200 & 24.00 & $1.418 \mathrm{E}-06$ & 672 & $3.252 \mathrm{E}-07$ & 93.15 \\
\hline 200 & 72.00 & $1.418 \mathrm{E}-06$ & 672 & $3.252 \mathrm{E}-07$ & 93.15 \\
\hline 200 & 144.00 & $1.418 \mathrm{E}-06$ & 958 & $2.449 \mathrm{E}-07$ & 100.00 \\
\hline 400 & 0.00 & $1.418 \mathrm{E}-06$ & - & $1.418 \mathrm{E}-06$ & 0.00 \\
\hline 400 & 0.25 & $1.418 \mathrm{E}-06$ & 337 & 7.696E-07 & 70.31 \\
\hline 400 & 2.00 & $1.418 \mathrm{E}-06$ & 477 & $6.468 \mathrm{E}-07$ & 83.63 \\
\hline 400 & 24.00 & $1.418 \mathrm{E}-06$ & 744 & $4.958 \mathrm{E}-07$ & 100.00 \\
\hline 400 & 72.00 & $1.418 \mathrm{E}-06$ & 643 & $5.438 \mathrm{E}-07$ & 94.79 \\
\hline 400 & 144.00 & $1.418 \mathrm{E}-06$ & 616 & $5.583 \mathrm{E}-07$ & 93.23 \\
\hline 1000 & 0.00 & $1.418 \mathrm{E}-06$ & - & $1.418 \mathrm{E}-06$ & 0.00 \\
\hline 1000 & 0.25 & $1.418 \mathrm{E}-06$ & 505 & $9.422 \mathrm{E}-07$ & 63.51 \\
\hline 1000 & 2.00 & $1.418 \mathrm{E}-06$ & 652 & $8.584 \mathrm{E}-07$ & 74.71 \\
\hline 1000 & 24.00 & $1.418 \mathrm{E}-06$ & 1120 & $6.689 \mathrm{E}-07$ & 100.00 \\
\hline 1000 & 72.00 & $1.418 \mathrm{E}-06$ & 1072 & $6.844 \mathrm{E}-07$ & 97.93 \\
\hline 1000 & 144.00 & $1.418 \mathrm{E}-06$ & 615 & $8.780 \mathrm{E}-07$ & 72.08 \\
\hline
\end{tabular}


Preliminary Ion Exchange Modeling for Removal of Cesium from

Table E-8. Key parameters measured or specified during the batch kinetics tests of Davidson et al. (1998) and used to establish the cesium isotherm used in VERSE-LC kinetic modeling.

\begin{tabular}{|c|c|c|c|c|}
\hline Parameter & $\begin{array}{c}\text { Parameter } \\
\text { setting for } \\
\text { phase ratio of } \\
100\end{array}$ & $\begin{array}{c}\text { Parameter } \\
\text { setting for } \\
\text { phase ratio of } \\
100\end{array}$ & $\begin{array}{c}\text { Parameter } \\
\text { setting for } \\
\text { phase ratio of } \\
100\end{array}$ & $\begin{array}{c}\text { Parameter } \\
\text { setting for } \\
\text { phase ratio of } \\
100\end{array}$ \\
\hline CST material & IE-910 & IE-910 & IE-910 & IE-910 \\
\hline Temperature & $25^{\circ} \mathrm{C}$ & $25^{\circ} \mathrm{C}$ & $25^{\circ} \mathrm{C}$ & $25^{\circ} \mathrm{C}$ \\
\hline Initial liquid $\mathrm{Na}^{+}$conc. & $? \mathrm{M}$ & $? \mathrm{M}$ & $? \mathrm{M}$ & $? \mathrm{M}$ \\
\hline Initial liquid $\mathrm{Cs}^{+}$conc. & $1.418 \times 10^{-4} \mathrm{M}$ & $1.418 \times 10^{-4} \mathrm{M}$ & $1.418 \times 10^{-4} \mathrm{M}$ & $1.418 \times 10^{-4} \mathrm{M}$ \\
\hline $\begin{array}{l}\text { Final liquid } \mathrm{Cs}^{+} \text {conc. } \\
\text { (actually at } 144 \text { hours) }\end{array}$ & $1.318 \times 10^{-7} \mathrm{M}$ & $2.449 \times 10^{-7} \mathrm{M}$ & $4.958 \times 10^{-7} \mathrm{M}$ & $6.689 \times 10^{-7} \mathrm{M}$ \\
\hline Initial liquid sample volume & $10.0 \mathrm{ml}$ & $10.0 \mathrm{ml}$ & $10.0 \mathrm{ml}$ & $10.0 \mathrm{ml}$ \\
\hline Initial resin mass & $0.1 \mathrm{~g}$ & $0.05 \mathrm{~g}$ & $0.025 \mathrm{~g}$ & $0.01 \mathrm{~g}$ \\
\hline Batch ID & na & na & na & na \\
\hline Solution composition & W-25 supernate & $\mathrm{W}-25$ supernate & $\mathrm{W}-25$ supernate & $\mathrm{W}-25$ supernate \\
\hline Particle porosity & 0.1 & 0.1 & 0.1 & 0.1 \\
\hline solid density & $1.520 \mathrm{~g} / \mathrm{ml}$ & $1.520 \mathrm{~g} / \mathrm{ml}$ & $1.520 \mathrm{~g} / \mathrm{ml}$ & $1.520 \mathrm{~g} / \mathrm{ml}$ \\
\hline solution density & $1.253 \mathrm{~g} / \mathrm{ml}$ & $1.253 \mathrm{~g} / \mathrm{ml}$ & $1.253 \mathrm{~g} / \mathrm{ml}$ & $1.253 \mathrm{~g} / \mathrm{ml}$ \\
\hline solution viscosity & na & na & na & na \\
\hline $\mathrm{F}$ factor & 0.86 & 0.86 & 0.86 & 0.86 \\
\hline Phase ratio & 100 & 200 & 400 & 1000 \\
\hline Bed density & $0.009935 \mathrm{~g} / \mathrm{ml}$ & $0.004984 \mathrm{~g} / \mathrm{ml}$ & $0.002496 \mathrm{~g} / \mathrm{ml}$ & $0.000999 \mathrm{~g} / \mathrm{ml}$ \\
\hline Bed porosity & 0.991400 & 0.995686 & 0.997839 & 0.999135 \\
\hline $\begin{array}{c}\text { Estimated beta value for cesium } \\
\text { isotherm }\end{array}$ & $4.03967 \times 10^{-4} \mathrm{M}$ & $4.11446 \times 10^{-4} \mathrm{M}$ & $5.29612 \times 10^{-4} \mathrm{M}$ & $3.51474 \times 10^{-4} \mathrm{M}$ \\
\hline
\end{tabular}




\section{VERSE Input for Powder Test (Brown et al., 1996)}

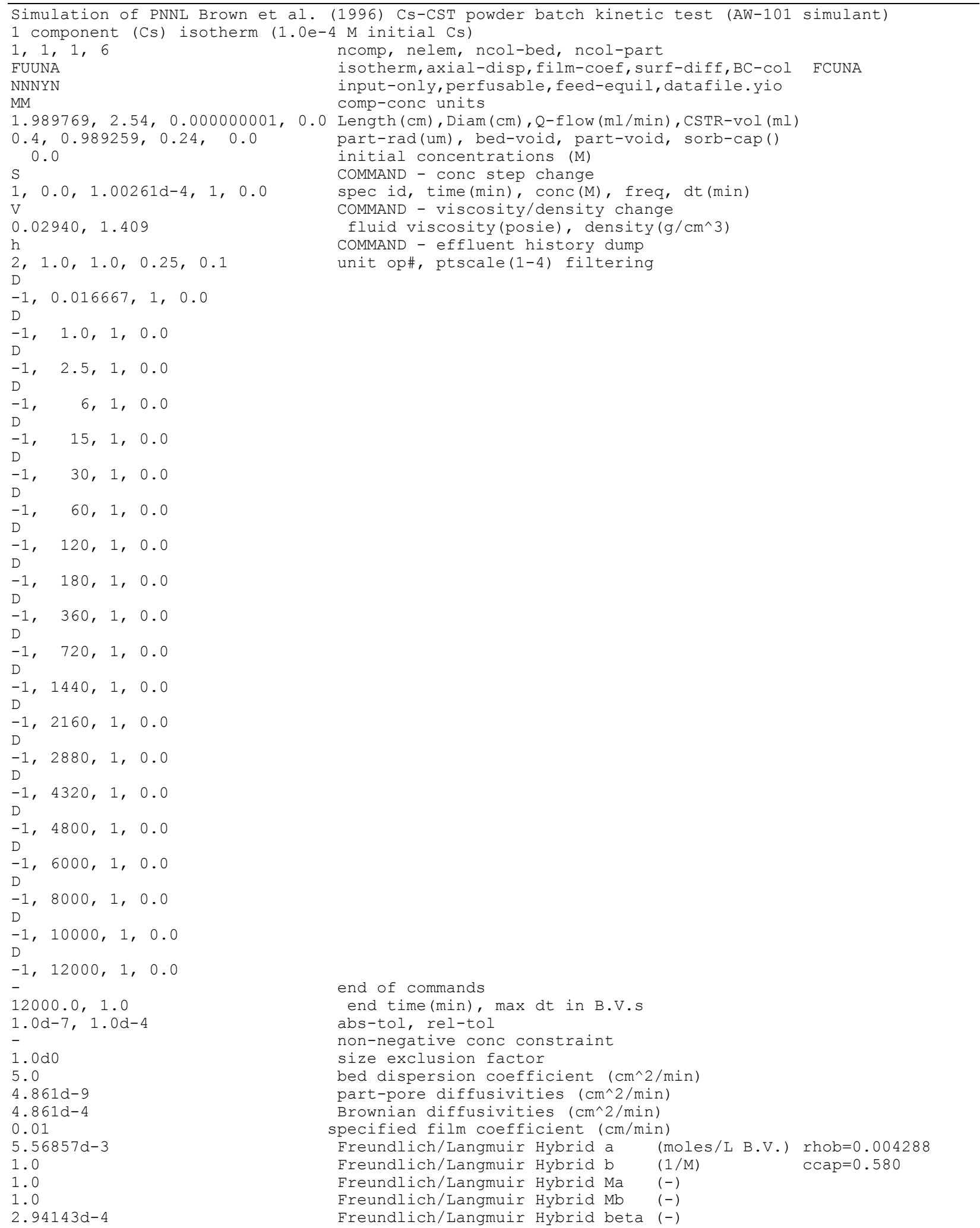




\section{VERSE Output for Powder Test (Brown et al., 1996)}

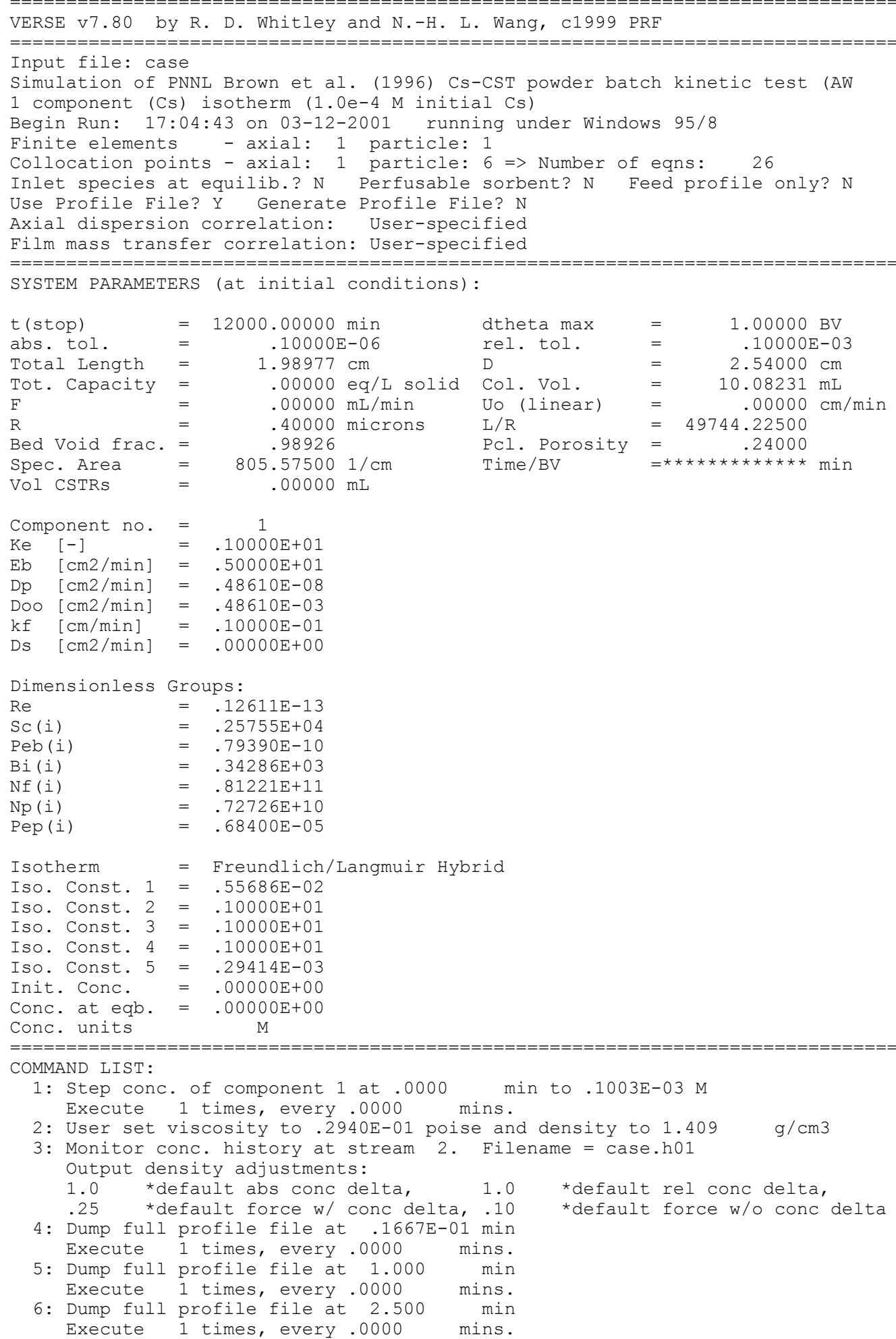


7: Dump full profile file at 6.000 Execute 1 times, every .0000

8: Dump full profile file at 15.00 Execute 1 times, every .0000

9: Dump full profile file at 30.00 Execute 1 times, every .0000

10: Dump full profile file at 60.00 Execute 1 times, every .0000

11: Dump full profile file at 120.0 Execute 1 times, every .0000

12: Dump full profile file at 180.0 Execute 1 times, every .0000

13: Dump full profile file at 360.0 Execute 1 times, every .0000

14: Dump full profile file at 720.0 Execute 1 times, every .0000

15: Dump full profile file at 1440. Execute 1 times, every .0000

16: Dump full profile file at 2160. Execute 1 times, every .0000

17: Dump full profile file at 2880. Execute 1 times, every .0000

18: Dump full profile file at 4320. Execute 1 times, every .0000

19: Dump full profile file at 4800 . Execute 1 times, every .0000

20: Dump full profile file at 6000 . Execute 1 times, every .0000

21: Dump full profile file at 8000 . Execute 1 times, every .0000

22: Dump full profile file at .1000E+05 min Execute 1 times, every .0000 mins.

23: Dump full profile file at .1200E+05 min Execute 1 times, every .0000 mins.

VERSE-LC finished in 241 steps. Average step size 49.79 minutes

End run: 17:04:44 on 03-12-2001

Integrated Areas in History Files:

case.h01 .614614E-01

\section{VERSE Input for Engineered -08 Test (Brown et al., 1996)}

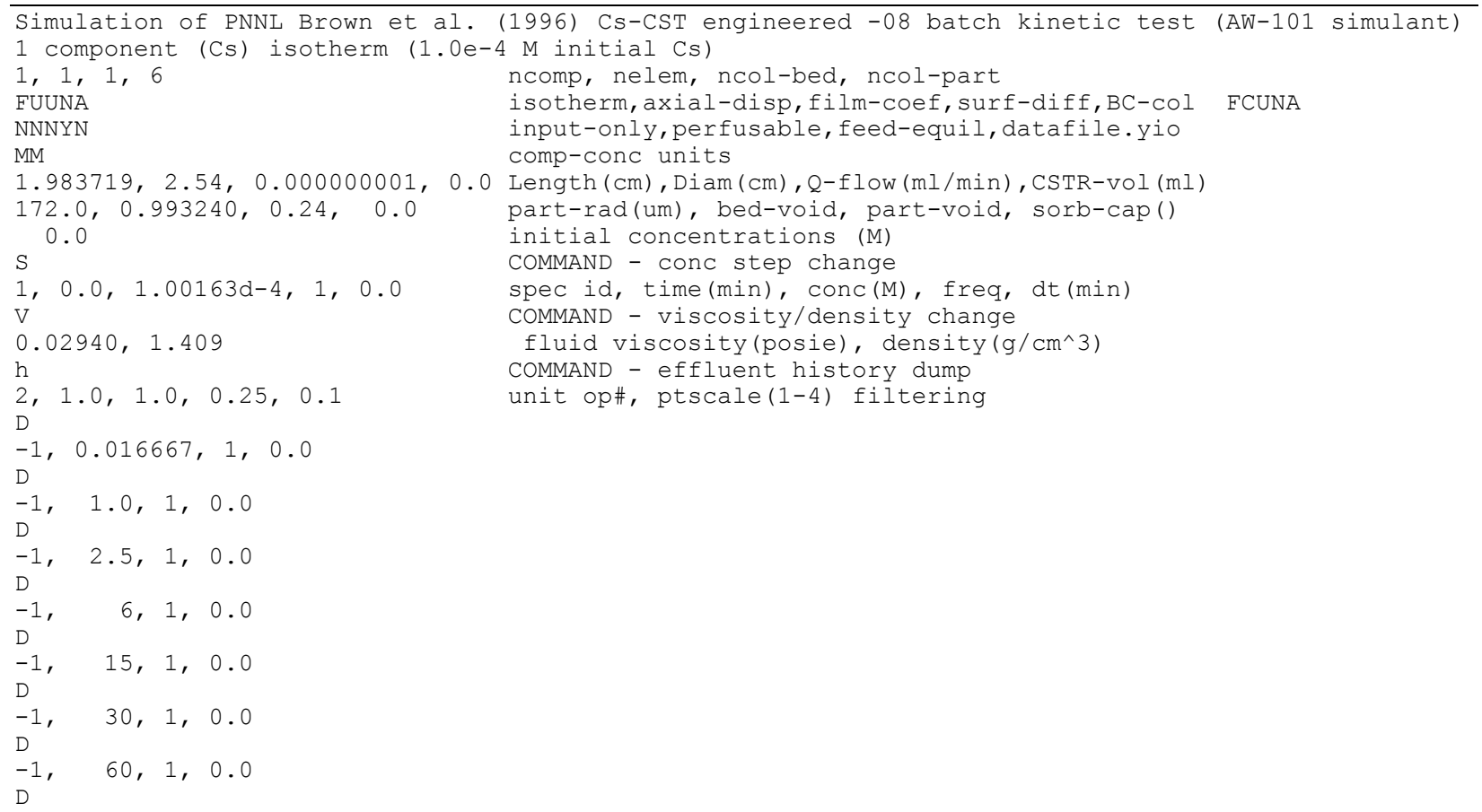


$-1,120,1,0.0$

$-1,180,1,0.0$

D

$-1,360,1,0.0$

$-1,720,1,0.0$

$-1,1440,1,0.0$

$\mathrm{D}$

$-1,2160,1,0.0$

$\mathrm{D}$

$-1,2880,1,0.0$

$\mathrm{D}$

$-1,4320,1,0.0$

$-1,4800,1,0.0$

D

$-1,6000,1,0.0$

D

$-1,8000,1,0.0$

D

$-1,10000,1,0.0$

$-1,12000,1,0.0$

$12000.0,1.0$

$1.0 d-7,1.0 d-4$

1. $0 \mathrm{dO}$

5.0

$2.431 d-5$

$4.861 d-4$

10.0

$3.48035 d-3$

1.0

1.0

1.0

3. $4921 d-4$ end of commands

end time(min), max dt in B.V.s

abs-tol, rel-tol

non-negative conc constraint

size exclusion factor

bed dispersion coefficient $\left(\mathrm{cm}^{\wedge} 2 / \mathrm{min}\right)$

part-pore diffusivities $\left(\mathrm{cm}^{\wedge} 2 / \mathrm{min}\right)$

Brownian diffusivities ( $\left.\mathrm{cm}^{\wedge} 2 / \mathrm{min}\right)$

specified film coefficient ( $\mathrm{cm} / \mathrm{min})$

Freundlich/Langmuir Hybrid a (moles/L B.V.) rhob=0.004288

Freundlich/Langmuir Hybrid b (1/M) ccap $=0.580$

Freundlich/Langmuir Hybrid $\mathrm{Ma} \quad(-)$

Freundlich/Langmuir Hybrid Mb (-)

Freundlich/Langmuir Hybrid beta (-)

\section{VERSE Input for Engineered -38b Test (Brown et al., 1996)}

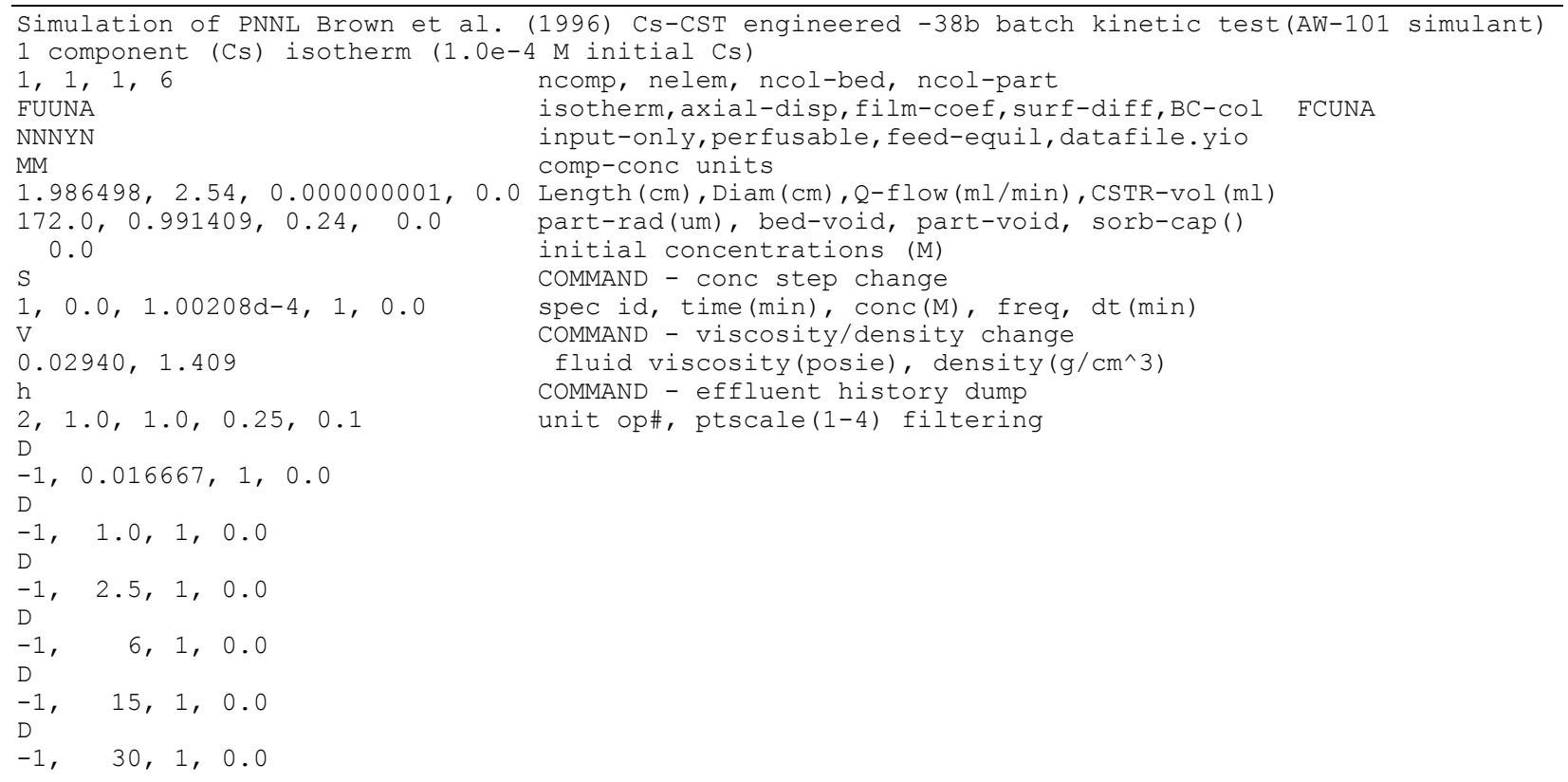


$-1, \quad 60,1,0.0$

$-1,120,1,0.0$

$-1,180,1,0.0$

D

$-1,360,1,0.0$

D

$-1,720,1,0.0$

$-1,1440,1,0.0$

$-1,2160,1,0.0$

D

$-1,2880,1,0.0$

D

$-1,4320,1,0.0$

D

$-1,4800,1,0.0$

D

$-1,6000,1,0.0$

D

$-1,8000,1,0.0$

D

$-1,10000,1,0.0$

$-1,12000,1,0.0$

$12000.0,1.0$

$1.0 \mathrm{~d}-7,1.0 \mathrm{~d}-4$

$1.0 \mathrm{~d} 0$

5.0

$2.431 d-5$

$4.861 d-4$

10.0

$3.52250 d-3$

1.0

1.0

1.0

$3.94901 d-4$ end of commands

end time (min), $\max d t$ in B.V.s

abs-tol, rel-tol

non-negative conc constraint

size exclusion factor

bed dispersion coefficient $\left(\mathrm{cm}^{\wedge} 2 / \mathrm{min}\right)$

part-pore diffusivities $\left(\mathrm{cm}^{\wedge} 2 / \mathrm{min}\right)$

Brownian diffusivities ( $\left.\mathrm{cm}^{\wedge} 2 / \mathrm{min}\right)$

specified film coefficient ( $\mathrm{cm} / \mathrm{min}$ )

Freundlich/Langmuir Hybrid a (moles/L B.V.) rhob=0.004288

Freundlich/Langmuir Hybrid b (1/M) ccap $=0.580$

Freundlich/Langmuir Hybrid Ma (-)

Freundlich/Langmuir Hybrid $\mathrm{Mb} \quad(-)$

Freundlich/Langmuir Hybrid beta (-)

\section{VERSE Input for Batch Kinetic Test (Fondeur et al., 2000)}

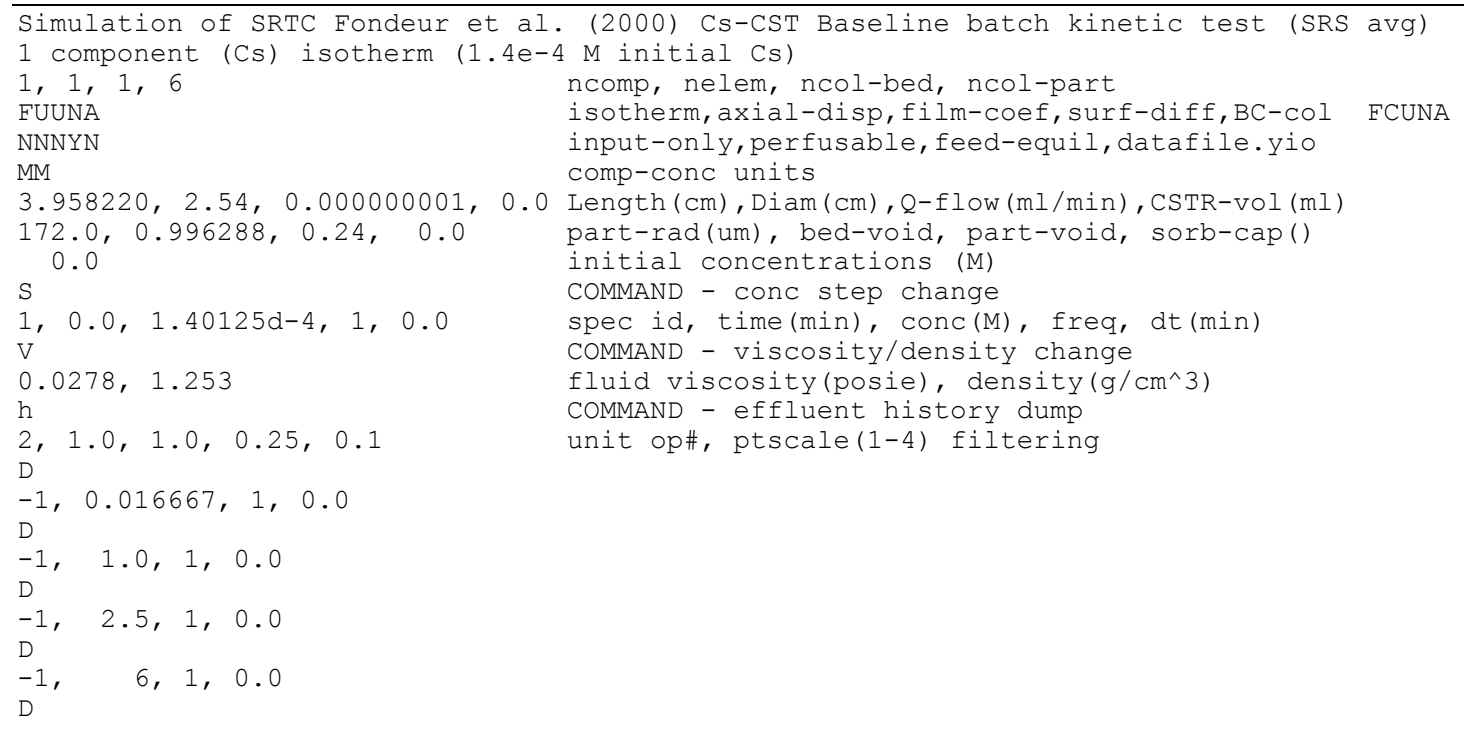


Preliminary Ion Exchange Modeling for Removal of Cesium from

Revision (Date):

$0(07 / 05 / 02)$

$-1, \quad 15,1,0.0$

$-1, \quad 30,1,0.0$

D

$-1, \quad 60,1,0.0$

$-1, \quad 120,1,0.0$

D

$-1, \quad 180,1,0.0$

$-1,360,1,0.0$

D

$-1,720,1,0.0$

$-1,1440,1,0.0$

$-1,2160,1,0.0$

D

$-1,2880,1,0.0$

D

$-1,4320,1,0.0$

D

$-1,4800,1,0.0$

D

$-1,6000,1,0.0$

D

$-1,8000,1,0.0$

D

$-1,10000,1,0.0$

$-1,12000,1,0.0$

$12000.0,1.0$

$1.0 d-7,1.0 d-4$

1. $0 \mathrm{~d} 0$

5.0

$2.486 d-5$

4. $972 d-4$

10.00

$2.48696 \mathrm{~d}-3$

1. 0

1.0

1.0

3. $53632 \mathrm{~d}-4$ end of commands

end time (min), $\max d t$ in B.V.s

abs-tol, rel-tol

non-negative conc constraint

size exclusion factor

bed dispersion coefficient $\left(\mathrm{cm}^{\wedge} 2 / \mathrm{min}\right)$

part-pore diffusivities $\left(\mathrm{cm}^{\wedge} 2 / \mathrm{min}\right)$ 5\% of free

Brownian diffusivities $\left(\mathrm{cm}^{\wedge} 2 / \mathrm{min}\right)$

specified film coefficient $(\mathrm{cm} / \mathrm{min})$

Freundlich/Langmuir Hybrid a (moles/L B.V.) rhob=0.004288

$\begin{array}{lll}\text { Freundlich/Langmuir Hybrid b } & (1 / \mathrm{M}) & \mathrm{ccap}=0.580\end{array}$

Freundlich/Langmuir Hybrid Ma (-)

Freundlich/Langmuir Hybrid Mb (-)

Freundlich/Langmuir Hybrid beta (-)

\section{VERSE Output for Batch Kinetic Test (Fondeur et al., 2000)}

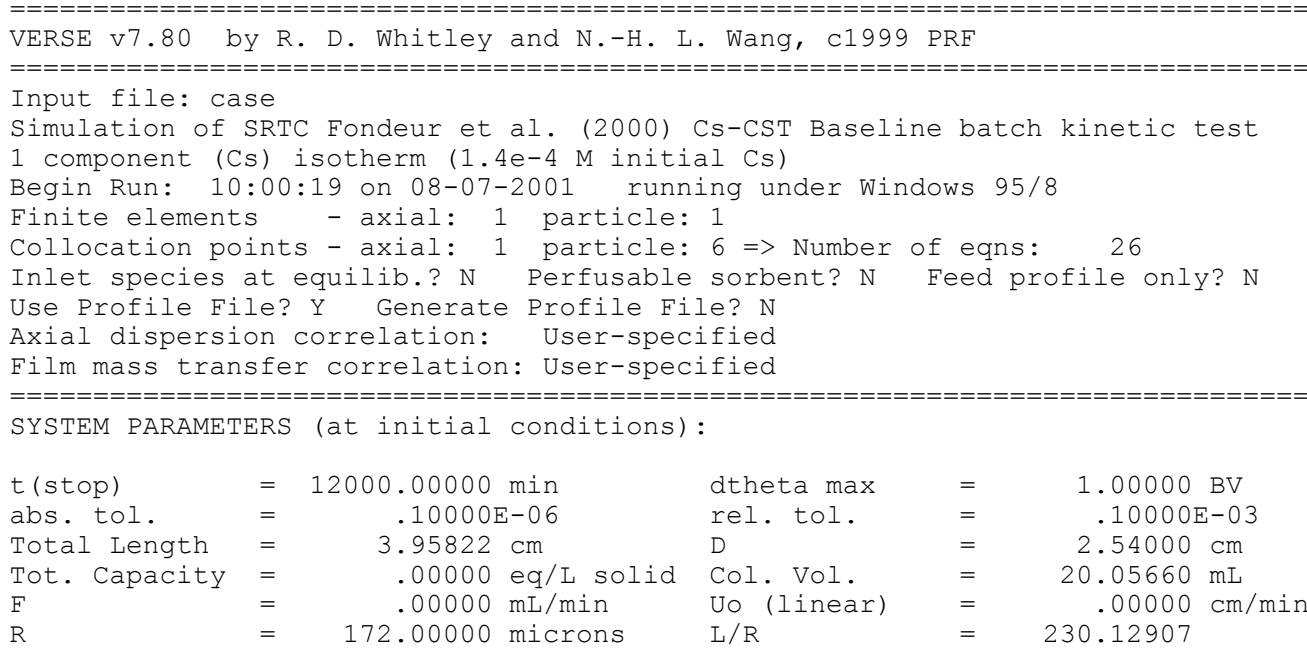

$1.00000 \mathrm{BV}$

$.10000 \mathrm{E}-03$

$2.54000 \mathrm{~cm}$

$20.05660 \mathrm{mI}$

$.00000 \mathrm{~cm} / \mathrm{min}$

230.12907 
WESTINGHOUSE SAVANNAH RIVER COMPANY

Report:

WSRC-TR-2001-00400

Preliminary Ion Exchange Modeling for Removal of Cesium from

Revision (Date):

$0(07 / 05 / 02)$

Hanford Waste Using Hydrous Crystalline Silicotitanate Material Page:

275 of 338

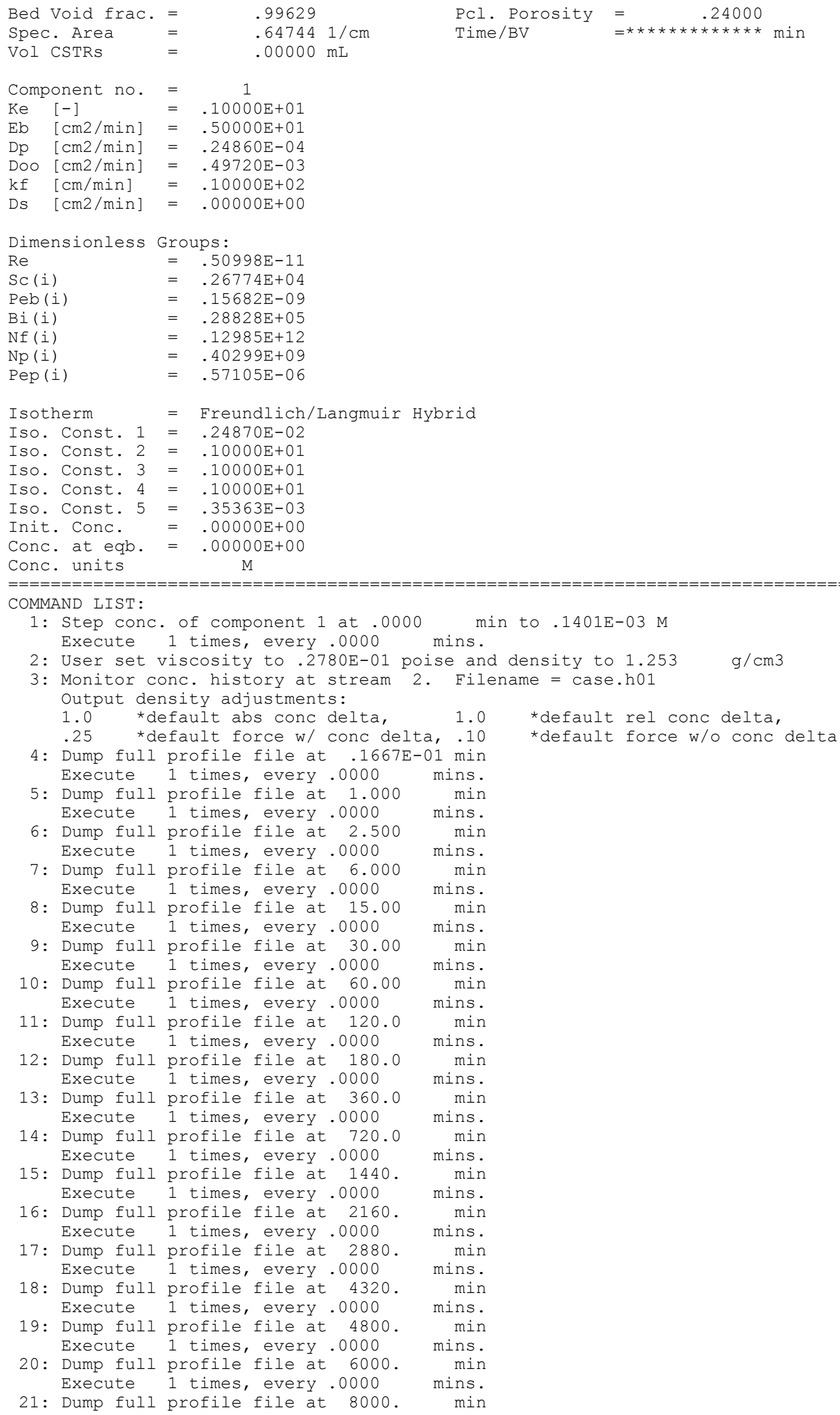


Preliminary Ion Exchange Modeling for Removal of Cesium from

22: Execute 1 times, every .0000 mins.
Execute 1 times, every.0000 m mins.
23: Dump full profile file at .1200E+05 min
Execute 1 times, every.0000 mins.
$============================================================$
VERSE-LC finished in 239 steps. Average step size $50.21 \quad$ minutes
End run: 10:00:20 on 08-07-2001
Integrated Areas in History Files:
case.h01

\section{VERSE Input for Cs Uptake Test (Anthony et al., 1996; $112 \mu \mathrm{m}$ )}

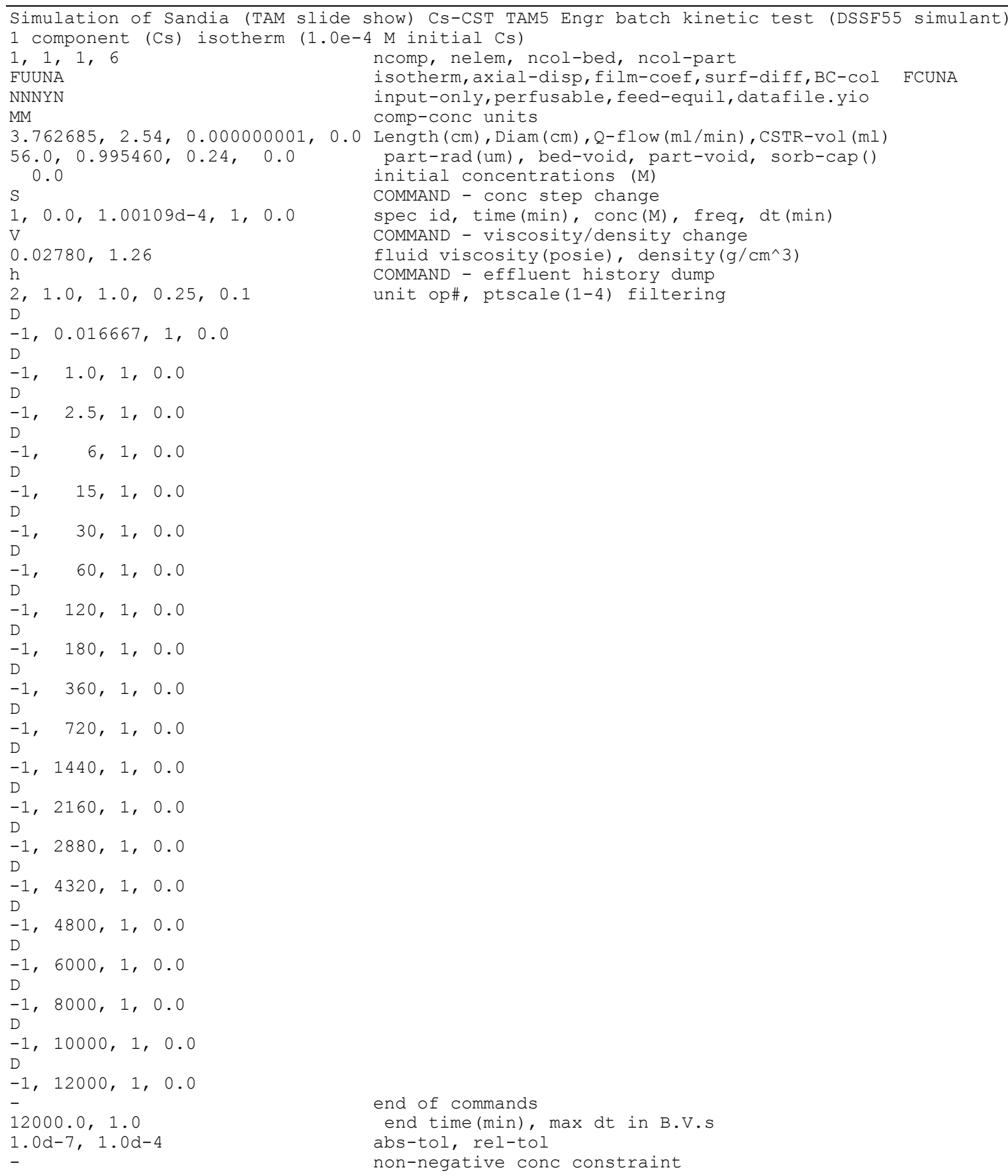


Preliminary Ion Exchange Modeling for Removal of Cesium from

$1.0 \mathrm{~d} 0$

5.0

$1.257 d-4$

$4.836 d-4$

10.0

$2.06863 d-3$

1.0

1.0

1.0

2. $89276 d-4$ size exclusion factor

bed dispersion coefficient $\left(\mathrm{cm}^{\wedge} 2 / \mathrm{min}\right)$

part-pore diffusivities $\left(\mathrm{cm}^{\wedge} 2 / \mathrm{min}\right)$

Brownian diffusivities ( $\left.\mathrm{cm}^{\wedge} 2 / \mathrm{min}\right)$

specified film coefficient ( $\mathrm{cm} / \mathrm{min}$ )

Freundlich/Langmuir Hybrid a (moles/L B.V.) rhob=0.004288

Freundlich/Langmuir Hybrid b (1/M) $\quad$ ccap $=0.580$

Freundlich/Langmuir Hybrid Ma (-)

Freundlich/Langmuir Hybrid Mb (-)

Freundlich/Langmuir Hybrid beta (-)

\section{VERSE Input for Cs Uptake Test (Anthony et al., 1996; $334 \mu \mathrm{m}$ )}

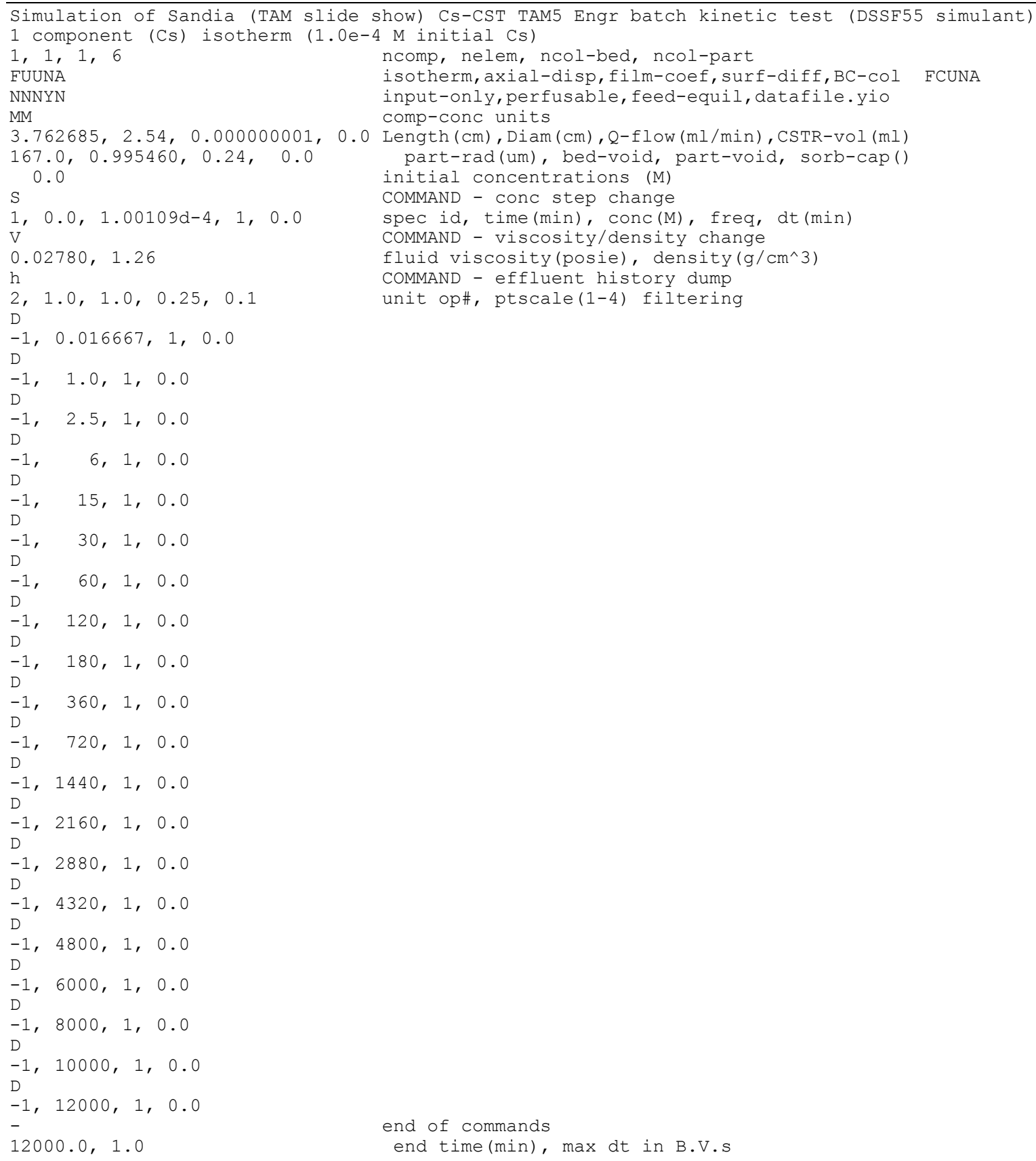


WESTINGHOUSE SAVANNAH RIVER COMPANY

Report: WSRC-TR-2001-00400

Preliminary Ion Exchange Modeling for Removal of Cesium from

Revision (Date):

$0(07 / 05 / 02)$

Hanford Waste Using Hydrous Crystalline Silicotitanate Material

Page:

278 of 338

$1.0 d-7,1.0 d-4$

abs-tol, rel-tol

non-negative conc constraint

$1.0 \mathrm{~d} 0$

5.0

size exclusion factor

bed dispersion coefficient $\left(\mathrm{cm}^{\wedge} 2 / \mathrm{min}\right)$

part-pore diffusivities $\left(\mathrm{cm}^{\wedge} 2 / \mathrm{min}\right)$

Brownian diffusivities ( $\left.\mathrm{cm}^{\wedge} 2 / \mathrm{min}\right)$

specified film coefficient $(\mathrm{cm} / \mathrm{min})$

10.0

$2.06863 d-3$

Freundlich/Langmuir Hybrid a

(moles/L B.V.) rhob $=0.004288$

1.0

Freundlich/Langmuir Hybrid b

$(1 / \mathrm{M}) \quad$ c cap $=0.580$

1.0

Freundlich/Langmuir Hybrid Ma

Freundlich/Langmuir Hybrid Mb

$(-)$

Freundlich/Langmuir Hybrid beta (-) 


\section{Appendix F (ZAM Code Description)}

The ZAM code is purchased commercial software developed at Texas A\&M University by Rayford G. Anthony and Zhixin Zheng. The ZAM code is a product of over several years development and research in Professor R. G. Anthony's Kinetics, Catalysis and Reaction Engineering Laboratory in the Department of Chemical Engineering Texas A\&M University. ZAM is written in FORTRAN 90 using the Microsoft Developer's Workbench. For applications performed at SRTC, PC based versions running under MS-DOS are used. No extensive user guide exists for ZAM; however, a brief user guide is available from Professor Anthony. A description of the current ZAM model is provided by Zheng et al. (1997). Supporting information and earlier modeling are provided by Zheng et al. (1995) and Zheng et al. (1996). Further information on ZAM is available by contacting Rayford G. Anthony at e-mail: RGANTHONY@TAMU.edu, Telephone (409)845-3370, or Fax (409)862-3266.

This appendix contains or references the information necessary for using version 4 or 5 of the ZAM code (i.e., executables referred to as CSTIEXV4 or CSTIEXV5; CST Ion Exchange Version $\underline{4}$ or $\underline{5}$ ). ZAM is commercial software designed to simulate ion-exchange equilibria of electrolytic solutions and crystalline silicotitanate solid in its powdered form (labeled as CST, TAM5, or IONSIV ${ }^{\circledR}$ IE-910).

In order to use ZAM for predicting the behavior of CST in its engineered forms, a correction factor accounting for the inert binding material must be considered (i.e., freferred to in this report as a dilution factor, $\eta_{\mathrm{df}}$ ). After the writing of this report a recent upgrade to ZAM was report by Anthony et al (2001), where several improvements pertinent to this work should be considered in any future modeling efforts.

\section{F.1 About the Model}

The ZAM model solves a set of equations for solid-liquid equilibrium. This model includes the competitive ion exchange at CST exchange sites between the following homovalent cations: $\mathrm{Na}^{+}$, $\mathrm{Cs}^{+}, \mathrm{H}^{+}, \mathrm{Rb}^{+}, \mathrm{K}^{+}$, and $\mathrm{SrOH}^{+}$. Non-idealities within the aqueous phase are handled using Bromley's model for calculating activity coefficients of the ions. Since Bromley (1973) only established modeling parameters for some of the most common ions up to an ionic strength of $\sim 6$ molal, errors may occur when one uses the ZAM code beyond 6 molal or when addressing solutions containing ions whose Bromley parameters do not exist. However, experience by the developers indicated that even at ionic strengths exceeding 6 molal good results were achieved for DSSF5, DSF7, NCAW, and other Hanford simulants (i.e., for Phase 1 inventories 10 molal and higher need to be addressed). A listing of the currently available ionic species contained within the ZAM database is provided in Table F-1.

Surface non-idealities on the solid phase CST material are handled by a supersite approach (see Zheng et al., 1997). The supersite approach involves three neighboring surface sites as shown below: 


\section{ZAM Supersite}

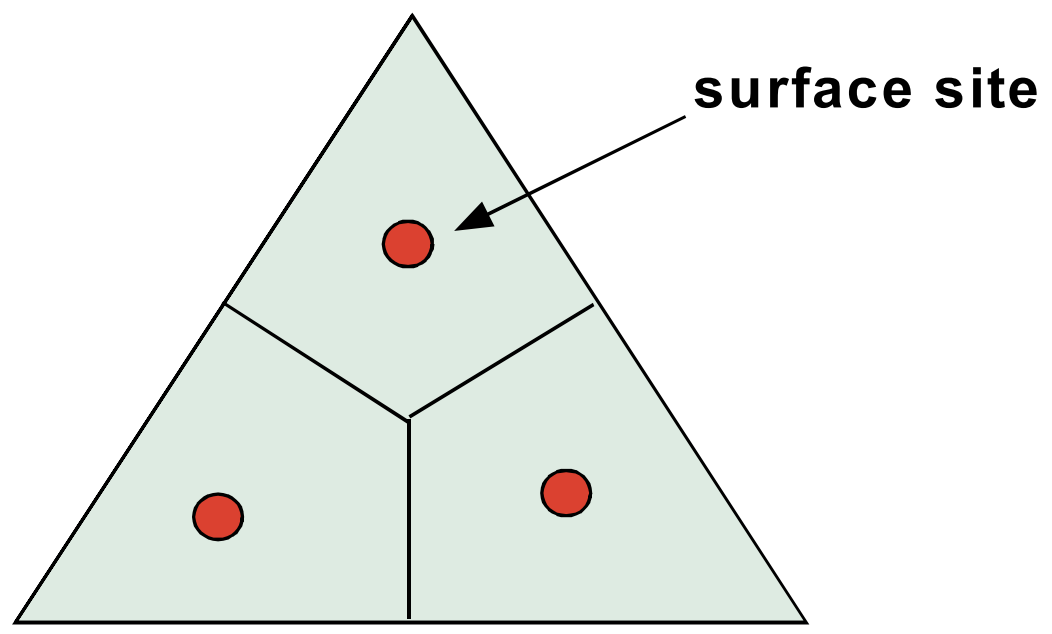

Here, experimental data indicates that a thermodynamically ideal solid phase can be achieved when the CST material is viewed on a supersite basis. Investigations made by Zheng et al. (1996) found step changes in binary ion exchange isotherms and that prior to these step changes, the solid phase was found to be ideal along the isotherms. For example, when viewing the ion exchange process between sodium and cesium we have the following three possible mass-action relationships to consider:

$$
\begin{gathered}
{\left[\mathrm{Na}_{3}^{+}\right]_{\text {lld }}+\left[\mathrm{Cs}^{+}\right]_{\text {liq }} \stackrel{\mathrm{K}_{\text {eq }, 1}}{\longleftrightarrow}\left[\mathrm{Na}_{2}^{+} \mathrm{Cs}^{+}\right]_{\text {lld }}+\left[\mathrm{Na}^{+}\right]_{\text {liq }},} \\
{\left[\mathrm{H}^{+} \mathrm{Na}_{2}^{+}\right]_{\text {lld }}+\left[\mathrm{Cs}^{+}\right]_{\text {liq }} \stackrel{\mathrm{K}_{\text {eq }, 2}}{\longleftrightarrow}\left[\mathrm{H}^{+} \mathrm{Na}^{+} \mathrm{Cs}^{+}\right]_{\text {lld }}+\left[\mathrm{Na}^{+}\right]_{\text {liq }},} \\
{\left[\mathrm{H}_{2}^{+} \mathrm{Na}^{+}\right]_{\text {lld }}+\left[\mathrm{Cs}^{+}\right]_{\text {liq }} \stackrel{\mathrm{K}_{\text {eq }, 3}}{\longleftrightarrow}\left[\mathrm{H}_{2}^{+} \mathrm{Cs}^{+}\right]_{\text {lld }}+\left[\mathrm{Na}^{+}\right]_{\text {liq }} .}
\end{gathered}
$$

Equations F-1, F-2, and F-3 represents the removal of a $\mathrm{Na}^{+}$cation at a surface site with a $\mathrm{Cs}^{+}$ cation. The three possible cases reflect the three possible states that the supersite might be in prior to the ion exchange (note that a supersite can not hold more than one $\mathrm{Cs}^{+}$cation at any point in time). The temperature dependence of the equilibrium constants for each of the above mass-action equations can be approximated by:

$$
\ln \left(\frac{\mathrm{K}_{\mathrm{eq}}\left(\mathrm{T}_{2}\right)}{\mathrm{K}_{\mathrm{eq}}\left(\mathrm{T}_{1}\right)}\right)=-\frac{\Delta \mathrm{H}_{\mathrm{IX}}}{\mathrm{R}}\left[\frac{1}{\mathrm{~T}_{2}}-\frac{1}{\mathrm{~T}_{1}}\right],
$$

where the heats of ion exchange under high alkaline conditions have been estimated to be:

$$
\begin{array}{ll}
\text { (Reaction 1) } & \Delta \mathrm{H}_{\mathrm{IX}}=-2.18 \times 10^{4} \mathrm{~J} / \text { gmole } \\
\text { (Reaction 2) } & \Delta \mathrm{H}_{\mathrm{IX}}=1.46 \times 10^{4} \mathrm{~J} / \text { gmole } \\
\text { (Reaction 3) } & \Delta \mathrm{H}_{\mathrm{IX}}=(\text { not recorded) }
\end{array}
$$


During the loading phase, the CST material will be in its Na-form with only trace amount of $\mathrm{H}^{+}$ present. Therefore, Eq. (F-1) will be the dominant ion exchange reaction taking place. Under these conditions the overall ion exchange process will be exothermic implying that higher column temperatures yields lower cesium loadings consistent with currently available batch contact data.

Looking at just the overall ion exchange reactions involving $\mathrm{Cs}^{+}$we have:

$$
\begin{gathered}
{\left[\mathrm{Na}^{+}\right]_{\text {lld }}+\left[\mathrm{Cs}^{+}\right]_{\text {liq }} \stackrel{\mathrm{K}_{13}}{\longleftrightarrow}\left[\mathrm{Cs}^{+}\right]_{\text {lld }}+\left[\mathrm{Na}^{+}\right]_{\text {liq }}\left(\mathrm{K}_{13} \sim 26,000\right)} \\
{\left[\mathrm{K}^{+}\right]_{\text {lld }}+\left[\mathrm{Cs}^{+}\right]_{\text {liq }} \stackrel{\mathrm{K}_{12}}{\longleftrightarrow}\left[\mathrm{Cs}^{+}\right]_{\text {lld }}+\left[\mathrm{K}^{+}\right]_{\text {liq }}\left(\mathrm{K}_{13} \sim 1,400\right)} \\
{\left[\mathrm{SrOH}^{+}\right]_{\text {lld }}+\left[\mathrm{Cs}^{+}\right]_{\text {liq }} \stackrel{\mathrm{K}_{14}}{\longleftrightarrow}\left[\mathrm{Cs}^{+}\right]_{\text {lld }}+\left[\mathrm{SrOH}^{+}\right]_{\text {liq }}\left(\mathrm{K}_{13} \sim 1\right)}
\end{gathered}
$$

where the selectivity coefficients $\left(\mathrm{K}_{1 \mathrm{j}}\right)$ listed reflect approximate estimates and provide insight into the selectivity of CST for cesium.

Mass-action relationships similar to those above are also written for each of the potential competitors $\left(\mathrm{Na}^{+}, \mathrm{Cs}^{+}, \mathrm{H}^{+}, \mathrm{Rb}^{+}, \mathrm{K}^{+}\right.$, and $\left.\mathrm{SrOH}^{+}\right)$. Also species material balance equations are written relating the amount of each species within the liquid and solid phases in the initial state to amounts in the final ("equilibrium") state. To obtain these material balances the mass of CST and mass of liquid (i.e., volume of liquid and its density) must also be specified. Solution of this set of nonlinear algebraic equations is achieved using a modified ("rate-limited") NewtonRaphson technique.

Two versions of ZAM have been used within SRTC (i.e., Version 4 and Version 5). Both versions model the effect of temperature on the system $\left(\mathrm{Na}^{+}, \mathrm{Cs}^{+}, \mathrm{H}^{+}, \mathrm{Rb}^{+}\right.$, and $\mathrm{K}^{+}$) when considering a basic solution of $\mathrm{pH}>12$. The newer version (Version 5) contains the following code improvements:

- Updates to aqueous phase strontium reaction $\left(\mathrm{Sr}^{+}, \mathrm{OH}^{-}\right.$, and $\left.\mathrm{SrOH}^{+}\right)$in basic solutions of $\mathrm{pH}>12$ has been added;

- Improvements were made in estimating the effect of $\mathrm{K}^{+}$on cesium distribution coefficients; and

- Bromley's parameters for $\mathrm{NO}_{2}{ }^{-}$and $\mathrm{Al}(\mathrm{OH})_{4}{ }^{-}$have been updated.

The actual impact as a result of these updates is small when considering the Phase 1 Law batch feeds as to be demonstrated by example below. For many of the Phase 1 LAW batch feed simulations required Version 5 had difficulty in converging. Since the final results from both versions are very similar, Version 4 was used the majority of the time.

The solid-liquid equilibrium model solves the various mass-action equations involving ion exchange in conjunction with the appropriate material balance equations. At a specified operating temperature $(\mathrm{K})$ and solution density $\left(\mathrm{g} / \mathrm{L}\right.$ or $\left.\mathrm{kg} / \mathrm{m}^{3}\right)$ ZAM performs a simulated batch 
contact (" $K_{d}$ ") test where the quantity of the following variables at their initial state must be specified:

- Initial composition of aqueous solution (gmole/L);

- Amount of aqueous solution present (L);

- Amount of CST material present (g); and

- Initial form of CST ( 0 for sodium or 1 for hydrogen).

Upon solving the simulated contact test, ZAM outputs the following final ("at equilibrium") state values for four of the competing cations $\left(\mathrm{Cs}^{+}, \mathrm{Rb}^{+}, \mathrm{SrOH}^{+}\right.$, and $\mathrm{K}^{+}$; note $\mathrm{Na}^{+}$and $\mathrm{H}^{+}$loading numbers are not provided):

- Final CST loading of cation (Q, mmole/g $\left.\mathrm{g}_{\mathrm{CST}}\right)$;

- Final aqueous phase concentration of cation (gmole/L); and

- Kd value of cation $\left(\mathrm{ml} / \mathrm{g}_{\mathrm{CST}}\right)$.

Note that the CST loading value is simply the product of the Kd value times the final concentration for each competitor. Also printed in output is the solutions $\mathrm{pH}$ and ionic strength values.

\section{F.2 I/O Files and Running the ZAM Code}

The program is accessed from the MS-DOS prompt. The executables are named "CSTIEXV4" for Version-4 and "CSTIEXV5" for Version-5. The standard input file is named "CSTIEXV.in" and contains all the necessary input to perform a single simulated $\mathrm{K}_{\mathrm{d}}$ test. Scripts can be used when multiple $K_{d}$ simulations are desired. The results of the simulated $K_{d}$ test are placed in the standard output file named "CSTIEXV.out".

Assuming that the above executables are located in a directory defined by your PATH or resides within the current directory, at the MS-DOS prompt entering the executable filename will execute the ZAM program if an input file named "CSTIEXV.in" exists within the current directory. The output file named "CSTIEXV.out" is placed into this current directory, as well. During execution ZAM may printout various runtime messages (i.e., progression of solver or error messages). If a $\mathrm{KNO}_{3}$ precipitation/solubility limit is reached, then ZAM prints out to the active MS-DOS window the new adjusted concentrations for $\mathrm{K}^{+}$and $\mathrm{NO}_{3}{ }^{-}$. ZAM is paused under these conditions requiring the user to enter a keystroke to continue the simulation if desired.

For computing several ZAM simulations together, a simple script such as the one below is useful. This script runs a comparison case where Version-4 and Version-5 results are generated based on the same input: 


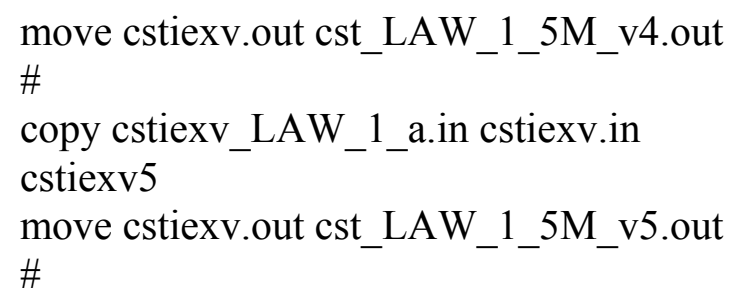

Note that the results of a simulation need to be moved or new results will overwrite the old results.

\section{F.3 Input File Structure}

The standard input file is named "CSTIEXV.in" and contains all the necessary input to perform a single simulated $\mathrm{K}_{\mathrm{d}}$ test. This file contains the information required prior to ion-exchange and the program then calculates the equilibrium based on this initial information. Briefly, you need to specify the choice of activity coefficient model, temperature, the title, the liquid molar density, the number of cations, the number of anions, cation concentrations, anion concentrations, amount of liquid, amount of solid, the initial solid form parameter, and the calculation adjustment parameter. The liquid phase concentrations must be charged balanced (i.e., ZAM assumes the solution to be charged balance to within a small tolerance). If the solution charge miss-balance exceed the tolerance an error message is printed and ZAM execution is terminated. Charge balancing should be performed using a species having a small overall impact like chlorine.

The following is a line-by-line description of the input needs for "CSTIEXV.in":

Line 1) Choice of activity model and temperature: Option 1 is the only activity model currently available and presents Bromley's model. Temperature is input in K units. The temperature dependent parameter within the ZAM model are based on experimental data taken within the range $298.15 \mathrm{~K}$ to $317.15 \mathrm{~K}$. The temperature effect in neutral to acidic solutions $(\mathrm{pH}<12)$ is not included, and the effect of temperature for strontium is not addressed.

Line 2) Title: The title inputted is printed out as a header in the output file and must be less than 33 characters long.

Line 3) Number of Cations: An integer whose number must exceed 6. A minimum of 7 cations is required because $\mathrm{Na}^{+}, \mathrm{Cs}^{+}, \mathrm{H}^{+}, \mathrm{Rb}^{+}, \mathrm{K}^{+}$, and $\mathrm{SrOH}^{+}$are competing cations for CST exchange sites and $\mathrm{Sr}^{2+}$ is in equilibrium with $\mathrm{SrOH}^{+}$in the aqueous phase.

Line 4) Number of Anions: An integer whose number must exceed 1. A minimum of 2 anions is required because $\mathrm{OH}^{-}$and $\mathrm{NO}_{3}{ }^{-}$are assumed to be always considered within the aqueous phase solution model formulation. 
Line 5) Solution Density $\left(\mathrm{g} / \mathbf{L ~ o r ~} \mathbf{k g} / \mathbf{m}^{3}\right)$ : The aqueous phase density is required to convert molar ion concentrations (gmole/L) into molal units (gmole $/ \mathrm{kg}_{\text {water }}$ ). Bromley's activity coefficient model is based on molal units.

Line 6) Cation Code IDs: These are integer ID numbers specifying which cations are being considered (see Table F-1). The first 7 cations (minimum number required) must be $\mathrm{Na}^{+}, \mathrm{Cs}^{+}, \mathrm{H}^{+}, \mathrm{Rb}^{+}, \mathrm{K}^{+}, \mathrm{SrOH}^{+}$, and $\mathrm{Sr}^{2+}$ in this order (i.e., 3, 6, 1,1, 5, 4, 40, 13). Additional cations can be added to the list starting in the $8^{\text {th }}$ position and beyond in any given order. Even under conditions where some of the first 7 cations do not exist within the aqueous phase, they must still be given with zero concentrations inputted.

Line 7) Anion Code IDs: These are integer ID numbers specifying which anions are being considered (see Table F-1). The first 2 anions (minimum number required) must be $\mathrm{OH}^{-}$and $\mathrm{NO}_{3}{ }^{-}$in this order (i.e., 13, 9). Additional anions can be added to the list starting in the $3^{\text {rd }}$ position and beyond in any given order. Even under conditions where some of the first 2 anions do not exist within the aqueous phase, they must still be given with zero concentrations inputted.

Line 8) Formula Weight: If you have chosen a ion not explicitly listed in Table F-1, then its molecular weight (g/gmole) must be supplied. If you have only requested ions contained within Table F-1, this line can be skipped. Three unlisted cations and three unlisted anions are optional. For cations the possible code IDs are 37, 38, and 39 (see Table F-1). For anions the possible code IDs are 24, 25, and 26 (see Table F-1).

Line 9) Cation Concentrations (M): Here the concentrations should be listed in the same order as the cation code IDs are listed. Zero concentrations can be used. However the concentration of $\mathrm{H}^{+}$must be greater than zero. For example, if the solution is basic use $\left[\mathrm{H}^{+}\right]\left[\mathrm{OH}^{-}\right]=1 \times 10^{-14}$ to estimate the $\mathrm{H}^{+}$concentration. The ZAM program will internally correct the $\mathrm{H}^{+}$concentration. If the solution is neutral use $1 \times 10^{-7}$. For strontium considerations, input zero concentration for $\mathrm{SrOH}^{+}$(cation \#40) and the actual concentration of total strontium as $\mathrm{Sr}^{2+}$ (cation \#13). The program will calculate the liquid-phase equilibrium between $\mathrm{SrOH}^{+}$and $\mathrm{Sr}^{2+}$ based on available free $\mathrm{OH}^{-}$.

Line 10) Anion Concentrations (M): Here the concentrations should be listed in the same order as the anion code IDs are listed. Zero concentrations can be used. However the concentration of $\mathrm{OH}^{-}$must be greater than zero. For example, if the solution is acidic use $\left[\mathrm{H}^{+}\right]\left[\mathrm{OH}^{-}\right]=1 \times 10^{-14}$ to estimate the $\mathrm{OH}^{-}$concentration. The ZAM program will internally correct the $\mathrm{OH}^{-}$concentration. If the solution is neutral use $1 \times 10^{-7}$.

Line 11) Liquid Volume (L) and Solid Mass (g): Enter the amount of volume of aqueous phase solution being placed in contact with CST. Enter the amount of mass of CST being considered. The phase ratio is the ratio of these two quantities.

Line 12) Initial form of solid: There are two initial forms for the CST material (its Na-form or its $\mathrm{H}$-form). Here it is assumed that all exchange sites are initially occupied by either $\mathrm{Na}^{+}$(option 0 ) or by $\mathrm{H}^{+}$(option 1 ). 
Line 13) Calculational Adjustment Parameter: Real number ranging from 0.0 to 1.0. This is an under-relaxation iteration parameter used to assist in convergence of the non-linear equation solver. The larger the number the faster (less under-relaxed) convergence is generally achieved (however, the larger the risk of divergence). The best overall value is around 0.7. The program has more difficulty in converging under near neutral conditions. Note that concentration ranges covering 5 to 10 orders in magnitude are typically being computed and the system of equations can become very stiff. This set of equilibrium and material balance equations are a very non-linear set of algebraic equations that can be difficult to solve for most standard solvers. Generally, low values of this adjustment parameter helps convergence but can greatly extend the overall runtime.

\section{F.4 Installation, Verification \& Validation}

The functional requirements placed on ZAM Version 4 or 5 are as follows:

1. Capability to reproduce the total ionic capacities of its cation competitors consistent with experimentally measured values.

Acceptance criterion: Successful prediction of total ionic capacity for $\mathrm{Cs}^{+}, \mathrm{K}^{+}, \mathrm{Rb}^{+}$, and $\mathrm{SrOH}^{+}$(in the judgement of the author(s) and independent technical reviewer).

2. Capability to reproduce the cesium $\mathrm{K}_{\mathrm{d}}$ and loading curves for Hanford AW-101 simulant and samples consistent with the experimentally measured values used in ZAM's original development phase.

Acceptance criterion: Successful prediction of the cesium $\mathrm{K}_{\mathrm{d}}$ and loading curves for Hanford AW-101 simulant and samples (in the judgement of the author(s) and independent technical reviewer).

PC based executable that has the capability to run on the following computer platform:

Computer platform chosen:

$\begin{array}{ll}\text { Platform: } & \text { Intel }^{\mathrm{TM}} \text { based Personal Computer } \\ \text { System: } & \text { Microsoft Windows-95 version 4.0 or higher } \\ \text { Compilers: } & \text { None required } \\ \text { Options: } & \text { Default settings } \\ \text { Acceptance criterion: } & \text { Successful installation testing }\end{array}$

The ZAM executables for this effort were loaded an IBM Personal Computer 300PL (with a PII 400 processor). Files corresponding to test cases were placed in shared folders while the ZAM code executables were kept in a protected file due to its proprietary nature.

Full path name: $\quad$ d:|versel

Cstiexv4.exe (protected; ZAM version 4) 


\section{Cstiexv5.exe (protected; ZAM version 5)}

The ZAM code was tested by running and comparing its output results to:

1. Isolated competitor to determine that ZAM will approach the appropriate total loading capacity at its limits (i.e., this is a verification activity checking that the version loaded onto the above PC is installed correctly and conforms to the expected behavior as published by its developers); and

2. Simulated and actual Hanford waste solutions that were originally used in ZAM's development (i.e., this is a validation activity checking that the version loaded onto the above $\mathrm{PC}$ is providing predictions that are consistent with those published by its developers for use with typical Hanford waste feeds).

\section{F.4.1 ZAM Verification of Total Ionic Capacities}

The ZAM model solves the solid-liquid equilibrium exchange and material balance equations that describe the ion exchange process between CST in its powdered-form in contact with a finite volume of liquid. In order to appropriately model this process, the number of "surface" sites (i.e., on a per mass basis of CST) at are available ("active") for a given cation competitor must be known and becomes an internally defined parameter within the equation set.

The total cation exchange capacity of the CST resin in its powder-form (batch IE-910) is species dependent. Two types of exchange sites exist on the CST solid. The total ion exchange capacity is stated to be $\sim 4.6 \mathrm{mmole} / \mathrm{g}_{\text {resin, }}$ but the cesium exchange capacity is much less indicating that not all sites are available for cesium exchange (see, Zheng et al., 1996). In the ideal solid region (i.e., the prior to the first step of the isotherm), the experimentally measured total capacities are $\sim 0.58 \mathrm{mmole} / \mathrm{g}_{\text {resin }}$ for $\mathrm{Cs}^{+}, \sim 1.2 \mathrm{mmole} / \mathrm{g}_{\text {resin }}$ for $\mathrm{K}^{+}, \sim 1.18 \mathrm{mmole} / \mathrm{g}_{\text {resin }}$ for $\mathrm{Rb}^{+}$, and $\sim 1.0$ $\mathrm{mmole} / \mathrm{g}_{\text {resin }}$ for $\mathrm{SrOH}^{+}$. For the expected feed concentrations it is anticipated that the entire columns will be operating within this ideal solid region.

To numerically compute these asymptotic cation capacities, the ZAM code was run under conditions where each cation competitor was individually set to a very high concentration (i.e., simple $5 \mathrm{M} \mathrm{Na}^{+}$and $2 \mathrm{M} \mathrm{OH}^{-}$solutions were used where $\mathrm{NO}_{3}{ }^{-}$concentrations were varied along with the given competitor for charge balance). The ZAM predicted results of these tests are:

- $\mathrm{Cs}^{+}$total ionic capacity $=0.5797 \mathrm{mmole} / \mathrm{g}_{\mathrm{CST}}$ (experimentally $\sim 0.58 ;-0.05 \%$ difference)

- $\mathrm{K}^{+}$total ionic capacity $=1.066 \mathrm{mmole} / \mathrm{g}_{\mathrm{CST}}$ (experimentally $\sim 1.2 ;-11.2 \%$ difference $)$

- $\mathrm{Rb}^{+}$total ionic capacity $=1.139 \mathrm{mmole} / \mathrm{g}_{\mathrm{CST}}$ (experimentally $\sim 1.18 ;-3.5 \%$ difference $)$

- $\mathrm{SrOH}^{+}$total ionic capacity $=1.159 \mathrm{mmole} / \mathrm{g}_{\mathrm{CST}}$ (experimentally $\sim 1.0 ;-15.9 \%$ difference)

As shown, the ZAM predicted total ionic capacities are all slightly under-predicting the measured values. However, these differences should be within experimental uncertainties and the ZAM results are considered acceptable. 


\section{F.4.2 ZAM Validation to Hanford LAW Solutions}

At the outset of development of ZAM, its intended purpose was to model the solid-liquid equilibrium exchange process of CST in its powdered-form in contact with high alkaline Hanford waste solutions (i.e., Cs removal from LAW solutions). Early on, validation of ZAMs ability to successfully model the ion exchange properties of CST in Hanford LAW was accomplished by direct comparison of ZAM predictions to experimental measurements. Here, we shall compare the ZAM results generated by our PC installed versions of ZAM to the original experimental database used in its creation.

Experimental batch contact tests ("K $\mathrm{K}_{\mathrm{d}}$ studies") by Brown et al. (1996) were performed placing CST (i.e., powder and engineered forms) in contact with both simulated and actual AW-101 Hanford waste solutions. In the simulated AW-101 studies the sodium concentration level was varied from $\sim 2$ to $\sim 7 \mathrm{M}$ over a large variation of cesium concentrations. For the smaller set of actual waste samples, a sodium concentration of $\sim 5 \mathrm{M}$ was tested.

A comparison of measured versus ZAM predicted $\mathrm{K}_{d}$ values for CST in its powder-form in contact with simulated AW-101 solutions is shown in Figure F-1. The measured data is provided in Table F-3. Sodium concentration levels varied from $2 \mathrm{M}$ up to $5 \mathrm{M}$ with all tests run at $\sim 25 \mathrm{C}$. Overall, good agreement is achieved with ZAM for the $\mathrm{K}_{\mathrm{d}}$ values corresponding to sodium levels of $1 \mathrm{M}$ and above. ZAM consistently under-predicts the measured $\mathrm{K}_{\mathrm{d}}$ values for the $0.2 \mathrm{M}$ sodium solutions. Our current applications focus primarily near $5 \mathrm{M}$ sodium solutions where adequate agreement exists. At $5 \mathrm{M}$ sodium conditions a comparison of measure $\mathrm{K}_{\mathrm{d}}$ values for CST powder in contact with simulated and actual AW-101 solutions is provided in Figure F2. Also shown in Figure F-2 is the corresponding ZAM prediction. The cesium $\mathrm{K}_{\mathrm{d}}$ values for the actual waste sample are systematically lower than ZAM or the simulated solution at increasing cesium concentrations. This may suggest that other species are present that affect $\mathrm{Cs}^{+}$ competition. The results shown in Figures F-1 and F-2 are consistent with the results provided by Zheng et al. (1997), indicating that our PC installed version of ZAM is operating consistent with its original release.

Brown et al. (1996) also performed batch contact tests using two CST engineered-form materials labeled as " $38 \mathrm{~b}$ " and " 08 " (i.e., these are some of the earlier-on engineered-forms made). Their contact data for these engineered-forms are given in Tables F-4 and F-5, respectively. Batch contact data ranging from $0.2 \mathrm{M}$ to $7 \mathrm{M}$ sodium were measured and are compared to ZAM predictions in Figures F-3 and F-4 for the "08" and " $38 \mathrm{~b}$ " engineered-forms, respectively. When comparing Figure F-1 to Figures F-3 and F-4, we see that in general ZAM slightly under-predicts CST powder performance while over-predicts CST engineered-form performance. This is a direct result of the presence of an inert binder (i.e., typically $20 \%$ to $30 \%$ by mass) used in making an engineered-form starting with CST powder.

For the engineered-form " $38 \mathrm{~b}$ " batch contact tests using actual AW-101 waste samples were performed at $5 \mathrm{M}$ sodium conditions. A comparison of measured cesium $\mathrm{K}_{\mathrm{d}}$ values for engineered-form " $38 \mathrm{~b}$ " in contact with simulant and actual waste samples is shown in Figure F5, along with the ZAM prediction. Similar to the trend mentioned above, when comparing Figures F-2 and F-5 we see that the engineered-forms have slightly degraded performance. 
Cesium loading data and ZAM predictions for the CST powder, engineered-form " 08 ", and engineered-form " $38 \mathrm{~b}$ " are shown in Figures F-6, F-7, and F-8, respectively. In all three figures significant data scatter is seen at low $\mathrm{Na}^{+} / \mathrm{Cs}^{+}$ratios (i.e., very high cesium concentrations). In Figure F-6 the data suggests that the total cesium capacity for CST powder varies with sodium concentration. However, at cesium and sodium concentrations levels of interest for Phase 1 inventories the ZAM predictions are reasonably consistent with the data.

Based on the comparisons made in this section of measured versus ZAM predicted cesium $\mathrm{K}_{\mathrm{d}}$ values and Cs loadings, we conclude that the ZAM model provides a reasonable estimate for CST powder (as shown in Figure F-9) but requires an adjustment (i.e., "dilution") factor when engineered-forms are being considered. For referencing, the ZAM predictions shown in Figures F-1 through F-9 are tabulated in Table F-6. The ZAM generated database is based on the AW101 compositions provided in Table F-7 where the cesium concentrations were varied from $1 \times 10^{-6} \mathrm{M}$ up to $0.1 \mathrm{M}$. To maintain charge balance $\mathrm{CsCl}$ was added where the original difference between $\mathrm{Cs}$ and $\mathrm{Cl}$ is maintained. Example $\mathrm{AW}-101$ input and output files for the $5 \mathrm{M}$ sodium conditions are provided in sections F.5 and F.6, respectively.

\section{F.4.3 Version-4 Versus Version-5 Comparison}

To see the impact of the improvements made in Version-5 when compared to results of Version4, both ZAM versions were run for the LAW-1 batch feed. For all Phase 1 LAW batch feeds, the ionic species considered in ZAM analyses are listed in Table F-2.

The input file for either version has the file structure as shown below in section F.5 for the Phase 1 LAW-1 batch feed. The test case considered for comparison has the CST initially in its sodium from where the equilibrium is calculated at $25 \mathrm{C}$. The LAW-1 feed adjusted to a $5 \mathrm{M}$ $\mathrm{Na}^{+}$basis with a solution density of $1.255 \mathrm{~g} / \mathrm{ml}$. At these conditions the solution has an ionic strength of $\sim 6.3$ molal and a $\mathrm{pH}$ of $\sim 14.6$. For convenience the phase ratio of the solid-liquid system is set to a very high value of $1 \times 10^{5} \mathrm{ml} / \mathrm{g}$ (i.e., $1000 \mathrm{~L}$ solution in contact with $0.01 \mathrm{~g}$ of CST). This is done so that the equilibrium point of interest will be very close to initial conditions (i.e., final cesium concentration close to its initial concentration).

Using the input file listed in section F.7 (file name: cstiexv.in), the results from Version-4 and Version-5 are provided in sections F.8 and F.9, respectively. The results are identical to within the level of accuracy provided (i.e., within round-off of the printed results; 4 digits for Version-4 and 3 digits for Version-5). As mentioned above, the use of a very high phase ratio resulted in the final $\mathrm{Cs}^{+}$solution concentration (i.e., $3.386 \times 10^{-4} \mathrm{M}$ ) being close to its initial concentration (i.e., $\left.3.433 \times 10^{-4} \mathrm{M}\right)$.

Comparisons made for other LAW batch feeds and for varying cesium concentrations (i.e., $\mathrm{CsCl}$ variations) show similar behavior. Basically, results based on versions 4 and 5 do not differ in any meaningful way. 
Preliminary Ion Exchange Modeling for Removal of Cesium from Revision (Date):

Table F-1. Ionic species available within the ZAM CST ion-exchange equilibrium model.

\begin{tabular}{|c|c|c|}
\hline ID & Cations & Anions \\
\hline 1 & $\mathrm{H}^{+}$ & $\mathrm{F}^{-}$ \\
\hline 2 & $\overline{\mathrm{Li}^{+}}$ & $\mathrm{Cl}^{-}$ \\
\hline 3 & $\mathrm{Na}^{+}$ & $\mathrm{Br}^{-}$ \\
\hline 4 & $\mathrm{~K}^{+}$ & $\mathrm{I}^{-}$ \\
\hline 5 & $\mathrm{Rb}^{+}$ & $\mathrm{ClO}_{3}{ }^{-}$ \\
\hline 6 & $\mathrm{Cs}^{+}$ & $\mathrm{ClO}_{4}^{-}$ \\
\hline 7 & $\mathrm{NH}_{4}^{+}$ & $\mathrm{BrO}_{3}^{-}$ \\
\hline 8 & $\mathrm{Tl}^{+}$ & $\mathrm{IO}_{3}^{-}$ \\
\hline 9 & $\mathrm{Ag}^{+}$ & $\mathrm{NO}_{3}^{-}$ \\
\hline 10 & $\mathrm{Be}^{2+}$ & $\mathrm{H}_{2} \mathrm{PO}_{4}{ }^{-}$ \\
\hline 11 & $\mathrm{Mg}^{2+}$ & $\mathrm{H}_{2} \mathrm{AsO}_{4}$ \\
\hline 12 & $\mathrm{Ca}^{2+}$ & $\mathrm{CNS}^{-}$ \\
\hline 13 & $\mathrm{Sr}^{2+}$ & $\mathrm{OH}^{-}$ \\
\hline 14 & $\mathrm{Ba}^{2+}$ & $\mathrm{CrO}_{4}{ }^{2-}$ \\
\hline 15 & $\mathrm{Mn}^{2+}$ & $\mathrm{SO}_{4}{ }^{2-}$ \\
\hline 16 & $\mathrm{Fe}^{2+}$ & $\mathrm{S}_{2} \mathrm{O}_{3}{ }^{2-}$ \\
\hline 17 & $\mathrm{Co}^{2+}$ & $\mathrm{HPO}_{4}{ }^{2-}$ \\
\hline 18 & $\mathrm{Ni}^{2+}$ & $\mathrm{HAsO}_{4}{ }^{2}$ \\
\hline 19 & $\mathrm{Cu}^{2+}$ & $\mathrm{CO}_{3}{ }^{2-}$ \\
\hline 20 & $\mathrm{Zn}^{2+}$ & $\mathrm{PO}_{4}{ }^{3-}$ \\
\hline
\end{tabular}

\begin{tabular}{|c|c|c|}
\hline ID & Cations & Anions \\
\hline 21 & $\mathrm{Cd}^{2+}$ & $\mathrm{AsO}_{4}{ }^{3--}$ \\
\hline 22 & $\mathrm{~Pb}^{2+}$ & $\mathrm{Fe}(\mathrm{CN})_{6}{ }^{3-}$ \\
\hline 23 & $\mathrm{UO}_{2}{ }^{2+}$ & $\mathrm{Mo}(\mathrm{CN})_{8}^{3-}$ \\
\hline 24 & $\mathrm{Cr}^{3+}$ & User defined $^{\text {a }}$ \\
\hline 25 & $\overline{\mathrm{Al}^{3+}}$ & User defined \\
\hline 26 & $\mathrm{Sc}^{3+}$ & User defined \\
\hline 27 & $\mathrm{Y}^{3+}$ & $\mathrm{NO}_{2}^{-}$ \\
\hline 28 & $\mathrm{La}^{3+}$ & $\mathrm{Al}(\mathrm{OH})_{4}^{-}$ \\
\hline 29 & $\mathrm{Ce}^{3+}$ & $\mathrm{na}^{\mathrm{b}}$ \\
\hline 30 & $\mathrm{Pr}^{3+}$ & na \\
\hline 31 & $\mathrm{Nd}^{3+}$ & na \\
\hline 32 & $\mathrm{Sm}^{3+}$ & na \\
\hline 33 & $\overline{\mathrm{Eu}^{3+}}$ & na \\
\hline 34 & $\mathrm{Ga}^{3+}$ & na \\
\hline 35 & $\mathrm{Co}^{3+}$ & na \\
\hline 36 & $\mathrm{Th}^{4+}$ & na \\
\hline 37 & User defined $^{\mathrm{a}}$ & na \\
\hline 38 & User defined & na \\
\hline 39 & User defined & na \\
\hline 40 & $\mathrm{SrOH}^{+}$ & na \\
\hline
\end{tabular}

${ }^{a}$ Array locations in storage that are available for user to specify additional species.

${ }^{\mathrm{b}}$ Array locations in storage that are currently unused. 
Table F-2. ZAM ionic species used in CST modeling of Phase 1 LAW batch feeds.

\begin{tabular}{|c|c|c|}
\hline ID & Cations & Anions \\
\hline 1 & $\mathrm{H}^{+}$ & $\mathrm{F}^{-}$ \\
\hline 2 & - & $\mathrm{Cl}^{-}$ \\
\hline 3 & $\mathrm{Na}^{+}$ & - \\
\hline 4 & $\mathrm{~K}^{+}$ & $\mathrm{I}^{-}$ \\
\hline 5 & $\mathrm{Rb}^{+}$ & - \\
\hline 6 & $\mathrm{Cs}^{+}$ & - \\
\hline 7 & - & - \\
\hline 8 & - & - \\
\hline 9 & - & $\mathrm{NO}_{3}^{-}$ \\
\hline 10 & - & - \\
\hline 11 & - & - \\
\hline 12 & $\mathrm{Ca}^{2+}$ & - \\
\hline 13 & $\mathrm{Sr}^{2+}$ & $\mathrm{OH}^{-}$ \\
\hline 14 & $\mathrm{Ba}^{2+}$ & - \\
\hline 15 & $\mathrm{Mn}^{2+}$ & $\mathrm{SO}_{4}{ }^{2-}$ \\
\hline 16 & $\mathrm{Fe}^{2+}$ & - \\
\hline 17 & - & - \\
\hline 18 & $\mathrm{Ni}^{2+}$ & - \\
\hline 19 & - & $\mathrm{CO}_{3}{ }^{2-}$ \\
\hline 20 & $\mathrm{Zn}^{2+}$ & $\mathrm{PO}_{4}{ }^{3-}$ \\
\hline
\end{tabular}

\begin{tabular}{|c|c|c|}
\hline ID & Cations & Anions \\
\hline 21 & $\mathrm{Cd}^{2+}$ & - \\
\hline 22 & $\mathrm{~Pb}^{2+}$ & - \\
\hline 23 & $\mathrm{UO}_{2}{ }^{2+}$ & - \\
\hline 24 & $\mathrm{Cr}^{3+}$ & \\
\hline 25 & $\mathrm{Al}^{3+}$ & \\
\hline 26 & - & \\
\hline 27 & - & $\mathrm{NO}_{2}{ }^{-}$ \\
\hline 28 & $\mathrm{La}^{3+}$ & $\mathrm{Al}(\mathrm{OH})_{4}$ \\
\hline 29 & $\mathrm{Ce}^{3+}$ & \\
\hline 30 & - & \\
\hline 31 & - & \\
\hline 32 & - & \\
\hline 33 & - & \\
\hline 34 & - & \\
\hline 35 & - & \\
\hline 36 & - & \\
\hline 37 & & \\
\hline 38 & & \\
\hline 39 & & \\
\hline 40 & $\mathrm{SrOH}^{+}$ & \\
\hline
\end{tabular}

Table F-3. Equilibrium data for cesium on CST powder (IE-910) based on batch contact tests performed at $25 \mathrm{C}$ in simulated and actual AW-101 solutions (Brown et al. (1996), $0.475 \mathrm{M}$ potassium). ${ }^{\mathrm{a}}$

\begin{tabular}{|c|c|c|c|c|c|}
\hline Sample Description & $\begin{array}{c}\mathbf{L i q u i d ~ N a}^{+} \\
{[\mathbf{M}]}\end{array}$ & $\begin{array}{c}\mathbf{L i q u i d}_{\mathbf{C s}}{ }^{+} \\
{[\mathbf{M}]}\end{array}$ & $\begin{array}{c}\mathbf{L i q u i d ~ N a}^{+} / \mathbf{C s}^{+} \\
{[\mathbf{r a t i o}]}\end{array}$ & $\begin{array}{c}\mathbf{K}_{\mathbf{d}} \\
{[\mathbf{m l} / \mathbf{g}]}\end{array}$ & $\begin{array}{c}\mathbf{C s}^{+} \text {loading } \\
{\left[\mathbf{m m o l e} / \mathbf{g}_{\text {CST }}\right]}\end{array}$ \\
\hline \hline POW-SIM-0.2 & 0.20 & $4.600 \mathrm{E}-04$ & $4.348 \mathrm{E}+02$ & 1857.00 & $8.542 \mathrm{E}-01$ \\
\hline POW-SIM-0.2 & 0.20 & $4.455 \mathrm{E}-04$ & $4.489 \mathrm{E}+02$ & 1923.00 & $8.568 \mathrm{E}-01$ \\
\hline POW-SIM-0.2 & 0.20 & $3.419 \mathrm{E}-06$ & $5.850 \mathrm{E}+04$ & 80630.00 & $2.757 \mathrm{E}-01$ \\
\hline POW-SIM-0.2 & 0.20 & $3.892 \mathrm{E}-06$ & $5.139 \mathrm{E}+04$ & 47780.00 & $1.860 \mathrm{E}-01$ \\
\hline POW-SIM-0.2 & 0.20 & $3.783 \mathrm{E}-07$ & $5.287 \mathrm{E}+05$ & 57100.00 & $2.160 \mathrm{E}-02$ \\
\hline POW-SIM-0.2 & 0.20 & $3.783 \mathrm{E}-07$ & $5.287 \mathrm{E}+05$ & 61570.00 & $2.329 \mathrm{E}-02$ \\
\hline POW-SIM-0.2 & 0.20 & $3.428 \mathrm{E}-08$ & $5.834 \mathrm{E}+06$ & 72750.00 & $2.494 \mathrm{E}-03$ \\
\hline POW-SIM-0.2 & 0.20 & $3.313 \mathrm{E}-08$ & $6.036 \mathrm{E}+06$ & 80260.00 & $2.659 \mathrm{E}-03$ \\
\hline POW-SIM-0.2 & 0.20 & $3.810 \mathrm{E}-09$ & $5.250 \mathrm{E}+07$ & 70840.00 & $2.699 \mathrm{E}-04$ \\
\hline POW-SIM-0.2 & 0.20 & $3.723 \mathrm{E}-09$ & $5.372 \mathrm{E}+07$ & 74930.00 & $2.790 \mathrm{E}-04$ \\
\hline POW-SIM-1.0 & 1.00 & $1.460 \mathrm{E}-02$ & $6.847 \mathrm{E}+01$ & 57.33 & $8.373 \mathrm{E}-01$ \\
\hline POW-SIM-1.0 & 1.00 & $1.463 \mathrm{E}-02$ & $6.836 \mathrm{E}+01$ & 56.89 & $8.322 \mathrm{E}-01$ \\
\hline POW-SIM-1.0 & 1.00 & $5.672 \mathrm{E}-05$ & $1.763 \mathrm{E}+04$ & 6463.00 & $3.666 \mathrm{E}-01$ \\
\hline POW-SIM-1.0 & 1.00 & $5.882 \mathrm{E}-05$ & $1.700 \mathrm{E}+04$ & 6243.00 & $3.672 \mathrm{E}-01$ \\
\hline POW-SIM-1.0 & 1.00 & $4.444 \mathrm{E}-06$ & $2.250 \mathrm{E}+05$ & 9493.00 & $4.219 \mathrm{E}-02$ \\
\hline
\end{tabular}




\begin{tabular}{|c|c|c|c|c|c|}
\hline Sample Description & $\begin{array}{c}\text { Liquid } \mathrm{Na}^{+} \\
{[\mathrm{M}]}\end{array}$ & $\begin{array}{c}\text { Liquid Cs }{ }^{+} \\
{[\mathrm{M}]}\end{array}$ & $\underset{\mid}{\text { Liquid } \mathrm{Na}^{+} / \mathrm{Cs}^{+}}$ & $\begin{array}{c}\mathbf{K}_{\mathbf{d}} \\
{[\mathbf{m l} / \mathbf{g}]}\end{array}$ & $\begin{array}{c}\mathrm{Cs}^{+} \text {loading } \\
{\left[\text { mmole/g } / \mathbf{g}_{\mathrm{CST}}\right]}\end{array}$ \\
\hline POW-SIM-1.0 & 1.00 & $4.521 \mathrm{E}-06$ & $2.212 \mathrm{E}+05$ & 9293.00 & $4.201 \mathrm{E}-02$ \\
\hline POW-SIM-1.0 & 1.00 & 4.448E-07 & $2.248 \mathrm{E}+06$ & 10100.00 & 4.493E-03 \\
\hline POW-SIM-1.0 & 1.00 & $4.305 \mathrm{E}-07$ & $2.323 \mathrm{E}+06$ & 10500.00 & $4.520 \mathrm{E}-03$ \\
\hline POW-SIM-1.0 & 1.00 & 4.715E-08 & $2.121 \mathrm{E}+07$ & 8875.00 & 4.184E-04 \\
\hline POW-SIM-1.0 & 1.00 & $4.531 \mathrm{E}-08$ & $2.207 \mathrm{E}+07$ & 9296.00 & 4.212E-04 \\
\hline POW-SIM-3.0 & 3.00 & $5.486 \mathrm{E}-02$ & $5.468 \mathrm{E}+01$ & 14.91 & $8.180 \mathrm{E}-01$ \\
\hline POW-SIM-3.0 & 3.00 & 5.489E-02 & $5.465 \mathrm{E}+01$ & 15.12 & $8.300 \mathrm{E}-01$ \\
\hline POW-SIM-3.0 & 3.00 & $1.569 \mathrm{E}-03$ & $1.912 \mathrm{E}+03$ & 340.70 & $5.346 \mathrm{E}-01$ \\
\hline POW-SIM-3.0 & 3.00 & $1.581 \mathrm{E}-03$ & $1.898 \mathrm{E}+03$ & 335.50 & 5.303E-01 \\
\hline POW-SIM-3.0 & 3.00 & $3.750 \mathrm{E}-05$ & $8.000 \mathrm{E}+04$ & 2709.00 & $1.016 \mathrm{E}-01$ \\
\hline POW-SIM-3.0 & 3.00 & $3.750 \mathrm{E}-05$ & $8.000 \mathrm{E}+04$ & 2681.00 & $1.005 \mathrm{E}-01$ \\
\hline POW-SIM-3.0 & 3.00 & $3.750 \mathrm{E}-06$ & $8.000 \mathrm{E}+05$ & 2845.00 & $1.067 \mathrm{E}-02$ \\
\hline POW-SIM-3.0 & 3.00 & $3.750 \mathrm{E}-06$ & $8.000 \mathrm{E}+05$ & 2735.00 & $1.026 \mathrm{E}-02$ \\
\hline POW-SIM-3.0 & 3.00 & $3.750 \mathrm{E}-07$ & $8.000 \mathrm{E}+06$ & 2749.00 & $1.031 \mathrm{E}-03$ \\
\hline POW-SIM-3.0 & 3.00 & $3.750 \mathrm{E}-07$ & $8.000 \mathrm{E}+06$ & 2791.00 & $1.047 \mathrm{E}-03$ \\
\hline POW-SIM-5.0 & 5.00 & 9.482E-02 & $5.273 \mathrm{E}+01$ & 8.44 & $8.002 \mathrm{E}-01$ \\
\hline POW-SIM-5.0 & 5.00 & $9.562 \mathrm{E}-02$ & $5.229 \mathrm{E}+01$ & 7.03 & $6.722 \mathrm{E}-01$ \\
\hline POW-SIM-5.0 & 5.00 & $6.007 \mathrm{E}-03$ & $8.323 \mathrm{E}+02$ & 103.60 & $6.224 \mathrm{E}-01$ \\
\hline POW-SIM-5.0 & 5.00 & $5.904 \mathrm{E}-03$ & $8.469 \mathrm{E}+02$ & 106.20 & $6.270 \mathrm{E}-01$ \\
\hline POW-SIM-5.0 & 5.00 & $1.089 \mathrm{E}-04$ & $4.590 \mathrm{E}+04$ & 1373.00 & $1.496 \mathrm{E}-01$ \\
\hline POW-SIM-5.0 & 5.00 & $1.114 \mathrm{E}-04$ & $4.489 \mathrm{E}+04$ & 1305.00 & $1.454 \mathrm{E}-01$ \\
\hline POW-SIM-5.0 & 5.00 & $9.690 \mathrm{E}-06$ & $5.160 \mathrm{E}+05$ & 1574.00 & $1.525 \mathrm{E}-02$ \\
\hline POW-SIM-5.0 & 5.00 & $9.705 \mathrm{E}-06$ & $5.152 \mathrm{E}+05$ & 1568.00 & $1.522 \mathrm{E}-02$ \\
\hline POW-SIM-5.0 & 5.00 & $9.850 \mathrm{E}-07$ & $5.076 \mathrm{E}+06$ & 1545.00 & $1.522 \mathrm{E}-03$ \\
\hline POW-SIM-5.0 & 5.00 & $9.766 \mathrm{E}-07$ & $5.120 \mathrm{E}+06$ & 1545.00 & $1.509 \mathrm{E}-03$ \\
\hline POW-ACT-5.0 & 5.01 & $4.246 \mathrm{E}-02$ & $1.180 \mathrm{E}+02$ & 18.90 & $8.024 \mathrm{E}-01$ \\
\hline POW-ACT-5.0 & 5.01 & $4.357 \mathrm{E}-02$ & $1.150 \mathrm{E}+02$ & 14.50 & $6.317 \mathrm{E}-01$ \\
\hline POW-ACT-5.0 & 5.01 & $1.681 \mathrm{E}-03$ & $2.980 \mathrm{E}+03$ & 276.00 & 4.640E-01 \\
\hline POW-ACT-5.0 & 5.01 & $1.710 \mathrm{E}-03$ & $2.930 \mathrm{E}+03$ & 269.00 & $4.600 \mathrm{E}-01$ \\
\hline POW-ACT-5.0 & 5.01 & $5.536 \mathrm{E}-05$ & $9.050 \mathrm{E}+04$ & 996.00 & 5.514E-02 \\
\hline POW-ACT-5.0 & 5.01 & 5.719E-05 & $8.760 \mathrm{E}+04$ & 959.00 & $5.485 \mathrm{E}-02$ \\
\hline POW-ACT-5.0 & 5.01 & $1.020 \mathrm{E}-05$ & $4.910 \mathrm{E}+05$ & 772.00 & 7.877E-03 \\
\hline POW-ACT-5.0 & 5.01 & $6.807 \mathrm{E}-06$ & $7.360 \mathrm{E}+05$ & 1230.00 & $8.373 \mathrm{E}-03$ \\
\hline
\end{tabular}

${ }^{\mathrm{a}}$ The concentrations listed are equilibrium values at the final state of the liquid solution.

Table F-4. Equilibrium data for cesium on CST engineered-form (IE-911 -38b) based on batch contact tests performed at $25 \mathrm{C}$ in simulated and actual AW-101 solutions (Brown et al. (1996), 0.475 M potassium). ${ }^{\mathrm{a}}$

\begin{tabular}{|c|c|r|r|r|r|}
\hline Sample Description & $\begin{array}{c}\text { Liquid Na } \\
{[\mathbf{M}]}\end{array}$ & $\begin{array}{c}\text { Liquid Cs } \\
{[\mathbf{M}]}\end{array}$ & $\begin{array}{c}\text { Liquid Na }^{+} / \mathbf{C s}^{+} \\
{[\mathbf{r a t i o}]}\end{array}$ & $\begin{array}{c}\mathbf{K}_{\mathbf{d}} \\
{[\mathbf{m l} / \mathbf{g}]}\end{array}$ & $\begin{array}{c}\mathbf{C s}^{+} \text {loading } \\
{[\mathbf{m m o l e} / \mathbf{g} \text { CsT] }}\end{array}$ \\
\hline \hline E38B-SIM-0.2 & 0.20 & $8.094 \mathrm{E}-04$ & $2.471 \mathrm{E}+02$ & 673.50 & $5.451 \mathrm{E}-01$ \\
\hline E38B-SIM-0.2 & 0.20 & $7.460 \mathrm{E}-04$ & $2.681 \mathrm{E}+02$ & 743.80 & $5.549 \mathrm{E}-01$ \\
\hline E38B-SIM-0.2 & 0.20 & $5.436 \mathrm{E}-06$ & $3.679 \mathrm{E}+04$ & 19190.00 & $1.043 \mathrm{E}-01$ \\
\hline E38B-SIM-0.2 & 0.20 & $5.167 \mathrm{E}-06$ & $3.871 \mathrm{E}+04$ & 20760.00 & $1.073 \mathrm{E}-01$ \\
\hline E38B-SIM-0.2 & 0.20 & $4.854 \mathrm{E}-07$ & $4.120 \mathrm{E}+05$ & 24620.00 & $1.195 \mathrm{E}-02$ \\
\hline
\end{tabular}


WESTINGHOUSE SAVANNAH RIVER COMPANY

Preliminary Ion Exchange Modeling for Removal of Cesium from Hanford Waste Using Hydrous Crystalline Silicotitanate Material
Report:

WSRC-TR-2001-00400

Revision (Date):

$0(07 / 05 / 02)$

Page:

\begin{tabular}{|c|c|c|c|c|c|}
\hline Sample Description & \begin{tabular}{|} 
Liquid $\mathrm{Na}^{+}$ \\
{$[\mathrm{M}]$}
\end{tabular} & \begin{tabular}{|} 
Liquid $\mathbf{C s}^{+}$ \\
{$[\mathrm{M}]$}
\end{tabular} & $\begin{array}{c}\text { Liquid } \mathrm{Na}^{+} / \mathrm{Cs}^{+} \\
\text {[ratio] }\end{array}$ & $\begin{array}{c}\mathbf{K}_{\mathbf{d}} \\
{[\mathrm{ml} / \mathbf{g}]}\end{array}$ & $\begin{array}{l}\mathrm{Cs}^{+} \text {loading } \\
{\left[\mathrm{mmole} / \mathrm{g}_{\mathrm{CST}}\right]}\end{array}$ \\
\hline E38B-SIM-0.2 & 0.20 & $6.129 \mathrm{E}-07$ & $3.263 \mathrm{E}+05$ & 16630.00 & $1.019 \mathrm{E}-02$ \\
\hline E38B-SIM-0.2 & 0.20 & $4.250 \mathrm{E}-08$ & $4.706 \mathrm{E}+06$ & 28700.00 & $1.220 \mathrm{E}-03$ \\
\hline E38B-SIM-0.2 & 0.20 & $4.277 \mathrm{E}-08$ & $4.676 \mathrm{E}+06$ & 28180.00 & $1.205 \mathrm{E}-03$ \\
\hline E38B-SIM-0.2 & 0.20 & 3.490E-09 & $5.731 \mathrm{E}+07$ & 43600.00 & $1.522 \mathrm{E}-04$ \\
\hline E38B-SIM-0.2 & 0.20 & 4.314E-09 & $4.636 \mathrm{E}+07$ & 28140.00 & $1.214 \mathrm{E}-04$ \\
\hline E38B-SIM-1.0 & 1.00 & $1.660 \mathrm{E}-02$ & $6.024 \mathrm{E}+01$ & 34.51 & $5.729 \mathrm{E}-01$ \\
\hline E38B-SIM-1.0 & 1.00 & $1.606 \mathrm{E}-02$ & $6.225 \mathrm{E}+01$ & 41.11 & $6.604 \mathrm{E}-01$ \\
\hline E38B-SIM-1.0 & 1.00 & $1.549 \mathrm{E}-04$ & $6.456 \mathrm{E}+03$ & 2159.00 & $3.344 \mathrm{E}-01$ \\
\hline E38B-SIM-1.0 & 1.00 & $1.473 \mathrm{E}-04$ & $6.791 \mathrm{E}+03$ & 2280.00 & $3.357 \mathrm{E}-01$ \\
\hline E38B-SIM-1.0 & 1.00 & $8.953 \mathrm{E}-06$ & $1.117 \mathrm{E}+05$ & 4125.00 & 3.693E-02 \\
\hline E38B-SIM-1.0 & 1.00 & $8.340 \mathrm{E}-06$ & $1.199 \mathrm{E}+05$ & 4493.00 & $3.747 \mathrm{E}-02$ \\
\hline E38B-SIM-1.0 & 1.00 & $6.305 \mathrm{E}-07$ & $1.586 \mathrm{E}+06$ & 6263.00 & 3.949E-03 \\
\hline E38B-SIM-1.0 & 1.00 & $6.116 \mathrm{E}-07$ & $1.635 \mathrm{E}+06$ & 6535.00 & 3.997E-03 \\
\hline E38B-SIM-1.0 & 1.00 & 7.369E-08 & $1.357 \mathrm{E}+07$ & 5056.00 & $3.726 \mathrm{E}-04$ \\
\hline E38B-SIM-1.0 & 1.00 & $7.210 \mathrm{E}-08$ & $1.387 \mathrm{E}+07$ & 5180.00 & $3.735 \mathrm{E}-04$ \\
\hline E38B-SIM-3.0 & 3.00 & $5.663 \mathrm{E}-02$ & $5.298 \mathrm{E}+01$ & 10.06 & $5.696 \mathrm{E}-01$ \\
\hline E38B-SIM-3.0 & 3.00 & 5.700E-02 & $5.263 \mathrm{E}+01$ & 8.79 & $5.008 \mathrm{E}-01$ \\
\hline E38B-SIM-3.0 & 3.00 & 3.422E-03 & $8.766 \mathrm{E}+02$ & 126.60 & 4.333E-01 \\
\hline E38B-SIM-3.0 & 3.00 & $3.429 \mathrm{E}-03$ & $8.748 \mathrm{E}+02$ & 127.00 & $4.355 \mathrm{E}-01$ \\
\hline E38B-SIM-3.0 & 3.00 & $6.241 \mathrm{E}-05$ & $4.807 \mathrm{E}+04$ & 1502.00 & $9.374 \mathrm{E}-02$ \\
\hline E38B-SIM-3.0 & 3.00 & $6.180 \mathrm{E}-05$ & $4.854 \mathrm{E}+04$ & 1519.00 & $9.388 \mathrm{E}-02$ \\
\hline E38B-SIM-3.0 & 3.00 & $6.537 \mathrm{E}-06$ & $4.589 \mathrm{E}+05$ & 1440.00 & $9.414 \mathrm{E}-03$ \\
\hline E38B-SIM-3.0 & 3.00 & 5.299E-06 & $5.661 \mathrm{E}+05$ & 1826.00 & $9.677 \mathrm{E}-03$ \\
\hline E38B-SIM-3.0 & 3.00 & $5.308 \mathrm{E}-07$ & $5.652 \mathrm{E}+06$ & 1853.00 & $9.835 \mathrm{E}-04$ \\
\hline E38B-SIM-3.0 & 3.00 & 3.779E-07 & $7.938 \mathrm{E}+06$ & 2773.00 & $1.048 \mathrm{E}-03$ \\
\hline E38B-SIM-5.0 & 5.00 & 9.619E-02 & $5.198 \mathrm{E}+01$ & 6.74 & $6.481 \mathrm{E}-01$ \\
\hline E38B-SIM-5.0 & 5.00 & $9.651 \mathrm{E}-02$ & $5.181 \mathrm{E}+01$ & 6.07 & $5.857 \mathrm{E}-01$ \\
\hline E38B-SIM-5.0 & 5.00 & $7.423 \mathrm{E}-03$ & $6.736 \mathrm{E}+02$ & 58.81 & $4.365 \mathrm{E}-01$ \\
\hline E38B-SIM-5.0 & 5.00 & 7.407E-03 & $6.750 \mathrm{E}+02$ & 59.17 & $4.383 \mathrm{E}-01$ \\
\hline E38B-SIM-5.0 & 5.00 & $1.987 \mathrm{E}-04$ & $2.516 \mathrm{E}+04$ & 700.30 & $1.392 \mathrm{E}-01$ \\
\hline E38B-SIM-5.0 & 5.00 & $1.962 \mathrm{E}-04$ & $2.549 \mathrm{E}+04$ & 711.40 & $1.395 \mathrm{E}-01$ \\
\hline E38B-SIM-5.0 & 5.00 & $1.486 \mathrm{E}-05$ & $3.365 \mathrm{E}+05$ & 998.40 & $1.484 \mathrm{E}-02$ \\
\hline E38B-SIM-5.0 & 5.00 & $1.493 \mathrm{E}-05$ & $3.350 \mathrm{E}+05$ & 1001.00 & $1.494 \mathrm{E}-02$ \\
\hline E38B-SIM-7.0 & 7.00 & 1.372E-01 & $5.101 \mathrm{E}+01$ & 3.40 & 4.662E-01 \\
\hline E38B-SIM-7.0 & 7.00 & $1.373 \mathrm{E}-01$ & $5.100 \mathrm{E}+01$ & 3.39 & $4.650 \mathrm{E}-01$ \\
\hline E38B-SIM-7.0 & 7.00 & $1.138 \mathrm{E}-02$ & $6.149 \mathrm{E}+02$ & 39.16 & $4.458 \mathrm{E}-01$ \\
\hline E38B-SIM-7.0 & 7.00 & $1.137 \mathrm{E}-02$ & $6.155 \mathrm{E}+02$ & 39.11 & $4.448 \mathrm{E}-01$ \\
\hline E38B-SIM-7.0 & 7.00 & 3.991E-04 & $1.754 \mathrm{E}+04$ & 434.80 & $1.735 \mathrm{E}-01$ \\
\hline E38B-SIM-7.0 & 7.00 & 3.919E-04 & $1.786 \mathrm{E}+04$ & 442.20 & $1.733 \mathrm{E}-01$ \\
\hline E38B-SIM-7.0 & 7.00 & $2.730 \mathrm{E}-05$ & $2.564 \mathrm{E}+05$ & 719.40 & $1.964 \mathrm{E}-02$ \\
\hline E38B-SIM-7.0 & 7.00 & $2.696 \mathrm{E}-05$ & $2.596 \mathrm{E}+05$ & 724.20 & $1.953 \mathrm{E}-02$ \\
\hline E38B-SIM-7.0 & 7.00 & $2.610 \mathrm{E}-06$ & $2.682 \mathrm{E}+06$ & 758.80 & $1.980 \mathrm{E}-03$ \\
\hline E38B-SIM-7.0 & 7.00 & $2.613 \mathrm{E}-06$ & $2.679 \mathrm{E}+06$ & 764.60 & $1.998 \mathrm{E}-03$ \\
\hline E38B-ACT-5.0 & 5.01 & 4.434E-02 & $1.130 \mathrm{E}+02$ & 12.60 & $5.586 \mathrm{E}-01$ \\
\hline E38B-ACT-5.0 & 5.01 & 4.514E-02 & $1.110 \mathrm{E}+02$ & 9.82 & 4.432E-01 \\
\hline E38B-ACT-5.0 & 5.01 & $2.518 \mathrm{E}-03$ & $1.990 \mathrm{E}+03$ & 146.00 & $3.676 \mathrm{E}-01$ \\
\hline
\end{tabular}




\begin{tabular}{|c|c|c|r|r|r|}
\hline Sample Description & $\begin{array}{c}\text { Liquid Na } \\
{[\mathbf{M}]}\end{array}$ & $\begin{array}{c}\text { Liquid Cs } \\
{[\mathbf{M}]}\end{array}$ & $\begin{array}{c}\mathbf{L i q u i d ~ N a}^{+} / \mathbf{C s}^{+} \\
{[\mathbf{r a t i o}]}\end{array}$ & $\begin{array}{c}\mathbf{K}_{\mathbf{d}} \\
{[\mathbf{m l} / \mathbf{g}]}\end{array}$ & $\begin{array}{c}\mathbf{C s}^{+} \text {loading } \\
{[\mathbf{m m o l e / g} / \mathbf{g S T}]}\end{array}$ \\
\hline E38B-ACT-5.0 & 5.01 & $2.665 \mathrm{E}-03$ & $1.880 \mathrm{E}+03$ & 129.00 & $3.438 \mathrm{E}-01$ \\
\hline E38B-ACT-5.0 & 5.01 & $9.210 \mathrm{E}-05$ & $5.440 \mathrm{E}+04$ & 646.00 & $5.949 \mathrm{E}-02$ \\
\hline E38B-ACT-5.0 & 5.01 & $9.093 \mathrm{E}-05$ & $5.510 \mathrm{E}+04$ & 656.00 & $5.965 \mathrm{E}-02$ \\
\hline E38B-ACT-5.0 & 5.01 & $1.136 \mathrm{E}-05$ & $4.410 \mathrm{E}+05$ & 782.00 & $8.884 \mathrm{E}-03$ \\
\hline E38B-ACT-5.0 & 5.01 & $1.116 \mathrm{E}-05$ & $4.490 \mathrm{E}+05$ & 797.00 & $8.893 \mathrm{E}-03$ \\
\hline
\end{tabular}

${ }^{\mathrm{a}}$ The concentrations listed are equilibrium values at the final state of the liquid solution.

Table F-5. Equilibrium data for cesium on CST engineered-form (IE-911 -08) based on batch contact tests performed at $25 \mathrm{C}$ in simulated AW-101 solutions (Brown et al. (1996), $0.475 \mathrm{M}$ potassium). ${ }^{\mathrm{a}}$

\begin{tabular}{|c|c|c|c|c|c|}
\hline Sample Description & $\begin{array}{c}\text { Liquid } \mathrm{Na}^{+} \\
{[\mathrm{M}]}\end{array}$ & $\begin{array}{c}\text { Liquid } \mathrm{Cs}^{+} \\
{[\mathrm{M}]}\end{array}$ & $\begin{array}{c}\text { Liquid } \mathrm{Na}^{+} / \mathrm{Cs}^{+} \\
\text {[ratio] }\end{array}$ & $\begin{array}{c}\mathbf{K}_{\mathbf{d}} \\
{[\mathrm{ml} / \mathrm{g}]}\end{array}$ & $\begin{array}{c}\mathrm{Cs}^{+} \text {loading } \\
\text { [mmole/g/gST] }\end{array}$ \\
\hline E08-SIM-0.2 & 0.20 & $3.982 \mathrm{E}-04$ & $5.022 \mathrm{E}+02$ & 1571.00 & $6.256 \mathrm{E}-01$ \\
\hline E08-SIM-0.2 & 0.20 & $3.131 \mathrm{E}-04$ & $6.387 \mathrm{E}+02$ & 2081.00 & $6.516 \mathrm{E}-01$ \\
\hline E08-SIM-0.2 & 0.20 & $4.240 \mathrm{E}-06$ & $4.717 \mathrm{E}+04$ & 29970.00 & $1.271 \mathrm{E}-01$ \\
\hline E08-SIM-0.2 & 0.20 & $4.327 \mathrm{E}-06$ & $4.622 \mathrm{E}+04$ & 28710.00 & $1.242 \mathrm{E}-01$ \\
\hline E08-SIM-0.2 & 0.20 & $4.442 \mathrm{E}-07$ & $4.502 \mathrm{E}+05$ & 31130.00 & $1.383 \mathrm{E}-02$ \\
\hline E08-SIM-0.2 & 0.20 & $4.239 \mathrm{E}-07$ & $4.718 \mathrm{E}+05$ & 34430.00 & $1.460 \mathrm{E}-02$ \\
\hline E08-SIM-0.2 & 0.20 & $3.706 \mathrm{E}-08$ & $5.397 \mathrm{E}+06$ & 39350.00 & $1.458 \mathrm{E}-03$ \\
\hline E08-SIM-0.2 & 0.20 & $3.599 \mathrm{E}-08$ & $5.557 \mathrm{E}+06$ & 42060.00 & $1.514 \mathrm{E}-03$ \\
\hline E08-SIM-0.2 & 0.20 & $4.337 \mathrm{E}-09$ & $4.612 \mathrm{E}+07$ & 23220.00 & $1.007 \mathrm{E}-04$ \\
\hline E08-SIM-0.2 & 0.20 & $4.521 \mathrm{E}-09$ & $4.424 \mathrm{E}+07$ & 21810.00 & $9.860 \mathrm{E}-05$ \\
\hline E08-SIM-1.0 & 1.00 & $1.597 \mathrm{E}-02$ & $6.261 \mathrm{E}+01$ & 41.91 & $6.694 \mathrm{E}-01$ \\
\hline E08-SIM-1.0 & 1.00 & $1.595 \mathrm{E}-02$ & $6.270 \mathrm{E}+01$ & 42.14 & $6.721 \mathrm{E}-01$ \\
\hline E08-SIM-1.0 & 1.00 & $9.881 \mathrm{E}-05$ & $1.012 \mathrm{E}+04$ & 3725.00 & $3.681 \mathrm{E}-01$ \\
\hline E08-SIM-1.0 & 1.00 & $1.022 \mathrm{E}-04$ & $9.781 \mathrm{E}+03$ & 3580.00 & $3.660 \mathrm{E}-01$ \\
\hline E08-SIM-1.0 & 1.00 & $6.460 \mathrm{E}-06$ & $1.548 \mathrm{E}+05$ & 6333.00 & $4.091 \mathrm{E}-02$ \\
\hline E08-SIM-1.0 & 1.00 & $6.623 \mathrm{E}-06$ & $1.510 \mathrm{E}+05$ & 6149.00 & $4.072 \mathrm{E}-02$ \\
\hline E08-SIM-1.0 & 1.00 & $6.301 \mathrm{E}-07$ & $1.587 \mathrm{E}+06$ & 6082.00 & $3.832 \mathrm{E}-03$ \\
\hline E08-SIM-1.0 & 1.00 & $6.321 \mathrm{E}-07$ & $1.582 \mathrm{E}+06$ & 6048.00 & $3.823 \mathrm{E}-03$ \\
\hline E08-SIM-1.0 & 1.00 & $6.192 \mathrm{E}-08$ & $1.615 \mathrm{E}+07$ & 6208.00 & $3.844 \mathrm{E}-04$ \\
\hline E08-SIM-1.0 & 1.00 & $6.414 \mathrm{E}-08$ & $1.559 \mathrm{E}+07$ & 5921.00 & $3.798 \mathrm{E}-04$ \\
\hline E08-SIM-3.0 & 3.00 & $5.640 \mathrm{E}-02$ & $5.319 \mathrm{E}+01$ & 10.66 & $6.012 \mathrm{E}-01$ \\
\hline E08-SIM-3.0 & 3.00 & $5.659 \mathrm{E}-02$ & $5.301 \mathrm{E}+01$ & 10.08 & $5.705 \mathrm{E}-01$ \\
\hline E08-SIM-3.0 & 3.00 & $2.910 \mathrm{E}-03$ & $1.031 \mathrm{E}+03$ & 177.90 & $5.177 \mathrm{E}-01$ \\
\hline E08-SIM-3.0 & 3.00 & $2.918 \mathrm{E}-03$ & $1.028 \mathrm{E}+03$ & 178.50 & $5.209 \mathrm{E}-01$ \\
\hline E08-SIM-3.0 & 3.00 & $5.393 \mathrm{E}-05$ & $5.563 \mathrm{E}+04$ & 1824.00 & $9.836 \mathrm{E}-02$ \\
\hline E08-SIM-3.0 & 3.00 & $5.568 \mathrm{E}-05$ & $5.388 \mathrm{E}+04$ & 1740.00 & $9.688 \mathrm{E}-02$ \\
\hline E08-SIM-3.0 & 3.00 & $5.054 \mathrm{E}-06$ & $5.936 \mathrm{E}+05$ & 1922.00 & $9.714 \mathrm{E}-03$ \\
\hline E08-SIM-3.0 & 3.00 & $5.165 \mathrm{E}-06$ & $5.808 \mathrm{E}+05$ & 1909.00 & $9.861 \mathrm{E}-03$ \\
\hline E08-SIM-3.0 & 3.00 & $5.512 \mathrm{E}-07$ & $5.443 \mathrm{E}+06$ & 1791.00 & $9.871 \mathrm{E}-04$ \\
\hline E08-SIM-3.0 & 3.00 & $5.376 \mathrm{E}-07$ & $5.580 \mathrm{E}+06$ & 1791.00 & $9.629 \mathrm{E}-04$ \\
\hline E08-SIM-5.0 & 5.00 & $9.625 \mathrm{E}-02$ & $5.195 \mathrm{E}+01$ & 6.67 & $6.422 \mathrm{E}-01$ \\
\hline E08-SIM-5.0 & 5.00 & $9.575 \mathrm{E}-02$ & $5.222 \mathrm{E}+01$ & 7.42 & $7.102 \mathrm{E}-01$ \\
\hline E08-SIM-5.0 & 5.00 & $6.780 \mathrm{E}-03$ & $7.375 \mathrm{E}+02$ & 77.63 & $5.263 \mathrm{E}-01$ \\
\hline E08-SIM-5.0 & 5.00 & $6.806 \mathrm{E}-03$ & $7.346 \mathrm{E}+02$ & 75.98 & $5.172 \mathrm{E}-01$ \\
\hline
\end{tabular}




\begin{tabular}{|c|c|c|c|c|c|}
\hline Sample Description & $\begin{array}{c}\text { Liquid Na }^{+} \\
{[\mathbf{M}]}\end{array}$ & $\begin{array}{c}\text { Liquid Cs }^{+} \\
{[\mathbf{M}]}\end{array}$ & $\begin{array}{c}\mathbf{L i q u i d ~ N a}^{+} / \mathbf{C s}^{+} \\
{[\mathbf{r a t i o}]}\end{array}$ & $\begin{array}{c}\mathbf{K}_{\mathbf{d}} \\
{[\mathbf{m l} / \mathbf{g}]}\end{array}$ & $\begin{array}{c}\mathbf{C s}^{+} \text {loading } \\
{[\mathbf{m m o l e / g} \mathbf{g} \mathbf{}]}\end{array}$ \\
\hline E08-SIM-5.0 & 5.00 & $1.612 \mathrm{E}-04$ & $3.102 \mathrm{E}+04$ & 867.30 & $1.398 \mathrm{E}-01$ \\
\hline E08-SIM-5.0 & 5.00 & $1.571 \mathrm{E}-04$ & $3.183 \mathrm{E}+04$ & 883.10 & $1.387 \mathrm{E}-01$ \\
\hline E08-SIM-5.0 & 5.00 & $1.417 \mathrm{E}-05$ & $3.528 \mathrm{E}+05$ & 1041.00 & $1.475 \mathrm{E}-02$ \\
\hline E08-SIM-5.0 & 5.00 & $1.454 \mathrm{E}-05$ & $3.438 \mathrm{E}+05$ & 1024.00 & $1.489 \mathrm{E}-02$ \\
\hline E08-SIM-7.0 & 7.00 & $1.368 \mathrm{E}-01$ & $5.118 \mathrm{E}+01$ & 3.91 & $5.345 \mathrm{E}-01$ \\
\hline E08-SIM-7.0 & 7.00 & $1.376 \mathrm{E}-01$ & $5.089 \mathrm{E}+01$ & 2.95 & $4.061 \mathrm{E}-01$ \\
\hline E08-SIM-7.0 & 7.00 & $1.103 \mathrm{E}-02$ & $6.347 \mathrm{E}+02$ & 44.94 & $4.956 \mathrm{E}-01$ \\
\hline E08-SIM-7.0 & 7.00 & $1.111 \mathrm{E}-02$ & $6.299 \mathrm{E}+02$ & 43.23 & $4.804 \mathrm{E}-01$ \\
\hline E08-SIM-7.0 & 7.00 & $3.302 \mathrm{E}-04$ & $2.120 \mathrm{E}+04$ & 553.20 & $1.827 \mathrm{E}-01$ \\
\hline E08-SIM-7.0 & 7.00 & $3.382 \mathrm{E}-04$ & $2.070 \mathrm{E}+04$ & 535.40 & $1.811 \mathrm{E}-01$ \\
\hline E08-SIM-7.0 & 7.00 & $2.510 \mathrm{E}-05$ & $2.789 \mathrm{E}+05$ & 791.80 & $1.987 \mathrm{E}-02$ \\
\hline E08-SIM-7.0 & 7.00 & $2.554 \mathrm{E}-05$ & $2.741 \mathrm{E}+05$ & 772.80 & $1.974 \mathrm{E}-02$ \\
\hline E08-SIM-7.0 & 7.00 & $2.647 \mathrm{E}-06$ & $2.645 \mathrm{E}+06$ & 728.70 & $1.929 \mathrm{E}-03$ \\
\hline E08-SIM-7.0 & 7.00 & $2.585 \mathrm{E}-06$ & $2.708 \mathrm{E}+06$ & 752.10 & $1.944 \mathrm{E}-03$ \\
\hline
\end{tabular}

${ }^{\mathrm{a}}$ The concentrations listed are equilibrium values at the final state of the liquid solution.

Table F-6. ZAM predictions for cesium on CST powder (IE-910) based on simulated batch contact tests performed at $25 \mathrm{C}$ in $\mathrm{AW}-101$ solutions. $^{\mathrm{a}}$

\begin{tabular}{|c|c|c|c|c|c|}
\hline Sample Description & $\begin{array}{c}\text { Liquid Na }^{+} \\
{[\mathbf{M}]}\end{array}$ & $\begin{array}{c}\text { Liquid Cs }^{+} \\
{[\mathbf{M}]}\end{array}$ & $\begin{array}{c}\text { Liquid Na }^{+} / \mathbf{C s}^{+} \\
{[\mathbf{r a t i o}]}\end{array}$ & $\begin{array}{c}\mathbf{K}_{\mathbf{d}} \\
{[\mathbf{m l} / \mathbf{g}]}\end{array}$ & $\begin{array}{c}\mathbf{C s}^{+} \text {loading } \\
{[\mathbf{m m o l e} / \mathbf{g} \text { csT }]}\end{array}$ \\
\hline \hline POW-ZAM-0.2 & 0.20 & $4.330 \mathrm{E}-09$ & $4.619 \mathrm{E}+07$ & 51400.00 & $2.226 \mathrm{E}-04$ \\
\hline POW-ZAM-0.2 & 0.20 & $4.350 \mathrm{E}-08$ & $4.598 \mathrm{E}+06$ & 51300.00 & $2.232 \mathrm{E}-03$ \\
\hline POW-ZAM-0.2 & 0.20 & $2.210 \mathrm{E}-07$ & $9.050 \mathrm{E}+05$ & 50500.00 & $1.116 \mathrm{E}-02$ \\
\hline POW-ZAM-0.2 & 0.20 & $4.510 \mathrm{E}-07$ & $4.435 \mathrm{E}+05$ & 49500.00 & $2.232 \mathrm{E}-02$ \\
\hline POW-ZAM-0.2 & 0.20 & $2.690 \mathrm{E}-06$ & $7.435 \mathrm{E}+04$ & 41500.00 & $1.116 \mathrm{E}-01$ \\
\hline POW-ZAM-0.2 & 0.20 & $7.040 \mathrm{E}-06$ & $2.841 \mathrm{E}+04$ & 31600.00 & $2.225 \mathrm{E}-01$ \\
\hline POW-ZAM-0.2 & 0.20 & $3.570 \mathrm{E}-05$ & $5.602 \mathrm{E}+03$ & 12300.00 & $4.391 \mathrm{E}-01$ \\
\hline POW-ZAM-0.2 & 0.20 & $1.260 \mathrm{E}-04$ & $1.587 \mathrm{E}+03$ & 4240.00 & $5.342 \mathrm{E}-01$ \\
\hline POW-ZAM-0.2 & 0.20 & $4.710 \mathrm{E}-04$ & $4.246 \mathrm{E}+02$ & 1200.00 & $5.652 \mathrm{E}-01$ \\
\hline POW-ZAM-0.2 & 0.20 & $2.420 \mathrm{E}-03$ & $8.264 \mathrm{E}+01$ & 238.00 & $5.760 \mathrm{E}-01$ \\
\hline POW-ZAM-1.0 & 1.00 & $2.280 \mathrm{E}-08$ & $4.386 \mathrm{E}+07$ & 9600.00 & $2.189 \mathrm{E}-04$ \\
\hline POW-ZAM-1.0 & 1.00 & $2.290 \mathrm{E}-07$ & $4.367 \mathrm{E}+06$ & 9570.00 & $2.192 \mathrm{E}-03$ \\
\hline POW-ZAM-1.0 & 1.00 & $1.160 \mathrm{E}-06$ & $8.621 \mathrm{E}+05$ & 9420.00 & $1.093 \mathrm{E}-02$ \\
\hline POW-ZAM-1.0 & 1.00 & $2.360 \mathrm{E}-06$ & $4.237 \mathrm{E}+05$ & 9240.00 & $2.181 \mathrm{E}-02$ \\
\hline POW-ZAM-1.0 & 1.00 & $1.400 \mathrm{E}-05$ & $7.143 \mathrm{E}+04$ & 7800.00 & $1.092 \mathrm{E}-01$ \\
\hline POW-ZAM-1.0 & 1.00 & $3.580 \mathrm{E}-05$ & $2.793 \mathrm{E}+04$ & 6020.00 & $2.155 \mathrm{E}-01$ \\
\hline POW-ZAM-1.0 & 1.00 & $1.510 \mathrm{E}-04$ & $6.623 \mathrm{E}+03$ & 2740.00 & $4.137 \mathrm{E}-01$ \\
\hline POW-ZAM-1.0 & 1.00 & $6.350 \mathrm{E}-04$ & $1.575 \mathrm{E}+03$ & 834.00 & $5.296 \mathrm{E}-01$ \\
\hline POW-ZAM-1.0 & 1.00 & $2.470 \mathrm{E}-03$ & $4.049 \mathrm{E}+02$ & 229.00 & $5.656 \mathrm{E}-01$ \\
\hline POW-ZAM-1.0 & 1.00 & $7.430 \mathrm{E}-03$ & $1.346 \mathrm{E}+02$ & 77.40 & $5.751 \mathrm{E}-01$ \\
\hline POW-ZAM-1.0 & 1.00 & $2.740 \mathrm{E}-02$ & $3.650 \mathrm{E}+01$ & 21.10 & $5.781 \mathrm{E}-01$ \\
\hline POW-ZAM-1.0 & 1.00 & $4.740 \mathrm{E}-02$ & $2.110 \mathrm{E}+01$ & 12.20 & $5.783 \mathrm{E}-01$ \\
\hline POW-ZAM-1.0 & 1.00 & $9.740 \mathrm{E}-02$ & $1.027 \mathrm{E}+01$ & 5.95 & $5.795 \mathrm{E}-01$ \\
\hline POW-ZAM-3.0 & 3.00 & $7.440 \mathrm{E}-08$ & $4.032 \mathrm{E}+07$ & 2790.00 & $2.076 \mathrm{E}-04$ \\
\hline
\end{tabular}


WESTINGHOUSE SAVANNAH RIVER COMPANY

Preliminary Ion Exchange Modeling for Removal of Cesium from

Hanford Waste Using Hydrous Crystalline Silicotitanate Material
Report:

WSRC-TR-2001-00400

Revision (Date):

$0(07 / 05 / 02)$

Page:

\begin{tabular}{|c|c|c|c|c|c|}
\hline Sample Description & $\begin{array}{c}\text { Liquid } \mathrm{Na}^{+} \\
{[\mathrm{M}]}\end{array}$ & $\begin{array}{l}\text { Liquid } \mathrm{Cs}^{+} \\
{[\mathrm{M}]}\end{array}$ & $\underset{\text { [ratio] }}{\text { Liquid } \mathrm{Na}^{+} / \mathrm{Cs}^{+}}$ & $\begin{array}{c}\mathbf{K}_{\mathbf{d}} \\
{[\mathrm{ml} / \mathbf{g}]}\end{array}$ & $\begin{array}{c}\mathrm{Cs}^{+} \text {loading } \\
{\left[\text { mmole/g/g } \text { g }_{\text {CST }}\right]}\end{array}$ \\
\hline POW-ZAM-3.0 & 3.00 & $7.460 \mathrm{E}-07$ & $4.021 \mathrm{E}+06$ & 2780.00 & $2.074 \mathrm{E}-03$ \\
\hline POW-ZAM-3.0 & 3.00 & $3.780 \mathrm{E}-06$ & $7.937 \mathrm{E}+05$ & 2740.00 & $1.036 \mathrm{E}-02$ \\
\hline POW-ZAM-3.0 & 3.00 & $7.690 \mathrm{E}-06$ & $3.901 \mathrm{E}+05$ & 2690.00 & $2.069 \mathrm{E}-02$ \\
\hline POW-ZAM-3.0 & 3.00 & $4.440 \mathrm{E}-05$ & $6.757 \mathrm{E}+04$ & 2300.00 & $1.021 \mathrm{E}-01$ \\
\hline POW-ZAM-3.0 & 3.00 & $1.090 \mathrm{E}-04$ & $2.752 \mathrm{E}+04$ & 1830.00 & $1.995 \mathrm{E}-01$ \\
\hline POW-ZAM-3.0 & 3.00 & $3.600 \mathrm{E}-04$ & $8.333 \mathrm{E}+03$ & 1020.00 & $3.672 \mathrm{E}-01$ \\
\hline POW-ZAM-3.0 & 3.00 & $8.970 \mathrm{E}-04$ & $3.344 \mathrm{E}+03$ & 524.00 & $4.700 \mathrm{E}-01$ \\
\hline POW-ZAM-3.0 & 3.00 & $2.600 \mathrm{E}-03$ & $1.154 \mathrm{E}+03$ & 206.00 & $5.356 \mathrm{E}-01$ \\
\hline POW-ZAM-3.0 & 3.00 & $7.480 \mathrm{E}-03$ & $4.011 \mathrm{E}+02$ & 75.40 & $5.640 \mathrm{E}-01$ \\
\hline POW-ZAM-3.0 & 3.00 & $2.740 \mathrm{E}-02$ & $1.095 \mathrm{E}+02$ & 21.00 & 5.754E-01 \\
\hline POW-ZAM-3.0 & 3.00 & $4.740 \mathrm{E}-02$ & $6.329 \mathrm{E}+01$ & 12.20 & $5.783 \mathrm{E}-01$ \\
\hline POW-ZAM-3.0 & 3.00 & $9.740 \mathrm{E}-02$ & $3.080 \mathrm{E}+01$ & 5.94 & $5.786 \mathrm{E}-01$ \\
\hline POW-ZAM-5.0 & 5.00 & $1.390 \mathrm{E}-07$ & $3.597 \mathrm{E}+07$ & 1390.00 & $1.932 \mathrm{E}-04$ \\
\hline POW-ZAM-5.0 & 5.00 & $1.390 \mathrm{E}-06$ & $3.597 \mathrm{E}+06$ & 1390.00 & $1.932 \mathrm{E}-03$ \\
\hline POW-ZAM-5.0 & 5.00 & $7.030 \mathrm{E}-06$ & $7.112 \mathrm{E}+05$ & 1370.00 & $9.631 \mathrm{E}-03$ \\
\hline POW-ZAM-5.0 & 5.00 & $1.430 \mathrm{E}-05$ & $3.497 \mathrm{E}+05$ & 1350.00 & $1.931 \mathrm{E}-02$ \\
\hline POW-ZAM-5.0 & 5.00 & $8.060 \mathrm{E}-05$ & $6.203 \mathrm{E}+04$ & 1170.00 & $9.430 \mathrm{E}-02$ \\
\hline POW-ZAM-5.0 & 5.00 & $1.900 \mathrm{E}-04$ & $2.632 \mathrm{E}+04$ & 956.00 & $1.816 \mathrm{E}-01$ \\
\hline POW-ZAM-5.0 & 5.00 & $5.390 \mathrm{E}-04$ & $9.276 \mathrm{E}+03$ & 606.00 & $3.266 \mathrm{E}-01$ \\
\hline POW-ZAM-5.0 & 5.00 & $1.120 \mathrm{E}-03$ & $4.464 \mathrm{E}+03$ & 378.00 & 4.234E-01 \\
\hline POW-ZAM-5.0 & 5.00 & $2.750 \mathrm{E}-03$ & $1.818 \mathrm{E}+03$ & 183.00 & $5.033 \mathrm{E}-01$ \\
\hline POW-ZAM-5.0 & 5.00 & $7.550 \mathrm{E}-03$ & $6.623 \mathrm{E}+02$ & 72.80 & $5.496 \mathrm{E}-01$ \\
\hline POW-ZAM-5.0 & 5.00 & $2.740 \mathrm{E}-02$ & $1.825 \mathrm{E}+02$ & 20.80 & $5.699 \mathrm{E}-01$ \\
\hline POW-ZAM-5.0 & 5.00 & $4.740 \mathrm{E}-02$ & $1.055 \mathrm{E}+02$ & 12.10 & $5.735 \mathrm{E}-01$ \\
\hline POW-ZAM-5.0 & 5.00 & $9.740 \mathrm{E}-02$ & $5.133 \mathrm{E}+01$ & 5.93 & $5.776 \mathrm{E}-01$ \\
\hline POW-ZAM-7.0 & 7.00 & $1.860 \mathrm{E}-07$ & $3.763 \mathrm{E}+07$ & 979.00 & $1.821 \mathrm{E}-04$ \\
\hline POW-ZAM-7.0 & 7.00 & $1.870 \mathrm{E}-06$ & $3.743 \mathrm{E}+06$ & 976.00 & $1.825 \mathrm{E}-03$ \\
\hline POW-ZAM-7.0 & 7.00 & $9.430 \mathrm{E}-06$ & $7.423 \mathrm{E}+05$ & 964.00 & $9.091 \mathrm{E}-03$ \\
\hline POW-ZAM-7.0 & 7.00 & $1.910 \mathrm{E}-05$ & $3.665 \mathrm{E}+05$ & 948.00 & $1.811 \mathrm{E}-02$ \\
\hline POW-ZAM-7.0 & 7.00 & $1.060 \mathrm{E}-04$ & $6.604 \mathrm{E}+04$ & 830.00 & $8.798 \mathrm{E}-02$ \\
\hline POW-ZAM-7.0 & 7.00 & $2.440 \mathrm{E}-04$ & $2.869 \mathrm{E}+04$ & 693.00 & $1.691 \mathrm{E}-01$ \\
\hline POW-ZAM-7.0 & 7.00 & $6.480 \mathrm{E}-04$ & $1.080 \mathrm{E}+04$ & 468.00 & $3.033 \mathrm{E}-01$ \\
\hline POW-ZAM-7.0 & 7.00 & $1.250 \mathrm{E}-03$ & $5.600 \mathrm{E}+03$ & 315.00 & $3.938 \mathrm{E}-01$ \\
\hline POW-ZAM-7.0 & 7.00 & $2.860 \mathrm{E}-03$ & $2.448 \mathrm{E}+03$ & 168.00 & $4.805 \mathrm{E}-01$ \\
\hline POW-ZAM-7.0 & 7.00 & $7.600 \mathrm{E}-03$ & $9.211 \mathrm{E}+02$ & 70.80 & $5.381 \mathrm{E}-01$ \\
\hline POW-ZAM-7.0 & 7.00 & $2.750 \mathrm{E}-02$ & $2.545 \mathrm{E}+02$ & 20.70 & $5.693 \mathrm{E}-01$ \\
\hline POW-ZAM-7.0 & 7.00 & $4.740 \mathrm{E}-02$ & $1.477 \mathrm{E}+02$ & 12.10 & $5.735 \mathrm{E}-01$ \\
\hline POW-ZAM-7.0 & 7.00 & $9.740 \mathrm{E}-02$ & $7.187 \mathrm{E}+01$ & 5.92 & $5.766 \mathrm{E}-01$ \\
\hline
\end{tabular}

${ }^{\mathrm{a}}$ The concentrations listed are equilibrium values at the final state of the liquid solution. 
Table F-7. AW-101 simulant and actual solution compositions used to perform ZAM predictions for cesium on CST powder (IE-910) at $25 \mathrm{C}^{\text {a }}$

\begin{tabular}{|c|c|c|c|c|c|c|c|}
\hline $\begin{array}{c}\text { Ion } \\
\text { Category }\end{array}$ & Species & $\begin{array}{c}\text { ZAM } \\
\text { ID }\end{array}$ & $\begin{array}{c}0.2 \mathrm{M} \mathrm{Na}^{+} \\
\text {Solution }\end{array}$ & $\begin{array}{l}1 \mathrm{M} \mathrm{Na}^{+} \\
\text {Solution }\end{array}$ & $\begin{array}{l}3 \mathrm{M} \mathrm{Na}^{+} \\
\text {Solution }\end{array}$ & $\begin{array}{l}5 \mathrm{M} \mathrm{Na}^{+} \\
\text {Solution }\end{array}$ & $\begin{array}{l}7 \mathrm{M} \mathrm{Na}^{+} \\
\text {Solution }\end{array}$ \\
\hline \multirow[t]{7}{*}{ Cations } & $\mathrm{Na}^{+}$ & 3 & $2.000 \mathrm{E}-01$ & $1.000 \mathrm{E}+00$ & $3.000 \mathrm{E}+00$ & $5.000 \mathrm{E}+00$ & $7.000 \mathrm{E}+00$ \\
\hline & $\mathrm{K}^{+}$ & 4 & $1.900 \mathrm{E}-02$ & $9.500 \mathrm{E}-02$ & $2.850 \mathrm{E}-01$ & $4.750 \mathrm{E}-01$ & $6.650 \mathrm{E}-01$ \\
\hline & $\mathrm{Cs}^{+}$(b) & 6 & varies & varies & Varies & varies & varies \\
\hline & $\mathrm{H}^{+}$ & 1 & $6.340 \mathrm{E}-17$ & $3.170 \mathrm{E}-16$ & $9.510 \mathrm{E}-16$ & $1.585 \mathrm{E}-15$ & $2.219 \mathrm{E}-15$ \\
\hline & $\mathrm{Ba}^{2+}$ & 14 & $2.364 \mathrm{E}-08$ & $1.182 \mathrm{E}-07$ & $3.546 \mathrm{E}-07$ & $5.910 \mathrm{E}-07$ & $8.274 \mathrm{E}-07$ \\
\hline & $\mathrm{Ca}^{2+}$ & 12 & $9.640 \mathrm{E}-06$ & $4.820 \mathrm{E}-05$ & $1.446 \mathrm{E}-04$ & $2.410 \mathrm{E}-04$ & $3.374 \mathrm{E}-04$ \\
\hline & $\mathrm{Sr}^{2+}$ & 13 & $2.856 \mathrm{E}-09$ & $1.428 \mathrm{E}-08$ & $4.284 \mathrm{E}-08$ & $7.140 \mathrm{E}-08$ & $9.996 \mathrm{E}-08$ \\
\hline \multirow[t]{9}{*}{ Anions } & $\mathrm{OH}^{-}$(free) & 13 & $8.400 \mathrm{E}-02$ & 4.200E-01 & $1.260 \mathrm{E}+00$ & $2.100 \mathrm{E}+00$ & $2.940 \mathrm{E}+00$ \\
\hline & $\mathrm{Cl}^{-(\mathrm{b})}$ & 2 & $\begin{array}{l}2.600 \mathrm{E}-03 \\
+ \text { Cs conc }\end{array}$ & $\begin{array}{l}1.300 \mathrm{E}-02 \\
+ \text { Cs conc }\end{array}$ & $\begin{array}{l}3.900 \mathrm{E}-02 \\
+ \text { Cs conc }\end{array}$ & $\begin{array}{l}6.500 \mathrm{E}-02 \\
+ \text { Cs conc }\end{array}$ & $\begin{array}{l}9.100 \mathrm{E}-02 \\
+ \text { Cs conc }\end{array}$ \\
\hline & $\mathrm{F}^{-}$ & 1 & $1.732 \mathrm{E}-03$ & $8.660 \mathrm{E}-03$ & $2.598 \mathrm{E}-02$ & $4.330 \mathrm{E}-02$ & $6.062 \mathrm{E}-02$ \\
\hline & $\mathrm{NO}_{2}^{-}$ & 27 & $3.760 \mathrm{E}-02$ & $1.880 \mathrm{E}-01$ & $5.640 \mathrm{E}-01$ & $9.400 \mathrm{E}-01$ & $1.316 \mathrm{E}+00$ \\
\hline & $\mathrm{NO}_{3}^{-}$ & 9 & $5.960 \mathrm{E}-02$ & $2.980 \mathrm{E}-01$ & $8.940 \mathrm{E}-01$ & $1.490 \mathrm{E}+00$ & $2.086 \mathrm{E}+00$ \\
\hline & $\mathrm{Al}(\mathrm{OH})_{4}^{-}$ & 28 & $1.988 \mathrm{E}-02$ & $9.940 \mathrm{E}-02$ & $2.982 \mathrm{E}-01$ & $4.970 \mathrm{E}-01$ & $6.958 \mathrm{E}-01$ \\
\hline & $\mathrm{SO}_{4}^{2-}$ & 15 & $5.040 \mathrm{E}-04$ & $2.520 \mathrm{E}-03$ & $7.560 \mathrm{E}-03$ & $1.260 \mathrm{E}-02$ & $1.764 \mathrm{E}-02$ \\
\hline & $\mathrm{CO}_{3}{ }^{2-}$ & 19 & $5.600 \mathrm{E}-03$ & $2.800 \mathrm{E}-02$ & $8.400 \mathrm{E}-02$ & $1.400 \mathrm{E}-01$ & $1.960 \mathrm{E}-01$ \\
\hline & $\mathrm{HPO}_{4}{ }^{2-}$ & 17 & $7.000 \mathrm{E}-04$ & $3.500 \mathrm{E}-03$ & $1.050 \mathrm{E}-02$ & $1.750 \mathrm{E}-02$ & $2.450 \mathrm{E}-02$ \\
\hline
\end{tabular}

${ }^{\mathrm{a}}$ The species concentrations for simulants at sodium concentrations other than $5 \mathrm{M}$ can be obtained by using a diluting factor (e.g., for a $2 \mathrm{M}$ sodium case multiply the $5 \mathrm{M}$ sodium simulant species concentrations by $2 / 5$ ).

${ }^{\mathrm{b}}$ Cesium and chlorine concentrations are varied in order to generate isotherm database over a wide cesium concentration range (i.e., $1 \times 10^{-6} \mathrm{M}$ to $0.1 \mathrm{M}$ ) where $\mathrm{CsCl}$ is added to maintain an overall charge balance. 


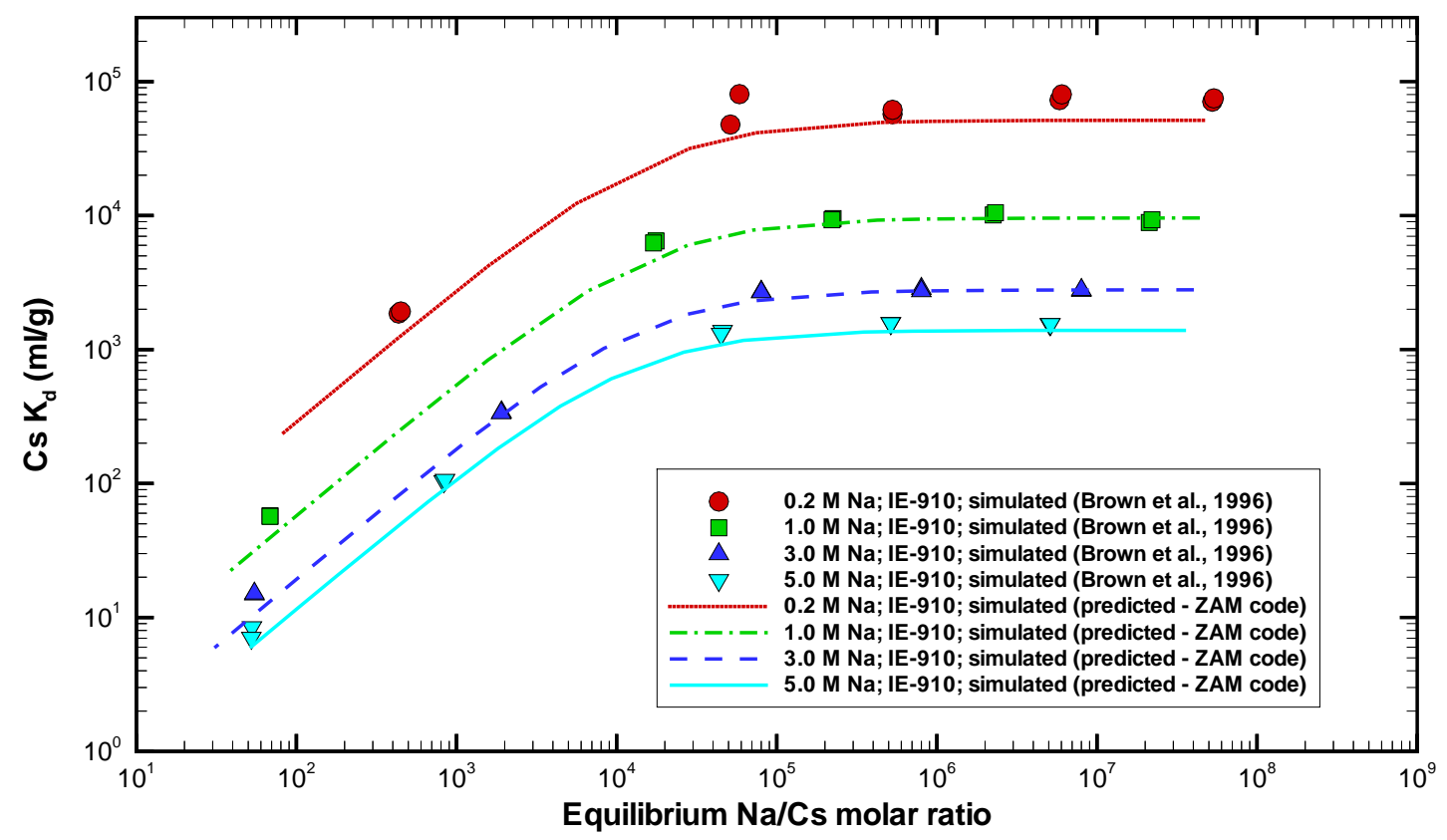

Figure F-1. A comparison of measured versus ZAM predicted cesium $\mathrm{K}_{\mathrm{d}}$ values for simulated AW-101 feed in contact with CST in its power-form (IE-910) over a range of sodium concentration levels [data by Brown et al. (1996)].

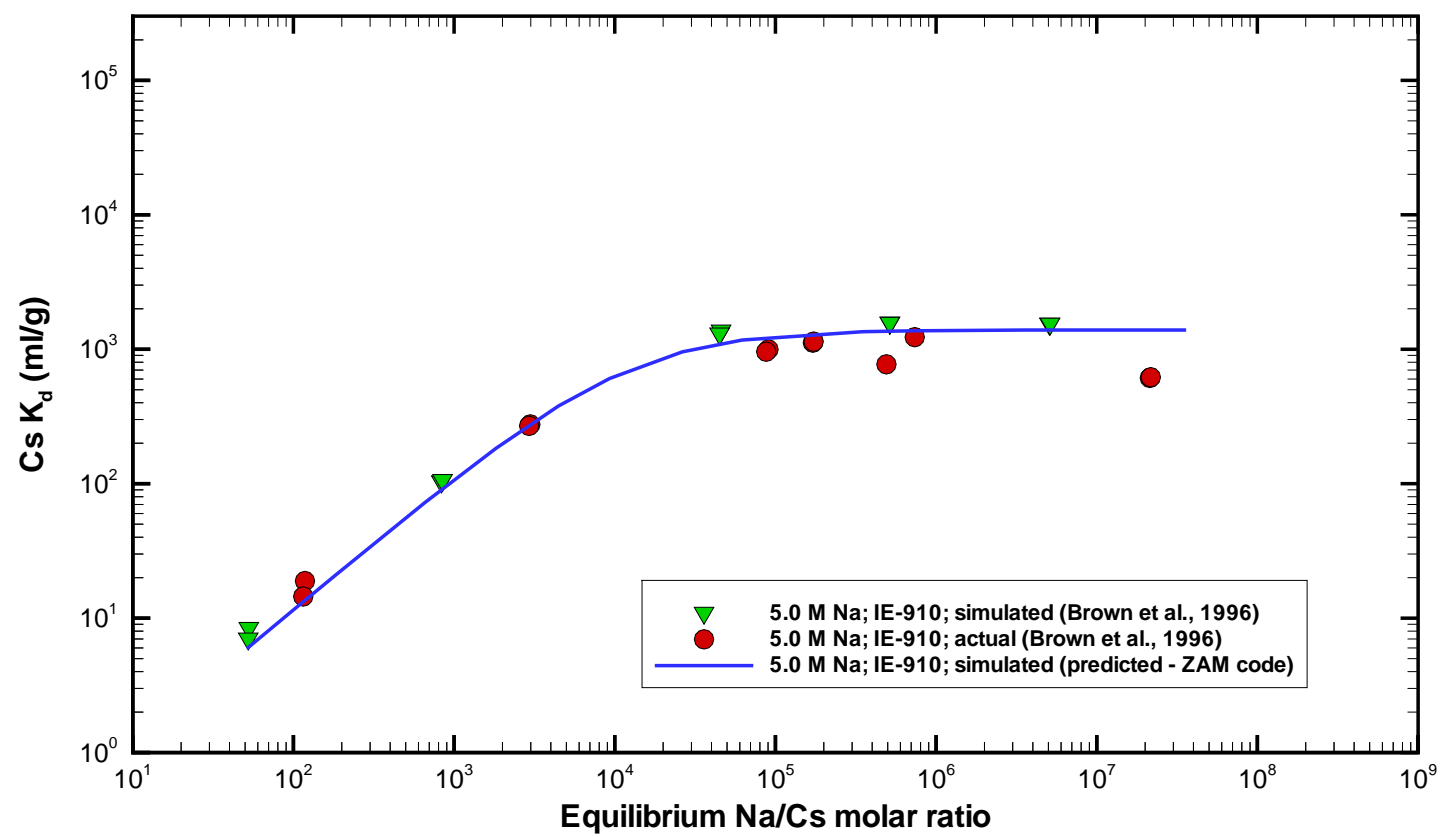

Figure F-2. A comparison of measured versus ZAM predicted cesium $\mathrm{K}_{\mathrm{d}}$ values for $5 \mathrm{M}$ sodium simulated and actual AW-101 feeds in contact with CST in its power-form (IE-910) [data by Brown et al. (1996)]. 


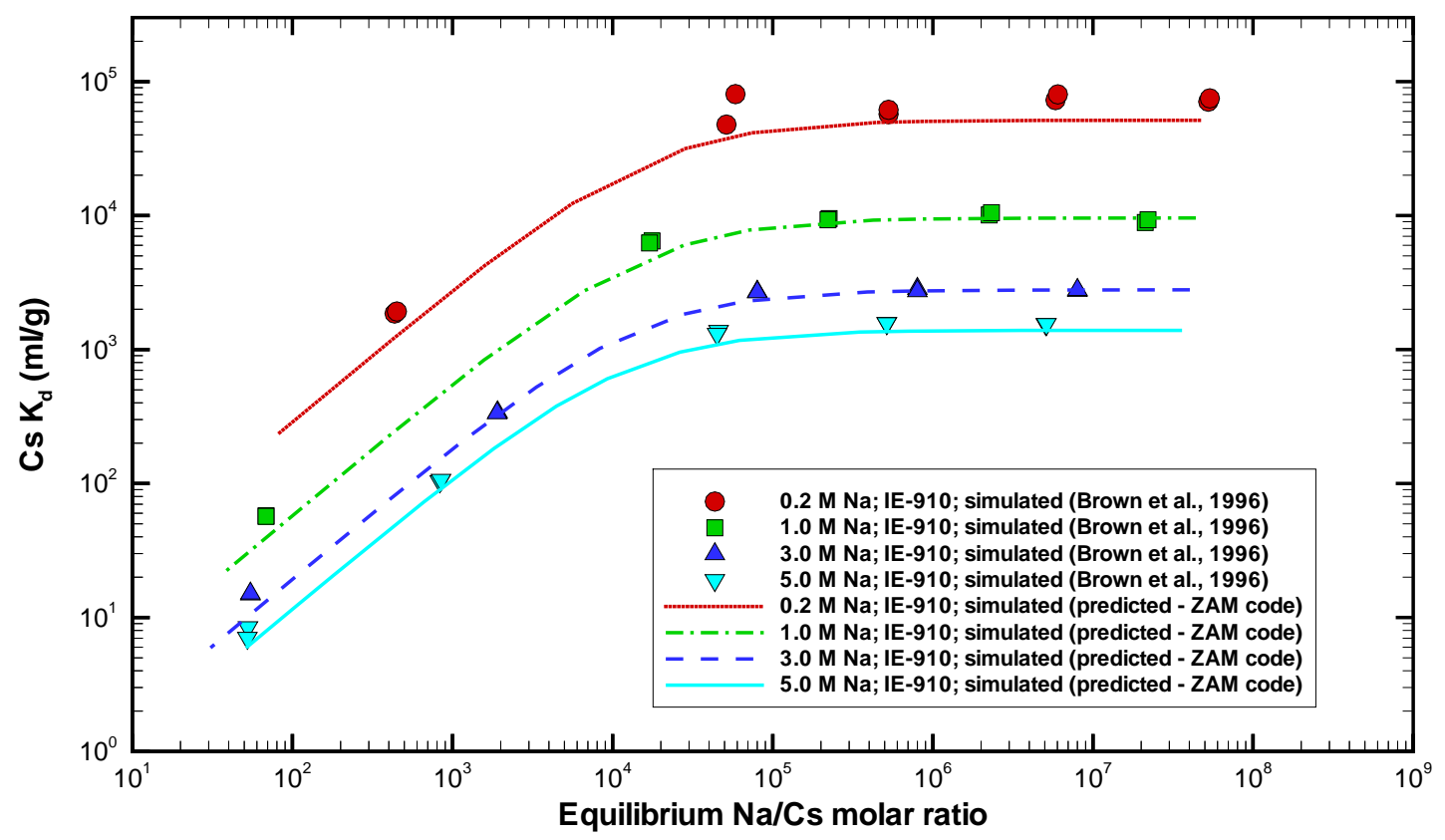

Figure F-3. A comparison of measured versus ZAM predicted cesium $\mathrm{K}_{\mathrm{d}}$ values for simulated AW-101 feed in contact with CST in one of its engineered-forms referred to as "08" (IE-911) over a range of sodium concentration levels [data by Brown et al. (1996)].

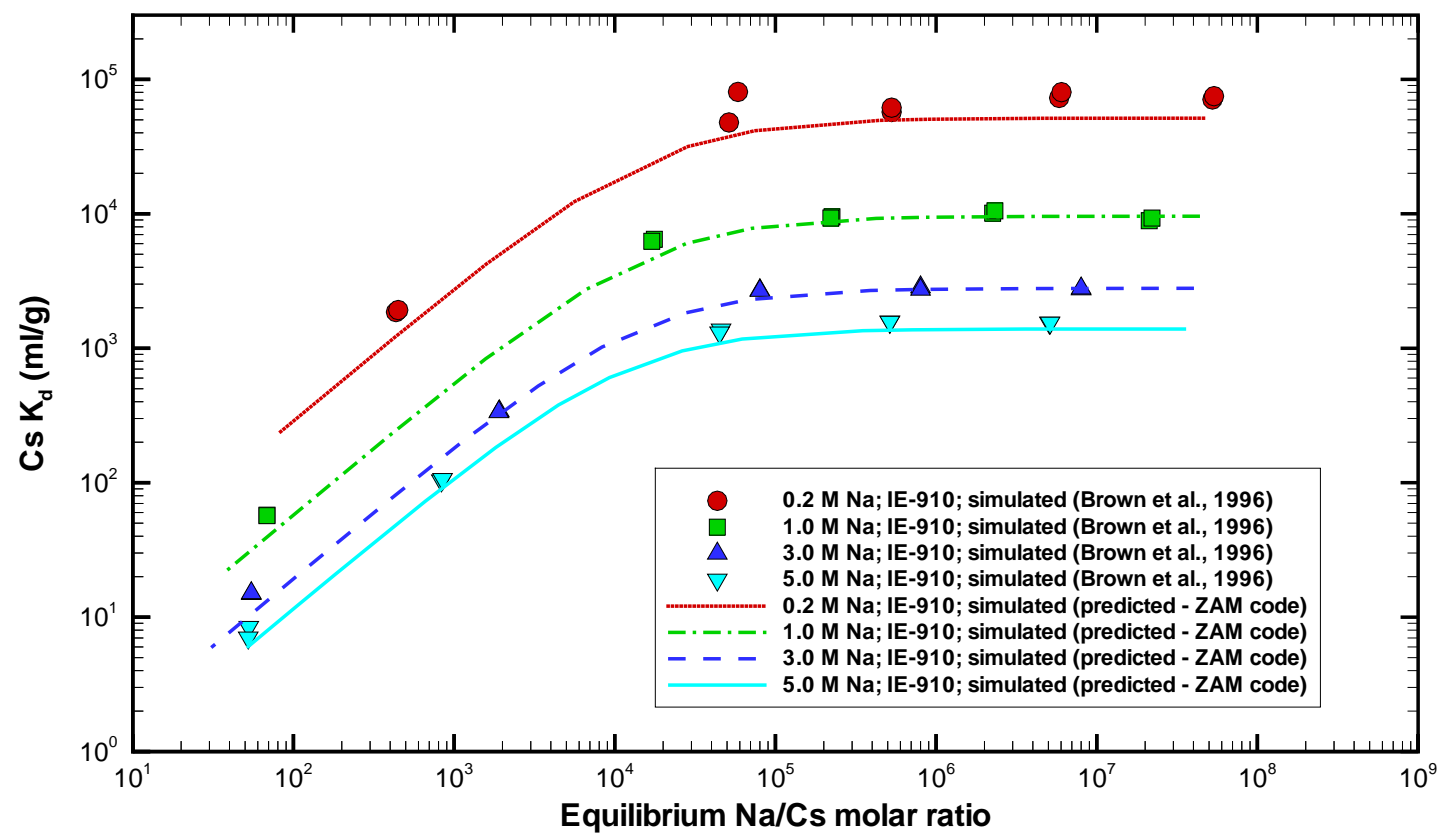

Figure F-4. A comparison of measured versus ZAM predicted cesium $\mathrm{K}_{\mathrm{d}}$ values for simulated AW-101 feed in contact with CST in one of its engineered-forms referred to as " $38 \mathrm{~b}$ " (IE-911) over a range of sodium concentration levels [data by Brown et al. (1996)]. 


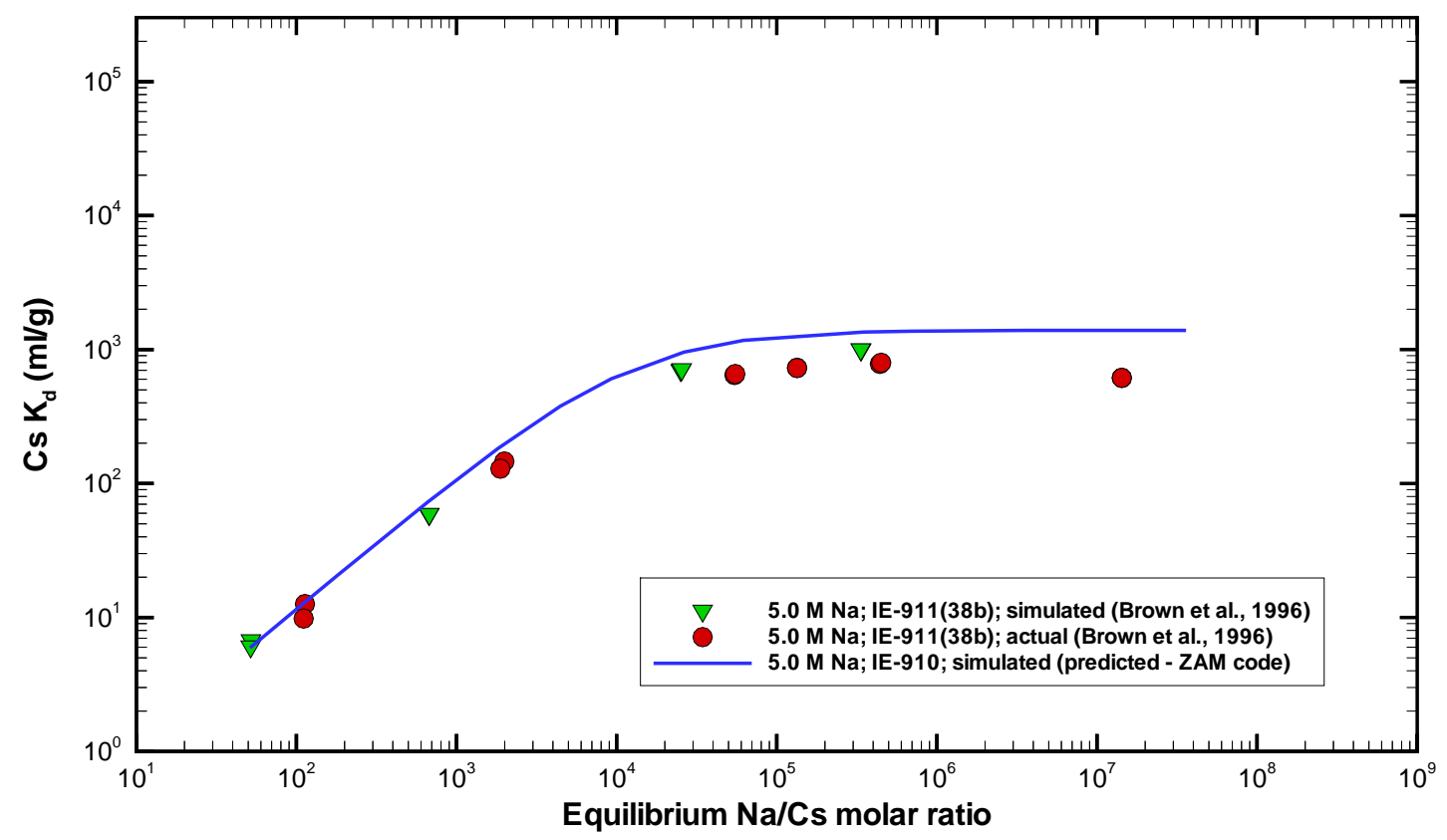

Figure F-5. A comparison of measured versus ZAM predicted cesium $\mathrm{K}_{\mathrm{d}}$ values for $5 \mathrm{M}$ sodium simulated and actual AW-101 feeds in contact with CST in one of its engineered-forms referred to as "038b" (IE-911) [data by Brown et al. (1996)].

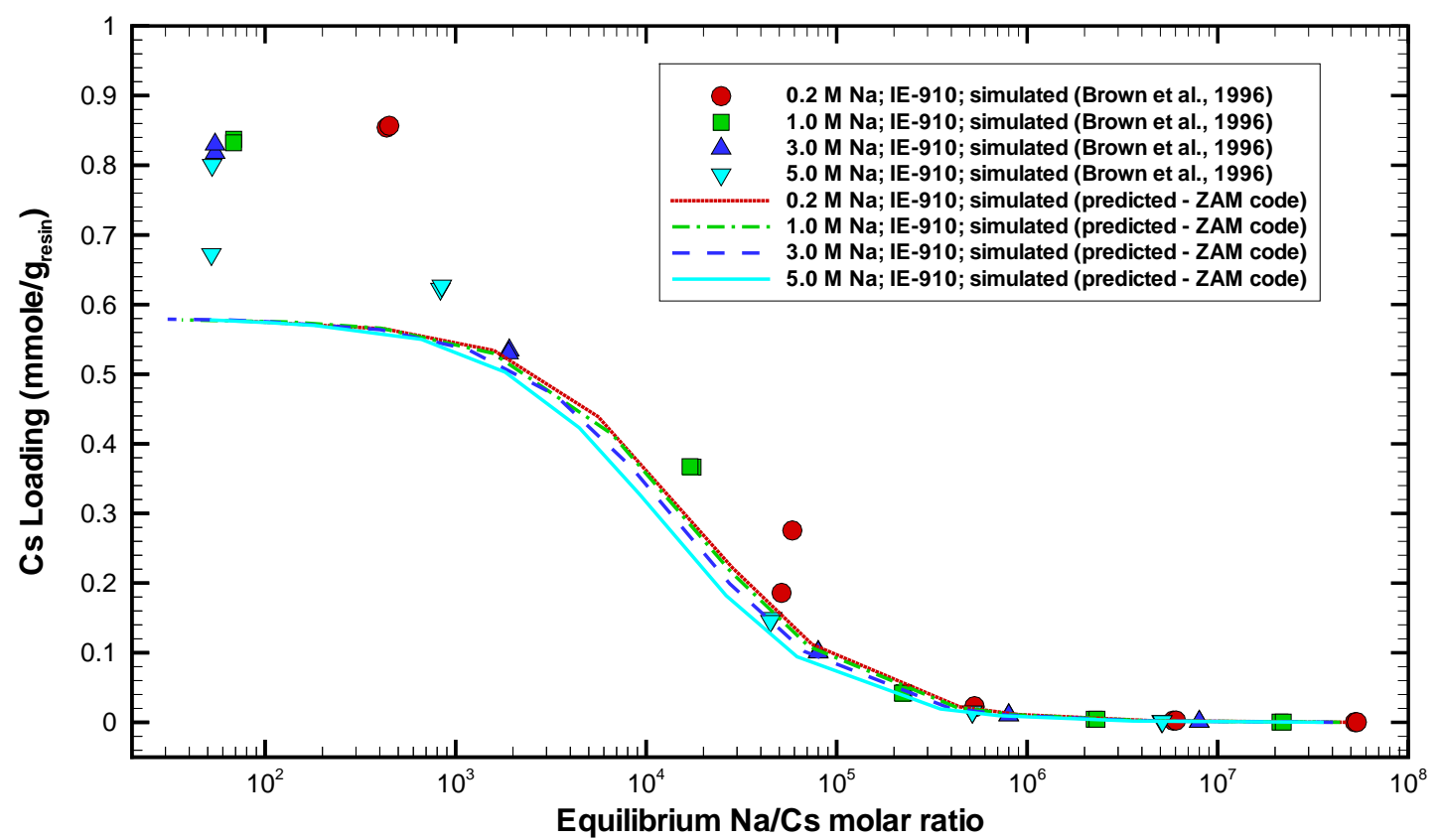

Figure F-6. A comparison of measured versus ZAM predicted cesium loadings for simulated AW-101 feed in contact with CST in its power-form (IE-910) over a range of sodium concentration levels [data by Brown et al. (1996)]. 


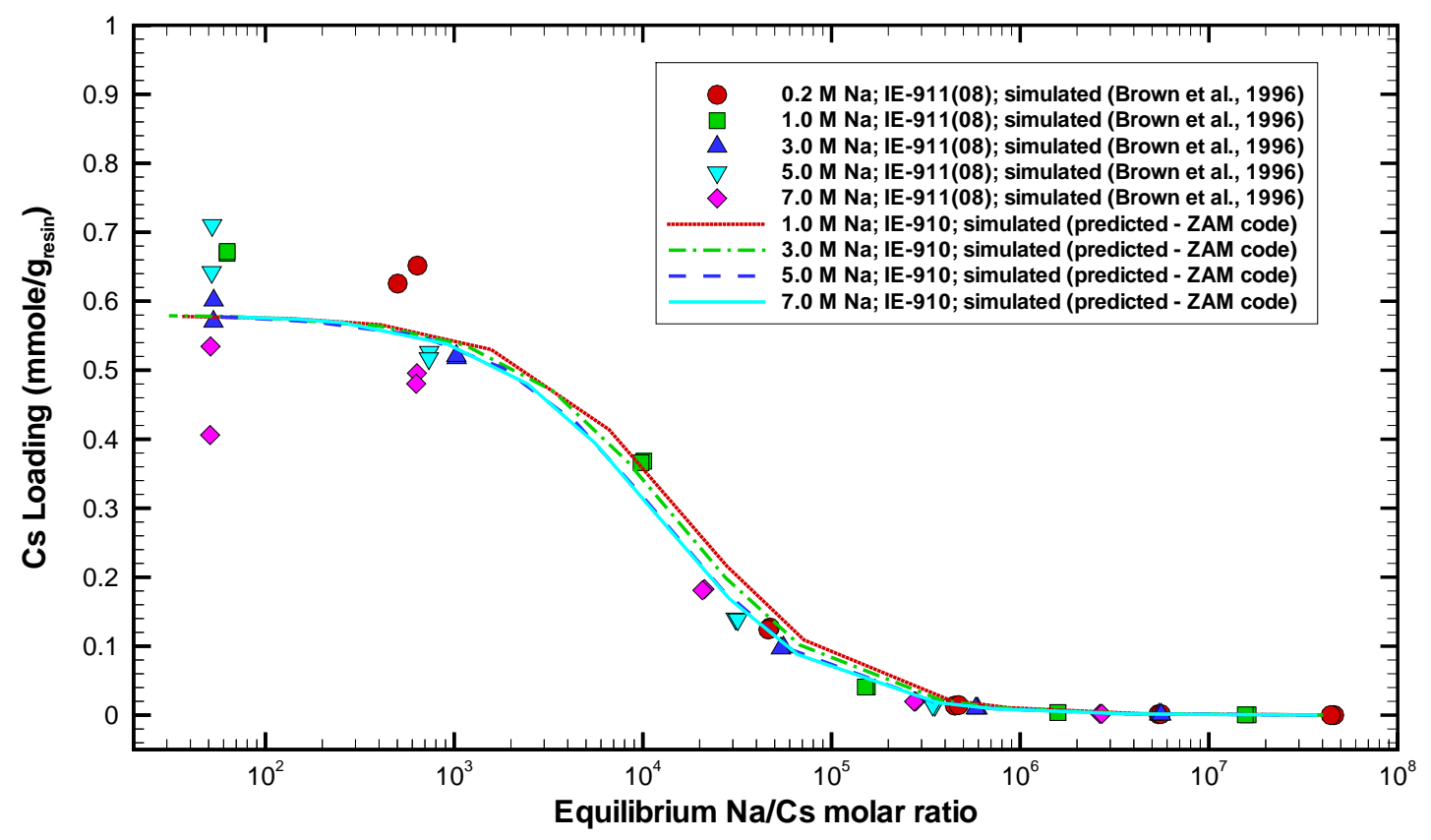

Figure F-7. A comparison of measured versus ZAM predicted cesium loadings for simulated AW-101 feed in contact with CST in one of its engineered-forms referred to as "08" (IE-911) over a range of sodium concentration levels [data by Brown et al. (1996)].

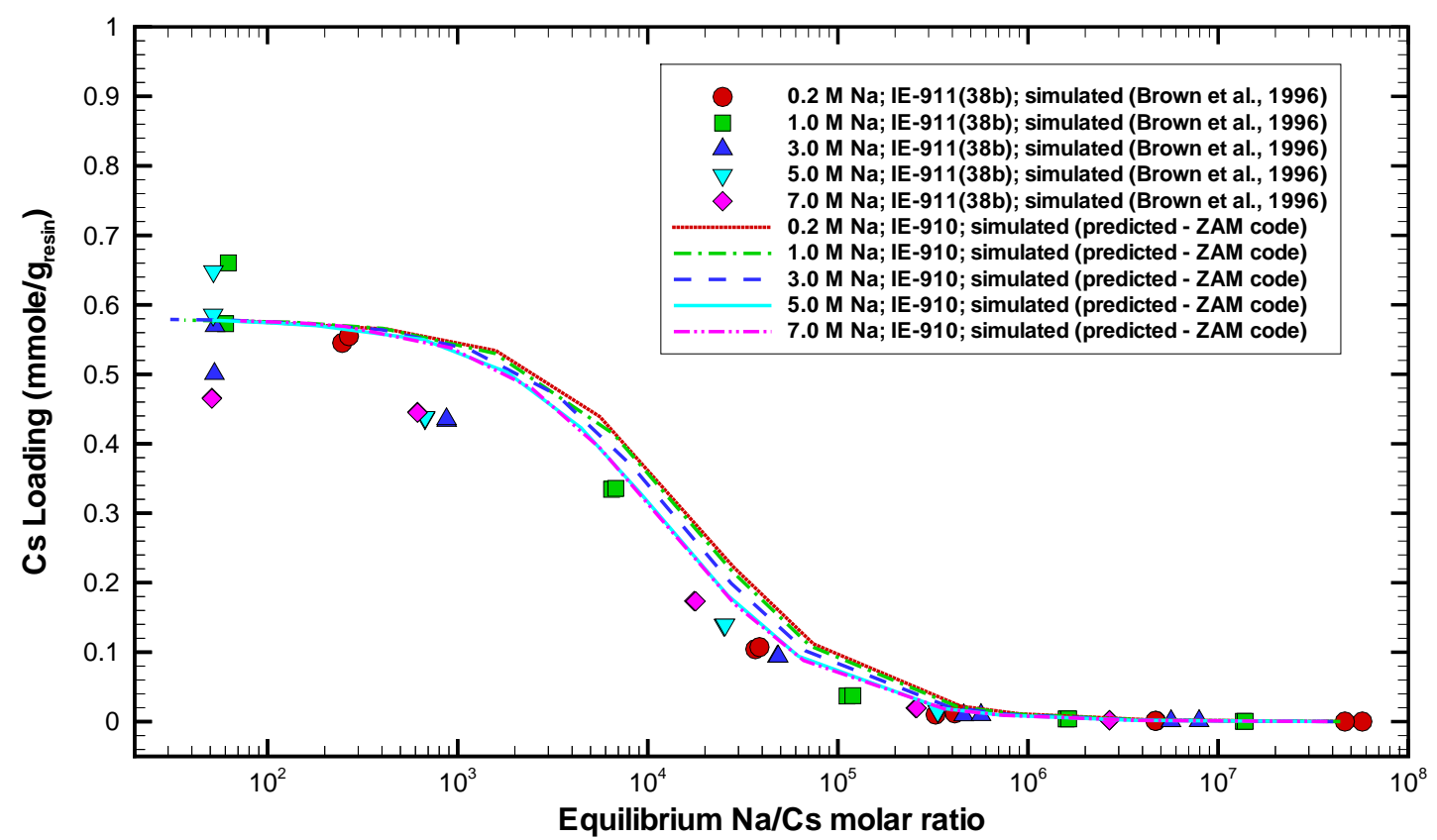

Figure F-8. A comparison of measured versus ZAM predicted cesium loadings for simulated AW-101 feed in contact with CST in one of its engineered-forms referred to as " $38 \mathrm{~b}$ " (IE-911) over a range of sodium concentration levels [data by Brown et al. (1996)]. 


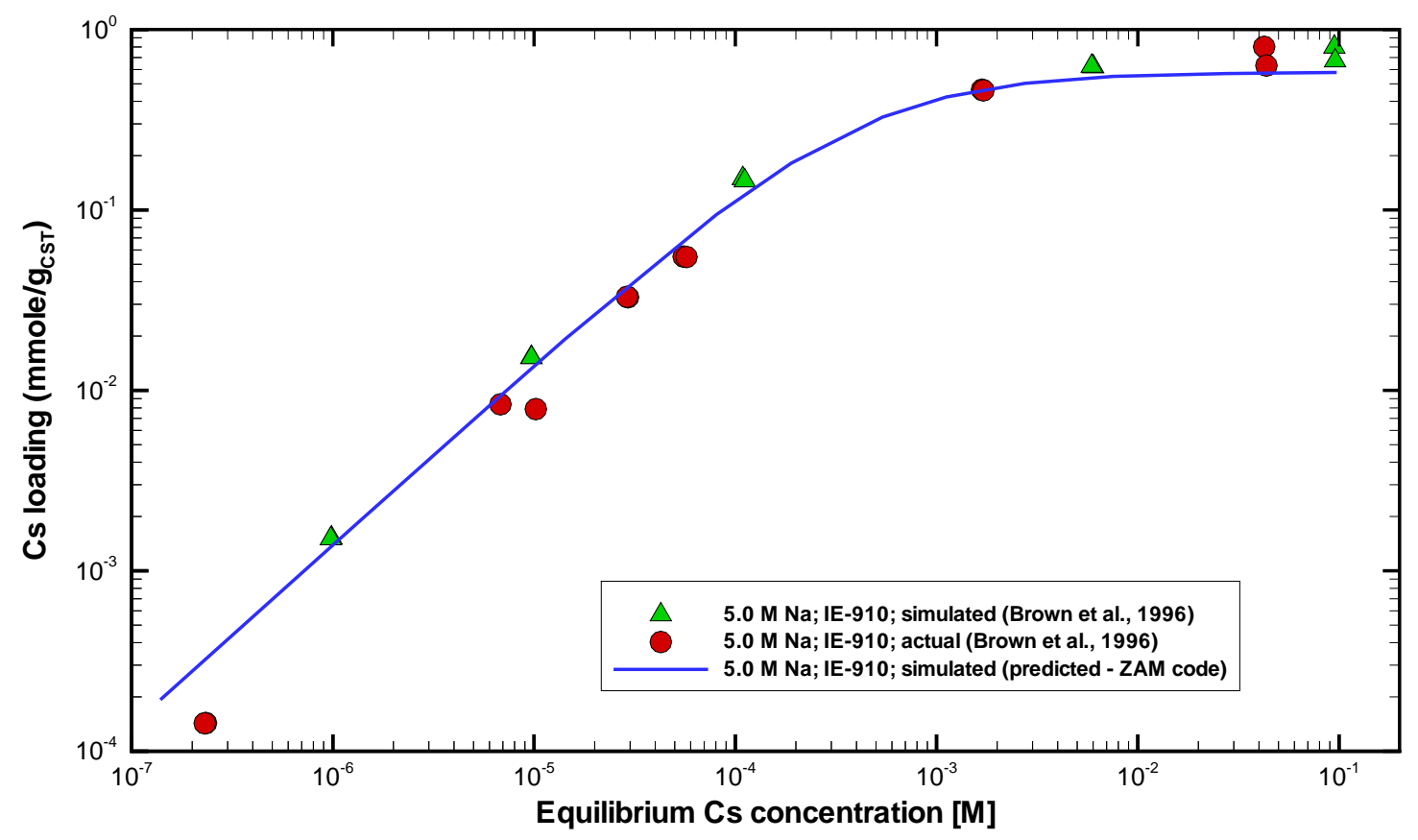

Figure F-9. A comparison of measured versus ZAM predicted cesium loading values for $5 \mathrm{M}$ sodium simulated and actual AW-101 feeds in contact with CST in its power-form (IE-910) [data by Brown et al. (1996)]. 


\section{F.5 ZAM Version-4 or Version-5 Input for 5 M Na AW-101 Feed)}

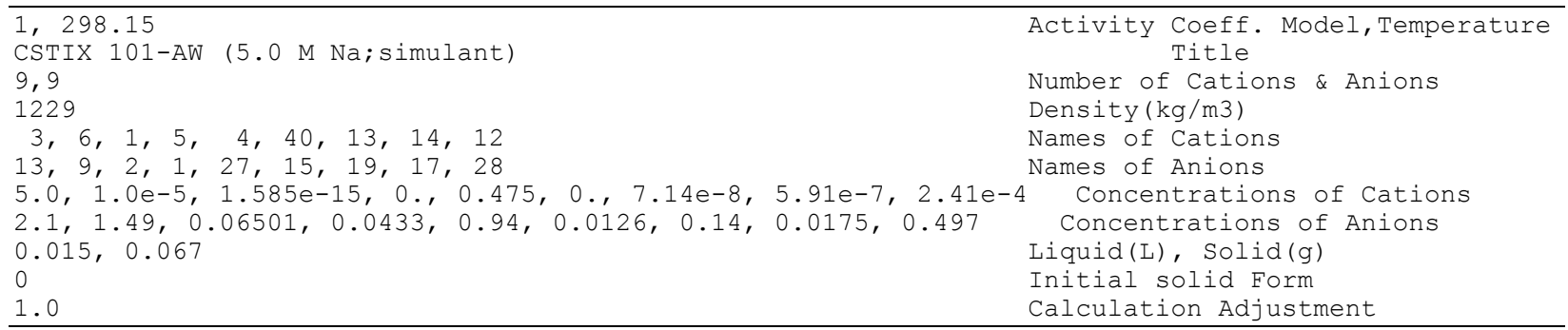

\section{F.6 ZAM Version-4 Output for 5 M Na AW-101 Feed)}

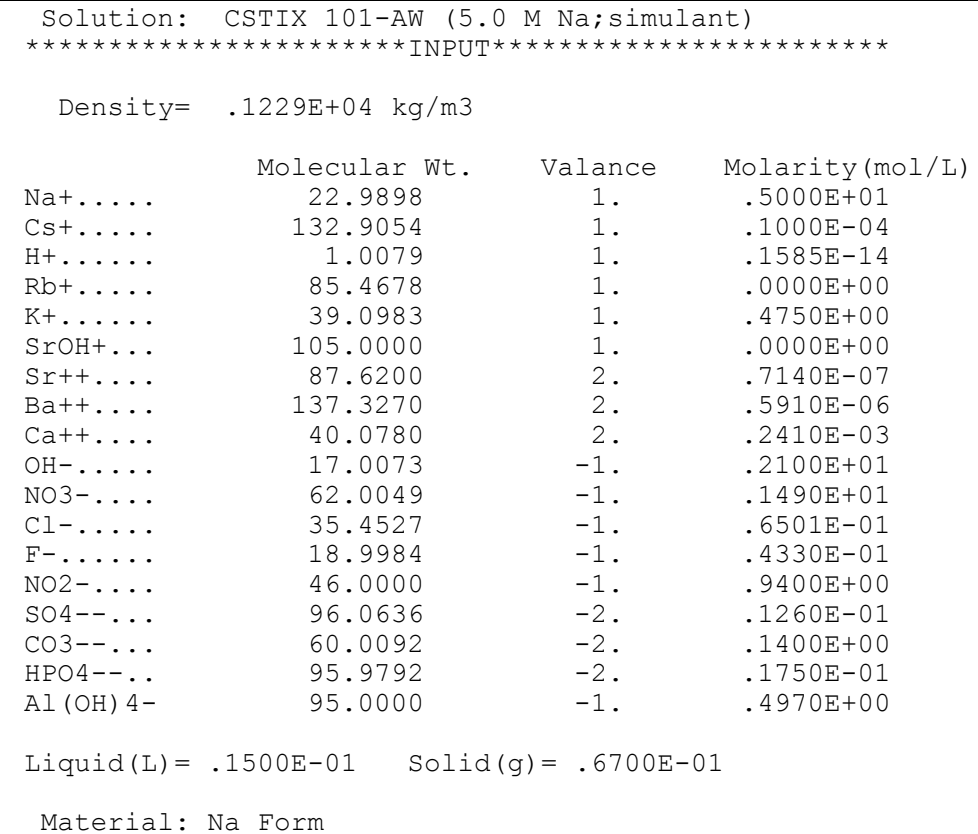

$\begin{array}{cccc}* * * * * * * * * * * * * * * * * * * * * * * & \text { OUTPUT } * * * * * * * * * * * * * * * * * * * * * * \\ \text { Ionic Strength }= & 6.665123171031650 \mathrm{~mol} / \mathrm{kg} \\ & \mathrm{Q}(\mathrm{mmol} / \mathrm{gCST}) & \mathrm{C}(\mathrm{mmol} / \mathrm{L}) & \mathrm{Kd} \mathrm{(ml/gCST)} \\ \mathrm{Cs} & .1928 \mathrm{E}-02 & .1390 \mathrm{E}-02 & .1386 \mathrm{E}+04 \\ \mathrm{Rb} & .0000 \mathrm{E}+00 & .0000 \mathrm{E}+00 & .0000 \mathrm{E}+00 \\ \mathrm{Sr} & .1593 \mathrm{E}-04 & .2566 \mathrm{E}-06 & .6208 \mathrm{E}+05 \\ \mathrm{~K} & .8634 \mathrm{E}+00 & .4711 \mathrm{E}+03 & .1832 \mathrm{E}+01\end{array}$

\section{F.7 ZAM Version-4 or Version-5 Input for Phase 1 LAW-1 Batch Feed)}

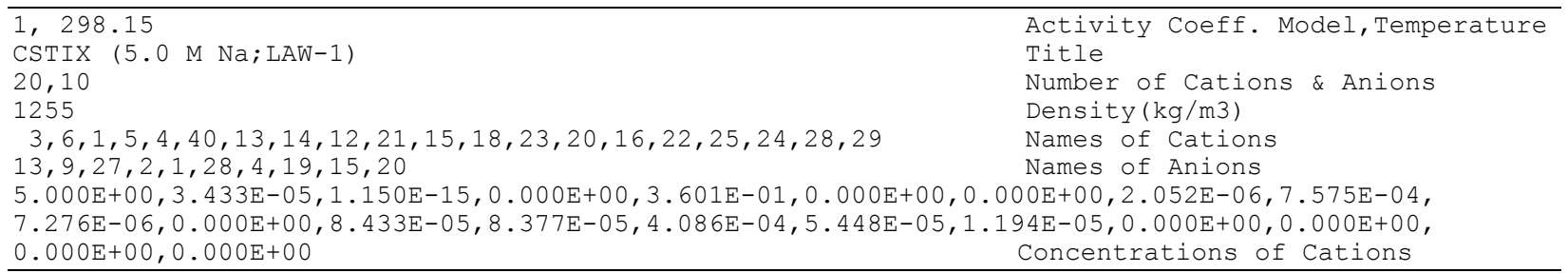


Preliminary Ion Exchange Modeling for Removal of Cesium from

$2.155 \mathrm{E}+00,1.666 \mathrm{E}+00,6.884 \mathrm{E}-01,2.910 \mathrm{E}-02,9.478 \mathrm{E}-02,2.834 \mathrm{E}-01,1.866 \mathrm{E}-06,1.828 \mathrm{E}-01,2.799 \mathrm{E}-02$,

$8.209 \mathrm{E}-03$

$1.0,0.01$

Concentrations of Anions

iquid(L), Solid $(g)$

0.95

Initial solid Form

Calculation Adjustment

\section{F.8 ZAM Version-4 Output for Phase 1 LAW-1 Batch Feed)}

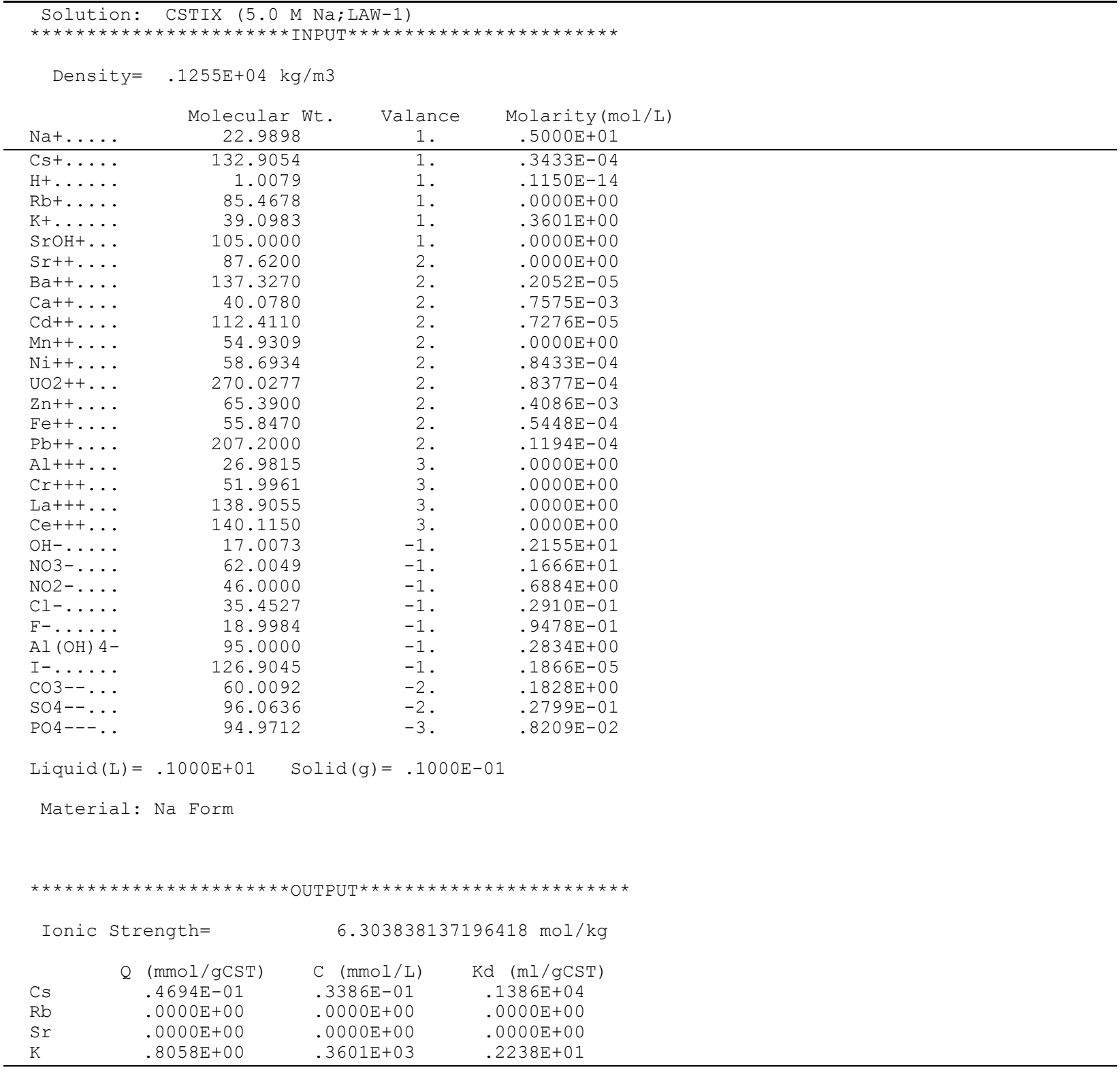

\section{F.9 ZAM Version-5 Output for Phase 1 LAW-1 Batch Feed)}

\footnotetext{
Solution: CSTIX (5.0 M Na; LAW-1)

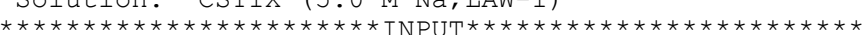

Density $=.1255 \mathrm{E}+04 \mathrm{~kg} / \mathrm{m} 3$

Molecular Wt. Valance Molarity (mol/L)

$\begin{array}{llll}\mathrm{Na}+\ldots . & 22.9898 & 1.5000 \mathrm{E}+01\end{array}$
} 
WESTINGHOUSE SAVANNAH RIVER COMPANY

Report: WSRC-TR-2001-00400

Preliminary Ion Exchange Modeling for Removal of Cesium from

Revision (Date):

$0(07 / 05 / 02)$

Hanford Waste Using Hydrous Crystalline Silicotitanate Material

Page:

304 of 338

\begin{tabular}{|c|c|c|c|}
\hline$C s+\ldots \ldots$ & 132.9054 & 1. & $.3433 E-04$ \\
\hline $\mathrm{H}+\ldots \ldots$ & 1.0079 & 1 . & $.1150 \mathrm{E}-14$ \\
\hline $\mathrm{Rb}+\ldots \ldots$ & 85.4678 & 1. & $.0000 \mathrm{E}+00$ \\
\hline$K+\ldots \ldots$ & 39.0983 & 1. & $.3601 \mathrm{E}+00$ \\
\hline $\mathrm{SrOH}+\ldots$ & 105.0000 & 1. & $.0000 \mathrm{E}+00$ \\
\hline $\mathrm{Sr}++\ldots$. & 87.6200 & 2. & $.0000 \mathrm{E}+\mathrm{C}$ \\
\hline $\mathrm{Ba}++\ldots$ & 137.3270 & 2 . & $.2052 \mathrm{E}-\mathrm{C}$ \\
\hline $\mathrm{Ca}++\ldots$ & 40.0780 & 2. & $.7575 \mathrm{E}-0$ \\
\hline $\mathrm{Cd}++\ldots$. & 112.4110 & 2 . & $.7276 \mathrm{E}-$ \\
\hline $\mathrm{Mn}++\ldots$. & 54.9309 & 2. & $.0000 \mathrm{E}+\mathrm{C}$ \\
\hline $\mathrm{Ni}++\ldots$. & 58.6934 & 2. & $.8433 \mathrm{E}-0$ \\
\hline UO2++ . . & 270.0277 & 2. & $.8377 \mathrm{E}-$ \\
\hline $\mathrm{Zn}++\ldots$ & 65.3900 & 2 . & $.4086 \mathrm{E}-\mathrm{C}$ \\
\hline $\mathrm{Fe}++\ldots$ & 55.8470 & 2 . & $.5448 \mathrm{E}-0$ \\
\hline $\mathrm{Pb}++\ldots$. & 207.2000 & 2 . & $.1194 \mathrm{E}-$ \\
\hline $\mathrm{Al}+++\ldots$ & 26.9815 & 3. & $.0000 \mathrm{E}+0$ \\
\hline $\mathrm{Cr}+++\ldots$ & 51.9961 & 3. & $.0000 \mathrm{E}+0$ \\
\hline $\mathrm{La}+++\ldots$ & 138.9055 & 3. & $.0000 \mathrm{E}+$ \\
\hline $\mathrm{Ce}+++\ldots$ & 140.1150 & 3. & $.0000 \mathrm{E}+\mathrm{C}$ \\
\hline $\mathrm{OH}-\ldots \ldots$ & 17.0073 & -1 . & $.2155 \mathrm{E}+\mathrm{C}$ \\
\hline NO3 $-\ldots$. & 62.0049 & -1 & $.1666 \mathrm{E}+$ \\
\hline $\mathrm{NO} 2-\ldots$. & 46.0000 & -1 . & $.6884 \mathrm{E}+$ \\
\hline $\mathrm{Cl}-\ldots$. & 35.4527 & -1. & $.2910 \mathrm{E}-$ \\
\hline$F-\ldots \ldots$ & 18.9984 & -1 . & $.9478 \mathrm{E}-1$ \\
\hline $\mathrm{Al}(\mathrm{OH}) 4-$ & 95.0000 & -1 . & $.2834 \mathrm{E}+$ \\
\hline$I-\ldots \ldots$ & 126.9045 & -1 & $.1866 \mathrm{E}-$ \\
\hline $\mathrm{CO} 3--\ldots$ & 60.0092 & -2 . & $.1828 \mathrm{E}+$ \\
\hline SO4--... & 96.0636 & -2 . & $.2799 \mathrm{E}-$ \\
\hline PO4---.. & 94.9712 & -3 & $.8209 \mathrm{E}-02$ \\
\hline
\end{tabular}

Material: Na Form

\begin{tabular}{|c|c|c|c|}
\hline \multicolumn{2}{|c|}{$\begin{array}{l}\text { Ionic Strength }= \\
\text { Equilibrium } \mathrm{pH}=\end{array}$} & \multicolumn{2}{|c|}{$\begin{array}{l}.630 \mathrm{E}+01 \mathrm{~mol} / \mathrm{kg} \\
14.6\end{array}$} \\
\hline & $\mathrm{Q} \quad(\mathrm{mmol} / \mathrm{gCST})$ & $\mathrm{C}(\mathrm{mmol} / \mathrm{L})$ & $\mathrm{Kd}(\mathrm{ml} / \mathrm{gCST})$ \\
\hline $\mathrm{Cs}$ & $.469 \mathrm{E}-01$ & $.339 \mathrm{E}-01$ & $.139 \mathrm{E}+04$ \\
\hline $\mathrm{Rb}$ & $.000 \mathrm{E}+00$ & $.000 \mathrm{E}+00$ & $.000 \mathrm{E}+00$ \\
\hline $\mathrm{SrOH}$ & $.000 \mathrm{E}+00$ & $.000 \mathrm{E}+00$ & $.000 \mathrm{E}+00$ \\
\hline & $.806 \mathrm{E}+00$ & $.360 \mathrm{E}+03$ & $.224 \mathrm{E}+01$ \\
\hline
\end{tabular}




\section{Appendix G (ZAM Input Files for Phase 1 Batch Feeds)}

For reference the ZAM input files for the 16 Phase 1 batch feeds are provided in this appendix. The input files for the nominal ZAM runs are provided. Other cesium concentration levels can be obtained by adding or reducing $\mathrm{CsCl}$ concentration within these input files. The ZAM results generated constitute our cesium isotherm database for the development of algebraic isotherm models for cesium loading that are used in VERSE-LC column transport simulation (i.e., fullscale column design studies). Also included are the ZAM input files for some of the sensitivity runs made. Note that nominal settings are $25 \mathrm{C}, 5 \mathrm{M} \mathrm{Na}^{+}, 0 \mathrm{M} \mathrm{Rb}^{+}$, and $0 \mathrm{M} \mathrm{Sr}^{+2}$.

Table E-1. Listing of ZAM input files provided in this appendix for reference.

\begin{tabular}{|c|c|}
\hline Feed Category & $\begin{array}{c}\text { Phase } 1 \text { LAW batch feeds } \\
\text { considered }\end{array}$ \\
\hline Nominal Envelope A & LAW-1 \\
& LAW-5 \\
& LAW-6 \\
& LAW-8 \\
& LAW-9 \\
& LAW-10 \\
& LAW-11 \\
& LAW-12 \\
& LAW-13 \\
& LAW-14 \\
& LAW-15 \\
\hline Nominal Envelope B & LAW-2a \\
& LAW-2b \\
\hline Nominal Envelope C & LAW-3 \\
& LAW-4 \\
& LAW-7 \\
\hline Sensitivity Envelope A & LAW-1 $\left(4 \mathrm{M} \mathrm{Na}{ }^{+}\right)$ \\
& LAW-1 $\left(6 \mathrm{M} \mathrm{Na}{ }^{+}\right)$ \\
\hline Sensitivity Envelope C & LAW-3 $\left(w i t h ~ \mathrm{Sr}^{+2}\right)$ \\
\hline
\end{tabular}




\section{ZAM Input for Phase 1 LAW-1 Batch Feed (Envelope A; nominal case)}

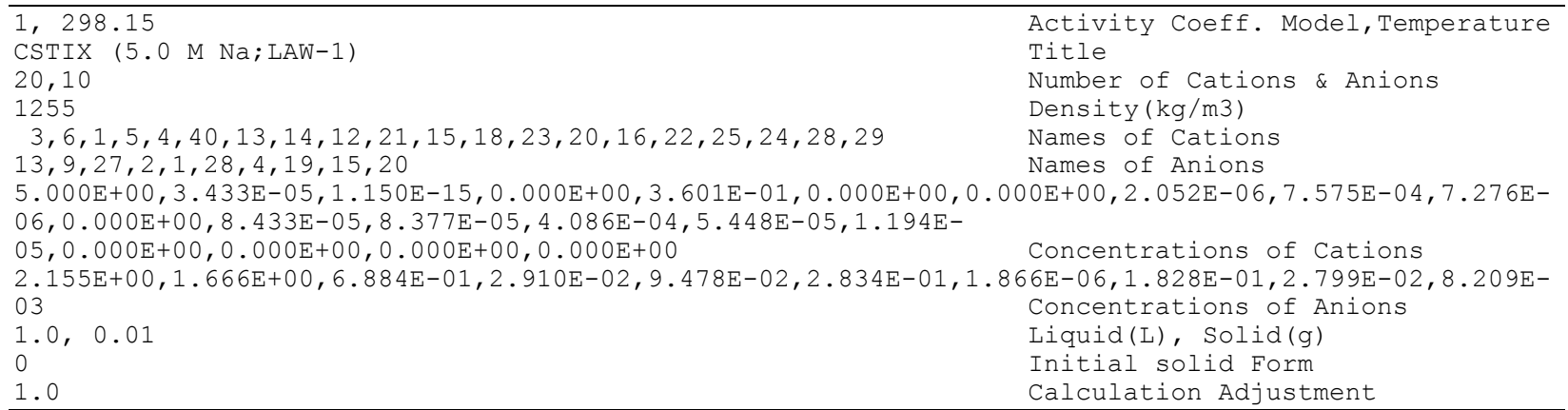

\section{ZAM Input for Phase 1 LAW-5 Batch Feed (Envelope A; nominal case)}

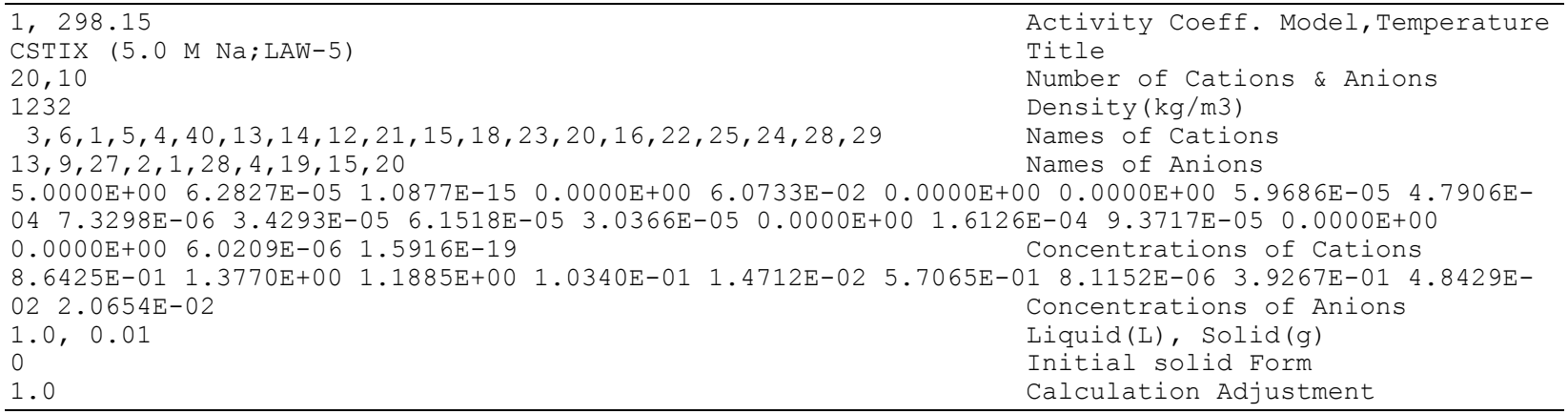

\section{ZAM Input for Phase 1 LAW-6 Batch Feed (Envelope A; nominal case)}

\begin{tabular}{ll}
\hline $1,298.15$ & Activity Coeff. Model, Temperature \\
CSTIX $(5.0 \mathrm{M} \mathrm{Na} ; \mathrm{LAW}-6)$ & Title \\
20,10 & Number of Cations \& Anions \\
1231 & Density (kg/m3) \\
$3,6,1,5,4,40,13,14,12,21,15,18,23,20,16,22,25,24,28,29$ & Names of Cations \\
$13,9,27,2,1,28,4,19,15,20$ & Names of Anions \\
$5.0000 \mathrm{E}+00,6.3277 \mathrm{E}-05,1.0890 \mathrm{E}-15,0.0000 \mathrm{E}+00,6.2429 \mathrm{E}-02,0.0000 \mathrm{E}+00,00000 \mathrm{E}+00,6.3559 \mathrm{E}-05,4.9718 \mathrm{E}-$ \\
$04,7.6271 \mathrm{E}-06,3.5876 \mathrm{E}-05,6.4689 \mathrm{E}-05,2.8192 \mathrm{E}-05,0.0000 \mathrm{E}+00,1.6949 \mathrm{E}-04,9.770 \mathrm{E}-$ \\
$05,0.0000 \mathrm{E}+00,0.0000 \mathrm{E}+00,6.4972 \mathrm{E}-06,1.7345 \mathrm{E}-19$ & Concentrations of Cations \\
$9.5427 \mathrm{E}-01,1.3588 \mathrm{E}+00,1.1921 \mathrm{E}+00,1.0311 \mathrm{E}-01,1.3362 \mathrm{E}-02,5.7606 \mathrm{E}-01,8.4746 \mathrm{E}-06,3.5876 \mathrm{E}-01,4.4915 \mathrm{E}-$ \\
$02,1.9802 \mathrm{E}-02$ & Concentrations of Anions \\
$1.0,0.01$ & Liquid(L), Solid(g) \\
0 & Initial Solid Form \\
1.0 & Calculation Adjustment
\end{tabular}

\section{ZAM Input for Phase 1 LAW-8 Batch Feed (Envelope A; nominal case)}

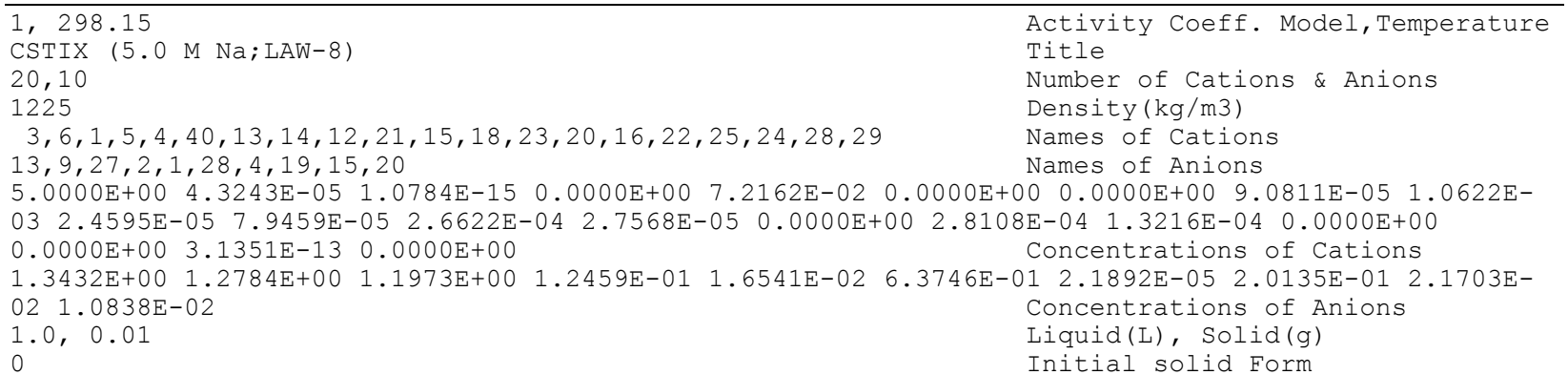




\section{ZAM Input for Phase 1 LAW-9 Batch Feed (Envelope A; nominal case)}

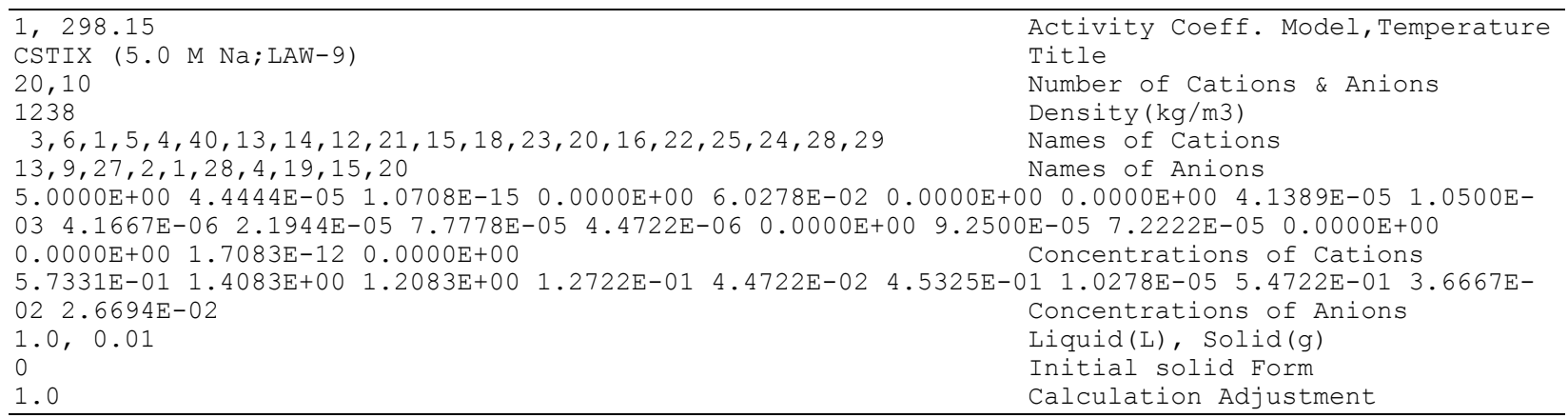

\section{ZAM Input for Phase 1 LAW-10 Batch Feed (Envelope A; nominal case)}

\begin{tabular}{ll}
\hline $1,298.15$ & Activity Coeff. Model, Temperature \\
CSTIX $(5.0 \mathrm{M} \mathrm{Na} ; \mathrm{LAW}-10)$ & Title \\
20,10 & Number of Cations \& Anions \\
1237 & Density (kg/m3) \\
$3,6,1,5,4,40,13,14,12,21,15,18,23,20,16,22,25,24,28,29$ & Names of Cations \\
$13,9,27,2,1,28,4,19,15,20$ & Names of Anions \\
$5.0 \mathrm{E}+00,3.6923 \mathrm{E}-05,1.6327 \mathrm{E}-15,0.0 \mathrm{E}+00,4.1923 \mathrm{E}-02,0.0 \mathrm{E}+00,0.0 \mathrm{E}+00,0.0 \mathrm{E}+00,1.6308 \mathrm{E}-$ \\
$03,0.0 \mathrm{E}+00,6.6923 \mathrm{E}-05,8.3462 \mathrm{E}-04,7.4231 \mathrm{E}-05,0.0 \mathrm{E}+00,1.7385 \mathrm{E}-03,1.5154 \mathrm{E}-$ \\
$03,0.0 \mathrm{E}+00,0.0 \mathrm{E}+00,2.0385 \mathrm{E}-05,2.5769 \mathrm{E}-05$ & Concentrations of Cations \\
$8.6970 \mathrm{E}-01,1.4308 \mathrm{E}+00,1.3115 \mathrm{E}+00,1.4000 \mathrm{E}-01,2.4962 \mathrm{E}-02,5.1500 \mathrm{E}-01,1.3077 \mathrm{E}-05,2.7846 \mathrm{E}-01,3.7269 \mathrm{E}-$ \\
$02,4.3462 \mathrm{E}-02$ & Concentrations of Anions \\
$1.0,0.01$ & Liquid(L), Solid(g) \\
0 & Initial solid Form \\
1.0 & Calculation Adjustment
\end{tabular}

\section{ZAM Input for Phase 1 LAW-11 Batch Feed (Envelope A; nominal case)}

\begin{tabular}{|c|c|}
\hline 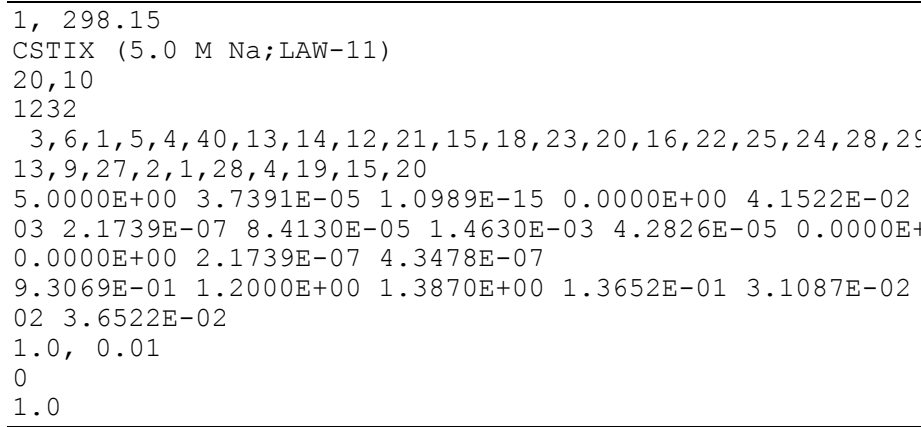 & $\begin{array}{l}\text { Activity Coeff. Model, Temperature } \\
\text { Title } \\
\text { Number of Cations \& Anions } \\
\text { Density(kg/m3) } \\
\text { Names of Cations } \\
\text { Names of Anions } \\
9 \quad 0.0000 \mathrm{E}+000.0000 \mathrm{E}+001.9565 \mathrm{E}-062.2174 \mathrm{E}- \\
+003.0870 \mathrm{E}-031.7370 \mathrm{E}-04 \quad 0.0000 \mathrm{E}+00 \\
\text { Concentrations of Cations } \\
4.9130 \mathrm{E}-017.3913 \mathrm{E}-063.6087 \mathrm{E}-012.3913 \mathrm{E}- \\
\text { Concentrations of Anions } \\
\text { Liquid(L), Solid(g) } \\
\text { Initial solid Form } \\
\text { Calculation Adjustment }\end{array}$ \\
\hline
\end{tabular}

\section{ZAM Input for Phase 1 LAW-12 Batch Feed (Envelope A; nominal case)}

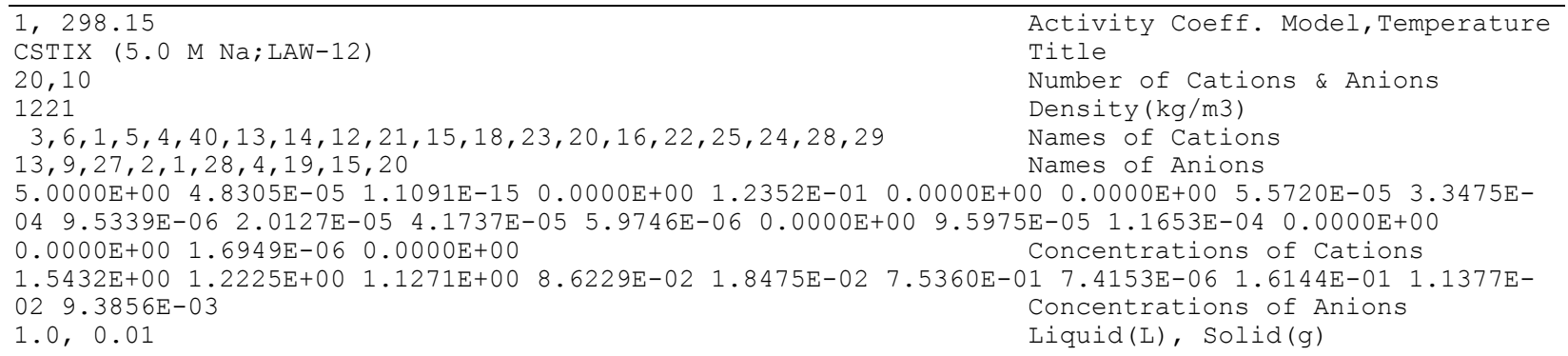




\section{ZAM Input for Phase 1 LAW-13 Batch Feed (Envelope A; nominal case)}

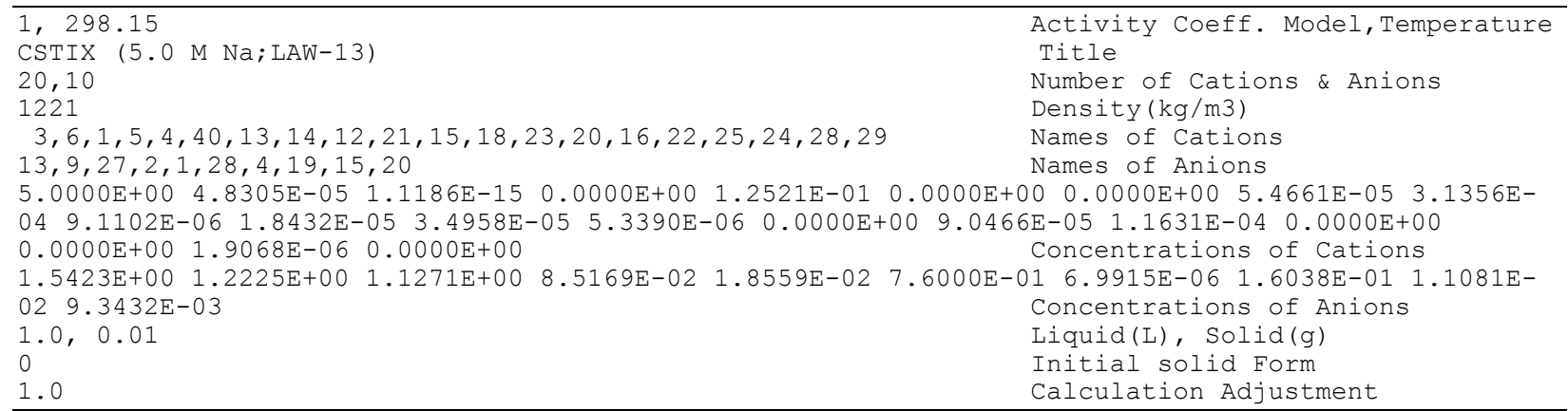

\section{ZAM Input for Phase 1 LAW-14 Batch Feed (Envelope A; nominal case)}

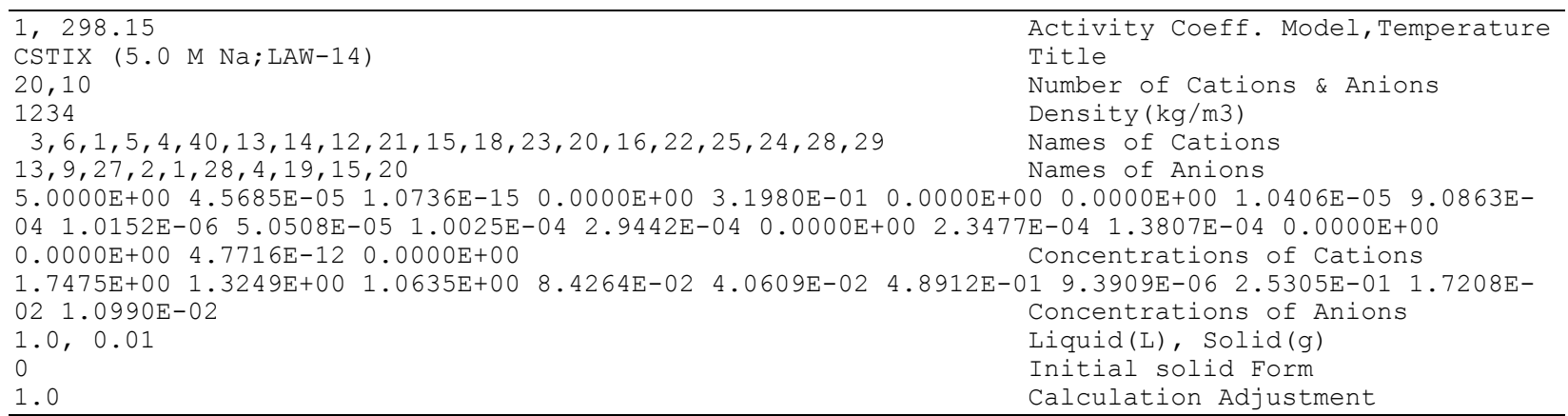

\section{ZAM Input for Phase 1 LAW-15 Batch Feed (Envelope A; nominal case)}

\begin{tabular}{ll}
\hline $1,298.15$ & Activity Coeff. Model, Temperature \\
CSTIX $(5.0 \mathrm{M} \mathrm{Na} ; \mathrm{LAW}-15)$ & Title \\
20,10 & Number of Cations \& Anions \\
1235 & Density (kg/m3) \\
$3,6,1,5,4,40,13,14,12,21,15,18,23,20,16,22,25,24,28,29$ & Names of Cations \\
$13,9,27,2,1,28,4,19,15,20$ & Names of Anions \\
$5.0000 \mathrm{E}+00,4.5522 \mathrm{E}-05,1.0746 \mathrm{E}-15,0.0000 \mathrm{E}+00,4.0672 \mathrm{E}-01,0.0000 \mathrm{E}+00,0.0000 \mathrm{E}+00,0.0000 \mathrm{E}+00,8.6007 \mathrm{E}-$ \\
$04,0.0000 \mathrm{E}+00,6.0261 \mathrm{E}-05,1.0802 \mathrm{E}-04,3.9552 \mathrm{E}-04,0.0000 \mathrm{E}+00,2.8358 \mathrm{E}-04,1.6063 \mathrm{E}-$ \\
$04,0.0000 \mathrm{E}+00,0.000 \mathrm{E}+00,5.8022 \mathrm{E}-12,0.0000 \mathrm{E}+00$ & Concentrations of Cations \\
$2.1409 \mathrm{E}+00,1.2985 \mathrm{E}+00,1.0149 \mathrm{E}+00,6.9776 \mathrm{E}-02,3.9366 \mathrm{E}-02,5.0132 \mathrm{E}-01,9.1418 \mathrm{E}-06,1.5373 \mathrm{E}-01,1.0616 \mathrm{E}-$ & Concentrations of Anions \\
$02,5.6716 \mathrm{E}-03$ & Liquid(L), Solid(g) \\
$1.0,0.01$ & Initial Solid Form \\
0 & Calculation Adjustment \\
1.0 &
\end{tabular}

\section{ZAM Input for Phase 1 LAW-2a Batch Feed (Envelope B; nominal case)}

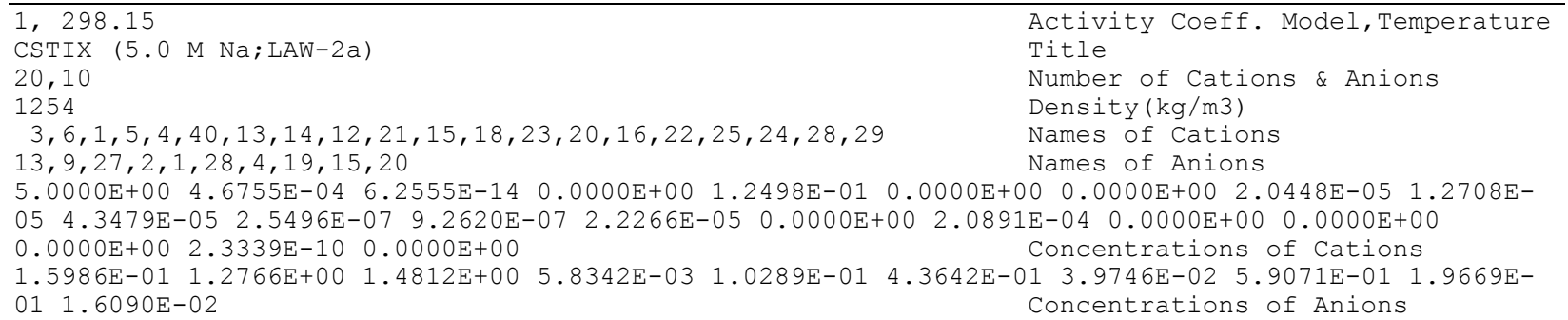




\section{ZAM Input for Phase 1 LAW-2b Batch Feed (Envelope B; nominal case)}

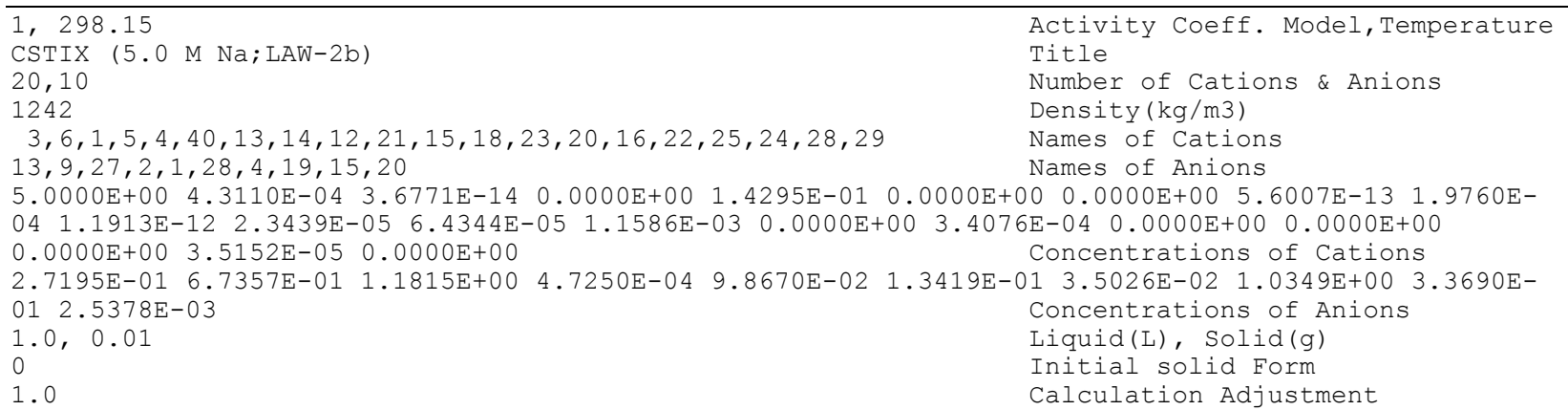

\section{ZAM Input for Phase 1 LAW-3 Batch Feed (Envelope C; nominal case)}

\begin{tabular}{|c|c|}
\hline 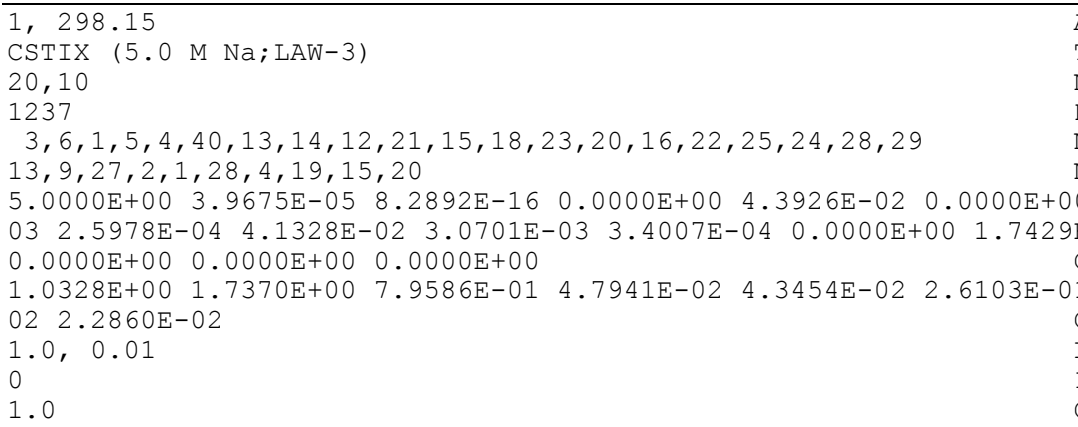 & $\begin{array}{l}\text { Activity Coeff. Model, Temperature } \\
\text { Title } \\
\text { Number of Cations \& Anions } \\
\text { Density(kg/m3) } \\
\text { Names of Cations } \\
\text { Names of Anions } \\
00.0000 \mathrm{E}+001.0013 \mathrm{E}-046.9195 \mathrm{E}- \\
0 \text {-03 4.2981E-04 0.0000E+00 } \\
\text { Concentrations of Cations } \\
1 \text { 1.5823E-05 5.1011E-01 7.2737E- } \\
\text { Concentrations of Anions } \\
\text { Liquid(L), Solid(g) } \\
\text { Initial solid Form } \\
\text { Calculation Adjustment }\end{array}$ \\
\hline
\end{tabular}

\section{ZAM Input for Phase 1 LAW-4 Batch Feed (Envelope C; nominal case)}

\begin{tabular}{|c|c|}
\hline 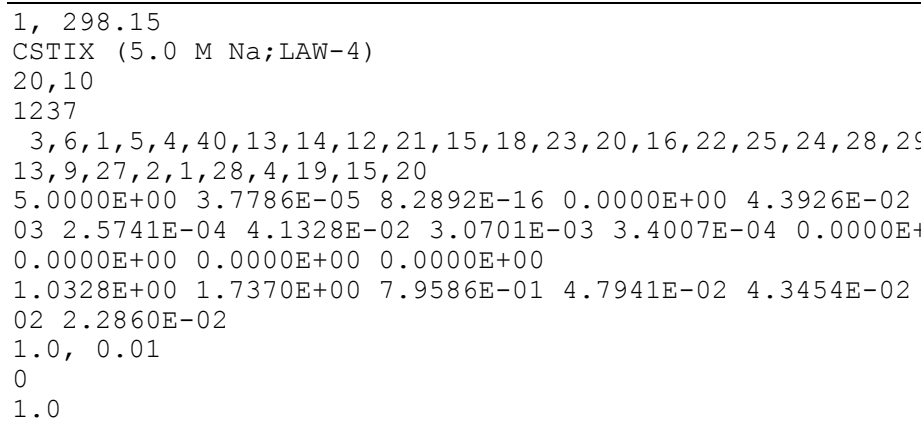 & $\begin{array}{l}\text { Activity Coeff. Model, Temperature } \\
\text { Title } \\
\text { Number of Cations \& Anions } \\
\text { Density (kg/m3) } \\
\text { Names of Cations } \\
\text { Names of Anions } \\
9.0000 \mathrm{E}+000.0000 \mathrm{E}+001.0013 \mathrm{E}-04 \quad 6.9195 \mathrm{E}- \\
+001.7429 \mathrm{E}-03 \quad 4.2981 \mathrm{E}-040.0000 \mathrm{E}+00 \\
\text { Concentrations of Cations } \\
\text { 2.6103E-01 } 1.5823 \mathrm{E}-05 \text { 5.1011E-01 7.2737E- } \\
\text { Concentrations of Anions } \\
\text { Liquid(L), Solid(g) } \\
\text { Initial solid Form } \\
\text { Calculation Adjustment }\end{array}$ \\
\hline
\end{tabular}

\section{ZAM Input for Phase 1 LAW-7 Batch Feed (Envelope C; nominal case)}

$1,298.15$

CSTIX (5.0 M Na;LAW-7)

20,10

1243

$3,6,1,5,4,40,13,14,12,21,15,18,23,20,16,22,25,24,28,29$

$13,9,27,2,1,28,4,19,15,20$

$03 \quad 0.0000 \mathrm{E}+00 \quad 4.0571 \mathrm{E}-02 \quad 4.4389 \mathrm{E}-03 \quad 3.1025 \mathrm{E}-04 \quad 0.0000 \mathrm{E}+00 \quad 2.6570 \mathrm{E}-02 \quad 1.0151 \mathrm{E}-03 \quad 0.0000 \mathrm{E}+00$

$0.0000 \mathrm{E}+00 \quad 1.3174 \mathrm{E}-04 \quad 0.0000 \mathrm{E}+00$

Number of Cations \& Anions
Activity Coeff. Model, Temperature Title

Density $(\mathrm{kg} / \mathrm{m} 3)$

Names of Cations

Names of Anions

Concentrations of Cations 


\section{ZAM Input for Phase 1 LAW-1 Batch Feed (Envelope A; with $4 \mathrm{M} \mathrm{Na}^{+}$)}

\begin{tabular}{ll}
\hline $1,298.15$ & Activity Coeff. Model, Temperature \\
CSTIX $(4.0 \mathrm{M} \mathrm{Na} ; \mathrm{LAW}-1)$ & Title \\
20,10 & Number of Cations \& Anions \\
1224 & Density (kg/m3) \\
$3,6,1,5,4,40,13,14,12,21,15,18,23,20,16,22,25,24,28,29$ & Names of Cations \\
$13,9,27,2,1,28,4,19,15,20$ & Names of Anions \\
$4.0000 \mathrm{E}+00,2.7463 \mathrm{E}-05,9.2015 \mathrm{E}-16,0.0000 \mathrm{E}+00,2.8806 \mathrm{E}-01,0.0000 \mathrm{E}+00,0.0000 \mathrm{E}+00,1.6418 \mathrm{E}-06,6.0597 \mathrm{E}-$ \\
$04,5.8209 \mathrm{E}-06,0.0000 \mathrm{E}+00,6.7463 \mathrm{E}-05,6.7015 \mathrm{E}-05,3.2687 \mathrm{E}-04,4.3582 \mathrm{E}-05,9.5522 \mathrm{E}-$ \\
$06,0.0000 \mathrm{E}+00,0.0000 \mathrm{E}+00,0.0000 \mathrm{E}+00,0.0000 \mathrm{E}+00$ & Concentrations of Cations \\
$1.7240 \mathrm{E}+00,1.3328 \mathrm{E}+00,5.5075 \mathrm{E}-01,2.3284 \mathrm{E}-02,7.5821 \mathrm{E}-02,2.2668 \mathrm{E}-01,1.4925 \mathrm{E}-06,1.4627 \mathrm{E}-01,2.2388 \mathrm{E}-$ \\
$02,6.5672 \mathrm{E}-03$ & Concentrations of Anions \\
$1.0,0.01$ & Liquid(L), Solid(g) \\
0 & Initial solid Form \\
1.0 & Calculation Adjustment \\
\hline
\end{tabular}

\section{ZAM Input for Phase $1 \mathrm{LAW}-1$ Batch Feed (Envelope A; with $6 \mathrm{M} \mathrm{Na}^{+}$)}

\begin{tabular}{ll}
\hline $1,298.15$ & Activity Coeff. Model, Temperature \\
CSTIX $(6.0 \mathrm{M} \mathrm{Na} ; \mathrm{LAW}-1)$ & Title \\
20,10 & Number of Cations \& Anions \\
1269 & Density(kg/m3) \\
$3,6,1,5,4,40,13,14,12,21,15,18,23,20,16,22,25,24,28,29$ & Names of Cations \\
$13,9,27,2,1,28,4,19,15,20$ & Names of Anions \\
$6.0000 \mathrm{E}+00,4.1194 \mathrm{E}-05,1.3802 \mathrm{E}-15,0.0000 \mathrm{E}+00,4.3209 \mathrm{E}-01,0.0000 \mathrm{E}+00,0.0000 \mathrm{E}+00,2.4627 \mathrm{E}-06,9.0896 \mathrm{E}-$ \\
$04,8.7313 \mathrm{E}-06,0.0000 \mathrm{E}+00,1.0119 \mathrm{E}-04,1.0052 \mathrm{E}-04,4.9030 \mathrm{E}-04,6.5373 \mathrm{E}-05,1.4328 \mathrm{E}-$ \\
$05,0.0000 \mathrm{E}+00,0.0000 \mathrm{E}+00,0.0000 \mathrm{E}+00,0.0000 \mathrm{E}+00$ & Concentrations of Cations \\
$2.5859 \mathrm{E}+00,1.9993 \mathrm{E}+00,8.2612 \mathrm{E}-01,3.4925 \mathrm{E}-02,1.1373 \mathrm{E}-01,3.4002 \mathrm{E}-01,2.2388 \mathrm{E}-06,2.1940 \mathrm{E}-01,3.3582 \mathrm{E}-$ \\
$02,9.8507 \mathrm{E}-03$ & Concentrations of Anions \\
$1.0,0.01$ & Liquid(L), Solid(g) \\
1.0 & Initial solid Form \\
1.0 & Calculation Adjustment \\
\hline
\end{tabular}

\section{ZAM Input for Phase 1 LAW-3 Batch Feed (Envelope C; with $\mathrm{Sr}^{+2}$ )}

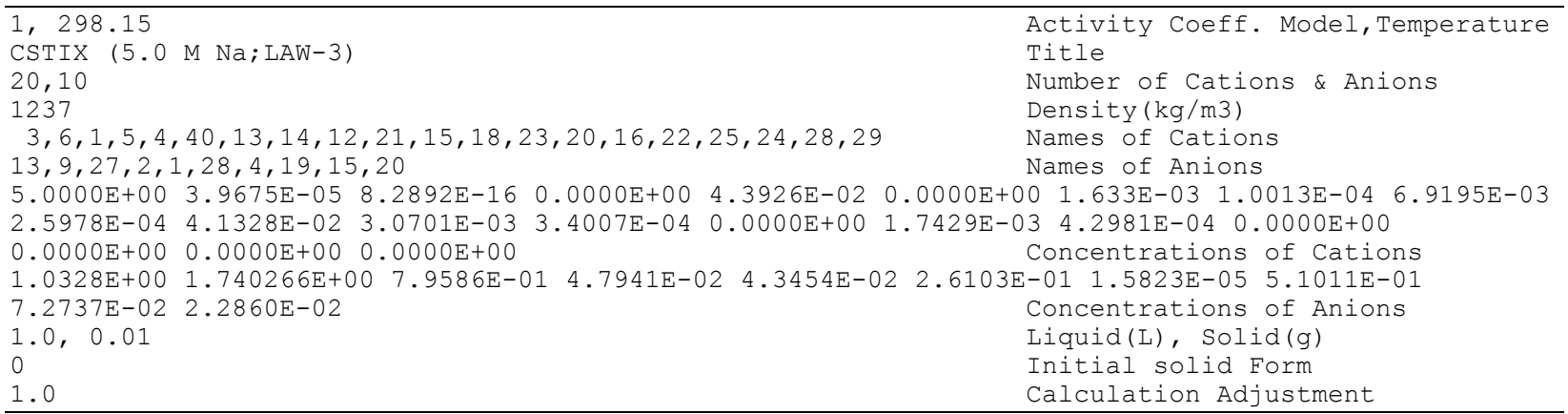




\section{Appendix H (Column Test Input Files)}

For reference the VERSE-LC input files for the laboratory-scale and pilot-scale test columns are provided in this appendix (output files are not shown but can be quickly generated based on the input files). The results of these VERSE-LC input files are discussed in Chapter 9. The column tests were performed using the single component modeling option (i.e., effective cesium isotherms and column transport simulations). Fifteen column simulation models were run. The input files for each case are listed below. Some of the key parameters and details of the experiments are listed in tables, along with the measured cesium breakthrough curves.

An "effective" single component transport analysis is used for all of the VERSE-LC column studies. When using this option a single component isotherm model for cesium loading is required. To estimate the cesium isotherms an algebraic model is fit to the ZAM generated database, where a beta factor for each column study is defined. The feed compositions for the column tests considered are listed in Table H-1.

In the majority of case runs a bed density of $1.0 \mathrm{~g} / \mathrm{ml}$ and a cesium total ionic capacity of 0.58 mmole/g $\mathrm{g}_{\text {CST }}$ were assumed. For assessment and optimization purposes several VERSE-LC simulation runs were made for each column test while varying the dilution factor (i.e., typical values of either $68 \%$ or $100 \%$ ) and the pore to free diffusion coefficient ratio (i.e., values of $10 \%, 20 \%, 26 \%, 30 \%$, and $35 \%$ ). The ratio value of $26 \%$ corresponds to pore diffusion coefficient values used in several earlier SRS analysis efforts.

\section{H.1 SRS Tank 44 Studies}

Walker et al. (1999) performed batch contact and column tests using CST IE-911 (Lot number 98-05) with actual diluted Tank 44 supernate. Some of the key column parameters for these tests are given in Table H-2. The cesium breakthrough data at two axial locations (i.e., sampling points) are tabulated in Table H-3. Two different VERSE-LC models were used to simulate this data. The only difference between the two models being the column size considered. Example input files for each model is also provided in this appendix. The beta parameter used for the cesium isotherm is $2.0486 \times 10^{-4} \mathrm{M}$ based on a fit of data generated using the ZAM code at $31 \mathrm{C}$.

\section{H.2 SRS Average Simulant Studies}

Several column tests using CST material were performed with SRS average simulant as the feed:

\section{Wilmarth et al. (1999) Data Sets}

Wilmarth et al. (1999) performed a series of column tests at $\sim 25 \mathrm{C}$ where the effects of CST pretreatment, superficial velocity, and the presence of organic constituents were considered. CST with Lot numbers 96-04 and 98-05 were tested. Some of the key column parameters for these tests are given in Table H-4. The cesium breakthrough data for these tests are tabulated in Tables H-5 and H-6. The beta parameter used for the cesium isotherm is $2.4145 \times 10^{-4} \mathrm{M}$ based on a fit of data generated using the ZAM code at $25 \mathrm{C}$. 
For the SRS-Avg-Test2 test both single component and ternary column simulations were performed, as discussed in Chapter 3 for justifying the use of the single component modeling approach. The input file of both approaches is proivded.

\section{Walker et al. (1998) Data Sets}

Walker et al. (1998) performed several batch contact tests and three column tests using CST IE911 (Lot numbers 96-02 and 96-04) with SRS-Avg simulant, as well. The column tests were performed at $\sim 22 \mathrm{C}$. The beta parameter used for the cesium isotherm is $2.4145 \times 10^{-4} \mathrm{M}$, the same value used for all of the seven SRS-Avg tests. The slight difference due to temperature was neglected. Some of the key column parameters for these tests are given in Table H-7. The cesium breakthrough data for the three column tests are tabulated in Table H-8. The VERSE-LC input file for each column test is also provided in this appendix.

\section{H.3 SRS High OH Simulant Studies}

Walker et al. (1999) also performed batch contact and column tests using CST IE-911 (Lot number 98-05) with SRS high OH simulant. These tests are basically repeat tests where the feed was changed from SRS Tank44 supernate to SRS high OH simulant. Some of the key column parameters for these tests are also given in Table H-2. The cesium breakthrough data at the earlier two axial locations (i.e., sampling points) are tabulated in Table H-9. No breakthrough was observed at the $85 \mathrm{~cm}$ axial location over the time period of the testing. Two different VERSE-LC models were used to simulate this data. The only difference between the two models being the column length considered. Example input files for each model is also provided in this appendix. The beta parameter used for the cesium isotherm is $2.0987 \times 10^{-4} \mathrm{M}$ based on a fit of data generated using the ZAM code at $31 \mathrm{C}$.

\section{H.4 PNNL AW-101 Sample Studies}

Hendrickson (1997) performed a column test using CST IE-911 (Lot number 96-01) with a diluted AW-101 sample. The column test was performed at $\sim 25 \mathrm{C}$ and the key features of these tests are listed in Table H-10. The cesium breakthrough data are tabulated in Table H-11. The beta parameter used for the cesium isotherm is $4.7414 \times 10^{-4} \mathrm{M}$ based on a fit of data generated using the ZAM code at $25 \mathrm{C}$.

\section{H.5 ORNL MVST Sample Tests}

Several column tests using CST material were performed with MVST waste as the feed:

\section{Lee et al. (1997) Data Sets}

Lee et al. (1997b) performed columns test using CST IE-911 (Lot number -38b) with a MVST $\mathrm{W}-27$ sample. The column test was performed at $\sim 25 \mathrm{C}$ and the key features of these tests are listed in Table H-12. The cesium breakthrough data are tabulated in Table H-13. The beta parameter used for the cesium isotherm is $9.3232 \times 10^{-4} \mathrm{M}$ based on a fit of data generated using the ZAM code at $25 \mathrm{C}$.

\section{Walker, Jr., et al. (1998) Data Sets}


Walker, Jr., et al. (1998) performed pilot-scale column testing using CST IE-911 (Lot number $38 \mathrm{~b}$ ) with a MVST W-29 sample. The column tests were performed at $\sim 25 \mathrm{C}$ and the key features of these tests are listed in Table H-14. The cesium breakthrough data are tabulated in Tables H-15, H-16, H-17, and H-18. Since the composition of the feeds was not well defined within the Walker, Jr., et al. (1998) report, the limited $\mathrm{K}_{\mathrm{d}}$ values reported were directly used to fit the isotherms where several beta values (feed dependent) were generated and used.

Table H-1. Ionic species molar concentrations for various simulated and actual waste solutions used in ZAM batch contact simulations to generate a cesium isotherm data used for estimating the beta factor.

\begin{tabular}{|c|c|c|c|c|c|}
\hline $\begin{array}{c}\text { Species } \\
\text { ID }\end{array}$ & $\begin{array}{c}\text { SRS } \\
\text { Average } \\
\text { simulant } \\
{[M]}\end{array}$ & $\begin{array}{c}\text { SRS High } \\
\text { OH }^{-} \\
\text {simulant } \\
{[M]}\end{array}$ & $\begin{array}{c}\text { SRS Tank } \\
44 \text { sample } \\
{[\mathrm{M}]}\end{array}$ & $\begin{array}{l}\text { PNNL AW- } \\
101 \text { sample } \\
\text { [M] }\end{array}$ & $\begin{array}{c}\text { ORNL } \\
\text { MVST W- }- \\
27 \text { sample } \\
\text { [M] }\end{array}$ \\
\hline \multicolumn{6}{|l|}{ Cations } \\
\hline $\mathrm{Na}^{+}$ & 5.6 & 5.6 & 5.4 & 5.64 & 5.119 \\
\hline $\mathrm{Cs}^{+}$ & $1.4 \times 10^{-4}$ & $3.7 \times 10^{-4}$ & $3.51 \times 10^{-4}$ & $7.26 \times 10^{-5}$ & $7.04 \times 10^{-6}$ \\
\hline $\mathrm{K}^{+}$ & 0.015 & 0.03 & 0.051 & 0.50 & 0.263 \\
\hline $\mathrm{H}^{+}$ & $5.2 \times 10^{-15}$ & $3.3 \times 10^{-15}$ & $2.3 \times 10^{-15}$ & $3.9 \times 10^{-15}$ & $1.4 \times 10^{-13}$ \\
\hline $\mathrm{Ca}^{+2}$ & - & - & - & - & $2.22 \times 10^{-3}$ \\
\hline \multicolumn{6}{|l|}{ Anions } \\
\hline $\mathrm{NO}_{3}{ }^{-}$ & 2.14 & 1.1 & 0.37 & 1.53 & 5.194 \\
\hline $\mathrm{NO}_{2}^{-}$ & 0.52 & 0.74 & 0.35 & 1.13 & - \\
\hline $\mathrm{Cl}^{-}$ & 0.025 & $3.637 \times 10^{-2}$ & 0.009 & 0.0883 & 0.09 \\
\hline $\mathrm{F}^{-}$ & 0.032 & 0.01 & - & 0.0334 & - \\
\hline $\mathrm{OH}^{-}$(free) & 1.938 & 3.05 & 4.3 & 2.541 & 0.0708 \\
\hline $\mathrm{Al}(\mathrm{OH})_{4}^{-}$ & 0.31 & 0.27 & 0.126 & 0.574 & $3.14 \times 10^{-5}$ \\
\hline $\mathrm{CO}_{3}^{2-}$ & 0.16 & 0.17 & 0.1412 & 0.11 & - \\
\hline $\mathrm{SO}_{4}{ }^{2-}$ & 0.15 & 0.03 & 0.001 & 3.28 & $1.60 \times 10^{-2}$ \\
\hline $\mathrm{PO}_{4}^{3-}$ & 0.01 & 0.008 & 0.0001 & $5.53 \times 10^{-3}$ & - \\
\hline Cations $=$ & 5.615 & 5.630 & 5.451 & 6.140 & 5.387 \\
\hline Anions $=$ & -5.615 & -5.630 & -5.451 & -6.140 & -5.387 \\
\hline Sum $=$ & 0.000 & 0.000 & 0.000 & 0.000 & 0.000 \\
\hline Beta $=$ & $2.4145 \times 10^{-4}$ & $2.0987 \times 10^{-4}$ & $2.0486 \times 10^{-4}$ & $4.7414 \times 10^{-4}$ & $9.3232 \times 10^{-4}$ \\
\hline
\end{tabular}


Table H-2. Key column parameters for CST IE-911 packed columns using SRS high OH simulant and SRS Tank 44 supernate waste taken by Walker et al. (1999) at 31. ${ }^{\text {a.b }}$

\begin{tabular}{|c|c|c||c|c|}
\hline Parameter & $\begin{array}{c}\text { SRS-High-OH- } \\
\text { Test1a }\end{array}$ & $\begin{array}{c}\text { SRS-High-OH- } \\
\text { Test1b }\end{array}$ & $\begin{array}{c}\text { SRS-Tank44- } \\
\text { Test1a }\end{array}$ & $\begin{array}{c}\text { SRS-Tank44- } \\
\text { Test1b }\end{array}$ \\
\hline $\begin{array}{c}\text { Column diameter } \\
\text { (cm) }\end{array}$ & 1.50 & 1.50 & 1.50 & 1.50 \\
\hline $\begin{array}{c}\text { Axial sampling } \\
\text { loaction (cm) }\end{array}$ & 10.0 & 85.0 & 10.0 & 85.0 \\
\hline CST (Lot \#) & $98-05$ & $98-05$ & $98-05$ & $98-05$ \\
\hline $\begin{array}{c}\text { Average } \\
\text { superficial } \\
\text { velocity (cm/min) }\end{array}$ & 5.43 & 5.43 & 5.32 & 3.32 \\
\hline $\begin{array}{c}\text { Average } \\
\text { temperature (C) }\end{array}$ & 31 & 31 & 31 & $3.51 \mathrm{E}-04$ \\
\hline $\begin{array}{c}\text { Avg. Cs feed } \\
\text { concentration } \\
\text { (M) }\end{array}$ & $3.70 \mathrm{E}-04$ & $3.70 \mathrm{E}-04$ & $3.51 \mathrm{E}-04$ & 150.2074 \\
\hline $\begin{array}{c}\text { Column volume } \\
\text { (ml) }\end{array}$ & 17.6714 & 150.2074 & 17.6714 & {$[3.8]$} \\
\hline $\begin{array}{c}\text { Flowrate Q } \\
\text { (ml/min) } \\
\text { [CV/hr] }\end{array}$ & 9.60 & 9.60 & 9.40 & {$[3.8]$} \\
\hline [32.6] & {$[32.6]$} & & \\
\hline
\end{tabular}

${ }^{a}$ The SRS high OH simulant and SRS Tank 44 waste feed compositions are provided in Appendix C.

b Same basic columns and sampling locations used for both feed types.

Table H-3. Cesium breakthrough data for CST IE-911 (Lot 98-05) and actual SRS Tank 44 waste taken by Walker et al. (1999) at $31 \mathrm{C}$.

\begin{tabular}{|c|c|c|c|}
\hline \multicolumn{2}{|c|}{$\begin{array}{l}\text { Effectively a Lead Column } \\
\text { (10 cm down column length) }\end{array}$} & \multicolumn{2}{|c|}{$\begin{array}{l}\text { Effectively a Lag Column } \\
\text { (85 cm down column length) }\end{array}$} \\
\hline $\begin{array}{l}\text { Time } \\
\text { (hrs) }\end{array}$ & $\begin{array}{l}\text { Normalized } \\
\text { cesium } \\
\text { breakthrough } \\
\left(\mathbf{c} / \mathbf{c}_{\mathbf{o}}\right)^{\text {a }}\end{array}$ & $\begin{array}{l}\text { Time } \\
\text { (hrs) }\end{array}$ & $\begin{array}{l}\text { Normalized } \\
\text { cesium } \\
\text { breakthrough } \\
\left(\mathbf{c} / \mathbf{c}_{\mathbf{o}}\right)^{\mathrm{a}}\end{array}$ \\
\hline 0 & 0.000 & 0 & $0.000 \mathrm{E}+00$ \\
\hline 1.1 & 0.007 & 1.1 & $4.700 \mathrm{E}-07$ \\
\hline 3.9 & 0.067 & 7.9 & $1.200 \mathrm{E}-06$ \\
\hline 7.9 & 0.174 & 15.6 & $4.300 \mathrm{E}-07$ \\
\hline 11.7 & 0.279 & 23.5 & $1.100 \mathrm{E}-06$ \\
\hline 15.6 & 0.381 & 31.4 & $4.500 \mathrm{E}-07$ \\
\hline 19.4 & 0.487 & 39.2 & $1.500 \mathrm{E}-06$ \\
\hline 23.5 & 0.554 & 46.9 & $1.400 \mathrm{E}-06$ \\
\hline 27.4 & 0.631 & 54.7 & $4.900 \mathrm{E}-06$ \\
\hline 31.4 & 0.710 & 62.5 & $1.100 \mathrm{E}-07$ \\
\hline 35.3 & 0.732 & 70.3 & $2.900 \mathrm{E}-05$ \\
\hline 39.2 & 0.770 & 78.1 & $7.600 \mathrm{E}-05$ \\
\hline 43.1 & 0.770 & 86.0 & $1.700 \mathrm{E}-04$ \\
\hline 46.9 & 0.817 & 93.9 & $5.300 \mathrm{E}-04$ \\
\hline 50.8 & 0.880 & 101.9 & $7.300 \mathrm{E}-04$ \\
\hline 54.7 & 0.899 & 109.8 & $1.200 \mathrm{E}-03$ \\
\hline 58.7 & 0.897 & 113.8 & $1.600 \mathrm{E}-03$ \\
\hline
\end{tabular}


Preliminary Ion Exchange Modeling for Removal of Cesium from

\begin{tabular}{|l|l|l|l|}
\hline 62.5 & 0.928 & 117.8 & $2.100 \mathrm{E}-03$ \\
\hline 66.4 & 0.918 & 125.7 & $3.400 \mathrm{E}-03$ \\
\hline 70.3 & 0.966 & & \\
\hline 74.2 & 0.961 & \\
\hline 78.1 & 0.955 & \\
\hline 82.0 & 0.955 & \\
\hline 86.0 & 0.976 & \\
\hline 90.0 & 0.946 & \\
\hline 93.9 & 0.974 & \\
\hline 97.9 & 0.997 & \\
\hline 101.9 & 1.025 & \\
\hline 105.9 & 0.992 \\
\hline 109.8 & 0.987 & \\
\hline 113.8 & 0.982 & \\
\hline 117.8 & 1.019 \\
\hline 121.7 & 1.221 & \\
\hline 125.7 & 0.999 \\
\hline
\end{tabular}

a The inlet cesium feed concentration (i.e., $3.51 \times 10^{-4} \mathrm{M}$ ) was used to normalize the breakthrough data.

Table H-4. Key column parameters for CST IE-911 packed columns using SRS-Avg simulant taken by Wilmarth et al. (1999) at $25 \mathrm{C}( \pm 5 \mathrm{C}){ }^{\mathrm{a}}$

\begin{tabular}{|c|c|c|c|c|}
\hline Parameter & SRS-Avg-Test 1 & SRS-Avg-Test 2 & $\begin{array}{c}\text { SRS-Avg-Test 3 } \\
\text { (a \& b) } \mathbf{b}\end{array}$ & SRS-Avg-Test 4 \\
\hline \hline $\begin{array}{c}\text { Column diameter } \\
\text { (cm) }\end{array}$ & 1.50 & 1.50 & 1.50 & 2.50 \\
\hline Column length (cm) & 10.0 & 10.0 & 10.0 & 10.0 \\
\hline CST (Lot \#) & $98-05$ & $98-05$ & $98-05$ & $98-05$ \\
\hline $\begin{array}{c}\text { Average superficial } \\
\text { velocity (cm/min) }\end{array}$ & 5.50 & 7.00 & 4.10 & 4.10 \\
\hline $\begin{array}{c}\text { Average temperature } \\
\text { (C) }\end{array}$ & 25 & 25 & 25 & 25 \\
\hline $\begin{array}{c}\text { Avg. Cs feed } \\
\text { concentration (M) }\end{array}$ & $1.30 \mathrm{E}-04$ & $1.24 \mathrm{E}-04$ & $1.43 \mathrm{E}-04$ & $1.366 \mathrm{E}-04$ \\
\hline $\begin{array}{c}\text { Column volume (ml) } \\
\text { Flowrate Q (ml/min) } \\
\text { [CV/hr] }\end{array}$ & 17.6714 & 17.6714 & 17.6714 & 49.0874 \\
\hline
\end{tabular}

${ }^{a}$ The SRS-Avg simulant feed composition is provided in Appendix C.

b Same basic column test where for Test3a the CST had prior exposure to humid air, while Test3b did not. 
Preliminary Ion Exchange Modeling for Removal of Cesium from

Table H-5. Cesium breakthrough data for CST IE-911 and SRS-Avg waste simulant taken by Wilmarth et al. (1999) at $\sim 25 \mathrm{C}$.

\begin{tabular}{|c|c|c|c|c|c|}
\hline \multicolumn{2}{|c|}{$\begin{array}{l}\text { SRS-Avg-Test1 } \\
\text { (CST lot 96-02) }\end{array}$} & \multicolumn{2}{|c|}{$\begin{array}{l}\text { SRS-Avg-Test2 } \\
\text { (CST lot 96-02) }\end{array}$} & \multicolumn{2}{|c|}{$\begin{array}{l}\text { SRS-Avg-Test4 } \\
\text { (CST lot 96-04) }\end{array}$} \\
\hline $\begin{array}{l}\text { Time } \\
\text { (hrs) }\end{array}$ & $\begin{array}{l}\text { Normalized } \\
\text { cesium } \\
\text { breakthrough } \\
\qquad\left(\mathrm{c} / \mathbf{c}_{\mathbf{o}}\right)^{\mathrm{a}}\end{array}$ & $\begin{array}{l}\text { Time } \\
\text { (hrs) }\end{array}$ & $\begin{array}{l}\text { Normalized } \\
\text { cesium } \\
\text { breakthrough } \\
\qquad\left(\mathrm{c} / \mathbf{c}_{\mathbf{o}}\right)^{\mathrm{a}}\end{array}$ & $\begin{array}{l}\text { Time } \\
\text { (hrs) }\end{array}$ & $\begin{array}{l}\text { Normalized } \\
\text { cesium } \\
\text { breakthrough } \\
\left(\mathbf{c} / \mathbf{c}_{\mathbf{o}}\right)^{\mathrm{a}}\end{array}$ \\
\hline 0.0 & 0.000 & 0.0 & 0.000 & 0.0 & 0.000 \\
\hline 4.0 & 0.184 & 8.0 & 0.390 & 0.0 & 0.033 \\
\hline 16.0 & 0.488 & 16.0 & 0.553 & 8.0 & 0.184 \\
\hline 28.0 & 0.574 & 20.0 & 0.615 & 20.0 & 0.307 \\
\hline 36.0 & 0.661 & 28.0 & 0.720 & 32.0 & 0.438 \\
\hline 44.0 & 0.701 & 36.0 & 0.756 & 44.0 & 0.534 \\
\hline 52.0 & 0.801 & 40.0 & 0.793 & 56.0 & 0.691 \\
\hline 60.0 & 0.857 & 48.0 & 0.824 & 68.0 & 0.736 \\
\hline 72.0 & 0.895 & 56.0 & 0.864 & 80.0 & 0.873 \\
\hline 84.0 & 1.039 & 60.0 & 0.867 & 92.0 & 0.694 \\
\hline 96.0 & 0.974 & 64.0 & 0.869 & 104.0 & 0.718 \\
\hline 108.0 & 1.031 & 68.0 & 0.875 & 116.0 & 0.742 \\
\hline 120.0 & 1.004 & 76.0 & 0.916 & 128.0 & 0.802 \\
\hline 132.0 & 1.004 & 80.0 & 0.886 & 140.0 & 0.754 \\
\hline \multirow[t]{7}{*}{140.0} & 1.018 & 88.0 & 0.882 & 152.0 & 0.823 \\
\hline & & 100.0 & 0.969 & 164.0 & 0.785 \\
\hline & & 104.0 & 0.936 & & \\
\hline & & 112.0 & 0.957 & & \\
\hline & & 120.0 & 0.950 & & \\
\hline & & 124.0 & 0.957 & & \\
\hline & & 132.0 & 0.900 & & \\
\hline
\end{tabular}

a The inlet cesium feed concentration (i.e., $1.30 \times 10^{-4} \mathrm{M}$ for Test1, $1.24 \times 10^{-4} \mathrm{M}$ for Test2, and $1.366 \times 10^{-4} \mathrm{M}$ for Test4) was used to normalize the breakthrough data. 
Preliminary Ion Exchange Modeling for Removal of Cesium from

Table H-6. Cesium breakthrough data for CST IE-911 (with and without prior exposure to humid air) and SRS-Avg waste simulant taken by Wilmarth et al. (1999) at $\sim 25$ C.

\begin{tabular}{|c|c|c|c|}
\hline \multicolumn{2}{|c|}{$\begin{array}{c}\text { SRS-Avg-Test3a } \\
\text { (CST lot 98-05) }\end{array}$} & \multicolumn{2}{|c|}{$\begin{array}{c}\text { SRS-Avg-Test3b } \\
\text { (CST lot 98-05) }\end{array}$} \\
\hline $\begin{array}{l}\text { Time } \\
\text { (hrs) }\end{array}$ & $\begin{array}{c}\text { Normalized Cs } \\
\text { breakthrough } \\
\left(\mathbf{c} / \mathbf{c}_{\mathrm{o}}\right)^{\mathrm{a}}\end{array}$ & $\begin{array}{l}\text { Time } \\
\text { (hrs) }\end{array}$ & $\begin{array}{c}\text { Normalized Cs } \\
\text { breakthrough } \\
\left(\mathbf{c} / \mathbf{c}_{\mathbf{o}}\right)^{\mathrm{a}}\end{array}$ \\
\hline 0.0 & 0.000 & 0.0 & 0.000 \\
\hline 2.0 & 0.020 & 2.0 & 0.001 \\
\hline 6.0 & 0.078 & 6.0 & 0.058 \\
\hline 10.0 & 0.146 & 10.0 & 0.114 \\
\hline 14.0 & 0.208 & 14.0 & 0.166 \\
\hline 18.0 & 0.261 & 18.0 & 0.217 \\
\hline 22.0 & 0.281 & 22.0 & 0.248 \\
\hline 26.0 & 0.279 & 26.0 & 0.307 \\
\hline 30.0 & 0.349 & 30.0 & 0.344 \\
\hline 34.0 & 0.333 & 34.0 & 0.371 \\
\hline 38.0 & 0.409 & 38.0 & 0.477 \\
\hline 42.0 & 0.379 & 42.0 & 0.636 \\
\hline 46.0 & 0.474 & 46.0 & 0.598 \\
\hline 50.0 & 0.510 & 50.0 & 0.726 \\
\hline 54.0 & 0.469 & 54.0 & 0.539 \\
\hline 58.0 & 0.630 & 58.0 & 0.545 \\
\hline 62.0 & 0.566 & 62.0 & 0.731 \\
\hline 66.0 & 0.603 & 66.0 & 0.539 \\
\hline 70.0 & 0.689 & 70.0 & 0.694 \\
\hline 74.0 & 0.678 & 74.0 & 0.833 \\
\hline 78.0 & 0.694 & 78.0 & 0.972 \\
\hline 82.0 & 0.683 & 82.0 & 0.902 \\
\hline 86.0 & 0.721 & 86.0 & 0.785 \\
\hline 90.0 & 0.705 & 90.0 & 0.747 \\
\hline 94.0 & 0.705 & 94.0 & 0.950 \\
\hline 98.0 & 0.668 & 98.0 & 0.859 \\
\hline & & 102.0 & 0.783 \\
\hline & & 106.0 & 0.755 \\
\hline & & 110.0 & 0.745 \\
\hline & & 114.0 & 0.772 \\
\hline & & 118.0 & 0.761 \\
\hline & & 122.0 & 0.826 \\
\hline & & 126.0 & 0.848 \\
\hline & & 130.0 & 0.837 \\
\hline & & 134.0 & 0.886 \\
\hline & & 138.0 & 0.853 \\
\hline & & 142.0 & 0.821 \\
\hline & & 146.0 & 0.821 \\
\hline & & 150.0 & 0.832 \\
\hline & & 154.0 & 0.929 \\
\hline & & 158.0 & 0.946 \\
\hline & & 162.0 & 0.929 \\
\hline & & 166.0 & 0.891 \\
\hline & & 170.0 & 0.984 \\
\hline & & 174.0 & 0.880 \\
\hline & & 178.0 & 0.902 \\
\hline & & 182.0 & 0.880 \\
\hline & & 184.0 & 0.940 \\
\hline & & 188.0 & 0.935 \\
\hline & & 192.0 & 0.891 \\
\hline & & 196.0 & 0.902 \\
\hline
\end{tabular}

a The inlet cesium feed concentration (i.e., $1.43 \times 10^{-4} \mathrm{M}$ for both tests) was used to normalize the breakthrough data. 
WESTINGHOUSE SAVANNAH RIVER COMPANY

Report:

WSRC-TR-2001-00400

Preliminary Ion Exchange Modeling for Removal of Cesium from

Hanford Waste Using Hydrous Crystalline Silicotitanate Material

Revision (Date):

$0(07 / 05 / 02)$

Page:

318 of 338

Table H-7. Key column parameters for CST IE-911 packed columns using SRS-Avg simulant taken by Walker et al. (1998) at 22 C. ${ }^{a, b}$

\begin{tabular}{|c|c|c|c|}
\hline Parameter & SRS-Avg-Test 5 & SRS-Avg-Test 6 & SRS-Avg-Test 7 \\
\hline $\begin{array}{c}\text { Column diameter } \\
\text { (cm) }\end{array}$ & 1.50 & 1.50 & 1.43 \\
\hline $\begin{array}{c}\text { Column length } \\
\text { (cm) }\end{array}$ & 10.0 & 10.0 & 11.0 \\
\hline CST (Lot \#) & $96-02$ & $96-02$ & $96-04$ \\
\hline $\begin{array}{c}\text { Average } \\
\text { superficial } \\
\text { velocity (cm/min) }\end{array}$ & 0.27 & 0.98 & 4.1 \\
\hline $\begin{array}{c}\text { Average } \\
\text { temperature (C) }\end{array}$ & 22 & 22 & $1.40 \mathrm{E}-04$ \\
\hline $\begin{array}{c}\text { Avg. Cs feed } \\
\text { concentration } \\
\text { (M) }\end{array}$ & $1.40 \mathrm{E}-04$ & $1.40 \mathrm{E}-04$ & 17.7 \\
\hline $\begin{array}{c}\text { column volume } \\
\text { (ml) }\end{array}$ & 17.7 & 17.7 & 6.53 \\
\hline $\begin{array}{c}\text { flowrate Q } \\
\text { (ml/min) } \\
\text { [CV/hr] }\end{array}$ & 0.47 & 1.73 & {$[22.1]$} \\
\hline
\end{tabular}

a These column tests were originally designed to only compare the effects of superficial velocity on CST column performance; however, due to availability limitations the third column had to be packed with a different CST material (i.e., a CST production-scale batch).

b The SRS-Avg simulant feed composition is provided in Appendix C.

Table H-8. Cesium breakthrough data for CST IE-911 and SRS-Avg waste simulant taken by Walker et al. (1998) at $\sim 22 \mathrm{C}$.

\begin{tabular}{|c|c|c|c|c|c|}
\hline \multicolumn{2}{|c|}{$\begin{array}{l}\text { SRS-Avg-Test5 } \\
\text { (CST lot 96-02) }\end{array}$} & \multicolumn{2}{|c|}{$\begin{array}{l}\text { SRS-Avg-Test6 } \\
\text { (CST lot 96-02) }\end{array}$} & \multicolumn{2}{|c|}{$\begin{array}{l}\text { SRS-Avg-Test7 } \\
\text { (CST lot 96-04) }\end{array}$} \\
\hline $\begin{array}{l}\text { Time } \\
\text { (hrs) }\end{array}$ & $\begin{array}{c}\text { Normalized } \\
\text { cesium } \\
\text { breakthrough } \\
\left(\mathbf{c} / \mathbf{c}_{\mathbf{o}}\right)^{\mathrm{a}} \\
\end{array}$ & $\begin{array}{l}\text { Time } \\
\text { (hrs) }\end{array}$ & $\begin{array}{c}\text { Normalized } \\
\text { cesium } \\
\text { breakthrough } \\
\left(\mathbf{c} / \mathbf{c}_{\mathbf{o}}\right)^{\mathrm{a}}\end{array}$ & $\begin{array}{l}\text { Time } \\
\text { (hrs) }\end{array}$ & $\begin{array}{c}\text { Normalized } \\
\text { cesium } \\
\text { breakthrough } \\
\left(\mathbf{c} / \mathbf{c}_{\mathbf{0}}\right)^{\mathrm{a}}\end{array}$ \\
\hline 0.0 & $0.000 \mathrm{E}+00$ & 0.0 & $1.571 \mathrm{E}-03$ & 0.0 & $0.000 \mathrm{E}+00$ \\
\hline 1.6 & $1.500 \mathrm{E}-05$ & 22.4 & $3.141 \mathrm{E}-03$ & 1.0 & $1.890 \mathrm{E}-02$ \\
\hline 7.5 & $1.100 \mathrm{E}-05$ & 57.3 & $1.518 \mathrm{E}-02$ & 3.9 & $4.730 \mathrm{E}-02$ \\
\hline 21.5 & $2.000 \mathrm{E}-06$ & 79.8 & $5.183 \mathrm{E}-02$ & 6.9 & $1.094 \mathrm{E}-01$ \\
\hline 55.8 & $3.400 \mathrm{E}-05$ & 103.2 & $1.005 \mathrm{E}-01$ & 9.8 & $1.702 \mathrm{E}-01$ \\
\hline 143.2 & $4.000 \mathrm{E}-06$ & 115.2 & $1.346 \mathrm{E}-01$ & 12.8 & $2.374 \mathrm{E}-01$ \\
\hline 153.7 & $7.000 \mathrm{E}-06$ & 138.9 & $1.671 \mathrm{E}-01$ & 15.7 & $2.820 \mathrm{E}-01$ \\
\hline 198.0 & $1.600 \mathrm{E}-05$ & 150.7 & $1.971 \mathrm{E}-01$ & 18.7 & $3.427 \mathrm{E}-01$ \\
\hline 222.7 & $2.900 \mathrm{E}-05$ & 162.6 & $2.551 \mathrm{E}-01$ & 21.6 & $3.552 \mathrm{E}-01$ \\
\hline 249.7 & $1.100 \mathrm{E}-04$ & 174.4 & $2.435 \mathrm{E}-01$ & 24.7 & 4.777E-01 \\
\hline 263.6 & $2.200 \mathrm{E}-04$ & 198.1 & $4.821 \mathrm{E}-01$ & 27.7 & $5.061 \mathrm{E}-01$ \\
\hline 304.4 & $4.600 \mathrm{E}-04$ & 210.0 & 4.101E-01 & 30.7 & $5.128 \mathrm{E}-01$ \\
\hline 312.6 & $8.500 \mathrm{E}-04$ & 221.9 & $5.809 \mathrm{E}-01$ & 33.7 & $5.973 \mathrm{E}-01$ \\
\hline 337.5 & $1.630 \mathrm{E}-03$ & 233.8 & $6.059 \mathrm{E}-01$ & 36.7 & $6.716 \mathrm{E}-01$ \\
\hline 361.8 & $2.560 \mathrm{E}-03$ & 245.6 & $6.558 \mathrm{E}-01$ & 39.7 & $6.757 \mathrm{E}-01$ \\
\hline 391.8 & $4.161 \mathrm{E}-03$ & 246.8 & $6.808 \mathrm{E}-01$ & 42.7 & $7.027 \mathrm{E}-01$ \\
\hline 409.5 & $6.411 \mathrm{E}-03$ & 259.9 & $6.246 \mathrm{E}-01$ & 45.7 & 7.297E-01 \\
\hline
\end{tabular}


WESTINGHOUSE SAVANNAH RIVER COMPANY

Report: WSRC-TR-2001-00400

Preliminary Ion Exchange Modeling for Removal of Cesium from

Revision (Date):

$0(07 / 05 / 02)$

Hanford Waste Using Hydrous Crystalline Silicotitanate Material

Page:

319 of 338

\begin{tabular}{|c|c|c|c|c|c|}
\hline 433.9 & $8.747 \mathrm{E}-03$ & 283.8 & $7.995 \mathrm{E}-01$ & 48.5 & $6.533 \mathrm{E}-01$ \\
\hline 457.9 & $1.220 \mathrm{E}-02$ & 295.8 & $8.182 \mathrm{E}-01$ & 51.5 & $7.181 \mathrm{E}-01$ \\
\hline 481.1 & $1.722 \mathrm{E}-02$ & 319.9 & 7.333E-01 & 54.5 & 7.049E-01 \\
\hline 504.8 & $2.222 \mathrm{E}-02$ & 344.0 & $7.641 \mathrm{E}-01$ & 57.5 & $7.335 \mathrm{E}-01$ \\
\hline 530.0 & $2.957 \mathrm{E}-02$ & 356.0 & $7.949 \mathrm{E}-01$ & 60.5 & $7.221 \mathrm{E}-01$ \\
\hline 550.9 & $3.366 \mathrm{E}-02$ & 367.9 & $6.513 \mathrm{E}-01$ & 63.5 & $8.223 \mathrm{E}-01$ \\
\hline \multirow[t]{33}{*}{575.2} & $4.455 \mathrm{E}-02$ & 392.3 & $8.513 \mathrm{E}-01$ & 66.5 & $7.450 \mathrm{E}-01$ \\
\hline & & 416.6 & $8.615 \mathrm{E}-01$ & 69.5 & $7.851 \mathrm{E}-01$ \\
\hline & & 429.0 & 7.385E-01 & 72.7 & 7.332E-01 \\
\hline & & 441.4 & $7.692 \mathrm{E}-01$ & 74.6 & $7.830 \mathrm{E}-01$ \\
\hline & & 465.9 & $8.772 \mathrm{E}-01$ & 77.6 & $7.880 \mathrm{E}-01$ \\
\hline & & 490.8 & $9.006 \mathrm{E}-01$ & 80.6 & $7.531 \mathrm{E}-01$ \\
\hline & & 516.0 & $8.713 \mathrm{E}-01$ & 83.5 & $7.930 \mathrm{E}-01$ \\
\hline & & 540.2 & $8.655 \mathrm{E}-01$ & 86.5 & $7.731 \mathrm{E}-01$ \\
\hline & & 552.4 & $9.474 \mathrm{E}-01$ & 89.5 & $8.080 \mathrm{E}-01$ \\
\hline & & & & 92.4 & $7.930 \mathrm{E}-01$ \\
\hline & & & & 94.4 & $8.922 \mathrm{E}-01$ \\
\hline & & & & 97.4 & $8.824 \mathrm{E}-01$ \\
\hline & & & & 100.4 & $8.971 \mathrm{E}-01$ \\
\hline & & & & 103.4 & $9.020 \mathrm{E}-01$ \\
\hline & & & & 106.4 & $9.265 \mathrm{E}-01$ \\
\hline & & & & 109.4 & $8.971 \mathrm{E}-01$ \\
\hline & & & & 112.3 & $8.725 \mathrm{E}-01$ \\
\hline & & & & 115.3 & $8.971 \mathrm{E}-01$ \\
\hline & & & & 117.3 & $9.375 \mathrm{E}-01$ \\
\hline & & & & 120.4 & $9.615 \mathrm{E}-01$ \\
\hline & & & & 123.4 & $1.024 \mathrm{E}+00$ \\
\hline & & & & 126.4 & $9.327 \mathrm{E}-01$ \\
\hline & & & & 129.5 & $9.038 \mathrm{E}-01$ \\
\hline & & & & 132.5 & $1.000 \mathrm{E}+00$ \\
\hline & & & & 135.5 & $9.135 \mathrm{E}-01$ \\
\hline & & & & 138.6 & $9.327 \mathrm{E}-01$ \\
\hline & & & & 140.6 & $9.417 \mathrm{E}-01$ \\
\hline & & & & 143.6 & $9.806 \mathrm{E}-01$ \\
\hline & & & & 146.6 & $1.015 \mathrm{E}+00$ \\
\hline & & & & 149.7 & $9.709 \mathrm{E}-01$ \\
\hline & & & & 152.7 & $1.000 \mathrm{E}+00$ \\
\hline & & & & 155.7 & $9.563 \mathrm{E}-01$ \\
\hline & & & & 158.8 & $1.005 \mathrm{E}+00$ \\
\hline
\end{tabular}

a The inlet cesium feed concentration (i.e., $1.40 \times 10^{-4} \mathrm{M}$ ) was used to normalize the breakthrough data. 
Table H-9. Cesium breakthrough data for CST IE-911 (Lot 98-05) and SRS high OH simulant taken by Walker et al. (1999) at $31 \mathrm{C}$.

\begin{tabular}{|c|c|c|c|}
\hline \multicolumn{2}{|c|}{$\begin{array}{l}\text { Effectively a Lead Column } \\
(10 \mathrm{~cm} \text { down column length) }\end{array}$} & \multicolumn{2}{|c|}{$\begin{array}{l}\text { Effectively a Lag Column } \\
\text { (85 cm down column length) }\end{array}$} \\
\hline $\begin{array}{l}\text { Time } \\
\text { (hrs) }\end{array}$ & $\begin{array}{l}\text { Normalized } \\
\text { cesium } \\
\text { breakthrough } \\
\left(\mathbf{c} / \mathbf{c}_{\mathbf{0}}\right)^{\mathrm{a}}\end{array}$ & $\begin{array}{l}\text { Time } \\
\text { (hrs) }\end{array}$ & $\begin{array}{l}\text { Normalized } \\
\text { cesium } \\
\text { breakthrough } \\
\left(\mathbf{c} / \mathbf{c}_{\mathbf{o}}\right)^{\mathrm{a}}\end{array}$ \\
\hline 0.0 & 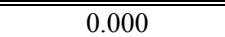 & 0.0 & 0.000 \\
\hline 2.9 & 0.042 & 2.9 & 0.000 \\
\hline 6.4 & 0.126 & 6.4 & 0.000 \\
\hline 10.1 & 0.262 & 10.1 & 0.000 \\
\hline 14.0 & 0.364 & 14.0 & 0.000 \\
\hline 17.8 & 0.424 & 17.8 & 0.000 \\
\hline 21.6 & 0.470 & 21.6 & 0.000 \\
\hline 25.3 & 0.550 & 25.3 & 0.000 \\
\hline 29.1 & 0.623 & 29.1 & 0.000 \\
\hline 32.8 & 0.622 & 32.8 & 0.000 \\
\hline 36.6 & 0.678 & 36.6 & 0.000 \\
\hline 40.5 & 0.748 & 40.5 & 0.000 \\
\hline 44.3 & 0.704 & 44.3 & 0.000 \\
\hline 48.1 & 0.741 & 48.1 & 0.000 \\
\hline 52.0 & 0.790 & 52.0 & 0.000 \\
\hline 55.7 & 0.827 & 55.7 & 0.000 \\
\hline 59.6 & 0.820 & 59.6 & 0.000 \\
\hline 63.4 & 0.850 & 63.4 & 0.000 \\
\hline 67.3 & 0.840 & 67.3 & 0.000 \\
\hline 72.6 & 0.852 & 72.6 & 0.000 \\
\hline
\end{tabular}

a The inlet cesium feed concentration (i.e., $3.70 \times 10^{-4} \mathrm{M}$ ) was used to normalize the breakthrough data. 
Table H-10. Key column parameters for CST IE-911 packed columns using PNNL diluted AW-101 sample taken by Hendrickson (1997) at 25 C. ${ }^{a}$

\begin{tabular}{|c|c|}
\hline Parameter & $\begin{array}{c}\text { PNNL-AW101- } \\
\text { Test 1 }\end{array}$ \\
\hline \hline $\begin{array}{c}\text { Column diameter } \\
\text { (cm) }\end{array}$ & 1.0 \\
\hline $\begin{array}{c}\text { Column length } \\
\text { (cm) }\end{array}$ & 10.0 \\
\hline CST (Lot \#) & $96-01$ \\
\hline $\begin{array}{c}\text { Average } \\
\text { superficial } \\
\text { velocity (cm/min) }\end{array}$ & 1.06 \\
\hline $\begin{array}{c}\text { Average } \\
\text { temperature (C) }\end{array}$ & 25 \\
\hline $\begin{array}{c}\text { Avg. Cs feed } \\
\text { concentration } \\
\text { (M) }\end{array}$ & $7.26 \mathrm{E}-05$ \\
\hline $\begin{array}{c}\text { Column volume } \\
\text { (ml) }\end{array}$ & 7.854 \\
\hline $\begin{array}{c}\text { Flowrate Q } \\
\text { (ml/min) } \\
\text { [CV/hr] }\end{array}$ & 0.83 \\
\hline $\begin{array}{c}\text { (cm } \\
\text { (cample feed comp }\end{array}$ & \\
\hline
\end{tabular}

${ }^{\text {a }}$ The PNNL AW-101 sample feed composition is provided in Appendix C.

Table H-11. Cesium breakthrough data for CST IE-911 and PNNL diluted AW-101 sample taken by Hendrickson (1997) at $\sim 25$ C.

\begin{tabular}{|c|c|}
\hline \multicolumn{2}{|c|}{$\begin{array}{l}\text { PNNL-AW101-Test1 } \\
\quad \text { (CST lot 96-01) }\end{array}$} \\
\hline $\begin{array}{l}\text { Time } \\
\text { (hrs) }\end{array}$ & $\begin{array}{l}\text { Normalized } \\
\text { cesium } \\
\text { breakthrough } \\
\left(\mathbf{c} / \mathbf{c}_{\mathbf{o}}\right)^{\mathrm{a}} \\
\end{array}$ \\
\hline 0.4 & 0.000 \\
\hline 5.8 & 0.000 \\
\hline 15.1 & 0.000 \\
\hline 19.6 & 0.000 \\
\hline 24.3 & 0.000 \\
\hline 28.7 & 0.001 \\
\hline 32.3 & 0.002 \\
\hline 36.0 & 0.005 \\
\hline 39.6 & 0.008 \\
\hline 43.6 & 0.013 \\
\hline 44.9 & 0.019 \\
\hline 51.4 & 0.027 \\
\hline 55.3 & 0.038 \\
\hline 59.6 & 0.056 \\
\hline 63.5 & 0.071 \\
\hline 67.5 & 0.102 \\
\hline 71.6 & 0.136 \\
\hline 75.6 & 0.163 \\
\hline
\end{tabular}


Preliminary Ion Exchange Modeling for Removal of Cesium from

\begin{tabular}{|c|c|}
\hline 80.0 & 0.182 \\
\hline 83.4 & 0.231 \\
\hline 87.8 & 0.263 \\
\hline 91.8 & 0.309 \\
\hline 95.7 & 0.347 \\
\hline 99.4 & 0.399 \\
\hline 103.7 & 0.430 \\
\hline 108.0 & 0.460 \\
\hline 111.8 & 0.470 \\
\hline 115.7 & 0.571 \\
\hline 119.6 & 0.682 \\
\hline 124.2 & 0.656 \\
\hline 128.3 & 0.674 \\
\hline
\end{tabular}

a The inlet cesium feed concentration (i.e., $7.26 \times 10^{-5} \mathrm{M}$ ) was used to normalize the breakthrough data.

Table H-12. Key column parameters for CST IE-911 packed columns using MVST W-27 sample taken by Lee et al. (1997b) at 25 C. a,b

\begin{tabular}{|c|c|c|}
\hline Parameter & ORNL-W27-Test 1 & ORNL-W27-Test 2 \\
\hline \hline $\begin{array}{c}\text { Column diameter } \\
\text { (cm) }\end{array}$ & 1.50 & 1.50 \\
\hline $\begin{array}{c}\text { Column length } \\
\text { (cm) }\end{array}$ & 5.659 & 5.659 \\
\hline CST (Lot \#) & $-38 \mathrm{~b}$ & $-38 \mathrm{~b}$ \\
\hline $\begin{array}{c}\text { Average } \\
\text { superficial } \\
\text { velocity (cm/min) }\end{array}$ & 0.283 & 0.566 \\
\hline $\begin{array}{c}\text { Average } \\
\text { temperature (C) }\end{array}$ & 25 & 25 \\
\hline $\begin{array}{c}\text { Avg. Cs feed } \\
\text { concentration } \\
\text { (M) }\end{array}$ & $7.04 \mathrm{E}-06$ & $7.04 \mathrm{E}-06$ \\
\hline $\begin{array}{c}\text { column volume } \\
\text { (ml) }\end{array}$ & 10.0 & 10.0 \\
\hline $\begin{array}{c}\text { Bed density } \\
\text { (g/ml) }\end{array}$ & 1.15 & 1.15 \\
\hline $\begin{array}{c}\text { Flowrate Q } \\
\text { (ml/min) } \\
\text { [CV/hr] }\end{array}$ & 0.5 & {$[6.0]$} \\
\hline
\end{tabular}

${ }^{\text {a }}$ The same column comparing the effect of superficial velocity on CST column performance.

${ }^{b}$ The MVST W-27 sample feed composition is provided in this appendix. 
Preliminary Ion Exchange Modeling for Removal of Cesium from

Revision (Date):

Table H-13. Cesium breakthrough data for CST IE-911 and MVST W-27 waste sample taken by Lee et al. (1997b) at $\sim 25 \mathrm{C}$.

\begin{tabular}{|c|c|c|c|}
\hline \multicolumn{2}{|c|}{$\begin{array}{l}\text { ORNL-W27-Test1 } \\
\text { (CST lot -38b) }\end{array}$} & \multicolumn{2}{|c|}{$\begin{array}{l}\text { ORNL-W27-Test2 } \\
\text { (CST lot-38b) }\end{array}$} \\
\hline $\begin{array}{l}\text { Time } \\
\text { (hrs) }\end{array}$ & $\begin{array}{l}\text { Normalized } \\
\text { cesium } \\
\text { breakthrough } \\
\left(\mathbf{c} / \mathbf{c}_{\mathbf{0}}\right)^{\mathrm{a}}\end{array}$ & $\begin{array}{l}\text { Time } \\
\text { (hrs) }\end{array}$ & $\begin{array}{l}\text { Normalized } \\
\text { cesium } \\
\text { breakthrough } \\
\left(\mathbf{c} / \mathbf{c}_{\mathbf{o}}\right)^{\mathrm{a}}\end{array}$ \\
\hline 0.0 & 0.000 & 0.0 & 0.000 \\
\hline 16.7 & 0.002 & 8.3 & 0.003 \\
\hline 33.3 & 0.005 & 10.0 & 0.004 \\
\hline 50.0 & 0.039 & 13.3 & 0.009 \\
\hline 66.7 & 0.121 & 16.7 & 0.019 \\
\hline 83.3 & 0.245 & 25.0 & 0.070 \\
\hline 100.0 & 0.389 & 33.3 & 0.160 \\
\hline 116.7 & 0.523 & 41.7 & 0.300 \\
\hline 133.3 & 0.631 & 50.0 & 0.400 \\
\hline 146.0 & 0.694 & 58.3 & 0.510 \\
\hline
\end{tabular}

${ }^{a}$ The inlet cesium feed concentration (i.e., $7.04 \times 10^{-6} \mathrm{M}$ ) was used to normalize the breakthrough data.

Table H-14. Key column parameters for CST IE-911 packed pilot-scale two-column carousel facility using MVST W-29 waste streams taken by Walker, Jr., et al. (1998) at 25 C. ${ }^{\text {a }}$

\begin{tabular}{|c|c|c|c|c|}
\hline Parameter & ORNL-CsRD-Run2 & ORNL-CsRD-Run3 & ORNL-CsRD-Run4a & ORNL-CsRD-Run4b \\
\hline $\begin{array}{c}\text { Column diameter } \\
(\mathrm{cm})\end{array}$ & 30.6 & 1.50 & 1.50 & 1.50 \\
\hline $\begin{array}{c}\text { Column length } \\
\text { (cm) }\end{array}$ & 51.672 & 5.659 & 5.659 & 5.659 \\
\hline CST (Lot \#) & $-38 b(96-01)$ & $-38 b(96-01)$ & $-38 b(96-01)$ & $-38 b(96-01)$ \\
\hline $\begin{array}{c}\text { Average } \\
\text { superficial } \\
\text { velocity }(\mathrm{cm} / \mathrm{min})\end{array}$ & 2.584 & 5.167 & 5.167 & 5.167 \\
\hline $\begin{array}{c}\text { Average } \\
\text { temperature (C) }\end{array}$ & 25 & 25 & 25 & 25 \\
\hline $\begin{array}{c}\text { Avg. Cs feed } \\
\text { concentration } \\
\text { (M) }\end{array}$ & $1.35 \mathrm{E}-05$ & $1.35 \mathrm{E}-05$ & $5.10 \mathrm{E}-06$ & $5.10 \mathrm{E}-06$ \\
\hline $\begin{array}{c}\text { Column volume } \\
\text { (L) }\end{array}$ & 38.0 & 38.0 & 38.0 & 38.0 \\
\hline $\begin{array}{l}\text { Bed density } \\
(\mathrm{g} / \mathrm{ml})\end{array}$ & 1.0 & 1.0 & 1.0 & 1.0 \\
\hline $\begin{array}{c}\text { Flowrate Q } \\
(\mathrm{ml} / \mathrm{min}) \\
{[\mathrm{CV} / \mathrm{hr}]}\end{array}$ & $\begin{array}{c}1900.0 \\
{[3.0]}\end{array}$ & $\begin{array}{c}3800.0 \\
{[6.0]}\end{array}$ & $\begin{array}{c}3800.0 \\
{[6.0]}\end{array}$ & $\begin{array}{c}3800.0 \\
{[6.0]}\end{array}$ \\
\hline
\end{tabular}


Table H-15. Cycle 1 ORNL-CsRD-Run2 cesium breakthrough data for CST IE-911 and MVST W-29 waste streams taken by Walker, Jr., et al. (1998) at $\sim 25$ C.

\begin{tabular}{|c|c|}
\hline \multicolumn{2}{|c|}{$\begin{array}{l}\text { ORNL-CsRD-Run2 } \\
\text { (fresh lead column) } \\
\text { (CST lot }-\mathbf{3 8 b})\end{array}$} \\
\hline $\begin{array}{l}\text { Time } \\
\text { (hrs) }\end{array}$ & $\begin{array}{c}\text { Normalized cesium } \\
\text { breakthrough } \\
\left(\mathbf{c} / \mathbf{c}_{\mathbf{o}}\right)^{\mathbf{a}}\end{array}$ \\
\hline 8.3 & $\begin{array}{l}0.014 \\
\end{array}$ \\
\hline 16.7 & 0.007 \\
\hline 25.0 & 0.014 \\
\hline 33.3 & 0.024 \\
\hline 41.7 & 0.043 \\
\hline 50.0 & 0.085 \\
\hline 58.3 & 0.113 \\
\hline 66.7 & 0.151 \\
\hline 75.0 & 0.193 \\
\hline 83.3 & 0.231 \\
\hline 91.7 & 0.113 \\
\hline 100.0 & 0.302 \\
\hline 108.3 & 0.293 \\
\hline 116.7 & 0.354 \\
\hline 125.0 & 0.425 \\
\hline 133.3 & 0.425 \\
\hline 141.7 & 0.472 \\
\hline 150.0 & 0.467 \\
\hline 158.3 & 0.509 \\
\hline
\end{tabular}

a The inlet cesium feed concentration (i.e., $1.35 \times 10^{-5} \mathrm{M}$ ) was used to normalize the breakthrough data. 
Table H-16. Cycle 1 ORNL-CsRD-Run3 cesium breakthrough data for CST IE-911 and MVST W-29 waste streams taken by Walker, Jr., et al. (1998) at 25 C.

\begin{tabular}{|c|c|c|c|}
\hline \multicolumn{2}{|c|}{$\begin{array}{l}\text { ORNL-CsRD-Run3 } \\
\text { (fresh lead column) } \\
\text { (CST lot }-\mathbf{3 8 b})\end{array}$} & \multicolumn{2}{|c|}{$\begin{array}{l}\text { ORNL-CsRD-Run3 } \\
\text { (fresh lag column) } \\
\text { (CST lot -38b) }\end{array}$} \\
\hline $\begin{array}{l}\text { Time } \\
\text { (hrs) }\end{array}$ & $\begin{array}{l}\text { Normalized } \\
\text { cesium } \\
\text { breakthrough } \\
\left(\mathbf{c} / \mathbf{c}_{\mathbf{0}}\right)^{\mathrm{a}} \\
\end{array}$ & $\begin{array}{l}\text { Time } \\
\text { (hrs) }\end{array}$ & $\begin{array}{l}\text { Normalized } \\
\text { cesium } \\
\text { breakthrough } \\
\left(\mathbf{c} / \mathbf{c}_{\mathbf{o}}\right)^{\mathrm{a}} \\
\end{array}$ \\
\hline 8.3 & 0.038 & 8.3 & 0.003 \\
\hline 16.7 & 0.114 & 16.7 & 0.006 \\
\hline 25.0 & 0.162 & 25.0 & 0.011 \\
\hline 33.3 & 0.210 & 33.3 & 0.020 \\
\hline 41.7 & 0.286 & 41.7 & 0.034 \\
\hline 50.0 & 0.371 & 50.0 & 0.057 \\
\hline 58.3 & 0.333 & 58.3 & 0.109 \\
\hline 66.7 & 0.324 & 66.7 & 0.097 \\
\hline 75.0 & 0.429 & 75.0 & 0.111 \\
\hline 83.3 & 0.424 & 83.3 & 0.143 \\
\hline 91.7 & 0.562 & 91.7 & 0.177 \\
\hline 100.0 & 0.543 & 100.0 & 0.211 \\
\hline 108.3 & 0.591 & 108.3 & 0.229 \\
\hline 116.7 & 0.657 & 116.7 & 0.269 \\
\hline 125.0 & 0.705 & 125.0 & 0.383 \\
\hline 133.3 & 0.700 & 133.3 & 0.360 \\
\hline 141.7 & 0.752 & 141.7 & 0.371 \\
\hline 150.0 & 0.743 & 150.0 & 0.394 \\
\hline 158.3 & 0.781 & 158.3 & 0.469 \\
\hline 166.7 & 0.733 & 166.7 & 0.451 \\
\hline 172.0 & 0.791 & 172.0 & 0.486 \\
\hline
\end{tabular}

${ }^{a}$ The inlet cesium feed concentration (i.e., $1.35 \times 10^{-5} \mathrm{M}$ ) was used to normalize the breakthrough data. 
Table H-17. Cycle 1 ORNL-CsRD-Run4 cesium breakthrough data for CST IE-911 and MVST W-29 waste streams taken by Walker, Jr., et al. (1998) at 25 C.

\begin{tabular}{|c|c|c|c|}
\hline \multicolumn{2}{|c|}{$\begin{array}{l}\text { ORNL-CsRD-Run4a } \\
\text { (fresh lead column) } \\
\text { (CST lot -38b) }\end{array}$} & \multicolumn{2}{|c|}{$\begin{array}{l}\text { ORNL-CsRD-Run4a } \\
\text { (fresh lag column) } \\
\text { (CST lot }-\mathbf{3 8 b})\end{array}$} \\
\hline $\begin{array}{l}\text { Time } \\
\text { (hrs) }\end{array}$ & $\begin{array}{c}\text { Normalized } \\
\text { cesium } \\
\text { breakthrough } \\
\left(\mathbf{c} / \mathbf{c}_{\mathbf{o}}\right)^{\mathrm{a}} \\
\end{array}$ & $\begin{array}{l}\text { Time } \\
\text { (hrs) }\end{array}$ & $\begin{array}{l}\text { Normalized } \\
\text { cesium } \\
\text { breakthrough } \\
\left(\mathbf{c} / \mathbf{c}_{\mathbf{0}}\right)^{\mathrm{a}} \\
\end{array}$ \\
\hline 8.3 & 0.014 & 8.3 & 0.002 \\
\hline 16.7 & 0.031 & 16.7 & 0.002 \\
\hline 25.0 & 0.071 & 25.0 & 0.004 \\
\hline 33.3 & 0.120 & 33.3 & 0.004 \\
\hline 41.7 & 0.186 & 41.7 & 0.011 \\
\hline 50.0 & 0.249 & 50.0 & 0.017 \\
\hline 58.3 & 0.291 & 58.3 & 0.031 \\
\hline 66.7 & 0.331 & 66.7 & 0.045 \\
\hline 75.0 & 0.411 & 75.0 & 0.069 \\
\hline 83.3 & 0.434 & 83.3 & 0.086 \\
\hline 91.7 & 0.474 & 91.7 & 0.116 \\
\hline 100.0 & 0.514 & 100.0 & 0.142 \\
\hline 108.3 & 0.534 & 108.3 & 0.174 \\
\hline 116.7 & 0.554 & 116.7 & 0.193 \\
\hline 124.3 & 0.555 & 124.3 & 0.205 \\
\hline
\end{tabular}

a The inlet cesium feed concentration (i.e., $5.10 \times 10^{-6} \mathrm{M}$ ) was used to normalize the breakthrough data.

Table H-18. Cycle 2 ORNL-CsRD-Run4 cesium breakthrough data for CST IE-911 and MVST W-29 waste streams taken by Walker, Jr., et al. (1998) at 25 C.

\begin{tabular}{|c|c|c|c|}
\hline \multicolumn{2}{|c|}{$\begin{array}{l}\text { ORNL-CsRD-Run4b } \\
\text { (lead column now cycle } 1 \\
\text { lag column) } \\
\text { (CST lot }-\mathbf{3 8 b})\end{array}$} & \multicolumn{2}{|c|}{$\begin{array}{l}\text { ORNL-CsRD-Run4b } \\
\text { (fresh lag column) } \\
\text { (CST lot -38b) }\end{array}$} \\
\hline $\begin{array}{l}\text { Time } \\
\text { (hrs) }\end{array}$ & $\begin{array}{l}\text { Normalized } \\
\text { cesium } \\
\text { breakthrough } \\
\left(\mathbf{c} / \mathbf{c}_{\mathbf{o}}\right)^{\mathrm{a}} \\
\end{array}$ & $\begin{array}{l}\text { Time } \\
\text { (hrs) }\end{array}$ & $\begin{array}{l}\text { Normalized } \\
\text { cesium } \\
\text { breakthrough } \\
\qquad\left(\mathbf{c} / \mathbf{c}_{\mathbf{0}}\right)^{\mathrm{a}} \\
\end{array}$ \\
\hline 8.3 & 0.014 & 8.3 & 0.002 \\
\hline 16.7 & 0.031 & 16.7 & 0.002 \\
\hline 25.0 & 0.071 & 25.0 & 0.004 \\
\hline 33.3 & 0.120 & 33.3 & 0.004 \\
\hline 41.7 & 0.186 & 41.7 & 0.011 \\
\hline 50.0 & 0.249 & 50.0 & 0.017 \\
\hline 58.3 & 0.291 & 58.3 & 0.031 \\
\hline 66.7 & 0.331 & 66.7 & 0.045 \\
\hline 75.0 & 0.411 & 75.0 & 0.069 \\
\hline 83.3 & 0.434 & 83.3 & 0.086 \\
\hline 91.7 & 0.474 & 91.7 & 0.116 \\
\hline 100.0 & 0.514 & 100.0 & 0.142 \\
\hline 108.3 & 0.534 & 108.3 & 0.174 \\
\hline 116.7 & 0.554 & 116.7 & 0.193 \\
\hline 124.3 & 0.555 & 124.3 & 0.205 \\
\hline
\end{tabular}

${ }^{\text {a }}$ The inlet cesium feed concentration (i.e., $5.10 \times 10^{-6} \mathrm{M}$ ) was used to normalize the breakthrough data. 


\section{VERSE Input for SRS-Tank44-Test1a}

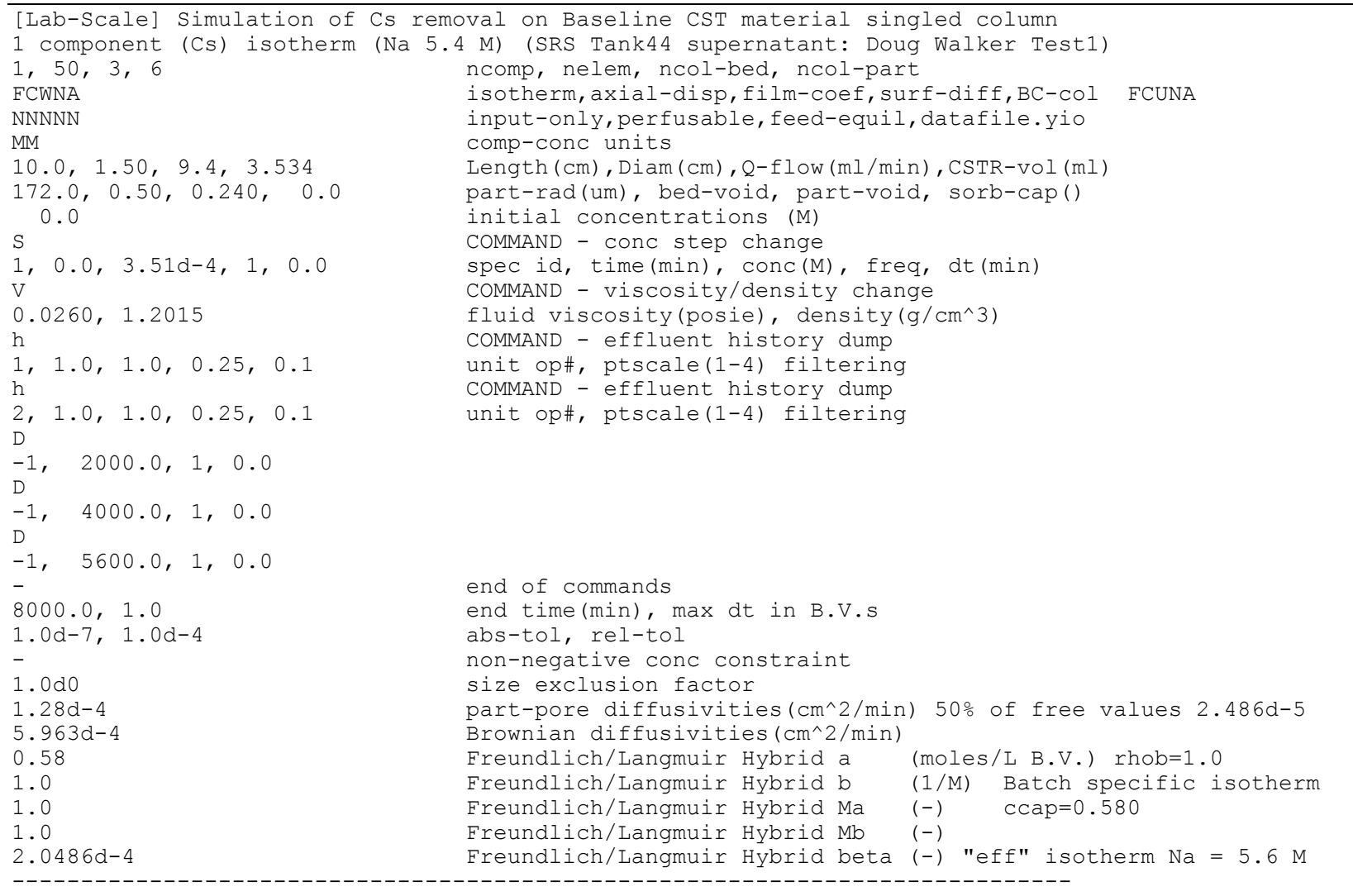

\section{VERSE Input for SRS-Tank44-Test1b}

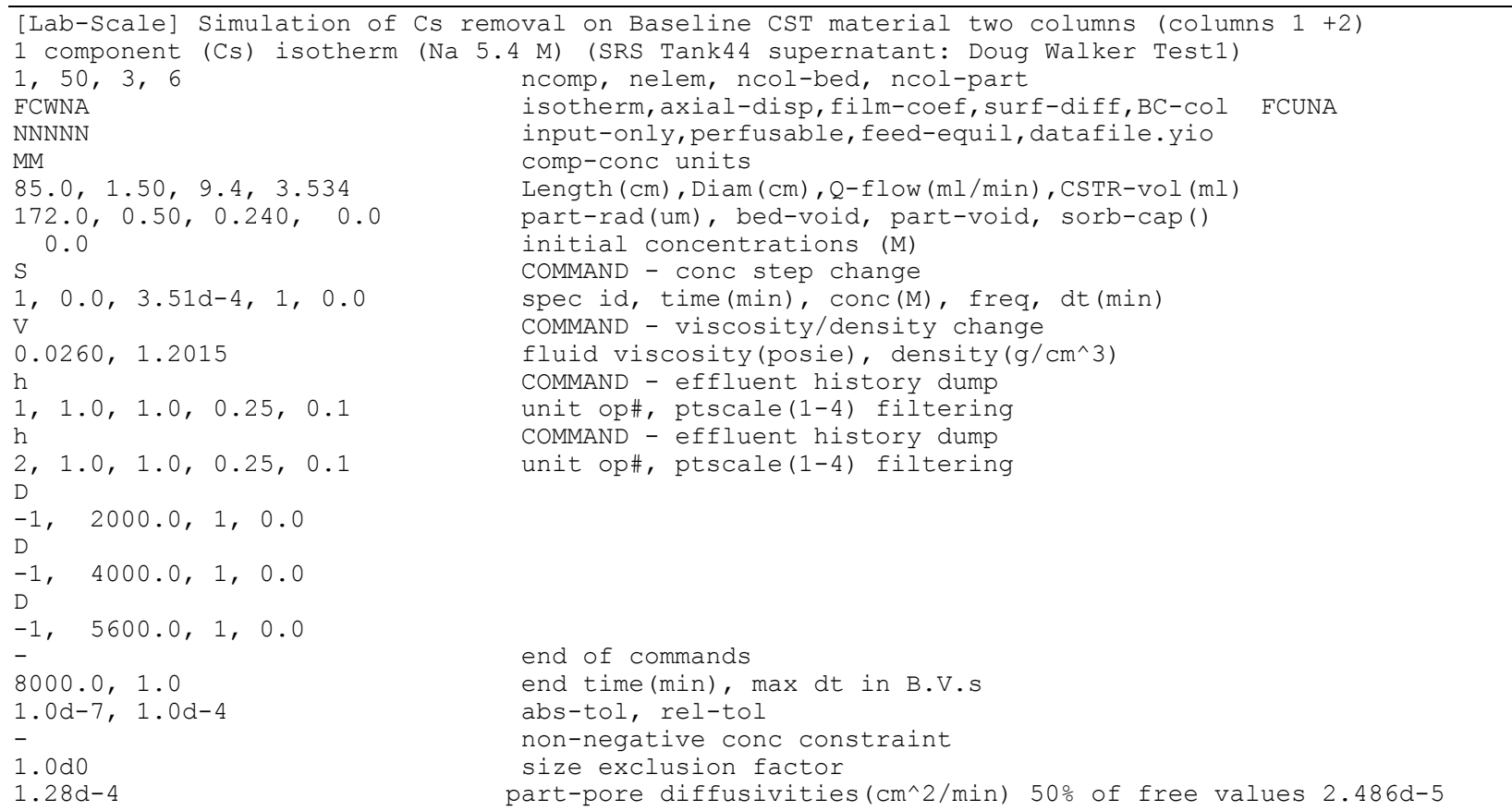




\begin{tabular}{|c|c|c|}
\hline $5.963 d-4$ & Brownian diffusivities $\left(\mathrm{cm}^{\wedge} 2 / \mathrm{min}\right.$ & \\
\hline 0.3944 & Freundlich/Langmuir Hybrid a & (moles/L B.V.) rhob $=1.0$ \\
\hline 1.0 & Freundlich/Langmuir Hybrid b & (1/M) Batch specific isotherm \\
\hline 1.0 & Freundlich/Langmuir Hybrid Ma & $\mathrm{ccap}=0.580$ \\
\hline 1.0 & Freundlich/Langmuir Hybrid Mb & $(-)$ \\
\hline $.0486 d-4$ & Freundlich/Langmuir Hybrid beta & "eff" isotherm $\mathrm{Na}$ \\
\hline
\end{tabular}

\section{VERSE Input for SRS-Avg-Test1}

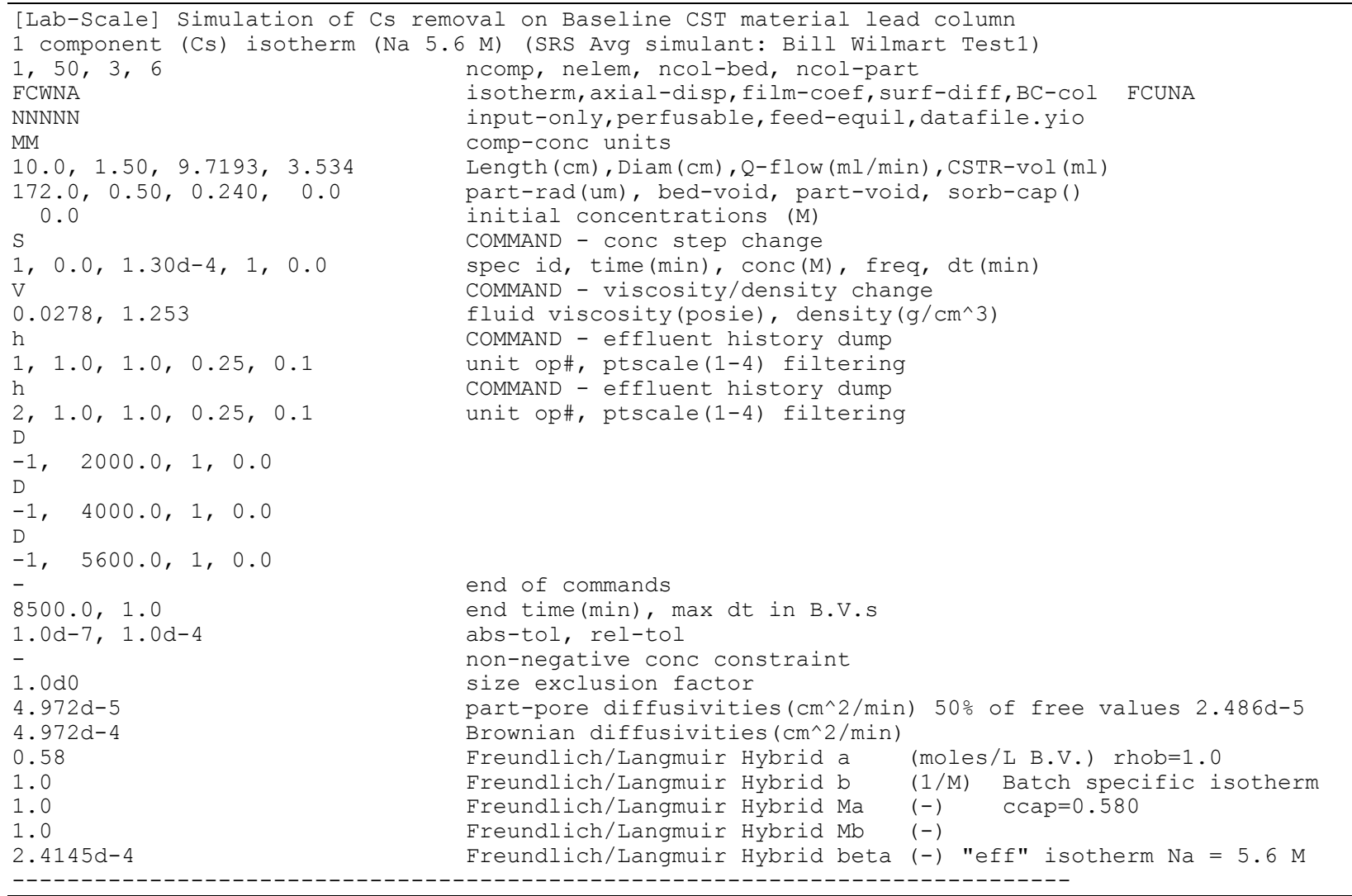

\section{VERSE Input for SRS-Avg-Test2 (single-component approach)}

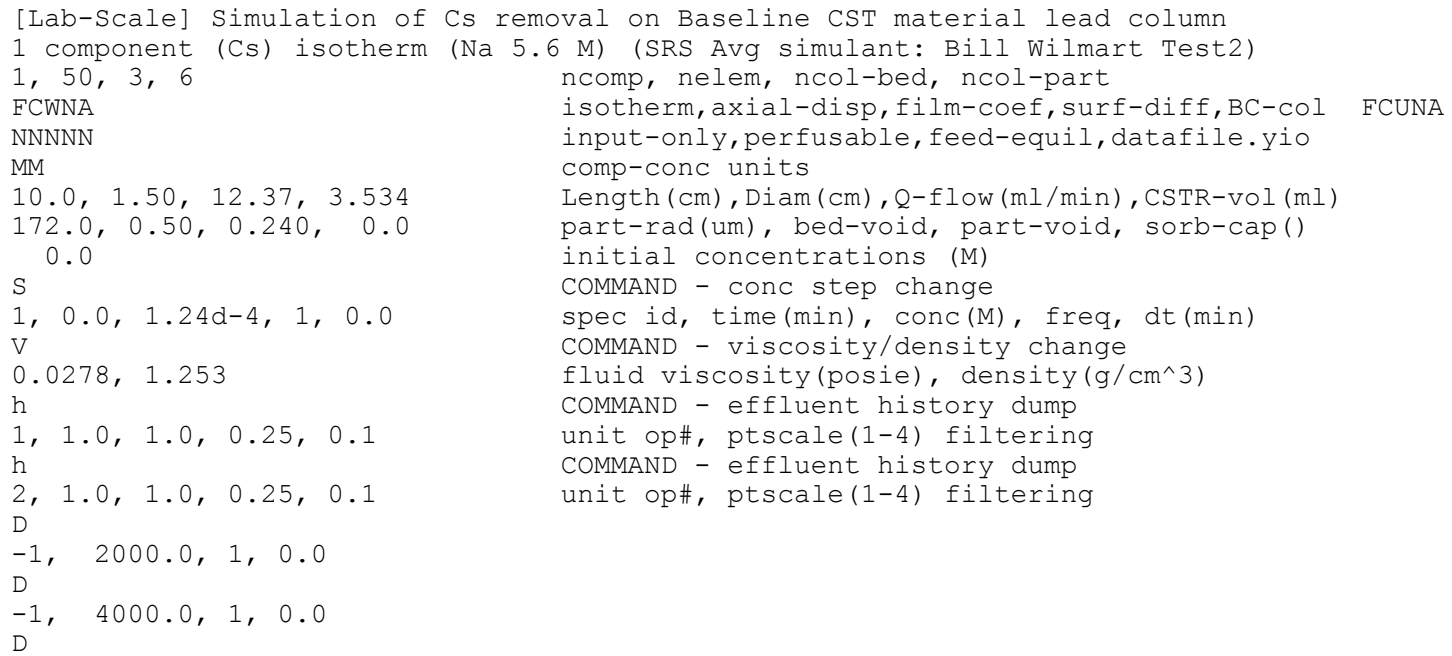




\section{VERSE Input for SRS-Avg-Test2 (ternary-component approach)}

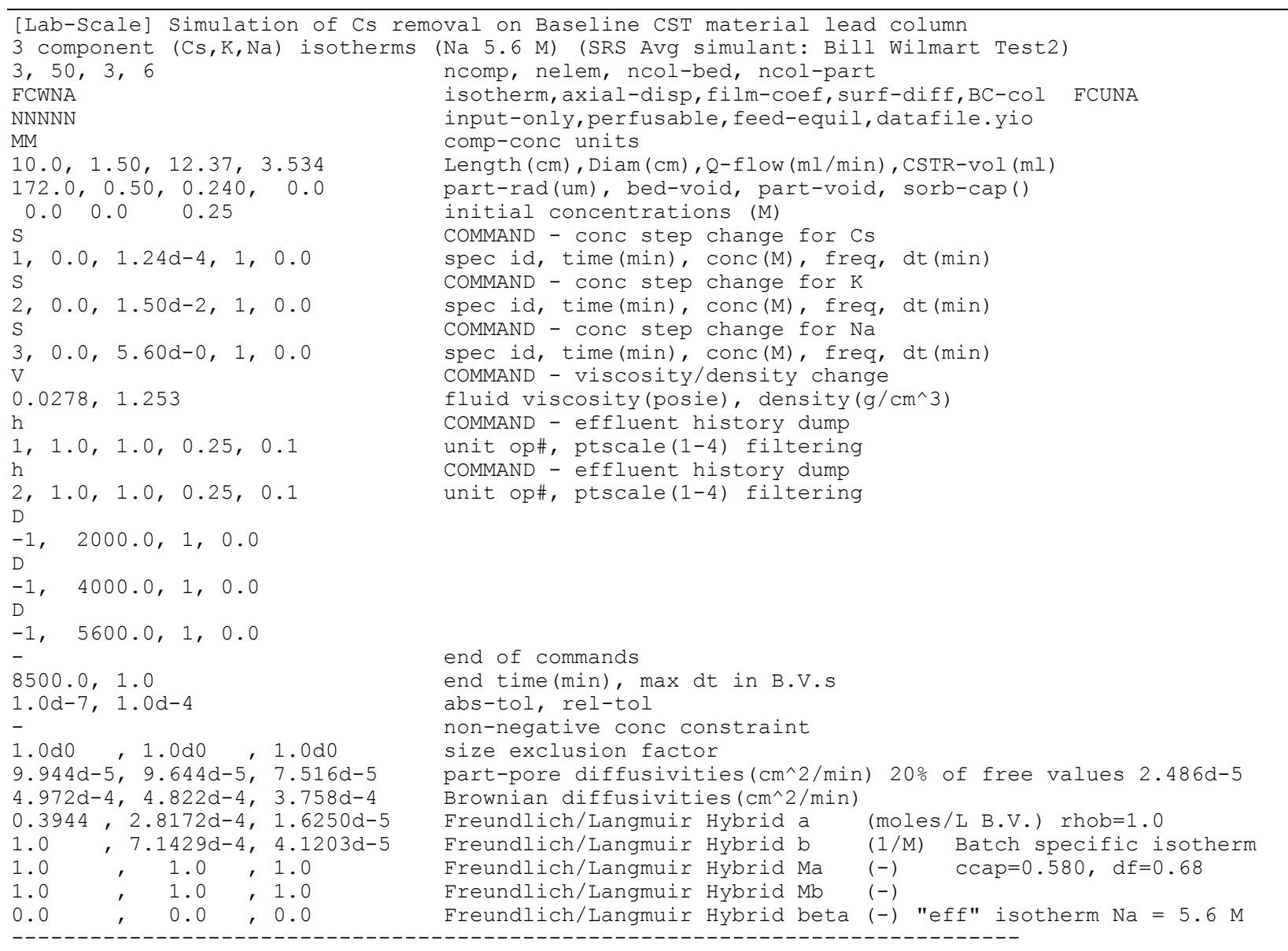

\section{VERSE Input for SRS-Avg-Test3}

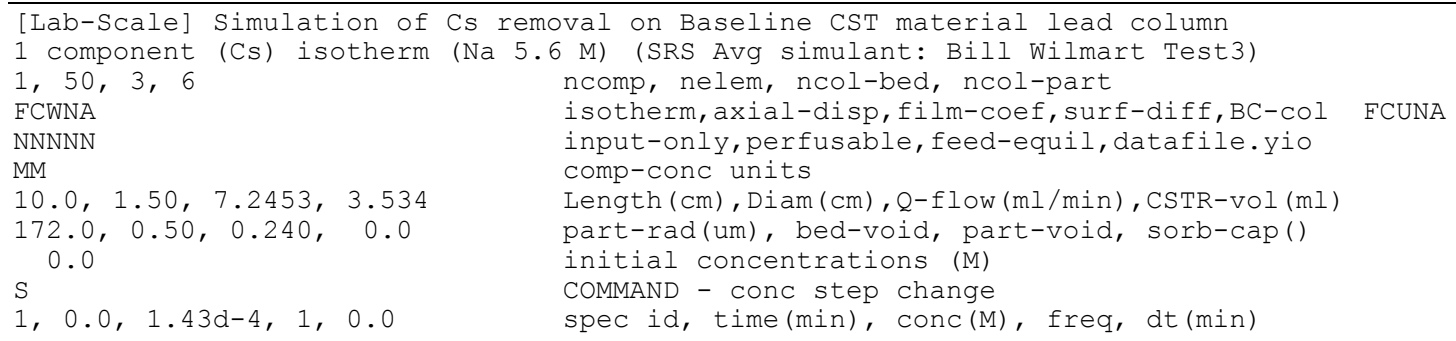


$\mathrm{V}$

$0.0278,1.253$

$1,1.0,1.0,0.25,0.1$

$\mathrm{h}$

$2,1.0,1.0,0.25,0.1$

$-1,2000.0,1,0.0$

$\mathrm{D}$

$-1, \quad 4000.0,1,0.0$

$-1, \quad 5600.0,1,0.0$

$12000.0,1.0$

$1.0 d-7,1.0 d-4$

$1.0 \mathrm{~d} 0$

1. $28 d-4$

$4.972 d-4$

0.58

1.0

1.0

1.0

$2.4145 d-4$
COMMAND - viscosity/density change

fluid viscosity (posie), density $\left(\mathrm{g} / \mathrm{cm}^{\wedge} 3\right)$

COMMAND - effluent history dump

unit op\#, ptscale(1-4) filtering

COMMAND - effluent history dump

unit op\#, ptscale(1-4) filtering

\section{VERSE Input for SRS-Avg-Test4}

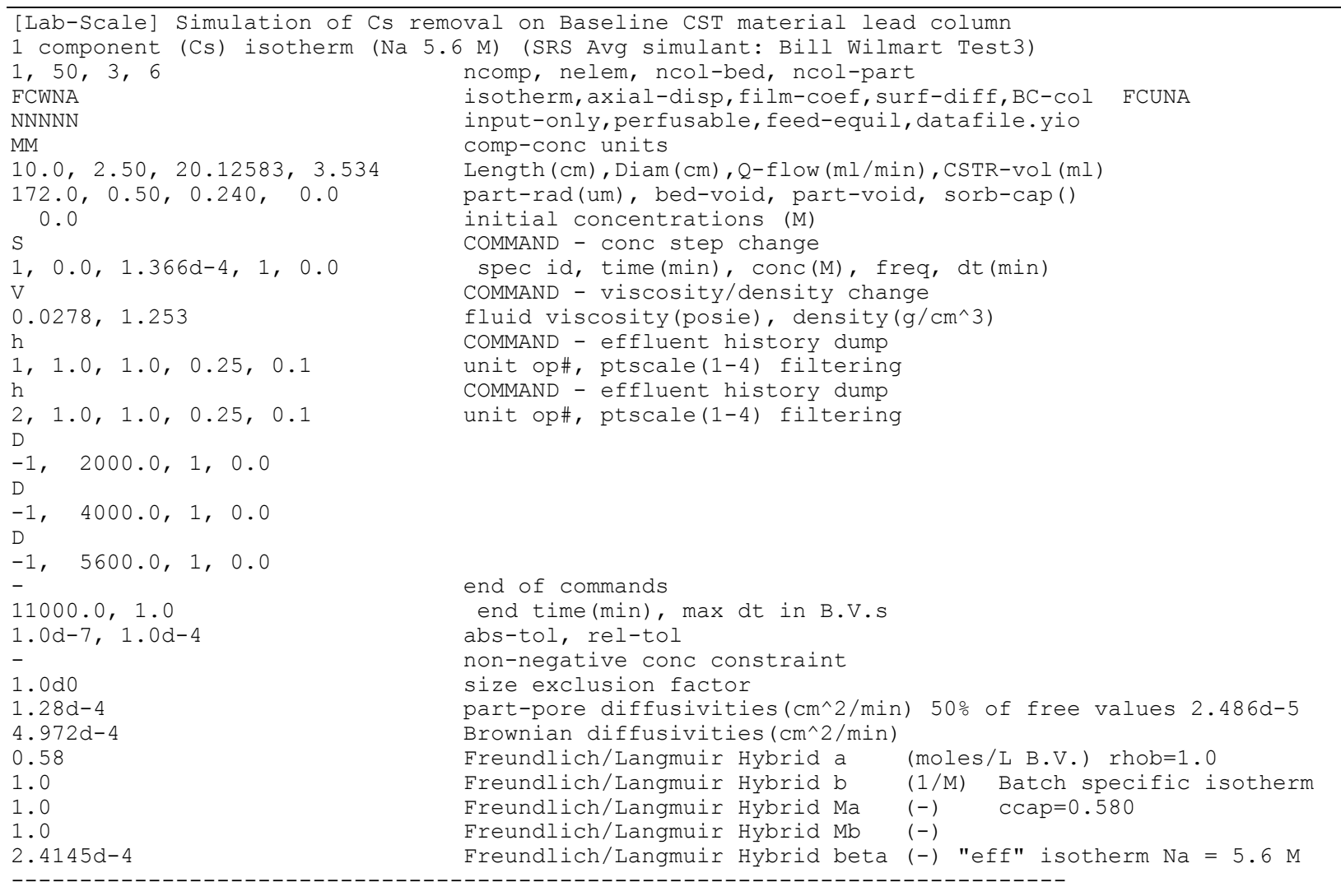

Length (cm), Diam (cm), Q-flow (ml/min), CSTR-vol (ml)

initial concentrations (M)

spec id, time(min), conc(M), freq, dt(min)

MAND - viscosity/density change

COMMAND - effluent history dump

it op\#, ptsale(1-4) filtering

COMMAND - effluent history dump

abs-tol, rel-tol

size exclusion factor

Brownian diffusivities ( $\left.\mathrm{cm}^{\wedge} 2 / \mathrm{min}\right)$

Freundlich/Langmuir Hybrid a

(1/M) Batch specific isotherm

(-)-------

\section{VERSE Input for SRS-Avg-Test5}

\footnotetext{
[Lab-Scale] Simulation of Cs removal on Baseline CST material lead column

1 component (CS) isotherm ( $\mathrm{Na} 5.6 \mathrm{M}$ ) (SRS Avg simulant: Doug Walker Test5 WSRC-TR-98-00344-r1)

$1,50,3,6$

FCWNA
}

end of commands

abs-tol, rel-tol

non-negative conc constraint

part-pore diffusivities $\left(\mathrm{cm}^{\wedge} 2 / \mathrm{min}\right) 50 \%$ of free values $2.486 \mathrm{~d}-5$

Brownian diffusivities $\left(\mathrm{cm}^{\wedge} 2 / \mathrm{min}\right)$

(1/M) Batch specific isotherm

$(-)$

(-) "eff" isotherm $\mathrm{Na}=5.6 \mathrm{M}$

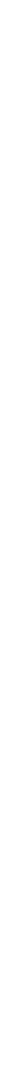




\section{NNNNN}

$\mathrm{MM}$

$10.0,1.50,0.47,3.534$

$172.0,0.50,0.240,0.0$ 0.0

$\mathrm{S}$

$1,0.0,1.40 d-4,1,0.0$

$0.0278,1.253$

$\mathrm{h}$

$1,1.0,1.0,0.25,0.1$

$\mathrm{h}$

$2,1.0,1.0,0.25,0.1$

$-1, \quad 2000.0,1,0.0$

D

$-1,4000.0,1,0.0$

$-1, \quad 5600.0,1,0.0$

$35000.0,1.0$

$1.0 d-7,1.0 d-4$

$-$

1. $0 \mathrm{~d} 0$

$4.972 d-5$

4. $972 d-4$

0.3944

1.0

1.0

1.0

2. $4145 d-4$ input-only, perfusable, feed-equil, datafile.yio

comp-conc units

Length (cm), Diam (cm), $\mathrm{Q}-\mathrm{flow}(\mathrm{ml} / \mathrm{min}), \mathrm{CSTR}-\mathrm{vol}(\mathrm{ml})$

part-rad(um), bed-void, part-void, sorb-cap()

initial concentrations (M)

COMMAND - conc step change

spec id, time(min), conc(M), freq, dt(min)

COMMAND - viscosity/density change

fluid viscosity (posie), density $\left(\mathrm{g} / \mathrm{cm}^{\wedge} 3\right)$

COMMAND - effluent history dump

unit op\#, ptscale(1-4) filtering

COMMAND - effluent history dump

unit op\#, ptscale(1-4) filtering

\section{VERSE Input for SRS-Avg-Test6}

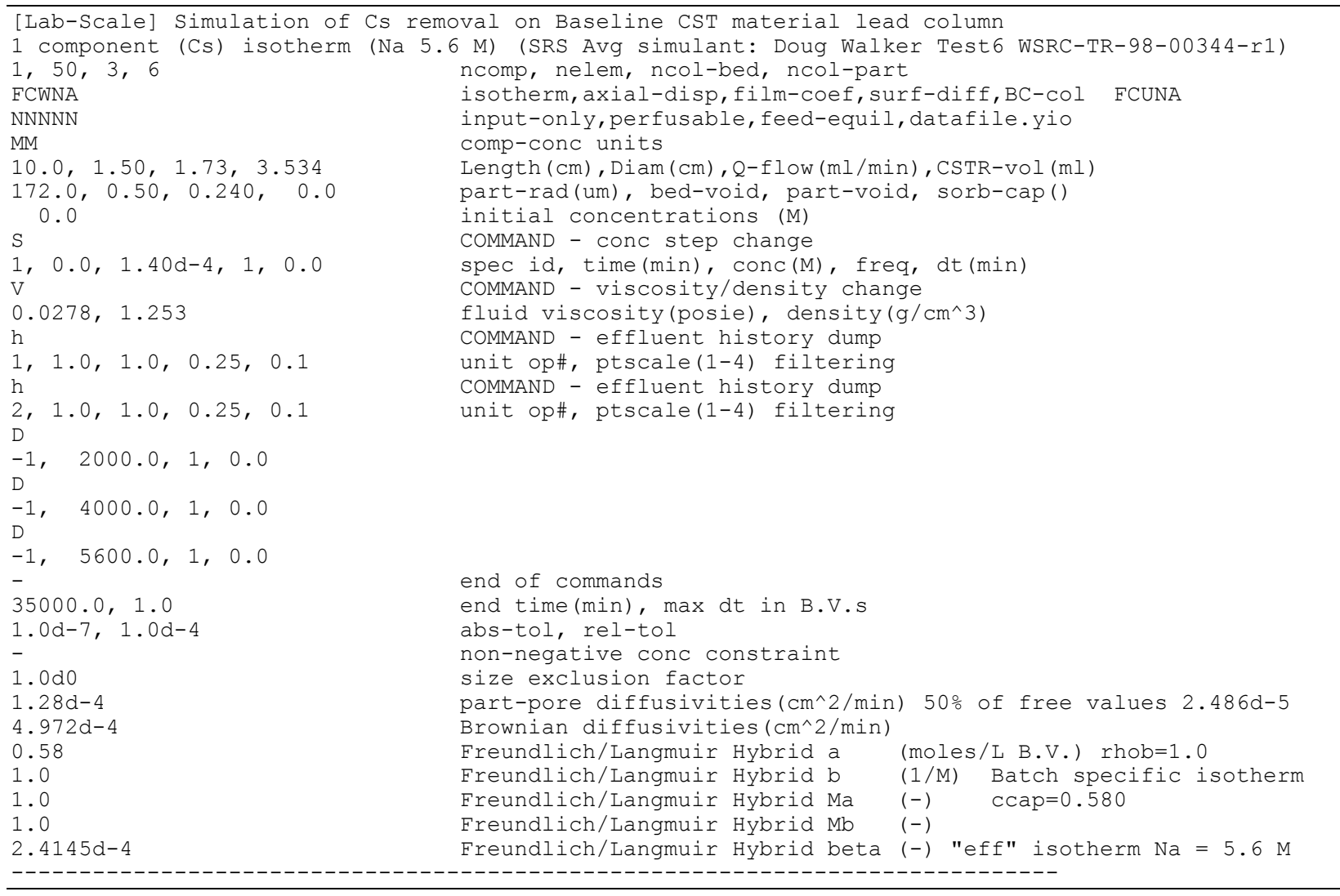




\section{VERSE Input for SRS-Avg-Test7}

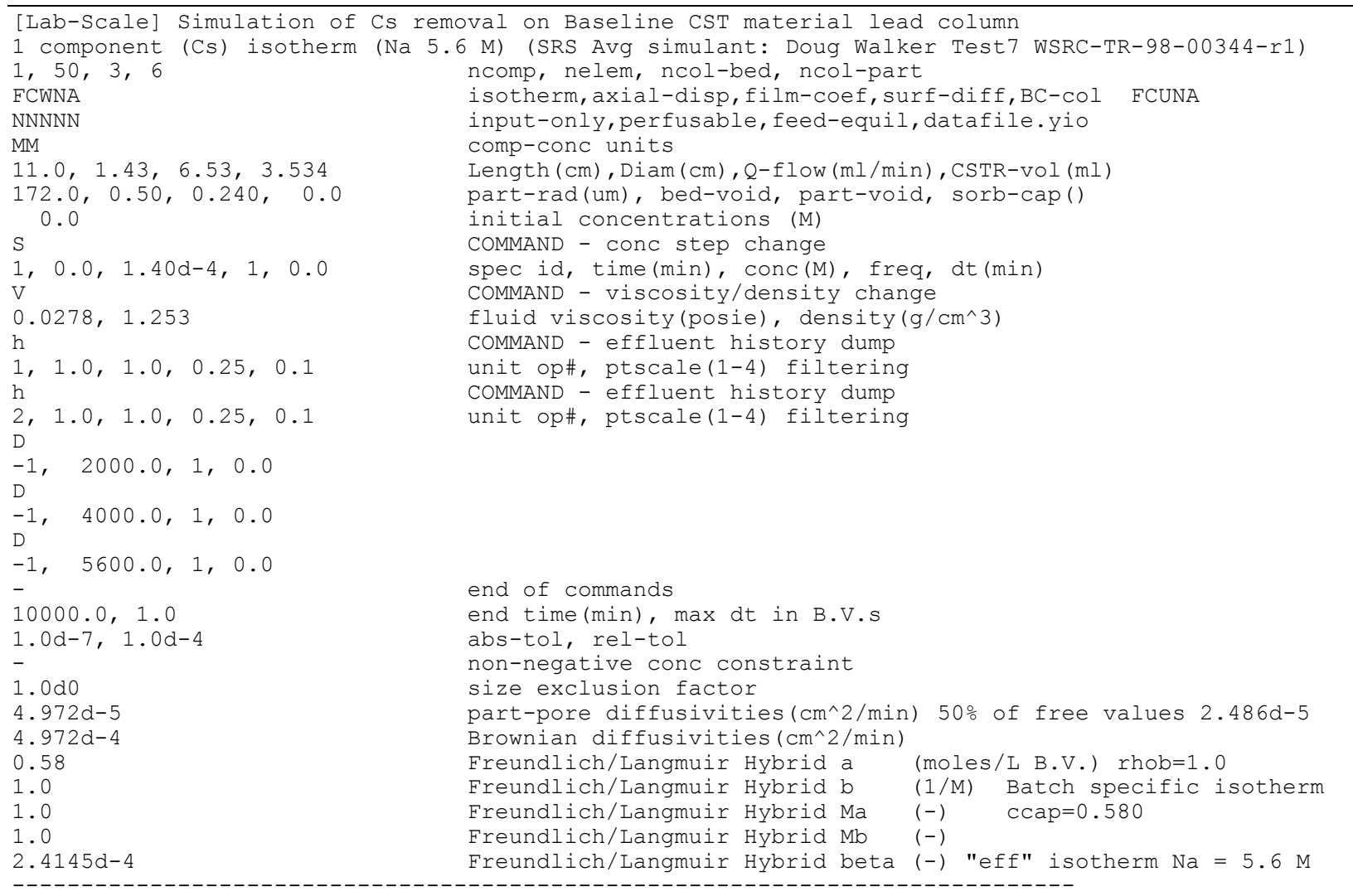

\section{VERSE Input for SRS-High-OH-Test1a}

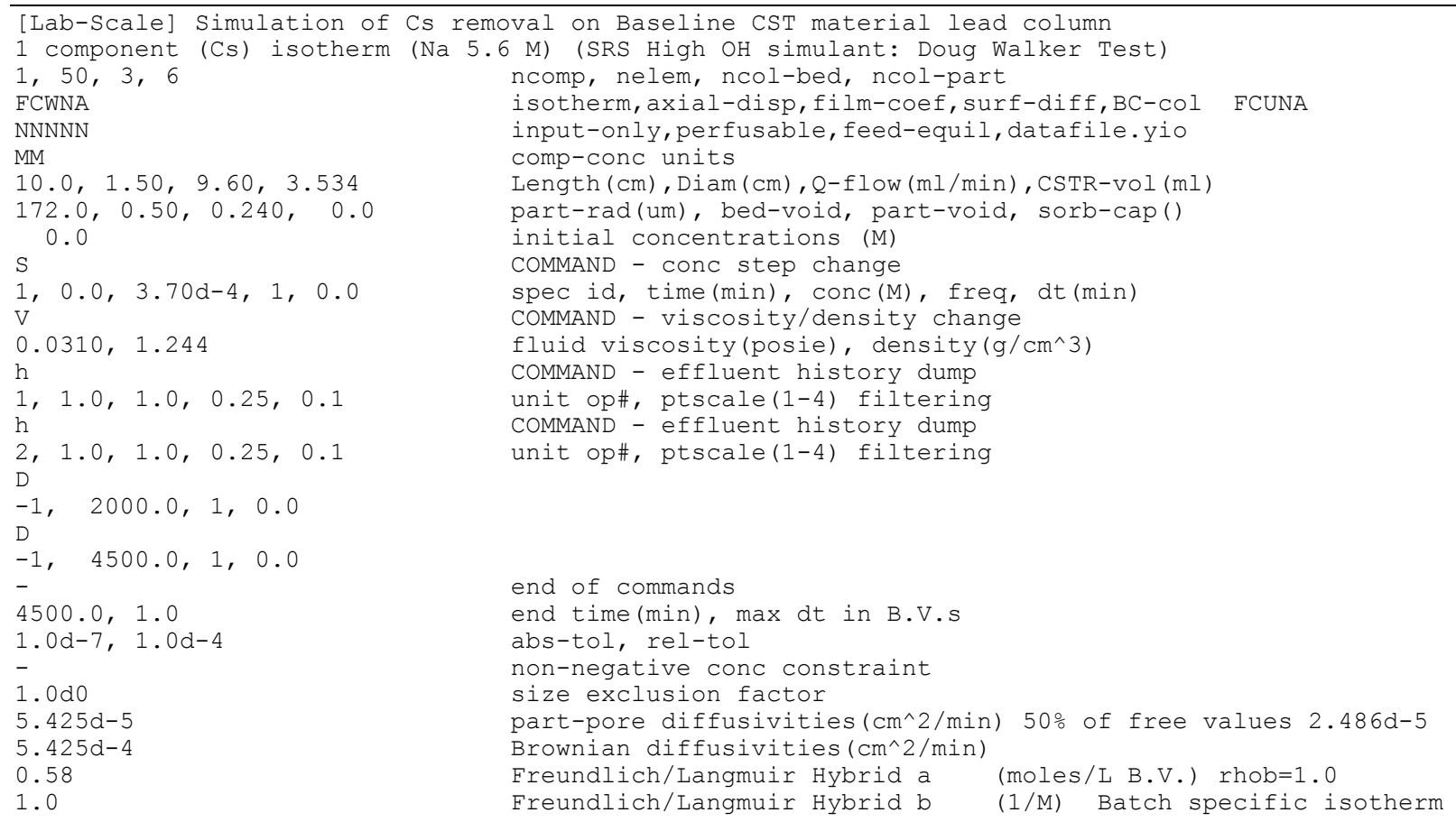




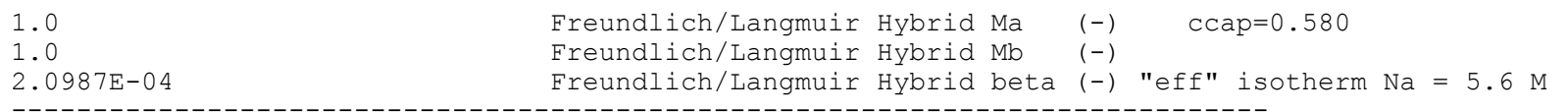

\section{VERSE Input for SRS-High-OH-Test1b}

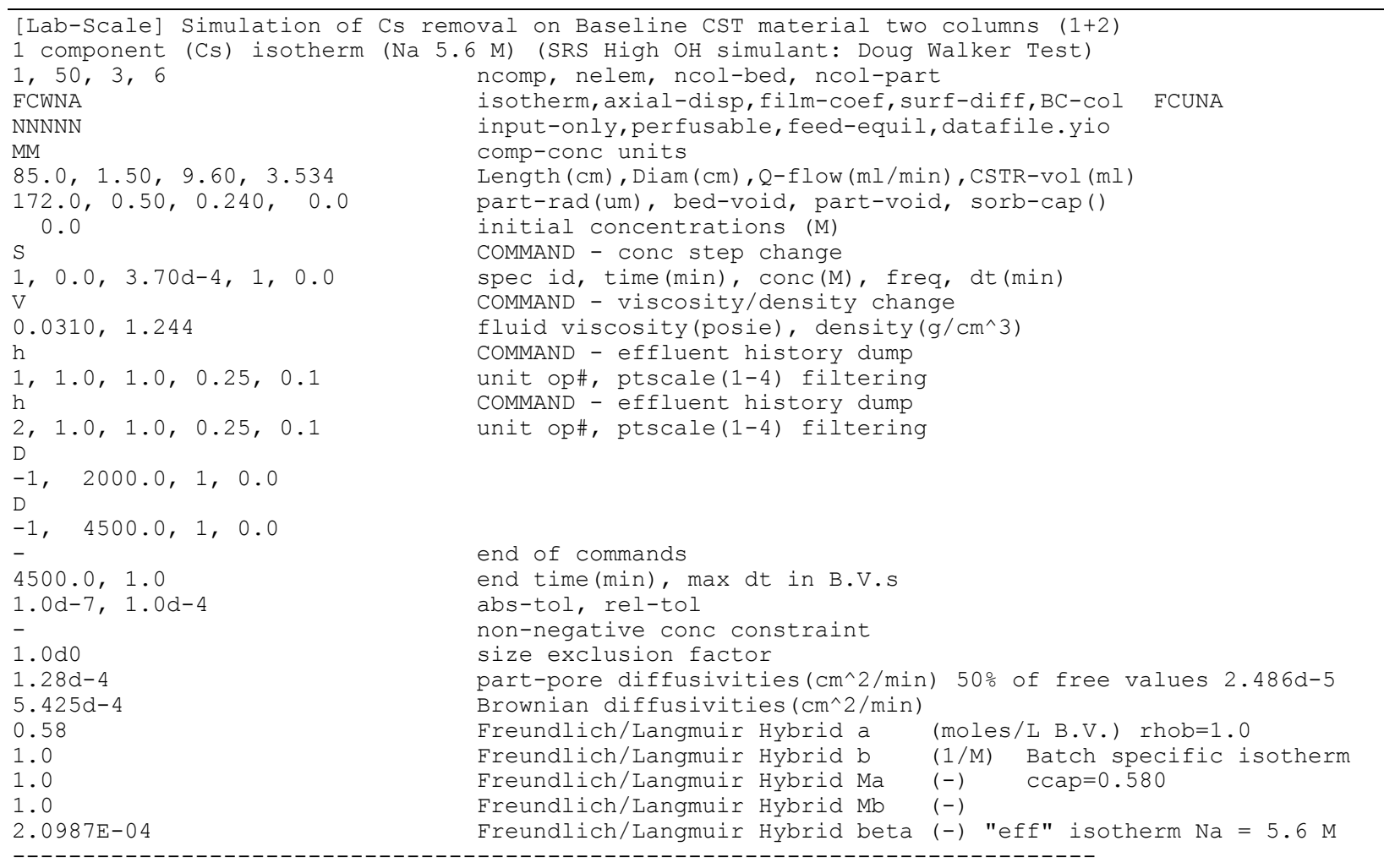

\section{VERSE Input for PNNL-AW101-Test1}

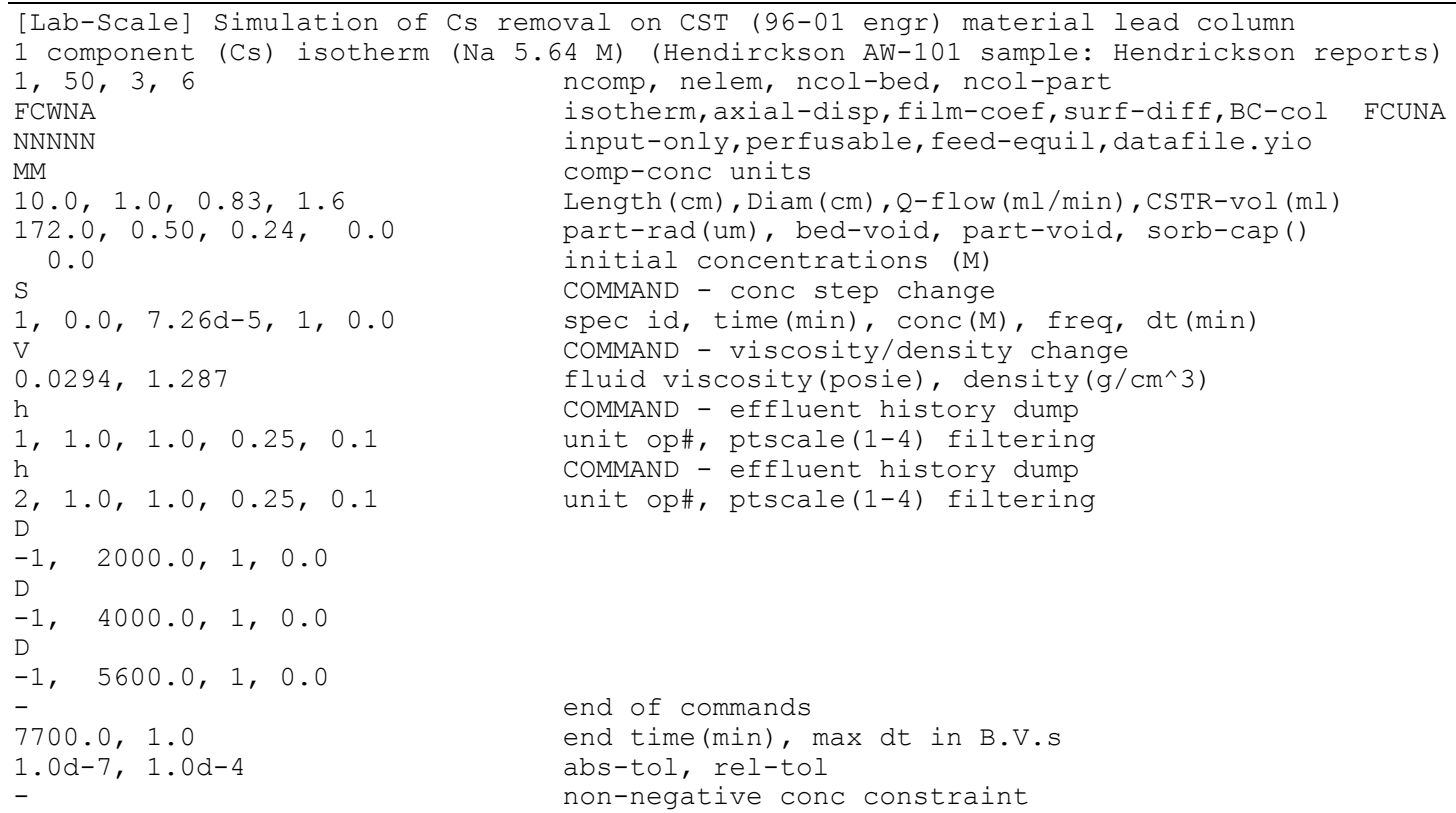




\section{VERSE Input for ORNL-W27-Test1}

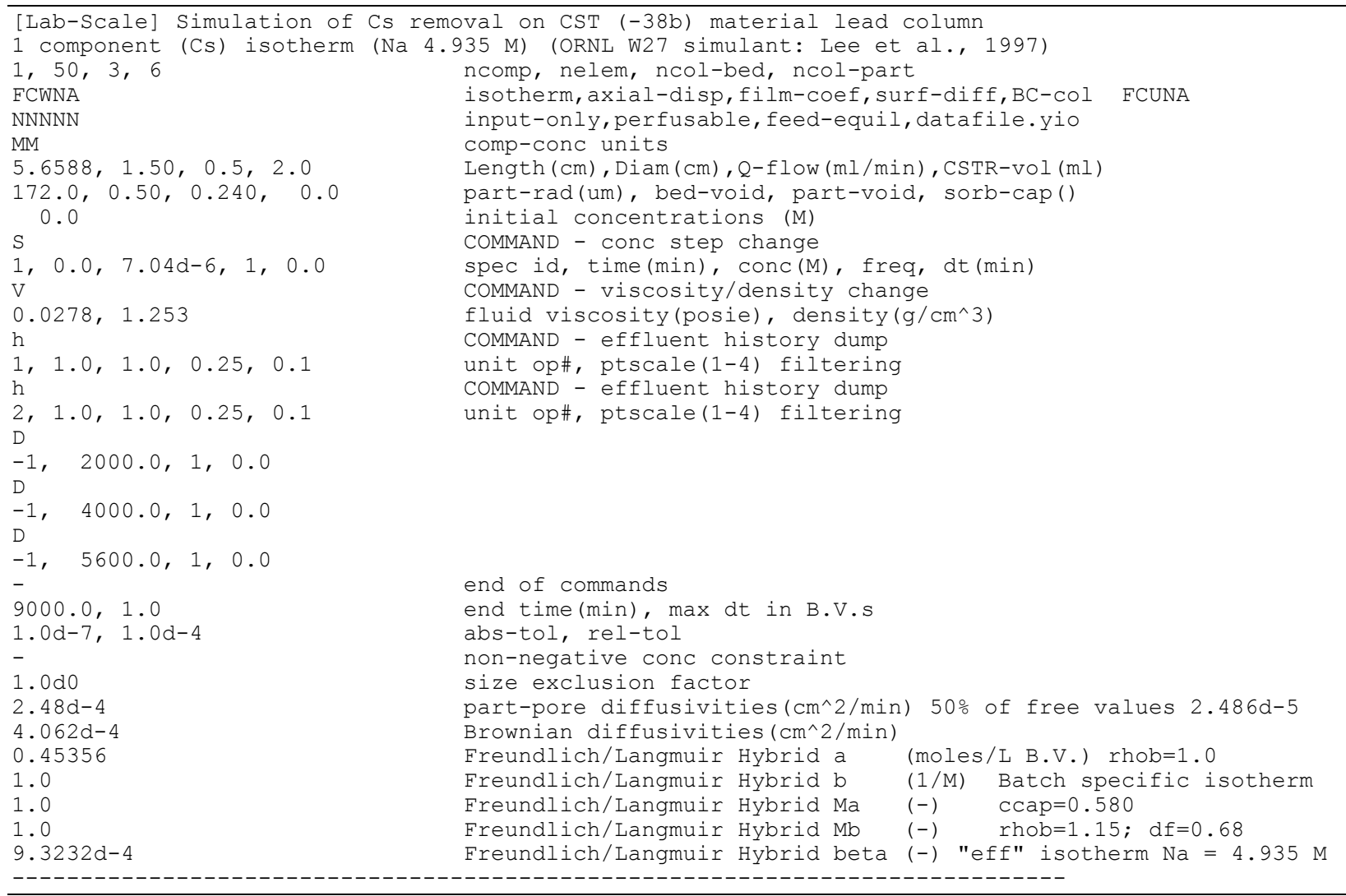

\section{VERSE Input for ORNL-W27-Test2}

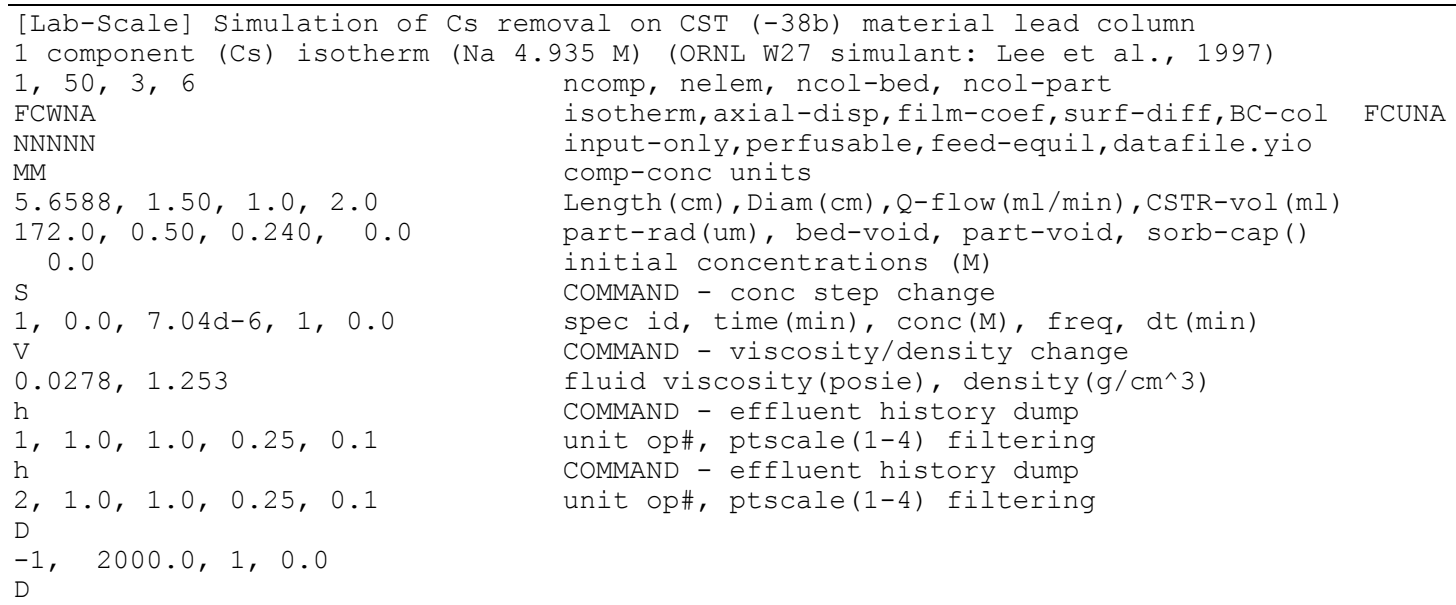


$-1, \quad 4000.0,1,0.0$

$-1, \quad 5600.0,1,0.0$

$9000.0,1.0$

end of commands

$1.0 d-7,1.0 d-4$

abs-tol, rel-tol

1. $0 \mathrm{~d} 0$

$2.48 d-4$

4. $062 d-4$

0.45356

1. 0

1. 0

1.0

abs-tol, rel-tol

non-negative conc constraint

size exclusion factor

part-pore diffusivities ( $\left.\mathrm{cm}^{\wedge} 2 / \mathrm{min}\right) \quad 50 \%$ of free values $2.486 \mathrm{~d}-5$

Brownian diffusivities ( $\left.\mathrm{cm}^{\wedge} 2 / \mathrm{min}\right)$

Freundlich/Langmuir Hybrid a (moles/L B.V.) rhob=1.0

Freundlich/Langmuir Hybrid b (1/M) Batch specific isotherm

Freundlich/Langmuir Hybrid Ma (-) ccap $=0.580$

Freundlich/Langmuir Hybrid $\mathrm{Mb} \quad(-) \quad$ rhob $=1.15 ; \mathrm{df}=0.68$

9. $3232 d-4$

Freundlich/Langmuir Hybrid beta (-) "eff" isotherm Na = $4.935 \mathrm{M}$

\section{VERSE Input for ORNL-CsRD-Run2}

[CsRD-Scale] Simulation of Cs removal on CST material (96-01) single column

1 component (CS) isotherm (feed point fit) (CsRD Run 2 avgerage waste supernatant: Walker Run 2)

$1,50,3,6$ ncomp, nelem, ncol-bed, ncol-part

FCWNA isotherm, axial-disp, film-coef, surf-diff, BC-Col FCUNA

NNNNN

$\mathrm{MM}$

input-only, perfusable, feed-equil, datafile.yio

comp-conc units

$51.672,30.6,1900.0,7600.0$

Length (cm), Diam (cm), $\mathrm{Q}-\mathrm{flow}(\mathrm{ml} / \mathrm{min}), \mathrm{CSTR}-\mathrm{vol}(\mathrm{ml})$

$172.0,0.50,0.240,0.0$

0.0

part-rad(um), bed-void, part-void, sorb-cap()

initial concentrations (M)

$1,0.0,1.35 d-5,1,0.0$

COMMAND - conc step change

spec id, time(min), conc(M), freq, dt(min)

COMMAND - viscosity/density change

$0.0278,1.253$

fluid viscosity (posie), density ( $\left.\mathrm{g} / \mathrm{cm}^{\wedge} 3\right)$

COMMAND - effluent history dump

unit op\#, ptscale(1-4) filtering

COMMAND - effluent history dump

$1,1.0,1.0,0.25,0.1$

$2,1.0,1.0,0.25,0.1$

unit op\#, ptscale(1-4) filtering

$-1, \quad 2000.0,1,0.0$

D

$-1,4000.0,1,0.0$

D

$-1, \quad 5600.0,1,0.0$

$10000.0,1.0$

$1.0 d-7,1.0 d-4$

$1.0 \mathrm{~d} 0$

$4.062 d-5$

4. $062 d-4$

0.3944

1.0

1.0

1.0

$6.50473 \mathrm{~d}-4$

end of commands

end time(min), max dt in B.V.s

abs-tol, rel-tol

non-negative conc constraint

size exclusion factor

part-pore diffusivities $\left(\mathrm{cm}^{\wedge} 2 / \mathrm{min}\right)$

Brownian diffusivities ( $\mathrm{cm}^{\wedge} 2 / \mathrm{min}$ )

Freundlich/Langmuir Hybrid a (moles/L B.V.) rhob=1.0

Freundlich/Langmuir Hybrid b (1/M) Batch specific isotherm

Freundlich/Langmuir Hybrid Ma $(-) \quad$ ccap $=0.580$

Freundlich/Langmuir Hybrid Mb (-)

Freundlich/Langmuir Hybrid beta (-) "eff" isotherm $\mathrm{Na}=$ unknown

\section{VERSE Input for ORNL-CsRD-Run3}

[CsRD-Scale] Simulation of Cs removal on CST material (96-01) two columns single cycle

1 component (CS) isotherm (feed point fit) (CsRD Run 3 avgerage waste supernatant: Walker Run 3)

$1,100,3,6$

FCWNA

NNNNN

$\mathrm{MM}$

$103.344,30.6,3800.0,7600.0$

$172.0,0.50,0.240,0.0$

0.0

$\mathrm{S}$

$1,0.0,1.35 d-5,1,0.0$

$\mathrm{V}$

$0.0278,1.253$

ncomp, nelem, ncol-bed, ncol-part

isotherm, axial-disp, film-coef, surf-diff,BC-col FCUNA

input-only, perfusable, feed-equil, datafile.yio

comp-conc units

Length (cm), Diam (cm), Q-flow (ml/min), CSTR-vol (ml)

part-rad(um), bed-void, part-void, sorb-cap()

initial concentrations (M)

COMMAND - conc step change

spec id, time(min), conc(M), freq, dt(min)

COMMAND - viscosity/density change

fluid viscosity (posie), density $\left(\mathrm{g} / \mathrm{cm}^{\wedge} 3\right)$ 
$-1, \quad 2000.0,1,0.0$

1.0

1.0

1.0

end of commands

end time (min), max dt in B.V.s

abs-tol, rel-tol

non-negative conc constraint

size exclusion factor

part-pore diffusivities $\left(\mathrm{cm}^{\wedge} 2 / \mathrm{min}\right)$

Brownian diffusivities ( $\left.\mathrm{cm}^{\wedge} 2 / \mathrm{min}\right)$

Freundlich/Langmuir Hybrid a (moles/L B.V.) rhob=1.0

Freundlich/Langmuir Hybrid b (1/M) Batch specific isotherm

Freundlich/Langmuir Hybrid Ma $\quad(-) \quad$ ccap $=0.580$

Freundlich/Langmuir Hybrid Mb (-)

Freundlich/Langmuir Hybrid beta (-) "eff" isotherm $\mathrm{Na}=$ unknown

\section{VERSE Input for ORNL-CsRD-Run4a}

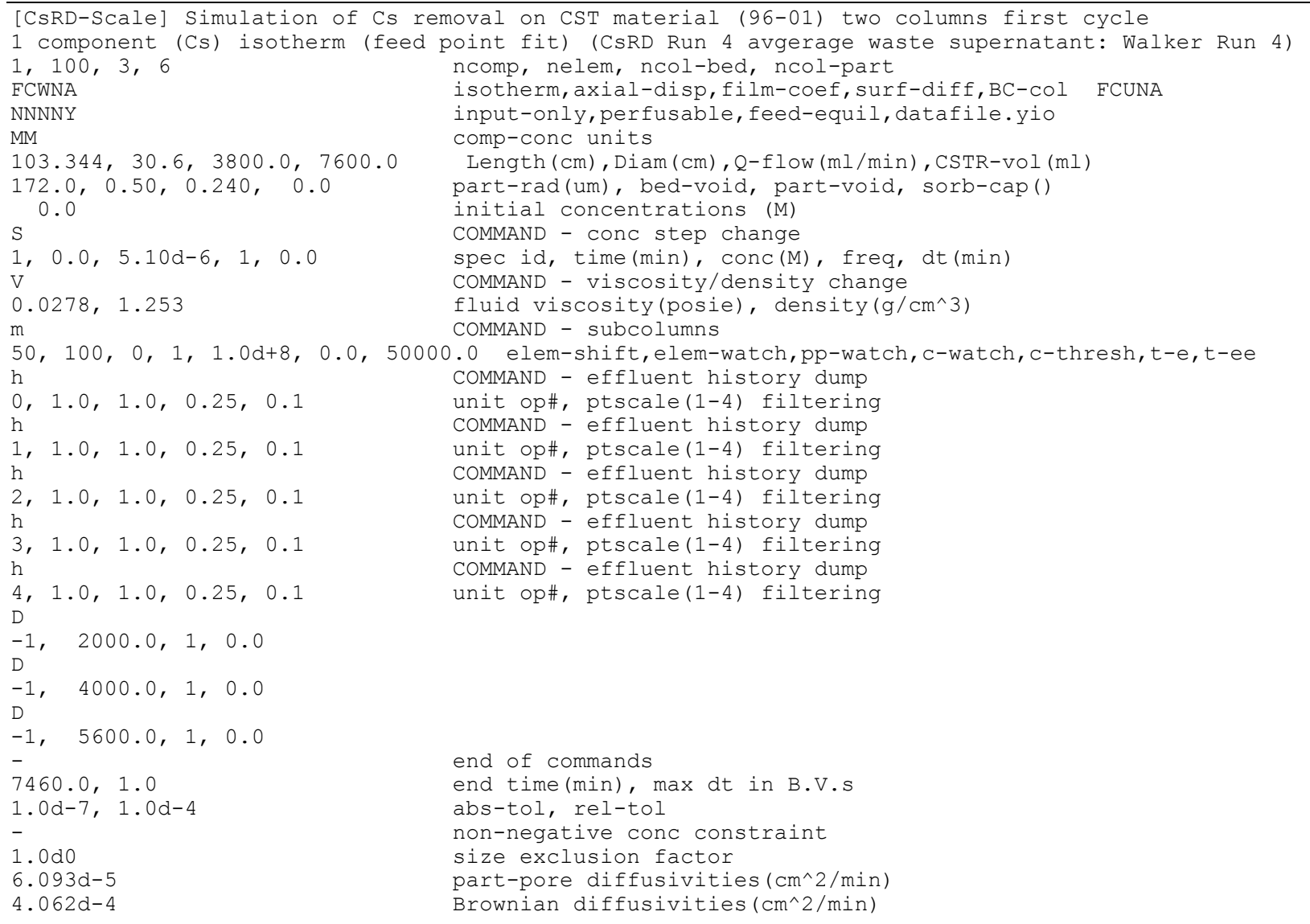




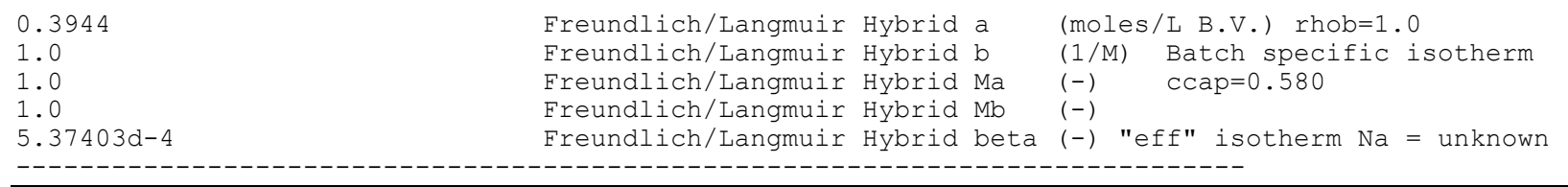

\section{VERSE Input for ORNL-CsRD-Run4b}

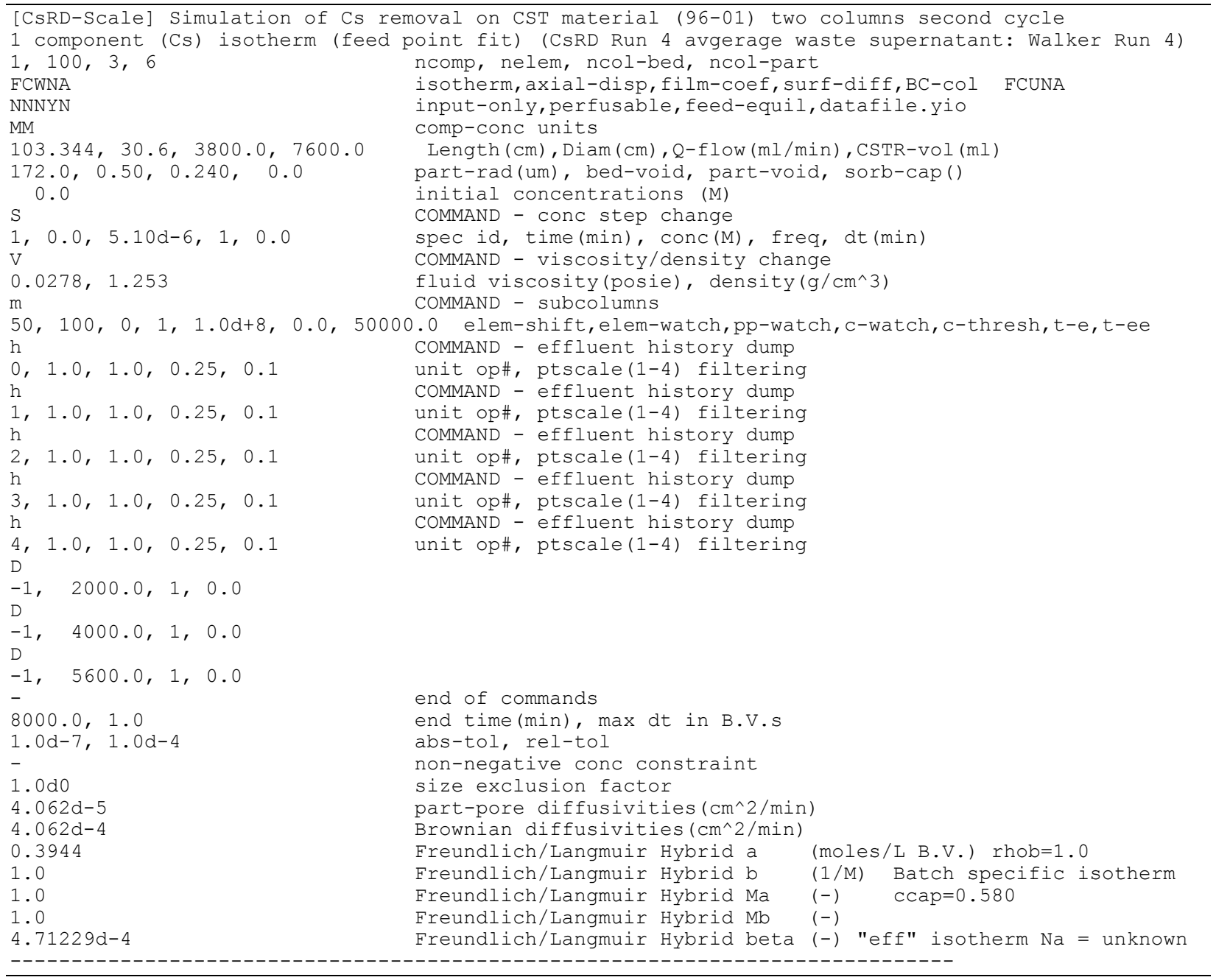


